REPORT S OF THE OAK RIDGE DOSE RECONSTRU CTION, V OI. 1A The R eport of Project T ask 1 • July 1999

lodine-131 $R$ eleases from $R$ adioactive $L$ anthanum Processing at the X-10 Site in 0 ak R idge, T ennessee (1944-1956) an A ssessment of $Q$ uantities $R$ eleased, $O$ ff-Site $R$ adiation D oses, and Potential Excess $R$ isks of $T$ hyroid $C$ ancer - APPENDICES -

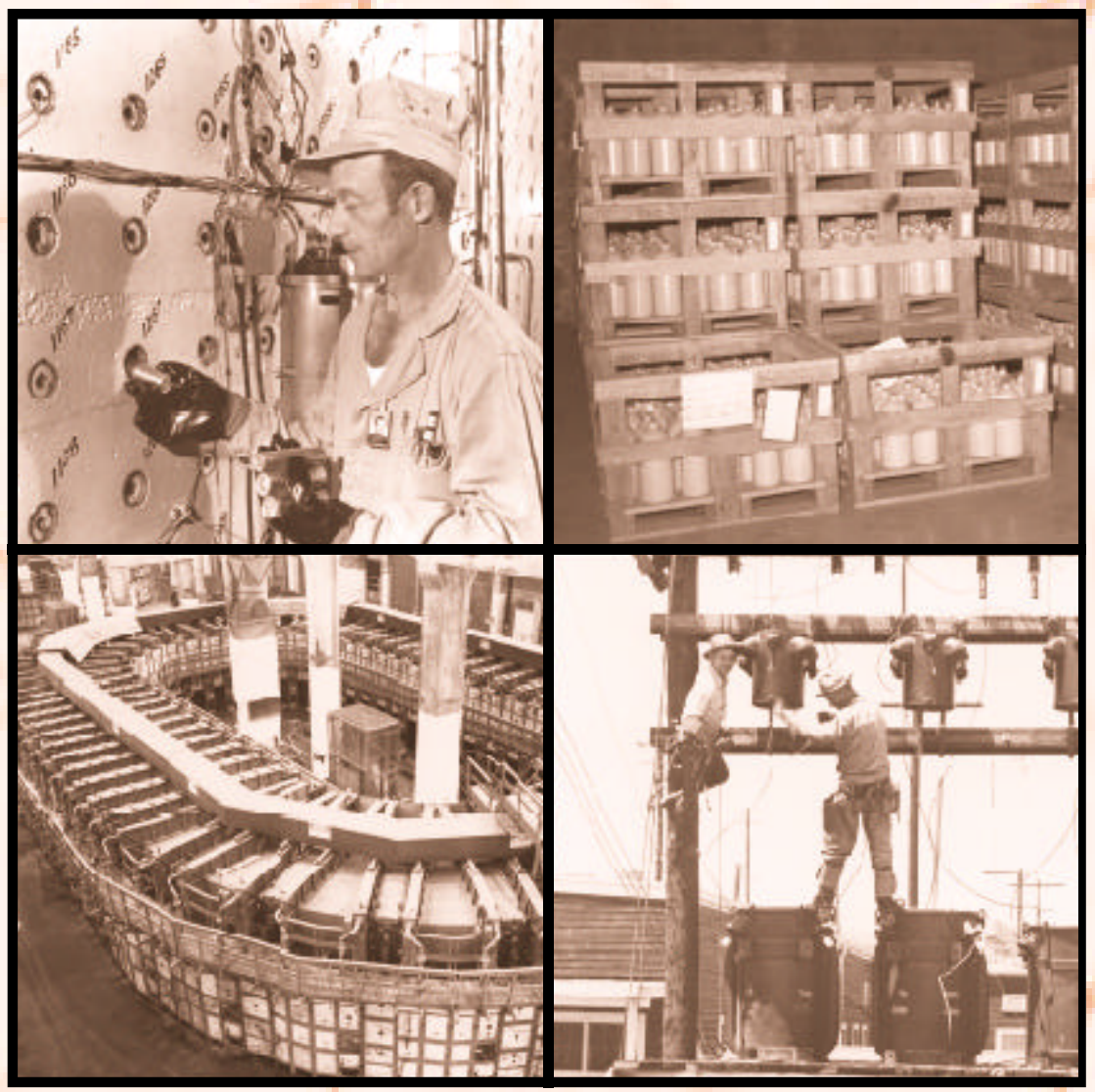

Submitted to the T ennessee D epartment of $\mathrm{H}$ ealth by

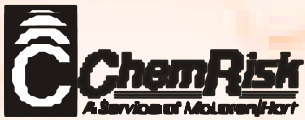




\author{
OAK RIDGE HEALTH STUDIES \\ OAK RIDGE DOSE RECONSTRUCTION
}

\title{
- TASK 1 REPORT -
}

IODINE-131 RELEASES FROM RADIOACTIVE LANTHANUM PROCESSING AT THE X-10 SITE IN OAK RIDGE, TENNESSEE (1944-1956) - AN ASSESSMENT OF QUANTITIES RELEASED, OFF-SITE RADIATION DOSES, AND POTENTIAL EXCESS RISKS OF THYROID CANCER

VOLUME 1A- APPENDICES

July 1999

Submitted to the Tennessee Department of Health by McLaren/Hart-ChemRisk

Prepared by
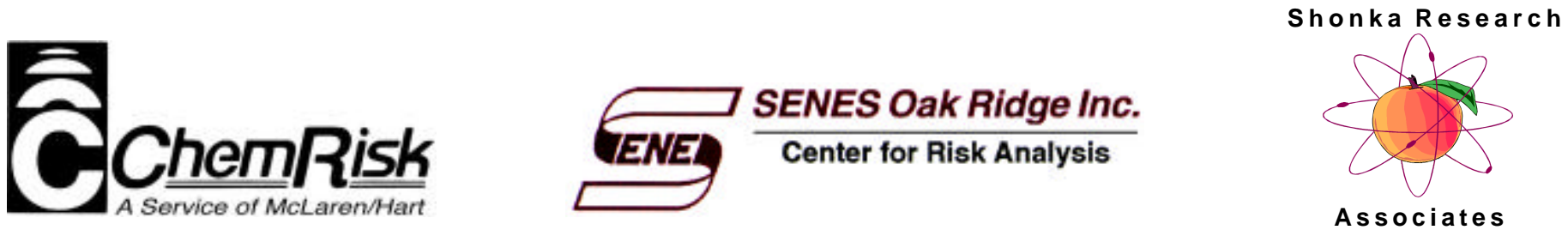
This document was prepared as a team effort by the following individuals:

\section{Project Manager}

Thomas E. Widner ${ }^{1}$

Task 1 Manager

F. Owen Hoffman ${ }^{2}$

\section{Principal Authors:}

A. Iulian Apostoaei ${ }^{2}$

Robert E. Burns ${ }^{3}$

F. Owen Hoffman

Talaat Ijaz ${ }^{4}$

Cathy J. Lewis ${ }^{2}$

Shyam K. Nair ${ }^{5}$

Thomas E. Widner
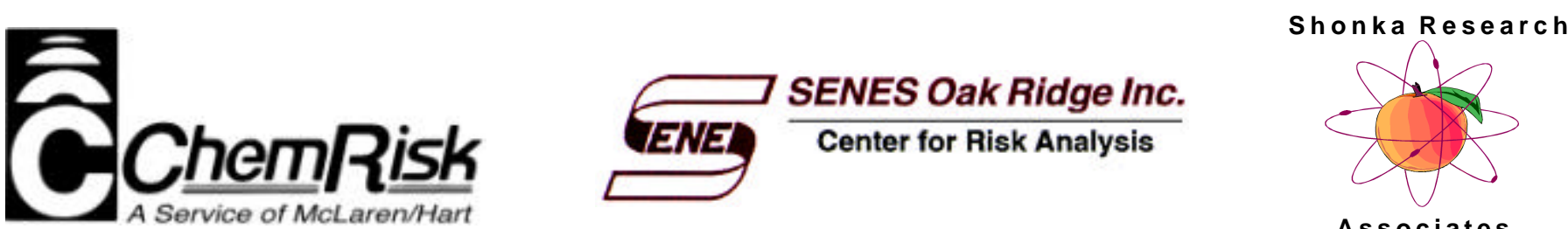

Associates

${ }^{1}$ ChemRisk ${ }^{\circledR}$ a Service of McLaren/Hart, Inc., 1320 Harbor Bay Parkway, Suite 100, Alameda, California 94502. (510) 521-5200. E-mail for the project team can be sent to: Tom_Widner@mclaren-hart.com.

2 SENES Oak Ridge, Inc., 102 Donner Drive, Oak Ridge, Tennessee 37830.

3 Shonka Research Associates, 4939 Lower Roswell Road, Suite 106, Marietta, Georgia 30068.

${ }_{5}^{4}$ ChemRisk, a Service of McLaren/Hart, Inc., 5900 Landerbrook Drive, Cleveland, Ohio, 44124.

${ }^{5}$ SENES Oak Ridge, Inc., 102 Donner Drive, Oak Ridge, Tennessee 37830. Dr. Nair is currently with The Cadmus Group, Inc. in Oak Ridge, Tennessee. 


\section{APPENDICES (Volume 1A)}

The numerals that occur before the letters in the appendix titles indicate the section of the main report text to which they are related. Not all sections have appendices.

APPENDIX 2A: DETAILED MATHEMATICAL APPROACH......................................... 2A-1

APPENDIX 3A： SAMPLE "PUSH DATA” FOR CLINTON PILE SLUGS ........................... 3A-1

APPENDIX 3B： SAMPLE HANFORD SLUG IRRADIATION CORRESPONDENCE.........3B-1

APPENDIX 3C: SAMPLE PAGES FROM 100 AREA (CLINTON PILE) LOG BOOKS.... 3C-1

APPENDIX 3D: SAMPLE PAGES FROM A CHEMICAL SEPARATIONS OPERATIONS LOG BOOK............................................................... 3D-1

APPENDIX 3E: A SAMPLE X-10 RALA RUN REPORT ................................................3E-1

APPENDIX 3F: A SAMPLE RALA RUN ANALYTICAL REPORT.......................................F-1

APPENDIX 3G: RALA DISSOLVING BATCHES CONDUCTED IN X-10's BUILDING 706-D ................................................................................ 3G-1

APPENDIX 3H: DETAILS OF BATCHES FOR WHICH NUMBERS OF SLUGS DISSOLVED WERE ESTIMATED ........................................................ $3 \mathrm{H}-1$

APPENDIX 3I: $\quad$ MEMO: FINAL INVENTORY VALUES FOR X-10 SLUGS …...................3I-1

APPENDIX 3J: MEMO: CHARACTERIZATION OF UNCERTAINTIES ASSOCIATED WITH THE CALCULATION OF DISCHARGE ${ }^{131}$ I INVENTORIES FOR X-SLUGS PUSHED FOR RALA PRODUCTION

APPENDIX 3K: MEMO: INVENTORY CALCULATIONS FOR W-SLUG RALA RUNS

APPENDIX 3L: MEMO: TUBE FACTORS FOR THE HANFORD PRODUCTION REACTORS

APPENDIX 3M: MEMO: SLUG FACTORS FOR THE HANFORD PRODUCTION REACTORS

APPENDIX 3N： MEMO: DECAY TIMES FOR W-SLUGS ............................................... 3N-1

APPENDIX 30: DOCUMENTS PERTAINING TO THE 1954 RALA ACCIDENT ..............30-1 APPENDIX 4A: AIR FLOW PATTERNS AT THE OAK RIDGE RESERVATION .............. 4A-1 APPENDIX 10A: THYROID CANCER INCIDENCE DATA............................................. 10A-1 
TASK 1 REPORT

July 1999

${ }^{131}$ I Releases from X-10 Radioactive Lanthanum Processing -

Page TOC-2

List of Appendices

APPENDIX 11A: RATIONALES FOR LOCATIONS AND EXPOSURE PATHWAYS ...... 11A-1 APPENDIX 11B: ESTIMATED IODINE-131 CONCENTRATIONS IN

ENVIRONMENTAL MEDIA 11B-1

APPENDIX 11C:DETAILED RESULTS OF THE DOSE RECONSTRUCTION

FOR IODINE-131 „.................................................................... 11C-1

APPENDIX 12A MILK PRODUCTION DATA.......................................................... 12A-1 
APPENDIX 2A

DETAILED MATHEMATICAL APPROACH 
TASK 1 REPORT

July 1999

Page 2A-2
${ }^{131}$ I Releases from X-10 Radioactive Lanthanum Processing Detailed Mathematical Approach

This page intentionally left blank 


\section{APPENDIX 2-A: DETAILED MATHEMATICAL APPROACH}

This study analyzes exposures to ${ }^{131}$ I released from the X10 facility due to ingestion of milk and cheese, leafy vegetables, meat, and eggs and from direct inhalation of contaminated air. A total of eight ingestion pathways have been analyzed: milk from backyard cows, milk from commercial sources, goat's milk, mother's milk, beef, leafy vegetables, cheese, and eggs. In addition, exposure through inhalation of contaminated air and exposure of a fetus via ingestion of ${ }^{131} \mathrm{I}$ by the mother are also analyzed. This appendix presents the equations used to estimate the total excess lifetime risks of thyroid cancer and the thyroid doses for the routine releases and for the 1954 accident, starting with ${ }^{131}$ I concentrations in air.

Individuals living near X-10 may have been exposed by more than one pathway at a time. Inhalation of contaminated air, for instance, is an exposure pathway that affected everyone in the contaminated areas. Three special exposure scenarios are designed to match the most likely dietary habits and lifestyles in the vicinity of the Oak Ridge Reservation. The first exposure scenario refers to individuals living in a "rural farm" setting (i.e., they own land and livestock; thus, they produce their own "backyard" cow's milk, cheese, vegetables and eggs). The second exposure scenario refers to individuals in a rural area who do not own their own dairy cows. However, they have some land and produce their own vegetables and eggs, and they purchase the necessary milk and cheese from farms located nearby. The third scenario refers to individuals in a more "urban" setting, who do not produce their own food products. These individuals buy milk and food products from the grocery store.

The total intake of ${ }^{131} \mathrm{I}$ for each exposure pathway is calculated according to the equations described in the following sections. From the intake via each pathway, or from the total intake for combined pathways, the dose and risk are calculated as follows:

$$
D=\sum_{i=N_{1}}^{N_{2}} I N T_{i} \cdot D C F_{i}
$$

and

$$
T E L R=\sum_{i=N_{1}}^{N_{2}} I N T_{i} \cdot D C F_{i} \cdot R F_{i}
$$

where

$$
\begin{array}{lll}
D & = & \text { dose to the thyroid due to intake of }{ }^{131} \mathrm{I}[\mathrm{Gy}] ; \\
T E L R & = & \text { total excess lifetime risk of thyroid cancer due to intake of }{ }^{131} \mathrm{I} \text { [unitless]; } \\
\mathrm{N}_{1}= & \text { age at which exposure began; } \\
\mathrm{N}_{2}= & \text { age at which exposure ended; } \\
I N T_{i}= & \text { age }(i) \text {-dependent intake of }{ }^{131} \mathrm{I}[\mathrm{Bq}] ; \\
D C F_{i}= & \text { thyroid dose per unit intake at age } i \text { (dose factor) }\left[\mathrm{Gy} \mathrm{Bq}^{-1}\right] \text { (Section 9); } \\
R F_{i}= & \text { the risk factor }=\text { excess lifetime risk of thyroid cancer per unit dose from } \\
i & = & \text { exposure at age } i\left[\mathrm{~Gy}^{-1}\right](\text { Section } 10) ; \text { and }
\end{array}
$$


The dose and risks are calculated separately for routine releases of ${ }^{131}$ I that occurred from 1944 to 1956 and for the ${ }^{131}$ I released from the April 29, 1954, accident. The amount of ${ }^{131}$ I released from X-10 during the accident is small compared to the total amount of ${ }^{131}$ I released during the entire year 1954. Thus, in the case called "routine releases," the source term for 1954 includes both the amount of ${ }^{131}$ I released during the 1954 accident and the amount released continuously during the rest of 1954. Thus, the doses and risks estimated for "routine releases" represent the health impact from the entire amount of ${ }^{131}$ I released from the RaLa facility.

\section{2-A.1 Ingestion of Contaminated Milk}

The approach described below applies to milk collected from backyard cows, commercial cows, and goats.

\section{Routine releases}

The generic equations used to estimate the intake of ${ }^{131} \mathrm{I}$ from the ingestion of contaminated milk are listed below.

and

$$
\begin{aligned}
I N T_{\text {milk,i }} & =C_{m m a, j} \cdot M D \cdot U_{m, i} \cdot \Delta t \\
C_{m m a, j} & =\sum_{k=1}^{3} C_{a a, j, k} \cdot A P_{k} \cdot P M
\end{aligned}
$$

where

$$
\begin{aligned}
& I N T_{\text {milk }, i}=\text { intake of }{ }^{131} \mathrm{I} \text { due to ingestion of contaminated milk for an individual } \\
& \text { of age } i[\mathrm{~Bq}] \text {; } \\
& C_{m m a, j}=\text { the annual average concentration of }{ }^{131} \mathrm{I} \text { in milk at milking in year } j \\
& {\left[\mathrm{~Bq} \mathrm{~L}^{-1}{ }_{\text {milk}}\right] \text {; }} \\
& j \quad=\quad \text { year of release: } j=1944+i \text {, for an individual born in 1944; } \\
& =1952+i \text {, for an individual born in 1952, etc; } \\
& M D=\text { milk distribution factor [unitless] (Section 7); } \\
& U_{m, i} \quad=\text { age }(i) \text {-dependent milk ingestion rate }\left[\mathrm{L} \mathrm{d}^{-1}\right] \text { (Sect. 8); } \\
& \Delta t \quad=\text { the time period corresponding to year } j[365 \mathrm{~d}] \text {; } \\
& k=\text { index for the physico-chemical forms of iodine present in air above a } \\
& \text { pasture: } k=1 \text { - elemental, } k=2 \text { - particulate iodine, } k=3 \text { - nonreactive } \\
& \text { form (assumed to be organic iodine); } \\
& C_{a a, j, k}=\text { annual average concentration in air of physico-chemical form } k\left[\mathrm{~Bq} \mathrm{~m}^{-3}{ }_{\text {air }}\right] \\
& \text { (Section 4); } \\
& A P_{k} \quad=\text { air-pasture transfer factor for physico-chemical form } k\left[\mathrm{~Bq} \mathrm{~kg}^{-1}\right. \text { dry mass } \\
& \text { per } \mathrm{Bq} \mathrm{m}^{-3}{ }_{\text {air }} \text { (Section 5); and } \\
& P M \quad=\text { the pasture-to-milk transfer factor }\left[\mathrm{Bq} \mathrm{L}^{-1} \text { milk per } \mathrm{Bq} \mathrm{kg}^{-1} \text { dry mass }\right] \text { (Sect. 6). }
\end{aligned}
$$


The solution to Equation 2-A.4 is dependent on the air-to-pasture transfer factor $\left(A P_{k}\right)$ for each chemical form of ${ }^{131} \mathrm{I} ; A P_{k}$ represents the ratio of annual average concentration of form $k$ in the pasture to that in air. Further discussions of individual parameters in Equations 2-A.3 and 2-A.4 are presented in the relevant sections of the main text of this report.

\section{Accident}

For the accidental release, the intake of ${ }^{131} \mathrm{I}$ from the ingestion of contaminated milk is estimated from the following equations.

$$
\begin{gathered}
I N T_{m i l k, i}=C_{m m, T} \cdot M D_{A} \cdot U_{m, i, A} \\
C_{m m, T}=\int_{0}^{T} C_{m m}(t) d t
\end{gathered}
$$

where

$$
\begin{aligned}
C_{m m, T}= & \text { the time-integrated concentration of }{ }^{131} \mathrm{I} \text { in milk at the time of milking } \\
& {\left[\mathrm{Bq} \cdot \mathrm{d} \mathrm{L}^{-1}\right. \text { milk]; }} \\
M D_{A}= & \text { milk distribution factor applicable during the accident [unitless]; } \\
U_{m, i, A}= & \text { age }(i) \text {-dependent milk ingestion rate at the time of the accident }\left[\mathrm{L} \mathrm{d}{ }^{-1}\right] \\
& \text { (Section 8); } \\
C_{m m}(t)= & \text { the concentration of }{ }^{131} \mathrm{I} \text { in milk at time } t \text { after the accident }\left[\mathrm{Bq} \mathrm{L}^{-1}{ }_{\text {milk }}\right] .
\end{aligned}
$$

Further discussions of individual parameters in Equations 2-A.4 through 2-A.6 are presented in the relevant sections of the main text of this report.

\section{2-A.2 Ingestion of Contaminated Beef}

\section{Routine Releases}

In this study, beef is considered a surrogate for various types of meat. Since meat is generally stored after slaughtering for a period of time longer than the half-life of ${ }^{131}$ I, consumption of contaminated meat is not expected to be an important contributor to the total dose and risk. The modeling approach for the ingestion of beef is similar to that for the ingestion of milk as shown in the following equations:

$$
\begin{gathered}
I N T_{b e e f, i}=C_{f s a, j} \cdot F D \cdot U_{f, i} \cdot \Delta t \\
C_{f s a, j}=\sum_{k=1}^{3} C_{a a, j, k} \cdot A P_{k} \cdot P F
\end{gathered}
$$


where

$$
\begin{aligned}
& I N T_{\text {beef }, i}=\text { intake of }{ }^{131} \mathrm{I} \text { due to ingestion of contaminated beef for an individual of } \\
& \left.C_{f s a, j}=\text { the annual average concentration of }{ }^{131} \mathrm{I} \text { in beef at slaughtering in year } \mathrm{f}\right) \\
& \text { [Bq kg }{ }^{-1} \text { beef]; } \\
& \text { FD = beef distribution factor [unitless] (Section 7); } \\
& U_{f, i} \quad=\text { age }(i) \text {-dependent beef ingestion rate }\left[\mathrm{kg} \mathrm{d}^{-1}\right](\text { Section } 8) ; \\
& \Delta t \quad=\text { the time period corresponding to year } j[365 \mathrm{~d}] \text {; } \\
& C_{a a, j, k}=\text { annual average concentration in air of physico-chemical form } k\left[\mathrm{~Bq} \mathrm{~m} \mathrm{~m}^{-3} \text { air }\right] \\
& \text { (Section 4); } \\
& \mathrm{AP}_{\mathrm{k}} \quad=\text { transfer factor of }{ }^{131} \mathrm{I} \text { from air to pasture grass for the physico-chemical } \\
& \text { form } k,\left[\mathrm{~Bq} \mathrm{~kg}^{-1} \text { dry mass per } \mathrm{Bq} \mathrm{m}^{-3}\right. \text { air] (Section 5); and } \\
& P F \quad=\text { the pasture-to-beef transfer factor }\left[\mathrm{Bq} \mathrm{kg}^{-1} \text { per } \mathrm{Bq} \mathrm{kg}^{-1} \text { dry mass }\right] \text { (Section 6). }
\end{aligned}
$$

Further discussions of individual parameters in Equations 2-A.7 through 2-A.8 are presented in the relevant sections of the main text of this report.

\section{Accident}

In the areas around the Oak Ridge Reservation, slaughtering of cattle is usually done in the fall, rather than spring, because the farmers use the cold weather to ensure that the meat does not spoil. The time between the accident in April 1954 and the normal period for cattle slaughtering was long enough that all of the ${ }^{131}$ I would have decayed away. Therefore, doses and risks from the ingestion of beef are not relevant for the analysis of the 1954 accident.

\section{2-A.3 Ingestion of Contaminated Leafy Vegetables}

\section{Routine Releases}

Contamination of "leafy" vegetables might be high because of the large surface of leaf exposed to the contaminated cloud. However, contamination is substantially reduced by washing and cooking. In addition, fresh vegetables are seasonal food products and are a source of exposure only during the harvest period. The governing equations for the estimation of ${ }^{131} \mathrm{I}$ intake from the ingestion of contaminated leafy vegetables are as follows:

$$
\begin{gathered}
I N T_{\text {leafy }, i}=C_{v h a, j} \cdot L D_{i} \cdot U_{L, i} \cdot \Delta t \\
C_{v h a, j}=\sum_{k=1}^{3} C_{a a, j, k} \cdot A V_{k}
\end{gathered}
$$

where

$I N T_{\text {leafy, } i}=$ intake of ${ }^{131} \mathrm{I}$ due to ingestion of contaminated leafy vegetables for an individual of age $i[\mathrm{~Bq}]$;

$C_{v h a, j}=$ the annual average concentration of ${ }^{131} \mathrm{I}$ in leafy vegetables at harvesting in year $(j)\left[\mathrm{Bq} \mathrm{kg}^{-1}\right.$ fresh mass];

$L D_{i} \quad=$ leafy vegetables distribution factor [unitless] (Section 7); 


$$
\begin{aligned}
& U_{f, i} \quad=\text { age }(i) \text {-dependent leafy vegetation ingestion rate }\left[\mathrm{kg}_{\text {fresh mass }} \mathrm{d}^{-1}\right] \\
& \text { (Section 8); } \\
& \Delta t \quad=\quad \text { the time period corresponding to year } j[365 \mathrm{~d}] \text {; } \\
& C_{a a, j, k}=\text { annual average concentration in air of physico-chemical form } \mathrm{k}\left[\mathrm{Bq} \mathrm{m}^{-3} \text { air }\right] \\
& \text { (Section 4); and } \\
& A V_{k}=\text { the air-to-vegetables transfer factor }\left[\mathrm{Bq} \mathrm{kg}^{-1} \text { fresh mass per } \mathrm{Bq} \mathrm{m}^{-3}\right] \text { for } \\
& \text { physico-chemical form } k \text { (Section 5). }
\end{aligned}
$$

\section{Accident}

The approach for the 1954 accident is similar to that presented in Section 2-A.1 for milk. The intake of ${ }^{131} \mathrm{I}$ from ingestion of leafy vegetables contaminated during 1954 accident is calculated as

$$
I N T_{\text {leafy, } i}=C_{v, T} \cdot L D_{A} \cdot U_{L, i, A}
$$

where

$$
\begin{aligned}
C_{v, T}= & \text { the time-integrated concentration of }{ }^{131} \mathrm{I} \text { in leafy vegetables at the time of } \\
& \text { harvesting }\left[\mathrm{Bq} @ \mathrm{~kg}^{-1} \text { fresh mass }\right] ; \\
L D_{A}= & \text { leafy vegetable distribution factor applicable during the accident } \\
& {[\text { dimensionless]; and }} \\
U_{L, i, A}= & \text { age }(i) \text {-dependent leafy vegetable ingestion rate at the time of the accident } \\
& {\left[\mathrm{kg}_{\text {fresh mass }} \mathrm{d}^{-1}\right] . }
\end{aligned}
$$

The time-integrated concentration $\left(C_{v, T}\right)$ is obtained by integrating the concentration of ${ }^{131} \mathrm{I}$ in vegetables $\left(C_{v}(t)\right)$ as a function of time after the accident. Further discussions of individual parameters in Equations 2-A.9 and 2-A.11 are presented in the relevant sections of the main text of this report.

\section{2-A.4 Ingestion of Contaminated Eggs}

Iodine-131 can accumulate in eggs if chickens are fed contaminated feed. In general, chicken feed is stored for periods of time longer than the half-life of ${ }^{131} \mathrm{I}$. If chickens are allowed to roam freely, however, they may consume small amounts of contaminated grass or soil. In this case, some ${ }^{131} \mathrm{I}$ is transferred to eggs. However, it has previously been shown that the dose from ingestion of contaminated eggs is much smaller than the dose from the ingestion of contaminated milk for similar concentrations of iodine in air, because of both the smaller amounts of eggs ingested as compared to the ingestion of fresh milk and the longer storage time of eggs (NCI, 1997). Therefore, a less realistic modeling approach was used in the present study. It is assumed that all eggs consumed by an individual are produced locally and therefore are contaminated. Eggs purchased from remote locations are either less contaminated with releases from X-10 or are stored for longer periods of time than the locally produced eggs. Thus, this approach eliminates the need to fully understand the local distribution system for eggs. Also, this approach is based on the observation that the concentration of ${ }^{131} \mathrm{I}$ in eggs is similar to that in cow's milk for a given deposition density of ${ }^{131}$ I (NCI, 1997). Therefore, the concentration in 
cows' milk is used as a surrogate for the concentration in eggs. For routine releases, the following equation applies:

$$
I N T_{e g g s, i}=C_{m m a, j} \cdot F_{g g} \cdot e^{-\lambda_{R} \cdot T_{g g}} \cdot U_{e g g, i} \cdot \Delta t
$$

where

$$
\begin{aligned}
I N T_{\text {eggs,i }}= & \text { intake of }{ }^{131} \mathrm{I} \text { due to ingestion of contaminated eggs for an individual of } \\
& \text { age } i[\mathrm{~Bq}] ; \\
C_{m m a, j} & \text { the annual average concentration in milk at milking in year } j \quad\left[\mathrm{~Bq} \mathrm{~L} \mathrm{~L}^{-1} \text { milk }\right] ; \\
F_{g g} & \text { parameter that accounts for the uncertainty in the assumption that the } \\
& \text { concentration in milk is a surrogate for the concentration in eggs; it is } \\
& \text { defined as the ratio between the average concentration in eggs and average } \\
& \text { concentration in milk for the same area deposition }\left[\mathrm{Lgg}^{-1}\right] \text { (Section 7); } \\
= & \text { the radionuclide decay constant for }{ }^{131} \mathrm{I}\left[0.0862 \mathrm{~d}^{-1}\right] ; \\
= & \text { delay between harvest and consumption of eggs }[\mathrm{d}](\text { Section } 7) ; \\
\lambda_{R} & \text { age }(i) \text {-dependent egg ingestion rate }\left[\mathrm{kg} \mathrm{d}^{-1}\right](\text { Section } 8) ; \text { and } \\
T_{g g} & \text { the time period corresponding to year } j[365 \mathrm{~d}] .
\end{aligned}
$$

Similarly, the following governing equation can be used for estimating the intake from the 1954 accident:

$$
I N T_{e g g s, i}=C_{m m, T} \cdot F_{g g} \cdot e^{-\lambda_{R} \cdot T_{g g}} \cdot U_{e g g A, i}
$$

where

$$
\begin{aligned}
C_{m m, T}= & \text { the time-integrated concentration of }{ }^{131} \mathrm{I} \text { in milk at the time of milking } \\
& {\left[\mathrm{Bq} \cdot \mathrm{d} \mathrm{L}^{-1}\right. \text { milk]; and }} \\
U_{e g g A, i}= & \text { age }(i) \text {-dependent egg ingestion rate }\left[\mathrm{kg} \mathrm{d} \mathrm{d}^{-1}\right] \text { at the time of the accident } \\
& (\text { Section } 8) .
\end{aligned}
$$

Further discussions of individual parameters in Equations 2-A.12 through 2-A.13 are presented in the relevant sections of the main text of this report.

\section{2-A.5 Ingestion of Contaminated Cheese}

Cheese produced from contaminated milk contains some amounts of ${ }^{131} \mathrm{I}$. Cheese is stored for longer times and is consumed in much lower quantities than milk. Contribution of cheese ingestion to the total dose and total excess lifetime risk has been found to be very small (NCI, 1997). Therefore, a less detailed approach was chosen for this exposure pathway. The simplifying assumptions are (a) only ingestion of cottage cheese is considered in this study because this type of cheese is usually consumed shortly after it is produced ${ }^{1}$, and (b) the entire amount of cheese consumed by an exposed individual is produced locally. For the routine releases, the intake of ${ }^{131} \mathrm{I}$ is estimated using the following equation:

\footnotetext{
${ }^{1}$ Other types of "hard" cheese are stored for longer periods of time that allow ${ }^{131}$ I to decay away.
} 


$$
I N T_{c c, i}=C_{m m a, j} \cdot F_{c c} \cdot e^{-\lambda_{R} \cdot T_{c c}} \cdot U_{c c, i} \cdot \Delta t
$$

where

$$
\begin{array}{ll}
I N T_{c c, i}= & \text { intake of }{ }^{131} \mathrm{I} \text { due to ingestion of contaminated cottage cheese for an } \\
\text { individual of age } i[\mathrm{~Bq}] ;
\end{array}
$$

The intake from the 1954 accident for the cheese pathway is estimated using the following equation:

where

$$
I N T_{c c, i}=C_{m m, T} \cdot F_{c c} \cdot e^{-\lambda_{R} T_{c c}} \cdot U_{c c c, i}
$$

$$
\begin{aligned}
& C_{m m, T}= \text { the time-integrated concentration of }{ }^{131} \mathrm{I} \text { in milk at the time of milking } \\
& {\left[\mathrm{Bq} \cdot \mathrm{d} \mathrm{L}^{-1} \text { milk }\right] \text {; and } } \\
& U_{c c A, i}=\begin{array}{l}
\text { age }(i) \text {-dependent cheese ingestion rate }\left[\mathrm{kg} \mathrm{d}^{-1}\right] \text { for the time of the accident } \\
\text { (Section 8). }
\end{array}
\end{aligned}
$$

Further discussions of individual parameters in Equations 2-A.14 and 2-A.15 are presented in the relevant sections of the main text of this report.

\section{2-A.6 Inhalation of Contaminated Air}

Atmospheric releases of ${ }^{131} \mathrm{I}$ produce an increased concentration of ${ }^{131} \mathrm{I}$ in air at ground level. Individual members of the public may be exposed from inhalation of contaminated air in addition to the ingestion of contaminated food. The inhalation pathway is described in detail in Section 8 of this report. The equations for this pathway are reproduced here. For he routine releases, the following equation applies:

where

$$
I N T_{i n h, i}=\left(\sum_{k=1}^{3}\left(f_{o}+\left(1-f_{o}\right) \cdot r_{i o}\right) \cdot C_{a a, i, k} \cdot B R_{i} \cdot D_{k}\right) \cdot \Delta t
$$

$$
\begin{aligned}
& I N T_{\text {inh }, i}=\text { intake of }{ }^{131} \mathrm{I} \text { due to inhalation of contaminated air for an individual of } \\
& \text { age } i[\mathrm{~Bq}] \text {; } \\
& f_{o} \quad=\quad \text { fraction of time spent outdoors [unitless]; } \\
& r_{i o}=\text { ratio of the indoor to outdoor concentrations of iodine in air [unitless]; } \\
& C_{a a, j, k}=\text { annual average concentration of physico-chemical form } k \text { in outside } \\
& \text { air }\left[\mathrm{Bq} \mathrm{m}^{-3}{ }_{\text {air }}\right] \text {; } \\
& B R_{i}=\text { breathing rate for an individual at age } i\left[\mathrm{~m}^{3} \text { air } \mathrm{d}^{-1}\right] \text {; }
\end{aligned}
$$



$D_{k} \quad=$ fraction of the total amount inhaled that deposits and is absorbed in different parts of the respiratory system (applies to each physico-chemical form $k$ ); and
$\Delta t \quad=$ the time period corresponding to year $j[365 \mathrm{~d}]$.

For the 1954 accident, the corresponding equation is as follows:

where

$$
I N T_{i n h, i}=\left(\sum_{k=1}^{3}\left(f_{o}+\left(1-f_{o}\right) \cdot r_{i o}\right) \cdot C_{a a, T, k} \cdot B R_{i A} \cdot D_{k}\right)_{i}
$$

$$
\begin{aligned}
C_{a a, T, k}= & \text { time-integrated concentration of iodine in chemical form } k \text { in the outside } \\
& \text { air }\left[\mathrm{Bq} \cdot \mathrm{d} \mathrm{m}^{-3} \text { air }\right] ; \text { and } \\
B R_{i A}= & \text { breathing rate for an individual at age } i \text { during the accident }\left[\mathrm{m}^{3} \text { air } \mathrm{d}^{-1}\right] .
\end{aligned}
$$

The time-integrated concentration of ${ }^{131} \mathrm{I}$ in chemical form $k$ is estimated from

$$
C_{a, T, k}=\int_{0}^{T} C_{a, k}(t) d t
$$

Further discussions of individual parameters in Equations 2-A.16 through 2-A.18 are presented in the relevant sections of the main text of this report.

\section{2-A.7 Exposure to Multiple Sources of Iodine}

Individuals living near the X-10 facility may have been exposed by more than one pathway. In this case, the total intake of ${ }^{131} \mathrm{I}$ is obtained by simply summing the intakes for individual pathways, as shown below. The summation is performed similarly for the case of routine releases or the 1954 accident.

$$
I N T_{i}=\left(I N T_{m i l k, i}+I N T_{b e e f, i}+I N T_{\text {leafy,i }}+I N T_{i n h, i}+\ldots\right)
$$

where

$$
\begin{aligned}
I N T_{i} & =\text { total intake of }{ }^{131} \mathrm{I} \text { at age } i \\
I N T_{\text {milk }, i}= & \text { intake of }{ }^{131} \mathrm{I} \text { due to ingestion of contaminated milk, at age } i[\mathrm{~Bq}] ; \\
I N T_{\text {beef }, i}= & \text { intake of }{ }^{131} \mathrm{I} \text { due to ingestion of contaminated beef, at age } i[\mathrm{~Bq}] \\
I N T_{\text {leafy, } i}= & \text { intake of }{ }^{131} \mathrm{I} \text { due to ingestion of contaminated leafy vegetables, } \\
& \text { at age } i[\mathrm{~Bq}] ; \text { and } \\
I N T_{\text {inh }, i}= & \text { intake of }{ }^{131} \mathrm{I} \text { due to inhalation of contaminated air, at age } i[\mathrm{~Bq}] .
\end{aligned}
$$

Three special exposure scenarios are designed to match the most likely dietary habits and lifestyles in the vicinity of the Oak Ridge Reservation. The first exposure scenario refers to individuals living in a "rural farm" setting (i.e., they own land and livestock and produce their own "backyard" cow milk, cheese, vegetables, and eggs). The intake for this exposure scenario is obtained by adding the intakes from inhalation and from the ingestion of backyard cow milk, 
beef, leafy vegetables, eggs, and cheese. The doses and risks for this exposure scenario are reported under the label "diet 1."

The second exposure scenario refers to individuals in a rural area who do not own their own dairy cows. However, they have some land and can produce their own vegetables and eggs, and they purchase the necessary milk and cheese from farms located nearby. The intake for this exposure scenario is obtained by adding the intakes from inhalation and from ingestion of locally produced commercial milk, beef, leafy vegetables, eggs, and cheese. The doses and risks for this exposure scenario are reported under the label "diet 2."

The third scenario refers to individuals in a more "urban" setting, who do not produce their own food products. They buy milk and food products from the grocery store. The intake for this exposure scenario is obtained by adding the intakes from inhalation and from ingestion of regionally averaged commercial milk. The contribution to the total thyroid dose and risk of cancer from other regionally mixed food products is assumed to be negligible compared to the contribution from ingestion of milk. The doses and risks for this exposure scenario are reported under the label "diet 3."

Given that the doses and risks from ingestion of goat's milk are substantially larger than the doses and risks from any other exposure pathway, these doses and risks are reported separately under the label "diet 4".

\section{2-A.8 Ingestion of Contaminated Mother's Milk by Breast-fed Infants}

If a lactating mother consumes food contaminated with ${ }^{131} \mathrm{I}$, a substantial fraction of the ingested iodine will be secreted to milk and supplied to her breast-fed infant. Infants also have a higher uptake of iodine by the thyroid than do people in other age groups. Fortunately, the amount of iodine ingested by an adult female (with respect to that consumed by grazing dairy animals) is such that the concentration in mother's milk is very low compared to the concentration in cow's or goat's milk. Previous studies (NCI, 1997) have shown that the contribution of the ingestion of contaminated mother's milk to the overall dose for a child exposed to contaminated cow's milk after birth is not of dominant importance.

Typically, lactating mothers are considered to be on "diet 1," composed of backyard cow milk, all other non-milk food products and inhalation. For locations where backyard cows are not present (e.g., the city of Oak Ridge), lactating mothers are considered to be on "diet 3," composed only of regionally mixed milk and inhalation.

\section{2-A.9 Exposure of a fetus}

If a pregnant woman consumes food contaminated with ${ }^{131} \mathrm{I}$, a fraction of the ingested iodine penetrates the placenta and is transferred to her fetus. Once the fetal thyroid is developed and starts functioning, it accumulates iodine based on the same physiological principles that apply to children and adults.

Typically, pregnant women are considered to be on "diet 1," composed of backyard cow milk, all other non-milk food products and inhalation. For locations where backyard cows are mt present (e.g., the city of Oak Ridge), pregnant women are considered to be on "diet 3," composed only of 
regionally mixed milk and inhalation. Given that the duration of the pregnancy is nine months, the intake of iodine by a pregnant woman was adjusted to (9/12) of the annual intake of a nonpregnant woman. The dose factor for the fetus $\left(D C F_{\text {fetus }}\right)$ represents the dose to the fetal thyroid for a unit intake of ${ }^{131} \mathrm{I}$ by the pregnant woman, as opposed to a fetal thyroid dose for a unit activity transferred through the placenta (Section 9).

\section{2-A.10 References}

NCI, (National Cancer Institute). Estimated Exposure and Thyroid Doses Received by the American People from Iodine-131 Fallout Following Nevada Atmospheric Nuclear Bomb Tests. U.S. Department of Health and Human Services. National Institute of Health. National Cancer Institute, October, 1997. 
APPENDIX 3A

SAMPLE “PUSH DATA” FOR CLINTON PILE SLUGS 
TASK 1 REPORT

July 1999

${ }^{131}$ I Releases from X-10 Radioactive Lanthanum Processing -

Page 3A-2

Appendices

This page intentionally left blank. 


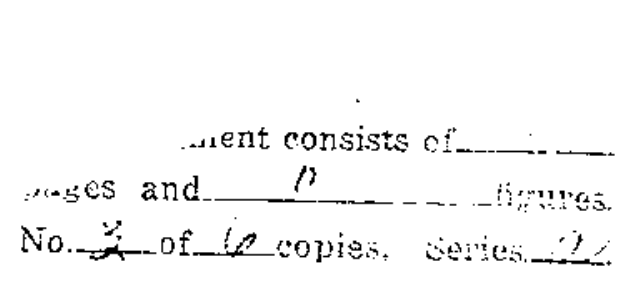

To: W. A. Rod cer

Froma

In Ro: SLing ton $706-\mathrm{y}$

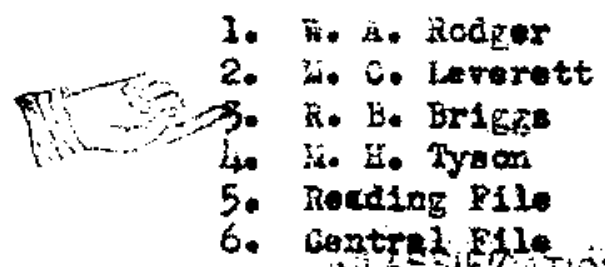

January 5. 1946 CATE

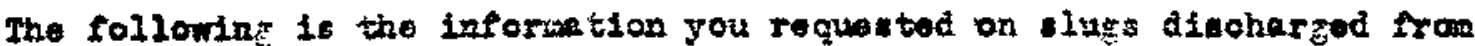
the pile on Decomber 31, 1945, Janumer 2, 3 and 5,1946 for use in the 706-D Euilding:

Accuigiand Xati

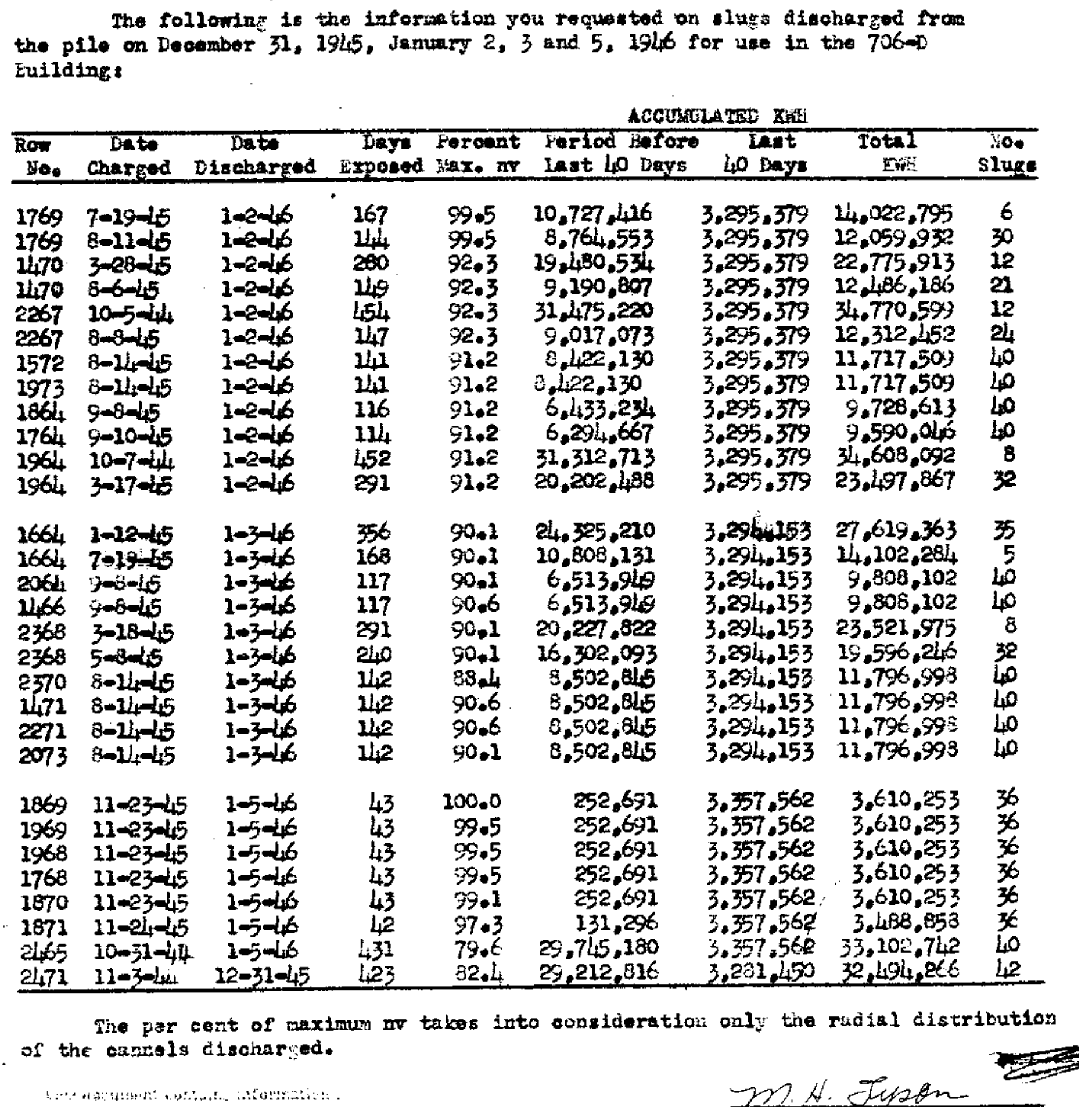

Tine par cent of maximum nv takos into consideration only the rudisl distribution of the cansels discharsed. 
TO: $\quad$ b. J. Witkowsk1

INRE: Data on Slugg D1 acharged Trom PIIO on Jü 13 for $\underline{706-D}$.
FROM: J. D. Knox

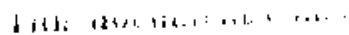

withes and lingues

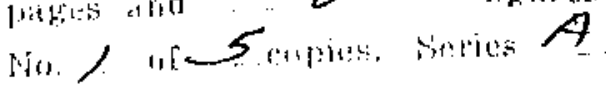

SLUGS FOR 706-D

Position

Factor

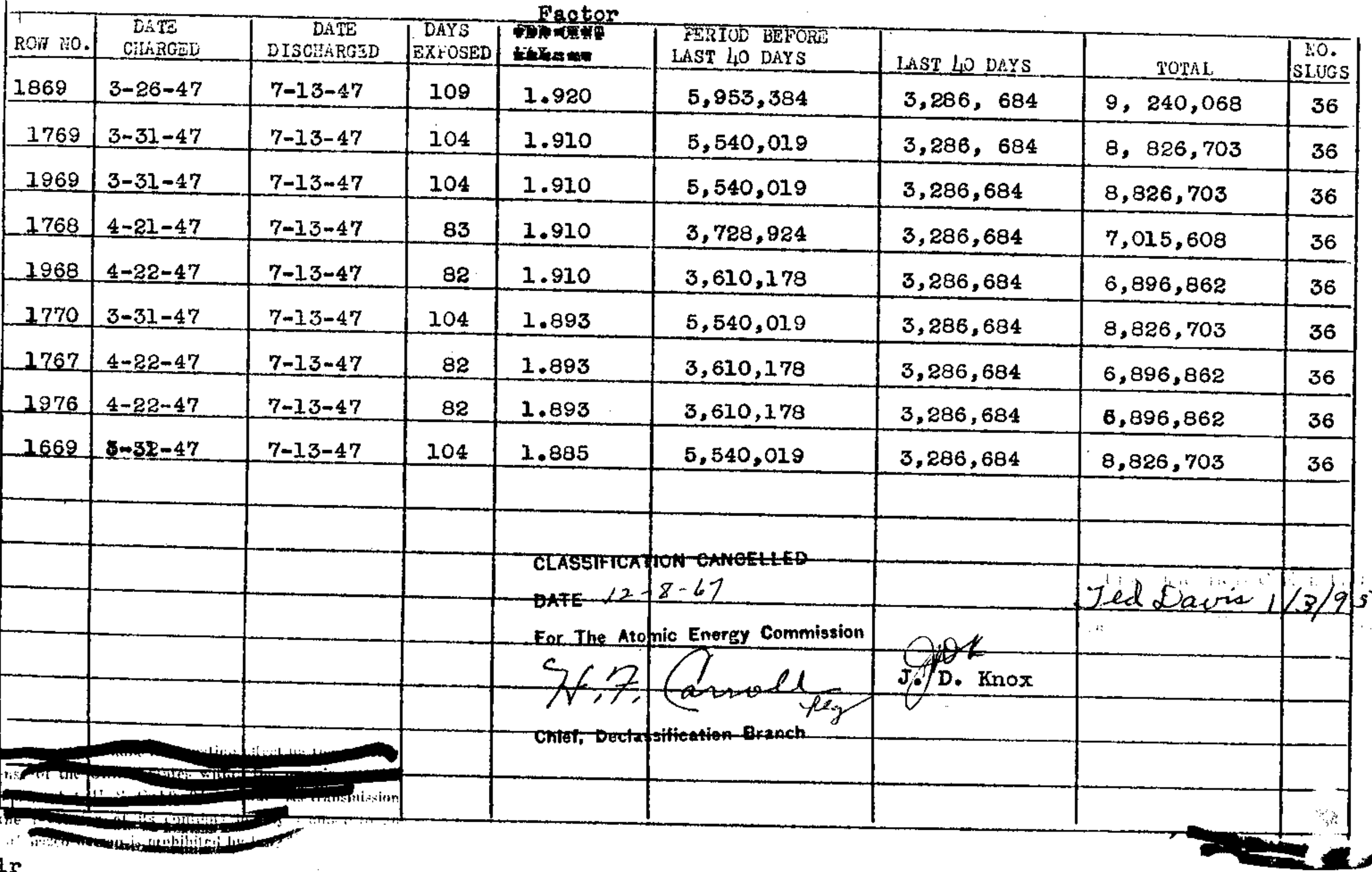

2. L. B. Enlot

3. F, R. Stuckey

4. Reading File

5. Central Files
July 13,1947 . 
APPENDIX 3B

SAMPLE HANFORD SLUG IRRADIATION CORRESPONDENCE 
TASK 1 REPORT

July 1999

${ }^{131}$ I Releases from X-10 Radioactive Lanthanum Processing -

Page 3B-2

Appendices

This page intentionally left blank. 


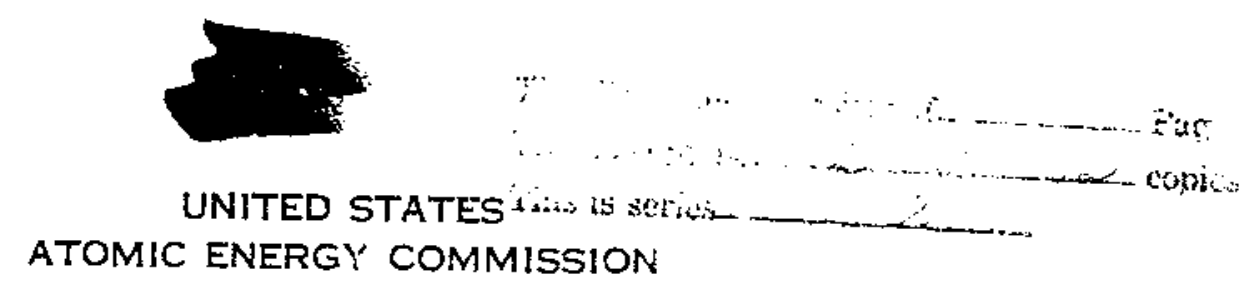

In Roply Lefer Do: Kh류 2 JS

Ciessification Carcelled

Ox-cinemento

Ex Authority of

Gim 3
Oak Ridge, Tounesset November 29, 1948

Carbicie and Carbon Chomicals Corporation

Post Office Box "n

Dak Elage, Temnesmoe

Attention: C. F. Ruoloer, Jr., icreeutive Director

Oak Rtige Iational Inboratory
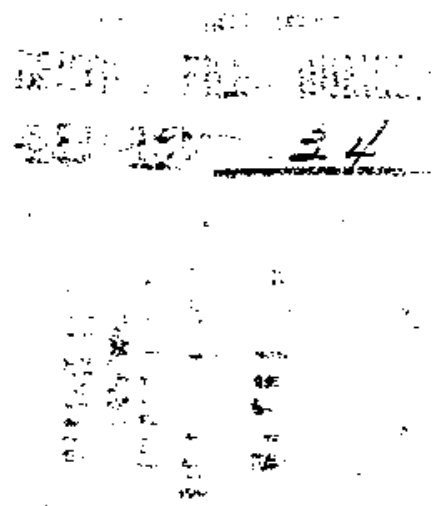

Geatlowen:

The following is an extract of a teletype mesage rooejved from the Oeflice of Finford Dirocted Operations in reply to our request for information, coneeraling the slugg usod in tho Rala run which began Nevembar 17 tis.

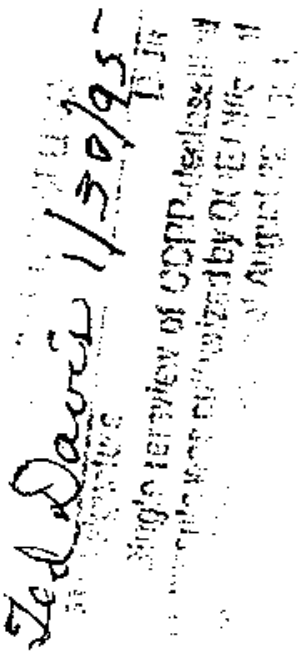

"Sluge were oharged August 17, 1942; discharged hovember 12, 2945. Tube used for woorure was 2379 of $B$ pilo. Bfrective duys of exposure during this period was equal to 70.3 or effeotive dentine equiralent to about ten (10) dajs. Fowwer, this domtime was not for hort periods for purpose of disoharging, but actually intolrad a prolonged aimtiow starting septerber 28 at 07,30 hours ma continuing until Spotenbar 24, midnight, when olle larted to operate at about $1 / 4$ nominal power levol. During period of septemier 25 to Ootobar 6 the power level wes raimed tncrementally to nominal ievel. Therefore, during this period the flux raried epprealably from ralue at nominal power levol. The pile was operating at low lovels at approximately the nalf way point of mosure poried during which the concentration lorel of product probabiy dropoed oonsiderably so thrie the resaining exposure thomel levels was intufeiciont to raise the conoentwation to the desired lorel. Total weight of uraniun wes 349.34 pounds."

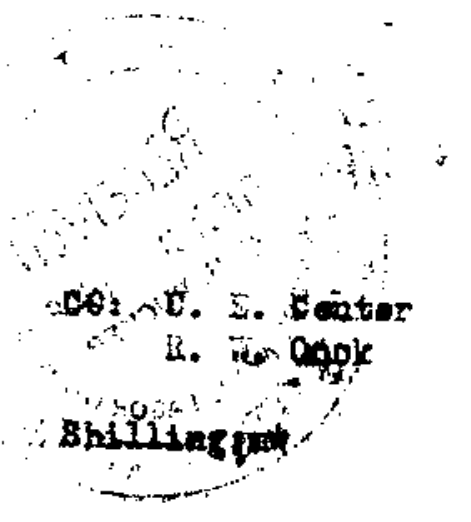

Sincerviy youre,

Mlbert i. Eolland, Jr., Xi.D. Director of Research and tuedicine

Page 3B-3 


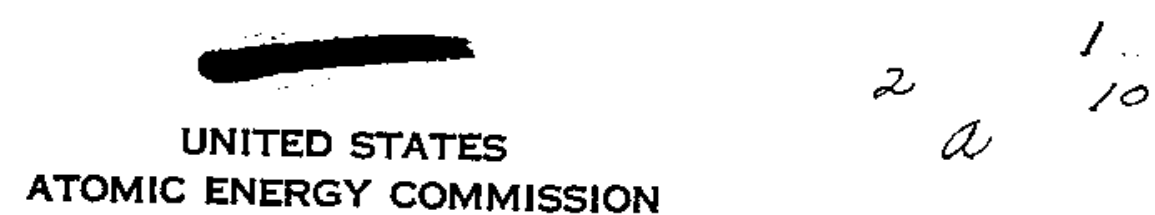

In Keply Rofor To:

RUR a JS

Clampitication chenged to

UNCLASSIFIED

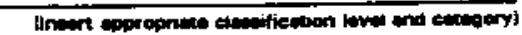

br whorrty of T, E. DAVIS 10-I8-93

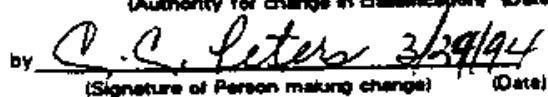

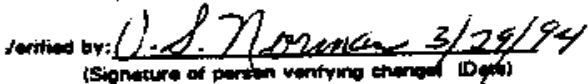

Gurbide and Carbon Chomicals Corporation

Post Oretce Bor "p"

Oak Ridge, Fanness

Oak Ridge, Toanes ase

Pebruary 16, 1949

Classification Cancelled

Consoroto

Ey Authority of $D x C$.

By EEP Datallif $30 \%$

sttention: C. F. Fucker, Jr.. Ecoutive Dirootor

Oak Ridge Hational Laboratory

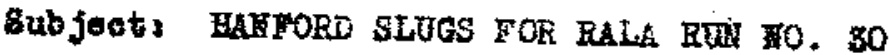

Gentl amax

Thirty-alght four froh Enford glug: wore delivered to you

Fobruary 24 for ues in the production of flale liun No. 50.

Shipunt was ande Eros tanford about 10,00 P.H. (PST), Tobruary 9. The alugs wore obtaincd from as near at poselble the centor of

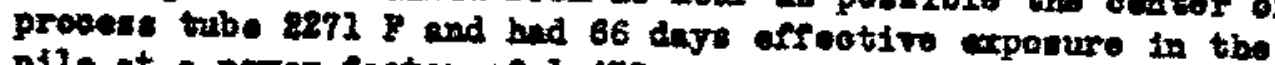
plle at a pewer factor of 1.478 .

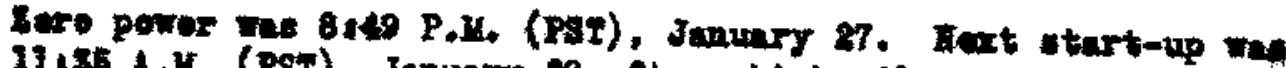
IIIst A.H. (PST), January 28 after whtoh $p 12$ e eperated at 125

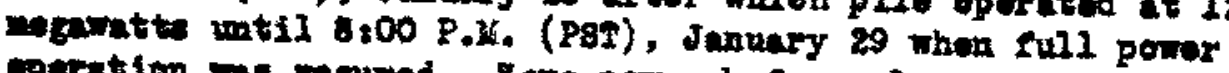
cperation wa romand. Zoro porror before elug dischargo was 2:20 A.K. (PST), February 8.

Sincerely yours,

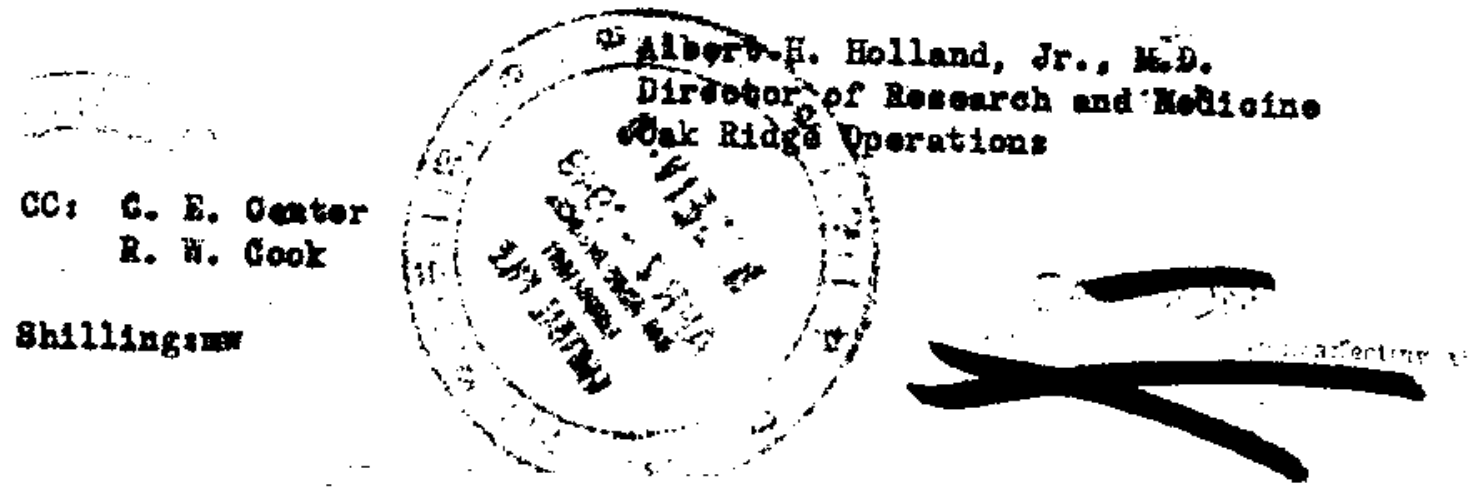

Page 3B-4 


\section{APPENDIX 3C}

SAMPLE PAGES FROM 100 AREA (CLINTON PILE) LOG BOOKS 
TASK 1 REPORT

July 1999

${ }^{131}$ I Releases from X-10 Radioactive Lanthanum Processing -

Page 3C-2

Appendices

This page intentionally left blank. 
54

Li lobets wants to make another sur at ite thernal colunen tonito Open shutters at $1200 \mathrm{Mn}$. An. Pobct will arrange to have then elozed in the norning.

Defore going ow in srex now before Dtornow.

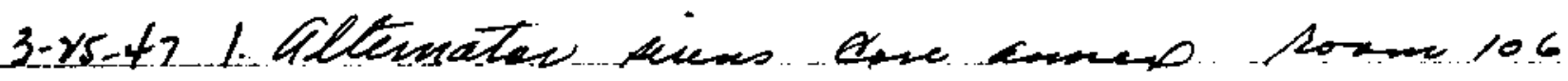
1238 the 19,0 Bedy $205 * 101$ ac

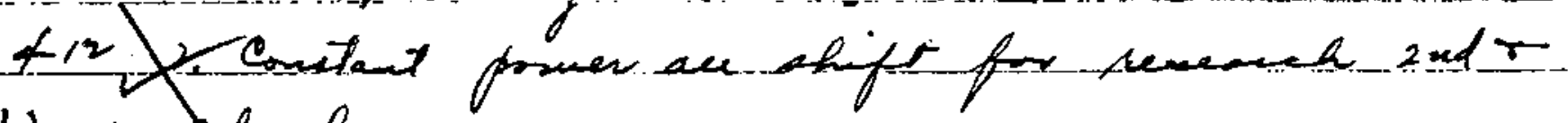
Weger hlevel.

3. Wollan operated at hale $22-4 \frac{50}{\mathrm{~F}} 12 \mathrm{gm}$ -

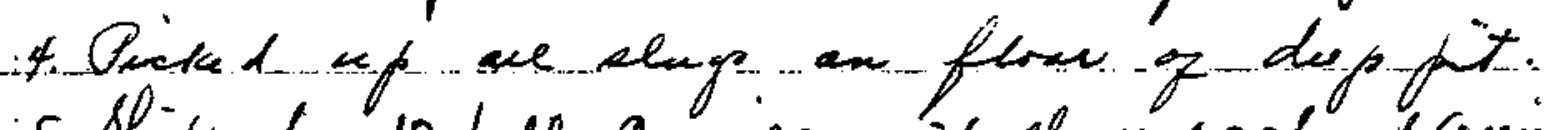
5. Shifped 10 full carriess 26 slugr wach Lamie Wwith 13 shego, to 706 D. Total shifped 273 gan hucket 18.3 rejects now in B...4s. 24 - elug shout of total puahed.

6. Picked sp slughes and smet elivator... 7. Changed Bellel's samples in decay Cuscer $303-114-3.03-115+303-116$.

8. Cut Coseal water $t$ mathers spray water

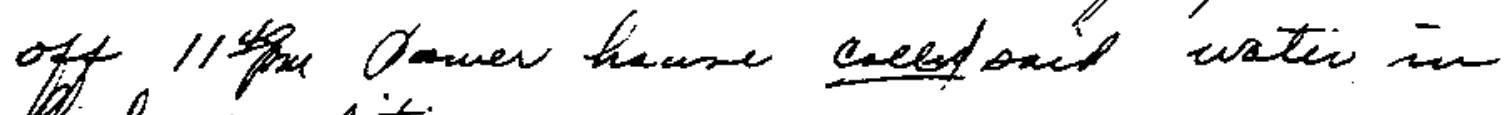
lid endition.

9. Ftrod hight suiteh for 115 had.

Page $3 \mathrm{C}-3$ 
Discharged and charged the following rover of slugs:-

Discharged into. Bucket 18 .

$1567 \quad 36$ Slug row .....

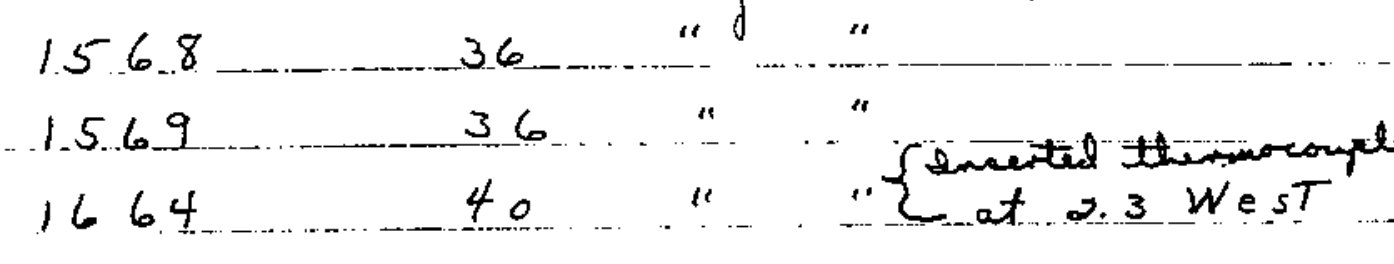

rat.

$1666 \ldots 36$

$16.70 \quad-36$

1671

36

1771

36

der

$1869 \quad 36$

$1872 \frac{36}{\text { TOTAL }}$

14. (Dot tim 917 ho total lat the 1.0.84 hoo.

15. I nothument department worked on internal Temp \#2 and changed some of the thermocouples. around. Check the new list which has been posted.

$-D$

Page $3 C-4$ 


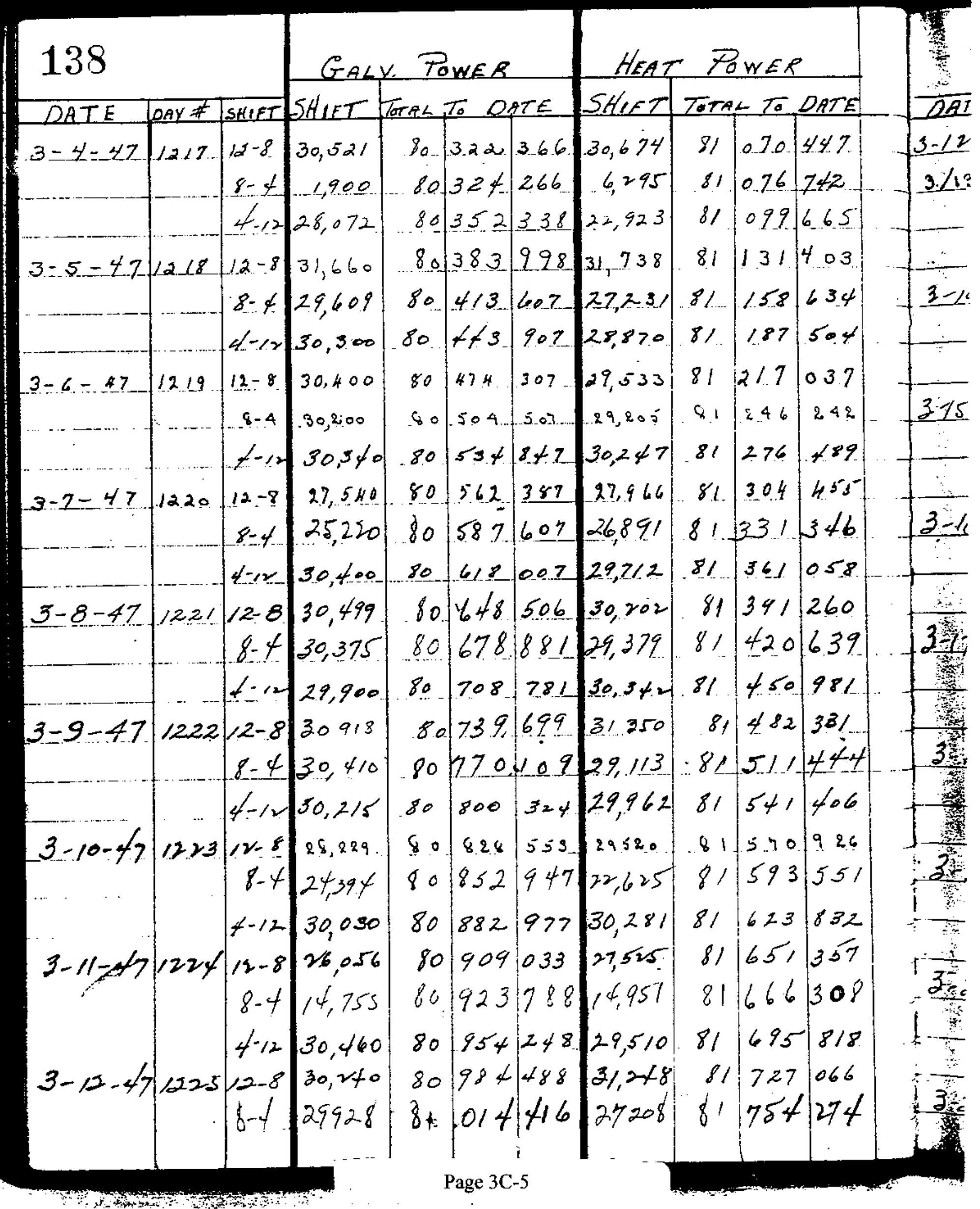


TASK 1 REPORT

July 1999

${ }^{131}$ I Releases from X-10 Radioactive Lanthanum Processing -

Page 3C-6

Appendices

This page intentionally left blank. 
APPENDIX 3D

SAMPLE PAGES FROM A CHEMICAL SEPARATIONS OPERATIONS LOG BOOK 
TASK 1 REPORT

July 1999

${ }^{131}$ I Releases from X-10 Radioactive Lanthanum Processing -

Page 3D-2

Appendices

This page intentionally left blank. 
4- Shat off the Ain Jet We-6 to Ne-5 5 W. 6 to W.S Sot is still ON. W-PLL ot 11.00 PM, 8111"

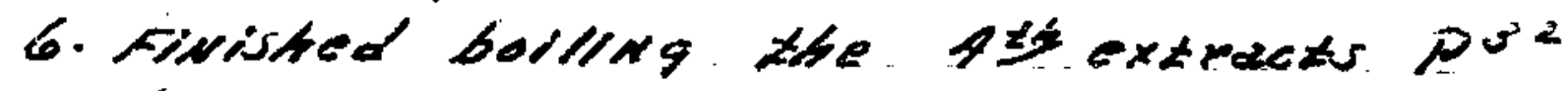
down in EN $\# 1$.

10-22-54 1. 166 TO W8 JeT is STiks Going.

$\because$ pters 12-8 Z. WASHED COTREELL Down.

ELEVINS. 3. DissoLved $5 \mathrm{cms}$ of Ba No in zoo ma of HO AND ADDED TO 200 CUA P.E. TANK THEN ADDED $5800 \mathrm{~mL}$ of $70 \%$ HNO, TO P.e. A 7Literes of Fumido: HNo 3 THEN FILTERED TO MW AVE. $30 \mathrm{~g}$ me/min. Before. THe Bano Test we Ran Two Rate Tosts WitH WATER AND Cor $420 \mathrm{~m} / \mathrm{min}$ EASH TEST.

THE PE TO MU FILTEK HAS ELEN EACK WASHED ANO FE. WASNED OUT GHG HEAD TO OFE DAS. iny HAS ASIO FIITRATION Waste.

tho to $10-22-541 . M . H .149$ brex in service.

mours 8-4 2 . Brovokt big truck back Frow Galabe, fH. H. however the reguested it be sewe backby Neyt WedNesdA $10-27-5 \%$.

3. Made uP ISt Pare of COATING removal IN Al.

4. SAMPLEC MW FNANd EMPEied M.W.

5. RAN I AMMONIA Biftu oride rinse throush F.E. Fiater then rivsed with Hzo Severs times.

P. SAmpred WDS ANd TURNed into LAb.

Page 3D-3 
20

7. Brovahx down I sher for I/31.

8. Removed Cone from Ch. HeAd CreANed ANd replaced baCI IN CArrier.

9. LoAded 16 savas to Al. CArrier is At pite bldG over shift chaviac.

10. Gased up bia truct.

$10-2$

12 .

BLEk

10/22/sal- Finisted loading the Rewainder of the f-12 8.6 slugs to $\mathrm{A}-1$. . Cleaned and stered R.L.N. SHUS MNOCKEN and Changen.

2.Two mone patrol Tobs carese in on this shift see Instructions los book.

3. had to shert stean beaden down att tonses Ml 141318 and CoOl Hull Tet With Haten befone it Hoold $\sqrt{3} t$.

4. The filten at W.G float Mantole is 10. sealed in Hith wax seal. seens to be tranti. 8 . 5 sampled MH. $22=39 \mathrm{sm} / \mathrm{m} / 3500=765 \mathrm{sm}$ and $0.0 . B=160$.

6- Ran two filter kate teit on P-Fingo filten

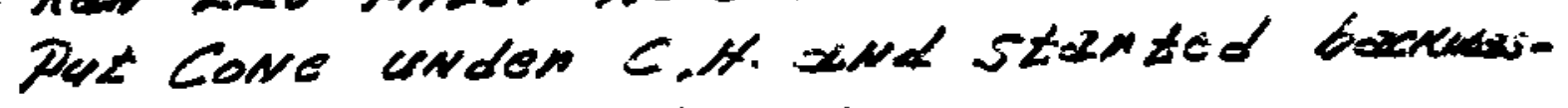
hivs with HNG \& Hezton.

7. Raz La Statkis.

A.l second Codtins Ticmoval ithellts COOlINS Q $Z$ IE:20 A,M.

milo $9 \%$ caratic.

$T .820 \%$ HNO.

$T-5$ 20\% HNE

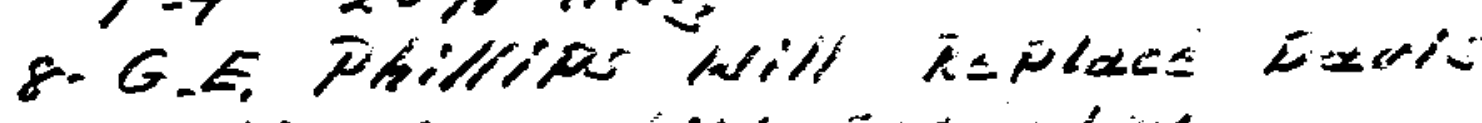
on the f.q ithits intwadidy.

Page 3D-4 
9-w-6 to HAj wet if still ox W ir 2. "II"

10-23-54 L. CUT W6 TOW 8 JET OFF AT S:COAM 7 STEAMED 12-8 THE LINE. W6 44. - $6^{\circ} 5^{\prime} \frac{1}{2}$ W $3<6 .-11^{\prime} 6 "$

3LEVINS. 2 WITH THE II ZOO CUB. P.E. TO MW. FILTER BACKWASHED TwO 18 Liter $H_{2}$ O FiLter TESTS were made OF WHICH $818 \mathrm{~mL} / \mathrm{m}$ IN KaTE WAS OBTANINED ON EACH.

3. RatA status:

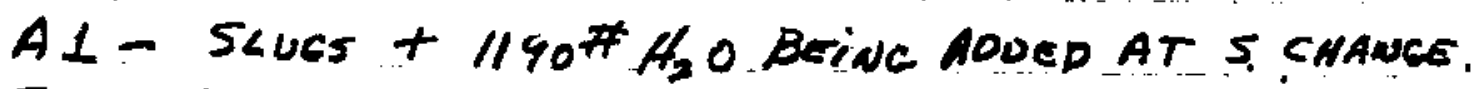

$T_{8}$ - $440 \pi H_{2} O$ For STCP 39 . ON Bonding Removal.

T9 - \$90 HNO FOR BATCH A Dissolving.

1C SAMPLE SHOWED . 123 SLUG.

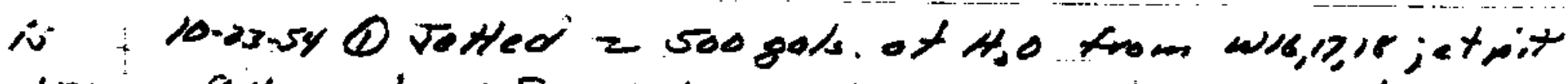

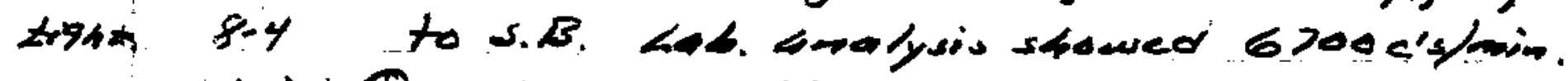

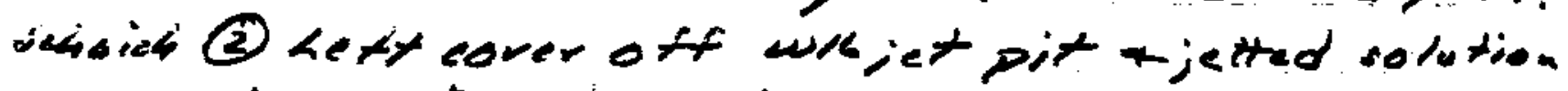

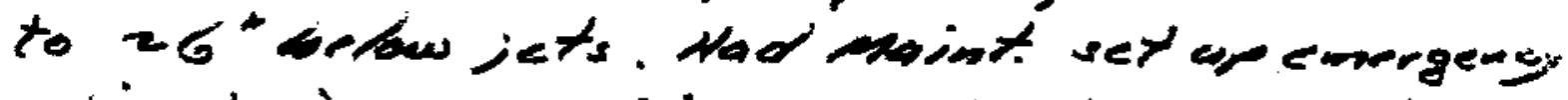
whines is case pit will not jet or solution gets top sot to go to sis. It too hot (ely/min)

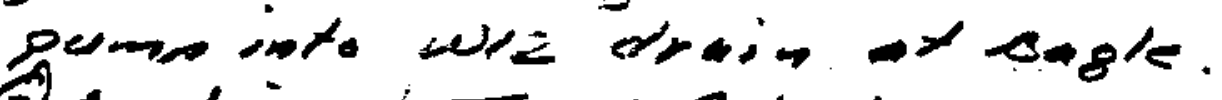

(3) Continued Ti. a Patrol.

(4) Ran stakes: Di-Batah D Diss.

$78-1 / 20$

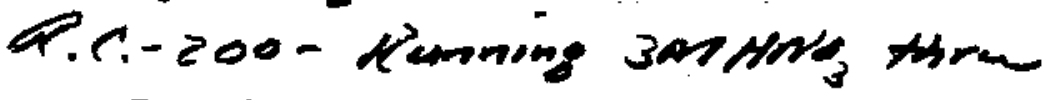
Resin.

E doit air count at 3:30pm. shat down cassation.

Page 3D-5 
$12 \%$

Misinal Ra la staters

UI A- Buter B Dissolvine.

ACN A-G Bater A Digestring until 12:00 M.N. A-8 Haxen.

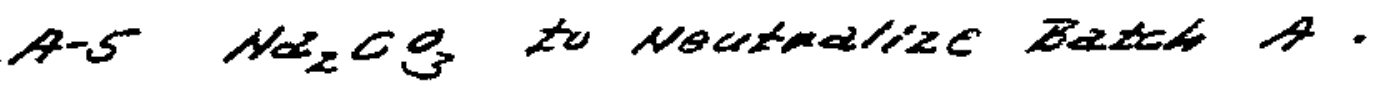

$M-1 M T$

INA Results 12,186 C's.

z. The 5P.GR. ON A-1 ONIY shows 1227 on the vituic fou dessoluins the $t$ is 1,36 A. bot the nolumes etrected Close atter

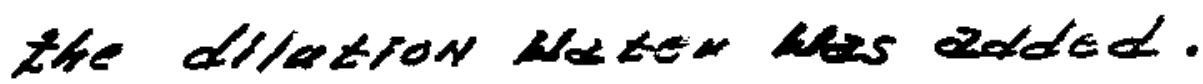

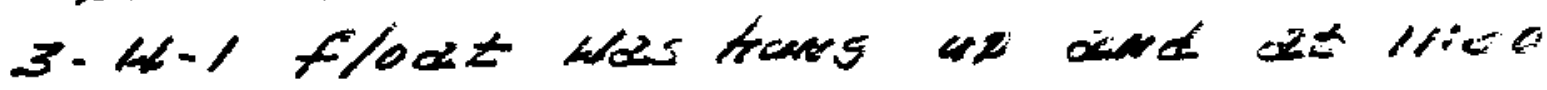
we toond $A-1$ twII

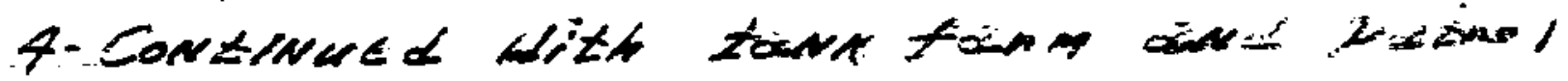

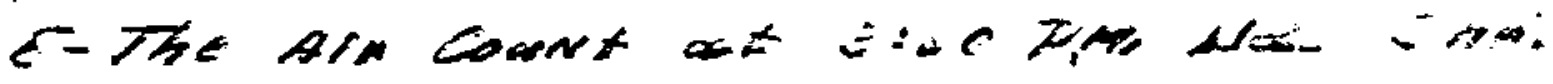

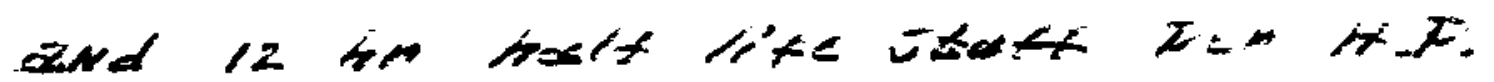

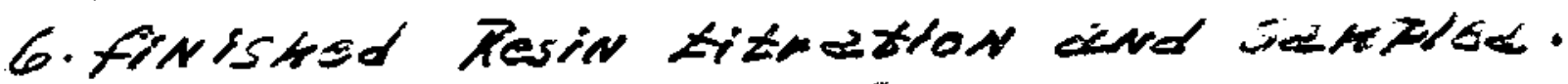
did Not Staint the lie gonenation.

3N HNG Samte thow

10-24.54 1. C.T. CARNEY THE INST. MAN CHECHED AL SP.GT. $12-8$ BuEvins. FOR GRROR BY USINE THE SMALL MANOMETER AND FOUND THE CHART TO EEAD HIGN SO HE PUT A WEIEHT ON THE LOW SIDE OF SP.GN INST.

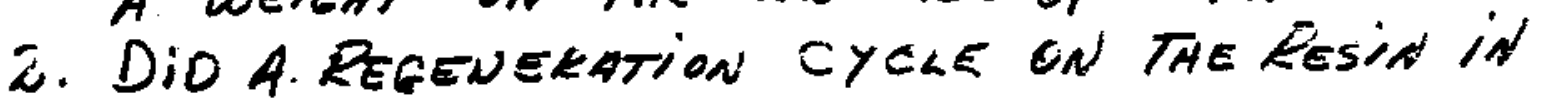
\#200 COB.

3. Rala Status:

A1- BatCH a cooline SampleE cileculatine it HAS BEAN DiLUTES.

A5, A9, AF, A11, A17, TY- T 
APPENDIX 3E

A SAMPLE X-10 RALA RUN REPORT 
TASK 1 REPORT

July 1999

${ }^{131}$ I Releases from X-10 Radioactive Lanthanum Processing -

Page 3E-2

Appendices

This page intentionally left blank. 


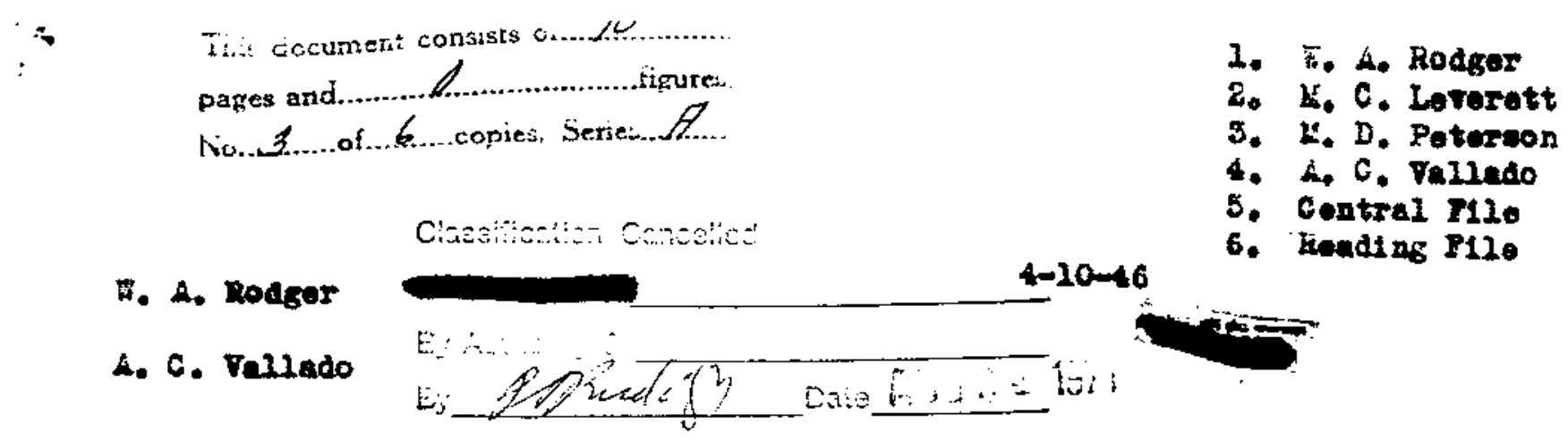

$\frac{\text { 706-D Produotion Kun } 19}{\text { (Bhiptent tip) }}$

\section{Loghani oal Obangas}

Iradiately upon the completion of ikun 48 , the decontantration of Oell $A$ was begun, and malntenance work atarted wing the tolerance lorels reached 00 to $100 \mathrm{mr} / \mathrm{hr}$. Bampling bllaters, Cell If and the flocr around the cubloled were decontaminated also. For a coplote coeount of

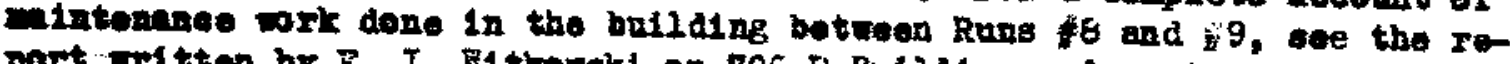

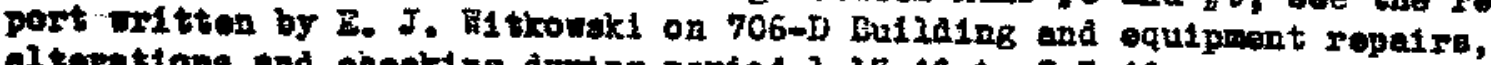
altarettons and ohooking awring pariod $1-13-46$ to $3-3-46$.

\section{Diacolver Operations}

ef rollowa:

The equ alngo charged to the diswolver for Run fo wero added

Dete Feghed end Chasged

$2-5$

g-6

$3-6$

$$
\text { SIugs Loaded CLASSIFICATION CANCELLED }
$$

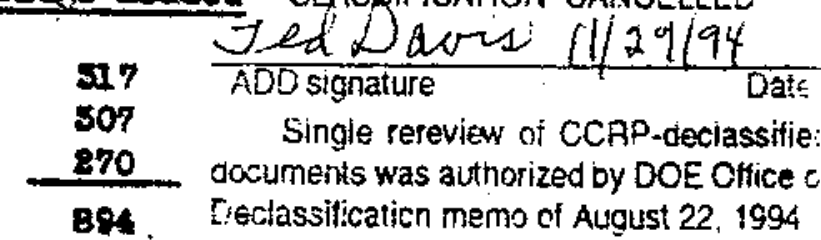

The alng londod on 5-3, 5-5 and 63 of the onas loaded on $3-6$

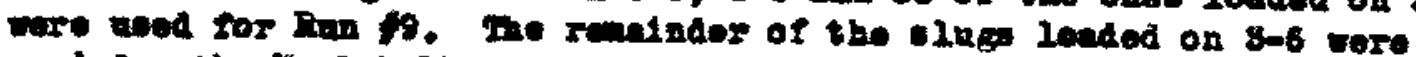

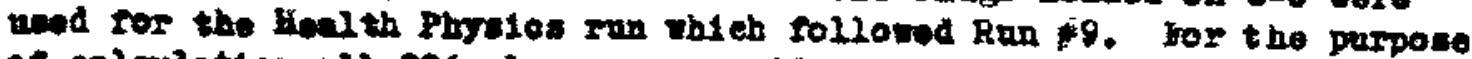

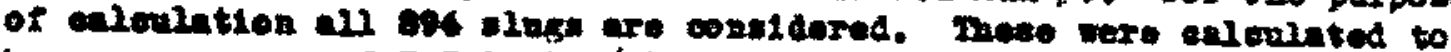

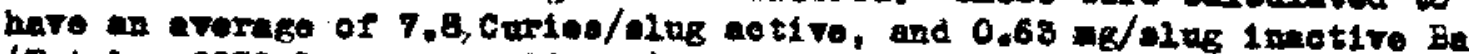
(Total: $8950 \mathrm{Cux}$ es and $585 \mathrm{mg}$ ) at tims of discharge.

The run was made in a Ingle ser18s, corsieting of eleven discolvinge ind extractions rollowed by motathoula, oati solutior, eleotro2Jole and volume redection in 36 . Tho Health Phyales men (reforred to a scoond sorien) whiah sollowed rue fo condsted of rour discolvings and extractions followed by metethesis, cake colution, electrolsata end rolume reduction in BI. This socond serios was primerily intended to sot

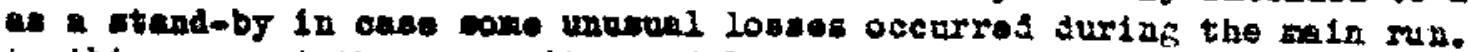
40 this was not the cano, it was doliverec to fiealth Phyaice.
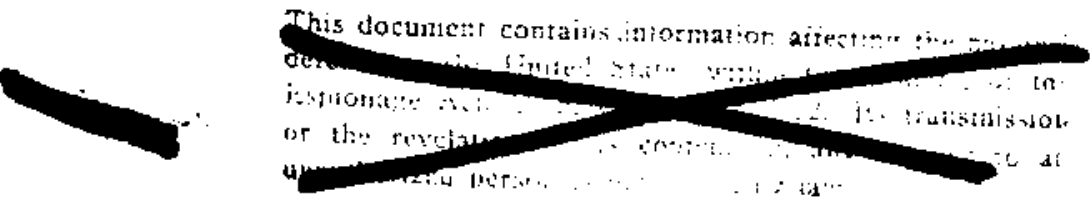
The mounte of reagents used for the coating romovals were $136 \# 60 \%$ सxNO $_{3}$, 688, of 20\% HaOF. The 20\% NaOH was uad 1nstead of the uswal $35 \%$, becauce the solntion ras stored in the outside tank $h=11$, where it could casliy rrease. For the metal solutions, the reagents were decreased about

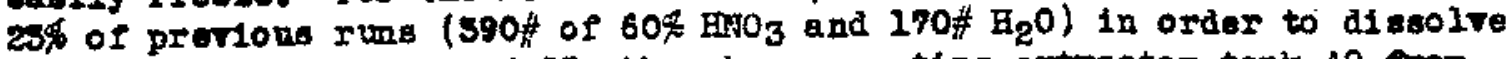
65 aluga/batch instead of 85, thereby preventing oxtraotor tank $A 9$ from overtlowing.

A total of ifteen (average 61.2 aluge distolred) motel solutlong and throe coating removal reactions wero carried out. Some diffioulty we enoounternd in malntalning an operating vaculm in the alsolver during the costing ramovel reactions. It Fos discorered that the off-gas efinter to A-1 was sealed off by a combination of the higher solution level in the disolver and the riolent reaction. Wha will be reotifled in the next Iun, by Increanlag the coscentration of baOR oolution. All motal colutione were earred ou withont difficutly.

\section{Extreotion}

the aponts of reagents for all fifteon extractiong vore decreased approximately $23 \%$ of provions flowaheet onentitiea for the amoller

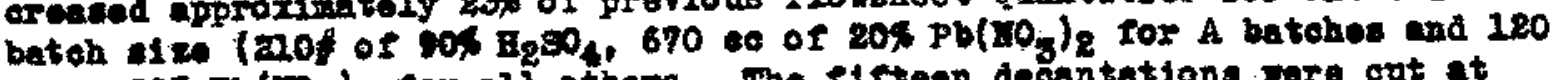

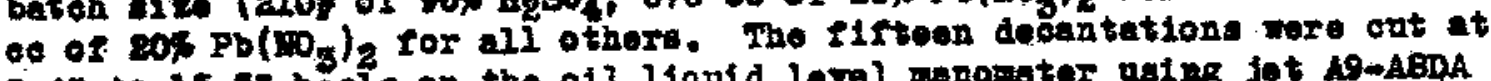

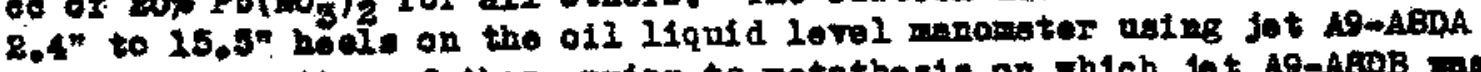
ith the erepption of those prior to motatheale on which jot A9-AROB was. noce. Inscrebel were left in extractor $A Q$, because the $A 9-A B D A$ suotion Ine wa sot elx 1nches off the bottom. Although extractor 19 ald not orernow throughout the $r$ w, and larger hoels were left bohind, the extraction losese wre mere than duping provions runs. The losen arexaged 35 ouries per axtruation or $12.8 \%$ of the product from sach individuel diveolving. A

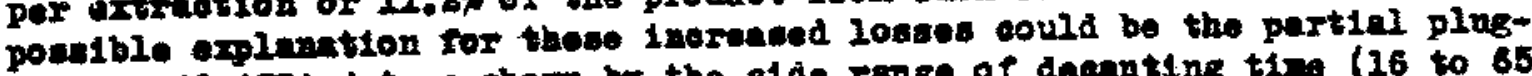
gling of A9-ABDA Jet an whom by the side range of deeanting time (16 to 65 minnter). Sos Iable II for complete extraction decantation reallo.

The extraction cakes from each of the two weried gaten the otandard one acld, fow wator wahes, all of 4 gallons rolume. Fach wash wes

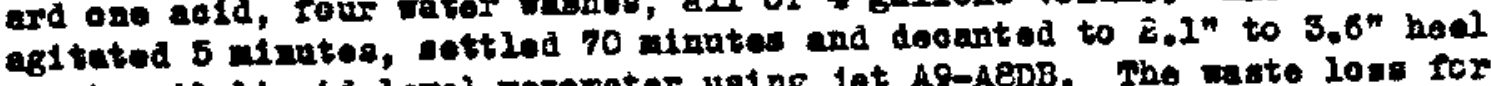
on tho oll 21 quid lerel manometer uaing Jat A9-ABDB. The wate loss fCr the conblend wahes ves $5.5 \mathrm{Cur} 1 \mathrm{es}$. (Seo Table I)

\section{Motathere}

Both notathos: wore wormal and wiform reagent ountities wore need. They were decanted to $A B$ asing jet $A 9-A B C B$ and cutting at 2.2" to 4.5" heels on the oll 11quid 20rel manometer. The comblned waste lose of the motathosis and metathes18 cake wash of the $f$ trut aeries was rether high (296 curtes). This 10 se was added to the furet extraction batch of the second nortes. Soe Iable III for motethesis conditions and loses.

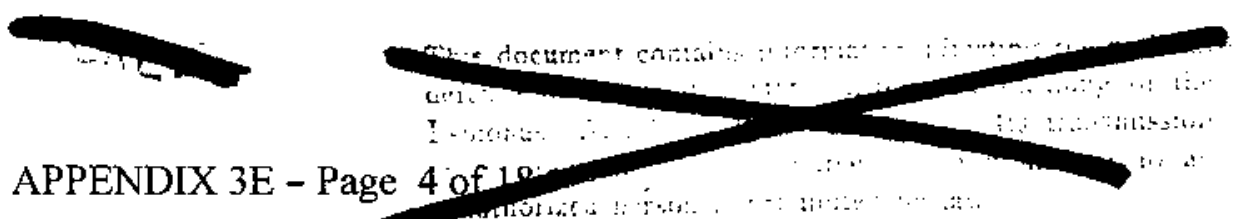


ii. in. Kodger

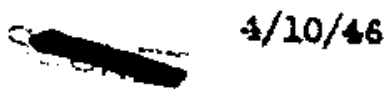

Some diffioulty we oxporionoed in tranarerring the oako solution of the p1ret antes to the crud pliter diug to the plugafng of the $A 9$ to Bas jet. Tho solution wea therefore tranaferred to BI2, then suaked brancremed to $\mathrm{Bl2}$ in thre ihota.

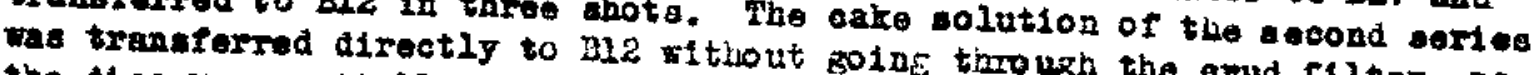
tho diso was partially plagged by thoto k0ine though the erud rilter, as

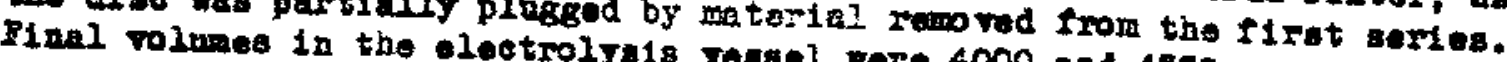
CeII B Oparation

The electrolysis time for the firat serios was increaged ono hour (3 boure at 15 anps, and 7 bours at 25 amps.) to finsure a 10 F $P$ value. The

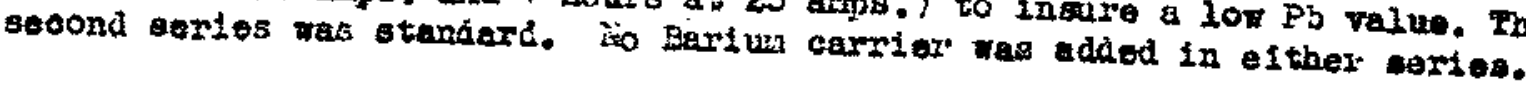

Operation of the glaseware was ressonably smooth. After olectroIyain the produet of the firat verises was tranarorres to preolpitator B6, ovagarated to Aryaess, diceolved in 2 litars of weter and reconeentratod to a 500 oo rolwa. The solution was tmansferred to B2lR by way of Bll

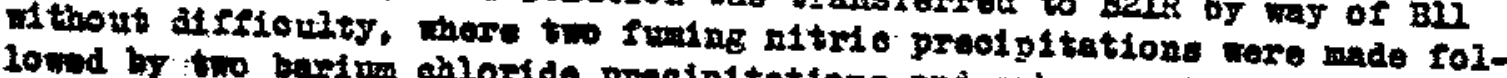

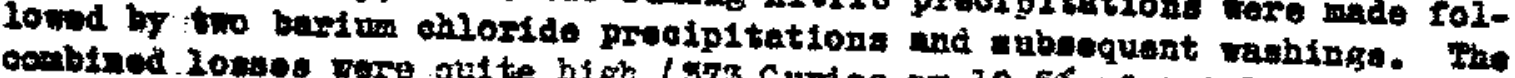

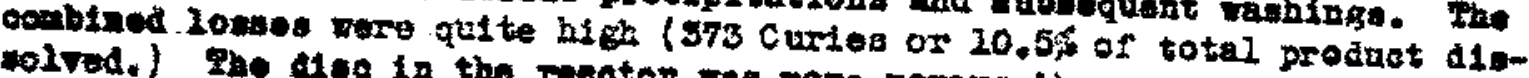
wolved.) The alse in the resotor was nore porous than ang provious ons. portevect. bet for eop reas ( broken off) 11 ante held mo in (probably due to faet thet the desp tip ras

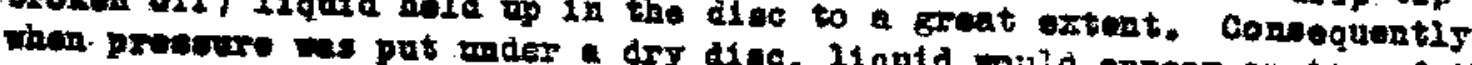

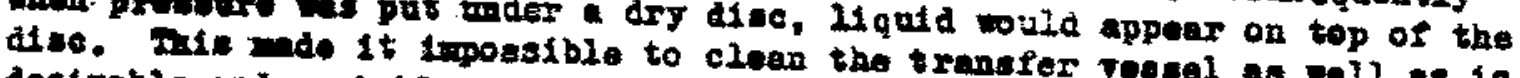
desizable and probubly sccousted for the the ransfer veasel as well as is

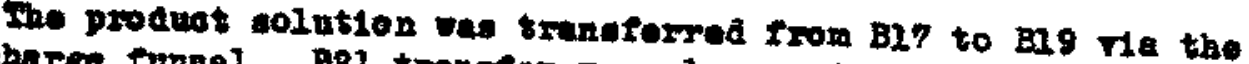

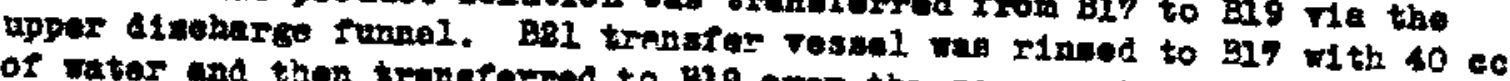

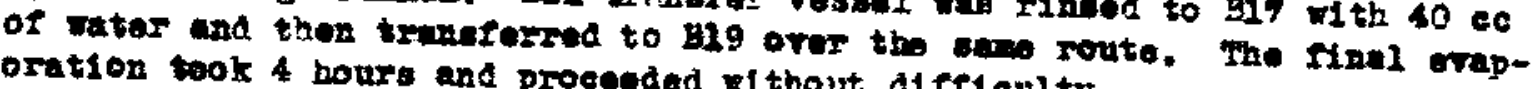
oration took 4 hours and proceoded pl thout diffoulty.

Fian analysis of run fo moduct was:

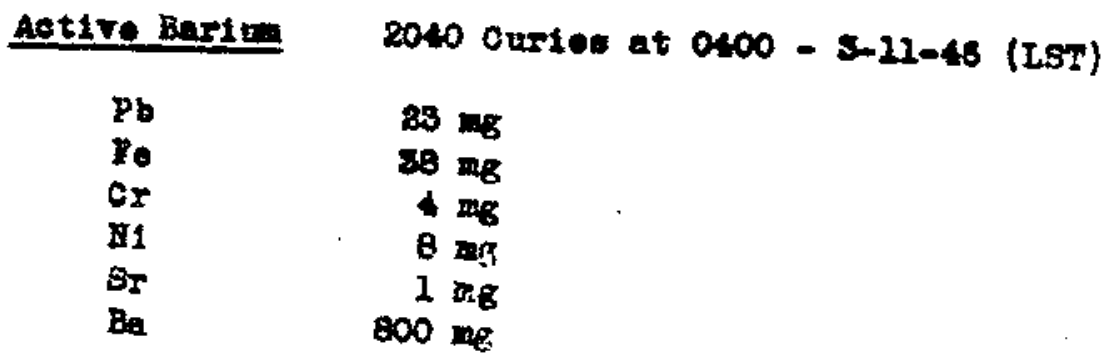

The product was womphat darker then ueval, but hed the ugual detribution in the cone.

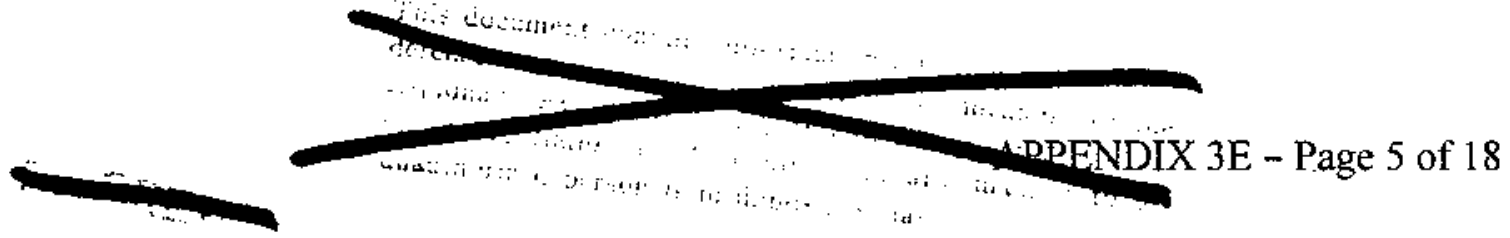


Serrod to Aftar electrolysia of the second series, the product was trabu-

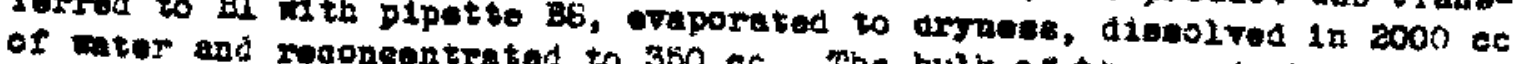
Ierred to BIs rie worble funal $\mathrm{Bz}$ and ovaporsted the product was trans-

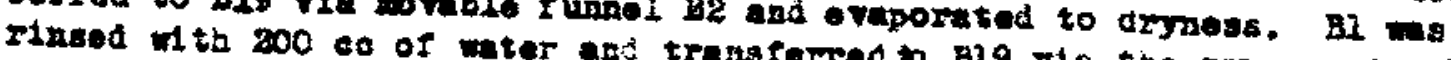
comblned Iinal ovaporation time was 10 \% ho uro.

The l-other wante solution was eraporated in a Pt-2inod cone

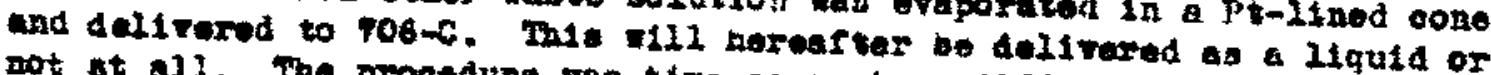

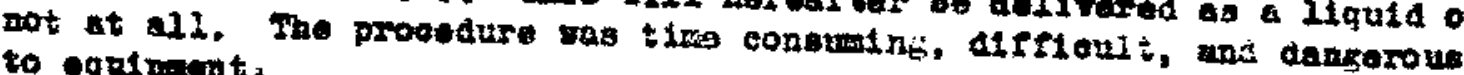
to eqatpenint

The ororall han fo was the most auccentul to date; a full 8000

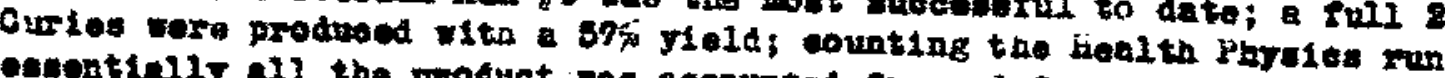

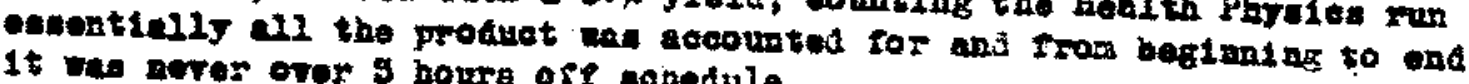
1t wa nerer over 9 houra ore cabedule.

\section{Aanlase:}

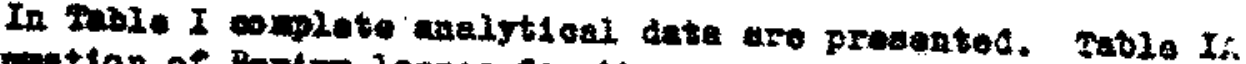

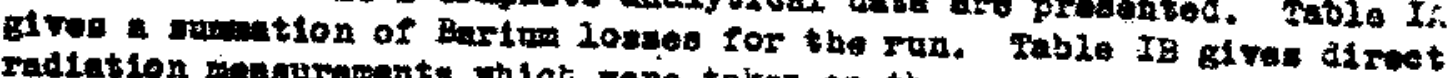
radiation moderverent: wich were taken on the open oone frow the top of

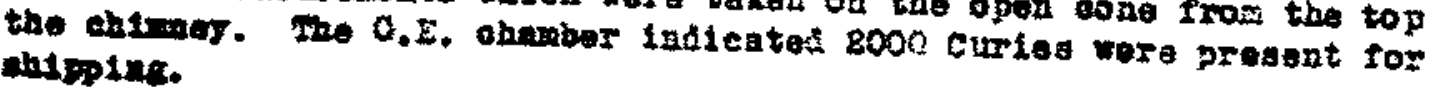

Conterstation and Redietion Exposure

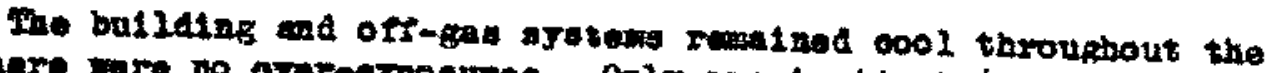
run and there were to oror-expoarres, Oaly one inctdent (deatribed in

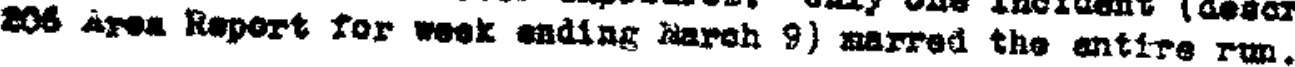

Doring the early part of the ren some inerease in alr counts ooewred on the third level when fotting. wolution from A8-AB; this dos-

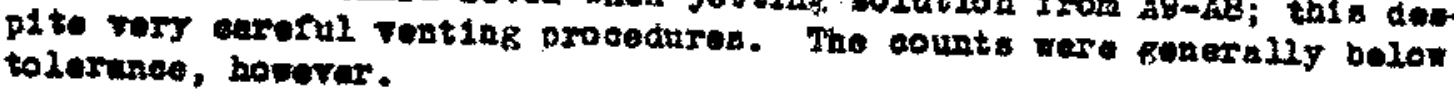

The firat two daye of the min, Iodine was colloaved from the trap between 43 and $A 4$. he the netal which was bol ge disolvod wes quite

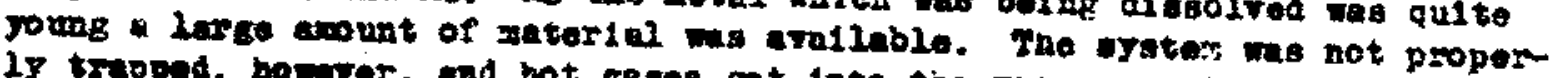

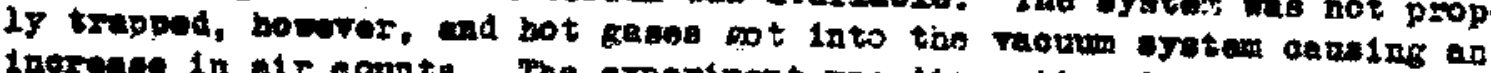

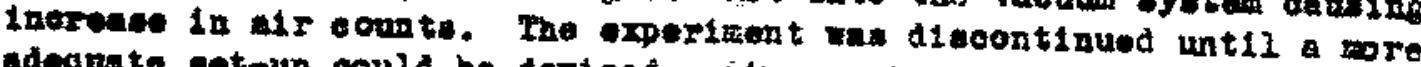
adeque te ent-up could bo derised. Als oolnts returnod to normal fit onoe.

No wrking area in the bul241 ate was ore: wieranoe exoept that between 83 and 86 bllatery. Thls area was juet over. The oell off-exo

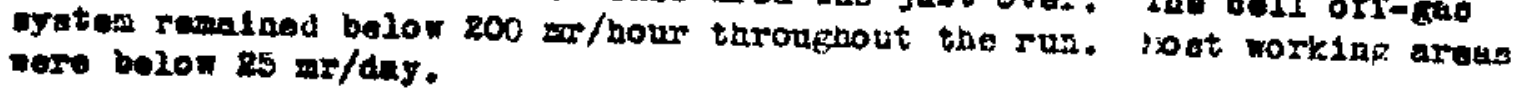

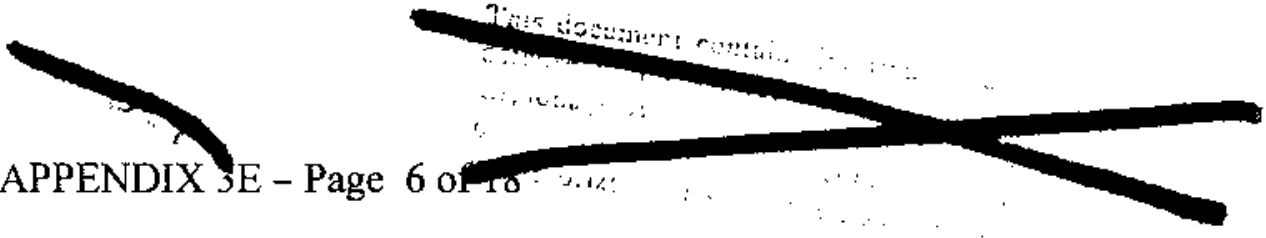


5. A. Nodfor

5

$4 / 20 / 46$

TABLE I

(AII OurioB are Calculated to $0400-3 / 22 / 46$ )

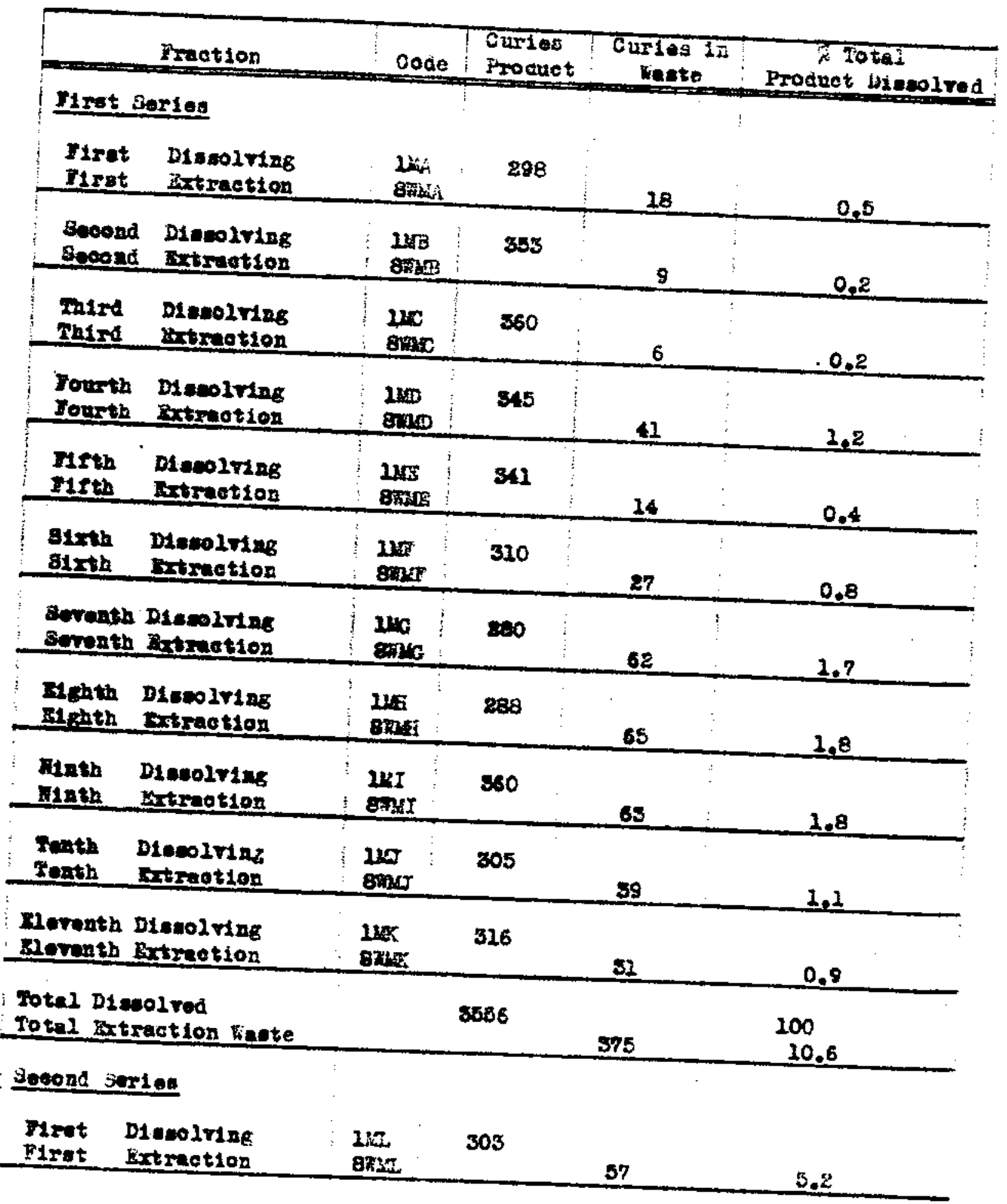


W. A. dodser

TABH ?

(Continued)

(A) Curteg are Caloulated to $0400-3 / 31 / 46)$

\begin{tabular}{|c|c|c|c|c|}
\hline Prection & Code & $\begin{array}{l}\text { Gorios } \\
\text { Product }\end{array}$ & $\begin{array}{c}\text { Gurios in } \\
\text { Hante }\end{array}$ & $\begin{array}{l}\text { Total } \\
\text { groduct Liseolred }\end{array}$ \\
\hline $\begin{array}{l}\text { Soeond Diseolving } \\
\text { Second nxtrection }\end{array}$ & $\begin{array}{l}\text { 210: } \\
\text { Bnins: }\end{array}$ & 276 & 37 & 3,4 \\
\hline 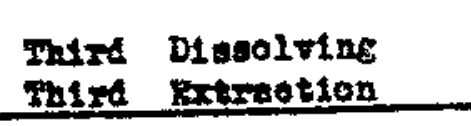 & $\begin{array}{l}12 \pi \\
\text { Bning: }\end{array}$ & 297 & 23 & 2.1 \\
\hline $\begin{array}{l}\text { Fowth Dincolving } \\
\text { Dourth Dtwation }\end{array}$ & $\begin{array}{l}1300 \\
\text { BIINO }\end{array}$ & 241 & 30 & 2.7 \\
\hline 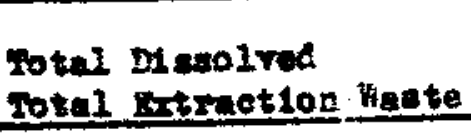 & & 1097 & 147 & $\begin{array}{l}100 \\
13.4 \\
\end{array}$ \\
\hline & & & & \\
\hline Btraetion Gaxe viah & $8 \%$ & & $\underline{6}$ & 0.1 \\
\hline 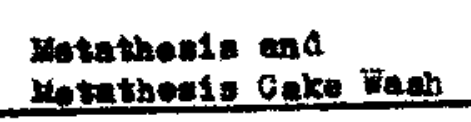 & 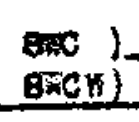 & & ins & 8.5 \\
\hline Iotel coll \& Loo & & & she & 19.0 \\
\hline I00002 $701=$ Lo: & SAPb & & 8 & 0.9 \\
\hline Ixoduct in $\mathrm{B} 6$ & $6 ?$ & 2850 & 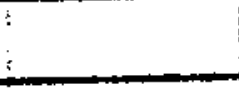 & \\
\hline B-6 B1en & & 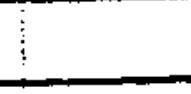 & 52 & 2.4 \\
\hline 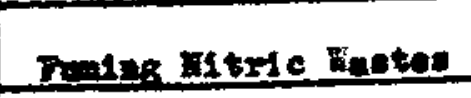 & Sint & & 21 & 0.6 \\
\hline El-otber pestes & & & 252 & 2.9 \\
\hline notel cell $B$ Lot: & & $\vdots$ & 457 & 12.E \\
\hline Mind Produet & 179 & 2040 & $i$ & \\
\hline Beond ier 1 es & : & & & \\
\hline numetron care neah & . & & 0.5 & 0.04 \\
\hline 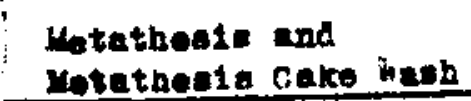 & $\begin{array}{l}\operatorname{Bnc} \\
\mathrm{Bnc}\end{array}$ & $1:-$ & $-\infty 91$ & 17.4 \\
\hline
\end{tabular}


5. A. Rodger

7

$4 / 10 / 46$

\section{TABLI I \\ (continued)}

(A21 Curies are calculated to $0400-3 / 11 / 46$ )

\begin{tabular}{|c|c|c|c|c|}
\hline Praction & Code & $\begin{array}{l}\text { Curies } \\
\text { Erodpet }\end{array}$ & $\begin{array}{l}\text { Curies in } \\
\text { Mepte }\end{array}$ & $\begin{array}{l}\text { Potal } \\
\text { Product Blssolved }\end{array}$ \\
\hline IoteI CelI A Ios & & & 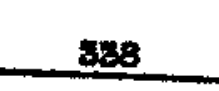 & 20.8 \\
\hline Heotrol zate Loes & & & 29 & 8.6 \\
\hline Produgt in B1 & $2 P$ & 1270 & & \\
\hline
\end{tabular}


7. A. Dodingr

B

$4 / 10 / 46$

TrAEL $Y$ is

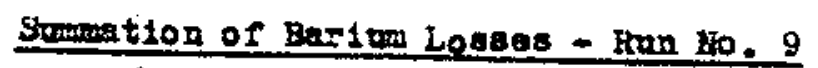

(Based on LST 0400 - $5 / 21 / 46$ )

\begin{tabular}{|c|c|c|}
\hline Dection & Caries & E \\
\hline 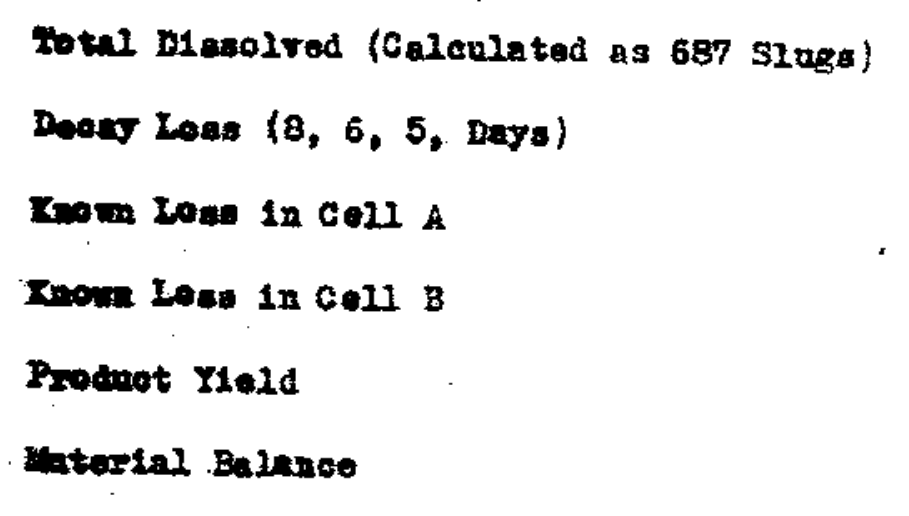 & $\begin{array}{r}5455 \\
1725 \\
676 \\
457 \\
2010\end{array}$ & $\begin{array}{r}100 \\
31.7 \\
12.4 \\
8.4 \\
37.4 \\
09.9\end{array}$ \\
\hline 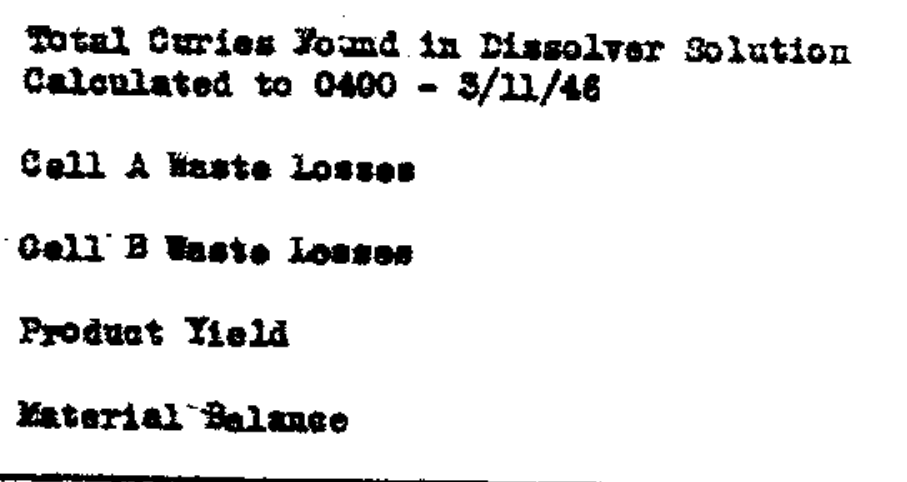 & $\begin{array}{r}3556 \\
676 \\
457 \\
2040 \\
5173\end{array}$ & $\begin{array}{l}100 \\
18.1 \\
18.8 \\
57.5 \\
89.4\end{array}$ \\
\hline
\end{tabular}


F. A.' Dentrex

9

$4 / 10 / 46$

TABLE IA

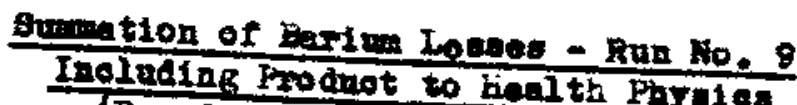

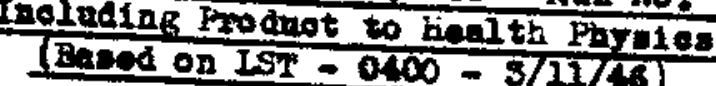

\begin{tabular}{|c|c|c|}
\hline Prection & corles & 4 \\
\hline 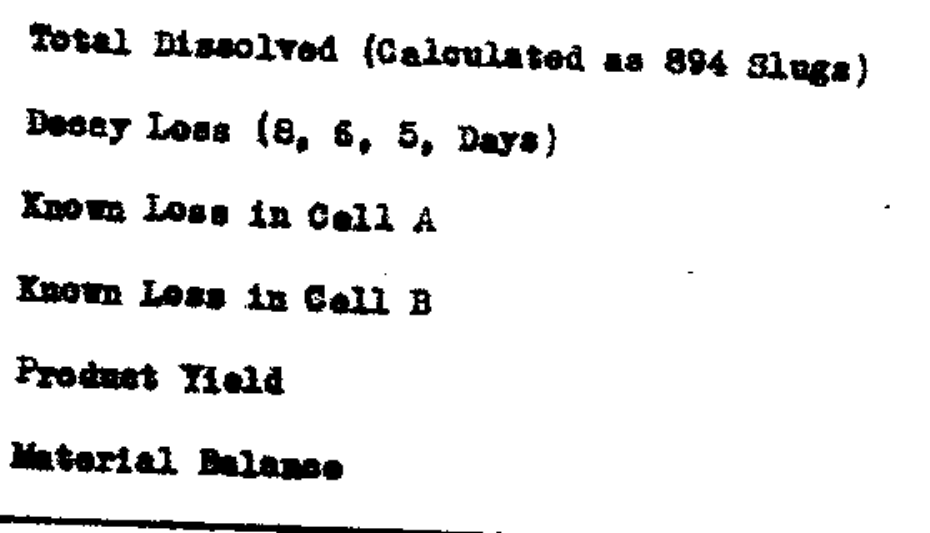 & $\begin{array}{r}6850 \\
2008 \\
1014 \\
486 \\
8518\end{array}$ & $\begin{array}{l}100 \\
20.0 \\
14.6 \\
7.0 \\
47.7 \\
99.3\end{array}$ \\
\hline 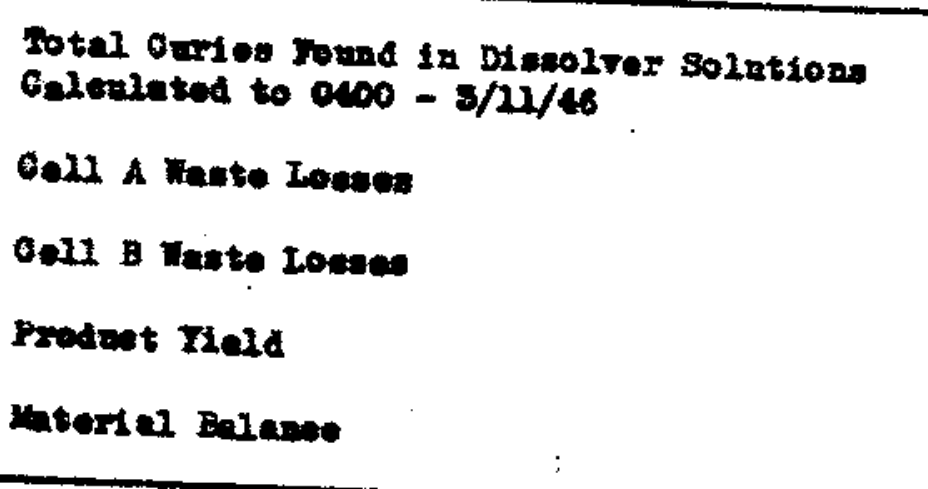 & $\begin{array}{l}1655 \\
2024 \\
486 \\
3815 \\
4815\end{array}$ & $\begin{array}{l}100 \\
21.8 \\
10.5 \\
71.2 \\
105.5\end{array}$ \\
\hline
\end{tabular}


F. A. Nadger

f

TABLF: IB

Direet Rediation Weasuremente For Run Ho. 9

\begin{tabular}{|c|c|c|}
\hline Eusles inalred & \multicolumn{2}{|c|}{8000} \\
\hline Inetroment & Hine 1n frours & 2/Eour \\
\hline c.3. Onoriber & $\begin{array}{l}16 \\
18 \\
86 \\
28\end{array}$ & $\begin{array}{l}16 \\
17 \\
21 \frac{1}{4} \\
28\end{array}$ \\
\hline 200 R Herter (1) & $\begin{array}{l}16 \\
86\end{array}$ & $\begin{array}{l}19 \\
82\end{array}$ \\
\hline 200 R. Neter $(2)$ & 86 & 20 \\
\hline
\end{tabular}


TABIEII

Extraction Decantations

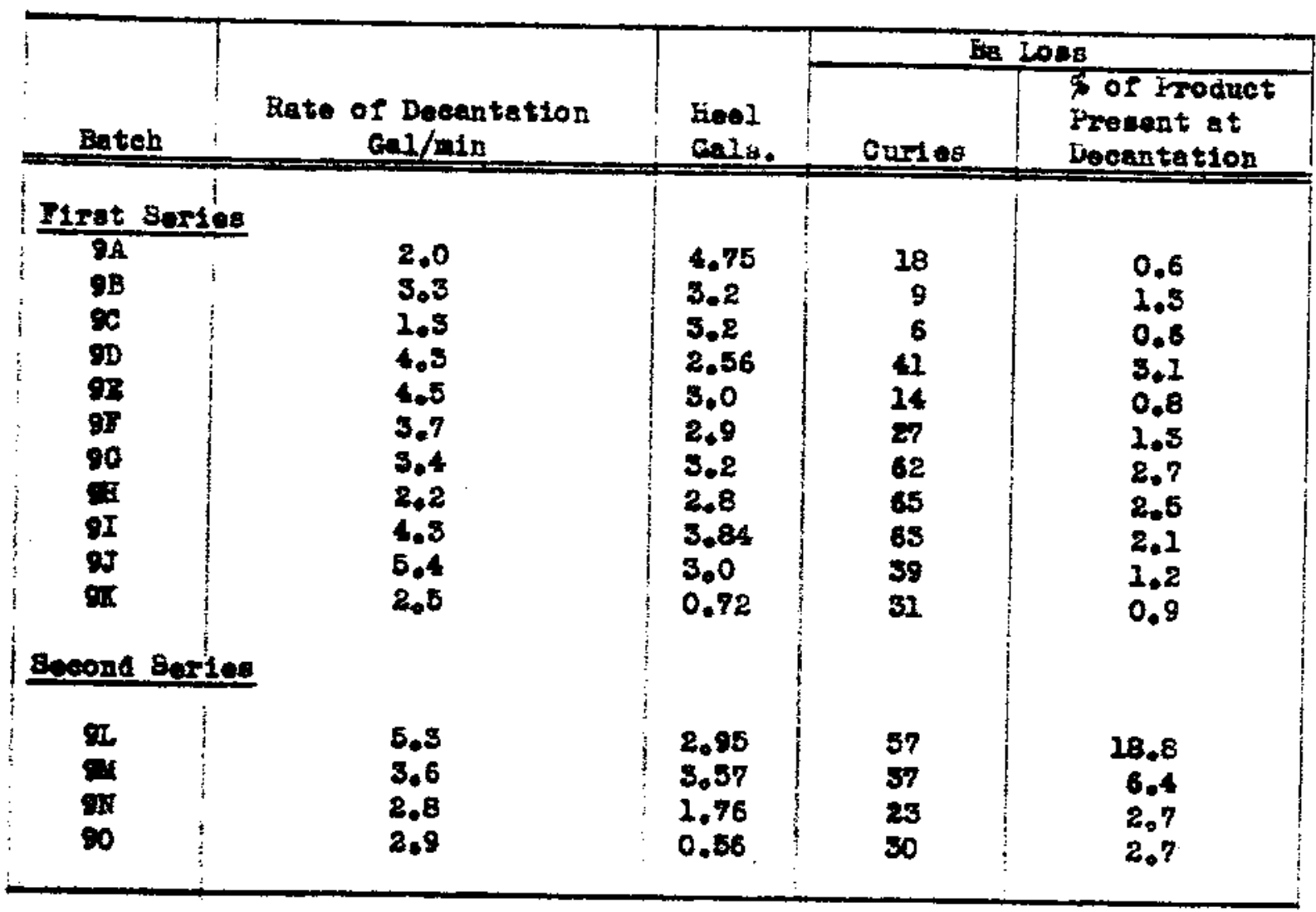


T. A. Dodger

12
$4 / 10 / 46$

TABLE III

Hetathea1s

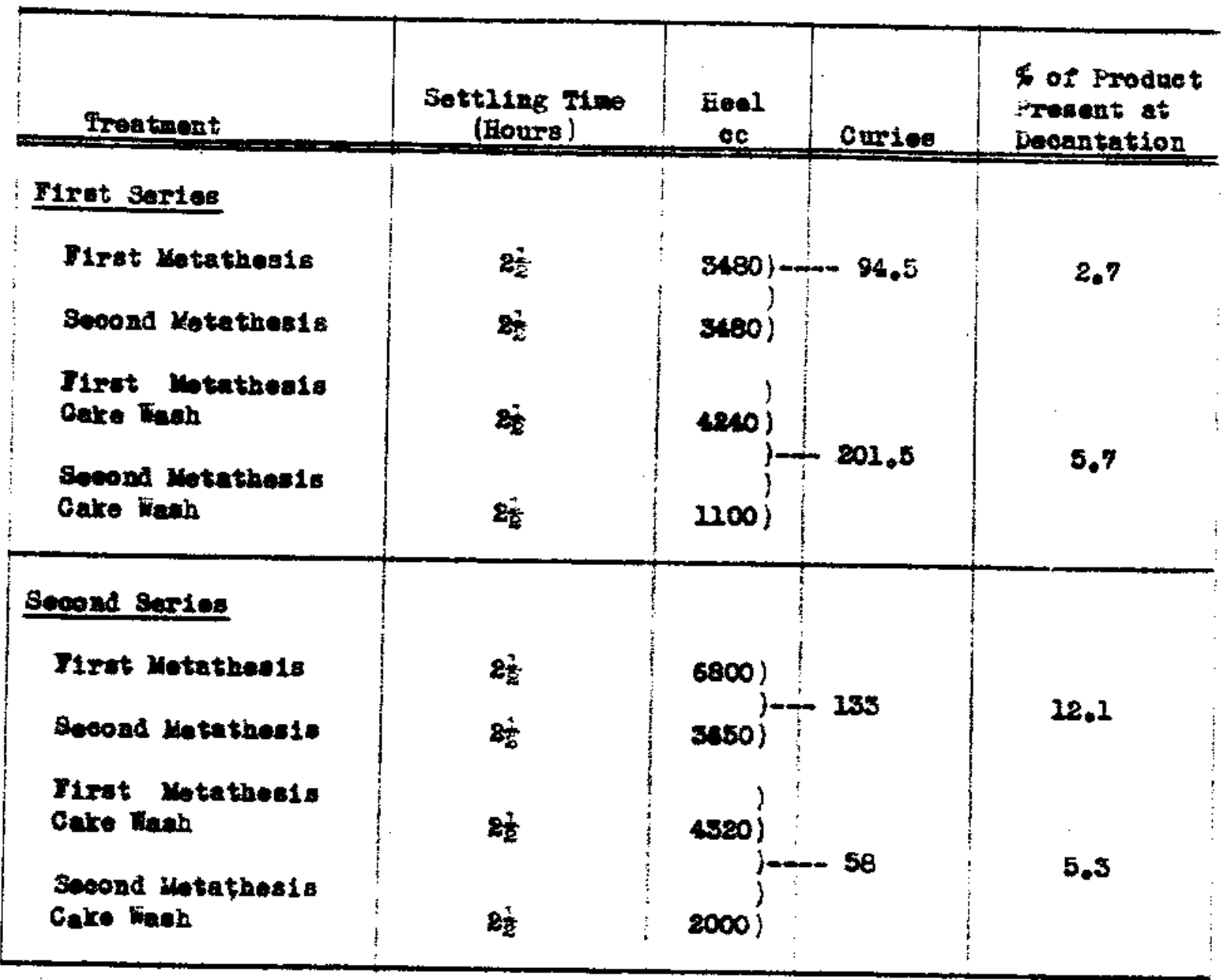


TABLE IV

Tim oroles

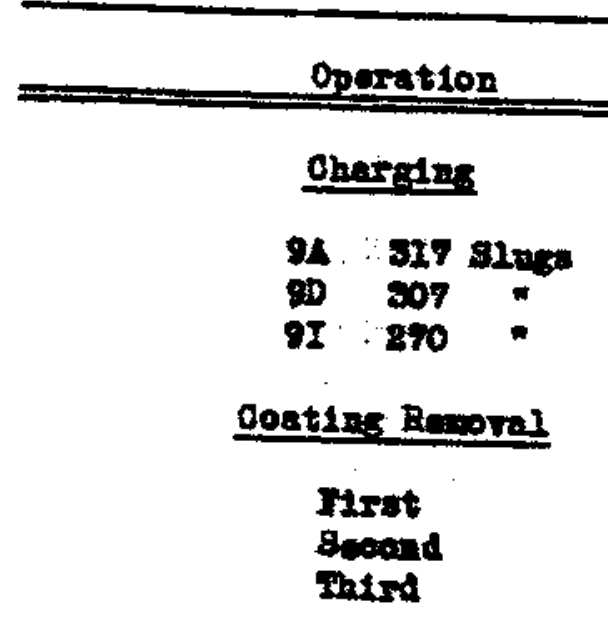

Eetel solntiog and frtisetion inet senes

$9 A$
$9 B$
90
90
$9 B$
92
$9 B$
97
91
97
$9 \pi$

(Socond Seried)

\section{Howrs Rogutrod}

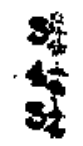


N. A, Dodger

TARLE TV

(continued)

$4 / 10 / 46$

7

\section{Ine Croles}

\section{Operation}

Eoure Required

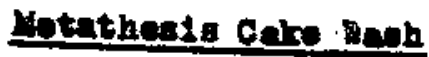

Iret serseo

Becond Sertea

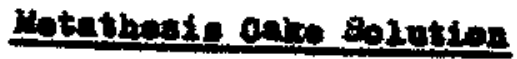

Mret Bureo

secosed serter

meatrotse

Itrat Barles

sweond sontes

Porinterial

Itzet Barto

Beoend Eartos

Dolve Reduat1on in 8

Firot serte

Fon manten in $\mathrm{gt}$

Beovend sares

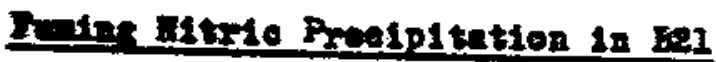

Itrat artes

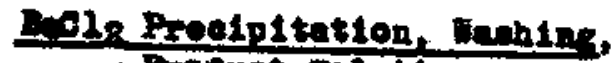

sroenet bivizen

Firet sonte:

Expling

Firet Barteo

gacond sarioc

Minal Produet Heportion

Fint 8exies

Booond Sertes

APPENDIX 3E - Page 16 of 18

Llines wore plugged.

* By-pasoed arad Plitor.

*** To abote.
20

9

4

No Data

8

$+$

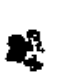

13

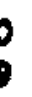

ate $\frac{8}{8}$
8

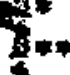

4 
T. 4. Podger

15

$4 / 10 / 46$

TAELE IY

(Continuod)

Time Croles

Operation Boura Reguired

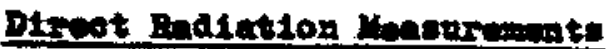

Misat Sortes

10

second Searies

10

zouasns

Itrut Earies

Beoond 8urtes 
APPENDIX 3F

A SAMPLE RALA RUN ANALYTICAL REPORT 
TASK 1 REPORT

July 1999

${ }^{131}$ I Releases from X-10 Radioactive Lanthanum Processing -

Page 3F-2

Appendices

This page intentionally left blank. 
inglotical Jata

$3 a$ Run 20

Shipment $; 32$

I. 3.m. 1500, 1-22-48

To: L.E. Frrlet

From: 3.A. Reynolds

This document consists of

gages and____ 0 ___figures.

Yo._Lor 4_copies, Serries A A

\begin{tabular}{|c|c|c|c|c|}
\hline Eatch & Guries & Slugs & $x-8$ & $\therefore-6$ \\
\hline $\bar{a}$ & 282 & $\overline{68.8}$ & 58 & 2 \\
\hline 3 & 315 & 84.1 & 25 & $-\infty$ \\
\hline$c$ & 270 & 76.3 & 55 & 7 \\
\hline$D$ & 261 & 69.6 & 43 & $-\ldots$ \\
\hline $\mathbf{I}$ & 301 & 72.0 & 20 & $\cdots$ \\
\hline$F$ & 264 & 64.7 & 242 & 11 \\
\hline$G$ & 248 & 75.0 & 207 & 12 \\
\hline$\ddot{z}$ & 218 & 69.1 & $4 \overline{50}$ & 10 \\
\hline I & 274 & 77.1 & 15 & $-\cdots$ \\
\hline$J$ & 277 & 76.9 & 218 & 12 \\
\hline$K$ & 307 & 75.4 & 79 & 10 \\
\hline I & 257 & 68.0 & 7 & 5 \\
\hline in & $15 \overline{5}$ & 43.1 & 411 & 13 \\
\hline $\mathrm{N}$-Feels & $\frac{4}{347}$ & $\frac{---}{30}$ & $\frac{--}{c+p}$ & --- \\
\hline
\end{tabular}

BENTRLL FILE YUMBPES

$48-1-405$

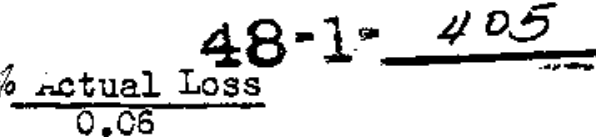

0.73

0.20

1.26

0.59

0.32

0.35

0.34

0.44

0.35

0.34

0.15

0.38

$\frac{--\pi}{5.42 \%}$

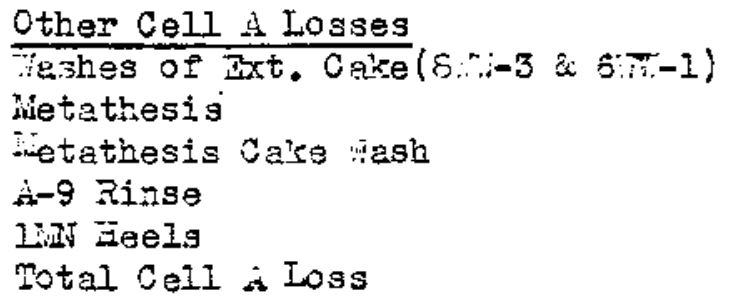

$\frac{\text { Curies }}{91}$

69

33

$\frac{4}{388}-$

Cell B Losses

ilectrolysis

D-6 Finse

Fumps itric tiosto

trCl-Jther Jaste

Total Loss (Cell i)

Product

Product in E-6

Shiment it 32

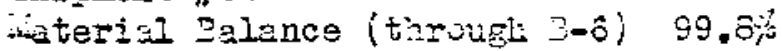

Radiation 3oacins (3krshine")

Injicstos epproximately 2750 curias

2016
6

Classiffcation Cancelled

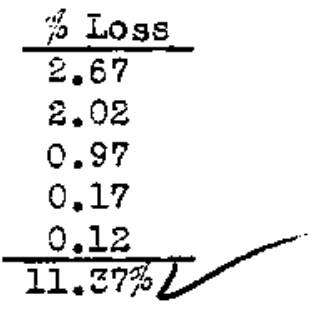

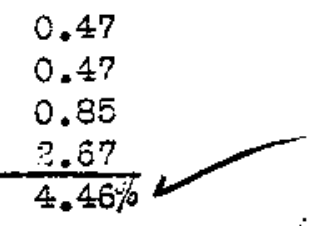

$88.4 ;$ Product

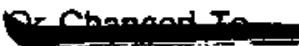

By Authority Of

By $P A$ Date AUG 26197
CLASSIFHCATION CANCELIED

OS Marosan $1-31-95$

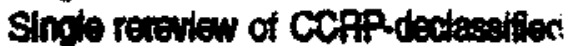

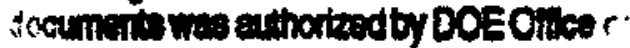

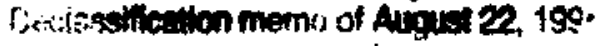

$$
\text { or cos } 24 / 28195
$$

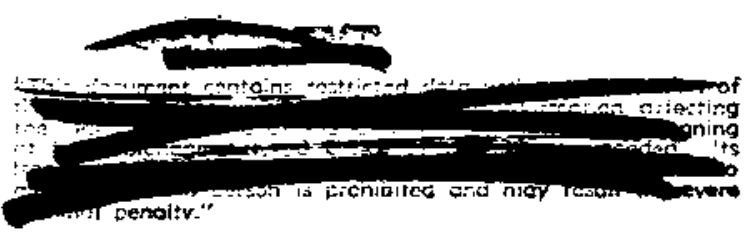

Page $3 F-3$ 
TASK 1 REPORT

July 1999

${ }^{131}$ I Releases from X-10 Radioactive Lanthanum Processing -

Page 3F-4

Appendices

This page intentionally left blank. 


\section{APPENDIX 3G}

\section{RALA DISSOLVING BATCHES CONDUCTED}

IN X-10'S BUILDING 706-D 
TASK 1 REPORT

July 1999

${ }^{131}$ I Releases from X-10 Radioactive Lanthanum Processing -

Page 3G-2

Appendices

This page intentionally left blank. 
Appendix 3G: RaLa Dissolving Batches Conducted in X-10's Building 706-D

\begin{tabular}{|c|c|c|c|c|c|c|c|c|}
\hline \multirow{2}{*}{$\begin{array}{l}\text { Batch } \\
\text { Name }\end{array}$} & \multicolumn{3}{|c|}{ Start of Dissolving } & \multicolumn{3}{|c|}{ No. of Slugs Dissolved } & \multirow{2}{*}{$\begin{array}{c}\text { Duration } \\
\text { (h) }\end{array}$} & \multirow{2}{*}{$\begin{array}{c}\text { Direct Release } \\
\text { Class } \\
\end{array}$} \\
\hline & Date & Year & Shift or Time & $\mathrm{X}-10$ & 4" Hanf. & 8" Hanf. & & \\
\hline$\overline{1 \mathrm{~A}}$ & 28-May & 1945 & "Night Shift, Beginning & $\overline{58}$ & 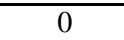 & 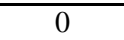 & $\overline{6}$ & $\overline{1}$ \\
\hline 1B & 28-May & 1945 & Day, Beginning & 58 & 0 & 0 & 6 & 1 \\
\hline $1 \mathrm{C}$ & 29-May & 1945 & Night, Beginning & 49 & 0 & 0 & 7 & 1 \\
\hline 1D & 29-May & 1945 & Day, Middle & 43 & 0 & 0 & 12 & 1 \\
\hline $2 \mathrm{AA}$ & $\overline{5-J u n}$ & 1945 & Night, Beginning & 52 & $\overline{0}$ & 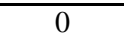 & 7 & 2 \\
\hline $2 \mathrm{AB}$ & 5-Jun & 1945 & Swing, Beginning & 52 & 0 & 0 & 7 & 2 \\
\hline $2 \mathrm{AC}$ & 6-Jun & 1945 & Night, Middle & 52 & 0 & 0 & 8 & 1 \\
\hline $2 \mathrm{AD}$ & 6-Jun & 1945 & Swing, Middle & 52 & 0 & 0 & 5 & 1 \\
\hline 2BA & 7-Jun & 1945 & Night, Middle & 52 & 0 & 0 & 6 & 1 \\
\hline $2 \mathrm{BB}$ & 8-Jun & 1945 & Swing, Beginning & 52 & 0 & 0 & 7 & 2 \\
\hline $2 \mathrm{BC}$ & 9-Jun & 1945 & Night, Beginning & 52 & 0 & 0 & 9 & 1 \\
\hline 2BD & 9-Jun & 1945 & Swing, Middle & 52 & 0 & 0 & 5 & 1 \\
\hline $2 \mathrm{CA}$ & 10-Jun & 1945 & Night, Middle & 52 & 0 & 0 & 6 & 1 \\
\hline $2 \mathrm{CB}$ & 11-Jun & 1945 & Day, Beginning & 52 & 0 & 0 & 8 & 1 \\
\hline $2 \mathrm{CC}$ & 11-Jun & 1945 & Swing, Beginning & 52 & 0 & 0 & 14 & 1 \\
\hline $2 \mathrm{CD}$ & 12-Jun & 1945 & Day & 52 & 0 & 0 & 4-14 U* & 1 \\
\hline $3 \mathrm{AA}$ & 12-Jul & 1945 & Swing, Beginning & 733.3 & 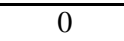 & 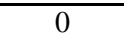 & $\overline{6} 6$ & 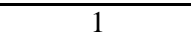 \\
\hline $3 \mathrm{AB}$ & 13-Jul & 1945 & Night, Middle & 73.3 & 0 & 0 & 6 & 1 \\
\hline $3 \mathrm{AC}$ & 13-Jul & 1945 & Swing, Beginning & 73.3 & 0 & 0 & 7 & 1 \\
\hline $3 \mathrm{AD}$ & 14-Jul & 1945 & Night, Beginning & 73.3 & 0 & 0 & 10 & 1 \\
\hline 3BA & 15-Jul & 1945 & Night & 73.3 & 0 & 0 & 9 & 1 \\
\hline $3 \mathrm{BB}$ & 15-Jul & 1945 & Swing & 73.3 & 0 & 0 & 5 & 1 \\
\hline $3 \mathrm{BC}$ & 16-Jul & 1945 & Day & 73.3 & 0 & 0 & 6 & 2 \\
\hline $3 B D$ & 16-Jul & 1945 & Swing & 73.3 & 0 & 0 & 9 & 2 \\
\hline $3 \mathrm{CA}$ & 17-Jul & 1945 & Swing & 73.3 & 0 & 0 & 6 & 2 \\
\hline $3 \mathrm{CB}$ & 18-Jul & 1945 & Day & 73.3 & 0 & 0 & 6 & 1 \\
\hline $3 \mathrm{CC}$ & 18-Jul & 1945 & Swing & 73.3 & 0 & 0 & 8 & 1 \\
\hline $3 \mathrm{CD}$ & 19-Jul & 1945 & Day & 25 & 0 & 0 & 4-14 U & 1 \\
\hline $4 \mathrm{AA}$ & 6-Aug & 1945 & Swing & $\overline{52}$ & $\overline{00}$ & 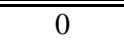 & $\overline{5}$ & 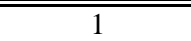 \\
\hline $4 \mathrm{AB}$ & 7-Aug & 1945 & Night & 52 & 0 & 0 & 6 & 1 \\
\hline $4 \mathrm{AC}$ & 7-Aug & 1945 & Swing & 52 & 0 & 0 & 8 & 1 \\
\hline $4 \mathrm{AD}$ & 8-Aug & 1945 & Night & 52 & 0 & 0 & 12 & 1 \\
\hline $4 \mathrm{AE}$ & 8-Aug & 1945 & Day & 52 & 0 & 0 & 4 & 1 \\
\hline $4 \mathrm{AF}$ & 9-Aug & 1945 & Night & 52 & 0 & 0 & 4 & 1 \\
\hline $4 \mathrm{BA}$ & 9-Aug & 1945 & Swing & 52 & 0 & 0 & 6 & 1 \\
\hline $4 \mathrm{BA}^{\prime}$ & 10-Aug & 1945 & Swing & 52 & 0 & 0 & 4-7 U & 1 \\
\hline $4 \mathrm{BB}$ & 11-Aug & 1945 & Swing & 52 & 0 & 0 & 3 & 1 \\
\hline $4 \mathrm{BC}$ & 12-Aug & 1945 & Night & 52 & 0 & 0 & 6 & 1 \\
\hline $4 \mathrm{BD}$ & 12-Aug & 1945 & Day & 52 & 0 & 0 & 4 & 1 \\
\hline $4 \mathrm{BE}$ & 13-Aug & 1945 & Night & 52 & 0 & 0 & 4 & 1 \\
\hline $4 \mathrm{BF}$ & 13-Aug & 1945 & Day & 52 & 0 & 0 & 5 & 1 \\
\hline $4 \mathrm{CA}$ & 15-Aug & 1945 & Night & 52 & 0 & 0 & 4 & 1 \\
\hline $4 \mathrm{CB}$ & 15-Aug & 1945 & Day & 52 & 0 & 0 & 4 & 1 \\
\hline $4 \mathrm{CC}$ & 15-Aug & 1945 & Swing & 52 & 0 & 0 & 4 & 1 \\
\hline $4 \mathrm{CD}$ & 16-Aug & 1945 & Day & 52 & 0 & 0 & 4 & 1 \\
\hline $4 \mathrm{CE}$ & 16-Aug & 1945 & Swing & 52 & 0 & 0 & 5 & 1 \\
\hline $4 \mathrm{CF}$ & 17-Aug & 1945 & Day & 52 & 0 & 0 & 4-7 U & 1 \\
\hline $4 \mathrm{CG}$ & 20-Aug & 1945 & Night & 52 & 0 & 0 & 11 & 1 \\
\hline $4 \mathrm{CH}$ & 20-Aug & 1945 & Day & 52 & 0 & 0 & 12 & 1 \\
\hline $4 \mathrm{CI}$ & 23-Aug & 1945 & Day & 52 & 0 & 0 & $9-22 \mathrm{U}$ & 1 \\
\hline
\end{tabular}

* Denotes a range between 4 and 14 hours, with each contained whole-number duration equally likely. 
Appendix 3G: RaLa Dissolving Batches Conducted in X-10's Building 706-D

\begin{tabular}{|c|c|c|c|c|c|c|c|c|}
\hline \multirow{2}{*}{$\begin{array}{l}\text { Batch } \\
\text { Name }\end{array}$} & \multicolumn{3}{|c|}{ Start of Dissolving } & \multicolumn{3}{|c|}{ No. of Slugs Dissolved } & \multirow{2}{*}{$\begin{array}{c}\text { Duration } \\
\text { (h) }\end{array}$} & \multirow{2}{*}{$\begin{array}{c}\text { Direct Release } \\
\text { Class } \\
\end{array}$} \\
\hline & Date & Year & Shift or Time & $\mathrm{X}-10$ & 4" Hanf. & 8" Hanf. & & \\
\hline $5 \mathrm{BA}$ & 4-Sep & 1945 & Swing & 61.5 & 0 & 0 & 6 & 1 \\
\hline $5 \mathrm{BB}$ & 5-Sep & 1945 & Night & 61.5 & 0 & 0 & 6 & 1 \\
\hline $5 \mathrm{BC}$ & 5-Sep & 1945 & Day & 61.5 & 0 & 0 & $4-14 \mathrm{U}$ & 1 \\
\hline $5 \mathrm{BD}$ & 6-Sep & 1945 & Day, Beginning & 61.5 & 0 & 0 & 5 & 1 \\
\hline $5 \mathrm{BE}$ & 6-Sep & 1945 & Day, End & 61.5 & 0 & 0 & 5 & 1 \\
\hline $5 \mathrm{BF}$ & 7-Sep & 1945 & Night, Beginning & 61.5 & 0 & 0 & 7 & 1 \\
\hline $5 \mathrm{BG}$ & 7-Sep & 1945 & Day & 61.5 & 0 & 0 & 6 & 1 \\
\hline $5 \mathrm{BH}$ & 8-Sep & 1945 & Night & 61.5 & 0 & 0 & 5 & 1 \\
\hline $5 \mathrm{BI}$ & 8-Sep & 1945 & Day & 61.5 & 0 & 0 & 4 & 1 \\
\hline $5 \mathrm{CA}$ & 9-Sep & 1945 & Night & 61.5 & 0 & 0 & 4 & 1 \\
\hline $5 \mathrm{CB}$ & 10-Sep & 1945 & Day & 61.5 & 0 & 0 & 3 & 1 \\
\hline $5 \mathrm{CC}$ & 10-Sep & 1945 & Swing, Beginning & 61.5 & 0 & 0 & 4 & 1 \\
\hline $5 \mathrm{CD}$ & 11-Sep & 1945 & Night & 61.5 & 0 & 0 & 5 & 1 \\
\hline $5 \mathrm{CE}$ & 11-Sep & 1945 & Swing, Beginning & 61.5 & 0 & 0 & 5 & 1 \\
\hline $5 \mathrm{CF}$ & 12-Sep & 1945 & Day & 61.5 & 0 & 0 & 7 & 1 \\
\hline $5 \mathrm{CG}$ & 12-Sep & 1945 & Swing & 61.5 & 0 & 0 & 9 & 1 \\
\hline $5 \mathrm{CH}$ heels & 13-Sep & 1945 & Night & 61.5 & 0 & 0 & $25-38 \mathrm{U}$ & 1 \\
\hline 5CI heels & 14-Sep & 1945 & Day & 61.5 & 0 & 0 & $41-54 \mathrm{U}$ & 1 \\
\hline 6A & 24-Nov & 1945 & Day, Beginning & 85 & 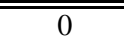 & 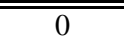 & $\overline{5}$ & 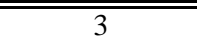 \\
\hline $6 \mathrm{~B}$ & 24-Nov & 1945 & Day, End & 85 & 0 & 0 & 4 & 1 \\
\hline $6 \mathrm{C}$ & 25-Nov & 1945 & Night & 85 & 0 & 0 & $4-14 \mathrm{U}$ & 2 \\
\hline $6 \mathrm{D}$ & 25-Nov & 1945 & Day & 85 & 0 & 0 & 4 & 1 \\
\hline $6 \mathrm{E}$ & 25-Nov & 1945 & 10:30 PM & 85 & 0 & 0 & 4 & 1 \\
\hline $6 \mathrm{~F}$ & 26-Nov & 1945 & Day & 85 & 0 & 0 & 5 & 1 \\
\hline $6 \mathrm{G}$ & 26-Nov & 1945 & Swing & 85 & 0 & 0 & 5 & 1 \\
\hline Heels & 28-Nov & 1945 & Swing & 9.3 & 0 & 0 & $25-38 \mathrm{U}$ & 3 \\
\hline $6 \mathrm{H}$ & 2-Dec & 1945 & Night & 60 & 0 & 0 & 4 & 2 \\
\hline $6 \mathrm{I}$ & 2-Dec & 1945 & Day & 60 & 0 & 0 & 4 & 2 \\
\hline $6 \mathrm{~J}$ & 2-Dec & 1945 & Swing & 60 & 0 & 0 & 5 & 2 \\
\hline $6 \mathrm{~K}$ heels & 3-Dec & 1945 & Day & 60 & 0 & 0 & 11 & 1 \\
\hline Heels & 3-Dec & 1945 & Swing & 9.3 & 0 & 0 & $17-30 \mathrm{U}$ & 1 \\
\hline $7 \mathrm{~A}$ & 14-Dec & 1945 & Swing & 66 & 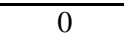 & 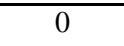 & 3 & 1 \\
\hline $7 \mathrm{~B}$ & 15-Dec & 1945 & Night & 66 & 0 & 0 & $4-14 \mathrm{U}$ & 3 \\
\hline $7 \mathrm{C}$ & 15-Dec & 1945 & Swing & 66 & 0 & 0 & 3 & 3 \\
\hline $7 \mathrm{D}$ & 16-Dec & 1945 & Night & 66 & 0 & 0 & 5 & 1 \\
\hline $7 \mathrm{E}$ & 16-Dec & 1945 & Day & 66 & 0 & 0 & 4 & 1 \\
\hline $7 F$ & 17-Dec & 1945 & Night, Beginning & 66 & 0 & 0 & 6 & 1 \\
\hline $7 \mathrm{G}$ & 17-Dec & 1945 & Day & 66 & 0 & 0 & 6 & 1 \\
\hline $7 \mathrm{H}$ & 18-Dec & 1945 & Swing & 66 & 0 & 0 & 5 & 3 \\
\hline $7 \mathrm{I}$ & 19-Dec & 1945 & Day & 66 & 0 & 0 & 8 & 1 \\
\hline $7 \mathrm{~J}$ & 20-Dec & 1945 & Night & 66 & 0 & 0 & 8 & 1 \\
\hline $7 \mathrm{~K}$ & 21-Dec & 1945 & Night & 66 & 0 & 0 & 10 & 1 \\
\hline $7 \mathrm{~L}$ & 21-Dec & 1945 & Swing & 66 & 0 & 0 & $4-14 \mathrm{U}$ & 1 \\
\hline $7 \mathrm{M}$ & 22-Dec & 1945 & Day & 66 & 0 & 0 & 4-14 U & 1 \\
\hline $7 \mathrm{~N}$ & 23-Dec & 1945 & Day & 66 & 0 & 0 & 4-14 U & 1 \\
\hline $8 \mathrm{~A}$ & 2-Jan & 1946 & Swing & 79.65 & 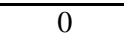 & 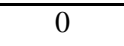 & 5 & 2 \\
\hline $8 \mathrm{~B}$ & 3-Jan & 1946 & Night & 79.65 & 0 & 0 & 5 & 1 \\
\hline $8 \mathrm{C}$ & 3-Jan & 1946 & Swing & 79.65 & 0 & 0 & 3 & 1 \\
\hline $8 \mathrm{D}$ & 4-Jan & 1946 & Night & 79.65 & 0 & 0 & 4 & 1 \\
\hline $8 \mathrm{E}$ & 4-Jan & 1946 & Day & 79.65 & 0 & 0 & 4 & 1 \\
\hline $8 \mathrm{~F}$ & 4-Jan & 1946 & Swing & 72 & 0 & 0 & 5 & 1 \\
\hline $8 \mathrm{G}$ & 5-Jan & 1946 & Day & 79.65 & 0 & 0 & 3 & 1 \\
\hline $8 \mathrm{H}$ & 5-Jan & 1946 & Swing & 79.65 & 0 & 0 & 3 & 1 \\
\hline $8 \mathrm{I}$ & 6-Jan & 1946 & Day & 79.65 & 0 & 0 & 5 & 1 \\
\hline $8 \mathrm{~J}$ & 7-Jan & 1946 & Swing & 79.65 & 0 & 0 & 3 & 1 \\
\hline $8 \mathrm{~K}$ & 8-Jan & 1946 & Night & 79.65 & 0 & 0 & 4 & 2 \\
\hline $8 \mathrm{~L}$ & 8-Jan & 1946 & Day & 79.65 & 0 & 0 & 4 & 2 \\
\hline $8 \mathrm{M}$ & 9-Jan & 1946 & Night & 79.65 & 0 & 0 & 3 & 1 \\
\hline
\end{tabular}


Appendix 3G: RaLa Dissolving Batches Conducted in X-10's Building 706-D

\begin{tabular}{|c|c|c|c|c|c|c|c|c|}
\hline \multirow{2}{*}{$\begin{array}{l}\text { Batch } \\
\text { Name }\end{array}$} & \multicolumn{3}{|c|}{ Start of Dissolving } & \multicolumn{3}{|c|}{ No. of Slugs Dissolved } & \multirow{2}{*}{$\begin{array}{c}\text { Duration } \\
\text { (h) }\end{array}$} & \multirow{2}{*}{$\begin{array}{c}\text { Direct Release } \\
\text { Class } \\
\end{array}$} \\
\hline & Date & Year & Shift or Time & $\mathrm{X}-10$ & 4" Hanf. & 8" Hanf. & & \\
\hline $8 \mathrm{~N}$ & 9-Jan & 1946 & Day & 79.65 & 0 & 0 & $4-14 \mathrm{U}$ & 1 \\
\hline 80 & 11-Jan & 1946 & Day & 79.65 & 0 & 0 & 4-7 U & 1 \\
\hline $8 \mathrm{P}$ & 13-Jan & 1946 & Night & 79.65 & 0 & 0 & $4-14 \mathrm{U}$ & 1 \\
\hline Heels & 13-Jan & 1946 & Day & 79.65 & 0 & 0 & $25-38 \mathrm{U}$ & 2 \\
\hline $9 \mathrm{~A}$ & 4-Mar & 1946 & Day & 61.2 & 0 & 0 & 3 & 2 \\
\hline $9 B$ & 4-Mar & 1946 & Day & 61.2 & 0 & 0 & 4 & 2 \\
\hline $9 \mathrm{C}$ & 4 or $5-\mathrm{Mar}$ & 1946 & Swing or Night & 61.2 & 0 & 0 & 3 & 2 \\
\hline 9D & 5-Mar & 1946 & Swing & 61.2 & 0 & 0 & 3 & 2 \\
\hline $9 \mathrm{E}$ & 5-Mar & 1946 & Swing & 61.2 & 0 & 0 & 3 & 2 \\
\hline $9 \mathrm{~F}$ & 6-Mar & 1946 & Day & 61.2 & 0 & 0 & 3 & 1 \\
\hline $9 \mathrm{G}$ & 6-Mar & 1946 & Swing & 61.2 & 0 & 0 & 3 & 1 \\
\hline $9 \mathrm{H}$ & 7-Mar & 1946 & Night & 61.2 & 0 & 0 & 4 & 1 \\
\hline 9I & 7-Mar & 1946 & Swing & 61.2 & 0 & 0 & 3 & 1 \\
\hline $9 \mathrm{~J}$ & 8-Mar & 1946 & Night & 61.2 & 0 & 0 & 3 & 1 \\
\hline $9 \mathrm{~K}$ & 8-Mar & 1946 & Day & 61.2 & 0 & 0 & 3 & 1 \\
\hline $9 \mathrm{~L}$ & 8-Mar & 1946 & Swing & 61.2 & 0 & 0 & 3 & 1 \\
\hline $9 \mathrm{M}$ & 10-Mar & 1946 & Day & 61.2 & 0 & 0 & 4 & 1 \\
\hline $9 \mathrm{~N}$ & 10-Mar & 1946 & Swing & 61.2 & 0 & 0 & 5 & 1 \\
\hline 90 & 11-Mar & 1946 & Night & 61.2 & 0 & 0 & 7 & 1 \\
\hline $10 \mathrm{~A}$ & 8-Apr & 1946 & Night & 67.2 & 0 & 0 & 3 & 1 \\
\hline 10B & 8-Apr & 1946 & Day & 67.2 & 0 & 0 & 4 & 1 \\
\hline $10 \mathrm{C}$ & 8-Apr & 1946 & Swing & 67.2 & 0 & 0 & 3 & 1 \\
\hline $10 \mathrm{D}$ & 9-Apr & 1946 & Day & 67.2 & 0 & 0 & 4 & 2 \\
\hline $10 \mathrm{E}$ & 9-Apr & 1946 & Swing & 67.2 & 0 & 0 & 4 & 2 \\
\hline $10 \mathrm{~F}$ & 10-Apr & 1946 & Night & 67.2 & 0 & 0 & 4 & 1 \\
\hline $10 \mathrm{G}$ & 10-Apr & 1946 & Swing & 67.2 & 0 & 0 & 3 & 1 \\
\hline $10 \mathrm{H}$ & 11-Apr & 1946 & Night & 67.2 & 0 & 0 & 4 & 1 \\
\hline $10 \mathrm{I}$ & 11-Apr & 1946 & Swing & 67.2 & 0 & 0 & 5 & 1 \\
\hline $10 \mathrm{~J}$ & 12-Apr & 1946 & Night & 67.2 & 0 & 0 & 5 & 1 \\
\hline $10 \mathrm{~K}$ & 12-Apr & 1946 & Day & 65.7 & 0 & 0 & 4 & 2 \\
\hline $10 \mathrm{~L}$ & 12-Apr & 1946 & Swing & 67.2 & 0 & 0 & 9 & 1 \\
\hline $10 \mathrm{M}$ & 13-Apr & 1946 & Day & 66.4 & 0 & 0 & 4-7 U & 1 \\
\hline $10 \mathrm{~N}$ & 14-Apr & 1946 & Day & 67.2 & 0 & 0 & $17-30 \mathrm{U}$ & 1 \\
\hline $11 \mathrm{~A}$ & 6-May & 1946 & Night & 65.9 & 0 & $\overline{0}$ & 4 & 1 \\
\hline $11 \mathrm{~B}$ & 6-May & 1946 & Day & 66.9 & 0 & 0 & 5 & 1 \\
\hline $11 \mathrm{C}$ & 6-May & 1946 & Swing & 66.9 & 0 & 0 & 5 & 1 \\
\hline $11 \mathrm{D}$ & 7-May & 1946 & Day & 66.9 & 0 & 0 & 5 & 1 \\
\hline $11 \mathrm{E}$ & 7-May & 1946 & Swing & 66.9 & 0 & 0 & 4 & 1 \\
\hline $11 \mathrm{~F}$ & 8-May & 1946 & Night & 66.9 & 0 & 0 & 4 & 1 \\
\hline $11 \mathrm{G}$ & 8-May & 1946 & Day & 66.3 & 0 & 0 & 4 & 1 \\
\hline $11 \mathrm{H}$ & 9-May & 1946 & Swing & 66.9 & 0 & 0 & 3 & 1 \\
\hline $11 \mathrm{I}$ & 9-May & 1946 & Swing & 66.9 & 0 & 0 & 3 & 1 \\
\hline $11 \mathrm{~J}$ & 10-May & 1946 & Day & 66.9 & 0 & 0 & 4 & 1 \\
\hline $11 \mathrm{~K}$ & 10-May & 1946 & Swing & 66.9 & 0 & 0 & 5 & 1 \\
\hline $11 \mathrm{~L}$ & 11-May & 1946 & Night & 66.9 & 0 & 0 & 16 & 1 \\
\hline $11 \mathrm{M}$ & 11-May & 1946 & Swing, Beginning & 66.9 & 0 & 0 & 11 & 1 \\
\hline $11 \mathrm{~N}$ & 13-May & 1946 & Day & 66.9 & 0 & 0 & 9 & 1 \\
\hline 110 & 13-May & 1946 & Swing & 23 & 0 & 0 & $17-30 \mathrm{U}$ & 1 \\
\hline $12 \mathrm{~A}$ & 10-Jun & 1946 & Night & 64.3 & 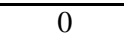 & 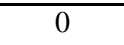 & $\overline{5}$ & 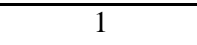 \\
\hline $12 \mathrm{~B}$ & 10-Jun & 1946 & Day & 65.6 & 0 & 0 & 6 & 1 \\
\hline $12 \mathrm{C}$ & 10-Jun & 1946 & Swing & 62.4 & 0 & 0 & 6 & 1 \\
\hline $12 \mathrm{D}$ & 11-Jun & 1946 & Day & 68.1 & 0 & 0 & 5 & 1 \\
\hline $12 \mathrm{E}$ & 11-Jun & 1946 & Swing & 63.5 & 0 & 0 & 5 & 1 \\
\hline $12 \mathrm{~F}$ & 12-Jun & 1946 & Night & 72.3 & 0 & 0 & 5 & 1 \\
\hline $12 \mathrm{G}$ & 12-Jun & 1946 & Swing & 64.3 & 0 & 0 & 5 & 1 \\
\hline $12 \mathrm{H}$ & 13-Jun & 1946 & Night & 64.3 & 0 & 0 & 7 & 2 \\
\hline $12 \mathrm{I}$ & 13-Jun & 1946 & 4:00 PM & 69.7 & 0 & 0 & 6 & 1 \\
\hline $12 \mathrm{~J}$ & 14-Jun & 1946 & Night & 66.6 & 0 & 0 & 4 & 1 \\
\hline
\end{tabular}


Appendix 3G: RaLa Dissolving Batches Conducted in X-10's Building 706-D

\begin{tabular}{|c|c|c|c|c|c|c|c|c|}
\hline \multirow{2}{*}{$\begin{array}{l}\text { Batch } \\
\text { Name }\end{array}$} & \multicolumn{3}{|c|}{ Start of Dissolving } & \multicolumn{3}{|c|}{ No. of Slugs Dissolved } & \multirow{2}{*}{$\begin{array}{c}\text { Duration } \\
\text { (h) }\end{array}$} & \multirow{2}{*}{$\begin{array}{c}\text { Direct Release } \\
\text { Class } \\
\end{array}$} \\
\hline & Date & Year & Shift or Time & $\mathrm{X}-10$ & 4" Hanf. & 8" Hanf. & & \\
\hline $12 \mathrm{~K}$ & 14-Jun & 1946 & Day & 64.3 & 0 & 0 & 6 & 1 \\
\hline $12 \mathrm{~L}$ & 14-Jun & 1946 & Swing & 64.3 & 0 & 0 & 4 & 1 \\
\hline $12 \mathrm{M}$ & 15-Jun & 1946 & Day & 63 & 0 & 0 & $4-14 \mathrm{U}$ & 1 \\
\hline $12 \mathrm{~N}$ & 16-Jun & 1946 & Day & 64.3 & 0 & 0 & $9-22 \mathrm{U}$ & 1 \\
\hline $13 \mathrm{~A}$ & 13-Aug & 1946 & Night & 65 & 0 & 0 & $4-14 \mathrm{U}$ & 1 \\
\hline $13 \mathrm{~B}$ & 13-Aug & 1946 & Day & 65 & 0 & 0 & 4-7 U & 1 \\
\hline $13 \mathrm{C}$ & 13-Aug & 1946 & Swing & 65 & 0 & 0 & 4-7 U & 2 \\
\hline $13 \mathrm{D}$ & 14-Aug & 1946 & Night & 65 & 0 & 0 & 4-7 U & 1 \\
\hline $13 \mathrm{E} ?$ & 14-Aug & 1946 & Unknown & 65 & 0 & 0 & 4-8 U & 4 \\
\hline $13 \mathrm{~F} ?$ & 15-Aug & 1946 & Unknown & 65 & 0 & 0 & $4-8 \mathrm{U}$ & 4 \\
\hline $13 \mathrm{G} ?$ & 15-Aug & 1946 & Unknown & 65 & 0 & 0 & 4-8 U & 4 \\
\hline $13 \mathrm{H} ?$ & 16-Aug & 1946 & Unknown & 65 & 0 & 0 & $4-8 \mathrm{U}$ & 4 \\
\hline $13 \mathrm{I} ?$ & 16-Aug & 1946 & Unknown & 65 & 0 & 0 & $4-8 \mathrm{U}$ & 4 \\
\hline $13 \mathrm{~J} ?$ & 17-Aug & 1946 & Unknown & 65 & 0 & 0 & 4-8 U & 4 \\
\hline $13 \mathrm{~K} ?$ & 17-Aug & 1946 & Unknown & 65 & 0 & 0 & $4-8 \mathrm{U}$ & 4 \\
\hline $13 \mathrm{~L} ?$ & 18-Aug & 1946 & Unknown & 65 & 0 & 0 & $4-8 \mathrm{U}$ & 4 \\
\hline Heels? & $\sim 18$-Aug & 1946 & Unknown & 32 & 0 & 0 & $4-8 \mathrm{U}$ & 4 \\
\hline $14 \mathrm{~A} ?$ & 3-Dec. & 1946 & Unknown & 65 & 0 & 0 & $4-8 \mathrm{U}$ & 4 \\
\hline $14 \mathrm{~B} ?$ & 3-Dec & 1946 & Unknown & 65 & 0 & 0 & $4-8 \mathrm{U}$ & 4 \\
\hline $14 \mathrm{C} ?$ & 4-Dec & 1946 & Unknown & 65 & 0 & 0 & $4-8 \mathrm{U}$ & 4 \\
\hline $14 \mathrm{D} ?$ & 5-Dec & 1946 & Unknown & 65 & 0 & 0 & $4-8 \mathrm{U}$ & 4 \\
\hline $14 \mathrm{E} ?$ & 6-Dec & 1946 & Unknown & 65 & 0 & 0 & $4-8 \mathrm{U}$ & 4 \\
\hline $14 \mathrm{~F} ?$ & 7-Dec & 1946 & Unknown & 65 & 0 & 0 & $4-8 \mathrm{U}$ & 4 \\
\hline $14 \mathrm{G} ?$ & 8-Dec & 1946 & Unknown & 65 & 0 & 0 & $4-8 \mathrm{U}$ & 4 \\
\hline $14 \mathrm{H} ?$ & 9-Dec. & 1946 & Unknown & 65 & 0 & 0 & $4-8 \mathrm{U}$ & 4 \\
\hline Heels & 11-Dec? & 1946 & $\sim$ Night & 44 & 0 & 0 & $25-38 \mathrm{U}$ & 1 \\
\hline $15 \mathrm{~A}$ & 15-Jan & 1947 & Night & 58.4 & 0 & 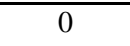 & 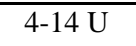 & 1 \\
\hline $15 B$ & 15-Jan & 1947 & Day & 56.8 & 0 & 0 & $4-14 \mathrm{U}$ & 1 \\
\hline $15 \mathrm{C}$ & 15-Jan & 1947 & Swing & 56.5 & 0 & 0 & $4-14 \mathrm{U}$ & 1 \\
\hline $15 \mathrm{D}$ & 16-Jan & 1947 & Day & 59 & 0 & 0 & 4-7 U & 1 \\
\hline $15 \mathrm{E}$ & 16-Jan & 1947 & Swing & 57 & 0 & 0 & 4-7 U & 1 \\
\hline $15 \mathrm{~F}$ & 17-Jan & 1947 & Night & 57 & 0 & 0 & $4-14 \mathrm{U}$ & 1 \\
\hline $15 \mathrm{G}$ & 17-Jan & 1947 & Swing & 57 & 0 & 0 & $4-14 \mathrm{U}$ & 1 \\
\hline $15 \mathrm{H}$ (heel) & 18-Jan & 1947 & Day & 27.6 & 0 & 0 & $25-38 \mathrm{U}$ & 1 \\
\hline $15 \mathrm{I}$ & 22-Jan & 1947 & 8:00:00 AM & 57 & 0 & 0 & 4-7 U & 1 \\
\hline $15 \mathrm{~J}$ & 22-Jan & 1947 & Swing & 57 & 0 & 0 & 4-14 U & 1 \\
\hline $15 \mathrm{~K}$ & 23-Jan & 1947 & Night & 54.4 & 0 & 0 & $4-14 \mathrm{U}$ & 1 \\
\hline $15 \mathrm{~L}$ & 23-Jan & 1947 & Day & 57 & 0 & 0 & 4-14 U & 1 \\
\hline heels & 23-Jan & 1947 & Swing & 30 & 0 & 0 & $17-30 \mathrm{U}$ & 1 \\
\hline $15 \mathrm{~A}-\mathrm{A}$ & $26-\mathrm{Feb}$ & 1947 & Day & 65.3 & 0 & 0 & $4-7 \mathrm{U}$ & 1 \\
\hline $15 \mathrm{~A}-\mathrm{B}$ & 26-Feb & 1947 & Day & 71.5 & 0 & 0 & $4-14 \mathrm{U}$ & 1 \\
\hline $15 \mathrm{~A}-\mathrm{C}$ & 26-Feb & 1947 & Swing & 60 & 0 & 0 & $4-7 \mathrm{U}$ & 1 \\
\hline $15 \mathrm{~A}-\mathrm{D}$ & 27-Feb & 1947 & Day & 75.3 & 0 & 0 & $4-7 \mathrm{U}$ & 1 \\
\hline $15 \mathrm{~A}-\mathrm{E}$ & 27-Feb & 1947 & Swing & 69.3 & 0 & 0 & $4-7 \mathrm{U}$ & 1 \\
\hline $15 \mathrm{~A}-\mathrm{F}$ & 28-Feb & 1947 & Night & 67 & 0 & 0 & 4-7 U & 1 \\
\hline $15 \mathrm{~A}-\mathrm{G}$ & 28-Feb & 1947 & Day & 62 & 0 & 0 & $4-14 \mathrm{U}$ & 1 \\
\hline $15 \mathrm{~A}-\mathrm{H}$ & 29-Feb & 1947 & Night & 67 & 0 & 0 & $4-7 \mathrm{U}$ & 1 \\
\hline 15A-I (heels) & 1-Mar & 1947 & Day & 32 & 0 & 0 & 4-14 U & 1 \\
\hline
\end{tabular}


Appendix 3G: RaLa Dissolving Batches Conducted in X-10's Building 706-D

\begin{tabular}{|c|c|c|c|c|c|c|c|c|}
\hline \multirow{2}{*}{$\begin{array}{l}\text { Batch } \\
\text { Name }\end{array}$} & \multicolumn{3}{|c|}{ Start of Dissolving } & \multicolumn{3}{|c|}{ No. of Slugs Dissolved } & \multirow{2}{*}{$\begin{array}{c}\text { Duration } \\
\text { (h) }\end{array}$} & \multirow{2}{*}{$\begin{array}{c}\text { Direct Release } \\
\text { Class } \\
\end{array}$} \\
\hline & Date & Year & Shift or Time & $\mathrm{X}-10$ & 4" Hanf. & 8" Hanf. & & \\
\hline $16-\mathrm{A}$ & 26-Mar & 1947 & 1:00 AM & 62.4 & 0 & 0 & 7 & 1 \\
\hline $16-\mathrm{B}$ & 26-Mar & 1947 & Night & 61.2 & 0 & 0 & $4-14 \mathrm{U}$ & 1 \\
\hline $16-\mathrm{C}$ & 26-Mar & 1947 & Day & 58.1 & 0 & 0 & $4-14 \mathrm{U}$ & 1 \\
\hline $16-\mathrm{D}$ & 27-Mar & 1947 & Day & 69.5 & 0 & 0 & 4-7 U & 1 \\
\hline $16-\mathrm{E}$ & 27-Mar & 1947 & Day & 69.3 & 0 & 0 & $4-14 \mathrm{U}$ & 1 \\
\hline $16-\mathrm{F}$ & 27-Mar & 1947 & Swing & 72.5 & 0 & 0 & 4-7 U & 1 \\
\hline $16-\mathrm{G}$ & 28-Mar & 1947 & Night & 59 & 0 & 0 & $4-14 \mathrm{U}$ & 1 \\
\hline $16-\mathrm{H}$ & 28-Mar & 1947 & $\sim 5: 00 \mathrm{PM}$ & 63 & 0 & 0 & $4-7 \mathrm{U}$ & 1 \\
\hline 16-I & 29-Mar & 1947 & Night & 60 & 0 & 0 & $4-14 \mathrm{U}$ & 1 \\
\hline 16-J heel & 29-Mar & 1947 & Day & 45 & 0 & 0 & 4-14 U & 1 \\
\hline $16-\mathrm{K}$ & 1-Apr & 1947 & Night & 62 & 0 & 0 & 4-7 U & 1 \\
\hline $16-\mathrm{L}$ & 2-Apr & 1947 & Night & 62 & 0 & 0 & 4-14 U & 2 \\
\hline 17-A & 21-Apr & 1947 & "Day & 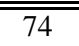 & 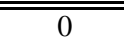 & 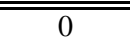 & 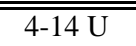 & $\overline{2}$ \\
\hline $17-B$ & 21-Apr & 1947 & Swing & 67.3 & 0 & 0 & $4-14 \mathrm{U}$ & 2 \\
\hline $17-\mathrm{C}$ & 22-Apr & 1947 & Night & 60 & 0 & 0 & $4-14 \mathrm{U}$ & 1 \\
\hline $17-\mathrm{D}$ & 22-Apr & 1947 & Swing & 66 & 0 & 0 & $4-7 \mathrm{U}$ & 1 \\
\hline $17-\mathrm{E}$ & 23-Apr & 1947 & Night & 63.7 & 0 & 0 & 4-7 U & 1 \\
\hline $17-\mathrm{F}$ & 23-Apr & 1947 & $\sim 4: 00 \mathrm{PM}$ & 66.1 & 0 & 0 & 4-7 U & 1 \\
\hline $17-\mathrm{G}$ & 24-Apr & 1947 & Night & 62 & 0 & 0 & 4-7 U & 1 \\
\hline $17-\mathrm{H}$ & 24-Apr & 1947 & Day & 55 & 0 & 0 & $4-14 \mathrm{U}$ & 1 \\
\hline $17-I$ & 25-Apr & 1947 & Night & 61 & 0 & 0 & 4-7 U & 1 \\
\hline $17-J$ & 25-Apr & 1947 & Day & 64 & 0 & 0 & $4-14 \mathrm{U}$ & 1 \\
\hline $17-\mathrm{K}$ & 25-Apr & 1947 & 11:00 PM & 65 & 0 & 0 & $4-14 \mathrm{U}$ & 1 \\
\hline 17-L & 26-Apr & 1947 & Day & 64 & 0 & 0 & 4-14 U & 2 \\
\hline 17-M (heels) & 28-Apr & 1947 & Day & 31 & 0 & 0 & 4-7 U & 1 \\
\hline 17-N (heels) & 29-Apr & 1947 & Day & 31 & 0 & 0 & $4-14 \mathrm{U}$ & 1 \\
\hline $17-0$ (heels) & 30-Apr & 1947 & Night & 31 & 0 & 0 & $9-22 \mathrm{U}$ & 1 \\
\hline $17 \mathrm{P}$ & 1-May & 1947 & Day & 31 & 0 & 0 & $17-30 \mathrm{U}$ & 1 \\
\hline $18 \mathrm{~A}$ & 9-Jun & 1947 & Day & 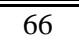 & 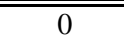 & 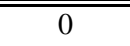 & 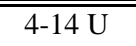 & $\overline{1} 1$ \\
\hline $18 \mathrm{~B}$ & 9-Jun & 1947 & Swing & 63 & 0 & 0 & $4-14 \mathrm{U}$ & 1 \\
\hline $18 \mathrm{C}$ & 10-Jun & 1947 & Night & 62 & 0 & 0 & $9-22 \mathrm{U}$ & 1 \\
\hline $18 \mathrm{D}$ & 10-Jun & 1947 & Swing & 69 & 0 & 0 & $4-14 \mathrm{U}$ & 1 \\
\hline $18 \mathrm{E}$ & 11-Jun & 1947 & Night & 59 & 0 & 0 & $4-14 \mathrm{U}$ & 1 \\
\hline $18 \mathrm{~F}$ & 11-Jun & 1947 & Day & 69 & 0 & 0 & 4-14 U & 1 \\
\hline $18 \mathrm{G}$ & 12-Jun & 1947 & Night & 68 & 0 & 0 & 4-7 U & 1 \\
\hline $18 \mathrm{H}$ & 12-Jun & 1947 & Swing & 70 & 0 & 0 & 4-7 U & 1 \\
\hline $18 \mathrm{I}$ & 13-Jun & 1947 & Night & 68 & 0 & 0 & 4-7 U & 1 \\
\hline $18 \mathrm{~J}$ & 13-Jun & 1947 & Day & 62 & 0 & 0 & $4-14 \mathrm{U}$ & 1 \\
\hline $18 \mathrm{~K}$ & 13-Jun & 1947 & Swing & 67 & 0 & 0 & 4-7 U & 1 \\
\hline $18 \mathrm{~L}$ & 14-Jun & 1947 & Day & 65 & 0 & 0 & 4-14 U & 1 \\
\hline $18 \mathrm{M}$ & 15-Jun & 1947 & Night & 61 & 0 & 0 & 4-14 U & 1 \\
\hline $19 \mathrm{~A}$ & "13-Jul & 1947 & Swing & 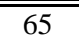 & 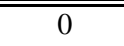 & 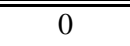 & 4-7 U & $\overline{1} 1$ \\
\hline $19 \mathrm{~B}$ & 14-Jul & 1947 & Night & 64.6 & 0 & 0 & 4-7 U & 1 \\
\hline $19 \mathrm{C}$ & 14-Jul & 1947 & Day & 62.9 & 0 & 0 & 4-7 U & 1 \\
\hline $19 \mathrm{D}$ & 15-Jul & 1947 & Night & 68 & 0 & 0 & 4-7 U & 1 \\
\hline $19 \mathrm{E}$ & 15-Jul & 1947 & Day & 64.2 & 0 & 0 & 4-7 U & 1 \\
\hline $19 \mathrm{~F}$ & 15-Jul & 1947 & Swing & 64 & 0 & 0 & 4-7 U & 1 \\
\hline $19 \mathrm{G}$ & 16-Jul & 1947 & Day & 59.3 & 0 & 0 & 4-7 U & 1 \\
\hline $19 \mathrm{H}$ & 16-Jul & 1947 & Swing & 70 & 0 & 0 & 4-7 U & 1 \\
\hline 19I & 17-Jul & 1947 & Night & 65 & 0 & 0 & 4-7 U & 1 \\
\hline $19 \mathrm{~J}$ & 17-Jul & 1947 & Day & 70 & 0 & 0 & 4-7 U & 1 \\
\hline $19 \mathrm{~K}$ & 17-Jul & 1947 & Swing & 65 & 0 & 0 & $4-14 \mathrm{U}$ & 1 \\
\hline $19 \mathrm{~L}$ & 18-Jul & 1947 & Day & 66.8 & 0 & 0 & 4-7 U & 1 \\
\hline $19 \mathrm{M}$ & 18-Jul & 1947 & Day & 59.2 & 0 & 0 & $4-14 \mathrm{U}$ & 1 \\
\hline $19 \mathrm{~N}$ & 19-Jul & 1947 & Day & 63 & 0 & 0 & $57-70 \mathrm{U}$ & 1 \\
\hline $20 \mathrm{~A}$ & 10-Aug & 1947 & Day & 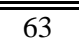 & 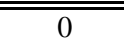 & 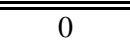 & "4-14 U & $\bar{~} 1$ \\
\hline $20 \mathrm{~B}$ & 10-Aug & 1947 & Swing & 61 & 0 & 0 & $4-14 \mathrm{U}$ & 1 \\
\hline $20 \mathrm{C}$ & 11-Aug & 1947 & Night & 57.9 & 0 & 0 & 4-14 U & 1 \\
\hline
\end{tabular}


Appendix 3G: RaLa Dissolving Batches Conducted in X-10's Building 706-D

\begin{tabular}{|c|c|c|c|c|c|c|c|c|}
\hline \multirow{2}{*}{$\begin{array}{l}\text { Batch } \\
\text { Name } \\
\end{array}$} & \multicolumn{3}{|c|}{ Start of Dissolving } & \multicolumn{3}{|c|}{ No. of Slugs Dissolved } & \multirow{2}{*}{$\begin{array}{c}\text { Duration } \\
\text { (h) }\end{array}$} & \multirow{2}{*}{$\begin{array}{c}\text { Direct Release } \\
\text { Class } \\
\end{array}$} \\
\hline & Date & Year & Shift or Time & $\mathrm{X}-10$ & 4" Hanf. & 8" Hanf. & & \\
\hline $20 \mathrm{D}$ & 11-Aug & 1947 & Swing & 68.2 & 0 & 0 & $4-14 \mathrm{U}$ & 1 \\
\hline $20 \mathrm{E}$ & 12-Aug & 1947 & Night & 62.6 & 0 & 0 & 4-7 U & 1 \\
\hline $20 \mathrm{~F}$ & 12-Aug & 1947 & Day & 63 & 0 & 0 & $4-14 \mathrm{U}$ & 1 \\
\hline $20 \mathrm{G}$ & 12-Aug & 1947 & Swing & 60 & 0 & 0 & $4-14 \mathrm{U}$ & 1 \\
\hline $20 \mathrm{H}$ & 13-Aug & 1947 & Swing & 67.1 & 0 & 0 & $4-14 \mathrm{U}$ & 1 \\
\hline $20 \mathrm{I}$ & 14-Aug & 1947 & Night & 67.3 & 0 & 0 & $4-7 \mathrm{U}$ & 1 \\
\hline $20 \mathrm{~J}$ & 14-Aug & 1947 & Night & 69 & 0 & 0 & $4-14 \mathrm{U}$ & 1 \\
\hline $20 \mathrm{~K}$ & 14-Aug & 1947 & Day & 62 & 0 & 0 & $4-14 \mathrm{U}$ & 1 \\
\hline $20 \mathrm{~L}$ & 15-Aug & 1947 & Night & 66 & 0 & 0 & 4-14 U & 1 \\
\hline $20 \mathrm{M}$ & 15-Aug & 1947 & Day & 67.3 & 0 & 0 & $4-14 \mathrm{U}$ & 1 \\
\hline $20 \mathrm{~N}$ & 15-Aug & 1947 & Swing & 64 & 0 & 0 & $4-14 \mathrm{U}$ & 1 \\
\hline 20-O (heels) & 16-Aug & 1947 & Swing & 57.3 & 0 & 0 & $65-78 \mathrm{U}$ & 3 \\
\hline $20 \mathrm{P}$ & 19-Aug & 1947 & Swing & 65.7 & 0 & 0 & $9-22 \mathrm{U}$ & 2 \\
\hline 20Q & 20-Aug & 1947 & Day & 65.7 & 0 & 0 & $25-38 \mathrm{U}$ & 1 \\
\hline $21 \mathrm{~A}$ & 5 -Oct & 1947 & Day & 66.0 & 0 & 0 & $4-14 \mathrm{U}$ & 1 \\
\hline $21 \mathrm{~B}$ & 5-Oct & 1947 & Swing & 78.0 & 0 & 0 & $4-14 \mathrm{U}$ & 1 \\
\hline $21 \mathrm{C}$ & 6-Oct & 1947 & Night & 58 & 0 & 0 & $4-14 \mathrm{U}$ & 1 \\
\hline $21 \mathrm{D}$ & 6-Oct & 1947 & Swing & 71 & 0 & 0 & $4-14 \mathrm{U}$ & 1 \\
\hline $21 \mathrm{E}$ & 7-Oct & 1947 & Night & 68 & 0 & 0 & $4-7 \mathrm{U}$ & 1 \\
\hline $21 \mathrm{~F}$ & 7-Oct & 1947 & Day & 56 & 0 & 0 & 4-7 U & 1 \\
\hline $21 \mathrm{G}$ & 7-Oct & 1947 & Swing & 70 & 0 & 0 & $4-14 \mathrm{U}$ & 1 \\
\hline $21 \mathrm{H}$ & 8-Oct & 1947 & Day & 70 & 0 & 0 & $4-14 \mathrm{U}$ & 1 \\
\hline $21 \mathrm{I}$ & 8-Oct & 1947 & Swing & 70 & 0 & 0 & $4-14 \mathrm{U}$ & 1 \\
\hline $21 \mathrm{~J}$ & 9-Oct & 1947 & Night & 65.4 & 0 & 0 & $4-14 \mathrm{U}$ & 1 \\
\hline $21 \mathrm{~K}$ & 9-Oct & 1947 & Swing & 64.7 & 0 & 0 & 4-7 U & 1 \\
\hline $21 \mathrm{~L}$ & 10-Oct & 1947 & Night & 64 & 0 & 0 & $4-14 \mathrm{U}$ & 1 \\
\hline $21 \mathrm{M}$ & 10-Oct & 1947 & Swing & 60.0 & 0 & 0 & $4-14 \mathrm{U}$ & 1 \\
\hline $21 N$ & $14-O c t$ & 1947 & Day & 70.6 & 0 & 0 & $4-14 \mathrm{U}$ & 1 \\
\hline 210 & $14-O c t$ & 1947 & Swing & 65.5 & 0 & 0 & $4-14 \mathrm{U}$ & 1 \\
\hline $21 \mathrm{P}$ & $15-$-Oct & 1947 & Night & 59 & 0 & 0 & $4-14 \mathrm{U}$ & 1 \\
\hline $21 Q$ & $15-O c t$ & 1947 & Day & 60.7 & 0 & 0 & $4-14 \mathrm{U}$ & 1 \\
\hline $21 R$ & $16-$ Oct & 1947 & Swing & 65.9 & 0 & 0 & 4-7 U & 1 \\
\hline $21 \mathrm{~S}$ & $17-$ Oct & 1947 & Day & 57 & 0 & 0 & $9-22 \mathrm{U}$ & 1 \\
\hline $21 \mathrm{~T}$ (heels) & 20-Oct & 1947 & Day & 7 & 0 & 0 & $9-22 \mathrm{U}$ & 1 \\
\hline $22 \mathrm{~A}$ & 9-Nov & 1947 & Day & 64.8 & 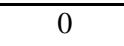 & 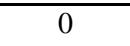 & 4-14 U & 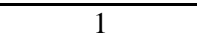 \\
\hline $22 \mathrm{~B}$ & 9-Nov & 1947 & Swing & 67.9 & 0 & 0 & $4-7 \mathrm{U}$ & 1 \\
\hline $22 \mathrm{C}$ & $10-\mathrm{Nov}$ & 1947 & Night & 66.5 & 0 & 0 & 4-14 U & 1 \\
\hline $22 \mathrm{D}$ & 10-Nov & 1947 & Swing, beginning & 70.2 & 0 & 0 & 4-7 U & 1 \\
\hline $22 \mathrm{E}$ & 10-Nov & 1947 & Swing & 66.0 & 0 & 0 & $4-14 \mathrm{U}$ & 1 \\
\hline $22 \mathrm{~F}$ & 11-Nov & 1947 & Day & 68.7 & 0 & 0 & 4-7 U & 1 \\
\hline $22 \mathrm{G}$ & 11-Nov & 1947 & Swing & 60.7 & 0 & 0 & 4-7 U & 1 \\
\hline $22 \mathrm{H}$ & 12-Nov & 1947 & Day & 67.4 & 0 & 0 & $4-14 \mathrm{U}$ & 1 \\
\hline $22 I$ & 12-Nov & 1947 & Swing & 78.0 & 0 & 0 & 4-7 U & 1 \\
\hline $22 \mathrm{~J}$ & 13-Nov & 1947 & Night & 69.8 & 0 & 0 & $4-14 \mathrm{U}$ & 1 \\
\hline $22 \mathrm{~K}$ & 13-Nov & 1947 & Day & 65.4 & 0 & 0 & $4-14 \mathrm{U}$ & 1 \\
\hline $22 \mathrm{~L}$ & 14-Nov & 1947 & Night & 78.0 & 0 & 0 & $4-14 \mathrm{U}$ & 1 \\
\hline $22 \mathrm{M}$ & 15-Nov & 1947 & Night & 65.6 & 0 & 0 & $4-14 \mathrm{U}$ & 1 \\
\hline $22 \mathrm{~N}$ & 15-Nov & 1947 & Day & 67.0 & 0 & 0 & $4-14 \mathrm{U}$ & 1 \\
\hline $22 \mathrm{O}$ & 15-Nov & 1947 & Swing & 57.2 & 0 & 0 & $4-14 \mathrm{U}$ & 1 \\
\hline $22 \mathrm{P}$ & 16-Nov & 1947 & Swing & 69.0 & 0 & 0 & 4-7 U & 1 \\
\hline $22 \mathrm{Q}$ & 17-Nov & 1947 & Night & 67.7 & 0 & 0 & 4-7 U & 1 \\
\hline $22 \mathrm{R}$ & 17-Nov & 1947 & Night & 60.0 & 0 & 0 & $4-14 \mathrm{U}$ & 1 \\
\hline $22 \mathrm{~S}$ & 17-Nov & 1947 & Swing & 68.2 & 0 & 0 & $4-14 \mathrm{U}$ & 1 \\
\hline $22 \mathrm{~T}$ & 18-Nov & 1947 & Night & 53.4 & 0 & 0 & $4-14 \mathrm{U}$ & 1 \\
\hline 22-U (heels) & 18-Nov & 1947 & Swing & 57.7 & 0 & 0 & $9-22 \mathrm{U}$ & 1 \\
\hline 22-V (heels) & 19-Nov & 1947 & Swing & 33 & 0 & 0 & $9-22 \mathrm{U}$ & 1 \\
\hline $23 \mathrm{~A}$ & (13-Jan. & 1948 & Unknown & 688.8 & 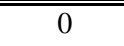 & 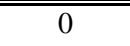 & 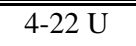 & 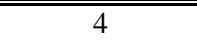 \\
\hline $23 \mathrm{~B}$ & 13-Jan & 1948 & Unknown & 84.1 & 0 & 0 & $4-22 \mathrm{U}$ & 4 \\
\hline
\end{tabular}


Appendix 3G: RaLa Dissolving Batches Conducted in X-10's Building 706-D

\begin{tabular}{|c|c|c|c|c|c|c|c|c|}
\hline \multirow{2}{*}{$\begin{array}{l}\text { Batch } \\
\text { Name } \\
\end{array}$} & \multicolumn{3}{|c|}{ Start of Dissolving } & \multicolumn{3}{|c|}{ No. of Slugs Dissolved } & \multirow{2}{*}{$\begin{array}{c}\text { Duration } \\
\text { (h) }\end{array}$} & \multirow{2}{*}{$\begin{array}{c}\text { Direct Release } \\
\text { Class }\end{array}$} \\
\hline & Date & Year & Shift or Time & $\mathrm{X}-10$ & 4" Hanf. & 8" Hanf. & & \\
\hline $23 \mathrm{C}$ & 14-Jan & 1948 & Unknown & 76.3 & 0 & 0 & $4-22 \mathrm{U}$ & 4 \\
\hline $23 \mathrm{D}$ & 14-Jan & 1948 & Unknown & 69.8 & 0 & 0 & $4-22 \mathrm{U}$ & 4 \\
\hline $23 \mathrm{E}$ & 15-Jan & 1948 & Unknown & 72.0 & 0 & 0 & $4-22 \mathrm{U}$ & 4 \\
\hline $23 \mathrm{~F}$ & 15-Jan & 1948 & Unknown & 64.7 & 0 & 0 & $4-22 \mathrm{U}$ & 4 \\
\hline $23 \mathrm{G}$ & 16-Jan & 1948 & Unknown & 75.0 & 0 & 0 & $4-22 \mathrm{U}$ & 4 \\
\hline $23 \mathrm{H}$ & 16-Jan & 1948 & Unknown & 69.1 & 0 & 0 & $4-22 \mathrm{U}$ & 4 \\
\hline $23 I$ & 17-Jan & 1948 & Unknown & 77.1 & 0 & 0 & $4-22 \mathrm{U}$ & 4 \\
\hline $23 \mathrm{~J}$ & 17-Jan & 1948 & Unknown & 76.9 & 0 & 0 & $4-22 \mathrm{U}$ & 4 \\
\hline $23 \mathrm{~K}$ & 18-Jan & 1948 & Unknown & 75.4 & 0 & 0 & $4-22 \mathrm{U}$ & 4 \\
\hline $23 \mathrm{~L}$ & 19-Jan & 1948 & Unknown & 68.0 & 0 & 0 & $4-22 \mathrm{U}$ & 4 \\
\hline $23 \mathrm{M}$ & 20-Jan & 1948 & Unknown & 43.1 & 0 & 0 & $4-30 \mathrm{U}$ & 4 \\
\hline $23 \mathrm{~N}$ & $\sim 21$-Jan. & 1948 & Unknown & 18 & 0 & 0 & 4-30 U & 4 \\
\hline$\overline{24 \mathrm{~A}}$ & 24-Feb & 1948 & " Unknown & 65.5 & 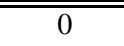 & 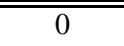 & 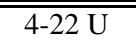 & 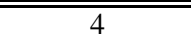 \\
\hline $24 \mathrm{~B}$ & 24-Feb & 1948 & Unknown & 68.0 & 0 & 0 & $4-22 \mathrm{U}$ & 4 \\
\hline $24 \mathrm{C}$ & 25-Feb & 1948 & Unknown & 60.7 & 0 & 0 & $4-22 \mathrm{U}$ & 4 \\
\hline $24 \mathrm{D}$ & 26-Feb & 1948 & Unknown & 72.3 & 0 & 0 & $4-22 \mathrm{U}$ & 4 \\
\hline $24 \mathrm{E}$ & 27-Feb & 1948 & Unknown & 68.8 & 0 & 0 & $4-22 \mathrm{U}$ & 4 \\
\hline $24 \mathrm{~F}$ & 27-Feb & 1948 & Unknown & 67.0 & 0 & 0 & $4-22 \mathrm{U}$ & 4 \\
\hline $24 \mathrm{G}$ & 28-Feb & 1948 & Unknown & 72.0 & 0 & 0 & $4-22 \mathrm{U}$ & 4 \\
\hline $24 \mathrm{H}$ & 28-Feb & 1948 & Unknown & 66.8 & 0 & 0 & $4-22 \mathrm{U}$ & 4 \\
\hline $24 \mathrm{I}$ & 1-Mar & 1948 & Unknown & 70.5 & 0 & 0 & $4-22 \mathrm{U}$ & 4 \\
\hline $24 \mathrm{~J}$ & 1-Mar & 1948 & Unknown & 66.3 & 0 & 0 & $4-22 \mathrm{U}$ & 4 \\
\hline $24 K$ & 2-Mar & 1948 & Unknown & 70.4 & 0 & 0 & $4-22 \mathrm{U}$ & 4 \\
\hline $24 \mathrm{~L}$ & 2-Mar & 1948 & Unknown & 60.7 & 0 & 0 & $4-22 \mathrm{U}$ & 4 \\
\hline $24 \mathrm{M}$ & 3-Mar & 1948 & Unknown & 50.2 & 0 & 0 & $4-22 \mathrm{U}$ & 4 \\
\hline $24 \mathrm{~N}$ & 4-Mar & 1948 & Unknown & 27.3 & 0 & 0 & $4-22 \mathrm{U}$ & 4 \\
\hline 240 & 5-Mar & 1948 & Unknown & 66.1 & 0 & 0 & $4-22 \mathrm{U}$ & 4 \\
\hline $24 \mathrm{P}$ & 6-Mar & 1948 & Unknown & 60.4 & 0 & 0 & $4-22 \mathrm{U}$ & 4 \\
\hline $24 Q$ & 7-Mar & 1948 & Unknown & 64.3 & 0 & 0 & $4-22 \mathrm{U}$ & 4 \\
\hline $24 \mathrm{R}$ & 8-Mar & 1948 & Unknown & 60.0 & 0 & 0 & $4-22 \mathrm{U}$ & 4 \\
\hline $24 \mathrm{~S}$ & 9-Mar & 1948 & Unknown & 63.6 & 0 & 0 & $4-22 \mathrm{U}$ & 4 \\
\hline $24 \mathrm{~T}$ & 10-Mar & 1948 & Unknown & 58.6 & 0 & 0 & $4-30 \mathrm{U}$ & 4 \\
\hline $24 \mathrm{U}$ & $\sim 11$-Mar. & 1948 & Unknown & 13.3 & 0 & 0 & $4-30 \mathrm{U}$ & 4 \\
\hline $25 \mathrm{~A}$ & " $\sim 6$-Jul & 1948 & " Unknown & 80.9 & 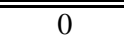 & 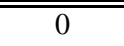 & 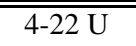 & 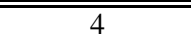 \\
\hline $25 B$ & 8-Jul & 1948 & Unknown & 80.2 & 0 & 0 & $4-22 \mathrm{U}$ & 4 \\
\hline $25 \mathrm{C}$ & 9-Jul & 1948 & Unknown & 67.1 & 0 & 0 & $4-22 \mathrm{U}$ & 4 \\
\hline $25 \mathrm{D}$ & 11-Jul & 1948 & Unknown & 37.9 & 0 & 0 & $4-22 \mathrm{U}$ & 4 \\
\hline $25 \mathrm{E}$ & 13-Jul & 1948 & Unknown & 66.3 & 0 & 0 & $4-22 \mathrm{U}$ & 4 \\
\hline $25 \mathrm{~F}$ & 14-Jul & 1948 & Unknown & 69.6 & 0 & 0 & $4-22 \mathrm{U}$ & 4 \\
\hline $25 \mathrm{G}$ & 15-Jul & 1948 & Unknown & 69.5 & 0 & 0 & $4-30 \mathrm{U}$ & 4 \\
\hline Heels & $\sim 17$-Jul & 1948 & Unknown & 58.5 & 0 & 0 & 4-30 U & 4 \\
\hline $26 \mathrm{~A}$ & " 19-Jul? & 1948 & Unknown & $\overline{770.7}$ & 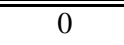 & 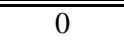 & 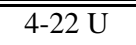 & 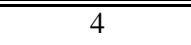 \\
\hline $26 \mathrm{~B}$ & 19-Jul & 1948 & Unknown & 72.7 & 0 & 0 & $4-22 \mathrm{U}$ & 4 \\
\hline $26 \mathrm{C}$ & 19-Jul & 1948 & Unknown & 57.0 & 0 & 0 & $4-22 \mathrm{U}$ & 4 \\
\hline $26 \mathrm{D}$ & 20-Jul & 1948 & Unknown & 77.6 & 0 & 0 & $4-22 \mathrm{U}$ & 4 \\
\hline $26 \mathrm{E}$ & 20-Jul & 1948 & Unknown & 72.7 & 0 & 0 & $4-22 \mathrm{U}$ & 4 \\
\hline $26 \mathrm{~F}$ & 20-Jul & 1948 & Unknown & 78.9 & 0 & 0 & $4-22 \mathrm{U}$ & 4 \\
\hline $26 \mathrm{G}$ & 21-Jul & 1948 & Unknown & 72.2 & 0 & 0 & $4-22 \mathrm{U}$ & 4 \\
\hline $26 \mathrm{H}$ & 21-Jul & 1948 & Unknown & 76.2 & 0 & 0 & $4-22 \mathrm{U}$ & 4 \\
\hline $26 \mathrm{I}$ & 21-Jul & 1948 & Unknown & 77.5 & 0 & 0 & $4-22 \mathrm{U}$ & 4 \\
\hline $26 \mathrm{~J}$ & 22-Jul & 1948 & Unknown & 75.1 & 0 & 0 & $4-22 \mathrm{U}$ & 4 \\
\hline $26 \mathrm{~K}$ & 22-Jul & 1948 & Unknown & 73.7 & 0 & 0 & $4-22 \mathrm{U}$ & 4 \\
\hline $26 \mathrm{~L}$ & 22-Jul & 1948 & Unknown & 78.4 & 0 & 0 & $4-22 \mathrm{U}$ & 4 \\
\hline $26 \mathrm{M}$ & 23-Jul & 1948 & Unknown & 70.6 & 0 & 0 & $4-22 \mathrm{U}$ & 4 \\
\hline Heels 2 & 23-Jul & 1948 & Unknown & 71.1 & 0 & 0 & $4-22 \mathrm{U}$ & 4 \\
\hline Heels 3 & 23-Jul & 1948 & Unknown & 66.5 & 0 & 0 & $4-22 \mathrm{U}$ & 4 \\
\hline Heels 4 & 24-Jul & 1948 & Unknown & 57.0 & 0 & 0 & $4-30 \mathrm{U}$ & 4 \\
\hline Heels 5 & 24-Jul & 1948 & Unknown & 8.6 & 0 & 0 & $4-30 \mathrm{U}$ & 4 \\
\hline
\end{tabular}


Appendix 3G: RaLa Dissolving Batches Conducted in X-10's Building 706-D

\begin{tabular}{|c|c|c|c|c|c|c|c|c|}
\hline \multirow{2}{*}{$\begin{array}{l}\text { Batch } \\
\text { Name }\end{array}$} & \multicolumn{3}{|c|}{ Start of Dissolving } & \multicolumn{3}{|c|}{ No. of Slugs Dissolved } & \multirow{2}{*}{$\begin{array}{c}\text { Duration } \\
\text { (h) }\end{array}$} & \multirow{2}{*}{$\begin{array}{c}\text { Direct Release } \\
\text { Class }\end{array}$} \\
\hline & Date & Year & Shift or Time & $\mathrm{X}-10$ & 4" Hanf. & 8" Hanf. & & \\
\hline $27 \mathrm{~A}$ & 25-Aug & 1948 & Swing & 57.5 & 0 & 0 & $4-14 \mathrm{U}$ & 1 \\
\hline 27B & 26-Aug & 1948 & Night & 62.5 & 0 & 0 & 4-7 U & 1 \\
\hline $27 \mathrm{C}$ & 26-Aug & 1948 & Night & 54.2 & 0 & 0 & $4-22 \mathrm{U}$ & 1 \\
\hline 27D & 26-Aug & 1948 & Swing & 62.5 & 0 & 0 & $4-14 \mathrm{U}$ & 1 \\
\hline $27 \mathrm{E}$ & 27-Aug & 1948 & Night & 60.3 & 0 & 0 & $4-14 \mathrm{U}$ & 1 \\
\hline $27 \mathrm{~F}$ & 27-Aug & 1948 & Day & 57.6 & 0 & 0 & $4-14 \mathrm{U}$ & 1 \\
\hline $27 \mathrm{G}$ & 27-Aug & 1948 & Swing & 60.2 & 0 & 0 & $4-14 \mathrm{U}$ & 1 \\
\hline $27 \mathrm{H}$ & 28-Aug & 1948 & Night & 63.4 & 0 & 0 & $4-22 \mathrm{U}$ & 1 \\
\hline $27 \mathrm{I}$ & 28-Aug & 1948 & Swing & 69.8 & 0 & 0 & $4-14 \mathrm{U}$ & 1 \\
\hline $27 \mathrm{~J}$ & 29-Aug & 1948 & Night & 58.8 & 0 & 0 & 4-14 U & 1 \\
\hline $27 \mathrm{~K}$ & 29-Aug & 1948 & Day & 58.1 & 0 & 0 & $4-14 \mathrm{U}$ & 1 \\
\hline $27 \mathrm{~L}$ & 29-Aug & 1948 & Swing & 55.6 & 0 & 0 & $4-14 \mathrm{U}$ & 1 \\
\hline $27 \mathrm{M}$ & 30-Aug & 1948 & Night & 61.7 & 0 & 0 & $4-22 \mathrm{U}$ & 1 \\
\hline $27 \mathrm{~N}$ & 30-Aug & 1948 & Swing & 52.6 & 0 & 0 & $4-14 \mathrm{U}$ & 1 \\
\hline 27-O (Heel) & 31-Aug & 1948 & Night & 3.1 & 0 & 0 & $4-22 \mathrm{U}$ & 1 \\
\hline $28 \mathrm{~A}$ & 18-Nov & 1948 & Night & 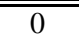 & 32.7 & 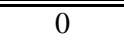 & $\overline{9-22 \mathrm{U}}$ & $\overline{1}$ \\
\hline heels & 19-Nov & 1948 & Day & 0 & 3.6 & 0 & $9-22 \mathrm{U}$ & 1 \\
\hline $29 \mathrm{~A}$ & 11-Jan & 1949 & $\overline{4: 25 \mathrm{AM}}$ & $\overline{0}$ & 27.7 & 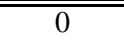 & $\overline{9-22 \mathrm{U}}$ & $\overline{1}$ \\
\hline $29 \mathrm{~B}$ & 12-Jan & 1949 & Night & 0 & 10.0 & 0 & 4-14 U & 1 \\
\hline$\overline{30 \mathrm{~A}}$ & 15-Feb & 1949 & $\overline{\text { Day }}$ & "59.3 & 3.9 & "0 & 4-7 U & $\bar{~} 1$ \\
\hline $30 \mathrm{~B}$ & 15-Feb & 1949 & Day & 56.6 & 3.7 & 0 & $4-14 \mathrm{U}$ & 1 \\
\hline $30 \mathrm{C}$ & 15-Feb & 1949 & Swing & 47.0 & 3.1 & 0 & $4-14 \mathrm{U}$ & 1 \\
\hline $30 \mathrm{D}$ & 16-Feb & 1949 & Day & 52.8 & 3.4 & 0 & $4-14 \mathrm{U}$ & 1 \\
\hline $30 \mathrm{E}$ & 16-Feb & 1949 & Swing & 56.1 & 3.6 & 0 & 4-7 U & 1 \\
\hline $30 \mathrm{~F}$ & 17-Feb & 1949 & Day & 37.9 & 2.5 & 0 & $9-22 \mathrm{U}$ & 1 \\
\hline heels & 19-Feb & 1949 & Day & 0.47 & 0.03 & 0 & $9-22 \mathrm{U}$ & 1 \\
\hline 30A-A & 22-Feb & 1949 & Night & 64.7 & 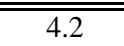 & 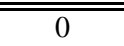 & 4-7 U & $\bar{~} 1$ \\
\hline 30A-B & 22-Feb & 1949 & 8:00 AM & 57.2 & 3.7 & 0 & 4-7 U & 1 \\
\hline $30 \mathrm{~A}-\mathrm{C}$ & 22-Feb & 1949 & Day, middle & 54.5 & 3.5 & 0 & 4-7 U & 1 \\
\hline 30A-D & 23-Feb & 1949 & Night & 54.0 & 3.5 & 0 & 2 & 2 \\
\hline $30 \mathrm{~A}-\mathrm{E}$ & 23-Feb & 1949 & Day & 50.4 & 3.3 & 0 & 4-7 U & 1 \\
\hline $30 \mathrm{~A}-\mathrm{F}$ & 24-Feb & 1949 & Day, beginning & 9.9 & 0.6 & 0 & $9-22 \mathrm{U}$ & 1 \\
\hline $31 \mathrm{~A}$ & 20-Mar & 1949 & "Day & 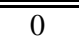 & 35.8 & 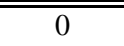 & 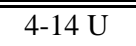 & 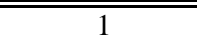 \\
\hline $31 \mathrm{~B}$ & 20-Mar & 1949 & Swing & 0 & 35.6 & 0 & $9-22 \mathrm{U}$ & 1 \\
\hline heels & 21-Mar & 1949 & Swing & 0 & 3.9 & 0 & $4-14 \mathrm{U}$ & 1 \\
\hline $32 \mathrm{~A}$ & 18-Apr & 1949 & Night & 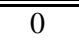 & 36.8 & 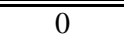 & 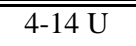 & 2 \\
\hline $32 \mathrm{~B}$ & 18-Apr & 1949 & Day & 0 & 32.9 & 0 & $9-22 \mathrm{U}$ & 2 \\
\hline heels & 19-Apr & 1949 & Night & 0 & 6.0 & 0 & $9-22 \mathrm{U}$ & 1 \\
\hline 33A & 24-May & 1949 & Night & "0 & "40.8 & "0 & 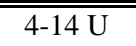 & 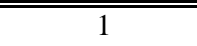 \\
\hline $33 \mathrm{~B}$ & 24-May & 1949 & Swing & 0 & 33.0 & 0 & $4-14 \mathrm{U}$ & 1 \\
\hline heels & 24-May & 1949 & Day & 0 & 4.6 & 0 & 4-14 U & 1 \\
\hline 34A & "10-Jul & 1949 & Swing 11 PM & "0 & 34.6 & "0 & 9-22 U & 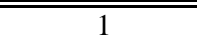 \\
\hline $34 \mathrm{~B}$ & 11-Jul & 1949 & Day & 0 & 32.0 & 0 & $4-14 \mathrm{U}$ & 1 \\
\hline heels & 12-Jul & 1949 & Night & 0 & 6.4 & 0 & 4-14 U & 1 \\
\hline
\end{tabular}


Appendix 3G: RaLa Dissolving Batches Conducted in X-10's Building 706-D

\begin{tabular}{|c|c|c|c|c|c|c|c|c|}
\hline \multirow{2}{*}{$\begin{array}{l}\text { Batch } \\
\text { Name } \\
\end{array}$} & \multicolumn{3}{|c|}{ Start of Dissolving } & \multicolumn{3}{|c|}{ No. of Slugs Dissolved } & \multirow{2}{*}{$\begin{array}{c}\text { Duration } \\
\text { (h) }\end{array}$} & \multirow{2}{*}{$\begin{array}{c}\text { Direct Release } \\
\text { Class }\end{array}$} \\
\hline & Date & Year & Shift or Time & $\mathrm{X}-10$ & 4" Hanf. & 8" Hanf. & & \\
\hline $35 \mathrm{~A}$ & 15-Aug & 1949 & Day & 0 & 35.9 & 0 & $4-14 \mathrm{U}$ & 1 \\
\hline $35 \mathrm{~B}$ & 15-Aug & 1949 & Swing & 0 & 35.1 & 0 & $9-22 \mathrm{U}$ & 1 \\
\hline heels & 16-Aug & 1949 & Day & 0 & 1.5 & 0 & 4-14 U & 1 \\
\hline $36 \mathrm{~A}$ & $10-$ Oct & 1949 & Night & 0 & 40.7 & 0 & $4-14 \mathrm{U}$ & 1 \\
\hline $36 \mathrm{~B}$ & 10-Oct & 1949 & Day & 0 & 30.7 & 0 & $9-22 \mathrm{U}$ & 1 \\
\hline heels & 11-Oct & 1949 & Night & 0 & 4.0 & 0 & $9-22 \mathrm{U}$ & 1 \\
\hline $37 \mathrm{~A}$ & 21-Nov & 1949 & Night & 0 & 34.8 & 0 & $4-14 \mathrm{U}$ & 1 \\
\hline 37B & 21-Nov & 1949 & Day & 0 & 30.9 & 0 & $9-22 \mathrm{U}$ & 1 \\
\hline heels & 22-Nov & 1949 & Night & 0 & 6.2 & 0 & $9-22 \mathrm{U}$ & 1 \\
\hline $38 \mathrm{~A}$ & 2-Jan & 1950 & Night, $\sim 7$ AM & 0 & 37 & 0 & 9 & 1 \\
\hline $38 B$ & 2-Jan & 1950 & Swing & 0 & 31 & 0 & 4-14 U & 1 \\
\hline heels & 3-Jan & 1950 & Day & 0 & 6.8 & 0 & 4-14 U & 1 \\
\hline $39 \mathrm{~A}$ & 16-Jan & 1950 & Day & 0 & 21.57 & $\overline{0}$ & $4-14 \mathrm{U}$ & 2 \\
\hline $39 B$ & 17-Jan & 1950 & Night? & 0 & 25.3 & 0 & 4-7 U & 1 \\
\hline $39 \mathrm{C}$ & 18-Jan & 1950 & Day & 0 & 29.1 & 0 & 4-14 U & 1 \\
\hline heels "B"? & 19-Jan & 1950 & Night? & 0 & 19 & 0 & $9-22 \mathrm{U}$ & 1 \\
\hline $40 \mathrm{~A}$ & 13-Mar & 1950 & Day, 4 PM & 0 & 40.85 & 0 & 8 & 1 \\
\hline $40 \mathrm{~B}$ & 14-Mar & 1950 & Night & 0 & 40.95 & 0 & $9-22 \mathrm{U}$ & 1 \\
\hline heels & 14-Mar & 1950 & Swing & 0 & 5 & 0 & $9-22 \mathrm{U}$ & 1 \\
\hline $41 \mathrm{~A}$ & 9-Apr & 1950 & Day & 0 & 36.8 & 0 & $9-22 \mathrm{U}$ & 1 \\
\hline $41 B$ & 10-Apr & 1950 & Night & 0 & 31.0 & 0 & 4-14 U & 1 \\
\hline heels & 10-Apr & 1950 & Swing & 0 & 6.5 & 0 & $17-30 \mathrm{U}$ & 1 \\
\hline $42 \mathrm{~A}$ & 11-Jun & 1950 & Swing & 0 & 36.6 & 0 & $4-14 \mathrm{U}$ & 1 \\
\hline $42 \mathrm{~B}$ & 12-Jun & 1950 & Day & 0 & 38.0 & 0 & 4-14 U & 1 \\
\hline $42 \mathrm{C}$ & 13-Jun & 1950 & Night & 0 & 20.5 & 0 & 4-14 U & 1 \\
\hline Heels & 13-Jun & 1950 & Swing & 0 & 1.2 & 0 & 4-14 U & 1 \\
\hline $43 \mathrm{~A}$ & 2-Apr & 1951 & Day & 58.6 & 0 & $\overline{0}$ & $9-22 \mathrm{U}$ & 1 \\
\hline heels & 3-Apr & 1951 & Night & 11.5 & 0 & 0 & $49-62 \mathrm{U}$ & 1 \\
\hline $44 \mathrm{~A}$ & 14-May & 1951 & Night & 0 & 38.1 & 0 & $4-14 \mathrm{U}$ & 1 \\
\hline $44 \mathrm{~B}$ & 14-May & 1951 & Swing & 0 & 29.5 & 0 & 4-14 U & 1 \\
\hline heels & 15-May & 1951 & Day, early & 0 & 1.9 & 0 & $4-22 \mathrm{U}$ & 1 \\
\hline $45 \mathrm{~A}$ & 13-Aug & 1951 & Day & 0 & 41.2 & 0 & 4-14 U & 1 \\
\hline $45 \mathrm{~B}$ & 3-Aug & 1951 & Swing & 0 & 41.9 & 0 & 4-14 U & 1 \\
\hline $45 \mathrm{C}$ & 14-Aug & 1951 & Night & 0 & 39.9 & 0 & $4-14 \mathrm{U}$ & 1 \\
\hline $45 \mathrm{D}$ & 15-Aug & 1951 & Day & 0 & 24.2 & 0 & $9-22 \mathrm{U}$ & 1 \\
\hline $46 \mathrm{~A}$ & 13-Jan & 1952 & Day & 0 & 39.4 & 0 & $4-14 \mathrm{U}$ & 1 \\
\hline $46 B$ & 13-Jan & 1952 & Swing & 0 & 38.9 & 0 & 4-14 U & 1 \\
\hline $46 \mathrm{C}$ & 14-Jan & 1952 & Night & 0 & 39.3 & 0 & 4-14 U & 1 \\
\hline 46D & 14-Jan & 1952 & Day & 0 & 36.7 & 0 & 4-14 U & 1 \\
\hline $46 \mathrm{E}$ & 14-Jan & 1952 & Swing, 11 PM & 0 & 22.1 & 0 & $9-22 \mathrm{U}$ & 1 \\
\hline $47 \mathrm{~A}$ & 1-Jun & 1952 & Day & 0 & 46.6 & 0 & $4-14 \mathrm{U}$ & 1 \\
\hline $47 \mathrm{~B}$ & 1-Jun & 1952 & Swing & 0 & 43.6 & 0 & 4-7 U & 1 \\
\hline $47 \mathrm{C}$ & 2-Jun & 1952 & Night, beginning & 0 & 41.2 & 0 & 4-7 U & 1 \\
\hline 47D & 2-Jun & 1952 & Night & 0 & 33.5 & 0 & 4-14 U & 1 \\
\hline $47 \mathrm{E}$ & 2-Jun & 1952 & Day & 0 & 39.1 & 0 & 4-14 U & 1 \\
\hline $47 \mathrm{~F}$ & 2-Jun & 1952 & Swing & 0 & 37.2 & 0 & 4-14 U & 1 \\
\hline 47-G (heels) & 3-Jun & 1952 & Night & 0 & 19.3 & 0 & $41-54 \mathrm{U}$ & 1 \\
\hline $48 \mathrm{~A}$ & 29-Jun & 1952 & Day & 0 & 42.6 & 0 & $4-7 \mathrm{U}$ & 1 \\
\hline $48 \mathrm{~B}$ & 29-Jun & 1952 & Swing, 11:30 PM & 0 & 40.3 & 0 & $4-14 \mathrm{U}$ & 1 \\
\hline $48 \mathrm{C}$ & 30-Jun & 1952 & Night, $\sim 8 \mathrm{AM}$ & 0 & 41.7 & 0 & 4-7 U & 1 \\
\hline $48 \mathrm{D}$ & 30-Jun & 1952 & Day & 0 & 37.5 & 0 & 4-14 U & 1 \\
\hline $48 \mathrm{E}$ & 30-Jun & 1952 & Swing & 0 & 32.0 & 0 & 4-14 U & 1 \\
\hline $48 \mathrm{~F}$ & 1-Jul & 1952 & Day & 0 & 19.5 & 0 & 4-14 U & 1 \\
\hline $49 \mathrm{~A}$ & 29-Jul & 1952 & Swing & 0 & 40.4 & 0 & $4-7 \mathrm{U}$ & 1 \\
\hline 49B & 30-Jul & 1952 & $12 \mathrm{MN}$ & 0 & 37.4 & 0 & 4-7 U & 1 \\
\hline
\end{tabular}


Appendix 3G: RaLa Dissolving Batches Conducted in X-10's Building 706-D

\begin{tabular}{|c|c|c|c|c|c|c|c|c|}
\hline \multirow{2}{*}{$\begin{array}{l}\text { Batch } \\
\text { Name } \\
\end{array}$} & \multicolumn{3}{|c|}{ Start of Dissolving } & \multicolumn{3}{|c|}{ No. of Slugs Dissolved } & \multirow{2}{*}{$\begin{array}{c}\text { Duration } \\
\text { (h) }\end{array}$} & \multirow{2}{*}{$\begin{array}{c}\text { Direct Release } \\
\text { Class } \\
\end{array}$} \\
\hline & Date & Year & Shift or Time & $\mathrm{X}-10$ & 4" Hanf. & 8" Hanf. & & \\
\hline $49 \mathrm{C}$ & 30-Jul & 1952 & Night & 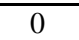 & 39.2 & 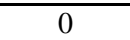 & 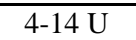 & 1 \\
\hline 49D & 30-Jul & 1952 & Day & 0 & 38.7 & 0 & $4-14 \mathrm{U}$ & 1 \\
\hline $49 \mathrm{E}$ & 30-Jul & 1952 & Swing & 0 & 38.5 & 0 & $4-14 \mathrm{U}$ & 1 \\
\hline $49 \mathrm{~F}$ & 31-Jul & 1952 & Night & 0 & 25.7 & 0 & $9-22 \mathrm{U}$ & 1 \\
\hline heels & 31-Jul & 1952 & Swing & 0 & 2.3 & 0 & $9-22 \mathrm{U}$ & 1 \\
\hline$\overline{50 \mathrm{~A}}$ & 19-Aug & 1952 & Swing & 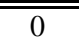 & 20.88 & 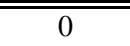 & 4-7 U & 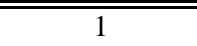 \\
\hline $50 \mathrm{~B}$ & 20-Aug & 1952 & Night & 0 & 20.5 & 0 & 4-7 U & 1 \\
\hline $50 \mathrm{C}$ & 20-Aug & 1952 & Night & 0 & 20.1 & 0 & $4-14 \mathrm{U}$ & 1 \\
\hline $50 \mathrm{D}$ & 20-Aug & 1952 & 4:00 PM & 0 & 18.3 & 0 & 8 & 1 \\
\hline $50 \mathrm{E}$ & 21-Aug & 1952 & Night & 0 & 14.7 & 0 & $4-14 \mathrm{U}$ & 1 \\
\hline $50 \mathrm{~F}$ & 21-Aug & 1952 & Day & 0 & 11.2 & 0 & $9-22 \mathrm{U}$ & 1 \\
\hline $51 \mathrm{~A}$ & 24-Sep & 1952 & Swing & & 18.48 & 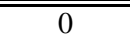 & 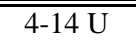 & $\overline{1}$ \\
\hline $51 \mathrm{~B}$ & 25-Sep & 1952 & Night & & 18.94 & 0 & $4-14 \mathrm{U}$ & 1 \\
\hline $51 \mathrm{C}$ & 25-Sep & 1952 & Day & & 20.42 & 0 & $4-14 \mathrm{U}$ & 1 \\
\hline $51 \mathrm{D}$ & 25-Sep & 1952 & Swing & & 18.60 & 0 & $4-14 \mathrm{U}$ & 1 \\
\hline $51 \mathrm{E}$ & 26-Sep & 1952 & Night & & 17.54 & 0 & 4-14 U & 1 \\
\hline $51 \mathrm{~F}$ & 26-Sep & 1952 & Swing, $\sim 6 \mathrm{PM}$ & & 12.36 & 0 & $4-14 \mathrm{U}$ & 1 \\
\hline heels & 27-Sep & 1952 & Night & & 1 & 0 & 4-14 U & 1 \\
\hline$\overline{\overline{52 \mathrm{~A}}}$ & 24-May & 1953 & Night & & & 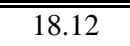 & 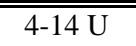 & 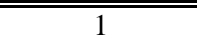 \\
\hline $52 \mathrm{~B}$ & 24-May & 1953 & 5:30 PM & & & 18.89 & 10 & 1 \\
\hline $52 \mathrm{C}$ & 25-May & 1953 & $6: 15 \mathrm{AM}$ & & & 16.65 & 9 & 1 \\
\hline $52 \mathrm{D}$ & 25-May & 1953 & 5:20 PM & & & 15.23 & 12 & 1 \\
\hline heels & 26-May & 1953 & Day & & & 4.27 & $9-22 \mathrm{U}$ & 1 \\
\hline 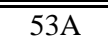 & 4-Jul & 1953 & $\overline{1: 30 \mathrm{PM}}$ & & & "17.1 & 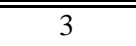 & 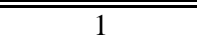 \\
\hline $53 \mathrm{~B}$ & 4-Jul & 1953 & 8:38 PM & & & 17.1 & $4-14 \mathrm{U}$ & 1 \\
\hline $53 \mathrm{C}$ & 5-Jul & 1953 & Night & & & 17.0 & $4-14 \mathrm{U}$ & 1 \\
\hline $53 \mathrm{D}$ & 5-Jul & 1953 & $9: 30 \mathrm{AM}$ & & & 20.3 & 8 & 1 \\
\hline 53A-E & 5-Jul & 1953 & $6: 20 \mathrm{PM}$ & & & 16.1 & 22 & 2 \\
\hline $53 \mathrm{~A}-\mathrm{F}$ & 6-Jul & 1953 & Swing & & & 20.3 & $4-14 \mathrm{U}$ & 1 \\
\hline $53 \mathrm{~A}-\mathrm{G}$ & 7-Jul & 1953 & Night & & & 16.8 & $15-23 \mathrm{U}$ & 1 \\
\hline$\overline{54 \mathrm{~A}}$ & 5-Nov & 1953 & $\overline{8: 20 \mathrm{PM}}$ & & 22.4 & 10.98 & 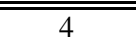 & $\overline{1.1}$ \\
\hline $54 \mathrm{~B}$ & 6-Nov & 1953 & Night & & 22.3 & 10.89 & $3-6 \mathrm{U}$ & 1 \\
\hline $54 \mathrm{C}$ & 6-Nov & 1953 & Night & & 24.1 & 11.79 & $4-14 \mathrm{U}$ & 1 \\
\hline $54 \mathrm{D}$ & 6-Nov & 1953 & Swing, beginning & & 24.7 & 12.06 & 4-7 U & 2 \\
\hline $54 \mathrm{E}$ & 11-Nov & 1953 & Night & & 19.5 & 9.54 & 4-7 U & 1 \\
\hline $54 \mathrm{~F}$ & 11-Nov & 1953 & Day & & 21.2 & 10.35 & $4-14 \mathrm{U}$ & 1 \\
\hline $54 \mathrm{G}$ & 18-Nov & 1953 & $20: 40$ & & 16.9 & 8.28 & 6 & 1 \\
\hline $54 \mathrm{H}$ & 19-Nov & 1953 & $8: 50$ & & 15.8 & 7.74 & 7 & 1 \\
\hline $54 \mathrm{I}$ & 19-Nov & 1953 & $22: 15$ & & 12.8 & 6.264 & 8 & 1 \\
\hline $54 \mathrm{~J}$ & 20-Nov & 1953 & $3: 40$ & & 4.0 & 1.962 & 15 & 1 \\
\hline 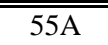 & 16-Jan & 1954 & $21: 00$ & & & 20.82 & 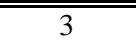 & 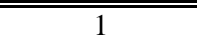 \\
\hline $55 \mathrm{~B}$ & 17-Jan & 1954 & 2:00 AM & & & 20.8 & 4-7 U & 1 \\
\hline $55 \mathrm{C}$ & 17-Jan & 1954 & Day & & & 20.3 & 4-7 U & 1 \\
\hline $55 \mathrm{D}$ & 17-Jan & 1954 & Day & & & 20.3 & $4-14 \mathrm{U}$ & 2 \\
\hline $55 \mathrm{E}$ & 18-Jan & 1954 & $22: 15$ & & & 16.29 & 4-14 U & 2 \\
\hline $55 \mathrm{~F}$ & 19-Jan & 1954 & Night & & & 20.3 & $4-14 \mathrm{U}$ & 1 \\
\hline $55 \mathrm{G}$ & 19-Jan & 1954 & Day & & & 20.3 & $4-14 \mathrm{U}$ & 1 \\
\hline $55 \mathrm{~A}-\mathrm{H}$ & 20-Jan & 1954 & $12: 00 \mathrm{MN}$ & & & 20.3 & 4-7 U & 1 \\
\hline 55A-I & 20-Jan & 1954 & Day & & & 17.51 & $4-14 \mathrm{U}$ & 1 \\
\hline $55 \mathrm{~A}-\mathrm{J}$ & 21-Jan & 1954 & Night & & & 20.3 & 4-14 U & 1 \\
\hline
\end{tabular}


Appendix 3G: RaLa Dissolving Batches Conducted in X-10's Building 706-D

\begin{tabular}{|c|c|c|c|c|c|c|c|c|}
\hline \multirow{2}{*}{$\begin{array}{l}\text { Batch } \\
\text { Name }\end{array}$} & \multicolumn{3}{|c|}{ Start of Dissolving } & \multicolumn{3}{|c|}{ No. of Slugs Dissolved } & \multirow{2}{*}{$\begin{array}{c}\text { Duration } \\
\text { (h) }\end{array}$} & \multirow{2}{*}{$\begin{array}{c}\text { Direct Release } \\
\text { Class } \\
\end{array}$} \\
\hline & Date & Year & Shift or Time & $\mathrm{X}-10$ & 4" Hanf. & 8" Hanf. & & \\
\hline $56 \mathrm{~A}$ & 26-Apr & 1954 & $19: 14$ & & & 18 & 4 & 4 \\
\hline $56 \mathrm{~B}$ & 27-Apr?? & 1954 & Unknown & & & 18 & $4-14 \mathrm{U}$ & 4 \\
\hline $56 \mathrm{C}$ & 28-Apr ?? & 1954 & Day? & & & 18 & $4-14 \mathrm{U}$ & 4 \\
\hline $56 \mathrm{E} ?$ & 2-May ?? & 1954 & Night? & & & 18 & $4-14 \mathrm{U}$ & 4 \\
\hline $56 \mathrm{~F} ?$ & 3-May ?? & 1954 & Unknown & & & 18 & $4-14 \mathrm{U}$ & 4 \\
\hline $56 \mathrm{G} ?$ & 3-May ?? & 1954 & Unknown & & & 18 & $4-14 \mathrm{U}$ & 4 \\
\hline $56 \mathrm{H} ?$ & 4-May ?? & 1954 & Unknown & & & 18 & $4-14 \mathrm{U}$ & 4 \\
\hline $56 \mathrm{I} ?$ & 4-May?? & 1954 & Unknown & & & 17 & 4-14 U & 4 \\
\hline $57 \mathrm{~A}$ & 17-Jul & 1954 & Day ( 4 PM) & & & 18.05 & 8 & 1 \\
\hline $57 \mathrm{~B}$ & 18-Jul & 1954 & 2:35 AM & & & 15.06 & $4-14 \mathrm{U}$ & 1 \\
\hline $57 \mathrm{C}$ & 18-Jul & 1954 & Day & & & 15.06 & $4-14 \mathrm{U}$ & 1 \\
\hline $57 \mathrm{D}$ & 19-Jul & 1954 & Night ( 2 AM) & & & 14 & $4-14 \mathrm{U}$ & 1 \\
\hline heels & 20-Jul & 1954 & Swing ( 11 PM) & & & 7.53 & 13 & 1 \\
\hline $58 \mathrm{~A}$ & 23 -Oct & 1954 & Day & & & 21.1 & $4-7 \mathrm{U}$ & 2 \\
\hline $58 \mathrm{~B}$ & 23-Oct & 1954 & Swing & & & 20.8 & $4-14 \mathrm{U}$ & 1 \\
\hline $58 \mathrm{C}$ & 24-Oct & 1954 & Day & & & 17.8 & $4-14 \mathrm{U}$ & 1 \\
\hline $58 \mathrm{D}$ & 24-Oct & 1954 & Swing & & & 15.0 & $4-14 \mathrm{U}$ & 1 \\
\hline $58 \mathrm{E}$ & 25-Oct & 1954 & Day & & & 11.4 & $4-14 \mathrm{U}$ & 1 \\
\hline $259 \mathrm{~A}$ & 2-Mar & 1955 & Day ( 4 PM) & & & 18.9 & 6 & 1 \\
\hline $59 B$ & 2-Mar & 1955 & $23: 10$ & & & 17.8 & $4-14 \mathrm{U}$ & 1 \\
\hline $59 \mathrm{C}$ & 3-Mar & 1955 & Night & & & 19.2 & $4-14 \mathrm{U}$ & 1 \\
\hline $59 \mathrm{D}$ & 3-Mar & 1955 & Swing & & & 19.2 & $4-14 \mathrm{U}$ & 1 \\
\hline $59 \mathrm{E}$ & 4-Mar & 1955 & 4:50 AM & & & 11.6 & $9-22 \mathrm{U}$ & 1 \\
\hline 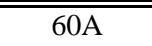 & 15-Apr & 1955 & "Swing & & & 18.05 & 44-7 U & 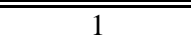 \\
\hline 60B & 16-Apr & 1955 & Night & & & 17.72 & 4-7 U & 1 \\
\hline $60 \mathrm{C}$ & 16-Apr & 1955 & Day & & & 17.72 & $4-14 \mathrm{U}$ & 2 \\
\hline $60 \mathrm{D}$ & 16-Apr & 1955 & Swing & & & 17.72 & $4-14 \mathrm{U}$ & 2 \\
\hline $60 \mathrm{E}$ & 17-Apr & 1955 & Night $(6: 30 \mathrm{AM})$ & & & 13.12 & $9-22 \mathrm{U}$ & 1 \\
\hline $61 \mathrm{~A}$ & 18-Jul & 1955 & $\overline{\text { Day (2:50 PM) }}$ & & & 2.48 & $9-22 \mathrm{U}$ & $\overline{1}$ \\
\hline heels & 19-Jul & 1955 & Day & & & 1.52 & $9-22 \mathrm{U}$ & 1 \\
\hline $62 \mathrm{~A}$ & 28-Aug & 1955 & Day & & & 14.95 & $9-22 \mathrm{U}$ & 1 \\
\hline $62 \mathrm{~B}$ & 29-Aug & 1955 & Night & & & 17.55 & $9-22 \mathrm{U}$ & 1 \\
\hline $62 \mathrm{C}$ & 29-Aug & 1955 & Swing & & & 16.44 & $4-14 \mathrm{U}$ & 1 \\
\hline $62 \mathrm{D}$ & 30-Aug & 1955 & Night & & & 18.66 & 4-14 U & 1 \\
\hline 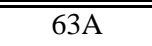 & 2-Oct & 1955 & SWwing ( 10 PM) & & & 21.84 & 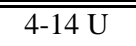 & 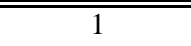 \\
\hline $63 \mathrm{~B}$ & 3-Oct & 1955 & Night & & & 21.04 & $4-14 \mathrm{U}$ & 1 \\
\hline $63 \mathrm{C}$ & 3-Oct & 1955 & Day & & & 18.56 & $4-14 \mathrm{U}$ & 1 \\
\hline $63 \mathrm{D}$ & 3-Oct & 1955 & Swing & & & 18.56 & $4-14 \mathrm{U}$ & 1 \\
\hline 63E (heels) & 4-Oct & 1955 & Night ( 7 AM) & & & 9 & $9-22 \mathrm{U}$ & 1 \\
\hline $64 \mathrm{~A}$ & 15-Jan & 1956 & Swing & & & 20.93 & $4-14 \mathrm{U}$ & 1 \\
\hline $64 \mathrm{~B}$ & 16-Jan & 1956 & Night & & & 20.93 & $4-14 \mathrm{U}$ & 1 \\
\hline $64 \mathrm{C}$ & 16-Jan & 1956 & Day & & & 20.93 & $4-14 \mathrm{U}$ & 1 \\
\hline $64 \mathrm{D}$ & 16-Jan & 1956 & Swing & & & 20.93 & $4-14 \mathrm{U}$ & 1 \\
\hline 64E? & 17-Jan? & 1956 & Assume Night & & & 9.3 & 4-14 U & 1 \\
\hline 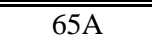 & 23-Mar & 1956 & "Day & & & 21.3 & 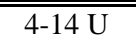 & 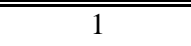 \\
\hline $65 B$ & 23-Mar & 1956 & Swing & & & 21.3 & $4-14 \mathrm{U}$ & 1 \\
\hline $65 \mathrm{C}$ & 24-Mar & 1956 & Night & & & 21.3 & $4-14 \mathrm{U}$ & 1 \\
\hline $65 \mathrm{D}$ & 24-Mar & 1956 & Day & & & 7.1 & $4-14 \mathrm{U}$ & 1 \\
\hline 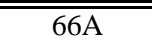 & 8-May & 1956 & Swing & & & 25.59 & 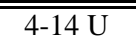 & $\bar{~} 1$ \\
\hline $66 \mathrm{~B}$ & 9-May & 1956 & Night & & & 25.59 & $4-14 \mathrm{U}$ & 1 \\
\hline $66 \mathrm{C}$ & 9-May & 1956 & Day & & & 25.59 & $4-14 \mathrm{U}$ & 1 \\
\hline $66 \mathrm{D}$ & 9-May & 1956 & Assume Swing & & & 8.53 & $9-30 \mathrm{U}$ & 1 \\
\hline
\end{tabular}


Appendix 3G: RaLa Dissolving Batches Conducted in X-10's Building 706-D

\begin{tabular}{|c|c|c|c|c|c|c|c|c|}
\hline \multirow{2}{*}{$\begin{array}{l}\text { Batch } \\
\text { Name } \\
\end{array}$} & \multicolumn{3}{|c|}{ Start of Dissolving } & \multicolumn{3}{|c|}{ No. of Slugs Dissolved } & \multirow{2}{*}{$\begin{array}{c}\text { Duration } \\
\text { (h) }\end{array}$} & \multirow{2}{*}{$\begin{array}{c}\text { Direct Release } \\
\text { Class } \\
\end{array}$} \\
\hline & Date & Year & Shift or Time & $\mathrm{X}-10$ & 4" Hanf. & 8" Hanf. & & \\
\hline $67 \mathrm{~A}$ & 9-Sep & 1956 & Swing (middle) & & & 19.38 & $4-14 \mathrm{U}$ & 1 \\
\hline $67 \mathrm{~B}$ & 10-Sep & 1956 & Night (middle) & & & 19.38 & $4-14 \mathrm{U}$ & 1 \\
\hline $67 \mathrm{C}$ & 10-Sep & 1956 & Day & & & 19.38 & $4-14 \mathrm{U}$ & 1 \\
\hline 67D & 10-Sep & 1956 & Day or Swing & & & 19.38 & $4-14 \mathrm{U}$ & 1 \\
\hline $67 \mathrm{E}$ & 10-Sep & 1956 & Assume Swing & & & 19.38 & $9-30 \mathrm{U}$ & 1 \\
\hline $67 \mathrm{~F}$ & 11-Sep & 1956 & Assume Night, End & & & 19.38 & $4-22 \mathrm{U}$ & 1 \\
\hline $67 \mathrm{G}$ & 11-Sep & 1956 & Swing & & & 12.92 & $4-14 \mathrm{U}$ & 1 \\
\hline $68 \mathrm{~A}$ & 21-Oct & 1956 & Swing & & & 21.54 & $4-14 \mathrm{U}$ & 1 \\
\hline $68 \mathrm{~B}$ & 22-Oct & 1956 & Night & & & 21.54 & $4-14 \mathrm{U}$ & 1 \\
\hline $68 \mathrm{C}$ & 22-Oct & 1956 & Day & & & 21.54 & $4-14 \mathrm{U}$ & 1 \\
\hline $68 \mathrm{D}$ & 22-Oct & 1956 & Swing & & & 7.18 & $4-22 \mathrm{U}$ & 1 \\
\hline
\end{tabular}


APPENDIX 3H

DETAILS OF BATCHES FOR WHICH NUMBERS OF SLUGS DISSOLVED WERE ESTIMATED 
TASK 1 REPORT

July 1999

${ }^{131}$ I Releases from X-10 Radioactive Lanthanum Processing -

Page 3H-2

Appendices

This page intentionally left blank. 


\section{Appendix 3-H: Details of Batches for which Numbers of Slugs Dissolved were Estimated}

Run 1: 208 slugs apportioned to 4 batches according to proportions of $\mathrm{Ci}$ Ba dissolved in those batches: $\mathrm{A}: 280 \mathrm{Ci}$ Ba; B: $281 \mathrm{Ci} \mathrm{Ba;} \mathrm{C:} 238 \mathrm{Ci} \mathrm{Ba;} \mathrm{D:} 208 \mathrm{Ci} \mathrm{Ba}$. Total = $1007 \mathrm{Ci} \mathrm{Ba}$.

Run 3: Based on 831 slugs charged. Assigned 25 slugs to $3 C D$ as reported, assigned remaining slugs evenly over remaining 11 batches. Report says -60 effective slugs per batch rather than $\sim 50$ in previous runs.

Run 4: CF45-8-219 says 12 batches $\times 50$ effective slugs per batch. Ops. log says there were 22 batches. $22 \times 50$ is in close agreement with the 1144 slugs reported charged. $1144 / 22=52$; assigned 52 to each batch.

Run 5: Reported 16 batches with average 61.5 effective slugs dissolved in each. Ops. log shows 18 batches; assigned 61.5 slugs to each.

Run 6: It is reported that the first 7 batches averaged 85 effective slugs dissolved; other 4 batches were at "normal levels." Assigned 60 slugs to the last 4 non-heel batches. Average over 11 batches reported as 77.6 slugs; $11 x$ $77.6=853$ slugs; $853-(7 \times 85+4 \times 60)=18 ; 18 / 2=9$ for each heel batch.

Run 7: Report indicates 7 batches $\times 86.3$ effective slugs dissolved in each. Ops. log shows 14 batches. The batches after the first 7 may not have been used for the product, but dissolvings appear to have occurred. Reported 924 slugs charged $/ 14$ batches $=66$ per batch.

Run 8: Reported 17 batches with average 79.2 effective slugs dissolved in each. It is also recorded that 72 eff. slugs were dissolved in Batch 8-F. Therefore: $[(79.2 \times 17)-(72)] / 16=79.65$ eff. slugs in each of the remaining 16 batches.

Run 9: Reported average 61.2 effective slugs dissolved in each of 15 batches.

Run 10: Reported 67.2 slugs averaged over 12 batches. But Ops. log indicates 14 dissolvings took place. Therefore, assigned 67.2 slugs to each batch for which we do not have reported slugs dissolved.

Run 11: Reported avg. 66.8 slugs dissolved over 14 batches (heels not included, but we have a reported eff. slugs dissolved for the heels).

Run 12: Reported average 64.3 slugs over 12 batches. But logs indicate 14 dissolvings occured. Therefore assigned 64.3 slugs to each batch with no report of slugs dissolved.

Run 13: 812 slugs dissolved per ORNL-246 Special. At 64.3 slugs/batch from previous run, would have been $\sim 12.6$ batches. Assigned 65 slugs to 12 batches and 32 to a heel batch.

Run 14: 564 slugs dissolved per ORNL-246 Special. At 64.3 slugs/batch from Run 12, would have been $\sim 8.77$ batches. Assigned 65 slugs to 8 batches and 44 to a heel batch.

Run 15: Where necessary, assigned the average of reported slugs/batch values from non-heel Run 15 batches. Average of 5 values $=57$ eff. slugs.

Run 16: Report says 10 batches at average 62.0 effective slugs dissolved per batch. Ops. log indicates 12 batches. Assigned 62 effective slugs to each batch for which we do not have an analysis value.

Run 17: ORNL-246 Special says 892 slugs were dissolved. $12 \times 64$ accounts for 768 ; the remaining 124 slugs were divided among the 4 final "heel" batches ( 31 to each).

Run 19: ORNL-246 Special says 905 slugs were dissolved. Available analyses account for 842 . $905-842=63$ slugs assigned to final Batch $19 \mathrm{~N}$. 


\section{Appendix 3-H: Details of Batches for which Numbers of Slugs Dissolved were Estimated}

Run 20: For Batches 20-P and 20-Q, set sluge dissolved equal to the average for the previous three non-heel batches.

Batch 22-V: Assumed number of slugs dissolved was equal to $50 \%$ of the average reported effective slugs per batch value for the run (66.1).

Batch 23-N: Assumed equal to $25 \%$ of the average reported effective slugs per batch value for the run (70.8). Analytical report says "N-Heels" and "-_" in the Slugs column.

Run 25: Analytical data per ORNL-CF-48-7-152, a 7/13/48 memo from Wyatt to Emlet. Poor copy- some values difficult to read, but batches add up to correct total.

Run 26: Analytical data indicate 1215 effective slugs were dissolved.

Batch 39-B: Assumed equal to average of effective slugs dissolved values for preceeding and subsequent batches.

Run 51: No slugs dissolved value given for heels. Total charged $=107$, minus 106.34 by analysis leaves $\sim 1$ effective slug for the heel batch.

Run 54: The 184 4" Hanford and 90 8" Hanford slugs used were apportioned according to distribution of $\mathrm{Ci}$ of $\mathrm{Ba}$ dissolved in batches $A$ thru $\mathrm{J}$ (A thru I from CF53-12-19, J from Ops log A-453 11/20/53 Swing shift). For each batch, the fraction of total Ba dissolved was multiplied times 184 to estimate the number of 4 "W slugs dissolved and by 90 to estimate the number of 8 "W slugs dissolved.

Run 55: The Ops. log indicates 197 slugs were charged to the dissolver. 75.42 are accounted for in analysis results we have. The remaining 121.58 were assigned evenly to the other 6 batches.

Run 56: The April 30, 1954 memo from Stanley to Larson indicates that three dissolvings of about 18 slugs each had occurred before the 4th cut was started. Logs show that 161 slugs were charged on 4/26 and 4/27 (this conflicts with Stanley's memo, which says 101 Hanford slugs were loaded). Assumed 18 slugs dissolved in Batches A-H, 17 in Batch 56-I. Releases from the Batch 56-D accident are handled separately.

Run 57: ORNL-CF-54-8-56 indicates 69.7 effective 8" Hanford slugs were dissolved. Analytical data is in Ops. logs for two batches; the remaining 37.65 effective slugs were apportioned to the other 3 batches as follows: $40 \%$ to each main batch and $20 \%$ to the heel batch.

Run 58: The Ops. log indicates 86 slugs were loaded. The Ops. $\log$ indicates Ci Ba for 4 of the 5 batches as 9829 , $9705,8291, \ldots, 5325$. A value of $7000 \mathrm{Ci}$ was estimated for Batch D to follow the observed trend. The 86 slugs reported dissolved were then apportioned to the 5 batches according to the fraction of total $\mathrm{Ci} \mathrm{Ba}$ dissolved in each.

Run 59: ORNL-CF-55-4-10 states that 86.6 effective 8" Hanford slugs were dissolved. The Ops. log gives Ci Ba and effective slugs values for Batch 59-A; this was used to determine $\mathrm{Ci} B$ a per slug as $7922 / 18.9=419.15$. Used this value to convert $7475 \mathrm{Ci} B a$ for Batch $59-\mathrm{B}$ to 17.8 slugs and $4860 \mathrm{Ci} \mathrm{Ba}$ for Batch $59-\mathrm{E}$ to 11.6 slugs. This left 38.3 slugs from the total 86.6 slugs; which were divided evenly across Batches $59-C$ and $-D$.

Run 60: ORNL-CF-55-5-82 indicates that 84.4 effective 8" Hanford slugs were dissolved. The Ops. logs attribute 18.05 slugs to Batch $60-A$ and 13.12 slugs to Batch $60-E$. This left 53.15 out of the 84.4 ; there were divided evenly between Batches 60-B, C, and D.

Run 61: ORNL-CF-55-8-7 indicates that 4 effective 8" Hanford slugs were dissolved. $50 \mathrm{X}$ slugs were also used, but they were "old ones". The Ops. log attributes $1423 \mathrm{Ci} B$ a to Batch 61-A and $870 \mathrm{Ci} B a$ to Batch 61-B (heels).

Assigned the 4 slugs to Batches 61-A and -B in same proportions. 


\section{Appendix 3-H: Details of Batches for which Numbers of Slugs Dissolved were Estimated}

Run 62: ORNL-CF-55-10-12 indicates that 67.6 effective 8" Hanford slugs were dissolved. The Ops. log gives the values shown for Batches 62-A, B, and C. They sum to 48.94, leaving 18.66 from the 67.6 for Batch 62-D.

Run 63: Logs show 89 slugs were charged. The Ops. $\log$ gives $\mathrm{Ci}$ Ba for Batches 63-A, B, C, and D. Assumed $90 \%$ of charged slugs ( 80 slugs) were dissolved in the 4 batches and $10 \%$ ( 9 slugs) in the heel batch. Assigned the 80 slugs to the 4 batches in same proportions as the $\mathrm{Ci}$ of $\mathrm{Ba}$ dissolved in each (i.e., 11,350;10,940;9.679;9,666).

Run 64: ORNL-CF-56-2-18 indicates that 93 effective 8" Hanford slugs were dissolved. Assumed $90 \%$ were dissolved in 4 batches and $10 \%$ in the heel batch.

Run 65: ORNL-CF-56-4-63 indicates that 71 8" Hanford slugs were dissolved. Assumed $90 \%$ were dissolved in the 3 batches and $10 \%$ were dissolved in the final batch.

Run 66: ORNL-CF-56-6-49 indicates that 85.3 effective 8" Hanford slugs were dissolved. Assumed $90 \%$ were dissolved in the first 3 batches and $10 \%$ in the final batch.

Run 67: ORNL-CF-56-9-104 indicates that 129.2 effective 8" Hanford slugs were dissolved. Assumed $90 \%$ were dissolved in the first 6 batches and $10 \%$ in the final batch.

Run 68: ORNL-CF-56-11-28 indicates that 71.8 effective 8 " Hanford slugs were dissolved. Assumed $90 \%$ were dissolved in the first 3 batches and $10 \%$ in the final batch. 
TASK 1 REPORT

July 1999

${ }^{131}$ I Releases from X-10 Radioactive Lanthanum Processing -

Page 3H-6

Appendices

This page intentionally left blank. 
Marietta, GA 30068

Phone: (770) 509-7606

FAX: (770) 509-7507

Internet: sra@crl.com

TO: $\quad$ Tom Widner

April 30, 1997

ChemRisk - Alameda

FROM: $\quad$ R. E. Burns, Jr.

Shonka Research Associates, Inc.

SUBJECT: Final inventory values for $\mathrm{X}$-slugs

MEMO NO: REB.002 C97

FINAL [X] DRAFT [ ]

Distribution:

Talaat Ijaz - ChemRisk - Cleveland

Joe Shonka - Shonka Research Associates, Inc.

References:

1. "Evaluation of an Expression for Computing the Relative Thermal Neutron Flux in Clinton Pile Fuel Channels Used for RaLa Production" SRA-95-003, Rev. 0 Shonka Research Associates, Inc. August, 1995.

2. "Fuel Elements For ORNL Graphite Reactor", memo from E. J. Boyle and C. D. Cagle to D. H. Gurinsky, ORNL central files number 52-6-10, June 3, 1952.

3. Cagle, C. D.; "The Oak Ridge National Laboratory Graphite Reactor", ORNL central files number 53-12-126, December, 1953.

4. "Clinton Pile Operating Manual", ORNL central files number 45-6-413, June, 1945.

5. Jones, H.; Watson, L. B.; Arnette, T.; Coveyou, I. "Neutron Distribution in the Clinton Pile" CP 2602, Oak Ridge National Laboratory, Oak Ridge, TN, February 27, 1945.

6. "Yield Calculations for $(\mathrm{n}, \gamma)$ Activities Produced in the Clinton Pile", memo to file from R. T. Overman, J. W. Jones and L. M. Gunning; ORNL central files number 47-4-21, April 23, 1947. 
7. Hsu, H. H.; Clement, R. S.; "Monte Carlo Calculations of LANL Graphite Pile Response Functions" Health Physics, Supplement to Vol. 70, No. 6, p S30, June, 1996.

8. Heeb, C. M.; "Uncertainties in Source Term Calculations Generated by the ORIGEN2 Computer Code for Hanford Production Reactors" PNL-7223 HEDR, Pacific Northwest Laboratory, March, 1991.

Memo:

This memo describes briefly the final calculations performed to establish the average inventory per slug at discharge for X-slugs irradiated in the Clinton pile for RaLa production, including treatment of uncertainties.

\section{Inventory Calculations}

Inventory calculations for $\mathrm{X}$-slugs proceeded as follows:

1. establish the operating power level for the pile for each eight hour shift from $7 / 27 / 44$ to $12 / 5 / 47$

2. establish the charge and discharge dates for all slugs pushed for each RaLa run

3. for each charge and discharge date, establish the shift on which the slugs were charged and discharged

4. calculate the peak slug inventory for each group pushed for each run

5. establish the position factors for each slug in each group pushed

6. sum up the total inventory for each group pushed for each run for selected isotopes (in terms of both grams and curies)

7. sum up total inventories for each isotope for each run (i.e., sum up the data for each channel)

8. divide the totals for each run by the number of slugs pushed and their mass to get the average content per slug and per kilogram of uranium

9. compute the average content per slug and per kilogram of uranium for each isotope over all RaLa runs in which X-slugs were used for which push data had been obtained

The operating power levels for the pile for each shift were computed by taking the accumulated power (in $\mathrm{kWh}$ ) recorded in the pile operations logbooks for each shift and dividing this value by 8.0 hours. This task required pile operations logbooks volumes 6 through 21. These data were compiled in a DOS file called SCHEDULE, an excerpt from which is shown in Fig. 1 below.

Charge and discharge dates for each channel pushed were taken directly from the push data for each run. There are two runs in the period from 1945 to 1947 for which we could not locate complete push data. These were runs 17 (April of 1947) and 20 (August of 1947). In addition, no push data was located for any of the X-slug runs conducted in 1948 (Runs 23 through 27). Note that in many cases the slugs pushed from a given channel for a given run were charged on different dates. When this occurred, group numbers were used to keep up with each set of slugs from the same channel that had a 
unique irradiation history. Channels for which all of the slugs had the same irradiation history were assigned the group number 1 by default.

Figure 1 Excerpt from the SCHEDULE file

\begin{tabular}{|lllll|}
\hline & SHIFT & POWER TO & AVERAGE POWER \\
DATE & SHIFT & POWER & DATE $(\mathrm{kWh})$ & FOR SHIFT (kW) \\
$7 / 27 / 44$ & $12-8$ & 24912 & 5724722 & 3114.000 \\
$7 / 27 / 44$ & $8-4$ & 7000 & 5731722 & 875.000 \\
$7 / 27 / 44$ & $4-12$ & 8942 & 5740664 & 1117.750 \\
$7 / 28 / 44$ & $12-8$ & 24905 & 5765569 & 3113.125 \\
$7 / 28 / 44$ & $8-4$ & 17655 & 5783224 & 2206.875 \\
$7 / 28 / 44$ & $4-12$ & 24160 & 5807384 & 3020.000 \\
$7 / 29 / 44$ & $12-8$ & 25146 & 5832530 & 3143.250 \\
$7 / 29 / 44$ & $8-4$ & 17206 & 5849736 & 3072.875 \\
$7 / 29 / 44$ & $4-12$ & 24583 & 5874319 & 3052.750 \\
$7 / 30 / 44$ & $12-8$ & 24422 & 5898741 & 2967.750 \\
$7 / 30 / 44$ & $8-4$ & 23742 & 5922483 & 3115.000 \\
$7 / 30 / 44$ & $4-12$ & 24268 & 5946751 & 2656.250 \\
$7 / 31 / 44$ & $12-8$ & 24920 & 5971671 & 2856.875 \\
$7 / 31 / 44$ & $8-4$ & 21250 & 5992921 & \\
$7 / 31 / 44$ & $4-12$ & 22855 & 6015776 & \\
\hline
\end{tabular}

While the push data retrieved for each run gave the charge and discharge dates for each group of slugs, it did not give the shift on which the charge or discharge took place. This information was taken from the pile operations logbooks for each group of slugs and was documented along with the push data. The complete set of charge and discharge data for all of the runs for which such was available was compiled in a DOS file called SLUGLIST. An excerpt from SLUGLIST is presented in Fig. 2 below.

Figure 2 Excerpt from the SLUGLIST file

\begin{tabular}{|llllllll|}
\hline PUSH DATA FOR & RALA RUN & 01 & & CHARGED & \multicolumn{2}{l|}{ DISCHARGED } \\
CHANNEL NO. & GROUP & SLUGS & DAYS & DATE & SHIFT & DATE & SHIFT \\
2069 & 1 & 29 & 302 & $7 / 27 / 44$ & -12 & $5 / 25 / 458-4$ \\
1967 & 1 & 30 & 232 & $10 / 5 / 448-4$ & $5 / 25 / 458-4$ \\
1968 & 1 & 30 & 232 & $10 / 5 / 448-4$ & $5 / 25 / 458-4$ \\
1969 & 1 & 30 & 232 & $10 / 5 / 448-4$ & $5 / 25 / 458-4$ \\
1970 & 1 & 30 & 232 & $10 / 5 / 448-4$ & $5 / 25 / 458-4$ \\
1767 & 1 & 30 & 232 & $10 / 5 / 448-4$ & $5 / 25 / 458-4$ \\
1771 & & 30 & 210 & $10 / 27 / 448-4$ & $5 / 25 / 458-4$ \\
\end{tabular}

After all of the needed irradiation history data had been compiled for each run (for which such was available), peak slug inventories were calculated for each group of slugs for each run. Peak slug inventory refers to the inventory that would be calculated for a slug located at the point of maximum flux in the Clinton pile that had the same irradiation history (time of charge and discharge) as a slug group of interest. The peak slug approach was chosen because it was best for calculating inventories for individual slugs based on the quantitative data we had for the Clinton pile. Specifically, we had a factor with which to convert from total pile power to peak flux and we had an expression for computing the relative flux anywhere in the pile with respect to the peak flux. Hence, all inventory calculations 
performed for X-slugs were carried out by computing the inventory for a slug located at the point of maximum flux, and then scaling this result down for the actual point of interest using the relative flux equation.

Peak slug inventories were computed using the ORIGEN2.1 computer code. A boiler-plate input file was created that computed the fission product inventories in both grams and curies for a single X-slug (1175 grams of natural uranium) using the CANDUNAU cross-section and fission product yield library file distributed with the code. The uranium was irradiated in eight hour increments starting with the shift on which a group of interest was charged and ending with the shift on which the slugs were discharged. For each eight hour increment, the fuel was irradiated at a flux level corresponding to the peak flux associated with the operating power level for the pile for that particular shift. Power levels were converted to flux using the factor $3.102 \times 10^{5}$ neutrons $\mathrm{cm}^{-2}$ second $^{-1}$ watt ${ }^{-1}$ to compute thermal flux, and then multiplying the thermal flux by 3.16 to get total flux for the CANDUNAU model. The basis for power-to-peak-flux factor is given in the section of this memo discussing its associated uncertainty. Calculation of the 3.16 conversion factor is described in SRA calculation SRA-95-003, Rev. 0 [1].

A computer program called ORIGENRS was developed to complete the task of running ORIGEN for each group of slugs for each RaLa run that had a unique irradiation history. Note that not every group of slugs for a given run had a unique history, as many channels would be charged at the same time. Hence, in many cases, a single calculation would provide the needed peak slug data for several groups of slugs. The purpose of the ORIGENRS program was to automate generation of the input files for each ORIGEN calculation and to extend the capability of the code to compute ingrowth over thousands of irradiation steps by running the code in a sequential manner, with the output from a previous run acting as the input for the next. ORIGEN is normally limited to a total of 150 irradiation steps in any given run. In the case of the calculations for Task 1 (where the fuel was irradiated in eight hour increments), this limited us to a 50 day irradiation duration for a single run, which was not sufficient in most cases. The ORIGENRS program read the irradiation history data from the SCHEDULE and SLUGLIST files for a given group of slugs, generated an ORIGEN input file for the first 40 days of irradiation, ran ORIGEN and then used the inventory data generated as the starting inventory in the input for the next 40 days. This cycle was repeated until the total irradiation duration was reached. In each case, ORIGEN was configured to give inventory results at the end of the irradiation cycle and at four hour increments thereafter up through 24 hours of decay time.

After all of the peak slug inventory calculations had been completed, a macro was written in the text editor program BRIEFTM to strip out the desired data and save it in DOS files having a standard format. The data stripped out were grams of each of the species ${ }^{127} \mathrm{I}$ (stable), ${ }^{129} \mathrm{I},{ }^{131} \mathrm{I},{ }^{132} \mathrm{I},{ }^{133} \mathrm{I},{ }^{134} \mathrm{I},{ }^{135} \mathrm{I},{ }^{140} \mathrm{Ba}$, total iodine, and total barium; and curies of these same species with the exception of ${ }^{127} \mathrm{I}$. Following this, another computer program was written that read these data files and inserted the data into spreadsheet files that summarized the peak slug inventory data for each group of slugs pushed for each run. These spreadsheets are 23 columns wide, so only a small portion of one of them (for RaLa Run $01)$ is presented in Fig. 3 below. 
Figure 3 Portion of a peak slug inventory spreadsheet

\begin{tabular}{|c|c|c|c|c|c|c|c|c|}
\hline \multirow{2}{*}{$\begin{array}{l}\text { Channel } \\
\text { Number }\end{array}$} & \multirow[b]{2}{*}{ Group } & \multirow{2}{*}{$\begin{array}{l}\text { Number } \\
\text { of Slugs }\end{array}$} & \multicolumn{2}{|c|}{ I-127 } & \multicolumn{2}{|c|}{$1-129$} & \multicolumn{2}{|c|}{$1-131$} \\
\hline & & & grams & Curies & grams & Curies & grams & Curies \\
\hline 2069 & 1 & 29 & 9.723E-05 & $N / A$ & 4.365E-04 & 7.709E-08 & 8.083E-05 & $1.002 E+01$ \\
\hline 1967 & 1 & 30 & 7.706E-05 & $N / A$ & 3.499E-04 & $6.180 \mathrm{E}-08$ & 8.060E-05 & $9.996 \mathrm{E}+00$ \\
\hline 1968 & 1 & 30 & 7.706E-05 & $N / A$ & 3.499E-04 & $6.180 \mathrm{E}-08$ & 8.060E-05 & $9.996 \mathrm{E}+00$ \\
\hline 1969 & 1 & 30 & 7.706E-05 & $N / A$ & 3.499E-04 & $6.180 \mathrm{E}-08$ & 8.060E-05 & $9.996 \mathrm{E}+00$ \\
\hline 1970 & 1 & 30 & 7.706E-05 & $N / A$ & 3.499E-04 & $6.180 \mathrm{E}-08$ & 8.060E-05 & $9.996 \mathrm{E}+00$ \\
\hline 1767 & 1 & 30 & 7.706E-05 & $\mathrm{N} / \mathrm{A}$ & 3.499E-04 & $6.180 \mathrm{E}-08$ & 8.060E-05 & $9.996 \mathrm{E}+00$ \\
\hline 1771 & 1 & 30 & 6.919E-05 & $\mathrm{N} / \mathrm{A}$ & 3.157E-04 & 5.576E-08 & $8.052 E-05$ & $9.986 E+00$ \\
\hline
\end{tabular}

After the peak slug inventories for each group had been compiled in spreadsheets, the next step was to define the positions of the slugs pushed from each channel for each run. This is straightforward in all but two cases, as the slugs pushed from a given channel were assumed to have been loaded symmetrically (in the axial direction) about the center of the channel. However, for RaLa Runs 01 and 02, not all of the slugs in some of the channels were pushed. Hence, the slug positions had to be chosen to account for the fact that only the west-most slugs were used in these cases. For the purpose of the final X-slug inventory calculations, all channels were assumed to have been charged in the previously named loadcentered configuration. Recall that the numbering convention for slug positions is that east slugs are numbered starting with - 1 for the first slug east of channel center and west slugs are numbered starting with 1 for the first slug west of center. For each group of slugs for a given channel, slug positions were chosen to reflect the position of the slugs in each group at the time of discharge.

After positions had been established for all of the slugs, the total discharge inventory for each group was computed for each run. A computer program was written that summed up the relative flux values for each slug in a given group and multiplied this sum by the corresponding peak slug inventory for that group. This product was then multiplied by a factor of 0.633 to correct for the fact the peak slug inventory is based on the flux corresponding to a pile experimental hole and not fuel. Two sets of spreadsheets were used for these calculations: one set for results in grams and one for results in curies. However, these are so large that not even an excerpt can be shown here.

After the total inventories for each group had been established for each run, a spreadsheet was created to sum up these data to provide totals for each run. The totals for each run were then divided by both the number of slugs discharged and their total mass to get the average content for each run per slug and per kilogram of uranium. (Mass was computed using the value $1.175 \mathrm{~kg}$ uranium per slug.) Finally, the average content data were averaged over all runs to obtain single values that could be used to establish the quantity of selected isotopes in a given number of slugs or mass of uranium for any RaLa campaign in which X-slugs were used. These results are provided in Figures 4 through 9 below. 
Figure 4 Total inventories in grams for X-slug RaLa runs

\begin{tabular}{|c|c|c|c|c|c|c|c|c|c|c|c|c|}
\hline \multirow[b]{2}{*}{$\begin{array}{c}\text { Run } \\
\text { Number }\end{array}$} & \multirow[b]{2}{*}{$\begin{array}{l}\text { Total } \\
\text { Slugs }\end{array}$} & \multirow[b]{2}{*}{$\begin{array}{c}\text { Total } \\
\text { Mass (kg) }\end{array}$} & \multicolumn{10}{|c|}{ Total Discharge Inventory (grams) for Run } \\
\hline & & & $\underline{\mathbf{1} 127}$ & $\underline{1-129}$ & $\underline{\mid-131}$ & $\underline{\mathbf{1}-132}$ & $\underline{1-133}$ & $\underline{\mathbf{1} 134}$ & $\underline{\mathbf{l}-135}$ & $\underline{\text { Ba-140 }}$ & $\begin{array}{r}\text { Total } \\
\text { lodine }\end{array}$ & $\begin{array}{c}\text { Total } \\
\text { Barium }\end{array}$ \\
\hline 1 & 209 & 245.575 & $7.572 \mathrm{E}-03$ & $3 . \overline{433 \mathrm{E}-02}$ & $7.733 \mathrm{E}-03$ & $1.366 \mathrm{E}-04$ & $1.690 \mathrm{E}-03$ & $1.916 \mathrm{E}-05$ & $3.467 \mathrm{E}-04$ & $2 . \overline{764 \mathrm{E}-02}$ & $5 . \overline{183 \mathrm{E}-02}$ & $3 . \overline{837 \mathrm{E}-01}$ \\
\hline 2 & 657 & 771.975 & $2.010 \mathrm{E}-02$ & $9.104 \mathrm{E}-02$ & $2.046 \mathrm{E}-02$ & 3.497E-04 & $4.940 \mathrm{E}-03$ & $1.724 \mathrm{E}-04$ & $1.371 \mathrm{E}-03$ & $7.536 \mathrm{E}-02$ & $1.384 \mathrm{E}-01$ & $1.022 \mathrm{E}+00$ \\
\hline 3 & 845 & 992.875 & $1.804 \mathrm{E}-02$ & $8.329 \mathrm{E}-02$ & 3.374E-02 & $6.229 \mathrm{E}-04$ & $8.551 \mathrm{E}-03$ & 2.663E-04 & $2.289 \mathrm{E}-03$ & 1.149E-01 & $1.468 \mathrm{E}-01$ & $9.904 \mathrm{E}-01$ \\
\hline 4 & 1182 & 1388.850 & $3.164 \mathrm{E}-02$ & $1.449 \mathrm{E}-01$ & 4.733E-02 & 8.536E-04 & $1.164 \mathrm{E}-02$ & $3.240 \mathrm{E}-04$ & $3.008 \mathrm{E}-03$ & $1.675 \mathrm{E}-01$ & 2.397E-01 & $1.686 \mathrm{E}+00$ \\
\hline 5 & 1054 & 1238.450 & $1.341 \mathrm{E}-02$ & 6.347E-02 & 3.954E-02 & $6.946 \mathrm{E}-04$ & $9.758 \mathrm{E}-03$ & 3.365E-04 & $2.743 \mathrm{E}-03$ & 1.39 & $1.300 \mathrm{E}-01$ & $8.235 E-01$ \\
\hline 6 & 816 & 958.800 & $1.475 \mathrm{E}-02$ & 6.922E-02 & 3.383E-02 & 6.047E-04 & $8.590 \mathrm{E}-03$ & 3.058E-04 & $2.426 \mathrm{E}-03$ & 1.215E-01 & 1.297E-01 & 8.622E-01 \\
\hline 7 & 933 & 1096.275 & $2.160 \mathrm{E}-02$ & $9.966 \mathrm{E}-02$ & $3.531 \mathrm{E}-02$ & $6.318 \mathrm{E}-04$ & 8.991E-03 & 3.576E-04 & $2.696 \mathrm{E}-03$ & $1.284 \mathrm{E}-01$ & $1.693 \mathrm{E}-01$ & $1.181 \mathrm{E}+00$ \\
\hline 8 & 1791 & 2104.425 & 4.950E-02 & $2.251 \mathrm{E}-01$ & 7.102E-02 & $1.321 \mathrm{E}-03$ & 1.891E-02 & 7.266E-04 & $5.481 \mathrm{E}-03$ & 2.464E-01 & $3.721 \mathrm{E}-01$ & $2.594 \mathrm{E}+00$ \\
\hline 9 & 897 & 1053.975 & $1.601 \mathrm{E}-02$ & 7.474E-02 & $3.890 \mathrm{E}-02$ & $7.021 \mathrm{E}-04$ & $9.824 \mathrm{E}-03$ & $3.911 \mathrm{E}-04$ & $2.868 \mathrm{E}-03$ & E-01 & $1.434 \mathrm{E}-01$ & $9.341 \mathrm{E}-01$ \\
\hline 10 & 866 & 1017.550 & 2.188E-02 & $1.008 \mathrm{E}-01$ & 3.672E-02 & 6.557E-04 & $9.269 \mathrm{E}-03$ & 3.777E-04 & $2.732 \mathrm{E}-03$ & 1.328E-01 & $1.725 \mathrm{E}-01$ & $1.197 \mathrm{E}+00$ \\
\hline 11 & 916 & 1076.300 & $1.464 \mathrm{E}-02$ & 6.849E-02 & $3.806 \mathrm{E}-02$ & $6.533 \mathrm{E}-04$ & 8.685E-03 & 4.033E-04 & $2.670 \mathrm{E}-03$ & 1.363E-01 & $1.336 \mathrm{E}-01$ & $8.710 \mathrm{E}-01$ \\
\hline 12 & 916 & 1076 & 1.495E-02 & $E-02$ & 3.374E-02 & $6.054 \mathrm{E}-04$ & $8.470 \mathrm{E}-03$ & 3.253E-04 & 2.437E-03 & 1.2 & $1.300 \mathrm{E}-01$ & $8.602 \mathrm{E}-01$ \\
\hline 13 & 819 & 962.325 & 2.386E-02 & $1.071 \mathrm{E}-01$ & $2.980 \mathrm{E}-02$ & $5.358 \mathrm{E}-04$ & $7.608 \mathrm{E}-03$ & $3.245 \mathrm{E}-04$ & $2.258 \mathrm{E}-03$ & $1.070 \mathrm{E}-01$ & $1.715 \mathrm{E}-01$ & $1.220 \mathrm{E}+00$ \\
\hline 14 & 643 & 755.525 & $1.721 \mathrm{E}-02$ & 7.897E-02 & 2.459E-02 & 4.214E-04 & $4.868 \mathrm{E}-03$ & $1.441 \mathrm{E}-04$ & $1.046 \mathrm{E}-03$ & $8.926 \mathrm{E}-02$ & $1.273 \mathrm{E}-01$ & $9.180 \mathrm{E}-01$ \\
\hline 15 & 819 & 962. & 1.662E-02 & 7.767E-02 & $3.511 \mathrm{E}-02$ & $6.403 E-04$ & 8.769E-03 & 3.483E-04 & $2.490 \mathrm{E}-03$ & 1.2 & 1.417E-01 & 9.445E-01 \\
\hline $15 \mathrm{~A}$ & 592 & 695.600 & 2.152E-02 & $9.698 \mathrm{E}-02$ & 2.395E-02 & $4.370 \mathrm{E}-04$ & $6.118 \mathrm{E}-03$ & 2.169E-04 & $1.702 \mathrm{E}-03$ & 8.587E-02 & $1.509 \mathrm{E}-01$ & $1.092 \mathrm{E}+00$ \\
\hline 16 & 784 & 921.200 & 1.365E-02 & 6.358E-02 & 3.107E-02 & $5.480 \mathrm{E}-04$ & 7.640E-03 & 2.894E-04 & 2.199E-03 & 1.115E-01 & 1.190E-01 & 7.896E-01 \\
\hline 18 & 916 & 1076.300 & 5.011E-02 & $2.180 \mathrm{E}-01$ & 3.459E-02 & $6.182 \mathrm{E}-04$ & 8.549E-03 & 3.139E-04 & $2.429 \mathrm{E}-03$ & 1.2 & $3.146 \mathrm{E}-01$ & $2.343 \mathrm{E}+00$ \\
\hline 19 & 889 & 1044.575 & 3.405E-02 & $1.488 \mathrm{E}-01$ & $3.157 \mathrm{E}-02$ & $5.291 \mathrm{E}-04$ & 6.707E-03 & $2.416 \mathrm{E}-04$ & $1.900 \mathrm{E}-03$ & 1.152E-01 & $2.238 \mathrm{E}-01$ & $1.641 \mathrm{E}+00$ \\
\hline 21 & 1181 & 1387.675 & 1.457E-02 & 7.003E-02 & 4.688E-02 & 8.503E-04 & 1.192E-02 & 4.910E-04 & $3.510 \mathrm{E}-03$ & 1.647E-01 & $1.483 \mathrm{E}-01$ & $9.261 \mathrm{E}-01$ \\
\hline $22 / 22 \mathrm{~A}$ & 1279 & 1502.825 & $2.422 \mathrm{E}-02$ & 1.113E-01 & 4.774E-02 & $8.652 \mathrm{E}-04$ & 1.265E-02 & $5.275 \mathrm{E}-04$ & 3.792E-03 & 1.682E-01 & $2.011 \mathrm{E}-01$ & $1.343 \mathrm{E}+00$ \\
\hline
\end{tabular}

Figure 5 Total inventories in curies for $\mathrm{X}$-slug RaLa runs

\begin{tabular}{|c|c|c|c|c|c|c|c|c|c|c|c|c|}
\hline \multirow[b]{2}{*}{$\begin{array}{c}\begin{array}{c}\text { Run } \\
\text { Number }\end{array} \\
\end{array}$} & \multirow[b]{2}{*}{$\begin{array}{l}\text { Total } \\
\text { Slugs }\end{array}$} & \multirow[b]{2}{*}{$\begin{array}{c}\text { Total } \\
\text { Mass }(\mathrm{kg}) \\
\end{array}$} & \multicolumn{10}{|c|}{ Total Discharge Inventory (curies) for Run } \\
\hline & & & $\underline{\mathrm{I}-127}$ & $\underline{\mathbf{1}-129}$ & I-131 & $\underline{\mathrm{I}-132}$ & I-133 & I-134 & $\underline{\mathrm{l}-135}$ & Ba-140 & $\begin{array}{r}\text { Total } \\
\text { lodine } \\
\end{array}$ & $\begin{array}{c}\begin{array}{c}\text { Total } \\
\text { Barium }\end{array} \\
\end{array}$ \\
\hline 1 & 209 & 245.575 & $\overline{\mathrm{N} / \mathrm{A}}$ & $6 . \overline{064 \mathrm{E}-06}$ & $9.5 \overline{90 \mathrm{E}+02}$ & $1 . \overline{411 \mathrm{E}+03}$ & $1.9 \overline{16 E}+03$ & $5.1 \overline{13 E}+02$ & $1.218 \mathrm{E}+03$ & $2 . \overline{016 \mathrm{E}+03}$ & $6 . \overline{863 E+03}$ & $4 . \overline{277 \mathrm{E}+03}$ \\
\hline 2 & 657 & 771.975 & $\mathrm{~N} / \mathrm{A}$ & $1.608 \mathrm{E}-05$ & $2.537 \mathrm{E}+03$ & $3.611 \mathrm{E}+03$ & $5.598 \mathrm{E}+03$ & $4.600 \mathrm{E}+03$ & $4.815 \mathrm{E}+03$ & $5.498 \mathrm{E}+03$ & $2.897 \mathrm{E}+04$ & $2.588 \mathrm{E}+04$ \\
\hline 3 & 845 & 992.875 & $N / A$ & $1.471 \mathrm{E}-05$ & $4.185 \mathrm{E}+03$ & $6.433 \mathrm{E}+03$ & $9.691 \mathrm{E}+03$ & $7.107 \mathrm{E}+03$ & $8.041 \mathrm{E}+03$ & $8.382 \mathrm{E}+03$ & $4.754 \mathrm{E}+04$ & $3.985 E+04$ \\
\hline 4 & 1182 & 1388.850 & $N / A$ & $2.560 \mathrm{E}-05$ & $5.870 E+03$ & $8.815 \mathrm{E}+03$ & $1.320 \mathrm{E}+04$ & $8.647 E+03$ & $1.057 \mathrm{E}+04$ & $1.222 \mathrm{E}+04$ & $6.176 \mathrm{E}+04$ & $5.049 \mathrm{E}+04$ \\
\hline 5 & 1054 & 1238.450 & $\mathrm{~N} / \mathrm{A}$ & $1.121 \mathrm{E}-05$ & $4.904 \mathrm{E}+03$ & $7.172 \mathrm{E}+03$ & $1.106 \mathrm{E}+04$ & $8.981 \mathrm{E}+03$ & $9.636 \mathrm{E}+03$ & $1.018 \mathrm{E}+04$ & $5.704 \mathrm{E}+04$ & $4.996 \mathrm{E}+04$ \\
\hline 6 & 816 & 958.800 & $\mathrm{~N} / \mathrm{A}$ & $1.222 \mathrm{E}-05$ & $4.195 \mathrm{E}+03$ & $6.244 \mathrm{E}+03$ & $9.735 E+03$ & $8.162 E+03$ & $8.523 E+03$ & $8.863 E+03$ & $5.074 \mathrm{E}+04$ & $4.501 E+04$ \\
\hline 7 & 933 & 1096.275 & $\mathrm{~N} / \mathrm{A}$ & $1.760 \mathrm{E}-05$ & $4.379 \mathrm{E}+03$ & $6.524 \mathrm{E}+03$ & $1.019 \mathrm{E}+04$ & $9.544 \mathrm{E}+03$ & $9.470 \mathrm{E}+03$ & $9.364 \mathrm{E}+03$ & $5.636 \mathrm{E}+04$ & $5.164 \mathrm{E}+04$ \\
\hline 8 & 1791 & 2104.425 & N/A & $3.976 \mathrm{E}-05$ & $8.808 \mathrm{E}+03$ & $1.364 \mathrm{E}+04$ & $2.143 E+04$ & $1.939 \mathrm{E}+04$ & $1.925 \mathrm{E}+04$ & $1.797 \mathrm{E}+04$ & $1.155 \mathrm{E}+05$ & $1.039 E+05$ \\
\hline 9 & 897 & 1053.975 & $\mathrm{~N} / \mathrm{A}$ & $1.320 \mathrm{E}-05$ & $4.824 \mathrm{E}+03$ & $7.251 \mathrm{E}+03$ & $1.113 E+04$ & $1.044 \mathrm{E}+04$ & $1.007 \mathrm{E}+04$ & $9.991 \mathrm{E}+03$ & $6.151 \mathrm{E}+04$ & $5.624 \mathrm{E}+04$ \\
\hline 10 & 866 & 1017.550 & $\mathrm{~N} / \mathrm{A}$ & $1.781 \mathrm{E}-05$ & $4.554 \mathrm{E}+03$ & $6.771 \mathrm{E}+03$ & $1.050 \mathrm{E}+04$ & $1.008 \mathrm{E}+04$ & $9.596 \mathrm{E}+03$ & $9.688 \mathrm{E}+03$ & $5.869 E+04$ & $5.435 E+04$ \\
\hline 11 & 916 & 1076.300 & N/A & $1.210 \mathrm{E}-05$ & $4.721 \mathrm{E}+03$ & $6.745 \mathrm{E}+03$ & $9.841 \mathrm{E}+03$ & $1.077 \mathrm{E}+04$ & $9.380 \mathrm{E}+03$ & $9.941 \mathrm{E}+03$ & $5.985 E+04$ & $5.768 \mathrm{E}+04$ \\
\hline 12 & 916 & 1076.300 & $\mathrm{~N} / \mathrm{A}$ & 1.227E-05 & $4.184 \mathrm{E}+03$ & $6.252 E+03$ & $9.598 \mathrm{E}+03$ & $8.682 E+03$ & $8.560 \mathrm{E}+03$ & $8.790 E+03$ & $5.207 \mathrm{E}+04$ & $4.726 \mathrm{E}+04$ \\
\hline 13 & 819 & 962.325 & $N / A$ & $1.892 \mathrm{E}-05$ & $3.695 \mathrm{E}+03$ & $5.532 \mathrm{E}+03$ & $8.622 E+03$ & $8.662 \mathrm{E}+03$ & $2 \mathrm{E}+03$ & $7.806 \mathrm{E}+03$ & $E+04$ & $4.620 \mathrm{E}+04$ \\
\hline 14 & 643 & 755.525 & $N / A$ & $1.395 \mathrm{E}-05$ & $3.049 \mathrm{E}+03$ & $4.352 \mathrm{E}+03$ & $5.518 \mathrm{E}+03$ & $3.846 \mathrm{E}+03$ & $3.674 \mathrm{E}+03$ & $6.512 \mathrm{E}+03$ & $2.701 \mathrm{E}+04$ & $2.361 \mathrm{E}+04$ \\
\hline 15 & 819 & 962.325 & $\mathrm{~N} / \mathrm{A}$ & $1.372 \mathrm{E}-05$ & $4.355 E+03$ & $6.613 E+03$ & $9.936 \mathrm{E}+03$ & $9.295 E+03$ & $8.746 E+03$ & $8.848 E+03$ & $5.479 \mathrm{E}+04$ & $5.005 E+04$ \\
\hline $15 \mathrm{~A}$ & 592 & 695.600 & $\mathrm{~N} / \mathrm{A}$ & 1.713E-05 & $2.971 \mathrm{E}+03$ & $4.512 \mathrm{E}+03$ & $6.933 E+03$ & $5.790 \mathrm{E}+03$ & $5.979 \mathrm{E}+03$ & $6.264 \mathrm{E}+03$ & $3.603 E+04$ & $3.191 \mathrm{E}+04$ \\
\hline 16 & 784 & 921.200 & $N / A$ & $1.123 \mathrm{E}-05$ & $3.853 E+03$ & $5.660 \mathrm{E}+03$ & $8.657 E+03$ & $7.724 \mathrm{E}+03$ & $7.726 \mathrm{E}+03$ & $8.132 \mathrm{E}+03$ & $4.677 \mathrm{E}+04$ & $4.236 \mathrm{E}+04$ \\
\hline 18 & 916 & 1076.300 & $N / A$ & 3.849E-05 & $4.289 \mathrm{E}+03$ & $6.383 \mathrm{E}+03$ & $9.689 \mathrm{E}+03$ & $8.376 \mathrm{E}+03$ & $8.534 \mathrm{E}+03$ & $9.052 \mathrm{E}+03$ & $5.149 \mathrm{E}+04$ & $4.615 \mathrm{E}+04$ \\
\hline 19 & 889 & 1044.575 & $\mathrm{~N} / \mathrm{A}$ & $2.629 \mathrm{E}-05$ & $3.916 \mathrm{E}+03$ & $5.463 \mathrm{E}+03$ & $7.601 \mathrm{E}+03$ & $6.449 E+03$ & $6.676 \mathrm{E}+03$ & $8.408 E+03$ & $4.105 \mathrm{E}+04$ & $3.699 \mathrm{E}+04$ \\
\hline 21 & 1181 & 1387.675 & $N / A$ & $1.237 \mathrm{E}-05$ & $5.814 \mathrm{E}+03$ & $8.780 \mathrm{E}+03$ & $1.351 \mathrm{E}+04$ & $1.310 \mathrm{E}+04$ & $1.233 E+04$ & $1.201 \mathrm{E}+04$ & $7.589 E+04$ & $7.011 \mathrm{E}+04$ \\
\hline $22 / 22 \mathrm{~A}$ & 1279 & 1502.825 & $\mathrm{~N} / \mathrm{A}$ & $1.966 \mathrm{E}-05$ & $5.921 \mathrm{E}+03$ & $8.936 \mathrm{E}+03$ & $1.433 E+04$ & $1.408 \mathrm{E}+04$ & $1.332 \mathrm{E}+04$ & $1.227 \mathrm{E}+04$ & $8.060 \mathrm{E}+04$ & $7.467 \mathrm{E}+04$ \\
\hline
\end{tabular}


Figure 6 Average inventory (grams per slug) for each RaLa run and for all runs combined

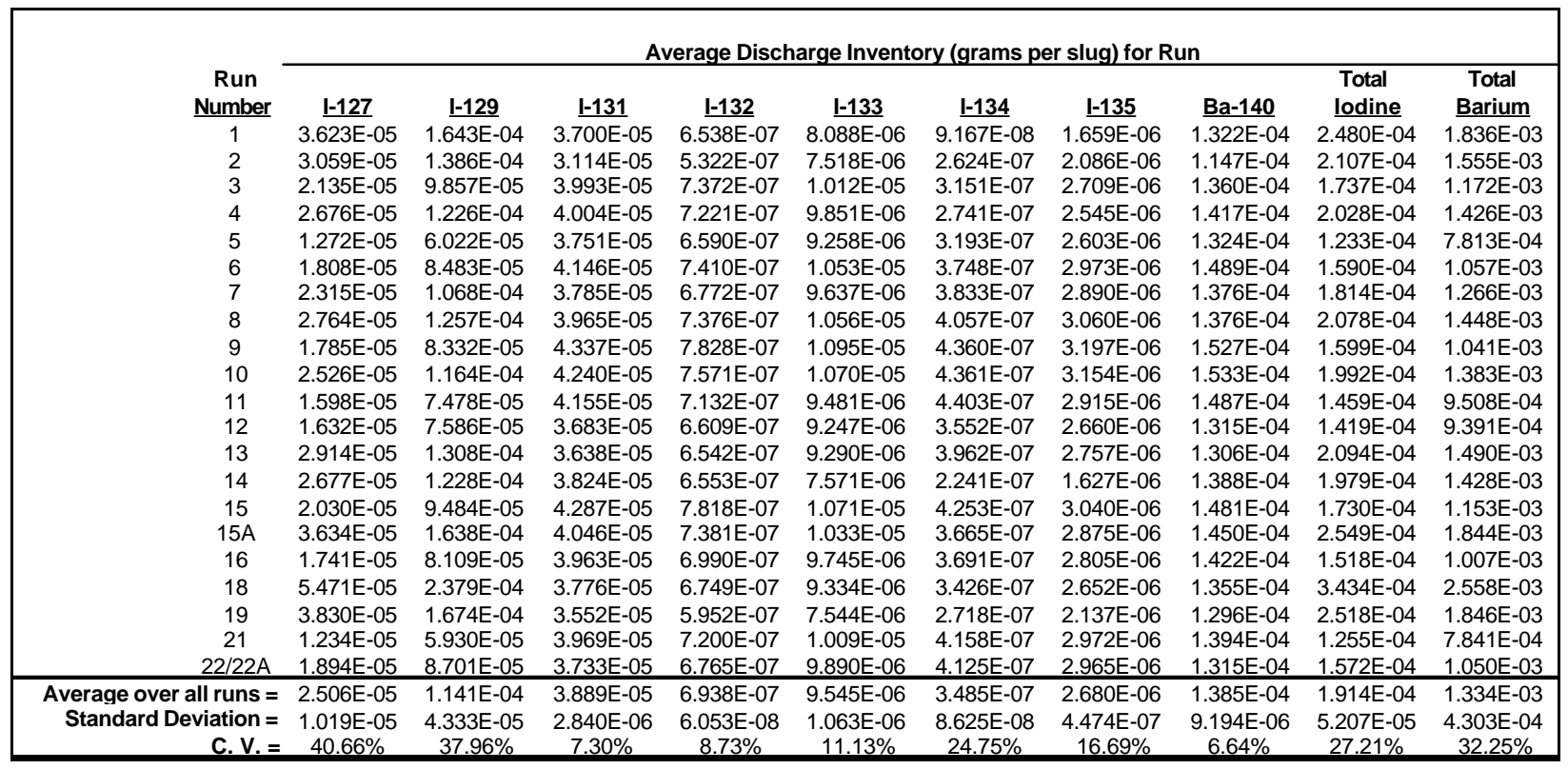

Figure 7 Average inventory (grams per kg uranium) for each RaLa run and for all runs combined

\begin{tabular}{|c|c|c|c|c|c|c|c|c|c|c|}
\hline \multirow[b]{2}{*}{$\begin{array}{c}\begin{array}{c}\text { Run } \\
\text { Number }\end{array} \\
\end{array}$} & \multicolumn{10}{|c|}{ Average Discharge Inventory (grams per kq uranium) for Run } \\
\hline & |-127 & I-129 & $\mid-131$ & $\mathrm{I}-132$ & I-133 & I-134 & I-135 & Ba-140 & $\begin{array}{l}\text { Total } \\
\text { lodine }\end{array}$ & $\begin{array}{c}\text { Total } \\
\text { Barium }\end{array}$ \\
\hline 1 & $3.083 \mathrm{E}-05$ & $1.398 \mathrm{E}-04$ & $3.149 \mathrm{E}-05$ & $5.564 \mathrm{E}-07$ & $6.884 \mathrm{E}-06$ & $7.801 \mathrm{E}-08$ & $1.412 \mathrm{E}-06$ & $1.125 \mathrm{E}-04$ & $2.111 \mathrm{E}-04$ & $1.563 \mathrm{E}-03$ \\
\hline 2 & 2.604E-05 & $1.179 \mathrm{E}-04$ & 2.650E-05 & $4.530 \mathrm{E}-07$ & 6.399E-06 & 2.233E-07 & $1.776 \mathrm{E}-06$ & $9.762 \mathrm{E}-05$ & 1.793E-04 & 1.323E-03 \\
\hline 3 & 1.817E-05 & 8.389E-05 & 3.398E-05 & $6.274 \mathrm{E}-07$ & 8.612E-06 & $2.682 \mathrm{E}-07$ & 2.305E-06 & 1.157E-04 & 1.479E-04 & 9.975E-04 \\
\hline 4 & $2.278 \mathrm{E}-05$ & $1.044 \mathrm{E}-04$ & 3.408E-05 & $6.146 \mathrm{E}-07$ & 8.384E-06 & 2.333E-07 & 2.166E-06 & $1.206 \mathrm{E}-04$ & $1.726 \mathrm{E}-04$ & $1.214 \mathrm{E}-03$ \\
\hline 5 & 1.083E-05 & $5.125 \mathrm{E}-05$ & 3.192E-05 & 5.608E-07 & 7.879E-06 & $2.717 \mathrm{E}-07$ & 2.215E-06 & 1.127E-04 & 1.049E-04 & 6.650E-04 \\
\hline 6 & $1.539 \mathrm{E}-05$ & 7.219E-05 & 3.529E-05 & 6.306E-07 & 8.960E-06 & $3.190 \mathrm{E}-07$ & $2.530 \mathrm{E}-06$ & 1.267E-04 & 1.353E-04 & 8.992E-04 \\
\hline 7 & $1.971 \mathrm{E}-05$ & $9.091 \mathrm{E}-05$ & 3.221E-05 & 5.763E-07 & 8.202E-06 & $3.262 \mathrm{E}-07$ & 2.459E-06 & $1.171 \mathrm{E}-04$ & $1.544 \mathrm{E}-04$ & 1.077E-03 \\
\hline 8 & $2.352 \mathrm{E}-05$ & $1.070 \mathrm{E}-04$ & 3.375E-05 & $6.278 \mathrm{E}-07$ & 8.988E-06 & 3.453E-07 & 2.604E-06 & $1.171 \mathrm{E}-04$ & $1.768 \mathrm{E}-04$ & 1.233E-03 \\
\hline 9 & 1.519E-05 & 7.091E-05 & 3.691E-05 & 6.662E-07 & $9.321 \mathrm{E}-06$ & $3.711 \mathrm{E}-07$ & $2.721 \mathrm{E}-06$ & $1.299 \mathrm{E}-04$ & $1.361 \mathrm{E}-04$ & 8.862E-04 \\
\hline 10 & $2.150 \mathrm{E}-05$ & $9.910 \mathrm{E}-05$ & 3.608E-05 & 6.444E-07 & $9.109 \mathrm{E}-06$ & 3.712E-07 & 2.685E-06 & $1.305 \mathrm{E}-04$ & $1.695 \mathrm{E}-04$ & 1.177E-03 \\
\hline 11 & 1.360E-05 & 6.364E-05 & 3.536E-05 & $6.070 \mathrm{E}-07$ & 8.069E-06 & 3.748E-07 & 2.481E-06 & $1.266 \mathrm{E}-04$ & $1.241 \mathrm{E}-04$ & 8.092E-04 \\
\hline 12 & 1.389E-05 & 6.456E-05 & 3.134E-05 & 5.625E-07 & 7.870E-06 & 3.023E-07 & 2.264E-06 & $1.120 \mathrm{E}-04$ & $1.208 \mathrm{E}-04$ & 7.992E-04 \\
\hline 13 & $2.480 \mathrm{E}-05$ & 1.113E-04 & 3.096E-05 & $5.568 \mathrm{E}-07$ & 7.906E-06 & 3.372E-07 & 2.347E-06 & $1.112 \mathrm{E}-04$ & $1.782 \mathrm{E}-04$ & $1.268 \mathrm{E}-03$ \\
\hline 14 & $2.278 \mathrm{E}-05$ & $1.045 \mathrm{E}-04$ & 3.254E-05 & 5.577E-07 & 6.444E-06 & 1.907E-07 & $1.385 \mathrm{E}-06$ & $1.181 \mathrm{E}-04$ & $1.684 \mathrm{E}-04$ & 1.215E-03 \\
\hline 15 & 1.727E-05 & 8.071E-05 & 3.649E-05 & 6.654E-07 & $9.112 \mathrm{E}-06$ & 3.619E-07 & 2.587E-06 & $1.260 \mathrm{E}-04$ & $1.472 \mathrm{E}-04$ & $9.815 \mathrm{E}-04$ \\
\hline $15 \mathrm{~A}$ & 3.093E-05 & $1.394 \mathrm{E}-04$ & 3.443E-05 & $6.282 \mathrm{E}-07$ & 8.795E-06 & $3.119 \mathrm{E}-07$ & 2.447E-06 & $1.234 \mathrm{E}-04$ & $2.170 \mathrm{E}-04$ & $1.569 \mathrm{E}-03$ \\
\hline 16 & $1.482 \mathrm{E}-05$ & $6.902 \mathrm{E}-05$ & 3.372E-05 & $5.949 \mathrm{E}-07$ & 8.294E-06 & $3.141 \mathrm{E}-07$ & 2.388E-06 & $1.210 \mathrm{E}-04$ & $1.292 \mathrm{E}-04$ & 8.571E-04 \\
\hline 18 & 4.656E-05 & 2.025E-04 & 3.213E-05 & 5.743E-07 & 7.943E-06 & 2.916E-07 & 2.257E-06 & 1.153E-04 & 2.923E-04 & 2.177E-03 \\
\hline 19 & $3.260 \mathrm{E}-05$ & $1.425 \mathrm{E}-04$ & 3.023E-05 & 5.065E-07 & $6.421 \mathrm{E}-06$ & 2.313E-07 & 1.819E-06 & 1.103E-04 & 2.143E-04 & $1.571 \mathrm{E}-03$ \\
\hline 21 & $1.050 \mathrm{E}-05$ & 5.047E-05 & 3.378E-05 & 6.127E-07 & $8.588 \mathrm{E}-06$ & 3.539E-07 & 2.529E-06 & 1.187E-04 & $1.068 \mathrm{E}-04$ & 6.674E-04 \\
\hline $22 / 22 \mathrm{~A}$ & $1.612 \mathrm{E}-05$ & $7.405 \mathrm{E}-05$ & 3.177E-05 & $5.757 \mathrm{E}-07$ & $8.417 \mathrm{E}-06$ & $3.510 \mathrm{E}-07$ & $2.523 \mathrm{E}-06$ & 1.119E-04 & $1.338 \mathrm{E}-04$ & 8.937E-04 \\
\hline all runs = & $2.132 \mathrm{E}-05$ & $9.714 \mathrm{E}-05$ & 3.309E-05 & $5.904 \mathrm{E}-07$ & $8.124 \mathrm{E}-06$ & $2.966 \mathrm{E}-07$ & $2.281 \mathrm{E}-06$ & 1.179E-04 & 1.629E-04 & $1.135 \mathrm{E}-03$ \\
\hline dard Deviation = & 8.670E-06 & 3.687E-05 & 2.417E-06 & $5.152 \mathrm{E}-08$ & $9.045 \mathrm{E}-07$ & $7.341 \mathrm{E}-08$ & 3.807E-07 & $7.825 \mathrm{E}-06$ & 4.432E-05 & $3.662 \mathrm{E}-04$ \\
\hline c. V. = & $40.66 \%$ & $37.96 \%$ & $7.30 \%$ & $8.73 \%$ & $11.13 \%$ & $24.75 \%$ & $16.69 \%$ & $6.64 \%$ & $27.21 \%$ & $32.25 \%$ \\
\hline
\end{tabular}


Figure 8 Average inventory (curies per slug) for each RaLa run and for all runs combined

\begin{tabular}{|c|c|c|c|c|c|c|c|c|c|c|}
\hline \multirow[b]{2}{*}{$\begin{array}{c}\text { Run } \\
\text { Number }\end{array}$} & \multicolumn{10}{|c|}{ Average Discharge Inventory (curies per slug) for Run } \\
\hline & $\underline{\mathrm{I}-127}$ & $\underline{\mathbf{l}-129}$ & $\underline{\mathbf{l}-131}$ & $\underline{\mathbf{l}-132}$ & $\underline{\mathbf{l}-133}$ & $\underline{\mathbf{I}-134}$ & $\underline{\mathbf{l}-135}$ & Ba-140 & $\begin{array}{l}\text { Total } \\
\text { lodine }\end{array}$ & $\begin{array}{c}\text { Total } \\
\text { Barium }\end{array}$ \\
\hline 1 & $\overline{N / A}$ & $2 . \overline{901 \mathrm{E}-08}$ & $4.5 \overline{89 \mathrm{E}+00}$ & $6.7 \overline{53 \mathrm{E}+00}$ & $9.1 \overline{67 \mathrm{E}+00}$ & $2.4 \overline{46 \mathrm{E}+00}$ & $5.8 \overline{29 E+00}$ & $9 . \overline{648 \mathrm{E}+00}$ & $3 . \overline{284 \mathrm{E}+01}$ & $2 . \overline{046 \mathrm{E}+01}$ \\
\hline 2 & $\mathrm{~N} / \mathrm{A}$ & $2.447 \mathrm{E}-08$ & $3.862 \mathrm{E}+00$ & $5.496 \mathrm{E}+00$ & $8.520 \mathrm{E}+00$ & $7.001 \mathrm{E}+00$ & $7.329 \mathrm{E}+00$ & $8.369 \mathrm{E}+00$ & $4.410 \mathrm{E}+01$ & $3.939 \mathrm{E}+01$ \\
\hline 3 & $\mathrm{~N} / \mathrm{A}$ & $1.741 \mathrm{E}-08$ & $4.952 \mathrm{E}+00$ & $7.613 \mathrm{E}+00$ & $1.147 \mathrm{E}+01$ & $8.411 \mathrm{E}+00$ & $9.516 \mathrm{E}+00$ & $9.920 \mathrm{E}+00$ & $5.626 \mathrm{E}+01$ & $4.716 \mathrm{E}+01$ \\
\hline 4 & $\mathrm{~N} / \mathrm{A}$ & $2.165 \mathrm{E}-08$ & $4.966 \mathrm{E}+00$ & $7.458 \mathrm{E}+00$ & $1.116 \mathrm{E}+01$ & $7.315 \mathrm{E}+00$ & $8.940 \mathrm{E}+00$ & $1.034 \mathrm{E}+01$ & $5.225 \mathrm{E}+01$ & $4.272 \mathrm{E}+01$ \\
\hline 5 & $N / A$ & $1.064 \mathrm{E}-08$ & $4.653 \mathrm{E}+00$ & $6.805 \mathrm{E}+00$ & $1.049 E+01$ & $8.521 \mathrm{E}+00$ & $9.142 \mathrm{E}+00$ & $9.660 \mathrm{E}+00$ & $5.412 \mathrm{E}+01$ & $4.740 \mathrm{E}+01$ \\
\hline 6 & $\mathrm{~N} / \mathrm{A}$ & $1.498 \mathrm{E}-08$ & $5.141 \mathrm{E}+00$ & $7.652 \mathrm{E}+00$ & $1.193 E+01$ & $1.000 \mathrm{E}+01$ & $1.045 \mathrm{E}+01$ & $1.086 \mathrm{E}+01$ & $6.219 \mathrm{E}+01$ & $5.516 \mathrm{E}+01$ \\
\hline 7 & $N / A$ & $1.887 \mathrm{E}-08$ & 4.694E+00 & $6.993 \mathrm{E}+00$ & $1.092 \mathrm{E}+01$ & $1.023 \mathrm{E}+01$ & $1.015 \mathrm{E}+01$ & $1.004 \mathrm{E}+01$ & $6.041 \mathrm{E}+01$ & $5.535 \mathrm{E}+01$ \\
\hline 8 & $\mathrm{~N} / \mathrm{A}$ & $2.220 \mathrm{E}-08$ & $4.918 \mathrm{E}+00$ & $7.617 \mathrm{E}+00$ & 1.197E+01 & $1.083 \mathrm{E}+01$ & $1.075 \mathrm{E}+01$ & $1.004 \mathrm{E}+01$ & $6.450 \mathrm{E}+01$ & $5.799 \mathrm{E}+01$ \\
\hline 9 & $\mathrm{~N} / \mathrm{A}$ & $1.472 \mathrm{E}-08$ & $5.378 \mathrm{E}+00$ & $8.083 \mathrm{E}+00$ & $1.241 \mathrm{E}+01$ & $1.164 \mathrm{E}+01$ & $1.123 E+01$ & $1.114 \mathrm{E}+01$ & $6.857 \mathrm{E}+01$ & $6.270 \mathrm{E}+01$ \\
\hline 10 & $\mathrm{~N} / \mathrm{A}$ & $2.056 \mathrm{E}-08$ & $5.258 \mathrm{E}+00$ & $7.818 \mathrm{E}+00$ & $1.213 E+01$ & $1.164 \mathrm{E}+01$ & $1.108 \mathrm{E}+01$ & $1.119 \mathrm{E}+01$ & $6.777 \mathrm{E}+01$ & $6.275 \mathrm{E}+01$ \\
\hline 11 & $\mathrm{~N} / \mathrm{A}$ & $1.321 \mathrm{E}-08$ & $5.154 \mathrm{E}+00$ & $7.364 \mathrm{E}+00$ & $1.074 \mathrm{E}+01$ & $1.175 \mathrm{E}+01$ & $1.024 \mathrm{E}+01$ & $1.085 \mathrm{E}+01$ & $6.534 \mathrm{E}+01$ & $6.297 \mathrm{E}+01$ \\
\hline 12 & $\mathrm{~N} / \mathrm{A}$ & $1.339 \mathrm{E}-08$ & $4.567 \mathrm{E}+00$ & $6.825 \mathrm{E}+00$ & $1.048 \mathrm{E}+01$ & $9.478 \mathrm{E}+00$ & $9.345 \mathrm{E}+00$ & $9.596 \mathrm{E}+00$ & $5.685 \mathrm{E}+01$ & $5.160 \mathrm{E}+01$ \\
\hline 13 & $\mathrm{~N} / \mathrm{A}$ & $2.310 \mathrm{E}-08$ & $4.512 \mathrm{E}+00$ & $6.755 \mathrm{E}+00$ & $1.053 E+01$ & $1.058 \mathrm{E}+01$ & $9.685 E+00$ & $9.531 \mathrm{E}+00$ & $6.008 \mathrm{E}+01$ & $5.640 \mathrm{E}+01$ \\
\hline 14 & $\mathrm{~N} / \mathrm{A}$ & $2.169 \mathrm{E}-08$ & $4.741 \mathrm{E}+00$ & $6.768 \mathrm{E}+00$ & $8.581 \mathrm{E}+00$ & $5.981 \mathrm{E}+00$ & $5.714 \mathrm{E}+00$ & $1.013 \mathrm{E}+01$ & $4.201 \mathrm{E}+01$ & $3.672 \mathrm{E}+01$ \\
\hline $\begin{array}{c}15 \\
15 A\end{array}$ & $\begin{array}{l}\text { N/A } \\
\text { N/A }\end{array}$ & $\begin{array}{l}1.675 \mathrm{E}-08 \\
2.893 \mathrm{E}-08\end{array}$ & $\begin{array}{l}5.317 \mathrm{E}+00 \\
5.018 \mathrm{E}+00\end{array}$ & $\begin{array}{l}8.074 \mathrm{E}+00 \\
7.621 \mathrm{E}+00\end{array}$ & $\begin{array}{l}1.213 \mathrm{E}+01 \\
1.171 \mathrm{E}+01\end{array}$ & $\begin{array}{l}1.135 \mathrm{E}+01 \\
9.780 \mathrm{E}+00\end{array}$ & $\begin{array}{l}1.068 \mathrm{E}+01 \\
1.010 \mathrm{E}+01\end{array}$ & $\begin{array}{l}1.080 \mathrm{E}+01 \\
1.058 \mathrm{E}+01\end{array}$ & $\begin{array}{l}6.690 \mathrm{E}+01 \\
6.085 \mathrm{E}+01\end{array}$ & $\begin{array}{l}6.112 \mathrm{E}+01 \\
5.390 \mathrm{E}+01\end{array}$ \\
\hline 16 & $\mathrm{~N} / \mathrm{A}$ & $1.432 \mathrm{E}-08$ & $4.914 \mathrm{E}+00$ & $7.219 \mathrm{E}+00$ & $1.104 \mathrm{E}+01$ & $9.852 \mathrm{E}+00$ & $9.855 E+00$ & $1.037 \mathrm{E}+01$ & $5.966 \mathrm{E}+01$ & $5.403 \mathrm{E}+01$ \\
\hline 18 & $\mathrm{~N} / \mathrm{A}$ & $4.202 \mathrm{E}-08$ & $4.683 \mathrm{E}+00$ & $6.968 \mathrm{E}+00$ & $1.058 \mathrm{E}+01$ & $9.144 \mathrm{E}+00$ & $9.317 \mathrm{E}+00$ & $9.882 \mathrm{E}+00$ & $5.621 \mathrm{E}+01$ & $5.038 \mathrm{E}+01$ \\
\hline 19 & $\mathrm{~N} / \mathrm{A}$ & $2.957 \mathrm{E}-08$ & 4.405E+00 & $6.145 \mathrm{E}+00$ & $8.550 \mathrm{E}+00$ & $7.254 \mathrm{E}+00$ & $7.509 E+00$ & $9.458 \mathrm{E}+00$ & $4.618 \mathrm{E}+01$ & $4.161 \mathrm{E}+01$ \\
\hline 21 & $\mathrm{~N} / \mathrm{A}$ & $1.047 \mathrm{E}-08$ & 4.923E+00 & $7.434 \mathrm{E}+00$ & $1.144 \mathrm{E}+01$ & $1.110 \mathrm{E}+01$ & $1.044 \mathrm{E}+01$ & $1.017 \mathrm{E}+01$ & $6.426 \mathrm{E}+01$ & $5.937 \mathrm{E}+01$ \\
\hline $22 / 22 \mathrm{~A}$ & $\mathrm{~N} / \mathrm{A}$ & $1.537 \mathrm{E}-08$ & $4.629 \mathrm{E}+00$ & $6.987 \mathrm{E}+00$ & $1.121 \mathrm{E}+01$ & $1.101 \mathrm{E}+01$ & $1.041 \mathrm{E}+01$ & $9.592 \mathrm{E}+00$ & $6.302 \mathrm{E}+01$ & $5.838 \mathrm{E}+01$ \\
\hline \multicolumn{2}{|c|}{ Average over all runs $=$} & $2.016 \mathrm{E}-08$ & $4.823 \mathrm{E}+00$ & $7.164 \mathrm{E}+00$ & $1.082 \mathrm{E}+01$ & $9.300 \mathrm{E}+00$ & $9.415 \mathrm{E}+00$ & $1.010 \mathrm{E}+01$ & $5.735 \mathrm{E}+01$ & $5.131 \mathrm{E}+01$ \\
\hline \multirow{2}{*}{\multicolumn{2}{|c|}{$\begin{array}{r}\text { Standard Deviation }= \\
\text { C. } \mathbf{V} .=\end{array}$}} & 7.652E-09 & 3.522E-01 & $6.251 \mathrm{E}-01$ & $1.204 \mathrm{E}+00$ & $2.302 \mathrm{E}+00$ & $1.571 \mathrm{E}+00$ & $6.709 \mathrm{E}-01$ & $9.366 \mathrm{E}+00$ & $1.056 \mathrm{E}+01$ \\
\hline & & $37.96 \%$ & $7.30 \%$ & $8.73 \%$ & $11.13 \%$ & $24.75 \%$ & $16.69 \%$ & $6.64 \%$ & $16.33 \%$ & $20.59 \%$ \\
\hline
\end{tabular}

Figure 9 Average inventory (curies per kg uranium) for each RaLa run and for all runs combined

\begin{tabular}{|c|c|c|c|c|c|c|c|c|c|c|}
\hline \multirow[b]{2}{*}{$\begin{array}{c}\text { Run } \\
\text { Number }\end{array}$} & \multicolumn{10}{|c|}{ Average Discharge Inventory (curies per kg uranium) for Run } \\
\hline & $\underline{\mid-127}$ & $\underline{\mathbf{l}-129}$ & $\mid-131$ & $\underline{\mathbf{1 - 1 3 2}}$ & $\underline{\mathbf{l}-133}$ & $\underline{\mathbf{l}-134}$ & $\underline{\mathbf{I}-135}$ & Ba-140 & $\begin{array}{l}\text { Total } \\
\text { lodine } \\
\end{array}$ & $\begin{array}{c}\text { Total } \\
\text { Barium }\end{array}$ \\
\hline 1 & $\mathrm{~N} / \mathrm{A}$ & $2.469 \mathrm{E}-08$ & $3.905 \mathrm{E}+00$ & $5.747 \mathrm{E}+00$ & $7.801 \mathrm{E}+00$ & $2.082 \mathrm{E}+00$ & $4.9 \overline{61 \mathrm{E}}+00$ & $8 . \overline{211 \mathrm{E}+00}$ & $2 . \overline{795 \mathrm{E}+01}$ & $1 . \overline{742 \mathrm{E}+01}$ \\
\hline 2 & $\mathrm{~N} / \mathrm{A}$ & $2.083 \mathrm{E}-08$ & $3.286 \mathrm{E}+00$ & 4.677E+00 & $7.251 \mathrm{E}+00$ & $5.959 E+00$ & $6.237 \mathrm{E}+00$ & $7.122 \mathrm{E}+00$ & $3.753 \mathrm{E}+01$ & $3.352 E+01$ \\
\hline 3 & $\mathrm{~N} / \mathrm{A}$ & $1.482 \mathrm{E}-08$ & $4.215 E+00$ & $6.479 \mathrm{E}+00$ & $9.761 E+00$ & $7.158 \mathrm{E}+00$ & $8.099 E+00$ & $8.442 \mathrm{E}+00$ & $4.788 \mathrm{E}+01$ & $4.014 \mathrm{E}+01$ \\
\hline 4 & $\mathrm{~N} / \mathrm{A}$ & $1.843 \mathrm{E}-08$ & $4.227 E+00$ & $6.347 \mathrm{E}+00$ & $9.502 \mathrm{E}+00$ & $6.226 \mathrm{E}+00$ & $7.609 E+00$ & $8.797 \mathrm{E}+00$ & $4.447 E+01$ & $3.636 \mathrm{E}+01$ \\
\hline 5 & $\mathrm{~N} / \mathrm{A}$ & $9.052 \mathrm{E}-09$ & $3.960 \mathrm{E}+00$ & $5.791 \mathrm{E}+00$ & $8.930 E+00$ & $7.252 \mathrm{E}+00$ & $7.780 \mathrm{E}+00$ & $8.221 \mathrm{E}+00$ & $4.606 \mathrm{E}+01$ & $4.034 \mathrm{E}+01$ \\
\hline 6 & $\mathrm{~N} / \mathrm{A}$ & $1.275 \mathrm{E}-08$ & $4.375 E+00$ & $6.512 \mathrm{E}+00$ & $1.015 E+01$ & $8.513 E+00$ & $8.889 E+00$ & $9.244 \mathrm{E}+00$ & $5.293 E+01$ & $4.694 \mathrm{E}+01$ \\
\hline 7 & $\mathrm{~N} / \mathrm{A}$ & $1.606 \mathrm{E}-08$ & $3.995 E+00$ & $5.951 \mathrm{E}+00$ & $9.295 \mathrm{E}+00$ & $8.706 \mathrm{E}+00$ & $8.639 E+00$ & $8.542 \mathrm{E}+00$ & $5.141 \mathrm{E}+01$ & $4.710 \mathrm{E}+01$ \\
\hline 8 & $\mathrm{~N} / \mathrm{A}$ & $1.889 \mathrm{E}-08$ & $4.186 \mathrm{E}+00$ & $6.483 E+00$ & $1.019 E+01$ & $9.214 \mathrm{E}+00$ & $9.148 \mathrm{E}+00$ & $8.541 \mathrm{E}+00$ & $5.490 \mathrm{E}+01$ & $4.936 \mathrm{E}+01$ \\
\hline 9 & $\mathrm{~N} / \mathrm{A}$ & $1.252 \mathrm{E}-08$ & $4.577 \mathrm{E}+00$ & $6.879 \mathrm{E}+00$ & $1.056 \mathrm{E}+01$ & $9.903 E+00$ & $9.558 \mathrm{E}+00$ & $9.479 \mathrm{E}+00$ & $5.836 \mathrm{E}+01$ & $5.336 \mathrm{E}+01$ \\
\hline $\begin{array}{l}10 \\
11\end{array}$ & $\begin{array}{l}\text { N/A } \\
\text { N/A }\end{array}$ & $\begin{array}{l}1.750 \mathrm{E}-08 \\
1.124 \mathrm{E}-08\end{array}$ & $\begin{array}{l}4.475 E+00 \\
4.386 E+00\end{array}$ & $\begin{array}{l}6.654 \mathrm{E}+00 \\
6.267 \mathrm{E}+00\end{array}$ & $\begin{array}{l}1.032 E+01 \\
9.144 E+00\end{array}$ & $\begin{array}{l}9.906 \mathrm{E}+00 \\
1.000 \mathrm{E}+01\end{array}$ & $\begin{array}{l}9.430 E+00 \\
8.715 E+00\end{array}$ & $\begin{array}{l}9.521 \mathrm{E}+00 \\
9.236 \mathrm{E}+00\end{array}$ & $\begin{array}{l}5.767 \mathrm{E}+01 \\
5.561 \mathrm{E}+01\end{array}$ & $\begin{array}{l}5.341 E+01 \\
5.359 E+01\end{array}$ \\
\hline 12 & $\mathrm{~N} / \mathrm{A}$ & $1.140 \mathrm{E}-08$ & $3.887 E+00$ & $5.809 E+00$ & $8.918 \mathrm{E}+00$ & $8.067 \mathrm{E}+00$ & $7.953 \mathrm{E}+00$ & $8.167 \mathrm{E}+00$ & $4.838 \mathrm{E}+01$ & $4.391 E+01$ \\
\hline $\begin{array}{l}13 \\
14\end{array}$ & $\begin{array}{l}\text { N/A } \\
\text { N/A }\end{array}$ & $\begin{array}{l}1.966 \mathrm{E}-08 \\
1.846 \mathrm{E}-08\end{array}$ & $\begin{array}{l}3.840 \mathrm{E}+00 \\
4.035 \mathrm{E}+00\end{array}$ & $\begin{array}{l}5.749 \mathrm{E}+00 \\
5.760 \mathrm{E}+00\end{array}$ & $\begin{array}{l}8.960 E+00 \\
7.303 E+00\end{array}$ & $\begin{array}{l}9.001 \mathrm{E}+00 \\
5.091 \mathrm{E}+00\end{array}$ & $\begin{array}{l}8.243 E+00 \\
4.863 E+00\end{array}$ & $\begin{array}{l}8.112 \mathrm{E}+00 \\
8.620 \mathrm{E}+00\end{array}$ & $\begin{array}{l}5.114 \mathrm{E}+01 \\
3.575 \mathrm{E}+01\end{array}$ & $\begin{array}{l}4.800 E+01 \\
3.125 E+01\end{array}$ \\
\hline 15 & $\mathrm{~N} / \mathrm{A}$ & $1.425 \mathrm{E}-08$ & $4.525 E+00$ & $6.872 \mathrm{E}+00$ & $1.033 E+01$ & $9.659 \mathrm{E}+00$ & $9.088 \mathrm{E}+00$ & $9.195 \mathrm{E}+00$ & $5.694 \mathrm{E}+01$ & $5.201 E+01$ \\
\hline $15 A$ & $\mathrm{~N} / \mathrm{A}$ & $2.462 \mathrm{E}-08$ & $4.271 \mathrm{E}+00$ & $6.486 \mathrm{E}+00$ & $9.967 \mathrm{E}+00$ & $8.323 E+00$ & $8.595 \mathrm{E}+00$ & $9.006 \mathrm{E}+00$ & $5.179 \mathrm{E}+01$ & $4.587 E+01$ \\
\hline 16 & $\mathrm{~N} / \mathrm{A}$ & $1.219 \mathrm{E}-08$ & $4.182 \mathrm{E}+00$ & $6.144 \mathrm{E}+00$ & $9.398 E+00$ & $8.384 \mathrm{E}+00$ & 8.387E+00 & $8.828 \mathrm{E}+00$ & $5.077 \mathrm{E}+01$ & $4.598 \mathrm{E}+01$ \\
\hline 18 & $\mathrm{~N} / \mathrm{A}$ & $3.576 \mathrm{E}-08$ & $3.985 E+00$ & $5.931 \mathrm{E}+00$ & $9.002 \mathrm{E}+00$ & $7.782 \mathrm{E}+00$ & $7.929 E+00$ & $8.410 \mathrm{E}+00$ & $4.784 \mathrm{E}+01$ & $4.288 \mathrm{E}+01$ \\
\hline 19 & $\mathrm{~N} / \mathrm{A}$ & $2.516 \mathrm{E}-08$ & $3.749 \mathrm{E}+00$ & $5.230 \mathrm{E}+00$ & $7.277 E+00$ & $6.173 E+00$ & $6.391 \mathrm{E}+00$ & $8.049 \mathrm{E}+00$ & $3.930 \mathrm{E}+01$ & $3.541 \mathrm{E}+01$ \\
\hline 21 & $\mathrm{~N} / \mathrm{A}$ & 8.913E-09 & $4.190 \mathrm{E}+00$ & $6.327 \mathrm{E}+00$ & $9.733 \mathrm{E}+00$ & $9.444 \mathrm{E}+00$ & $8.884 \mathrm{E}+00$ & $8.658 \mathrm{E}+00$ & $5.469 \mathrm{E}+01$ & $5.052 \mathrm{E}+01$ \\
\hline $22 / 22 \mathrm{~A}$ & $\mathrm{~N} / \mathrm{A}$ & $1.308 \mathrm{E}-08$ & $3.940 \mathrm{E}+00$ & $5.946 \mathrm{E}+00$ & $9.539 E+00$ & $9.368 \mathrm{E}+00$ & $8.862 \mathrm{E}+00$ & $8.164 \mathrm{E}+00$ & $5.364 \mathrm{E}+01$ & $4.969 \mathrm{E}+01$ \\
\hline \multirow{2}{*}{\multicolumn{2}{|c|}{$\begin{array}{r}\text { Average over all runs }= \\
\text { Standard Deviation }= \\
\text { C. } \text { V. }=\end{array}$}} & $1.716 \mathrm{E}-08$ & $4.104 \mathrm{E}+00$ & $6.097 \mathrm{E}+00$ & $9.206 \mathrm{E}+00$ & $7.915 \mathrm{E}+00$ & $8.012 \mathrm{E}+00$ & $8.598 \mathrm{E}+00$ & $4.881 \mathrm{E}+01$ & $4.367 \mathrm{E}+01$ \\
\hline & & $\begin{array}{c}6.512 \mathrm{E}-09 \\
37.96 \%\end{array}$ & $\begin{array}{c}2.998 \mathrm{E}-01 \\
7.30 \%\end{array}$ & $\begin{array}{c}5.320 \mathrm{E}-01 \\
8.73 \%\end{array}$ & $\begin{array}{c}1.025 \mathrm{E}+00 \\
11.13 \%\end{array}$ & $\begin{array}{c}1.959 \mathrm{E}+00 \\
24.75 \%\end{array}$ & $\begin{array}{c}1.337 \mathrm{E}+00 \\
16.69 \%\end{array}$ & $\begin{array}{c}5.710 \mathrm{E}-01 \\
6.64 \%\end{array}$ & $\begin{array}{c}7.971 \mathrm{E}+00 \\
16.33 \%\end{array}$ & $\begin{array}{c}8.990 \mathrm{E}+00 \\
20.59 \%\end{array}$ \\
\hline
\end{tabular}




\section{Treatment of Uncertainties}

The following uncertain parameters associated with the X-slug inventory calculations described above have been identified and characterized:

- variability in the mass of uranium metal contained in an individual slug

- physical constants (fission yields, recoverable energy per fission, etc.)

- pile power

- pile power-to-peak-flux conversion factor

- relative flux values

Variability in the mass of uranium metal contained in an individual slug

From 52-6-10, the original specifications for X-slugs (prior to the switch to the bonded alpha-slugs in the 1950 's) was length $=4.000 \pm 0.010$ inches and diameter $=1.100 \pm 0.002$ inches [2]. These tolerances result in a maximum dispersion in the slug mass of $\pm 0.6 \%$.

As an aside, note that the theoretical mass of uranium (theoretical density $=19.05 \mathrm{~g} \mathrm{~cm}^{-3}$ ) calculated for a cylindrical volume 1.1 inches in diameter and 4.0 inches long is 11.67 grams higher than the reference value for the mass of uranium in an X-slug (1175 grams). A mass of 1175 grams corresponds to a density of $18.86 \mathrm{~g} \mathrm{~cm}^{-3}$.

Physical constants:

The ORIGEN2.1 computer code used to perform the inventory calculations for Task 1 has seen decades of widespread use throughout the nuclear industry. It was used in the previously completed Hanford Environmental Dose Reconstruction (HEDR) effort to calculate quantities of ${ }^{131} \mathrm{I}$ present in fuel dissolved at the Hanford reservation for the purpose of plutonium production. Formal calculations performed under the HEDR study to assess the uncertainties associated with using the ORIGEN2 code showed the maximum errors to be within $\pm 5 \%$ for the nuclides considered in the Task 1 source term [8]. Since the calculations performed under the HEDR study are nearly identical to those performed for the current effort, the $\pm 5 \%$ dispersion from the HEDR study should be equally applicable our own.

\section{Pile power:}

In the case of calculations performed for Task 1, the pile power is used only as a proxy for computing the thermal flux. The absolute accuracy of the asserted power level is not of consequence. It is only important that the variability in the asserted power levels be addressed.

The pile staff recorded two values for the total pile power for each of the three shifts each day: the accumulated galvanometer power and the accumulated heat power. Since the galvanometer power was considered the reference value and was directly proportional to the neutron flux, this was the information used for all X-slug inventory calculations. Heat power, on the other hand, was prone to bias from the 
variability in pile heat capacity with ambient temperature and from other sources. For this reason, the average of the heat power data over several days was used when calibrating the galvanometers [3].

The galvanometer reading was the net current that resulted from a pair of ion chambers located at one of the pile's shield walls. Both ion chambers were photon sensitive, but only one was neutron sensitive. The current output from the photon-only chamber was bucked against the current from the neutronplus-photon chamber, with the resulting current being read out on an optical galvanometer in the pile control room $[3,4]$.

Power for each shift was determined by dividing the accumulated power recorded in the pile operations logbooks for each shift (in $\mathrm{kWh}$ ) by the nominal duration of each shift (eight hours). Accumulated power for each shift was determined by the pile operators from the accumulated $\mathrm{kWh}$ meter in the control room. It is assumed for the purpose of calculating the average pile power level for each shift that the accumulated power readings recorded in the logbooks correspond to changeover for each shift and thus correspond to a time interval of exactly 8.0 hours. However, as it is doubtful this was actually the case, an uncertainty exists in the shift power data that results from the difference between the actual time interval that each accumulated power reading corresponds to and the assumed interval ( 8.0 hours). This uncertainty was judged to be at most fifteen minutes, which corresponds to a dispersion of $\pm 3 \%$ about an 8.0 hour interval.

In addition to the uncertainty in the shift power data from the actual versus assumed time interval, any variability in the performance of the galvanometer circuit would also contribute to the uncertainty in the asserted shift power values. However, it is believed the galvanometer circuit would have given very consistent results on a day-to-day basis. The ion chamber bias was supplied by dry-cell batteries and thus would have been quite stable. In addition, the response of ion chambers is not sensitive to small changes in bias voltage, unlike other types of counters. Further, the galvanometer power was calibrated against the heat power at least monthly [3], hence drift would likely have not been an issue. In light of these facts, it was decided that extending the uncertainty assigned to the shift power data to account for additional uncertainty contributed by variability in the galvanometer circuit was not necessary. Hence, the uncertainty assigned to the shift power data should be left at $\pm 3 \%$.

\section{Pile power-to-peak-flux conversion factor:}

The power-to-peak-flux conversion factor used in the final inventory calculations for X-slugs was 3.102 $\times 10^{5}$ neutrons $\mathrm{cm}^{-2}$ second $^{-1}$ watt ${ }^{-1}$. This value was derived by multiplying the value for this factor given in CP 2602 [5] $\left(3.234 \times 10^{5}\right)$ by the ratio of the fuel-to-air flux factor determined using MCNP (0.633) to that determined using the original power-to-peak-flux factor in conjunction with the relative flux equation (0.66). There are two inherent assumptions here: 1) that the difference in the two assessments of the fuel-to-air flux factor is due solely to bias in the power-to-peak-flux factor and not any bias in the relative flux expression; and 2) that the MCNP result is absolutely correct. The assumption that the difference in the flux ratios is due to bias in the power-to-flux factor is supported by MCNP calculations that show excellent agreement with values computed using the relative flux expression. The assumption that the MCNP result is accurate is one of convenience, as the uncertainties associated with this result 
are judged to be minimal and hence the effort required to address these would not be warranted given they would have no appreciable impact on the overall results. The precision of the MCNP result was $\pm 1.3 \%$, confirming it was statistically reliable.

The power-to-peak-flux factor determined by the X-10 pile physicists in 1945 was computed by multiplying the so-called $\phi$-value, which is the conversion factor between the total pile power and the neutron density at the pile center, by the neutron speed at thermal equilibrium $\left(2.2 \times 10^{5} \mathrm{~cm} \mathrm{~second}^{-1}\right)$. Dimensions of the $\phi$-value are neutrons $\mathrm{cm}^{-3}$ watt ${ }^{-1}$. The $\phi$-value was determined by physical measurements using the so-called "standard graphite pile", which was a graphite matrix into which a neutron source could be installed. Though specific details are not available, it is presumed a neutron source having a known emission rate was installed in the standard pile. The reference galvanometer (for measuring pile thermal power) was then used to correlate the neutron density at the pile center to thermal power with the results scaled up based on the relative size of the standard pile with respect to the Clinton pile. Hence, the measurement of the $\phi$-value depends on the uncertainty in the neutron emission rate of the reference source used and on the uncertainty in the calibration of the reference galvanometer against thermal power. The $\phi$-value determined by the pile physicists in February of 1945 was 1.47 neutrons $\mathrm{cm}^{-3}$ watt ${ }^{-1}$ [5]. Multiplying this value by the thermal neutron speed gives the powerto-peak-flux factor $3.234 \times 10^{5}$ neutrons $\mathrm{cm}^{-2}$ second $^{-1}$ watt $^{-1}$.

Subsequent measurements of the $\phi$-value conducted after February of 1945 resulted in a revised reference value of 1.45 neutrons $\mathrm{cm}^{-3}$ watt ${ }^{-1}$ being given in 1947 [6]. This value is $1.4 \%$ lower than the original value determined in early 1945. More recently, researchers at Los Alamos used MCNP to assess response functions for LANL's standard pile, which was built in the early 1950's. The LANL pile had a different use than X-10's in that it served as a reference geometry for the absolute calibration of neutron sources. However, the results for this assessment are still useful in that they show the assumption used by the X-10 physicists in the mid-1940's that the neutrons emitted from the X-10 standard pile were completely thermalized appears valid. The LANL study showed the response functions for the Los Alamos pile to be relatively flat with respect to energy and that detection efficiencies for $\mathrm{a} \mathrm{BF}_{3}$ neutron detector located at the center of the pile varied by only $5 \%$ over a range of neutron energies [7].

The results from the MCNP calculations performed for the Los Alamos pile are consistent with the 4\% bias that exists between the results from the MCNP calculations performed for the Clinton pile and the physical measurements performed by the Clinton pile physicists in 1945 . The $4 \%$ bias is therefore seen to be reasonable. However, this does not address the issue of the uncertainty (dispersion) in the value of the power-to-peak-flux conversion factor used in the final X-slug inventory calculations. The precision of the MCNP calculation performed to yield the fuel-to-air flux ratio for the Clinton Pile was $\pm 1.3 \%$. Thus, the overall uncertainty in this result is the precision plus any bias between the MCNP model and the actual system. An example of a source of bias would be impurities in the graphite or fuel slugs that were neglected in the MCNP model (though the levels of impurities in the Clinton pile had to be kept to an absolute minimum to be able to achieve a self-sustaining chain reaction). Whatever sources of bias may exist between the MCNP model and the actual system, there is no way to quantitatively account for them short of performing physical benchmarking experiments with actual 
material from the Clinton pile. Hence, a subjective judgment is required to quantitatively address the uncertainty in the power-to-peak-flux conversion factor. Given the precision of the MCNP calculations, the power-to-peak-flux factor was assigned an uncertainty of $\pm 5 \%$.

\section{$\underline{\text { Relative flux values: }}$}

The relative flux distribution in the pile was characterized by running 0.020 inch diameter silver wires through eleven of the piles experimental holes, irradiating them for the same effective exposure time, allowing them to decay for two weeks and then counting them on an end-window GM counter [5]. The wires were cut into one inch segments at eight inch intervals and weighed before counting. Count results were normalized to the mass of each sample. Corrections were also made for counter dead-time and geometry. The relative flux distribution in the pile was determined by simply taking the ratio of the counting results from the wire segments as a function of position. No absolute quantification of flux was required. Thus, the uncertainty in the relative flux data is that associated with the counting of the individual wire segments. The components of this uncertainty are: counting statistics, reproducibility of geometry, dead-time corrections, and sample mass. There is no way to quantitatively address each of these components individually. However, there is information with which an assessment can be made of the overall uncertainty in the counting data.

CP 2602 speaks of a 5\% ripple seen in the silver wire measurements corresponding to where the wire passed over the fuel channels [5]. Thus, it is reasonable to assume that the observed variability in their counting results was less than $5 \%$ and hence the overall error associated with the counting of the activated silver segments would have been within this range $( \pm 5 \%)$.

\section{$\underline{\text { Summary of uncertain parameters: }}$}

The uncertain parameters described above are summarized in Table 1 below. I think the best way to apply these to the inventory calculation results is to treat each as a normal distribution having a mean of unity and a standard deviation equal to the dispersion assigned in Table 1.

Table 1 Summary of uncertain parameters identified for the calculation of discharge inventories for X-slugs used for RaLa production

\begin{tabular}{|c|c|}
\hline Parameter & Assigned Dispersion \\
\hline Slug mass & $\pm 0.6 \%$ \\
\hline Physical constants & $\pm 5 \%$ \\
\hline pile power & $\pm 3 \%$ \\
\hline power-to-peak-flux conversion factor & $\pm 5 \%$ \\
\hline relative flux values & $\pm 5 \%$ \\
\hline
\end{tabular}


4939 Lower Roswell Road, Suite 106

Marietta, GA 30068

Phone: (770) 509-7606

FAX: (770) 509-7507

Internet: sra@crl.com

May 8, 1996

TO: $\quad$ Tom Widner

ChemRisk

FROM: $\quad$ R. E. Burns, Jr.

Shonka Research Associates, Inc.

SUBJECT: Characterization of uncertainties associated with the calculation of discharge ${ }^{131} \mathrm{I}$ inventories for X-slugs pushed for RaLa production

MEMO NO: REB.004 C96

FINAL $[\mathbf{X}] \quad$ DRAFT [ ]

Distribution:

Project File - SRA

Joe Shonka - SRA

References:

1. Jones, H.; Watson, L. B.; Arnette, T.; Coveyou, I. "Neutron Distribution in the Clinton Pile" CP 2602, Oak Ridge National Laboratory, Oak Ridge, TN, February 27, 1945.

2. "Evaluation of an Expression for Computing the Relative Thermal Neutron Flux in Clinton Pile Fuel Channels Used for RaLa Production" SRA-95-003, Rev. 0 Shonka Research Associates, Inc. August, 1995.

3. Heeb, C. M. "Uncertainties in Source Term Calculations Generated by the ORIGEN2 Computer Code for Hanford Production Reactors" PNL-7223 HEDR, Battelle/Pacific Northwest Laboratories, Richland, WA March, 1991.

Memo:

Calculation of the ${ }^{131} \mathrm{I}$ inventory contained in X-slugs pushed from the Clinton Pile for RaLa production will be accomplished in three basic steps:

1. Calculate the peak slug inventories for each channel pushed,

2. Establish the slug position factor for each individual slug in a set, and 
3. Calculate the inventory distribution for each slug pushed from a given channel and then sum these distributions to establish distributions for ${ }^{131}$ I inventory for the entire channel and for the entire push

Each of these steps is discussed below, followed by identification of the uncertain parameters associated with each step and the means that will be used to characterize each parameter so the contribution of each to the overall uncertainty can be assessed. The overall approach to establishing distributions for the ${ }^{131}$ I inventory at discharge for X-slugs pushed from the Clinton Pile for RaLa will be to embed the methodology described above in a spreadsheet model and use the Crystal Ball ${ }^{\circledR}$ add-in package to propagate uncertainties. The result will be frequency distributions for each RaLa push that represent the total ${ }^{131}$ I inventory that was available for dissolving at the time the slugs were pushed.

\section{Step 1: Calculate the peak slug inventories for each channel pushed.}

The peak slug inventory (PSI) for a given set of slugs is the ${ }^{131}$ I inventory calculated (using the ORIGEN2.1 code) for an arbitrary slug located at the center of the Clinton Pile having the same irradiation history as the slugs of interest. Note that not all of the slugs pushed at a specific time from a given fuel channel had the same irradiation history. Channels were often loaded incrementally in the earlier years of RaLa production at X-10, i.e., not all of the slugs in a channel were loaded at the same time. Thus, all of the slugs making up the total pushed from a specific channel could have made up of individual sets of slugs having the same irradiation history, i.e., the same charge and discharge dates. PSI's are therefore calculated for each set of slugs within a specific channel pushed at a specific time. Multi-set channels were common through RaLa Run 18 (June, 1947).

As said, PSI's are calculated using the ORIGEN2.1 code. The code is set up to calculate the nuclide inventory contained in a single X-slug (1175 grams of natural uranium) based on the irradiation history for the set of slugs of interest. The irradiation history for each set of slugs is derived from the push data for each RaLa discharge, which establishes the charge and discharge dates, and the pile operating logs, which establish the actual shift when the slugs were charged and discharged. The slug irradiation history is established down to the shift level as this is the precision to which we know the pile's power history. We have retrieved the shiftly power history for the Clinton Pile for the entire period of interest. Thus, the power history for each set of slugs is known to the nearest eight hours over their entire irradiation period. The pile power data for each shift is converted, using the expression from CP 2602 [1], to a peak thermal flux value. This value is then multiplied by 3.16 to convert it to total flux [2]. Thus, the ORIGEN2.1 code is run for a single X-slug located at the point of maximum pile flux over the period of time corresponding to that for the set of slugs of interest. The resulting ${ }^{131}$ I inventory calculated is then the PSI for the set of slugs of interest. Once the PSI for a set of slugs has been calculated, the actual inventories for each slug in the set are computed based on the position of the slugs within the pile relative to the pile center. 


\section{Step 2: Establish the slug position factor for each individual slug in a set.}

The slug position factor represents the relative value of the pile flux at the position of interest to the peak flux at the pile center. Position factors are determined using the expression given in CP 2602 for this purpose and assuming that the slugs were loaded so that they were centered in the channel in the axial direction. Multiplying the position factor value for an individual slug by the PSI for its set gives the uncorrected inventory for that slug. (Uncorrected inventory refers to the fact that this value needs to be corrected for the fact that the expression used to calculate the position factors was developed for the purpose of calculating the flux in the pile experimental holes and therefore overestimates the flux in the fuel regions [2]). For channels with more than one set of slugs, position factors will have to be chosen carefully so as to accurately reflect the position of the slugs within the channel. This is particularly true for Runs 1 and 2, as not all of the slugs in a channel were pushed for these runs.

Note that treating the slugs as being "load-centered" (entire charge of slugs is centered in the channel) or "slug-centered" (middle slug in a charge is located at the center of the channel) makes no appreciable difference in the value of the slug position factors. For a 36 slug charge, the difference in the position factor between the two assumptions for the slug on the east end is less than $4 \%(3.6 \%)$ These differences for individual slugs decrease as one approaches the center of the channel. In terms of the effect on the total inventory for a channel, the differences in the position factors between the two assumptions are negligible. As shown in SRA-95-003 [2], treating the slugs as load-centered or slugcentered makes no difference when the contributions from all slugs are considered together.

Step 3: Calculate the inventory distribution for each slug pushed from a given channel.

Each slug in a channel will have its own position factor. Hence, the inventory for each slug is assessed by multiplying the position factor distribution by the appropriate PSI distribution for the set from which each slug came. This product is then multiplied by the power factor used to correct for the overestimation of the pile flux by the relative flux expression (taken from [2]). The result will be a distribution of possible ${ }^{131}$ I inventories for each slug in a channel that can then be summed to yield the distribution for the channel as a whole. These channel distributions can then also be summed to yield the distribution for an entire push. (In actuality, all of this summing of distributions will have to be done simultaneously, as the distributions are established through Monte Carlo sampling).

\section{Uncertain parameters associated with calculation of the PSI for each set of slugs:}

The uncertain parameters associated with the calculation of the peak slug inventory (PSI) are as follows:

- mass of uranium metal in an X-slug

- ${ }^{235} \mathrm{U}$ content per unit mass of uranium metal in each slug

- total pile power (i.e., change in peak flux over time)

- total pile power to peak flux conversion factor 
- change in flux over the length of the slug

- ORIGEN cross-sections and half-lives

Of the parameters listed above, the only ones likely to be of any consequence will be the uncertainties associated with the total pile power and the power to peak flux conversion factor. Uncertainties in the total pile power will likely have to be established on a subjective basis, as it is doubtful that any analysis of this nature was ever done by the pile staff. The uncertainty associated with the power to flux conversion will be established by developing a computer model of the Clinton Pile using a code such as MCNP 4A.

The uncertainty associated with variances in the mass of uranium contained in X-slugs will have to be established from historical records from X-10. At Hanford, there were strict requirements on slug quality implemented in the operational practices for the 300 area. Since X-10 did not produce their own slugs, responsibility for slug quality rested with the vendor (ALCOA). I would presume that X-10 did provide ALCOA with acceptance criteria for slugs, but I do not recall coming across anything like this in our records to date. Thus, additional records searches will need to be conducted for the purpose of establishing the bounds on the mass of metal used in Clinton Pile slugs.

The uncertainty in the ${ }^{235} \mathrm{U}$ content per unit mass of uranium metal in each slug is of no appreciable consequence for our purpose and will be neglected. Since the slugs used for RaLa were irradiated to such low burnup, the ${ }^{235} \mathrm{U}$ content of the fuel is inconsequential since almost all the power is coming from the ${ }^{235} \mathrm{U}$ and very little from ingrowth of ${ }^{239} \mathrm{Pu}$. (In cases where fuel is irradiated to higher burnups, the contribution to the total power from ${ }^{239} \mathrm{Pu}$ becomes substantial). Thus, there is no significant change in the fission yield over time and therefore no need to account for variability in ${ }^{235} \mathrm{U}$ content. To demonstrate this, three runs were made using the ORIGEN2.1 code to irradiate 1175 grams of uranium having enrichments of $0.3 \%$, natural $(0.71 \%)$ and $3 \%{ }^{235} \mathrm{U}$. In each case, the uranium was irradiated for 100 days at a power of 400 watts. The resulting ${ }^{131} \mathrm{I}$ inventories at the end of the 100 days were $9.854 \mathrm{Ci}$ for the $0.3 \%$ enriched fuel, $9.654 \mathrm{Ci}$ for the natural uranium and 9.554 $\mathrm{Ci}$ for the $3 \%$ enriched metal. As is seen, the difference between the $0.3 \%$ case and the $3 \%$ case is $3 \%$. As any credible assessment of the variability in the ${ }^{235} \mathrm{U}$ content of the uranium metal used in the $\mathrm{X}$-slugs would be on the order of parts per million, it is clear that the variability in the ${ }^{235} \mathrm{U}$ abundance in the X-slug metal can be neglected. For the record, the reason for the difference in the ${ }^{131}$ I produced in the three test cases is the amount of ${ }^{239} \mathrm{Pu}$ produced in each case during the 100 day irradiation. Even with these very low burnups, there is $86 \mathrm{mg}$ of ${ }^{239} \mathrm{Pu}$ produced in the slug with the depleted metal $(0.3 \%$ enriched) and only $9 \mathrm{mg}$ produced in the enriched case (3\%). The ${ }^{131} \mathrm{I}$ fission yield from ${ }^{239} \mathrm{Pu}$ fission is three times that of ${ }^{235} \mathrm{U}$, thus a relatively small quantity of ${ }^{239} \mathrm{Pu}$ present in the slug can have a significant impact on the quantity of ${ }^{131}$ I produced. Note that our method of using the actual slug power histories for calculating X-slug inventories fully accounts for this effect and thus eliminates it as a source of uncertainty.

For the purposes of calculating the ${ }^{131}$ I content of individual slugs in a Clinton Pile channel, the slug position factor used has been the value that corresponds to the geometric center of the slug, and this value has been used to compute the inventory for the slug's total volume. Thus, the value for the center of the slug is treated as an average value that is applied over the slug's entire length. To assess the 
magnitude of any uncertainty resulting from this practice, one need only look at the change in the calculated values of the slug position factor over the length of a slug (four inches) at positions where the flux gradient in the axial direction would be the greatest, i.e., the ends of the channels. If the difference between the position factors at one of the slug and the other are not symmetric about the value at the center, then applying the center value over the entire length will introduce an uncertainty that would need to be addressed. If the values are symmetric, however, then no such uncertainty exists. To establish if the position factor values were symmetric over the length of an X-slug for axial positions of interest in the Task 1 effort, the position factors were calculated for locations corresponding to the middle and two ends of a slug located at the east and west end of pile channel 1868 containing a total of 36 slugs. For the east slug, the position factor values for the two ends were 0.529 and 0.566 , and the value for the center was 0.548. Likewise, the values for the two ends of the west slug were 0.528 and 0.490 , with a center value of 0.509 . In both cases, the average of the values for the two ends equals the value for the center. Thus, it is determined that there is no appreciable uncertainty contributed from the practice of treating the position factor value corresponding to the center of a slug as an average for the total slug volume. (There is no need to perform calculations for other channel, as the axial distribution of the flux does not change with channel location, i.e., radial position).

Using the ORIGEN2.1 computer code to assess the quantities of ${ }^{131}$ I contained in slugs discharged for RaLa processing introduces some minor uncertainties to the calculated values. These uncertainties stem from uncertainties in the various constants and conversion factors used by the code to calculate isotope production and depletion. These factors include reaction cross-sections, fission product yields, recoverable heat energy per unit fission, decay constants, etc. Of these, the largest contributors to uncertainty are the values for the spectrum averaged reaction cross-sections used by the code to compute fission, activation, etc. These values are specific to the neutron energy distribution in the reactor system of interest and therefore vary with the reactor type being considered. However, the importance of these differences with respect to the accuracy of results calculated by the ORIGEN code depends on the specific nuclides of interest. In the case of ${ }^{131} \mathrm{I}$, production is directly proportional to the number of fissions that have occurred, since this nuclide is a fission product and does not have a significant activation cross-section in comparison with its half-life. Thus, differences in the reaction cross-sections for ${ }^{131}$ I for different reactor models are largely insignificant (as long as the fuel makeup stays the same), since ${ }^{131}$ I production depends for the most part on reactor power and not the neutron energy spectrum. The neutron energy spectrum can be of consequence in the case of higher burnups, as the spectrum has an effect on plutonium ingrowth, which can substantially impact ${ }^{131}$ I production (as discussed above). However, this situation is of minor concern with respect to ORIGEN calculations for Task 1, as the burnups of interest are small. The impact of the choice of the cross-section and fission product yield library on the quantities of ${ }^{131}$ I calculated by the ORIGEN2.1 code for a natural uranium fuel for two different burnups is illustrated in Table 1.

Table $1{ }^{131}$ I content for one X-slug calculated by ORIGEN2.1 using two different crosssection and fission product yield libraries

\begin{tabular}{|c|c|c|}
\hline Reactor Model & ${ }^{131}$ I Content at 100 days & ${ }^{131}$ I Content at 1000 days \\
\hline CANDUNAU & $9.654 \mathrm{Ci}$ & $9.849 \mathrm{Ci}$ \\
\hline PWRUS & $10.21 \mathrm{Ci}$ & $10.57 \mathrm{Ci}$ \\
\hline
\end{tabular}


The table shows the calculated ${ }^{131}$ I inventory contained in 1175 grams of natural uranium (one X-slug) irradiated for 100 days and for 1000 days using two different cross-section and fission product yield libraries: the CANDUNAU library for CANDU reactors using natural uranium fuel and the PWRUS library for a three-cycle PWR using 3.2\% enriched fuel at a standard burnup of 33,000 MWd/MTHM. The neutron energy spectrum for the Clinton Pile would lie somewhere in between the spectra for the two cases considered (CANDU and PWR). As is seen, the inventories calculated using the PWR library are greater than those for the same irradiation using the CANDU data. The reason for this is that the harder spectrum found in the PWR results in higher production of ${ }^{239} \mathrm{Pu}$, thus resulting in more production of ${ }^{131} \mathrm{I}$. The difference is $5.7 \%$ at 100 days and $7.3 \%$ at 1000 days.

For the Hanford dose reconstruction study, Cal Heeb performed a similar comparison between the CANDU library and a library that had been developed for the Hanford $\mathrm{N}$ reactor to assess the magnitude of the uncertainty from using the $\mathrm{N}$ reactor library to perform calculations for the other Hanford piles (which were more heavily moderated than the $\mathrm{N}$ reactor and thus more thermal) [3]. Heeb found the average difference between the ${ }^{131}$ I content calculated using the CANDU and N reactor libraries was 3.6\% for burnups between 100 and 1000 MWD/Ton (i.e., $\mathrm{N}$ reactor inventories were higher) [3]. Given this, Heeb concluded that the uncertainty contributed by the ORIGEN code to calculations for ${ }^{131}$ I was negligible in comparison with uncertainties in release fractions and atmospheric transport [3]. Given that the neutron energy spectrum for the Clinton Pile had to be very similar to that for the Hanford piles and that the fuel burnups we are concerned with for RaLa are much smaller than those experienced at Hanford, I think it is safe to draw the same conclusion that any uncertainty contributed to the ${ }^{131}$ I inventory calculations for X-slugs from the ORIGEN2.1 code can be neglected for the purpose of this study.

Note that the conclusion that uncertainties contributed by the ORIGEN2.1 code to the overall uncertainty in the discharge inventory calculations can be neglected is not based solely on comparisons of ORIGEN results with each other. The ORIGEN code is widely used in the nuclear industry and has been extensively benchmarked against physical measurements made with irradiated reactor fuel. Heeb discusses such comparisons in section 2 of PNL-7223 HEDR [3].

\section{Uncertain parameters associated with the position factor for each slug in a set:}

The uncertain parameters associated with establishing the slug position factor for each slug in a set are as follows.

- uncertainty in the relative flux equation

- uncertainty from assuming slugs spent their entire irradiation duration in their final position (not applicable for channels with only one set)

Uncertainty in the relative flux equation will be assessed using the same analysis used to establish the uncertainty in the power to peak flux conversion factor. However, one should recognize that the flux equation is being applied on a relative basis and thus the associated uncertainty should be small. 
The uncertainty associated with treating slugs as if they spent their entire irradition cycle in one position (their final position) was assessed by using the ORIGEN2.1 code to calculate the difference between the ${ }^{131}$ I inventories calculated for slugs using their actual power histories (i.e., accounting for slug movement) and inventories calculated for the same time duration, but at the power level corresponding to that at the slug's final position. Calculations were performed for the eastmost and westmost slugs of the initial charge for several RaLa discharges thought to represent worst case histories. A total of nine cases were considered. In seven of these, the initial charge was only moved once (slugs had two positions). In one case, the slugs were moved twice and in another they were moved three times. The results from these experiments are shown in Table 2. The values in the "Error" columns are the differences between the inventory calculated with the slug irradiated in its final position only and the inventory calculated accounting for the time the slug spent in each position it was moved to during irradiation for the slugs on the two ends of the initial charge.

Table 2 Errors resulting from the assumption that X-slugs did not move during their irradiation cycle for selected cases

\begin{tabular}{|c|c|c|c|}
\hline \multirow{2}{*}{ Run Number } & \multirow{2}{*}{ Channel } & \multicolumn{2}{|c|}{ Error (assumed position vs. actual) } \\
\cline { 2 - 4 } & 1566 & East Slug & West Slug \\
\hline 2 & 1766 & $0.0 \%$ & $-0.3 \%$ \\
\hline 3 & 2269 & $0.0 \%$ & $-1.2 \%$ \\
\hline 3 & 1665 & $0.1 \%$ & $-1.1 \%$ \\
\hline 4 & 1766 & $0.0 \%$ & $-0.3 \%$ \\
\hline 4 & 2065 & $-0.3 \%$ & $-3.6 \%$ \\
\hline 4 & 1669 & $-0.1 \%$ & $-0.4 \%$ \\
\hline 5 & 1672 & $-0.1 \%$ & $-0.6 \%$ \\
\hline 8 & 1767 & $0.1 \%$ & $-0.7 \%$ \\
\hline 9 & & $-1.2 \%$ & $-1.4 \%$ \\
\hline Average & & $\mathbf{- 0 . 2 \%}$ & $\mathbf{- 1 . 1 \%}$ \\
\hline
\end{tabular}

As is seen, the impact is trivial for the slug on the east end of the charge. In general, the east slug experiences only a minor change in relative power level when it is moved by the charging of additional slugs to the same channel since the change in power level per unit change in axial position decreases as the slug moves from east to west. It would be rare for the charging of new slugs to result in the east slug being moved to a position of lower power than where it was before. This would only happen if there were only a few slugs in the initial charge, which was the case for channel 1767 pushed for RaLa Run 9. As seen, the error for the east slug in this case is significantly higher than for the other cases (although still quite small as a whole).

The error values for the west slugs are higher than those for the east slugs since the west slug is always moving from higher to lower power as new slugs are added. However, even in the case of the west slugs, the effect of assuming the slug did not move is quite small, with an average error of $-1.1 \%$ and a maximum of $-3.6 \%$ for the cases considered. Thus, in consideration of the data in Table 2, uncertainty from the practice of treating X-slugs as if they spent their entire irradiation cycle in their final position will be neglected. 


\section{Summary of Uncertain Parameters}

From the above discussions, it follows that there are only three of the uncertain parameters identified as associated with calculation of the ${ }^{131}$ I inventory at discharge for X-slugs pushed for RaLa that need to be considered in such calculations. These are

- the mass of uranium metal in each slug,

- the pile power to peak flux conversion factor, and

- the slug position factors (relative flux values).

The bounds for the mass of uranium metal contained in X-slugs will have to be established from the available historical literature. We intend to use the appropriate modules from the SCALE4.3 code package to address the uncertainties for the power to flux conversion and the relative flux as a function of position.

\section{Spreadsheet model:}

Once evaluations for all of the relevant uncertain parameters identified above have been completed, the distributions established for each parameter will be incorporated into a spreadsheet model that will, utilizing the Crystal Ball ${ }^{\circledR}$ add in package for Excel $^{\mathrm{TM}}$, allow us to establish frequency distributions for the following:

- ${ }^{131}$ I inventory for individual slugs at discharge,

- ${ }^{131}$ I inventory for each channel at discharge, and

- ${ }^{131}$ I inventory for an entire push.

Analyses will be performed for each push that was made for each RaLa run, with a push defined as a group of channels discharged at one time for the purpose of obtaining slugs for RaLa. Inventory calculations will be performed for each individual slug in each channel discharged for a specific push, with the distributions for each slug summed to yield the distribution for the channel. The inventory distributions for each channel would then be summed to get the distribution for the push as a whole. An example of what the spreadsheets for the calculation of the channel inventories should look like is presented in Fig. 1. The spreadsheet shows the calculation of the individual slug and channel discharge inventory for channel 1967 pushed for RaLa Run 1. The PSI and power factor are accurate, however, the position factors are not, as the 30 slugs pushed from this channel were not all of the slugs available, i.e., only the westmost 30 slugs were pushed. The uncertain parameters have been set to 1.0, as it is anticipated that these will be treated as distributions about unity once they have been characterized. In reality, the values under the " 131 I Inventory" column would be forecast cells, with the inventory values for each slug being frequency distributions for the ${ }^{131}$ I inventory based on the distributions defined for the uncertain parameters. The "Sum" cell would be an aggregate distribution made up of the individual values determined for the distributions for the inventories for each slug. There would be one additional forecast cell that is not shown, that being the sum of all of the sum distributions for each channel that would be the distribution for the entire push. The push distribution is what would then be used as input to the plant model, i.e., whatever means we decide to use to go from discharge inventory to releases. 
Figure 1 Example spreadsheet for the calculation of channel inventories, including propagation of uncertainties

\begin{tabular}{|c|c|c|c|c|c|c|c|c|c|}
\hline \multicolumn{10}{|c|}{${ }^{131}$ I Inventorv Calculations for RaLa Run 1} \\
\hline \multirow[b]{2}{*}{ Channel } & \multirow[b]{2}{*}{ Slug } & \multirow[b]{2}{*}{ PSI } & \multirow[b]{2}{*}{$\begin{array}{c}\text { Position } \\
\text { Factor }\end{array}$} & \multirow[b]{2}{*}{$\begin{array}{l}\text { Power } \\
\text { Factor }\end{array}$} & \multicolumn{4}{|c|}{ Uncertainty Distributions } & \multirow[b]{2}{*}{${ }^{131}$ I Inventorv } \\
\hline & & & & & $\begin{array}{l}\text { Slug } \\
\text { Mass }\end{array}$ & $\begin{array}{l}\text { Pile } \\
\text { Power }\end{array}$ & $\begin{array}{l}\text { Power to } \\
\text { Flux Factor }\end{array}$ & $\begin{array}{l}\text { Relative } \\
\text { Flux }\end{array}$ & \\
\hline 1967 & -15 & 10.72 & 0.641 & 0.66 & 1.0 & 1.0 & 1.0 & 1.0 & 4.5 \\
\hline 1967 & -14 & 10.72 & 0.671 & 0.66 & 1.0 & 1.0 & 1.0 & 1.0 & 4.7 \\
\hline 1967 & -13 & 10.72 & 0.699 & 0.66 & 1.0 & 1.0 & 1.0 & 1.0 & 4.9 \\
\hline 1967 & -12 & 10.72 & 0.725 & 0.66 & 1.0 & 1.0 & 1.0 & 1.0 & 5.1 \\
\hline 1967 & -11 & 10.72 & 0.749 & 0.66 & 1.0 & 1.0 & 1.0 & 1.0 & 5.3 \\
\hline 1967 & -10 & 10.72 & 0.771 & 0.66 & 1.0 & 1.0 & 1.0 & 1.0 & 5.5 \\
\hline 1967 & -9 & 10.72 & 0.791 & 0.66 & 1.0 & 1.0 & 1.0 & 1.0 & 5.6 \\
\hline 1967 & -8 & 10.72 & 0.809 & 0.66 & 1.0 & 1.0 & 1.0 & 1.0 & 5.7 \\
\hline 1967 & -7 & 10.72 & 0.824 & 0.66 & 1.0 & 1.0 & 1.0 & 1.0 & 5.8 \\
\hline 1967 & -6 & 10.72 & 0.838 & 0.66 & 1.0 & 1.0 & 1.0 & 1.0 & 5.9 \\
\hline 1967 & -5 & 10.72 & 0.848 & 0.66 & 1.0 & 1.0 & 1.0 & 1.0 & 6.0 \\
\hline 1967 & -4 & 10.72 & 0.857 & 0.66 & 1.0 & 1.0 & 1.0 & 1.0 & 6.1 \\
\hline 1967 & -3 & 10.72 & 0.863 & 0.66 & 1.0 & 1.0 & 1.0 & 1.0 & 6.1 \\
\hline 1967 & -2 & 10.72 & 0.866 & 0.66 & 1.0 & 1.0 & 1.0 & 1.0 & 6.1 \\
\hline 1967 & -1 & 10.72 & 0.867 & 0.66 & 1.0 & 1.0 & 1.0 & 1.0 & 6.1 \\
\hline 1967 & 1 & 10.72 & 0.866 & 0.66 & 1.0 & 1.0 & 1.0 & 1.0 & 6.1 \\
\hline 1967 & 2 & 10.72 & 0.863 & 0.66 & 1.0 & 1.0 & 1.0 & 1.0 & 6.1 \\
\hline 1967 & 3 & 10.72 & 0.856 & 0.66 & 1.0 & 1.0 & 1.0 & 1.0 & 6.1 \\
\hline 1967 & 4 & 10.72 & 0.848 & 0.66 & 1.0 & 1.0 & 1.0 & 1.0 & 6.0 \\
\hline 1967 & 5 & 10.72 & 0.837 & 0.66 & 1.0 & 1.0 & 1.0 & 1.0 & 5.9 \\
\hline 1967 & 6 & 10.72 & 0.824 & 0.66 & 1.0 & 1.0 & 1.0 & 1.0 & 5.8 \\
\hline 1967 & 7 & 10.72 & 0.808 & 0.66 & 1.0 & 1.0 & 1.0 & 1.0 & 5.7 \\
\hline 1967 & 8 & 10.72 & 0.791 & 0.66 & 1.0 & 1.0 & 1.0 & 1.0 & 5.6 \\
\hline 1967 & 9 & 10.72 & 0.771 & 0.66 & 1.0 & 1.0 & 1.0 & 1.0 & 5.5 \\
\hline 1967 & 10 & 10.72 & 0.749 & 0.66 & 1.0 & 1.0 & 1.0 & 1.0 & 5.3 \\
\hline 1967 & 11 & 10.72 & 0.724 & 0.66 & 1.0 & 1.0 & 1.0 & 1.0 & 5.1 \\
\hline 1967 & 12 & 10.72 & 0.698 & 0.66 & 1.0 & 1.0 & 1.0 & 1.0 & 4.9 \\
\hline 1967 & 13 & 10.72 & 0.670 & 0.66 & 1.0 & 1.0 & 1.0 & 1.0 & 4.7 \\
\hline 1967 & 14 & 10.72 & 0.640 & 0.66 & 1.0 & 1.0 & 1.0 & 1.0 & 4.5 \\
\hline \multirow[t]{2}{*}{1967} & 15 & 10.72 & 0.608 & 0.66 & 1.0 & 1.0 & 1.0 & 1.0 & 4.3 \\
\hline & & & & & & & & Sum & $165.4 \mathrm{Ci}$ \\
\hline
\end{tabular}


Marietta, GA 30068

Phone: (770) 509-7606

FAX: (770) 509-7507

Internet: sra@crl.com

TO: $\quad$ Tom Widner

May 19, 1997

ChemRisk - Alameda

FROM: $\quad$ R. E. Burns, Jr.

Shonka Research Associates, Inc.

SUBJECT: Inventory calculations for $\mathrm{W}$-slug RaLa runs

MEMO NO: REB.010 C97

FINAL [X] DRAFT [ ]

Distribution:

References:

1. Heeb, C. M.; Bates, D. J. "Radionuclide Releases to the Columbia River from Hanford Operations, 1944-1971” PNWD-2223 HEDR, Battelle/Pacific Northwest Laboratories, Richland, WA January, 1994.

2. Barnard, M. G. “100 Areas Monthly Reports - 1949” Hanford 45828, Hanford Operations Division, 1949.

3. Jaech, J. L. "Monthly Summary of Dissolver Data" FTS-CLVI-73, undated.

4. Heeb, C. M. "Radionuclide Releases to the Atmosphere from Hanford Operations, 1944-1972”' PNWD-2222 HEDR, Battelle/Pacific Northwest Laboratories, Richland, WA January, 1994.

5. REB.007 C97, "Slug factors for the Hanford production reactors", memo from R. E. Burns, Jr. to Tom Widner dated April 28, 1997.

6. REB.005 C97, "Tube factors for the Hanford production reactors", memo from R. E. Burns, Jr. to Tom Widner dated April 25, 1997.

7. REB.002 C97, "Final inventory values for X-slugs", memo from R. E. Burns, Jr. to Tom Widner dated April 30, 1997. 
8. Heeb, C. M.; "Uncertainties in Source Term Calculations Generated by the ORIGEN2 Computer Code for Hanford Production Reactors” PNL-7223 HEDR, Pacific Northwest Laboratory, March, 1991.

Memo:

Inventory calculations have been completed for all RaLa runs in which $\mathrm{W}$-slugs were used. These were Runs 28 through 68, with the exception of Run 43, which was a low-level break in run in which only Xslugs were used (approximately 60).

Inventories have been calculated for each run for ${ }^{131} \mathrm{I},{ }^{140} \mathrm{Ba}$ and total iodine content at the time of reactor shutdown. The inventory values for ${ }^{131} \mathrm{I}$ and ${ }^{140} \mathrm{Ba}$ are given in terms of curies of activity per unit mass of uranium shipped (in kilograms). Total iodine values are given in terms of mass of total iodine (in grams) per unit mass of uranium. Again, these values are per unit mass of uranium shipped and not per mass of uranium pushed. The calculations account for the fact that only the center-most slugs were used from each tube pushed.

Inventories were calculated using the ORIGEN2.1 computer code with the CANDUNAU crosssection and fission product yield library. The calculations were carried out for continuous irradiation, with corrections for saturation applied afterwards. In cases were the irradiation time was not known, two ORIGEN calculations were performed to bound the possible inventory. The irradiation time used for the upper bound calculation was always 100 days. 100 days was chosen to guarantee saturation for all radio-iodines and for ${ }^{140} \mathrm{Ba}$ without being excessively long. For the lower bound calculations, one of two different irradiation times was used depending on the year in which the run took place. Examination of the runs for which the irradiation time was known showed that for runs prior to 1951, a lower bound of 50 days seemed appropriate, whereas a lower bound of 14 days appeared correct for runs from 1951 on. Known irradiation times are given in Table 1. Different irradiation times for the same run number correspond to different groups of slugs in the same discharge (i.e., different charge dates).

Once the desired irradiation times were established, ORIGEN calculations were carried out for a mass of natural uranium equivalent to that in one tube. The tubes in the Hanford piles were charged with either 32 eight inch (W-8) slugs or 64 four inch (W-4) slugs. At $3.564 \mathrm{~kg}$ per W-8 slug, this is a total mass of $114.048 \mathrm{~kg}{ }^{\text {nat }} \mathrm{U}$. The power levels used for each reactor were the average tube power for the appropriate time period, taken to the nearest month. The average tube power for each month was computed by dividing the average reactor power for the month of interest by the total number of tubes. All of the Hanford reactors used for RaLa contained 2004 tubes. The same average tube power was used for all days in a given month. The average reactor power for each month was taken from Appendix A of PNWD-2223 HEDR [1]. Note that for several of the W-slug runs, inventory calculations had to be carried out for more than one reactor since the reactor the slugs came from was unknown. In cases where there was overlap in the power levels for the possible reactors of origin, the inventory values reflect the average across all possible source reactors. However, there were cases where the reactor power levels did not overlap. In these cases, individual inventory distributions for the two reactors representing the most likely source and worst case source were established. 
Table 1 Known irradiation times for W-slug RaLa runs

\begin{tabular}{|c|c|c|}
\hline Run Number & Date & Irradiation Time (days) \\
\hline 28 & November, 1948 & 87 \\
\hline 31 & March, 1949 & 69 \\
\hline 31 & March, 1949 & 46 \\
\hline 38 & January, 1950 & 98 \\
\hline 40 & March, 1950 & 99 \\
\hline 41 & April, 1950 & 90 \\
\hline 42 & June, 1950 & 50 \\
\hline 51 & September, 1953 & 302 \\
\hline 53 & July, 1953 & 40 \\
\hline 55 & January, 1954 & 63 \\
\hline 55 & January, 1954 & 29 \\
\hline 57 & July, 1954 & 33 \\
\hline 58 & October, 1954 & 21 \\
\hline 59 & March, 1955 & 14 \\
\hline 68 & October, 1956 & 43 \\
\hline
\end{tabular}

In cases where the reactor of origin (source reactor) for the slugs pushed for a given run was established, this information was obtained from one of three sources. For several of the runs, the teletypes that were sent from Hanford to X-10 notifying them that shipment had been made were found. Some of these (but by no means all of them) stated the reactor from which the slugs had come from (along with additional irradiation data). For all of the runs that took place in 1949, Ref. [2] was used to establish the source reactor. A third document used to establish source reactor was Ref. [3], which was used in cases where both the charge and discharge dates for a set of slugs was known. In these cases, the dissolver summary data were reviewed to identify the reactor for which routine discharges that uniquely matched both the charge and discharge dates had occurred. If such a match could be made, then that reactor was considered to be the source for the RaLa slugs.

Following each ORIGEN run, the values for curies of ${ }^{131} \mathrm{I}$ and ${ }^{140} \mathrm{Ba}$ and for grams of total iodine were corrected for saturation. Saturation correction factors were computed on the basis that the Hanford piles maintained a $90 \%$ capacity factor. $90 \%$ capacity was the value established by Heeb during the HEDR study [4] and is consistent with what was observed during this current effort.

Saturation factors were computed for the three quantities of concern for the three values used for irradiation time (14, 50 and 100 days). The factors were determined using ORIGEN to calculate inventories for equivalent durations of continuous and discontinuous operation. For the 14 day period, the saturation fractions are based on a continuous operating interval of 13 days compared to a cycle of 6 days on, one day off and 6 days on. For the 50 and 100 day intervals, the comparisons are between 49 and 99 days of continuous operation and cycles of 9 days on and one day off repeated 5 and 10 times, respectively. The results from these assessments are given in Table 2. 
Table 2 Saturation factors for the three irradiation intervals used

\begin{tabular}{|c|c|c|c|}
\hline & \multicolumn{3}{|c|}{ Saturation Factor (ratio of discontinuous to continuous operation) } \\
\hline Species & 14 Days & 50 Days & 100 Days \\
\hline${ }^{131} \mathrm{I}$ & 0.924 & 0.933 & 0.932 \\
\hline${ }^{140} \mathrm{Ba}$ & 0.925 & 0.926 & 0.923 \\
\hline total iodine & 0.943 & 0.935 & 0.922 \\
\hline
\end{tabular}

For runs where the actual irradiation time was known, the saturation factors used were those for the irradiation time from Table 2 closest to the actual duration.

After the ORIGEN results were corrected for saturation, they were then corrected so that the inventory reflected that contained only in the 38 center-most slugs (in the case of W-4 tubes) or 18 center-most slugs (in the case of W-8 tubes). The center 38 slugs in a tube of $64 \mathrm{~W}-4$ slugs yield $74.9 \%$ of the tube's power and the center 18 slugs in a tube containing $32 \mathrm{~W}-8$ slugs provide $71.8 \%$ of the power [5]. Thus, the inventories for a given tube are multiplied by either 0.749 or 0.718 , depending on the slug type, to get the inventory contained in the slugs shipped to X-10 from that particular tube. Note that, at this point, the inventory values still represent the average for all tubes in the reactor - adjustments for radial power variation have yet to be made.

After the inventory results were corrected to give that contained only in the slugs shipped, tube factors were established to adjust the inventory to reflect the actual rather than the average tube power. In cases where the tube factor for a given discharge was known, the value was simply multiplied by the average inventory value. If the tube factor was not known, an appropriate Beta distribution was established from which tube factor values could be sampled. A detailed discussion of tube factors and how they are sampled for Task 1 calculations is given in REB.005 C97 [6].

After tube factors were established for each reactor of concern for a given RaLa run, how the inventory calculation was completed depended upon what information was available. The inventory calculations can be broken up into seven different cases:

1. runs where the reactor of origin, irradiation time and tube factor are all known;

2. runs where the reactor of origin is known, but tube factor and irradiation time are not;

3. runs where reactor of origin and tube factor are known, but irradiation time is not;

4. runs where reactor of origin, tube factor and irradiation time are all unknown, but power levels among the possible source reactors overlap;

5. runs where reactor of origin and irradiation time are known, but tube factor is not;

6. runs where reactor of origin, tube factor and irradiation time are all unknown, and power levels among the possible source reactors do not overlap; and

7. runs where irradiation time is known, but source reactor and tube factors are unknown and reactor power levels do not overlap. 
The breakdown of the $\mathrm{W}$-slug runs with respect to the seven categories above is as follows:

Category 1: Runs 28 through 31 and 40 through 42;

Category 2: Runs 32 through 37;

Category 3: Run 38

Category 4: Runs 39 and 44 through 50;

Category 5: Runs 51, 53, 55, 57, 59 and 68;

Category 6: Runs 52, 54, 56 and 60 through 67; and

Category 7: Run 58.

For the runs in Category 1, no sampling of any kind is required, and the result is simply the product of the corrected ORIGEN data and the tube factor divided by the mass of $38 \mathrm{~W}-4$ slugs. (No runs in this category involved W-8 slugs.)

For the runs in Category 2, Monte Carlo sampling is performed on tube factor and the corrected ORIGEN results for the upper and lower bounds for irradiation time. Tube factor is sampled as a Beta distribution and the ORIGEN data are sampled uniformly between the lower and upper bounds. For all runs in Category 2, the lower bound for irradiation time is 50 days, as all of these runs took place prior to 1951 .

For the runs in Category 3, only the ORIGEN results for the upper and lower bounds for irradiation time are sampled. This gives a trivial result of a uniform distribution bounded between the product of the lower and upper ORIGEN results and the tube factor (divided by the appropriate mass).

For the runs in Category 4, the calculation is the same as those in Category 2 for each individual reactor, but the individual results are averaged to establish uniform sampling across the possible reactors of origin as well.

For runs in Category 5, sampling is performed only on the tube factor.

Like Category 4, the runs in Category 6 are addressed through sampling the tube factor and average tube inventory. However, since the power levels for the possible piles of origin do not overlap, a single distribution representing the possible inventory values cannot be established. Thus, individual distributions for the most likely and worst case source reactors were established instead. For all runs in Category 6, the most likely source was deemed the $\mathrm{H}$ reactor and the worst case (highest power) was the $\mathrm{C}$ reactor.

The inventory calculation for the run in Category 7 was completed through sampling the tube factor distribution for the most likely and worst case source reactors ( $\mathrm{H}$ and $\mathrm{C}$, respectively). 
In all of the inventory calculations described above except for those in Category 1, uncertainty in the results is reflected in the frequency distributions that are generated in each calculation. However, there are some additional sources of uncertainty in these calculations that should be mentioned. The results from the ORIGEN computer code have a small uncertainty due to uncertainty in the reaction crosssection and fission product yield values. There is also uncertainty associated with the correction of the ORIGEN results for actual reactor operation (saturation). A value of $\pm 5 \%$ was recommended previously to address the uncertainty associated with the use of the ORIGEN code and other physical constants in the inventory calculations completed for the X-slug RaLa runs [7]. This value represented a maximum uncertainty for the nuclides of interest in this current effort, and was based on an assessment performed by Heeb during the Hanford study [8]. Given that the $\pm 5 \%$ dispersion is a maximum value and that there is little variability seen in the saturation factors computed for the W-slug calculations, an uncertainty of $\pm 5 \%$ is recommended to account for the combined uncertainties from physical constants and reactor power variation.

An additional source of uncertainty in the W-slug calculations is that associated with reactor power and asserted tube factors. In the Hanford study, Heeb used a value of 5\% for the uncertainty in the recorded pile power levels [1]. This is likely an overstatement given that pile power was recorded to much greater precision, hence an uncertainty of $\pm 5 \%$ should be adequate for the combined uncertainties in pile power and asserted tube factors.

To give an idea of the magnitude of the potential releases of ${ }^{131}$ I from RaLa processing at X-10, the mean values from the most likely distributions for each $\mathrm{W}$-slug RaLa run were used to generate the data in Fig. 1. Totals are given for the time of reactor shutdown prior to discharge and for six days of decay time, where decay time is defined as the time between reactor shutdown and the start of the first dissolving. As seen, the most likely total for all of the runs at six days of decay is $638,498 \mathrm{Ci}$ of ${ }^{131} \mathrm{I}$. 
Figure 1 Total ${ }^{131} \mathrm{I}$ inventories for each W-slug RaLa run based on most likely results

\begin{tabular}{|c|c|c|c|c|c|c|c|c|c|c|}
\hline Run No. & $\begin{array}{c}\text { W-8 } \\
\text { Slugs } \\
\text { Charqed }\end{array}$ & $\begin{array}{c}\text { W-4 } \\
\text { Slugs } \\
\text { Charqed }\end{array}$ & $\begin{array}{c}\text { Total } \\
\text { Mass (kq) } \\
\text { W-8 }\end{array}$ & $\begin{array}{c}\text { Total } \\
\text { Mass (kq) } \\
\text { W-4 }\end{array}$ & $\begin{array}{c}\text { Mean }^{131} \text { I at } \\
\text { shutdown } \\
\text { W-8 }(\mathrm{Ci} / \mathrm{kq})\end{array}$ & $\begin{array}{c}\text { Mean }^{131} \mathrm{I} \text { at } \\
6 \text { days } \\
\mathrm{W}-8(\mathrm{Ci} / \mathrm{kq})\end{array}$ & $\begin{array}{c}\text { Mean }{ }^{131} \mathrm{I} \text { at } \\
\text { discharge } \\
\mathrm{W}-4(\mathrm{Ci} / \mathrm{kq})\end{array}$ & $\begin{array}{c}\text { Mean }^{131} \text { I at } \\
6 \text { days } \\
\mathrm{W}-4(\mathrm{Ci} / \mathrm{kq})\end{array}$ & $\begin{array}{l}\text { Total }^{131} \mathrm{I} \text { at } \\
\text { shutdown } \\
\text { (Ci) }\end{array}$ & $\begin{array}{c}\text { Total }{ }^{131} \mathrm{I} \text { at } \\
6 \text { days } \\
\text { (Ci) }\end{array}$ \\
\hline 28 & 0 & 36 & 0.000 & $6 \overline{4.152}$ & $0.000 \mathrm{E}+00$ & $0.000 \mathrm{E}+00$ & $5.288 \mathrm{E}+01$ & $3.152 \mathrm{E}+01$ & $3.392 E+03$ & $2.022 \mathrm{E}+03$ \\
\hline 29 & 0 & 38 & 0.000 & 67.716 & $0.000 \mathrm{E}+00$ & $0.000 \mathrm{E}+00$ & $5.180 \mathrm{E}+01$ & $3.088 \mathrm{E}+01$ & $3.508 E+03$ & 2.091E+03 \\
\hline 30 & 0 & 38 & 0.000 & 67.716 & $0.000 \mathrm{E}+00$ & $0.000 E+00$ & 4.937E+01 & $2.943 E+01$ & $3.343 E+03$ & $1.993 E+03$ \\
\hline 31 & 0 & 76 & 0.000 & 135.432 & $0.000 E+00$ & $0.000 E+00$ & $5.251 \mathrm{E}+01$ & $3.130 E+01$ & $7.112 \mathrm{E}+03$ & 4.239E+03 \\
\hline 32 & 0 & 76 & 0.000 & 135.432 & $0.000 \mathrm{E}+00$ & $0.000 \mathrm{E}+00$ & 4.687E+01 & $2.794 \mathrm{E}+01$ & $6.348 \mathrm{E}+03$ & $3.784 \mathrm{E}+03$ \\
\hline 33 & 0 & 76 & 0.000 & 135.432 & $0.000 \mathrm{E}+00$ & $0.000 \mathrm{E}+00$ & 4.889E+01 & $2.914 \mathrm{E}+01$ & $6.621 \mathrm{E}+03$ & 3.947E+03 \\
\hline 34 & 0 & 76 & 0.000 & 135.432 & $0.000 E+00$ & $0.000 E+00$ & $5.114 \mathrm{E}+01$ & $3.048 E+01$ & $6.925 E+03$ & $4.128 \mathrm{E}+03$ \\
\hline 35 & 0 & 74 & 0.000 & 131.868 & $0.000 E+00$ & $0.000 E+00$ & $4.640 \mathrm{E}+01$ & $2.766 \mathrm{E}+01$ & $6.118 \mathrm{E}+03$ & 3.647E+03 \\
\hline 36 & 0 & 74 & 0.000 & 131.868 & $0.000 \mathrm{E}+00$ & $0.000 E+00$ & 4.643E+01 & $2.767 \mathrm{E}+01$ & $6.122 \mathrm{E}+03$ & $3.649 \mathrm{E}+03$ \\
\hline 37 & 0 & 74 & 0.000 & 131.868 & $0.000 \mathrm{E}+00$ & $0.000 \mathrm{E}+00$ & 4.689E+01 & $2.795 E+01$ & $6.183 \mathrm{E}+03$ & $3.686 \mathrm{E}+03$ \\
\hline 38 & 0 & 74 & 0.000 & 131.868 & $0.000 E+00$ & $0.000 \mathrm{E}+00$ & 4.419E+01 & $2.634 \mathrm{E}+01$ & $5.828 \mathrm{E}+03$ & $3.474 \mathrm{E}+03$ \\
\hline 39 & 0 & 74 & 0.000 & 131.868 & $0.000 E+00$ & $0.000 E+00$ & 4.994E+01 & 2.977E+01 & $6.585 E+03$ & $3.925 E+03$ \\
\hline 40 & 0 & 73 & 0.000 & 130.086 & $0.000 E+00$ & $0.000 E+00$ & $5.033 E+01$ & $3.000 E+01$ & 6.547E+03 & $3.902 E+03$ \\
\hline 41 & 0 & 74 & 0.000 & 131.868 & $0.000 \mathrm{E}+00$ & $0.000 E+00$ & 4.999E+01 & $2.980 E+01$ & $6.592 \mathrm{E}+03$ & $3.929 \mathrm{E}+03$ \\
\hline 42 & 0 & 74 & 0.000 & 131.868 & $0.000 \mathrm{E}+00$ & $0.000 \mathrm{E}+00$ & $4.549 \mathrm{E}+01$ & $2.712 \mathrm{E}+01$ & $5.999 \mathrm{E}+03$ & $3.576 \mathrm{E}+03$ \\
\hline 44 & 0 & 73 & 0.000 & 130.086 & $0.000 \mathrm{E}+00$ & $0.000 \mathrm{E}+00$ & $6.211 \mathrm{E}+01$ & $3.703 E+01$ & $8.080 E+03$ & 4.817E+03 \\
\hline 45 & 0 & 150 & 0.000 & 267.300 & $0.000 \mathrm{E}+00$ & $0.000 \mathrm{E}+00$ & $6.673 E+01$ & $3.978 E+01$ & 1.784E+04 & $1.063 \mathrm{E}+04$ \\
\hline 46 & 0 & 176 & 0.000 & 313.632 & $0.000 \mathrm{E}+00$ & $0.000 \mathrm{E}+00$ & $6.673 \mathrm{E}+01$ & $3.978 \mathrm{E}+01$ & 2.093E+04 & $1.248 \mathrm{E}+04$ \\
\hline 47 & 0 & 231 & 0.000 & 411.642 & $0.000 \mathrm{E}+00$ & $0.000 \mathrm{E}+00$ & $7.519 \mathrm{E}+01$ & $4.482 \mathrm{E}+01$ & $3.095 E+04$ & $1.845 \mathrm{E}+04$ \\
\hline 48 & 0 & 214 & 0.000 & 381.348 & $0.000 E+00$ & $0.000 E+00$ & $7.659 \mathrm{E}+01$ & $4.565 \mathrm{E}+01$ & $2.921 \mathrm{E}+04$ & $1.741 \mathrm{E}+04$ \\
\hline 49 & 0 & 226 & 0.000 & 402.732 & $0.000 \mathrm{E}+00$ & $0.000 \mathrm{E}+00$ & $7.755 \mathrm{E}+01$ & 4.623E+01 & $3.123 E+04$ & 1.862E+04 \\
\hline 50 & 107 & 0 & 381.348 & 0.000 & $7.684 \mathrm{E}+01$ & $4.581 \mathrm{E}+01$ & $0.000 E+00$ & $0.000 E+00$ & $2.930 E+04$ & 1.747E+04 \\
\hline 51 & 107 & 0 & 381.348 & 0.000 & 1.043E+02 & $6.220 \mathrm{E}+01$ & $0.000 \mathrm{E}+00$ & $0.000 \mathrm{E}+00$ & 3.979E+04 & $2.372 \mathrm{E}+04$ \\
\hline 52 & 71 & 0 & 253.044 & 0.000 & $8.456 \mathrm{E}+01$ & $5.041 \mathrm{E}+01$ & $0.000 E+00$ & $0.000 E+00$ & $2.140 \mathrm{E}+04$ & $1.275 \mathrm{E}+04$ \\
\hline 53 & 125 & 0 & 445.500 & 0.000 & $1.423 \mathrm{E}+02$ & $8.481 \mathrm{E}+01$ & $0.000 \mathrm{E}+00$ & $0.000 \mathrm{E}+00$ & $6.338 \mathrm{E}+04$ & $3.778 \mathrm{E}+04$ \\
\hline 54 & 90 & 184 & 320.760 & 327.888 & $1.001 \mathrm{E}+02$ & $5.965 \mathrm{E}+01$ & $1.044 \mathrm{E}+02$ & $6.223 E+01$ & $6.633 \mathrm{E}+04$ & $3.954 \mathrm{E}+04$ \\
\hline 55 & 197 & 0 & 702.108 & 0.000 & 1.337E+02 & 7.967E+01 & $0.000 \mathrm{E}+00$ & $0.000 E+00$ & $9.384 \mathrm{E}+04$ & $5.594 \mathrm{E}+04$ \\
\hline 56 & 161 & 0 & 573.804 & 0.000 & 1.267E+02 & $7.552 \mathrm{E}+01$ & $0.000 \mathrm{E}+00$ & $0.000 \mathrm{E}+00$ & $7.269 \mathrm{E}+04$ & 4.333E+04 \\
\hline 57 & 71 & 0 & 253.044 & 0.000 & $1.438 \mathrm{E}+02$ & $8.572 E+01$ & $0.000 E+00$ & $0.000 E+00$ & $3.639 \mathrm{E}+04$ & $2.169 E+04$ \\
\hline 58 & 86 & 0 & 306.504 & 0.000 & $1.012 \mathrm{E}+02$ & $6.033 \mathrm{E}+01$ & $0.000 \mathrm{E}+00$ & $0.000 \mathrm{E}+00$ & $3.102 E+04$ & 1.849E+04 \\
\hline 59 & 89 & 0 & 317.196 & 0.000 & 1.123E+02 & $6.694 \mathrm{E}+01$ & $0.000 \mathrm{E}+00$ & $0.000 \mathrm{E}+00$ & $3.562 \mathrm{E}+04$ & $2.123 E+04$ \\
\hline 60 & 84 & 0 & 299.376 & 0.000 & 1.332E+02 & $7.942 \mathrm{E}+01$ & $0.000 E+00$ & $0.000 E+00$ & $3.989 E+04$ & $2.378 E+04$ \\
\hline 61 & 4 & 0 & 14.256 & 0.000 & 1.376E+02 & $8.200 \mathrm{E}+01$ & $0.000 \mathrm{E}+00$ & $0.000 \mathrm{E}+00$ & $1.961 \mathrm{E}+03$ & 1.169E+03 \\
\hline 62 & 71 & 0 & 253.044 & 0.000 & 1.331E+02 & $7.932 \mathrm{E}+01$ & $0.000 E+00$ & $0.000 E+00$ & 3.367E+04 & 2.007E+04 \\
\hline 63 & 89 & 0 & 317.196 & 0.000 & $1.372 \mathrm{E}+02$ & $8.178 E+01$ & $0.000 E+00$ & $0.000 E+00$ & 4.352E+04 & $2.594 \mathrm{E}+04$ \\
\hline 64 & 100 & 0 & 356.400 & 0.000 & $1.422 \mathrm{E}+02$ & 8.477E+01 & $0.000 E+00$ & $0.000 E+00$ & $5.068 \mathrm{E}+04$ & $3.021 \mathrm{E}+04$ \\
\hline 65 & 71 & 0 & 253.044 & 0.000 & $1.482 E+02$ & $8.835 E+01$ & $0.000 \mathrm{E}+00$ & $0.000 \mathrm{E}+00$ & $3.750 \mathrm{E}+04$ & $2.236 \mathrm{E}+04$ \\
\hline 66 & 87 & 0 & 310.068 & 0.000 & $1.566 \mathrm{E}+02$ & $9.336 \mathrm{E}+01$ & $0.000 E+00$ & $0.000 E+00$ & $4.856 \mathrm{E}+04$ & $2.895 E+04$ \\
\hline 67 & 130 & 0 & 463.320 & 0.000 & 1.427E+02 & 8.507E+01 & $0.000 E+00$ & $0.000 E+00$ & $6.612 \mathrm{E}+04$ & $3.941 \mathrm{E}+04$ \\
\hline 68 & 70 & 0 & 249.480 & 0.000 & 1.363E+02 & $8.124 \mathrm{E}+01$ & $0.000 \mathrm{E}+00$ & $0.000 E+00$ & $3.400 \mathrm{E}+04$ & 2.027E+04 \\
\hline TOTALS & 1810 & 2261 & 6450.84 & \multicolumn{5}{|l|}{4029.102} & 1071125.9 & 638498.2 \\
\hline \multicolumn{11}{|c|}{$\begin{array}{l}\text { NOTES: } \\
\text { All results above represent the most-likely case. } \\
\text { In Run } 30 / 30 A, 585 \text { X-slugs were dissolved in addition to the W-slugs. The data above reflect only the } \\
\text { contribution from the W-slugs. }\end{array}$} \\
\hline \multicolumn{11}{|c|}{$\begin{array}{l}\text { The number of slugs charged for Runs } 41,42 \text { and } 53 \text { are estimated based on the known number shipped. } \\
\text { (The normal practice was to use two of the W-4 slugs or one of the W-8 slugs shipped for RaLa for iodine production.) }\end{array}$} \\
\hline \multicolumn{11}{|c|}{ Run 43 was a low-level break in run - only around $60 \mathrm{X}$-slugs were dissolved. } \\
\hline
\end{tabular}




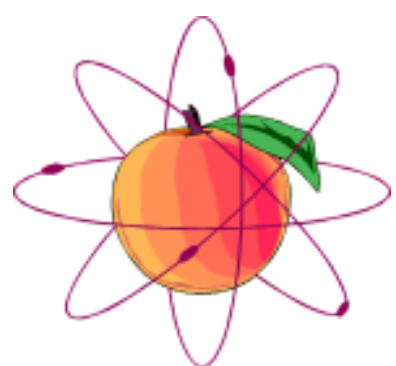

SRA

4939 Lower Roswell Road, Suite 106

Marietta, GA 30068

Phone: (770) 509-7606

FAX: (770) 509-7507

Internet: sra@crl.com

April 25, 1997

TO: $\quad$ Tom Widner

ChemRisk - Alameda

FROM: $\quad$ R. E. Burns, Jr.

Shonka Research Associates, Inc.

SUBJECT: Tube factors for the Hanford production reactors

MEMO NO: REB.005 C97

FINAL $[\mathbf{X}] \quad$ DRAFT [ ]

Distribution:

Talaat Ijaz - ChemRisk - Cleveland

Joe Shonka - Shonka Research Associates, Inc.

References:

Due to the large number of references involved in the development of this information, direct references are made in text to the appropriate documents without summarizing them here. Documents from ORNL Central Files are referred to using the standard $\mathrm{XX}-\mathrm{YY}-\mathrm{ZZZ}$ format, where $\mathrm{XX}$ is the calendar year, $\mathrm{YY}$ is the month an ZZZ is the sequential document number. Teletypes from Hanford are identified with the prefix "TT". Memo reports from Hanford are identified with the prefix "HW".

Memo:

The available references regarding RaLa operations at X-10 were reviewed for the purpose of compiling what we know about tube factors for the Hanford production reactors with respect to estimating radionuclide inventories for $\mathrm{W}$-slugs used in RaLa production. The intent was to improve our basis for the tube factor bounds established previously and to revise these bounds and the model used to describe the distribution of factors between them as necessary. Recall that the previous approach for tube factors was to treat them as a triangular distribution between 1.1 and 1.6, with a most likely value of 1.4 (Burns, R. E.; "Bounds for the average values of ${ }^{131}$ I content per W-slug pushed for RaLa", REB.003 C96, April 12, 1996).

The tube factor is the relative power of a given tube with respect to the average for all tubes in a given reactor. Note that the tube factor is a measure of only the radial variation in power and does not account for axial variation. Axial power variation was addressed by using what the 
Hanford staff referred to as "slug factors" (Moon, M. R.; Brugge, R. O.; "Slug and Tube Factors", HW-31848, May 13, 1954). These will be the subject of a forthcoming document and are not addressed here.

All of the Hanford production reactors considered in the Task 1 effort (i.e., the B, D, F, H, DR and C reactors) had a total of 2004 process tubes on a 8.375 inch pitch. Thus, there would have been little variability in tube factors between the different piles as long as the fuel and poison loading patterns were kept consistent among them. The central tubes in the piles were loaded with poisons to flatten out the power distribution.

From review of the available references (teletypes) we have to date regarding irradiation histories for $\mathrm{W}$-slugs used for RaLa production at X-10, we have tube factor values for 11 individual tubes. We also have tables of pile performance data that I extracted as handwritten notes from classified monthly production reports during a records search effort conducted at the DOE - Richland Records Holding Area in October of 1996. (These were submitted for classification review and were released to me before I left.) I extracted a total of eight of these summary tables for the months December of 1953, January of 1954, July of 1954, January of 1955, July of 1955, January of 1956, July of 1956 and October of 1956. (October of 1956 is the date of the last RaLa run conducted at X-10.)

The eleven tube factor values that we have (see Table 1) are only for tubes in the original three piles (B, $\mathrm{D}$ and F), and do not date beyond June of 1950. Thus, this information does not tell us how the tube factors may have differed for the later piles (H, DR and C) or how they may have changed over time. However, it does show us that RaLa slugs appear to have always been selected from tubes having a tube factor of at least 1.3, and thus the previous lower bound of 1.1 was likely too low. Even if the flattening for the B, D and F piles was increased in later years (resulting in a lower peak tube factor and less change with position), the fact that the tube factor data we have show that tubes having a factor greater than 1.3 were always used indicates that RaLa slugs were always selected from tubes in the central zone. (The tubes in the Hanford production reactors were broken up into three zones, with each zone characterized by a different tube inlet orifice diameter.) (Note that there is an error in document 50-4-9 in that one of the tube numbers is incomplete. I was not able to figure out what the number was supposed to be.)

While the information in Table 1 gives us an indication of the distribution in tube factors for RaLa slugs discharged from the original three piles through 1950, it does not tell us how this distribution might have changed beyond this time (due to changes in pile flattening or fuel loading), or what the distribution looked like for the later piles (H, DR and C). To address these issues, we need to look at the maximum tube factors for the various piles over time. This will tell us if there were any differences in power distribution between the different units, and if the distribution for a given unit changed over time.

The summary tables (see Fig. 1) contain information that allow us to estimate maximum tube factors for the piles for a given month. By assuming that the maximum power level that a given reactor achieved during a month corresponded to the maximum allowable tube power, one can compute the maximum tube factor by dividing the maximum allowable tube power by the average tube power. Under this 
assumption, the tube factors computed represent upper limits for a given month, as any higher factor would have resulted in exceeding the allowable tube power for a given pile.

Table 1 Known tube factors for the Hanford production piles

\begin{tabular}{|c|c|c|c|c|}
\hline Push Date & Reference & Tube Number & Pile & Tube Factor \\
\hline January, 1949 & $49-2-190$ & 2271 & F & 1.473 \\
\hline March, 1949 & TT 00456, TT00487 & 2268 & F & 1.563 \\
\hline March, 1949 & TT 00456, TT00487 & 2269 & F & 1.586 \\
\hline December, 1949 & $49-12-149$ & 3283 & B & 1.334 \\
\hline December, 1949 & $49-12-149$ & 3562 & B & 1.304 \\
\hline March, 1950 & $50-3-35$ & 2964 & D & 1.339 \\
\hline March, 1950 & $50-3-35$ & 3083 & D & 1.347 \\
\hline April, 1950 & $50-4-9$ & 2464 & D & 1.329 \\
\hline April, 1950 & $50-4-9$ & $? 488 ?$ & D & 1.336 \\
\hline June, 1950 & $50-6-49$ & 1178 & B & 1.304 \\
\hline June, 1950 & $50-6-49$ & 3364 & B & 1.301 \\
\hline
\end{tabular}

Fig. 2 shows the spreadsheet used for the calculation of the maximum (limiting) tube factor for each pile for each month where performance summary data were available. Note that the data in Fig. 2 do not address the period prior to December of 1953. (The H reactor came on line in October of 1949, the $\mathrm{DR}$ reactor in October of 1950 and the $\mathrm{C}$ reactor in November of 1952). Also, the B reactor was shut down during October and November of 1956.

The data in Fig. 2 show the $\mathrm{H}$ and $\mathrm{C}$ reactors had flatter power distributions than the other piles in the interval between December of 1953 and October of 1956, meaning these two reactors had lower peak tube factors than the others and that the factors changed less with distance from the pile center. The flatter power distribution seen for the $\mathrm{H}$ and $\mathrm{C}$ reactors is due to the use of slightly enriched fuel in the outer tubes, a practice began in January of 1953 (Ballinger, M. Y.; Hall, R. B.; "A History of Major Hanford Facilities and Processes Involving Radioactive Material”, PNL-6964 HEDR, Pacific Northwest Laboratory, March, 1991).

The data also show that the maximum tube factors for the other four piles were in many cases below the upper bound of 1.6 used previously. Given these observations, the following approach was established for choosing the upper bound for tube factors for the various Hanford reactors over the time period of interest for the purpose of computing radionuclide inventories per slug:

- $\quad$ prior to December of 1953, and for any month thereafter for which we do not have a known upper limit for tube factors, the upper bound for tube factors for the B, D, F and DR reactors will be set to 1.6;

- after December of 1953, in any cases where the upper limit for tube factor exceeds 1.6 for the B, D, F or DR reactors, an upper bound of 1.6 will be used; 
Figure 1 Example of pile performance data extracted from Hanford monthly production reports

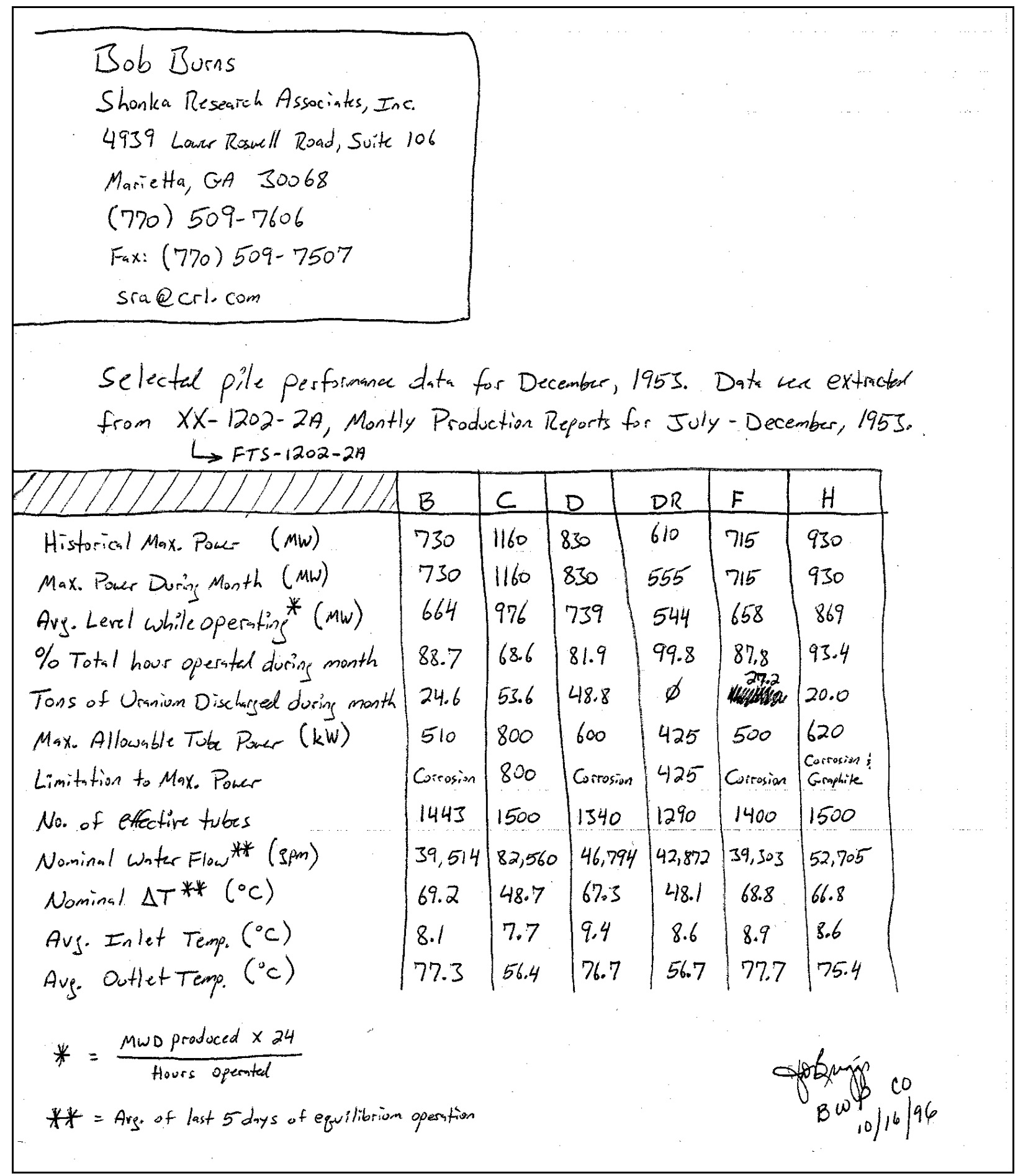


Figure 2 Spreadsheet for the calculation of maximum tube factors from the monthly pile performance data

\begin{tabular}{|c|c|c|c|c|c|c|c|c|c|}
\hline \multirow[b]{2}{*}{ Period } & \multicolumn{4}{|c|}{ B Reactor (power data in kW) } & \multirow[b]{2}{*}{ Period } & \multicolumn{4}{|c|}{ H Reactor (power data in kW) } \\
\hline & $\begin{array}{l}\text { Max. Power } \\
\text { During Month }\end{array}$ & $\begin{array}{l}\text { Avg. Power } \\
\text { per Tube }\end{array}$ & $\begin{array}{l}\text { Max. Tube } \\
\text { Power }\end{array}$ & $\begin{array}{l}\text { Max. Tube } \\
\text { Factor }\end{array}$ & & $\begin{array}{l}\text { Max. Power } \\
\text { During Month }\end{array}$ & $\begin{array}{l}\text { Avg. Power } \\
\text { per Tube }\end{array}$ & $\begin{array}{l}\text { Max. Tube } \\
\text { Power }\end{array}$ & $\begin{array}{l}\text { Max. Tube } \\
\text { Factor }\end{array}$ \\
\hline December, 1953 & 730000 & 364.271 & 510 & $\overline{1.400}$ & December, 1953 & 930000 & 464.072 & $\overline{620}$ & 1.336 \\
\hline January, 1954 & 747000 & 372.754 & 525 & 1.408 & January, 1954 & 930000 & 464.072 & 620 & 1.336 \\
\hline July, 1954 & 905000 & 451.597 & 675 & 1.495 & July, 1954 & 950000 & 474.052 & 570 & 1.202 \\
\hline January, 1955 & 1035000 & 516.467 & 805 & 1.559 & January, 1955 & 1035000 & 516.467 & 670 & 1.297 \\
\hline July, 1955 & 925000 & 461.577 & 740 & 1.603 & July, 1955 & 960000 & 479.042 & 640 & 1.336 \\
\hline January, 1956 & 935000 & 466.567 & 810 & 1.736 & January, 1956 & 1156000 & 576.846 & 795 & 1.378 \\
\hline July, 1956 & 850000 & 424.152 & 635 & 1.497 & July, 1956 & 1075000 & 536.427 & 663 & 1.236 \\
\hline \multirow[t]{2}{*}{ October, 1956} & $\mathrm{~N} / \mathrm{A}$ & $\mathrm{N} / \mathrm{A}$ & $\mathrm{N} / \mathrm{A}$ & $\mathrm{N} / \mathrm{A}$ & October, 1956 & 1095000 & 546.407 & 699 & 1.279 \\
\hline & \multicolumn{4}{|c|}{ D Reactor (power data in kW) } & & \multicolumn{4}{|c|}{ DR Reactor (power data in kW) } \\
\hline Period & $\begin{array}{l}\text { Max. Power } \\
\text { During Month }\end{array}$ & $\begin{array}{l}\text { Avg. Power } \\
\text { per Tube }\end{array}$ & $\begin{array}{c}\text { Max. Tube } \\
\text { Power }\end{array}$ & $\begin{array}{l}\text { Max. Tube } \\
\text { Factor }\end{array}$ & Period & $\begin{array}{l}\text { Max. Power } \\
\text { During Month }\end{array}$ & $\begin{array}{l}\text { Avg. Power } \\
\text { per Tube }\end{array}$ & $\begin{array}{l}\text { Max. Tube } \\
\text { Power }\end{array}$ & $\begin{array}{l}\text { Max. Tube } \\
\text { Factor }\end{array}$ \\
\hline December, 1953 & 830000 & 414.172 & 600 & 1.449 & December, 1953 & 555000 & 276.946 & 425 & 1.535 \\
\hline January, 1954 & 848000 & 423.154 & 640 & 1.512 & January, 1954 & 665000 & 331.836 & 525 & 1.582 \\
\hline July, 1954 & 775000 & 386.727 & 550 & 1.422 & July, 1954 & 697000 & 347.804 & 575 & 1.653 \\
\hline January, 1955 & 1125000 & 561.377 & 810 & 1.443 & January, 1955 & 780000 & 389.222 & 630 & 1.619 \\
\hline July, 1955 & 965000 & 481.537 & 710 & 1.474 & July, 1955 & 935000 & 466.567 & 675 & 1.447 \\
\hline January, 1956 & 1060000 & 528.942 & 820 & 1.550 & January, 1956 & 1075000 & 536.427 & 750 & 1.398 \\
\hline July, 1956 & 925000 & 461.577 & 635 & 1.376 & July, 1956 & 945000 & 471.557 & 636 & 1.349 \\
\hline \multirow[t]{2}{*}{ October, 1956} & 920000 & 459.082 & 668 & 1.455 & October, 1956 & 965000 & 481.537 & 663 & 1.377 \\
\hline & \multicolumn{4}{|c|}{ F Reactor (power data in kW) } & & \multicolumn{4}{|c|}{ C Reactor (power data in kW) } \\
\hline Period & $\begin{array}{c}\text { Max. Power } \\
\text { During Month }\end{array}$ & $\begin{array}{l}\text { Avg. Power } \\
\text { per Tube }\end{array}$ & $\begin{array}{l}\text { Max. Tube } \\
\text { Power }\end{array}$ & $\begin{array}{l}\text { Max. Tube } \\
\text { Factor }\end{array}$ & Period & $\begin{array}{l}\text { Max. Power } \\
\text { During Month }\end{array}$ & $\begin{array}{l}\text { Avg. Power } \\
\text { per Tube }\end{array}$ & $\begin{array}{l}\text { Max. Tube } \\
\text { Power }\end{array}$ & $\begin{array}{l}\text { Max. Tube } \\
\text { Factor }\end{array}$ \\
\hline December, 1953 & 715000 & 356.786 & 500 & 1.401 & December, 1953 & 1160000 & 578.842 & 800 & 1.382 \\
\hline January, 1954 & 725000 & 361.776 & 525 & 1.451 & January, 1954 & 1300000 & 648.703 & 800 & 1.233 \\
\hline July, 1954 & 824000 & 411.178 & 615 & 1.496 & July, 1954 & 1365000 & 681.138 & 950 & 1.395 \\
\hline January, 1955 & 980000 & 489.022 & 800 & 1.636 & January, 1955 & 1545000 & 770.958 & 1090 & 1.414 \\
\hline July, 1955 & 910000 & 454.092 & 720 & 1.586 & July, 1955 & 1500000 & 748.503 & 975 & 1.303 \\
\hline January, 1956 & 985000 & 491.517 & 840 & 1.709 & January, 1956 & 1725000 & 860.778 & 1125 & 1.307 \\
\hline July, 1956 & 855000 & 426.647 & 613 & 1.437 & July, 1956 & 1600000 & 798.403 & 951 & 1.191 \\
\hline October, 1956 & 905000 & 451.597 & 665 & 1.473 & October, 1956 & 1355000 & 676.148 & 885 & 1.309 \\
\hline
\end{tabular}

- between January and December of 1953, and for any month thereafter for which we do not have a known upper limit for tube factors, the upper bound for tube factors for the $\mathrm{C}$ and $\mathrm{H}$ reactors will be set to 1.4;

- $\quad$ prior to January of 1953 , the upper bound for tube factors for the H reactor will be set to 1.6;

- $\quad$ in cases where an upper limit is available for a given reactor and that values does not exceed 1.6, then that value will be used as the upper bound for tube factors.

The reason for choosing a upper bound of 1.6 for the months where the upper limit value in Fig. 2 exceeds 1.6 is that the upper limit values are estimates contingent on the assumption that the maximum power achieved by a given reactor during a month corresponded to a maximum tube power equal to the maximum allowable tube power. As this would likely not be the case, the upper limits given in Fig. 2 are therefore conservatively high. This is presumably why some of the upper limit values exceed 1.6. 1.6 is considered to be the highest value tube factors ever would have actually reached given it is unlikely that pile flattening would have ever been reduced for any reason. As time went on, efforts (such as using slightly enriched metal in the outer tubes) were made toward increasing the degree of flattening. Hence, a decrease in flattening was deemed unlikely. 
Note the approach outlined above for choosing upper bounds for tube factors does not address the first two months of operation for the $\mathrm{C}$ reactor (November and December of 1952). This was intentional, as it is known that no RaLa slugs came from the $\mathrm{C}$ reactor during this time.

At this point we have established upper bounds for tube factors for all of the piles over the time period of interest, but with respect to corresponding lower bounds, we only have such information for the B, D and $F$ reactors through June of 1950. Recall that a decrease in peak tube factor means an increase in pile flattening, and thus less change in tube factor as a function of distance from the center of a given pile. Hence, while a lower bound of 1.3 may be appropriate in cases where the peak tube factor is 1.6, a higher lower bound may be required in cases where the peak tube factor is lower.

Document HW-31848 contains both slug and tube factors calculated for the Hanford piles under the assumption of a cylindrical reactor model and that power distributions follow a cosine function outside of the central, flattened region. In the case of the tube factors, values are calculated using different flattening zone radii. These data can be used to establish appropriate lower bounds for tube factors based on corresponding upper bounds, as the upper bound is indicative of the degree of pile flattening, which in turn dictates the change in tube factor as a function of radius.

HW-31848 gives tube factors for eight cases, corresponding to eight different assumptions for flattening zone areas. These areas are given in terms of radius, where a radius of one equals the fuel channel pitch (8.375 inches). The eight radii are $0,6,10,12,14,16,18$ and 20 units. The entire reactor is modeled as a cylinder having a radius such that its area equals that of the active pile lattice, i.e., $(8.375)^{2} \times 2004$ tubes $=140,561.813$ square inches. Using this value, one finds a circle having a radius of 25.256 pitch units (211.519 inches) represents the same area.

Four sets of data from HW-31848 are shown in Fig.'s 3, 4 and 5 below. These data are the calculated tube factors (under the cylindrical assumption) for the 2004 tube piles for four different flattening zone radii: $0,6,10$ and 12 units. 
Figure 3 Tube factors from HW-31848 for a flattening zone radius of 0

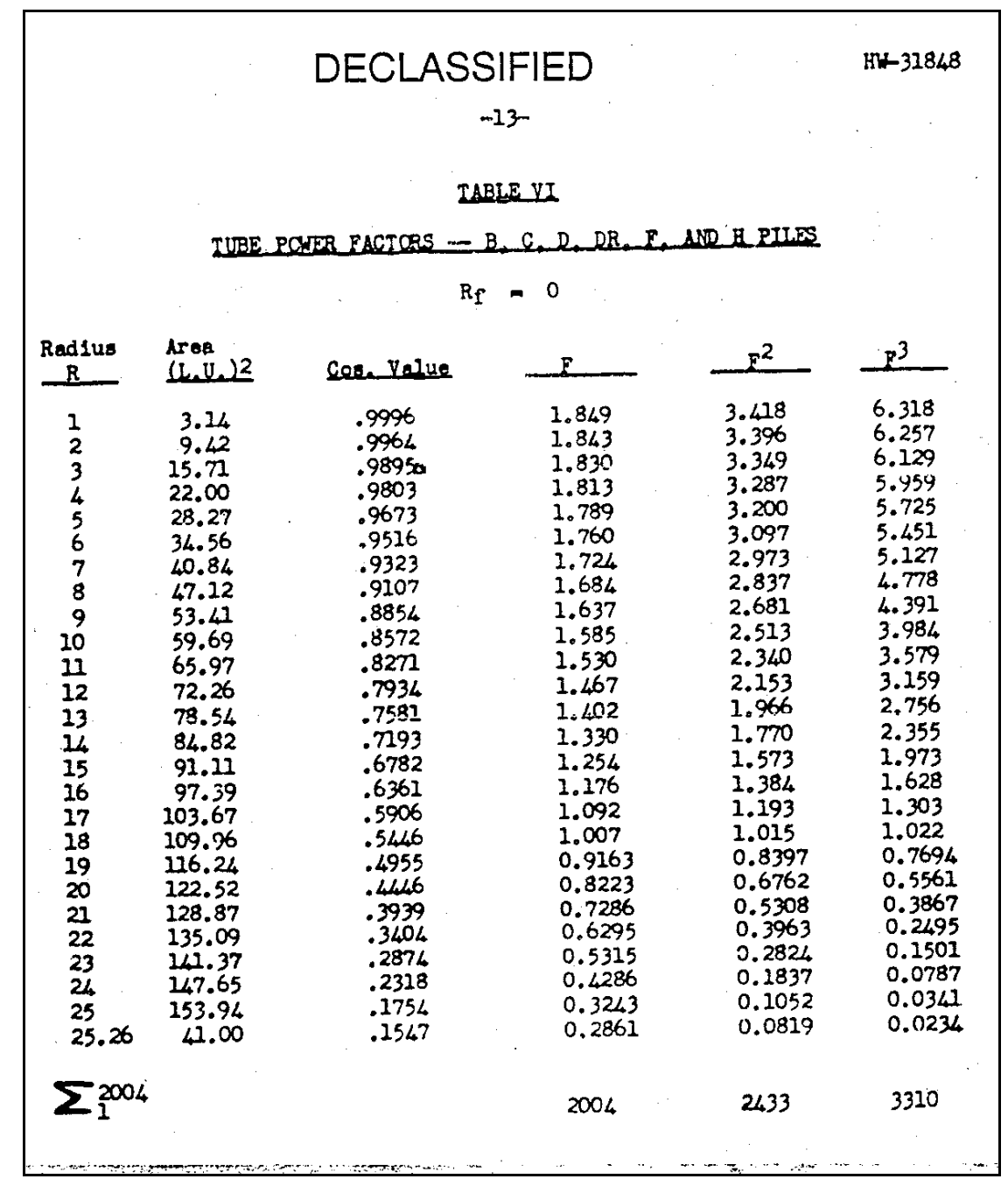


Figure 4 Tube factors from HW-31848 for a flattening zone radius of 6

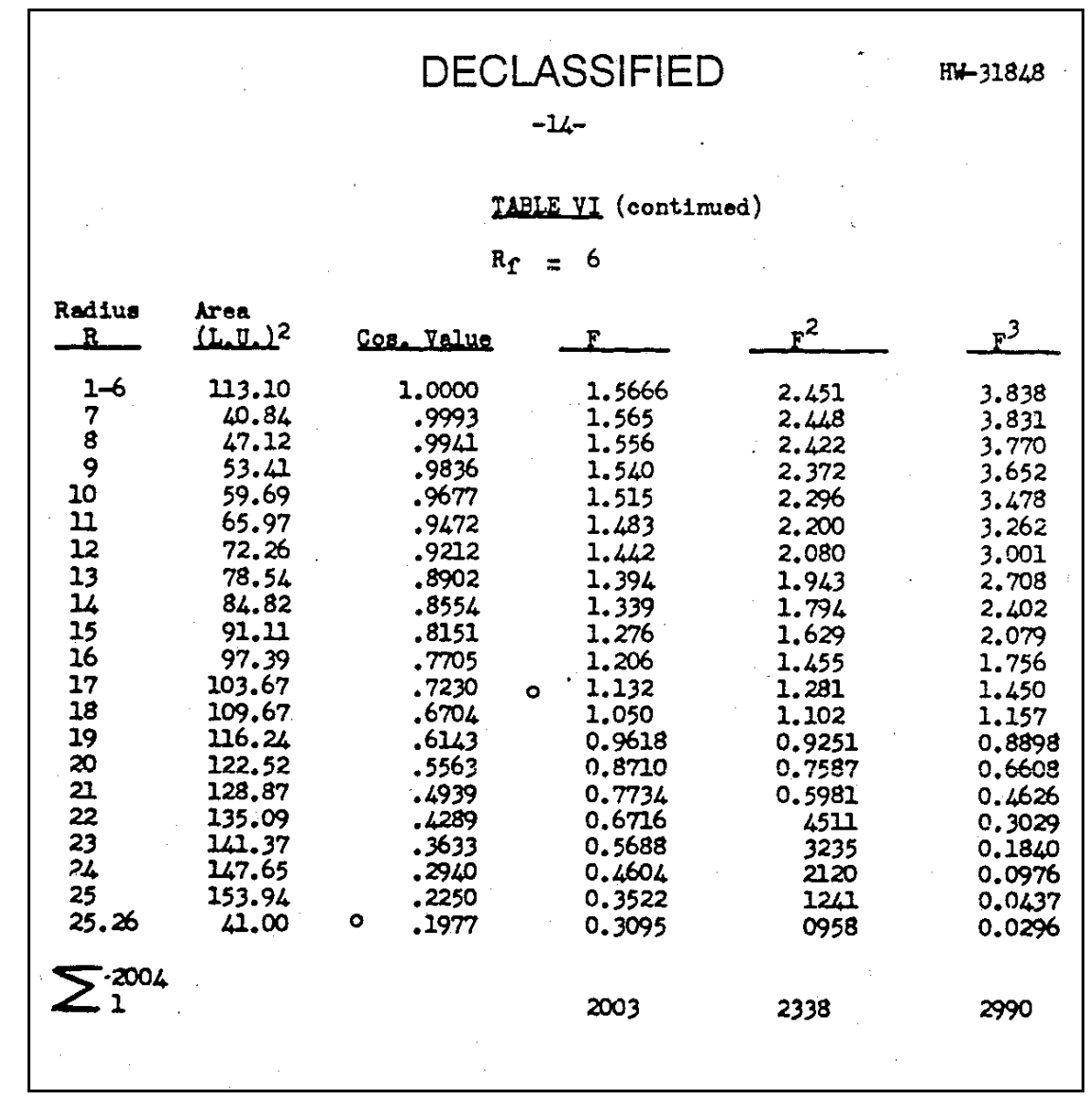


Figure 5 Tube factors from HW-31848 for flattening zone radii of 10 and 12

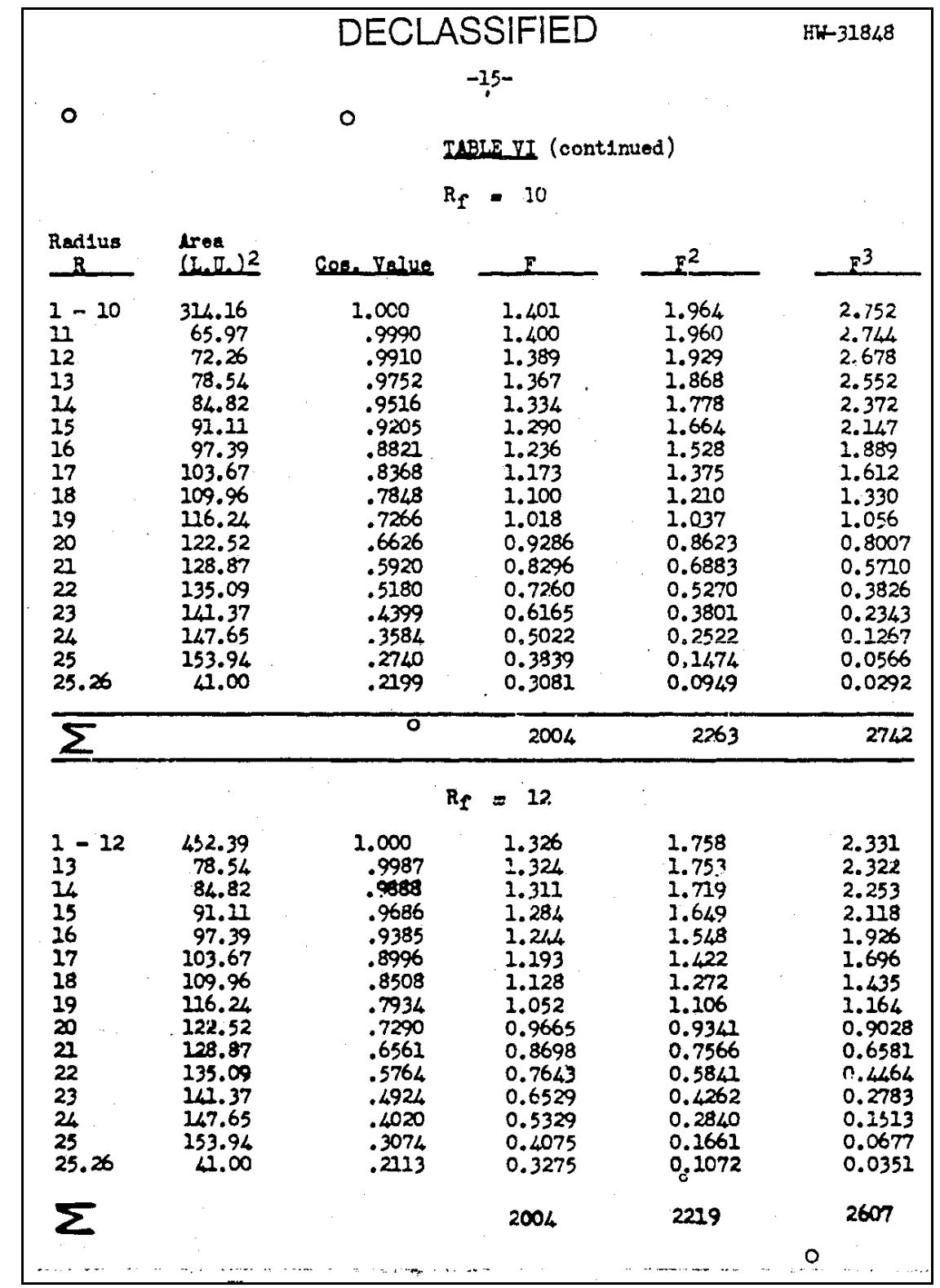

From inspection of the data in Fig.'s 3 through 5, one can see that a peak tube factor of 1.6 approximately corresponds to a flattening zone radius of 6 pitch units. [Calculation of the peak tube factor for a flattening zone radius of 5 gives a value greater than 1.6 (1.613).] The lower bound of 1.3 associated with the upper bound of 1.6 then approximately corresponds to a radius of 15 units. Thus, one can assert that this 15 unit radius corresponds to the set of tubes from which RaLa slugs were always chosen. Lower bounds for tube factors can then be chosen by determining the degree of pile flattening (in terms of cylindrical radius) based on the upper bound value and then setting the lower bound to the tube factor corresponding to a radius of 15 units. Using this approach, the following data 
(Table 2) were compiled using the derived tube factors from HW-31848. Note all values were rounded to two significant digits. The lower bound value for the upper bound of 1.5 was calculated, as a flattening zone radius of 8 was not included in HW-31848.

Table 2 Lower bounds for tube factors as a function of upper bound value

\begin{tabular}{|c|c|c|}
\hline Upper Bound for Tube Factor & Flattening Zone Radius & Lower Bound for Tube Factor \\
\hline 1.6 & 6 & 1.3 \\
\hline 1.5 & 8 & 1.3 \\
\hline 1.4 & 10 & 1.3 \\
\hline 1.3 & 12 & 1.3 \\
\hline 1.2 & 16 & 1.2 \\
\hline
\end{tabular}

Now that both upper and lower bounds for tube factors have been established, the question becomes how does one characterize the frequency distribution for tube power in cases where the pile of origin for RaLa slugs is unknown? In the case where the pile of origin is not known, there is then an equal chance that the slugs came from any of the operating piles. This then defines a uniform distribution. Further, since the monthly operating power level is known for all piles during the period of interest, it is easy to establish which units represent the lower and upper bounds for tube power. However, the question remains what the distribution of tube power looks like between these upper and lower bounds. To address this correctly, one must recognize there are two factors at play: overlap in operating power levels for the piles and the fact that the data in Table 1 indicate that RaLa slugs were more often selected from tubes farther from the pile center and only rarely from tubes closer in.

Overlap in pile power levels would bias the frequency distribution for tube factors in that the probability associated with an overlapped region would be increased. Conversely, if there were not overlap between power levels, the tube power distribution would be discontinuous. To examine whether or not there was overlap between possible tube power levels for the piles, the power levels over the period of interest were reviewed to look for cases where there were large differences between the lowest power and highest power piles. Power levels were examined between 1951 and 1956, as piles of origin are fairly well known prior to 1951, and the pile power levels were quite consistent. For each year between 1951 and 1956, the month corresponding to the lowest power level for any of the operating piles was established. The power levels for all of the operating piles were then examined to see if the corresponding tube powers overlapped when tube factors were considered. The months examined were January of 1951, December of 1952, August of 1953, February of 1954, April of 1955 and February of 1956. In all of these cases except January of 1951, there was not continuous overlap between tube power levels. Thus, the distribution of tube powers cannot be established as a continuous distribution between the lowest and highest powered piles. In addition, while there is generally not continuous overlap between tube power levels over a given time period, overlap does typically exist between a few of the piles. This results in an increase in the likelihood of a given tube power occurring, an effect that must be accounted for when assessing the overall distribution of tube powers. 
In light of the discussion above, it seems the only acceptable approach for accounting for the distribution in tube powers when computing radionuclide inventories for W-slugs for Task 1 will be to establish individual distributions for each possible pile of origin, and then uniformly sample from these to establish the aggregate distribution that represents the combined data set. To properly establish the distribution of tube powers for each possible pile of origin, the distribution of tubes chosen for RaLa slugs must be considered.

With respect to the distribution in tubes chosen for RaLa slugs, the data from Table 1 can be used. Inspection of these data show that the tubes chosen for RaLa slugs from the original three piles more often had tube factors in the range between 1.3 and 1.35, as there are only three cases where higher tube factors are seen. If one treats the data in Table 1 as six individual sets (corresponding to the six RaLa discharges they represent), it is seen that four out of the six times (66\%) tubes with factors less than 1.35 were chosen, while tubes having factors near the maximum value were used two out of the six times $(33 \%)$ - half the frequency of the lower-factor tubes. This means that the choice of tubes for RaLa slugs was either significantly biased toward tubes further from the pile center or that tubes close to poison columns were always selected. The later explanation is most unreasonable, and is easily dismissed if one computes the radial distance from the center tube for each tube in Table 1 and sorts by increasing radius.

The radial distance ( $\mathrm{r}$ ) from the center tube is computed by establishing the distance of each tube of interest from the pile center in both the $\mathrm{x}$ - and $\mathrm{z}$-directions and then using the expression

$$
r=\sqrt{(x)^{2}+(z)^{2}}
$$

Distance is determined using the known pitch for the process tubes (8.375 inches). The $\mathrm{x}$-direction is defined as going to the left or right from the center tube when facing the pile loading face, with left being negative and right being positive. The z-direction is defined as up and down, with up being positive. The radii for each tube in Table 1 and the corresponding tube factor are shown in Table 3. Note the data for the unknown tube number were omitted.

Table 3 Known tube factors as a function of radius from pile center

\begin{tabular}{|c|c|c|c|c|c|}
\hline Tube & Pile & Factor & $\underline{x}$ (inches) & $\underline{z \text { (inches) }}$ & $\underline{r}$ (inches) \\
\hline 2271 & $\mathrm{~F}$ & 1.473 & -20.9375 & -12.5625 & 24.417 \\
\hline 2669 & $\mathrm{~F}$ & 1.586 & -37.6875 & 20.9375 & 43.113 \\
\hline 2668 & $\mathrm{~F}$ & 1.563 & -46.0625 & 20.9375 & 50.598 \\
\hline 2464 & D & 1.329 & -79.5625 & 4.1875 & 79.673 \\
\hline 2964 & D & 1.339 & -79.5625 & 46.0625 & 91.934 \\
\hline 3083 & D & 1.347 & 79.5625 & 54.4375 & 96.403 \\
\hline 3283 & $B$ & 1.334 & 79.5625 & 71.1875 & 106.761 \\
\hline 1178 & $B$ & 1.304 & 37.6875 & -104.6875 & 111.265 \\
\hline 3364 & B & 1.301 & 79.5625 & -79.5625 & 112.518 \\
\hline 3562 & B & 1.304 & -96.3125 & 96.3125 & 136.206 \\
\hline
\end{tabular}


The data in Table 3 clearly show a correlation between tube factor and radius from the pile center ${ }^{1}$, meaning the choice of tubes for RaLa slugs was significantly biased toward tubes further out.

Conversely, an arbitrary selection process would have resulted in a much different distribution. This can be illustrated by establishing an expression for tube factor as a function of radius and then sampling radius uniformly across its range.

Tube factors ranging between 1.3 and 1.6 can be described by the function

$$
T F(r)=1.6 \cos \left[\frac{\pi}{2}(r)\right]
$$

where $r$ is empirically defined as the interval $0 \leq r \leq 0.3962$. If one uniformly samples $r$ on the defined interval, the distribution in Fig. 6 is obtained.

\section{Figure 6 Tube factors for arbitrary selection of tubes}

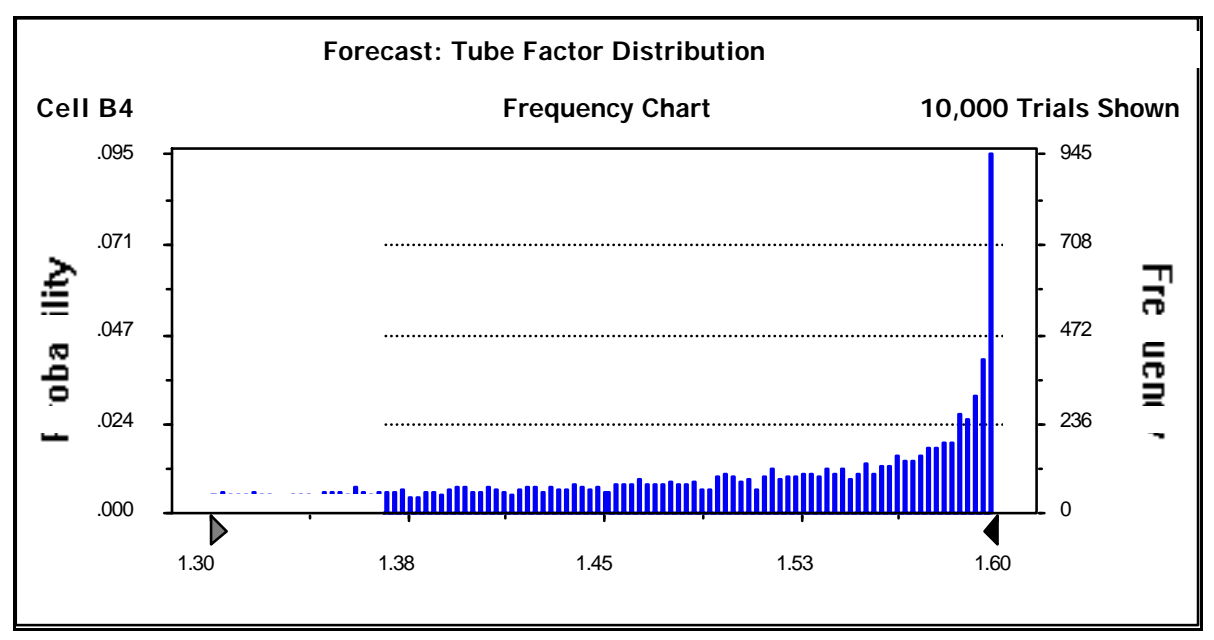

This distribution is clearly contrary to the observed distribution of tube factors from Tables 1 and 3 , confirming that selection of RaLa tubes was biased toward tubes further from the pile center (but still within the central zone defined by a radius of 15 pitch units under the cylindrical model). Thus, it appears tube factors (and consequently, tube powers) for a given pile should be established through sampling of a cosine function that gives tube factor as a function of position, where the sampling is biased so that outer positions are selected with twice the frequency of the inner ones.

Through trial-and-error, it was found that defining the radius parameter (r) as a Beta distribution bounded between 0 and 39.62 and having parameters alpha and beta equal to 4.25 and 1.00, respectively, would represent the desired distribution of tube factors. The parameter $r$ from Eq. 2 was

\footnotetext{
${ }^{1}$ It is presumed the factor for tube $2271 \mathrm{~F}$ is lower than those for tubes $2268 \mathrm{~F}$ and $2269 \mathrm{~F}$ because it was so near the flattening region.
} 
multiplied by 100 because Crystal Ball ${ }^{\mathrm{TM}}$ only allows values to two decimal places. Eq. 2 was therefore modified to

$$
T F(r)=1.6 \cos \left[\frac{\pi}{2}\left(\frac{r}{100}\right)\right]
$$

The desired value of the alpha parameter was established by comparing the ratio of the area under the tube factor probability curve between 1.30 and 1.35 to that for the interval 1.47 to 1.60 . Alpha was varied until this ratio achieved the value 2.0. For alpha $=4.25$ and beta $=1.00$, the probability associated with the range 1.30 to 1.35 was $35.31 \%$, versus a probability of $17.65 \%$ for the range 1.47 to 1.60. The entire frequency distribution is shown in Fig. 7 below.

Figure 7 Frequency distribution for tube factors represented using a Beta distribution

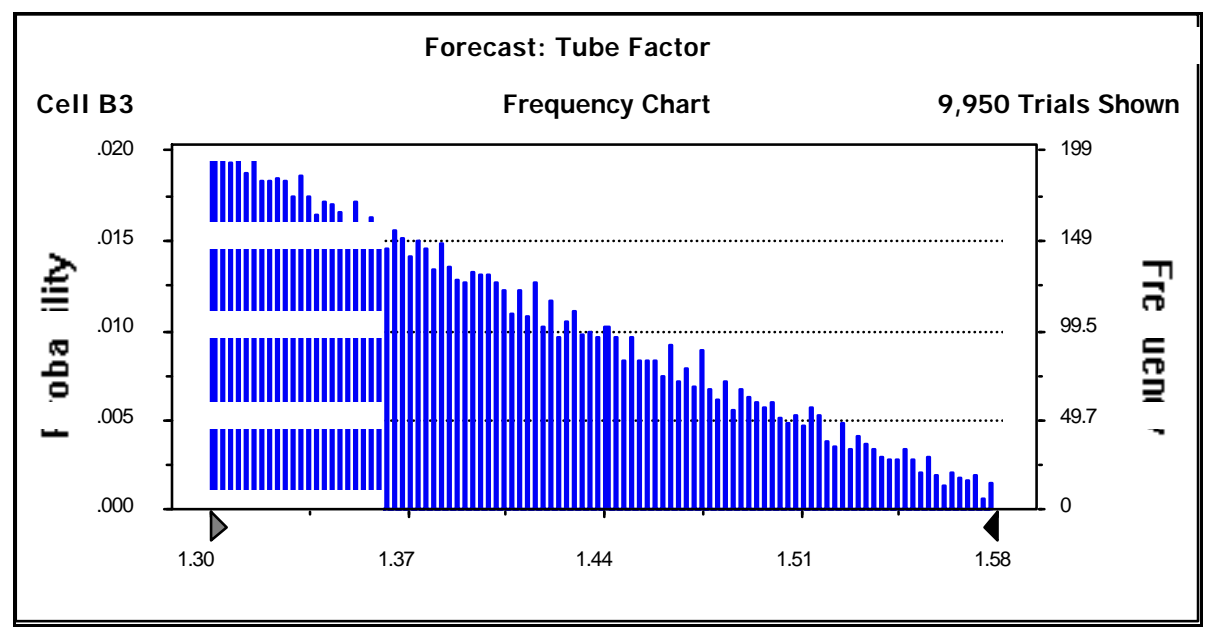

It is shown that applying Eq. 3 with $\mathrm{r}$ sampled as a Beta distribution bounded between 0 and 39.62 and with alpha and beta equal to 4.25 and 1.00, respectively, provides an adequate representation of the observed selection of tubes for RaLa slugs for tube factors bounded between 1.3 and 1.6. Hence, distributions for the other tube factor ranges given in Table 2 are defined by simply changing the bounds on $\mathrm{r}$ and $\mathrm{TF}_{\max }$ to give the desired range, where $\mathrm{TF}_{\max }$ is the upper bound of the tube factor range. The Beta distribution parameters alpha and beta do not change. Eq. 4 is then used to establish the frequency distribution for tube factors for any of the ranges given in Table 2. (Obviously, there is no need to establish distributions in cases where the piles are flattened to such an extent there is no distinction between the upper and lower tube factor bounds.)

$$
T F(r)=T F_{\max } \cos \left[\frac{\pi}{2}\left(\frac{r}{100}\right)\right]
$$

Table 4 gives the appropriate bounds for $\mathrm{r}$ for each tube factor range from Table 2. 
Table 4 Beta distribution parameters for establishing frequency distributions for tube factors for W-slug inventory calculations

\begin{tabular}{|c|c|c|c|c|}
\hline TF Range & TF $_{\text {max }}$ & Bounds for $\mathbf{r}$ & Alpha & Beta \\
\hline $1.3-1.6$ & 1.6 & $0.00-39.62$ & 4.25 & 1.00 \\
\hline $1.3-1.5$ & 1.5 & $0.00-33.25$ & 4.25 & 1.00 \\
\hline $1.3-1.4$ & 1.4 & $0.00-24.21$ & 4.25 & 1.00 \\
\hline
\end{tabular}

The one issue that remains to be addressed with respect to tube factors is tube factors in cases where a tube number is known, but the actual tube factor is not. This occurs a few times for runs between 1948 and 1950. Thus, the data from Table 3 (tube factor versus radius) can be used to establish appropriate tube factor values. The radius for the tube of interest would be calculated and compared with the radii from Table 3. A range of tube factors would then be established based on where the radius for the tube of interest fell with respect to those for which tube factors are known. 
Marietta, GA 30068

Phone: (770) 509-7606

FAX: (770) 509-7507

Internet: sra@crl.com

TO: $\quad$ Tom Widner

April 28, 1997

ChemRisk - Alameda

FROM: $\quad$ R. E. Burns, Jr.

Shonka Research Associates, Inc.

SUBJECT: Slug factors for the Hanford production reactors

MEMO NO: REB.007 C97

FINAL [X] DRAFT [ ]

Distribution:

Talaat Ijaz - ChemRisk - Cleveland

Joe Shonka - Shonka Research Associates, Inc.

References:

1. REB.005 C97, "Tube factors for the Hanford production reactors" memo from R. E. Burns, Jr. to Tom Widner dated April 25, 1997.

2. Moon, M. R.; Brugge, R. O.; "Slug and Tube Factors", HW-31848, May 13, 1954.

3. REB.003 C96, "Bounds for average values of ${ }^{131} \mathrm{I}$ content per W-slug pushed for RaLa" memo from R. E. Burns, Jr. to Tom Widner dated April 12, 1996.

Memo:

A memo was issued previously that addressed tube factors for the Hanford production reactors for the purpose of computing radionuclide inventories for $\mathrm{W}$-slugs used in RaLa production at X-10 [1]. That memo made reference to the fact a subsequent memo would be provided describing the basis for slug factors for the Hanford piles of interest. Thus, this memo (REB.007 C97) was developed to serve that purpose. It also provides fractions of total tube power contributed by a given number of slugs taken from the center of a tube. This fractional tube power information is needed because only the highest power slugs pushed from a given tube were actually used for RaLa.

The slug factor is the fraction of the total power for a given tube produced by a specific slug. Tube factors then describe the axial variation in power along a tube, and are a function of axial 
position. Conversely, a tube factor is the relative power of a given tube with respect to the average for all tubes in a given reactor. Tube factors then represent the radial variation in tube power for a given reactor.

Ref. [2] provides tabulated slug factors for the six Hanford reactors of interest with respect to RaLa production at X-10 between 1948 and 1956. All six of these reactors (the B, D, F, H, DR and C units) contained 2004 process tubes on a 8.375 inch pitch. As the standard charge (number of slugs per tube) for all six reactors was the same, there was no difference in slug factors among them. The slug factors given in Ref. [2] were computed assuming the distribution of power along a tube was a symmetric cosine function having its peak value at the tube's center. They are computed for a standard charge of 32 eight-inch slugs, with slug number 1 being the upstream slug (i.e., the one closest to the loading face of the pile). The tabulated slug factors (f) from HW-31848 are presented in Fig. 1.

As Fig. 1 only contained slug factors for channels containing 32 eight-inch slugs, factors for channels containing 64 four-inch slugs had to be calculated. The calculated factors are shown in Fig. 2.

Figure 1 Slug factors (f) for Hanford pile tubes containing 32 eight-inch slugs

\begin{tabular}{|c|c|c|c|c|c|c|c|c|}
\hline \multirow[b]{2}{*}{ Slug No. } & \multicolumn{7}{|c|}{$\begin{array}{c}\text { DECLASSIFIED } \\
\text { TAELE II } \\
\text { 32-SLUO COLUMN } \\
\text { SLU, FACIORS. }\end{array}$} & \multirow[t]{2}{*}{$E H-31848$} \\
\hline & Cos. Value & 1 & $\sum^{N}, \varepsilon$ & $?^{2}$ & $\underline{F}^{2}$ & $\leq \sum^{N}, F^{2}$ & $\sum^{\pi}, x^{2} / \leq^{\pi}, 2$ & \\
\hline $\begin{array}{l}1 \\
2 \\
3 \\
4 \\
5 \\
6 \\
7 \\
8 \\
9 \\
10 \\
11 \\
12 \\
13 \\
14 \\
15 \\
16 \\
17 \\
18 \\
19 \\
20 \\
21 \\
22 \\
23 \\
24 \\
25 \\
26 \\
27 \\
28 \\
29 \\
30 \\
31 \\
32\end{array}$ & $\begin{array}{l}.2164 \\
.3007 \\
.3827 \\
.4617 \\
.5373 \\
.6088 \\
.6756 \\
.7373 \\
.7934 \\
.8434 \\
.8860 \\
.9239 \\
.9537 \\
.9763 \\
.9914 \\
.9990 \\
.9990 \\
.9914 \\
.9763 \\
.9537 \\
.9939 \\
.8860 \\
.8434 \\
.7932 \\
.7373 \\
.6756 \\
.6088 \\
.5373 \\
.4617 \\
.3827 \\
.3007 \\
.2164\end{array}$ & $\begin{array}{l}.0095 \\
.0133 \\
.0169 \\
.0204 \\
.0237 \\
.0268 \\
.0298 \\
.0325 \\
.0350 \\
.0372 \\
.0391 \\
.0407 \\
.0420 \\
.0430 \\
.0437 \\
.0440 \\
.0440 \\
.0437 \\
.0430 \\
.0420 \\
.0407 \\
.0391 \\
.0372 \\
.0350 \\
.0325 \\
.0298 \\
.0268 \\
.0237 \\
.0204 \\
.0169 \\
.0133 \\
.0095\end{array}$ & $\begin{array}{l}.0095 \\
.0228 \\
.0397 \\
.0601 \\
.0838 \\
.1106 \\
.1404 \\
.1729 \\
.0079 \\
.2451 \\
.2842 \\
.3249 \\
.3669 \\
.4099 \\
.4536 \\
.4976 \\
.5416 \\
.5853 \\
.6283 \\
.6703 \\
.7110 \\
.7501 \\
.7873 \\
.8223 \\
.8548 \\
.8846 \\
.9114 \\
.9351 \\
.9555 \\
.9724 \\
.9857 \\
.9952\end{array}$ & $\begin{array}{l}.00009 \\
.00018 \\
.00029 \\
.00042 \\
.00056 \\
.00072 \\
.00089 \\
.00106 \\
.00123 \\
.00138 \\
.00152 \\
.00166 \\
.00176 \\
.00185 \\
.00191 \\
.001 \% \\
.00194 \\
.00191 \\
.00185 \\
.00176 \\
.00166 \\
.00152 \\
.00138 \\
.00123 \\
.00106 \\
.00089 \\
.00072 \\
.00056 \\
.00042 \\
.00029 \\
.00018 \\
.00009\end{array}$ & $\begin{array}{l}.0026 \\
.0052 \\
.0083 \\
.0120 \\
.0160 \\
.0206 \\
.0255 \\
.0304 \\
.0352 \\
.0395 \\
.0435 \\
.0475 \\
.0504 \\
.0530 \\
.0547 \\
.0556 \\
.0556 \\
.0547 \\
.0530 \\
.0504 \\
.0475 \\
.0435 \\
.0395 \\
.0352 \\
.0304 \\
.0255 \\
.0206 \\
.0160 \\
.0120 \\
.0083 \\
.0052 \\
.0026\end{array}$ & $\begin{array}{l}.0026 \\
.0078 \\
.0161 \\
.0281 \\
.0441 \\
.0647 \\
.0902 \\
.1206 \\
.1558 \\
.1952 \\
.2388 \\
.2863 \\
.3367 \\
.3897 \\
.4444 \\
.5000 \\
.5556 \\
.6103 \\
.6533 \\
.7137 \\
.7612 \\
.8047 \\
.0442 \\
.8794 \\
.9098 \\
.9353 \\
.9559 \\
.99719 \\
.9839 \\
.9992 \\
.9974 \\
1.000\end{array}$ & $\begin{array}{r}.2737 \\
.3421 \\
.4056 \\
.4676 \\
.5263 \\
.5850 \\
.6425 \\
.6975 \\
.7494 \\
.7968 \\
.8403 \\
.8812 \\
.9177 \\
.9507 \\
.9797 \\
1.005 \\
1.026 \\
1.043 \\
1.056 \\
1.065 \\
1.071 \\
1.073 \\
1.072 \\
1.069 \\
1.064 \\
1.057 \\
1.049 \\
1.039 \\
.1 .030 \\
1.020 \\
1.012 \\
1.005\end{array}$ & \\
\hline
\end{tabular}


Figure 2 Slug factors for Hanford pile tubes containing 64 four-inch slugs

\begin{tabular}{|cccccccc|}
\hline $\begin{array}{c}\text { Slug } \\
\text { Number }\end{array}$ & $\begin{array}{ccccccc}\text { Increment } \\
1\end{array}$ & $\begin{array}{c}\text { Cosine } \\
\text { Value }\end{array}$ & $\begin{array}{c}\text { Slug } \\
\text { Factor }\end{array}$ & $\begin{array}{c}\text { Slug } \\
\text { Number }\end{array}$ & $\begin{array}{c}\text { Cosine } \\
\text { Increment }\end{array}$ & $\begin{array}{c}\text { Salue } \\
\text { Factor }\end{array}$ \\
2 & 31.5 & 0.1951 & 0.0043 & 33 & 0.5 & 0.9998 & 0.0221 \\
3 & 29.5 & 0.2377 & 0.0053 & 34 & 1.5 & 0.9979 & 0.0221 \\
4 & 28.5 & 0.3214 & 0.0071 & 36 & 3.5 & 0.9884 & 0.0219 \\
5 & 27.5 & 0.3624 & 0.0080 & 37 & 4.5 & 0.9808 & 0.0217 \\
6 & 26.5 & 0.4027 & 0.0089 & 38 & 5.5 & 0.9713 & 0.0215 \\
7 & 25.5 & 0.4423 & 0.0098 & 39 & 6.5 & 0.9600 & 0.0213 \\
8 & 24.5 & 0.4810 & 0.0107 & 40 & 7.5 & 0.9469 & 0.0210 \\
9 & 23.5 & 0.5188 & 0.0115 & 41 & 8.5 & 0.9320 & 0.0206 \\
10 & 22.5 & 0.5556 & 0.0123 & 42 & 9.5 & 0.9153 & 0.0203 \\
11 & 21.5 & 0.5913 & 0.0131 & 43 & 10.5 & 0.8969 & 0.0199 \\
12 & 20.5 & 0.6259 & 0.0139 & 44 & 11.5 & 0.8767 & 0.0194 \\
13 & 19.5 & 0.6593 & 0.0146 & 45 & 12.5 & 0.8549 & 0.0189 \\
14 & 18.5 & 0.6915 & 0.0153 & 46 & 13.5 & 0.8315 & 0.0184 \\
15 & 17.5 & 0.7224 & 0.0160 & 47 & 14.5 & 0.8064 & 0.0179 \\
16 & 16.5 & 0.7518 & 0.0167 & 48 & 15.5 & 0.7799 & 0.0173 \\
17 & 15.5 & 0.7799 & 0.0173 & 49 & 16.5 & 0.7518 & 0.0167 \\
18 & 14.5 & 0.8064 & 0.0179 & 50 & 17.5 & 0.7224 & 0.0160 \\
19 & 13.5 & 0.8315 & 0.0184 & 51 & 18.5 & 0.6915 & 0.0153 \\
20 & 12.5 & 0.8549 & 0.0189 & 52 & 19.5 & 0.6593 & 0.0146 \\
21 & 11.5 & 0.8767 & 0.0194 & 53 & 20.5 & 0.6259 & 0.0139 \\
22 & 10.5 & 0.8969 & 0.0199 & 54 & 21.5 & 0.5913 & 0.0131 \\
23 & 9.5 & 0.9153 & 0.0203 & 55 & 22.5 & 0.5556 & 0.0123 \\
24 & 8.5 & 0.9320 & 0.0206 & 56 & 23.5 & 0.5188 & 0.0115 \\
25 & 7.5 & 0.9469 & 0.0210 & 57 & 24.5 & 0.4810 & 0.0107 \\
26 & 6.5 & 0.9600 & 0.0213 & 58 & 25.5 & 0.4423 & 0.0098 \\
27 & 5.5 & 0.9713 & 0.0215 & 59 & 26.5 & 0.4027 & 0.0089 \\
28 & 4.5 & 0.9808 & 0.0217 & 60 & 27.5 & 0.3624 & 0.0080 \\
29 & 3.5 & 0.9884 & 0.0219 & 61 & 28.5 & 0.3214 & 0.0071 \\
30 & 2.5 & 0.9941 & 0.0220 & 62 & 29.5 & 0.2798 & 0.0062 \\
31 & 1.5 & 0.9979 & 0.0221 & 63 & 30.5 & 0.2377 & 0.0053 \\
32 & 0.5 & 0.9998 & 0.0221 & 64 & 31.5 & 0.1951 & 0.0043 \\
\hline
\end{tabular}

The data in Fig.'s 1 and 2 can be used to calculate the power contributed by an individual slug in the case where the total tube power in known. However, for the purpose of calculating radionuclide inventories for RaLa slugs, it is more useful to use these data to compute the fraction of the total tube power contained in a given set of slugs, e.g., a fixed number of slugs from the center of the tube. This fraction is simply the sum of the cosine values for the slugs of interest divided by the sum for the whole channel.

Not all slugs pushed from a given tube were actually used for RaLa production. Instead, only the highest power slugs from a given tube were shipped with the rest going to waste. (The burnups were typically too low for them to have significant plutonium content.) Hence, two assumptions were established previously: 1) for 32 slug tubes (eight-inch slugs), only the center 18 were used for RaLa; and 2) for 64 slug tubes (four-inch slugs), only the center 38 slugs were used [3]. 
Referring to Fig. 1, the center 18 slugs from a 32 slug tube would be slug numbers 8 through 25 . By summing the cosine values for these slugs and dividing by the sum for all slugs, one finds that the center 18 slugs from a 32 slug tube provide $71.8 \%$ of the tube's power. This value can be used to scale radionuclide inventory results computed from an entire tube to get the inventory contained in just the center 18 slugs.

Referring to Fig. 2, one sees that the center 38 slugs for a 64 slug tube would be numbers 14 through 51. The fraction of the total tube power contained in these 38 slugs is computed to be $74.9 \%$.

If there are cases where it is known that a different number of center slugs were used from a particular tube, then the data in Fig.'s 1 or 2 (as appropriate) would be used to compute the fraction of total tube power produced by the slugs of interest in the same manner as above. 


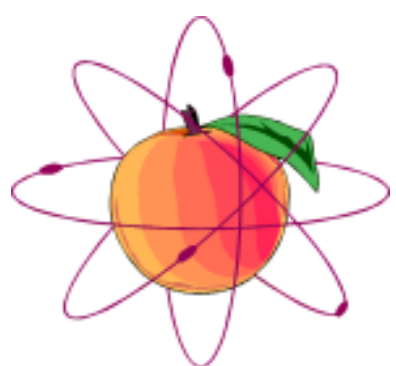

SRA

4939 Lower Roswell Road, Suite 106

Marietta, GA 30068

Phone: (770) 509-7606

FAX: (770) 509-7507

Internet: sra@crl.com

May 19, 1997

TO: $\quad$ Tom Widner

ChemRisk - Alameda

FROM: $\quad$ R. E. Burns, Jr.

Shonka Research Associates, Inc.

SUBJECT: Decay times for $\mathrm{W}$-slugs

MEMO NO: REB.004 C97

FINAL [X] DRAFT [ ]

Distribution:

Talaat Ijaz - ChemRisk - Cleveland

Joe Shonka - Shonka Research Associates, Inc.

References:

Due to the large number of references involved in the development of this information, direct references are made in text to the appropriate documents without being summarized here. Document HANFORD 45828 is a collection of 100 area monthly reports for the Hanford site for the calendar year 1949. These reports provide detailed summaries of pile operations for each pile from January through November of that year. Documents from the $\mathrm{X}-10$ Central Files are referred to using the standard XX-YY-ZZZ format, where XX is the calendar year, YY is the month an $\mathrm{ZZZ}$ is the sequential document number. Likewise, logbooks are referred to by their assigned logbook numbers.

Memo:

The available references regarding RaLa operations at X-10 were reviewed for the purpose of compiling what we know about decay times for fuel slugs discharged from the various Hanford production reactors and shipped to X-10 for RaLa production. Note that the start of the decay time for RaLa slugs is when the reactor was shut down prior to discharge, and not the time when discharge actually occurred. In the case of Hanford pile operations, the time between shutdown for discharge and subsequent restart was around 24 hours. Hence, the elapsed time between shutdown and actual discharge needs to be accounted for. Likewise, the end of the decay interval is defined as the start of the first dissolving, and not the start of coating removal or slug charging. 
Review of the available information resulted in two sets of data being compiled: cases where time of pile shutdown was known, and cases where only a push date was known. Both sets of data are summarized below by RaLa run number, followed by conclusions. Note that in some cases, the time when the first cut began is known exactly, and in others, this time is known only approximately. I have used the word "around" to denote judgment on my part based on my familiarity with RaLa operations at X-10. Precision for the given decay times is to the nearest 12 hours ( 0.5 day). Also note that all times and dates have been adjusted to the eastern time zone.

\section{RaLa runs for which the time of pile shutdown is known:}

$\underline{\text { Run 29: }}$

Pile shutdown time: 22:20 hours on 1/4/49 [HANFORD 45828]

First cut: 1/10/49 [49-1-184]

Decay time: around 5.5 days

$\underline{\text { Run 30: }}$

Pile shutdown time: 05:20 hours on 2/8/49 [HANFORD 45828]

First cut: 2/14/49 [49-4-35]

Decay time: around 6.5 days

$\underline{\text { Run 31: }}$

Pile shutdown time: 00:20 hours on 3/15/49 [HANFORD 45828]

First cut: 3/20/49, morning [logbook A-37 part I]

Decay time: around 5.5 days

$\underline{\text { Run 32: }}$

Pile shutdown time: 03:50 hours on 4/12/49 [HANFORD 45828]

First cut: 4/18/49, around 02:00 hours [logbook A-37 part I]

Decay time: 6.0 days

Run 33:

Pile shutdown time: 04:00 hours on 5/18/49 [HANFORD 45828]

First cut: 5/24/49, around 06:00 hours [logbook A-37 part I]

Decay time: 6.0 days

$\underline{\text { Run 34: }}$

Pile shutdown time: 22:31 hours on 7/5/49 [HANFORD 45828]

First cut: 7/11/49, midnight [logbook A-37 part I]

Decay time: 6.0 days 
$\underline{\text { Run 35: }}$

Pile shutdown time: $01: 20$ hours on 8/10/49 [HANFORD 45828]

First cut: 8/15/49, around 10:00 hours [logbook A-37 part I]

Decay time: 5.5 days

$\underline{\text { Run 36: }}$

Pile shutdown time: $02: 35$ hours on 10/4/49 [HANFORD 45828]

First cut: 10/10/49, around 02:00 hours [logbook A-37 part II]

Decay time: 6.0 days

$\underline{\text { Run 37: }}$

Pile shutdown time: $07: 50$ hours on 11/16/49 [HANFORD 45828]

First cut: 11/21/49, around 02:00 hours [logbook A-37 part II]

Decay time: 5.0 days

$\underline{\text { Data summary for runs where pile shutdown time is known: }}$

\begin{tabular}{|c|c|}
\hline Run Number & Decay Time \\
\hline 29 & around 6.5 days \\
\hline 30 & around 5.5 days \\
\hline 31 & around 6.0 days \\
\hline 32 & 6.0 days \\
\hline 33 & 6.0 days \\
\hline 34 & 6.0 days \\
\hline 35 & 5.5 days \\
\hline 36 & 6.0 days \\
\hline 37 & 5.0 days \\
\hline
\end{tabular}

\section{RaLa runs for which only the push date is known:}

The estimated decay times given below include time to account for the decay between the time of pile shutdown and the time the slugs were pushed. A nominal interval of 12 hours was used. For Runs 51 and 53, there were problems with slugs getting stuck in chute during loading that resulted in extended decay times. For Run 55, there was a long delay between slug discharge and shipping that resulted in a much longer than usual decay time.

\section{$\underline{\text { Run 51: }}$}

Push date: 9/18/52 [52-9-113]

First cut: 9/25/52, around 00:00 hours [logbook A-379]

Decay time: around 7.0 days 
$\underline{\text { Run 53: }}$

Push date: 6/27/53 [53-6-211]

First cut: 7/4/53 at 13:30 hours [logbook A-429]

Decay time: around 7.5 days

$\underline{\text { Run 55: }}$

Push date: 1/5/54 [54-1-42]

First cut: 1/16/54 at 21:00 hours [logbook A-429]

Decay time: around 11 days

$\underline{\text { Run 57: }}$

Push date: 7/12/54 [54-7-61]

First cut: 7/17/54 at around 16:00 hours [logbook A-616]

Decay time: around 5.5 days

$\underline{\text { Run 58: }}$

Push date: 10/18/54 [54-10-85]

First cut: 10/23/54, afternoon [logbooks A-429 and A-616]

Decay time: around 5.5 days

Run 59:

Push date: $02 / 25 / 55$ [55-3-9]

First cut: 03/02/55 around 16:00 hours [logbook A-664]

Decay time: around 5.5 days

$\underline{\text { Run 68: }}$

Push date: 10/14/56 [56-10-57]

First cut: 10/21/56, around 17:00 hours [logbook A-429]

Decay time: around 7.0 days 
Data summary for runs where only the push date is known:

\begin{tabular}{|c|c|}
\hline Run Number & Decay Time \\
\hline 51 & around 7.0 days \\
\hline 53 & around 7.5 days \\
\hline 55 & around 11.0 days \\
\hline 57 & around 5.5 days \\
\hline 58 & around 5.5 days \\
\hline 59 & around 5.5 days \\
\hline 68 & around 7.0 days \\
\hline
\end{tabular}

\section{Conclusions:}

The two sets of decay times detailed above are combined below.

\begin{tabular}{|c|c|}
\hline Run Number & Decay Time \\
\hline 29 & around 6.5 days \\
\hline 30 & around 5.5 days \\
\hline 31 & around 6.0 days \\
\hline 32 & 6.0 days \\
\hline 33 & 6.0 days \\
\hline 34 & 6.0 days \\
\hline 35 & 5.5 days \\
\hline 36 & 6.0 days \\
\hline 37 & 5.0 days \\
\hline 51 & around 7.0 days \\
\hline 53 & around 7.5 days \\
\hline 55 & around 11.0 days \\
\hline 57 & around 5.5 days \\
\hline 58 & around 5.5 days \\
\hline 59 & around 5.5 days \\
\hline 68 & around 7.0 days \\
\hline
\end{tabular}

If one treats the above data as a single set and ignores the subjective nature of many of the points, the following distribution is obtained: 


\begin{tabular}{|c|c|}
\hline Decay Time & Number of Occurrences \\
\hline 5.0 days & 1 \\
\hline 5.5 days & 5 \\
\hline 6.0 days & 5 \\
\hline 6.5 days & 1 \\
\hline 7.0 days & 2 \\
\hline 7.5 days & 1 \\
\hline 11.0 days & 1 \\
\hline
\end{tabular}

Thus, for the sixteen cases for which decay time can be established, the most likely decay interval between reactor shutdown and the start of dissolving was 5.5 to 6.0 days (10 out of 16 times or $62 \%$ ). Eleven out of the sixteen intervals (69\%) occur in the range from 5.0 to 6.0 days. There are three intervals (19\%) in the range 6.0 to 7.0 days and two that are greater than 7.0 days (12\%).

Recognize that the distribution data described above are days of decay time, and not the magnitude of the decay itself. The magnitude of decay as a function of decay time is obviously a function of the nuclide or combination of nuclides of interest, and is inherently logarithmic in terms of frequency distribution.

Conversely, the distribution for days of decay time should be treated as uniform between the appropriate lower and upper bounds in cases where the actual decay interval is unknown. (There was an error made in the Hanford study where decay was inappropriately sampled uniformly rather than as a logarithmic distribution) (PNWD-2222 HEDR, page 4.17, item 4).

For nuclides that do not experience significant ingrowth from precursors, the magnitude of decay is computed by simply applying the known half-life of the species of interest. With respect to decay calculations for Task 1, this would apply for both ${ }^{131} \mathrm{I}$ and ${ }^{140} \mathrm{Ba}$. However, for the total iodine values, simple decay is not the case. The presence of the stable fission product ${ }^{127} \mathrm{I}$ in the total iodine values makes it necessary to establish decay expressions that are a function of irradiation time. This is due to the fact there is no decay associated with the stable iodine. Hence, the quantity present in irradiated nuclear fuel always increases with increased irradiation time. There is never any decay, and no equilibrium condition can be established (loss from neutron absorption reactions is being negated here.) The quantitative expression for computing the decay of the total iodine inventory versus time is therefore a strong function of the amount of ${ }^{127} \mathrm{I}$ present in the fuel at discharge, since, in limit, the decay expression reaches an asymptote at this amount when time becomes large with respect to the effective half life of the radioactive fission product iodines.

In the inventory calculations performed for the $\mathrm{W}$-slug RaLa runs, there were in general three irradiation durations used to compute the ingrowth of fission products in the slugs. For the earlier W-slug runs, irradiation time was bounded between 50 and 100 days. A larger interval of 14 to 100 days was used for runs that took place after 1951. There were also runs where the actual irradiation time was known, however, in all of these cases the durations were close enough to one of the three "standard" durations that no distinction was deemed necessary for the purpose of computing percent saturation or decay. Thus, three expressions are needed to compute decay of the total iodine results from the W-slug inventory calculations, corresponding to the three irradiation durations 14, 50 and 100 days. 
The general expression for computing decay for the total iodine inventories is

$$
f(t)=a e^{b t}+c e^{d t}
$$

where $f(t)$ is the fraction remaining at time $t$,

$t$ is the elapsed time in days since reactor shutdown, and

$\mathrm{a}, \mathrm{b}, \mathrm{c}$ and $\mathrm{d}$ are constant coefficients.

The coefficients required to compute decay for the total iodine values for each irradiation duration are given in the table below. These values were obtained through fitting total iodine results from ORIGEN2.1 as a function of time for the three irradiation times of interest.

\begin{tabular}{|c|c|c|c|}
\hline & \multicolumn{3}{|c|}{ Irradiation Duration } \\
\hline Coefficient & 14 days & 50 days & 100 days \\
\hline a & 0.6810 & 0.7971 & 0.8741 \\
\hline b & -0.0362 & -0.0203 & -0.0104 \\
\hline c & 0.3190 & 0.2029 & 0.1259 \\
\hline d & -0.4477 & -0.4749 & -0.4433 \\
\hline
\end{tabular}




\section{APPENDIX 30 \\ DOCUMENTS PERTAINING TO THE 1954 RALA ACCIDENT}

1. April 30, 1954 memorandum from W. M. Stanley Jr., Laboratory Shift Supervisor, to C. E. Larson, ORNL Director; Subject: "Area Contamination on April 29, 1954." (partial copy, 2 pages).

2. May 3, 1954 memorandum from W. M. Stanley Jr., Laboratory Shift Supervisor, to M. E. Ramsey; Subject: “3026 Incident 4/29/54 4:48 p.m.” (2 pages).

3. May 10, 1954 memorandum from W. M. Stanley Jr., Laboratory Shift Supervisor, to M. E. Ramsey; Subject: “3026-D Incident of April 29, 1954” (4 pages).

4. Pages from X-10 Health Physics notebook A-569 for 4/29/54 (4 pages).

5. "RaLa Production - 1954" by A. F. Rupp and E. J. Witkowski. ORNL Central Files Number 55-1-211 (Partial copy, 3 pages).

6. June 2, 1954 memorandum from C.E. Larson to K. A. Kasschau of the USAEC; Subject: "RaLa Production." (4 pages). 
( $\left.\begin{array}{c}\text { INSERT } \\ \text { NAME }\end{array}\right)$

NAME COMPANY

C. R. Inteos

Lochtion Bullatis 4500

ATTENTION

$\begin{aligned} \text { Copy to K. 7. Morganat } & \text { A. F. Rupp }\end{aligned}$
INTER-GOMPANY CORRESPONDENCE

OAK RIOGE NATIONAL IABORATORY

Operated By

CARBDE AND CARBON CHEMTCALS COMPANY LOCATION

Post office Bor D OAK RIDGE, THNN 124

DATE Awell 30, 195t, 8,00 ant. ANSWERING LETTER DATE

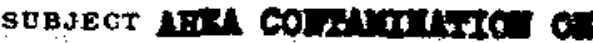
Arite, 29, 195k

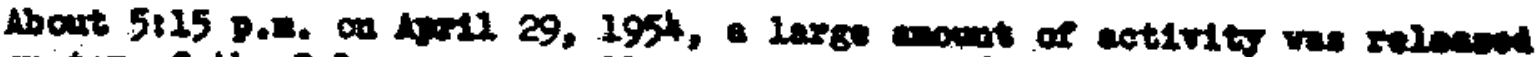

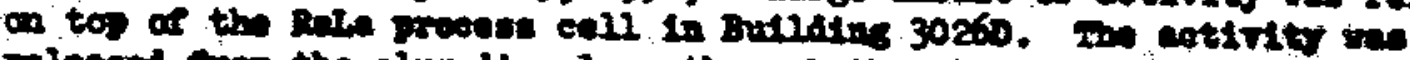

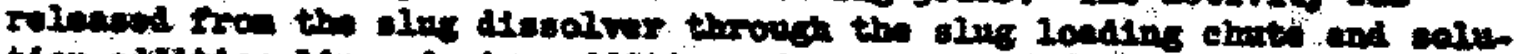

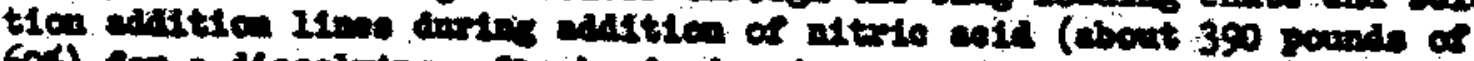

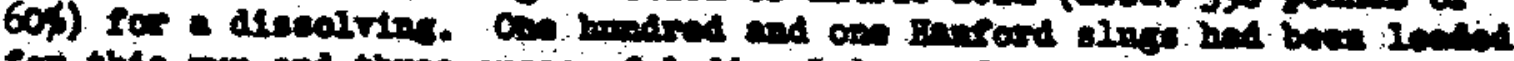

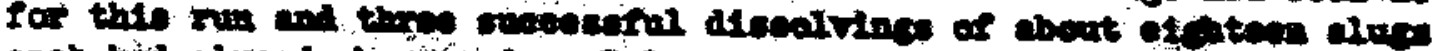

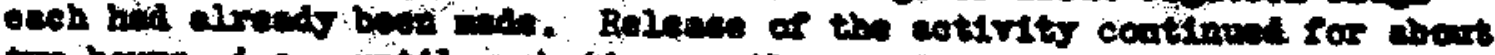

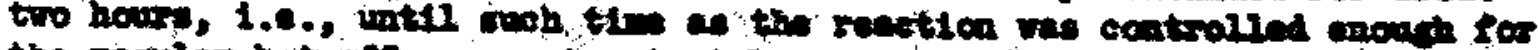

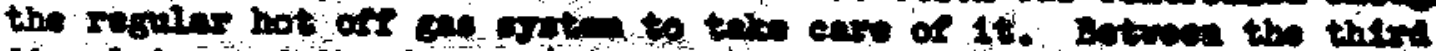

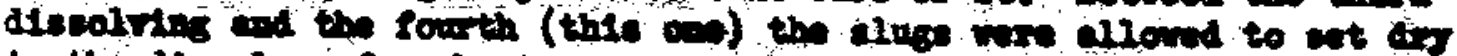

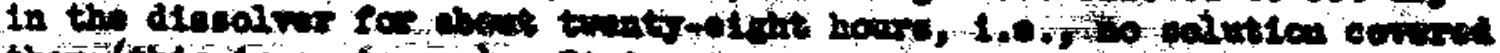

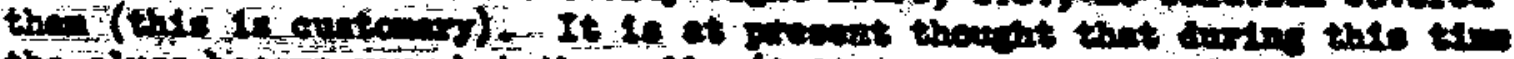

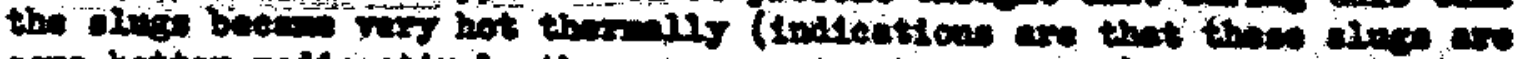

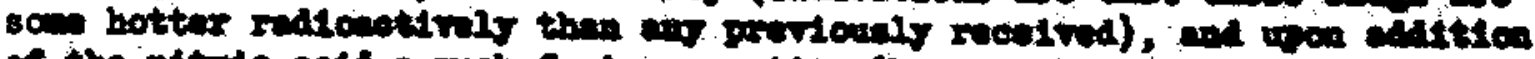

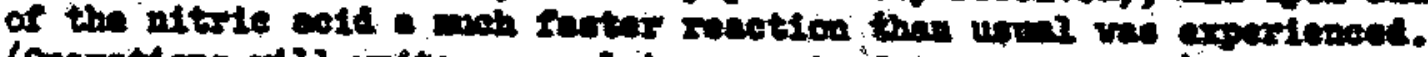

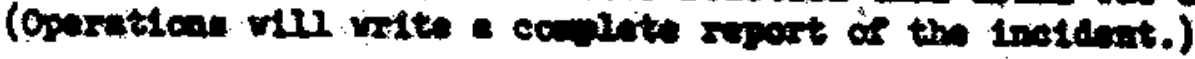

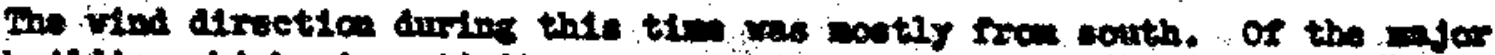

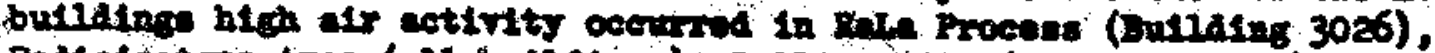

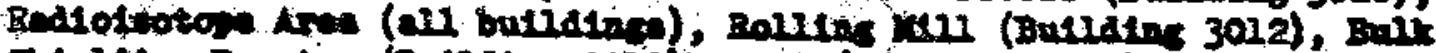

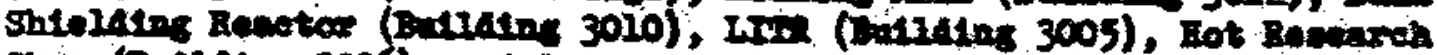

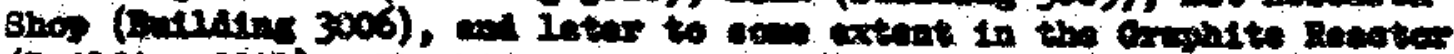

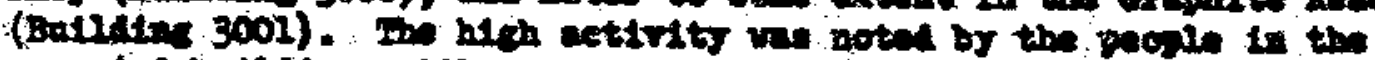

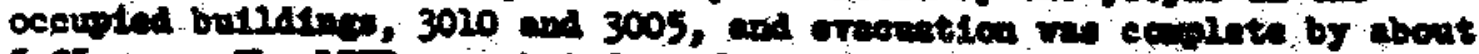

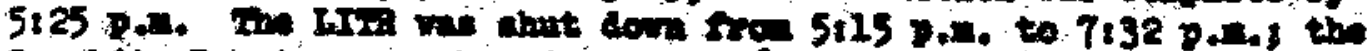

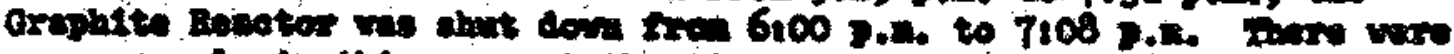

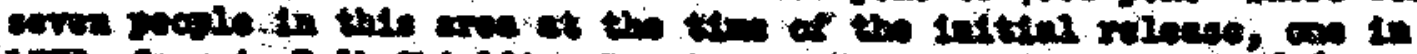

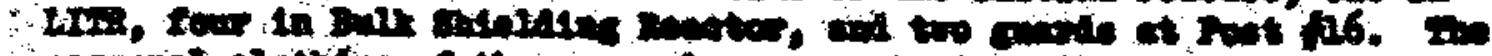

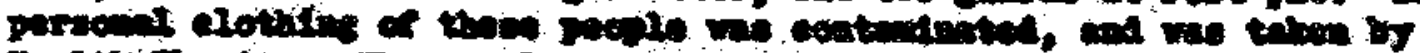

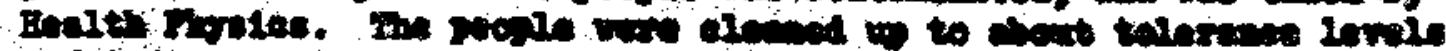

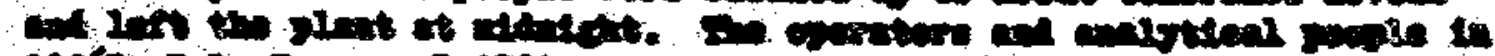

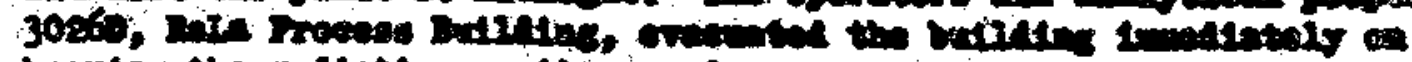

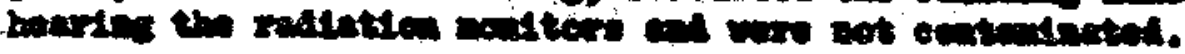

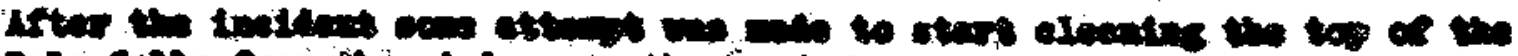

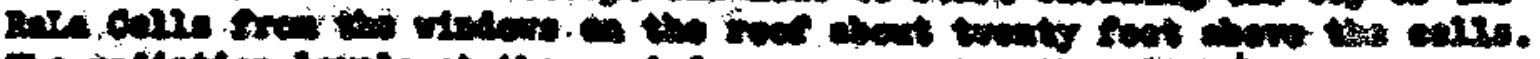

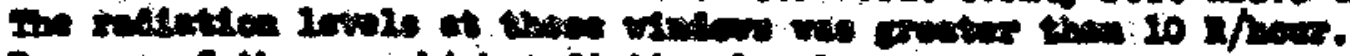

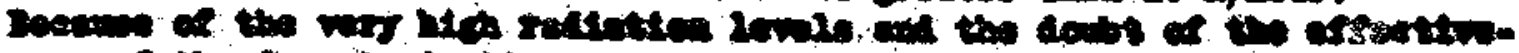

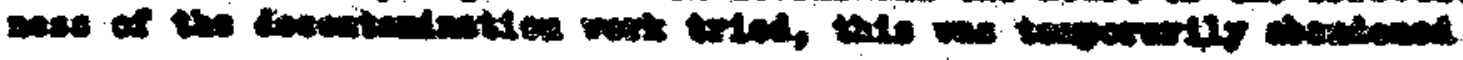


and the exfort res concentrated on the othex mojo building: 10volved.

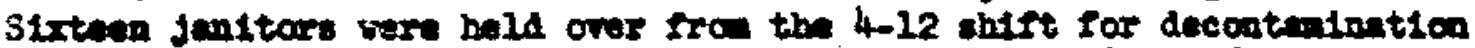
rort. ill horlecatal swefeces in Busldsing 3037, 3038, 3006, 3005, and 3010 wre cleand and Indsenticen at 7130 e.2. wre that they are below

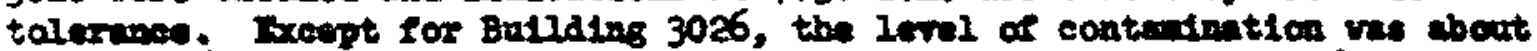

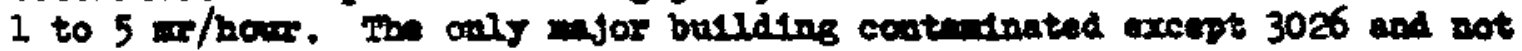
cleased by 8,00 a.n. Is the Rolling ull, Eullding 3012. The roadrare in the ares involved rexs rashod and all sew to be belor talornace levels st 8,00 a. $\mathrm{E}$.

Al poople Involved in the Incident and later in the htgh level decontenelnation vort are belng given the standard IP check tocluding urine checks, ote. A preliminary check of san of the Plin bedees is attached.

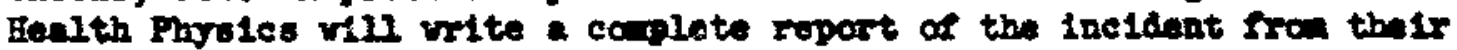
angle.

Seples rexw taken of the 8attling Baslet orthet at 7:00 a.m. showed 235 counts; Inlet solp shove 651 counte st 7,00 p.1., 10,335 counte at

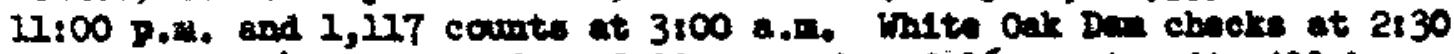

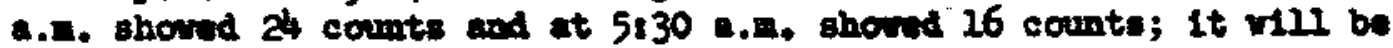
several boters before this activity-reaches the dean.

Present indientions are thet the ectivity has a half life of about tinety hours, Radiation seare to be genarells vers hard.

Radiation levels in Bullalng 30260 resend was then $100 \mathrm{R} / \mathrm{h}$ ow on the third level. At 7:00 a.1. the efret floor ves ebort $100 \mathrm{~m} / \mathrm{hour}$ and atr ectifity ves below tolerance lovis. Proceseling of the first part of the

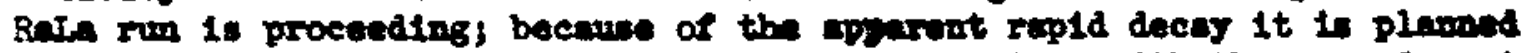
to valt until sunder night before trying to contimu with the seccond part.

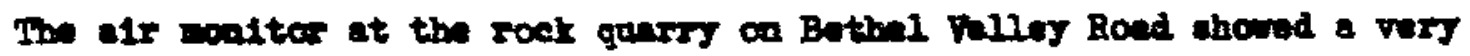
slight rise artar the incident.

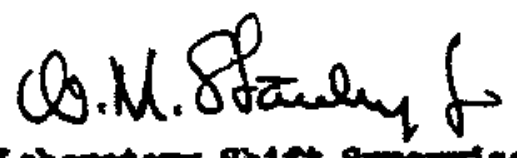

\section{Attecturat}

Fip Cook nationd

sow.

2.132 
To H. B. Ramey

LOCATION

ATTENTION

COPY To
Buslatns 4500

DATE Hay 3, 1954

ANSWERING LETTER DATE

suBJECT 3026 Ineldent $4 / 29 / 54$

4.48 p.?.

At approxdrately 5:00 this orfice vas inforind of a radiation 1netant in 3026 Buileing by Guard Fendquartens. On arrlvel it vas found that a diesolving

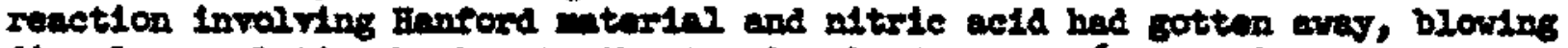
desolver solutlon beck anto the tog level of the 3026 eell bloot. A plum of nitric funs could be seen blllowing from the third level window, cast stde.

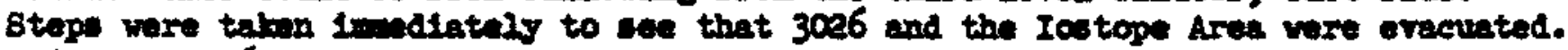

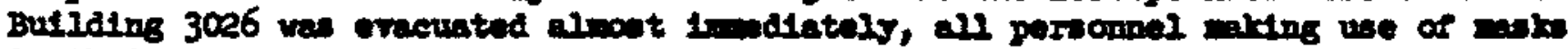

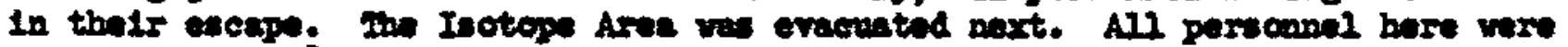
In 3037 and 3038. A the reaction earsing the activity vas itill going personnel

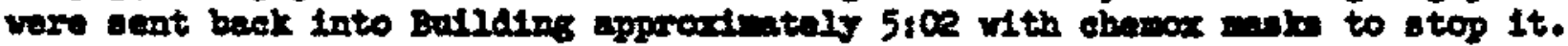

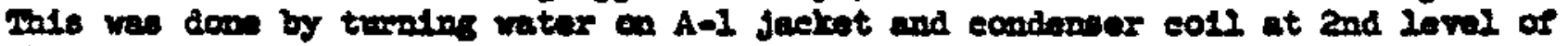

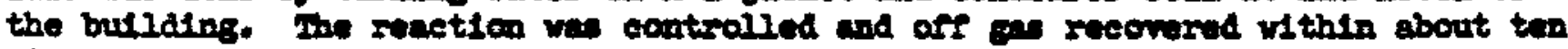

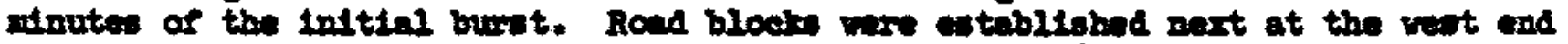

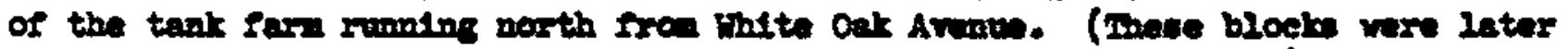

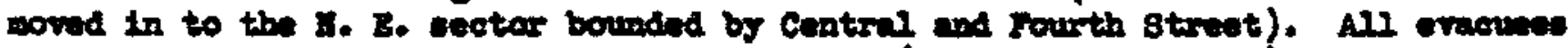

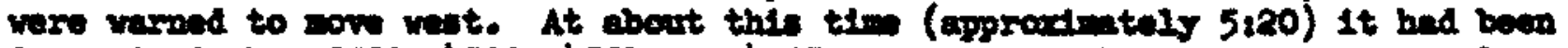
deteryined that $3550,4500,4501$ and 4505 wor not invelved and thene people advised that they could ranin in thely butlding but to be ready to leave.

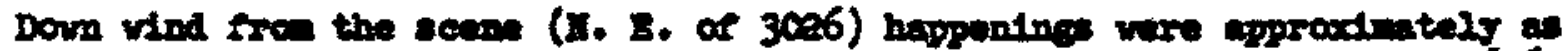

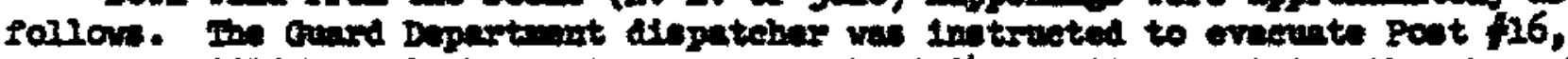

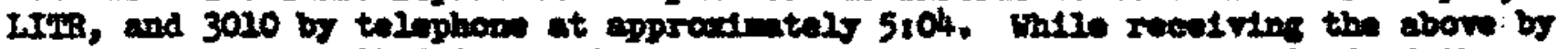

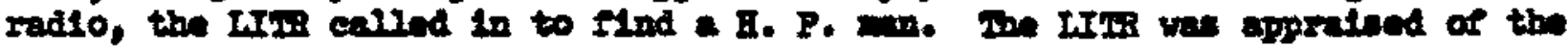

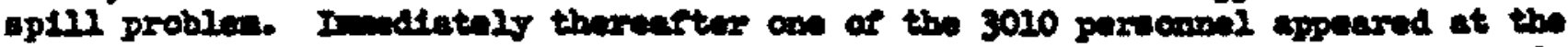

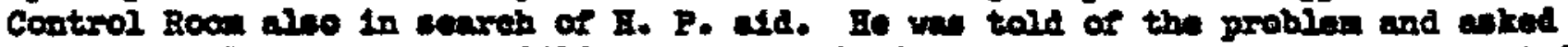

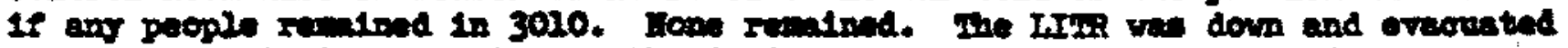

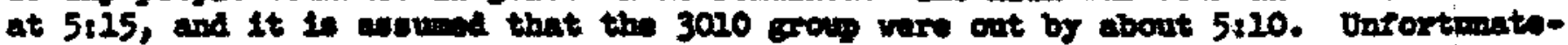

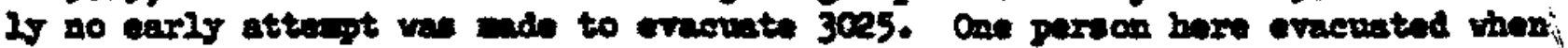

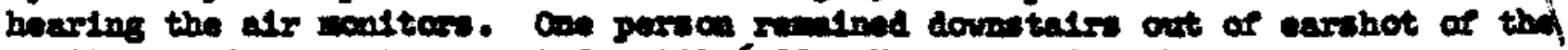

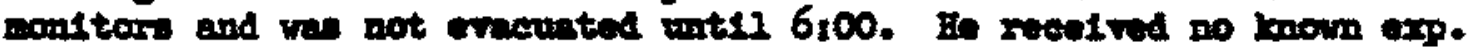

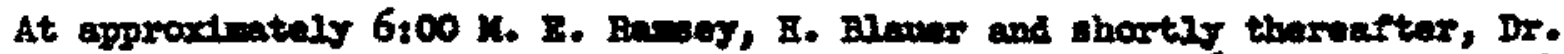

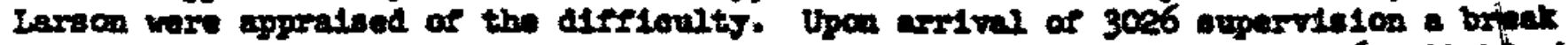

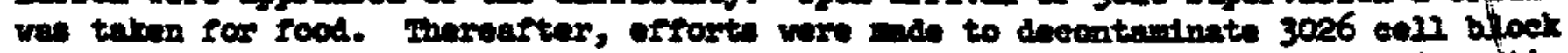

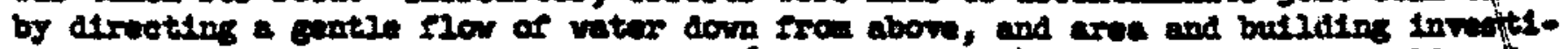

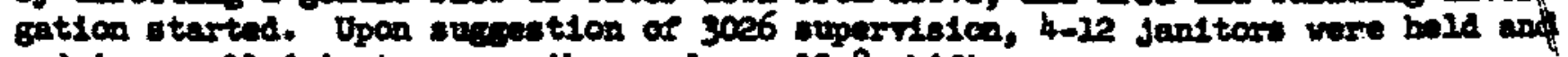
a driver called in to sprar the roude on 12.8 shift.

Unfortumataly serwel inclasnts were noted that could not be considered the

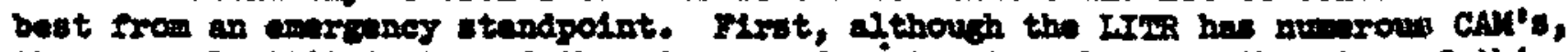

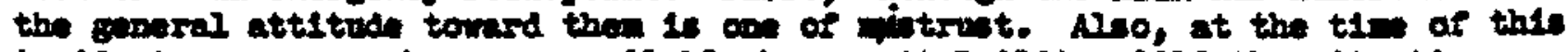

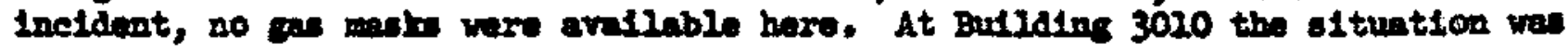




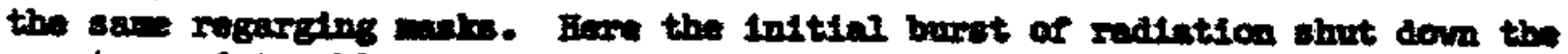

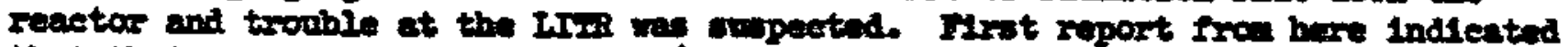

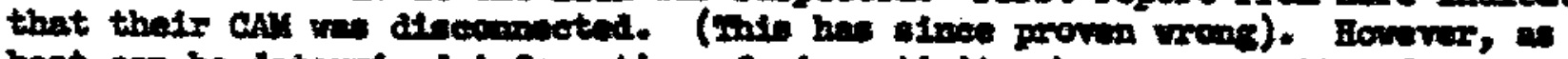

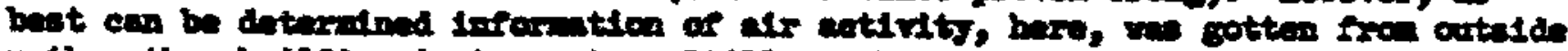

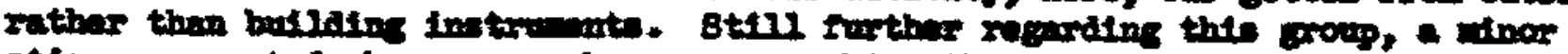

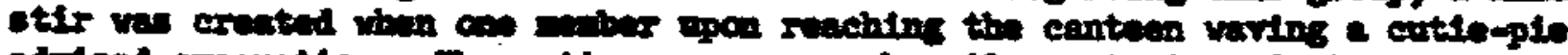

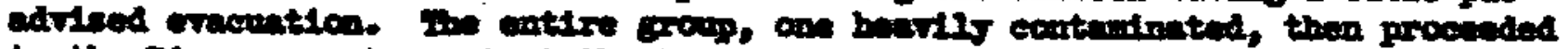
to the Dippandery to centact the B. 8. 8. for afd.

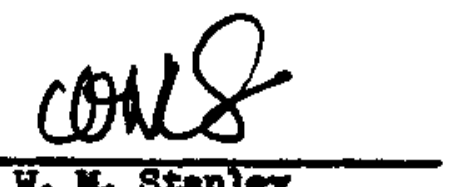

W. L. Stentry 


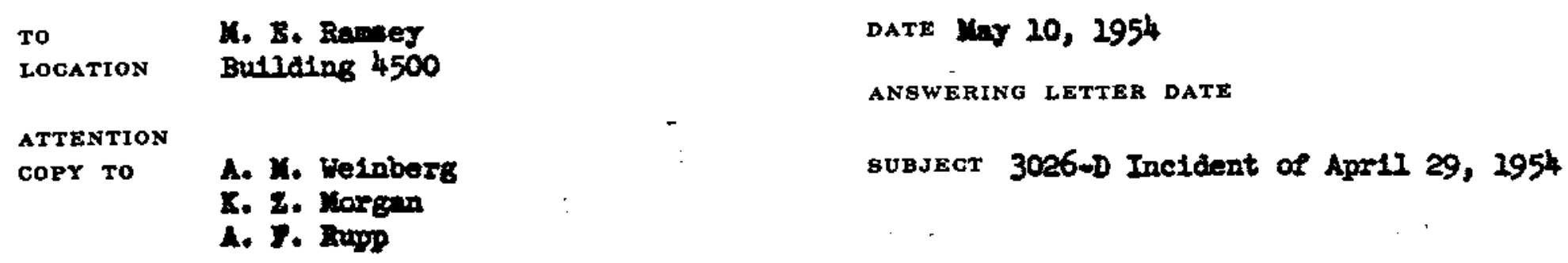

At 4:58 p.w on the eternoon of April 29, 1954, andintion inclaent oceurred

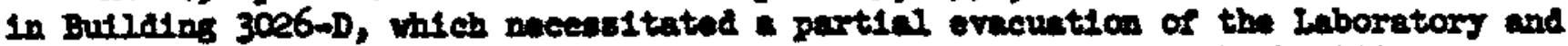
wich contaminated considerable ares north and noxtheset of the building. An account of the evente just prior to ind efter the incident are al follow:

At about 4:56 p.s. (estiwnted) one of the operstors on aut in Butialng 3026-D went to the thind level (top) of the procese celi, started adation of attrie acta to the dissolvar, and went beck to the second level (Inttronent panel). Upon the

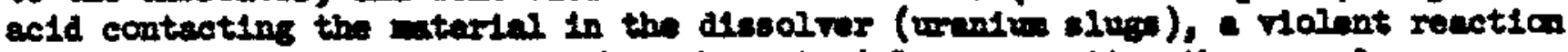
cocurred. It is nor belioved that the waterial, was active than wivel, ves:

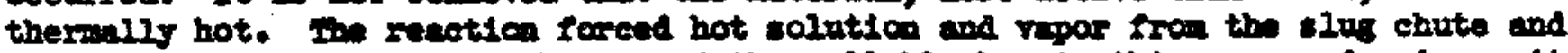
solutica edditica lines to the top of the cell block. is this oceurred, ais monitors

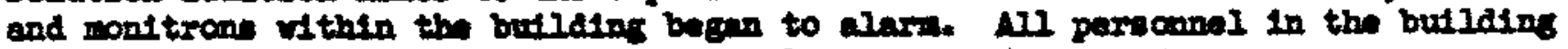
doned gen mole and eracustod iriediately.

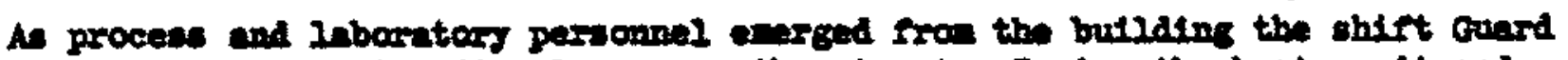

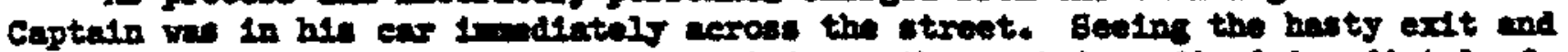

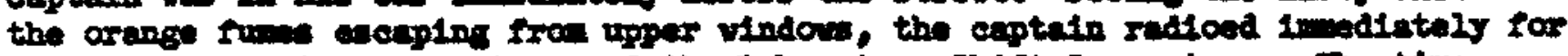

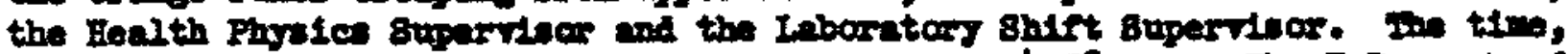

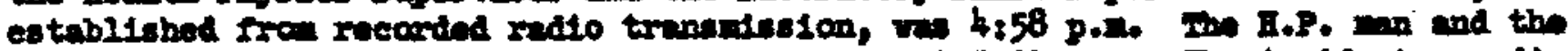

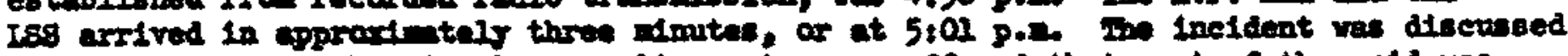
and the fact that the desolver cooling witer wa ofe and thet unt of the acid vas

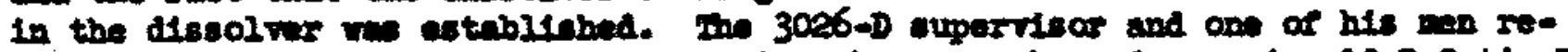

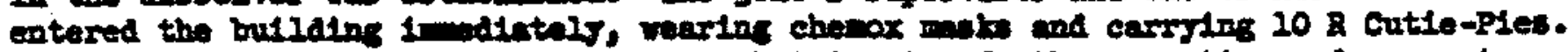

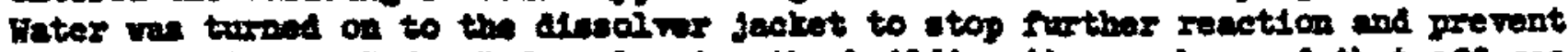
coliderlestion of tin. Bafor learing the bullating it was observed that ort-gad

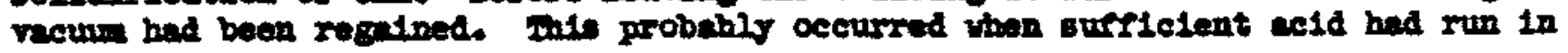
to cool the aterinl.

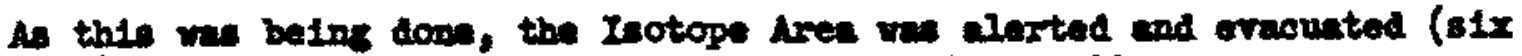

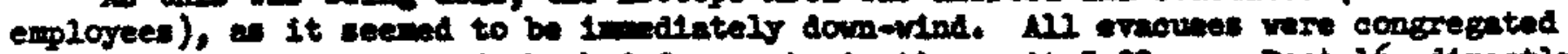

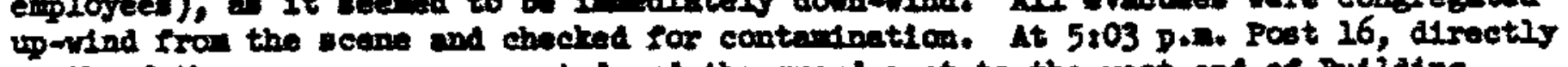
north of the scens, we evacusted and the guard eent to the rest end of Busliting

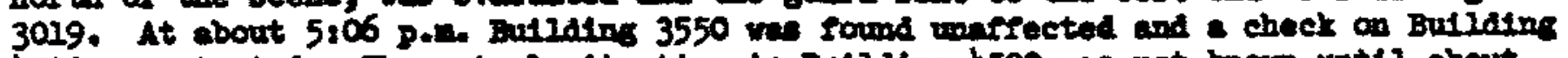

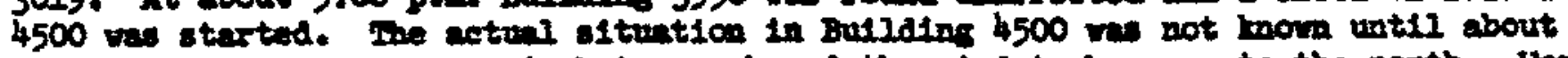
$5: 20$ p.e.; however, evente is between showed the vind to be wore to the north. Word val fiven to the shift group hore, hoverer, to alert then. 


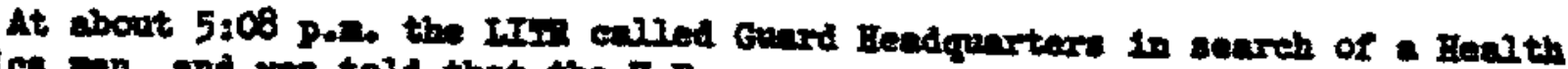

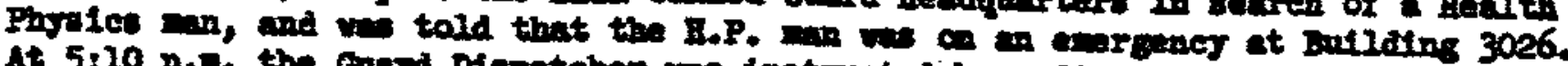

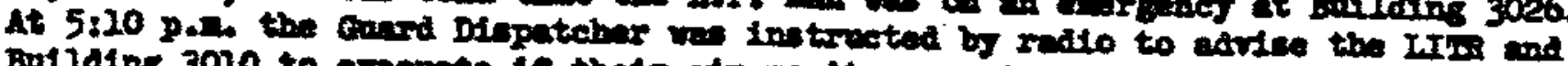

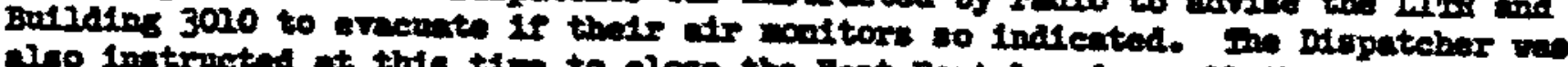

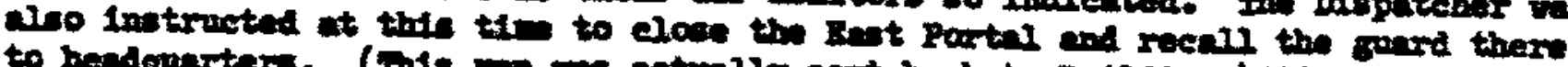

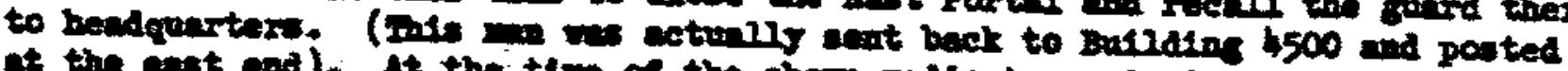

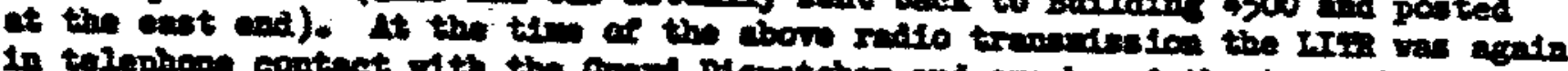

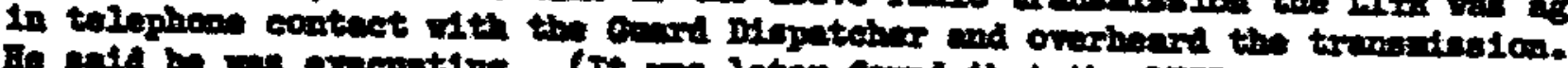

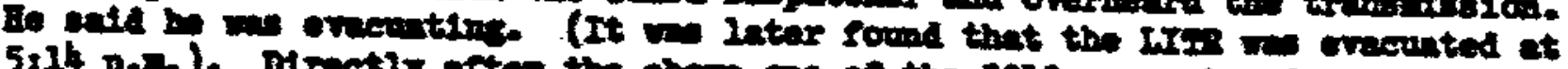

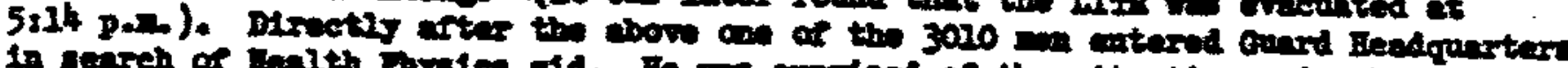

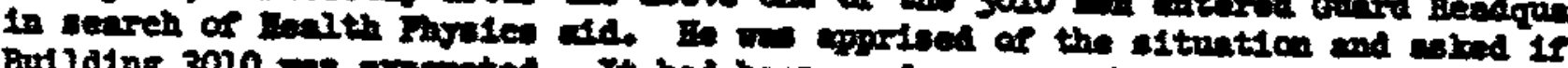

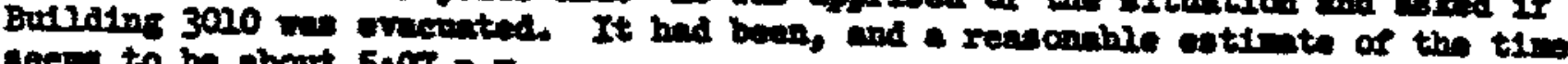

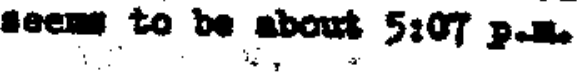

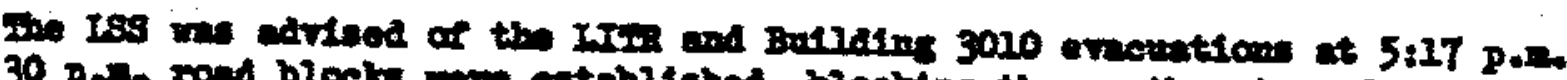

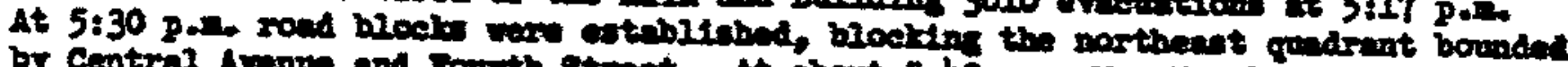

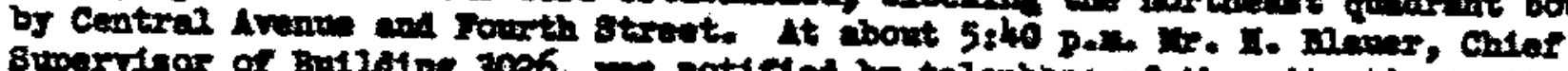

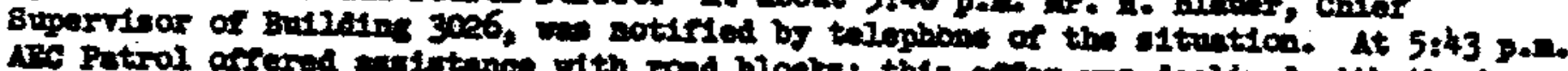

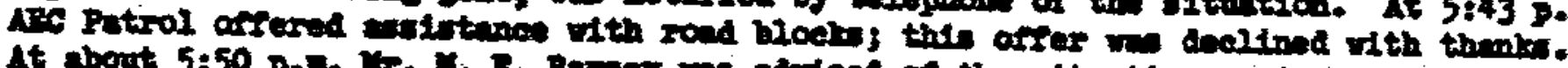

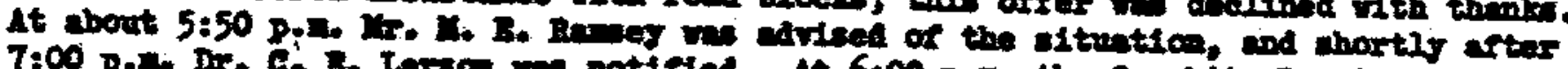

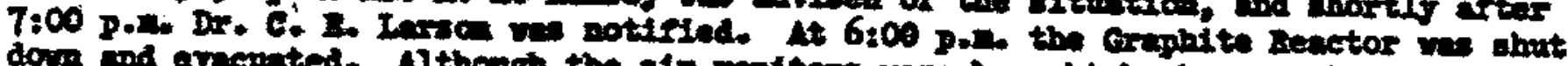

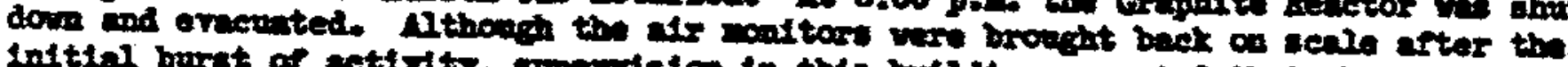

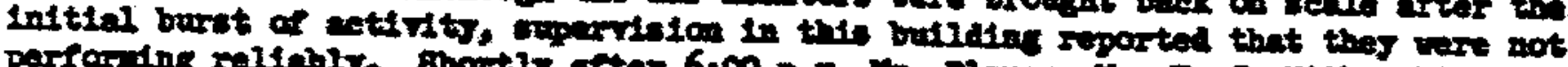

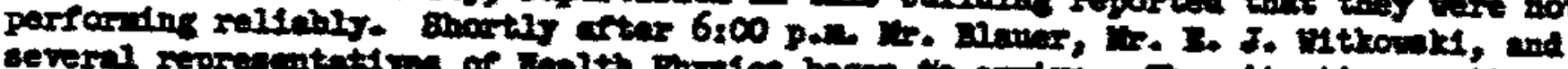

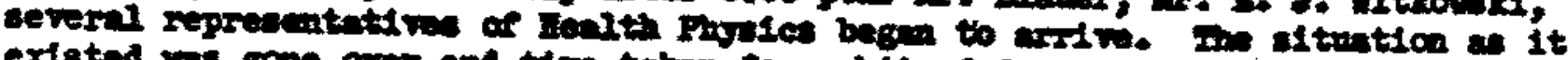

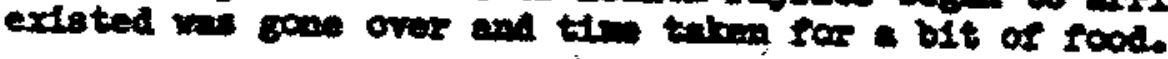

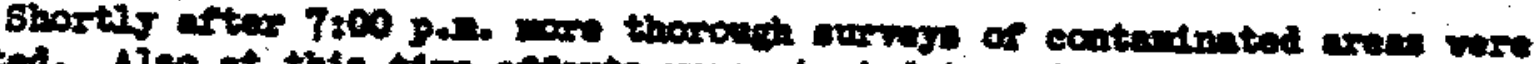

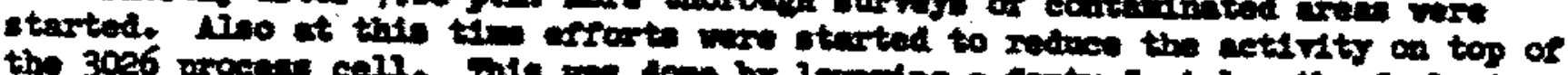

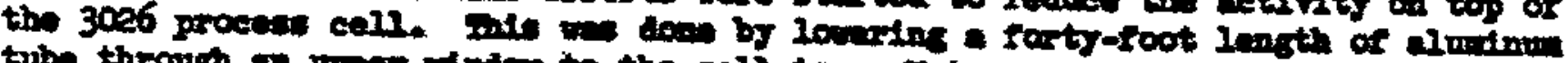

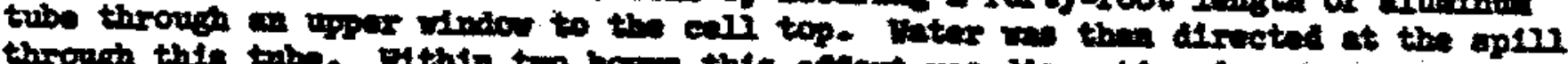

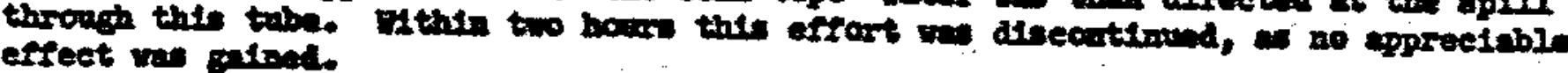

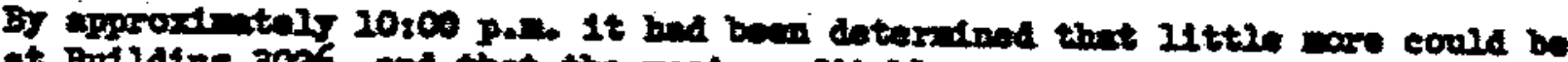

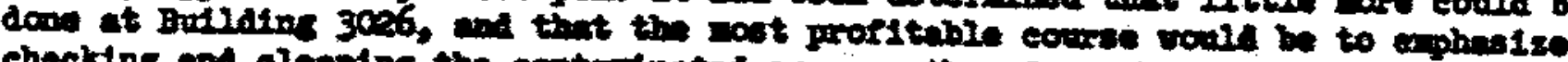

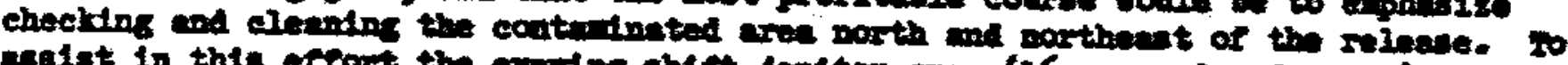

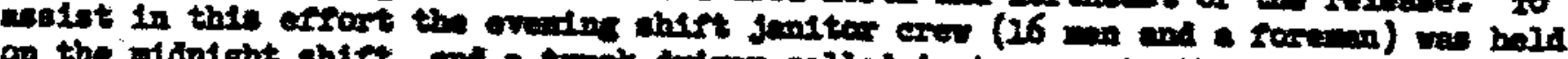

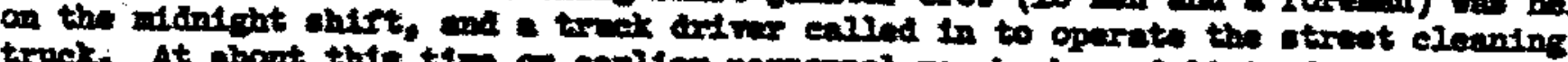

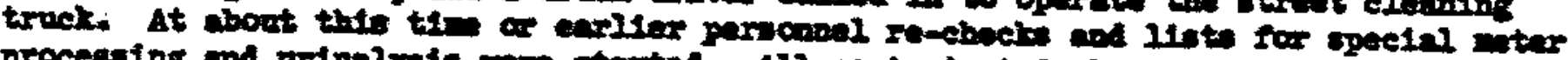

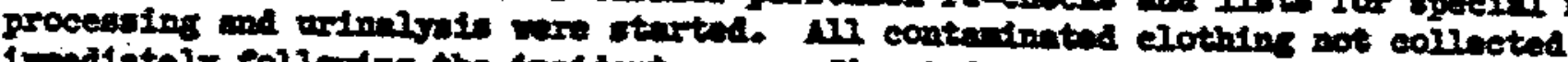

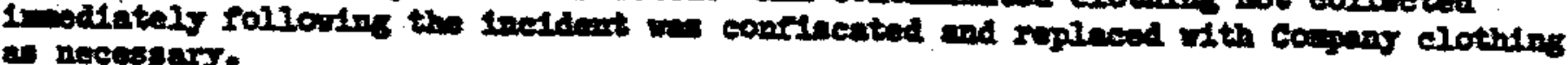

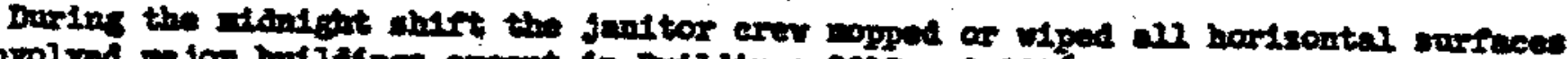

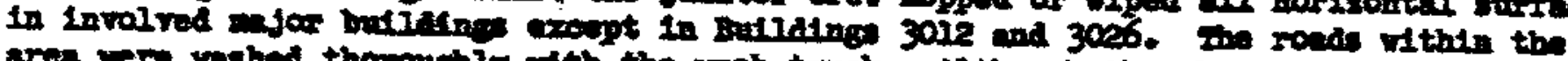

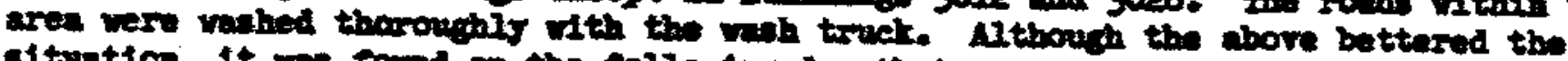

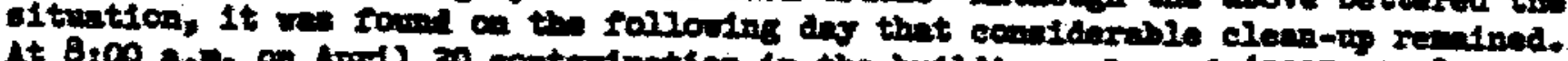

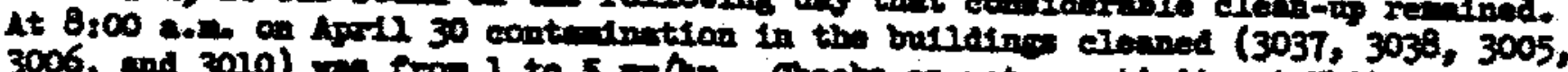

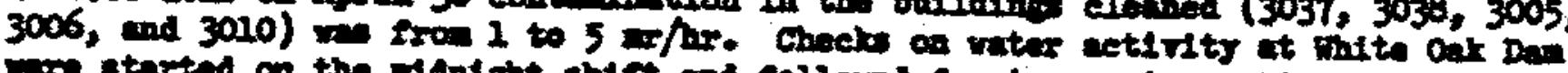

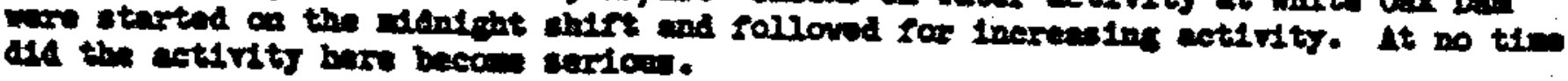




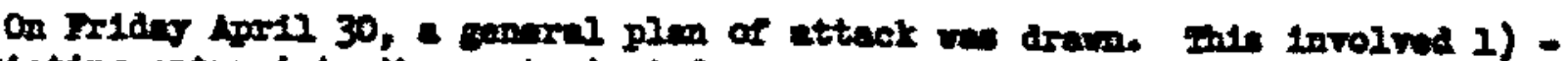

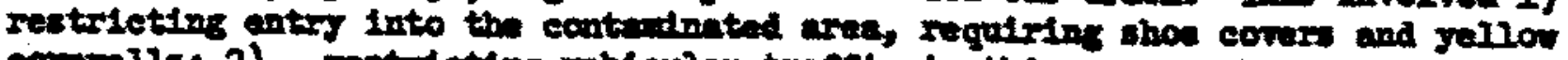

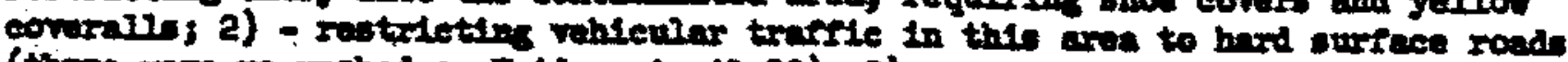

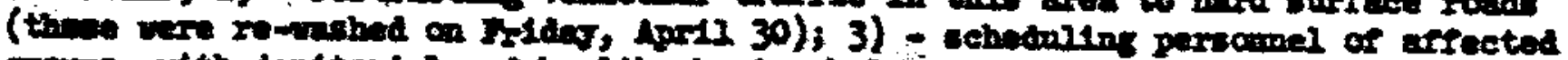

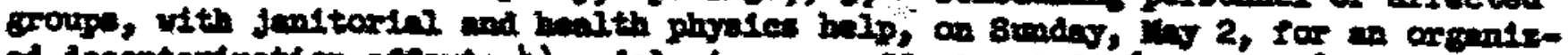

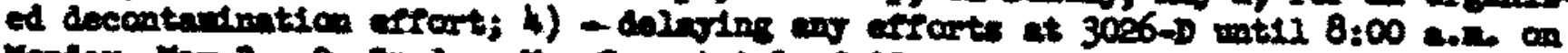

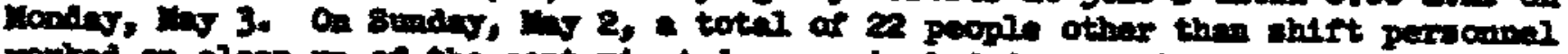

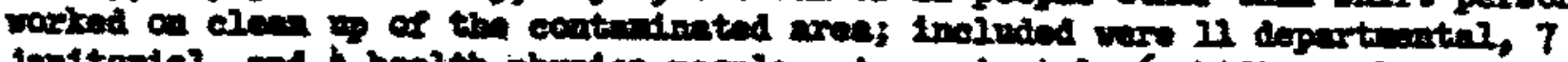

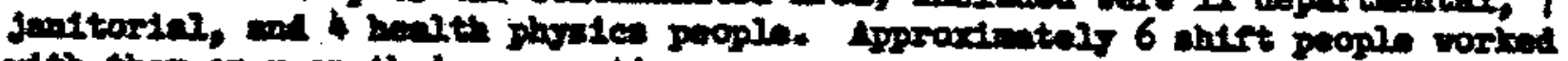

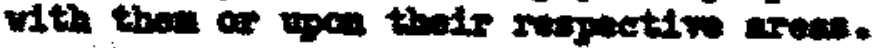

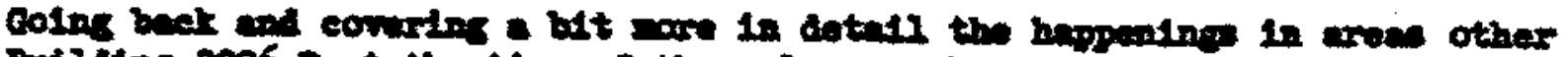

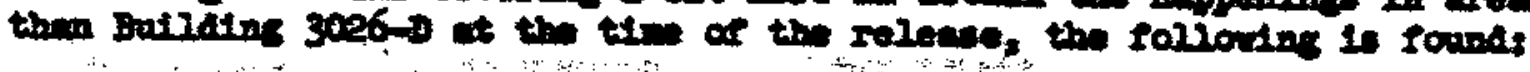

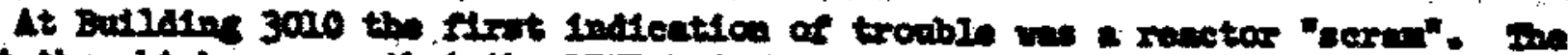

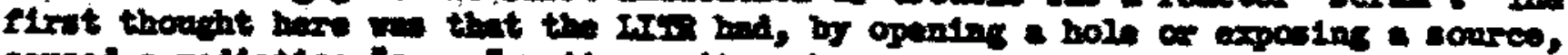

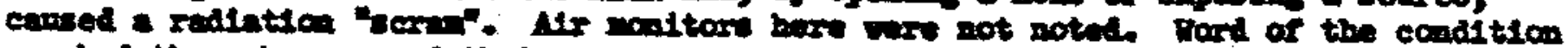

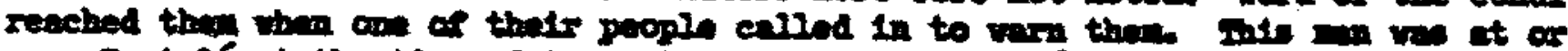

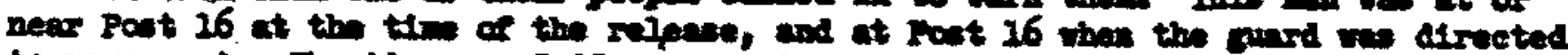

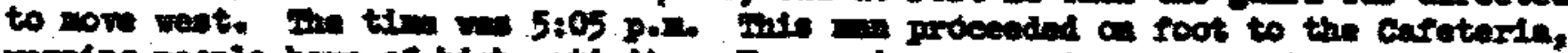

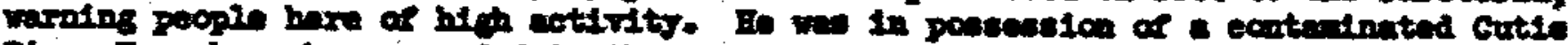

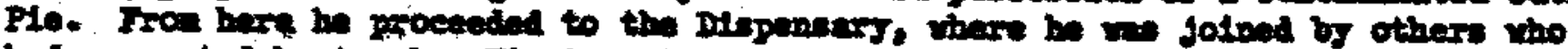

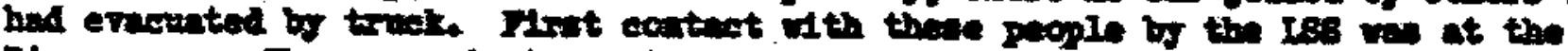

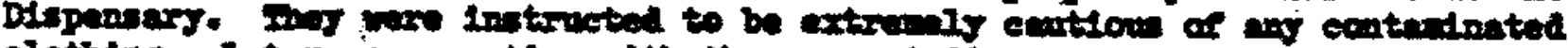

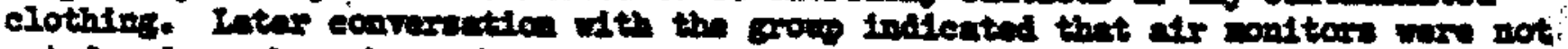

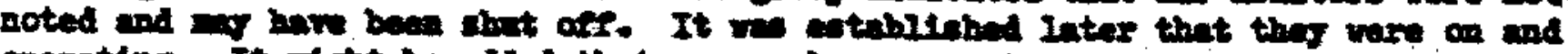

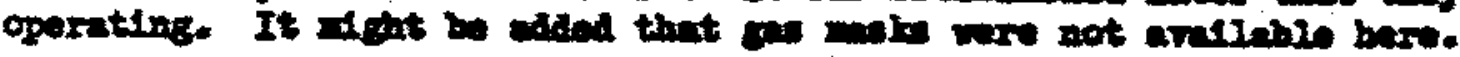

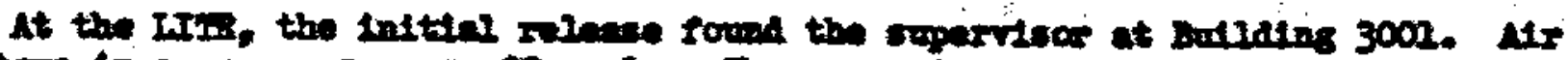

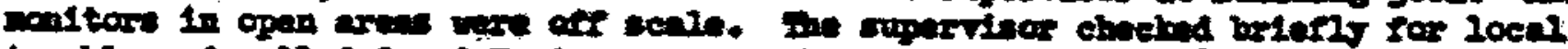

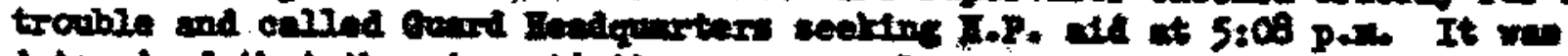

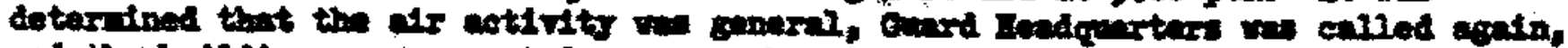

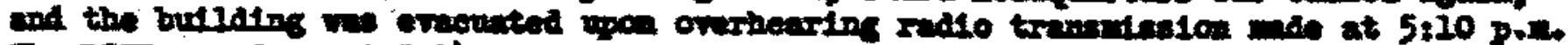

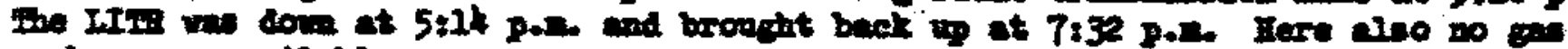
Dave rere aratlable.

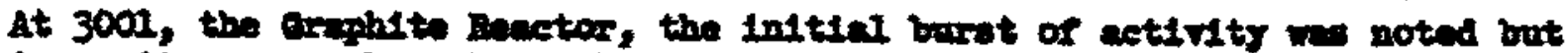

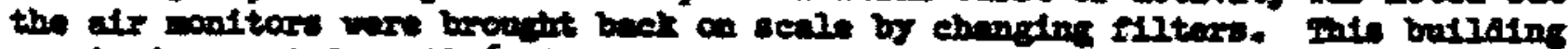

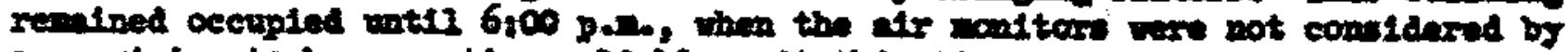

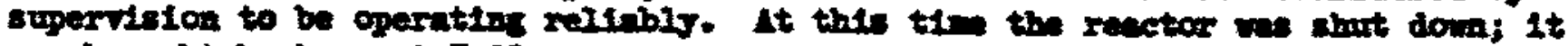
in brosight back ap at 7:00 pars

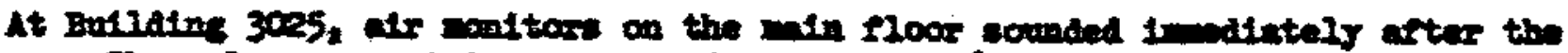

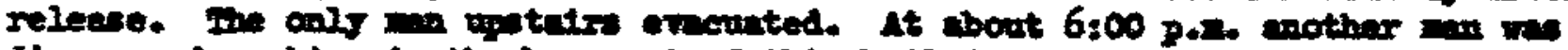

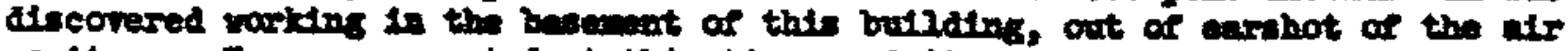

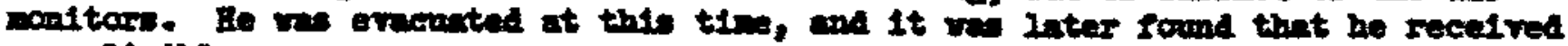
- negliglbie expostre. 


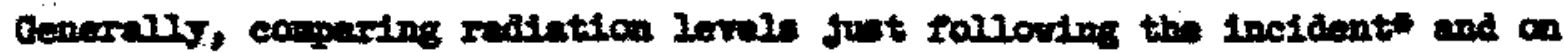

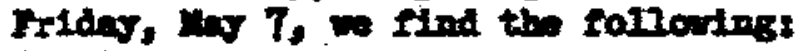

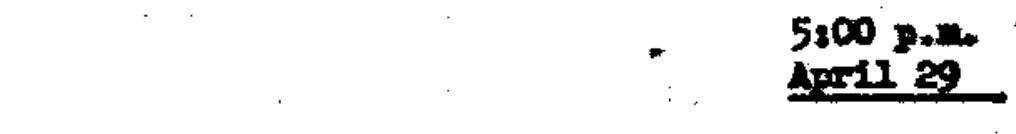

Butlding $3006-3$

Herst Iavi

gecond Ievial

mird Inel (8ptul arow)

Boadways Dorthenet of 3006

Butlasing Iortheant of 3006

Exterio: mills

rable tope, otoe, Inide

$$
\begin{gathered}
750 \mathrm{~m} / \mathrm{hr} \\
7-8 \mathrm{r} / \mathrm{mx} \\
N 100 \mathrm{r} / \mathrm{hr} \\
5-10 \mathrm{mr} / \mathrm{hr} \\
10-20 \mathrm{~m} / \mathrm{hx} \\
4-5 \mathrm{hx}
\end{gathered}
$$

$<2 \pi / \operatorname{mr}$

< I W/hr(ouly were decontrualn:

$$
\text { 4:30 p.: }
$$$$
17
$$

$$
\begin{aligned}
& 100 \mathrm{r} / \mathrm{hr} \\
& 150 \mathrm{~m} / \mathrm{hr} \\
& 10-20 \mathrm{r} / \mathrm{hr}
\end{aligned}
$$

Belor backround

Crans and Growing Fortheant

$$
\text { of } 3006
$$

$10-15=2 \times$

$$
4-5 \mathrm{~m} / \mathrm{hr}
$$

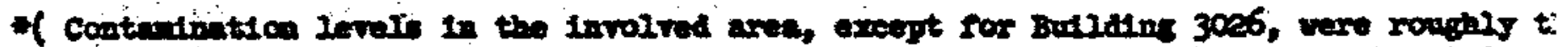

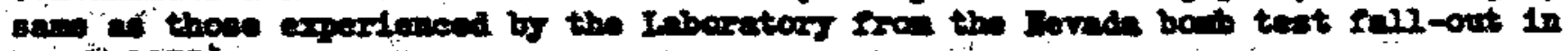
Mareit 2953).

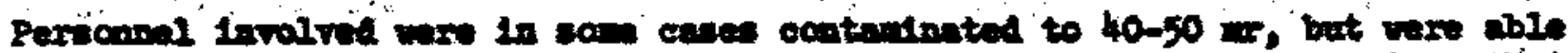

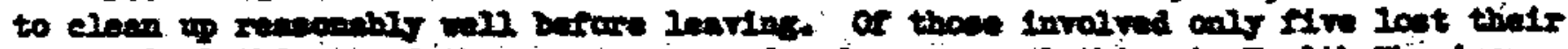

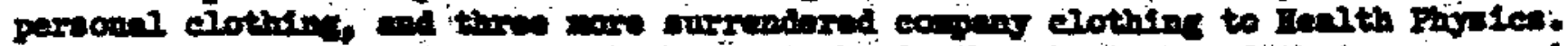

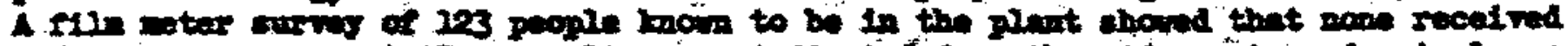

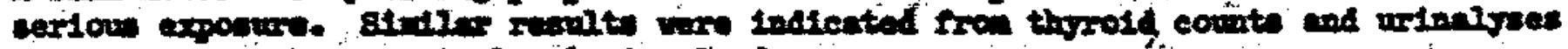

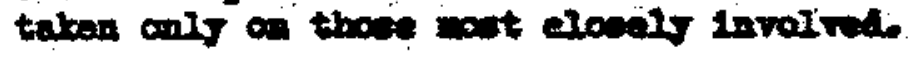

W: :bb

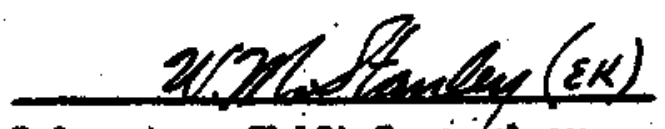

Ieboratory shift Bupuifisor 
Shiff men:

$109623 \%$

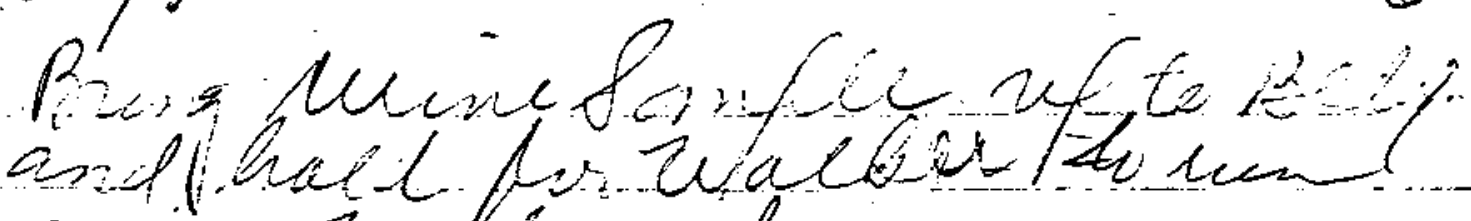

onder dufo end

sid

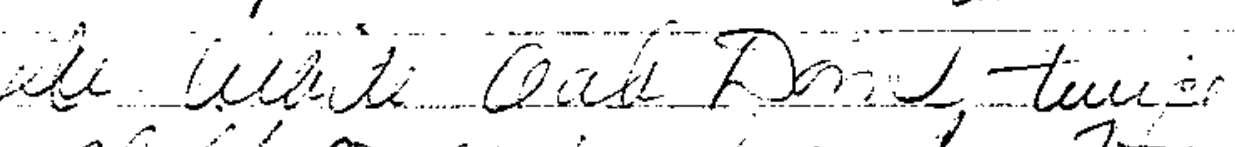

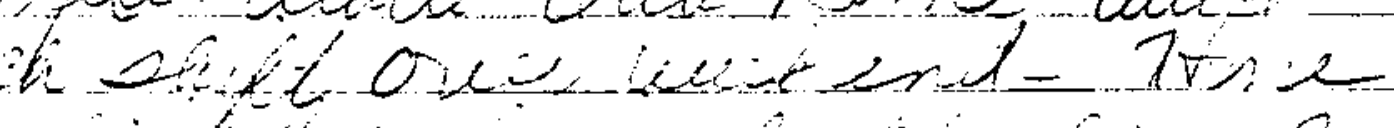

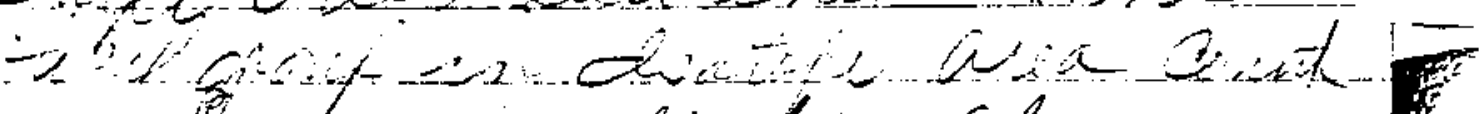

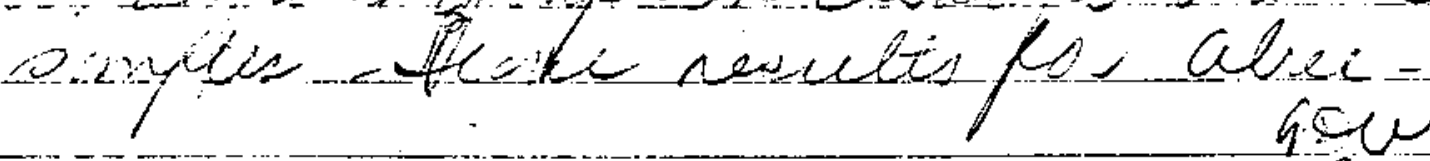

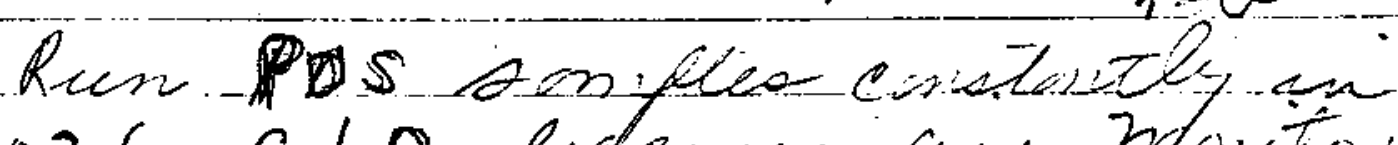

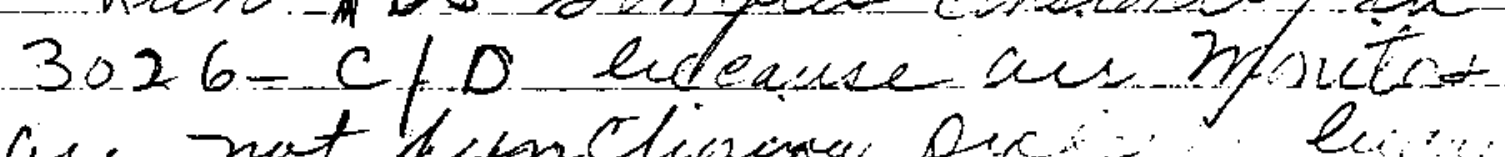

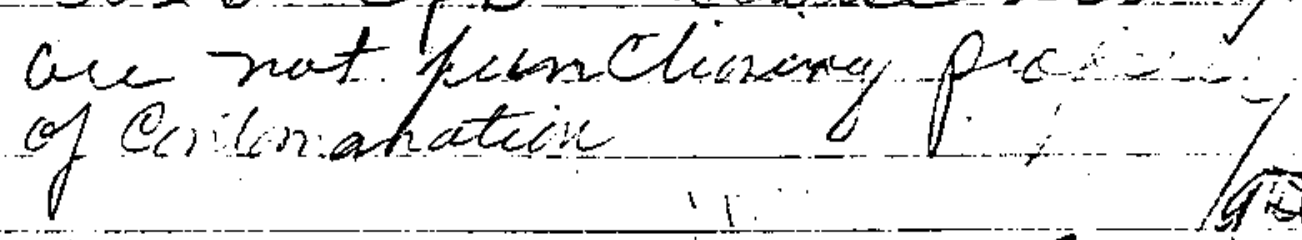

4-29-54 4-12

Goin

At $\sim$ sts pM this date while acid was berif odded to dissoluen in 3026-0 all movitrous $t$ chas sudderly begar atoming. In a few seconds Acid fumes wese noticied on the thind level- At this porix kel Personval in 3026.0 loxt the buillewis the following Personvel were in $7026-0$ It the time of the incident: Dailey $* 7566$ Terny *780x hampton *7676 and Bnify * 2387 - those vamed were extuemo's alent and resulted in very linfle over exposume of the time the, içident occunned by leaving the building immedidtely. 
il

$A t \sim 50 m$ veny heovy gaid

funes courd be seen enenging fiem the tol of 3.26.0. No Pekseccel weap in the buitleng ax this time-Bivia Q2382 and Lamplow 7676 domed Chemox masks and entezed the builderiy to stop the flow of zeid to the dessilura

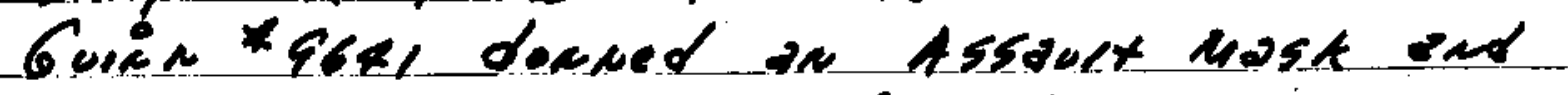
evtened $3026 . \mathrm{C}$ and found 411 Chas

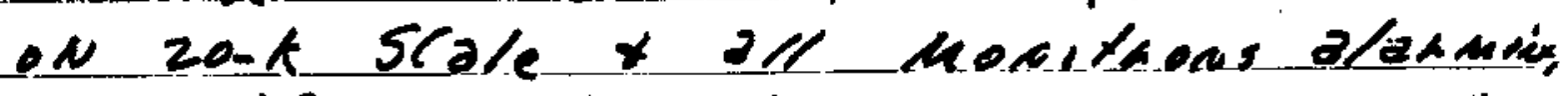
An ith chect et qoo hed hevedlet All CAMs o horitans alasming. At this same time 3o-1, L1XR $x$ 3oro heported 4 U chus alarming-Buibrig $355^{\circ}$, 3505, tankfasm AReP and Kle BB Shop ChMS were \&ll NeLMal on only skowing slitikx actionty = ywo Ain semples were hur at thes hisher the rood south of 30r6-0 and hith 7 istiation of 4 ir detinity was noted the Bact laoud isuthe Rood south ox

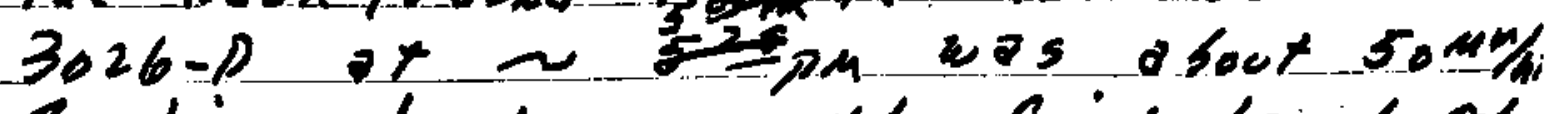
Reading taken on the finit level of $3026-0$ 2x. this time wexe $\sim 800 \mathrm{mr} / \mathrm{m}$ the thinl level read gheoten thow

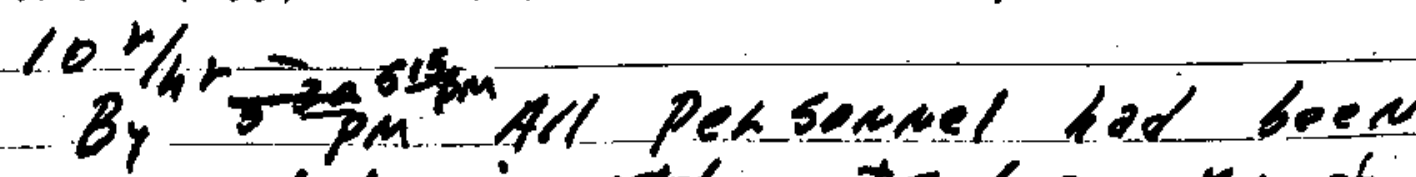
evacuated in Dxe tsotope ruatan tolether with Pessownel faem 3oz6-0 
(I)

gsipm

Nouth of boilding 3515.. By $=\frac{25}{7 \%}$ all Peasocnel in 3001, 3005, and 3010 hod a/so lext thein wespectice aleasAt 6 pm H.P. wes requested ot nedicil to Check Hunfenford \& hoce 8088 , Heake"ush Dailey *1566 + Kirby *1349. All Ktere lidividuals hod sevesal preres of Coutonminted cloxhing which becone contanineded while in 30ro - All coctoninated xtens wesp confiscaled these 7 tens left at blat. 2012Bailey $\$ 2387+$ bamptow $=7676$ becane cecfaminated to n_ $100 \mathrm{mr} / \mathrm{hr}$ aftea enteding 3026-D. they weze cleaned UP olmost inmediafely-

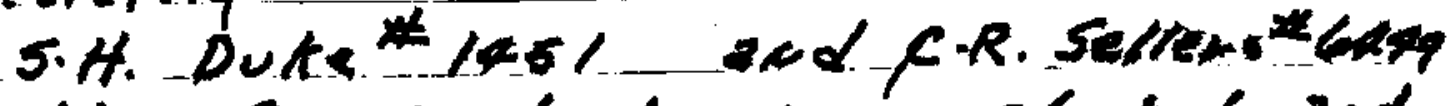
of the Guard dept wede chected pat

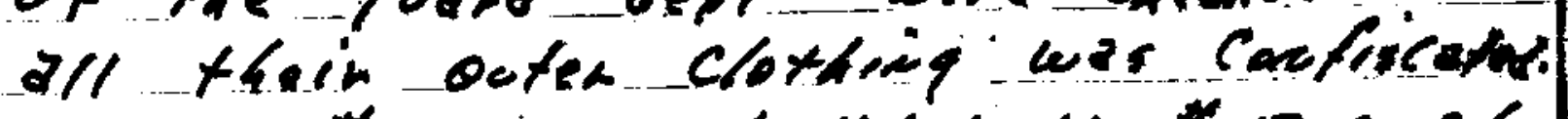

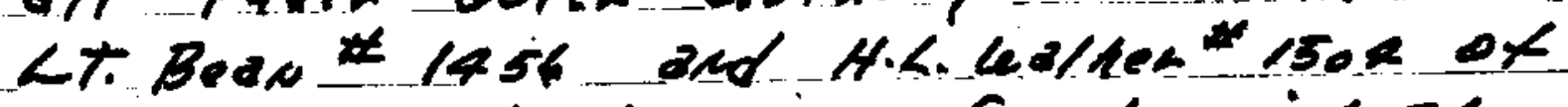
the quand bept wete foudd cuith shoes readich oboue tolekdore- Both cheaced thain shops to toleapere -

R.D. Hulen 1877 wes found woth contouinated skint and it cas cacfistated. By $7 \mathrm{PM}$ it was deter miced thet the following placest wexe contaninated:3005, 3006, 3004, 3010, 3012, 3550, 3000.(substation), entide 7 sotope ahee, and tow Sone Contamination in and acount baols 30.25 and Post 16 Guand shock-1 H/50 if wos detenmired thox the groond in) 
W.

- Gevenor NE driection from 30z6-s

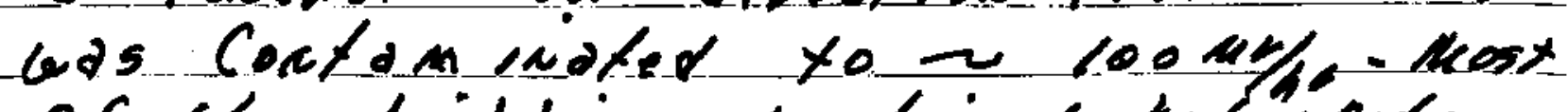
of the toildings. wentiene tod spots heading to ro ur/s wixh sceneced spets to $30.040 \mathrm{~m} / \mathrm{ha}=$

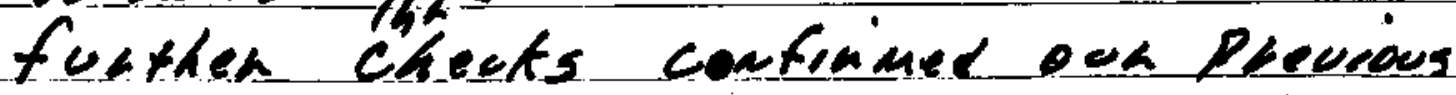
beleit thot pethrig west of $3024-0$

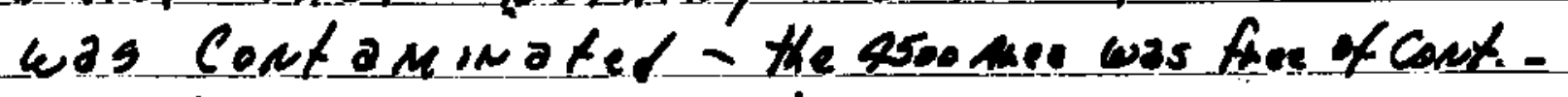
A chew of Jarikens leas put ro woht ahount 12 nid Niglt and they brought the levels of coutominitio: down- $3026-0$ was repro off ond is still in a highly Gontaminated state A moar A couplete ond comptebousim vepont is being plepoted to roun such thems do, unindlysis reponts, pecroud

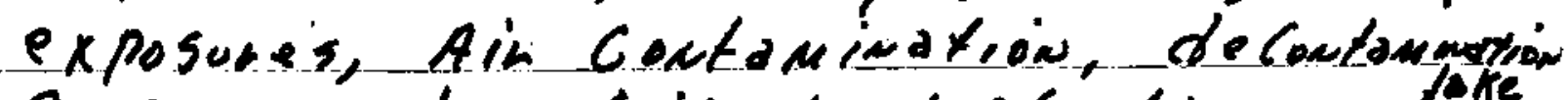

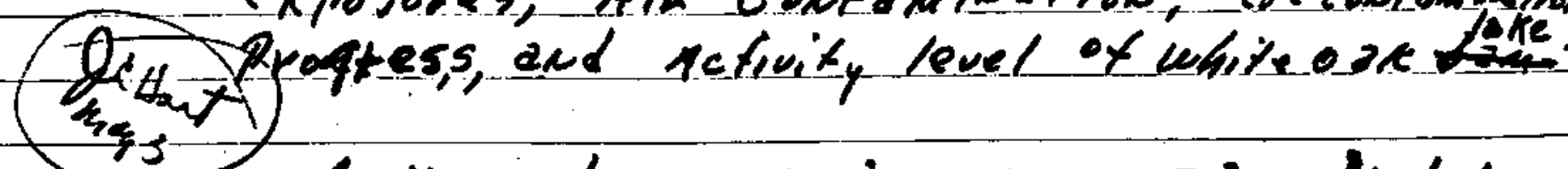

A vehy heary hain zt u $7 \mathrm{Pm}$ lhobly cleder the stares etc. 9hently=

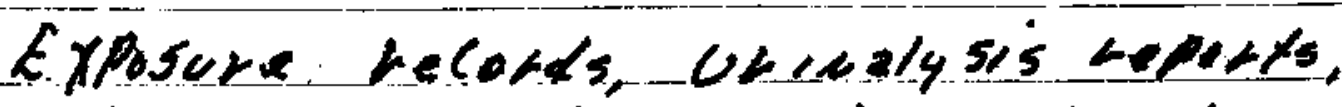
thytoik coonts and a neview of the entide 3020-p episode cocting ock beleit ex the time of the ricident thot vo ove received o dosale hith pcouph to couse ony a latm 25 to rhet idiciduls health- 


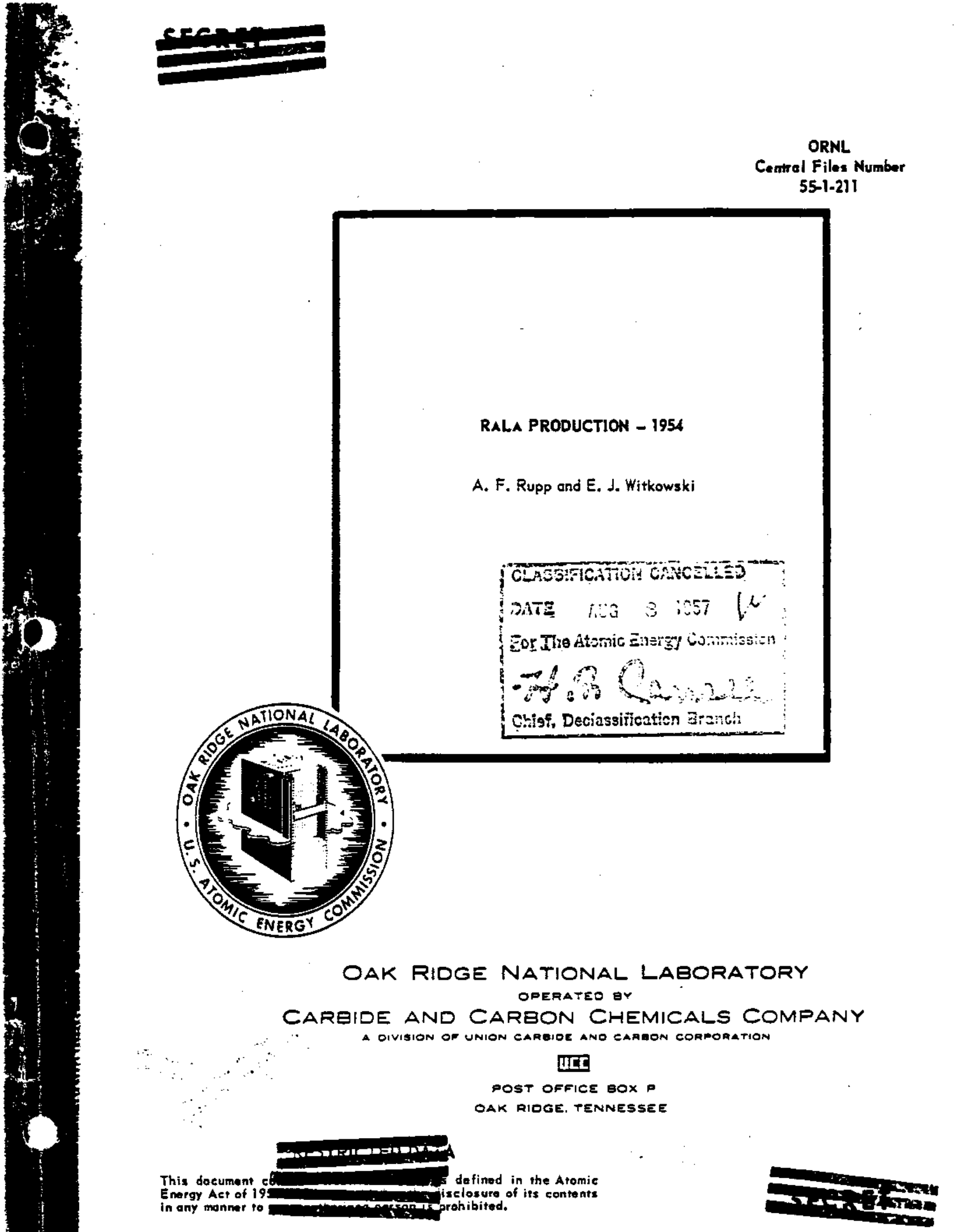


volume of $\mathrm{K}_{2} \mathrm{CO}_{3}$ solution from 9 to 50 liters in on effort to ovoid the incomplete reactions ex. perienced in some previous runs.

Extractor and Filter Rinse. Product was left behind in the extractor tank and on the process filters after it was transferred from the extractor to the resin column feed tonk. It is impossible to make complete tronsfers becouse the relatively small size of the feed tank limits the volume of solutions that can be used for cleoning out the large extractor tank.

Fuming Nitric Acid Fittration. The highest loss experienced in this step was incurred in run No. 55, the only run processed through cubicle No. 300; this cubicle has a product evaporator and filter of the old design, and it is believed that the high loss was caused by cracks in the filter resulting from fabrication difficulties.

Product Evaporator Rinse. Some product was left behind in the evaporotor ofter the moin portion of the product solution had been transferred to the shipping cone. The volume of water used for the dissolution and transfer of the product is limited by the size of the cone and is too small for an effective transfer. Attempts to wash the tank with more woter and to transfer the water to the shipping cone after the first portion had evaporated resulted in higher losses from the cone into the off-gas line.

In addition to the losses determined by the analysis of waste solutions, it is estimated that more than $15 \%$ of the starting product was lost through the off-gas line during the drying operation in the shipping cone. The largest single loss occurred in run No. 55. Since ORNL does not have the facility for making o radiochemical analysis of the product after it is put in the drying cone, such an analysis was made of Los Alamos; their analysis was lower by 20,000 curies than the measurement made of ORNL after the fuming nitric acid step.

\section{UNUSUAL INCIDENTS}

The equipment was operoted far above its designed capacity (500 curies in dissolver and extraction section and 10,000 curies in the purification section) in every run. This condition led to the most serious accidental release of activity ever experienced in the history of the process, which mode it necessary to abondon a run and to shut down the building for decontamination for a period of 11 weeks.
The slugs looded into the dissolver for this run contained approximately 100,000 curies of product. The quantity of starting material was unusually large so that a very large shipment could be produced and so that the chance of a poor ship. ment resulting from losses coused by the usual processing difficulties could be reduced. It was planned to make the run in two parts and two separate shipments.

Dissolvings for the first part were discontinued ofter the third batch, instead of ofter the fourth batch os had been planned, because the UNH analysis showed that the slugs contoined more than the expected amount of activity. The partially dissolved uranium slugs left in the dissolver therefore were hotter than in ony previous run. The incident occurred at the beginning of the addition of $60 \% \mathrm{HNO}_{3}$ for the second port of the run. Becouse of their intense radioactivity, the siugs apparently had become very (thermally) hot during the period of time that they were not covered with liquid between the dissolvings for the two parts of the run and had reacted violently with the $\mathrm{HNO}_{3}$. The solution contoining the radioactivity was blown into the operoting oreo through the slug chute and the solution addition lines.

Following the incident, the radiation levels in the RaLa building itself were too high to permit entrance for effective decontamination. Work was started after the short-lived activity was allowed to decay for a period of ten days. Several other Laboratory areas north of the RoLo building were also contaminated, principally with short-lived iodine. Fortunately, the incident occurred during the 4-12 shift, when these areas were not occupied; by the use of emergency crews, most of the contamination was cleaned up before personnel reported for work ot 8:00 AM the next doy.

The incident caused a great deal of inconverience in Laboratory operations and some lost time, but there was no serious overexposure of Laboratory personnel. The operators who were in the most vulnerable position at the time of the accident were quick to recognize the hazard and to protect themsetves, being well practiced because of the frequent use of the Rolo emergency procedures.

As a result of this incident and of the frequent hazords encountered in the production of the large batches of RaLa, Los Alomos was requested to review its requirements and to reduce the number 
and size of the batches requested, if possible. An agreement was reached that the Laboratory will attempt no more double runs and that only four runs per year will be made. It is believed that the new schedule will substantially reduce the operating hazords.

Direct pipe connections between the process vesseis in the cells ond the operating orea (which are considered by the Operations Division to be obsolete design) were responsible for several other backups of activity into the operating orea. The most serious of these occurred during run No. 55, in Januory, when activity bocked up to the operating panel board through a steam line servicing $a$ jot in cell $A$. While an ottempt was being made to decontaminate the line with steam, a small valve at the steam pressure gage failed and activity wos blown into the operating area. The building was contominated, and two operators received some radiation overexposure - fortunately, not a serious amount.

General air contomination that was experienced through a large portion of the Laboratory on three occosions during two of the three successful runs was attributed to the RoLa operations; however, there is a good possibility that some of this air activity came from the experimemtal reactor areo. The condition was presumably caused by a high discharge from the stack; in one case the caustic line to the dissolver off-gos scrubber piugged, and in another case, during an otmospheric inversion, the scrubbers operated normally but not effectively enough.

\section{EQUIPMENT}

The equipment used for the production of RaLo has alwoys been operated for above its design capacity; changes to allow for higher levels of production were made only in the final purification stages, and they were inadequate for very large batches. The last alterations in the final purification equipment were made in order to produce 10,000-eurie betches; however, before the equipment was completed, a new goal of 30,000 curies was set, and 50,000-curie batehes were actually produced. Operation of these very high radiation levels has materially increased the hazards, and the equipment has deteriorated over a period of years.

It was decided several years ago that it would not be practical to further alter the equipment to increase the capacity, and a proposal to build new, adequate facilities was submitted to the Atomic Energy Commission. However, it was decided this year to build the new plant at Arco; the new plant is expected to be in operation by July 1, 1956, and the existing RaLa plant will be permonently shut down at that time.

Mointenence of the equipment has continued to be difficult and expensive. For long-term operations, it would be more practical to replace or to rebuild some parts than to moke the excessive repairs that would be needed. However, since the present plans are to produce only six more batches, no replacement of equipment or rebuilding will be done except where absolutely necessary.

The only major maintenance job undertaken this year was to rebuild one of the cubicles which hold the ion-exchange equipment. This was necessory because of a leak in the product evaporat or thermo. couple well and the failure of the sampling valves. The evaporator was replaced by ane of a greater capacity and of an improved design that permits much closer control of the evaporation operation.

The RaLa equipment of the present time is in only foir condition. The cell A transfor lines are known to have many leaks, and the thermocouples in the dissolver have been inoperable for years. Decontomination and repairs in this cell ore inadvisable and will not be done unless some major piece of equipment foils completely. The high level of contamination on the concrete walls and floor would require the removal of 0 large quantity of highly radioactive concrete, ond it would be impossible to complete the work fast enough to meet the Los Alamos schedule. Also, a major decontamination job in this cell would be very expensive and would require o great deal of radiation exposure to operating and maintenance personnel. It is hoped that this work can be avoided, since RaLa operations at the Laboratory are to be permanently discontinued.

The main operating difficulties with the purification equipment are the erratic operation and the frequent failure of the sampling plug valves; both conditions are caused by the deterioration of the Tefion seats.

It is believed that one more decontamination of one of the cubicles will be required to complete the last six runs scheduled at the Laboratory.

\section{PROCESS IMPROVEMENTS}

Severol important changes were made in the process this year. One was the addition (to the 


\section{SECREI COVER SHEET}

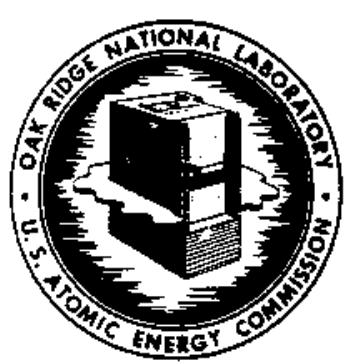

DATE: June 2, 1954

SUBJECT: RAIA FRODUCTION

TO: X. A. Kasschau

FROM: C. E. Larson
OAK RIDGE NATIONAL LABORATORY

Operated By

CARBIDE AND CARBON CHEMICALS COMPANY

TटE

POST OFFICE BOX P

OA K RIDGE, TENNESSEE

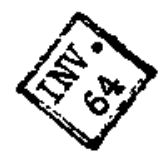

Copy 5 of 9 copies. Series A
This document consists of 3 pages.

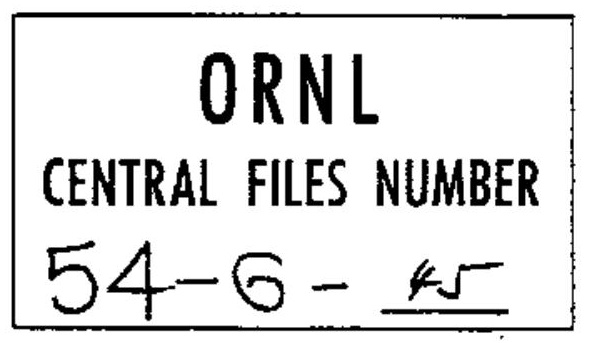

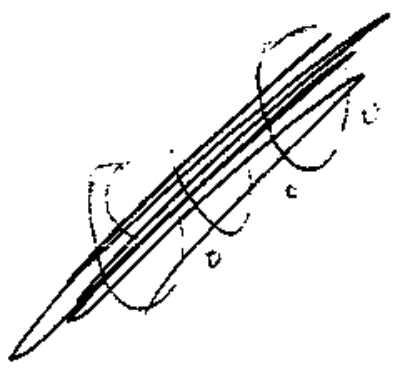

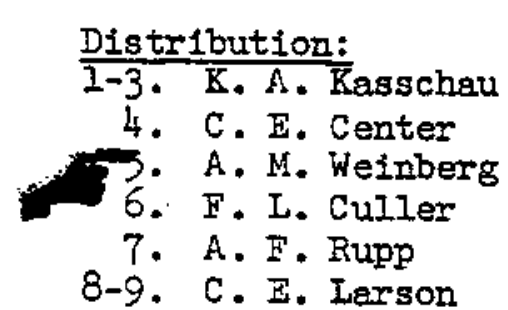

Distr1bution:

-3. K. A. Kasschau

. F. L. Culler

8-9. C. $\mathbf{E}$. Iarson

This document has been approved for telease to the public by.

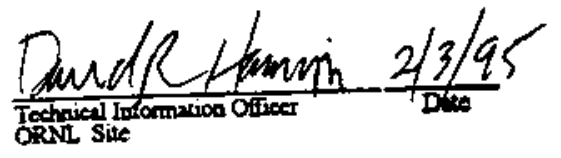




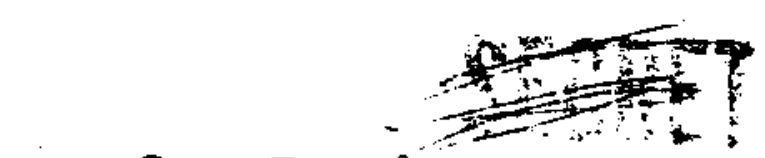

\section{OAK RIDGE NATIONAL LaBoratory OPERATED BY}

Carmide and Carbon Chemicals Company

A DIVISION OF UNION CARBITE AND CARBON CORPORATION

प]EC

POST OFFICE BOX $P$

OAK RIDGE, TENN.

June 2,295

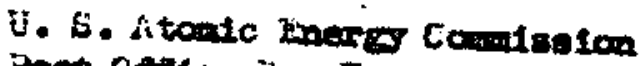

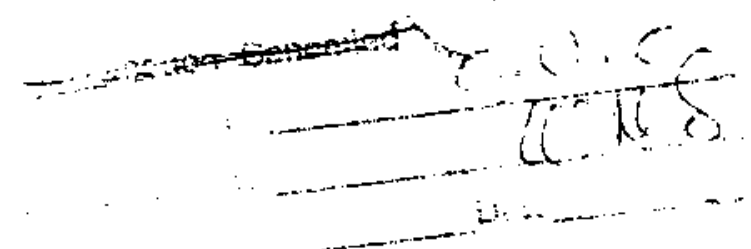

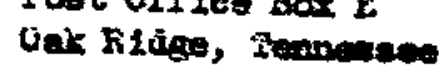

Attertion: Ka. Kenneth K. Zneachin

Subject: FAIA ERortorymo:

Cootheron:

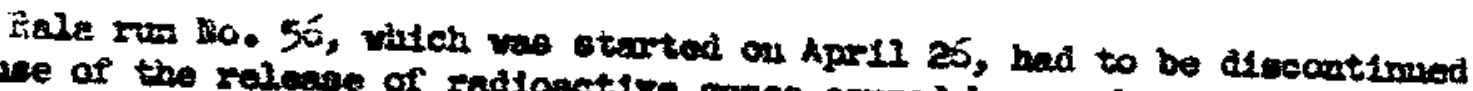

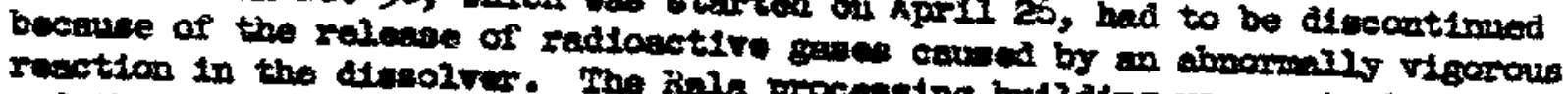

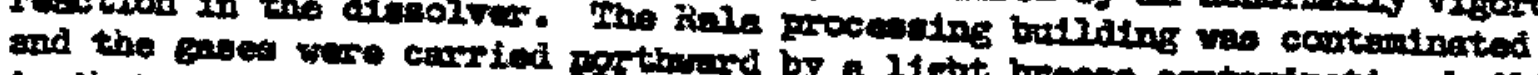

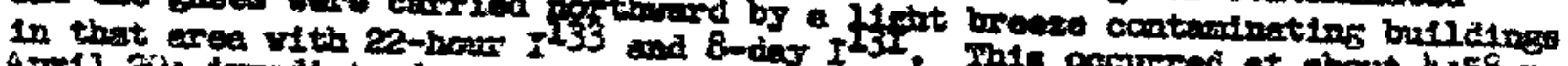

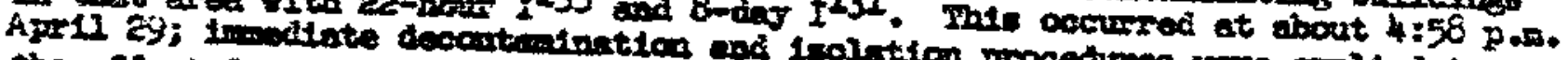

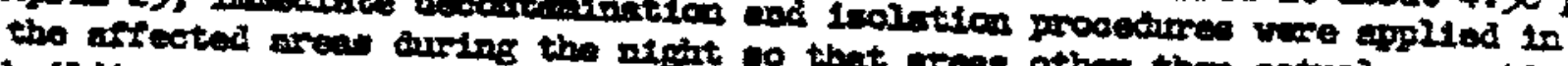
bulliting were in fairly good ninge by borning.

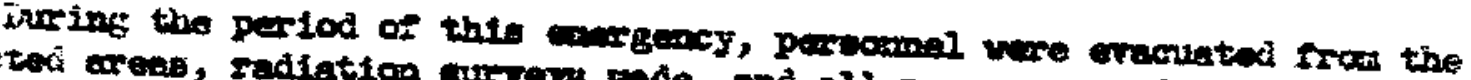

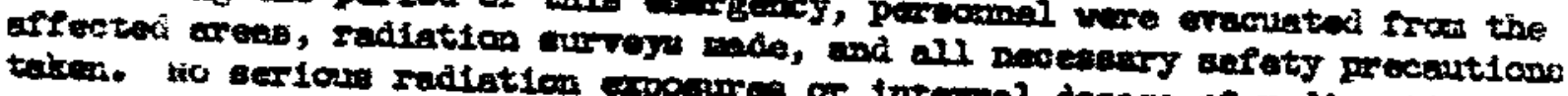

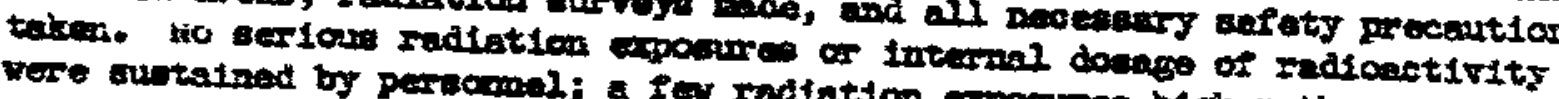

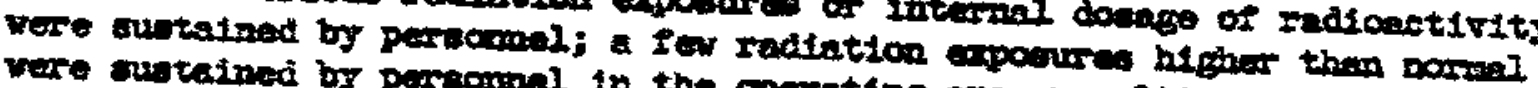

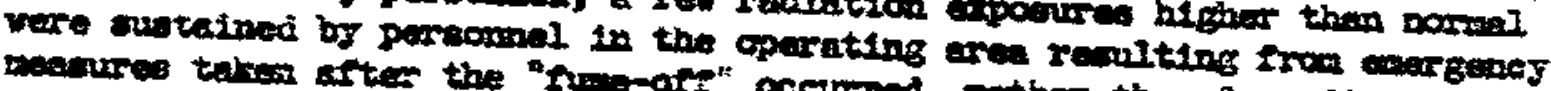

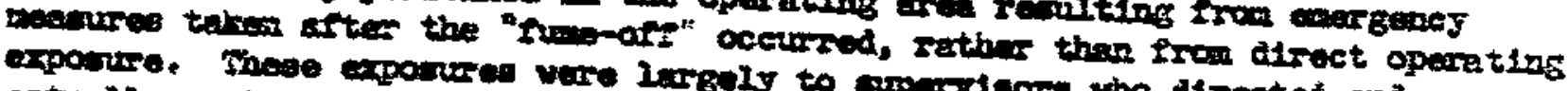

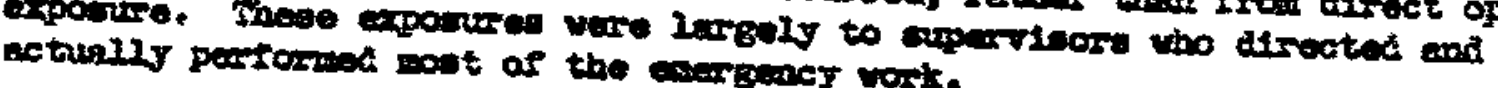

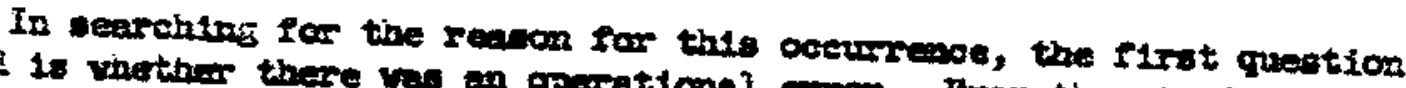

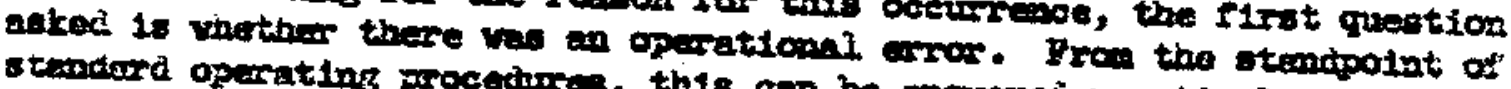

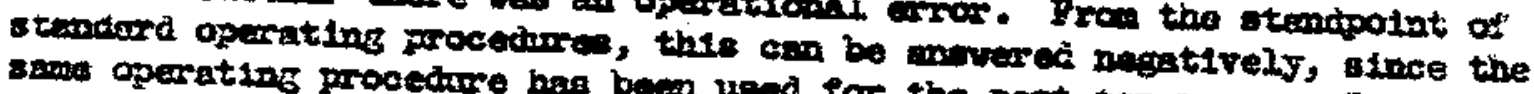

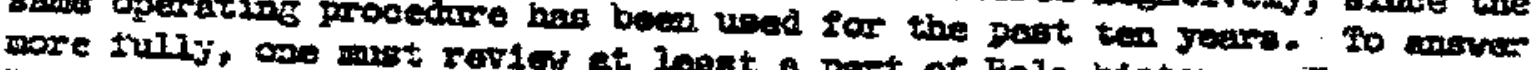

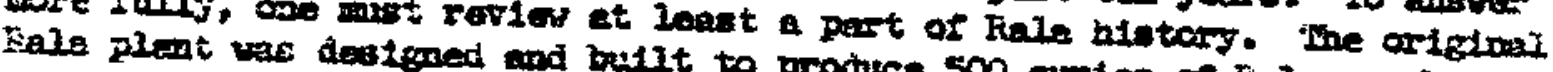

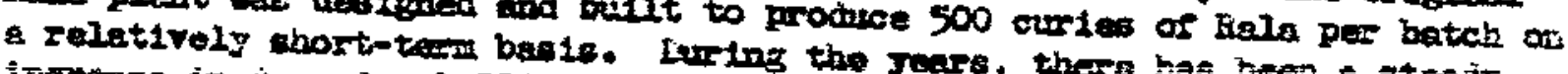

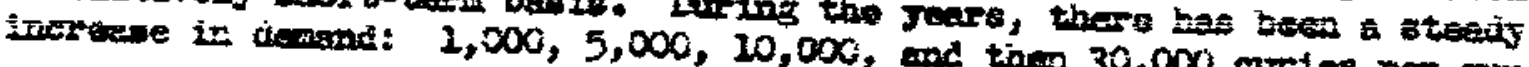
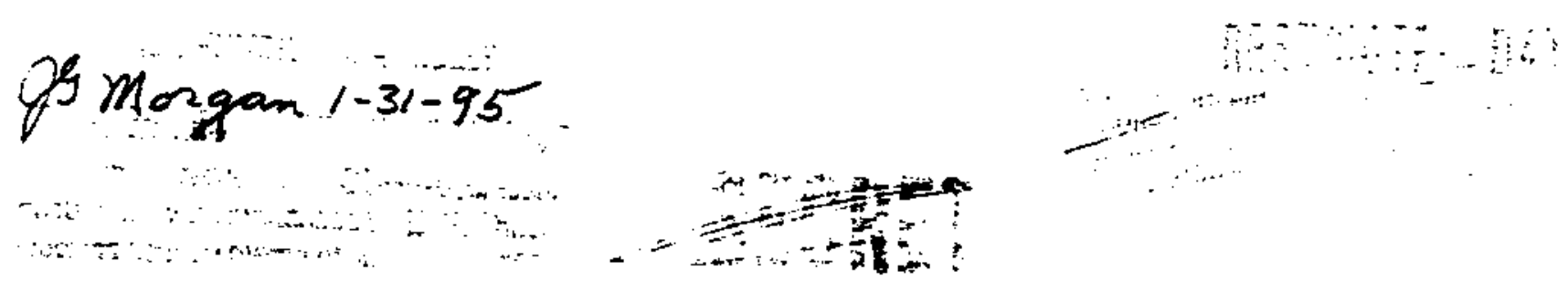


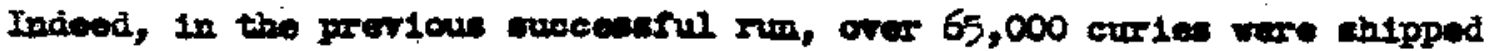
in two batches. At the 30,000 eurie level, this ropreante an 1noreace in production of orer 60 tines the demigned eapacity of the plent: It is true that the Ininhing scetion of the plent ras rebullt in 1950 to lncrease the capacity to 10,000 eurles (1 matiately insreaned to 30,000 curlec), but tho

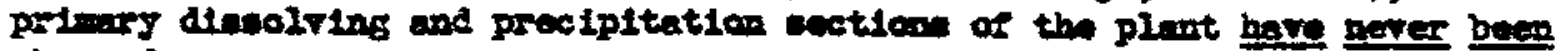
changed.

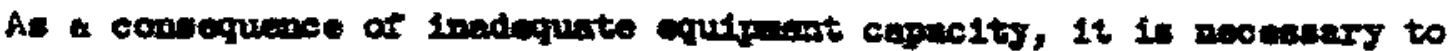

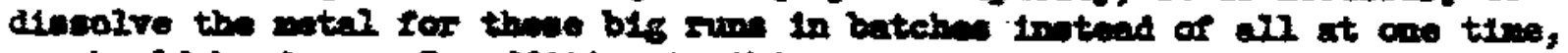

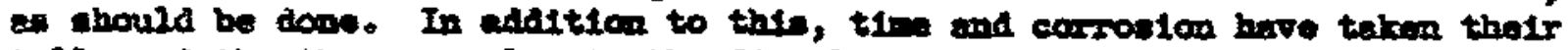

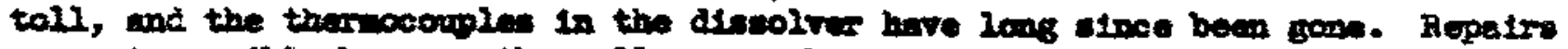
are not possfble becoune the cell cemot be entared, wd decontendnation vould

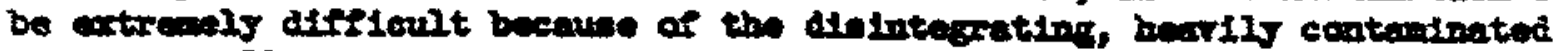
concrote valle and eokroded, leakting oruiprount.

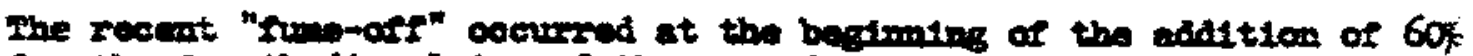

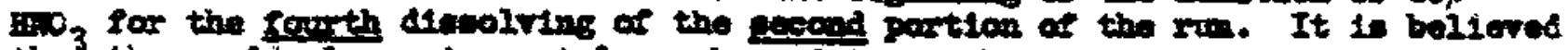

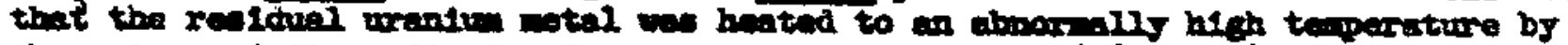

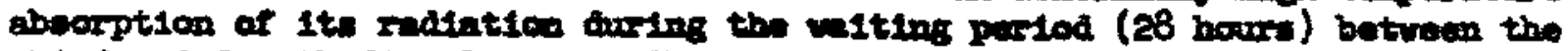

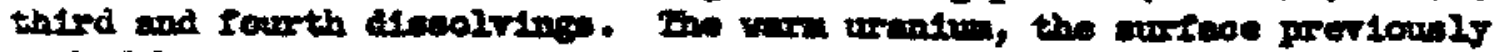

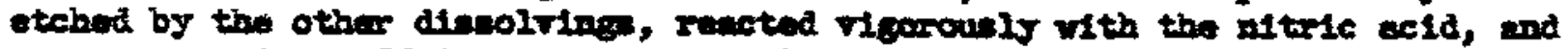

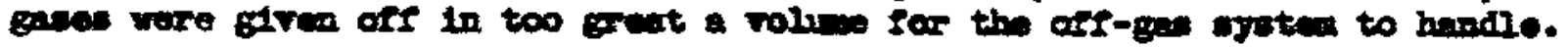

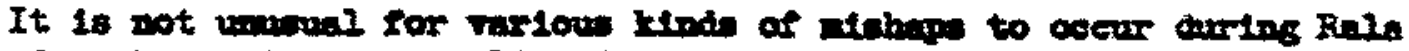

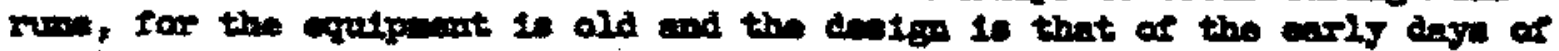

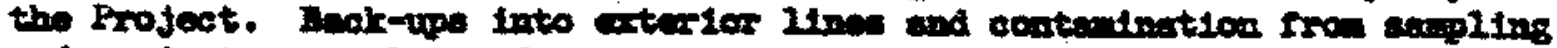

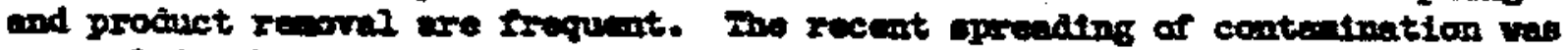

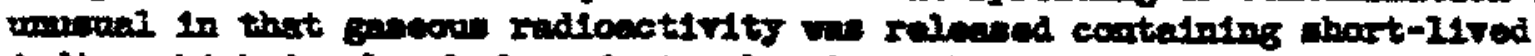

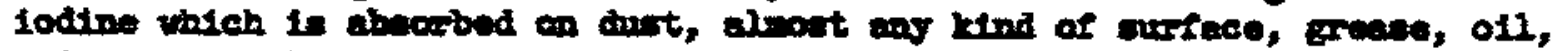

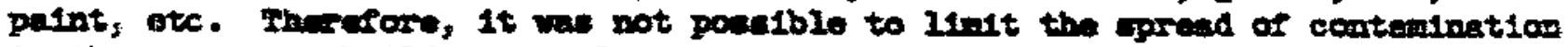
to the operating bullating 1teals.

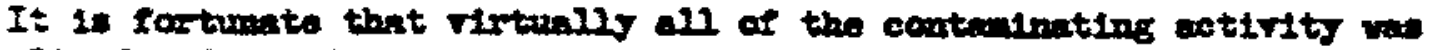
chort-21ved radiolodin, since the rapld duces halped during decenterination

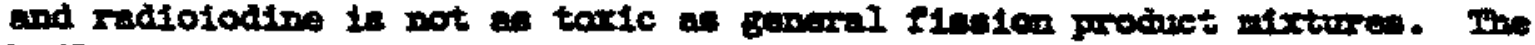

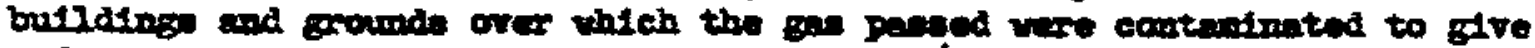
radiation reading renging $150 \mathrm{~m} 0.5$ to 20 ur/hr. and averading roughy

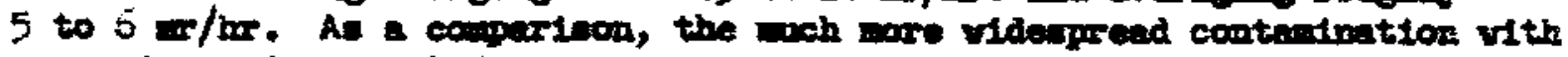

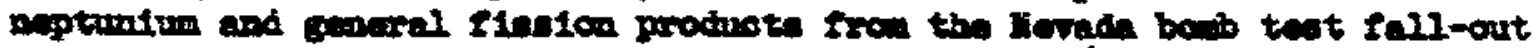
In March 1953 gare sadiatien reading of about $2 \mathrm{~m} / \mathrm{w}$. Whis corpartivon is

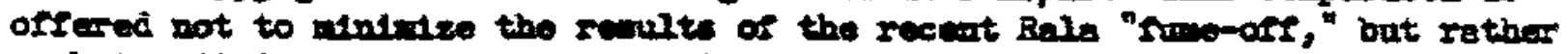
to bring it into 1 te true perepective.

It If Dot necenaexy to c1te the wany atatenouts wade to the ABC by the

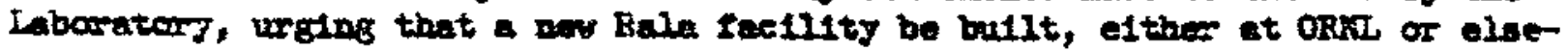

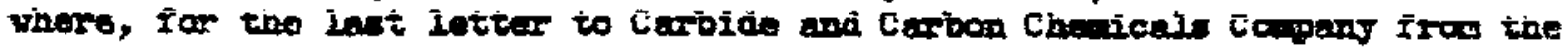




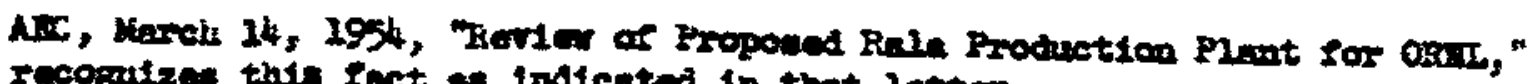
recogutzes this lact as indicated in that ietrer.

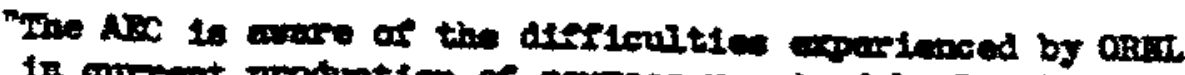

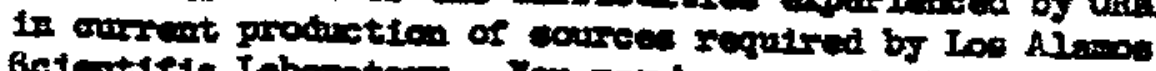

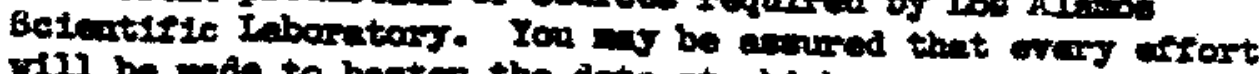
will bo made to basten the date at viseh jow antiquated prodinotiod plent can be rutired."

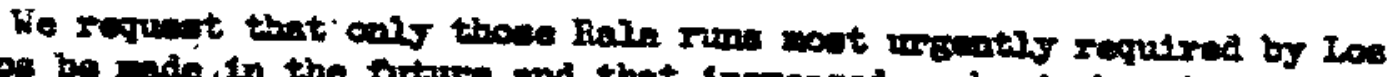

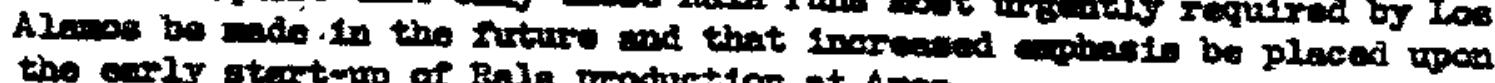
the corly atedt-m of Rale production at Aroo.

Very turf goom,

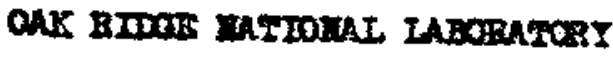

C. Z. Inteon

Director

Cintarasbg

Distrelbatica:

1-3. K. 3. Xatwehent

4. C. I. Conter

5. A. H. Watrbors

6. F. L. Cw110.

7. 4. $\mathrm{Y} \cdot \mathrm{Bum}$

8-9. C. E. Heatwo

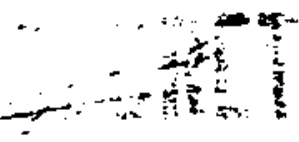


APPENDIX 4A

AIRFLOW PATTERNS AT THE OAK RIDGE RESERVATION 
TASK 1 REPORT

July 1999

Page 4A-2

${ }^{131}$ I Releases from X-10 Radioactive Lanthanum Processing Atmospheric Dispersion/Radioiodine Chemistry

This page intentionally left blank 


\section{APPENDIX 4A}

\section{AIRFLOW PATTERNS AT THE OAK RIDGE RESERVATION}

\section{4-A.1 Air Flow Patterns at the Oak Ridge Reservation}

The dispersion of contaminants in the atmosphere is influenced primarily by the prevailing winds, meteorology, and terrain conditions. Some of the key factors influencing the atmospheric dispersion of contaminants from X-10 are presented here to provide an understanding of the airflow patterns at the Oak Ridge Reservation. The discussion presented below is based on a review of several relevant documents (Holland, 1953; Gifford, 1953; Hanna et al., 1974; Nappo et al., 1978; Eckman et al., 1992; Porch et al. 1991).

The Oak Ridge Reservation is situated in the Southern Appalachian Valley between the Great Smoky Mountains (1800 m MSL, maximum elevation) on the east and the Cumberland Plateau (1000 m MSL, maximum elevation) on the west (Figure 4A.1). The orientation of the valley is northeast-southwest in the vicinity of the Oak Ridge Reservation, and the valley floor slopes gently from the northeast to the southwest. Within the Southern Appalachian Valley, several smaller ridges and valleys run parallel to each other and to the larger valley. The Oak Ridge Reservation is located among these smaller ridges and valleys, where ridges rise to over $350 \mathrm{~m}$ MSL, and the valley floors are at elevations of $240 \mathrm{~m}$ MSL. The average distance between two adjacent ridges is roughly $1.6 \mathrm{~km}$.

The influence of the topography on prevailing winds in the Oak Ridge Reservation, particularly for a mid-valley location consistent with the location of the X-10 site within Bethel Valley, can be clearly seen in the wind roses (Figure 4-A.2). The predominant wind direction has clearly shifted from a west-to-east orientation in the upper layers of the atmosphere to southwest-tonortheast and northeast-to-southwest directions in layers close to the surface. During the day, the wind direction is predominantly up-valley, from southwest to northeast, and during the night, it is predominantly down-valley, from northeast to southwest. 


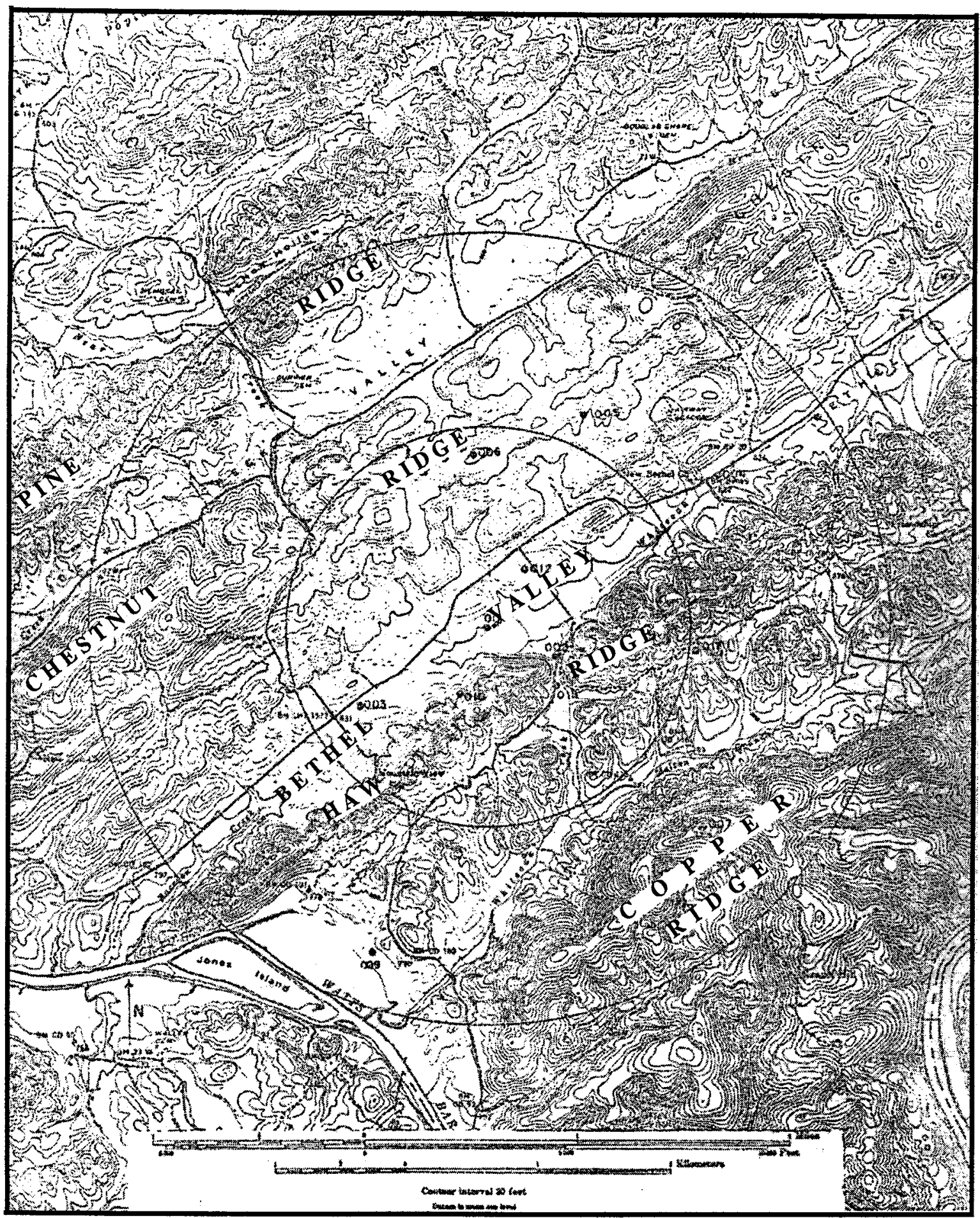

Figure 4-A.1 General topography of the region surrounding Oak Ridge. 


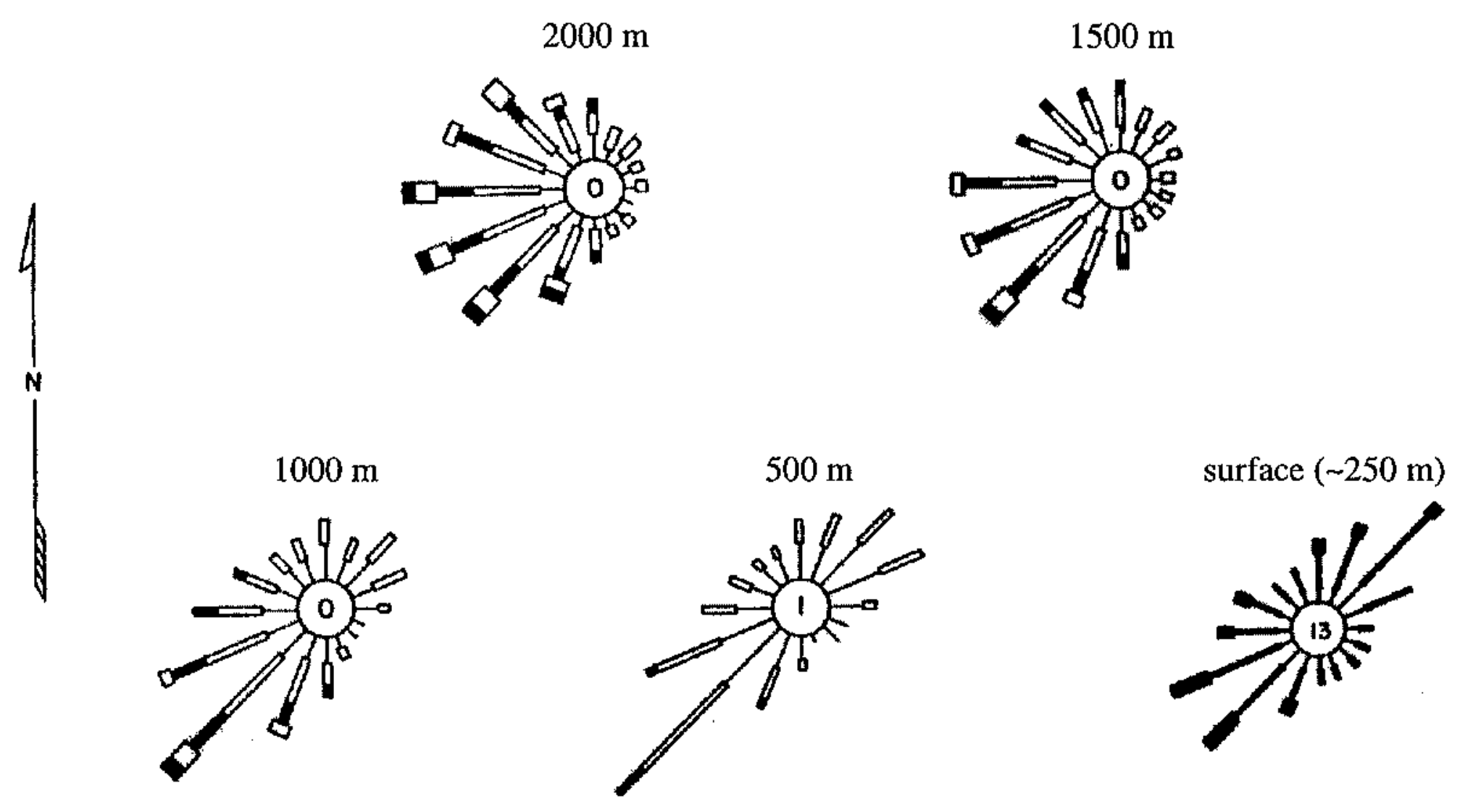

Figure 4-A.2 Wind roses at different elevations above mean sea level for a mid-valley location within the Bethel Valley. 
The long, low ridges within the Oak Ridge Reservation are broken by several narrow gaps (e.g., the White Oak Gap in Haw Ridge, which separates Bethel and Melton Valleys). The flow near the White Oak Gap does not indicate that there is any exchange of air between the two valleys. During the day, flows from both sides converge at the gap, resulting in a flow that resembles the up-slope characteristics of typical daytime flow along the unbroken parts of Haw Ridge. During the night, flow on the Bethel Valley side of White Oak Gap resembles the downward drainage flows on other unbroken parts of Haw Ridge. The flow pattern within Bethel Valley in a vertical extent of up to $100 \mathrm{~m}$ from the valley floors is summarized in the schematic of Figure 4-A.3.

A study of the annual average wind rose from a $10-\mathrm{m}$ monitoring station in the middle of Bethel Valley (Figure 4-A.4) reveals that there is always a significant air flow in a cross-valley direction. These cross-valley flow components at lower elevations represent the typical daytime up-slope and nighttime down-slope flow characteristics. In other words, these cross-valley components may not necessarily translate into flows from one valley to the next, consistent with the schematic in Figure 4-A.5. However, it must be recognized that under conditions of low wind speeds, cross-valley exchange of winds can occur over the ridges as indicated by the experiments conducted by Gifford (1953).

Gifford (1953) analyzed a series of experiments in which neutrally buoyant balloons were released and tracked during day and night and under light and strong wind conditions. His study indicated that under very light wind conditions, it is sometimes possible for a balloon released in one valley to rise above the ridges and travel to the ground in an adjacent valley with a simultaneous equidistant downwind (along-valley direction) transport. However, there were more cases in which the balloons would be caught in cross-valley, upper layer winds and travel several kilometers across several valleys without any descent. These two observations suggest that for locations of concern along cross-valley directions in valleys adjacent to the Bethel Valley, wind-speeds with low magnitudes are more important than those with larger magnitudes for the transport of contaminants.

The cross-valley exchange of air at low wind speeds across the ridge tops is a significant process for the analysis of dispersion across ridges. Since the predominant direction of wind flow in Bethel Valley is along the direction of the valley orientation, the frequencies of winds in crossvalley directions are lower than those for winds in the direction of valley orientation. However, if these cross-valley flows were dominated by winds of low magnitude, the movement of contaminants across the valley would be very slow, resulting in higher concentrations of contaminants along this flow path. Surface level winds in Figures 4A.2 and 4-A.4 in the crossvalley direction do seem to be dominated by winds of low magnitudes (up to a maximum of about $3 \mathrm{~m} \mathrm{~s}^{-1}$ from Figure 4-A.4). 


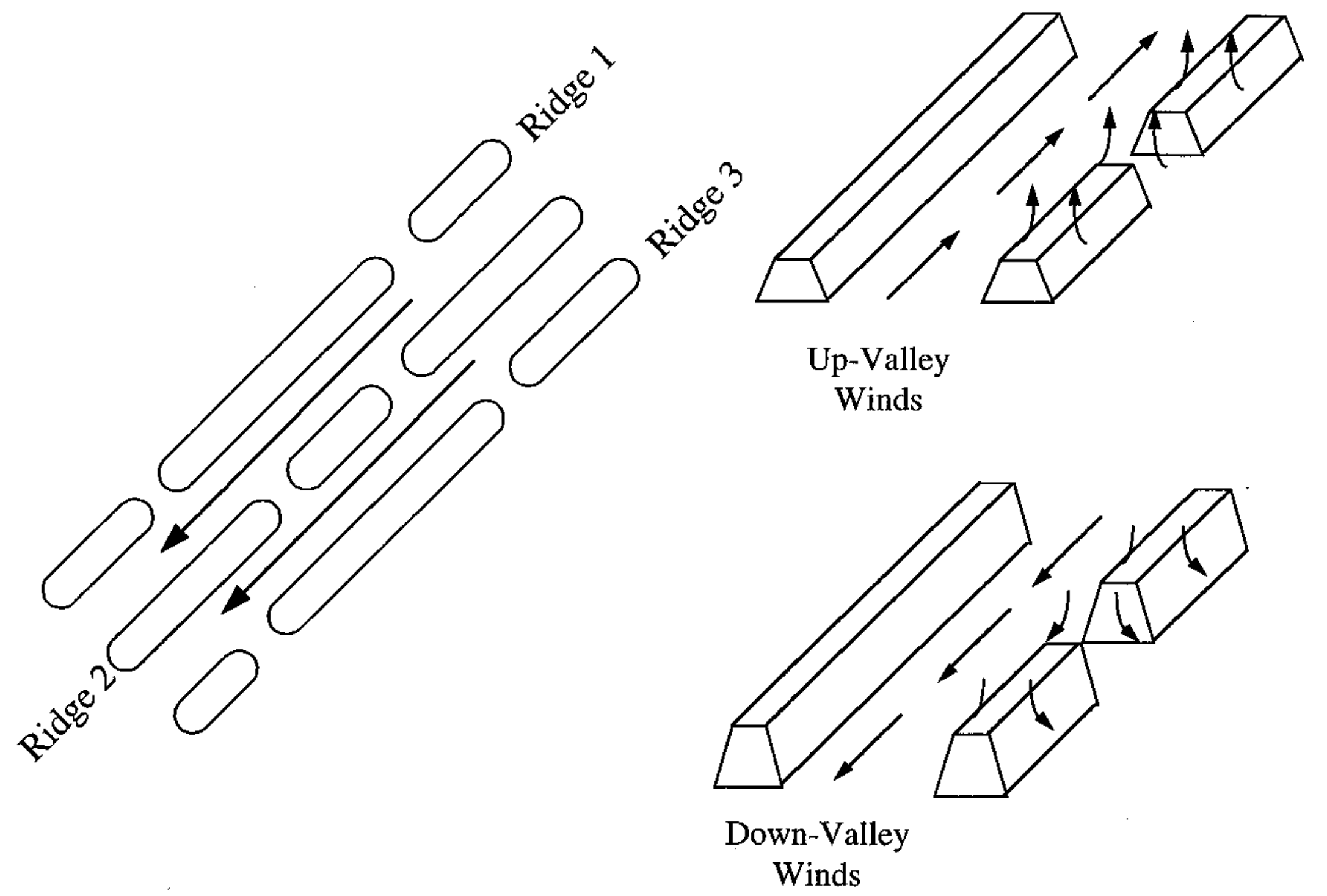

Figure 4-A.3 Flow patterns within the valley-ridge region surrounding the radioactive lantanum processing facility. 


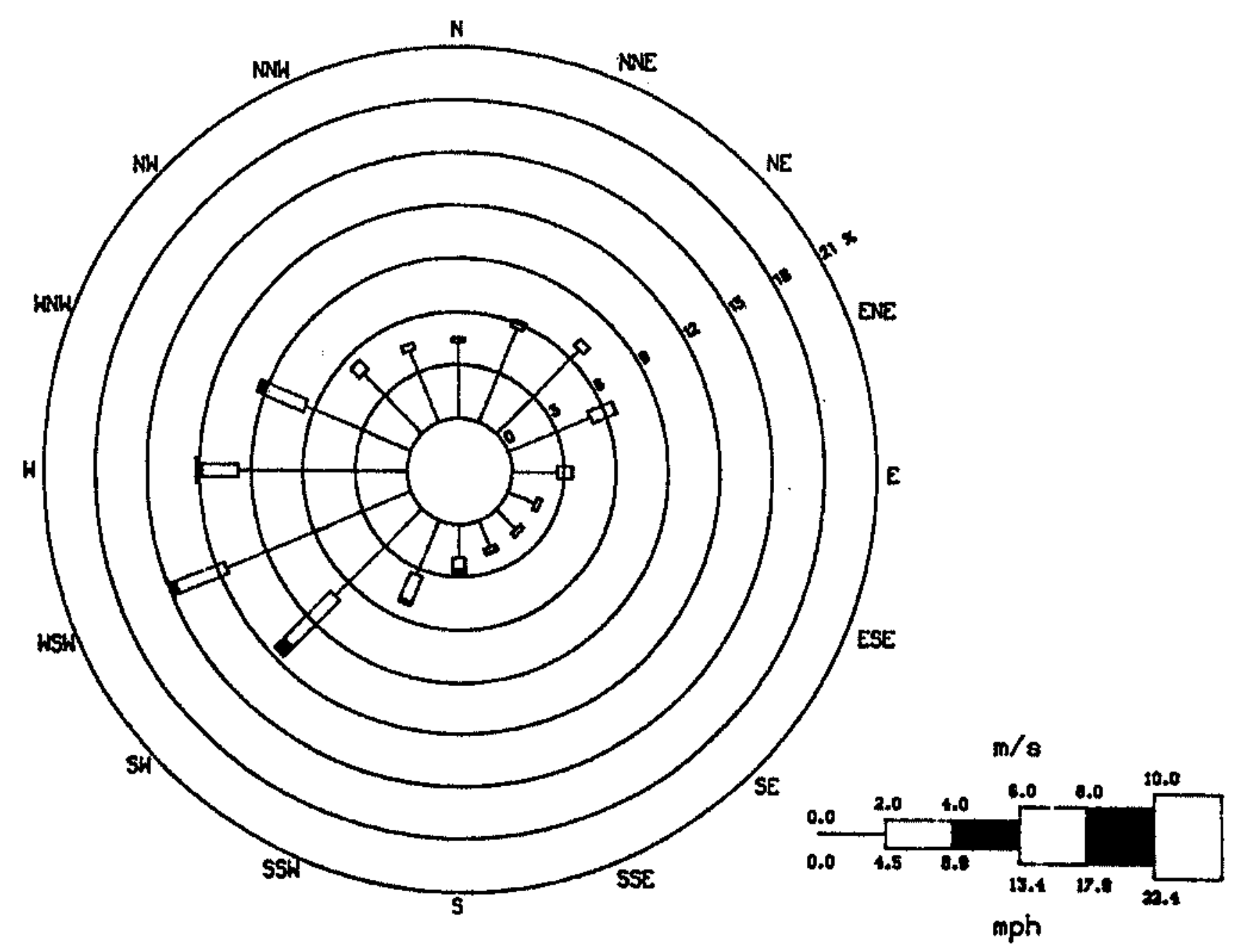

Figure 4-A.4 Annual wind rose for Tower C (at $10 \mathrm{~m}$ elevation) for 1993. 

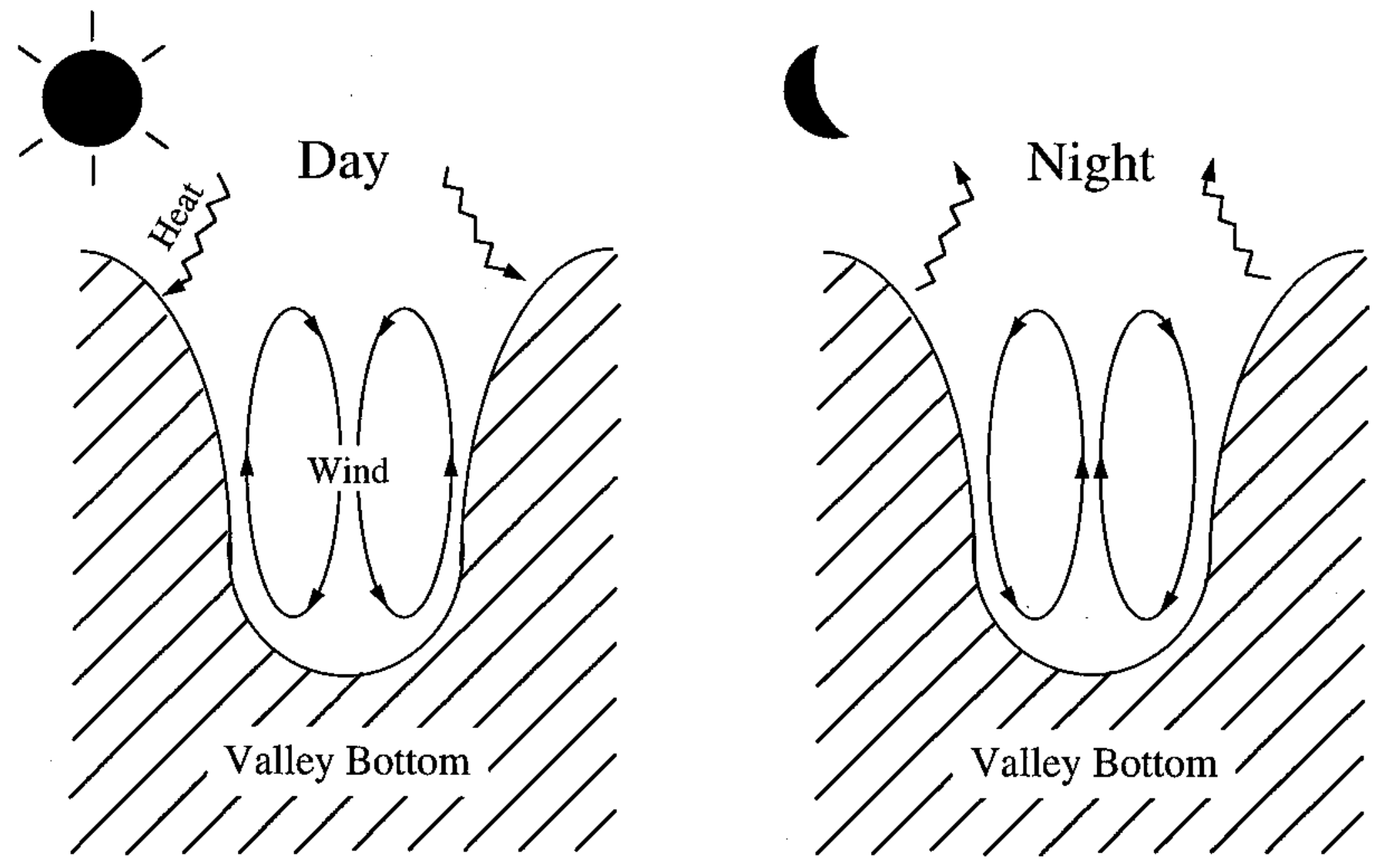

Figure 4-A.5 Cross-valley component of the mid-valley flow. 
Because direct measurements at ridge-top levels from ridges adjacent to Bethel Valley are not available, it is not clear how much of the mid-valley winds of low magnitudes in cross-valley directions would translate into real cross-valley flows. Accurate quantification of the frequency and speed of winds across the ridges would, therefore, be a very difficult exercise requiring the establishment of correlations among meteorological parameters measured at mid-valley, upslope, and ridge-top stations. After the establishment of correlations, a spatial and temporal distribution of the wind-field can be developed. However, such an exercise is clearly out of the scope of this study. Furthermore, it was determined very early in this study that the uncertainties in the estimates of doses and risks to individuals exposed to ${ }^{131} \mathrm{I}$ are dominated by contributions from processes other than the atmospheric dispersion of ${ }^{131} \mathrm{I}$ (e.g., dose-response relationship, internal dosimetry, and pasture-milk transfer coefficients). Therefore, it was decided to use an atmospheric dispersion model in this study that used the meteorology from a mid-valley location and validate the model using field data on releases and measured concentrations of ${ }^{131} \mathrm{I}$. The results of the validation study, as shown in Section 4, clearly indicate that it was not necessary to account explicitly in the model for the effects of local terrain features.

\section{4-A.2 References}

Eckman, R. M., Dobosy, R. J., and Pendergrass, W. R. "Preliminary Analysis of Wind Data for the Oak Ridge Site.” NOAA/ATDD TM/ERL/ARI-193, 1992.

Gifford, F. A., Jr. "A Study of Low Level Air Trajectories at Oak Ridge," Tenn. Monthly Weather Review 18 (7):179-192. July 1953.

Hanna, S. R., Nappo, C. J., Hosker, R. P., and Briggs, G. A. "Description of the Eastern Tennessee Trajectory Experiment (ETTEX).” ATDL Contribution File No. 103. 1974.

Holland, J.Z. "A Meteorological Survey of the Oak Ridge Area: Final Report Covering the Period 1948-1952." NOAA/ATDD Report ORO-99. Oak Ridge, TN. 1953. (ChemRisk Repository No. 1211)

Nappo, C. J., Jr., Hosker, R. P., and Hanna, S. R. "The Eastern Tennessee Trajectory Experiment (ETTEX): Description and Data Summary." NOAA Technical Memorandum ERL ARL-71, 1978.

Porch, W., Clements, W., Grant, T., and Coulter, R. "Comparison of Gap and Tributary Interactions with Nighttime Valley Flows from the ASCOT 1990 and 1988 Experiments." LAUR-91-764, ASCOT-91-1. 1991. 
APPENDIX 10A

THYROID CANCER INCIDENCE DATA 
TASK 1 REPORT

July 1999

${ }^{131}$ I Releases from X-10 Radioactive Lanthanum Processing Page 10A-2 Thyroid Cancer Incidence Data

This page intentionally left blank. 


\section{APPENDIX 10A THYROID CANCER INCIDENCE DATA}

Exposure to radiation can increase a person's risk of getting thyroid cancer. Epidemiological evidence (Ron et al., 1995) shows that the increased risk of thyroid cancer depends on the background incidence of thyroid cancer of the population of interest, the gender of the exposed individual, and the age at the time of first exposure. The "background incidence" in his study is defined as the incidence in a population not exposed to ${ }^{131}$ I released from X-10.

This appendix presents the thyroid cancer incidence data used in this study to estimate the excess lifetime risk of cancer from exposure to ${ }^{131}$ I. Section 10-A.1 summarizes the background cancer incidence rates for the state of Tennessee. Section 10-A.2 describes the derivation of the "lifetime background" risk of thyroid cancer incidence, which is the quantity used to estimate the excess "lifetime" risk of thyroid cancer for an exposed individual (Section 10.2).

The background incidence of cancer in Tennessee exhibits two important properties: (a) large differences between people of different ethnic backgrounds, and (b) an increase with time during the last two decades. Sections 10-A.3 and 10-A.4 investigate the effect of these two properties on the final risk estimates reported in this study.

Individuals living in the state of Tennessee between 1951 and 1958 were exposed to ${ }^{131}$ I from nuclear weapons testing performed at the Nevada Test Site (NTS). This exposure elevates their background incidence of cancer. In this study, a comparison is provided between the risk of cancer from exposure to ${ }^{131} \mathrm{I}$ released from X-10 and the risk of cancer from exposure to ${ }^{131} \mathrm{I}$ from NTS fallout. To estimate the risk from exposure to NTS fallout, the reported "background incidence" must be corrected for the contribution of NTS fallout. Section 10-A.5 describes the method used for this correction.

\section{A.1 Thyroid cancer incidence rates for the state of Tennessee}

The age-specific thyroid cancer incidence rates for Tennessee were provided by the Tennessee Department of Health in Nashville (Turri, 1998). These data are gender-specific, and they were provided in three geographical groups: (a) incidence rates for all Tennessee counties (Table 10A.1), (b) incidence rates for Anderson, Roane, Loudon and Knox counties (Table 10-A.2), and (c) incidence rates for all counties in Tennessee other than Anderson, Roane, Loudon and Knox (Table 10-A.3.) The last data set was used for estimation of the background risk of thyroid cancer because it is considered to be specific for Tennessee areas while representing people that most probably were not exposed to ${ }^{131}$ I released from the X-10 processing plant. Data were also separated by ethnic background into two categories (Tables 10-A.4 through 10-A.9): (a) whites, and (b) other races. In Tennessee, the category "other races" is dominated by individuals of African-American ethnic background. 
TASK 1 REPORT

July 1999

${ }^{131}$ I Releases from X-10 Radioactive Lanthanum Processing -

Page 10A-4

Thyroid Cancer Incidence Data

Table 10-A.1 Thyroid cancer incidence data (1988 to 1995) for people of all races residing in the entire state of Tennessee (Turri, 1998).

\begin{tabular}{|c|c|c|c|}
\hline \multicolumn{4}{|c|}{ FEMALES } \\
\hline Age & Cases & Population $^{\mathrm{a}}$ & Age-specific rate \\
\hline $0-4$ & 1 & $1,343,239$ & 0.1 \\
\hline $5-9$ & 1 & $1,361,900$ & 0.1 \\
\hline $10-14$ & 5 & $1,361,356$ & 0.4 \\
\hline $15-19$ & 35 & $1,442,875$ & 2.4 \\
\hline $20-24$ & 48 & $1,493,258$ & 3.2 \\
\hline $25-29$ & 73 & $1,625,259$ & 4.5 \\
\hline $30-34$ & 139 & $1,660,664$ & 8.4 \\
\hline $35-39$ & 138 & $1,609,513$ & 8.6 \\
\hline $40-44$ & 126 & $1,496,264$ & 8.4 \\
\hline $45-49$ & 109 & $1,250,494$ & 8.7 \\
\hline $50-54$ & 92 & $1,086,455$ & 8.5 \\
\hline $55-59$ & 81 & 995,148 & 8.1 \\
\hline $60-64$ & 75 & 946,657 & 7.9 \\
\hline $65-69$ & 90 & 905,859 & 9.9 \\
\hline 70-74 & 50 & 752,240 & 6.6 \\
\hline $75-79$ & 44 & 633,749 & 6.9 \\
\hline $80-84$ & 42 & 438,173 & 9.6 \\
\hline $85+$ & 24 & 368,431 & 6.5 \\
\hline \multirow{2}{*}{\multicolumn{4}{|c|}{$\begin{array}{c}1173 \mathbf{2 0 , 7 7 1 , 5 3 4} \\
\text { MALES }\end{array}$}} \\
\hline & & & \\
\hline $0-4$ & 1 & $1,407,329$ & 0.1 \\
\hline $5-9$ & 1 & $1,433,174$ & 0.1 \\
\hline $10-14$ & 2 & $1,431,410$ & 0.1 \\
\hline $15-19$ & 1 & $1,524,739$ & 0.1 \\
\hline $20-24$ & 8 & $1,486,906$ & 0.5 \\
\hline $25-29$ & 27 & $1,572,468$ & 1.7 \\
\hline $30-34$ & 37 & $1,569,621$ & 2.4 \\
\hline $35-39$ & 31 & $1,524,145$ & 2.0 \\
\hline $40-44$ & 34 & $1,422,288$ & 2.4 \\
\hline $45-49$ & 37 & $1,186,734$ & 3.1 \\
\hline $50-54$ & 47 & $1,006,636$ & 4.7 \\
\hline $55-59$ & 31 & 882,440 & 3.5 \\
\hline $60-64$ & 31 & 803,538 & 3.9 \\
\hline $65-69$ & 28 & 714,163 & 3.9 \\
\hline $70-74$ & 23 & 535,913 & 4.3 \\
\hline $75-79$ & 20 & 385,733 & 5.2 \\
\hline $80-84$ & 9 & 219,069 & 4.1 \\
\hline $85+$ & 7 & 137,466 & 5.1 \\
\hline TOTAL & 375 & $19,243,772$ & 1.9 \\
\hline
\end{tabular}

${ }^{\text {a }}$ Population figures are cumulative over 8 years (1988 to 1995).

${ }^{\mathrm{b}}$ per 100,000 per year. 
Table 10-A.2 Thyroid cancer incidence data (1988 to 1995) for people of all races residing in Anderson, Roane, Loudon or Knox counties in Tennessee (Turri, 1998).

\begin{tabular}{|c|c|c|c|}
\hline \multicolumn{4}{|c|}{ FEMALES } \\
\hline Age & Cases & Population $^{\mathrm{a}}$ & Age-specific rate ${ }^{b}$ \\
\hline $0-4$ & $\overline{0}$ & 121,686 & 0.0 \\
\hline $5-9$ & 0 & 124,646 & 0.0 \\
\hline $10-14$ & 0 & 122,781 & 0.0 \\
\hline $15-19$ & 3 & 140,737 & 2.1 \\
\hline $20-24$ & 6 & 155,847 & 3.8 \\
\hline $25-29$ & 11 & 158,567 & 6.9 \\
\hline $30-34$ & 17 & 164,111 & 10.4 \\
\hline $35-39$ & 20 & 161,154 & 12.4 \\
\hline $40-44$ & 9 & 151,206 & 6.0 \\
\hline $45-49$ & 10 & 126,497 & 7.9 \\
\hline $50-54$ & 10 & 109,351 & 9.1 \\
\hline $55-59$ & 9 & 102,613 & 8.8 \\
\hline $60-64$ & 11 & 101,703 & 10.8 \\
\hline $65-69$ & 13 & 99,415 & 13.1 \\
\hline $70-74$ & 6 & 80,648 & 7.4 \\
\hline $75-79$ & 2 & 67,460 & 3.0 \\
\hline $80-84$ & 6 & 46,095 & 13.0 \\
\hline $85+$ & 1 & 37,552 & 2.7 \\
\hline \multirow{2}{*}{\multicolumn{4}{|c|}{$\frac{134 \mathbf{2 , 0 7 2 , 0 6 9}}{\text { MALES }}$}} \\
\hline & & & \\
\hline$\overline{0-4}$ & 0 & 127,229 & 0.0 \\
\hline $5-9$ & 0 & 129,014 & 0.0 \\
\hline $10-14$ & 0 & 129,187 & 0.0 \\
\hline $15-19$ & 0 & 146,073 & 0.0 \\
\hline $20-24$ & 1 & 158,469 & 0.6 \\
\hline $25-29$ & 1 & 152,329 & 0.7 \\
\hline $30-34$ & 5 & 156,404 & 3.2 \\
\hline $35-39$ & 2 & 152,216 & 1.3 \\
\hline $40-44$ & 4 & 141,904 & 2.8 \\
\hline $45-49$ & 6 & 120,584 & 5.0 \\
\hline $50-54$ & 9 & 101,430 & 8.9 \\
\hline $55-59$ & 4 & 88,818 & 4.5 \\
\hline $60-64$ & 2 & 83,861 & 2.4 \\
\hline $65-69$ & 2 & 75,865 & 2.6 \\
\hline $70-74$ & 1 & 55,631 & 1.8 \\
\hline $75-79$ & 4 & 38,945 & 10.3 \\
\hline $80-84$ & 1 & 21,643 & 4.6 \\
\hline $85+$ & 2 & 12,434 & 16.1 \\
\hline TOTAL & 44 & $\mathbf{1 , 8 9 2 , 0 3 6}$ & 2.3 \\
\hline
\end{tabular}

${ }^{\text {a }}$ Population figures are cumulative over 8 years (1988 to 1995).

${ }^{\mathrm{b}}$ per 100,000 per year. 
TASK 1 REPORT

July 1999

${ }^{131}$ I Releases from X-10 Radioactive Lanthanum Processing -

Page 10A-6

Thyroid Cancer Incidence Data

Table 10-A.3 Thyroid cancer incidence data (1988 to 1995) for people of all races residing in Tennessee counties other than Anderson, Roane, Loudon or Knox (Turri, 1998).

\begin{tabular}{|c|c|c|c|}
\hline \multicolumn{4}{|c|}{ FEMALES } \\
\hline Age & Cases & Population $^{\mathrm{a}}$ & Age-specific rate ${ }^{b}$ \\
\hline $0-4$ & 1 & $1,221,553$ & 0.1 \\
\hline $5-9$ & 1 & $1,237,254$ & 0.1 \\
\hline $10-14$ & 5 & $1,238,575$ & 0.4 \\
\hline $15-19$ & 32 & $1,302,138$ & 2.5 \\
\hline $20-24$ & 42 & $1,337,411$ & 3.1 \\
\hline $25-29$ & 62 & $1,466,692$ & 4.2 \\
\hline $30-34$ & 122 & $1,496,553$ & 8.2 \\
\hline $35-39$ & 118 & $1,448,359$ & 8.1 \\
\hline $40-44$ & 117 & $1,345,058$ & 8.7 \\
\hline $45-49$ & 99 & $1,123,997$ & 8.8 \\
\hline $50-54$ & 82 & 977,104 & 8.4 \\
\hline $55-59$ & 72 & 892,535 & 8.1 \\
\hline $60-64$ & 64 & 844,954 & 7.6 \\
\hline $65-69$ & 77 & 806,444 & 9.5 \\
\hline $70-74$ & 44 & 671,592 & 6.6 \\
\hline $75-79$ & 42 & 566,289 & 7.4 \\
\hline $80-84$ & 36 & 392,078 & 9.2 \\
\hline $85+$ & 23 & 330,879 & 7.0 \\
\hline \multirow{2}{*}{\multicolumn{4}{|c|}{$\begin{array}{c}1039 \text { 18,699,465 } \\
\text { MALES }\end{array}$}} \\
\hline & & & \\
\hline $0-4$ & 1 & $1,280,100$ & 0.1 \\
\hline $5-9$ & 1 & $1,304,160$ & 0.1 \\
\hline $10-14$ & 2 & $1,302,223$ & 0.2 \\
\hline $15-19$ & 1 & $1,378,666$ & 0.1 \\
\hline $20-24$ & 7 & $1,328,437$ & 0.5 \\
\hline $25-29$ & 26 & $1,420,139$ & 1.8 \\
\hline $30-34$ & 32 & $1,413,217$ & 2.3 \\
\hline $35-39$ & 29 & $1,371,929$ & 2.1 \\
\hline $40-44$ & 30 & $1,280,384$ & 2.3 \\
\hline $45-49$ & 31 & $1,066,150$ & 2.9 \\
\hline $50-54$ & 38 & 905,206 & 4.2 \\
\hline $55-59$ & 27 & 793,622 & 3.4 \\
\hline $60-64$ & 29 & 719,677 & 4.0 \\
\hline $65-69$ & 26 & 638,298 & 4.1 \\
\hline $70-74$ & 22 & 480,282 & 4.6 \\
\hline $75-79$ & 16 & 346,788 & 4.6 \\
\hline $80-84$ & 8 & 197,426 & 4.1 \\
\hline $85+$ & 5 & 125,032 & 4.0 \\
\hline TOTAL & 331 & $17,351,736$ & 1.9 \\
\hline
\end{tabular}

${ }^{\text {a }}$ Population figures are cumulative over 8 years (1988 to 1995).

${ }^{\mathrm{b}}$ per 100,000 per year. 
Table 10-A.4 Thyroid cancer incidence data (1988 to 1995) for white people residing in the entire state of Tennessee (Turri, 1998).

\begin{tabular}{|c|c|c|c|}
\hline \multicolumn{4}{|c|}{ FEMALES-WHITE } \\
\hline Age & Cases & Population $^{\mathrm{a}}$ & Age-specific rate \\
\hline $0-4$ & 1 & 1029505 & 0.1 \\
\hline $5-9$ & 1 & 1056941 & 0.1 \\
\hline $10-14$ & 5 & 1062538 & 0.5 \\
\hline $15-19$ & 35 & 1137105 & 3.1 \\
\hline $20-24$ & 48 & 1188611 & 4.0 \\
\hline $25-29$ & 71 & 1312434 & 5.4 \\
\hline $30-34$ & 138 & 1343990 & 10.3 \\
\hline $35-39$ & 138 & 1318069 & 10.5 \\
\hline $40-44$ & 126 & 1258719 & 10 \\
\hline $45-49$ & 107 & 1069860 & 10 \\
\hline $50-54$ & 91 & 936518 & 9.7 \\
\hline $55-59$ & 80 & 865909 & 9.2 \\
\hline $60-64$ & 75 & 827761 & 9.1 \\
\hline $65-69$ & 88 & 795065 & 11.1 \\
\hline $70-74$ & 50 & 660648 & 7.6 \\
\hline $75-79$ & 44 & 556258 & 7.9 \\
\hline $80-84$ & 42 & 385588 & 10.9 \\
\hline $85+$ & 23 & 324004 & 7.1 \\
\hline \multirow{2}{*}{\multicolumn{4}{|c|}{$\frac{116317, \mathbf{1 2 9 , 5 2 3}}{\text { MALES-WHITE }}$}} \\
\hline & & & \\
\hline $0-4$ & 1 & 1083362 & 0.1 \\
\hline $5-9$ & 1 & 1117526 & 0.1 \\
\hline $10-14$ & 2 & 1123466 & 0.2 \\
\hline $15-19$ & 1 & 1213367 & 0.1 \\
\hline $20-24$ & 7 & 1204816 & 0.6 \\
\hline $25-29$ & 27 & 1301752 & 2.1 \\
\hline $30-34$ & 37 & 1312547 & 2.8 \\
\hline $35-39$ & 31 & 1289631 & 2.4 \\
\hline $40-44$ & 32 & 1229105 & 2.6 \\
\hline $45-49$ & 37 & 1043617 & 3.5 \\
\hline $50-54$ & 46 & 891205 & 5.2 \\
\hline $55-59$ & 31 & 788907 & 3.9 \\
\hline $60-64$ & 31 & 719272 & 4.3 \\
\hline $65-69$ & 28 & 638364 & 4.4 \\
\hline $70-74$ & 22 & 476731 & 4.6 \\
\hline $75-79$ & 20 & 340091 & 5.9 \\
\hline $80-84$ & c & 190639 & 4.7 \\
\hline $85+$ & 6 & 116854 & 5.1 \\
\hline TOTAL & 369 & $16,081,252$ & 2.3 \\
\hline
\end{tabular}

${ }^{\text {a }}$ Population figures are cumulative over 8 years (1988 to 1995).

${ }^{\mathrm{b}}$ per 100,000 per year. 
TASK 1 REPORT

July 1999

${ }^{131}$ I Releases from X-10 Radioactive Lanthanum Processing -

Page 10A-8

Thyroid Cancer Incidence Data

Table 10-A.5 Thyroid cancer incidence data (1988 to 1995) for white people residing in Anderson, Roane, Loudon or Knox counties in Tennessee (Turri, 1998).

\begin{tabular}{|c|c|c|c|}
\hline \multicolumn{4}{|c|}{ FEMALES-WHITE } \\
\hline Age & Cases & Population $^{\mathrm{a}}$ & Age-specific rate ${ }^{b}$ \\
\hline $0-4$ & & 107837 & \\
\hline $5-9$ & & 110210 & \\
\hline $10-14$ & & 109185 & \\
\hline $15-19$ & 3 & 125086 & 2.4 \\
\hline $20-24$ & 6 & 139668 & 4.3 \\
\hline $25-29$ & 10 & 143580 & 7 \\
\hline $30-34$ & 17 & 148556 & 11.4 \\
\hline $35-39$ & 20 & 146912 & 13.6 \\
\hline $40-44$ & 9 & 139080 & 6.5 \\
\hline $45-49$ & 9 & 117375 & 7.7 \\
\hline $50-54$ & 10 & 101685 & 9.8 \\
\hline $55-59$ & 9 & 96408 & 9.3 \\
\hline $60-64$ & 11 & 95497 & 11.5 \\
\hline $65-69$ & 13 & 93575 & 13.9 \\
\hline $70-74$ & 6 & 75697 & 7.9 \\
\hline $75-79$ & 2 & 63232 & 3.2 \\
\hline $80-84$ & 6 & 43162 & 13.9 \\
\hline $85+$ & 1 & 35395 & 2.8 \\
\hline TOTAL & & & 7.0 \\
\hline \multicolumn{4}{|c|}{ MALES-WHITE } \\
\hline $0-4$ & & 113129 & \\
\hline $5-9$ & & 114546 & \\
\hline $10-14$ & & 114909 & \\
\hline $15-19$ & & 129987 & \\
\hline $20-24$ & 1 & 142756 & 0.7 \\
\hline $25-29$ & 1 & 139636 & 0.7 \\
\hline $30-34$ & 5 & 143656 & 3.5 \\
\hline $35-39$ & 2 & 141064 & 1.4 \\
\hline $40-44$ & 3 & 132540 & 2.3 \\
\hline $45-49$ & 6 & 112784 & 5.3 \\
\hline $50-54$ & 9 & 95706 & 9.4 \\
\hline $55-59$ & 4 & 83991 & 4.8 \\
\hline $60-64$ & 2 & 79721 & 2.5 \\
\hline $65-69$ & 2 & 71969 & 2.8 \\
\hline $70-74$ & 1 & 52719 & 1.9 \\
\hline $75-79$ & 4 & 36625 & 10.9 \\
\hline $80-84$ & 1 & 20208 & 4.9 \\
\hline $85+$ & 1 & 11458 & 8.7 \\
\hline TOTAL & 42 & $1,737,404$ & 2.4 \\
\hline
\end{tabular}

${ }^{\text {a }}$ Population figures are cumulative over 8 years (1988 to 1995).

${ }^{\mathrm{b}}$ per 100,000 per year. 
Table 10-A.6 Thyroid cancer incidence data (1988 to 1995) for white people residing in Tennessee counties other than Anderson, Roane, Loudon or Knox counties (Turri, 1998).

\begin{tabular}{|c|c|c|c|}
\hline \multicolumn{4}{|c|}{ FEMALES-WHITE } \\
\hline Age & Cases & Population $^{\mathrm{a}}$ & Age-specific rate ${ }^{b}$ \\
\hline $0-4$ & 1 & 921668 & 0.1 \\
\hline $5-9$ & 1 & 946731 & 0.1 \\
\hline $10-14$ & 5 & 953353 & 0.5 \\
\hline $15-19$ & 32 & 1012019 & 3.2 \\
\hline $20-24$ & 42 & 1048943 & 4.0 \\
\hline $25-29$ & 61 & 1168854 & 5.2 \\
\hline $30-34$ & 121 & 1195434 & 10.1 \\
\hline $35-39$ & 118 & 1171157 & 10.1 \\
\hline $40-44$ & 117 & 1119639 & 10.4 \\
\hline $45-49$ & 98 & 952485 & 10.3 \\
\hline $50-54$ & 81 & 834833 & 9.7 \\
\hline $55-59$ & 71 & 769501 & 9.2 \\
\hline $60-64$ & 62 & 732264 & 8.7 \\
\hline $65-69$ & 75 & 701490 & 10.7 \\
\hline $70-74$ & 4 & 584951 & 7.5 \\
\hline $75-79$ & 42 & 493026 & 8.5 \\
\hline $80-84$ & 36 & 342426 & 10.5 \\
\hline $85+$ & 22 & 288609 & 7.6 \\
\hline \multirow{2}{*}{\multicolumn{4}{|c|}{$\begin{array}{c}\mathbf{1 0 3 1} \mathbf{1 5 , 2 3 7 , 3 8 3} \\
\text { MALES-WHITE }\end{array}$}} \\
\hline & & & \\
\hline $0-4$ & 1 & 970233 & 0.1 \\
\hline $5-9$ & 1 & 1002980 & 0.1 \\
\hline $10-14$ & 2 & 1008557 & 0.2 \\
\hline $15-19$ & 1 & 1083380 & 0.1 \\
\hline $20-24$ & 6 & 1062060 & 0.6 \\
\hline $25-29$ & 26 & 1162116 & 2.2 \\
\hline $30-34$ & 32 & 1168891 & 2.7 \\
\hline $35-39$ & 29 & 1148567 & 2.5 \\
\hline $40-44$ & 29 & 1096565 & 2.6 \\
\hline $45-49$ & 31 & 930833 & 3.3 \\
\hline $50-54$ & 37 & 795499 & 4.7 \\
\hline $55-59$ & 27 & 704916 & 3.8 \\
\hline $60-64$ & 29 & 639551 & 4.5 \\
\hline $65-69$ & 26 & 566395 & 4.6 \\
\hline $70-74$ & 21 & 424012 & 5 \\
\hline $75-79$ & 16 & 303466 & 5.3 \\
\hline $80-84$ & $\varepsilon$ & 170431 & 4.7 \\
\hline $85+$ & 5 & 105396 & 4.7 \\
\hline TOTAL & 327 & $14,343,848$ & 2.3 \\
\hline
\end{tabular}

${ }^{a}$ Population figures are cumulative over 8 years (1988 to 1995).

${ }^{\mathrm{b}}$ per 100,000 per year. 
TASK 1 REPORT

July 1999

${ }^{131}$ I Releases from X-10 Radioactive Lanthanum Processing -

Page 10A-10

Thyroid Cancer Incidence Data

Table 10-A.7 Thyroid cancer incidence data (1988 to 1995) for people of other races than white residing in the entire state of Tennessee (Turri, 1998).

\begin{tabular}{|c|c|c|c|}
\hline \multicolumn{4}{|c|}{ FEMALES - Other races } \\
\hline Age & Cases & Population $^{\mathrm{a}}$ & Age-specific rate \\
\hline $0-4$ & & 313734 & \\
\hline $5-9$ & & 304959 & \\
\hline $10-14$ & & 298818 & \\
\hline $15-19$ & & 305770 & \\
\hline $20-24$ & & 304647 & \\
\hline $25-29$ & 2 & 312825 & 0.6 \\
\hline $30-34$ & 1 & 316674 & 0.3 \\
\hline $35-39$ & & 291444 & \\
\hline $40-44$ & & 237545 & \\
\hline $45-49$ & 2 & 180634 & 1.1 \\
\hline $50-54$ & 1 & 149937 & 0.7 \\
\hline $55-59$ & 1 & 129239 & 0.8 \\
\hline $60-64$ & & 118896 & \\
\hline $65-69$ & 2 & 110794 & 1.8 \\
\hline 70-74 & & 91592 & \\
\hline $75-79$ & & 77491 & \\
\hline $80-84$ & & 52585 & \\
\hline $85+$ & 1 & 44427 & 2.3 \\
\hline TOTAL & 10 & $3,642,011$ & 0.3 \\
\hline \multicolumn{4}{|c|}{ MALES - Other races } \\
\hline $0-4$ & & 323967 & \\
\hline $5-9$ & & 315648 & \\
\hline $10-14$ & & 307944 & \\
\hline $15-19$ & & 311372 & \\
\hline $20-24$ & 1 & 282090 & 0.4 \\
\hline $25-29$ & & 270716 & \\
\hline $30-34$ & & 257074 & \\
\hline $35-39$ & & 234514 & \\
\hline $40-44$ & 2 & 193183 & 1 \\
\hline $45-49$ & & 143117 & \\
\hline $50-54$ & 1 & 115431 & 0.9 \\
\hline $55-59$ & & 93533 & \\
\hline $60-64$ & & 84266 & \\
\hline $65-69$ & & 75799 & \\
\hline $70-74$ & 1 & 59182 & 1.7 \\
\hline $75-79$ & & 45642 & \\
\hline $80-84$ & & 28430 & \\
\hline $85+$ & 1 & 20612 & 4.9 \\
\hline TOTAL & 6 & $3,162,520$ & 0.2 \\
\hline
\end{tabular}

${ }^{\text {a }}$ Population figures are cumulative over 8 years (1988 to 1995).

${ }^{\mathrm{b}}$ per 100,000 per year. 
Table 10-A.8 Thyroid cancer incidence data (1988 to 1995) for people of other races than white residing in Anderson, Roane, Loudon or Knox counties in Tennessee (Turri, 1998).

\begin{tabular}{|c|c|c|c|}
\hline \multicolumn{4}{|c|}{ FEMALES - Other races } \\
\hline Age & Cases & Population $^{\mathrm{a}}$ & Age-specific rate \\
\hline $0-4$ & & 13849 & \\
\hline $5-9$ & & 14436 & \\
\hline $10-14$ & & 13596 & \\
\hline $15-19$ & & 15651 & \\
\hline $20-24$ & & 16179 & \\
\hline $25-29$ & 1 & 14987 & 6.7 \\
\hline $30-34$ & & 15555 & \\
\hline $35-39$ & & 14242 & \\
\hline $40-44$ & & 12126 & \\
\hline $45-49$ & 1 & 9122 & 11 \\
\hline $50-54$ & & 7666 & \\
\hline $55-59$ & & 6205 & \\
\hline $60-64$ & & 6206 & \\
\hline $65-69$ & & 5840 & \\
\hline $70-74$ & & 4951 & \\
\hline $75-79$ & & 4228 & \\
\hline $80-84$ & & 2933 & \\
\hline $85+$ & & 2157 & \\
\hline TOTAL & 2 & 179,929 & 1.1 \\
\hline \multicolumn{4}{|c|}{ MALES - Other races } \\
\hline $0-4$ & & 14100 & \\
\hline $5-9$ & & 14468 & \\
\hline $10-14$ & & 14278 & \\
\hline $15-19$ & & 16086 & \\
\hline $20-24$ & & 15713 & \\
\hline $25-29$ & & 12693 & \\
\hline $30-34$ & & 12748 & \\
\hline $35-39$ & & 11152 & \\
\hline $40-44$ & 1 & 9364 & 10.7 \\
\hline $45-49$ & & 7800 & \\
\hline $50-54$ & & 5724 & \\
\hline $55-59$ & & 4827 & \\
\hline $60-64$ & & 4140 & \\
\hline $65-69$ & & 3896 & \\
\hline $70-74$ & & 2912 & \\
\hline $75-79$ & & 2320 & \\
\hline $80-84$ & & 1435 & \\
\hline $85+$ & 1 & 976 & 102.5 \\
\hline TOTAL & 2 & 154,632 & 1.3 \\
\hline
\end{tabular}

${ }^{\text {a }}$ Population figures are cumulative over 8 years (1988 to 1995).

${ }^{\mathrm{b}}$ per 100,000 per year. 
TASK 1 REPORT

July 1999

${ }^{131}$ I Releases from X-10 Radioactive Lanthanum Processing -

Page 10A-12

Thyroid Cancer Incidence Data

Table 10-A.9 Thyroid cancer incidence data (1988 to 1995) for people of other races than white residing in Tennessee counties other than Anderson, Roane, Loudon or Knox counties (Turri, 1998).

\begin{tabular}{|c|c|c|c|}
\hline \multicolumn{4}{|c|}{ FEMALES - Other races } \\
\hline Age & Cases & Population $^{\mathrm{a}}$ & Age-specific rate ${ }^{b}$ \\
\hline $0-4$ & & 299885 & \\
\hline $5-9$ & & 290523 & \\
\hline $10-14$ & & 285222 & \\
\hline $15-19$ & & 290119 & \\
\hline $20-24$ & & 288468 & \\
\hline $25-29$ & 1 & 297838 & 0.3 \\
\hline $30-34$ & 1 & 301119 & 0.3 \\
\hline $35-39$ & & 277202 & \\
\hline $40-44$ & & 225419 & \\
\hline $45-49$ & 1 & 171512 & 0.6 \\
\hline $50-54$ & 1 & 142271 & 0.7 \\
\hline $55-59$ & 1 & 123034 & 0.8 \\
\hline $60-64$ & & 112690 & \\
\hline $65-69$ & 2 & 104954 & 1.9 \\
\hline $70-74$ & & 86641 & \\
\hline $75-79$ & & 73263 & \\
\hline $80-84$ & & 49652 & \\
\hline $85+$ & 1 & 42270 & 2.4 \\
\hline TOTAL & 8 & $3,462,082$ & 0.2 \\
\hline \multicolumn{4}{|c|}{ MALES - Other races } \\
\hline $0-4$ & & 309867 & \\
\hline $5-9$ & & 301180 & \\
\hline $10-14$ & & 293666 & \\
\hline $15-19$ & & 295286 & \\
\hline $20-24$ & 1 & 266377 & 0.4 \\
\hline $25-29$ & & 258023 & \\
\hline $30-34$ & & 244326 & \\
\hline $35-39$ & & 223362 & \\
\hline $40-44$ & 1 & 183819 & 0.5 \\
\hline $45-49$ & & 135317 & \\
\hline $50-54$ & 1 & 109707 & 0.9 \\
\hline $55-59$ & & 88706 & \\
\hline $60-64$ & & 80126 & \\
\hline $65-69$ & & 71903 & \\
\hline $70-74$ & 1 & 56270 & 1.8 \\
\hline $75-79$ & & 43322 & \\
\hline $80-84$ & & 26995 & \\
\hline $85+$ & & 19636 & \\
\hline TOTAL & 4 & $\mathbf{3 , 0 0 7 , 8 8 8}$ & 0.1 \\
\hline
\end{tabular}

${ }^{\text {a }}$ Population figures are cumulative over 8 years (1988 to 1995).

${ }^{\mathrm{b}}$ per 100,000 per year. 
The differences in the risk estimates between the ethnic groups of African-Americans and European-Americans may be due to possible different dose-response relationships, as well as to different background incidence rates of thyroid cancer (Equation 10.1). To date, there is no established radiation dose - thyroid cancer relationship for African-Americans. If the available dose-response relationship (Ron et al., 1995) is valid for both blacks and whites, the differences in the risk estimates will be due only to the differences in the background incidence rates.

\section{A.2 Derivation of the lifetime "background" risk of thyroid cancer incidence}

An individual of age " $i$ " has a baseline risk of getting thyroid cancer during his or her remaining life. This baseline risk is called "lifetime background risk," and it depends on the age " $i$," and on the life expectancy of the individual. In this study, the exposed individual is an average individual having an average lifetime of 70 years. Also, by definition, the individual has no thyroid cancer at age "i."

The lifetime background risk of thyroid cancer for an individual can be estimated as follows:

\section{$\underline{\text { In the case of exposure to radiation at age " } i "}$}

Let $R_{o, k}$ be the incidence rate of thyroid cancer for a nonirradiated population of age $k$ (new cases per 100,000 per year); this quantity is known as the background risk of thyroid cancer. Let $R_{l, k, i}$ be the excess incidence rate of thyroid cancer for a population of age $k$ exposed to a dose $D$ at age $i$; that is, $R_{l, k, i}$ represents the excess incidence rate produced by the radiation only (new cases per 100,000 per year). The total incidence rate of thyroid cancer $R_{k}$ for a population of age $k$ is given by

$$
R_{k}=R_{o, k}+R_{l, k, i}
$$

For an individual exposed to a dose $D$ at age $i$, the probability of surviving through age $k$ without a thyroid cancer can be expressed as:

$$
L_{k}=L_{k-1} \cdot\left(1-R_{k} \cdot \Delta t\right) \quad k=i+1, i+2, \ldots
$$

To attain age $k$ without acquiring a thyroid cancer means attaining age $k-1$ with no thyroid cancer $\left(L_{k-1}\right)$ and getting no cancer in year $k$. The individual is assumed to have had no thyroid cancer before the age $i$ when exposure takes place, thus $L_{i}=1$. The time step for which incidence of thyroid cancer is detected is $\Delta \mathrm{t}=1 \mathrm{yr}$.

For an individual exposed to a dose $D$ at age $i$, the probability of acquiring a thyroid cancer at age $k$, for any reason, can be expressed as

$$
T_{k}=L_{k-1} \cdot\left(R_{k} \cdot \Delta t\right) \quad k=i+1, i+2, \ldots
$$

Moreover, for the same individual, the probability of acquiring a radiation-induced thyroid cancer at age $k$ is:

$$
T R I_{k}=L_{k-1} \cdot\left(R_{l, k, i} \cdot \Delta t\right) \quad k=i+1, i+2, \ldots
$$


Thus, the lifetime probability of acquiring a radiation-induced thyroid cancer (or the excess lifetime risk $(E L R)$ of thyroid cancer for an individual exposed to a dose $D$ at age $i$ ) can be written as

$$
\begin{aligned}
\operatorname{ELR}\left(D_{i}\right) & =\sum_{k=i}^{\max a g e} L_{k-1} \cdot\left(R_{1, k, i} \cdot \Delta t\right) \\
& =\sum_{k=i}^{\max a g e}\left\{1 \cdot\left[1-R_{1} \cdot \Delta t\right] \cdot\left[1-R_{2} \cdot \Delta t\right] \cdot \ldots \cdot\left[1-R_{k-1} \cdot \Delta t\right]\right\} \cdot\left(R_{1, k, i} \cdot \Delta t\right)
\end{aligned}
$$

The risk of acquiring thyroid cancer from exposure to a radiation dose $D$ can be expressed by a linear relative model (Ron et al., 1995)

$$
R=R_{0} \cdot h=R_{0} \cdot(1+\beta \cdot D)
$$

where

$$
\begin{array}{lll}
R & = & \text { total risk; } \\
R_{0} & = & \text { background risk; } \\
h & = & \text { dose-response function; } \\
\beta & = & \text { excess relative risk per unit dose; and } \\
D & = & \text { radiation dose }
\end{array}
$$

Using this model, one can identify

$$
\begin{aligned}
& R-R_{0}=\text { the excess (absolute) risk (EAR) } \\
& \left(R-R_{0}\right) / R_{0}=\text { the excess relative risk (ERR) } \\
& \beta=\left(R-R_{0}\right) /\left(R_{0} D\right)=\text { the excess relative risk per unit dose. }
\end{aligned}
$$

Further, assuming a linear dependence of the dose-response function, the incidence rate of radiation-induced thyroid cancer can be written as:

$$
\begin{aligned}
R_{l, k, i} & =0 & & \text { for } i \leq k<i+5 \\
& =R_{o, k} \cdot \beta_{i} \cdot D_{i} & & \text { for } k \geq i+5
\end{aligned}
$$

This expression is based on experimental evidence (Ron et al., 1995) suggesting that (a) there is no observed effect in the first 5 years after the exposure, and (b) the radiation-induced thyroid cancer rate is proportional to the exposure dose $(D)$ and to the background rate $\left(R_{o, k}\right)$ at the age of interest $(k)$. The proportionality constant $(\beta)$ is assumed to depend only on the age at exposure (i). 
The excess lifetime risk (ELR) of thyroid cancer after an exposure at age $i$ can be computed using Equation 10-A.1. The terms $\left(1-R_{k} \Delta t\right)$ are less than but very close to 1 , because the incidence rates $R_{k}$ are less than $10^{-2}$ per year. Thus, a good approximation for estimating $E L R$ is

$$
\begin{aligned}
\operatorname{ELR}\left(D_{i}\right) & \cong \sum_{k=i}^{\text {lifime }}\left(R_{1, k, i} \cdot \Delta t\right)=\sum_{k=i}^{\text {lifetime }}\left(R_{0, k} \cdot \beta_{i} \cdot D_{i} \cdot \Delta t\right) \\
& =\beta_{i} \cdot D_{i} \cdot \sum_{k=i}^{\text {lifetime }}\left(R_{0, k} \cdot \Delta t\right)=\beta_{i} \cdot D_{i} \cdot B_{i}
\end{aligned}
$$

where $B_{i}=$ the lifetime background risk of thyroid cancer from an exposure at age $i$.

Equation 10-A.2 will always indicate a larger risk than equation 10-A.1, but the relative difference is less than $10 \%$ for doses of about 500 rad, and less than $1 \%$ for doses of about 50 rad.

From Equation 10-A.2, the quantity $B_{i}$ (called "lifetime background risk of thyroid cancer") is calculated as

$$
B_{i}=\sum_{k=i}^{\text {lifetime }}\left(R_{0, k} \cdot \Delta t\right)
$$

where $R_{0, k} \quad=$ the age-specific thyroid cancer incidence rate for age "k."

In the absence of exposure to radiation

In this case, the lifetime risk of acquiring (thyroid) cancer for an individual at age $i$ is given by the probability of attaining age $i$ without contracting a cancer (or dying from some other cause), and by the probability of contracting cancer in the years following age $i$.

$$
\begin{aligned}
B_{i} & =\sum_{k=i}^{\max a g e} L_{k-1} \cdot\left(R_{k} \cdot \Delta t\right) \\
& =\sum_{k=i}^{\max a g e}\left\{1 \cdot\left[1-R_{1} \cdot \Delta t\right] \cdot\left[1-R_{2} \cdot \Delta t\right] \cdot \ldots\left[1-R_{k-1} \cdot \Delta t\right]\right\} \cdot\left(R_{k} \cdot \Delta t\right)
\end{aligned}
$$

Again, the terms $\left(1-R_{k} \Delta t\right)$ are less than but very close to 1 , because the incidence rates $R_{k}$ are less than $10^{-2}$ per year. Thus, an approximation of the lifetime risk of acquiring cancer from natural causes for an individual of age $i$ is

$$
B_{i}=\sum_{k=i}^{\text {lifetime }}\left(R_{k} \cdot \Delta t\right)=\sum_{k=i}^{\text {lifetime }}\left(R_{0, k} \cdot \Delta t\right)
$$


In this case, $R_{k}=R_{0, k}$, because no exposure to radiation occurred. Equations 10-A.3 and 10-A.5 are identical.

\section{A.3 Influence of ethnic background on the estimates of thyroid cancer risk}

The racial differences in the thyroid risk estimates (if any) are given by possible different doseresponse relationships and by different background incidence rates of thyroid cancer (Equation 10.1) between blacks and whites. To date, there is no dose-response relationship determined for thyroid cancer in blacks. Assuming that the available dose-response relationship (Ron et al., 1995) would apply the same to both blacks and whites, the differences in the risk estimates will be due only to the differences in the background incidence rates.

Data collected across the United States (Figure 10-A.1) show that blacks have a lower incidence of thyroid cancer than do whites. This difference seems to be more pronounced for people in the state of Tennessee. However, when analyzing the statistics for blacks in the state of Tennessee, one must keep in mind the very low number of observed cases between 1988 and 1995: 6 for males and 10 females over the entire state, including 4 for males and 8 for females in all counties in Tennessee other than Anderson, Roane, Loudon and Knox counties (Tables 10-A.4 through 10-A.9; Figure 10-A.2).

Because of the low number of thyroid cancer cases for blacks, a "lifetime" background incidence of cancer $\left(B_{i}\right)$, cannot be estimated. However, this quantity can be estimated for whites only, and then compared to the estimate for a population formed of all races (currently used to produce the risk estimates presented in this report). The estimates are made using the method presented in Section 10-A.2. The background for whites is larger than the background for all races combined by about 18 to $19 \%$ for females and by $14 \%$ for males. That is, if risk estimates were produced for the white population only, the current risk estimates would increase by $19 \%$ for females and by $14 \%$ for males.

Since the "lifetime" background incidence of cancer cannot be estimated for blacks, a rough estimate of the change in the risk estimate can be obtained using the total number of people in the area of interest (i.e., all counties in Tennessee other than Anderson, Roane, Loudon and Knox). For females, these numbers (summed from 1988 to 1995) are (a) 15,237,383 white females, (b) 3,462,082 black females, and (c) 18,699,465 females of all races. Given that the background for white females is larger by only $19 \%$ than the background for females all races $\left(4.61 \times 10^{-3}\right.$ versus $\left.3.88 \times 10^{-3}\right)$, the background for black females must be about 16 times lower than the background for females of all races combined. That is, the risk estimates for black females should be a factor of 16 lower than the risk estimates for females of all races reported in this study. 


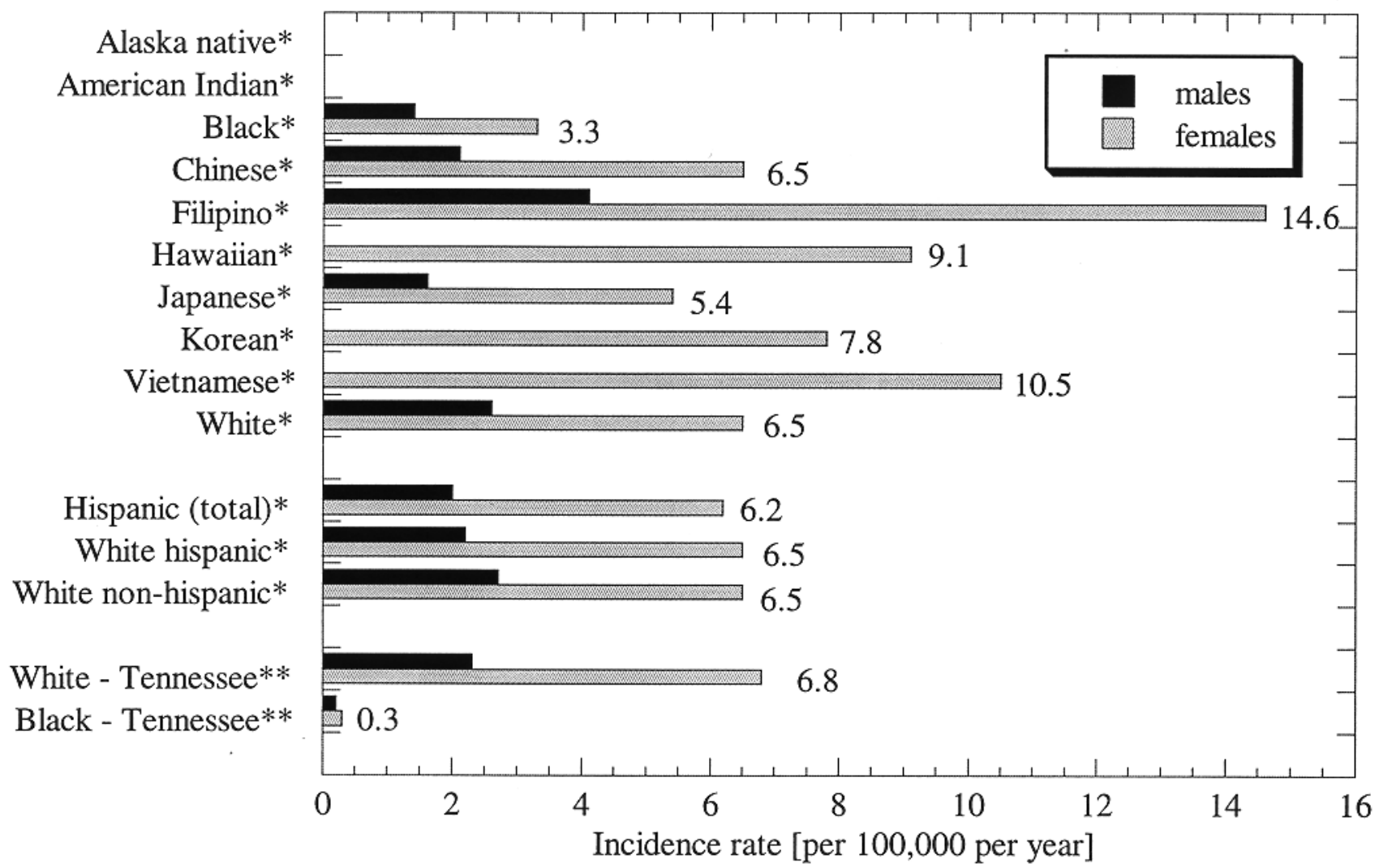

Figure 10-A.1 Influence of ethnic background on the incidence of thyroid cancer in the United States. Sources: (*) SEER program of the National Cancer Institute; Reporting period 1988 - 1992 (Miller et al., 1996); and (**) Tennessee Dept. of Health; Reporting period 1988-1995 (Turri, 1998). 

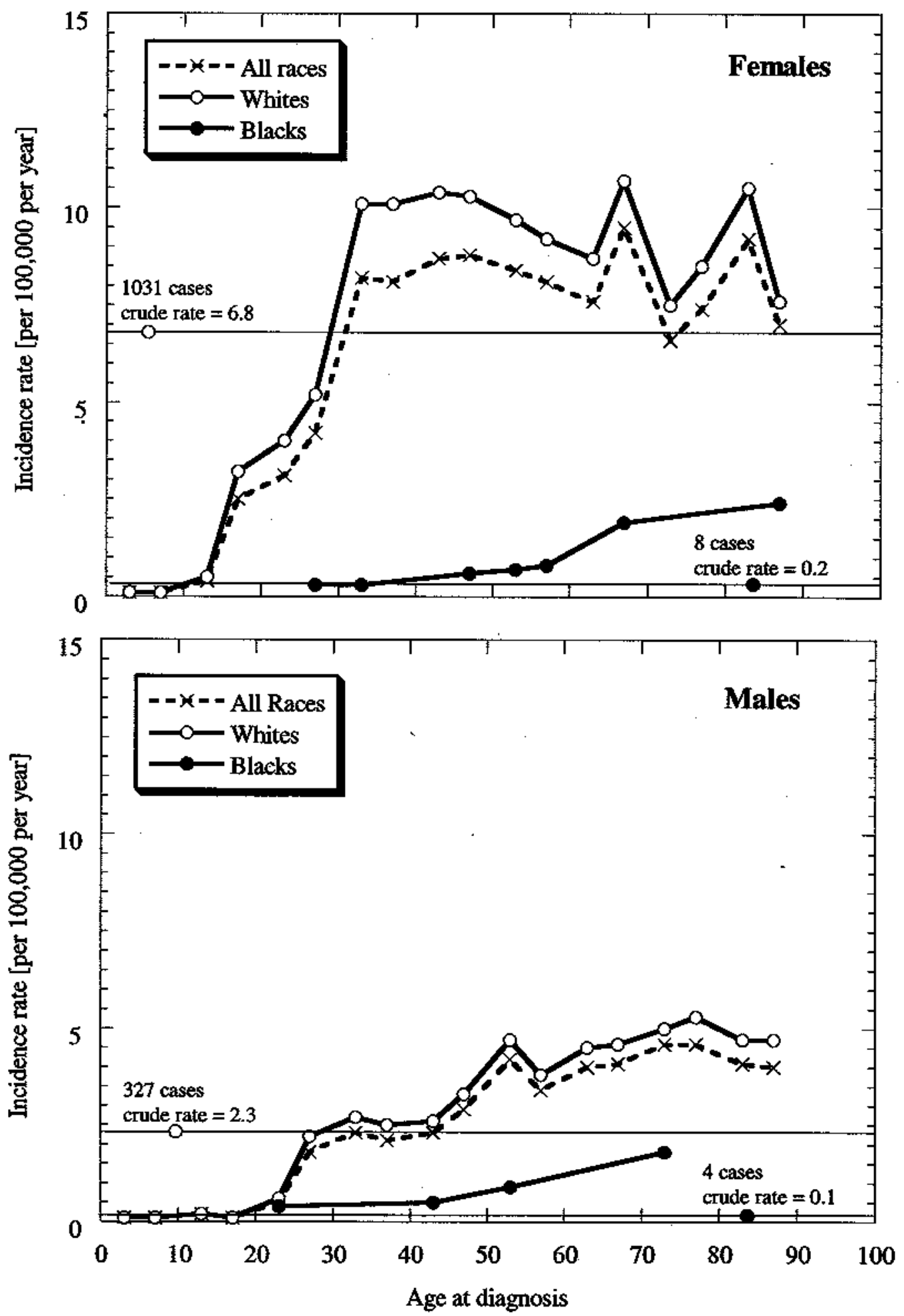

Figure 10-A.2 Thyroid cancer incidence rates for all Tennessee counties other than Anderson, Roane, Loudon and Knox counties. The reporting period is 1988 - 1995. Source: Tennessee Dept. of Health (Turri, 1998). 
Similarly, in the region of interest, there are (a) 14,343,848 white males, (b) 3,007,888 black males, and (c) 17,351,736 males of all races (population numbers are summed from 1988 to 1995). Given that the background for white males is larger by only $14 \%$ than the background for males of all races combined $\left(1.6 \times 10^{-3}\right.$ versus $\left.1.4 \times 10^{-3}\right)$, the background for black males must be about 28 times lower than the background for males of all races. That is, the risk estimates for black males should be a factor of 28 lower than the risk estimates for males of all races reported in this study.

\section{A.4 Effect on the risk estimates of the changes in background incidence of thyroid cancer over the last few decades}

In the state of Tennessee, a systematic collection of data on thyroid cancer incidence has been performed only since the late 1980s. However, the incidence of thyroid cancer has been followed up for many years in selected regions of the United States under the Surveillance, Epidemiology, and End Results (SEER) Program of the National Cancer Institute. Data have been collected on a routine basis from 9 designated population-based cancer registries since 1973. The nine "standard" registries are the states of Connecticut, Iowa, New Mexico, Utah, and Hawaii and the metropolitan areas of Detroit, San Francisco, Seattle-Puget Sound, and Atlanta.

These data show that the thyroid cancer incidence rates increase with time (Figure 10-A.3). During 1973-1995, the rate of increase is about $10.7 \%$ per year for white females, but only $0.3 \%$ for black women. For males the rate of increase is about $3.4 \%$ per year for whites and $1.5 \%$ per year for blacks. This finding is very important for the understanding of the differences between individuals of different ethnic backgrounds. If the increasing trend were due only to the improvement of health care and cancer reporting systems, then the trend should be about the same for both blacks and whites. The large difference between the increasing background rates is an indication that there may be true ethnic differences in cancer risk induction.

If the thyroid cancer incidence varies with time, the risk estimates produced in this study (which are based on 1988-1995 data) might be biased. Therefore, the effect of the variation in incidence rates must be analyzed. For simplicity, let us make the following assumptions:

a) the incidence rates change by a constant amount every year; that is, the rate of change is constant over time.

b) the same rate of change applies to all age groups. That is, if the incidence rate for age group $k$ is larger in one year than in the previous year by a certain amount, then this amount is the same for all age groups.

c) If, in a given year, the incidence rate for the age group $k$ is $R_{0, k}$, then the incidence rate for the same age group $n$ years later is $R_{0, k}(1+\alpha n)$, where $\alpha$ is the rate of change in the incidence rate [per year]. 

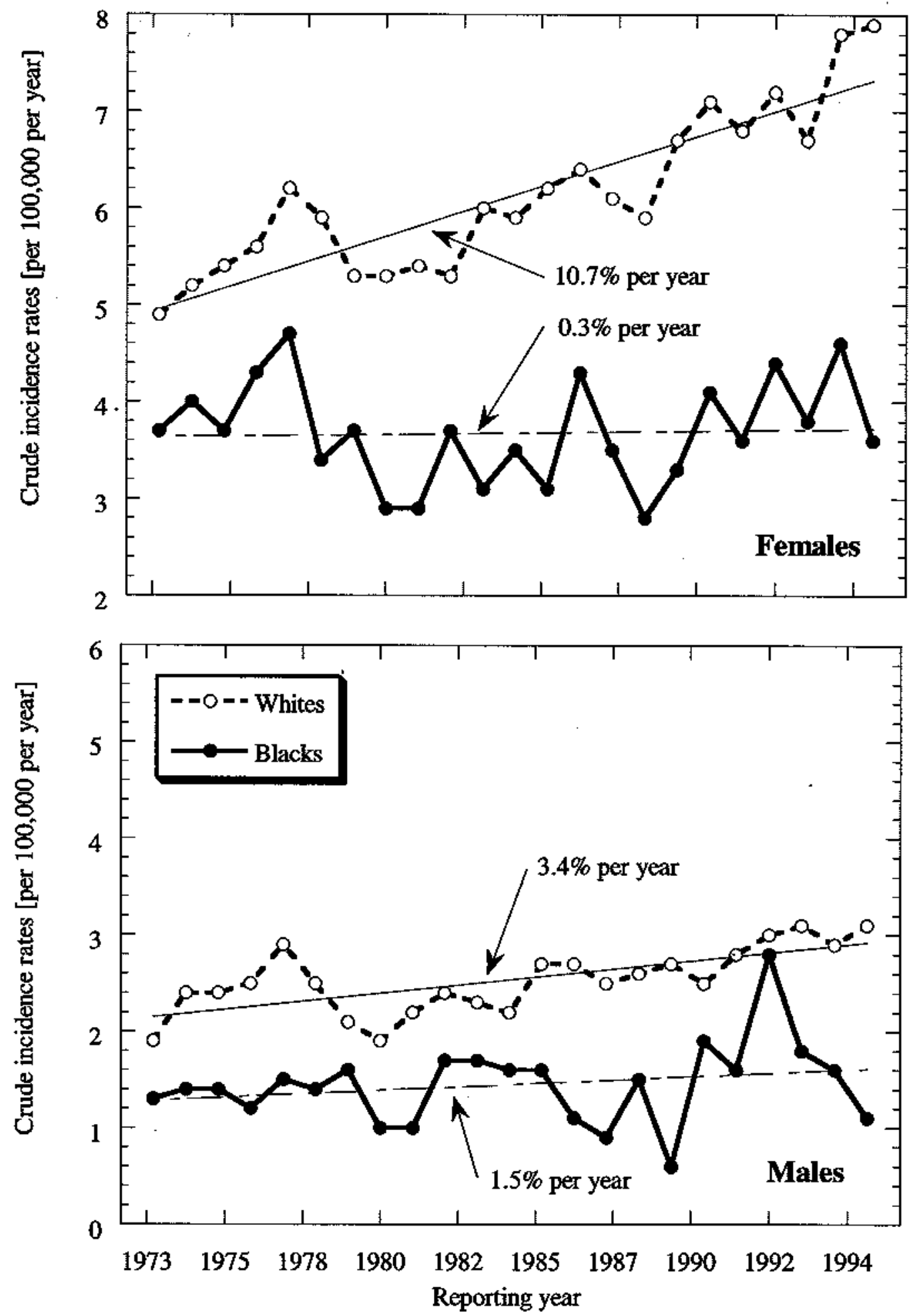

Figure 10-A.3 Increase in the thyroid cancer incidence rates in the United States as a function of time. Source: SEER program of the National Cancer Institute (Ries et al., 1998). 
Taking the above assumptions into account, the excess lifetime risk of thyroid cancer in Equation 10-A. 2 can be modified to

$$
\operatorname{ELR}\left(D_{i}\right) \cong \sum_{k=i}^{\text {lifime }}\left(R_{1, k, i} \cdot \Delta t\right)=\beta_{i} \cdot D_{i} \cdot \sum_{k=i+5}^{\text {lifetime }}\left(R_{0, k} \cdot(1+\alpha \cdot n) \cdot \Delta t\right)=\beta_{i} \cdot D_{i} \cdot B_{i} *
$$

and the new (adjusted) lifetime background risk of thyroid cancer $\left(B_{i}^{*}\right)$ will be given by:

$$
B_{i}^{*}=\sum_{k=i+5}^{\text {lifetime }}\left(R_{0, k} \cdot(1+\alpha \cdot n) \cdot \Delta t\right)=\sum_{k=i+5}^{\text {lifetime }}\left(R_{0, k} \cdot \Delta t\right)+\alpha \cdot \sum_{k=i+5}^{\text {lifetime }}\left(R_{0, k} \cdot n \cdot \Delta t\right)
$$

If an individual is exposed at age $i$, and he or she is of age $k$ at the moment of the data collection $(k>i)$, there are $(k-i)$ years that have passed from the moment of exposure to the moment of the data collection. If the incidence rate data $\left(R_{0, k}\right)$ are available for the year of exposure (corresponding to age $i$ of the individual), then the parameter $n$ in the previous equation should be set to $n=(k-i)$.

If the incidence rate data $\left(R_{0, k}\right)$ are available for another year (e.g., when the individual is of age $m, m>k>i)$, then parameter $n$ in the previous equation must be set to $n=((m-i)-k)>0$; that is, $n^{1}$ must be set back to estimate the incidence rate in the year when the individual was of age $i$.

In this study, the incidence rates of thyroid cancer are available for the 1988-1995 reporting period (Turri, 1998). Exposures to ${ }^{131}$ I released from X-10 took place from 1944 to 1956. Thus, there is a difference $(m-i)$ of about 40 years from the exposure period to the period when the incidence rates are available.

The rate of change $(\alpha)$ in the incidence rates is unknown for the 1950 - 1990 period. Rates of change $(\alpha)$ are available for 1973 - 1995 period: $10.7 \% \mathrm{y}^{1}$ for white females, $0.3 \% \mathrm{y}^{1}$ for black females, $3.4 \%$ for white males, and $1.5 \%$ for black males (Figure 10-A.3).

The estimates of the excess lifetime risk in the case of a constant background $\left(B_{i}\right)$ were then compared to the estimates of the excess lifetime risk for the case when background is changing $\left(B_{i}{ }^{*}\right)$. For the latter case, the rate of change in the background $(\alpha)$ was allowed to vary between $0.3 \% \mathrm{y}^{1}$ and $10.7 \% \mathrm{y}^{1}$. In both cases, the background cancer incidence rates for females in all Tennessee counties other than Anderson, Roane, Loudon or Knox counties (Table 10-A.2) were used as a starting point. The comparison is performed in a relative manner: $\left[\left(B_{i}{ }^{*}-B_{i}\right) /\left(B_{i}\right)\right]$, expressed in percent.

The results of this analysis are presented in Table 10-A.10. The table should be read in the following manner: If the rate of change in the background incidence of cancer was $0.3 \% \mathrm{y}^{-1}$ for the entire period from 1950 to 1990, then the current excess lifetime risk estimates will decrease by $0.1 \%$ for an individual born in 1950 , by $0.3 \%$ for an individual of age 10 in 1950 , and so on.

\footnotetext{
${ }^{1}$ Note that, in the actual calculation, parameter $n$ is taken to be negative, since the incidence rates are calculated at a past moment in time, when they should be lower.
} 
Table 10-A.10 The effects of change with time of the background incidence of cancer on the current estimates of the excess lifetime risk of thyroid cancer.

\begin{tabular}{cccccc}
\hline & & \multicolumn{5}{c}{ Relative change [\%] } \\
\hline year of birth & $\begin{array}{c}\text { age at exposure } \\
\text { (in 1950) }\end{array}$ & \multicolumn{4}{c}{$\begin{array}{c}\text { Variation rate in the background } \\
\text { [\% per year] }\end{array}$} \\
\hline 1950 & 0 & $-0.30 \%$ & $1.5 \%$ & $3.4 \%$ & $10.7 \%$ \\
1940 & 10 & $-0.1 \%$ & $-0.2 \%$ & $0.3 \%$ & $2.4 \%$ \\
1930 & 20 & $-0.5 \%$ & $-2.5 \%$ & $-5.6 \%$ & $-17.5 \%$ \\
\hline
\end{tabular}

By implementing the variation with time of the background cancer incidence, the changes in the risk estimates will be very small $(<5 \%)$ in most cases. For the case when the rate of change in the incidence rate is $3.4 \%$ and is constant for 60 years, the estimated risks will decrease by $5.6 \%$. When the rate of change in the incidence rate was set to $10.7 \%$, the estimated bias in the risk estimate increased up to $18 \%$. However, a rate of $10.7 \%$ per year was observed for white females only, and it does not apply for mixed races or for males. Also, it is unlikely that the rate of change is $10.7 \%$ for every year, because, starting with the incidence rates in the 1990s, the estimated incidence rates for the 1940s would approach zero.

Therefore, we conclude that although the incidence of thyroid cancer increases with time, this increasing trend has practically no effect on the risk estimates based on the data for 1988-1995. An explanation for this behavior is illustrated in Figure 10-A.4. Our lifetime risk estimates are produced assuming an average life expectancy of 70 years. A person born in 1950 will be 40 years of age in 1990, and thus will have, on average, 30 more years to live (until year 2020). Assuming that the incidence rate of thyroid cancer increased continuously for the entire period between 1950 and 2020, the value of the incidence rate measured in 1990 will overestimate the values for the 1950-1990 period, but will underestimate the values for 1990-2020 period. Thus, the overall bias in the estimates of total lifetime risk is negligible. If, for instance, the incidence rate were measured in year 2010, and if the same trend is maintained, then a significant overestimate of risk would be obtained by using those incidence rates.

In conclusion, in the risk estimates produced in this study, it is not necessary to account explicitly for the variation with time of the incidence of thyroid cancer. 


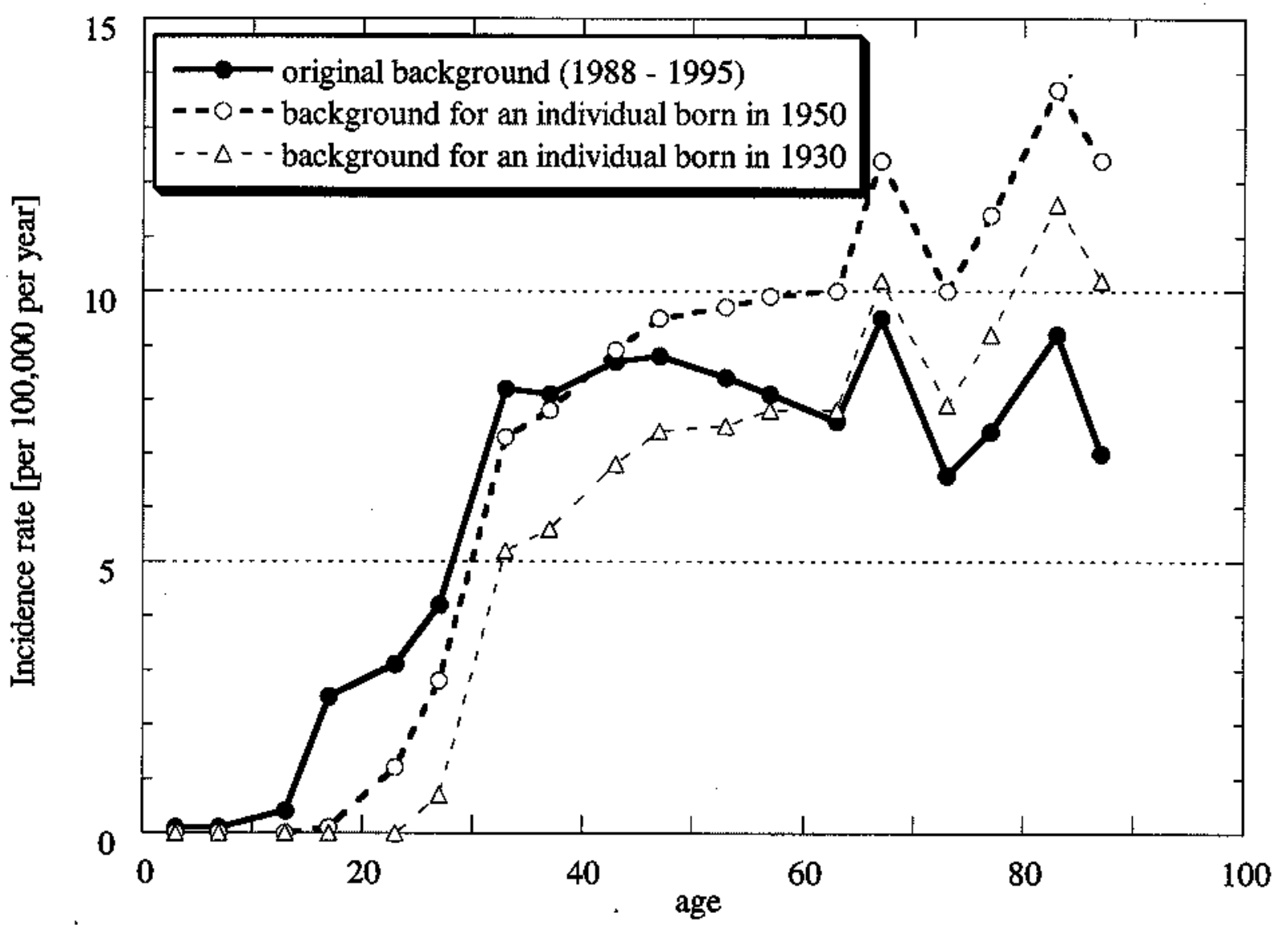

Figure 10-A.4 Adjustment of the background incidence rates for the effect of variation with time of the incidence rates of thyroid cancer. A variation in the incidence rates of $10.7 \%$ per year was assumed for all years. 
10A.5 Correction of the lifetime "background" risk of thyroid cancer incidence for the contribution from the Nevada Test Site Fallout

The problem of correcting the cancer incidence rate observed in the 1990s for the contribution from cases induced by NTS fallout is conceptually simple. Assuming a linear relative model, the incidence rate measured in the 1990s is

$$
R=R_{0} \cdot h=R_{0} \cdot(1+\beta \cdot D),
$$

where

$R=$ the cancer incidence rate including the contribution from cases induced by the NTS fallout (i.e., the current observed values presented in Section 10-A.1);

$R_{0}=$ the cancer incidence rate in the absence of NTS fallout;

$h=$ the dose-response function;

$\beta=$ the excess relative risk per unit dose for ${ }^{131} \mathrm{I}$; and

$D=$ the radiation dose from NTS fallout.

To correct for the contribution of NTS fallout to the background incidence of cancer as observed in the $1990 \mathrm{~s}$, one must determine $R_{0}$ from the previous equation. That is,

$$
R_{0}=\frac{R_{1}}{(1+\beta \cdot D)}
$$

However, the problem becomes very complicated because NTS fallout occurred over 8 years (1951 to 1958), and the 1990s incidence rates were also collected over 8 years (1988 to 1995). Given this situation, one must take into account the age dependency of the excess relative risk per unit dose $(\beta)$ and the year in which the exposed individual was born.

Individuals of certain ages diagnosed in any year between 1988-1995 have an increased risk of thyroid cancer from exposure to NTS fallout. This increased risk can be estimated in terms of an excess relative risk $(E R R)$ from the thyroid doses received in the 1950s due to NTS fallout.

$$
\operatorname{ERR}_{\left(\text {age }_{1}, \text { year }\right)}=\sum_{\text {ibirth }=1935}^{1958} \sum_{\text {ifallou } \in 1951}^{1958}\left(\frac{E R R_{1 S v}\left(\text { age }_{2}\right)}{\delta}\right) \cdot \varepsilon\left(\text { age }_{2}\right) \cdot D(\text { ib, ir })
$$

where

year $=$ year when cancer incidence is detected (1988 through 1995);

age $_{1}=$ age in the year of incidence detection (it varies from 30 to 60 years);

ibirth $=$ year of birth for people exposed as children to NTS fallout (1935-1958); 


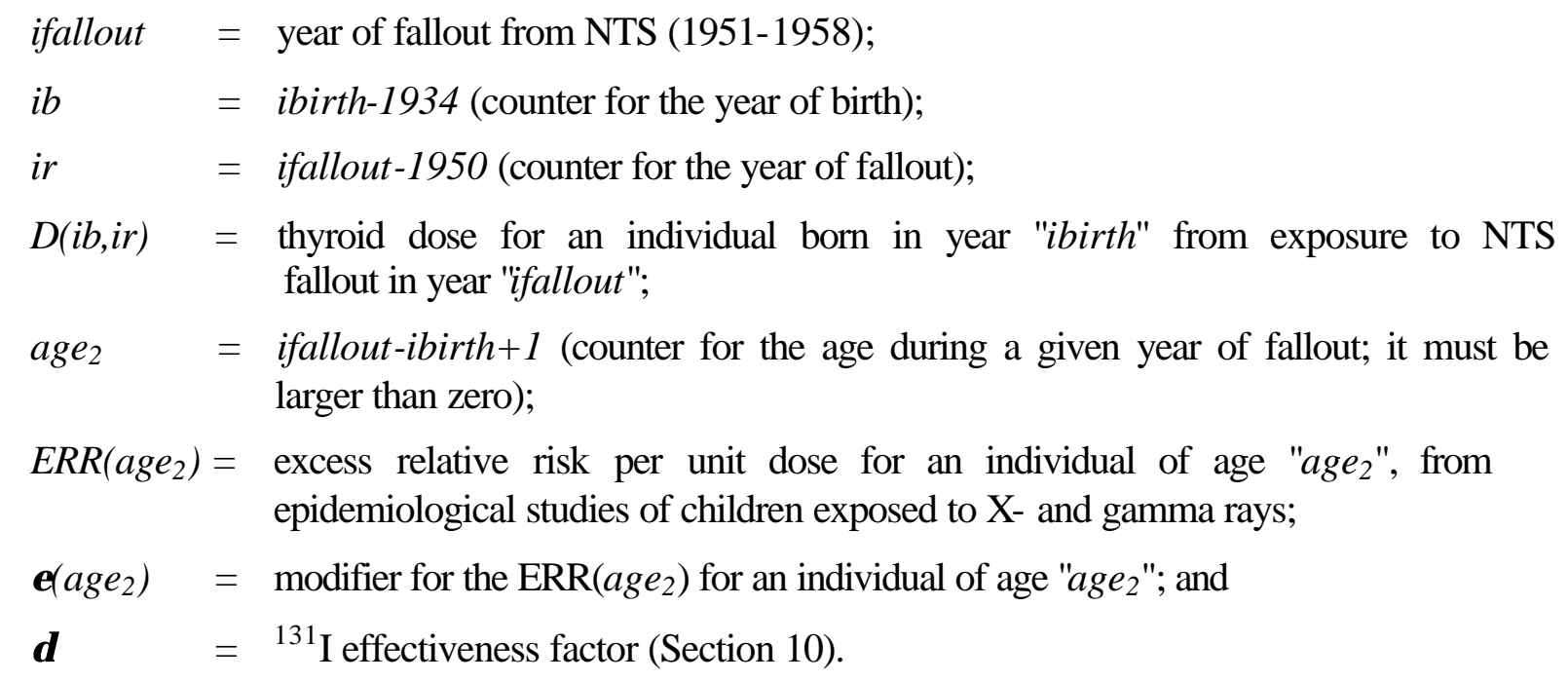

Averaging over all years of detection ( 8 years) and over all ages in an age group of the epidemiological data (5 years), one obtains

$$
\operatorname{ERR}\left(\text { age }_{1}\right)=\frac{1}{8} \sum_{\text {year }=1988}^{1995} \operatorname{ERR}\left(\text { age }_{1}, \text { year }\right)
$$

and

$$
R R(\text { age group })=1+\frac{1}{5} \sum_{\text {age }_{1}}^{\operatorname{age}_{1}+5} \operatorname{ERR}\left(\text { age }_{1}\right)
$$

The corrected incidence rate $(C I R)$ for a given age group is determined as a function of the observed incidence rate $(I R)$ :

$$
C I R(\text { age group })=I R(\text { age group }) / R R(\text { age group })
$$

This approach was applied for the females exposed during 1951-1958. The estimated thyroid doses received by females living in Anderson County, Tennessee, who were on an average diet of milk are presented in Table 10-A.11. The measured (Table 10-A.3) and the corrected thyroid cancer incidence rates are presented in Table 10-A.12 and in Figure 10-A.5.

The corrected incidence rates must then be integrated by using Equation 10-A.3 to obtain a corrected "lifetime" background incidence of thyroid cancer. The overall impact in the risk estimates is obtained by comparing the original and the corrected lifetime background of thyroid cancer incidence (Figures 10-A.5 and 10-A.6).

The difference between the two lifetime backgrounds is less than 6\% (Figures 10-A.5 and 10A.6; Table 10-A.12). That is, the contribution of NTS fallout in changing the background incidence of cancer produces a negligible impact on the lifetime risk estimates produced in this study. 
Table 10-A.11 Thyroid doses from ${ }^{131} \mathrm{I}$ in Nevada Test Site fallout for females living in Anderson Co., Tennessee, who had average consumption of cow's milk (NCI, 1997).

\begin{tabular}{|c|c|c|c|c|c|c|c|c|c|}
\hline \multirow[b]{2}{*}{ Nuclear Test Series } & \multirow{2}{*}{$\begin{array}{l}\text { year of } \\
\text { release }\end{array}$} & \multicolumn{8}{|c|}{ year of birth } \\
\hline & & 1935 & 1936 & 1937 & 1938 & 1939 & 1940 & 1941 & 1942 \\
\hline Ranger (28 Jan 1951 - 6 Feb 1951) and Buster-Jangle (28 Oct. 1951 - 29 Nov. 1951) & 1951 & 0.015 & 0.015 & 0.02 & 0.02 & 0.02 & 0.02 & 0.02 & 0.026 \\
\hline Tumbler-Snapper (1 Apr. 1952 - 5 Jun 1952) & 1952 & 0.33 & 0.33 & 0.33 & 0.45 & 0.45 & 0.45 & 0.45 & 0.45 \\
\hline Upshot-Knothole (15 Mar 1953 - 4 Jun 1953) & 1953 & 0.37 & 0.37 & 0.37 & 0.37 & 0.5 & 0.5 & 0.5 & 0.5 \\
\hline No Tests & 1954 & 0.0 & 0.0 & 0.0 & 0.0 & 0.0 & 0.0 & 0.0 & 0.0 \\
\hline Teapot (18 Feb 1955 - 15 May 1955) & 1955 & 0.14 & 0.35 & 0.35 & 0.35 & 0.35 & 0.35 & 0.46 & 0.46 \\
\hline No Tests & 1956 & 0.0 & 0.0 & 0.0 & 0.0 & 0.0 & 0.0 & 0.0 & 0.0 \\
\hline Plumbbob (28 May 1957 - 7 Oct 1957) & 1957 & 0.2 & 0.2 & 0.2 & 0.47 & 0.47 & 0.47 & 0.47 & 0.47 \\
\hline Hardtrack Phase II (19 Sep 1958 - 26 Oct 1958) & 1958 & 0.0 & 0.0 & 0.0 & 0.0 & 0.0 & 0.0 & 0.0 & 0.0 \\
\hline Nuclear Test Series & & 1943 & 1944 & 1945 & 1946 & 1947 & 1948 & 1949 & 1950 \\
\hline Ranger (28 Jan 1951 - 6 Feb 1951) and Buster-Jangle (28 Oct. 1951 - 29 Nov. 1951) & 1951 & 0.026 & 0.026 & 0.026 & 0.026 & 0.026 & 0.036 & 0.036 & 0.036 \\
\hline Tumbler-Snapper (1 Apr. 1952 - 5 Jun 1952) & 1952 & 0.66 & 0.66 & 0.66 & 0.66 & 0.66 & 0.66 & 0.94 & 0.94 \\
\hline Upshot-Knothole (15 Mar 1953 - 4 Jun 1953) & 1953 & 0.5 & 0.7 & 0.7 & 0.7 & 0.7 & 0.7 & 0.7 & 1.0 \\
\hline No Tests & 1954 & 0.0 & 0.0 & 0.0 & 0.0 & 0.0 & 0.0 & 0.0 & 0.0 \\
\hline Teapot (18 Feb 1955 - 15 May 1955) & 1955 & 0.46 & 0.46 & 0.46 & 0.66 & 0.66 & 0.66 & 0.66 & 0.66 \\
\hline No Tests & 1956 & 0.0 & 0.0 & 0.0 & 0.0 & 0.0 & 0.0 & 0.0 & 0.0 \\
\hline Plumbbob (28 May 1957 - 7 Oct 1957) & 1957 & 0.64 & 0.64 & 0.64 & 0.64 & 0.64 & 0.9 & 0.9 & 0.9 \\
\hline Hardtrack Phase II (19 Sep 1958 - 26 Oct 1958) & 1958 & 0.0 & 0.0 & 0.0 & 0.0 & 0.0 & 0.0 & 0.0 & 0.0 \\
\hline
\end{tabular}


Table 10-A.11 (continued)

\begin{tabular}{|c|c|c|c|c|c|c|c|c|c|}
\hline \multirow[b]{2}{*}{ Nuclear Test Series } & \multirow{2}{*}{$\begin{array}{l}\text { year of } \\
\text { release }\end{array}$} & \multicolumn{8}{|c|}{ year of birth } \\
\hline & & 1951 & 1952 & 1953 & 1954 & 1955 & 1956 & 1957 & 1958 \\
\hline Ranger (28 Jan 1951 - 6 Feb 1951) and Buster-Jangle (28 Oct. 1951 - 29 Nov. 1951) & 1951 & 0.058 & & & & & & & \\
\hline Tumbler-Snapper (1 Apr. 1952 - 5 Jun 1952) & 1952 & 0.94 & 1.9 & & & & & & \\
\hline Upshot-Knothole (15 Mar 1953 - 4 Jun 1953) & 1953 & 1.0 & 1.0 & 2.1 & & & & & \\
\hline No Tests & 1954 & 0.0 & 0.0 & 0.0 & 0.0 & & & & \\
\hline Teapot (18 Feb 1955 - 15 May 1955) & 1955 & 0.66 & 0.96 & 0.96 & 0.96 & 2.0 & & & \\
\hline No Tests & 1956 & 0.0 & 0.0 & 0.0 & 0.0 & 0.0 & 0.0 & & \\
\hline Plumbbob (28 May 1957 - 7 Oct 1957) & 1957 & 0.9 & 0.9 & 0.9 & 1.3 & 1.3 & 1.3 & 2.6 & \\
\hline Hardtrack Phase II (19 Sep 1958 - 26 Oct 1958) & 1958 & 0.0 & 0.0 & 0.0 & 0.0 & 0.0 & 0.0 & 0.0 & 0.0 \\
\hline
\end{tabular}


TASK 1 REPORT

July 1999

${ }^{131}$ I Releases from X-10 Radioactive Lanthanum Processing -

Page 10A-28

Thyroid Cancer Incidence Data

Table 10-A.12 Comparison between the thyroid cancer incidence rates observed during 19881995 in Tennessee and the thyroid cancer incidence rates corrected for the contribution of NTS fallout. ${ }^{\mathrm{a}}$

\begin{tabular}{lccc}
\hline age-group & $\begin{array}{c}\text { Observed } \\
\text { incidence } \\
\text { rates }\end{array}$ & $\begin{array}{c}\text { Corrected } \\
\text { incidence } \\
\text { rates }\end{array}$ & \\
\hline $0-4$ & 0.1 & 0.1 & \\
$5-9$ & 0.1 & 0.1 & \\
$10-14$ & 0.4 & 0.4 & \\
\hline $15-19$ & 2.5 & 2.5 & \\
$20-24$ & 3.1 & 3.1 & \\
$25-29$ & 4.2 & 4.2 & \\
\hline $30-34$ & 8.2 & $\mathbf{8 . 1}$ & \\
$35-39$ & 8.1 & $\mathbf{7 . 9}$ & Age groups \\
$40-44$ & 8.7 & $\mathbf{8 . 0}$ & affected by \\
\hline $45-49$ & 8.8 & $\mathbf{7 . 3}$ & correction for \\
$50-54$ & 8.4 & $\mathbf{7 . 0}$ & NTS fallout \\
$55-59$ & 8.1 & $\mathbf{7 . 4}$ & \\
\hline $60-64$ & 7.6 & 7.6 & \\
$65-69$ & 9.5 & 9.5 & \\
$70-74$ & 6.6 & 6.6 & \\
\hline $75-79$ & 7.4 & 7.4 & \\
$80-84$ & 9.2 & 9.2 & \\
$85+$ & 7.0 & 7.0 & \\
\hline
\end{tabular}

${ }^{a}$ Age groups affected by correction for NTS fallout include 30-34, 35-39, 40-44, 45-49, 50-54, and 55-59. 


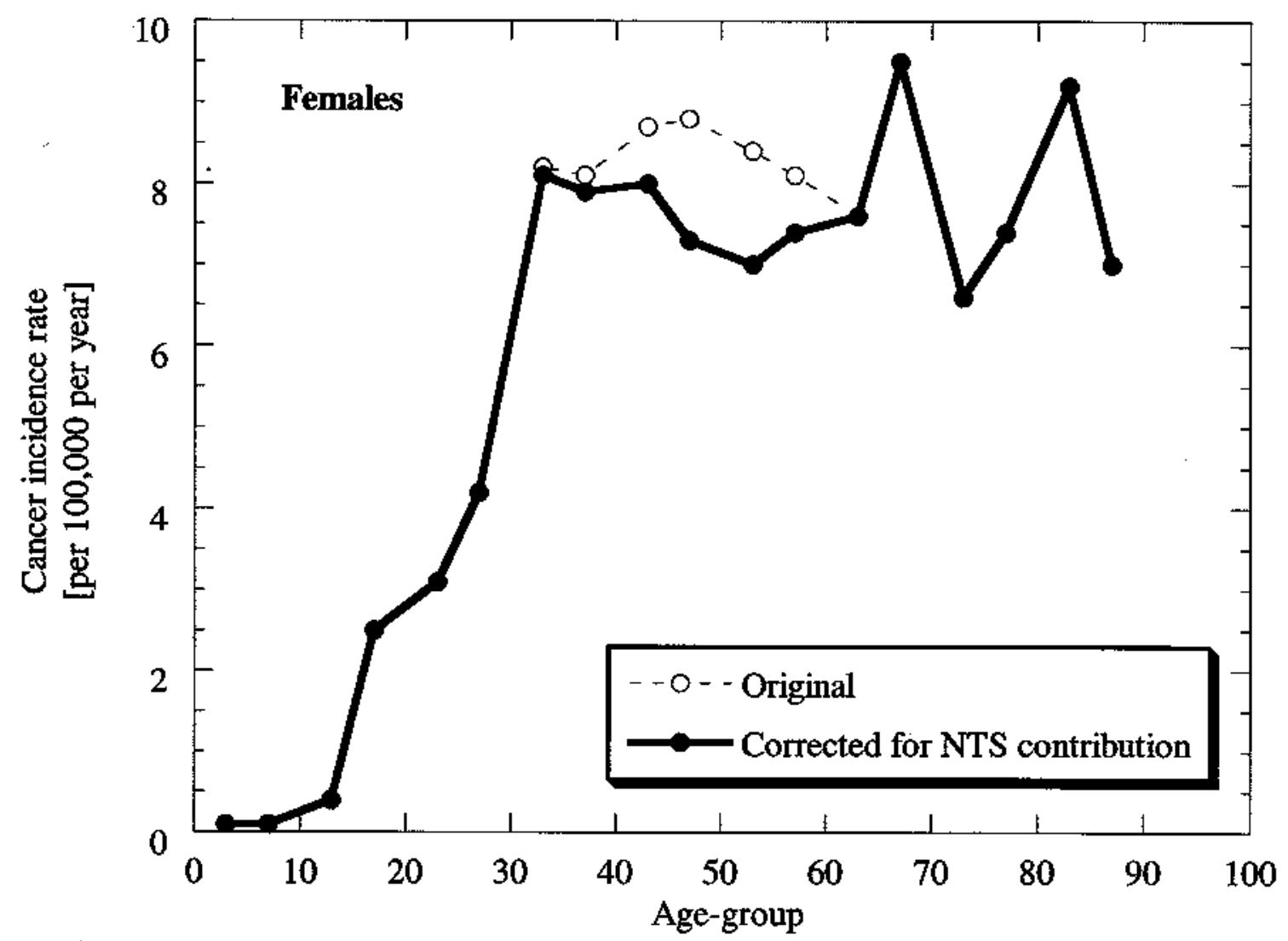

Figure 10-A.5 Comparison between the observed thyroid cancer incidence rates and the rates corrected for the contribution of NTS fallout. The reporting period for the data is $1988-1995$ (Turri, 1998). 


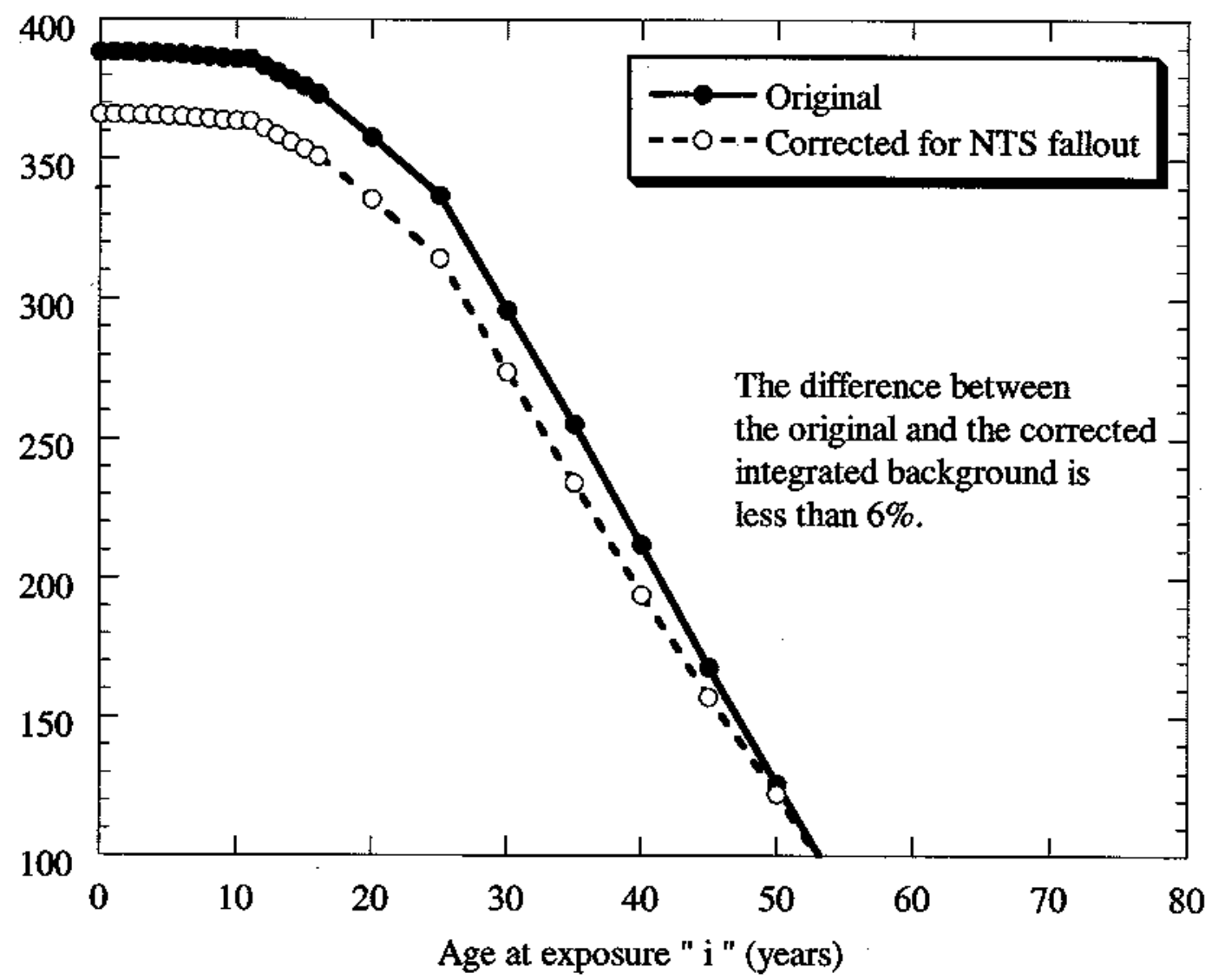

Figure 10-A.6 Comparison between the original background lifetime risk of thyroid cancer and the same background corrected for the contribution of NTS fallout. The data represent females in all counties in Tennessee other than Anderson, Roane, Loudon and Knox counties, as reported between 1988 and 1995 (Turri, 1998). 


\section{References}

Miller, B.A., Kolonel, L.N., Bernstein, L., Young, J.L., Jr., Swanson, G.M., West, D., Key, C.R., Liff, J.M., Glover C.S., Alexander, G.A. et al. (editors). 1996. Racial/Ethnic Patterns in the United States 1988-1992. National Cancer Institute, NIH Pub. No. 96-4104, Bethesda, MD.

NCI (National Cancer Institute). 1997. Estimated Exposure and Thyroid Doses Received by the American People from Iodine-131 Fallout Following Nevada Atmospheric Nuclear Bomb Tests. U.S. Department of Health and Human Services. National Institute of Health. National Cancer Institute, October, 1997.

Ries, L.A.G., Kosaty, C.L., Hankey, B.F., Miller, B.A., and Edwards, B.K. (editors) 1998. SEER Cancer Statistics Review, 1973-1995, National Cancer Institute, Bethesda, MD.

Ron, E., Lubin, J.H., Shore, R.E., Mabuchi, K., Modan, B., Pottern, L.M., Schneider, A.B., Tucker, M.A., and Boice, J.D., Jr. 1995. Thyroid Cancer after Exposure to External Radiation: A Pooled Analysis of Seven Studies. Radiation Research 141:259-277.

Turri, P. 1998. Personal Communication. Tennessee Department of Health, Cordell Hull Building, 425 5th Avenue North, Nashville, TN 37247-5261. 
APPENDIX 11A

RATIONALE FOR LOCATIONS

AND EXPOSURE PATHWAYS 
TASK 1 REPORT

July 1999

${ }^{131}$ I Releases from X-10 Radioactive Lanthanum Processing-

Page 11A-2

Locations/Exposure Pathways

This page intentionally left blank. 


\section{APPENDIX 11A: RATIONALE FOR LOCATIONS AND EXPOSURE PATHWAYS}

The location of individuals exposed to ${ }^{131} \mathrm{I}$ releases from X-10 due to RaLa operations is an important element of the dose reconstruction for Task 1 . Each exposure pathway must be evaluated with respect to specific population clusters that might have been affected. For ${ }^{131} \mathrm{I}$, the primary human exposure pathways considered in this analysis are direct inhalation; ingestion of milk (backyard cow's, commercial, and goat's), cottage cheese, and meat from cattle grazing on contaminated pasture grass; ingestion of eggs; ingestion of leafy vegetables; and ingestion of breast milk. The period of interest for the analysis of the local population described here spans from 1944 to 1956 . Figure 11.1 presents the locations within a $38-\mathrm{km}$ circle around the RaLa processing facility at which doses and risks were evaluated. The rationales for the choice of these locations are provided below.

The milk and meat exposure pathways generally affected the same populations because most rural farms in Anderson, Blount, Knox, Loudon, Morgan, and Roane counties raised both beef and dairy cattle (Waller,1996; Stokes, 1996; Prichard, 1997; Clark, 1997; Harkins, 1998; Lowe, 1997; Hackett, 1998; Hudson, 1998; Zirkle, 1996; Wade, 1996; Idom, 1997; Jenkins, 1997; Mickey, 1996). If backyard cows were present, cottage cheese could have been produced and consumed; therefore the cottage cheese pathway is considered for the same locations as the backyard cow. The egg consumption and vegetable consumption pathways were also considered for these same locations due to the high probability that each farm raised a few chickens and a vegetable garden. All locations were considered for the inhalation, commercial milk, and breast milk pathways. Goat's milk was considered for those areas where confirmed usage existed as well as for those areas that were predominantly rural and agricultural. This study included various locations in Oak Ridge (Woodland, Scarboro, Oak Ridge High School, and East Fork Poplar Creek), Norwood, Oliver Springs, Jonesville, Dyllis, Wartburg, Oakdale, Harriman, Rockwood, Sugar Grove, Lawnville, Kingston, Barnardsville, Bradbury, Hines Valley Community, Lenoir City, Loudon, Sweetwater, Hope Creek Community, Greenback, Friendsville, Louisville, Maryville, Rockford, Farragut, Cedar Bluff, Buttermilk Road Community, Gallaher Bend Community, Hardin Valley, Solway, Karns, Knoxville, Claxton, Clinton, Dutch Valley, Cedar Grove, and Lake City.

The City of Oak Ridge is a population cluster of interest in which five local points were monitored for

${ }^{131}$ I and other radionuclides in air (see Section 4). Within the City of Oak Ridge, several locations were considered representative population clusters: the Oak Ridge High School area, the Scarboro Community, the Woodland Community, and the Oak Ridge Town Site area (see Figure 11.1). Although the residents in these areas may not have grown produce or raised animals, their potential for obtaining locally grown food was high due to the practices of "truck farming" and "rolling stores." "Truck farming" and "rolling stores" involved individuals who grew their own produce and sold it out of their trucks (Wade, 1998; Harkins, 1998). These practices were common in the Oak Ridge and Knoxville areas.

Within Oak Ridge, potential exposure pathways include inhalation, ingestion of milk and meat from animals grazing on contaminated pasture grass, and ingestion of leafy vegetables from gardens. The consumption of human breast milk also occurred. Personal communications with several former 
residents (e.g., Hileman, Clark, Vowell, Brooks) contributed to the following overview of possible exposures.

Interviews indicated that beef and dairy cattle were kept in small numbers along East Fork Poplar Creek (EFPC). At least two of the 3 or 4 families living along EFPC had dairy cattle for their own family use over a period of several years in the 1950s. However, no evidence of commercial dairies or the sale of local milk to commercial dairies was found. There was also no indication of any dairy goats in the Oak Ridge area.

Approximately half the land along EFPC on the south side of the Oak Ridge Turnpike was used for grazing beef cattle, starting in the 1950s. Four or five families slaughtered at least one beef cow a year for personal use; the rest were raised for resale. No grain was raised except for livestock feed. One family had chickens and sold them locally for about 5 years, and other interviewees mentioned hearing or seeing chickens. It is possible, though not confirmed through interviews, that eggs from local chickens were available.

At least one, perhaps more, vegetable gardens were grown near EFPC, and occasional vegetable gardens were mentioned in connection with other sections of Oak Ridge (Jenkins, 1997). Tomatoes and corn from local land may have been sold at a grocery story near the Oak Ridge Turnpike. Other occasional wild food gathering activities (blackberries, walnuts, mushrooms and grapes, as well as honey collection) could have been minor contributors to exposure.

Oliver Springs and Norwood are also communities potentially affected by the ${ }^{131}$ I releases from X-10. Oliver Springs was once a coal mining town, while Norwood is a small agricultural community located to the southeast of Oliver Springs. Beef and dairy cattle were raised in these areas for personal consumption (Lowe, 1997).

Clinton and Claxton are population clusters located northeast of X-10. These areas were of concern due to the presence of dairy and beef cattle, in addition to chickens, pigs, and goats (Clark, 1997). In the Claxton area, one family was known to consume goat's milk as well as cow's milk (Clark, 1997). The goat's milk was not sold commercially in this area. Backyard cow's milk, however, was sold to neighbors, along with cream, butter, and eggs (Clark, 1997). Vegetable gardens were also grown in these two areas.

Lake City is the farthest northerly location selected. Despite its being a rural mountainous area, beef cattle, pigs, and chickens were raised in Lake City, along with vegetable gardens. Produce frequently grown in this area included beans, onions, tomatoes, and potatoes. These items were generally consumed by extended families and neighbors.

Cedar Grove and Dutch Valley are located to the northeast of the X-10 facility. These rural communities are predominantly agricultural. Corn and hay are a few of the crops grown in these areas (Courier-News, 1998). Presently, dairy cattle are limited to a few farms, but beef cattle dot the landscape on most larger farms. Although not confirmed through interviews, vegetable gardens were considered to have been grown by most individuals in the Cedar Grove and Dutch Valley areas. 
Wartburg and Oakdale are rural communities located in Morgan County in the Cumberland Mountains. These areas have regions in which farming is practiced, but most of the land is too rugged to have large farming tracts. Vegetable gardens are grown by most individuals in this area, with the most popular crops being tomatoes and beans. Beef cattle grazed the mountainsides, while dairy cattle were kept close to the house. Most individuals were assumed to have backyard cows. No large commercial dairies were located in these areas, but local sale of milk cannot be excluded. Oak Ridge and Harriman are the closest urban areas.

Agriculture has been and is still practiced in small communities in Roane County, such as Dyllis and Jonesville. The communities in these locations continue to raise beef cattle and to plant crops for production (grain and hay) and for family consumption. The number of grocery stores in Dyllis and Jonesville was limited; therefore, residents often produced their own food or traveled to nearby cities (e.g., Harriman, Oliver Springs, or Oak Ridge) to purchase groceries.

Harriman was another population cluster where urban and rural individuals raised livestock and grew crops (Lowe, 1997). The crops and livestock were used primarily for family consumption, but were also shared with friends and neighbors. Harriman was also serviced by local milk deliveries from the Bradbury community (Prichard, 1997) and by local dairies (Lowe, 1997).

Sugar Grove lies in the Sugar Grove Valley, which is approximately due west of the X-10 facility. This small rural community was an area in which beef cattle grazed the hillsides and vegetable gardens were planted. Hay, tobacco, onions, potatoes, beans, tomatoes, berries, corn, and other grains were grown as well (Adkins, 1997; Lowe, 1997).

Rockwood is located to the southwest of $\mathrm{X}-10$ and is predominantly rural, with several farms. Hay, tobacco, and other grains are produced in this location. If acreage was owned, vegetable gardens were planted. Tomatoes, beans, potatoes, and other root crops were grown in this area, and beef cattle were also raised for family consumption.

For the Kingston area, parcels of farm land were located both within and outside the city limits. Raising livestock and planting gardens were an integral part of life in this area. Beef and dairy cattle were raised, in addition to chickens, pigs, sheep, and goats (Prichard, 1997). Several residents in the rural Kingston area consumed goat's milk (Prichard, 1997). During interviews that were conducted with local residents, one farm was identified as raising goats. The farm raised approximately 60-70 goats for milk production and for land clearing purposes (Prichard, 1997). Goat's milk was not sold commercially in Kingston; however, family members and neighbors who preferred goat's milk to cow's milk utilized the milk (Prichard, 1997).

Individuals living within the city limits of Kingston could have had access to farm-raised milk (through creameries and local deliveries) and meat (through local grocers and meat companies). For example, residents of the Bradbury community often delivered milk to the homes of residents in Kingston, Harriman, Oakdale, and Oak Ridge until the 1950s (Prichard, 1997). Local grocery stores in Kingston carried farm-raised beef, and slaughterhouses were also present in this area (Stokes, 1997). In addition, excess local milk was often sold to local grocery stores after refrigeration became available (Adkins, 1997). 
The communities living along Gallaher Road in Roane County (Gallaher/Lawnville communities) were also considered in this analysis. Gallaher Road is located between Pine and Black Oak Ridges and is predominantly agricultural. Beef and dairy cattle were raised in this area, along with vegetable gardens. Hay and grain crops were also produced in this area.

Barnardsville is located in the southwestern section of Roane County and is predominantly rural. Beef cattle can still be seen in this area today. Hay and tobacco were also grown in this area. Vegetable gardens and dairy cattle were assumed to have been raised here as well.

For the Bradbury community (located near Jones Island in Loudon and Roane counties), beef cattle were the primary livestock; however, each farm had its own dairy cattle to produce milk for the family (Waller, 1996). Local meat (beef) and milk were not sold commercially in the Bradbury area due to the lack of refrigeration (Waller, 1996).

The closest urban area to the Bradbury community was Lenoir City. Even though this area was considered urban, farms were located within the city limits. These farms raised livestock (beef and dairy cattle, chickens, and pigs) and continue to raise a limited amount of livestock today. Small gardens were planted, since the land was predominantly used for grazing and the production of grain and hay.

Hines Valley is also an agricultural community. Pigs, beef, and dairy cattle were raised in this area for local consumption (Idom, 1997). Hay and other grains were grown in this area, in addition to vegetable gardens.

Loudon and Sweetwater are also considered urban areas, but farms and agricultural land are predominant in these areas. Beef and dairy cattle, along with pigs were prominently raised in this area. The Sweetwater area was supplied with feeder pigs from the Hines Valley area (Idom, 1997). Large gardens (corn and soybeans) can still be viewed in these areas today, especially along the Tennessee River.

Greenback, Friendsville, and Louisville are located southeast of the X-10 facility and were affected by the releases of ${ }^{131}$ I. These areas are primarily agricultural, with the closest urban areas being Lenoir City and Maryville. Tobacco, corn, hay, and soybeans were the primary crops. Beef and dairy cattle were also raised in these areas. Maryville is considered an urban area, but large farming tracts of land are located on the outskirts of the city. Beef cattle were the predominant animals raised; some vegetable gardens were grown as well.

Rockford is another small community that lies south of Knoxville. This was a predominantly agricultural area and continues to be the home of a large portion of the University of Tennessee's Agricultural Experiment Station. Holstein cattle can still be seen grazing this land. Vegetable gardens and hay crops are also grown in this community.

The Buttermilk, Hardin Valley, Gallaher Bend, and Hope Creek communities are predominantly agricultural areas. Beef and dairy cattle were raised here along with chickens and pigs, as early as the Civil War (Ward, 1998; Hackett, 1998; Hudson, 1998). One family in the Gallaher Bend area raised goats and consumed the milk (Hackett, 1998; Hudson, 1998). Grain, hay, and tobacco were the 
largest crops grown in these four areas; however, vegetables gardens were also grown. These four communities are still predominantly agricultural areas.

Solway was an area in which beef and dairy cattle were present in the 1940s and 1950s (Wade, 1996). Goats, pigs, and chickens were also raised in this area. Vegetable gardens were grown for family consumption as well as for resale. Individuals known as "truck farmers" took excess vegetables to the Knoxville Farmer's Market on Market Street in downtown Knoxville and to Oak Ridge markets for resale (Wade, 1996). Excess milk was sold to other local farmers for use as food for pigs (Wade, 1996).

Knoxville was considered important for this study due to the presence of cattle in areas surrounding downtown Knoxville, such as north and west Knoxville. Large farms existed in the Farragut, Karns, and Cedar Bluff areas (Harkins, 1998). These farms raised beef and dairy cattle, in addition to vegetable gardens, from which excess produce was taken to Market Square and the Forrest Avenue Farmer's Market in Knoxville. Milk from the Cedar Bluff area was sold commercially to larger dairies in the area (Harkins, 1998). Beef cattle are still raised on the outskirts of Knoxville today. The Knoxville area also received vegetables grown in the Oak Ridge area and outlying regions via the Farmer's Market, which was originally located on Market Street (Wade, 1997), and the Forrest Avenue Farmer's Market.

Table 11-A.1 provides a summary of the locations of interest and the exposure pathways of concern. The table locations are arranged in order of increasing distance from the X-10 facility. The pathways that were considered relevant for a specific location are identified by a number "1." Those pathways not considered applicable to residents at a given location are described with a " 0 ." Inhalation of ${ }^{131} \mathrm{I}$ was considered at all sites. The consumption patterns of the individuals considered to be reference individuals consisted of three diets. Diet 1 consisted of the backyard milk pathway in addition to consumption of vegetables, meat, cheese, and eggs. Ingestion of local commercial milk, and ingestion of meat, eggs, vegetables, and cheese make up Diet 2. Diet 3 describes an individual who consumed regional commercial milk. 
Table 11-A.1 Summary of the locations of interest and the exposure pathways selected for dose and risk estimation.

\begin{tabular}{|c|c|c|c|c|c|c|c|c|c|c|c|c|c|c|c|c|c|}
\hline Location & $\begin{array}{c}\text { Distance } \\
\text { from X-10 } \\
(\mathrm{km})\end{array}$ & $\begin{array}{c}\text { Angle from } \\
\text { North } \\
\text { Direction } \\
\text { (degrees) }\end{array}$ & 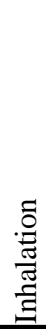 & 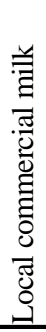 & 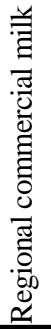 & 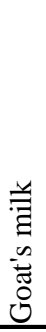 & 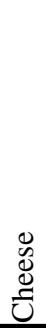 & $\begin{array}{l}\breve{d} \\
\stackrel{\Delta}{~} \\
\end{array}$ & 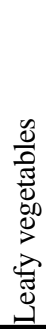 & . & 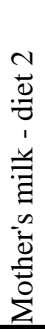 & 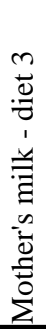 & 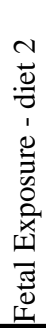 & 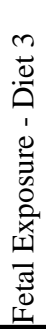 & $\stackrel{\vec{\Perp}}{\ddot{\theta}}$ & $\begin{array}{l}N \\
\stackrel{\oplus}{\theta} \\
\end{array}$ & $\stackrel{m}{\stackrel{0}{\theta}}$ \\
\hline Bradbury & 6000 & 228 & 1 & 1 & 1 & 1 & 1 & 1 & 1 & 1 & 1 & 1 & 1 & 1 & 1 & 1 & 1 \\
\hline Gallaher Bend & 6300 & 89 & 1 & 1 & 1 & 1 & 1 & 1 & 1 & 1 & 1 & 1 & 1 & 1 & 1 & 1 & 1 \\
\hline EFPC & 7600 & 7 & 1 & 1 & 1 & 0 & 1 & 1 & 1 & 1 & 1 & 1 & 1 & 1 & 1 & 1 & 1 \\
\hline Hope Creek & 7600 & 119 & 1 & 1 & 1 & 0 & 1 & 1 & 1 & 1 & 1 & 1 & 1 & 1 & 1 & 1 & 1 \\
\hline Buttermilk Rd. & 8400 & 106 & 1 & 1 & 1 & 1 & 1 & 1 & 1 & 1 & 1 & 1 & 1 & 1 & 1 & 1 & 1 \\
\hline Jonesville & 9000 & 312 & 1 & 1 & 1 & 1 & 1 & 1 & 1 & 1 & 1 & 1 & 1 & 1 & 1 & 1 & 1 \\
\hline OR Scarboro & 9300 & 32 & 1 & 0 & 1 & 0 & 0 & 0 & 1 & 0 & 0 & 1 & 0 & 1 & 0 & 0 & 1 \\
\hline Lawnville/Gallaher & 9300 & 245 & 1 & 1 & 1 & 1 & 1 & 1 & 1 & 1 & 1 & 1 & 1 & 1 & 1 & 1 & 1 \\
\hline Dyllis & 10100 & 293 & 1 & 1 & 1 & 1 & 1 & 1 & 1 & 1 & 1 & 1 & 1 & 1 & 1 & 1 & 1 \\
\hline OR High School Area & 11200 & 27 & 1 & 0 & 1 & 0 & 0 & 0 & 1 & 0 & 0 & 1 & 0 & 1 & 0 & 0 & 1 \\
\hline Norwood & 11300 & 355 & 1 & 1 & 1 & 1 & 1 & 1 & 1 & 1 & 1 & 1 & 1 & 1 & 1 & 1 & 1 \\
\hline Woodland & 11500 & 35 & 1 & 0 & 1 & 0 & 0 & 0 & 1 & 0 & 0 & 1 & 0 & 1 & 0 & 0 & 1 \\
\hline Hardin Valley & 12000 & 85 & 1 & 1 & 1 & 1 & 1 & 1 & 1 & 1 & 1 & 1 & 1 & 1 & 1 & 1 & 1 \\
\hline Oliver Springs & 13300 & 350 & 1 & 1 & 1 & 1 & 1 & 1 & 1 & 1 & 1 & 1 & 1 & 1 & 1 & 1 & 1 \\
\hline Solway & 13500 & 61 & 1 & 1 & 1 & 1 & 1 & 1 & 1 & 1 & 1 & 1 & 1 & 1 & 1 & 1 & 1 \\
\hline Sugar Grove & 13600 & 263 & 1 & 1 & 1 & 1 & 1 & 1 & 1 & 1 & 1 & 1 & 1 & 1 & 1 & 1 & 1 \\
\hline OR Townsite & 13800 & 31 & 1 & 0 & 1 & 0 & 0 & 0 & 1 & 0 & 0 & 1 & 0 & 1 & 0 & 0 & 1 \\
\hline Hines Valley & 14500 & 185 & 1 & 1 & 1 & 1 & 1 & 1 & 1 & 1 & 1 & 1 & 1 & 1 & 1 & 1 & 1 \\
\hline Farragut & 15100 & 108 & 1 & 1 & 1 & 1 & 1 & 1 & 1 & 1 & 1 & 1 & 1 & 1 & 1 & 1 & 1 \\
\hline Lenoir City & 15500 & 155 & 1 & 1 & 1 & 0 & 1 & 1 & 1 & 1 & 1 & 1 & 1 & 1 & 1 & 1 & 1 \\
\hline Kingston & 19000 & 250 & 1 & 1 & 1 & 1 & 1 & 1 & 1 & 1 & 1 & 1 & 1 & 1 & 1 & 1 & 1 \\
\hline Karns & 19500 & 73 & 1 & 1 & 1 & 1 & 1 & 1 & 1 & 1 & 1 & 1 & 1 & 1 & 1 & 1 & 1 \\
\hline Loudon & 19500 & 192 & 1 & 1 & 1 & 1 & 1 & 1 & 1 & 1 & 1 & 1 & 1 & 1 & 1 & 1 & 1 \\
\hline Harriman & 20500 & 271 & 1 & 1 & 1 & 1 & 1 & 1 & 1 & 1 & 1 & 1 & 1 & 1 & 1 & 1 & 1 \\
\hline Cedar Bluff & 21100 & 95 & 1 & 1 & 1 & 1 & 1 & 1 & 1 & 1 & 1 & 1 & 1 & 1 & 1 & 1 & 1 \\
\hline Oakdale & 21100 & 286 & 1 & 1 & 1 & 1 & 1 & 1 & 1 & 1 & 1 & 1 & 1 & 1 & 1 & 1 & 1 \\
\hline Claxton & 22700 & 54 & 1 & 1 & 1 & 1 & 1 & 1 & 1 & 1 & 1 & 1 & 1 & 1 & 1 & 1 & 1 \\
\hline Dutch Valley & 24000 & 27 & 1 & 1 & 1 & 1 & 1 & 1 & 1 & 1 & 1 & 1 & 1 & 1 & 1 & 1 & 1 \\
\hline Clinton & 25400 & 38 & 1 & 1 & 1 & 1 & 1 & 1 & 1 & 1 & 1 & 1 & 1 & 1 & 1 & 1 & 1 \\
\hline Friendsville & 26400 & 138 & 1 & 1 & 1 & 1 & 1 & 1 & 1 & 1 & 1 & 1 & 1 & 1 & 1 & 1 & 1 \\
\hline Wartburg & 27000 & 317 & 1 & 1 & 1 & 1 & 1 & 1 & 1 & 1 & 1 & 1 & 1 & 1 & 1 & 1 & 1 \\
\hline Rockwood & 28000 & 258 & 1 & 1 & 1 & 1 & 1 & 1 & 1 & 1 & 1 & 1 & 1 & 1 & 1 & 1 & 1 \\
\hline Louisville & 28200 & 125 & 1 & 1 & 1 & 1 & 1 & 1 & 1 & 1 & 1 & 1 & 1 & 1 & 1 & 1 & 1 \\
\hline Barnardville & 31400 & 232 & 1 & 1 & 1 & 1 & 1 & 1 & 1 & 1 & 1 & 1 & 1 & 1 & 1 & 1 & 1 \\
\hline Greenback & 32300 & 156 & 1 & 1 & 1 & 1 & 1 & 1 & 1 & 1 & 1 & 1 & 1 & 1 & 1 & 1 & 1 \\
\hline Rockford & 33300 & 104 & 1 & 1 & 1 & 1 & 1 & 1 & 1 & 1 & 1 & 1 & 1 & 1 & 1 & 1 & 1 \\
\hline Lake City & 35000 & 27 & 1 & 1 & 1 & 1 & 1 & 1 & 1 & 1 & 1 & 1 & 1 & 1 & 1 & 1 & 1 \\
\hline Sweetwater & 35100 & 204 & 1 & 1 & 1 & 1 & 1 & 1 & 1 & 1 & 1 & 1 & 1 & 1 & 1 & 1 & 1 \\
\hline Knoxville & 35800 & 80 & 1 & 1 & 1 & 1 & 1 & 1 & 1 & 1 & 1 & 1 & 1 & 1 & 1 & 1 & 1 \\
\hline Maryville & 36200 & 136 & 1 & 1 & 1 & 1 & 1 & 1 & 1 & 1 & 1 & 1 & 1 & 1 & 1 & 1 & 1 \\
\hline Cedar Grove & 36200 & 54 & 1 & 1 & 1 & 1 & 1 & 1 & 1 & 1 & 1 & 1 & 1 & 1 & 1 & 1 & 1 \\
\hline
\end{tabular}




\section{References}

Adkins, P. 1997. Former Kingston, TN resident. Interview with C. Lewis, January 29, 1997.

Brooks, A. 1996. Lifetime Oak Ridge, TN resident. Personal communication with C. DaMassa.

Clark, C. 1997. Child raised in the Claxton, TN community. Interview with C. Lewis, April 25, 1997.

The Courier-News. 1998. Clinton, TN, community newspaper. Section B, 1. Clinton, Tennessee. July 19-20, 1998.

Hackett, B. 1998. Resident of Hickory Creek/Gallaher Bend Community who consumed goat milk as a child. Telephone interview with C. Lewis. May 29, 1998.

Harkins, S. 1998. Grandchild of a dairy and beef farmer, whose farm was on Kingston Pike in Knoxville, Tennessee. Telephone interview with C. Lewis. August 25, 1998.

Hileman, E. 1996. Oak Ridge, TN resident who lived along East Fork Poplar Creek. Telephone interview with E. W. Reed.

Hudson, J. 1998. Resident of Hickory Creek/Gallaher Bend Community who consumed goat milk as a child. Telephone interview with C. Lewis. May 19, 1998.

Idom, N. 1997. Lifetime resident and farmer in Loudon County. Interview with C. Lewis. April 30, 1997.

Jenkins, M. 1997. Nephew of an Oak Ridge, TN, resident who raised a vegetable garden in his backyard. Telephone interview with C. Lewis. April 25, 1997.

Lowe, E. 1997. Lifetime resident of Harriman, TN. Interview with C. Lewis, April 25, 1997.

Mickey, J. 1996. Son of a lifetime resident and farmer in Roane County. Telephone interview with C. Lewis. November 12, 1996.

Prichard, D. 1997. Dairy farmer and milk delivery route owner in Kingston, TN. Personal interview with C. Lewis, G. Blaylock, K. Thiessen, and W. Reed, April 8, 1997.

Stokes, J. 1996. Former Kingston, TN resident. Interview with C. Lewis, October 16, 1996.

Vowell, B. 1996. Oliver Springs resident. Telephone interview with E. W. Reed.

Wade, A. 1996. Farm owner in Solway, TN. Interview with C. Lewis, October 10, 1996.

Waller, M., Jr. 1996. Child raised on land grant farm in the Bradbury community. Personal interview with C. Lewis, September 25, 1996. 
Ward, T. 1998. Family history of the Ward Family, lifetime residents of Loudon County. Unpublished data.

Zirkle, J. 1996. Lifetime resident of Kingston, Tennessee. Telephone interview with C. Lewis, November 21, 1996. 
APPENDIX 11B

\section{ESTIMATED ${ }^{131}$ I CONCENTRATIONS}

IN ENVIRONMENTAL MEDIA 
TASK 1 REPORT

July 1999

Page 11B-2

${ }^{131}$ I Releases from X-10 Radioactive Lanthanum ProcessingEstimated ${ }^{131}$ I Concentrations in Environmental Media

This page intentionally left blank. 


\section{APPENDIX 11B ESTIMATED ${ }^{131}$ I CONCENTRATIONS IN ENVIRONMENTAL MEDIA}

This Appendix presents the estimated concentrations of ${ }^{131} \mathrm{I}$ in various environmental media and food products as a result of the 1944-1956 releases from the X-10 radioactive lanthanum processing facility located in Oak Ridge, Tennessee. These concentrations represent timeaverages over the entire 13 years of releases and include the effects of both routine releases and the accidental release of April 29, 1954.

The results are presented separately for each of the 41 locations of interest (Appendix 11A) within a 38-km radius around the RaLa facility. For each location, the following concentrations are given:

- Ground-level concentrations of ${ }^{131} \mathrm{I}$ in air (the concentrations represent total iodine, or all three physico-chemical forms combined)

- Concentrations of ${ }^{131} \mathrm{I}$ in leafy vegetables

- Concentrations of ${ }^{131} \mathrm{I}$ in pasture grass

- Concentrations of ${ }^{131}$ I in milk of "backyard" cows

- Concentrations of ${ }^{131}$ I in milk of "commercial" cows (concentrations are given separately for local and regional distribution of commercially produced milk)

- Concentrations of ${ }^{131} \mathrm{I}$ in milk of goats

- Concentrations of ${ }^{131} \mathrm{I}$ in locally produced meat

- Concentrations of ${ }^{131} \mathrm{I}$ in cheese produced from contaminated milk

- Concentrations of ${ }^{131} \mathrm{I}$ in eggs from chickens fed contaminated feed

- Concentrations of ${ }^{131} \mathrm{I}$ in human breast milk

The results are presented as 95\% subjective confidence intervals (2.5th and 97.5th percentiles) and a central estimate (50th percentile). A 95\% subjective confidence interval is expected, at the 95th percent level of confidence, to encompass the true but unknown concentration of ${ }^{131} \mathrm{I}$. The confidence intervals are called "subjective" because they are based in part on assumptions made using expert judgment and on professional analysis of the exposure situation. Other investigators may come to slightly different results, but the final conclusions are not expected to change significantly.

At some locations, the concentration for an endpoint may be zero (e.g., no cows were present in downtown Oak Ridge during the 1940s or 1950s). For these locations, a dash "-." is shown in the tables. 
${ }^{131}$ I Releases from X-10 Radioactive Lanthanum Processing

TASK 1 REPORT

Appendix $11 B$

July 1999

Page 11B-4

\section{Location: Barnardville}

\begin{tabular}{lcccc}
\hline Environmental media or food product & Units & \multicolumn{3}{c}{13 years-average concentration } \\
& & lower limit & central estimate & upper limit \\
\hline Air (all physico-chemical forms) & $\mathrm{Bq} \mathrm{m}^{-3}$ & 0.013 & 0.021 & 0.035 \\
Pasture grass & $\mathrm{Bq} \mathrm{kg}^{-1}$ dry mass & 51 & 150 & 440 \\
Backyard cow milk (locally produced) & $\mathrm{Bq} \mathrm{L}^{-1}$ & 2.6 & 8.6 & 32 \\
Commercial milk (locally produced) & $\mathrm{Bq} \mathrm{L}^{-1}$ & 1.7 & 5.2 & 15 \\
Commercial milk (regional mixed) & $\mathrm{Bq} \mathrm{L}^{-1}$ & 2.2 & 5.8 & 16 \\
Goat milk (locally produced) & $\mathrm{Bq} \mathrm{L}^{-1}$ & 9 & 41 & 160 \\
Beef & $\mathrm{Bq} \mathrm{kg}^{-1}$ & 0.42 & 2.5 & 16 \\
Leafy vegetables & $\mathrm{Bq} \mathrm{kg} \mathrm{fresh} \mathrm{mass}^{-1}$ & 3.4 & 10.0 & 33 \\
Cottage Cheese & $\mathrm{Bq} \mathrm{kg}^{-1}$ & 0.8 & 4.8 & 31 \\
Eggs & $\mathrm{Bq} \mathrm{kg}^{-1}$ & 1.4 & 5.8 & 25 \\
Mother's milk* & $\mathrm{Bq} \mathrm{L}^{-1}$ & 0.18 & 1.4 & 10.0 \\
\hline
\end{tabular}

*Mother on Diet 3 - Regionally mixed commercial milk + inhalation

\section{Location: Bradbury}

\begin{tabular}{lcccc}
\hline Environmental media or food product & Units & \multicolumn{3}{c}{13 years-average concentration } \\
& & lower limit & central estimate & upper limit \\
\hline Air (all physico-chemical forms) & $\mathrm{Bq} \mathrm{m}^{-3}$ & 0.074 & 0.110 & 0.160 \\
Pasture grass & $\mathrm{Bq} \mathrm{kg}^{-1}$ dry mass & 470 & 1300 & 3300 \\
Backyard cow milk (locally produced) & $\mathrm{Bq} \mathrm{L}^{-1}$ & 24.0 & 74.0 & 250 \\
Commercial milk (locally produced) & $\mathrm{Bq} \mathrm{L}^{-1}$ & 15.0 & 43.0 & 120 \\
Commercial milk (regional mixed) & $\mathrm{Bq} \mathrm{L}^{-1}$ & 2.2 & 5.8 & 16 \\
Goat milk (locally produced) & $\mathrm{Bq} \mathrm{L}^{-1}$ & 79 & 340 & 1300 \\
Beef & $\mathrm{Bq} \mathrm{kg}^{-1}$ & 3.80 & 22.0 & 140 \\
Leafy vegetables & $\mathrm{Bq} \mathrm{kg} \mathrm{fresh} \mathrm{mass}^{-1}$ & 30.0 & 88.0 & 250 \\
Cottage Cheese & $\mathrm{Bq} \mathrm{kg}^{-1}$ & 6.9 & 39.0 & 240 \\
Eggs & $\mathrm{Bq} \mathrm{kg}^{-1}$ & 12.0 & 49.0 & 200 \\
Mother's milk* & $\mathrm{Bq} \mathrm{L}^{-1}$ & 0.38 & 2.6 & 15.0 \\
\hline
\end{tabular}

*Mother on Diet 3 - Regionally mixed commercial milk + inhalation 
${ }^{131}$ I Releases from X-10 Radioactive Lanthanum Processing

TASK 1 REPORT

Appendix $11 B$

July 1999

Page 11B-5

\section{Location: Buttermilk Rd.}

\begin{tabular}{lcccc}
\hline Environmental media or food product & Units & \multicolumn{3}{c}{13 years-average concentration } \\
& & lower limit & central estimate & upper limit \\
\hline Air (all physico-chemical forms) & $\mathrm{Bq} \mathrm{m}^{-3}$ & 0.057 & 0.082 & 0.120 \\
Pasture grass & $\mathrm{Bq} \mathrm{kg}^{-1}$ dry mass & 320 & 870 & 2300 \\
Backyard cow milk (locally produced) & $\mathrm{Bq} \mathrm{L}^{-1}$ & 17.0 & 51.0 & 190 \\
Commercial milk (locally produced) & $\mathrm{Bq} \mathrm{L}^{-1}$ & 10.0 & 30.0 & 85 \\
Commercial milk (regional mixed) & $\mathrm{Bq} \mathrm{L}^{-1}$ & 2.2 & 5.8 & 16 \\
Goat milk (locally produced) & $\mathrm{Bq} \mathrm{L}^{-1}$ & 53 & 240 & 900 \\
Beef & $\mathrm{Bq} \mathrm{kg}^{-1}$ & 2.50 & 16.0 & 92 \\
Leafy vegetables & $\mathrm{Bq} \mathrm{kg} \mathrm{fresh} \mathrm{mass}^{-1}$ & 20.0 & 59.0 & 170 \\
Cottage Cheese & $\mathrm{Bq} \mathrm{kg}^{-1}$ & 5.1 & 28.0 & 180 \\
Eggs & $\mathrm{Bq} \mathrm{kg}^{-1}$ & 8.6 & 34.0 & 140 \\
Mother's milk* & $\mathrm{Bq} \mathrm{L}^{-1}$ & 0.33 & 2.2 & 13.0 \\
\hline
\end{tabular}

*Mother on Diet 3 - Regionally mixed commercial milk + inhalation

\section{Location: Cedar Bluff}

\begin{tabular}{lcccc}
\hline Environmental media or food product & Units & \multicolumn{3}{c}{13 years-average concentration } \\
& & lower limit & central estimate & upper limit \\
\hline Air (all physico-chemical forms) & $\mathrm{Bq} \mathrm{m}^{-3}$ & 0.028 & 0.041 & 0.060 \\
Pasture grass & $\mathrm{Bq} \mathrm{kg}^{-1}$ dry mass & 130 & 340 & 910 \\
Backyard cow milk (locally produced) & $\mathrm{Bq} \mathrm{L}^{-1}$ & 6.4 & 20.0 & 68 \\
Commercial milk (locally produced) & $\mathrm{Bq} \mathrm{L}^{-1}$ & 4.0 & 12.0 & 33 \\
Commercial milk (regional mixed) & $\mathrm{Bq} \mathrm{L}^{-1}$ & 2.2 & 5.8 & 16 \\
Goat milk (locally produced) & $\mathrm{Bq} \mathrm{L}^{-1}$ & 21 & 93 & 340 \\
Beef & $\mathrm{Bq} \mathrm{kg}^{-1}$ & 1.00 & 6.0 & 37 \\
Leafy vegetables & $\mathrm{Bq} \mathrm{kg} \mathrm{fresh} \mathrm{mass}^{-1}$ & 8.1 & 23.0 & 69 \\
Cottage Cheese & $\mathrm{Bq} \mathrm{kg}^{-1}$ & 1.9 & 11.0 & 64 \\
Eggs & $\mathrm{Bq} \mathrm{kg}^{-1}$ & 3.2 & 13.0 & 56 \\
Mother's milk* & $\mathrm{Bq} \mathrm{L}^{-1}$ & 0.23 & 1.7 & 11.0 \\
\hline
\end{tabular}

*Mother on Diet 3 - Regionally mixed commercial milk + inhalation 
${ }^{131}$ I Releases from X-10 Radioactive Lanthanum Processing

TASK 1 REPORT

Appendix $11 B$

July 1999

Page 11B-6

\section{Location: Cedar Grove}

\begin{tabular}{lcccc}
\hline Environmental media or food product & Units & \multicolumn{3}{c}{13 years-average concentration } \\
& & lower limit & central estimate & upper limit \\
\hline Air (all physico-chemical forms) & $\mathrm{Bq} \mathrm{m}^{-3}$ & 0.013 & 0.020 & 0.031 \\
Pasture grass & $\mathrm{Bq} \mathrm{kg}^{-1}$ dry mass & 54 & 150 & 390 \\
Backyard cow milk (locally produced) & $\mathrm{Bq} \mathrm{L}^{-1}$ & 2.9 & 8.8 & 31 \\
Commercial milk (locally produced) & $\mathrm{Bq} \mathrm{L}^{-1}$ & 1.8 & 5.1 & 15 \\
Commercial milk (regional mixed) & $\mathrm{Bq} \mathrm{L}^{-1}$ & 2.2 & 5.8 & 16 \\
Goat milk (locally produced) & $\mathrm{Bq} \mathrm{L}^{-1}$ & 9 & 41 & 160 \\
Beef & $\mathrm{Bq} \mathrm{kg}^{-1}$ & 0.46 & 2.6 & 16 \\
Leafy vegetables & $\mathrm{Bq} \mathrm{kg} \mathrm{fresh} \mathrm{mass}^{-1}$ & 3.6 & 10.0 & 31 \\
Cottage Cheese & $\mathrm{Bq} \mathrm{kg}^{-1}$ & 0.9 & 4.8 & 31 \\
Eggs & $\mathrm{Bq} \mathrm{kg}^{-1}$ & 1.5 & 5.8 & 24 \\
Mother's milk* & $\mathrm{Bq} \mathrm{L}^{-1}$ & 0.18 & 1.4 & 10.0 \\
\hline
\end{tabular}

*Mother on Diet 3 - Regionally mixed commercial milk + inhalation

\section{Location: Claxton}

\begin{tabular}{lcccc}
\hline Environmental media or food product & Units & \multicolumn{3}{c}{13 years-average concentration } \\
& & lower limit & central estimate & upper limit \\
\hline Air (all physico-chemical forms) & $\mathrm{Bq} \mathrm{m}^{-3}$ & 0.023 & 0.034 & 0.049 \\
Pasture grass & $\mathrm{Bq} \mathrm{kg}^{-1}$ dry mass & 110 & 280 & 740 \\
Backyard cow milk (locally produced) & $\mathrm{Bq} \mathrm{L}^{-1}$ & 5.5 & 17.0 & 57 \\
Commercial milk (locally produced) & $\mathrm{Bq} \mathrm{L}^{-1}$ & 3.4 & 9.7 & 28 \\
Commercial milk (regional mixed) & $\mathrm{Bq} \mathrm{L}^{-1}$ & 2.2 & 5.8 & 16 \\
Goat milk (locally produced) & $\mathrm{Bq} \mathrm{L}^{-1}$ & 17 & 78 & 290 \\
Beef & $\mathrm{Bq} \mathrm{kg}^{-1}$ & 0.85 & 5.1 & 31 \\
Leafy vegetables & $\mathrm{Bq} \mathrm{kg} \mathrm{fresh} \mathrm{mass}^{-1}$ & 6.7 & 19.0 & 56 \\
Cottage Cheese & $\mathrm{Bq} \mathrm{kg}^{-1}$ & 1.6 & 9.1 & 55 \\
Eggs & $\mathrm{Bq} \mathrm{kg}^{-1}$ & 2.8 & 11.0 & 46 \\
Mother's milk* & $\mathrm{Bq} \mathrm{L}^{-1}$ & 0.21 & 1.6 & 11.0 \\
\hline
\end{tabular}

*Mother on Diet 3 - Regionally mixed commercial milk + inhalation 
${ }^{131}$ I Releases from X-10 Radioactive Lanthanum Processing

TASK 1 REPORT

Appendix $11 B$

July 1999

Page 11B-7

\section{Location: Clinton}

\begin{tabular}{lcccc}
\hline Environmental media or food product & Units & \multicolumn{3}{c}{13 years-average concentration } \\
& & lower limit & central estimate & upper limit \\
\hline Air (all physico-chemical forms) & $\mathrm{Bq} \mathrm{m}^{-3}$ & 0.018 & 0.026 & 0.039 \\
Pasture grass & $\mathrm{Bq} \mathrm{kg}^{-1}$ dry mass & 82 & 220 & 580 \\
Backyard cow milk (locally produced) & $\mathrm{Bq} \mathrm{L}^{-1}$ & 4.3 & 13.0 & 45 \\
Commercial milk (locally produced) & $\mathrm{Bq} \mathrm{L}^{-1}$ & 2.6 & 7.4 & 22 \\
Commercial milk (regional mixed) & $\mathrm{Bq} \mathrm{L}^{-1}$ & 2.2 & 5.8 & 16 \\
Goat milk (locally produced) & $\mathrm{Bq} \mathrm{L}^{-1}$ & 13 & 60 & 210 \\
Beef & $\mathrm{Bq} \mathrm{kg}^{-1}$ & 0.64 & 3.9 & 23 \\
Leafy vegetables & $\mathrm{Bq} \mathrm{kg} \mathrm{fresh} \mathrm{mass}^{-1}$ & 5.4 & 15.0 & 45 \\
Cottage Cheese & $\mathrm{Bq} \mathrm{kg}^{-1}$ & 1.3 & 7.1 & 44 \\
Eggs & $\mathrm{Bq} \mathrm{kg}^{-1}$ & 2.1 & 8.5 & 36 \\
Mother's milk* & $\mathrm{Bq} \mathrm{L}^{-1}$ & 0.19 & 1.5 & 11.0 \\
\hline
\end{tabular}

*Mother on Diet 3 - Regionally mixed commercial milk + inhalation

\section{Location: Dutch Valley}

\begin{tabular}{lcccc}
\hline Environmental media or food product & Units & \multicolumn{3}{c}{13 years-average concentration } \\
& & lower limit & central estimate & upper limit \\
\hline Air (all physico-chemical forms) & $\mathrm{Bq} \mathrm{m}^{-3}$ & 0.010 & 0.016 & 0.025 \\
Pasture grass & $\mathrm{Bq} \mathrm{kg}^{-1}$ dry mass & 51 & 140 & 360 \\
Backyard cow milk (locally produced) & $\mathrm{Bq} \mathrm{L}^{-1}$ & 2.7 & 8.1 & 29 \\
Commercial milk (locally produced) & $\mathrm{Bq} \mathrm{L}^{-1}$ & 1.6 & 4.8 & 13 \\
Commercial milk (regional mixed) & $\mathrm{Bq} \mathrm{L}^{-1}$ & 2.2 & 5.8 & 16 \\
Goat milk (locally produced) & $\mathrm{Bq} \mathrm{L}^{-1}$ & 8 & 36 & 150 \\
Beef & $\mathrm{Bq} \mathrm{kg}^{-1}$ & 0.38 & 2.4 & 15 \\
Leafy vegetables & $\mathrm{Bq} \mathrm{kg} \mathrm{fresh} \mathrm{mass}^{-1}$ & 3.3 & 9.4 & 27 \\
Cottage Cheese & $\mathrm{Bq} \mathrm{kg}^{-1}$ & 0.8 & 4.3 & 29 \\
Eggs & $\mathrm{Bq} \mathrm{kg}^{-1}$ & 1.3 & 5.3 & 23 \\
Mother's milk* & $\mathrm{Bq} \mathrm{L}^{-1}$ & 0.17 & 1.4 & 10.0 \\
\hline
\end{tabular}

*Mother on Diet 3 - Regionally mixed commercial milk + inhalation 
${ }^{131}$ I Releases from X-10 Radioactive Lanthanum Processing

TASK 1 REPORT

Appendix $11 B$

July 1999

Page 11B-8

\section{Location: Dyllis}

\begin{tabular}{lcccc}
\hline Environmental media or food product & Units & \multicolumn{3}{c}{13 years-average concentration } \\
& & lower limit & central estimate & upper limit \\
\hline Air (all physico-chemical forms) & $\mathrm{Bq} \mathrm{m}^{-3}$ & 0.010 & 0.017 & 0.030 \\
Pasture grass & $\mathrm{Bq} \mathrm{kg}^{-1}$ dry mass & 56 & 170 & 470 \\
Backyard cow milk (locally produced) & $\mathrm{Bq} \mathrm{L}^{-1}$ & 3.0 & 9.8 & 39 \\
Commercial milk (locally produced) & $\mathrm{Bq} \mathrm{L}^{-1}$ & 2.0 & 5.7 & 18 \\
Commercial milk (regional mixed) & $\mathrm{Bq} \mathrm{L}^{-1}$ & 2.2 & 5.8 & 16 \\
Goat milk (locally produced) & $\mathrm{Bq} \mathrm{L}^{-1}$ & 9 & 47 & 190 \\
Beef & $\mathrm{Bq} \mathrm{kg}^{-1}$ & 0.47 & 3.0 & 18 \\
Leafy vegetables & $\mathrm{Bq} \mathrm{kg} \mathrm{fresh} \mathrm{mass}^{-1}$ & 3.6 & 12.0 & 36 \\
Cottage Cheese & $\mathrm{Bq} \mathrm{kg}^{-1}$ & 0.9 & 5.4 & 39 \\
Eggs & $\mathrm{Bq} \mathrm{kg}^{-1}$ & 1.6 & 6.6 & 30 \\
Mother's milk* & $\mathrm{Bq} \mathrm{L}^{-1}$ & 0.17 & 1.4 & 10.0 \\
\hline
\end{tabular}

*Mother on Diet 3 - Regionally mixed commercial milk + inhalation

\section{Location: EFPC}

\begin{tabular}{lcccc}
\hline Environmental media or food product & Units & \multicolumn{3}{c}{13 years-average concentration } \\
& & lower limit & central estimate & upper limit \\
\hline Air (all physico-chemical forms) & $\mathrm{Bq} \mathrm{m}^{-3}$ & 0.018 & 0.030 & 0.047 \\
Pasture grass & $\mathrm{Bq} \mathrm{kg}^{-1}$ dry mass & 120 & 330 & 910 \\
Backyard cow milk (locally produced) & $\mathrm{Bq} \mathrm{L}^{-1}$ & 5.9 & 19.0 & 66 \\
Commercial milk (locally produced) & $\mathrm{Bq} \mathrm{L}^{-1}$ & 3.7 & 11.0 & 33 \\
Commercial milk (regional mixed) & $\mathrm{Bq} \mathrm{L}^{-1}$ & 2.2 & 5.8 & 16 \\
Goat milk (locally produced) & $\mathrm{Bq} \mathrm{L}^{-1}$ & -- & -- & -- \\
Beef & $\mathrm{Bq} \mathrm{kg}^{-1}$ & 0.91 & 5.6 & 39 \\
Leafy vegetables & $\mathrm{Bq} \mathrm{kg} \mathrm{fresh} \mathrm{mass}^{-1}$ & 7.4 & 22.0 & 65 \\
Cottage Cheese & $\mathrm{Bq} \mathrm{kg}^{-1}$ & 1.8 & 10.0 & 65 \\
Eggs & $\mathrm{Bq} \mathrm{kg}^{-1}$ & 3.1 & 13.0 & 51 \\
Mother's milk* & $\mathrm{Bq} \mathrm{L}^{-1}$ & 0.21 & 1.6 & 11.0 \\
\hline
\end{tabular}

*Mother on Diet 3 - Regionally mixed commercial milk + inhalation 
${ }^{131}$ I Releases from X-10 Radioactive Lanthanum Processing

TASK 1 REPORT

Appendix $11 B$

July 1999

Page 11B-9

\section{Location: Farragut}

\begin{tabular}{lcccc}
\hline Environmental media or food product & Units & \multicolumn{3}{c}{13 years-average concentration } \\
& & lower limit & central estimate & upper limit \\
\hline Air (all physico-chemical forms) & $\mathrm{Bq} \mathrm{m}^{-3}$ & 0.029 & 0.045 & 0.067 \\
Pasture grass & $\mathrm{Bq} \mathrm{kg}^{-1}$ dry mass & 150 & 410 & 1200 \\
Backyard cow milk (locally produced) & $\mathrm{Bq} \mathrm{L}^{-1}$ & 7.9 & 24.0 & 84 \\
Commercial milk (locally produced) & $\mathrm{Bq} \mathrm{L}^{-1}$ & 4.7 & 14.0 & 44 \\
Commercial milk (regional mixed) & $\mathrm{Bq} \mathrm{L}^{-1}$ & 2.2 & 5.8 & 16 \\
Goat milk (locally produced) & $\mathrm{Bq} \mathrm{L}^{-1}$ & 25 & 110 & 440 \\
Beef & $\mathrm{Bq} \mathrm{kg}^{-1}$ & 1.20 & 7.2 & 46 \\
Leafy vegetables & $\mathrm{Bq} \mathrm{kg} \mathrm{fresh} \mathrm{mass}^{-1}$ & 9.5 & 28.0 & 87 \\
Cottage Cheese & $\mathrm{Bq} \mathrm{kg}^{-1}$ & 2.2 & 13.0 & 80 \\
Eggs & $\mathrm{Bq} \mathrm{kg}^{-1}$ & 3.8 & 16.0 & 69 \\
Mother's milk* & $\mathrm{Bq} \mathrm{L}^{-1}$ & 0.24 & 1.8 & 12.0 \\
\hline
\end{tabular}

*Mother on Diet 3 - Regionally mixed commercial milk + inhalation

\section{Location: Friendsville}

\begin{tabular}{lcccc}
\hline Environmental media or food product & Units & \multicolumn{3}{c}{13 years-average concentration } \\
& & lower limit & central estimate & upper limit \\
\hline Air (all physico-chemical forms) & $\mathrm{Bq} \mathrm{m}^{-3}$ & 0.011 & 0.018 & 0.030 \\
Pasture grass & $\mathrm{Bq} \mathrm{kg}^{-1}$ dry mass & 40 & 120 & 400 \\
Backyard cow milk (locally produced) & $\mathrm{Bq} \mathrm{L}^{-1}$ & 2.2 & 7.2 & 28 \\
Commercial milk (locally produced) & $\mathrm{Bq} \mathrm{L}^{-1}$ & 1.2 & 4.3 & 15 \\
Commercial milk (regional mixed) & $\mathrm{Bq} \mathrm{L}^{-1}$ & 2.2 & 5.8 & 16 \\
Goat milk (locally produced) & $\mathrm{Bq} \mathrm{L}^{-1}$ & 7 & 34 & 150 \\
Beef & $\mathrm{Bq} \mathrm{kg}^{-1}$ & 0.36 & 2.1 & 14 \\
Leafy vegetables & $\mathrm{Bq} \mathrm{kg} \mathrm{fresh} \mathrm{mass}^{-1}$ & 2.6 & 8.8 & 30 \\
Cottage Cheese & $\mathrm{Bq} \mathrm{kg}^{-1}$ & 0.6 & 4.2 & 27 \\
Eggs & $\mathrm{Bq} \mathrm{kg}^{-1}$ & 1.1 & 4.8 & 23 \\
Mother's milk* & $\mathrm{Bq} \mathrm{L}^{-1}$ & 0.18 & 1.4 & 10.0 \\
\hline
\end{tabular}

*Mother on Diet 3 - Regionally mixed commercial milk + inhalation 
${ }^{131}$ I Releases from X-10 Radioactive Lanthanum Processing

TASK 1 REPORT

Appendix $11 B$

July 1999

Page 11B-10

\section{Location: Gallaher Bend}

\begin{tabular}{lcccc}
\hline Environmental media or food product & Units & \multicolumn{3}{c}{13 years-average concentration } \\
& & lower limit & central estimate & upper limit \\
\hline Air (all physico-chemical forms) & $\mathrm{Bq} \mathrm{m}^{-3}$ & 0.096 & 0.140 & 0.200 \\
Pasture grass & $\mathrm{Bq} \mathrm{kg}^{-1}$ dry mass & 580 & 1600 & 3900 \\
Backyard cow milk (locally produced) & $\mathrm{Bq} \mathrm{L}^{-1}$ & 31.0 & 92.0 & 290 \\
Commercial milk (locally produced) & $\mathrm{Bq} \mathrm{L}^{-1}$ & 19.0 & 54.0 & 140 \\
Commercial milk (regional mixed) & $\mathrm{Bq} \mathrm{L}^{-1}$ & 2.2 & 5.8 & 16 \\
Goat milk (locally produced) & $\mathrm{Bq} \mathrm{L}^{-1}$ & 94 & 420 & 1700 \\
Beef & $\mathrm{Bq} \mathrm{kg}^{-1}$ & 4.50 & 26.0 & 160 \\
Leafy vegetables & $\mathrm{Bq} \mathrm{kg} \mathrm{fresh} \mathrm{mass}^{-1}$ & 35.0 & 110.0 & 290 \\
Cottage Cheese & $\mathrm{Bq} \mathrm{kg}^{-1}$ & 8.3 & 48.0 & 300 \\
Eggs & $\mathrm{Bq} \mathrm{kg}^{-1}$ & 15.0 & 59.0 & 240 \\
Mother's milk* & $\mathrm{Bq} \mathrm{L}^{-1}$ & 0.42 & 2.9 & 16.0 \\
\hline
\end{tabular}

*Mother on Diet 3 - Regionally mixed commercial milk + inhalation

\section{Location: Greenback}

\begin{tabular}{lcccc}
\hline Environmental media or food product & Units & \multicolumn{3}{c}{13 years-average concentration } \\
& & lower limit & central estimate & upper limit \\
\hline Air (all physico-chemical forms) & $\mathrm{Bq} \mathrm{m}^{-3}$ & 0.008 & 0.013 & 0.022 \\
Pasture grass & $\mathrm{Bq} \mathrm{kg}^{-1}$ dry mass & 26 & 80 & 270 \\
Backyard cow milk (locally produced) & $\mathrm{Bq} \mathrm{L}^{-1}$ & 1.4 & 5.0 & 20 \\
Commercial milk (locally produced) & $\mathrm{Bq} \mathrm{L}^{-1}$ & 0.9 & 2.8 & 10 \\
Commercial milk (regional mixed) & $\mathrm{Bq} \mathrm{L}^{-1}$ & 2.2 & 5.8 & 16 \\
Goat milk (locally produced) & $\mathrm{Bq} \mathrm{L}^{-1}$ & 4 & 22 & 95 \\
Beef & $\mathrm{Bq} \mathrm{kg}^{-1}$ & 0.23 & 1.4 & 10 \\
Leafy vegetables & $\mathrm{Bq} \mathrm{kg} \mathrm{fresh} \mathrm{mass}^{-1}$ & 1.7 & 5.7 & 20 \\
Cottage Cheese & $\mathrm{Bq} \mathrm{kg}^{-1}$ & 0.4 & 2.7 & 20 \\
Eggs & $\mathrm{Bq} \mathrm{kg}^{-1}$ & 0.8 & 3.2 & 15 \\
Mother's milk* & $\mathrm{Bq} \mathrm{L}^{-1}$ & 0.16 & 1.3 & 10.0 \\
\hline
\end{tabular}

*Mother on Diet 3 - Regionally mixed commercial milk + inhalation 
${ }^{131}$ I Releases from X-10 Radioactive Lanthanum Processing

TASK 1 REPORT

Appendix $11 B$

July 1999

Page 11B-11

\section{Location: Hardin Valley}

\begin{tabular}{lcccc}
\hline Environmental media or food product & Units & \multicolumn{3}{c}{13 years-average concentration } \\
& & lower limit & central estimate & upper limit \\
\hline Air (all physico-chemical forms) & $\mathrm{Bq} \mathrm{m}^{-3}$ & 0.054 & 0.080 & 0.110 \\
Pasture grass & $\mathrm{Bq} \mathrm{kg}^{-1}$ dry mass & 300 & 780 & 2000 \\
Backyard cow milk (locally produced) & $\mathrm{Bq} \mathrm{L}^{-1}$ & 15.0 & 47.0 & 160 \\
Commercial milk (locally produced) & $\mathrm{Bq} \mathrm{L}^{-1}$ & 9.3 & 27.0 & 77 \\
Commercial milk (regional mixed) & $\mathrm{Bq} \mathrm{L}^{-1}$ & 2.2 & 5.8 & 16 \\
Goat milk (locally produced) & $\mathrm{Bq} \mathrm{L}^{-1}$ & 49 & 220 & 800 \\
Beef & $\mathrm{Bq} \mathrm{kg}^{-1}$ & 2.40 & 14.0 & 79 \\
Leafy vegetables & $\mathrm{Bq} \mathrm{kg} \mathrm{fresh} \mathrm{mass}^{-1}$ & 19.0 & 54.0 & 150 \\
Cottage Cheese & $\mathrm{Bq} \mathrm{kg}^{-1}$ & 4.6 & 25.0 & 160 \\
Eggs & $\mathrm{Bq} \mathrm{kg}^{-1}$ & 7.6 & 31.0 & 130 \\
Mother's milk* & $\mathrm{Bq} \mathrm{L}^{-1}$ & 0.32 & 2.2 & 13.0 \\
\hline
\end{tabular}

*Mother on Diet 3 - Regionally mixed commercial milk + inhalation

\section{Location: Harriman}

\begin{tabular}{lcccc}
\hline Environmental media or food product & Units & \multicolumn{3}{c}{13 years-average concentration } \\
& & lower limit & central estimate & upper limit \\
\hline Air (all physico-chemical forms) & $\mathrm{Bq} \mathrm{m}^{-3}$ & 0.010 & 0.016 & 0.025 \\
Pasture grass & $\mathrm{Bq} \mathrm{kg}^{-1}$ dry mass & 55 & 140 & 370 \\
Backyard cow milk (locally produced) & $\mathrm{Bq} \mathrm{L}^{-1}$ & 2.9 & 8.5 & 30 \\
Commercial milk (locally produced) & $\mathrm{Bq} \mathrm{L}^{-1}$ & 1.8 & 4.9 & 14 \\
Commercial milk (regional mixed) & $\mathrm{Bq} \mathrm{L}^{-1}$ & 2.2 & 5.8 & 16 \\
Goat milk (locally produced) & $\mathrm{Bq} \mathrm{L}^{-1}$ & 8 & 38 & 160 \\
Beef & $\mathrm{Bq} \mathrm{kg}^{-1}$ & 0.40 & 2.6 & 15 \\
Leafy vegetables & $\mathrm{Bq} \mathrm{kg} \mathrm{fresh} \mathrm{mass}^{-1}$ & 3.6 & 9.6 & 28 \\
Cottage Cheese & $\mathrm{Bq} \mathrm{kg}^{-1}$ & 0.8 & 4.5 & 31 \\
Eggs & $\mathrm{Bq} \mathrm{kg}^{-1}$ & 1.4 & 5.5 & 26 \\
Mother's milk* & $\mathrm{Bq} \mathrm{L}^{-1}$ & 0.16 & 1.4 & 10.0 \\
\hline
\end{tabular}

*Mother on Diet 3 - Regionally mixed commercial milk + inhalation 
${ }^{131}$ I Releases from X-10 Radioactive Lanthanum Processing

TASK 1 REPORT

Appendix $11 B$

July 1999

Page 11B-12

\section{Location: Hines Valley}

\begin{tabular}{lcccc}
\hline Environmental media or food product & Units & \multicolumn{3}{c}{13 years-average concentration } \\
& & lower limit & central estimate & upper limit \\
\hline Air (all physico-chemical forms) & $\mathrm{Bq} \mathrm{m}^{-3}$ & 0.027 & 0.041 & 0.060 \\
Pasture grass & $\mathrm{Bq} \mathrm{kg}^{-1}$ dry mass & 130 & 370 & 980 \\
Backyard cow milk (locally produced) & $\mathrm{Bq} \mathrm{L}^{-1}$ & 6.6 & 21.0 & 76 \\
Commercial milk (locally produced) & $\mathrm{Bq} \mathrm{L}^{-1}$ & 4.1 & 12.0 & 37 \\
Commercial milk (regional mixed) & $\mathrm{Bq} \mathrm{L}^{-1}$ & 2.2 & 5.8 & 16 \\
Goat milk (locally produced) & $\mathrm{Bq} \mathrm{L}^{-1}$ & 22 & 97 & 390 \\
Beef & $\mathrm{Bq} \mathrm{kg}^{-1}$ & 1.00 & 6.4 & 39 \\
Leafy vegetables & $\mathrm{Bq} \mathrm{kg} \mathrm{fresh} \mathrm{mass}^{-1}$ & 8.0 & 25.0 & 76 \\
Cottage Cheese & $\mathrm{Bq} \mathrm{kg}^{-1}$ & 2.1 & 12.0 & 70 \\
Eggs & $\mathrm{Bq} \mathrm{kg}^{-1}$ & 3.3 & 14.0 & 58 \\
Mother's milk* & $\mathrm{Bq} \mathrm{L}^{-1}$ & 0.23 & 1.7 & 11.0 \\
\hline
\end{tabular}

*Mother on Diet 3 - Regionally mixed commercial milk + inhalation

\section{Location: Hope Creek}

\begin{tabular}{lcccc}
\hline Environmental media or food product & Units & \multicolumn{3}{c}{13 years-average concentration } \\
& & lower limit & central estimate & upper limit \\
\hline Air (all physico-chemical forms) & $\mathrm{Bq} \mathrm{m}^{-3}$ & 0.056 & 0.082 & 0.120 \\
Pasture grass & $\mathrm{Bq} \mathrm{kg}^{-1}$ dry mass & 320 & 900 & 2300 \\
Backyard cow milk (locally produced) & $\mathrm{Bq} \mathrm{L}^{-1}$ & 17.0 & 52.0 & 190 \\
Commercial milk (locally produced) & $\mathrm{Bq} \mathrm{L}^{-1}$ & 10.0 & 30.0 & 87 \\
Commercial milk (regional mixed) & $\mathrm{Bq} \mathrm{L}^{-1}$ & 2.2 & 5.8 & 16 \\
Goat milk (locally produced) & $\mathrm{Bq} \mathrm{L}^{-1}$ & -- & -- & -- \\
Beef & $\mathrm{Bq} \mathrm{kg}^{-1}$ & 2.60 & 16.0 & 97 \\
Leafy vegetables & $\mathrm{Bq} \mathrm{kg} \mathrm{fresh} \mathrm{mass}^{-1}$ & 20.0 & 61.0 & 180 \\
Cottage Cheese & $\mathrm{Bq} \mathrm{kg}^{-1}$ & 5.0 & 28.0 & 170 \\
Eggs & $\mathrm{Bq} \mathrm{kg}^{-1}$ & 8.6 & 34.0 & 140 \\
Mother's milk* & $\mathrm{Bq} \mathrm{L}^{-1}$ & 0.33 & 2.2 & 13.0 \\
\hline
\end{tabular}

*Mother on Diet 3 - Regionally mixed commercial milk + inhalation 
${ }^{131}$ I Releases from X-10 Radioactive Lanthanum Processing

TASK 1 REPORT

Appendix $11 B$

July 1999

Page 11B-13

\section{Location: Jonesville}

\begin{tabular}{lcccc}
\hline Environmental media or food product & Units & \multicolumn{3}{c}{13 years-average concentration } \\
& & lower limit & central estimate & upper limit \\
\hline Air (all physico-chemical forms) & $\mathrm{Bq} \mathrm{m}^{-3}$ & 0.007 & 0.012 & 0.022 \\
Pasture grass & $\mathrm{Bq} \mathrm{kg}^{-1}$ dry mass & 40 & 120 & 380 \\
Backyard cow milk (locally produced) & $\mathrm{Bq} \mathrm{L}^{-1}$ & 2.2 & 7.4 & 29 \\
Commercial milk (locally produced) & $\mathrm{Bq} \mathrm{L}^{-1}$ & 1.3 & 4.3 & 14 \\
Commercial milk (regional mixed) & $\mathrm{Bq} \mathrm{L}^{-1}$ & 2.2 & 5.8 & 16 \\
Goat milk (locally produced) & $\mathrm{Bq} \mathrm{L}^{-1}$ & 6 & 34 & 150 \\
Beef & $\mathrm{Bq} \mathrm{kg}^{-1}$ & 0.32 & 2.3 & 16 \\
Leafy vegetables & $\mathrm{Bq} \mathrm{kg} \mathrm{fresh} \mathrm{mass}^{-1}$ & 2.6 & 8.5 & 28 \\
Cottage Cheese & $\mathrm{Bq} \mathrm{kg}^{-1}$ & 0.6 & 4.1 & 29 \\
Eggs & $\mathrm{Bq} \mathrm{kg}^{-1}$ & 1.2 & 4.8 & 23 \\
Mother's milk* & $\mathrm{Bq} \mathrm{L}^{-1}$ & 0.16 & 1.3 & 10.0 \\
\hline
\end{tabular}

*Mother on Diet 3 - Regionally mixed commercial milk + inhalation

\section{Location: Karns}

\begin{tabular}{lcccc}
\hline Environmental media or food product & Units & \multicolumn{3}{c}{13 years-average concentration } \\
& & lower limit & central estimate & upper limit \\
\hline Air (all physico-chemical forms) & $\mathrm{Bq} \mathrm{m}^{-3}$ & 0.034 & 0.050 & 0.073 \\
Pasture grass & $\mathrm{Bq} \mathrm{kg}^{-1}$ dry mass & 160 & 440 & 1200 \\
Backyard cow milk (locally produced) & $\mathrm{Bq} \mathrm{L}^{-1}$ & 8.5 & 26.0 & 82 \\
Commercial milk (locally produced) & $\mathrm{Bq} \mathrm{L}^{-1}$ & 5.1 & 15.0 & 44 \\
Commercial milk (regional mixed) & $\mathrm{Bq} \mathrm{L}^{-1}$ & 2.2 & 5.8 & 16 \\
Goat milk (locally produced) & $\mathrm{Bq} \mathrm{L}^{-1}$ & 25 & 120 & 460 \\
Beef & $\mathrm{Bq} \mathrm{kg}^{-1}$ & 1.30 & 7.4 & 48 \\
Leafy vegetables & $\mathrm{Bq} \mathrm{kg} \mathrm{fresh} \mathrm{mass}^{-1}$ & 9.9 & 30.0 & 87 \\
Cottage Cheese & $\mathrm{Bq} \mathrm{kg}^{-1}$ & 2.4 & 14.0 & 84 \\
Eggs & $\mathrm{Bq} \mathrm{kg}^{-1}$ & 4.2 & 17.0 & 69 \\
Mother's milk* & $\mathrm{Bq} \mathrm{L}^{-1}$ & 0.25 & 1.8 & 12.0 \\
\hline
\end{tabular}

*Mother on Diet 3 - Regionally mixed commercial milk + inhalation 
${ }^{131}$ I Releases from X-10 Radioactive Lanthanum Processing

TASK 1 REPORT

Appendix $11 B$

July 1999

Page 11B-14

\section{Location: Kingston}

\begin{tabular}{lcccc}
\hline Environmental media or food product & Units & \multicolumn{3}{c}{13 years-average concentration } \\
& & lower limit & central estimate & upper limit \\
\hline Air (all physico-chemical forms) & $\mathrm{Bq} \mathrm{m}^{-3}$ & 0.025 & 0.037 & 0.057 \\
Pasture grass & $\mathrm{Bq} \mathrm{kg}^{-1}$ dry mass & 110 & 320 & 850 \\
Backyard cow milk (locally produced) & $\mathrm{Bq} \mathrm{L}^{-1}$ & 5.9 & 19.0 & 64 \\
Commercial milk (locally produced) & $\mathrm{Bq} \mathrm{L}^{-1}$ & 3.6 & 11.0 & 32 \\
Commercial milk (regional mixed) & $\mathrm{Bq} \mathrm{L}^{-1}$ & 2.2 & 5.8 & 16 \\
Goat milk (locally produced) & $\mathrm{Bq} \mathrm{L}^{-1}$ & 20 & 88 & 330 \\
Beef & $\mathrm{Bq} \mathrm{kg}^{-1}$ & 0.90 & 5.5 & 33 \\
Leafy vegetables & $\mathrm{Bq} \mathrm{kg} \mathrm{fresh} \mathrm{mass}^{-1}$ & 7.4 & 22.0 & 66 \\
Cottage Cheese & $\mathrm{Bq} \mathrm{kg}^{-1}$ & 1.6 & 10.0 & 68 \\
Eggs & $\mathrm{Bq} \mathrm{kg}^{-1}$ & 2.9 & 12.0 & 54 \\
Mother's milk* & $\mathrm{Bq} \mathrm{L}^{-1}$ & 0.22 & 1.6 & 11.0 \\
\hline
\end{tabular}

*Mother on Diet 3 - Regionally mixed commercial milk + inhalation

\section{Location: Knoxville}

\begin{tabular}{lcccc}
\hline Environmental media or food product & Units & \multicolumn{3}{c}{13 years-average concentration } \\
& & lower limit & central estimate & upper limit \\
\hline Air (all physico-chemical forms) & $\mathrm{Bq} \mathrm{m}^{-3}$ & 0.015 & 0.024 & 0.036 \\
Pasture grass & $\mathrm{Bq} \mathrm{kg}^{-1}$ dry mass & 59 & 160 & 440 \\
Backyard cow milk (locally produced) & $\mathrm{Bq} \mathrm{L}^{-1}$ & 2.9 & 9.6 & 33 \\
Commercial milk (locally produced) & $\mathrm{Bq} \mathrm{L}^{-1}$ & 1.9 & 5.6 & 16 \\
Commercial milk (regional mixed) & $\mathrm{Bq} \mathrm{L}^{-1}$ & 2.2 & 5.8 & 16 \\
Goat milk (locally produced) & $\mathrm{Bq} \mathrm{L}^{-1}$ & 10 & 44 & 170 \\
Beef & $\mathrm{Bq} \mathrm{kg}^{-1}$ & 0.48 & 2.9 & 17 \\
Leafy vegetables & $\mathrm{Bq} \mathrm{kg} \mathrm{fresh} \mathrm{mass}^{-1}$ & 3.9 & 11.0 & 34 \\
Cottage Cheese & $\mathrm{Bq} \mathrm{kg}^{-1}$ & 1.0 & 5.3 & 35 \\
Eggs & $\mathrm{Bq} \mathrm{kg}^{-1}$ & 1.5 & 6.4 & 26 \\
Mother's milk* & $\mathrm{Bq} \mathrm{L}^{-1}$ & 0.19 & 1.5 & 10.0 \\
\hline
\end{tabular}

*Mother on Diet 3 - Regionally mixed commercial milk + inhalation 
${ }^{131}$ I Releases from X-10 Radioactive Lanthanum Processing

TASK 1 REPORT

Appendix $11 B$

July 1999

Page 11B-15

\section{Location: Lake City}

\begin{tabular}{lcccc}
\hline Environmental media or food product & Units & \multicolumn{3}{c}{13 years-average concentration } \\
& & lower limit & central estimate & upper limit \\
\hline Air (all physico-chemical forms) & $\mathrm{Bq} \mathrm{m}^{-3}$ & 0.006 & 0.010 & 0.016 \\
Pasture grass & $\mathrm{Bq} \mathrm{kg}^{-1}$ dry mass & 28 & 80 & 210 \\
Backyard cow milk (locally produced) & $\mathrm{Bq} \mathrm{L}^{-1}$ & 1.3 & 4.7 & 16 \\
Commercial milk (locally produced) & $\mathrm{Bq} \mathrm{L}^{-1}$ & 0.9 & 2.8 & 8 \\
Commercial milk (regional mixed) & $\mathrm{Bq} \mathrm{L}^{-1}$ & 2.2 & 5.8 & 16 \\
Goat milk (locally produced) & $\mathrm{Bq} \mathrm{L}^{-1}$ & 5 & 22 & 92 \\
Beef & $\mathrm{Bq} \mathrm{kg}^{-1}$ & 0.23 & 1.4 & 9 \\
Leafy vegetables & $\mathrm{Bq} \mathrm{kg} \mathrm{fresh} \mathrm{mass}^{-1}$ & 1.7 & 5.4 & 16 \\
Cottage Cheese & $\mathrm{Bq} \mathrm{kg}^{-1}$ & 0.4 & 2.5 & 17 \\
Eggs & $\mathrm{Bq} \mathrm{kg}^{-1}$ & 0.7 & 3.1 & 13 \\
Mother's milk* & $\mathrm{Bq} \mathrm{L}^{-1}$ & 0.15 & 1.3 & 10.0 \\
\hline
\end{tabular}

*Mother on Diet 3 - Regionally mixed commercial milk + inhalation

\section{Location: Lawnville/Gallaher}

\begin{tabular}{lcccc}
\hline Environmental media or food product & Units & \multicolumn{3}{c}{13 years-average concentration } \\
& & lower limit & central estimate & upper limit \\
\hline Air (all physico-chemical forms) & $\mathrm{Bq} \mathrm{m}^{-3}$ & 0.055 & 0.080 & 0.120 \\
Pasture grass & $\mathrm{Bq} \mathrm{kg}^{-1}$ dry mass & 330 & 820 & 2100 \\
Backyard cow milk (locally produced) & $\mathrm{Bq} \mathrm{L}^{-1}$ & 18.0 & 49.0 & 170 \\
Commercial milk (locally produced) & $\mathrm{Bq} \mathrm{L}^{-1}$ & 11.0 & 29.0 & 75 \\
Commercial milk (regional mixed) & $\mathrm{Bq} \mathrm{L}^{-1}$ & 2.2 & 5.8 & 16 \\
Goat milk (locally produced) & $\mathrm{Bq} \mathrm{L}^{-1}$ & 51 & 220 & 920 \\
Beef & $\mathrm{Bq} \mathrm{kg}^{-1}$ & 2.40 & 15.0 & 84 \\
Leafy vegetables & $\mathrm{Bq} \mathrm{kg} \mathrm{fresh} \mathrm{mass}^{-1}$ & 21.0 & 57.0 & 150 \\
Cottage Cheese & $\mathrm{Bq} \mathrm{kg}^{-1}$ & 4.9 & 26.0 & 180 \\
Eggs & $\mathrm{Bq} \mathrm{kg}^{-1}$ & 8.8 & 32.0 & 130 \\
Mother's milk* & $\mathrm{Bq} \mathrm{L}^{-1}$ & 0.31 & 2.2 & 13.0 \\
\hline
\end{tabular}

*Mother on Diet 3 - Regionally mixed commercial milk + inhalation 
${ }^{131}$ I Releases from X-10 Radioactive Lanthanum Processing

TASK 1 REPORT

Appendix $11 B$

July 1999

Page 11B-16

\section{Location: Lenoir City}

\begin{tabular}{lcccc}
\hline Environmental media or food product & Units & \multicolumn{3}{c}{13 years-average concentration } \\
& & lower limit & central estimate & upper limit \\
\hline Air (all physico-chemical forms) & $\mathrm{Bq} \mathrm{m}^{-3}$ & 0.018 & 0.029 & 0.047 \\
Pasture grass & $\mathrm{Bq} \mathrm{kg}^{-1}$ dry mass & 95 & 250 & 670 \\
Backyard cow milk (locally produced) & $\mathrm{Bq} \mathrm{L}^{-1}$ & 5.1 & 15.0 & 56 \\
Commercial milk (locally produced) & $\mathrm{Bq} \mathrm{L}^{-1}$ & 3.0 & 8.6 & 25 \\
Commercial milk (regional mixed) & $\mathrm{Bq} \mathrm{L}^{-1}$ & 2.2 & 5.8 & 16 \\
Goat milk (locally produced) & $\mathrm{Bq} \mathrm{L}^{-1}$ & -- & -- & -- \\
Beef & $\mathrm{Bq} \mathrm{kg}^{-1}$ & 0.68 & 4.5 & 27 \\
Leafy vegetables & $\mathrm{Bq} \mathrm{kg} \mathrm{fresh} \mathrm{mass}^{-1}$ & 6.3 & 17.0 & 49 \\
Cottage Cheese & $\mathrm{Bq} \mathrm{kg}^{-1}$ & 1.4 & 7.8 & 55 \\
Eggs & $\mathrm{Bq} \mathrm{kg}^{-1}$ & 2.5 & 9.7 & 47 \\
Mother's milk* & $\mathrm{Bq} \mathrm{L}^{-1}$ & 0.19 & 1.5 & 11.0 \\
\hline
\end{tabular}

*Mother on Diet 3 - Regionally mixed commercial milk + inhalation

\section{Location: Loudon}

\begin{tabular}{lcccc}
\hline Environmental media or food product & Units & \multicolumn{3}{c}{13 years-average concentration } \\
& & lower limit & central estimate & upper limit \\
\hline Air (all physico-chemical forms) & $\mathrm{Bq} \mathrm{m}^{-3}$ & 0.019 & 0.030 & 0.047 \\
Pasture grass & $\mathrm{Bq} \mathrm{kg}^{-1}$ dry mass & 86 & 240 & 680 \\
Backyard cow milk (locally produced) & $\mathrm{Bq} \mathrm{L}^{-1}$ & 4.4 & 14.0 & 52 \\
Commercial milk (locally produced) & $\mathrm{Bq} \mathrm{L}^{-1}$ & 2.8 & 8.3 & 25 \\
Commercial milk (regional mixed) & $\mathrm{Bq} \mathrm{L}^{-1}$ & 2.2 & 5.8 & 16 \\
Goat milk (locally produced) & $\mathrm{Bq} \mathrm{L}^{-1}$ & 14 & 65 & 260 \\
Beef & $\mathrm{Bq} \mathrm{kg}^{-1}$ & 0.69 & 4.2 & 27 \\
Leafy vegetables & $\mathrm{Bq} \mathrm{kg} \mathrm{fresh} \mathrm{mass}^{-1}$ & 5.5 & 17.0 & 50 \\
Cottage Cheese & $\mathrm{Bq} \mathrm{kg}^{-1}$ & 1.3 & 7.7 & 49 \\
Eggs & $\mathrm{Bq} \mathrm{kg}^{-1}$ & 2.3 & 9.4 & 39 \\
Mother's milk* & $\mathrm{Bq} \mathrm{L}^{-1}$ & 0.20 & 1.5 & 11.0 \\
\hline
\end{tabular}

*Mother on Diet 3 - Regionally mixed commercial milk + inhalation 
${ }^{131}$ I Releases from X-10 Radioactive Lanthanum Processing

TASK 1 REPORT

Appendix $11 B$

July 1999

Page 11B-17

\section{Location: Louisville}

\begin{tabular}{lcccc}
\hline Environmental media or food product & Units & \multicolumn{3}{c}{13 years-average concentration } \\
& & lower limit & central estimate & upper limit \\
\hline Air (all physico-chemical forms) & $\mathrm{Bq} \mathrm{m}^{-3}$ & 0.011 & 0.019 & 0.031 \\
Pasture grass & $\mathrm{Bq} \mathrm{kg}^{-1}$ dry mass & 43 & 130 & 380 \\
Backyard cow milk (locally produced) & $\mathrm{Bq} \mathrm{L}^{-1}$ & 2.2 & 7.7 & 29 \\
Commercial milk (locally produced) & $\mathrm{Bq} \mathrm{L}^{-1}$ & 1.5 & 4.6 & 14 \\
Commercial milk (regional mixed) & $\mathrm{Bq} \mathrm{L}^{-1}$ & 2.2 & 5.8 & 16 \\
Goat milk (locally produced) & $\mathrm{Bq} \mathrm{L}^{-1}$ & 7 & 36 & 160 \\
Beef & $\mathrm{Bq} \mathrm{kg}^{-1}$ & 0.37 & 2.3 & 14 \\
Leafy vegetables & $\mathrm{Bq} \mathrm{kg} \mathrm{fresh} \mathrm{mass}^{-1}$ & 2.9 & 9.0 & 29 \\
Cottage Cheese & $\mathrm{Bq} \mathrm{kg}^{-1}$ & 0.7 & 4.2 & 29 \\
Eggs & $\mathrm{Bq} \mathrm{kg}^{-1}$ & 1.2 & 5.1 & 24 \\
Mother's milk* & $\mathrm{Bq} \mathrm{L}^{-1}$ & 0.17 & 1.4 & 10.0 \\
\hline
\end{tabular}

*Mother on Diet 3 - Regionally mixed commercial milk + inhalation

\section{Location: Maryville}

\begin{tabular}{lcccc}
\hline Environmental media or food product & Units & \multicolumn{3}{c}{13 years-average concentration } \\
& & lower limit & central estimate & upper limit \\
\hline Air (all physico-chemical forms) & $\mathrm{Bq} \mathrm{m}^{-3}$ & 0.007 & 0.013 & 0.023 \\
Pasture grass & $\mathrm{Bq} \mathrm{kg}^{-1}$ dry mass & 26 & 80 & 240 \\
Backyard cow milk (locally produced) & $\mathrm{Bq} \mathrm{L}^{-1}$ & 1.3 & 4.7 & 18 \\
Commercial milk (locally produced) & $\mathrm{Bq} \mathrm{L}^{-1}$ & 0.9 & 2.8 & 9 \\
Commercial milk (regional mixed) & $\mathrm{Bq} \mathrm{L}^{-1}$ & 2.2 & 5.8 & 16 \\
Goat milk (locally produced) & $\mathrm{Bq} \mathrm{L}^{-1}$ & 4 & 22 & 91 \\
Beef & $\mathrm{Bq} \mathrm{kg}^{-1}$ & 0.23 & 1.3 & 9 \\
Leafy vegetables & $\mathrm{Bq} \mathrm{kg} \mathrm{fresh} \mathrm{mass}^{-1}$ & 1.7 & 5.6 & 17 \\
Cottage Cheese & $\mathrm{Bq} \mathrm{kg}^{-1}$ & 0.4 & 2.5 & 16 \\
Eggs & $\mathrm{Bq} \mathrm{kg}^{-1}$ & 0.7 & 3.1 & 14 \\
Mother's milk* & $\mathrm{Bq} \mathrm{L}^{-1}$ & 0.16 & 1.3 & 10.0 \\
\hline
\end{tabular}

*Mother on Diet 3 - Regionally mixed commercial milk + inhalation 
${ }^{131}$ I Releases from X-10 Radioactive Lanthanum Processing

TASK 1 REPORT

Appendix $11 B$

July 1999

Page 11B-18

\section{Location: Norwood}

\begin{tabular}{lcccc}
\hline Environmental media or food product & Units & \multicolumn{3}{c}{13 years-average concentration } \\
& & lower limit & central estimate & upper limit \\
\hline Air (all physico-chemical forms) & $\mathrm{Bq} \mathrm{m}^{-3}$ & 0.010 & 0.016 & 0.028 \\
Pasture grass & $\mathrm{Bq} \mathrm{kg}^{-1}$ dry mass & 51 & 160 & 490 \\
Backyard cow milk (locally produced) & $\mathrm{Bq} \mathrm{L}^{-1}$ & 3.0 & 9.4 & 37 \\
Commercial milk (locally produced) & $\mathrm{Bq} \mathrm{L}^{-1}$ & 1.7 & 5.6 & 19 \\
Commercial milk (regional mixed) & $\mathrm{Bq} \mathrm{L}^{-1}$ & 2.2 & 5.8 & 16 \\
Goat milk (locally produced) & $\mathrm{Bq} \mathrm{L}^{-1}$ & 9 & 44 & 190 \\
Beef & $\mathrm{Bq} \mathrm{kg}^{-1}$ & 0.44 & 2.8 & 18 \\
Leafy vegetables & $\mathrm{Bq} \mathrm{kg} \mathrm{fresh} \mathrm{mass}^{-1}$ & 3.4 & 11.0 & 37 \\
Cottage Cheese & $\mathrm{Bq} \mathrm{kg}^{-1}$ & 0.8 & 5.1 & 34 \\
Eggs & $\mathrm{Bq} \mathrm{kg}^{-1}$ & 1.4 & 6.3 & 29 \\
Mother's milk* & $\mathrm{Bq} \mathrm{L}^{-1}$ & 0.17 & 1.4 & 10.0 \\
\hline
\end{tabular}

*Mother on Diet 3 - Regionally mixed commercial milk + inhalation

\section{Location: Oakdale}

\begin{tabular}{lcccc}
\hline Environmental media or food product & Units & \multicolumn{3}{c}{13 years-average concentration } \\
& & lower limit & central estimate & upper limit \\
\hline Air (all physico-chemical forms) & $\mathrm{Bq} \mathrm{m}^{-3}$ & 0.005 & 0.009 & 0.016 \\
Pasture grass & $\mathrm{Bq} \mathrm{kg}^{-1}$ dry mass & 27 & 79 & 240 \\
Backyard cow milk (locally produced) & $\mathrm{Bq} \mathrm{L}^{-1}$ & 1.4 & 4.6 & 18 \\
Commercial milk (locally produced) & $\mathrm{Bq} \mathrm{L}^{-1}$ & 0.9 & 2.7 & 9 \\
Commercial milk (regional mixed) & $\mathrm{Bq} \mathrm{L}^{-1}$ & 2.2 & 5.8 & 16 \\
Goat milk (locally produced) & $\mathrm{Bq} \mathrm{L}^{-1}$ & 5 & 22 & 90 \\
Beef & $\mathrm{Bq} \mathrm{kg}^{-1}$ & 0.22 & 1.4 & 9 \\
Leafy vegetables & $\mathrm{Bq} \mathrm{kg} \mathrm{fresh} \mathrm{mass}^{-1}$ & 1.8 & 5.4 & 18 \\
Cottage Cheese & $\mathrm{Bq} \mathrm{kg}^{-1}$ & 0.5 & 2.6 & 18 \\
Eggs & $\mathrm{Bq} \mathrm{kg}^{-1}$ & 0.7 & 3.1 & 14 \\
Mother's milk* & $\mathrm{Bq} \mathrm{L}^{-1}$ & 0.15 & 1.3 & 10.0 \\
\hline
\end{tabular}

*Mother on Diet 3 - Regionally mixed commercial milk + inhalation 
${ }^{131}$ I Releases from X-10 Radioactive Lanthanum Processing

TASK 1 REPORT

Appendix $11 B$

July 1999

Page 11B-19

\section{Location: Oliver Springs}

\begin{tabular}{lcccc}
\hline Environmental media or food product & Units & \multicolumn{3}{c}{13 years-average concentration } \\
& & lower limit & central estimate & upper limit \\
\hline Air (all physico-chemical forms) & $\mathrm{Bq} \mathrm{m}^{-3}$ & 0.007 & 0.012 & 0.022 \\
Pasture grass & $\mathrm{Bq} \mathrm{kg}^{-1}$ dry mass & 38 & 120 & 400 \\
Backyard cow milk (locally produced) & $\mathrm{Bq} \mathrm{L}^{-1}$ & 2.0 & 7.1 & 29 \\
Commercial milk (locally produced) & $\mathrm{Bq} \mathrm{L}^{-1}$ & 1.3 & 4.2 & 15 \\
Commercial milk (regional mixed) & $\mathrm{Bq} \mathrm{L}^{-1}$ & 2.2 & 5.8 & 16 \\
Goat milk (locally produced) & $\mathrm{Bq} \mathrm{L}^{-1}$ & 7 & 33 & 140 \\
Beef & $\mathrm{Bq} \mathrm{kg}^{-1}$ & 0.30 & 2.2 & 14 \\
Leafy vegetables & $\mathrm{Bq} \mathrm{kg} \mathrm{fresh} \mathrm{mass}^{-1}$ & 2.4 & 8.3 & 30 \\
Cottage Cheese & $\mathrm{Bq} \mathrm{kg}^{-1}$ & 0.7 & 3.9 & 27 \\
Eggs & $\mathrm{Bq} \mathrm{kg}^{-1}$ & 1.0 & 4.7 & 22 \\
Mother's milk* & $\mathrm{Bq} \mathrm{L}^{-1}$ & 0.16 & 1.3 & 10.0 \\
\hline
\end{tabular}

*Mother on Diet 3 - Regionally mixed commercial milk + inhalation

\section{Location: OR High School Area}

\begin{tabular}{lcccc}
\hline Environmental media or food product & Units & \multicolumn{3}{c}{13 years-average concentration } \\
& & lower limit & central estimate & upper limit \\
\hline Air (all physico-chemical forms) & $\mathrm{Bq} \mathrm{m}^{-3}$ & 0.024 & 0.037 & 0.053 \\
Pasture grass & $\mathrm{Bq} \mathrm{kg}^{-1}$ dry mass & 130 & 360 & 940 \\
Backyard cow milk (locally produced) & $\mathrm{Bq} \mathrm{L}^{-1}$ & -- & -- & -- \\
Commercial milk (locally produced) & $\mathrm{Bq} \mathrm{L}^{-1}$ & -- & -- & -- \\
Commercial milk (regional mixed) & $\mathrm{Bq} \mathrm{L}^{-1}$ & 2.2 & 5.8 & 16 \\
Goat milk (locally produced) & $\mathrm{Bq} \mathrm{L}^{-1}$ & -- & -- & -- \\
Beef & $\mathrm{Bq} \mathrm{kg}^{-1}$ & -- & -- & -- \\
Leafy vegetables & $\mathrm{Bq} \mathrm{kg} \mathrm{fresh} \mathrm{mass}^{-1}$ & 8.5 & 25.0 & 70 \\
Cottage Cheese & $\mathrm{Bq} \mathrm{kg}^{-1}$ & -- & -- & -- \\
Eggs & $\mathrm{Bq} \mathrm{kg}^{-1}$ & -- & -- & -- \\
Mother's milk* & $\mathrm{Bq} \mathrm{L}^{-1}$ & 0.22 & 1.7 & 11.0 \\
\hline
\end{tabular}

*Mother on Diet 3 - Regionally mixed commercial milk + inhalation 


\section{Location: OR Scarboro}

\begin{tabular}{lcccc}
\hline Environmental media or food product & Units & \multicolumn{3}{c}{13 years-average concentration } \\
& & lower limit & central estimate & upper limit \\
\hline Air (all physico-chemical forms) & $\mathrm{Bq} \mathrm{m}^{-3}$ & 0.041 & 0.060 & 0.089 \\
Pasture grass & $\mathrm{Bq} \mathrm{kg}^{-1}$ dry mass & 240 & 650 & 1700 \\
Backyard cow milk (locally produced) & $\mathrm{Bq} \mathrm{L}^{-1}$ & -- & -- & -- \\
Commercial milk (locally produced) & $\mathrm{Bq} \mathrm{L}^{-1}$ & -- & -- & -- \\
Commercial milk (regional mixed) & $\mathrm{Bq} \mathrm{L}^{-1}$ & 2.2 & 5.8 & 16 \\
Goat milk (locally produced) & $\mathrm{Bq} \mathrm{L}^{-1}$ & -- & -- & -- \\
Beef & $\mathrm{Bq} \mathrm{kg}^{-1}$ & -- & -- & -- \\
Leafy vegetables & $\mathrm{Bq} \mathrm{kg} \mathrm{fresh} \mathrm{mass}^{-1}$ & 15.0 & 45.0 & 130 \\
Cottage Cheese & $\mathrm{Bq} \mathrm{kg}^{-1}$ & -- & -- & -- \\
Eggs & $\mathrm{Bq} \mathrm{kg}^{-1}$ & -- & -- & -- \\
Mother's milk* & $\mathrm{Bq} \mathrm{L}^{-1}$ & 0.27 & 2.0 & 12.0 \\
\hline
\end{tabular}

*Mother on Diet 3 - Regionally mixed commercial milk + inhalation

\section{Location: OR Townsite}

\begin{tabular}{lcccc}
\hline Environmental media or food product & Units & \multicolumn{3}{c}{13 years-average concentration } \\
& & lower limit & central estimate & upper limit \\
\hline Air (all physico-chemical forms) & $\mathrm{Bq} \mathrm{m}^{-3}$ & 0.023 & 0.034 & 0.050 \\
Pasture grass & $\mathrm{Bq} \mathrm{kg}^{-1}$ dry mass & 120 & 330 & 850 \\
Backyard cow milk (locally produced) & $\mathrm{Bq} \mathrm{L}^{-1}$ & -- & -- & -- \\
Commercial milk (locally produced) & $\mathrm{Bq} \mathrm{L}^{-1}$ & -- & -- & -- \\
Commercial milk (regional mixed) & $\mathrm{Bq} \mathrm{L}^{-1}$ & 2.2 & 5.8 & 16 \\
Goat milk (locally produced) & $\mathrm{Bq} \mathrm{L}^{-1}$ & -- & -- & -- \\
Beef & $\mathrm{Bq} \mathrm{kg}^{-1}$ & -- & -- & -- \\
Leafy vegetables & $\mathrm{Bq} \mathrm{kg} \mathrm{fresh} \mathrm{mass}^{-1}$ & 7.7 & 22.0 & 65 \\
Cottage Cheese & $\mathrm{Bq} \mathrm{kg}^{-1}$ & -- & -- & -- \\
Eggs & $\mathrm{Bq} \mathrm{kg}^{-1}$ & -- & -- & -- \\
Mother's milk* & $\mathrm{Bq} \mathrm{L}^{-1}$ & 0.21 & 1.6 & 11.0 \\
\hline
\end{tabular}

*Mother on Diet 3 - Regionally mixed commercial milk + inhalation 
${ }^{131}$ I Releases from X-10 Radioactive Lanthanum Processing

TASK 1 REPORT

Appendix $11 B$

July 1999

Page 11B-21

\section{Location: Rockford}

\begin{tabular}{lcccc}
\hline Environmental media or food product & Units & \multicolumn{3}{c}{13 years-average concentration } \\
& & lower limit & central estimate & upper limit \\
\hline Air (all physico-chemical forms) & $\mathrm{Bq} \mathrm{m}^{-3}$ & 0.012 & 0.019 & 0.029 \\
Pasture grass & $\mathrm{Bq} \mathrm{kg}^{-1}$ dry mass & 45 & 130 & 360 \\
Backyard cow milk (locally produced) & $\mathrm{Bq} \mathrm{L}^{-1}$ & 2.5 & 7.6 & 27 \\
Commercial milk (locally produced) & $\mathrm{Bq} \mathrm{L}^{-1}$ & 1.5 & 4.4 & 14 \\
Commercial milk (regional mixed) & $\mathrm{Bq} \mathrm{L}^{-1}$ & 2.2 & 5.8 & 16 \\
Goat milk (locally produced) & $\mathrm{Bq} \mathrm{L}^{-1}$ & 7 & 35 & 140 \\
Beef & $\mathrm{Bq} \mathrm{kg}^{-1}$ & 0.39 & 2.3 & 14 \\
Leafy vegetables & $\mathrm{Bq} \mathrm{kg} \mathrm{fresh} \mathrm{mass}^{-1}$ & 3.0 & 8.8 & 28 \\
Cottage Cheese & $\mathrm{Bq} \mathrm{kg}^{-1}$ & 0.7 & 4.2 & 28 \\
Eggs & $\mathrm{Bq} \mathrm{kg}^{-1}$ & 1.3 & 5.0 & 21 \\
Mother's milk* & $\mathrm{Bq} \mathrm{L}^{-1}$ & 0.18 & 1.4 & 10.0 \\
\hline
\end{tabular}

*Mother on Diet 3 - Regionally mixed commercial milk + inhalation

\section{Location: Rockwood}

\begin{tabular}{lcccc}
\hline Environmental media or food product & Units & \multicolumn{3}{c}{13 years-average concentration } \\
& & lower limit & central estimate & upper limit \\
\hline Air (all physico-chemical forms) & $\mathrm{Bq} \mathrm{m}^{-3}$ & 0.012 & 0.018 & 0.029 \\
Pasture grass & $\mathrm{Bq} \mathrm{kg}^{-1}$ dry mass & 51 & 140 & 410 \\
Backyard cow milk (locally produced) & $\mathrm{Bq} \mathrm{L}^{-1}$ & 2.7 & 8.3 & 31 \\
Commercial milk (locally produced) & $\mathrm{Bq} \mathrm{L}^{-1}$ & 1.6 & 5.0 & 15 \\
Commercial milk (regional mixed) & $\mathrm{Bq} \mathrm{L}^{-1}$ & 2.2 & 5.8 & 16 \\
Goat milk (locally produced) & $\mathrm{Bq} \mathrm{L}^{-1}$ & 9 & 39 & 150 \\
Beef & $\mathrm{Bq} \mathrm{kg}^{-1}$ & 0.42 & 2.5 & 16 \\
Leafy vegetables & $\mathrm{Bq} \mathrm{kg} \mathrm{fresh} \mathrm{mass}^{-1}$ & 3.3 & 9.9 & 31 \\
Cottage Cheese & $\mathrm{Bq} \mathrm{kg}^{-1}$ & 0.8 & 4.5 & 30 \\
Eggs & $\mathrm{Bq} \mathrm{kg}^{-1}$ & 1.4 & 5.5 & 23 \\
Mother's milk* & $\mathrm{Bq} \mathrm{L}^{-1}$ & 0.17 & 1.4 & 10.0 \\
\hline
\end{tabular}

*Mother on Diet 3 - Regionally mixed commercial milk + inhalation 
${ }^{131}$ I Releases from X-10 Radioactive Lanthanum Processing

TASK 1 REPORT

Appendix $11 B$

July 1999

Page 11B-22

\section{Location: Solway}

\begin{tabular}{lcccc}
\hline Environmental media or food product & Units & \multicolumn{3}{c}{13 years-average concentration } \\
& & lower limit & central estimate & upper limit \\
\hline Air (all physico-chemical forms) & $\mathrm{Bq} \mathrm{m}^{-3}$ & 0.052 & 0.074 & 0.100 \\
Pasture grass & $\mathrm{Bq} \mathrm{kg}^{-1}$ dry mass & 280 & 710 & 1800 \\
Backyard cow milk (locally produced) & $\mathrm{Bq} \mathrm{L}^{-1}$ & 14.0 & 42.0 & 140 \\
Commercial milk (locally produced) & $\mathrm{Bq} \mathrm{L}^{-1}$ & 8.9 & 24.0 & 69 \\
Commercial milk (regional mixed) & $\mathrm{Bq} \mathrm{L}^{-1}$ & 2.2 & 5.8 & 16 \\
Goat milk (locally produced) & $\mathrm{Bq} \mathrm{L}^{-1}$ & 44 & 200 & 720 \\
Beef & $\mathrm{Bq} \mathrm{kg}^{-1}$ & 2.20 & 13.0 & 73 \\
Leafy vegetables & $\mathrm{Bq} \mathrm{kg} \mathrm{fresh} \mathrm{mass}^{-1}$ & 17.0 & 48.0 & 140 \\
Cottage Cheese & $\mathrm{Bq} \mathrm{kg}^{-1}$ & 4.2 & 23.0 & 140 \\
Eggs & $\mathrm{Bq} \mathrm{kg}^{-1}$ & 7.3 & 27.0 & 110 \\
Mother's milk* & $\mathrm{Bq} \mathrm{L}^{-1}$ & 0.30 & 2.1 & 13.0 \\
\hline
\end{tabular}

*Mother on Diet 3 - Regionally mixed commercial milk + inhalation

\section{Location: Sugar Grove}

\begin{tabular}{lcccc}
\hline Environmental media or food product & Units & \multicolumn{3}{c}{13 years-average concentration } \\
& & lower limit & central estimate & upper limit \\
\hline Air (all physico-chemical forms) & $\mathrm{Bq} \mathrm{m}^{-3}$ & 0.020 & 0.031 & 0.046 \\
Pasture grass & $\mathrm{Bq} \mathrm{kg}^{-1}$ dry mass & 110 & 300 & 800 \\
Backyard cow milk (locally produced) & $\mathrm{Bq} \mathrm{L}^{-1}$ & 5.8 & 18.0 & 64 \\
Commercial milk (locally produced) & $\mathrm{Bq} \mathrm{L}^{-1}$ & 3.5 & 10.0 & 30 \\
Commercial milk (regional mixed) & $\mathrm{Bq} \mathrm{L}^{-1}$ & 2.2 & 5.8 & 16 \\
Goat milk (locally produced) & $\mathrm{Bq} \mathrm{L}^{-1}$ & 18 & 82 & 320 \\
Beef & $\mathrm{Bq} \mathrm{kg}^{-1}$ & 0.92 & 5.4 & 33 \\
Leafy vegetables & $\mathrm{Bq} \mathrm{kg} \mathrm{fresh} \mathrm{mass}^{-1}$ & 7.0 & 21.0 & 60 \\
Cottage Cheese & $\mathrm{Bq} \mathrm{kg}^{-1}$ & 1.7 & 9.5 & 64 \\
Eggs & $\mathrm{Bq} \mathrm{kg}^{-1}$ & 3.0 & 12.0 & 49 \\
Mother's milk* & $\mathrm{Bq} \mathrm{L}^{-1}$ & 0.21 & 1.6 & 11.0 \\
\hline
\end{tabular}

*Mother on Diet 3 - Regionally mixed commercial milk + inhalation 
${ }^{131}$ I Releases from X-10 Radioactive Lanthanum Processing

TASK 1 REPORT

Appendix $11 B$

July 1999

Page 11B-23

\section{Location: Sweetwater}

\begin{tabular}{lcccc}
\hline Environmental media or food product & Units & \multicolumn{3}{c}{13 years-average concentration } \\
& & lower limit & central estimate & upper limit \\
\hline Air (all physico-chemical forms) & $\mathrm{Bq} \mathrm{m}^{-3}$ & 0.009 & 0.015 & 0.025 \\
Pasture grass & $\mathrm{Bq} \mathrm{kg}^{-1}$ dry mass & 31 & 97 & 290 \\
Backyard cow milk (locally produced) & $\mathrm{Bq} \mathrm{L}^{-1}$ & 1.6 & 5.7 & 22 \\
Commercial milk (locally produced) & $\mathrm{Bq} \mathrm{L}^{-1}$ & 1.0 & 3.4 & 11 \\
Commercial milk (regional mixed) & $\mathrm{Bq} \mathrm{L}^{-1}$ & 2.2 & 5.8 & 16 \\
Goat milk (locally produced) & $\mathrm{Bq} \mathrm{L}^{-1}$ & 5 & 26 & 110 \\
Beef & $\mathrm{Bq} \mathrm{kg}^{-1}$ & 0.28 & 1.6 & 11 \\
Leafy vegetables & $\mathrm{Bq} \mathrm{kg} \mathrm{fresh} \mathrm{mass}^{-1}$ & 2.1 & 6.6 & 22 \\
Cottage Cheese & $\mathrm{Bq} \mathrm{kg}^{-1}$ & 0.5 & 3.0 & 21 \\
Eggs & $\mathrm{Bq} \mathrm{kg}^{-1}$ & 0.9 & 3.7 & 17 \\
Mother's milk* & $\mathrm{Bq} \mathrm{L}^{-1}$ & 0.16 & 1.4 & 10.0 \\
\hline
\end{tabular}

*Mother on Diet 3 - Regionally mixed commercial milk + inhalation

\section{Location: Wartburg}

\begin{tabular}{lcccc}
\hline Environmental media or food product & Units & \multicolumn{3}{c}{13 years-average concentration } \\
& & lower limit & central estimate & upper limit \\
\hline Air (all physico-chemical forms) & $\mathrm{Bq} \mathrm{m}^{-3}$ & 0.002 & 0.004 & 0.008 \\
Pasture grass & $\mathrm{Bq} \mathrm{kg}^{-1}$ dry mass & 9 & 35 & 120 \\
Backyard cow milk (locally produced) & $\mathrm{Bq} \mathrm{L}^{-1}$ & 0.5 & 2.0 & 8 \\
Commercial milk (locally produced) & $\mathrm{Bq} \mathrm{L}^{-1}$ & 0.3 & 1.2 & 4 \\
Commercial milk (regional mixed) & $\mathrm{Bq} \mathrm{L}^{-1}$ & 2.2 & 5.8 & 16 \\
Goat milk (locally produced) & $\mathrm{Bq} \mathrm{L}^{-1}$ & 2 & 9 & 46 \\
Beef & $\mathrm{Bq} \mathrm{kg}^{-1}$ & 0.08 & 0.6 & 4 \\
Leafy vegetables & $\mathrm{Bq} \mathrm{kg} \mathrm{fresh} \mathrm{mass}^{-1}$ & 0.6 & 2.3 & 9 \\
Cottage Cheese & $\mathrm{Bq} \mathrm{kg}^{-1}$ & 0.2 & 1.1 & 8 \\
Eggs & $\mathrm{Bq} \mathrm{kg}^{-1}$ & 0.2 & 1.3 & 7 \\
Mother's milk* & $\mathrm{Bq} \mathrm{L}^{-1}$ & 0.13 & 1.2 & 9.8 \\
\hline
\end{tabular}

*Mother on Diet 3 - Regionally mixed commercial milk + inhalation 
${ }^{131}$ I Releases from X-10 Radioactive Lanthanum Processing

TASK 1 REPORT Appendix $11 B$

July 1999

Page 11B-24

\section{Location: Woodland}

\begin{tabular}{lcccc}
\hline Environmental media or food product & Units & \multicolumn{3}{c}{13 years-average concentration } \\
& & lower limit & central estimate & upper limit \\
\hline Air (all physico-chemical forms) & $\mathrm{Bq} \mathrm{m}^{-3}$ & 0.037 & 0.055 & 0.078 \\
Pasture grass & $\mathrm{Bq} \mathrm{kg}^{-1}$ dry mass & 210 & 550 & 1400 \\
Backyard cow milk (locally produced) & $\mathrm{Bq} \mathrm{L}^{-1}$ & -- & -- & -- \\
Commercial milk (locally produced) & $\mathrm{Bq} \mathrm{L}^{-1}$ & -- & -- \\
Commercial milk (regional mixed) & $\mathrm{Bq} \mathrm{L}^{-1}$ & 2.2 & 5.8 & 16 \\
Goat milk (locally produced) & $\mathrm{Bq} \mathrm{L}^{-1}$ & -- & -- & -- \\
Beef & $\mathrm{Bq} \mathrm{kg}^{-1}$ & -- & -- & -- \\
Leafy vegetables & $\mathrm{Bq} \mathrm{kg} \mathrm{fresh} \mathrm{mass}^{-1}$ & 13.0 & 38.0 & 100 \\
Cottage Cheese & $\mathrm{Bq} \mathrm{kg}^{-1}$ & -- & -- & -- \\
Eggs & $\mathrm{Bq} \mathrm{kg}^{-1}$ & -- & -- & -- \\
Mother's milk* & $\mathrm{Bq} \mathrm{L}^{-1}$ & 0.26 & 1.9 & 12.0 \\
\hline
\end{tabular}

*Mother on Diet 3 - Regionally mixed commercial milk + inhalation 
APPENDIX 11C

DETAILED RESULTS OF THE

DOSE RECONSTRUCTION FOR IODINE-131 
TASK 1 REPORT

July 1999

Page 11C-2

${ }^{131}$ I Releases from X-10 Radioactive Lanthanum ProcessingDetailed Results of the Dose Reconstruction

This page intentionally left blank. 


\section{APPENDIX 11C}

\section{DETAILED RESULTS OF THE DOSE RECONSTRUCTION FOR IODINE-131}

This Appendix presents the estimated thyroid doses, excess lifetime risks, and relative risks of thyroid cancer from exposure to ${ }^{131} \mathrm{I}$ released from the X-10 Radioactive Lanthanum Processing facility located in Oak Ridge, Tennessee. These results represent the effects of both routine releases and the April 29, 1954, accident. In addition, this Appendix presents estimates for the probability of causation, which is defined as the probability that a manifested thyroid cancer was induced by the person's exposure to ${ }^{131} \mathrm{I}$ released from X-10.

The dose is defined as the energy deposited in the thyroid tissue from the decay of ${ }^{131} \mathrm{I}$ accumulated in the thyroid. The deposited energy induces modifications in the thyroid cells that may manifest as thyroid cancer or other abnormalities. The risk values represent the probability (or the chance) that an exposed individual will contract a thyroid cancer during his or her remaining lifetime. The excess lifetime risks presented in this Appendix represent the radiationinduced thyroid cancer incidence in excess of the natural background incidence of thyroid cancer. The relative difference between the incidence of cancer in the case of exposure and the incidence of cancer in the absence of exposure (background incidence) when divided by the background incidence of cancer provides the quantity called "relative risk." The relative risk for a given individual represents the factor by which his or her background incidence of cancer has been increased by exposure to ${ }^{131}$ I released from X-10.

The results are presented as ranges of values, which at the $95^{\text {th }}$ percent level of confidence ${ }^{1}$ encompass the true but unknown doses or risks for an exposed individual. Central values $\left(50^{\text {th }}\right.$ percentiles) are also presented.

The results are presented separately for each of the 41 locations of interest (Appendix 11A) within a 38- $\mathrm{km}$ radius around the RaLa facility. Doses and risks are estimated for reference individuals of nine age categories exposed at these locations. The difference between genders is addressed explicitly. For each location and each exposed individual, doses from the most relevant exposure pathways are included. At some locations, not all the exposure pathways are applicable. For instance, no cows were present in downtown Oak Ridge during 1940s or 1950s. For these locations a dash "-" is shown in the tables, to indicate that the exposure pathway is not valid for that particular location. Also, for some individuals, exposure from ingestion of mother's milk is zero, meaning that even though infants living at that location were breast-fed, mother's milk was not contaminated because ${ }^{131} \mathrm{I}$ was not released from X10 in the year when the infant was born. For similar reasons, some of the doses from prenatal exposures are zero.

1 The ranges cover a 95\% subjective confidence interval. The confidence intervals are called "subjective" because they are based on assumptions made using expert judgement and on professional analysis of the exposure situation. Other investigators may come to slightly different results, but the final conclusions would not change significantly. 
Most of the doses are estimated based on the ingestion of locally produced food items. However, doses are also estimated for the specific case of ingestion of commercial milk representative of the entire affected region. The latter dose estimates are based on a regionally averaged concentration in milk.

Individuals living around X-10 may have been exposed via more than one exposure pathway at a time. Inhalation of contaminated air, for instance, is an exposure pathway that affected everyone in the contaminated area. Three special exposure scenarios are designed to match the most likely dietary habits and lifestyles in the vicinity of the Oak Ridge Reservation. The first exposure scenario refers to individuals living in a "rural farm" setting: that is, they own land and livestock and produce their own "backyard" cow milk, cheese, vegetables, and eggs. The intake for this exposure scenario is obtained by adding the intakes from inhalation and from ingestion of backyard cow's milk, beef, leafy vegetables, eggs, and cheese. The doses and risks for this exposure scenario are reported under the label "diet 1."

The second exposure scenario refers to individuals in a rural area who do not own their own dairy cows. However, they have some land and can produce their own vegetables and eggs, but they purchase the necessary milk and cheese from nearby farms. The intake for this exposure scenario is obtained by adding the intakes from inhalation and from ingestion of locally produced commercial milk, beef, leafy vegetables, eggs, and cheese. The doses and risks for this exposure scenario are reported under the label "diet 2."

The third scenario refers to individuals in a more "urban" setting, who do not produce their own food products. They buy milk and food products from the grocery store. The intake for this exposure scenario is obtained by adding the intakes from ingestion of regionally averaged commercial milk and from inhalation. The contribution to the total thyroid dose and risk of cancer of other regionally mixed food products is negligible compared to the contribution of ingestion of milk. The doses and risks for this exposure scenario are reported under the label "diet 3."

Given that the doses and risks from ingestion of goat's milk are substantially larger than the doses and risks from any other exposure pathway, these results are reported separately under the label "diet 4".

Typically, pregnant women and lactating mothers are considered to be on "diet 1," composed of backyard cow's milk, all other nonmilk food products, and inhalation. For locations where backyard cows are not present (e.g., the city of Oak Ridge), pregnant women and lactating mothers are considered to be on "diet 3," composed only of regionally mixed milk and inhalation. For a complete list of locations, and for the rationale on the choices of the exposure pathways at a given location, the reader should consult Appendix 11A. Figure 11-C.1 shows the locations of the reference locations used in the dose reconstruction for ${ }^{131} \mathrm{I}$. 


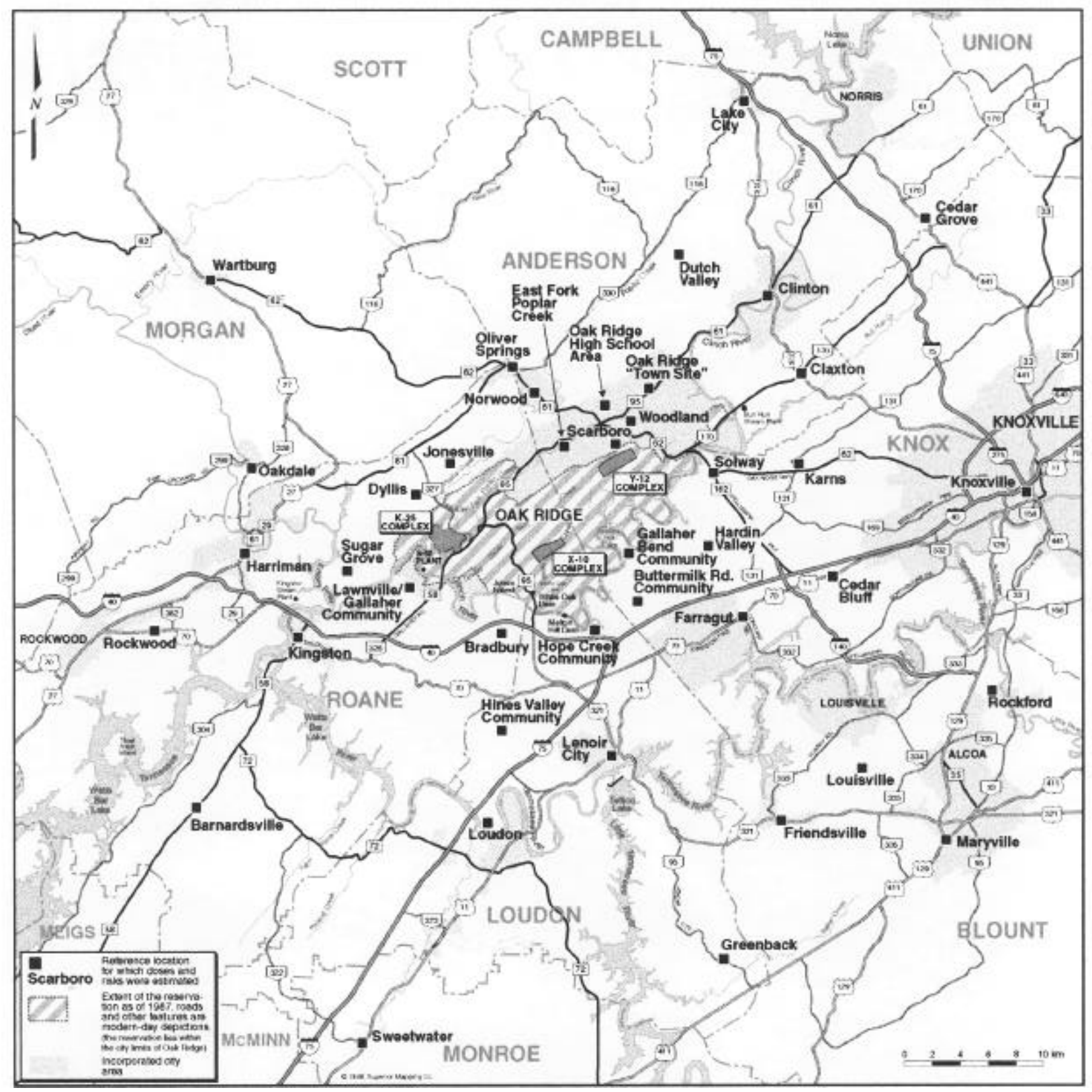

Figure 11C.1: Map Showing the 41 Reference Locations for the ${ }^{131}$ I Dose Reconstruction 
Index of page numbers for results within Appendix 11-C for specific combinations of location and birth year

\begin{tabular}{|c|c|c|c|c|c|c|c|c|c|}
\hline \multirow[b]{2}{*}{ Location } & \multicolumn{9}{|c|}{ Year of Birth } \\
\hline & 1920 & 1930 & 1935 & 1940 & 1944 & 1950 & 1952 & 1954 & 1956 \\
\hline Barnardville & 73 & 155 & 237 & 319 & 401 & 4883 & 565 & 6477 & 729 \\
\hline Bradbury & 7 & 89 & 171 & 253 & 335 & 417 & 499 & 581 & 663 \\
\hline Buttermilk Rd. & 15 & 97 & 179 & 261 & 343 & 425 & 507 & 589 & 671 \\
\hline Cedar Bluff & 55 & 137 & 219 & 301 & 383 & 465 & 547 & 629 & 711 \\
\hline Cedar Grove & 87 & 169 & 251 & 333 & 415 & 497 & 579 & 661 & 743 \\
\hline Claxton & 59 & 141 & 223 & 305 & 387 & 469 & 551 & 633 & 715 \\
\hline Clinton & 63 & 145 & 227 & 309 & 391 & 473 & 555 & 637 & 719 \\
\hline Dutch Valley & 61 & 143 & 225 & 307 & 389 & 471 & 553 & 635 & 717 \\
\hline Dyllis & 23 & 105 & 187 & 269 & 351 & 433 & 515 & 597 & 679 \\
\hline EFPC & 11 & 93 & 175 & 257 & 339 & 421 & 503 & 585 & 667 \\
\hline Farragut & 43 & 125 & 207 & 289 & 371 & 453 & 535 & 617 & 699 \\
\hline Friendsville & 65 & 147 & 229 & 311 & 393 & 475 & 557 & 639 & 721 \\
\hline Gallaher Bend & 9 & 91 & 173 & 255 & 337 & 419 & 501 & 583 & 665 \\
\hline Greenback & 75 & 157 & 239 & 321 & 403 & 485 & 567 & 649 & 731 \\
\hline Hardin Valley & 31 & 113 & 195 & 277 & 359 & 441 & 523 & 605 & 687 \\
\hline Harriman & 53 & 135 & 217 & 299 & 381 & 463 & 545 & 627 & 709 \\
\hline Hines Valley & 41 & 123 & 205 & 287 & 369 & 451 & 533 & 615 & 697 \\
\hline Hope Creek & 13 & 95 & 177 & 259 & 341 & 423 & 505 & 587 & 669 \\
\hline Jonesville & 17 & 99 & 181 & 263 & 345 & 427 & 509 & 591 & 673 \\
\hline Karns & 49 & 131 & 213 & 295 & 377 & 459 & 541 & 623 & 705 \\
\hline Kingston & 47 & 129 & 211 & 293 & 375 & 457 & 539 & 621 & 703 \\
\hline Knoxville & 83 & 165 & 247 & 329 & 411 & 493 & 575 & 657 & 739 \\
\hline Lake City & 79 & 161 & 243 & 325 & 407 & 489 & 571 & 653 & 735 \\
\hline Lawnville/Gallaher & 21 & 103 & 185 & 267 & 349 & 431 & 513 & 595 & 677 \\
\hline Lenoir City & 45 & 127 & 209 & 291 & 373 & 455 & 537 & 619 & 701 \\
\hline Loudon & 51 & 133 & 215 & 297 & 379 & 461 & 543 & 625 & 707 \\
\hline Louisville & 71 & 153 & 235 & 317 & 399 & 481 & 563 & 645 & 727 \\
\hline Maryville & 85 & 167 & 249 & 331 & 413 & 495 & 577 & 659 & 741 \\
\hline Norwood & 27 & 109 & 191 & 273 & 355 & 437 & 519 & 601 & 683 \\
\hline Oakdale & 57 & 139 & 221 & 303 & 385 & 467 & 549 & 631 & 713 \\
\hline Oliver Springs & 33 & 115 & 197 & 279 & 361 & 443 & 525 & 607 & 689 \\
\hline OR High School Area & 25 & 107 & 189 & 271 & 353 & 435 & 517 & 599 & 681 \\
\hline OR Scarboro & 19 & 101 & 183 & 265 & 347 & 429 & 511 & 593 & 675 \\
\hline OR Townsite & 39 & 121 & 203 & 285 & 367 & 449 & 531 & 613 & 695 \\
\hline Rockford & 77 & 159 & 241 & 323 & 405 & 487 & 569 & 651 & 733 \\
\hline Rockwood & 69 & 151 & 233 & 315 & 397 & 479 & 561 & 643 & 725 \\
\hline Solway & 35 & 117 & 199 & 281 & 363 & 445 & 527 & 609 & 691 \\
\hline Sugar Grove & 37 & 119 & 201 & 283 & 365 & 447 & 529 & 611 & 693 \\
\hline Sweetwater & 81 & 163 & 245 & 327 & 409 & 491 & 573 & 655 & 737 \\
\hline Wartburg & 67 & 149 & 231 & 313 & 395 & 477 & 559 & 641 & 723 \\
\hline Woodland & 29 & 111 & 193 & 275 & 357 & 439 & 521 & 603 & 685 \\
\hline
\end{tabular}


Location: Bradbury

Receptor: Female born in 1920

\begin{tabular}{lccc}
\hline & \multicolumn{3}{c}{ Thyroid Dose [cGy] } \\
Exposure Pathway & \multicolumn{2}{c}{$95 \%$ Subjective Confidence Interval } \\
\hline Backyard Cow Milk & 1.4 & 7.2 & 41 \\
Commercial Milk (locally produced) & 0.23 & 1.5 & 10 \\
Commercial Milk (regionally mixed) & 0.037 & 0.21 & 1.3 \\
Goat Milk (locally produced) & 2.4 & 18 & 120 \\
Beef (locally produced) & 0.0036 & 0.06 & 1.2 \\
Leafy Vegetables (locally produced) & 0.0013 & 0.015 & 0.13 \\
Eggs (locally produced) & 0.087 & 0.61 & 3.8 \\
Cottage Cheese (locally produced) & 0.0033 & 0.036 & 0.37 \\
Inhalation & 0.063 & 0.2 & 0.7 \\
Mother's milk (mother on Diet 1) & -- & -- & -- \\
Prenatal exposure (mother on Diet 1) & -- & -- & -- \\
Diet 1 & 1.7 & 8.4 & 46 \\
Diet 2 & 0.5 & 2.7 & 15 \\
Diet 3 & 0.12 & 0.43 & 2 \\
\hline
\end{tabular}

Excess Lifetime Risk [ ]

\begin{tabular}{llcc}
\hline Diet 1 & $2.0 \mathrm{E}-06$ & $3.2 \mathrm{E}-05$ & $4.8 \mathrm{E}-04$ \\
Diet 2 & $6.3 \mathrm{E}-07$ & $1.0 \mathrm{E}-05$ & $1.3 \mathrm{E}-04$ \\
Diet 3 & $1.5 \mathrm{E}-07$ & $1.6 \mathrm{E}-06$ & $1.7 \mathrm{E}-05$ \\
Diet 4 & $4.4 \mathrm{E}-06$ & $7.4 \mathrm{E}-05$ & $1.0 \mathrm{E}-03$ \\
\hline
\end{tabular}

\section{Relative Risk [ ]}

\begin{tabular}{lccc}
\hline Diet 1 & 1.0013 & 1.012 & 1.14 \\
Diet 2 & 1.00041 & 1.0037 & 1.051 \\
Diet 3 & 1.000084 & 1.00063 & 1.0062 \\
Diet 4 & 1.0026 & 1.026 & 1.4 \\
\hline
\end{tabular}

\section{Probability of Causation [\%]}

\begin{tabular}{llcc}
\hline Diet 1 & 0.125 & 1.147 & 12.14 \\
Diet 2 & 0.041 & 0.373 & 4.86 \\
Diet 3 & 0.008 & 0.063 & 0.61 \\
Diet 4 & 0.263 & 2.512 & 28.51 \\
\hline
\end{tabular}

Diet 1 - Backyard cow milk + all other locally produced non-milk exposure pathways

Diet 2 - Locally produced commercial milk + all other locally produced non-milk exposure pathways

Diet 3 - Regionally mixed commercial milk + inhalation (other regionally mixed food items are minor contributors to the total)

Diet 4 - Goat milk (all other exposure pathways are of negligible importance compared to goat milk) 


\section{Location: Bradbury}

Receptor: Male born in 1920

\begin{tabular}{lccc}
\hline & \multicolumn{3}{c}{ Thyroid Dose [cGy] } \\
& \multicolumn{2}{c}{$95 \%$ Subjective Confidence Interval } \\
Exposure Pathway & lower limit & central estimate & upper limit \\
\hline Backyard Cow Milk & 1.7 & 8.9 & 49 \\
Commercial Milk (locally produced) & 0.29 & 1.9 & 12 \\
Commercial Milk (regionally mixed) & 0.045 & 0.25 & 1.5 \\
Goat Milk (locally produced) & 3.3 & 24 & 160 \\
Beef (locally produced) & 0.0061 & 0.093 & 2 \\
Leafy Vegetables (locally produced) & 0.0014 & 0.015 & 0.14 \\
Eggs (locally produced) & 0.094 & 0.66 & 4.5 \\
Cottage Cheese (locally produced) & 0.0036 & 0.036 & 0.39 \\
Inhalation & 0.076 & 0.26 & 0.88 \\
Mother's milk (mother on Diet 1) & -- & -- & - \\
Prenatal exposure (mother on Diet 1) & -- & -- & - \\
Diet 1 & 2 & 10 & 56 \\
Diet 2 & 0.62 & 3.2 & 18 \\
Diet 3 & 0.14 & 0.55 & 2.3 \\
\hline
\end{tabular}

Excess Lifetime Risk [ ]

\begin{tabular}{lllc}
\hline Diet 1 & $2.2 \mathrm{E}-07$ & $1.2 \mathrm{E}-05$ & $3.5 \mathrm{E}-04$ \\
Diet 2 & $8.8 \mathrm{E}-08$ & $3.7 \mathrm{E}-06$ & $1.4 \mathrm{E}-04$ \\
Diet 3 & $1.8 \mathrm{E}-08$ & $6.7 \mathrm{E}-07$ & $1.8 \mathrm{E}-05$ \\
Diet 4 & $5.3 \mathrm{E}-07$ & $2.6 \mathrm{E}-05$ & $1.1 \mathrm{E}-03$ \\
\hline
\end{tabular}

Relative Risk [ ]

\begin{tabular}{lccc}
\hline Diet 1 & 1.00045 & 1.0095 & 1.28 \\
Diet 2 & 1.00013 & 1.003 & 1.086 \\
Diet 3 & 1.000027 & 1.00051 & 1.012 \\
Diet 4 & 1.00073 & 1.023 & 1.75 \\
\hline
\end{tabular}

\section{Probability of Causation [\%]}

\begin{tabular}{llcc}
\hline Diet 1 & 0.045 & 0.940 & 21.63 \\
Diet 2 & 0.013 & 0.300 & 7.88 \\
Diet 3 & 0.003 & 0.051 & 1.20 \\
Diet 4 & 0.073 & 2.257 & 42.69 \\
\hline
\end{tabular}

Diet 1 - Backyard cow milk + all other locally produced non-milk exposure pathways

Diet 2 - Locally produced commercial milk + all other locally produced non-milk exposure pathways

Diet 3 - Regionally mixed commercial milk + inhalation (other regionally mixed food items are minor contributors to the total)

Diet 4 - Goat milk (all other exposure pathways are of negligible importance compared to goat milk) 
Location: Gallaher Bend

Receptor: Female born in 1920

\begin{tabular}{lccc}
\hline & \multicolumn{3}{c}{ Thyroid Dose [cGy] } \\
Exposure Pathway & \multicolumn{2}{c}{$95 \%$ Subjective Confidence Interval } \\
Backyard Cow Milk & lower limit & central estimate & upper limit \\
Commercial Milk (locally produced) & 1.7 & 9.2 & 48 \\
Commercial Milk (regionally mixed) & 0.32 & 1.9 & 12 \\
Goat Milk (locally produced) & 0.037 & 0.2 & 1.3 \\
Beef (locally produced) & 2.8 & 22 & 160 \\
Leafy Vegetables (locally produced) & 0.0041 & 0.072 & 1.5 \\
Eggs (locally produced) & 0.0016 & 0.019 & 0.16 \\
Cottage Cheese (locally produced) & 0.12 & 0.77 & 4.5 \\
Inhalation & 0.0045 & 0.044 & 0.43 \\
Mother's milk (mother on Diet 1) & 0.074 & 0.25 & 0.83 \\
Prenatal exposure (mother on Diet 1) & -- & -- & -- \\
Diet 1 & -- & -- & -- \\
Diet 2 & 2 & 10 & 56 \\
Diet 3 & 0.63 & 3.3 & 18 \\
\hline
\end{tabular}

Excess Lifetime Risk [ ]

\begin{tabular}{lllc}
\hline Diet 1 & $2.2 \mathrm{E}-06$ & $4.0 \mathrm{E}-05$ & $5.8 \mathrm{E}-04$ \\
Diet 2 & $7.7 \mathrm{E}-07$ & $1.2 \mathrm{E}-05$ & $1.6 \mathrm{E}-04$ \\
Diet 3 & $1.6 \mathrm{E}-07$ & $1.8 \mathrm{E}-06$ & $1.8 \mathrm{E}-05$ \\
Diet 4 & $5.1 \mathrm{E}-06$ & $8.7 \mathrm{E}-05$ & $1.4 \mathrm{E}-03$ \\
\hline
\end{tabular}

\section{Relative Risk [ ]}

\begin{tabular}{lccc}
\hline Diet 1 & 1.0016 & 1.014 & 1.16 \\
Diet 2 & 1.00049 & 1.0047 & 1.057 \\
Diet 3 & 1.0001 & 1.00069 & 1.0067 \\
Diet 4 & 1.003 & 1.032 & 1.49 \\
\hline
\end{tabular}

\section{Probability of Causation [\%]}

\begin{tabular}{lccc}
\hline Diet 1 & 0.164 & 1.414 & 13.72 \\
Diet 2 & 0.049 & 0.467 & 5.37 \\
Diet 3 & 0.010 & 0.069 & 0.66 \\
Diet 4 & 0.302 & 3.055 & 33.02 \\
\hline
\end{tabular}

Diet 1 - Backyard cow milk + all other locally produced non-milk exposure pathways

Diet 2 - Locally produced commercial milk + all other locally produced non-milk exposure pathways

Diet 3 - Regionally mixed commercial milk + inhalation (other regionally mixed food items are minor contributors to the total)

Diet 4 - Goat milk (all other exposure pathways are of negligible importance compared to goat milk) 


\section{Location: Gallaher Bend}

Receptor: Male born in 1920

\begin{tabular}{lccc}
\hline & \multicolumn{3}{c}{ Thyroid Dose [cGy] } \\
& \multicolumn{2}{c}{$95 \%$ Subjective Confidence Interval } \\
Exposure Pathway & lower limit & central estimate & upper limit \\
\hline Backyard Cow Milk & 2 & 11 & 58 \\
Commercial Milk (locally produced) & 0.4 & 2.3 & 14 \\
Commercial Milk (regionally mixed) & 0.045 & 0.25 & 1.5 \\
Goat Milk (locally produced) & 3.9 & 30 & 200 \\
Beef (locally produced) & 0.0068 & 0.12 & 2.5 \\
Leafy Vegetables (locally produced) & 0.0017 & 0.019 & 0.16 \\
Eggs (locally produced) & 0.12 & 0.84 & 5.3 \\
Cottage Cheese (locally produced) & 0.0045 & 0.044 & 0.44 \\
Inhalation & 0.094 & 0.33 & 1.1 \\
Mother's milk (mother on Diet 1) & -- & -- & - \\
Prenatal exposure (mother on Diet 1) & -- & -- & - \\
Diet 1 & 2.3 & 12 & 66 \\
Diet 2 & 0.78 & 4 & 20 \\
Diet 3 & 0.15 & 0.61 & 2.5 \\
\hline
\end{tabular}

Excess Lifetime Risk [ ]

\begin{tabular}{llll}
\hline Diet 1 & $2.8 \mathrm{E}-07$ & $1.5 \mathrm{E}-05$ & $3.9 \mathrm{E}-04$ \\
Diet 2 & $9.8 \mathrm{E}-08$ & $4.8 \mathrm{E}-06$ & $1.6 \mathrm{E}-04$ \\
Diet 3 & $2.0 \mathrm{E}-08$ & $7.4 \mathrm{E}-07$ & $1.9 \mathrm{E}-05$ \\
Diet 4 & $6.6 \mathrm{E}-07$ & $3.4 \mathrm{E}-05$ & $1.3 \mathrm{E}-03$ \\
\hline
\end{tabular}

Relative Risk [ ]

\begin{tabular}{lccc}
\hline Diet 1 & 1.00051 & 1.011 & 1.32 \\
Diet 2 & 1.00016 & 1.0036 & 1.11 \\
Diet 3 & 1.000031 & 1.00057 & 1.013 \\
Diet 4 & 1.00094 & 1.029 & 1.85 \\
\hline
\end{tabular}

\section{Probability of Causation [\%]}

\begin{tabular}{lccc}
\hline Diet 1 & 0.051 & 1.112 & 24.45 \\
Diet 2 & 0.016 & 0.359 & 9.64 \\
Diet 3 & 0.003 & 0.057 & 1.27 \\
Diet 4 & 0.094 & 2.840 & 45.81 \\
\hline
\end{tabular}

Diet 1 - Backyard cow milk + all other locally produced non-milk exposure pathways

Diet 2 - Locally produced commercial milk + all other locally produced non-milk exposure pathways

Diet 3 - Regionally mixed commercial milk + inhalation (other regionally mixed food items are minor contributors to the total)

Diet 4 - Goat milk (all other exposure pathways are of negligible importance compared to goat milk) 


\section{Location: EFPC}

Receptor: Female born in 1920

\begin{tabular}{lccc}
\hline & \multicolumn{3}{c}{ Thyroid Dose [cGy] } \\
Exposure Pathway & \multicolumn{2}{c}{$95 \%$ Subjective Confidence Interval } \\
\hline Backyard Cow Milk & lower limit & central estimate & upper limit \\
Commercial Milk (locally produced) & 0.36 & 1.9 & 11 \\
Commercial Milk (regionally mixed) & 0.06 & 0.4 & 2.7 \\
Goat Milk (locally produced) & 0.037 & 0.21 & 1.3 \\
Beef (locally produced) & -- & -- & -- \\
Leafy Vegetables (locally produced) & 0.00097 & 0.016 & 0.35 \\
Eggs (locally produced) & 0.00034 & 0.0039 & 0.033 \\
Cottage Cheese (locally produced) & 0.023 & 0.16 & 1 \\
Inhalation & 0.0009 & 0.0092 & 0.096 \\
Mother's milk (mother on Diet 1) & 0.016 & 0.055 & 0.19 \\
Prenatal exposure (mother on Diet 1) & -- & -- & - \\
Diet 1 & -- & -- & -- \\
Diet 2 & 0.42 & 2.2 & 12 \\
Diet 3 & 0.13 & 0.69 & 3.9 \\
\hline
\end{tabular}

Excess Lifetime Risk [ ]

\begin{tabular}{lccc}
\hline Diet 1 & $5.0 \mathrm{E}-07$ & $8.0 \mathrm{E}-06$ & $1.2 \mathrm{E}-04$ \\
Diet 2 & $1.7 \mathrm{E}-07$ & $2.6 \mathrm{E}-06$ & $3.5 \mathrm{E}-05$ \\
Diet 3 & $7.7 \mathrm{E}-08$ & $9.9 \mathrm{E}-07$ & $1.3 \mathrm{E}-05$ \\
Diet 4 & -- & -- & -- \\
\hline
\end{tabular}

Relative Risk [ ]

\begin{tabular}{lccc}
\hline Diet 1 & 1.00031 & 1.0031 & 1.035 \\
Diet 2 & 1.00011 & 1.00097 & 1.012 \\
Diet 3 & 1.000046 & 1.00039 & 1.0043 \\
Diet 4 & -- & -- & -- \\
\hline
\end{tabular}

Probability of Causation [\%]

\begin{tabular}{lccc}
\hline Diet 1 & 0.031 & 0.307 & 3.33 \\
Diet 2 & 0.011 & 0.097 & 1.21 \\
Diet 3 & 0.005 & 0.039 & 0.43 \\
Diet 4 & -- & -- & - \\
Diet 1 - Backyard cow milk + all other locally produced non-milk exposure pathways & \\
Diet 2 - Locally produced commercial milk + all other locally produced non-milk exposure pathways \\
Diet 3 - Regionally mixed commercial milk + inhalation (other regionally mixed food items are minor contributors to the total) \\
Diet 4 - Goat milk (all other exposure pathways are of negligible importance compared to goat milk)
\end{tabular}




\section{Location: EFPC}

\section{Receptor: Male born in 1920}

\begin{tabular}{lccc}
\hline & \multicolumn{3}{c}{ Thyroid Dose [cGy] } \\
& \multicolumn{2}{c}{$95 \%$ Subjective Confidence Interval } \\
Exposure Pathway & lower limit & central estimate & upper limit \\
\hline Backyard Cow Milk & 0.4 & 2.3 & 13 \\
Commercial Milk (locally produced) & 0.076 & 0.49 & 3.2 \\
Commercial Milk (regionally mixed) & 0.045 & 0.25 & 1.5 \\
Goat Milk (locally produced) & -- & -- & -- \\
Beef (locally produced) & 0.0016 & 0.025 & 0.55 \\
Leafy Vegetables (locally produced) & 0.00036 & 0.0039 & 0.037 \\
Eggs (locally produced) & 0.025 & 0.17 & 1.2 \\
Cottage Cheese (locally produced) & 0.001 & 0.0091 & 0.095 \\
Inhalation & 0.019 & 0.071 & 0.24 \\
Mother's milk (mother on Diet 1) & -- & -- & - \\
Prenatal exposure (mother on Diet 1) & -- & -- & - \\
Diet 1 & 0.48 & 2.6 & 14 \\
Diet 2 & 0.16 & 0.84 & 4.7 \\
Diet 3 & 0.075 & 0.33 & 1.7 \\
\hline
\end{tabular}

Excess Lifetime Risk [ ]

\begin{tabular}{lccc}
\hline Diet 1 & $5.4 \mathrm{E}-08$ & $3.1 \mathrm{E}-06$ & $9.9 \mathrm{E}-05$ \\
Diet 2 & $2.2 \mathrm{E}-08$ & $1.0 \mathrm{E}-06$ & $3.5 \mathrm{E}-05$ \\
Diet 3 & $1.0 \mathrm{E}-08$ & $4.1 \mathrm{E}-07$ & $1.3 \mathrm{E}-05$ \\
Diet 4 & -- & -- & -- \\
\hline
\end{tabular}

\section{Relative Risk [ ]}

\begin{tabular}{lccc}
\hline Diet 1 & 1.00011 & 1.0024 & 1.079 \\
Diet 2 & 1.000033 & 1.00077 & 1.024 \\
Diet 3 & 1.000013 & 1.0003 & 1.0094 \\
Diet 4 & -- & -- & -- \\
\hline
\end{tabular}

\begin{tabular}{lccc}
\hline Diet 1 & 0.011 & 0.236 & 7.28 \\
Diet 2 & 0.003 & 0.077 & 2.33 \\
Diet 3 & 0.001 & 0.030 & 0.93 \\
Diet 4 & -- & -- & - \\
Diet 1 - Backyard cow milk + all other locally produced non-milk exposure pathways & \\
Diet 2 - Locally produced commercial milk + all other locally produced non-milk exposure pathways \\
Diet 3 - Regionally mixed commercial milk + inhalation (other regionally mixed food items are minor contributors to the total) \\
Diet 4 - Goat milk (all other exposure pathways are of negligible importance compared to goat milk)
\end{tabular}




\section{Location: Hope Creek}

Receptor: Female born in 1920

\begin{tabular}{lccc}
\hline & \multicolumn{3}{c}{ Thyroid Dose [cGy] } \\
& \multicolumn{2}{c}{$95 \%$ Subjective Confidence Interval } \\
Exposure Pathway & lower limit & central estimate & upper limit \\
\hline Backyard Cow Milk & 0.95 & 5.1 & 29 \\
Commercial Milk (locally produced) & 0.17 & 1 & 7.4 \\
Commercial Milk (regionally mixed) & 0.037 & 0.2 & 1.3 \\
Goat Milk (locally produced) & -- & -- & -- \\
Beef (locally produced) & 0.0024 & 0.043 & 0.96 \\
Leafy Vegetables (locally produced) & 0.001 & 0.011 & 0.097 \\
Eggs (locally produced) & 0.065 & 0.44 & 2.8 \\
Cottage Cheese (locally produced) & 0.0026 & 0.025 & 0.27 \\
Inhalation & 0.045 & 0.15 & 0.51 \\
Mother's milk (mother on Diet 1) & -- & -- & -- \\
Prenatal exposure (mother on Diet 1) & -- & -- & -- \\
Diet 1 & 1.1 & 5.9 & 34 \\
Diet 2 & 0.37 & 1.8 & 11 \\
Diet 3 & 0.097 & 0.37 & 1.8 \\
\hline
\end{tabular}

Excess Lifetime Risk [ ]

\begin{tabular}{lccc}
\hline Diet 1 & $1.3 \mathrm{E}-06$ & $2.2 \mathrm{E}-05$ & $3.5 \mathrm{E}-04$ \\
Diet 2 & $4.7 \mathrm{E}-07$ & $6.9 \mathrm{E}-06$ & $9.9 \mathrm{E}-05$ \\
Diet 3 & $1.2 \mathrm{E}-07$ & $1.4 \mathrm{E}-06$ & $1.6 \mathrm{E}-05$ \\
Diet 4 & -- & -- & -- \\
\hline
\end{tabular}

Relative Risk [ ]

\begin{tabular}{lccc}
\hline Diet 1 & 1.00082 & 1.0085 & 1.091 \\
Diet 2 & 1.00029 & 1.0026 & 1.035 \\
Diet 3 & 1.000071 & 1.00054 & 1.0054 \\
Diet 4 & -- & -- & -- \\
\hline
\end{tabular}

\begin{tabular}{lccc}
\hline Diet 1 & 0.082 & 0.843 & 8.34 \\
Diet 2 & 0.029 & 0.263 & 3.35 \\
Diet 3 & 0.007 & 0.054 & 0.53 \\
Diet 4 & -- & -- & -- \\
\hline Diet 1 - Backyard cow milk + all other locally produced non-milk exposure pathways & \\
Diet 2 - Locally produced commercial milk + all other locally produced non-milk exposure pathways \\
Diet 3 - Regionally mixed commercial milk + inhalation (other regionally mixed food items are minor contributors to the total) \\
Diet 4 - Goat milk (all other exposure pathways are of negligible importance compared to goat milk)
\end{tabular}




\section{Location: Hope Creek}

Receptor: Male born in 1920

\begin{tabular}{lccc}
\hline & \multicolumn{3}{c}{ Thyroid Dose [cGy] } \\
& \multicolumn{2}{c}{$95 \%$ Subjective Confidence Interval } \\
Exposure Pathway & lower limit & central estimate & upper limit \\
\hline Backyard Cow Milk & 1.1 & 6.1 & 36 \\
Commercial Milk (locally produced) & 0.22 & 1.3 & 9.3 \\
Commercial Milk (regionally mixed) & 0.045 & 0.25 & 1.5 \\
Goat Milk (locally produced) & -- & -- & -- \\
Beef (locally produced) & 0.004 & 0.067 & 1.5 \\
Leafy Vegetables (locally produced) & 0.001 & 0.011 & 0.099 \\
Eggs (locally produced) & 0.069 & 0.47 & 3.2 \\
Cottage Cheese (locally produced) & 0.0026 & 0.026 & 0.26 \\
Inhalation & 0.054 & 0.19 & 0.62 \\
Mother's milk (mother on Diet 1) & -- & -- & -- \\
Prenatal exposure (mother on Diet 1) & -- & -- & -- \\
Diet 1 & 1.3 & 7 & 40 \\
Diet 2 & 0.45 & 2.2 & 13 \\
Diet 3 & 0.12 & 0.47 & 2.1 \\
\hline
\end{tabular}

Excess Lifetime Risk [ ]

\begin{tabular}{lccc}
\hline Diet 1 & $1.5 \mathrm{E}-07$ & $8.3 \mathrm{E}-06$ & $2.5 \mathrm{E}-04$ \\
Diet 2 & $5.7 \mathrm{E}-08$ & $2.8 \mathrm{E}-06$ & $9.3 \mathrm{E}-05$ \\
Diet 3 & $1.5 \mathrm{E}-08$ & $5.6 \mathrm{E}-07$ & $1.5 \mathrm{E}-05$ \\
Diet 4 & -- & -- & -- \\
\hline
\end{tabular}

\section{Relative Risk [ ]}

\begin{tabular}{lccc}
\hline Diet 1 & 1.0003 & 1.0064 & 1.18 \\
Diet 2 & 1.000087 & 1.0021 & 1.058 \\
Diet 3 & 1.000021 & 1.00043 & 1.011 \\
Diet 4 & -- & -- & -- \\
\hline
\end{tabular}

\section{Probability of Causation [\%]}

\begin{tabular}{lccc}
\hline Diet 1 & 0.030 & 0.635 & 15.06 \\
Diet 2 & 0.009 & 0.206 & 5.44 \\
Diet 3 & 0.002 & 0.043 & 1.12 \\
Diet 4 & -- & -- & -- \\
\hline Diet 1 - Backyard cow milk + all other locally produced non-milk exposure pathways & \\
Diet 2 - Locally produced commercial milk + all other locally produced non-milk exposure pathways \\
Diet 3 - Regionally mixed commercial milk + inhalation (other regionally mixed food items are minor contributors to the total) \\
Diet 4 - Goat milk (all other exposure pathways are of negligible importance compared to goat milk)
\end{tabular}


Location: Buttermilk Rd.

Receptor: Female born in 1920

\begin{tabular}{lccc}
\hline & \multicolumn{3}{c}{ Thyroid Dose [cGy] } \\
Exposure Pathway & $95 \%$ Subjective Confidence Interval \\
\hline Backyard Cow Milk & lower limit & central estimate & upper limit \\
Commercial Milk (locally produced) & 0.92 & 5.1 & 29 \\
Commercial Milk (regionally mixed) & 0.17 & 1 & 7.3 \\
Goat Milk (locally produced) & 0.037 & 0.21 & 1.3 \\
Beef (locally produced) & 1.6 & 12 & 88 \\
Leafy Vegetables (locally produced) & 0.0024 & 0.042 & 0.9 \\
Eggs (locally produced) & 0.001 & 0.011 & 0.092 \\
Cottage Cheese (locally produced) & 0.064 & 0.43 & 2.7 \\
Inhalation & 0.0026 & 0.024 & 0.25 \\
Mother's milk (mother on Diet 1) & 0.046 & 0.15 & 0.52 \\
Prenatal exposure (mother on Diet 1) & -- & -- & -- \\
Diet 1 & -- & -- & -- \\
Diet 2 & 1.1 & 5.8 & 34 \\
Diet 3 & 0.36 & 1.8 & 11 \\
\hline
\end{tabular}

Excess Lifetime Risk [ ]

\begin{tabular}{lllc}
\hline Diet 1 & $1.4 \mathrm{E}-06$ & $2.2 \mathrm{E}-05$ & $3.5 \mathrm{E}-04$ \\
Diet 2 & $4.4 \mathrm{E}-07$ & $6.8 \mathrm{E}-06$ & $9.3 \mathrm{E}-05$ \\
Diet 3 & $1.2 \mathrm{E}-07$ & $1.4 \mathrm{E}-06$ & $1.6 \mathrm{E}-05$ \\
Diet 4 & $3.0 \mathrm{E}-06$ & $5.0 \mathrm{E}-05$ & $7.8 \mathrm{E}-04$ \\
\hline
\end{tabular}

Relative Risk [ ]

\begin{tabular}{lccc}
\hline Diet 1 & 1.00079 & 1.0084 & 1.091 \\
Diet 2 & 1.00028 & 1.0027 & 1.032 \\
Diet 3 & 1.00007 & 1.00055 & 1.0053 \\
Diet 4 & 1.0018 & 1.018 & 1.29 \\
\hline
\end{tabular}

\section{Probability of Causation [\%]}

\begin{tabular}{llcc}
\hline Diet 1 & 0.079 & 0.829 & 8.32 \\
Diet 2 & 0.027 & 0.266 & 3.12 \\
Diet 3 & 0.007 & 0.054 & 0.53 \\
Diet 4 & 0.176 & 1.740 & 22.25 \\
\hline
\end{tabular}

Diet 1 - Backyard cow milk + all other locally produced non-milk exposure pathways

Diet 2 - Locally produced commercial milk + all other locally produced non-milk exposure pathways

Diet 3 - Regionally mixed commercial milk + inhalation (other regionally mixed food items are minor contributors to the total)

Diet 4 - Goat milk (all other exposure pathways are of negligible importance compared to goat milk) 
Location: Buttermilk Rd.

Receptor: Male born in 1920

\begin{tabular}{lccc}
\hline & \multicolumn{3}{c}{ Thyroid Dose [cGy] } \\
& \multicolumn{2}{c}{$95 \%$ Subjective Confidence Interval } \\
Exposure Pathway & lower limit & central estimate & upper limit \\
\hline Backyard Cow Milk & 1.1 & 6 & 37 \\
Commercial Milk (locally produced) & 0.21 & 1.3 & 8.9 \\
Commercial Milk (regionally mixed) & 0.045 & 0.25 & 1.5 \\
Goat Milk (locally produced) & 2.2 & 17 & 110 \\
Beef (locally produced) & 0.0038 & 0.066 & 1.4 \\
Leafy Vegetables (locally produced) & 0.001 & 0.011 & 0.094 \\
Eggs (locally produced) & 0.067 & 0.47 & 3 \\
Cottage Cheese (locally produced) & 0.0026 & 0.026 & 0.26 \\
Inhalation & 0.054 & 0.19 & 0.63 \\
Mother's milk (mother on Diet 1) & -- & -- & -- \\
Prenatal exposure (mother on Diet 1) & -- & -- & - \\
Diet 1 & 1.3 & 6.9 & 40 \\
Diet 2 & 0.44 & 2.2 & 12 \\
Diet 3 & 0.12 & 0.48 & 2.1 \\
\hline
\end{tabular}

Excess Lifetime Risk [ ]

\begin{tabular}{lllc}
\hline Diet 1 & $1.3 \mathrm{E}-07$ & $8.4 \mathrm{E}-06$ & $2.3 \mathrm{E}-04$ \\
Diet 2 & $5.4 \mathrm{E}-08$ & $2.8 \mathrm{E}-06$ & $9.2 \mathrm{E}-05$ \\
Diet 3 & $1.5 \mathrm{E}-08$ & $5.7 \mathrm{E}-07$ & $1.5 \mathrm{E}-05$ \\
Diet 4 & $3.3 \mathrm{E}-07$ & $1.9 \mathrm{E}-05$ & $7.5 \mathrm{E}-04$ \\
\hline
\end{tabular}

\section{Relative Risk [ ]}

\begin{tabular}{lccc}
\hline Diet 1 & 1.00031 & 1.0063 & 1.18 \\
Diet 2 & 1.000087 & 1.002 & 1.058 \\
Diet 3 & 1.000021 & 1.00043 & 1.011 \\
Diet 4 & 1.00049 & 1.016 & 1.49 \\
\hline
\end{tabular}

\section{Probability of Causation [\%]}

\begin{tabular}{lccc}
\hline Diet 1 & 0.031 & 0.631 & 15.07 \\
Diet 2 & 0.009 & 0.197 & 5.46 \\
Diet 3 & 0.002 & 0.043 & 1.12 \\
Diet 4 & 0.049 & 1.599 & 32.72 \\
\hline
\end{tabular}

Diet 1 - Backyard cow milk + all other locally produced non-milk exposure pathways

Diet 2 - Locally produced commercial milk + all other locally produced non-milk exposure pathways

Diet 3 - Regionally mixed commercial milk + inhalation (other regionally mixed food items are minor contributors to the total)

Diet 4 - Goat milk (all other exposure pathways are of negligible importance compared to goat milk) 


\section{Location: Jonesville}

Receptor: Female born in 1920

\begin{tabular}{lccc}
\hline & \multicolumn{3}{c}{ Thyroid Dose [cGy] } \\
Exposure Pathway & \multicolumn{2}{c}{$95 \%$ Subjective Confidence Interval } \\
\hline Backyard Cow Milk & lower limit & central estimate & upper limit \\
Commercial Milk (locally produced) & 0.12 & 0.68 & 4.4 \\
Commercial Milk (regionally mixed) & 0.024 & 0.15 & 1 \\
Goat Milk (locally produced) & 0.037 & 0.2 & 1.3 \\
Beef (locally produced) & 0.21 & 1.8 & 15 \\
Leafy Vegetables (locally produced) & 0.00033 & 0.0061 & 0.13 \\
Eggs (locally produced) & 0.00013 & 0.0015 & 0.014 \\
Cottage Cheese (locally produced) & 0.0082 & 0.058 & 0.4 \\
Inhalation & 0.00033 & 0.0035 & 0.043 \\
Mother's milk (mother on Diet 1) & 0.0066 & 0.022 & 0.085 \\
Prenatal exposure (mother on Diet 1) & -- & -- & -- \\
Diet 1 & -- & -- & -- \\
Diet 2 & 0.16 & 0.8 & 4.9 \\
Diet 3 & 0.05 & 0.26 & 1.6 \\
\hline
\end{tabular}

Excess Lifetime Risk [ ]

\begin{tabular}{llll}
\hline Diet 1 & $2.0 \mathrm{E}-07$ & $3.1 \mathrm{E}-06$ & $5.4 \mathrm{E}-05$ \\
Diet 2 & $6.6 \mathrm{E}-08$ & $9.9 \mathrm{E}-07$ & $1.6 \mathrm{E}-05$ \\
Diet 3 & $5.9 \mathrm{E}-08$ & $8.5 \mathrm{E}-07$ & $1.3 \mathrm{E}-05$ \\
Diet 4 & $4.2 \mathrm{E}-07$ & $6.8 \mathrm{E}-06$ & $1.2 \mathrm{E}-04$ \\
\hline
\end{tabular}

Relative Risk [ ]

\begin{tabular}{lccc}
\hline Diet 1 & 1.00011 & 1.0011 & 1.015 \\
Diet 2 & 1.00004 & 1.00036 & 1.0048 \\
Diet 3 & 1.000036 & 1.00034 & 1.0039 \\
Diet 4 & 1.00022 & 1.0024 & 1.047 \\
\hline
\end{tabular}

Probability of Causation [\%]

\begin{tabular}{llcc}
\hline Diet 1 & 0.011 & 0.112 & 1.43 \\
Diet 2 & 0.004 & 0.036 & 0.48 \\
Diet 3 & 0.004 & 0.034 & 0.39 \\
Diet 4 & 0.022 & 0.239 & 4.52 \\
\hline
\end{tabular}

Diet 1 - Backyard cow milk + all other locally produced non-milk exposure pathways

Diet 2 - Locally produced commercial milk + all other locally produced non-milk exposure pathways

Diet 3 - Regionally mixed commercial milk + inhalation (other regionally mixed food items are minor contributors to the total)

Diet 4 - Goat milk (all other exposure pathways are of negligible importance compared to goat milk) 


\section{Location: Jonesville}

\section{Receptor: Male born in 1920}

\begin{tabular}{lccc}
\hline & \multicolumn{3}{c}{ Thyroid Dose [cGy] } \\
& \multicolumn{2}{c}{$95 \%$ Subjective Confidence Interval } \\
Exposure Pathway & lower limit & central estimate & upper limit \\
\hline Backyard Cow Milk & 0.16 & 0.84 & 5.1 \\
Commercial Milk (locally produced) & 0.027 & 0.19 & 1.3 \\
Commercial Milk (regionally mixed) & 0.045 & 0.25 & 1.5 \\
Goat Milk (locally produced) & 0.31 & 2.4 & 19 \\
Beef (locally produced) & 0.00052 & 0.0097 & 0.2 \\
Leafy Vegetables (locally produced) & 0.00013 & 0.0015 & 0.016 \\
Eggs (locally produced) & 0.0095 & 0.063 & 0.47 \\
Cottage Cheese (locally produced) & 0.0003 & 0.0036 & 0.045 \\
Inhalation & 0.0085 & 0.028 & 0.11 \\
Mother's milk (mother on Diet 1) & -- & -- & - \\
Prenatal exposure (mother on Diet 1) & -- & -- & -- \\
Diet 1 & 0.19 & 0.97 & 5.9 \\
Diet 2 & 0.063 & 0.32 & 2 \\
Diet 3 & 0.06 & 0.28 & 1.6 \\
\hline
\end{tabular}

Excess Lifetime Risk [ ]

\begin{tabular}{llll}
\hline Diet 1 & $1.9 \mathrm{E}-08$ & $1.2 \mathrm{E}-06$ & $3.9 \mathrm{E}-05$ \\
Diet 2 & $7.8 \mathrm{E}-09$ & $3.7 \mathrm{E}-07$ & $1.4 \mathrm{E}-05$ \\
Diet 3 & $8.4 \mathrm{E}-09$ & $3.4 \mathrm{E}-07$ & $1.3 \mathrm{E}-05$ \\
Diet 4 & $5.4 \mathrm{E}-08$ & $2.6 \mathrm{E}-06$ & $1.1 \mathrm{E}-04$ \\
\hline
\end{tabular}

Relative Risk [ ]

\begin{tabular}{lccc}
\hline Diet 1 & 1.000039 & 1.00095 & 1.028 \\
Diet 2 & 1.000012 & 1.00031 & 1.009 \\
Diet 3 & 1.000011 & 1.00026 & 1.0081 \\
Diet 4 & 1.000069 & 1.0024 & 1.073 \\
\hline
\end{tabular}

\section{Probability of Causation [\%]}

\begin{tabular}{lllc}
\hline Diet 1 & 0.004 & 0.095 & 2.69 \\
Diet 2 & 0.001 & 0.031 & 0.89 \\
Diet 3 & 0.001 & 0.026 & 0.81 \\
Diet 4 & 0.007 & 0.235 & 6.75 \\
\hline
\end{tabular}

Diet 1 - Backyard cow milk + all other locally produced non-milk exposure pathways

Diet 2 - Locally produced commercial milk + all other locally produced non-milk exposure pathways

Diet 3 - Regionally mixed commercial milk + inhalation (other regionally mixed food items are minor contributors to the total)

Diet 4 - Goat milk (all other exposure pathways are of negligible importance compared to goat milk) 


\section{Location: OR Scarboro}

Receptor: Female born in 1920

\begin{tabular}{lccc}
\hline & \multicolumn{3}{c}{ Thyroid Dose [cGy] } \\
Exposure Pathway & \multicolumn{2}{c}{ 95\% Subjective Confidence Interval } \\
\hline Backyard Cow Milk & lower limit & central estimate & upper limit \\
Commercial Milk (locally produced) & -- & -- & -- \\
Commercial Milk (regionally mixed) & -- & -- & -- \\
Goat Milk (locally produced) & 0.037 & 0.21 & 1.3 \\
Beef (locally produced) & -- & -- & -- \\
Leafy Vegetables (locally produced) & -- & -- & -- \\
Eggs (locally produced) & -- & -- & -- \\
Cottage Cheese (locally produced) & -- & -- & -- \\
Inhalation & 0.0018 & 0.019 & 0.18 \\
Mother's milk (mother on Diet 3) & 0.033 & 0.11 & 0.37 \\
Prenatal exposure (mother on Diet 3) & -- & -- & -- \\
Diet 1 & -- & -- & -- \\
Diet 2 & -- & -- & -- \\
Diet 3 & -- & -- & -- \\
\hline
\end{tabular}

Excess Lifetime Risk [ ]

\begin{tabular}{lccc}
\hline Diet 1 & -- & -- & -- \\
Diet 2 & -- & -- & -- \\
Diet 3 & $1.0 \mathrm{E}-07$ & $1.2 \mathrm{E}-06$ & $1.5 \mathrm{E}-05$ \\
Diet 4 & -- & -- & -- \\
\hline
\end{tabular}

\section{Relative Risk [ ]}

\begin{tabular}{lccc}
\hline Diet 1 & -- & -- & -- \\
Diet 2 & -- & -- & -- \\
Diet 3 & 1.000061 & 1.00049 & 1.0049 \\
Diet 4 & -- & -- & -- \\
\hline
\end{tabular}

\begin{tabular}{lccc}
\hline Diet 1 & -- & -- & -- \\
Diet 2 & -- & -- & -- \\
Diet 3 & 0.006 & 0.049 & 0.49 \\
Diet 4 & -- & -- & - \\
\hline Diet 1 - Backyard cow milk + all other locally produced non-milk exposure pathways & \\
Diet 2 - Locally produced commercial milk + all other locally produced non-milk exposure pathways \\
Diet 3 - Regionally mixed commercial milk + inhalation (other regionally mixed food items are minor contributors to the total) \\
Diet 4 - Goat milk (all other exposure pathways are of negligible importance compared to goat milk)
\end{tabular}




\section{Location: OR Scarboro}

Receptor: Male born in 1920

\begin{tabular}{lccc}
\hline & \multicolumn{3}{c}{ Thyroid Dose [cGy] } \\
Exposure Pathway & \multicolumn{2}{c}{$95 \%$ Subjective Confidence Interval } \\
\hline Backyard Cow Milk & lower limit & central estimate & upper limit \\
Commercial Milk (locally produced) & -- & -- & -- \\
Commercial Milk (regionally mixed) & -- & -- & -- \\
Goat Milk (locally produced) & 0.045 & 0.25 & 1.5 \\
Beef (locally produced) & -- & -- & -- \\
Leafy Vegetables (locally produced) & -- & -- & -- \\
Eggs (locally produced) & -- & -- & -- \\
Cottage Cheese (locally produced) & -- & -- \\
Inhalation & 0.0019 & 0.019 & 0.19 \\
Mother's milk (mother on Diet 3) & 0.04 & 0.14 & 0.47 \\
Prenatal exposure (mother on Diet 3) & -- & -- & -- \\
Diet 1 & -- & -- & -- \\
Diet 2 & -- & -- & -- \\
Diet 3 & -- & -- & -- \\
\hline
\end{tabular}

Excess Lifetime Risk [ ]

\begin{tabular}{lccc}
\hline Diet 1 & -- & -- & -- \\
Diet 2 & -- & -- & -- \\
Diet 3 & $1.3 \mathrm{E}-08$ & $5.0 \mathrm{E}-07$ & $1.5 \mathrm{E}-05$ \\
Diet 4 & -- & -- & -- \\
\hline
\end{tabular}

\section{Relative Risk [ ]}

\begin{tabular}{lccc}
\hline Diet 1 & -- & -- & -- \\
Diet 2 & -- & -- & -- \\
Diet 3 & 1.000018 & 1.00037 & 1.011 \\
Diet 4 & -- & -- & -- \\
\hline
\end{tabular}

\begin{tabular}{lccc}
\hline Diet 1 & -- & -- & -- \\
Diet 2 & -- & -- & -- \\
Diet 3 & 0.002 & 0.037 & 1.04 \\
Diet 4 & -- & -- & - \\
\hline Diet 1 - Backyard cow milk + all other locally produced non-milk exposure pathways & \\
Diet 2 - Locally produced commercial milk + all other locally produced non-milk exposure pathways \\
Diet 3 - Regionally mixed commercial milk + inhalation (other regionally mixed food items are minor contributors to the total) \\
Diet 4 - Goat milk (all other exposure pathways are of negligible importance compared to goat milk)
\end{tabular}




\section{Location: Lawnville/Gallaher}

Receptor: Female born in 1920

\begin{tabular}{lccc}
\hline & \multicolumn{3}{c}{ Thyroid Dose [cGy] } \\
& \multicolumn{2}{c}{$95 \%$ Subjective Confidence Interval } \\
Exposure Pathway & lower limit & central estimate & upper limit \\
\hline Backyard Cow Milk & 0.98 & 4.8 & 25 \\
Commercial Milk (locally produced) & 0.18 & 1 & 6.9 \\
Commercial Milk (regionally mixed) & 0.037 & 0.2 & 1.3 \\
Goat Milk (locally produced) & 1.5 & 12 & 83 \\
Beef (locally produced) & 0.0022 & 0.041 & 0.79 \\
Leafy Vegetables (locally produced) & 0.00099 & 0.011 & 0.083 \\
Eggs (locally produced) & 0.067 & 0.41 & 2.5 \\
Cottage Cheese (locally produced) & 0.0026 & 0.024 & 0.22 \\
Inhalation & 0.045 & 0.14 & 0.5 \\
Mother's milk (mother on Diet 1) & -- & -- & -- \\
Prenatal exposure (mother on Diet 1) & -- & -- & -- \\
Diet 1 & 1.2 & 5.5 & 29 \\
Diet 2 & 0.36 & 1.8 & 9.8 \\
Diet 3 & 0.097 & 0.36 & 1.8 \\
\hline
\end{tabular}

Excess Lifetime Risk [ ]

\begin{tabular}{llll}
\hline Diet 1 & $1.3 \mathrm{E}-06$ & $2.1 \mathrm{E}-05$ & $3.4 \mathrm{E}-04$ \\
Diet 2 & $4.2 \mathrm{E}-07$ & $6.6 \mathrm{E}-06$ & $8.8 \mathrm{E}-05$ \\
Diet 3 & $1.2 \mathrm{E}-07$ & $1.4 \mathrm{E}-06$ & $1.6 \mathrm{E}-05$ \\
Diet 4 & $3.0 \mathrm{E}-06$ & $4.5 \mathrm{E}-05$ & $7.3 \mathrm{E}-04$ \\
\hline
\end{tabular}

Relative Risk [ ]

\begin{tabular}{lccc}
\hline Diet 1 & 1.00088 & 1.008 & 1.082 \\
Diet 2 & 1.00027 & 1.0025 & 1.03 \\
Diet 3 & 1.000073 & 1.00054 & 1.0053 \\
Diet 4 & 1.0017 & 1.017 & 1.24 \\
\hline
\end{tabular}

\begin{tabular}{|c|c|c|c|}
\hline Diet 1 & 0.088 & 0.793 & 7.60 \\
\hline Diet 2 & 0.027 & 0.253 & 2.93 \\
\hline Diet 3 & 0.007 & 0.054 & 0.53 \\
\hline Diet 4 & 0.172 & 1.673 & 19.13 \\
\hline
\end{tabular}

Diet 1 - Backyard cow milk + all other locally produced non-milk exposure pathways

Diet 2 - Locally produced commercial milk + all other locally produced non-milk exposure pathways

Diet 3 - Regionally mixed commercial milk + inhalation (other regionally mixed food items are minor contributors to the total)

Diet 4 - Goat milk (all other exposure pathways are of negligible importance compared to goat milk) 


\section{Location: Lawnville/Gallaher}

Receptor: Male born in 1920

\begin{tabular}{lccc}
\hline & \multicolumn{3}{c}{ Thyroid Dose [cGy] } \\
& \multicolumn{2}{c}{$95 \%$ Subjective Confidence Interval } \\
Exposure Pathway & lower limit & central estimate & upper limit \\
\hline Backyard Cow Milk & 1.2 & 5.7 & 30 \\
Commercial Milk (locally produced) & 0.21 & 1.2 & 8.5 \\
Commercial Milk (regionally mixed) & 0.045 & 0.25 & 1.5 \\
Goat Milk (locally produced) & 2.1 & 16 & 100 \\
Beef (locally produced) & 0.0035 & 0.065 & 1.3 \\
Leafy Vegetables (locally produced) & 0.0011 & 0.01 & 0.081 \\
Eggs (locally produced) & 0.069 & 0.44 & 2.6 \\
Cottage Cheese (locally produced) & 0.0024 & 0.023 & 0.24 \\
Inhalation & 0.054 & 0.18 & 0.66 \\
Mother's milk (mother on Diet 1) & -- & -- & - \\
Prenatal exposure (mother on Diet 1) & -- & -- & - \\
Diet 1 & 1.4 & 6.5 & 35 \\
Diet 2 & 0.43 & 2.1 & 12 \\
Diet 3 & 0.12 & 0.47 & 2.1 \\
\hline
\end{tabular}

Excess Lifetime Risk [ ]

\begin{tabular}{llll}
\hline Diet 1 & $1.3 \mathrm{E}-07$ & $7.9 \mathrm{E}-06$ & $2.3 \mathrm{E}-04$ \\
Diet 2 & $5.3 \mathrm{E}-08$ & $2.5 \mathrm{E}-06$ & $7.7 \mathrm{E}-05$ \\
Diet 3 & $1.4 \mathrm{E}-08$ & $5.6 \mathrm{E}-07$ & $1.5 \mathrm{E}-05$ \\
Diet 4 & $3.2 \mathrm{E}-07$ & $1.7 \mathrm{E}-05$ & $6.3 \mathrm{E}-04$ \\
\hline
\end{tabular}

Relative Risk [ ]

\begin{tabular}{lccc}
\hline Diet 1 & 1.00029 & 1.006 & 1.16 \\
Diet 2 & 1.000087 & 1.0019 & 1.057 \\
Diet 3 & 1.000021 & 1.00042 & 1.011 \\
Diet 4 & 1.00046 & 1.016 & 1.45 \\
\hline
\end{tabular}

\section{Probability of Causation [\%]}

\begin{tabular}{lccc}
\hline Diet 1 & 0.029 & 0.600 & 13.99 \\
Diet 2 & 0.009 & 0.191 & 5.41 \\
Diet 3 & 0.002 & 0.042 & 1.10 \\
Diet 4 & 0.046 & 1.570 & 30.97 \\
\hline Diet 1 Bas
\end{tabular}

Diet 1 - Backyard cow milk + all other locally produced non-milk exposure pathways

Diet 2 - Locally produced commercial milk + all other locally produced non-milk exposure pathways

Diet 3 - Regionally mixed commercial milk + inhalation (other regionally mixed food items are minor contributors to the total)

Diet 4 - Goat milk (all other exposure pathways are of negligible importance compared to goat milk) 


\section{Location: Dyllis}

Receptor: Female born in 1920

\begin{tabular}{lccc}
\hline & \multicolumn{3}{c}{ Thyroid Dose [cGy] } \\
Exposure Pathway & \multicolumn{2}{c}{$95 \%$ Subjective Confidence Interval } \\
\hline Backyard Cow Milk & lower limit & central estimate & upper limit \\
Commercial Milk (locally produced) & 0.16 & 0.97 & 5.5 \\
Commercial Milk (regionally mixed) & 0.03 & 0.2 & 1.5 \\
Goat Milk (locally produced) & 0.037 & 0.2 & 1.3 \\
Beef (locally produced) & 0.31 & 2.4 & 20 \\
Leafy Vegetables (locally produced) & 0.00053 & 0.008 & 0.16 \\
Eggs (locally produced) & 0.00019 & 0.0021 & 0.019 \\
Cottage Cheese (locally produced) & 0.012 & 0.084 & 0.6 \\
Inhalation & 0.00047 & 0.005 & 0.056 \\
Mother's milk (mother on Diet 1) & 0.0087 & 0.03 & 0.11 \\
Prenatal exposure (mother on Diet 1) & -- & -- & -- \\
Diet 1 & -- & -- & -- \\
Diet 2 & 0.2 & 1.1 & 6.3 \\
Diet 3 & 0.071 & 0.36 & 2.2 \\
\hline
\end{tabular}

Excess Lifetime Risk [ ]

\begin{tabular}{llll}
\hline Diet 1 & $2.8 \mathrm{E}-07$ & $4.1 \mathrm{E}-06$ & $6.8 \mathrm{E}-05$ \\
Diet 2 & $8.3 \mathrm{E}-08$ & $1.4 \mathrm{E}-06$ & $1.8 \mathrm{E}-05$ \\
Diet 3 & $6.4 \mathrm{E}-08$ & $8.9 \mathrm{E}-07$ & $1.3 \mathrm{E}-05$ \\
Diet 4 & $6.0 \mathrm{E}-07$ & $9.9 \mathrm{E}-06$ & $1.4 \mathrm{E}-04$ \\
\hline
\end{tabular}

\section{Relative Risk [ ]}

\begin{tabular}{lccc}
\hline Diet 1 & 1.00017 & 1.0016 & 1.018 \\
Diet 2 & 1.000063 & 1.0005 & 1.0066 \\
Diet 3 & 1.00004 & 1.00035 & 1.004 \\
Diet 4 & 1.00035 & 1.0034 & 1.055 \\
\hline
\end{tabular}

\section{Probability of Causation [\%]}

\begin{tabular}{llll}
\hline Diet 1 & 0.017 & 0.156 & 1.80 \\
Diet 2 & 0.006 & 0.050 & 0.65 \\
Diet 3 & 0.004 & 0.035 & 0.40 \\
Diet 4 & 0.035 & 0.336 & 5.19 \\
\hline
\end{tabular}

Diet 1 - Backyard cow milk + all other locally produced non-milk exposure pathways

Diet 2 - Locally produced commercial milk + all other locally produced non-milk exposure pathways

Diet 3 - Regionally mixed commercial milk + inhalation (other regionally mixed food items are minor contributors to the total)

Diet 4 - Goat milk (all other exposure pathways are of negligible importance compared to goat milk) 


\section{Location: Dyllis}

\section{Receptor: Male born in 1920}

\begin{tabular}{lccc}
\hline & \multicolumn{3}{c}{ Thyroid Dose [cGy] } \\
& \multicolumn{2}{c}{$95 \%$ Subjective Confidence Interval } \\
Exposure Pathway & lower limit & central estimate & upper limit \\
\hline Backyard Cow Milk & 0.19 & 1.1 & 6.6 \\
Commercial Milk (locally produced) & 0.042 & 0.24 & 1.8 \\
Commercial Milk (regionally mixed) & 0.045 & 0.25 & 1.5 \\
Goat Milk (locally produced) & 0.44 & 3.2 & 24 \\
Beef (locally produced) & 0.00081 & 0.013 & 0.26 \\
Leafy Vegetables (locally produced) & 0.00019 & 0.0021 & 0.02 \\
Eggs (locally produced) & 0.012 & 0.089 & 0.68 \\
Cottage Cheese (locally produced) & 0.00044 & 0.0047 & 0.053 \\
Inhalation & 0.011 & 0.04 & 0.14 \\
Mother's milk (mother on Diet 1) & -- & -- & - \\
Prenatal exposure (mother on Diet 1) & -- & -- & -- \\
Diet 1 & 0.23 & 1.3 & 7.4 \\
Diet 2 & 0.09 & 0.43 & 2.8 \\
Diet 3 & 0.067 & 0.3 & 1.6 \\
\hline
\end{tabular}

Excess Lifetime Risk [ ]

\begin{tabular}{llll}
\hline Diet 1 & $2.8 \mathrm{E}-08$ & $1.7 \mathrm{E}-06$ & $5.3 \mathrm{E}-05$ \\
Diet 2 & $1.1 \mathrm{E}-08$ & $5.3 \mathrm{E}-07$ & $1.9 \mathrm{E}-05$ \\
Diet 3 & $9.1 \mathrm{E}-09$ & $3.6 \mathrm{E}-07$ & $1.3 \mathrm{E}-05$ \\
Diet 4 & $6.8 \mathrm{E}-08$ & $3.6 \mathrm{E}-06$ & $1.3 \mathrm{E}-04$ \\
\hline
\end{tabular}

\section{Relative Risk [ ]}

\begin{tabular}{lccc}
\hline Diet 1 & 1.000054 & 1.0012 & 1.038 \\
Diet 2 & 1.000015 & 1.00043 & 1.012 \\
Diet 3 & 1.000012 & 1.00027 & 1.0084 \\
Diet 4 & 1.0001 & 1.0032 & 1.091 \\
\hline
\end{tabular}

\section{Probability of Causation [\%]}

\begin{tabular}{llcc}
\hline Diet 1 & 0.005 & 0.125 & 3.62 \\
Diet 2 & 0.001 & 0.043 & 1.21 \\
Diet 3 & 0.001 & 0.027 & 0.83 \\
Diet 4 & 0.010 & 0.314 & 8.30 \\
\hline
\end{tabular}

Diet 1 - Backyard cow milk + all other locally produced non-milk exposure pathways

Diet 2 - Locally produced commercial milk + all other locally produced non-milk exposure pathways

Diet 3 - Regionally mixed commercial milk + inhalation (other regionally mixed food items are minor contributors to the total)

Diet 4 - Goat milk (all other exposure pathways are of negligible importance compared to goat milk) 


\section{Location: OR High School Area}

Receptor: Female born in 1920

\begin{tabular}{lccc}
\hline & \multicolumn{3}{c}{ Thyroid Dose [cGy] } \\
Exposure Pathway & \multicolumn{2}{c}{$9 \%$ Subjective Confidence Interval } \\
\hline Backyard Cow Milk & lower limit & central estimate & upper limit \\
Commercial Milk (locally produced) & -- & -- & -- \\
Commercial Milk (regionally mixed) & -- & -- & -- \\
Goat Milk (locally produced) & 0.037 & 0.2 & 1.3 \\
Beef (locally produced) & -- & -- & -- \\
Leafy Vegetables (locally produced) & -- & -- & -- \\
Eggs (locally produced) & -- & -- & -- \\
Cottage Cheese (locally produced) & -- & -- & -- \\
Inhalation & 0.0011 & 0.011 & 0.1 \\
Mother's milk (mother on Diet 3) & 0.02 & 0.063 & 0.23 \\
Prenatal exposure (mother on Diet 3) & -- & -- & -- \\
Diet 1 & -- & -- & -- \\
Diet 2 & -- & -- & -- \\
Diet 3 & -- & -- & -- \\
\hline
\end{tabular}

Excess Lifetime Risk [ ]

\begin{tabular}{lccc}
\hline Diet 1 & -- & -- & -- \\
Diet 2 & -- & -- & -- \\
Diet 3 & $7.8 \mathrm{E}-08$ & $1.0 \mathrm{E}-06$ & $1.4 \mathrm{E}-05$ \\
Diet 4 & -- & -- & -- \\
\hline
\end{tabular}

\section{Relative Risk [ ]}

\begin{tabular}{lccc}
\hline Diet 1 & -- & -- & -- \\
Diet 2 & -- & -- & -- \\
Diet 3 & 1.00005 & 1.00041 & 1.0044 \\
Diet 4 & -- & -- & -- \\
\hline
\end{tabular}

\begin{tabular}{lccc}
\hline Diet 1 & -- & -- & -- \\
Diet 2 & -- & -- & -- \\
Diet 3 & 0.005 & 0.041 & 0.44 \\
Diet 4 & -- & -- & - \\
\hline Diet 1 - Backyard cow milk + all other locally produced non-milk exposure pathways & \\
Diet 2 - Locally produced commercial milk + all other locally produced non-milk exposure pathways \\
Diet 3 - Regionally mixed commercial milk + inhalation (other regionally mixed food items are minor contributors to the total) \\
Diet 4 - Goat milk (all other exposure pathways are of negligible importance compared to goat milk)
\end{tabular}




\section{Location: OR High School Area}

Receptor: Male born in 1920

\begin{tabular}{lccc}
\hline & \multicolumn{3}{c}{ Thyroid Dose [cGy] } \\
Exposure Pathway & \multicolumn{2}{c}{$95 \%$ Subjective Confidence Interval } \\
\hline Backyard Cow Milk & lower limit & central estimate & upper limit \\
Commercial Milk (locally produced) & -- & -- & -- \\
Commercial Milk (regionally mixed) & -- & -- & -- \\
Goat Milk (locally produced) & 0.045 & 0.25 & 1.5 \\
Beef (locally produced) & -- & -- & -- \\
Leafy Vegetables (locally produced) & -- & -- & -- \\
Eggs (locally produced) & -- & -- & -- \\
Cottage Cheese (locally produced) & -- & -- \\
Inhalation & 0.0012 & 0.011 & 0.094 \\
Mother's milk (mother on Diet 3) & 0.025 & 0.082 & 0.28 \\
Prenatal exposure (mother on Diet 3) & -- & -- & -- \\
Diet 1 & -- & -- & -- \\
Diet 2 & -- & -- & -- \\
Diet 3 & -- & -- & -- \\
\hline
\end{tabular}

Excess Lifetime Risk [ ]

\begin{tabular}{lccc}
\hline Diet 1 & -- & -- & -- \\
Diet 2 & -- & -- & -- \\
Diet 3 & $1.1 \mathrm{E}-08$ & $4.2 \mathrm{E}-07$ & $1.4 \mathrm{E}-05$ \\
Diet 4 & -- & -- & -- \\
\hline
\end{tabular}

\section{Relative Risk [ ]}

\begin{tabular}{lccc}
\hline Diet 1 & -- & -- & -- \\
Diet 2 & -- & -- & -- \\
Diet 3 & 1.000014 & 1.00031 & 1.0096 \\
Diet 4 & -- & -- & -- \\
\hline
\end{tabular}

\begin{tabular}{lccc}
\hline Diet 1 & -- & -- & -- \\
Diet 2 & -- & -- & -- \\
Diet 3 & 0.001 & 0.031 & -95 \\
Diet 4 & -- & -- & \\
\hline Diet 1 - Backyard cow milk + all other locally produced non-milk exposure pathways \\
Diet 2 - Locally produced commercial milk + all other locally produced non-milk exposure pathways \\
Diet 3 - Regionally mixed commercial milk + inhalation (other regionally mixed food items are minor contributors to the total) \\
Diet 4 - Goat milk (all other exposure pathways are of negligible importance compared to goat milk)
\end{tabular}




\section{Location: Norwood}

Receptor: Female born in 1920

\begin{tabular}{lccc}
\hline & \multicolumn{3}{c}{ Thyroid Dose [cGy] } \\
& \multicolumn{2}{c}{$95 \%$ Subjective Confidence Interval } \\
Exposure Pathway & lower limit & central estimate & upper limit \\
\hline Backyard Cow Milk & 0.16 & 0.89 & 5.8 \\
Commercial Milk (locally produced) & 0.028 & 0.19 & 1.4 \\
Commercial Milk (regionally mixed) & 0.037 & 0.2 & 1.3 \\
Goat Milk (locally produced) & 0.28 & 2.3 & 21 \\
Beef (locally produced) & 0.00045 & 0.0077 & 0.18 \\
Leafy Vegetables (locally produced) & 0.0002 & 0.002 & 0.018 \\
Eggs (locally produced) & 0.01 & 0.077 & 0.54 \\
Cottage Cheese (locally produced) & 0.0004 & 0.0046 & 0.055 \\
Inhalation & 0.0082 & 0.029 & 0.11 \\
Mother's milk (mother on Diet 1) & -- & -- & -- \\
Prenatal exposure (mother on Diet 1) & -- & -- & -- \\
Diet 1 & 0.19 & 1 & 6.6 \\
Diet 2 & 0.061 & 0.34 & 2.2 \\
Diet 3 & 0.049 & 0.23 & 1.4 \\
\hline
\end{tabular}

Excess Lifetime Risk [ ]

\begin{tabular}{llll}
\hline Diet 1 & $2.6 \mathrm{E}-07$ & $4.2 \mathrm{E}-06$ & $6.7 \mathrm{E}-05$ \\
Diet 2 & $7.8 \mathrm{E}-08$ & $1.3 \mathrm{E}-06$ & $1.9 \mathrm{E}-05$ \\
Diet 3 & $6.2 \mathrm{E}-08$ & $8.8 \mathrm{E}-07$ & $1.3 \mathrm{E}-05$ \\
Diet 4 & $5.6 \mathrm{E}-07$ & $9.0 \mathrm{E}-06$ & $1.6 \mathrm{E}-04$ \\
\hline
\end{tabular}

\section{Relative Risk [ ]}

\begin{tabular}{lccc}
\hline Diet 1 & 1.00016 & 1.0015 & 1.017 \\
Diet 2 & 1.000051 & 1.00047 & 1.0061 \\
Diet 3 & 1.000039 & 1.00035 & 1.004 \\
Diet 4 & 1.0003 & 1.0032 & 1.051 \\
\hline
\end{tabular}

\section{Probability of Causation [\%]}

\begin{tabular}{llll}
\hline Diet 1 & 0.016 & 0.148 & 1.69 \\
Diet 2 & 0.005 & 0.047 & 0.61 \\
Diet 3 & 0.004 & 0.035 & 0.40 \\
Diet 4 & 0.030 & 0.321 & 4.81 \\
\hline
\end{tabular}

Diet 1 - Backyard cow milk + all other locally produced non-milk exposure pathways

Diet 2 - Locally produced commercial milk + all other locally produced non-milk exposure pathways

Diet 3 - Regionally mixed commercial milk + inhalation (other regionally mixed food items are minor contributors to the total)

Diet 4 - Goat milk (all other exposure pathways are of negligible importance compared to goat milk) 


\section{Location: Norwood}

\section{Receptor: Male born in 1920}

\begin{tabular}{lccc}
\hline & \multicolumn{3}{c}{ Thyroid Dose [cGy] } \\
& \multicolumn{2}{c}{$95 \%$ Subjective Confidence Interval } \\
Exposure Pathway & lower limit & central estimate & upper limit \\
\hline Backyard Cow Milk & 0.19 & 1.1 & 6.9 \\
Commercial Milk (locally produced) & 0.037 & 0.24 & 1.8 \\
Commercial Milk (regionally mixed) & 0.045 & 0.25 & 1.5 \\
Goat Milk (locally produced) & 0.39 & 3 & 24 \\
Beef (locally produced) & 0.00074 & 0.012 & 0.28 \\
Leafy Vegetables (locally produced) & 0.00021 & 0.002 & 0.019 \\
Eggs (locally produced) & 0.011 & 0.083 & 0.59 \\
Cottage Cheese (locally produced) & 0.00044 & 0.0046 & 0.055 \\
Inhalation & 0.0098 & 0.037 & 0.13 \\
Mother's milk (mother on Diet 1) & -- & -- & - \\
Prenatal exposure (mother on Diet 1) & -- & -- & -- \\
Diet 1 & 0.23 & 1.2 & 8.2 \\
Diet 2 & 0.074 & 0.41 & 2.7 \\
Diet 3 & 0.064 & 0.3 & 1.6 \\
\hline
\end{tabular}

Excess Lifetime Risk [ ]

\begin{tabular}{llll}
\hline Diet 1 & $2.7 \mathrm{E}-08$ & $1.4 \mathrm{E}-06$ & $4.9 \mathrm{E}-05$ \\
Diet 2 & $9.8 \mathrm{E}-09$ & $4.6 \mathrm{E}-07$ & $1.7 \mathrm{E}-05$ \\
Diet 3 & $8.7 \mathrm{E}-09$ & $3.5 \mathrm{E}-07$ & $1.3 \mathrm{E}-05$ \\
Diet 4 & $6.4 \mathrm{E}-08$ & $3.3 \mathrm{E}-06$ & $1.2 \mathrm{E}-04$ \\
\hline
\end{tabular}

\section{Relative Risk [ ]}

\begin{tabular}{lccc}
\hline Diet 1 & 1.000051 & 1.0012 & 1.037 \\
Diet 2 & 1.000015 & 1.00039 & 1.013 \\
Diet 3 & 1.000011 & 1.00027 & 1.0085 \\
Diet 4 & 1.000092 & 1.003 & 1.098 \\
\hline
\end{tabular}

\section{Probability of Causation [\%]}

\begin{tabular}{llll}
\hline Diet 1 & 0.005 & 0.115 & 3.59 \\
Diet 2 & 0.002 & 0.039 & 1.26 \\
Diet 3 & 0.001 & 0.027 & 0.84 \\
Diet 4 & 0.009 & 0.294 & 8.93 \\
\hline
\end{tabular}

Diet 1 - Backyard cow milk + all other locally produced non-milk exposure pathways

Diet 2 - Locally produced commercial milk + all other locally produced non-milk exposure pathways

Diet 3 - Regionally mixed commercial milk + inhalation (other regionally mixed food items are minor contributors to the total)

Diet 4 - Goat milk (all other exposure pathways are of negligible importance compared to goat milk) 
Location: Woodland

Receptor: Female born in 1920

\begin{tabular}{lccc}
\hline & \multicolumn{3}{c}{ Thyroid Dose [cGy] } \\
Exposure Pathway & \multicolumn{2}{c}{ 95\% Subjective Confidence Interval } \\
\hline Backyard Cow Milk & lower limit & central estimate & upper limit \\
Commercial Milk (locally produced) & -- & -- & -- \\
Commercial Milk (regionally mixed) & -- & -- & -- \\
Goat Milk (locally produced) & 0.037 & 0.2 & 1.3 \\
Beef (locally produced) & -- & -- & -- \\
Leafy Vegetables (locally produced) & -- & -- & -- \\
Eggs (locally produced) & -- & -- & -- \\
Cottage Cheese (locally produced) & -- & -- & -- \\
Inhalation & 0.0017 & 0.016 & 0.15 \\
Mother's milk (mother on Diet 3) & 0.03 & 0.097 & 0.34 \\
Prenatal exposure (mother on Diet 3) & -- & -- & -- \\
Diet 1 & -- & -- & -- \\
Diet 2 & -- & -- & -- \\
Diet 3 & -- & -- & -- \\
\hline
\end{tabular}

Excess Lifetime Risk [ ]

\begin{tabular}{lccc}
\hline Diet 1 & -- & -- & -- \\
Diet 2 & -- & -- & -- \\
Diet 3 & $9.5 \mathrm{E}-08$ & $1.2 \mathrm{E}-06$ & $1.5 \mathrm{E}-05$ \\
Diet 4 & -- & -- & -- \\
\hline
\end{tabular}

\section{Relative Risk [ ]}

\begin{tabular}{lccc}
\hline Diet 1 & -- & -- & -- \\
Diet 2 & -- & -- & -- \\
Diet 3 & 1.000059 & 1.00047 & 1.0048 \\
Diet 4 & -- & -- & -- \\
\hline
\end{tabular}

\begin{tabular}{lccc}
\hline Diet 1 & -- & -- & -- \\
Diet 2 & -- & -- & -- \\
Diet 3 & 0.006 & 0.047 & -- \\
Diet 4 & -- & -- & \\
\hline Diet 1 - Backyard cow milk + all other locally produced non-milk exposure pathways \\
Diet 2 - Locally produced commercial milk + all other locally produced non-milk exposure pathways \\
Diet 3 - Regionally mixed commercial milk + inhalation (other regionally mixed food items are minor contributors to the total) \\
Diet 4 - Goat milk (all other exposure pathways are of negligible importance compared to goat milk)
\end{tabular}


Location: Woodland

Receptor: Male born in 1920

\begin{tabular}{lccc}
\hline & \multicolumn{3}{c}{ Thyroid Dose [cGy] } \\
Exposure Pathway & \multicolumn{2}{c}{ 95\% Subjective Confidence Interval } \\
\hline Backyard Cow Milk & lower limit & central estimate & upper limit \\
Commercial Milk (locally produced) & -- & -- & -- \\
Commercial Milk (regionally mixed) & -- & -- & -- \\
Goat Milk (locally produced) & 0.045 & 0.25 & 1.5 \\
Beef (locally produced) & -- & -- & -- \\
Leafy Vegetables (locally produced) & -- & -- & -- \\
Eggs (locally produced) & -- & -- & -- \\
Cottage Cheese (locally produced) & -- & -- & -- \\
Inhalation & 0.0016 & 0.016 & 0.15 \\
Mother's milk (mother on Diet 3) & 0.037 & 0.12 & 0.43 \\
Prenatal exposure (mother on Diet 3) & -- & -- & -- \\
Diet 1 & -- & -- & -- \\
Diet 2 & -- & -- & -- \\
Diet 3 & -- & -- & -- \\
\hline
\end{tabular}

Excess Lifetime Risk [ ]

\begin{tabular}{lccc}
\hline Diet 1 & -- & -- & -- \\
Diet 2 & -- & -- & -- \\
Diet 3 & $1.2 \mathrm{E}-08$ & $4.8 \mathrm{E}-07$ & $1.4 \mathrm{E}-05$ \\
Diet 4 & -- & -- & -- \\
\hline
\end{tabular}

\section{Relative Risk [ ]}

\begin{tabular}{lccc}
\hline Diet 1 & -- & -- & -- \\
Diet 2 & -- & -- & -- \\
Diet 3 & 1.000017 & 1.00036 & 1.01 \\
Diet 4 & -- & -- & -- \\
\hline
\end{tabular}

\begin{tabular}{lccc}
\hline Diet 1 & -- & -- & -- \\
Diet 2 & -- & -- & -- \\
Diet 3 & 0.002 & 0.036 & 1.01 \\
Diet 4 & -- & -- & - \\
\hline Diet 1 - Backyard cow milk + all other locally produced non-milk exposure pathways \\
Diet 2 - Locally produced commercial milk + all other locally produced non-milk exposure pathways \\
Diet 3 - Regionally mixed commercial milk + inhalation (other regionally mixed food items are minor contributors to the total) \\
Diet 4 - Goat milk (all other exposure pathways are of negligible importance compared to goat milk)
\end{tabular}


Location: Hardin Valley

Receptor: Female born in 1920

\begin{tabular}{lccc}
\hline & \multicolumn{3}{c}{ Thyroid Dose [cGy] } \\
Exposure Pathway & $95 \%$ Subjective Confidence Interval \\
\hline Backyard Cow Milk & lower limit & central estimate & upper limit \\
Commercial Milk (locally produced) & 0.85 & 4.6 & 25 \\
Commercial Milk (regionally mixed) & 0.16 & 0.94 & 6.4 \\
Goat Milk (locally produced) & 0.037 & 0.2 & 1.3 \\
Beef (locally produced) & 1.5 & 11 & 81 \\
Leafy Vegetables (locally produced) & 0.0023 & 0.037 & 0.75 \\
Eggs (locally produced) & 0.0009 & 0.0096 & 0.08 \\
Cottage Cheese (locally produced) & 0.061 & 0.39 & 2.4 \\
Inhalation & 0.0023 & 0.022 & 0.22 \\
Mother's milk (mother on Diet 1) & 0.043 & 0.14 & 0.5 \\
Prenatal exposure (mother on Diet 1) & -- & -- & -- \\
Diet 1 & -- & -- & -- \\
Diet 2 & 1 & 5.3 & 29 \\
Diet 3 & 0.33 & 1.7 & 9.8 \\
\hline
\end{tabular}

Excess Lifetime Risk [ ]

\begin{tabular}{lllc}
\hline Diet 1 & $1.3 \mathrm{E}-06$ & $1.9 \mathrm{E}-05$ & $2.9 \mathrm{E}-04$ \\
Diet 2 & $4.1 \mathrm{E}-07$ & $6.2 \mathrm{E}-06$ & $8.0 \mathrm{E}-05$ \\
Diet 3 & $1.2 \mathrm{E}-07$ & $1.3 \mathrm{E}-06$ & $1.6 \mathrm{E}-05$ \\
Diet 4 & $2.6 \mathrm{E}-06$ & $4.6 \mathrm{E}-05$ & $6.3 \mathrm{E}-04$ \\
\hline
\end{tabular}

\section{Relative Risk [ ]}

\begin{tabular}{lccc}
\hline Diet 1 & 1.00082 & 1.0073 & 1.079 \\
Diet 2 & 1.00027 & 1.0023 & 1.027 \\
Diet 3 & 1.000069 & 1.00054 & 1.0053 \\
Diet 4 & 1.0017 & 1.016 & 1.25 \\
\hline
\end{tabular}

\begin{tabular}{llcc} 
& \multicolumn{3}{c}{ Probability of Causation [\%] } \\
\hline Diet 1 & 0.082 & 0.727 & 7.29 \\
Diet 2 & 0.027 & 0.228 & 2.61 \\
Diet 3 & 0.007 & 0.054 & 0.53 \\
Diet 4 & 0.166 & 1.545 & 19.75 \\
\hline
\end{tabular}

Diet 1 - Backyard cow milk + all other locally produced non-milk exposure pathways

Diet 2 - Locally produced commercial milk + all other locally produced non-milk exposure pathways

Diet 3 - Regionally mixed commercial milk + inhalation (other regionally mixed food items are minor contributors to the total)

Diet 4 - Goat milk (all other exposure pathways are of negligible importance compared to goat milk) 
Location: Hardin Valley

Receptor: Male born in 1920

\begin{tabular}{lccc}
\hline & \multicolumn{3}{c}{ Thyroid Dose [cGy] } \\
& \multicolumn{2}{c}{$95 \%$ Subjective Confidence Interval } \\
Exposure Pathway & lower limit & central estimate & upper limit \\
\hline Backyard Cow Milk & 1 & 5.5 & 30 \\
Commercial Milk (locally produced) & 0.21 & 1.2 & 7.7 \\
Commercial Milk (regionally mixed) & 0.045 & 0.25 & 1.5 \\
Goat Milk (locally produced) & 2.2 & 15 & 97 \\
Beef (locally produced) & 0.0036 & 0.058 & 1.2 \\
Leafy Vegetables (locally produced) & 0.00089 & 0.0096 & 0.083 \\
Eggs (locally produced) & 0.06 & 0.41 & 2.8 \\
Cottage Cheese (locally produced) & 0.0023 & 0.023 & 0.22 \\
Inhalation & 0.054 & 0.18 & 0.61 \\
Mother's milk (mother on Diet 1) & -- & -- & -- \\
Prenatal exposure (mother on Diet 1) & -- & -- & - \\
Diet 1 & 1.2 & 6.4 & 35 \\
Diet 2 & 0.42 & 2 & 11 \\
Diet 3 & 0.12 & 0.47 & 2.1 \\
\hline
\end{tabular}

Excess Lifetime Risk [ ]

\begin{tabular}{lllc}
\hline Diet 1 & $1.3 \mathrm{E}-07$ & $7.6 \mathrm{E}-06$ & $2.1 \mathrm{E}-04$ \\
Diet 2 & $5.1 \mathrm{E}-08$ & $2.4 \mathrm{E}-06$ & $8.2 \mathrm{E}-05$ \\
Diet 3 & $1.4 \mathrm{E}-08$ & $5.6 \mathrm{E}-07$ & $1.6 \mathrm{E}-05$ \\
Diet 4 & $3.1 \mathrm{E}-07$ & $1.7 \mathrm{E}-05$ & $6.1 \mathrm{E}-04$ \\
\hline
\end{tabular}

Relative Risk [ ]

\begin{tabular}{lccc}
\hline Diet 1 & 1.00028 & 1.0057 & 1.16 \\
Diet 2 & 1.000081 & 1.0019 & 1.056 \\
Diet 3 & 1.000022 & 1.00042 & 1.011 \\
Diet 4 & 1.00044 & 1.014 & 1.44 \\
\hline
\end{tabular}

\begin{tabular}{lccc}
\hline Diet 1 & 0.028 & 0.567 & 13.63 \\
Diet 2 & 0.008 & 0.185 & 5.30 \\
Diet 3 & 0.002 & 0.042 & 1.11 \\
Diet 4 & 0.044 & 1.406 & 30.41 \\
\hline
\end{tabular}

Diet 1 - Backyard cow milk + all other locally produced non-milk exposure pathways

Diet 2 - Locally produced commercial milk + all other locally produced non-milk exposure pathways

Diet 3 - Regionally mixed commercial milk + inhalation (other regionally mixed food items are minor contributors to the total)

Diet 4 - Goat milk (all other exposure pathways are of negligible importance compared to goat milk) 


\section{Location: Oliver Springs}

Receptor: Female born in 1920

\begin{tabular}{lccc}
\hline & \multicolumn{3}{c}{ Thyroid Dose [cGy] } \\
Exposure Pathway & \multicolumn{2}{c}{$95 \%$ Subjective Confidence Interval } \\
\hline Backyard Cow Milk & lower limit & central estimate & upper limit \\
Commercial Milk (locally produced) & 0.13 & 0.68 & 4 \\
Commercial Milk (regionally mixed) & 0.022 & 0.15 & 1 \\
Goat Milk (locally produced) & 0.037 & 0.2 & 1.3 \\
Beef (locally produced) & 0.21 & 1.8 & 14 \\
Leafy Vegetables (locally produced) & 0.00034 & 0.006 & 0.13 \\
Eggs (locally produced) & 0.00013 & 0.0015 & 0.014 \\
Cottage Cheese (locally produced) & 0.0089 & 0.059 & 0.43 \\
Inhalation & 0.00033 & 0.0035 & 0.038 \\
Mother's milk (mother on Diet 1) & 0.0067 & 0.022 & 0.085 \\
Prenatal exposure (mother on Diet 1) & -- & -- & -- \\
Diet 1 & -- & -- & -- \\
Diet 2 & 0.15 & 0.79 & 4.7 \\
Diet 3 & 0.051 & 0.26 & 1.6 \\
\hline
\end{tabular}

Excess Lifetime Risk [ ]

\begin{tabular}{lllc}
\hline Diet 1 & $1.8 \mathrm{E}-07$ & $3.0 \mathrm{E}-06$ & $5.3 \mathrm{E}-05$ \\
Diet 2 & $6.2 \mathrm{E}-08$ & $9.7 \mathrm{E}-07$ & $1.5 \mathrm{E}-05$ \\
Diet 3 & $6.0 \mathrm{E}-08$ & $8.5 \mathrm{E}-07$ & $1.3 \mathrm{E}-05$ \\
Diet 4 & $4.0 \mathrm{E}-07$ & $6.7 \mathrm{E}-06$ & $1.2 \mathrm{E}-04$ \\
\hline
\end{tabular}

\section{Relative Risk [ ]}

\begin{tabular}{lccc}
\hline Diet 1 & 1.00011 & 1.0011 & 1.013 \\
Diet 2 & 1.000037 & 1.00036 & 1.0049 \\
Diet 3 & 1.000037 & 1.00034 & 1.004 \\
Diet 4 & 1.00022 & 1.0025 & 1.04 \\
\hline
\end{tabular}

\section{Probability of Causation [\%]}

\begin{tabular}{llll}
\hline Diet 1 & 0.011 & 0.115 & 1.30 \\
Diet 2 & 0.004 & 0.036 & 0.49 \\
Diet 3 & 0.004 & 0.034 & 0.40 \\
Diet 4 & 0.022 & 0.254 & 3.87 \\
\hline
\end{tabular}

Diet 1 - Backyard cow milk + all other locally produced non-milk exposure pathways

Diet 2 - Locally produced commercial milk + all other locally produced non-milk exposure pathways

Diet 3 - Regionally mixed commercial milk + inhalation (other regionally mixed food items are minor contributors to the total)

Diet 4 - Goat milk (all other exposure pathways are of negligible importance compared to goat milk) 


\section{Location: Oliver Springs}

\section{Receptor: Male born in 1920}

\begin{tabular}{lccc}
\hline & \multicolumn{3}{c}{ Thyroid Dose [cGy] } \\
& \multicolumn{2}{c}{$95 \%$ Subjective Confidence Interval } \\
Exposure Pathway & lower limit & central estimate & upper limit \\
\hline Backyard Cow Milk & 0.15 & 0.82 & 4.8 \\
Commercial Milk (locally produced) & 0.028 & 0.18 & 1.3 \\
Commercial Milk (regionally mixed) & 0.045 & 0.25 & 1.5 \\
Goat Milk (locally produced) & 0.29 & 2.3 & 17 \\
Beef (locally produced) & 0.00055 & 0.0093 & 0.2 \\
Leafy Vegetables (locally produced) & 0.00013 & 0.0015 & 0.014 \\
Eggs (locally produced) & 0.0092 & 0.063 & 0.44 \\
Cottage Cheese (locally produced) & 0.00032 & 0.0035 & 0.037 \\
Inhalation & 0.0082 & 0.029 & 0.11 \\
Mother's milk (mother on Diet 1) & -- & -- & -- \\
Prenatal exposure (mother on Diet 1) & -- & -- & -- \\
Diet 1 & 0.17 & 0.95 & 5.6 \\
Diet 2 & 0.06 & 0.31 & 2 \\
Diet 3 & 0.058 & 0.29 & 1.6 \\
\hline
\end{tabular}

Excess Lifetime Risk [ ]

\begin{tabular}{lllc}
\hline Diet 1 & $2.0 \mathrm{E}-08$ & $1.2 \mathrm{E}-06$ & $3.8 \mathrm{E}-05$ \\
Diet 2 & $7.6 \mathrm{E}-09$ & $3.7 \mathrm{E}-07$ & $1.3 \mathrm{E}-05$ \\
Diet 3 & $8.6 \mathrm{E}-09$ & $3.4 \mathrm{E}-07$ & $1.3 \mathrm{E}-05$ \\
Diet 4 & $4.7 \mathrm{E}-08$ & $2.8 \mathrm{E}-06$ & $9.5 \mathrm{E}-05$ \\
\hline
\end{tabular}

\section{Relative Risk [ ]}

\begin{tabular}{lccc}
\hline Diet 1 & 1.00004 & 1.00091 & 1.026 \\
Diet 2 & 1.000012 & 1.00031 & 1.009 \\
Diet 3 & 1.000011 & 1.00026 & 1.0083 \\
Diet 4 & 1.000065 & 1.0023 & 1.071 \\
\hline
\end{tabular}

\section{Probability of Causation [\%]}

\begin{tabular}{llll}
\hline Diet 1 & 0.004 & 0.091 & 2.50 \\
Diet 2 & 0.001 & 0.031 & 0.89 \\
Diet 3 & 0.001 & 0.026 & 0.82 \\
Diet 4 & 0.006 & 0.229 & 6.59 \\
\hline
\end{tabular}

Diet 1 - Backyard cow milk + all other locally produced non-milk exposure pathways

Diet 2 - Locally produced commercial milk + all other locally produced non-milk exposure pathways

Diet 3 - Regionally mixed commercial milk + inhalation (other regionally mixed food items are minor contributors to the total)

Diet 4 - Goat milk (all other exposure pathways are of negligible importance compared to goat milk) 


\section{Location: Solway}

Receptor: Female born in 1920

\begin{tabular}{lccc}
\hline & \multicolumn{3}{c}{ Thyroid Dose [cGy] } \\
Exposure Pathway & \multicolumn{2}{c}{$95 \%$ Subjective Confidence Interval } \\
\hline Backyard Cow Milk & lower limit & central estimate & upper limit \\
Commercial Milk (locally produced) & 0.82 & 4.2 & 23 \\
Commercial Milk (regionally mixed) & 0.15 & 0.86 & 5.9 \\
Goat Milk (locally produced) & 0.037 & 0.2 & 1.3 \\
Beef (locally produced) & 1.3 & 10 & 69 \\
Leafy Vegetables (locally produced) & 0.002 & 0.034 & 0.69 \\
Eggs (locally produced) & 0.00086 & 0.009 & 0.071 \\
Cottage Cheese (locally produced) & 0.055 & 0.34 & 2.1 \\
Inhalation & 0.0021 & 0.02 & 0.21 \\
Mother's milk (mother on Diet 1) & 0.041 & 0.13 & 0.45 \\
Prenatal exposure (mother on Diet 1) & -- & -- & -- \\
Diet 1 & -- & -- & -- \\
Diet 2 & 0.98 & 4.8 & 26 \\
Diet 3 & 0.3 & 1.5 & 8.6 \\
\hline
\end{tabular}

Excess Lifetime Risk [ ]

\begin{tabular}{lllc}
\hline Diet 1 & $1.2 \mathrm{E}-06$ & $1.8 \mathrm{E}-05$ & $2.7 \mathrm{E}-04$ \\
Diet 2 & $3.7 \mathrm{E}-07$ & $5.7 \mathrm{E}-06$ & $7.0 \mathrm{E}-05$ \\
Diet 3 & $1.1 \mathrm{E}-07$ & $1.3 \mathrm{E}-06$ & $1.6 \mathrm{E}-05$ \\
Diet 4 & $2.4 \mathrm{E}-06$ & $4.0 \mathrm{E}-05$ & $6.2 \mathrm{E}-04$ \\
\hline
\end{tabular}

Relative Risk [ ]

\begin{tabular}{lccc}
\hline Diet 1 & 1.00068 & 1.0067 & 1.069 \\
Diet 2 & 1.00023 & 1.0022 & 1.025 \\
Diet 3 & 1.000065 & 1.00053 & 1.0051 \\
Diet 4 & 1.0016 & 1.014 & 1.24 \\
\hline
\end{tabular}

\section{Probability of Causation [\%]}

\begin{tabular}{lccc}
\hline Diet 1 & 0.068 & 0.668 & 6.47 \\
Diet 2 & 0.023 & 0.218 & 2.42 \\
Diet 3 & 0.006 & 0.053 & 0.51 \\
Diet 4 & 0.158 & 1.424 & 19.48 \\
\hline
\end{tabular}

Diet 1 - Backyard cow milk + all other locally produced non-milk exposure pathways

Diet 2 - Locally produced commercial milk + all other locally produced non-milk exposure pathways

Diet 3 - Regionally mixed commercial milk + inhalation (other regionally mixed food items are minor contributors to the total)

Diet 4 - Goat milk (all other exposure pathways are of negligible importance compared to goat milk) 


\section{Location: Solway}

\section{Receptor: Male born in 1920}

\begin{tabular}{lccc}
\hline & \multicolumn{3}{c}{ Thyroid Dose [cGy] } \\
& \multicolumn{2}{c}{$95 \%$ Subjective Confidence Interval } \\
Exposure Pathway & lower limit & central estimate & upper limit \\
\hline Backyard Cow Milk & 0.95 & 5.1 & 28 \\
Commercial Milk (locally produced) & 0.18 & 1.1 & 6.8 \\
Commercial Milk (regionally mixed) & 0.045 & 0.25 & 1.5 \\
Goat Milk (locally produced) & 2 & 14 & 91 \\
Beef (locally produced) & 0.0032 & 0.054 & 1.1 \\
Leafy Vegetables (locally produced) & 0.00084 & 0.0088 & 0.078 \\
Eggs (locally produced) & 0.059 & 0.38 & 2.5 \\
Cottage Cheese (locally produced) & 0.002 & 0.021 & 0.22 \\
Inhalation & 0.051 & 0.17 & 0.55 \\
Mother's milk (mother on Diet 1) & -- & -- & - \\
Prenatal exposure (mother on Diet 1) & -- & -- & - \\
Diet 1 & 1.1 & 5.9 & 33 \\
Diet 2 & 0.39 & 1.8 & 10 \\
Diet 3 & 0.12 & 0.45 & 2 \\
\hline
\end{tabular}

Excess Lifetime Risk [ ]

\begin{tabular}{llll}
\hline Diet 1 & $1.2 \mathrm{E}-07$ & $6.8 \mathrm{E}-06$ & $1.8 \mathrm{E}-04$ \\
Diet 2 & $4.7 \mathrm{E}-08$ & $2.2 \mathrm{E}-06$ & $8.1 \mathrm{E}-05$ \\
Diet 3 & $1.4 \mathrm{E}-08$ & $5.5 \mathrm{E}-07$ & $1.5 \mathrm{E}-05$ \\
Diet 4 & $2.7 \mathrm{E}-07$ & $1.5 \mathrm{E}-05$ & $6.1 \mathrm{E}-04$ \\
\hline
\end{tabular}

\section{Relative Risk [ ]}

\begin{tabular}{lccc}
\hline Diet 1 & 1.00024 & 1.0054 & 1.15 \\
Diet 2 & 1.000073 & 1.0017 & 1.049 \\
Diet 3 & 1.000021 & 1.00042 & 1.011 \\
Diet 4 & 1.00038 & 1.013 & 1.42 \\
\hline
\end{tabular}

\begin{tabular}{lccc}
\hline Diet 1 & 0.024 & 0.533 & 13.00 \\
Diet 2 & 0.007 & 0.165 & 4.71 \\
Diet 3 & 0.002 & 0.042 & 1.09 \\
Diet 4 & 0.038 & 1.285 & 29.39 \\
\hline
\end{tabular}

Diet 1 - Backyard cow milk + all other locally produced non-milk exposure pathways

Diet 2 - Locally produced commercial milk + all other locally produced non-milk exposure pathways

Diet 3 - Regionally mixed commercial milk + inhalation (other regionally mixed food items are minor contributors to the total)

Diet 4 - Goat milk (all other exposure pathways are of negligible importance compared to goat milk) 
Location: Sugar Grove

Receptor: Female born in 1920

\begin{tabular}{lccc}
\hline & \multicolumn{3}{c}{ Thyroid Dose [cGy] } \\
Exposure Pathway & $95 \%$ Subjective Confidence Interval \\
\hline Backyard Cow Milk & lower limit & central estimate & upper limit \\
Commercial Milk (locally produced) & 0.33 & 1.7 & 10 \\
Commercial Milk (regionally mixed) & 0.059 & 0.36 & 2.5 \\
Goat Milk (locally produced) & 0.037 & 0.2 & 1.3 \\
Beef (locally produced) & 0.56 & 4.2 & 31 \\
Leafy Vegetables (locally produced) & 0.00086 & 0.015 & 0.29 \\
Eggs (locally produced) & 0.00034 & 0.0037 & 0.032 \\
Cottage Cheese (locally produced) & 0.023 & 0.14 & 0.92 \\
Inhalation & 0.00084 & 0.0088 & 0.091 \\
Mother's milk (mother on Diet 1) & 0.016 & 0.054 & 0.19 \\
Prenatal exposure (mother on Diet 1) & -- & -- & - \\
Diet 1 & -- & -- & -- \\
Diet 2 & 0.4 & 2 & 11 \\
Diet 3 & 0.13 & 0.65 & 4 \\
\hline
\end{tabular}

Excess Lifetime Risk [ ]

\begin{tabular}{llll}
\hline Diet 1 & $4.6 \mathrm{E}-07$ & $7.5 \mathrm{E}-06$ & $1.2 \mathrm{E}-04$ \\
Diet 2 & $1.6 \mathrm{E}-07$ & $2.4 \mathrm{E}-06$ & $3.2 \mathrm{E}-05$ \\
Diet 3 & $7.8 \mathrm{E}-08$ & $9.9 \mathrm{E}-07$ & $1.3 \mathrm{E}-05$ \\
Diet 4 & $1.1 \mathrm{E}-06$ & $1.7 \mathrm{E}-05$ & $2.6 \mathrm{E}-04$ \\
\hline
\end{tabular}

\section{Relative Risk [ ]}

\begin{tabular}{lccc}
\hline Diet 1 & 1.0003 & 1.0028 & 1.03 \\
Diet 2 & 1.0001 & 1.00089 & 1.011 \\
Diet 3 & 1.000047 & 1.00039 & 1.0043 \\
Diet 4 & 1.00062 & 1.006 & 1.095 \\
\hline
\end{tabular}

\begin{tabular}{llcc} 
& \multicolumn{3}{c}{ Probability of Causation [\%] } \\
\hline Diet 1 & 0.030 & 0.279 & 2.94 \\
Diet 2 & 0.010 & 0.089 & 1.05 \\
Diet 3 & 0.005 & 0.039 & 0.42 \\
Diet 4 & 0.062 & 0.595 & 8.54 \\
\hline
\end{tabular}

Diet 1 - Backyard cow milk + all other locally produced non-milk exposure pathways

Diet 2 - Locally produced commercial milk + all other locally produced non-milk exposure pathways

Diet 3 - Regionally mixed commercial milk + inhalation (other regionally mixed food items are minor contributors to the total)

Diet 4 - Goat milk (all other exposure pathways are of negligible importance compared to goat milk) 
Location: Sugar Grove

Receptor: Male born in 1920

\begin{tabular}{lccc}
\hline & \multicolumn{3}{c}{ Thyroid Dose [cGy] } \\
& \multicolumn{2}{c}{$95 \%$ Subjective Confidence Interval } \\
Exposure Pathway & lower limit & central estimate & upper limit \\
\hline Backyard Cow Milk & 0.38 & 2.1 & 12 \\
Commercial Milk (locally produced) & 0.074 & 0.45 & 3.1 \\
Commercial Milk (regionally mixed) & 0.045 & 0.25 & 1.5 \\
Goat Milk (locally produced) & 0.83 & 5.8 & 40 \\
Beef (locally produced) & 0.0014 & 0.023 & 0.47 \\
Leafy Vegetables (locally produced) & 0.00034 & 0.0036 & 0.034 \\
Eggs (locally produced) & 0.024 & 0.16 & 1.1 \\
Cottage Cheese (locally produced) & 0.00084 & 0.0086 & 0.091 \\
Inhalation & 0.02 & 0.071 & 0.24 \\
Mother's milk (mother on Diet 1) & -- & -- & -- \\
Prenatal exposure (mother on Diet 1) & -- & -- & - \\
Diet 1 & 0.46 & 2.5 & 13 \\
Diet 2 & 0.16 & 0.77 & 4.7 \\
Diet 3 & 0.078 & 0.33 & 1.7 \\
\hline
\end{tabular}

Excess Lifetime Risk [ ]

\begin{tabular}{lllc}
\hline Diet 1 & $5.0 \mathrm{E}-08$ & $2.9 \mathrm{E}-06$ & $8.3 \mathrm{E}-05$ \\
Diet 2 & $2.0 \mathrm{E}-08$ & $9.1 \mathrm{E}-07$ & $3.2 \mathrm{E}-05$ \\
Diet 3 & $1.0 \mathrm{E}-08$ & $4.1 \mathrm{E}-07$ & $1.4 \mathrm{E}-05$ \\
Diet 4 & $1.2 \mathrm{E}-07$ & $6.3 \mathrm{E}-06$ & $2.5 \mathrm{E}-04$ \\
\hline
\end{tabular}

\section{Relative Risk [ ]}

\begin{tabular}{lccc}
\hline Diet 1 & 1.000098 & 1.0023 & 1.063 \\
Diet 2 & 1.00003 & 1.00072 & 1.022 \\
Diet 3 & 1.000014 & 1.0003 & 1.0091 \\
Diet 4 & 1.00016 & 1.0054 & 1.17 \\
\hline
\end{tabular}

\begin{tabular}{lccc} 
& \multicolumn{3}{c}{ Probability of Causation [\%] } \\
\hline Diet 1 & 0.010 & 0.227 & 5.91 \\
Diet 2 & 0.003 & 0.072 & 2.11 \\
Diet 3 & 0.001 & 0.030 & 0.91 \\
Diet 4 & 0.016 & 0.541 & 14.28 \\
\hline
\end{tabular}

Diet 1 - Backyard cow milk + all other locally produced non-milk exposure pathways

Diet 2 - Locally produced commercial milk + all other locally produced non-milk exposure pathways

Diet 3 - Regionally mixed commercial milk + inhalation (other regionally mixed food items are minor contributors to the total)

Diet 4 - Goat milk (all other exposure pathways are of negligible importance compared to goat milk) 


\section{Location: OR Townsite}

Receptor: Female born in 1920

\begin{tabular}{lccc}
\hline & \multicolumn{3}{c}{ Thyroid Dose [cGy] } \\
Exposure Pathway & \multicolumn{2}{c}{$9 \%$ Subjective Confidence Interval } \\
\hline Backyard Cow Milk & lower limit & central estimate & upper limit \\
Commercial Milk (locally produced) & -- & -- & -- \\
Commercial Milk (regionally mixed) & -- & -- & -- \\
Goat Milk (locally produced) & 0.037 & 0.2 & 1.3 \\
Beef (locally produced) & -- & -- & -- \\
Leafy Vegetables (locally produced) & -- & -- & -- \\
Eggs (locally produced) & -- & -- & -- \\
Cottage Cheese (locally produced) & -- & -- & -- \\
Inhalation & 0.00098 & 0.0092 & 0.093 \\
Mother's milk (mother on Diet 3) & 0.018 & 0.06 & 0.21 \\
Prenatal exposure (mother on Diet 3) & -- & -- & -- \\
Diet 1 & -- & -- & -- \\
Diet 2 & -- & -- & -- \\
Diet 3 & -- & -- & -- \\
\hline
\end{tabular}

Excess Lifetime Risk [ ]

\begin{tabular}{lccc}
\hline Diet 1 & -- & -- & -- \\
Diet 2 & -- & -- & -- \\
Diet 3 & $7.9 \mathrm{E}-08$ & $1.0 \mathrm{E}-06$ & $1.3 \mathrm{E}-05$ \\
Diet 4 & -- & -- & -- \\
\hline
\end{tabular}

\section{Relative Risk [ ]}

\begin{tabular}{lccc}
\hline Diet 1 & -- & -- & -- \\
Diet 2 & -- & -- & -- \\
Diet 3 & 1.000048 & 1.0004 & 1.0043 \\
Diet 4 & -- & -- & -- \\
\hline
\end{tabular}

\begin{tabular}{lccc}
\hline Diet 1 & -- & -- & -- \\
Diet 2 & -- & -- & -- \\
Diet 3 & 0.005 & 0.040 & 0.43 \\
Diet 4 & -- & -- & - \\
\hline Diet 1 - Backyard cow milk + all other locally produced non-milk exposure pathways & \\
Diet 2 - Locally produced commercial milk + all other locally produced non-milk exposure pathways \\
Diet 3 - Regionally mixed commercial milk + inhalation (other regionally mixed food items are minor contributors to the total) \\
Diet 4 - Goat milk (all other exposure pathways are of negligible importance compared to goat milk)
\end{tabular}




\section{Location: OR Townsite}

\section{Receptor: Male born in 1920}

\begin{tabular}{lccc}
\hline & \multicolumn{3}{c}{ Thyroid Dose [cGy] } \\
Exposure Pathway & \multicolumn{2}{c}{$9 \%$ Subjective Confidence Interval } \\
\hline Backyard Cow Milk & lower limit & central estimate & upper limit \\
Commercial Milk (locally produced) & -- & -- & -- \\
Commercial Milk (regionally mixed) & -- & -- & -- \\
Goat Milk (locally produced) & 0.045 & 0.25 & 1.5 \\
Beef (locally produced) & -- & -- & -- \\
Leafy Vegetables (locally produced) & -- & -- & -- \\
Eggs (locally produced) & -- & -- & -- \\
Cottage Cheese (locally produced) & -- & -- & -- \\
Inhalation & 0.00095 & 0.0096 & 0.094 \\
Mother's milk (mother on Diet 3) & 0.022 & 0.076 & 0.26 \\
Prenatal exposure (mother on Diet 3) & -- & -- & -- \\
Diet 1 & -- & -- & -- \\
Diet 2 & -- & -- & -- \\
Diet 3 & -- & -- & -- \\
\hline
\end{tabular}

Excess Lifetime Risk [ ]

\begin{tabular}{lccc}
\hline Diet 1 & -- & -- & -- \\
Diet 2 & -- & -- & -- \\
Diet 3 & $1.0 \mathrm{E}-08$ & $4.2 \mathrm{E}-07$ & $1.4 \mathrm{E}-05$ \\
Diet 4 & -- & -- & -- \\
\hline
\end{tabular}

\section{Relative Risk [ ]}

\begin{tabular}{lccc}
\hline Diet 1 & -- & -- & -- \\
Diet 2 & -- & -- & -- \\
Diet 3 & 1.000014 & 1.00031 & 1.0095 \\
Diet 4 & -- & -- & -- \\
\hline
\end{tabular}

\begin{tabular}{lccc}
\hline Diet 1 & -- & -- & -- \\
Diet 2 & -- & -- & -- \\
Diet 3 & 0.001 & 0.031 & 0.94 \\
Diet 4 & -- & -- & - \\
\hline Diet 1 - Backyard cow milk + all other locally produced non-milk exposure pathways & \\
Diet 2 - Locally produced commercial milk + all other locally produced non-milk exposure pathways \\
Diet 3 - Regionally mixed commercial milk + inhalation (other regionally mixed food items are minor contributors to the total) \\
Diet 4 - Goat milk (all other exposure pathways are of negligible importance compared to goat milk)
\end{tabular}


Location: Hines Valley

Receptor: Female born in 1920

\begin{tabular}{lccc}
\hline & \multicolumn{3}{c}{ Thyroid Dose [cGy] } \\
Exposure Pathway & $95 \%$ Subjective Confidence Interval \\
\hline Backyard Cow Milk & lower limit & central estimate & upper limit \\
Commercial Milk (locally produced) & 0.41 & 2.1 & 12 \\
Commercial Milk (regionally mixed) & 0.073 & 0.43 & 3 \\
Goat Milk (locally produced) & 0.037 & 0.21 & 1.3 \\
Beef (locally produced) & 0.65 & 5 & 37 \\
Leafy Vegetables (locally produced) & 0.00098 & 0.017 & 0.38 \\
Eggs (locally produced) & 0.00044 & 0.0044 & 0.04 \\
Cottage Cheese (locally produced) & 0.027 & 0.18 & 1.1 \\
Inhalation & 0.001 & 0.01 & 0.11 \\
Mother's milk (mother on Diet 1) & 0.022 & 0.072 & 0.24 \\
Prenatal exposure (mother on Diet 1) & -- & -- & - \\
Diet 1 & -- & -- & -- \\
Diet 2 & 0.51 & 2.4 & 13 \\
Diet 3 & 0.16 & 0.76 & 4.4 \\
\hline
\end{tabular}

Excess Lifetime Risk [ ]

\begin{tabular}{lllc}
\hline Diet 1 & $5.4 \mathrm{E}-07$ & $8.8 \mathrm{E}-06$ & $1.4 \mathrm{E}-04$ \\
Diet 2 & $2.0 \mathrm{E}-07$ & $2.9 \mathrm{E}-06$ & $4.6 \mathrm{E}-05$ \\
Diet 3 & $8.2 \mathrm{E}-08$ & $1.1 \mathrm{E}-06$ & $1.4 \mathrm{E}-05$ \\
Diet 4 & $1.3 \mathrm{E}-06$ & $2.0 \mathrm{E}-05$ & $3.1 \mathrm{E}-04$ \\
\hline
\end{tabular}

Relative Risk [ ]

\begin{tabular}{lccc}
\hline Diet 1 & 1.00037 & 1.0035 & 1.035 \\
Diet 2 & 1.00012 & 1.0011 & 1.014 \\
Diet 3 & 1.000051 & 1.00042 & 1.0045 \\
Diet 4 & 1.00073 & 1.0073 & 1.12 \\
\hline
\end{tabular}

\begin{tabular}{llcc} 
& \multicolumn{3}{c}{ Probability of Causation [\%] } \\
\hline Diet 1 & 0.037 & 0.349 & 3.37 \\
Diet 2 & 0.012 & 0.109 & 1.35 \\
Diet 3 & 0.005 & 0.042 & 0.44 \\
Diet 4 & 0.072 & 0.728 & 10.32 \\
\hline
\end{tabular}

Diet 1 - Backyard cow milk + all other locally produced non-milk exposure pathways

Diet 2 - Locally produced commercial milk + all other locally produced non-milk exposure pathways

Diet 3 - Regionally mixed commercial milk + inhalation (other regionally mixed food items are minor contributors to the total)

Diet 4 - Goat milk (all other exposure pathways are of negligible importance compared to goat milk) 
Location: Hines Valley

Receptor: Male born in 1920

\begin{tabular}{lccc}
\hline & \multicolumn{3}{c}{ Thyroid Dose [cGy] } \\
& \multicolumn{2}{c}{$95 \%$ Subjective Confidence Interval } \\
Exposure Pathway & lower limit & central estimate & upper limit \\
\hline Backyard Cow Milk & 0.49 & 2.5 & 14 \\
Commercial Milk (locally produced) & 0.091 & 0.53 & 3.8 \\
Commercial Milk (regionally mixed) & 0.045 & 0.25 & 1.5 \\
Goat Milk (locally produced) & 0.94 & 6.8 & 47 \\
Beef (locally produced) & 0.0016 & 0.027 & 0.62 \\
Leafy Vegetables (locally produced) & 0.00047 & 0.0045 & 0.041 \\
Eggs (locally produced) & 0.027 & 0.19 & 1.2 \\
Cottage Cheese (locally produced) & 0.001 & 0.011 & 0.11 \\
Inhalation & 0.026 & 0.091 & 0.31 \\
Mother's milk (mother on Diet 1) & -- & -- & -- \\
Prenatal exposure (mother on Diet 1) & -- & -- & - \\
Diet 1 & 0.57 & 2.9 & 16 \\
Diet 2 & 0.19 & 0.91 & 5.3 \\
Diet 3 & 0.085 & 0.36 & 1.8 \\
\hline
\end{tabular}

Excess Lifetime Risk [ ]

\begin{tabular}{llll}
\hline Diet 1 & $6.4 \mathrm{E}-08$ & $3.5 \mathrm{E}-06$ & $1.2 \mathrm{E}-04$ \\
Diet 2 & $2.6 \mathrm{E}-08$ & $1.2 \mathrm{E}-06$ & $4.1 \mathrm{E}-05$ \\
Diet 3 & $1.1 \mathrm{E}-08$ & $4.3 \mathrm{E}-07$ & $1.4 \mathrm{E}-05$ \\
Diet 4 & $1.5 \mathrm{E}-07$ & $7.8 \mathrm{E}-06$ & $3.0 \mathrm{E}-04$ \\
\hline
\end{tabular}

\section{Relative Risk [ ]}

\begin{tabular}{lccc}
\hline Diet 1 & 1.00012 & 1.0027 & 1.075 \\
Diet 2 & 1.000036 & 1.00087 & 1.023 \\
Diet 3 & 1.000015 & 1.00033 & 1.0098 \\
Diet 4 & 1.00018 & 1.0069 & 1.21 \\
\hline
\end{tabular}

\begin{tabular}{lccc} 
& \multicolumn{3}{c}{ Probability of Causation [\%] } \\
\hline Diet 1 & 0.012 & 0.266 & 6.95 \\
Diet 2 & 0.004 & 0.087 & 2.25 \\
Diet 3 & 0.001 & 0.033 & 0.97 \\
Diet 4 & 0.018 & 0.680 & 17.20 \\
\hline
\end{tabular}

Diet 1 - Backyard cow milk + all other locally produced non-milk exposure pathways

Diet 2 - Locally produced commercial milk + all other locally produced non-milk exposure pathways

Diet 3 - Regionally mixed commercial milk + inhalation (other regionally mixed food items are minor contributors to the total)

Diet 4 - Goat milk (all other exposure pathways are of negligible importance compared to goat milk) 


\section{Location: Farragut}

Receptor: Female born in 1920

\begin{tabular}{lccc}
\hline & \multicolumn{3}{c}{ Thyroid Dose [cGy] } \\
& \multicolumn{2}{c}{$95 \%$ Subjective Confidence Interval } \\
Exposure Pathway & lower limit & central estimate & upper limit \\
\hline Backyard Cow Milk & 0.46 & 2.3 & 13 \\
Commercial Milk (locally produced) & 0.078 & 0.49 & 3.3 \\
Commercial Milk (regionally mixed) & 0.037 & 0.21 & 1.3 \\
Goat Milk (locally produced) & 0.79 & 6 & 44 \\
Beef (locally produced) & 0.0012 & 0.02 & 0.47 \\
Leafy Vegetables (locally produced) & 0.00042 & 0.0051 & 0.043 \\
Eggs (locally produced) & 0.03 & 0.2 & 1.3 \\
Cottage Cheese (locally produced) & 0.0011 & 0.012 & 0.11 \\
Inhalation & 0.024 & 0.08 & 0.28 \\
Mother's milk (mother on Diet 1) & -- & -- & -- \\
Prenatal exposure (mother on Diet 1) & -- & -- & - \\
Diet 1 & 0.56 & 2.7 & 15 \\
Diet 2 & 0.18 & 0.86 & 4.7 \\
Diet 3 & 0.073 & 0.29 & 1.6 \\
\hline
\end{tabular}

Excess Lifetime Risk [ ]

\begin{tabular}{llll}
\hline Diet 1 & $6.4 \mathrm{E}-07$ & $1.0 \mathrm{E}-05$ & $1.7 \mathrm{E}-04$ \\
Diet 2 & $2.2 \mathrm{E}-07$ & $3.3 \mathrm{E}-06$ & $4.1 \mathrm{E}-05$ \\
Diet 3 & $8.6 \mathrm{E}-08$ & $1.1 \mathrm{E}-06$ & $1.4 \mathrm{E}-05$ \\
Diet 4 & $1.4 \mathrm{E}-06$ & $2.4 \mathrm{E}-05$ & $3.5 \mathrm{E}-04$ \\
\hline
\end{tabular}

Relative Risk [ ]

\begin{tabular}{lccc}
\hline Diet 1 & 1.00039 & 1.0038 & 1.04 \\
Diet 2 & 1.00014 & 1.0012 & 1.015 \\
Diet 3 & 1.000054 & 1.00044 & 1.0045 \\
Diet 4 & 1.00088 & 1.0083 & 1.13 \\
\hline
\end{tabular}

\section{Probability of Causation [\%]}

\begin{tabular}{llcc}
\hline Diet 1 & 0.039 & 0.379 & 3.83 \\
Diet 2 & 0.014 & 0.124 & 1.52 \\
Diet 3 & 0.005 & 0.044 & 0.45 \\
Diet 4 & 0.088 & 0.823 & 11.35 \\
\hline
\end{tabular}

Diet 1 - Backyard cow milk + all other locally produced non-milk exposure pathways

Diet 2 - Locally produced commercial milk + all other locally produced non-milk exposure pathways

Diet 3 - Regionally mixed commercial milk + inhalation (other regionally mixed food items are minor contributors to the total)

Diet 4 - Goat milk (all other exposure pathways are of negligible importance compared to goat milk) 


\section{Location: Farragut}

\section{Receptor: Male born in 1920}

\begin{tabular}{lccc}
\hline & \multicolumn{3}{c}{ Thyroid Dose [cGy] } \\
& \multicolumn{2}{c}{$95 \%$ Subjective Confidence Interval } \\
Exposure Pathway & lower limit & central estimate & upper limit \\
\hline Backyard Cow Milk & 0.54 & 2.8 & 16 \\
Commercial Milk (locally produced) & 0.1 & 0.6 & 3.8 \\
Commercial Milk (regionally mixed) & 0.045 & 0.25 & 1.5 \\
Goat Milk (locally produced) & 1.1 & 8.1 & 55 \\
Beef (locally produced) & 0.0019 & 0.031 & 0.76 \\
Leafy Vegetables (locally produced) & 0.00046 & 0.005 & 0.046 \\
Eggs (locally produced) & 0.032 & 0.21 & 1.5 \\
Cottage Cheese (locally produced) & 0.0012 & 0.012 & 0.11 \\
Inhalation & 0.028 & 0.1 & 0.34 \\
Mother's milk (mother on Diet 1) & -- & -- & - \\
Prenatal exposure (mother on Diet 1) & -- & -- & - \\
Diet 1 & 0.65 & 3.2 & 18 \\
Diet 2 & 0.22 & 1 & 5.6 \\
Diet 3 & 0.089 & 0.37 & 1.8 \\
\hline
\end{tabular}

Excess Lifetime Risk [ ]

\begin{tabular}{llll}
\hline Diet 1 & $7.2 \mathrm{E}-08$ & $3.9 \mathrm{E}-06$ & $1.3 \mathrm{E}-04$ \\
Diet 2 & $3.0 \mathrm{E}-08$ & $1.2 \mathrm{E}-06$ & $4.8 \mathrm{E}-05$ \\
Diet 3 & $1.1 \mathrm{E}-08$ & $4.5 \mathrm{E}-07$ & $1.4 \mathrm{E}-05$ \\
Diet 4 & $1.7 \mathrm{E}-07$ & $8.2 \mathrm{E}-06$ & $3.7 \mathrm{E}-04$ \\
\hline
\end{tabular}

\section{Relative Risk [ ]}

\begin{tabular}{lccc}
\hline Diet 1 & 1.00015 & 1.003 & 1.087 \\
Diet 2 & 1.000044 & 1.00097 & 1.029 \\
Diet 3 & 1.000015 & 1.00033 & 1.0099 \\
Diet 4 & 1.00024 & 1.0075 & 1.23 \\
\hline
\end{tabular}

\section{Probability of Causation [\%]}

\begin{tabular}{lccc}
\hline Diet 1 & 0.015 & 0.296 & 8.02 \\
Diet 2 & 0.004 & 0.097 & 2.79 \\
Diet 3 & 0.002 & 0.033 & 0.98 \\
Diet 4 & 0.024 & 0.745 & 18.82 \\
\hline
\end{tabular}

Diet 1 - Backyard cow milk + all other locally produced non-milk exposure pathways

Diet 2 - Locally produced commercial milk + all other locally produced non-milk exposure pathways

Diet 3 - Regionally mixed commercial milk + inhalation (other regionally mixed food items are minor contributors to the total)

Diet 4 - Goat milk (all other exposure pathways are of negligible importance compared to goat milk) 
Location: Lenoir City

Receptor: Female born in 1920

\begin{tabular}{lccc}
\hline & \multicolumn{3}{c}{ Thyroid Dose [cGy] } \\
Exposure Pathway & $95 \%$ Subjective Confidence Interval \\
\hline Backyard Cow Milk & lower limit & central estimate & upper limit \\
Commercial Milk (locally produced) & 0.29 & 1.4 & 7.9 \\
Commercial Milk (regionally mixed) & 0.051 & 0.3 & 2 \\
Goat Milk (locally produced) & 0.037 & 0.2 & 1.3 \\
Beef (locally produced) & -- & -- & -- \\
Leafy Vegetables (locally produced) & 0.00066 & 0.012 & 0.23 \\
Eggs (locally produced) & 0.00035 & 0.0031 & 0.025 \\
Cottage Cheese (locally produced) & 0.019 & 0.12 & 0.83 \\
Inhalation & 0.00071 & 0.0073 & 0.07 \\
Mother's milk (mother on Diet 1) & 0.015 & 0.05 & 0.18 \\
Prenatal exposure (mother on Diet 1) & -- & -- & -- \\
Diet 1 & -- & -- & -- \\
Diet 2 & 0.34 & 1.7 & 8.7 \\
Diet 3 & 0.12 & 0.51 & 2.8 \\
\hline
\end{tabular}

Excess Lifetime Risk [ ]

\begin{tabular}{lccc}
\hline Diet 1 & $3.9 \mathrm{E}-07$ & $6.0 \mathrm{E}-06$ & $1.1 \mathrm{E}-04$ \\
Diet 2 & $1.5 \mathrm{E}-07$ & $2.0 \mathrm{E}-06$ & $3.1 \mathrm{E}-05$ \\
Diet 3 & $7.5 \mathrm{E}-08$ & $9.7 \mathrm{E}-07$ & $1.3 \mathrm{E}-05$ \\
Diet 4 & -- & -- & -- \\
\hline
\end{tabular}

Relative Risk [ ]

\begin{tabular}{lccc}
\hline Diet 1 & 1.00028 & 1.0024 & 1.025 \\
Diet 2 & 1.000088 & 1.00076 & 1.0091 \\
Diet 3 & 1.000046 & 1.00039 & 1.0042 \\
Diet 4 & -- & -- & -- \\
\hline
\end{tabular}

\begin{tabular}{lccc}
\hline Diet 1 & 0.028 & 0.238 & 2.47 \\
Diet 2 & 0.009 & 0.076 & 0.90 \\
Diet 3 & 0.005 & 0.039 & 0.42 \\
Diet 4 & -- & -- & -- \\
\hline Diet 1 - Backyard cow milk + all other locally produced non-milk exposure pathways & \\
Diet 2 - Locally produced commercial milk + all other locally produced non-milk exposure pathways \\
Diet 3 - Regionally mixed commercial milk + inhalation (other regionally mixed food items are minor contributors to the total) \\
Diet 4 - Goat milk (all other exposure pathways are of negligible importance compared to goat milk)
\end{tabular}




\section{Location: Lenoir City}

\section{Receptor: Male born in 1920}

\begin{tabular}{lccc}
\hline & \multicolumn{3}{c}{ Thyroid Dose [cGy] } \\
Exposure Pathway & \multicolumn{2}{c}{$95 \%$ Subjective Confidence Interval } \\
\hline Backyard Cow Milk & lower limit & central estimate & upper limit \\
Commercial Milk (locally produced) & 0.35 & 1.7 & 8.8 \\
Commercial Milk (regionally mixed) & 0.061 & 0.37 & 2.4 \\
Goat Milk (locally produced) & 0.045 & 0.25 & 1.5 \\
Beef (locally produced) & -- & -- & -- \\
Leafy Vegetables (locally produced) & 0.0011 & 0.019 & 0.36 \\
Eggs (locally produced) & 0.00034 & 0.003 & 0.024 \\
Cottage Cheese (locally produced) & 0.021 & 0.13 & 0.79 \\
Inhalation & 0.0007 & 0.0072 & 0.074 \\
Mother's milk (mother on Diet 1) & 0.019 & 0.063 & 0.23 \\
Prenatal exposure (mother on Diet 1) & -- & -- & -- \\
Diet 1 & -- & -- & -- \\
Diet 2 & 0.41 & 2 & 9.9 \\
Diet 3 & 0.14 & 0.62 & 3.4 \\
\hline
\end{tabular}

Excess Lifetime Risk [ ]

\begin{tabular}{lccc}
\hline Diet 1 & $3.7 \mathrm{E}-08$ & $2.4 \mathrm{E}-06$ & $6.9 \mathrm{E}-05$ \\
Diet 2 & $1.6 \mathrm{E}-08$ & $7.7 \mathrm{E}-07$ & $2.3 \mathrm{E}-05$ \\
Diet 3 & $9.9 \mathrm{E}-09$ & $4.1 \mathrm{E}-07$ & $1.3 \mathrm{E}-05$ \\
Diet 4 & -- & -- & -- \\
\hline
\end{tabular}

\section{Relative Risk [ ]}

\begin{tabular}{lccc}
\hline Diet 1 & 1.000092 & 1.0018 & 1.045 \\
Diet 2 & 1.000027 & 1.00061 & 1.018 \\
Diet 3 & 1.000014 & 1.00029 & 1.0093 \\
Diet 4 & -- & -- & -- \\
\hline
\end{tabular}

\begin{tabular}{lccc}
\hline Diet 1 & 0.009 & 0.180 & 4.28 \\
Diet 2 & 0.003 & 0.061 & 1.73 \\
Diet 3 & 0.001 & 0.029 & 0.92 \\
Diet 4 & -- & -- & -- \\
\hline Diet 1 - Backyard cow milk + all other locally produced non-milk exposure pathways & \\
Diet 2 - Locally produced commercial milk + all other locally produced non-milk exposure pathways \\
Diet 3 - Regionally mixed commercial milk + inhalation (other regionally mixed food items are minor contributors to the total) \\
Diet 4 - Goat milk (all other exposure pathways are of negligible importance compared to goat milk)
\end{tabular}




\section{Location: Kingston}

Receptor: Female born in 1920

\begin{tabular}{lccc}
\hline & \multicolumn{3}{c}{ Thyroid Dose [cGy] } \\
Exposure Pathway & \multicolumn{2}{c}{$95 \%$ Subjective Confidence Interval } \\
\hline Backyard Cow Milk & lower limit & central estimate & upper limit \\
Commercial Milk (locally produced) & 0.34 & 1.9 & 10 \\
Commercial Milk (regionally mixed) & 0.062 & 0.38 & 2.5 \\
Goat Milk (locally produced) & 0.037 & 0.2 & 1.3 \\
Beef (locally produced) & 0.59 & 4.6 & 33 \\
Leafy Vegetables (locally produced) & 0.00096 & 0.016 & 0.33 \\
Eggs (locally produced) & 0.00037 & 0.0039 & 0.033 \\
Cottage Cheese (locally produced) & 0.023 & 0.15 & 0.98 \\
Inhalation & 0.00095 & 0.0092 & 0.084 \\
Mother's milk (mother on Diet 1) & 0.02 & 0.065 & 0.23 \\
Prenatal exposure (mother on Diet 1) & -- & -- & -- \\
Diet 1 & -- & -- & -- \\
Diet 2 & 0.42 & 2.2 & 12 \\
Diet 3 & 0.15 & 0.68 & 4 \\
\hline
\end{tabular}

Excess Lifetime Risk [ ]

\begin{tabular}{llll}
\hline Diet 1 & $4.7 \mathrm{E}-07$ & $7.8 \mathrm{E}-06$ & $1.2 \mathrm{E}-04$ \\
Diet 2 & $1.6 \mathrm{E}-07$ & $2.6 \mathrm{E}-06$ & $3.4 \mathrm{E}-05$ \\
Diet 3 & $8.1 \mathrm{E}-08$ & $1.0 \mathrm{E}-06$ & $1.4 \mathrm{E}-05$ \\
Diet 4 & $1.2 \mathrm{E}-06$ & $1.7 \mathrm{E}-05$ & $2.8 \mathrm{E}-04$ \\
\hline
\end{tabular}

Relative Risk [ ]

\begin{tabular}{lccc}
\hline Diet 1 & 1.00034 & 1.003 & 1.031 \\
Diet 2 & 1.00011 & 1.00098 & 1.011 \\
Diet 3 & 1.00005 & 1.00041 & 1.0043 \\
Diet 4 & 1.0007 & 1.0065 & 1.092 \\
\hline
\end{tabular}

Probability of Causation [\%]

\begin{tabular}{llll}
\hline Diet 1 & 0.034 & 0.300 & 3.01 \\
Diet 2 & 0.011 & 0.097 & 1.06 \\
Diet 3 & 0.005 & 0.041 & 0.43 \\
Diet 4 & 0.070 & 0.648 & 8.41 \\
\hline
\end{tabular}

Diet 1 - Backyard cow milk + all other locally produced non-milk exposure pathways

Diet 2 - Locally produced commercial milk + all other locally produced non-milk exposure pathways

Diet 3 - Regionally mixed commercial milk + inhalation (other regionally mixed food items are minor contributors to the total)

Diet 4 - Goat milk (all other exposure pathways are of negligible importance compared to goat milk) 


\section{Location: Kingston}

\section{Receptor: Male born in 1920}

\begin{tabular}{lccc}
\hline & \multicolumn{3}{c}{ Thyroid Dose [cGy] } \\
Exposure Pathway & \multicolumn{2}{c}{ 95\% } & Subjective Confidence Interval \\
Backyard Cow Milk & lower limit & central estimate & upper limit \\
Commercial Milk (locally produced) & 0.4 & 2.2 & 12 \\
Commercial Milk (regionally mixed) & 0.083 & 0.47 & 3 \\
Goat Milk (locally produced) & 0.045 & 0.25 & 1.5 \\
Beef (locally produced) & 0.85 & 6.3 & 41 \\
Leafy Vegetables (locally produced) & 0.0015 & 0.024 & 0.52 \\
Eggs (locally produced) & 0.00041 & 0.0039 & 0.034 \\
Cottage Cheese (locally produced) & 0.025 & 0.17 & 1 \\
Inhalation & 0.00096 & 0.0094 & 0.088 \\
Mother's milk (mother on Diet 1) & 0.024 & 0.083 & 0.29 \\
Prenatal exposure (mother on Diet 1) & -- & -- & - \\
Diet 1 & -- & -- & - \\
Diet 2 & 0.49 & 2.6 & 14 \\
Diet 3 & 0.18 & 0.82 & 4.7 \\
\hline
\end{tabular}

Excess Lifetime Risk [ ]

\begin{tabular}{llll}
\hline Diet 1 & $5.5 \mathrm{E}-08$ & $3.0 \mathrm{E}-06$ & $9.3 \mathrm{E}-05$ \\
Diet 2 & $2.2 \mathrm{E}-08$ & $1.0 \mathrm{E}-06$ & $3.2 \mathrm{E}-05$ \\
Diet 3 & $1.1 \mathrm{E}-08$ & $4.2 \mathrm{E}-07$ & $1.4 \mathrm{E}-05$ \\
Diet 4 & $1.1 \mathrm{E}-07$ & $6.9 \mathrm{E}-06$ & $2.7 \mathrm{E}-04$ \\
\hline
\end{tabular}

\section{Relative Risk [ ]}

\begin{tabular}{lccc}
\hline Diet 1 & 1.0001 & 1.0024 & 1.065 \\
Diet 2 & 1.000032 & 1.00074 & 1.023 \\
Diet 3 & 1.000015 & 1.00032 & 1.0097 \\
Diet 4 & 1.00017 & 1.0057 & 1.19 \\
\hline
\end{tabular}

\section{Probability of Causation [\%]}

\begin{tabular}{lccc}
\hline Diet 1 & 0.010 & 0.235 & 6.13 \\
Diet 2 & 0.003 & 0.074 & 2.26 \\
Diet 3 & 0.001 & 0.032 & 0.96 \\
Diet 4 & 0.017 & 0.572 & 15.61 \\
\hline
\end{tabular}

Diet 1 - Backyard cow milk + all other locally produced non-milk exposure pathways

Diet 2 - Locally produced commercial milk + all other locally produced non-milk exposure pathways

Diet 3 - Regionally mixed commercial milk + inhalation (other regionally mixed food items are minor contributors to the total)

Diet 4 - Goat milk (all other exposure pathways are of negligible importance compared to goat milk) 


\section{Location: Karns}

Receptor: Female born in 1920

\begin{tabular}{lccc}
\hline & \multicolumn{3}{c}{ Thyroid Dose [cGy] } \\
& \multicolumn{2}{c}{$95 \%$ Subjective Confidence Interval } \\
Exposure Pathway & lower limit & central estimate & upper limit \\
\hline Backyard Cow Milk & 0.48 & 2.5 & 14 \\
Commercial Milk (locally produced) & 0.088 & 0.52 & 3.2 \\
Commercial Milk (regionally mixed) & 0.037 & 0.2 & 1.3 \\
Goat Milk (locally produced) & 0.79 & 6.3 & 45 \\
Beef (locally produced) & 0.0012 & 0.021 & 0.43 \\
Leafy Vegetables (locally produced) & 0.00048 & 0.0054 & 0.043 \\
Eggs (locally produced) & 0.032 & 0.21 & 1.3 \\
Cottage Cheese (locally produced) & 0.0012 & 0.013 & 0.12 \\
Inhalation & 0.027 & 0.088 & 0.3 \\
Mother's milk (mother on Diet 1) & -- & -- & -- \\
Prenatal exposure (mother on Diet 1) & -- & -- & - \\
Diet 1 & 0.59 & 2.9 & 16 \\
Diet 2 & 0.19 & 0.93 & 4.9 \\
Diet 3 & 0.076 & 0.3 & 1.6 \\
\hline
\end{tabular}

Excess Lifetime Risk [ ]

\begin{tabular}{lllc}
\hline Diet 1 & $6.6 \mathrm{E}-07$ & $1.1 \mathrm{E}-05$ & $1.7 \mathrm{E}-04$ \\
Diet 2 & $2.4 \mathrm{E}-07$ & $3.5 \mathrm{E}-06$ & $4.5 \mathrm{E}-05$ \\
Diet 3 & $9.1 \mathrm{E}-08$ & $1.2 \mathrm{E}-06$ & $1.4 \mathrm{E}-05$ \\
Diet 4 & $1.5 \mathrm{E}-06$ & $2.5 \mathrm{E}-05$ & $4.0 \mathrm{E}-04$ \\
\hline
\end{tabular}

Relative Risk [ ]

\begin{tabular}{lccc}
\hline Diet 1 & 1.00046 & 1.0041 & 1.042 \\
Diet 2 & 1.00016 & 1.0014 & 1.015 \\
Diet 3 & 1.000058 & 1.00045 & 1.0046 \\
Diet 4 & 1.00094 & 1.0087 & 1.13 \\
\hline
\end{tabular}

\section{Probability of Causation [\%]}

\begin{tabular}{lccc}
\hline Diet 1 & 0.046 & 0.404 & 4.01 \\
Diet 2 & 0.016 & 0.136 & 1.51 \\
Diet 3 & 0.006 & 0.045 & 0.46 \\
Diet 4 & 0.094 & 0.865 & 11.82 \\
\hline
\end{tabular}

Diet 1 - Backyard cow milk + all other locally produced non-milk exposure pathways

Diet 2 - Locally produced commercial milk + all other locally produced non-milk exposure pathways

Diet 3 - Regionally mixed commercial milk + inhalation (other regionally mixed food items are minor contributors to the total)

Diet 4 - Goat milk (all other exposure pathways are of negligible importance compared to goat milk) 


\section{Location: Karns}

\section{Receptor: Male born in 1920}

\begin{tabular}{lccc}
\hline & \multicolumn{3}{c}{ Thyroid Dose [cGy] } \\
& \multicolumn{2}{c}{$95 \%$ Subjective Confidence Interval } \\
Exposure Pathway & lower limit & central estimate & upper limit \\
\hline Backyard Cow Milk & 0.57 & 3 & 15 \\
Commercial Milk (locally produced) & 0.11 & 0.64 & 3.9 \\
Commercial Milk (regionally mixed) & 0.045 & 0.25 & 1.5 \\
Goat Milk (locally produced) & 1.1 & 8.4 & 58 \\
Beef (locally produced) & 0.002 & 0.032 & 0.71 \\
Leafy Vegetables (locally produced) & 0.00049 & 0.0054 & 0.045 \\
Eggs (locally produced) & 0.035 & 0.23 & 1.5 \\
Cottage Cheese (locally produced) & 0.0013 & 0.012 & 0.11 \\
Inhalation & 0.032 & 0.11 & 0.38 \\
Mother's milk (mother on Diet 1) & -- & -- & -- \\
Prenatal exposure (mother on Diet 1) & -- & -- & -- \\
Diet 1 & 0.68 & 3.5 & 17 \\
Diet 2 & 0.24 & 1.1 & 5.8 \\
Diet 3 & 0.093 & 0.39 & 1.9 \\
\hline
\end{tabular}

Excess Lifetime Risk [ ]

\begin{tabular}{llll}
\hline Diet 1 & $7.7 \mathrm{E}-08$ & $4.3 \mathrm{E}-06$ & $1.2 \mathrm{E}-04$ \\
Diet 2 & $3.0 \mathrm{E}-08$ & $1.3 \mathrm{E}-06$ & $4.6 \mathrm{E}-05$ \\
Diet 3 & $1.2 \mathrm{E}-08$ & $4.7 \mathrm{E}-07$ & $1.4 \mathrm{E}-05$ \\
Diet 4 & $1.8 \mathrm{E}-07$ & $9.0 \mathrm{E}-06$ & $3.8 \mathrm{E}-04$ \\
\hline
\end{tabular}

\section{Relative Risk [ ]}

\begin{tabular}{lccc}
\hline Diet 1 & 1.00015 & 1.0033 & 1.088 \\
Diet 2 & 1.000045 & 1.001 & 1.029 \\
Diet 3 & 1.000016 & 1.00035 & 1.01 \\
Diet 4 & 1.00027 & 1.008 & 1.24 \\
\hline
\end{tabular}

\section{Probability of Causation [\%]}

\begin{tabular}{lccc}
\hline Diet 1 & 0.015 & 0.326 & 8.08 \\
Diet 2 & 0.005 & 0.101 & 2.80 \\
Diet 3 & 0.002 & 0.035 & 0.99 \\
Diet 4 & 0.027 & 0.791 & 19.25 \\
\hline
\end{tabular}

Diet 1 - Backyard cow milk + all other locally produced non-milk exposure pathways

Diet 2 - Locally produced commercial milk + all other locally produced non-milk exposure pathways

Diet 3 - Regionally mixed commercial milk + inhalation (other regionally mixed food items are minor contributors to the total)

Diet 4 - Goat milk (all other exposure pathways are of negligible importance compared to goat milk) 


\section{Location: Loudon}

Receptor: Female born in 1920

\begin{tabular}{lccc}
\hline & \multicolumn{3}{c}{ Thyroid Dose [cGy] } \\
Exposure Pathway & \multicolumn{2}{c}{$95 \%$ Subjective Confidence Interval } \\
\hline Backyard Cow Milk & lower limit & central estimate & upper limit \\
Commercial Milk (locally produced) & 0.27 & 1.4 & 7.7 \\
Commercial Milk (regionally mixed) & 0.045 & 0.29 & 2 \\
Goat Milk (locally produced) & 0.037 & 0.21 & 1.3 \\
Beef (locally produced) & 0.46 & 3.4 & 24 \\
Leafy Vegetables (locally produced) & 0.00072 & 0.012 & 0.24 \\
Eggs (locally produced) & 0.00028 & 0.0029 & 0.025 \\
Cottage Cheese (locally produced) & 0.018 & 0.12 & 0.73 \\
Inhalation & 0.00067 & 0.007 & 0.07 \\
Mother's milk (mother on Diet 1) & 0.016 & 0.052 & 0.18 \\
Prenatal exposure (mother on Diet 1) & -- & -- & - \\
Diet 1 & -- & -- & -- \\
Diet 2 & 0.34 & 1.6 & 8.8 \\
Diet 3 & 0.11 & 0.52 & 2.9 \\
\hline
\end{tabular}

Excess Lifetime Risk [ ]

\begin{tabular}{llll}
\hline Diet 1 & $3.7 \mathrm{E}-07$ & $6.1 \mathrm{E}-06$ & $9.7 \mathrm{E}-05$ \\
Diet 2 & $1.3 \mathrm{E}-07$ & $2.0 \mathrm{E}-06$ & $2.8 \mathrm{E}-05$ \\
Diet 3 & $7.4 \mathrm{E}-08$ & $9.7 \mathrm{E}-07$ & $1.3 \mathrm{E}-05$ \\
Diet 4 & $8.7 \mathrm{E}-07$ & $1.4 \mathrm{E}-05$ & $2.1 \mathrm{E}-04$ \\
\hline
\end{tabular}

Relative Risk [ ]

\begin{tabular}{lccc}
\hline Diet 1 & 1.00025 & 1.0022 & 1.023 \\
Diet 2 & 1.000084 & 1.00073 & 1.0082 \\
Diet 3 & 1.000045 & 1.00038 & 1.0043 \\
Diet 4 & 1.00049 & 1.0048 & 1.077 \\
\hline
\end{tabular}

\begin{tabular}{llcc} 
& \multicolumn{3}{c}{ Probability of Causation [\%] } \\
\hline Diet 1 & 0.025 & 0.222 & 2.25 \\
Diet 2 & 0.008 & 0.073 & 0.81 \\
Diet 3 & 0.005 & 0.038 & 0.42 \\
Diet 4 & 0.049 & 0.479 & 7.15 \\
\hline
\end{tabular}

Diet 1 - Backyard cow milk + all other locally produced non-milk exposure pathways

Diet 2 - Locally produced commercial milk + all other locally produced non-milk exposure pathways

Diet 3 - Regionally mixed commercial milk + inhalation (other regionally mixed food items are minor contributors to the total)

Diet 4 - Goat milk (all other exposure pathways are of negligible importance compared to goat milk) 


\section{Location: Loudon}

\section{Receptor: Male born in 1920}

\begin{tabular}{lccc}
\hline & \multicolumn{3}{c}{ Thyroid Dose [cGy] } \\
& \multicolumn{2}{c}{$95 \%$ Subjective Confidence Interval } \\
Exposure Pathway & lower limit & central estimate & upper limit \\
\hline Backyard Cow Milk & 0.33 & 1.7 & 9 \\
Commercial Milk (locally produced) & 0.059 & 0.37 & 2.3 \\
Commercial Milk (regionally mixed) & 0.045 & 0.25 & 1.5 \\
Goat Milk (locally produced) & 0.66 & 4.7 & 32 \\
Beef (locally produced) & 0.0012 & 0.018 & 0.38 \\
Leafy Vegetables (locally produced) & 0.00029 & 0.0029 & 0.027 \\
Eggs (locally produced) & 0.019 & 0.13 & 0.81 \\
Cottage Cheese (locally produced) & 0.00067 & 0.0069 & 0.072 \\
Inhalation & 0.019 & 0.067 & 0.23 \\
Mother's milk (mother on Diet 1) & -- & -- & - \\
Prenatal exposure (mother on Diet 1) & -- & -- & - \\
Diet 1 & 0.39 & 2 & 11 \\
Diet 2 & 0.13 & 0.64 & 3.5 \\
Diet 3 & 0.075 & 0.33 & 1.7 \\
\hline
\end{tabular}

Excess Lifetime Risk [ ]

\begin{tabular}{lllc}
\hline Diet 1 & $4.2 \mathrm{E}-08$ & $2.3 \mathrm{E}-06$ & $6.8 \mathrm{E}-05$ \\
Diet 2 & $1.8 \mathrm{E}-08$ & $7.3 \mathrm{E}-07$ & $2.5 \mathrm{E}-05$ \\
Diet 3 & $1.0 \mathrm{E}-08$ & $4.0 \mathrm{E}-07$ & $1.3 \mathrm{E}-05$ \\
Diet 4 & $1.1 \mathrm{E}-07$ & $5.1 \mathrm{E}-06$ & $2.1 \mathrm{E}-04$ \\
\hline
\end{tabular}

\section{Relative Risk [ ]}

\begin{tabular}{lccc}
\hline Diet 1 & 1.000078 & 1.0019 & 1.049 \\
Diet 2 & 1.000025 & 1.0006 & 1.017 \\
Diet 3 & 1.000014 & 1.0003 & 1.0092 \\
Diet 4 & 1.00013 & 1.0046 & 1.13 \\
\hline
\end{tabular}

\begin{tabular}{lccc} 
& \multicolumn{3}{c}{ Probability of Causation [\%] } \\
\hline Diet 1 & 0.008 & 0.187 & 4.68 \\
Diet 2 & 0.003 & 0.060 & 1.63 \\
Diet 3 & 0.001 & 0.030 & 0.91 \\
Diet 4 & 0.013 & 0.458 & 11.37 \\
\hline
\end{tabular}

Diet 1 - Backyard cow milk + all other locally produced non-milk exposure pathways

Diet 2 - Locally produced commercial milk + all other locally produced non-milk exposure pathways

Diet 3 - Regionally mixed commercial milk + inhalation (other regionally mixed food items are minor contributors to the total)

Diet 4 - Goat milk (all other exposure pathways are of negligible importance compared to goat milk) 
Location: Harriman

Receptor: Female born in 1920

\begin{tabular}{lccc}
\hline & \multicolumn{3}{c}{ Thyroid Dose [cGy] } \\
Exposure Pathway & \multicolumn{2}{c}{$95 \%$ Subjective Confidence Interval } \\
\hline Backyard Cow Milk & lower limit & central estimate & upper limit \\
Commercial Milk (locally produced) & 0.17 & 0.82 & 4.5 \\
Commercial Milk (regionally mixed) & 0.03 & 0.17 & 1.2 \\
Goat Milk (locally produced) & 0.037 & 0.2 & 1.3 \\
Beef (locally produced) & 0.25 & 2 & 14 \\
Leafy Vegetables (locally produced) & 0.00039 & 0.0071 & 0.13 \\
Eggs (locally produced) & 0.0002 & 0.0018 & 0.015 \\
Cottage Cheese (locally produced) & 0.011 & 0.068 & 0.46 \\
Inhalation & 0.00042 & 0.004 & 0.039 \\
Mother's milk (mother on Diet 1) & 0.0085 & 0.028 & 0.1 \\
Prenatal exposure (mother on Diet 1) & -- & -- & -- \\
Diet 1 & -- & -- & - \\
Diet 2 & 0.2 & 0.96 & 5 \\
Diet 3 & 0.066 & 0.29 & 1.7 \\
\hline
\end{tabular}

Excess Lifetime Risk [ ]

\begin{tabular}{lllc}
\hline Diet 1 & $2.3 \mathrm{E}-07$ & $3.5 \mathrm{E}-06$ & $5.9 \mathrm{E}-05$ \\
Diet 2 & $8.4 \mathrm{E}-08$ & $1.2 \mathrm{E}-06$ & $1.7 \mathrm{E}-05$ \\
Diet 3 & $6.4 \mathrm{E}-08$ & $8.9 \mathrm{E}-07$ & $1.3 \mathrm{E}-05$ \\
Diet 4 & $5.4 \mathrm{E}-07$ & $7.9 \mathrm{E}-06$ & $1.3 \mathrm{E}-04$ \\
\hline
\end{tabular}

Relative Risk [ ]

\begin{tabular}{lccc}
\hline Diet 1 & 1.00015 & 1.0013 & 1.014 \\
Diet 2 & 1.00005 & 1.00044 & 1.0052 \\
Diet 3 & 1.000038 & 1.00035 & 1.004 \\
Diet 4 & 1.00029 & 1.0028 & 1.04 \\
\hline
\end{tabular}

\section{Probability of Causation [\%]}

\begin{tabular}{llll}
\hline Diet 1 & 0.015 & 0.135 & 1.42 \\
Diet 2 & 0.005 & 0.044 & 0.52 \\
Diet 3 & 0.004 & 0.035 & 0.40 \\
Diet 4 & 0.029 & 0.283 & 3.85 \\
\hline
\end{tabular}

Diet 1 - Backyard cow milk + all other locally produced non-milk exposure pathways

Diet 2 - Locally produced commercial milk + all other locally produced non-milk exposure pathways

Diet 3 - Regionally mixed commercial milk + inhalation (other regionally mixed food items are minor contributors to the total)

Diet 4 - Goat milk (all other exposure pathways are of negligible importance compared to goat milk) 


\section{Location: Harriman}

\section{Receptor: Male born in 1920}

\begin{tabular}{lccc}
\hline & \multicolumn{3}{c}{ Thyroid Dose [cGy] } \\
& \multicolumn{2}{c}{$95 \%$ Subjective Confidence Interval } \\
Exposure Pathway & lower limit & central estimate & upper limit \\
\hline Backyard Cow Milk & 0.2 & 0.98 & 5.1 \\
Commercial Milk (locally produced) & 0.035 & 0.21 & 1.4 \\
Commercial Milk (regionally mixed) & 0.045 & 0.25 & 1.5 \\
Goat Milk (locally produced) & 0.38 & 2.8 & 18 \\
Beef (locally produced) & 0.00063 & 0.011 & 0.21 \\
Leafy Vegetables (locally produced) & 0.00019 & 0.0017 & 0.014 \\
Eggs (locally produced) & 0.012 & 0.075 & 0.46 \\
Cottage Cheese (locally produced) & 0.00041 & 0.0041 & 0.042 \\
Inhalation & 0.011 & 0.035 & 0.13 \\
Mother's milk (mother on Diet 1) & -- & -- & - \\
Prenatal exposure (mother on Diet 1) & -- & -- & -- \\
Diet 1 & 0.24 & 1.1 & 5.9 \\
Diet 2 & 0.08 & 0.36 & 2 \\
Diet 3 & 0.061 & 0.29 & 1.6 \\
\hline
\end{tabular}

Excess Lifetime Risk [ ]

\begin{tabular}{lllc}
\hline Diet 1 & $2.1 \mathrm{E}-08$ & $1.4 \mathrm{E}-06$ & $4.0 \mathrm{E}-05$ \\
Diet 2 & $9.0 \mathrm{E}-09$ & $4.4 \mathrm{E}-07$ & $1.4 \mathrm{E}-05$ \\
Diet 3 & $8.6 \mathrm{E}-09$ & $3.6 \mathrm{E}-07$ & $1.3 \mathrm{E}-05$ \\
Diet 4 & $5.6 \mathrm{E}-08$ & $3.1 \mathrm{E}-06$ & $1.1 \mathrm{E}-04$ \\
\hline
\end{tabular}

Relative Risk [ ]

\begin{tabular}{lccc}
\hline Diet 1 & 1.000051 & 1.001 & 1.026 \\
Diet 2 & 1.000015 & 1.00035 & 1.01 \\
Diet 3 & 1.000012 & 1.00027 & 1.0085 \\
Diet 4 & 1.000077 & 1.0027 & 1.07 \\
\hline
\end{tabular}

\section{Probability of Causation [\%]}

\begin{tabular}{llll}
\hline Diet 1 & 0.005 & 0.104 & 2.56 \\
Diet 2 & 0.002 & 0.035 & 0.99 \\
Diet 3 & 0.001 & 0.027 & 0.85 \\
Diet 4 & 0.008 & 0.271 & 6.54 \\
\hline
\end{tabular}

Diet 1 - Backyard cow milk + all other locally produced non-milk exposure pathways

Diet 2 - Locally produced commercial milk + all other locally produced non-milk exposure pathways

Diet 3 - Regionally mixed commercial milk + inhalation (other regionally mixed food items are minor contributors to the total)

Diet 4 - Goat milk (all other exposure pathways are of negligible importance compared to goat milk) 


\section{Location: Cedar Bluff}

Receptor: Female born in 1920

\begin{tabular}{lccc}
\hline & \multicolumn{3}{c}{ Thyroid Dose [cGy] } \\
Exposure Pathway & \multicolumn{2}{c}{$95 \%$ Subjective Confidence Interval } \\
\hline Backyard Cow Milk & lower limit & central estimate & upper limit \\
Commercial Milk (locally produced) & 0.39 & 1.9 & 11 \\
Commercial Milk (regionally mixed) & 0.072 & 0.4 & 2.8 \\
Goat Milk (locally produced) & 0.037 & 0.21 & 1.3 \\
Beef (locally produced) & 0.62 & 4.7 & 35 \\
Leafy Vegetables (locally produced) & 0.00092 & 0.017 & 0.36 \\
Eggs (locally produced) & 0.00042 & 0.0042 & 0.035 \\
Cottage Cheese (locally produced) & 0.026 & 0.17 & 1 \\
Inhalation & 0.001 & 0.0097 & 0.098 \\
Mother's milk (mother on Diet 1) & 0.022 & 0.072 & 0.24 \\
Prenatal exposure (mother on Diet 1) & -- & -- & -- \\
Diet 1 & -- & -- & -- \\
Diet 2 & 0.47 & 2.3 & 13 \\
Diet 3 & 0.15 & 0.72 & 4 \\
\hline
\end{tabular}

Excess Lifetime Risk [ ]

\begin{tabular}{llcc}
\hline Diet 1 & $5.1 \mathrm{E}-07$ & $8.3 \mathrm{E}-06$ & $1.4 \mathrm{E}-04$ \\
Diet 2 & $1.8 \mathrm{E}-07$ & $2.8 \mathrm{E}-06$ & $4.1 \mathrm{E}-05$ \\
Diet 3 & $8.2 \mathrm{E}-08$ & $1.1 \mathrm{E}-06$ & $1.4 \mathrm{E}-05$ \\
Diet 4 & $1.2 \mathrm{E}-06$ & $1.9 \mathrm{E}-05$ & $2.8 \mathrm{E}-04$ \\
\hline
\end{tabular}

Relative Risk [ ]

\begin{tabular}{lccc}
\hline Diet 1 & 1.00036 & 1.0033 & 1.032 \\
Diet 2 & 1.00012 & 1.001 & 1.012 \\
Diet 3 & 1.000052 & 1.00042 & 1.0044 \\
Diet 4 & 1.0007 & 1.0069 & 1.11 \\
\hline
\end{tabular}

Probability of Causation [\%]

\begin{tabular}{llll}
\hline Diet 1 & 0.036 & 0.331 & 3.12 \\
Diet 2 & 0.012 & 0.104 & 1.17 \\
Diet 3 & 0.005 & 0.042 & 0.44 \\
Diet 4 & 0.070 & 0.686 & 9.72 \\
\hline
\end{tabular}

Diet 1 - Backyard cow milk + all other locally produced non-milk exposure pathways

Diet 2 - Locally produced commercial milk + all other locally produced non-milk exposure pathways

Diet 3 - Regionally mixed commercial milk + inhalation (other regionally mixed food items are minor contributors to the total)

Diet 4 - Goat milk (all other exposure pathways are of negligible importance compared to goat milk) 


\section{Location: Cedar Bluff}

Receptor: Male born in 1920

\begin{tabular}{lccc}
\hline & \multicolumn{3}{c}{ Thyroid Dose [cGy] } \\
& \multicolumn{2}{c}{$95 \%$ Subjective Confidence Interval } \\
Exposure Pathway & lower limit & central estimate & upper limit \\
\hline Backyard Cow Milk & 0.46 & 2.4 & 13 \\
Commercial Milk (locally produced) & 0.09 & 0.51 & 3.4 \\
Commercial Milk (regionally mixed) & 0.045 & 0.25 & 1.5 \\
Goat Milk (locally produced) & 0.9 & 6.4 & 42 \\
Beef (locally produced) & 0.0015 & 0.026 & 0.6 \\
Leafy Vegetables (locally produced) & 0.00044 & 0.0042 & 0.036 \\
Eggs (locally produced) & 0.027 & 0.18 & 1.1 \\
Cottage Cheese (locally produced) & 0.001 & 0.0098 & 0.1 \\
Inhalation & 0.026 & 0.092 & 0.31 \\
Mother's milk (mother on Diet 1) & -- & -- & - \\
Prenatal exposure (mother on Diet 1) & -- & -- & - \\
Diet 1 & 0.53 & 2.7 & 14 \\
Diet 2 & 0.18 & 0.87 & 4.9 \\
Diet 3 & 0.086 & 0.36 & 1.8 \\
\hline
\end{tabular}

Excess Lifetime Risk [ ]

\begin{tabular}{llll}
\hline Diet 1 & $5.8 \mathrm{E}-08$ & $3.3 \mathrm{E}-06$ & $9.9 \mathrm{E}-05$ \\
Diet 2 & $2.4 \mathrm{E}-08$ & $1.1 \mathrm{E}-06$ & $3.6 \mathrm{E}-05$ \\
Diet 3 & $1.1 \mathrm{E}-08$ & $4.3 \mathrm{E}-07$ & $1.4 \mathrm{E}-05$ \\
Diet 4 & $1.4 \mathrm{E}-07$ & $7.5 \mathrm{E}-06$ & $2.7 \mathrm{E}-04$ \\
\hline
\end{tabular}

Relative Risk [ ]

\begin{tabular}{lccc}
\hline Diet 1 & 1.00012 & 1.0025 & 1.069 \\
Diet 2 & 1.000036 & 1.00081 & 1.021 \\
Diet 3 & 1.000014 & 1.00033 & 1.0098 \\
Diet 4 & 1.00018 & 1.0062 & 1.19 \\
\hline
\end{tabular}

\section{Probability of Causation [\%]}

\begin{tabular}{llcc}
\hline Diet 1 & 0.012 & 0.249 & 6.41 \\
Diet 2 & 0.004 & 0.081 & 2.10 \\
Diet 3 & 0.001 & 0.033 & 0.97 \\
Diet 4 & 0.018 & 0.616 & 15.92 \\
\hline
\end{tabular}

Diet 1 - Backyard cow milk + all other locally produced non-milk exposure pathways

Diet 2 - Locally produced commercial milk + all other locally produced non-milk exposure pathways

Diet 3 - Regionally mixed commercial milk + inhalation (other regionally mixed food items are minor contributors to the total)

Diet 4 - Goat milk (all other exposure pathways are of negligible importance compared to goat milk) 


\section{Location: Oakdale}

Receptor: Female born in 1920

\begin{tabular}{lccc}
\hline & \multicolumn{3}{c}{ Thyroid Dose [cGy] } \\
Exposure Pathway & \multicolumn{2}{c}{$95 \%$ Subjective Confidence Interval } \\
\hline Backyard Cow Milk & lower limit & central estimate & upper limit \\
Commercial Milk (locally produced) & 0.083 & 0.46 & 2.8 \\
Commercial Milk (regionally mixed) & 0.015 & 0.099 & 0.66 \\
Goat Milk (locally produced) & 0.037 & 0.21 & 1.3 \\
Beef (locally produced) & 0.13 & 1.1 & 8 \\
Leafy Vegetables (locally produced) & 0.00023 & 0.0039 & 0.079 \\
Eggs (locally produced) & 0.000094 & 0.001 & 0.0081 \\
Cottage Cheese (locally produced) & 0.0054 & 0.04 & 0.25 \\
Inhalation & 0.00023 & 0.0023 & 0.023 \\
Mother's milk (mother on Diet 1) & 0.0049 & 0.016 & 0.061 \\
Prenatal exposure (mother on Diet 1) & -- & -- & -- \\
Diet 1 & -- & -- & -- \\
Diet 2 & 0.1 & 0.54 & 3.1 \\
Diet 3 & 0.034 & 0.17 & 0.98 \\
\hline
\end{tabular}

Excess Lifetime Risk [ ]

\begin{tabular}{llll}
\hline Diet 1 & $1.2 \mathrm{E}-07$ & $2.0 \mathrm{E}-06$ & $3.2 \mathrm{E}-05$ \\
Diet 2 & $4.4 \mathrm{E}-08$ & $6.5 \mathrm{E}-07$ & $9.4 \mathrm{E}-06$ \\
Diet 3 & $5.6 \mathrm{E}-08$ & $8.3 \mathrm{E}-07$ & $1.3 \mathrm{E}-05$ \\
Diet 4 & $2.5 \mathrm{E}-07$ & $4.4 \mathrm{E}-06$ & $7.3 \mathrm{E}-05$ \\
\hline
\end{tabular}

Relative Risk [ ]

\begin{tabular}{lccc}
\hline Diet 1 & 1.000072 & 1.00077 & 1.0081 \\
Diet 2 & 1.000026 & 1.00025 & 1.0029 \\
Diet 3 & 1.000035 & 1.00033 & 1.0039 \\
Diet 4 & 1.00015 & 1.0016 & 1.024 \\
\hline
\end{tabular}

\section{Probability of Causation [\%]}

\begin{tabular}{llcc}
\hline Diet 1 & 0.007 & 0.077 & 0.81 \\
Diet 2 & 0.003 & 0.025 & 0.29 \\
Diet 3 & 0.003 & 0.033 & 0.39 \\
Diet 4 & 0.015 & 0.159 & 2.32 \\
\hline
\end{tabular}

Diet 1 - Backyard cow milk + all other locally produced non-milk exposure pathways

Diet 2 - Locally produced commercial milk + all other locally produced non-milk exposure pathways

Diet 3 - Regionally mixed commercial milk + inhalation (other regionally mixed food items are minor contributors to the total)

Diet 4 - Goat milk (all other exposure pathways are of negligible importance compared to goat milk) 


\section{Location: Oakdale}

\section{Receptor: Male born in 1920}

\begin{tabular}{lccc}
\hline & \multicolumn{3}{c}{ Thyroid Dose [cGy] } \\
& \multicolumn{2}{c}{$95 \%$ Subjective Confidence Interval } \\
Exposure Pathway & lower limit & central estimate & upper limit \\
\hline Backyard Cow Milk & 0.098 & 0.55 & 3.3 \\
Commercial Milk (locally produced) & 0.019 & 0.12 & 0.8 \\
Commercial Milk (regionally mixed) & 0.045 & 0.25 & 1.5 \\
Goat Milk (locally produced) & 0.18 & 1.5 & 11 \\
Beef (locally produced) & 0.00036 & 0.0061 & 0.13 \\
Leafy Vegetables (locally produced) & 0.000094 & 0.001 & 0.0088 \\
Eggs (locally produced) & 0.0057 & 0.043 & 0.28 \\
Cottage Cheese (locally produced) & 0.00023 & 0.0024 & 0.026 \\
Inhalation & 0.0059 & 0.021 & 0.075 \\
Mother's milk (mother on Diet 1) & -- & -- & -- \\
Prenatal exposure (mother on Diet 1) & -- & -- & -- \\
Diet 1 & 0.12 & 0.64 & 3.8 \\
Diet 2 & 0.042 & 0.21 & 1.2 \\
Diet 3 & 0.055 & 0.28 & 1.6 \\
\hline
\end{tabular}

Excess Lifetime Risk [ ]

\begin{tabular}{llll}
\hline Diet 1 & $1.2 \mathrm{E}-08$ & $7.7 \mathrm{E}-07$ & $2.4 \mathrm{E}-05$ \\
Diet 2 & $5.1 \mathrm{E}-09$ & $2.5 \mathrm{E}-07$ & $8.6 \mathrm{E}-06$ \\
Diet 3 & $8.0 \mathrm{E}-09$ & $3.2 \mathrm{E}-07$ & $1.3 \mathrm{E}-05$ \\
Diet 4 & $3.1 \mathrm{E}-08$ & $1.7 \mathrm{E}-06$ & $7.0 \mathrm{E}-05$ \\
\hline
\end{tabular}

\section{Relative Risk [ ]}

\begin{tabular}{lccc}
\hline Diet 1 & 1.000028 & 1.0006 & 1.017 \\
Diet 2 & 1.0000077 & 1.00019 & 1.0055 \\
Diet 3 & 1.00001 & 1.00025 & 1.0079 \\
Diet 4 & 1.000045 & 1.0015 & 1.044 \\
\hline
\end{tabular}

\section{Probability of Causation [\%]}

\begin{tabular}{llll}
\hline Diet 1 & 0.003 & 0.060 & 1.72 \\
Diet 2 & 0.001 & 0.019 & 0.54 \\
Diet 3 & 0.001 & 0.025 & 0.78 \\
Diet 4 & 0.005 & 0.154 & 4.16 \\
\hline
\end{tabular}

Diet 1 - Backyard cow milk + all other locally produced non-milk exposure pathways

Diet 2 - Locally produced commercial milk + all other locally produced non-milk exposure pathways

Diet 3 - Regionally mixed commercial milk + inhalation (other regionally mixed food items are minor contributors to the total)

Diet 4 - Goat milk (all other exposure pathways are of negligible importance compared to goat milk) 


\section{Location: Claxton}

Receptor: Female born in 1920

\begin{tabular}{lccc}
\hline & \multicolumn{3}{c}{ Thyroid Dose [cGy] } \\
Exposure Pathway & 95\% & Subjective Confidence Interval \\
Backyard Cow Milk & lower limit & central estimate & upper limit \\
Commercial Milk (locally produced) & 0.32 & 1.6 & 9.1 \\
Commercial Milk (regionally mixed) & 0.06 & 0.34 & 2.3 \\
Goat Milk (locally produced) & 0.037 & 0.2 & 1.3 \\
Beef (locally produced) & 0.55 & 4 & 29 \\
Leafy Vegetables (locally produced) & 0.00078 & 0.014 & 0.3 \\
Eggs (locally produced) & 0.00034 & 0.0036 & 0.029 \\
Cottage Cheese (locally produced) & 0.022 & 0.14 & 0.85 \\
Inhalation & 0.00084 & 0.008 & 0.082 \\
Mother's milk (mother on Diet 1) & 0.018 & 0.059 & 0.2 \\
Prenatal exposure (mother on Diet 1) & -- & -- & - \\
Diet 1 & -- & -- & -- \\
Diet 2 & 0.39 & 1.9 & 10 \\
Diet 3 & 0.13 & 0.6 & 3.3 \\
\hline
\end{tabular}

Excess Lifetime Risk [ ]

\begin{tabular}{llll}
\hline Diet 1 & $4.5 \mathrm{E}-07$ & $7.1 \mathrm{E}-06$ & $1.1 \mathrm{E}-04$ \\
Diet 2 & $1.6 \mathrm{E}-07$ & $2.3 \mathrm{E}-06$ & $3.4 \mathrm{E}-05$ \\
Diet 3 & $7.8 \mathrm{E}-08$ & $1.0 \mathrm{E}-06$ & $1.3 \mathrm{E}-05$ \\
Diet 4 & $9.9 \mathrm{E}-07$ & $1.6 \mathrm{E}-05$ & $2.4 \mathrm{E}-04$ \\
\hline
\end{tabular}

Relative Risk [ ]

\begin{tabular}{lccc}
\hline Diet 1 & 1.00029 & 1.0027 & 1.027 \\
Diet 2 & 1.000099 & 1.00087 & 1.0098 \\
Diet 3 & 1.000048 & 1.0004 & 1.0043 \\
Diet 4 & 1.0006 & 1.0057 & 1.087 \\
\hline
\end{tabular}

\begin{tabular}{lccc} 
& \multicolumn{3}{c}{ Probability of Causation [\%] } \\
\hline Diet 1 & 0.029 & 0.274 & 2.61 \\
Diet 2 & 0.010 & 0.087 & 0.97 \\
Diet 3 & 0.005 & 0.040 & 0.43 \\
Diet 4 & 0.060 & 0.569 & 8.02 \\
\hline
\end{tabular}

Diet 1 - Backyard cow milk + all other locally produced non-milk exposure pathways

Diet 2 - Locally produced commercial milk + all other locally produced non-milk exposure pathways

Diet 3 - Regionally mixed commercial milk + inhalation (other regionally mixed food items are minor contributors to the total)

Diet 4 - Goat milk (all other exposure pathways are of negligible importance compared to goat milk) 


\section{Location: Claxton}

\section{Receptor: Male born in 1920}

\begin{tabular}{lccc}
\hline & \multicolumn{3}{c}{ Thyroid Dose [cGy] } \\
& \multicolumn{2}{c}{$95 \%$ Subjective Confidence Interval } \\
Exposure Pathway & lower limit & central estimate & upper limit \\
\hline Backyard Cow Milk & 0.39 & 2 & 11 \\
Commercial Milk (locally produced) & 0.075 & 0.42 & 2.8 \\
Commercial Milk (regionally mixed) & 0.045 & 0.25 & 1.5 \\
Goat Milk (locally produced) & 0.78 & 5.4 & 36 \\
Beef (locally produced) & 0.0013 & 0.022 & 0.49 \\
Leafy Vegetables (locally produced) & 0.00036 & 0.0035 & 0.03 \\
Eggs (locally produced) & 0.023 & 0.15 & 0.93 \\
Cottage Cheese (locally produced) & 0.00085 & 0.0082 & 0.08 \\
Inhalation & 0.022 & 0.077 & 0.25 \\
Mother's milk (mother on Diet 1) & -- & -- & - \\
Prenatal exposure (mother on Diet 1) & -- & -- & - \\
Diet 1 & 0.45 & 2.3 & 12 \\
Diet 2 & 0.16 & 0.73 & 4 \\
Diet 3 & 0.08 & 0.34 & 1.7 \\
\hline
\end{tabular}

Excess Lifetime Risk [ ]

\begin{tabular}{llll}
\hline Diet 1 & $4.9 \mathrm{E}-08$ & $2.7 \mathrm{E}-06$ & $8.3 \mathrm{E}-05$ \\
Diet 2 & $2.0 \mathrm{E}-08$ & $9.0 \mathrm{E}-07$ & $3.1 \mathrm{E}-05$ \\
Diet 3 & $1.1 \mathrm{E}-08$ & $4.2 \mathrm{E}-07$ & $1.3 \mathrm{E}-05$ \\
Diet 4 & $1.2 \mathrm{E}-07$ & $6.2 \mathrm{E}-06$ & $2.2 \mathrm{E}-04$ \\
\hline
\end{tabular}

\section{Relative Risk [ ]}

\begin{tabular}{lccc}
\hline Diet 1 & 1.000096 & 1.0021 & 1.056 \\
Diet 2 & 1.00003 & 1.00068 & 1.018 \\
Diet 3 & 1.000014 & 1.00031 & 1.0095 \\
Diet 4 & 1.00015 & 1.0052 & 1.16 \\
\hline
\end{tabular}

\section{Probability of Causation [\%]}

\begin{tabular}{lccc}
\hline Diet 1 & 0.010 & 0.208 & 5.29 \\
Diet 2 & 0.003 & 0.068 & 1.81 \\
Diet 3 & 0.001 & 0.031 & 0.95 \\
Diet 4 & 0.015 & 0.519 & 13.74 \\
\hline
\end{tabular}

Diet 1 - Backyard cow milk + all other locally produced non-milk exposure pathways

Diet 2 - Locally produced commercial milk + all other locally produced non-milk exposure pathways

Diet 3 - Regionally mixed commercial milk + inhalation (other regionally mixed food items are minor contributors to the total)

Diet 4 - Goat milk (all other exposure pathways are of negligible importance compared to goat milk) 
Location: Dutch Valley

Receptor: Female born in 1920

\begin{tabular}{lccc}
\hline & \multicolumn{3}{c}{ Thyroid Dose [cGy] } \\
Exposure Pathway & $95 \%$ Subjective Confidence Interval \\
\hline Backyard Cow Milk & lower limit & central estimate & upper limit \\
Commercial Milk (locally produced) & 0.15 & 0.79 & 4.3 \\
Commercial Milk (regionally mixed) & 0.029 & 0.17 & 1.1 \\
Goat Milk (locally produced) & 0.037 & 0.21 & 1.3 \\
Beef (locally produced) & 0.23 & 1.9 & 14 \\
Leafy Vegetables (locally produced) & 0.00036 & 0.0066 & 0.12 \\
Eggs (locally produced) & 0.00017 & 0.0017 & 0.014 \\
Cottage Cheese (locally produced) & 0.01 & 0.067 & 0.43 \\
Inhalation & 0.00039 & 0.004 & 0.038 \\
Mother's milk (mother on Diet 1) & 0.0083 & 0.028 & 0.099 \\
Prenatal exposure (mother on Diet 1) & -- & -- & -- \\
Diet 1 & -- & -- & -- \\
Diet 2 & 0.18 & 0.93 & 4.7 \\
Diet 3 & 0.062 & 0.29 & 1.5 \\
\hline
\end{tabular}

Excess Lifetime Risk [ ]

\begin{tabular}{llcc}
\hline Diet 1 & $2.0 \mathrm{E}-07$ & $3.4 \mathrm{E}-06$ & $5.4 \mathrm{E}-05$ \\
Diet 2 & $7.6 \mathrm{E}-08$ & $1.1 \mathrm{E}-06$ & $1.6 \mathrm{E}-05$ \\
Diet 3 & $6.3 \mathrm{E}-08$ & $8.8 \mathrm{E}-07$ & $1.3 \mathrm{E}-05$ \\
Diet 4 & $4.6 \mathrm{E}-07$ & $7.7 \mathrm{E}-06$ & $1.3 \mathrm{E}-04$ \\
\hline
\end{tabular}

Relative Risk [ ]

\begin{tabular}{lccc}
\hline Diet 1 & 1.00016 & 1.0013 & 1.013 \\
Diet 2 & 1.000049 & 1.00043 & 1.005 \\
Diet 3 & 1.000039 & 1.00035 & 1.004 \\
Diet 4 & 1.00027 & 1.0028 & 1.042 \\
\hline
\end{tabular}

\begin{tabular}{llcc} 
& \multicolumn{3}{c}{ Probability of Causation [\%] } \\
\hline Diet 1 & 0.016 & 0.129 & 1.31 \\
Diet 2 & 0.005 & 0.043 & 0.50 \\
Diet 3 & 0.004 & 0.035 & 0.40 \\
Diet 4 & 0.027 & 0.276 & 3.99 \\
\hline
\end{tabular}

Diet 1 - Backyard cow milk + all other locally produced non-milk exposure pathways

Diet 2 - Locally produced commercial milk + all other locally produced non-milk exposure pathways

Diet 3 - Regionally mixed commercial milk + inhalation (other regionally mixed food items are minor contributors to the total)

Diet 4 - Goat milk (all other exposure pathways are of negligible importance compared to goat milk) 


\section{Location: Dutch Valley}

Receptor: Male born in 1920

\begin{tabular}{lccc}
\hline & \multicolumn{3}{c}{ Thyroid Dose [cGy] } \\
& \multicolumn{2}{c}{$95 \%$ Subjective Confidence Interval } \\
Exposure Pathway & lower limit & central estimate & upper limit \\
\hline Backyard Cow Milk & 0.18 & 0.95 & 4.8 \\
Commercial Milk (locally produced) & 0.034 & 0.2 & 1.3 \\
Commercial Milk (regionally mixed) & 0.045 & 0.25 & 1.5 \\
Goat Milk (locally produced) & 0.33 & 2.6 & 18 \\
Beef (locally produced) & 0.00061 & 0.01 & 0.2 \\
Leafy Vegetables (locally produced) & 0.00017 & 0.0017 & 0.014 \\
Eggs (locally produced) & 0.011 & 0.073 & 0.44 \\
Cottage Cheese (locally produced) & 0.0004 & 0.0039 & 0.039 \\
Inhalation & 0.01 & 0.036 & 0.13 \\
Mother's milk (mother on Diet 1) & -- & -- & -- \\
Prenatal exposure (mother on Diet 1) & -- & -- & - \\
Diet 1 & 0.21 & 1.1 & 5.5 \\
Diet 2 & 0.073 & 0.35 & 1.9 \\
Diet 3 & 0.061 & 0.3 & 1.6 \\
\hline
\end{tabular}

Excess Lifetime Risk [ ]

\begin{tabular}{llll}
\hline Diet 1 & $2.3 \mathrm{E}-08$ & $1.3 \mathrm{E}-06$ & $3.6 \mathrm{E}-05$ \\
Diet 2 & $9.1 \mathrm{E}-09$ & $4.3 \mathrm{E}-07$ & $1.3 \mathrm{E}-05$ \\
Diet 3 & $8.8 \mathrm{E}-09$ & $3.6 \mathrm{E}-07$ & $1.3 \mathrm{E}-05$ \\
Diet 4 & $5.9 \mathrm{E}-08$ & $2.9 \mathrm{E}-06$ & $1.1 \mathrm{E}-04$ \\
\hline
\end{tabular}

\section{Relative Risk [ ]}

\begin{tabular}{lccc}
\hline Diet 1 & 1.000048 & 1.001 & 1.026 \\
Diet 2 & 1.000015 & 1.00033 & 1.0094 \\
Diet 3 & 1.000012 & 1.00027 & 1.0085 \\
Diet 4 & 1.000074 & 1.0026 & 1.068 \\
\hline
\end{tabular}

\section{Probability of Causation [\%]}

\begin{tabular}{llcc}
\hline Diet 1 & 0.005 & 0.102 & 2.56 \\
Diet 2 & 0.001 & 0.033 & 0.93 \\
Diet 3 & 0.001 & 0.027 & 0.84 \\
Diet 4 & 0.007 & 0.259 & 6.33 \\
\hline
\end{tabular}

Diet 1 - Backyard cow milk + all other locally produced non-milk exposure pathways

Diet 2 - Locally produced commercial milk + all other locally produced non-milk exposure pathways

Diet 3 - Regionally mixed commercial milk + inhalation (other regionally mixed food items are minor contributors to the total)

Diet 4 - Goat milk (all other exposure pathways are of negligible importance compared to goat milk) 


\section{Location: Clinton}

Receptor: Female born in 1920

\begin{tabular}{lccc}
\hline & \multicolumn{3}{c}{ Thyroid Dose [cGy] } \\
& \multicolumn{2}{c}{$95 \%$ Subjective Confidence Interval } \\
Exposure Pathway & lower limit & central estimate & upper limit \\
\hline Backyard Cow Milk & 0.24 & 1.3 & 7.3 \\
Commercial Milk (locally produced) & 0.045 & 0.26 & 1.8 \\
Commercial Milk (regionally mixed) & 0.037 & 0.2 & 1.3 \\
Goat Milk (locally produced) & 0.41 & 3.1 & 22 \\
Beef (locally produced) & 0.0006 & 0.011 & 0.23 \\
Leafy Vegetables (locally produced) & 0.00026 & 0.0028 & 0.022 \\
Eggs (locally produced) & 0.017 & 0.11 & 0.66 \\
Cottage Cheese (locally produced) & 0.00066 & 0.0062 & 0.059 \\
Inhalation & 0.014 & 0.046 & 0.16 \\
Mother's milk (mother on Diet 1) & -- & -- & -- \\
Prenatal exposure (mother on Diet 1) & -- & -- & -- \\
Diet 1 & 0.3 & 1.5 & 8.3 \\
Diet 2 & 0.093 & 0.47 & 2.5 \\
Diet 3 & 0.058 & 0.25 & 1.5 \\
\hline
\end{tabular}

Excess Lifetime Risk [ ]

\begin{tabular}{llll}
\hline Diet 1 & $3.4 \mathrm{E}-07$ & $5.6 \mathrm{E}-06$ & $8.9 \mathrm{E}-05$ \\
Diet 2 & $1.2 \mathrm{E}-07$ & $1.8 \mathrm{E}-06$ & $2.4 \mathrm{E}-05$ \\
Diet 3 & $7.0 \mathrm{E}-08$ & $9.6 \mathrm{E}-07$ & $1.3 \mathrm{E}-05$ \\
Diet 4 & $7.2 \mathrm{E}-07$ & $1.2 \mathrm{E}-05$ & $1.8 \mathrm{E}-04$ \\
\hline
\end{tabular}

Relative Risk [ ]

\begin{tabular}{lccc}
\hline Diet 1 & 1.00021 & 1.0021 & 1.021 \\
Diet 2 & 1.000072 & 1.00068 & 1.0074 \\
Diet 3 & 1.000043 & 1.00038 & 1.0042 \\
Diet 4 & 1.00048 & 1.0044 & 1.07 \\
\hline
\end{tabular}

\begin{tabular}{llcc} 
& \multicolumn{3}{c}{ Probability of Causation [\%] } \\
\hline Diet 1 & 0.021 & 0.214 & 2.09 \\
Diet 2 & 0.007 & 0.068 & 0.74 \\
Diet 3 & 0.004 & 0.038 & 0.41 \\
Diet 4 & 0.048 & 0.442 & 6.56 \\
\hline
\end{tabular}

Diet 1 - Backyard cow milk + all other locally produced non-milk exposure pathways

Diet 2 - Locally produced commercial milk + all other locally produced non-milk exposure pathways

Diet 3 - Regionally mixed commercial milk + inhalation (other regionally mixed food items are minor contributors to the total)

Diet 4 - Goat milk (all other exposure pathways are of negligible importance compared to goat milk) 


\section{Location: Clinton}

\section{Receptor: Male born in 1920}

\begin{tabular}{lccc}
\hline & \multicolumn{3}{c}{ Thyroid Dose [cGy] } \\
& \multicolumn{2}{c}{$95 \%$ Subjective Confidence Interval } \\
Exposure Pathway & lower limit & central estimate & upper limit \\
\hline Backyard Cow Milk & 0.28 & 1.5 & 8.6 \\
Commercial Milk (locally produced) & 0.056 & 0.33 & 2.1 \\
Commercial Milk (regionally mixed) & 0.045 & 0.25 & 1.5 \\
Goat Milk (locally produced) & 0.56 & 4.2 & 27 \\
Beef (locally produced) & 0.001 & 0.016 & 0.37 \\
Leafy Vegetables (locally produced) & 0.00027 & 0.0027 & 0.023 \\
Eggs (locally produced) & 0.017 & 0.12 & 0.74 \\
Cottage Cheese (locally produced) & 0.00065 & 0.0064 & 0.064 \\
Inhalation & 0.017 & 0.058 & 0.2 \\
Mother's milk (mother on Diet 1) & -- & -- & - \\
Prenatal exposure (mother on Diet 1) & -- & -- & -- \\
Diet 1 & 0.33 & 1.8 & 9.8 \\
Diet 2 & 0.12 & 0.57 & 3.1 \\
Diet 3 & 0.072 & 0.32 & 1.7 \\
\hline
\end{tabular}

Excess Lifetime Risk [ ]

\begin{tabular}{llll}
\hline Diet 1 & $3.5 \mathrm{E}-08$ & $2.2 \mathrm{E}-06$ & $6.1 \mathrm{E}-05$ \\
Diet 2 & $1.5 \mathrm{E}-08$ & $7.1 \mathrm{E}-07$ & $2.4 \mathrm{E}-05$ \\
Diet 3 & $9.5 \mathrm{E}-09$ & $3.9 \mathrm{E}-07$ & $1.3 \mathrm{E}-05$ \\
Diet 4 & $8.7 \mathrm{E}-08$ & $4.9 \mathrm{E}-06$ & $1.8 \mathrm{E}-04$ \\
\hline
\end{tabular}

\section{Relative Risk [ ]}

\begin{tabular}{lccc}
\hline Diet 1 & 1.00008 & 1.0016 & 1.045 \\
Diet 2 & 1.000023 & 1.00051 & 1.015 \\
Diet 3 & 1.000013 & 1.00029 & 1.0091 \\
Diet 4 & 1.00012 & 1.004 & 1.12 \\
\hline
\end{tabular}

\begin{tabular}{lccc}
\hline Diet 1 & 0.008 & 0.162 & 4.34 \\
Diet 2 & 0.002 & 0.051 & 1.49 \\
Diet 3 & 0.001 & 0.029 & 0.91 \\
Diet 4 & 0.012 & 0.395 & 10.86 \\
\hline
\end{tabular}

Diet 1 - Backyard cow milk + all other locally produced non-milk exposure pathways

Diet 2 - Locally produced commercial milk + all other locally produced non-milk exposure pathways

Diet 3 - Regionally mixed commercial milk + inhalation (other regionally mixed food items are minor contributors to the total)

Diet 4 - Goat milk (all other exposure pathways are of negligible importance compared to goat milk) 
Location: Friendsville

Receptor: Female born in 1920

\begin{tabular}{lccc}
\hline & \multicolumn{3}{c}{ Thyroid Dose [cGy] } \\
Exposure Pathway & \multicolumn{2}{c}{$95 \%$ Subjective Confidence Interval } \\
\hline Backyard Cow Milk & lower limit & central estimate & upper limit \\
Commercial Milk (locally produced) & 0.13 & 0.71 & 4.1 \\
Commercial Milk (regionally mixed) & 0.024 & 0.15 & 1 \\
Goat Milk (locally produced) & 0.037 & 0.2 & 1.3 \\
Beef (locally produced) & 0.21 & 1.7 & 15 \\
Leafy Vegetables (locally produced) & 0.00035 & 0.006 & 0.14 \\
Eggs (locally produced) & 0.00014 & 0.0016 & 0.013 \\
Cottage Cheese (locally produced) & 0.0087 & 0.064 & 0.42 \\
Inhalation & 0.00031 & 0.0036 & 0.035 \\
Mother's milk (mother on Diet 1) & 0.0088 & 0.031 & 0.12 \\
Prenatal exposure (mother on Diet 1) & -- & -- & -- \\
Diet 1 & -- & -- & - \\
Diet 2 & 0.17 & 0.84 & 4.6 \\
Diet 3 & 0.053 & 0.28 & 1.4 \\
\hline
\end{tabular}

Excess Lifetime Risk [ ]

\begin{tabular}{lllc}
\hline Diet 1 & $2.1 \mathrm{E}-07$ & $3.2 \mathrm{E}-06$ & $5.4 \mathrm{E}-05$ \\
Diet 2 & $7.6 \mathrm{E}-08$ & $1.1 \mathrm{E}-06$ & $1.3 \mathrm{E}-05$ \\
Diet 3 & $6.4 \mathrm{E}-08$ & $8.9 \mathrm{E}-07$ & $1.3 \mathrm{E}-05$ \\
Diet 4 & $4.1 \mathrm{E}-07$ & $6.9 \mathrm{E}-06$ & $1.2 \mathrm{E}-04$ \\
\hline
\end{tabular}

\section{Relative Risk [ ]}

\begin{tabular}{lccc}
\hline Diet 1 & 1.00013 & 1.0012 & 1.012 \\
Diet 2 & 1.000048 & 1.0004 & 1.0045 \\
Diet 3 & 1.00004 & 1.00035 & 1.004 \\
Diet 4 & 1.00026 & 1.0026 & 1.038 \\
\hline
\end{tabular}

\section{Probability of Causation [\%]}

\begin{tabular}{llll}
\hline Diet 1 & 0.013 & 0.120 & 1.19 \\
Diet 2 & 0.005 & 0.040 & 0.45 \\
Diet 3 & 0.004 & 0.035 & 0.40 \\
Diet 4 & 0.026 & 0.259 & 3.65 \\
\hline
\end{tabular}

Diet 1 - Backyard cow milk + all other locally produced non-milk exposure pathways

Diet 2 - Locally produced commercial milk + all other locally produced non-milk exposure pathways

Diet 3 - Regionally mixed commercial milk + inhalation (other regionally mixed food items are minor contributors to the total)

Diet 4 - Goat milk (all other exposure pathways are of negligible importance compared to goat milk) 
Location: Friendsville

Receptor: Male born in 1920

\begin{tabular}{lccc}
\hline & \multicolumn{3}{c}{ Thyroid Dose [cGy] } \\
& \multicolumn{2}{c}{$95 \%$ Subjective Confidence Interval } \\
Exposure Pathway & lower limit & central estimate & upper limit \\
\hline Backyard Cow Milk & 0.16 & 0.85 & 4.6 \\
Commercial Milk (locally produced) & 0.03 & 0.19 & 1.1 \\
Commercial Milk (regionally mixed) & 0.045 & 0.25 & 1.5 \\
Goat Milk (locally produced) & 0.31 & 2.4 & 18 \\
Beef (locally produced) & 0.0006 & 0.0095 & 0.21 \\
Leafy Vegetables (locally produced) & 0.00014 & 0.0016 & 0.013 \\
Eggs (locally produced) & 0.0099 & 0.065 & 0.47 \\
Cottage Cheese (locally produced) & 0.00034 & 0.0035 & 0.035 \\
Inhalation & 0.01 & 0.04 & 0.13 \\
Mother's milk (mother on Diet 1) & -- & -- & -- \\
Prenatal exposure (mother on Diet 1) & -- & -- & -- \\
Diet 1 & 0.19 & 0.99 & 5.2 \\
Diet 2 & 0.064 & 0.33 & 1.6 \\
Diet 3 & 0.065 & 0.3 & 1.6 \\
\hline
\end{tabular}

Excess Lifetime Risk [ ]

\begin{tabular}{llll}
\hline Diet 1 & $2.3 \mathrm{E}-08$ & $1.3 \mathrm{E}-06$ & $3.9 \mathrm{E}-05$ \\
Diet 2 & $9.0 \mathrm{E}-09$ & $3.9 \mathrm{E}-07$ & $1.4 \mathrm{E}-05$ \\
Diet 3 & $9.0 \mathrm{E}-09$ & $3.6 \mathrm{E}-07$ & $1.3 \mathrm{E}-05$ \\
Diet 4 & $5.5 \mathrm{E}-08$ & $2.7 \mathrm{E}-06$ & $1.2 \mathrm{E}-04$ \\
\hline
\end{tabular}

\section{Relative Risk [ ]}

\begin{tabular}{lccc}
\hline Diet 1 & 1.000043 & 1.00094 & 1.026 \\
Diet 2 & 1.000013 & 1.00031 & 1.0086 \\
Diet 3 & 1.000011 & 1.00027 & 1.0085 \\
Diet 4 & 1.000081 & 1.0024 & 1.07 \\
\hline
\end{tabular}

\section{Probability of Causation [\%]}

\begin{tabular}{llll}
\hline Diet 1 & 0.004 & 0.094 & 2.52 \\
Diet 2 & 0.001 & 0.031 & 0.85 \\
Diet 3 & 0.001 & 0.027 & 0.84 \\
Diet 4 & 0.008 & 0.235 & 6.57 \\
\hline
\end{tabular}

Diet 1 - Backyard cow milk + all other locally produced non-milk exposure pathways

Diet 2 - Locally produced commercial milk + all other locally produced non-milk exposure pathways

Diet 3 - Regionally mixed commercial milk + inhalation (other regionally mixed food items are minor contributors to the total)

Diet 4 - Goat milk (all other exposure pathways are of negligible importance compared to goat milk) 
Location: Wartburg

Receptor: Female born in 1920

\begin{tabular}{lccc}
\hline & \multicolumn{3}{c}{ Thyroid Dose [cGy] } \\
Exposure Pathway & \multicolumn{2}{c}{$95 \%$ Subjective Confidence Interval } \\
\hline Backyard Cow Milk & lower limit & central estimate & upper limit \\
Commercial Milk (locally produced) & 0.033 & 0.2 & 1.2 \\
Commercial Milk (regionally mixed) & 0.0059 & 0.042 & 0.3 \\
Goat Milk (locally produced) & 0.037 & 0.2 & 1.3 \\
Beef (locally produced) & 0.061 & 0.51 & 3.8 \\
Leafy Vegetables (locally produced) & 0.00011 & 0.0017 & 0.039 \\
Eggs (locally produced) & 0.000039 & 0.00041 & 0.004 \\
Cottage Cheese (locally produced) & 0.0021 & 0.017 & 0.11 \\
Inhalation & 0.00008 & 0.001 & 0.01 \\
Mother's milk (mother on Diet 1) & 0.002 & 0.0073 & 0.03 \\
Prenatal exposure (mother on Diet 1) & -- & -- & -- \\
Diet 1 & -- & -- & -- \\
Diet 2 & 0.042 & 0.23 & 1.3 \\
Diet 3 & 0.014 & 0.075 & 0.48 \\
\hline
\end{tabular}

Excess Lifetime Risk [ ]

\begin{tabular}{lllc}
\hline Diet 1 & $4.8 \mathrm{E}-08$ & $8.4 \mathrm{E}-07$ & $1.7 \mathrm{E}-05$ \\
Diet 2 & $1.7 \mathrm{E}-08$ & $2.8 \mathrm{E}-07$ & $4.1 \mathrm{E}-06$ \\
Diet 3 & $5.3 \mathrm{E}-08$ & $7.9 \mathrm{E}-07$ & $1.3 \mathrm{E}-05$ \\
Diet 4 & $1.0 \mathrm{E}-07$ & $1.8 \mathrm{E}-06$ & $3.1 \mathrm{E}-05$ \\
\hline
\end{tabular}

\section{Relative Risk [ ]}

\begin{tabular}{lccc}
\hline Diet 1 & 1.000034 & 1.00031 & 1.0041 \\
Diet 2 & 1.000012 & 1.00011 & 1.0015 \\
Diet 3 & 1.000032 & 1.00032 & 1.0038 \\
Diet 4 & 1.000068 & 1.00069 & 1.011 \\
\hline
\end{tabular}

\section{Probability of Causation [\%]}

\begin{tabular}{llcc}
\hline Diet 1 & 0.003 & 0.032 & 0.41 \\
Diet 2 & 0.001 & 0.011 & 0.15 \\
Diet 3 & 0.003 & 0.032 & 0.38 \\
Diet 4 & 0.007 & 0.069 & 1.09 \\
\hline
\end{tabular}

Diet 1 - Backyard cow milk + all other locally produced non-milk exposure pathways

Diet 2 - Locally produced commercial milk + all other locally produced non-milk exposure pathways

Diet 3 - Regionally mixed commercial milk + inhalation (other regionally mixed food items are minor contributors to the total)

Diet 4 - Goat milk (all other exposure pathways are of negligible importance compared to goat milk) 


\section{Location: Wartburg}

\section{Receptor: Male born in 1920}

\begin{tabular}{lccc}
\hline & \multicolumn{3}{c}{ Thyroid Dose [cGy] } \\
Exposure Pathway & 95\% Subjective Confidence Interval \\
\hline Backyard Cow Milk & lower limit & central estimate & upper limit \\
Commercial Milk (locally produced) & 0.04 & 0.23 & 1.3 \\
Commercial Milk (regionally mixed) & 0.008 & 0.053 & 0.37 \\
Goat Milk (locally produced) & 0.045 & 0.25 & 1.5 \\
Beef (locally produced) & 0.088 & 0.69 & 4.8 \\
Leafy Vegetables (locally produced) & 0.00018 & 0.0025 & 0.059 \\
Eggs (locally produced) & 0.000038 & 0.00043 & 0.004 \\
Cottage Cheese (locally produced) & 0.0022 & 0.018 & 0.12 \\
Inhalation & 0.000084 & 0.001 & 0.01 \\
Mother's milk (mother on Diet 1) & 0.0025 & 0.0095 & 0.037 \\
Prenatal exposure (mother on Diet 1) & -- & -- & -- \\
Diet 1 & -- & -- & -- \\
Diet 2 & 0.049 & 0.27 & 1.5 \\
Diet 3 & 0.016 & 0.088 & 0.55 \\
\hline
\end{tabular}

Excess Lifetime Risk [ ]

\begin{tabular}{llll}
\hline Diet 1 & $5.8 \mathrm{E}-09$ & $3.3 \mathrm{E}-07$ & $1.1 \mathrm{E}-05$ \\
Diet 2 & $2.2 \mathrm{E}-09$ & $1.1 \mathrm{E}-07$ & $3.5 \mathrm{E}-06$ \\
Diet 3 & $7.6 \mathrm{E}-09$ & $3.0 \mathrm{E}-07$ & $1.3 \mathrm{E}-05$ \\
Diet 4 & $1.4 \mathrm{E}-08$ & $7.3 \mathrm{E}-07$ & $2.9 \mathrm{E}-05$ \\
\hline
\end{tabular}

\section{Relative Risk [ ]}

\begin{tabular}{lccc}
\hline Diet 1 & 1.0000099 & 1.00026 & 1.0065 \\
Diet 2 & 1.0000029 & 1.000082 & 1.0024 \\
Diet 3 & 1.0000099 & 1.00024 & 1.0074 \\
Diet 4 & 1.000018 & 1.00064 & 1.019 \\
\hline
\end{tabular}

\section{Probability of Causation [\%]}

\begin{tabular}{lccc}
\hline Diet 1 & 0.001 & 0.026 & 0.65 \\
Diet 2 & -- & 0.008 & 0.24 \\
Diet 3 & 0.001 & 0.024 & 0.73 \\
Diet 4 & 0.002 & 0.064 & 1.83 \\
\hline
\end{tabular}

Diet 1 - Backyard cow milk + all other locally produced non-milk exposure pathways

Diet 2 - Locally produced commercial milk + all other locally produced non-milk exposure pathways

Diet 3 - Regionally mixed commercial milk + inhalation (other regionally mixed food items are minor contributors to the total)

Diet 4 - Goat milk (all other exposure pathways are of negligible importance compared to goat milk) 
Location: Rockwood

Receptor: Female born in 1920

\begin{tabular}{lccc}
\hline & \multicolumn{3}{c}{ Thyroid Dose [cGy] } \\
Exposure Pathway & \multicolumn{2}{c}{$95 \%$ Subjective Confidence Interval } \\
\hline Backyard Cow Milk & lower limit & central estimate & upper limit \\
Commercial Milk (locally produced) & 0.16 & 0.84 & 4.6 \\
Commercial Milk (regionally mixed) & 0.028 & 0.17 & 1.2 \\
Goat Milk (locally produced) & 0.037 & 0.21 & 1.3 \\
Beef (locally produced) & 0.28 & 2 & 15 \\
Leafy Vegetables (locally produced) & 0.00043 & 0.0069 & 0.16 \\
Eggs (locally produced) & 0.00017 & 0.0018 & 0.015 \\
Cottage Cheese (locally produced) & 0.011 & 0.069 & 0.45 \\
Inhalation & 0.00041 & 0.0043 & 0.039 \\
Mother's milk (mother on Diet 1) & 0.0095 & 0.032 & 0.11 \\
Prenatal exposure (mother on Diet 1) & -- & -- & -- \\
Diet 1 & -- & -- & - \\
Diet 2 & 0.19 & 0.98 & 5.2 \\
Diet 3 & 0.065 & 0.31 & 1.7 \\
\hline
\end{tabular}

Excess Lifetime Risk [ ]

\begin{tabular}{llll}
\hline Diet 1 & $2.2 \mathrm{E}-07$ & $3.6 \mathrm{E}-06$ & $5.8 \mathrm{E}-05$ \\
Diet 2 & $7.6 \mathrm{E}-08$ & $1.2 \mathrm{E}-06$ & $1.6 \mathrm{E}-05$ \\
Diet 3 & $6.4 \mathrm{E}-08$ & $8.8 \mathrm{E}-07$ & $1.3 \mathrm{E}-05$ \\
Diet 4 & $5.1 \mathrm{E}-07$ & $8.3 \mathrm{E}-06$ & $1.2 \mathrm{E}-04$ \\
\hline
\end{tabular}

\section{Relative Risk [ ]}

\begin{tabular}{lccc}
\hline Diet 1 & 1.00016 & 1.0013 & 1.014 \\
Diet 2 & 1.000051 & 1.00044 & 1.0048 \\
Diet 3 & 1.00004 & 1.00035 & 1.004 \\
Diet 4 & 1.0003 & 1.0029 & 1.042 \\
\hline
\end{tabular}

\section{Probability of Causation [\%]}

\begin{tabular}{llll}
\hline Diet 1 & 0.016 & 0.135 & 1.34 \\
Diet 2 & 0.005 & 0.044 & 0.47 \\
Diet 3 & 0.004 & 0.035 & 0.40 \\
Diet 4 & 0.030 & 0.287 & 3.99 \\
\hline
\end{tabular}

Diet 1 - Backyard cow milk + all other locally produced non-milk exposure pathways

Diet 2 - Locally produced commercial milk + all other locally produced non-milk exposure pathways

Diet 3 - Regionally mixed commercial milk + inhalation (other regionally mixed food items are minor contributors to the total)

Diet 4 - Goat milk (all other exposure pathways are of negligible importance compared to goat milk) 


\section{Location: Rockwood}

Receptor: Male born in 1920

\begin{tabular}{lccc}
\hline & \multicolumn{3}{c}{ Thyroid Dose [cGy] } \\
& \multicolumn{2}{c}{$95 \%$ Subjective Confidence Interval } \\
Exposure Pathway & lower limit & central estimate & upper limit \\
\hline Backyard Cow Milk & 0.19 & 1 & 5.4 \\
Commercial Milk (locally produced) & 0.038 & 0.22 & 1.4 \\
Commercial Milk (regionally mixed) & 0.045 & 0.25 & 1.5 \\
Goat Milk (locally produced) & 0.39 & 2.8 & 19 \\
Beef (locally produced) & 0.00069 & 0.011 & 0.24 \\
Leafy Vegetables (locally produced) & 0.00017 & 0.0017 & 0.016 \\
Eggs (locally produced) & 0.011 & 0.075 & 0.47 \\
Cottage Cheese (locally produced) & 0.00044 & 0.0041 & 0.04 \\
Inhalation & 0.011 & 0.042 & 0.14 \\
Mother's milk (mother on Diet 1) & -- & -- & - \\
Prenatal exposure (mother on Diet 1) & -- & -- & -- \\
Diet 1 & 0.22 & 1.2 & 6.2 \\
Diet 2 & 0.082 & 0.37 & 2 \\
Diet 3 & 0.064 & 0.3 & 1.6 \\
\hline
\end{tabular}

Excess Lifetime Risk [ ]

\begin{tabular}{llll}
\hline Diet 1 & $2.6 \mathrm{E}-08$ & $1.3 \mathrm{E}-06$ & $4.2 \mathrm{E}-05$ \\
Diet 2 & $1.1 \mathrm{E}-08$ & $4.3 \mathrm{E}-07$ & $1.6 \mathrm{E}-05$ \\
Diet 3 & $9.0 \mathrm{E}-09$ & $3.5 \mathrm{E}-07$ & $1.3 \mathrm{E}-05$ \\
Diet 4 & $6.3 \mathrm{E}-08$ & $2.9 \mathrm{E}-06$ & $1.2 \mathrm{E}-04$ \\
\hline
\end{tabular}

\section{Relative Risk [ ]}

\begin{tabular}{lccc}
\hline Diet 1 & 1.000048 & 1.0011 & 1.03 \\
Diet 2 & 1.000016 & 1.00035 & 1.01 \\
Diet 3 & 1.000012 & 1.00027 & 1.0086 \\
Diet 4 & 1.000071 & 1.0027 & 1.079 \\
\hline
\end{tabular}

\begin{tabular}{llcc} 
& \multicolumn{3}{c}{ Probability of Causation [\%] } \\
\hline Diet 1 & 0.005 & 0.106 & 2.91 \\
Diet 2 & 0.002 & 0.035 & 1.01 \\
Diet 3 & 0.001 & 0.027 & 0.85 \\
Diet 4 & 0.007 & 0.269 & 7.27 \\
\hline
\end{tabular}

Diet 1 - Backyard cow milk + all other locally produced non-milk exposure pathways

Diet 2 - Locally produced commercial milk + all other locally produced non-milk exposure pathways

Diet 3 - Regionally mixed commercial milk + inhalation (other regionally mixed food items are minor contributors to the total)

Diet 4 - Goat milk (all other exposure pathways are of negligible importance compared to goat milk) 
Location: Louisville

Receptor: Female born in 1920

\begin{tabular}{lccc}
\hline & \multicolumn{3}{c}{ Thyroid Dose [cGy] } \\
Exposure Pathway & $95 \%$ Subjective Confidence Interval \\
\hline Backyard Cow Milk & lower limit & central estimate & upper limit \\
Commercial Milk (locally produced) & 0.14 & 0.77 & 4 \\
Commercial Milk (regionally mixed) & 0.026 & 0.16 & 1.1 \\
Goat Milk (locally produced) & 0.037 & 0.2 & 1.3 \\
Beef (locally produced) & 0.23 & 1.9 & 14 \\
Leafy Vegetables (locally produced) & 0.00039 & 0.0064 & 0.13 \\
Eggs (locally produced) & 0.00016 & 0.0016 & 0.014 \\
Cottage Cheese (locally produced) & 0.0094 & 0.064 & 0.42 \\
Inhalation & 0.00036 & 0.0038 & 0.036 \\
Mother's milk (mother on Diet 1) & 0.0093 & 0.032 & 0.12 \\
Prenatal exposure (mother on Diet 1) & -- & -- & -- \\
Diet 1 & -- & -- & -- \\
Diet 2 & 0.17 & 0.9 & 4.6 \\
Diet 3 & 0.06 & 0.29 & 1.6 \\
\hline
\end{tabular}

Excess Lifetime Risk [ ]

\begin{tabular}{lllr}
\hline Diet 1 & $2.0 \mathrm{E}-07$ & $3.3 \mathrm{E}-06$ & $5.3 \mathrm{E}-05$ \\
Diet 2 & $7.6 \mathrm{E}-08$ & $1.1 \mathrm{E}-06$ & $1.6 \mathrm{E}-05$ \\
Diet 3 & $6.5 \mathrm{E}-08$ & $9.0 \mathrm{E}-07$ & $1.3 \mathrm{E}-05$ \\
Diet 4 & $4.3 \mathrm{E}-07$ & $7.1 \mathrm{E}-06$ & $1.3 \mathrm{E}-04$ \\
\hline
\end{tabular}

\section{Relative Risk [ ]}

\begin{tabular}{lccc}
\hline Diet 1 & 1.00015 & 1.0012 & 1.013 \\
Diet 2 & 1.00005 & 1.00041 & 1.0047 \\
Diet 3 & 1.000041 & 1.00035 & 1.004 \\
Diet 4 & 1.00027 & 1.0027 & 1.038 \\
\hline
\end{tabular}

\begin{tabular}{llcc} 
& \multicolumn{3}{c}{ Probability of Causation [\%] } \\
\hline Diet 1 & 0.015 & 0.124 & 1.27 \\
Diet 2 & 0.005 & 0.041 & 0.46 \\
Diet 3 & 0.004 & 0.035 & 0.40 \\
Diet 4 & 0.027 & 0.266 & 3.65 \\
\hline
\end{tabular}

Diet 1 - Backyard cow milk + all other locally produced non-milk exposure pathways

Diet 2 - Locally produced commercial milk + all other locally produced non-milk exposure pathways

Diet 3 - Regionally mixed commercial milk + inhalation (other regionally mixed food items are minor contributors to the total)

Diet 4 - Goat milk (all other exposure pathways are of negligible importance compared to goat milk) 


\section{Location: Louisville}

\section{Receptor: Male born in 1920}

\begin{tabular}{lccc}
\hline & \multicolumn{3}{c}{ Thyroid Dose [cGy] } \\
& \multicolumn{2}{c}{$95 \%$ Subjective Confidence Interval } \\
Exposure Pathway & lower limit & central estimate & upper limit \\
\hline Backyard Cow Milk & 0.16 & 0.9 & 4.6 \\
Commercial Milk (locally produced) & 0.032 & 0.2 & 1.2 \\
Commercial Milk (regionally mixed) & 0.045 & 0.25 & 1.5 \\
Goat Milk (locally produced) & 0.33 & 2.6 & 18 \\
Beef (locally produced) & 0.00062 & 0.01 & 0.2 \\
Leafy Vegetables (locally produced) & 0.00016 & 0.0016 & 0.014 \\
Eggs (locally produced) & 0.01 & 0.07 & 0.44 \\
Cottage Cheese (locally produced) & 0.00036 & 0.0038 & 0.037 \\
Inhalation & 0.012 & 0.041 & 0.15 \\
Mother's milk (mother on Diet 1) & -- & -- & - \\
Prenatal exposure (mother on Diet 1) & -- & -- & -- \\
Diet 1 & 0.2 & 1.1 & 5.3 \\
Diet 2 & 0.073 & 0.35 & 1.9 \\
Diet 3 & 0.064 & 0.3 & 1.6 \\
\hline
\end{tabular}

Excess Lifetime Risk [ ]

\begin{tabular}{llll}
\hline Diet 1 & $2.3 \mathrm{E}-08$ & $1.3 \mathrm{E}-06$ & $3.7 \mathrm{E}-05$ \\
Diet 2 & $9.3 \mathrm{E}-09$ & $4.2 \mathrm{E}-07$ & $1.3 \mathrm{E}-05$ \\
Diet 3 & $9.0 \mathrm{E}-09$ & $3.7 \mathrm{E}-07$ & $1.3 \mathrm{E}-05$ \\
Diet 4 & $5.4 \mathrm{E}-08$ & $2.8 \mathrm{E}-06$ & $1.1 \mathrm{E}-04$ \\
\hline
\end{tabular}

\section{Relative Risk [ ]}

\begin{tabular}{lccc}
\hline Diet 1 & 1.000042 & 1.001 & 1.025 \\
Diet 2 & 1.000014 & 1.00032 & 1.0094 \\
Diet 3 & 1.000012 & 1.00027 & 1.0087 \\
Diet 4 & 1.00007 & 1.0025 & 1.066 \\
\hline
\end{tabular}

\section{Probability of Causation [\%]}

\begin{tabular}{llcc}
\hline Diet 1 & 0.004 & 0.099 & 2.45 \\
Diet 2 & 0.001 & 0.032 & 0.93 \\
Diet 3 & 0.001 & 0.027 & 0.86 \\
Diet 4 & 0.007 & 0.247 & 6.15 \\
\hline
\end{tabular}

Diet 1 - Backyard cow milk + all other locally produced non-milk exposure pathways

Diet 2 - Locally produced commercial milk + all other locally produced non-milk exposure pathways

Diet 3 - Regionally mixed commercial milk + inhalation (other regionally mixed food items are minor contributors to the total)

Diet 4 - Goat milk (all other exposure pathways are of negligible importance compared to goat milk) 
Location: Barnardville

Receptor: Female born in 1920

\begin{tabular}{lccc}
\hline & \multicolumn{3}{c}{ Thyroid Dose [cGy] } \\
Exposure Pathway & \multicolumn{2}{c}{ 95\% } & Subjective Confidence Interval \\
Backyard Cow Milk & lower limit & central estimate & upper limit \\
Commercial Milk (locally produced) & 0.17 & 0.88 & 4.8 \\
Commercial Milk (regionally mixed) & 0.029 & 0.18 & 1.2 \\
Goat Milk (locally produced) & 0.037 & 0.21 & 1.3 \\
Beef (locally produced) & 0.27 & 2.1 & 16 \\
Leafy Vegetables (locally produced) & 0.00045 & 0.0073 & 0.15 \\
Eggs (locally produced) & 0.00017 & 0.0018 & 0.015 \\
Cottage Cheese (locally produced) & 0.011 & 0.073 & 0.45 \\
Inhalation & 0.00042 & 0.0043 & 0.04 \\
Mother's milk (mother on Diet 1) & 0.011 & 0.037 & 0.14 \\
Prenatal exposure (mother on Diet 1) & -- & -- & -- \\
Diet 1 & -- & -- & -- \\
Diet 2 & 0.2 & 1 & 5.5 \\
Diet 3 & 0.068 & 0.33 & 1.8 \\
\hline
\end{tabular}

Excess Lifetime Risk [ ]

\begin{tabular}{llcc}
\hline Diet 1 & $2.5 \mathrm{E}-07$ & $3.8 \mathrm{E}-06$ & $6.1 \mathrm{E}-05$ \\
Diet 2 & $8.5 \mathrm{E}-08$ & $1.3 \mathrm{E}-06$ & $1.7 \mathrm{E}-05$ \\
Diet 3 & $6.7 \mathrm{E}-08$ & $9.1 \mathrm{E}-07$ & $1.3 \mathrm{E}-05$ \\
Diet 4 & $5.2 \mathrm{E}-07$ & $8.2 \mathrm{E}-06$ & $1.4 \mathrm{E}-04$ \\
\hline
\end{tabular}

\section{Relative Risk [ ]}

\begin{tabular}{lccc}
\hline Diet 1 & 1.00017 & 1.0014 & 1.014 \\
Diet 2 & 1.000056 & 1.00046 & 1.0049 \\
Diet 3 & 1.000042 & 1.00036 & 1.0041 \\
Diet 4 & 1.00031 & 1.0031 & 1.044 \\
\hline
\end{tabular}

\section{Probability of Causation [\%]}

\begin{tabular}{llll}
\hline Diet 1 & 0.017 & 0.139 & 1.34 \\
Diet 2 & 0.006 & 0.046 & 0.49 \\
Diet 3 & 0.004 & 0.036 & 0.41 \\
Diet 4 & 0.031 & 0.312 & 4.25 \\
\hline
\end{tabular}

Diet 1 - Backyard cow milk + all other locally produced non-milk exposure pathways

Diet 2 - Locally produced commercial milk + all other locally produced non-milk exposure pathways

Diet 3 - Regionally mixed commercial milk + inhalation (other regionally mixed food items are minor contributors to the total)

Diet 4 - Goat milk (all other exposure pathways are of negligible importance compared to goat milk) 


\section{Location: Barnardville}

Receptor: Male born in 1920

\begin{tabular}{lccc}
\hline & \multicolumn{3}{c}{ Thyroid Dose [cGy] } \\
& \multicolumn{2}{c}{$95 \%$ Subjective Confidence Interval } \\
Exposure Pathway & lower limit & central estimate & upper limit \\
\hline Backyard Cow Milk & 0.2 & 1 & 5.5 \\
Commercial Milk (locally produced) & 0.038 & 0.22 & 1.4 \\
Commercial Milk (regionally mixed) & 0.045 & 0.25 & 1.5 \\
Goat Milk (locally produced) & 0.4 & 2.9 & 20 \\
Beef (locally produced) & 0.0007 & 0.011 & 0.23 \\
Leafy Vegetables (locally produced) & 0.00018 & 0.0018 & 0.017 \\
Eggs (locally produced) & 0.011 & 0.079 & 0.5 \\
Cottage Cheese (locally produced) & 0.0004 & 0.0044 & 0.042 \\
Inhalation & 0.013 & 0.048 & 0.17 \\
Mother's milk (mother on Diet 1) & -- & -- & - \\
Prenatal exposure (mother on Diet 1) & -- & -- & -- \\
Diet 1 & 0.24 & 1.2 & 6.4 \\
Diet 2 & 0.086 & 0.4 & 2.1 \\
Diet 3 & 0.066 & 0.31 & 1.6 \\
\hline
\end{tabular}

Excess Lifetime Risk [ ]

\begin{tabular}{llll}
\hline Diet 1 & $2.7 \mathrm{E}-08$ & $1.5 \mathrm{E}-06$ & $4.3 \mathrm{E}-05$ \\
Diet 2 & $1.1 \mathrm{E}-08$ & $4.7 \mathrm{E}-07$ & $1.5 \mathrm{E}-05$ \\
Diet 3 & $9.1 \mathrm{E}-09$ & $3.7 \mathrm{E}-07$ & $1.3 \mathrm{E}-05$ \\
Diet 4 & $6.3 \mathrm{E}-08$ & $3.2 \mathrm{E}-06$ & $1.3 \mathrm{E}-04$ \\
\hline
\end{tabular}

\section{Relative Risk [ ]}

\begin{tabular}{lccc}
\hline Diet 1 & 1.000047 & 1.0012 & 1.033 \\
Diet 2 & 1.000017 & 1.00037 & 1.011 \\
Diet 3 & 1.000012 & 1.00028 & 1.0088 \\
Diet 4 & 1.000086 & 1.0028 & 1.08 \\
\hline
\end{tabular}

\section{Probability of Causation [\%]}

\begin{tabular}{llll}
\hline Diet 1 & 0.005 & 0.115 & 3.17 \\
Diet 2 & 0.002 & 0.037 & 1.09 \\
Diet 3 & 0.001 & 0.028 & 0.88 \\
Diet 4 & 0.009 & 0.277 & 7.40 \\
\hline
\end{tabular}

Diet 1 - Backyard cow milk + all other locally produced non-milk exposure pathways

Diet 2 - Locally produced commercial milk + all other locally produced non-milk exposure pathways

Diet 3 - Regionally mixed commercial milk + inhalation (other regionally mixed food items are minor contributors to the total)

Diet 4 - Goat milk (all other exposure pathways are of negligible importance compared to goat milk) 
Location: Greenback

Receptor: Female born in 1920

\begin{tabular}{lccc}
\hline & \multicolumn{3}{c}{ Thyroid Dose [cGy] } \\
& \multicolumn{2}{c}{$95 \%$ Subjective Confidence Interval } \\
Exposure Pathway & lower limit & central estimate & upper limit \\
\hline Backyard Cow Milk & 0.089 & 0.48 & 2.7 \\
Commercial Milk (locally produced) & 0.016 & 0.098 & 0.67 \\
Commercial Milk (regionally mixed) & 0.037 & 0.2 & 1.3 \\
Goat Milk (locally produced) & 0.14 & 1.1 & 9.4 \\
Beef (locally produced) & 0.00024 & 0.004 & 0.085 \\
Leafy Vegetables (locally produced) & 0.0001 & 0.00099 & 0.0089 \\
Eggs (locally produced) & 0.0056 & 0.039 & 0.27 \\
Cottage Cheese (locally produced) & 0.00024 & 0.0024 & 0.027 \\
Inhalation & 0.0063 & 0.022 & 0.081 \\
Mother's milk (mother on Diet 1) & -- & -- & -- \\
Prenatal exposure (mother on Diet 1) & -- & -- & -- \\
Diet 1 & 0.11 & 0.55 & 3.1 \\
Diet 2 & 0.038 & 0.18 & 1.1 \\
Diet 3 & 0.049 & 0.23 & 1.4 \\
\hline
\end{tabular}

Excess Lifetime Risk [ ]

\begin{tabular}{llll}
\hline Diet 1 & $1.2 \mathrm{E}-07$ & $2.0 \mathrm{E}-06$ & $3.5 \mathrm{E}-05$ \\
Diet 2 & $5.1 \mathrm{E}-08$ & $7.0 \mathrm{E}-07$ & $9.9 \mathrm{E}-06$ \\
Diet 3 & $5.9 \mathrm{E}-08$ & $8.5 \mathrm{E}-07$ & $1.3 \mathrm{E}-05$ \\
Diet 4 & $2.7 \mathrm{E}-07$ & $4.5 \mathrm{E}-06$ & $8.5 \mathrm{E}-05$ \\
\hline
\end{tabular}

\section{Relative Risk [ ]}

\begin{tabular}{lccc}
\hline Diet 1 & 1.000084 & 1.00075 & 1.0085 \\
Diet 2 & 1.000031 & 1.00026 & 1.0032 \\
Diet 3 & 1.000037 & 1.00034 & 1.0039 \\
Diet 4 & 1.00016 & 1.0016 & 1.029 \\
\hline
\end{tabular}

\section{Probability of Causation [\%]}

\begin{tabular}{llcc}
\hline Diet 1 & 0.008 & 0.075 & 0.84 \\
Diet 2 & 0.003 & 0.026 & 0.32 \\
Diet 3 & 0.004 & 0.034 & 0.39 \\
Diet 4 & 0.016 & 0.158 & 2.82 \\
\hline
\end{tabular}

Diet 1 - Backyard cow milk + all other locally produced non-milk exposure pathways

Diet 2 - Locally produced commercial milk + all other locally produced non-milk exposure pathways

Diet 3 - Regionally mixed commercial milk + inhalation (other regionally mixed food items are minor contributors to the total)

Diet 4 - Goat milk (all other exposure pathways are of negligible importance compared to goat milk) 
Location: Greenback

Receptor: Male born in 1920

\begin{tabular}{lccc}
\hline & \multicolumn{3}{c}{ Thyroid Dose [cGy] } \\
& \multicolumn{2}{c}{$95 \%$ Subjective Confidence Interval } \\
Exposure Pathway & lower limit & central estimate & upper limit \\
\hline Backyard Cow Milk & 0.11 & 0.57 & 3.3 \\
Commercial Milk (locally produced) & 0.02 & 0.12 & 0.81 \\
Commercial Milk (regionally mixed) & 0.045 & 0.25 & 1.5 \\
Goat Milk (locally produced) & 0.2 & 1.6 & 11 \\
Beef (locally produced) & 0.00039 & 0.0064 & 0.14 \\
Leafy Vegetables (locally produced) & 0.0001 & 0.00099 & 0.0095 \\
Eggs (locally produced) & 0.0063 & 0.042 & 0.28 \\
Cottage Cheese (locally produced) & 0.00021 & 0.0023 & 0.028 \\
Inhalation & 0.0083 & 0.027 & 0.11 \\
Mother's milk (mother on Diet 1) & -- & -- & -- \\
Prenatal exposure (mother on Diet 1) & -- & -- & -- \\
Diet 1 & 0.13 & 0.67 & 3.8 \\
Diet 2 & 0.047 & 0.22 & 1.2 \\
Diet 3 & 0.059 & 0.28 & 1.6 \\
\hline
\end{tabular}

Excess Lifetime Risk [ ]

\begin{tabular}{llll}
\hline Diet 1 & $1.4 \mathrm{E}-08$ & $7.6 \mathrm{E}-07$ & $2.3 \mathrm{E}-05$ \\
Diet 2 & $5.4 \mathrm{E}-09$ & $2.5 \mathrm{E}-07$ & $8.3 \mathrm{E}-06$ \\
Diet 3 & $8.3 \mathrm{E}-09$ & $3.3 \mathrm{E}-07$ & $1.3 \mathrm{E}-05$ \\
Diet 4 & $4.0 \mathrm{E}-08$ & $1.7 \mathrm{E}-06$ & $6.8 \mathrm{E}-05$ \\
\hline
\end{tabular}

\section{Relative Risk [ ]}

\begin{tabular}{lccc}
\hline Diet 1 & 1.000024 & 1.00063 & 1.018 \\
Diet 2 & 1.000008 & 1.00022 & 1.0062 \\
Diet 3 & 1.000011 & 1.00026 & 1.008 \\
Diet 4 & 1.000041 & 1.0016 & 1.045 \\
\hline
\end{tabular}

\section{Probability of Causation [\%]}

\begin{tabular}{llcc}
\hline Diet 1 & 0.002 & 0.063 & 1.73 \\
Diet 2 & 0.001 & 0.022 & 0.62 \\
Diet 3 & 0.001 & 0.026 & 0.79 \\
Diet 4 & 0.004 & 0.157 & 4.31 \\
\hline
\end{tabular}

Diet 1 - Backyard cow milk + all other locally produced non-milk exposure pathways

Diet 2 - Locally produced commercial milk + all other locally produced non-milk exposure pathways

Diet 3 - Regionally mixed commercial milk + inhalation (other regionally mixed food items are minor contributors to the total)

Diet 4 - Goat milk (all other exposure pathways are of negligible importance compared to goat milk) 


\section{Location: Rockford}

Receptor: Female born in 1920

\begin{tabular}{lccc}
\hline & \multicolumn{3}{c}{ Thyroid Dose [cGy] } \\
Exposure Pathway & \multicolumn{2}{c}{$95 \%$ Subjective Confidence Interval } \\
\hline Backyard Cow Milk & lower limit & central estimate & upper limit \\
Commercial Milk (locally produced) & 0.15 & 0.74 & 4 \\
Commercial Milk (regionally mixed) & 0.026 & 0.16 & 0.98 \\
Goat Milk (locally produced) & 0.037 & 0.2 & 1.3 \\
Beef (locally produced) & 0.25 & 1.8 & 13 \\
Leafy Vegetables (locally produced) & 0.00038 & 0.0063 & 0.13 \\
Eggs (locally produced) & 0.00015 & 0.0016 & 0.013 \\
Cottage Cheese (locally produced) & 0.0093 & 0.063 & 0.4 \\
Inhalation & 0.00037 & 0.0037 & 0.036 \\
Mother's milk (mother on Diet 1) & 0.01 & 0.032 & 0.11 \\
Prenatal exposure (mother on Diet 1) & -- & -- & -- \\
Diet 1 & -- & -- & -- \\
Diet 2 & 0.18 & 0.87 & 4.6 \\
Diet 3 & 0.06 & 0.28 & 1.5 \\
\hline
\end{tabular}

Excess Lifetime Risk [ ]

\begin{tabular}{llll}
\hline Diet 1 & $2.2 \mathrm{E}-07$ & $3.3 \mathrm{E}-06$ & $4.7 \mathrm{E}-05$ \\
Diet 2 & $7.7 \mathrm{E}-08$ & $1.1 \mathrm{E}-06$ & $1.5 \mathrm{E}-05$ \\
Diet 3 & $6.6 \mathrm{E}-08$ & $9.0 \mathrm{E}-07$ & $1.3 \mathrm{E}-05$ \\
Diet 4 & $4.0 \mathrm{E}-07$ & $7.2 \mathrm{E}-06$ & $1.2 \mathrm{E}-04$ \\
\hline
\end{tabular}

Relative Risk [ ]

\begin{tabular}{lccc}
\hline Diet 1 & 1.00013 & 1.0012 & 1.012 \\
Diet 2 & 1.000047 & 1.00041 & 1.0043 \\
Diet 3 & 1.000039 & 1.00035 & 1.004 \\
Diet 4 & 1.00028 & 1.0026 & 1.04 \\
\hline
\end{tabular}

\section{Probability of Causation [\%]}

\begin{tabular}{llcc}
\hline Diet 1 & 0.013 & 0.124 & 1.18 \\
Diet 2 & 0.005 & 0.041 & 0.43 \\
Diet 3 & 0.004 & 0.035 & 0.40 \\
Diet 4 & 0.028 & 0.256 & 3.81 \\
\hline
\end{tabular}

Diet 1 - Backyard cow milk + all other locally produced non-milk exposure pathways

Diet 2 - Locally produced commercial milk + all other locally produced non-milk exposure pathways

Diet 3 - Regionally mixed commercial milk + inhalation (other regionally mixed food items are minor contributors to the total)

Diet 4 - Goat milk (all other exposure pathways are of negligible importance compared to goat milk) 


\section{Location: Rockford}

Receptor: Male born in 1920

\begin{tabular}{lccc}
\hline & \multicolumn{3}{c}{ Thyroid Dose [cGy] } \\
Exposure Pathway & \multicolumn{2}{c}{$95 \%$ Subjective Confidence Interval } \\
\hline Backyard Cow Milk & lower limit & central estimate & upper limit \\
Commercial Milk (locally produced) & 0.18 & 0.89 & 4.8 \\
Commercial Milk (regionally mixed) & 0.032 & 0.19 & 1.2 \\
Goat Milk (locally produced) & 0.045 & 0.25 & 1.5 \\
Beef (locally produced) & 0.34 & 2.5 & 17 \\
Leafy Vegetables (locally produced) & 0.00059 & 0.0098 & 0.21 \\
Eggs (locally produced) & 0.00016 & 0.0016 & 0.013 \\
Cottage Cheese (locally produced) & 0.01 & 0.069 & 0.41 \\
Inhalation & 0.00037 & 0.0037 & 0.039 \\
Mother's milk (mother on Diet 1) & 0.012 & 0.041 & 0.15 \\
Prenatal exposure (mother on Diet 1) & -- & -- & - \\
Diet 1 & -- & -- & -- \\
Diet 2 & 0.21 & 1 & 5.5 \\
Diet 3 & 0.077 & 0.35 & 1.8 \\
\hline
\end{tabular}

Excess Lifetime Risk [ ]

\begin{tabular}{lllc}
\hline Diet 1 & $2.2 \mathrm{E}-08$ & $1.2 \mathrm{E}-06$ & $3.8 \mathrm{E}-05$ \\
Diet 2 & $8.9 \mathrm{E}-09$ & $4.2 \mathrm{E}-07$ & $1.4 \mathrm{E}-05$ \\
Diet 3 & $8.9 \mathrm{E}-09$ & $3.6 \mathrm{E}-07$ & $1.3 \mathrm{E}-05$ \\
Diet 4 & $5.6 \mathrm{E}-08$ & $2.7 \mathrm{E}-06$ & $1.1 \mathrm{E}-04$ \\
\hline
\end{tabular}

\section{Relative Risk [ ]}

\begin{tabular}{lccc}
\hline Diet 1 & 1.000044 & 1.00098 & 1.028 \\
Diet 2 & 1.000013 & 1.00032 & 1.0095 \\
Diet 3 & 1.000012 & 1.00028 & 1.0086 \\
Diet 4 & 1.000069 & 1.0024 & 1.071 \\
\hline
\end{tabular}

\section{Probability of Causation [\%]}

\begin{tabular}{llll}
\hline Diet 1 & 0.004 & 0.098 & 2.72 \\
Diet 2 & 0.001 & 0.032 & 0.95 \\
Diet 3 & 0.001 & 0.028 & 0.85 \\
Diet 4 & 0.007 & 0.239 & 6.60 \\
\hline
\end{tabular}

Diet 1 - Backyard cow milk + all other locally produced non-milk exposure pathways

Diet 2 - Locally produced commercial milk + all other locally produced non-milk exposure pathways

Diet 3 - Regionally mixed commercial milk + inhalation (other regionally mixed food items are minor contributors to the total)

Diet 4 - Goat milk (all other exposure pathways are of negligible importance compared to goat milk) 
Location: Lake City

Receptor: Female born in 1920

\begin{tabular}{lccc}
\hline & \multicolumn{3}{c}{ Thyroid Dose [cGy] } \\
& \multicolumn{2}{c}{$95 \%$ Subjective Confidence Interval } \\
Exposure Pathway & lower limit & central estimate & upper limit \\
\hline Backyard Cow Milk & 0.089 & 0.45 & 2.3 \\
Commercial Milk (locally produced) & 0.015 & 0.096 & 0.63 \\
Commercial Milk (regionally mixed) & 0.037 & 0.21 & 1.3 \\
Goat Milk (locally produced) & 0.16 & 1.1 & 8.2 \\
Beef (locally produced) & 0.00023 & 0.0039 & 0.087 \\
Leafy Vegetables (locally produced) & 0.0001 & 0.00099 & 0.0083 \\
Eggs (locally produced) & 0.0056 & 0.038 & 0.24 \\
Cottage Cheese (locally produced) & 0.00023 & 0.0023 & 0.022 \\
Inhalation & 0.0051 & 0.017 & 0.065 \\
Mother's milk (mother on Diet 1) & -- & -- & -- \\
Prenatal exposure (mother on Diet 1) & -- & -- & -- \\
Diet 1 & 0.11 & 0.52 & 2.6 \\
Diet 2 & 0.038 & 0.17 & 0.97 \\
Diet 3 & 0.045 & 0.22 & 1.4 \\
\hline
\end{tabular}

Excess Lifetime Risk [ ]

\begin{tabular}{llll}
\hline Diet 1 & $1.1 \mathrm{E}-07$ & $2.0 \mathrm{E}-06$ & $3.2 \mathrm{E}-05$ \\
Diet 2 & $4.6 \mathrm{E}-08$ & $6.5 \mathrm{E}-07$ & $9.3 \mathrm{E}-06$ \\
Diet 3 & $5.7 \mathrm{E}-08$ & $8.3 \mathrm{E}-07$ & $1.3 \mathrm{E}-05$ \\
Diet 4 & $2.6 \mathrm{E}-07$ & $4.4 \mathrm{E}-06$ & $6.9 \mathrm{E}-05$ \\
\hline
\end{tabular}

\section{Relative Risk [ ]}

\begin{tabular}{lccc}
\hline Diet 1 & 1.000087 & 1.00074 & 1.0077 \\
Diet 2 & 1.00003 & 1.00025 & 1.003 \\
Diet 3 & 1.000035 & 1.00033 & 1.0039 \\
Diet 4 & 1.00016 & 1.0016 & 1.023 \\
\hline
\end{tabular}

\section{Probability of Causation [\%]}

\begin{tabular}{llcc}
\hline Diet 1 & 0.009 & 0.074 & 0.76 \\
Diet 2 & 0.003 & 0.025 & 0.30 \\
Diet 3 & 0.003 & 0.033 & 0.39 \\
Diet 4 & 0.016 & 0.165 & 2.27 \\
\hline
\end{tabular}

Diet 1 - Backyard cow milk + all other locally produced non-milk exposure pathways

Diet 2 - Locally produced commercial milk + all other locally produced non-milk exposure pathways

Diet 3 - Regionally mixed commercial milk + inhalation (other regionally mixed food items are minor contributors to the total)

Diet 4 - Goat milk (all other exposure pathways are of negligible importance compared to goat milk) 
Location: Lake City

Receptor: Male born in 1920

\begin{tabular}{lccc}
\hline & \multicolumn{3}{c}{ Thyroid Dose [cGy] } \\
Exposure Pathway & 95\% Subjective Confidence Interval \\
\hline Backyard Cow Milk & lower limit & central estimate & upper limit \\
Commercial Milk (locally produced) & 0.11 & 0.54 & 2.7 \\
Commercial Milk (regionally mixed) & 0.02 & 0.12 & 0.76 \\
Goat Milk (locally produced) & 0.045 & 0.25 & 1.5 \\
Beef (locally produced) & 0.23 & 1.5 & 11 \\
Leafy Vegetables (locally produced) & 0.00037 & 0.0061 & 0.14 \\
Eggs (locally produced) & 0.0001 & 0.00097 & 0.0084 \\
Cottage Cheese (locally produced) & 0.0058 & 0.042 & 0.25 \\
Inhalation & 0.00023 & 0.0023 & 0.023 \\
Mother's milk (mother on Diet 1) & 0.0064 & 0.023 & 0.078 \\
Prenatal exposure (mother on Diet 1) & -- & -- & -- \\
Diet 1 & -- & -- & -- \\
Diet 2 & 0.13 & 0.63 & 3 \\
Diet 3 & 0.045 & 0.21 & 1.1 \\
\hline
\end{tabular}

Excess Lifetime Risk [ ]

\begin{tabular}{lllc}
\hline Diet 1 & $1.4 \mathrm{E}-08$ & $7.7 \mathrm{E}-07$ & $2.4 \mathrm{E}-05$ \\
Diet 2 & $6.0 \mathrm{E}-09$ & $2.6 \mathrm{E}-07$ & $8.2 \mathrm{E}-06$ \\
Diet 3 & $8.2 \mathrm{E}-09$ & $3.3 \mathrm{E}-07$ & $1.3 \mathrm{E}-05$ \\
Diet 4 & $3.4 \mathrm{E}-08$ & $1.7 \mathrm{E}-06$ & $6.3 \mathrm{E}-05$ \\
\hline
\end{tabular}

Relative Risk [ ]

\begin{tabular}{lccc}
\hline Diet 1 & 1.000024 & 1.00057 & 1.015 \\
Diet 2 & 1.0000076 & 1.00019 & 1.0054 \\
Diet 3 & 1.00001 & 1.00025 & 1.0079 \\
Diet 4 & 1.000039 & 1.0015 & 1.043 \\
\hline
\end{tabular}

\section{Probability of Causation [\%]}

\begin{tabular}{llcc}
\hline Diet 1 & 0.002 & 0.057 & 1.46 \\
Diet 2 & 0.001 & 0.019 & 0.53 \\
Diet 3 & 0.001 & 0.025 & 0.79 \\
Diet 4 & 0.004 & 0.149 & 4.10 \\
\hline
\end{tabular}

Diet 1 - Backyard cow milk + all other locally produced non-milk exposure pathways

Diet 2 - Locally produced commercial milk + all other locally produced non-milk exposure pathways

Diet 3 - Regionally mixed commercial milk + inhalation (other regionally mixed food items are minor contributors to the total)

Diet 4 - Goat milk (all other exposure pathways are of negligible importance compared to goat milk) 
Location: Sweetwater

Receptor: Female born in 1920

\begin{tabular}{lccc}
\hline & \multicolumn{3}{c}{ Thyroid Dose [cGy] } \\
Exposure Pathway & \multicolumn{2}{c}{$95 \%$ Subjective Confidence Interval } \\
\hline Backyard Cow Milk & lower limit & central estimate & upper limit \\
Commercial Milk (locally produced) & 0.098 & 0.56 & 2.9 \\
Commercial Milk (regionally mixed) & 0.02 & 0.12 & 0.82 \\
Goat Milk (locally produced) & 0.037 & 0.2 & 1.3 \\
Beef (locally produced) & 0.15 & 1.3 & 10 \\
Leafy Vegetables (locally produced) & 0.00028 & 0.0046 & 0.088 \\
Eggs (locally produced) & 0.00012 & 0.0012 & 0.0099 \\
Cottage Cheese (locally produced) & 0.0066 & 0.047 & 0.3 \\
Inhalation & 0.00026 & 0.0028 & 0.026 \\
Mother's milk (mother on Diet 1) & 0.0071 & 0.025 & 0.089 \\
Prenatal exposure (mother on Diet 1) & -- & -- & -- \\
Diet 1 & -- & -- & - \\
Diet 2 & 0.12 & 0.67 & 3.3 \\
Diet 3 & 0.043 & 0.22 & 1.2 \\
\hline
\end{tabular}

Excess Lifetime Risk [ ]

\begin{tabular}{llll}
\hline Diet 1 & $1.5 \mathrm{E}-07$ & $2.5 \mathrm{E}-06$ & $3.9 \mathrm{E}-05$ \\
Diet 2 & $5.7 \mathrm{E}-08$ & $8.3 \mathrm{E}-07$ & $1.2 \mathrm{E}-05$ \\
Diet 3 & $6.2 \mathrm{E}-08$ & $8.6 \mathrm{E}-07$ & $1.3 \mathrm{E}-05$ \\
Diet 4 & $3.0 \mathrm{E}-07$ & $5.3 \mathrm{E}-06$ & $1.1 \mathrm{E}-04$ \\
\hline
\end{tabular}

\section{Relative Risk [ ]}

\begin{tabular}{lccc}
\hline Diet 1 & 1.00011 & 1.00093 & 1.0092 \\
Diet 2 & 1.000039 & 1.0003 & 1.0035 \\
Diet 3 & 1.000039 & 1.00034 & 1.004 \\
Diet 4 & 1.00018 & 1.0019 & 1.029 \\
\hline
\end{tabular}

\section{Probability of Causation [\%]}

\begin{tabular}{llll}
\hline Diet 1 & 0.011 & 0.093 & 0.91 \\
Diet 2 & 0.004 & 0.030 & 0.35 \\
Diet 3 & 0.004 & 0.034 & 0.40 \\
Diet 4 & 0.018 & 0.194 & 2.86 \\
\hline
\end{tabular}

Diet 1 - Backyard cow milk + all other locally produced non-milk exposure pathways

Diet 2 - Locally produced commercial milk + all other locally produced non-milk exposure pathways

Diet 3 - Regionally mixed commercial milk + inhalation (other regionally mixed food items are minor contributors to the total)

Diet 4 - Goat milk (all other exposure pathways are of negligible importance compared to goat milk) 
Location: Sweetwater

Receptor: Male born in 1920

\begin{tabular}{lccc}
\hline & \multicolumn{3}{c}{ Thyroid Dose [cGy] } \\
Exposure Pathway & 95\% Subjective Confidence Interval \\
\hline Backyard Cow Milk & lower limit & central estimate & upper limit \\
Commercial Milk (locally produced) & 0.12 & 0.65 & 3.3 \\
Commercial Milk (regionally mixed) & 0.023 & 0.15 & 0.92 \\
Goat Milk (locally produced) & 0.045 & 0.25 & 1.5 \\
Beef (locally produced) & 0.22 & 1.9 & 14 \\
Leafy Vegetables (locally produced) & 0.00047 & 0.0073 & 0.14 \\
Eggs (locally produced) & 0.00012 & 0.0012 & 0.0099 \\
Cottage Cheese (locally produced) & 0.0072 & 0.05 & 0.33 \\
Inhalation & 0.00026 & 0.0028 & 0.027 \\
Mother's milk (mother on Diet 1) & 0.009 & 0.033 & 0.12 \\
Prenatal exposure (mother on Diet 1) & -- & -- & -- \\
Diet 1 & -- & -- & -- \\
Diet 2 & 0.14 & 0.78 & 3.8 \\
Diet 3 & 0.052 & 0.26 & 1.4 \\
\hline
\end{tabular}

Excess Lifetime Risk [ ]

\begin{tabular}{llll}
\hline Diet 1 & $1.7 \mathrm{E}-08$ & $9.9 \mathrm{E}-07$ & $2.6 \mathrm{E}-05$ \\
Diet 2 & $7.2 \mathrm{E}-09$ & $3.1 \mathrm{E}-07$ & $9.9 \mathrm{E}-06$ \\
Diet 3 & $8.8 \mathrm{E}-09$ & $3.5 \mathrm{E}-07$ & $1.3 \mathrm{E}-05$ \\
Diet 4 & $4.3 \mathrm{E}-08$ & $2.0 \mathrm{E}-06$ & $7.3 \mathrm{E}-05$ \\
\hline
\end{tabular}

Relative Risk [ ]

\begin{tabular}{lccc}
\hline Diet 1 & 1.00003 & 1.00076 & 1.02 \\
Diet 2 & 1.00001 & 1.00024 & 1.0067 \\
Diet 3 & 1.000011 & 1.00026 & 1.0084 \\
Diet 4 & 1.00005 & 1.0019 & 1.047 \\
\hline
\end{tabular}

\section{Probability of Causation [\%]}

\begin{tabular}{llcc}
\hline Diet 1 & 0.003 & 0.076 & 1.94 \\
Diet 2 & 0.001 & 0.024 & 0.66 \\
Diet 3 & 0.001 & 0.026 & 0.83 \\
Diet 4 & 0.005 & 0.188 & 4.49 \\
\hline
\end{tabular}

Diet 1 - Backyard cow milk + all other locally produced non-milk exposure pathways

Diet 2 - Locally produced commercial milk + all other locally produced non-milk exposure pathways

Diet 3 - Regionally mixed commercial milk + inhalation (other regionally mixed food items are minor contributors to the total)

Diet 4 - Goat milk (all other exposure pathways are of negligible importance compared to goat milk) 


\section{Location: Knoxville}

Receptor: Female born in 1920

\begin{tabular}{lccc}
\hline & \multicolumn{3}{c}{ Thyroid Dose [cGy] } \\
& \multicolumn{2}{c}{$95 \%$ Subjective Confidence Interval } \\
Exposure Pathway & lower limit & central estimate & upper limit \\
\hline Backyard Cow Milk & 0.18 & 0.95 & 5.1 \\
Commercial Milk (locally produced) & 0.033 & 0.2 & 1.3 \\
Commercial Milk (regionally mixed) & 0.037 & 0.21 & 1.3 \\
Goat Milk (locally produced) & 0.33 & 2.3 & 17 \\
Beef (locally produced) & 0.00048 & 0.0079 & 0.16 \\
Leafy Vegetables (locally produced) & 0.0002 & 0.002 & 0.017 \\
Eggs (locally produced) & 0.012 & 0.08 & 0.5 \\
Cottage Cheese (locally produced) & 0.00049 & 0.0047 & 0.044 \\
Inhalation & 0.012 & 0.04 & 0.15 \\
Mother's milk (mother on Diet 1) & -- & -- & -- \\
Prenatal exposure (mother on Diet 1) & -- & -- & - \\
Diet 1 & 0.21 & 1.1 & 5.9 \\
Diet 2 & 0.072 & 0.36 & 2 \\
Diet 3 & 0.055 & 0.24 & 1.5 \\
\hline
\end{tabular}

Excess Lifetime Risk [ ]

\begin{tabular}{llll}
\hline Diet 1 & $2.6 \mathrm{E}-07$ & $4.0 \mathrm{E}-06$ & $6.1 \mathrm{E}-05$ \\
Diet 2 & $9.2 \mathrm{E}-08$ & $1.4 \mathrm{E}-06$ & $1.9 \mathrm{E}-05$ \\
Diet 3 & $7.0 \mathrm{E}-08$ & $9.3 \mathrm{E}-07$ & $1.3 \mathrm{E}-05$ \\
Diet 4 & $5.3 \mathrm{E}-07$ & $9.2 \mathrm{E}-06$ & $1.4 \mathrm{E}-04$ \\
\hline
\end{tabular}

\section{Relative Risk [ ]}

\begin{tabular}{lccc}
\hline Diet 1 & 1.00018 & 1.0016 & 1.015 \\
Diet 2 & 1.000062 & 1.00051 & 1.0051 \\
Diet 3 & 1.000042 & 1.00037 & 1.0041 \\
Diet 4 & 1.00035 & 1.0033 & 1.048 \\
\hline
\end{tabular}

\section{Probability of Causation [\%]}

\begin{tabular}{llcc}
\hline Diet 1 & 0.018 & 0.155 & 1.47 \\
Diet 2 & 0.006 & 0.051 & 0.50 \\
Diet 3 & 0.004 & 0.036 & 0.41 \\
Diet 4 & 0.035 & 0.331 & 4.55 \\
\hline
\end{tabular}

Diet 1 - Backyard cow milk + all other locally produced non-milk exposure pathways

Diet 2 - Locally produced commercial milk + all other locally produced non-milk exposure pathways

Diet 3 - Regionally mixed commercial milk + inhalation (other regionally mixed food items are minor contributors to the total)

Diet 4 - Goat milk (all other exposure pathways are of negligible importance compared to goat milk) 


\section{Location: Knoxville}

\section{Receptor: Male born in 1920}

\begin{tabular}{lccc}
\hline & \multicolumn{3}{c}{ Thyroid Dose [cGy] } \\
& \multicolumn{2}{c}{$95 \%$ Subjective Confidence Interval } \\
Exposure Pathway & lower limit & central estimate & upper limit \\
\hline Backyard Cow Milk & 0.21 & 1.1 & 6 \\
Commercial Milk (locally produced) & 0.042 & 0.25 & 1.6 \\
Commercial Milk (regionally mixed) & 0.045 & 0.25 & 1.5 \\
Goat Milk (locally produced) & 0.45 & 3.1 & 20 \\
Beef (locally produced) & 0.00077 & 0.012 & 0.26 \\
Leafy Vegetables (locally produced) & 0.0002 & 0.002 & 0.017 \\
Eggs (locally produced) & 0.013 & 0.087 & 0.55 \\
Cottage Cheese (locally produced) & 0.00048 & 0.0047 & 0.048 \\
Inhalation & 0.015 & 0.051 & 0.18 \\
Mother's milk (mother on Diet 1) & -- & -- & - \\
Prenatal exposure (mother on Diet 1) & -- & -- & -- \\
Diet 1 & 0.26 & 1.3 & 6.9 \\
Diet 2 & 0.092 & 0.44 & 2.4 \\
Diet 3 & 0.07 & 0.31 & 1.7 \\
\hline
\end{tabular}

Excess Lifetime Risk [ ]

\begin{tabular}{llll}
\hline Diet 1 & $2.6 \mathrm{E}-08$ & $1.6 \mathrm{E}-06$ & $4.7 \mathrm{E}-05$ \\
Diet 2 & $1.1 \mathrm{E}-08$ & $5.4 \mathrm{E}-07$ & $1.7 \mathrm{E}-05$ \\
Diet 3 & $9.4 \mathrm{E}-09$ & $3.8 \mathrm{E}-07$ & $1.3 \mathrm{E}-05$ \\
Diet 4 & $6.5 \mathrm{E}-08$ & $3.5 \mathrm{E}-06$ & $1.3 \mathrm{E}-04$ \\
\hline
\end{tabular}

Relative Risk [ ]

\begin{tabular}{lccc}
\hline Diet 1 & 1.000057 & 1.0012 & 1.033 \\
Diet 2 & 1.000018 & 1.00039 & 1.012 \\
Diet 3 & 1.000012 & 1.00028 & 1.0089 \\
Diet 4 & 1.000089 & 1.003 & 1.085 \\
\hline
\end{tabular}

\section{Probability of Causation [\%]}

\begin{tabular}{lllc}
\hline Diet 1 & 0.006 & 0.121 & 3.17 \\
Diet 2 & 0.002 & 0.039 & 1.18 \\
Diet 3 & 0.001 & 0.028 & 0.89 \\
Diet 4 & 0.009 & 0.301 & 7.86 \\
\hline
\end{tabular}

Diet 1 - Backyard cow milk + all other locally produced non-milk exposure pathways

Diet 2 - Locally produced commercial milk + all other locally produced non-milk exposure pathways

Diet 3 - Regionally mixed commercial milk + inhalation (other regionally mixed food items are minor contributors to the total)

Diet 4 - Goat milk (all other exposure pathways are of negligible importance compared to goat milk) 
Location: Maryville

Receptor: Female born in 1920

\begin{tabular}{lccc}
\hline & \multicolumn{3}{c}{ Thyroid Dose [cGy] } \\
Exposure Pathway & \multicolumn{2}{c}{$95 \%$ Subjective Confidence Interval } \\
\hline Backyard Cow Milk & lower limit & central estimate & upper limit \\
Commercial Milk (locally produced) & 0.089 & 0.46 & 2.6 \\
Commercial Milk (regionally mixed) & 0.015 & 0.096 & 0.6 \\
Goat Milk (locally produced) & 0.037 & 0.2 & 1.3 \\
Beef (locally produced) & 0.15 & 1.1 & 8.2 \\
Leafy Vegetables (locally produced) & 0.00025 & 0.0038 & 0.08 \\
Eggs (locally produced) & 0.000091 & 0.00098 & 0.0081 \\
Cottage Cheese (locally produced) & 0.0055 & 0.039 & 0.23 \\
Inhalation & 0.00021 & 0.0023 & 0.022 \\
Mother's milk (mother on Diet 1) & 0.0061 & 0.021 & 0.085 \\
Prenatal exposure (mother on Diet 1) & -- & -- & -- \\
Diet 1 & -- & -- & -- \\
Diet 2 & 0.11 & 0.54 & 2.9 \\
Diet 3 & 0.037 & 0.18 & 0.94 \\
\hline
\end{tabular}

Excess Lifetime Risk [ ]

\begin{tabular}{llll}
\hline Diet 1 & $1.3 \mathrm{E}-07$ & $2.1 \mathrm{E}-06$ & $3.6 \mathrm{E}-05$ \\
Diet 2 & $4.7 \mathrm{E}-08$ & $7.1 \mathrm{E}-07$ & $1.1 \mathrm{E}-05$ \\
Diet 3 & $5.9 \mathrm{E}-08$ & $8.4 \mathrm{E}-07$ & $1.3 \mathrm{E}-05$ \\
Diet 4 & $2.8 \mathrm{E}-07$ & $4.6 \mathrm{E}-06$ & $7.1 \mathrm{E}-05$ \\
\hline
\end{tabular}

\section{Relative Risk [ ]}

\begin{tabular}{lccc}
\hline Diet 1 & 1.000089 & 1.00071 & 1.0073 \\
Diet 2 & 1.000029 & 1.00024 & 1.0027 \\
Diet 3 & 1.000036 & 1.00034 & 1.004 \\
Diet 4 & 1.00015 & 1.0017 & 1.023 \\
\hline
\end{tabular}

\section{Probability of Causation [\%]}

\begin{tabular}{llll}
\hline Diet 1 & 0.009 & 0.070 & 0.72 \\
Diet 2 & 0.003 & 0.024 & 0.27 \\
Diet 3 & 0.004 & 0.034 & 0.40 \\
Diet 4 & 0.015 & 0.168 & 2.26 \\
\hline
\end{tabular}

Diet 1 - Backyard cow milk + all other locally produced non-milk exposure pathways

Diet 2 - Locally produced commercial milk + all other locally produced non-milk exposure pathways

Diet 3 - Regionally mixed commercial milk + inhalation (other regionally mixed food items are minor contributors to the total)

Diet 4 - Goat milk (all other exposure pathways are of negligible importance compared to goat milk) 


\section{Location: Maryville}

\section{Receptor: Male born in 1920}

\begin{tabular}{lccc}
\hline & \multicolumn{3}{c}{ Thyroid Dose [cGy] } \\
Exposure Pathway & \multicolumn{2}{c}{$95 \%$ Subjective Confidence Interval } \\
\hline Backyard Cow Milk & lower limit & central estimate & upper limit \\
Commercial Milk (locally produced) & 0.11 & 0.55 & 2.9 \\
Commercial Milk (regionally mixed) & 0.019 & 0.12 & 0.78 \\
Goat Milk (locally produced) & 0.045 & 0.25 & 1.5 \\
Beef (locally produced) & 0.2 & 1.6 & 10 \\
Leafy Vegetables (locally produced) & 0.0004 & 0.0061 & 0.13 \\
Eggs (locally produced) & 0.000092 & 0.00098 & 0.0086 \\
Cottage Cheese (locally produced) & 0.0061 & 0.042 & 0.27 \\
Inhalation & 0.00021 & 0.0023 & 0.021 \\
Mother's milk (mother on Diet 1) & 0.0077 & 0.027 & 0.1 \\
Prenatal exposure (mother on Diet 1) & -- & -- & -- \\
Diet 1 & -- & -- & -- \\
Diet 2 & 0.13 & 0.65 & 3.3 \\
Diet 3 & 0.046 & 0.22 & 1.2 \\
\hline
\end{tabular}

Excess Lifetime Risk [ ]

\begin{tabular}{llll}
\hline Diet 1 & $1.4 \mathrm{E}-08$ & $8.0 \mathrm{E}-07$ & $2.3 \mathrm{E}-05$ \\
Diet 2 & $6.6 \mathrm{E}-09$ & $2.6 \mathrm{E}-07$ & $8.2 \mathrm{E}-06$ \\
Diet 3 & $8.4 \mathrm{E}-09$ & $3.3 \mathrm{E}-07$ & $1.3 \mathrm{E}-05$ \\
Diet 4 & $3.8 \mathrm{E}-08$ & $1.7 \mathrm{E}-06$ & $7.0 \mathrm{E}-05$ \\
\hline
\end{tabular}

\section{Relative Risk [ ]}

\begin{tabular}{lccc}
\hline Diet 1 & 1.000026 & 1.00063 & 1.017 \\
Diet 2 & 1.0000087 & 1.00021 & 1.0056 \\
Diet 3 & 1.000011 & 1.00026 & 1.0081 \\
Diet 4 & 1.000052 & 1.0015 & 1.045 \\
\hline
\end{tabular}

\section{Probability of Causation [\%]}

\begin{tabular}{llll}
\hline Diet 1 & 0.003 & 0.063 & 1.70 \\
Diet 2 & 0.001 & 0.021 & 0.56 \\
Diet 3 & 0.001 & 0.026 & 0.80 \\
Diet 4 & 0.005 & 0.154 & 4.35 \\
\hline
\end{tabular}

Diet 1 - Backyard cow milk + all other locally produced non-milk exposure pathways

Diet 2 - Locally produced commercial milk + all other locally produced non-milk exposure pathways

Diet 3 - Regionally mixed commercial milk + inhalation (other regionally mixed food items are minor contributors to the total)

Diet 4 - Goat milk (all other exposure pathways are of negligible importance compared to goat milk) 
Location: Cedar Grove

Receptor: Female born in 1920

\begin{tabular}{lccc}
\hline & \multicolumn{3}{c}{ Thyroid Dose [cGy] } \\
Exposure Pathway & \multicolumn{2}{c}{$95 \%$ Subjective Confidence Interval } \\
\hline Backyard Cow Milk & lower limit & central estimate & upper limit \\
Commercial Milk (locally produced) & 0.17 & 0.87 & 4.6 \\
Commercial Milk (regionally mixed) & 0.031 & 0.18 & 1.1 \\
Goat Milk (locally produced) & 0.037 & 0.2 & 1.3 \\
Beef (locally produced) & 0.31 & 2.1 & 15 \\
Leafy Vegetables (locally produced) & 0.00044 & 0.0072 & 0.15 \\
Eggs (locally produced) & 0.00017 & 0.0018 & 0.015 \\
Cottage Cheese (locally produced) & 0.011 & 0.072 & 0.45 \\
Inhalation & 0.00044 & 0.0043 & 0.041 \\
Mother's milk (mother on Diet 1) & 0.011 & 0.035 & 0.12 \\
Prenatal exposure (mother on Diet 1) & -- & -- & -- \\
Diet 1 & -- & -- & -- \\
Diet 2 & 0.21 & 1 & 5.4 \\
Diet 3 & 0.069 & 0.32 & 1.8 \\
\hline
\end{tabular}

Excess Lifetime Risk [ ]

\begin{tabular}{llll}
\hline Diet 1 & $2.6 \mathrm{E}-07$ & $3.8 \mathrm{E}-06$ & $5.3 \mathrm{E}-05$ \\
Diet 2 & $8.6 \mathrm{E}-08$ & $1.2 \mathrm{E}-06$ & $1.7 \mathrm{E}-05$ \\
Diet 3 & $6.6 \mathrm{E}-08$ & $9.2 \mathrm{E}-07$ & $1.3 \mathrm{E}-05$ \\
Diet 4 & $4.9 \mathrm{E}-07$ & $8.6 \mathrm{E}-06$ & $1.3 \mathrm{E}-04$ \\
\hline
\end{tabular}

\section{Relative Risk [ ]}

\begin{tabular}{lccc}
\hline Diet 1 & 1.00015 & 1.0014 & 1.014 \\
Diet 2 & 1.000055 & 1.00047 & 1.0049 \\
Diet 3 & 1.00004 & 1.00036 & 1.0041 \\
Diet 4 & 1.00032 & 1.003 & 1.043 \\
\hline
\end{tabular}

\section{Probability of Causation [\%]}

\begin{tabular}{llll}
\hline Diet 1 & 0.015 & 0.141 & 1.35 \\
Diet 2 & 0.005 & 0.047 & 0.48 \\
Diet 3 & 0.004 & 0.036 & 0.41 \\
Diet 4 & 0.032 & 0.297 & 4.15 \\
\hline
\end{tabular}

Diet 1 - Backyard cow milk + all other locally produced non-milk exposure pathways

Diet 2 - Locally produced commercial milk + all other locally produced non-milk exposure pathways

Diet 3 - Regionally mixed commercial milk + inhalation (other regionally mixed food items are minor contributors to the total)

Diet 4 - Goat milk (all other exposure pathways are of negligible importance compared to goat milk) 
Location: Cedar Grove

Receptor: Male born in 1920

\begin{tabular}{lccc}
\hline & \multicolumn{3}{c}{ Thyroid Dose [cGy] } \\
Exposure Pathway & \multicolumn{2}{c}{$95 \%$ Subjective Confidence Interval } \\
\hline Backyard Cow Milk & lower limit & central estimate & upper limit \\
Commercial Milk (locally produced) & 0.21 & 1 & 5.5 \\
Commercial Milk (regionally mixed) & 0.039 & 0.22 & 1.3 \\
Goat Milk (locally produced) & 0.045 & 0.25 & 1.5 \\
Beef (locally produced) & 0.42 & 2.9 & 19 \\
Leafy Vegetables (locally produced) & 0.00069 & 0.011 & 0.24 \\
Eggs (locally produced) & 0.00018 & 0.0018 & 0.015 \\
Cottage Cheese (locally produced) & 0.012 & 0.079 & 0.48 \\
Inhalation & 0.00043 & 0.0042 & 0.041 \\
Mother's milk (mother on Diet 1) & 0.013 & 0.045 & 0.15 \\
Prenatal exposure (mother on Diet 1) & -- & -- & - \\
Diet 1 & -- & -- & -- \\
Diet 2 & 0.25 & 1.2 & 6.3 \\
Diet 3 & 0.089 & 0.4 & 2.1 \\
\hline
\end{tabular}

Excess Lifetime Risk [ ]

\begin{tabular}{llll}
\hline Diet 1 & $2.6 \mathrm{E}-08$ & $1.4 \mathrm{E}-06$ & $4.3 \mathrm{E}-05$ \\
Diet 2 & $1.0 \mathrm{E}-08$ & $4.7 \mathrm{E}-07$ & $1.7 \mathrm{E}-05$ \\
Diet 3 & $9.1 \mathrm{E}-09$ & $3.7 \mathrm{E}-07$ & $1.3 \mathrm{E}-05$ \\
Diet 4 & $6.1 \mathrm{E}-08$ & $3.2 \mathrm{E}-06$ & $1.2 \mathrm{E}-04$ \\
\hline
\end{tabular}

\section{Relative Risk [ ]}

\begin{tabular}{lccc}
\hline Diet 1 & 1.000052 & 1.0011 & 1.03 \\
Diet 2 & 1.000016 & 1.00036 & 1.011 \\
Diet 3 & 1.000012 & 1.00028 & 1.0087 \\
Diet 4 & 1.000081 & 1.0028 & 1.081 \\
\hline
\end{tabular}

\begin{tabular}{llcc} 
& \multicolumn{3}{c}{ Probability of Causation [\%] } \\
\hline Diet 1 & 0.005 & 0.113 & 2.92 \\
Diet 2 & 0.002 & 0.036 & 1.05 \\
Diet 3 & 0.001 & 0.028 & 0.86 \\
Diet 4 & 0.008 & 0.281 & 7.51 \\
\hline
\end{tabular}

Diet 1 - Backyard cow milk + all other locally produced non-milk exposure pathways

Diet 2 - Locally produced commercial milk + all other locally produced non-milk exposure pathways

Diet 3 - Regionally mixed commercial milk + inhalation (other regionally mixed food items are minor contributors to the total)

Diet 4 - Goat milk (all other exposure pathways are of negligible importance compared to goat milk) 
Location: Bradbury

Receptor: Female born in 1930

\begin{tabular}{lccc}
\hline & \multicolumn{3}{c}{ Thyroid Dose [cGy] } \\
Exposure Pathway & \multicolumn{2}{c}{$95 \%$ Subjective Confidence Interval } \\
\hline Backyard Cow Milk & 1.5 & 8 & 49 \\
Commercial Milk (locally produced) & 0.28 & 1.8 & 12 \\
Commercial Milk (regionally mixed) & 0.043 & 0.25 & 1.5 \\
Goat Milk (locally produced) & 2.8 & 21 & 140 \\
Beef (locally produced) & 0.0042 & 0.064 & 1.3 \\
Leafy Vegetables (locally produced) & 0.0015 & 0.017 & 0.15 \\
Eggs (locally produced) & 0.094 & 0.69 & 4.5 \\
Cottage Cheese (locally produced) & 0.0035 & 0.038 & 0.46 \\
Inhalation & 0.069 & 0.22 & 0.76 \\
Mother's milk (mother on Diet 1) & -- & -- & -- \\
Prenatal exposure (mother on Diet 1) & -- & -- & -- \\
Diet 1 & 1.8 & 9.2 & 55 \\
Diet 2 & 0.57 & 3.1 & 17 \\
Diet 3 & 0.14 & 0.5 & 2.2 \\
\hline
\end{tabular}

Excess Lifetime Risk [ ]

\begin{tabular}{llll}
\hline Diet 1 & $4.7 \mathrm{E}-06$ & $7.0 \mathrm{E}-05$ & $9.5 \mathrm{E}-04$ \\
Diet 2 & $1.7 \mathrm{E}-06$ & $2.5 \mathrm{E}-05$ & $3.0 \mathrm{E}-04$ \\
Diet 3 & $3.8 \mathrm{E}-07$ & $3.7 \mathrm{E}-06$ & $4.0 \mathrm{E}-05$ \\
Diet 4 & $1.2 \mathrm{E}-05$ & $1.7 \mathrm{E}-04$ & $2.4 \mathrm{E}-03$ \\
\hline
\end{tabular}

\section{Relative Risk [ ]}

\begin{tabular}{lccc}
\hline Diet 1 & 1.002 & 1.019 & 1.21 \\
Diet 2 & 1.00077 & 1.0067 & 1.084 \\
Diet 3 & 1.00014 & 1.0011 & 1.011 \\
Diet 4 & 1.0049 & 1.044 & 1.63 \\
\hline
\end{tabular}

\section{Probability of Causation [\%]}

\begin{tabular}{lccc}
\hline Diet 1 & 0.200 & 1.820 & 17.42 \\
Diet 2 & 0.077 & 0.665 & 7.73 \\
Diet 3 & 0.014 & 0.107 & 1.08 \\
Diet 4 & 0.491 & 4.222 & 38.39 \\
\hline
\end{tabular}

Diet 1 - Backyard cow milk + all other locally produced non-milk exposure pathways

Diet 2 - Locally produced commercial milk + all other locally produced non-milk exposure pathways

Diet 3 - Regionally mixed commercial milk + inhalation (other regionally mixed food items are minor contributors to the total)

Diet 4 - Goat milk (all other exposure pathways are of negligible importance compared to goat milk) 


\section{Location: Bradbury}

Receptor: Male born in 1930

\begin{tabular}{lccc}
\hline & \multicolumn{3}{c}{ Thyroid Dose [cGy] } \\
& \multicolumn{2}{c}{$95 \%$ Subjective Confidence Interval } \\
Exposure Pathway & lower limit & central estimate & upper limit \\
\hline Backyard Cow Milk & 1.9 & 9.9 & 58 \\
Commercial Milk (locally produced) & 0.37 & 2.3 & 14 \\
Commercial Milk (regionally mixed) & 0.056 & 0.3 & 2 \\
Goat Milk (locally produced) & 3.9 & 28 & 180 \\
Beef (locally produced) & 0.0065 & 0.1 & 2.1 \\
Leafy Vegetables (locally produced) & 0.0015 & 0.016 & 0.15 \\
Eggs (locally produced) & 0.1 & 0.74 & 4.8 \\
Cottage Cheese (locally produced) & 0.0032 & 0.038 & 0.41 \\
Inhalation & 0.078 & 0.27 & 0.91 \\
Mother's milk (mother on Diet 1) & -- & -- & - \\
Prenatal exposure (mother on Diet 1) & -- & -- & - \\
Diet 1 & 2.2 & 11 & 66 \\
Diet 2 & 0.71 & 3.8 & 21 \\
Diet 3 & 0.15 & 0.62 & 2.5 \\
\hline
\end{tabular}

Excess Lifetime Risk [ ]

\begin{tabular}{lllc}
\hline Diet 1 & $4.9 \mathrm{E}-07$ & $2.2 \mathrm{E}-05$ & $6.4 \mathrm{E}-04$ \\
Diet 2 & $1.8 \mathrm{E}-07$ & $7.7 \mathrm{E}-06$ & $2.5 \mathrm{E}-04$ \\
Diet 3 & $3.7 \mathrm{E}-08$ & $1.2 \mathrm{E}-06$ & $3.4 \mathrm{E}-05$ \\
Diet 4 & $1.4 \mathrm{E}-06$ & $5.3 \mathrm{E}-05$ & $1.8 \mathrm{E}-03$ \\
\hline
\end{tabular}

Relative Risk [ ]

\begin{tabular}{lccc}
\hline Diet 1 & 1.00078 & 1.016 & 1.41 \\
Diet 2 & 1.00026 & 1.0053 & 1.12 \\
Diet 3 & 1.000048 & 1.00082 & 1.017 \\
Diet 4 & 1.0015 & 1.039 & 1.98 \\
\hline
\end{tabular}

\section{Probability of Causation [\%]}

\begin{tabular}{lccc}
\hline Diet 1 & 0.078 & 1.529 & 28.96 \\
Diet 2 & 0.026 & 0.526 & 10.91 \\
Diet 3 & 0.005 & 0.082 & 1.69 \\
Diet 4 & 0.146 & 3.791 & 49.45 \\
\hline
\end{tabular}

Diet 1 - Backyard cow milk + all other locally produced non-milk exposure pathways

Diet 2 - Locally produced commercial milk + all other locally produced non-milk exposure pathways

Diet 3 - Regionally mixed commercial milk + inhalation (other regionally mixed food items are minor contributors to the total)

Diet 4 - Goat milk (all other exposure pathways are of negligible importance compared to goat milk) 
Location: Gallaher Bend

Receptor: Female born in 1930

\begin{tabular}{lccc}
\hline & \multicolumn{3}{c}{ Thyroid Dose [cGy] } \\
Exposure Pathway & $95 \%$ & Subjective Confidence Interval \\
Backyard Cow Milk & lower limit & central estimate & upper limit \\
Commercial Milk (locally produced) & 1.8 & 10 & 60 \\
Commercial Milk (regionally mixed) & 0.4 & 2.3 & 14 \\
Goat Milk (locally produced) & 0.043 & 0.25 & 1.5 \\
Beef (locally produced) & 3.2 & 26 & 180 \\
Leafy Vegetables (locally produced) & 0.0049 & 0.078 & 1.7 \\
Eggs (locally produced) & 0.0019 & 0.021 & 0.17 \\
Cottage Cheese (locally produced) & 0.13 & 0.83 & 5.2 \\
Inhalation & 0.0043 & 0.047 & 0.52 \\
Mother's milk (mother on Diet 1) & 0.083 & 0.27 & 0.92 \\
Prenatal exposure (mother on Diet 1) & -- & -- & -- \\
Diet 1 & -- & -- & -- \\
Diet 2 & 2.1 & 12 & 69 \\
Diet 3 & 0.74 & 3.8 & 19 \\
\hline
\end{tabular}

Excess Lifetime Risk [ ]

\begin{tabular}{llll}
\hline Diet 1 & $5.2 \mathrm{E}-06$ & $8.7 \mathrm{E}-05$ & $1.1 \mathrm{E}-03$ \\
Diet 2 & $2.1 \mathrm{E}-06$ & $2.9 \mathrm{E}-05$ & $3.7 \mathrm{E}-04$ \\
Diet 3 & $4.3 \mathrm{E}-07$ & $4.2 \mathrm{E}-06$ & $4.4 \mathrm{E}-05$ \\
Diet 4 & $1.4 \mathrm{E}-05$ & $2.0 \mathrm{E}-04$ & $2.8 \mathrm{E}-03$ \\
\hline
\end{tabular}

\section{Relative Risk [ ]}

\begin{tabular}{lccc}
\hline Diet 1 & 1.0024 & 1.023 & 1.27 \\
Diet 2 & 1.00095 & 1.0086 & 1.097 \\
Diet 3 & 1.00016 & 1.0012 & 1.012 \\
Diet 4 & 1.0059 & 1.057 & 1.79 \\
\hline
\end{tabular}

\begin{tabular}{llcc} 
& \multicolumn{3}{c}{ Probability of Causation [\%] } \\
\hline Diet 1 & 0.238 & 2.218 & 20.96 \\
Diet 2 & 0.095 & 0.853 & 8.80 \\
Diet 3 & 0.016 & 0.117 & 1.14 \\
Diet 4 & 0.587 & 5.378 & 44.05 \\
\hline
\end{tabular}

Diet 1 - Backyard cow milk + all other locally produced non-milk exposure pathways

Diet 2 - Locally produced commercial milk + all other locally produced non-milk exposure pathways

Diet 3 - Regionally mixed commercial milk + inhalation (other regionally mixed food items are minor contributors to the total)

Diet 4 - Goat milk (all other exposure pathways are of negligible importance compared to goat milk) 
Location: Gallaher Bend

Receptor: Male born in 1930

\begin{tabular}{lccc}
\hline & \multicolumn{3}{c}{ Thyroid Dose [cGy] } \\
& \multicolumn{2}{c}{$95 \%$ Subjective Confidence Interval } \\
Exposure Pathway & lower limit & central estimate & upper limit \\
\hline Backyard Cow Milk & 2.3 & 12 & 65 \\
Commercial Milk (locally produced) & 0.5 & 2.7 & 16 \\
Commercial Milk (regionally mixed) & 0.056 & 0.3 & 2 \\
Goat Milk (locally produced) & 4.3 & 35 & 220 \\
Beef (locally produced) & 0.0075 & 0.13 & 2.7 \\
Leafy Vegetables (locally produced) & 0.0018 & 0.021 & 0.17 \\
Eggs (locally produced) & 0.14 & 0.89 & 5.6 \\
Cottage Cheese (locally produced) & 0.0042 & 0.048 & 0.47 \\
Inhalation & 0.096 & 0.34 & 1.1 \\
Mother's milk (mother on Diet 1) & -- & -- & -- \\
Prenatal exposure (mother on Diet 1) & -- & -- & -- \\
Diet 1 & 2.6 & 14 & 74 \\
Diet 2 & 0.87 & 4.6 & 24 \\
Diet 3 & 0.17 & 0.7 & 2.7 \\
\hline
\end{tabular}

Excess Lifetime Risk [ ]

\begin{tabular}{lllc}
\hline Diet 1 & $6.3 \mathrm{E}-07$ & $2.8 \mathrm{E}-05$ & $7.2 \mathrm{E}-04$ \\
Diet 2 & $2.1 \mathrm{E}-07$ & $9.7 \mathrm{E}-06$ & $3.1 \mathrm{E}-04$ \\
Diet 3 & $4.1 \mathrm{E}-08$ & $1.3 \mathrm{E}-06$ & $3.6 \mathrm{E}-05$ \\
Diet 4 & $1.8 \mathrm{E}-06$ & $6.4 \mathrm{E}-05$ & $2.1 \mathrm{E}-03$ \\
\hline
\end{tabular}

Relative Risk [ ]

\begin{tabular}{lccc}
\hline Diet 1 & 1.00095 & 1.018 & 1.46 \\
Diet 2 & 1.00032 & 1.0063 & 1.15 \\
Diet 3 & 1.000052 & 1.00092 & 1.018 \\
Diet 4 & 1.0017 & 1.05 & 2.2 \\
\hline
\end{tabular}

\section{Probability of Causation [\%]}

\begin{tabular}{llcc}
\hline Diet 1 & 0.095 & 1.740 & 31.53 \\
Diet 2 & 0.032 & 0.628 & 12.82 \\
Diet 3 & 0.005 & 0.092 & 1.77 \\
Diet 4 & 0.168 & 4.770 & 54.40 \\
\hline
\end{tabular}

Diet 1 - Backyard cow milk + all other locally produced non-milk exposure pathways

Diet 2 - Locally produced commercial milk + all other locally produced non-milk exposure pathways

Diet 3 - Regionally mixed commercial milk + inhalation (other regionally mixed food items are minor contributors to the total)

Diet 4 - Goat milk (all other exposure pathways are of negligible importance compared to goat milk) 


\section{Location: EFPC}

Receptor: Female born in 1930

\begin{tabular}{lccc}
\hline & \multicolumn{3}{c}{ Thyroid Dose [cGy] } \\
Exposure Pathway & $95 \%$ Subjective Confidence Interval \\
\hline Backyard Cow Milk & lower limit & central estimate & upper limit \\
Commercial Milk (locally produced) & 0.39 & 2.1 & 13 \\
Commercial Milk (regionally mixed) & 0.07 & 0.48 & 3.1 \\
Goat Milk (locally produced) & 0.043 & 0.25 & 1.5 \\
Beef (locally produced) & -- & -- & -- \\
Leafy Vegetables (locally produced) & 0.0011 & 0.017 & 0.38 \\
Eggs (locally produced) & 0.00038 & 0.0044 & 0.039 \\
Cottage Cheese (locally produced) & 0.024 & 0.18 & 1.1 \\
Inhalation & 0.00093 & 0.0099 & 0.13 \\
Mother's milk (mother on Diet 1) & 0.018 & 0.06 & 0.2 \\
Prenatal exposure (mother on Diet 1) & -- & -- & -- \\
Diet 1 & -- & -- & -- \\
Diet 2 & 0.46 & 2.4 & 15 \\
Diet 3 & 0.14 & 0.81 & 4.6 \\
\hline
\end{tabular}

Excess Lifetime Risk [ ]

\begin{tabular}{lccc}
\hline Diet 1 & $1.2 \mathrm{E}-06$ & $1.8 \mathrm{E}-05$ & $2.4 \mathrm{E}-04$ \\
Diet 2 & $4.6 \mathrm{E}-07$ & $6.3 \mathrm{E}-06$ & $7.7 \mathrm{E}-05$ \\
Diet 3 & $2.0 \mathrm{E}-07$ & $2.5 \mathrm{E}-06$ & $3.1 \mathrm{E}-05$ \\
Diet 4 & -- & -- & -- \\
\hline
\end{tabular}

Relative Risk [ ]

\begin{tabular}{lccc}
\hline Diet 1 & 1.00049 & 1.0048 & 1.057 \\
Diet 2 & 1.00019 & 1.0017 & 1.023 \\
Diet 3 & 1.000087 & 1.00072 & 1.0084 \\
Diet 4 & -- & -- & -- \\
\hline
\end{tabular}

Probability of Causation [\%]

\begin{tabular}{lccc}
\hline Diet 1 & 0.049 & 0.477 & 5.33 \\
Diet 2 & 0.019 & 0.175 & 2.25 \\
Diet 3 & 0.009 & 0.072 & 0.83 \\
Diet 4 & -- & -- & -- \\
\hline Diet 1 - Backyard cow milk + all other locally produced non-milk exposure pathways & \\
Diet 2 - Locally produced commercial milk + all other locally produced non-milk exposure pathways \\
Diet 3 - Regionally mixed commercial milk + inhalation (other regionally mixed food items are minor contributors to the total) \\
Diet 4 - Goat milk (all other exposure pathways are of negligible importance compared to goat milk)
\end{tabular}




\section{Location: EFPC}

\section{Receptor: Male born in 1930}

\begin{tabular}{lccc}
\hline & \multicolumn{3}{c}{ Thyroid Dose [cGy] } \\
Exposure Pathway & $95 \%$ Subjective Confidence Interval \\
\hline Backyard Cow Milk & lower limit & central estimate & upper limit \\
Commercial Milk (locally produced) & 0.48 & 2.6 & 15 \\
Commercial Milk (regionally mixed) & 0.095 & 0.59 & 3.8 \\
Goat Milk (locally produced) & 0.056 & 0.3 & 2 \\
Beef (locally produced) & -- & -- & -- \\
Leafy Vegetables (locally produced) & 0.0017 & 0.027 & 0.58 \\
Eggs (locally produced) & 0.0004 & 0.0042 & 0.037 \\
Cottage Cheese (locally produced) & 0.026 & 0.19 & 1.2 \\
Inhalation & 0.00085 & 0.0099 & 0.11 \\
Mother's milk (mother on Diet 1) & 0.019 & 0.073 & 0.25 \\
Prenatal exposure (mother on Diet 1) & -- & -- & - \\
Diet 1 & -- & -- & -- \\
Diet 2 & 0.56 & 3 & 17 \\
Diet 3 & 0.18 & 0.97 & 5.4 \\
\hline
\end{tabular}

Excess Lifetime Risk [ ]

\begin{tabular}{lccc}
\hline Diet 1 & $1.2 \mathrm{E}-07$ & $5.8 \mathrm{E}-06$ & $1.7 \mathrm{E}-04$ \\
Diet 2 & $4.5 \mathrm{E}-08$ & $2.1 \mathrm{E}-06$ & $6.4 \mathrm{E}-05$ \\
Diet 3 & $2.2 \mathrm{E}-08$ & $8.2 \mathrm{E}-07$ & $2.6 \mathrm{E}-05$ \\
Diet 4 & -- & -- & -- \\
\hline
\end{tabular}

\section{Relative Risk [ ]}

\begin{tabular}{lccc}
\hline Diet 1 & 1.00019 & 1.0039 & 1.11 \\
Diet 2 & 1.000067 & 1.0014 & 1.033 \\
Diet 3 & 1.000028 & 1.00054 & 1.012 \\
Diet 4 & -- & -- & -- \\
\hline
\end{tabular}

\begin{tabular}{lccc}
\hline Diet 1 & 0.019 & 0.385 & 9.69 \\
Diet 2 & 0.007 & 0.135 & 3.21 \\
Diet 3 & 0.003 & 0.054 & 1.23 \\
Diet 4 & -- & -- & -- \\
\hline Diet 1 - Backyard cow milk + all other locally produced non-milk exposure pathways & \\
Diet 2 - Locally produced commercial milk + all other locally produced non-milk exposure pathways \\
Diet 3 - Regionally mixed commercial milk + inhalation (other regionally mixed food items are minor contributors to the total) \\
Diet 4 - Goat milk (all other exposure pathways are of negligible importance compared to goat milk)
\end{tabular}




\section{Location: Hope Creek}

Receptor: Female born in 1930

\begin{tabular}{lccc}
\hline & \multicolumn{3}{c}{ Thyroid Dose [cGy] } \\
& \multicolumn{2}{c}{$95 \%$ Subjective Confidence Interval } \\
Exposure Pathway & lower limit & central estimate & upper limit \\
\hline Backyard Cow Milk & 1 & 5.7 & 33 \\
Commercial Milk (locally produced) & 0.2 & 1.3 & 8.5 \\
Commercial Milk (regionally mixed) & 0.043 & 0.25 & 1.5 \\
Goat Milk (locally produced) & -- & -- & -- \\
Beef (locally produced) & 0.0027 & 0.046 & 1 \\
Leafy Vegetables (locally produced) & 0.0011 & 0.012 & 0.1 \\
Eggs (locally produced) & 0.07 & 0.47 & 3 \\
Cottage Cheese (locally produced) & 0.0026 & 0.028 & 0.33 \\
Inhalation & 0.049 & 0.16 & 0.55 \\
Mother's milk (mother on Diet 1) & -- & -- & -- \\
Prenatal exposure (mother on Diet 1) & -- & -- & - \\
Diet 1 & 1.2 & 6.6 & 38 \\
Diet 2 & 0.42 & 2.1 & 12 \\
Diet 3 & 0.11 & 0.43 & 2 \\
\hline
\end{tabular}

Excess Lifetime Risk [ ]

\begin{tabular}{lccc}
\hline Diet 1 & $3.0 \mathrm{E}-06$ & $4.7 \mathrm{E}-05$ & $6.7 \mathrm{E}-04$ \\
Diet 2 & $1.2 \mathrm{E}-06$ & $1.7 \mathrm{E}-05$ & $2.2 \mathrm{E}-04$ \\
Diet 3 & $3.1 \mathrm{E}-07$ & $3.3 \mathrm{E}-06$ & $3.7 \mathrm{E}-05$ \\
Diet 4 & -- & -- & -- \\
\hline
\end{tabular}

Relative Risk [ ]

\begin{tabular}{lccc}
\hline Diet 1 & 1.0013 & 1.013 & 1.14 \\
Diet 2 & 1.0005 & 1.0048 & 1.061 \\
Diet 3 & 1.00012 & 1.00094 & 1.0096 \\
Diet 4 & -- & -- & -- \\
\hline
\end{tabular}

\section{Probability of Causation [\%]}

\begin{tabular}{|c|c|c|c|}
\hline$\overline{\text { Diet } 1}$ & 0.125 & 1.329 & 12.18 \\
\hline Diet 2 & 0.050 & 0.482 & 5.72 \\
\hline Diet 3 & 0.012 & 0.094 & 0.95 \\
\hline Diet 4 & -- & -- & -- \\
\hline
\end{tabular}




\section{Location: Hope Creek}

\section{Receptor: Male born in 1930}

\begin{tabular}{lccc}
\hline & \multicolumn{3}{c}{ Thyroid Dose [cGy] } \\
& \multicolumn{2}{c}{$95 \%$ Subjective Confidence Interval } \\
Exposure Pathway & lower limit & central estimate & upper limit \\
\hline Backyard Cow Milk & 1.3 & 6.9 & 42 \\
Commercial Milk (locally produced) & 0.26 & 1.6 & 11 \\
Commercial Milk (regionally mixed) & 0.056 & 0.3 & 2 \\
Goat Milk (locally produced) & -- & -- & -- \\
Beef (locally produced) & 0.0043 & 0.073 & 1.7 \\
Leafy Vegetables (locally produced) & 0.0011 & 0.012 & 0.1 \\
Eggs (locally produced) & 0.07 & 0.51 & 3.3 \\
Cottage Cheese (locally produced) & 0.0024 & 0.028 & 0.31 \\
Inhalation & 0.056 & 0.19 & 0.64 \\
Mother's milk (mother on Diet 1) & -- & -- & -- \\
Prenatal exposure (mother on Diet 1) & -- & -- & -- \\
Diet 1 & 1.5 & 7.9 & 46 \\
Diet 2 & 0.51 & 2.6 & 15 \\
Diet 3 & 0.14 & 0.54 & 2.4 \\
\hline
\end{tabular}

Excess Lifetime Risk [ ]

\begin{tabular}{lccc}
\hline Diet 1 & $3.1 \mathrm{E}-07$ & $1.5 \mathrm{E}-05$ & $4.5 \mathrm{E}-04$ \\
Diet 2 & $1.2 \mathrm{E}-07$ & $5.6 \mathrm{E}-06$ & $1.6 \mathrm{E}-04$ \\
Diet 3 & $3.2 \mathrm{E}-08$ & $1.1 \mathrm{E}-06$ & $3.0 \mathrm{E}-05$ \\
Diet 4 & -- & -- & -- \\
\hline
\end{tabular}

\section{Relative Risk [ ]}

\begin{tabular}{lccc}
\hline Diet 1 & 1.00055 & 1.01 & 1.27 \\
Diet 2 & 1.00017 & 1.0037 & 1.084 \\
Diet 3 & 1.000041 & 1.00072 & 1.016 \\
Diet 4 & -- & -- & -- \\
\hline
\end{tabular}

Probability of Causation [\%]

\begin{tabular}{lccc}
\hline Diet 1 & 0.055 & 1.036 & 21.41 \\
Diet 2 & 0.017 & 0.371 & 7.72 \\
Diet 3 & 0.004 & 0.072 & 1.57 \\
Diet 4 & -- & -- & - \\
\hline Diet 1 - Backyard cow milk + all other locally produced non-milk exposure pathways & \\
Diet 2 - Locally produced commercial milk + all other locally produced non-milk exposure pathways \\
Diet 3 - Regionally mixed commercial milk + inhalation (other regionally mixed food items are minor contributors to the total) \\
Diet 4 - Goat milk (all other exposure pathways are of negligible importance compared to goat milk)
\end{tabular}


Location: Buttermilk Rd.

Receptor: Female born in 1930

\begin{tabular}{lccc}
\hline & \multicolumn{3}{c}{ Thyroid Dose [cGy] } \\
Exposure Pathway & \multicolumn{2}{c}{$95 \%$ Subjective Confidence Interval } \\
Backyard Cow Milk & 1 & 5.7 & 33 \\
Commercial Milk (locally produced) & 0.21 & 1.3 & 8.3 \\
Commercial Milk (regionally mixed) & 0.043 & 0.25 & 1.5 \\
Goat Milk (locally produced) & 1.8 & 14 & 100 \\
Beef (locally produced) & 0.0027 & 0.045 & 0.96 \\
Leafy Vegetables (locally produced) & 0.0011 & 0.012 & 0.098 \\
Eggs (locally produced) & 0.069 & 0.47 & 2.9 \\
Cottage Cheese (locally produced) & 0.0025 & 0.027 & 0.3 \\
Inhalation & 0.05 & 0.16 & 0.56 \\
Mother's milk (mother on Diet 1) & -- & -- & -- \\
Prenatal exposure (mother on Diet 1) & -- & -- & -- \\
Diet 1 & 1.2 & 6.5 & 38 \\
Diet 2 & 0.41 & 2.1 & 12 \\
Diet 3 & 0.11 & 0.43 & 2 \\
\hline
\end{tabular}

Excess Lifetime Risk [ ]

\begin{tabular}{llll}
\hline Diet 1 & $2.9 \mathrm{E}-06$ & $4.8 \mathrm{E}-05$ & $6.9 \mathrm{E}-04$ \\
Diet 2 & $1.2 \mathrm{E}-06$ & $1.7 \mathrm{E}-05$ & $2.1 \mathrm{E}-04$ \\
Diet 3 & $3.1 \mathrm{E}-07$ & $3.3 \mathrm{E}-06$ & $3.7 \mathrm{E}-05$ \\
Diet 4 & $7.8 \mathrm{E}-06$ & $1.2 \mathrm{E}-04$ & $1.6 \mathrm{E}-03$ \\
\hline
\end{tabular}

\section{Relative Risk [ ]}

\begin{tabular}{lccc}
\hline Diet 1 & 1.0013 & 1.013 & 1.14 \\
Diet 2 & 1.00051 & 1.0048 & 1.056 \\
Diet 3 & 1.00012 & 1.00094 & 1.0097 \\
Diet 4 & 1.0033 & 1.03 & 1.46 \\
\hline
\end{tabular}

\section{Probability of Causation [\%]}

\begin{tabular}{lccc}
\hline Diet 1 & 0.125 & 1.299 & 12.01 \\
Diet 2 & 0.051 & 0.477 & 5.30 \\
Diet 3 & 0.012 & 0.094 & 0.96 \\
Diet 4 & 0.326 & 2.931 & 31.56 \\
\hline
\end{tabular}

Diet 1 - Backyard cow milk + all other locally produced non-milk exposure pathways

Diet 2 - Locally produced commercial milk + all other locally produced non-milk exposure pathways

Diet 3 - Regionally mixed commercial milk + inhalation (other regionally mixed food items are minor contributors to the total)

Diet 4 - Goat milk (all other exposure pathways are of negligible importance compared to goat milk) 
Location: Buttermilk Rd.

Receptor: Male born in 1930

\begin{tabular}{lccc}
\hline & \multicolumn{3}{c}{ Thyroid Dose [cGy] } \\
Exposure Pathway & $95 \%$ Subjective Confidence Interval \\
\hline Backyard Cow Milk & lower limit & central estimate & upper limit \\
Commercial Milk (locally produced) & 1.3 & 6.8 & 42 \\
Commercial Milk (regionally mixed) & 0.27 & 1.5 & 10 \\
Goat Milk (locally produced) & 0.056 & 0.3 & 2 \\
Beef (locally produced) & 2.6 & 20 & 120 \\
Leafy Vegetables (locally produced) & 0.0042 & 0.071 & 1.5 \\
Eggs (locally produced) & 0.0011 & 0.012 & 0.098 \\
Cottage Cheese (locally produced) & 0.071 & 0.5 & 3.3 \\
Inhalation & 0.0024 & 0.027 & 0.28 \\
Mother's milk (mother on Diet 1) & 0.056 & 0.19 & 0.65 \\
Prenatal exposure (mother on Diet 1) & -- & -- & -- \\
Diet 1 & -- & -- & -- \\
Diet 2 & 1.5 & 7.8 & 46 \\
Diet 3 & 0.51 & 2.5 & 15 \\
\hline
\end{tabular}

Excess Lifetime Risk [ ]

\begin{tabular}{llcc}
\hline Diet 1 & $3.1 \mathrm{E}-07$ & $1.5 \mathrm{E}-05$ & $4.3 \mathrm{E}-04$ \\
Diet 2 & $1.1 \mathrm{E}-07$ & $5.6 \mathrm{E}-06$ & $1.7 \mathrm{E}-04$ \\
Diet 3 & $3.1 \mathrm{E}-08$ & $1.1 \mathrm{E}-06$ & $3.0 \mathrm{E}-05$ \\
Diet 4 & $9.3 \mathrm{E}-07$ & $3.6 \mathrm{E}-05$ & $1.2 \mathrm{E}-03$ \\
\hline
\end{tabular}

Relative Risk [ ]

\begin{tabular}{lccc}
\hline Diet 1 & 1.00055 & 1.01 & 1.26 \\
Diet 2 & 1.00018 & 1.0036 & 1.081 \\
Diet 3 & 1.000041 & 1.00072 & 1.016 \\
Diet 4 & 1.00092 & 1.027 & 1.68 \\
\hline
\end{tabular}

Probability of Causation [\%]

\begin{tabular}{lccc}
\hline Diet 1 & 0.055 & 0.999 & 20.46 \\
Diet 2 & 0.018 & 0.359 & 7.45 \\
Diet 3 & 0.004 & 0.072 & 1.56 \\
Diet 4 & 0.092 & 2.670 & 40.28 \\
\hline
\end{tabular}

Diet 1 - Backyard cow milk + all other locally produced non-milk exposure pathways

Diet 2 - Locally produced commercial milk + all other locally produced non-milk exposure pathways

Diet 3 - Regionally mixed commercial milk + inhalation (other regionally mixed food items are minor contributors to the total)

Diet 4 - Goat milk (all other exposure pathways are of negligible importance compared to goat milk) 
Location: Jonesville

Receptor: Female born in 1930

\begin{tabular}{lccc}
\hline & \multicolumn{3}{c}{ Thyroid Dose [cGy] } \\
Exposure Pathway & \multicolumn{2}{c}{$95 \%$ Subjective Confidence Interval } \\
\hline Backyard Cow Milk & lower limit & central estimate & upper limit \\
Commercial Milk (locally produced) & 0.14 & 0.78 & 4.9 \\
Commercial Milk (regionally mixed) & 0.026 & 0.19 & 1.3 \\
Goat Milk (locally produced) & 0.043 & 0.25 & 1.5 \\
Beef (locally produced) & 0.26 & 2.1 & 17 \\
Leafy Vegetables (locally produced) & 0.00039 & 0.0066 & 0.14 \\
Eggs (locally produced) & 0.00015 & 0.0017 & 0.016 \\
Cottage Cheese (locally produced) & 0.0092 & 0.068 & 0.44 \\
Inhalation & 0.00033 & 0.0039 & 0.049 \\
Mother's milk (mother on Diet 1) & 0.0071 & 0.024 & 0.092 \\
Prenatal exposure (mother on Diet 1) & -- & -- & -- \\
Diet 1 & -- & -- & -- \\
Diet 2 & 0.17 & 0.91 & 5.6 \\
Diet 3 & 0.058 & 0.31 & 2 \\
\hline
\end{tabular}

Excess Lifetime Risk [ ]

\begin{tabular}{llll}
\hline Diet 1 & $4.7 \mathrm{E}-07$ & $6.9 \mathrm{E}-06$ & $1.0 \mathrm{E}-04$ \\
Diet 2 & $1.8 \mathrm{E}-07$ & $2.5 \mathrm{E}-06$ & $3.5 \mathrm{E}-05$ \\
Diet 3 & $1.7 \mathrm{E}-07$ & $2.2 \mathrm{E}-06$ & $3.0 \mathrm{E}-05$ \\
Diet 4 & $1.2 \mathrm{E}-06$ & $1.6 \mathrm{E}-05$ & $2.5 \mathrm{E}-04$ \\
\hline
\end{tabular}

Relative Risk [ ]

\begin{tabular}{lccc}
\hline Diet 1 & 1.00017 & 1.0018 & 1.024 \\
Diet 2 & 1.00007 & 1.00064 & 1.01 \\
Diet 3 & 1.000074 & 1.00064 & 1.008 \\
Diet 4 & 1.00042 & 1.0043 & 1.076 \\
\hline
\end{tabular}

\begin{tabular}{llcc} 
& \multicolumn{3}{c}{ Probability of Causation [\%] } \\
\hline Diet 1 & 0.017 & 0.181 & 2.34 \\
Diet 2 & 0.007 & 0.064 & 1.03 \\
Diet 3 & 0.007 & 0.064 & 0.79 \\
Diet 4 & 0.042 & 0.426 & 7.08 \\
\hline
\end{tabular}

Diet 1 - Backyard cow milk + all other locally produced non-milk exposure pathways

Diet 2 - Locally produced commercial milk + all other locally produced non-milk exposure pathways

Diet 3 - Regionally mixed commercial milk + inhalation (other regionally mixed food items are minor contributors to the total)

Diet 4 - Goat milk (all other exposure pathways are of negligible importance compared to goat milk) 


\section{Location: Jonesville}

\section{Receptor: Male born in 1930}

\begin{tabular}{lccc}
\hline & \multicolumn{3}{c}{ Thyroid Dose [cGy] } \\
Exposure Pathway & \multicolumn{2}{c}{$95 \%$ Subjective Confidence Interval } \\
\hline Backyard Cow Milk & lower limit & central estimate & upper limit \\
Commercial Milk (locally produced) & 0.18 & 0.96 & 6 \\
Commercial Milk (regionally mixed) & 0.034 & 0.22 & 1.6 \\
Goat Milk (locally produced) & 0.056 & 0.3 & 2 \\
Beef (locally produced) & 0.33 & 2.7 & 21 \\
Leafy Vegetables (locally produced) & 0.00056 & 0.01 & 0.21 \\
Eggs (locally produced) & 0.00015 & 0.0017 & 0.016 \\
Cottage Cheese (locally produced) & 0.0099 & 0.07 & 0.51 \\
Inhalation & 0.00032 & 0.0037 & 0.045 \\
Mother's milk (mother on Diet 1) & 0.0087 & 0.029 & 0.11 \\
Prenatal exposure (mother on Diet 1) & -- & -- & - \\
Diet 1 & -- & -- & -- \\
Diet 2 & 0.22 & 1.1 & 6.9 \\
Diet 3 & 0.07 & 0.37 & 2.4 \\
\hline
\end{tabular}

Excess Lifetime Risk [ ]

\begin{tabular}{llll}
\hline Diet 1 & $4.3 \mathrm{E}-08$ & $2.2 \mathrm{E}-06$ & $7.0 \mathrm{E}-05$ \\
Diet 2 & $1.7 \mathrm{E}-08$ & $7.5 \mathrm{E}-07$ & $2.7 \mathrm{E}-05$ \\
Diet 3 & $1.9 \mathrm{E}-08$ & $7.2 \mathrm{E}-07$ & $2.4 \mathrm{E}-05$ \\
Diet 4 & $1.3 \mathrm{E}-07$ & $5.0 \mathrm{E}-06$ & $1.9 \mathrm{E}-04$ \\
\hline
\end{tabular}

Relative Risk [ ]

\begin{tabular}{lccc}
\hline Diet 1 & 1.000071 & 1.0016 & 1.039 \\
Diet 2 & 1.000023 & 1.00055 & 1.013 \\
Diet 3 & 1.000024 & 1.00048 & 1.011 \\
Diet 4 & 1.00014 & 1.0041 & 1.1 \\
\hline
\end{tabular}

\begin{tabular}{llcc} 
& \multicolumn{3}{c}{ Probability of Causation [\%] } \\
\hline Diet 1 & 0.007 & 0.158 & 3.72 \\
Diet 2 & 0.002 & 0.055 & 1.27 \\
Diet 3 & 0.002 & 0.048 & 1.11 \\
Diet 4 & 0.014 & 0.411 & 9.47 \\
\hline
\end{tabular}

Diet 1 - Backyard cow milk + all other locally produced non-milk exposure pathways

Diet 2 - Locally produced commercial milk + all other locally produced non-milk exposure pathways

Diet 3 - Regionally mixed commercial milk + inhalation (other regionally mixed food items are minor contributors to the total)

Diet 4 - Goat milk (all other exposure pathways are of negligible importance compared to goat milk) 


\section{Location: OR Scarboro}

Receptor: Female born in 1930

\begin{tabular}{lccc}
\hline & \multicolumn{3}{c}{ Thyroid Dose [cGy] } \\
Exposure Pathway & \multicolumn{2}{c}{$9 \%$ Subjective Confidence Interval } \\
\hline Backyard Cow Milk & lower limit & central estimate & upper limit \\
Commercial Milk (locally produced) & -- & -- & -- \\
Commercial Milk (regionally mixed) & -- & -- & -- \\
Goat Milk (locally produced) & 0.043 & 0.25 & 1.5 \\
Beef (locally produced) & -- & -- & -- \\
Leafy Vegetables (locally produced) & -- & -- & -- \\
Eggs (locally produced) & -- & -- & -- \\
Cottage Cheese (locally produced) & -- & -- & -- \\
Inhalation & 0.0018 & 0.02 & 0.23 \\
Mother's milk (mother on Diet 3) & 0.036 & 0.12 & 0.41 \\
Prenatal exposure (mother on Diet 3) & -- & -- & -- \\
Diet 1 & -- & -- & -- \\
Diet 2 & -- & -- & -- \\
Diet 3 & -- & -- & -- \\
\hline
\end{tabular}

Excess Lifetime Risk [ ]

\begin{tabular}{lccc}
\hline Diet 1 & -- & -- & -- \\
Diet 2 & -- & -- & -- \\
Diet 3 & $2.7 \mathrm{E}-07$ & $3.0 \mathrm{E}-06$ & $3.4 \mathrm{E}-05$ \\
Diet 4 & -- & -- & -- \\
\hline
\end{tabular}

\section{Relative Risk [ ]}

\begin{tabular}{lccc}
\hline Diet 1 & -- & -- & -- \\
Diet 2 & -- & -- & -- \\
Diet 3 & 1.00011 & 1.00084 & 1.0092 \\
Diet 4 & -- & -- & -- \\
\hline
\end{tabular}

\begin{tabular}{lccc}
\hline Diet 1 & -- & -- & -- \\
Diet 2 & -- & -- & -- \\
Diet 3 & 0.011 & 0.084 & 0.92 \\
Diet 4 & -- & -- & -- \\
\hline Diet 1 - Backyard cow milk + all other locally produced non-milk exposure pathways & \\
Diet 2 - Locally produced commercial milk + all other locally produced non-milk exposure pathways \\
Diet 3 - Regionally mixed commercial milk + inhalation (other regionally mixed food items are minor contributors to the total) \\
Diet 4 - Goat milk (all other exposure pathways are of negligible importance compared to goat milk)
\end{tabular}




\section{Location: OR Scarboro}

Receptor: Male born in 1930

\begin{tabular}{lccc}
\hline & \multicolumn{3}{c}{ Thyroid Dose [cGy] } \\
Exposure Pathway & \multicolumn{2}{c}{ 95\% Subjective Confidence Interval } \\
\hline Backyard Cow Milk & lower limit & central estimate & upper limit \\
Commercial Milk (locally produced) & -- & -- & -- \\
Commercial Milk (regionally mixed) & -- & -- & -- \\
Goat Milk (locally produced) & 0.056 & 0.3 & 2 \\
Beef (locally produced) & -- & -- & -- \\
Leafy Vegetables (locally produced) & -- & -- & -- \\
Eggs (locally produced) & -- & -- & -- \\
Cottage Cheese (locally produced) & -- & -- & -- \\
Inhalation & 0.0017 & 0.02 & 0.21 \\
Mother's milk (mother on Diet 3) & 0.041 & 0.15 & 0.49 \\
Prenatal exposure (mother on Diet 3) & -- & -- & -- \\
Diet 1 & -- & -- & -- \\
Diet 2 & -- & -- & -- \\
Diet 3 & -- & -- & -- \\
\hline
\end{tabular}

Excess Lifetime Risk [ ]

\begin{tabular}{lccc}
\hline Diet 1 & -- & -- & -- \\
Diet 2 & -- & -- & -- \\
Diet 3 & $2.8 \mathrm{E}-08$ & $9.6 \mathrm{E}-07$ & $2.9 \mathrm{E}-05$ \\
Diet 4 & -- & -- & -- \\
\hline
\end{tabular}

\section{Relative Risk [ ]}

\begin{tabular}{lccc}
\hline Diet 1 & -- & -- & -- \\
Diet 2 & -- & -- & -- \\
Diet 3 & 1.000036 & 1.00065 & 1.014 \\
Diet 4 & -- & -- & -- \\
\hline
\end{tabular}

\begin{tabular}{lccc}
\hline Diet 1 & -- & -- & -- \\
Diet 2 & -- & -- & -- \\
Diet 3 & 0.004 & -- & -41 \\
Diet 4 & -- & - \\
\hline Diet 1 - Backyard cow milk + all other locally produced non-milk exposure pathways & \\
Diet 2 - Locally produced commercial milk + all other locally produced non-milk exposure pathways \\
Diet 3 - Regionally mixed commercial milk + inhalation (other regionally mixed food items are minor contributors to the total) \\
Diet 4 - Goat milk (all other exposure pathways are of negligible importance compared to goat milk)
\end{tabular}


Location: Lawnville/Gallaher Receptor: Female born in 1930

\begin{tabular}{lccc}
\hline & \multicolumn{3}{c}{ Thyroid Dose [cGy] } \\
& \multicolumn{2}{c}{$95 \%$ Subjective Confidence Interval } \\
Exposure Pathway & lower limit & central estimate & upper limit \\
\hline Backyard Cow Milk & 1 & 5.3 & 29 \\
Commercial Milk (locally produced) & 0.21 & 1.2 & 8.3 \\
Commercial Milk (regionally mixed) & 0.042 & 0.25 & 1.5 \\
Goat Milk (locally produced) & 1.8 & 13 & 95 \\
Beef (locally produced) & 0.0026 & 0.044 & 0.86 \\
Leafy Vegetables (locally produced) & 0.0012 & 0.011 & 0.091 \\
Eggs (locally produced) & 0.072 & 0.44 & 2.8 \\
Cottage Cheese (locally produced) & 0.0023 & 0.026 & 0.26 \\
Inhalation & 0.05 & 0.16 & 0.54 \\
Mother's milk (mother on Diet 1) & -- & -- & - \\
Prenatal exposure (mother on Diet 1) & -- & -- & -- \\
Diet 1 & 1.2 & 6.1 & 33 \\
Diet 2 & 0.42 & 2 & 11 \\
Diet 3 & 0.11 & 0.43 & 2 \\
\hline
\end{tabular}

Excess Lifetime Risk [ ]

\begin{tabular}{llll}
\hline Diet 1 & $2.9 \mathrm{E}-06$ & $4.5 \mathrm{E}-05$ & $6.7 \mathrm{E}-04$ \\
Diet 2 & $1.1 \mathrm{E}-06$ & $1.6 \mathrm{E}-05$ & $2.0 \mathrm{E}-04$ \\
Diet 3 & $3.1 \mathrm{E}-07$ & $3.3 \mathrm{E}-06$ & $3.5 \mathrm{E}-05$ \\
Diet 4 & $7.9 \mathrm{E}-06$ & $1.1 \mathrm{E}-04$ & $1.7 \mathrm{E}-03$ \\
\hline
\end{tabular}

Relative Risk [ ]

\begin{tabular}{lccc}
\hline Diet 1 & 1.0012 & 1.012 & 1.13 \\
Diet 2 & 1.0005 & 1.0046 & 1.055 \\
Diet 3 & 1.00013 & 1.00093 & 1.0099 \\
Diet 4 & 1.0033 & 1.03 & 1.4 \\
\hline
\end{tabular}

Probability of Causation [\%]

\begin{tabular}{lccc}
\hline Diet 1 & 0.123 & 1.216 & 11.61 \\
Diet 2 & 0.050 & 0.454 & 5.22 \\
Diet 3 & 0.013 & 0.093 & 0.98 \\
Diet 4 & 0.327 & 2.943 & 28.73 \\
\hline
\end{tabular}

Diet 1 - Backyard cow milk + all other locally produced non-milk exposure pathways

Diet 2 - Locally produced commercial milk + all other locally produced non-milk exposure pathways

Diet 3 - Regionally mixed commercial milk + inhalation (other regionally mixed food items are minor contributors to the total)

Diet 4 - Goat milk (all other exposure pathways are of negligible importance compared to goat milk) 


\section{Location: Lawnville/Gallaher}

Receptor: Male born in 1930

\begin{tabular}{lccc}
\hline & \multicolumn{3}{c}{ Thyroid Dose [cGy] } \\
& \multicolumn{2}{c}{$95 \%$ Subjective Confidence Interval } \\
Exposure Pathway & lower limit & central estimate & upper limit \\
\hline Backyard Cow Milk & 1.3 & 6.4 & 34 \\
Commercial Milk (locally produced) & 0.26 & 1.4 & 9.4 \\
Commercial Milk (regionally mixed) & 0.056 & 0.3 & 2 \\
Goat Milk (locally produced) & 2.6 & 19 & 110 \\
Beef (locally produced) & 0.0039 & 0.069 & 1.4 \\
Leafy Vegetables (locally produced) & 0.0012 & 0.011 & 0.089 \\
Eggs (locally produced) & 0.072 & 0.48 & 3 \\
Cottage Cheese (locally produced) & 0.0023 & 0.025 & 0.24 \\
Inhalation & 0.055 & 0.18 & 0.69 \\
Mother's milk (mother on Diet 1) & -- & -- & - \\
Prenatal exposure (mother on Diet 1) & -- & -- & - \\
Diet 1 & 1.5 & 7.3 & 38 \\
Diet 2 & 0.5 & 2.4 & 14 \\
Diet 3 & 0.13 & 0.54 & 2.3 \\
\hline
\end{tabular}

\section{Excess Lifetime Risk [ ]}

\begin{tabular}{llll}
\hline Diet 1 & $3.0 \mathrm{E}-07$ & $1.5 \mathrm{E}-05$ & $4.0 \mathrm{E}-04$ \\
Diet 2 & $1.0 \mathrm{E}-07$ & $5.0 \mathrm{E}-06$ & $1.5 \mathrm{E}-04$ \\
Diet 3 & $3.0 \mathrm{E}-08$ & $1.1 \mathrm{E}-06$ & $3.0 \mathrm{E}-05$ \\
Diet 4 & $8.7 \mathrm{E}-07$ & $3.3 \mathrm{E}-05$ & $1.1 \mathrm{E}-03$ \\
\hline
\end{tabular}

\section{Relative Risk [ ]}

\begin{tabular}{lccc}
\hline Diet 1 & 1.0005 & 1.0098 & 1.23 \\
Diet 2 & 1.00017 & 1.0035 & 1.08 \\
Diet 3 & 1.000041 & 1.0007 & 1.015 \\
Diet 4 & 1.001 & 1.027 & 1.67 \\
\hline
\end{tabular}

Probability of Causation [\%]

\begin{tabular}{lccc}
\hline Diet 1 & 0.050 & 0.968 & 18.77 \\
Diet 2 & 0.017 & 0.348 & 7.45 \\
Diet 3 & 0.004 & 0.070 & 1.52 \\
Diet 4 & 0.102 & 2.613 & 40.15 \\
\hline
\end{tabular}

Diet 1 - Backyard cow milk + all other locally produced non-milk exposure pathways

Diet 2 - Locally produced commercial milk + all other locally produced non-milk exposure pathways

Diet 3 - Regionally mixed commercial milk + inhalation (other regionally mixed food items are minor contributors to the total)

Diet 4 - Goat milk (all other exposure pathways are of negligible importance compared to goat milk) 


\section{Location: Dyllis}

Receptor: Female born in 1930

\begin{tabular}{lccc}
\hline & \multicolumn{3}{c}{ Thyroid Dose [cGy] } \\
& \multicolumn{2}{c}{$95 \%$ Subjective Confidence Interval } \\
Exposure Pathway & lower limit & central estimate & upper limit \\
\hline Backyard Cow Milk & 0.18 & 1.1 & 6.3 \\
Commercial Milk (locally produced) & 0.038 & 0.24 & 1.8 \\
Commercial Milk (regionally mixed) & 0.042 & 0.25 & 1.5 \\
Goat Milk (locally produced) & 0.38 & 2.8 & 21 \\
Beef (locally produced) & 0.00057 & 0.0086 & 0.18 \\
Leafy Vegetables (locally produced) & 0.00021 & 0.0023 & 0.021 \\
Eggs (locally produced) & 0.012 & 0.092 & 0.67 \\
Cottage Cheese (locally produced) & 0.00049 & 0.0051 & 0.063 \\
Inhalation & 0.0095 & 0.033 & 0.13 \\
Mother's milk (mother on Diet 1) & -- & -- & -- \\
Prenatal exposure (mother on Diet 1) & -- & -- & -- \\
Diet 1 & 0.21 & 1.2 & 7.3 \\
Diet 2 & 0.081 & 0.42 & 2.8 \\
Diet 3 & 0.062 & 0.28 & 1.6 \\
\hline
\end{tabular}

Excess Lifetime Risk [ ]

\begin{tabular}{llll}
\hline Diet 1 & $6.0 \mathrm{E}-07$ & $9.0 \mathrm{E}-06$ & $1.2 \mathrm{E}-04$ \\
Diet 2 & $2.1 \mathrm{E}-07$ & $3.3 \mathrm{E}-06$ & $4.3 \mathrm{E}-05$ \\
Diet 3 & $1.8 \mathrm{E}-07$ & $2.3 \mathrm{E}-06$ & $3.0 \mathrm{E}-05$ \\
Diet 4 & $1.5 \mathrm{E}-06$ & $2.4 \mathrm{E}-05$ & $3.3 \mathrm{E}-04$ \\
\hline
\end{tabular}

Relative Risk [ ]

\begin{tabular}{lccc}
\hline Diet 1 & 1.00025 & 1.0024 & 1.03 \\
Diet 2 & 1.00011 & 1.00091 & 1.014 \\
Diet 3 & 1.00008 & 1.00066 & 1.0079 \\
Diet 4 & 1.00069 & 1.0058 & 1.095 \\
\hline
\end{tabular}

\begin{tabular}{llcc} 
& \multicolumn{3}{c}{ Probability of Causation [\%] } \\
\hline Diet 1 & 0.025 & 0.243 & 2.91 \\
Diet 2 & 0.011 & 0.091 & 1.36 \\
Diet 3 & 0.008 & 0.066 & 0.78 \\
Diet 4 & 0.069 & 0.576 & 8.65 \\
\hline
\end{tabular}

Diet 1 - Backyard cow milk + all other locally produced non-milk exposure pathways

Diet 2 - Locally produced commercial milk + all other locally produced non-milk exposure pathways

Diet 3 - Regionally mixed commercial milk + inhalation (other regionally mixed food items are minor contributors to the total)

Diet 4 - Goat milk (all other exposure pathways are of negligible importance compared to goat milk) 


\section{Location: Dyllis}

\section{Receptor: Male born in 1930}

\begin{tabular}{lccc}
\hline & \multicolumn{3}{c}{ Thyroid Dose [cGy] } \\
& \multicolumn{2}{c}{$95 \%$ Subjective Confidence Interval } \\
Exposure Pathway & lower limit & central estimate & upper limit \\
\hline Backyard Cow Milk & 0.21 & 1.3 & 7.4 \\
Commercial Milk (locally produced) & 0.049 & 0.3 & 2.1 \\
Commercial Milk (regionally mixed) & 0.056 & 0.3 & 2 \\
Goat Milk (locally produced) & 0.5 & 3.8 & 27 \\
Beef (locally produced) & 0.00091 & 0.014 & 0.28 \\
Leafy Vegetables (locally produced) & 0.00021 & 0.0023 & 0.021 \\
Eggs (locally produced) & 0.013 & 0.097 & 0.69 \\
Cottage Cheese (locally produced) & 0.00048 & 0.0051 & 0.057 \\
Inhalation & 0.011 & 0.041 & 0.15 \\
Mother's milk (mother on Diet 1) & -- & -- & - \\
Prenatal exposure (mother on Diet 1) & -- & -- & -- \\
Diet 1 & 0.26 & 1.5 & 8.3 \\
Diet 2 & 0.11 & 0.5 & 3.3 \\
Diet 3 & 0.075 & 0.34 & 2 \\
\hline
\end{tabular}

Excess Lifetime Risk [ ]

\begin{tabular}{llll}
\hline Diet 1 & $6.0 \mathrm{E}-08$ & $2.9 \mathrm{E}-06$ & $8.8 \mathrm{E}-05$ \\
Diet 2 & $2.3 \mathrm{E}-08$ & $1.1 \mathrm{E}-06$ & $3.5 \mathrm{E}-05$ \\
Diet 3 & $2.0 \mathrm{E}-08$ & $7.5 \mathrm{E}-07$ & $2.5 \mathrm{E}-05$ \\
Diet 4 & $1.7 \mathrm{E}-07$ & $6.8 \mathrm{E}-06$ & $2.4 \mathrm{E}-04$ \\
\hline
\end{tabular}

\section{Relative Risk [ ]}

\begin{tabular}{lccc}
\hline Diet 1 & 1.0001 & 1.002 & 1.059 \\
Diet 2 & 1.000032 & 1.00075 & 1.017 \\
Diet 3 & 1.000025 & 1.00049 & 1.011 \\
Diet 4 & 1.0002 & 1.0055 & 1.13 \\
\hline
\end{tabular}

\section{Probability of Causation [\%]}

\begin{tabular}{lccc}
\hline Diet 1 & 0.010 & 0.200 & 5.55 \\
Diet 2 & 0.003 & 0.075 & 1.66 \\
Diet 3 & 0.002 & 0.049 & 1.12 \\
Diet 4 & 0.020 & 0.546 & 11.19 \\
\hline
\end{tabular}

Diet 1 - Backyard cow milk + all other locally produced non-milk exposure pathways

Diet 2 - Locally produced commercial milk + all other locally produced non-milk exposure pathways

Diet 3 - Regionally mixed commercial milk + inhalation (other regionally mixed food items are minor contributors to the total)

Diet 4 - Goat milk (all other exposure pathways are of negligible importance compared to goat milk) 
Location: OR High School Area

Receptor: Female born in 1930

\begin{tabular}{lccc}
\hline & \multicolumn{3}{c}{ Thyroid Dose [cGy] } \\
Exposure Pathway & \multicolumn{2}{c}{ 95\% Subjective Confidence Interval } \\
\hline Backyard Cow Milk & lower limit & central estimate & upper limit \\
Commercial Milk (locally produced) & -- & -- & -- \\
Commercial Milk (regionally mixed) & -- & -- & -- \\
Goat Milk (locally produced) & 0.043 & 0.25 & 1.5 \\
Beef (locally produced) & -- & -- & -- \\
Leafy Vegetables (locally produced) & -- & -- & -- \\
Eggs (locally produced) & -- & -- & -- \\
Cottage Cheese (locally produced) & -- & -- & -- \\
Inhalation & 0.0011 & 0.012 & 0.13 \\
Mother's milk (mother on Diet 3) & 0.021 & 0.069 & 0.25 \\
Prenatal exposure (mother on Diet 3) & -- & -- & -- \\
Diet 1 & -- & -- & -- \\
Diet 2 & -- & -- & -- \\
Diet 3 & -- & -- & -- \\
\hline
\end{tabular}

Excess Lifetime Risk [ ]

\begin{tabular}{lccc}
\hline Diet 1 & -- & -- & -- \\
Diet 2 & -- & -- & -- \\
Diet 3 & $2.1 \mathrm{E}-07$ & $2.6 \mathrm{E}-06$ & $3.2 \mathrm{E}-05$ \\
Diet 4 & -- & -- & -- \\
\hline
\end{tabular}

\section{Relative Risk [ ]}

\begin{tabular}{lccc}
\hline Diet 1 & -- & -- & -- \\
Diet 2 & -- & -- & -- \\
Diet 3 & 1.000094 & 1.00074 & 1.0084 \\
Diet 4 & -- & -- & -- \\
\hline
\end{tabular}

\begin{tabular}{lccc}
\hline Diet 1 & -- & -- & -- \\
Diet 2 & -- & -- & -- \\
Diet 3 & 0.009 & 0.074 & 0.84 \\
Diet 4 & -- & -- & - \\
\hline Diet 1 - Backyard cow milk + all other locally produced non-milk exposure pathways \\
Diet 2 - Locally produced commercial milk + all other locally produced non-milk exposure pathways \\
Diet 3 - Regionally mixed commercial milk + inhalation (other regionally mixed food items are minor contributors to the total) \\
Diet 4 - Goat milk (all other exposure pathways are of negligible importance compared to goat milk)
\end{tabular}




\section{Location: OR High School Area}

Receptor: Male born in 1930

\begin{tabular}{lccc}
\hline & \multicolumn{3}{c}{ Thyroid Dose [cGy] } \\
Exposure Pathway & \multicolumn{2}{c}{$9 \%$ Subjective Confidence Interval } \\
\hline Backyard Cow Milk & lower limit & central estimate & upper limit \\
Commercial Milk (locally produced) & -- & -- & -- \\
Commercial Milk (regionally mixed) & -- & -- & -- \\
Goat Milk (locally produced) & 0.056 & 0.3 & 2 \\
Beef (locally produced) & -- & -- & -- \\
Leafy Vegetables (locally produced) & -- & -- & -- \\
Eggs (locally produced) & -- & -- & -- \\
Cottage Cheese (locally produced) & -- & -- & -- \\
Inhalation & 0.0011 & 0.012 & 0.12 \\
Mother's milk (mother on Diet 3) & 0.025 & 0.084 & 0.29 \\
Prenatal exposure (mother on Diet 3) & -- & -- & -- \\
Diet 1 & -- & -- & -- \\
Diet 2 & -- & -- & -- \\
Diet 3 & -- & -- & -- \\
\hline
\end{tabular}

Excess Lifetime Risk [ ]

\begin{tabular}{lccc}
\hline Diet 1 & -- & -- & -- \\
Diet 2 & -- & -- & -- \\
Diet 3 & $2.3 \mathrm{E}-08$ & $8.5 \mathrm{E}-07$ & $2.6 \mathrm{E}-05$ \\
Diet 4 & -- & -- & -- \\
\hline
\end{tabular}

\section{Relative Risk [ ]}

\begin{tabular}{lccc}
\hline Diet 1 & -- & -- & -- \\
Diet 2 & -- & -- & -- \\
Diet 3 & 1.00003 & 1.00056 & 1.013 \\
Diet 4 & -- & -- & -- \\
\hline
\end{tabular}

\begin{tabular}{lccc}
\hline Diet 1 & -- & -- & -- \\
Diet 2 & -- & -- & -- \\
Diet 3 & 0.003 & 0.056 & 1.27 \\
Diet 4 & -- & -- & -- \\
\hline Diet 1 - Backyard cow milk + all other locally produced non-milk exposure pathways \\
Diet 2 - Locally produced commercial milk + all other locally produced non-milk exposure pathways \\
Diet 3 - Regionally mixed commercial milk + inhalation (other regionally mixed food items are minor contributors to the total) \\
Diet 4 - Goat milk (all other exposure pathways are of negligible importance compared to goat milk)
\end{tabular}


${ }^{131}$ I Releases from X-10 Radioactive Lanthanum Processing

TASK 1 REPORT

Appendix 11-C

July 1999

Location: Norwood

Receptor: Female born in 1930

\begin{tabular}{lccc}
\hline & \multicolumn{3}{c}{ Thyroid Dose [cGy] } \\
Exposure Pathway & \multicolumn{2}{c}{$95 \%$ Subjective Confidence Interval } \\
\hline Backyard Cow Milk & lower limit & central estimate & upper limit \\
Commercial Milk (locally produced) & 0.17 & 1 & 7.2 \\
Commercial Milk (regionally mixed) & 0.036 & 0.23 & 1.7 \\
Goat Milk (locally produced) & 0.043 & 0.25 & 1.5 \\
Beef (locally produced) & 0.33 & 2.7 & 22 \\
Leafy Vegetables (locally produced) & 0.0005 & 0.0083 & 0.19 \\
Eggs (locally produced) & 0.00022 & 0.0021 & 0.018 \\
Cottage Cheese (locally produced) & 0.011 & 0.086 & 0.57 \\
Inhalation & 0.0004 & 0.0052 & 0.07 \\
Mother's milk (mother on Diet 1) & 0.009 & 0.032 & 0.12 \\
Prenatal exposure (mother on Diet 1) & -- & -- & - \\
Diet 1 & -- & -- & -- \\
Diet 2 & 0.21 & 1.2 & 7.9 \\
Diet 3 & 0.074 & 0.39 & 2.5 \\
\hline
\end{tabular}

Excess Lifetime Risk [ ]

\begin{tabular}{llll}
\hline Diet 1 & $5.6 \mathrm{E}-07$ & $8.8 \mathrm{E}-06$ & $1.4 \mathrm{E}-04$ \\
Diet 2 & $2.3 \mathrm{E}-07$ & $3.2 \mathrm{E}-06$ & $4.3 \mathrm{E}-05$ \\
Diet 3 & $1.7 \mathrm{E}-07$ & $2.3 \mathrm{E}-06$ & $3.0 \mathrm{E}-05$ \\
Diet 4 & $1.5 \mathrm{E}-06$ & $2.2 \mathrm{E}-05$ & $3.7 \mathrm{E}-04$ \\
\hline
\end{tabular}

Relative Risk [ ]

\begin{tabular}{lccc}
\hline Diet 1 & 1.00023 & 1.0023 & 1.029 \\
Diet 2 & 1.000095 & 1.00084 & 1.011 \\
Diet 3 & 1.000077 & 1.00066 & 1.008 \\
Diet 4 & 1.00064 & 1.0056 & 1.085 \\
\hline
\end{tabular}

\begin{tabular}{lccc} 
& \multicolumn{3}{c}{ Probability of Causation [\%] } \\
\hline Diet 1 & 0.023 & 0.230 & 2.81 \\
Diet 2 & 0.010 & 0.084 & 1.10 \\
Diet 3 & 0.008 & 0.066 & 0.80 \\
Diet 4 & 0.064 & 0.557 & 7.84 \\
\hline
\end{tabular}

Diet 1 - Backyard cow milk + all other locally produced non-milk exposure pathways

Diet 2 - Locally produced commercial milk + all other locally produced non-milk exposure pathways

Diet 3 - Regionally mixed commercial milk + inhalation (other regionally mixed food items are minor contributors to the total)

Diet 4 - Goat milk (all other exposure pathways are of negligible importance compared to goat milk) 


\section{Location: Norwood}

\section{Receptor: Male born in 1930}

\begin{tabular}{lccc}
\hline & \multicolumn{3}{c}{ Thyroid Dose [cGy] } \\
Exposure Pathway & \multicolumn{2}{c}{$95 \%$ Subjective Confidence Interval } \\
\hline Backyard Cow Milk & lower limit & central estimate & upper limit \\
Commercial Milk (locally produced) & 0.21 & 1.2 & 8.3 \\
Commercial Milk (regionally mixed) & 0.046 & 0.28 & 2.1 \\
Goat Milk (locally produced) & 0.056 & 0.3 & 2 \\
Beef (locally produced) & 0.45 & 3.5 & 27 \\
Leafy Vegetables (locally produced) & 0.00077 & 0.013 & 0.32 \\
Eggs (locally produced) & 0.00022 & 0.0021 & 0.019 \\
Cottage Cheese (locally produced) & 0.012 & 0.092 & 0.65 \\
Inhalation & 0.00044 & 0.0049 & 0.059 \\
Mother's milk (mother on Diet 1) & 0.01 & 0.038 & 0.14 \\
Prenatal exposure (mother on Diet 1) & -- & -- & - \\
Diet 1 & -- & -- & -- \\
Diet 2 & 0.25 & 1.4 & 9.4 \\
Diet 3 & 0.086 & 0.47 & 3 \\
\hline
\end{tabular}

Excess Lifetime Risk [ ]

\begin{tabular}{llll}
\hline Diet 1 & $6.0 \mathrm{E}-08$ & $2.7 \mathrm{E}-06$ & $8.6 \mathrm{E}-05$ \\
Diet 2 & $2.2 \mathrm{E}-08$ & $9.6 \mathrm{E}-07$ & $2.8 \mathrm{E}-05$ \\
Diet 3 & $1.9 \mathrm{E}-08$ & $7.5 \mathrm{E}-07$ & $2.5 \mathrm{E}-05$ \\
Diet 4 & $1.7 \mathrm{E}-07$ & $6.5 \mathrm{E}-06$ & $2.5 \mathrm{E}-04$ \\
\hline
\end{tabular}

\section{Relative Risk [ ]}

\begin{tabular}{lccc}
\hline Diet 1 & 1.000092 & 1.0019 & 1.054 \\
Diet 2 & 1.00003 & 1.0007 & 1.017 \\
Diet 3 & 1.000024 & 1.00049 & 1.012 \\
Diet 4 & 1.00021 & 1.0051 & 1.14 \\
\hline
\end{tabular}

\section{Probability of Causation [\%]}

\begin{tabular}{llcc}
\hline Diet 1 & 0.009 & 0.188 & 5.11 \\
Diet 2 & 0.003 & 0.070 & 1.68 \\
Diet 3 & 0.002 & 0.049 & 1.14 \\
Diet 4 & 0.021 & 0.506 & 12.49 \\
\hline
\end{tabular}

Diet 1 - Backyard cow milk + all other locally produced non-milk exposure pathways

Diet 2 - Locally produced commercial milk + all other locally produced non-milk exposure pathways

Diet 3 - Regionally mixed commercial milk + inhalation (other regionally mixed food items are minor contributors to the total)

Diet 4 - Goat milk (all other exposure pathways are of negligible importance compared to goat milk) 
Location: Woodland

Receptor: Female born in 1930

\begin{tabular}{lccc}
\hline & \multicolumn{3}{c}{ Thyroid Dose [cGy] } \\
Exposure Pathway & \multicolumn{2}{c}{ 95\% Subjective Confidence Interval } \\
\hline Backyard Cow Milk & lower limit & central estimate & upper limit \\
Commercial Milk (locally produced) & -- & -- & -- \\
Commercial Milk (regionally mixed) & -- & -- & -- \\
Goat Milk (locally produced) & 0.043 & 0.25 & 1.5 \\
Beef (locally produced) & -- & -- & -- \\
Leafy Vegetables (locally produced) & -- & -- & -- \\
Eggs (locally produced) & -- & -- & -- \\
Cottage Cheese (locally produced) & -- & -- & -- \\
Inhalation & 0.0016 & 0.017 & 0.18 \\
Mother's milk (mother on Diet 3) & 0.034 & 0.11 & 0.37 \\
Prenatal exposure (mother on Diet 3) & -- & -- & -- \\
Diet 1 & -- & -- & -- \\
Diet 2 & -- & -- & -- \\
Diet 3 & -- & -- & -- \\
\hline
\end{tabular}

Excess Lifetime Risk [ ]

\begin{tabular}{lccc}
\hline Diet 1 & -- & -- & -- \\
Diet 2 & -- & -- & -- \\
Diet 3 & $2.6 \mathrm{E}-07$ & $2.9 \mathrm{E}-06$ & $3.3 \mathrm{E}-05$ \\
Diet 4 & -- & -- & -- \\
\hline
\end{tabular}

\section{Relative Risk [ ]}

\begin{tabular}{lccc}
\hline Diet 1 & -- & -- & -- \\
Diet 2 & -- & -- & -- \\
Diet 3 & 1.00011 & 1.00082 & 1.009 \\
Diet 4 & -- & -- & -- \\
\hline
\end{tabular}

\begin{tabular}{lccc}
\hline Diet 1 & -- & -- & -- \\
Diet 2 & -- & -- & -- \\
Diet 3 & 0.011 & 0.082 & - \\
Diet 4 & -- & -- & - \\
\hline Diet 1 - Backyard cow milk + all other locally produced non-milk exposure pathways \\
Diet 2 - Locally produced commercial milk + all other locally produced non-milk exposure pathways \\
Diet 3 - Regionally mixed commercial milk + inhalation (other regionally mixed food items are minor contributors to the total) \\
Diet 4 - Goat milk (all other exposure pathways are of negligible importance compared to goat milk)
\end{tabular}


Location: Woodland

Receptor: Male born in 1930

\begin{tabular}{lccc}
\hline & \multicolumn{3}{c}{ Thyroid Dose [cGy] } \\
Exposure Pathway & \multicolumn{2}{c}{ 95\% Subjective Confidence Interval } \\
\hline Backyard Cow Milk & lower limit & central estimate & upper limit \\
Commercial Milk (locally produced) & -- & -- & -- \\
Commercial Milk (regionally mixed) & -- & -- & -- \\
Goat Milk (locally produced) & 0.056 & 0.3 & 2 \\
Beef (locally produced) & -- & -- & -- \\
Leafy Vegetables (locally produced) & -- & -- & -- \\
Eggs (locally produced) & -- & -- & -- \\
Cottage Cheese (locally produced) & -- & -- & -- \\
Inhalation & 0.0016 & 0.017 & 0.17 \\
Mother's milk (mother on Diet 3) & 0.038 & 0.13 & 0.45 \\
Prenatal exposure (mother on Diet 3) & -- & -- & -- \\
Diet 1 & -- & -- & -- \\
Diet 2 & -- & -- & -- \\
Diet 3 & -- & -- & -- \\
\hline
\end{tabular}

Excess Lifetime Risk [ ]

\begin{tabular}{lccc}
\hline Diet 1 & -- & -- & -- \\
Diet 2 & -- & -- & -- \\
Diet 3 & $2.6 \mathrm{E}-08$ & $9.4 \mathrm{E}-07$ & $2.8 \mathrm{E}-05$ \\
Diet 4 & -- & -- & -- \\
\hline
\end{tabular}

\section{Relative Risk [ ]}

\begin{tabular}{lccc}
\hline Diet 1 & -- & -- & -- \\
Diet 2 & -- & -- & -- \\
Diet 3 & 1.000035 & 1.00062 & 1.014 \\
Diet 4 & -- & -- & -- \\
\hline
\end{tabular}

\begin{tabular}{lccc}
\hline Diet 1 & -- & -- & -- \\
Diet 2 & -- & -- & -- \\
Diet 3 & 0.003 & 0.062 & -38 \\
Diet 4 & -- & -- & - \\
\hline Diet 1 - Backyard cow milk + all other locally produced non-milk exposure pathways \\
Diet 2 - Locally produced commercial milk + all other locally produced non-milk exposure pathways \\
Diet 3 - Regionally mixed commercial milk + inhalation (other regionally mixed food items are minor contributors to the total) \\
Diet 4 - Goat milk (all other exposure pathways are of negligible importance compared to goat milk)
\end{tabular}


Location: Hardin Valley

Receptor: Female born in 1930

\begin{tabular}{lccc}
\hline & \multicolumn{3}{c}{ Thyroid Dose [cGy] } \\
Exposure Pathway & $95 \%$ Subjective Confidence Interval \\
\hline Backyard Cow Milk & lower limit & central estimate & upper limit \\
Commercial Milk (locally produced) & 0.92 & 5.1 & 28 \\
Commercial Milk (regionally mixed) & 0.19 & 1.2 & 7.6 \\
Goat Milk (locally produced) & 0.043 & 0.25 & 1.5 \\
Beef (locally produced) & 1.9 & 13 & 90 \\
Leafy Vegetables (locally produced) & 0.0026 & 0.04 & 0.79 \\
Eggs (locally produced) & 0.001 & 0.011 & 0.088 \\
Cottage Cheese (locally produced) & 0.061 & 0.42 & 2.6 \\
Inhalation & 0.0023 & 0.025 & 0.26 \\
Mother's milk (mother on Diet 1) & 0.047 & 0.15 & 0.55 \\
Prenatal exposure (mother on Diet 1) & -- & -- & -- \\
Diet 1 & -- & -- & -- \\
Diet 2 & 1.1 & 5.8 & 33 \\
Diet 3 & 0.38 & 2 & 11 \\
\hline
\end{tabular}

Excess Lifetime Risk [ ]

\begin{tabular}{lllc}
\hline Diet 1 & $2.8 \mathrm{E}-06$ & $4.2 \mathrm{E}-05$ & $5.4 \mathrm{E}-04$ \\
Diet 2 & $1.1 \mathrm{E}-06$ & $1.5 \mathrm{E}-05$ & $2.0 \mathrm{E}-04$ \\
Diet 3 & $3.2 \mathrm{E}-07$ & $3.3 \mathrm{E}-06$ & $3.6 \mathrm{E}-05$ \\
Diet 4 & $7.2 \mathrm{E}-06$ & $1.1 \mathrm{E}-04$ & $1.5 \mathrm{E}-03$ \\
\hline
\end{tabular}

Relative Risk [ ]

\begin{tabular}{lccc}
\hline Diet 1 & 1.0012 & 1.012 & 1.12 \\
Diet 2 & 1.00051 & 1.0042 & 1.052 \\
Diet 3 & 1.00012 & 1.00093 & 1.0096 \\
Diet 4 & 1.0031 & 1.027 & 1.4 \\
\hline
\end{tabular}

Probability of Causation [\%]

\begin{tabular}{lccc}
\hline Diet 1 & 0.122 & 1.170 & 11.06 \\
Diet 2 & 0.051 & 0.415 & 4.90 \\
Diet 3 & 0.012 & 0.093 & 0.95 \\
Diet 4 & 0.313 & 2.588 & 28.73 \\
\hline
\end{tabular}

Diet 1 - Backyard cow milk + all other locally produced non-milk exposure pathways

Diet 2 - Locally produced commercial milk + all other locally produced non-milk exposure pathways

Diet 3 - Regionally mixed commercial milk + inhalation (other regionally mixed food items are minor contributors to the total)

Diet 4 - Goat milk (all other exposure pathways are of negligible importance compared to goat milk) 
Location: Hardin Valley

Receptor: Male born in 1930

\begin{tabular}{lccc}
\hline & \multicolumn{3}{c}{ Thyroid Dose [cGy] } \\
& \multicolumn{2}{c}{$95 \%$ Subjective Confidence Interval } \\
Exposure Pathway & lower limit & central estimate & upper limit \\
\hline Backyard Cow Milk & 1.1 & 6.2 & 34 \\
Commercial Milk (locally produced) & 0.25 & 1.4 & 9.2 \\
Commercial Milk (regionally mixed) & 0.056 & 0.3 & 2 \\
Goat Milk (locally produced) & 2.5 & 18 & 110 \\
Beef (locally produced) & 0.0039 & 0.063 & 1.3 \\
Leafy Vegetables (locally produced) & 0.00099 & 0.011 & 0.089 \\
Eggs (locally produced) & 0.065 & 0.45 & 3 \\
Cottage Cheese (locally produced) & 0.0023 & 0.023 & 0.25 \\
Inhalation & 0.055 & 0.19 & 0.63 \\
Mother's milk (mother on Diet 1) & -- & -- & - \\
Prenatal exposure (mother on Diet 1) & -- & -- & - \\
Diet 1 & 1.3 & 7.1 & 39 \\
Diet 2 & 0.48 & 2.3 & 14 \\
Diet 3 & 0.14 & 0.53 & 2.3 \\
\hline
\end{tabular}

Excess Lifetime Risk [ ]

\begin{tabular}{llll}
\hline Diet 1 & $2.9 \mathrm{E}-07$ & $1.4 \mathrm{E}-05$ & $3.7 \mathrm{E}-04$ \\
Diet 2 & $1.1 \mathrm{E}-07$ & $5.0 \mathrm{E}-06$ & $1.5 \mathrm{E}-04$ \\
Diet 3 & $3.0 \mathrm{E}-08$ & $1.1 \mathrm{E}-06$ & $3.0 \mathrm{E}-05$ \\
Diet 4 & $8.6 \mathrm{E}-07$ & $3.2 \mathrm{E}-05$ & $1.0 \mathrm{E}-03$ \\
\hline
\end{tabular}

Relative Risk [ ]

\begin{tabular}{lccc}
\hline Diet 1 & 1.00051 & 1.009 & 1.24 \\
Diet 2 & 1.00016 & 1.0033 & 1.075 \\
Diet 3 & 1.00004 & 1.00071 & 1.015 \\
Diet 4 & 1.00088 & 1.025 & 1.62 \\
\hline
\end{tabular}

\section{Probability of Causation [\%]}

\begin{tabular}{lccc}
\hline Diet 1 & 0.050 & 0.894 & 19.53 \\
Diet 2 & 0.016 & 0.325 & 6.94 \\
Diet 3 & 0.004 & 0.070 & 1.51 \\
Diet 4 & 0.088 & 2.407 & 38.37 \\
\hline
\end{tabular}

Diet 1 - Backyard cow milk + all other locally produced non-milk exposure pathways

Diet 2 - Locally produced commercial milk + all other locally produced non-milk exposure pathways

Diet 3 - Regionally mixed commercial milk + inhalation (other regionally mixed food items are minor contributors to the total)

Diet 4 - Goat milk (all other exposure pathways are of negligible importance compared to goat milk) 


\section{Location: Oliver Springs}

Receptor: Female born in 1930

\begin{tabular}{lccc}
\hline & \multicolumn{3}{c}{ Thyroid Dose [cGy] } \\
& \multicolumn{2}{c}{$95 \%$ Subjective Confidence Interval } \\
Exposure Pathway & lower limit & central estimate & upper limit \\
\hline Backyard Cow Milk & 0.13 & 0.77 & 4.9 \\
Commercial Milk (locally produced) & 0.028 & 0.18 & 1.3 \\
Commercial Milk (regionally mixed) & 0.042 & 0.25 & 1.5 \\
Goat Milk (locally produced) & 0.25 & 2 & 15 \\
Beef (locally produced) & 0.00038 & 0.0064 & 0.14 \\
Leafy Vegetables (locally produced) & 0.00014 & 0.0017 & 0.016 \\
Eggs (locally produced) & 0.009 & 0.065 & 0.45 \\
Cottage Cheese (locally produced) & 0.00032 & 0.0038 & 0.046 \\
Inhalation & 0.0072 & 0.024 & 0.093 \\
Mother's milk (mother on Diet 1) & -- & -- & -- \\
Prenatal exposure (mother on Diet 1) & -- & -- & - \\
Diet 1 & 0.15 & 0.89 & 5.5 \\
Diet 2 & 0.059 & 0.31 & 1.8 \\
Diet 3 & 0.056 & 0.28 & 1.6 \\
\hline
\end{tabular}

Excess Lifetime Risk [ ]

\begin{tabular}{llll}
\hline Diet 1 & $4.0 \mathrm{E}-07$ & $6.4 \mathrm{E}-06$ & $9.9 \mathrm{E}-05$ \\
Diet 2 & $1.8 \mathrm{E}-07$ & $2.3 \mathrm{E}-06$ & $3.3 \mathrm{E}-05$ \\
Diet 3 & $1.7 \mathrm{E}-07$ & $2.2 \mathrm{E}-06$ & $3.0 \mathrm{E}-05$ \\
Diet 4 & $1.0 \mathrm{E}-06$ & $1.6 \mathrm{E}-05$ & $2.7 \mathrm{E}-04$ \\
\hline
\end{tabular}

Relative Risk [ ]

\begin{tabular}{lccc}
\hline Diet 1 & 1.00017 & 1.0018 & 1.022 \\
Diet 2 & 1.000067 & 1.00064 & 1.009 \\
Diet 3 & 1.000075 & 1.00064 & 1.0079 \\
Diet 4 & 1.00042 & 1.0043 & 1.069 \\
\hline
\end{tabular}

\begin{tabular}{llcc} 
& \multicolumn{3}{c}{ Probability of Causation [\%] } \\
\hline Diet 1 & 0.017 & 0.179 & 2.17 \\
Diet 2 & 0.007 & 0.064 & 0.89 \\
Diet 3 & 0.007 & 0.064 & 0.79 \\
Diet 4 & 0.042 & 0.433 & 6.42 \\
\hline
\end{tabular}

Diet 1 - Backyard cow milk + all other locally produced non-milk exposure pathways

Diet 2 - Locally produced commercial milk + all other locally produced non-milk exposure pathways

Diet 3 - Regionally mixed commercial milk + inhalation (other regionally mixed food items are minor contributors to the total)

Diet 4 - Goat milk (all other exposure pathways are of negligible importance compared to goat milk) 


\section{Location: Oliver Springs}

\section{Receptor: Male born in 1930}

\begin{tabular}{lccc}
\hline & \multicolumn{3}{c}{ Thyroid Dose [cGy] } \\
& \multicolumn{2}{c}{$95 \%$ Subjective Confidence Interval } \\
Exposure Pathway & lower limit & central estimate & upper limit \\
\hline Backyard Cow Milk & 0.16 & 0.93 & 5.7 \\
Commercial Milk (locally produced) & 0.037 & 0.22 & 1.6 \\
Commercial Milk (regionally mixed) & 0.056 & 0.3 & 2 \\
Goat Milk (locally produced) & 0.34 & 2.6 & 21 \\
Beef (locally produced) & 0.00057 & 0.01 & 0.21 \\
Leafy Vegetables (locally produced) & 0.00015 & 0.0016 & 0.015 \\
Eggs (locally produced) & 0.01 & 0.07 & 0.5 \\
Cottage Cheese (locally produced) & 0.00032 & 0.0038 & 0.041 \\
Inhalation & 0.0084 & 0.029 & 0.11 \\
Mother's milk (mother on Diet 1) & -- & -- & - \\
Prenatal exposure (mother on Diet 1) & -- & -- & -- \\
Diet 1 & 0.19 & 1.1 & 6.4 \\
Diet 2 & 0.069 & 0.36 & 2.3 \\
Diet 3 & 0.069 & 0.33 & 2 \\
\hline
\end{tabular}

Excess Lifetime Risk [ ]

\begin{tabular}{llll}
\hline Diet 1 & $4.2 \mathrm{E}-08$ & $2.1 \mathrm{E}-06$ & $6.1 \mathrm{E}-05$ \\
Diet 2 & $1.6 \mathrm{E}-08$ & $7.5 \mathrm{E}-07$ & $2.3 \mathrm{E}-05$ \\
Diet 3 & $1.8 \mathrm{E}-08$ & $7.2 \mathrm{E}-07$ & $2.4 \mathrm{E}-05$ \\
Diet 4 & $1.2 \mathrm{E}-07$ & $5.1 \mathrm{E}-06$ & $1.7 \mathrm{E}-04$ \\
\hline
\end{tabular}

\section{Relative Risk [ ]}

\begin{tabular}{lccc}
\hline Diet 1 & 1.000071 & 1.0015 & 1.039 \\
Diet 2 & 1.000024 & 1.00053 & 1.013 \\
Diet 3 & 1.000023 & 1.00048 & 1.011 \\
Diet 4 & 1.00013 & 1.0038 & 1.1 \\
\hline
\end{tabular}

\begin{tabular}{llcc} 
& \multicolumn{3}{c}{ Probability of Causation [\%] } \\
\hline Diet 1 & 0.007 & 0.147 & 3.70 \\
Diet 2 & 0.002 & 0.053 & 1.27 \\
Diet 3 & 0.002 & 0.048 & 1.12 \\
Diet 4 & 0.013 & 0.383 & 9.36 \\
\hline
\end{tabular}

Diet 1 - Backyard cow milk + all other locally produced non-milk exposure pathways

Diet 2 - Locally produced commercial milk + all other locally produced non-milk exposure pathways

Diet 3 - Regionally mixed commercial milk + inhalation (other regionally mixed food items are minor contributors to the total)

Diet 4 - Goat milk (all other exposure pathways are of negligible importance compared to goat milk) 


\section{Location: Solway}

Receptor: Female born in 1930

\begin{tabular}{lccc}
\hline & \multicolumn{3}{c}{ Thyroid Dose [cGy] } \\
& \multicolumn{2}{c}{$95 \%$ Subjective Confidence Interval } \\
Exposure Pathway & lower limit & central estimate & upper limit \\
\hline Backyard Cow Milk & 0.87 & 4.6 & 26 \\
Commercial Milk (locally produced) & 0.17 & 1.1 & 6.8 \\
Commercial Milk (regionally mixed) & 0.042 & 0.25 & 1.5 \\
Goat Milk (locally produced) & 1.6 & 12 & 80 \\
Beef (locally produced) & 0.0022 & 0.038 & 0.71 \\
Leafy Vegetables (locally produced) & 0.00093 & 0.0099 & 0.082 \\
Eggs (locally produced) & 0.059 & 0.38 & 2.3 \\
Cottage Cheese (locally produced) & 0.0021 & 0.022 & 0.24 \\
Inhalation & 0.045 & 0.14 & 0.49 \\
Mother's milk (mother on Diet 1) & -- & -- & -- \\
Prenatal exposure (mother on Diet 1) & -- & -- & -- \\
Diet 1 & 1 & 5.3 & 30 \\
Diet 2 & 0.36 & 1.7 & 10 \\
Diet 3 & 0.11 & 0.41 & 2 \\
\hline
\end{tabular}

Excess Lifetime Risk [ ]

\begin{tabular}{llll}
\hline Diet 1 & $2.5 \mathrm{E}-06$ & $4.0 \mathrm{E}-05$ & $5.2 \mathrm{E}-04$ \\
Diet 2 & $1.0 \mathrm{E}-06$ & $1.4 \mathrm{E}-05$ & $1.8 \mathrm{E}-04$ \\
Diet 3 & $3.0 \mathrm{E}-07$ & $3.2 \mathrm{E}-06$ & $3.5 \mathrm{E}-05$ \\
Diet 4 & $6.6 \mathrm{E}-06$ & $9.6 \mathrm{E}-05$ & $1.4 \mathrm{E}-03$ \\
\hline
\end{tabular}

Relative Risk [ ]

\begin{tabular}{lccc}
\hline Diet 1 & 1.0011 & 1.011 & 1.12 \\
Diet 2 & 1.00044 & 1.0039 & 1.048 \\
Diet 3 & 1.00012 & 1.00091 & 1.0097 \\
Diet 4 & 1.0028 & 1.025 & 1.37 \\
\hline
\end{tabular}

Probability of Causation [\%]

\begin{tabular}{lccc}
\hline Diet 1 & 0.109 & 1.064 & 10.47 \\
Diet 2 & 0.044 & 0.388 & 4.55 \\
Diet 3 & 0.012 & 0.091 & 0.96 \\
Diet 4 & 0.283 & 2.437 & 27.14 \\
\hline
\end{tabular}

Diet 1 - Backyard cow milk + all other locally produced non-milk exposure pathways

Diet 2 - Locally produced commercial milk + all other locally produced non-milk exposure pathways

Diet 3 - Regionally mixed commercial milk + inhalation (other regionally mixed food items are minor contributors to the total)

Diet 4 - Goat milk (all other exposure pathways are of negligible importance compared to goat milk) 


\section{Location: Solway}

\section{Receptor: Male born in 1930}

\begin{tabular}{lccc}
\hline & \multicolumn{3}{c}{ Thyroid Dose [cGy] } \\
& \multicolumn{2}{c}{$95 \%$ Subjective Confidence Interval } \\
Exposure Pathway & 1.1 & 5.7 & 31 \\
\hline Backyard Cow Milk & 0.22 & 1.3 & 8.4 \\
Commercial Milk (locally produced) & 0.056 & 0.3 & 2 \\
Commercial Milk (regionally mixed) & 2.2 & 16 & 99 \\
Goat Milk (locally produced) & 0.0033 & 0.059 & 1.2 \\
Beef (locally produced) & 0.00095 & 0.0097 & 0.082 \\
Leafy Vegetables (locally produced) & 0.06 & 0.42 & 2.5 \\
Eggs (locally produced) & 0.002 & 0.022 & 0.22 \\
Cottage Cheese (locally produced) & 0.052 & 0.17 & 0.57 \\
Inhalation & -- & -- & -- \\
Mother's milk (mother on Diet 1) & -- & -- & -- \\
Prenatal exposure (mother on Diet 1) & 1.3 & 6.5 & 36 \\
Diet 1 & 0.45 & 2.1 & 12 \\
Diet 2 & 0.13 & 0.51 & 2.3 \\
Diet 3 & & upper limit \\
\hline
\end{tabular}

Excess Lifetime Risk [ ]

\begin{tabular}{llll}
\hline Diet 1 & $2.9 \mathrm{E}-07$ & $1.2 \mathrm{E}-05$ & $3.5 \mathrm{E}-04$ \\
Diet 2 & $1.0 \mathrm{E}-07$ & $4.4 \mathrm{E}-06$ & $1.5 \mathrm{E}-04$ \\
Diet 3 & $2.9 \mathrm{E}-08$ & $1.0 \mathrm{E}-06$ & $2.9 \mathrm{E}-05$ \\
Diet 4 & $7.6 \mathrm{E}-07$ & $2.8 \mathrm{E}-05$ & $9.9 \mathrm{E}-04$ \\
\hline
\end{tabular}

\section{Relative Risk [ ]}

\begin{tabular}{lccc}
\hline Diet 1 & 1.00041 & 1.0084 & 1.21 \\
Diet 2 & 1.00014 & 1.003 & 1.065 \\
Diet 3 & 1.00004 & 1.0007 & 1.015 \\
Diet 4 & 1.00076 & 1.023 & 1.58 \\
\hline
\end{tabular}

\section{Probability of Causation [\%]}

\begin{tabular}{lccc}
\hline Diet 1 & 0.041 & 0.834 & 17.56 \\
Diet 2 & 0.014 & 0.296 & 6.06 \\
Diet 3 & 0.004 & 0.070 & 1.48 \\
Diet 4 & 0.076 & 2.272 & 36.61 \\
\hline
\end{tabular}

Diet 1 - Backyard cow milk + all other locally produced non-milk exposure pathways

Diet 2 - Locally produced commercial milk + all other locally produced non-milk exposure pathways

Diet 3 - Regionally mixed commercial milk + inhalation (other regionally mixed food items are minor contributors to the total)

Diet 4 - Goat milk (all other exposure pathways are of negligible importance compared to goat milk) 
Location: Sugar Grove

Receptor: Female born in 1930

\begin{tabular}{lccc}
\hline & \multicolumn{3}{c}{ Thyroid Dose [cGy] } \\
Exposure Pathway & $95 \%$ & Subjective Confidence Interval \\
Backyard Cow Milk & lower limit & central estimate & upper limit \\
Commercial Milk (locally produced) & 0.35 & 1.9 & 11 \\
Commercial Milk (regionally mixed) & 0.072 & 0.45 & 3 \\
Goat Milk (locally produced) & 0.043 & 0.25 & 1.5 \\
Beef (locally produced) & 0.71 & 4.9 & 36 \\
Leafy Vegetables (locally produced) & 0.00099 & 0.016 & 0.31 \\
Eggs (locally produced) & 0.00038 & 0.0041 & 0.035 \\
Cottage Cheese (locally produced) & 0.024 & 0.16 & 1 \\
Inhalation & 0.00088 & 0.0095 & 0.1 \\
Mother's milk (mother on Diet 1) & 0.018 & 0.059 & 0.21 \\
Prenatal exposure (mother on Diet 1) & -- & -- & -- \\
Diet 1 & -- & -- & -- \\
Diet 2 & 0.42 & 2.2 & 13 \\
Diet 3 & 0.15 & 0.74 & 4.6 \\
\hline
\end{tabular}

Excess Lifetime Risk [ ]

\begin{tabular}{lllc}
\hline Diet 1 & $1.1 \mathrm{E}-06$ & $1.6 \mathrm{E}-05$ & $2.3 \mathrm{E}-04$ \\
Diet 2 & $4.3 \mathrm{E}-07$ & $6.0 \mathrm{E}-06$ & $7.5 \mathrm{E}-05$ \\
Diet 3 & $2.1 \mathrm{E}-07$ & $2.5 \mathrm{E}-06$ & $3.1 \mathrm{E}-05$ \\
Diet 4 & $2.8 \mathrm{E}-06$ & $4.0 \mathrm{E}-05$ & $5.8 \mathrm{E}-04$ \\
\hline
\end{tabular}

Relative Risk [ ]

\begin{tabular}{lccc}
\hline Diet 1 & 1.00047 & 1.0045 & 1.052 \\
Diet 2 & 1.00019 & 1.0016 & 1.021 \\
Diet 3 & 1.000089 & 1.00072 & 1.0084 \\
Diet 4 & 1.0012 & 1.01 & 1.16 \\
\hline
\end{tabular}

\begin{tabular}{lccc}
\hline Diet 1 & 0.047 & 0.445 & 4.89 \\
Diet 2 & 0.019 & 0.161 & 2.02 \\
Diet 3 & 0.009 & 0.072 & 0.83 \\
Diet 4 & 0.115 & 1.011 & 13.88 \\
\hline
\end{tabular}

Diet 1 - Backyard cow milk + all other locally produced non-milk exposure pathways

Diet 2 - Locally produced commercial milk + all other locally produced non-milk exposure pathways

Diet 3 - Regionally mixed commercial milk + inhalation (other regionally mixed food items are minor contributors to the total)

Diet 4 - Goat milk (all other exposure pathways are of negligible importance compared to goat milk) 
Location: Sugar Grove

Receptor: Male born in 1930

\begin{tabular}{lccc}
\hline & \multicolumn{3}{c}{ Thyroid Dose [cGy] } \\
& \multicolumn{2}{c}{$95 \%$ Subjective Confidence Interval } \\
Exposure Pathway & lower limit & central estimate & upper limit \\
\hline Backyard Cow Milk & 0.44 & 2.4 & 14 \\
Commercial Milk (locally produced) & 0.093 & 0.54 & 3.7 \\
Commercial Milk (regionally mixed) & 0.056 & 0.3 & 2 \\
Goat Milk (locally produced) & 0.93 & 6.7 & 43 \\
Beef (locally produced) & 0.0015 & 0.025 & 0.51 \\
Leafy Vegetables (locally produced) & 0.00038 & 0.0041 & 0.036 \\
Eggs (locally produced) & 0.025 & 0.18 & 1.1 \\
Cottage Cheese (locally produced) & 0.00083 & 0.0092 & 0.098 \\
Inhalation & 0.021 & 0.073 & 0.25 \\
Mother's milk (mother on Diet 1) & -- & -- & -- \\
Prenatal exposure (mother on Diet 1) & -- & -- & -- \\
Diet 1 & 0.51 & 2.7 & 16 \\
Diet 2 & 0.19 & 0.89 & 5.5 \\
Diet 3 & 0.088 & 0.38 & 2.1 \\
\hline
\end{tabular}

Excess Lifetime Risk [ ]

\begin{tabular}{llcc}
\hline Diet 1 & $1.1 \mathrm{E}-07$ & $5.2 \mathrm{E}-06$ & $1.5 \mathrm{E}-04$ \\
Diet 2 & $4.3 \mathrm{E}-08$ & $1.8 \mathrm{E}-06$ & $6.0 \mathrm{E}-05$ \\
Diet 3 & $2.2 \mathrm{E}-08$ & $8.1 \mathrm{E}-07$ & $2.6 \mathrm{E}-05$ \\
Diet 4 & $3.4 \mathrm{E}-07$ & $1.2 \mathrm{E}-05$ & $4.1 \mathrm{E}-04$ \\
\hline
\end{tabular}

Relative Risk [ ]

\begin{tabular}{lccc}
\hline Diet 1 & 1.00018 & 1.0037 & 1.097 \\
Diet 2 & 1.000057 & 1.0013 & 1.03 \\
Diet 3 & 1.000029 & 1.00054 & 1.012 \\
Diet 4 & 1.00032 & 1.0098 & 1.23 \\
\hline
\end{tabular}

\section{Probability of Causation [\%]}

\begin{tabular}{lccc}
\hline Diet 1 & 0.018 & 0.366 & 8.72 \\
Diet 2 & 0.006 & 0.126 & 2.91 \\
Diet 3 & 0.003 & 0.054 & 1.19 \\
Diet 4 & 0.032 & 0.967 & 18.51 \\
\hline
\end{tabular}

Diet 1 - Backyard cow milk + all other locally produced non-milk exposure pathways

Diet 2 - Locally produced commercial milk + all other locally produced non-milk exposure pathways

Diet 3 - Regionally mixed commercial milk + inhalation (other regionally mixed food items are minor contributors to the total)

Diet 4 - Goat milk (all other exposure pathways are of negligible importance compared to goat milk) 


\section{Location: OR Townsite}

Receptor: Female born in 1930

\begin{tabular}{lccc}
\hline & \multicolumn{3}{c}{ Thyroid Dose [cGy] } \\
Exposure Pathway & \multicolumn{2}{c}{$9 \%$ Subjective Confidence Interval } \\
\hline Backyard Cow Milk & lower limit & central estimate & upper limit \\
Commercial Milk (locally produced) & -- & -- & -- \\
Commercial Milk (regionally mixed) & -- & -- & -- \\
Goat Milk (locally produced) & 0.042 & 0.25 & 1.5 \\
Beef (locally produced) & -- & -- & -- \\
Leafy Vegetables (locally produced) & -- & -- & -- \\
Eggs (locally produced) & -- & -- & -- \\
Cottage Cheese (locally produced) & -- & -- & -- \\
Inhalation & 0.00093 & 0.01 & 0.11 \\
Mother's milk (mother on Diet 3) & 0.02 & 0.065 & 0.23 \\
Prenatal exposure (mother on Diet 3) & -- & -- & -- \\
Diet 1 & -- & -- & -- \\
Diet 2 & -- & -- & -- \\
Diet 3 & -- & -- & -- \\
\hline
\end{tabular}

Excess Lifetime Risk [ ]

\begin{tabular}{lccc}
\hline Diet 1 & -- & -- & -- \\
Diet 2 & -- & -- & -- \\
Diet 3 & $2.2 \mathrm{E}-07$ & $2.6 \mathrm{E}-06$ & $3.2 \mathrm{E}-05$ \\
Diet 4 & -- & -- & -- \\
\hline
\end{tabular}

\section{Relative Risk [ ]}

\begin{tabular}{lccc}
\hline Diet 1 & -- & -- & -- \\
Diet 2 & -- & -- & -- \\
Diet 3 & 1.00009 & 1.00073 & 1.0083 \\
Diet 4 & -- & -- & -- \\
\hline
\end{tabular}

\begin{tabular}{lccc}
\hline Diet 1 & -- & -- & -- \\
Diet 2 & -- & -- & -- \\
Diet 3 & 0.009 & 0.073 & 0.83 \\
Diet 4 & -- & -- & - \\
\hline Diet 1 - Backyard cow milk + all other locally produced non-milk exposure pathways & \\
Diet 2 - Locally produced commercial milk + all other locally produced non-milk exposure pathways \\
Diet 3 - Regionally mixed commercial milk + inhalation (other regionally mixed food items are minor contributors to the total) \\
Diet 4 - Goat milk (all other exposure pathways are of negligible importance compared to goat milk)
\end{tabular}




\section{Location: OR Townsite}

\section{Receptor: Male born in 1930}

\begin{tabular}{lccc}
\hline & \multicolumn{3}{c}{ Thyroid Dose [cGy] } \\
Exposure Pathway & \multicolumn{2}{c}{$9 \%$ Subjective Confidence Interval } \\
\hline Backyard Cow Milk & lower limit & central estimate & upper limit \\
Commercial Milk (locally produced) & -- & -- & -- \\
Commercial Milk (regionally mixed) & -- & -- & -- \\
Goat Milk (locally produced) & 0.056 & 0.3 & 2 \\
Beef (locally produced) & -- & -- & -- \\
Leafy Vegetables (locally produced) & -- & -- & -- \\
Eggs (locally produced) & -- & -- & -- \\
Cottage Cheese (locally produced) & -- & -- & -- \\
Inhalation & 0.00093 & 0.0099 & 0.11 \\
Mother's milk (mother on Diet 3) & 0.023 & 0.079 & 0.27 \\
Prenatal exposure (mother on Diet 3) & -- & -- & -- \\
Diet 1 & -- & -- & -- \\
Diet 2 & -- & -- & -- \\
Diet 3 & -- & -- & -- \\
\hline
\end{tabular}

Excess Lifetime Risk [ ]

\begin{tabular}{lccc}
\hline Diet 1 & -- & -- & -- \\
Diet 2 & -- & -- & -- \\
Diet 3 & $2.2 \mathrm{E}-08$ & $8.3 \mathrm{E}-07$ & $2.6 \mathrm{E}-05$ \\
Diet 4 & -- & -- & -- \\
\hline
\end{tabular}

\section{Relative Risk [ ]}

\begin{tabular}{lccc}
\hline Diet 1 & -- & -- & -- \\
Diet 2 & -- & -- & -- \\
Diet 3 & 1.000029 & 1.00055 & 1.012 \\
Diet 4 & -- & -- & -- \\
\hline
\end{tabular}

\begin{tabular}{lccc}
\hline Diet 1 & -- & -- & -- \\
Diet 2 & -- & -- & -- \\
Diet 3 & 0.003 & 0.055 & 1.22 \\
Diet 4 & -- & -- & - \\
\hline Diet 1 - Backyard cow milk + all other locally produced non-milk exposure pathways & \\
Diet 2 - Locally produced commercial milk + all other locally produced non-milk exposure pathways \\
Diet 3 - Regionally mixed commercial milk + inhalation (other regionally mixed food items are minor contributors to the total) \\
Diet 4 - Goat milk (all other exposure pathways are of negligible importance compared to goat milk)
\end{tabular}


Location: Hines Valley

Receptor: Female born in 1930

\begin{tabular}{lccc}
\hline & \multicolumn{3}{c}{ Thyroid Dose [cGy] } \\
Exposure Pathway & $95 \%$ Subjective Confidence Interval \\
\hline Backyard Cow Milk & lower limit & central estimate & upper limit \\
Commercial Milk (locally produced) & 0.44 & 2.3 & 14 \\
Commercial Milk (regionally mixed) & 0.081 & 0.53 & 3.5 \\
Goat Milk (locally produced) & 0.043 & 0.25 & 1.5 \\
Beef (locally produced) & 0.82 & 5.9 & 41 \\
Leafy Vegetables (locally produced) & 0.0011 & 0.019 & 0.41 \\
Eggs (locally produced) & 0.00051 & 0.0049 & 0.043 \\
Cottage Cheese (locally produced) & 0.027 & 0.19 & 1.2 \\
Inhalation & 0.0011 & 0.011 & 0.13 \\
Mother's milk (mother on Diet 1) & 0.023 & 0.078 & 0.26 \\
Prenatal exposure (mother on Diet 1) & -- & -- & -- \\
Diet 1 & -- & -- & -- \\
Diet 2 & 0.52 & 2.7 & 15 \\
Diet 3 & 0.18 & 0.89 & 4.8 \\
\hline
\end{tabular}

Excess Lifetime Risk [ ]

\begin{tabular}{lllc}
\hline Diet 1 & $1.3 \mathrm{E}-06$ & $1.9 \mathrm{E}-05$ & $2.8 \mathrm{E}-04$ \\
Diet 2 & $5.4 \mathrm{E}-07$ & $7.1 \mathrm{E}-06$ & $9.1 \mathrm{E}-05$ \\
Diet 3 & $2.2 \mathrm{E}-07$ & $2.6 \mathrm{E}-06$ & $3.2 \mathrm{E}-05$ \\
Diet 4 & $3.4 \mathrm{E}-06$ & $4.9 \mathrm{E}-05$ & $6.7 \mathrm{E}-04$ \\
\hline
\end{tabular}

Relative Risk [ ]

\begin{tabular}{lccc}
\hline Diet 1 & 1.00053 & 1.0056 & 1.055 \\
Diet 2 & 1.00021 & 1.002 & 1.024 \\
Diet 3 & 1.000094 & 1.00077 & 1.0084 \\
Diet 4 & 1.0013 & 1.013 & 1.18 \\
\hline
\end{tabular}

\section{Probability of Causation [\%]}

\begin{tabular}{llcc}
\hline Diet 1 & 0.053 & 0.556 & 5.21 \\
Diet 2 & 0.021 & 0.199 & 2.30 \\
Diet 3 & 0.009 & 0.077 & 0.84 \\
Diet 4 & 0.130 & 1.272 & 15.28 \\
\hline
\end{tabular}

Diet 1 - Backyard cow milk + all other locally produced non-milk exposure pathways

Diet 2 - Locally produced commercial milk + all other locally produced non-milk exposure pathways

Diet 3 - Regionally mixed commercial milk + inhalation (other regionally mixed food items are minor contributors to the total)

Diet 4 - Goat milk (all other exposure pathways are of negligible importance compared to goat milk) 
Location: Hines Valley

Receptor: Male born in 1930

\begin{tabular}{lccc}
\hline & \multicolumn{3}{c}{ Thyroid Dose [cGy] } \\
Exposure Pathway & $95 \%$ & Subjective Confidence Interval \\
Backyard Cow Milk & lower limit & central estimate & upper limit \\
Commercial Milk (locally produced) & 0.55 & 2.9 & 16 \\
Commercial Milk (regionally mixed) & 0.11 & 0.65 & 4.2 \\
Goat Milk (locally produced) & 0.056 & 0.3 & 2 \\
Beef (locally produced) & 1.1 & 8 & 50 \\
Leafy Vegetables (locally produced) & 0.0017 & 0.031 & 0.66 \\
Eggs (locally produced) & 0.00048 & 0.0048 & 0.046 \\
Cottage Cheese (locally produced) & 0.028 & 0.21 & 1.3 \\
Inhalation & 0.001 & 0.012 & 0.13 \\
Mother's milk (mother on Diet 1) & 0.027 & 0.094 & 0.32 \\
Prenatal exposure (mother on Diet 1) & -- & -- & -- \\
Diet 1 & -- & -- & -- \\
Diet 2 & 0.65 & 3.3 & 18 \\
Diet 3 & 0.22 & 1.1 & 6 \\
\hline
\end{tabular}

Excess Lifetime Risk [ ]

\begin{tabular}{lllc}
\hline Diet 1 & $1.3 \mathrm{E}-07$ & $6.3 \mathrm{E}-06$ & $2.1 \mathrm{E}-04$ \\
Diet 2 & $5.6 \mathrm{E}-08$ & $2.3 \mathrm{E}-06$ & $7.3 \mathrm{E}-05$ \\
Diet 3 & $2.3 \mathrm{E}-08$ & $8.5 \mathrm{E}-07$ & $2.6 \mathrm{E}-05$ \\
Diet 4 & $4.2 \mathrm{E}-07$ & $1.5 \mathrm{E}-05$ & $4.7 \mathrm{E}-04$ \\
\hline
\end{tabular}

\section{Relative Risk [ ]}

\begin{tabular}{lccc}
\hline Diet 1 & 1.0002 & 1.0044 & 1.11 \\
Diet 2 & 1.000069 & 1.0016 & 1.032 \\
Diet 3 & 1.000031 & 1.00057 & 1.013 \\
Diet 4 & 1.00038 & 1.011 & 1.29 \\
\hline
\end{tabular}

\section{Probability of Causation [\%]}

\begin{tabular}{llcc}
\hline Diet 1 & 0.020 & 0.435 & 10.05 \\
Diet 2 & 0.007 & 0.156 & 3.15 \\
Diet 3 & 0.003 & 0.057 & 1.26 \\
Diet 4 & 0.038 & 1.134 & 22.21 \\
\hline
\end{tabular}

Diet 1 - Backyard cow milk + all other locally produced non-milk exposure pathways

Diet 2 - Locally produced commercial milk + all other locally produced non-milk exposure pathways

Diet 3 - Regionally mixed commercial milk + inhalation (other regionally mixed food items are minor contributors to the total)

Diet 4 - Goat milk (all other exposure pathways are of negligible importance compared to goat milk) 


\section{Location: Farragut}

Receptor: Female born in 1930

\begin{tabular}{lccc}
\hline & \multicolumn{3}{c}{ Thyroid Dose [cGy] } \\
Exposure Pathway & \multicolumn{2}{c}{$95 \%$ Subjective Confidence Interval } \\
\hline Backyard Cow Milk & lower limit & central estimate & upper limit \\
Commercial Milk (locally produced) & 0.49 & 2.6 & 15 \\
Commercial Milk (regionally mixed) & 0.095 & 0.61 & 3.6 \\
Goat Milk (locally produced) & 0.043 & 0.25 & 1.5 \\
Beef (locally produced) & 0.95 & 6.7 & 49 \\
Leafy Vegetables (locally produced) & 0.0013 & 0.021 & 0.48 \\
Eggs (locally produced) & 0.00052 & 0.0057 & 0.05 \\
Cottage Cheese (locally produced) & 0.033 & 0.22 & 1.4 \\
Inhalation & 0.0012 & 0.013 & 0.14 \\
Mother's milk (mother on Diet 1) & 0.026 & 0.087 & 0.3 \\
Prenatal exposure (mother on Diet 1) & -- & -- & -- \\
Diet 1 & -- & -- & -- \\
Diet 2 & 0.6 & 3 & 17 \\
Diet 3 & 0.21 & 1 & 5.4 \\
\hline
\end{tabular}

Excess Lifetime Risk [ ]

\begin{tabular}{llll}
\hline Diet 1 & $1.5 \mathrm{E}-06$ & $2.2 \mathrm{E}-05$ & $3.2 \mathrm{E}-04$ \\
Diet 2 & $5.8 \mathrm{E}-07$ & $8.0 \mathrm{E}-06$ & $9.4 \mathrm{E}-05$ \\
Diet 3 & $2.3 \mathrm{E}-07$ & $2.7 \mathrm{E}-06$ & $3.3 \mathrm{E}-05$ \\
Diet 4 & $3.6 \mathrm{E}-06$ & $5.5 \mathrm{E}-05$ & $7.5 \mathrm{E}-04$ \\
\hline
\end{tabular}

Relative Risk [ ]

\begin{tabular}{lccc}
\hline Diet 1 & 1.00064 & 1.0061 & 1.064 \\
Diet 2 & 1.00026 & 1.0022 & 1.027 \\
Diet 3 & 1.000099 & 1.00078 & 1.0086 \\
Diet 4 & 1.0016 & 1.014 & 1.21 \\
\hline
\end{tabular}

Probability of Causation [\%]

\begin{tabular}{lccc}
\hline Diet 1 & 0.064 & 0.609 & 5.97 \\
Diet 2 & 0.026 & 0.223 & 2.62 \\
Diet 3 & 0.010 & 0.078 & 0.85 \\
Diet 4 & 0.159 & 1.427 & 16.98 \\
\hline
\end{tabular}

Diet 1 - Backyard cow milk + all other locally produced non-milk exposure pathways

Diet 2 - Locally produced commercial milk + all other locally produced non-milk exposure pathways

Diet 3 - Regionally mixed commercial milk + inhalation (other regionally mixed food items are minor contributors to the total)

Diet 4 - Goat milk (all other exposure pathways are of negligible importance compared to goat milk) 


\section{Location: Farragut}

\section{Receptor: Male born in 1930}

\begin{tabular}{lccc}
\hline & \multicolumn{3}{c}{ Thyroid Dose [cGy] } \\
& \multicolumn{2}{c}{$95 \%$ Subjective Confidence Interval } \\
Exposure Pathway & lower limit & central estimate & upper limit \\
\hline Backyard Cow Milk & 0.63 & 3.2 & 17 \\
Commercial Milk (locally produced) & 0.13 & 0.74 & 4.3 \\
Commercial Milk (regionally mixed) & 0.056 & 0.3 & 2 \\
Goat Milk (locally produced) & 1.3 & 9.2 & 60 \\
Beef (locally produced) & 0.002 & 0.034 & 0.85 \\
Leafy Vegetables (locally produced) & 0.00047 & 0.0054 & 0.049 \\
Eggs (locally produced) & 0.035 & 0.24 & 1.6 \\
Cottage Cheese (locally produced) & 0.0011 & 0.012 & 0.12 \\
Inhalation & 0.028 & 0.11 & 0.35 \\
Mother's milk (mother on Diet 1) & -- & -- & -- \\
Prenatal exposure (mother on Diet 1) & -- & -- & -- \\
Diet 1 & 0.76 & 3.6 & 20 \\
Diet 2 & 0.25 & 1.2 & 6.5 \\
Diet 3 & 0.1 & 0.43 & 2.2 \\
\hline
\end{tabular}

Excess Lifetime Risk [ ]

\begin{tabular}{llll}
\hline Diet 1 & $1.7 \mathrm{E}-07$ & $7.0 \mathrm{E}-06$ & $2.3 \mathrm{E}-04$ \\
Diet 2 & $6.2 \mathrm{E}-08$ & $2.4 \mathrm{E}-06$ & $8.2 \mathrm{E}-05$ \\
Diet 3 & $2.5 \mathrm{E}-08$ & $8.8 \mathrm{E}-07$ & $2.7 \mathrm{E}-05$ \\
Diet 4 & $4.3 \mathrm{E}-07$ & $1.7 \mathrm{E}-05$ & $6.1 \mathrm{E}-04$ \\
\hline
\end{tabular}

\section{Relative Risk [ ]}

\begin{tabular}{lccc}
\hline Diet 1 & 1.00026 & 1.0049 & 1.12 \\
Diet 2 & 1.000089 & 1.0017 & 1.041 \\
Diet 3 & 1.000032 & 1.00057 & 1.013 \\
Diet 4 & 1.00053 & 1.013 & 1.31 \\
\hline
\end{tabular}

\section{Probability of Causation [\%]}

\begin{tabular}{lccc}
\hline Diet 1 & 0.026 & 0.486 & 10.95 \\
Diet 2 & 0.009 & 0.174 & 3.96 \\
Diet 3 & 0.003 & 0.057 & 1.31 \\
Diet 4 & 0.053 & 1.291 & 23.31 \\
\hline
\end{tabular}

Diet 1 - Backyard cow milk + all other locally produced non-milk exposure pathways

Diet 2 - Locally produced commercial milk + all other locally produced non-milk exposure pathways

Diet 3 - Regionally mixed commercial milk + inhalation (other regionally mixed food items are minor contributors to the total)

Diet 4 - Goat milk (all other exposure pathways are of negligible importance compared to goat milk) 
Location: Lenoir City

Receptor: Female born in 1930

\begin{tabular}{lccc}
\hline & \multicolumn{3}{c}{ Thyroid Dose [cGy] } \\
Exposure Pathway & $95 \%$ Subjective Confidence Interval \\
\hline Backyard Cow Milk & lower limit & central estimate & upper limit \\
Commercial Milk (locally produced) & 0.32 & 1.6 & 8.5 \\
Commercial Milk (regionally mixed) & 0.06 & 0.37 & 2.4 \\
Goat Milk (locally produced) & 0.043 & 0.25 & 1.5 \\
Beef (locally produced) & -- & -- & -- \\
Leafy Vegetables (locally produced) & 0.00079 & 0.013 & 0.23 \\
Eggs (locally produced) & 0.00037 & 0.0035 & 0.027 \\
Cottage Cheese (locally produced) & 0.021 & 0.14 & 0.85 \\
Inhalation & 0.00065 & 0.0076 & 0.085 \\
Mother's milk (mother on Diet 1) & 0.016 & 0.055 & 0.2 \\
Prenatal exposure (mother on Diet 1) & -- & -- & -- \\
Diet 1 & -- & -- & -- \\
Diet 2 & 0.37 & 1.9 & 9.3 \\
Diet 3 & 0.13 & 0.6 & 3.4 \\
\hline
\end{tabular}

Excess Lifetime Risk [ ]

\begin{tabular}{lccc}
\hline Diet 1 & $9.6 \mathrm{E}-07$ & $1.3 \mathrm{E}-05$ & $2.1 \mathrm{E}-04$ \\
Diet 2 & $3.8 \mathrm{E}-07$ & $4.9 \mathrm{E}-06$ & $7.0 \mathrm{E}-05$ \\
Diet 3 & $2.0 \mathrm{E}-07$ & $2.5 \mathrm{E}-06$ & $3.1 \mathrm{E}-05$ \\
Diet 4 & -- & -- & -- \\
\hline
\end{tabular}

Relative Risk [ ]

\begin{tabular}{lccc}
\hline Diet 1 & 1.00039 & 1.0037 & 1.038 \\
Diet 2 & 1.00015 & 1.0013 & 1.017 \\
Diet 3 & 1.000088 & 1.00071 & 1.0084 \\
Diet 4 & -- & -- & -- \\
\hline
\end{tabular}

Probability of Causation [\%]

\begin{tabular}{lccc}
\hline Diet 1 & 0.039 & 0.366 & 3.67 \\
Diet 2 & 0.016 & 0.134 & 1.67 \\
Diet 3 & 0.009 & 0.071 & 0.83 \\
Diet 4 & -- & -- & -- \\
\hline Diet 1 - Backyard cow milk + all other locally produced non-milk exposure pathways & \\
Diet 2 - Locally produced commercial milk + all other locally produced non-milk exposure pathways \\
Diet 3 - Regionally mixed commercial milk + inhalation (other regionally mixed food items are minor contributors to the total) \\
Diet 4 - Goat milk (all other exposure pathways are of negligible importance compared to goat milk)
\end{tabular}




\section{Location: Lenoir City}

Receptor: Male born in 1930

\begin{tabular}{lccc}
\hline & \multicolumn{3}{c}{ Thyroid Dose [cGy] } \\
Exposure Pathway & $95 \%$ Subjective Confidence Interval \\
\hline Backyard Cow Milk & lower limit & central estimate & upper limit \\
Commercial Milk (locally produced) & 0.38 & 2 & 9.6 \\
Commercial Milk (regionally mixed) & 0.076 & 0.43 & 2.9 \\
Goat Milk (locally produced) & 0.056 & 0.3 & 2 \\
Beef (locally produced) & -- & -- & -- \\
Leafy Vegetables (locally produced) & 0.0012 & 0.021 & 0.41 \\
Eggs (locally produced) & 0.00037 & 0.0034 & 0.026 \\
Cottage Cheese (locally produced) & 0.021 & 0.15 & 0.9 \\
Inhalation & 0.00067 & 0.0076 & 0.076 \\
Mother's milk (mother on Diet 1) & 0.02 & 0.065 & 0.24 \\
Prenatal exposure (mother on Diet 1) & -- & -- & - \\
Diet 1 & -- & -- & -- \\
Diet 2 & 0.45 & 2.2 & 11 \\
Diet 3 & 0.16 & 0.71 & 4 \\
\hline
\end{tabular}

Excess Lifetime Risk [ ]

\begin{tabular}{lccc}
\hline Diet 1 & $8.8 \mathrm{E}-08$ & $4.4 \mathrm{E}-06$ & $1.2 \mathrm{E}-04$ \\
Diet 2 & $2.9 \mathrm{E}-08$ & $1.5 \mathrm{E}-06$ & $4.3 \mathrm{E}-05$ \\
Diet 3 & $2.1 \mathrm{E}-08$ & $8.3 \mathrm{E}-07$ & $2.6 \mathrm{E}-05$ \\
Diet 4 & -- & -- & -- \\
\hline
\end{tabular}

Relative Risk [ ]

\begin{tabular}{lccc}
\hline Diet 1 & 1.00015 & 1.0029 & 1.059 \\
Diet 2 & 1.000053 & 1.0011 & 1.025 \\
Diet 3 & 1.000029 & 1.00054 & 1.012 \\
Diet 4 & -- & -- & -- \\
\hline
\end{tabular}

\begin{tabular}{lccc}
\hline Diet 1 & 0.015 & 0.293 & 5.58 \\
Diet 2 & 0.005 & 0.106 & 2.44 \\
Diet 3 & 0.003 & 0.054 & 1.21 \\
Diet 4 & -- & -- & -- \\
\hline Diet 1 - Backyard cow milk + all other locally produced non-milk exposure pathways & \\
Diet 2 - Locally produced commercial milk + all other locally produced non-milk exposure pathways \\
Diet 3 - Regionally mixed commercial milk + inhalation (other regionally mixed food items are minor contributors to the total) \\
Diet 4 - Goat milk (all other exposure pathways are of negligible importance compared to goat milk)
\end{tabular}




\section{Location: Kingston}

Receptor: Female born in 1930

\begin{tabular}{lccc}
\hline & \multicolumn{3}{c}{ Thyroid Dose [cGy] } \\
Exposure Pathway & \multicolumn{2}{c}{$95 \%$ Subjective Confidence Interval } \\
\hline Backyard Cow Milk & lower limit & central estimate & upper limit \\
Commercial Milk (locally produced) & 0.35 & 2.1 & 12 \\
Commercial Milk (regionally mixed) & 0.079 & 0.47 & 2.9 \\
Goat Milk (locally produced) & 0.042 & 0.25 & 1.5 \\
Beef (locally produced) & 0.75 & 5.5 & 38 \\
Leafy Vegetables (locally produced) & 0.001 & 0.017 & 0.35 \\
Eggs (locally produced) & 0.00043 & 0.0043 & 0.04 \\
Cottage Cheese (locally produced) & 0.025 & 0.17 & 1.1 \\
Inhalation & 0.00095 & 0.0098 & 0.11 \\
Mother's milk (mother on Diet 1) & 0.022 & 0.071 & 0.25 \\
Prenatal exposure (mother on Diet 1) & -- & -- & -- \\
Diet 1 & -- & -- & -- \\
Diet 2 & 0.43 & 2.4 & 13 \\
Diet 3 & 0.16 & 0.79 & 4.4 \\
\hline
\end{tabular}

Excess Lifetime Risk [ ]

\begin{tabular}{lllc}
\hline Diet 1 & $1.2 \mathrm{E}-06$ & $1.7 \mathrm{E}-05$ & $2.4 \mathrm{E}-04$ \\
Diet 2 & $4.4 \mathrm{E}-07$ & $6.2 \mathrm{E}-06$ & $8.1 \mathrm{E}-05$ \\
Diet 3 & $2.1 \mathrm{E}-07$ & $2.7 \mathrm{E}-06$ & $3.2 \mathrm{E}-05$ \\
Diet 4 & $3.1 \mathrm{E}-06$ & $4.2 \mathrm{E}-05$ & $6.4 \mathrm{E}-04$ \\
\hline
\end{tabular}

Relative Risk [ ]

\begin{tabular}{lccc}
\hline Diet 1 & 1.00052 & 1.0048 & 1.052 \\
Diet 2 & 1.0002 & 1.0017 & 1.021 \\
Diet 3 & 1.000094 & 1.00073 & 1.0085 \\
Diet 4 & 1.0013 & 1.011 & 1.16 \\
\hline
\end{tabular}

\section{Probability of Causation [\%]}

\begin{tabular}{llcc}
\hline Diet 1 & 0.052 & 0.479 & 4.95 \\
Diet 2 & 0.020 & 0.172 & 2.09 \\
Diet 3 & 0.009 & 0.073 & 0.84 \\
Diet 4 & 0.126 & 1.130 & 14.08 \\
\hline
\end{tabular}

Diet 1 - Backyard cow milk + all other locally produced non-milk exposure pathways

Diet 2 - Locally produced commercial milk + all other locally produced non-milk exposure pathways

Diet 3 - Regionally mixed commercial milk + inhalation (other regionally mixed food items are minor contributors to the total)

Diet 4 - Goat milk (all other exposure pathways are of negligible importance compared to goat milk) 


\section{Location: Kingston}

\section{Receptor: Male born in 1930}

\begin{tabular}{lccc}
\hline & \multicolumn{3}{c}{ Thyroid Dose [cGy] } \\
Exposure Pathway & $95 \%$ Subjective Confidence Interval \\
\hline Backyard Cow Milk & lower limit & central estimate & upper limit \\
Commercial Milk (locally produced) & 0.45 & 2.5 & 14 \\
Commercial Milk (regionally mixed) & 0.1 & 0.58 & 3.7 \\
Goat Milk (locally produced) & 0.056 & 0.3 & 2 \\
Beef (locally produced) & 0.97 & 7.3 & 45 \\
Leafy Vegetables (locally produced) & 0.0016 & 0.026 & 0.57 \\
Eggs (locally produced) & 0.0004 & 0.0042 & 0.038 \\
Cottage Cheese (locally produced) & 0.027 & 0.18 & 1.2 \\
Inhalation & 0.00086 & 0.01 & 0.1 \\
Mother's milk (mother on Diet 1) & 0.024 & 0.086 & 0.3 \\
Prenatal exposure (mother on Diet 1) & -- & -- & - \\
Diet 1 & -- & -- & - \\
Diet 2 & 0.53 & 2.9 & 15 \\
Diet 3 & 0.2 & 0.96 & 5.7 \\
\hline
\end{tabular}

Excess Lifetime Risk [ ]

\begin{tabular}{llll}
\hline Diet 1 & $1.2 \mathrm{E}-07$ & $5.5 \mathrm{E}-06$ & $1.7 \mathrm{E}-04$ \\
Diet 2 & $4.6 \mathrm{E}-08$ & $2.0 \mathrm{E}-06$ & $6.1 \mathrm{E}-05$ \\
Diet 3 & $2.4 \mathrm{E}-08$ & $8.5 \mathrm{E}-07$ & $2.6 \mathrm{E}-05$ \\
Diet 4 & $3.4 \mathrm{E}-07$ & $1.3 \mathrm{E}-05$ & $4.5 \mathrm{E}-04$ \\
\hline
\end{tabular}

\section{Relative Risk [ ]}

\begin{tabular}{lccc}
\hline Diet 1 & 1.00018 & 1.0039 & 1.089 \\
Diet 2 & 1.00006 & 1.0014 & 1.033 \\
Diet 3 & 1.00003 & 1.00057 & 1.013 \\
Diet 4 & 1.00035 & 1.0099 & 1.29 \\
\hline
\end{tabular}

\section{Probability of Causation [\%]}

\begin{tabular}{lccc}
\hline Diet 1 & 0.018 & 0.387 & 8.19 \\
Diet 2 & 0.006 & 0.136 & 3.15 \\
Diet 3 & 0.003 & 0.057 & 1.27 \\
Diet 4 & 0.035 & 0.981 & 22.22 \\
\hline
\end{tabular}

Diet 1 - Backyard cow milk + all other locally produced non-milk exposure pathways

Diet 2 - Locally produced commercial milk + all other locally produced non-milk exposure pathways

Diet 3 - Regionally mixed commercial milk + inhalation (other regionally mixed food items are minor contributors to the total)

Diet 4 - Goat milk (all other exposure pathways are of negligible importance compared to goat milk) 


\section{Location: Karns}

Receptor: Female born in 1930

\begin{tabular}{lccc}
\hline & \multicolumn{3}{c}{ Thyroid Dose [cGy] } \\
& \multicolumn{2}{c}{$95 \%$ Subjective Confidence Interval } \\
Exposure Pathway & lower limit & central estimate & upper limit \\
\hline Backyard Cow Milk & 0.51 & 2.8 & 16 \\
Commercial Milk (locally produced) & 0.11 & 0.65 & 3.6 \\
Commercial Milk (regionally mixed) & 0.042 & 0.25 & 1.5 \\
Goat Milk (locally produced) & 0.99 & 7.1 & 51 \\
Beef (locally produced) & 0.0013 & 0.022 & 0.47 \\
Leafy Vegetables (locally produced) & 0.00056 & 0.0059 & 0.05 \\
Eggs (locally produced) & 0.034 & 0.23 & 1.5 \\
Cottage Cheese (locally produced) & 0.0013 & 0.013 & 0.14 \\
Inhalation & 0.029 & 0.095 & 0.33 \\
Mother's milk (mother on Diet 1) & -- & -- & -- \\
Prenatal exposure (mother on Diet 1) & -- & -- & -- \\
Diet 1 & 0.63 & 3.2 & 18 \\
Diet 2 & 0.23 & 1.1 & 5.4 \\
Diet 3 & 0.088 & 0.36 & 1.8 \\
\hline
\end{tabular}

Excess Lifetime Risk [ ]

\begin{tabular}{llll}
\hline Diet 1 & $1.6 \mathrm{E}-06$ & $2.4 \mathrm{E}-05$ & $3.3 \mathrm{E}-04$ \\
Diet 2 & $6.2 \mathrm{E}-07$ & $8.5 \mathrm{E}-06$ & $1.0 \mathrm{E}-04$ \\
Diet 3 & $2.4 \mathrm{E}-07$ & $2.8 \mathrm{E}-06$ & $3.3 \mathrm{E}-05$ \\
Diet 4 & $3.8 \mathrm{E}-06$ & $5.6 \mathrm{E}-05$ & $7.8 \mathrm{E}-04$ \\
\hline
\end{tabular}

\section{Relative Risk [ ]}

\begin{tabular}{lccc}
\hline Diet 1 & 1.00067 & 1.0063 & 1.067 \\
Diet 2 & 1.00028 & 1.0024 & 1.028 \\
Diet 3 & 1.00011 & 1.0008 & 1.0089 \\
Diet 4 & 1.0018 & 1.016 & 1.22 \\
\hline
\end{tabular}

\section{Probability of Causation [\%]}

\begin{tabular}{lccc}
\hline Diet 1 & 0.067 & 0.631 & 6.32 \\
Diet 2 & 0.028 & 0.243 & 2.69 \\
Diet 3 & 0.011 & 0.080 & 0.88 \\
Diet 4 & 0.177 & 1.551 & 18.08 \\
\hline
\end{tabular}

Diet 1 - Backyard cow milk + all other locally produced non-milk exposure pathways

Diet 2 - Locally produced commercial milk + all other locally produced non-milk exposure pathways

Diet 3 - Regionally mixed commercial milk + inhalation (other regionally mixed food items are minor contributors to the total)

Diet 4 - Goat milk (all other exposure pathways are of negligible importance compared to goat milk) 


\section{Location: Karns}

\section{Receptor: Male born in 1930}

\begin{tabular}{lccc}
\hline & \multicolumn{3}{c}{ Thyroid Dose [cGy] } \\
& \multicolumn{2}{c}{$95 \%$ Subjective Confidence Interval } \\
Exposure Pathway & lower limit & central estimate & upper limit \\
\hline Backyard Cow Milk & 0.67 & 3.4 & 17 \\
Commercial Milk (locally produced) & 0.14 & 0.79 & 4.3 \\
Commercial Milk (regionally mixed) & 0.056 & 0.3 & 2 \\
Goat Milk (locally produced) & 1.3 & 9.7 & 62 \\
Beef (locally produced) & 0.0021 & 0.036 & 0.76 \\
Leafy Vegetables (locally produced) & 0.00053 & 0.0058 & 0.049 \\
Eggs (locally produced) & 0.037 & 0.25 & 1.6 \\
Cottage Cheese (locally produced) & 0.0012 & 0.013 & 0.13 \\
Inhalation & 0.033 & 0.12 & 0.39 \\
Mother's milk (mother on Diet 1) & -- & -- & - \\
Prenatal exposure (mother on Diet 1) & -- & -- & - \\
Diet 1 & 0.79 & 3.9 & 19 \\
Diet 2 & 0.27 & 1.3 & 6.8 \\
Diet 3 & 0.1 & 0.44 & 2.2 \\
\hline
\end{tabular}

Excess Lifetime Risk [ ]

\begin{tabular}{llll}
\hline Diet 1 & $1.8 \mathrm{E}-07$ & $7.6 \mathrm{E}-06$ & $2.2 \mathrm{E}-04$ \\
Diet 2 & $6.3 \mathrm{E}-08$ & $2.7 \mathrm{E}-06$ & $8.4 \mathrm{E}-05$ \\
Diet 3 & $2.5 \mathrm{E}-08$ & $8.9 \mathrm{E}-07$ & $2.8 \mathrm{E}-05$ \\
Diet 4 & $4.7 \mathrm{E}-07$ & $1.8 \mathrm{E}-05$ & $6.3 \mathrm{E}-04$ \\
\hline
\end{tabular}

\section{Relative Risk [ ]}

\begin{tabular}{lccc}
\hline Diet 1 & 1.00026 & 1.0052 & 1.13 \\
Diet 2 & 1.000089 & 1.0019 & 1.041 \\
Diet 3 & 1.000033 & 1.0006 & 1.014 \\
Diet 4 & 1.00052 & 1.014 & 1.32 \\
\hline
\end{tabular}

\section{Probability of Causation [\%]}

\begin{tabular}{lccc}
\hline Diet 1 & 0.026 & 0.516 & 11.49 \\
Diet 2 & 0.009 & 0.185 & 3.96 \\
Diet 3 & 0.003 & 0.060 & 1.34 \\
Diet 4 & 0.052 & 1.364 & 24.04 \\
\hline
\end{tabular}

Diet 1 - Backyard cow milk + all other locally produced non-milk exposure pathways

Diet 2 - Locally produced commercial milk + all other locally produced non-milk exposure pathways

Diet 3 - Regionally mixed commercial milk + inhalation (other regionally mixed food items are minor contributors to the total)

Diet 4 - Goat milk (all other exposure pathways are of negligible importance compared to goat milk) 


\section{Location: Loudon}

Receptor: Female born in 1930

\begin{tabular}{lccc}
\hline & \multicolumn{3}{c}{ Thyroid Dose [cGy] } \\
Exposure Pathway & \multicolumn{2}{c}{$95 \%$ Subjective Confidence Interval } \\
\hline Backyard Cow Milk & lower limit & central estimate & upper limit \\
Commercial Milk (locally produced) & 0.29 & 1.5 & 8.8 \\
Commercial Milk (regionally mixed) & 0.054 & 0.36 & 2.3 \\
Goat Milk (locally produced) & 0.043 & 0.25 & 1.5 \\
Beef (locally produced) & 0.54 & 4 & 29 \\
Leafy Vegetables (locally produced) & 0.00081 & 0.012 & 0.26 \\
Eggs (locally produced) & 0.00032 & 0.0033 & 0.028 \\
Cottage Cheese (locally produced) & 0.019 & 0.13 & 0.8 \\
Inhalation & 0.0007 & 0.0075 & 0.084 \\
Mother's milk (mother on Diet 1) & 0.017 & 0.056 & 0.2 \\
Prenatal exposure (mother on Diet 1) & -- & -- & - \\
Diet 1 & -- & -- & -- \\
Diet 2 & 0.35 & 1.8 & 10 \\
Diet 3 & 0.12 & 0.6 & 3.4 \\
\hline
\end{tabular}

Excess Lifetime Risk [ ]

\begin{tabular}{llll}
\hline Diet 1 & $9.3 \mathrm{E}-07$ & $1.3 \mathrm{E}-05$ & $1.9 \mathrm{E}-04$ \\
Diet 2 & $3.5 \mathrm{E}-07$ & $4.9 \mathrm{E}-06$ & $6.4 \mathrm{E}-05$ \\
Diet 3 & $2.0 \mathrm{E}-07$ & $2.5 \mathrm{E}-06$ & $3.1 \mathrm{E}-05$ \\
Diet 4 & $2.3 \mathrm{E}-06$ & $3.3 \mathrm{E}-05$ & $4.8 \mathrm{E}-04$ \\
\hline
\end{tabular}

Relative Risk [ ]

\begin{tabular}{lccc}
\hline Diet 1 & 1.00038 & 1.0035 & 1.039 \\
Diet 2 & 1.00015 & 1.0013 & 1.016 \\
Diet 3 & 1.000086 & 1.0007 & 1.0083 \\
Diet 4 & 1.00091 & 1.0084 & 1.13 \\
\hline
\end{tabular}

Probability of Causation [\%]

\begin{tabular}{llcc}
\hline Diet 1 & 0.038 & 0.352 & 3.78 \\
Diet 2 & 0.015 & 0.130 & 1.55 \\
Diet 3 & 0.009 & 0.070 & 0.83 \\
Diet 4 & 0.091 & 0.834 & 11.30 \\
\hline
\end{tabular}

Diet 1 - Backyard cow milk + all other locally produced non-milk exposure pathways

Diet 2 - Locally produced commercial milk + all other locally produced non-milk exposure pathways

Diet 3 - Regionally mixed commercial milk + inhalation (other regionally mixed food items are minor contributors to the total)

Diet 4 - Goat milk (all other exposure pathways are of negligible importance compared to goat milk) 


\section{Location: Loudon}

\section{Receptor: Male born in 1930}

\begin{tabular}{lccc}
\hline & \multicolumn{3}{c}{ Thyroid Dose [cGy] } \\
Exposure Pathway & $95 \%$ Subjective Confidence Interval \\
\hline Backyard Cow Milk & lower limit & central estimate & upper limit \\
Commercial Milk (locally produced) & 0.37 & 1.9 & 11 \\
Commercial Milk (regionally mixed) & 0.07 & 0.44 & 2.9 \\
Goat Milk (locally produced) & 0.056 & 0.3 & 2 \\
Beef (locally produced) & 0.72 & 5.5 & 35 \\
Leafy Vegetables (locally produced) & 0.0012 & 0.02 & 0.4 \\
Eggs (locally produced) & 0.0003 & 0.0032 & 0.029 \\
Cottage Cheese (locally produced) & 0.02 & 0.14 & 0.86 \\
Inhalation & 0.00062 & 0.0074 & 0.076 \\
Mother's milk (mother on Diet 1) & 0.02 & 0.069 & 0.24 \\
Prenatal exposure (mother on Diet 1) & -- & -- & -- \\
Diet 1 & -- & -- & -- \\
Diet 2 & 0.44 & 2.2 & 12 \\
Diet 3 & 0.15 & 0.74 & 4.1 \\
\hline
\end{tabular}

Excess Lifetime Risk [ ]

\begin{tabular}{llll}
\hline Diet 1 & $9.5 \mathrm{E}-08$ & $4.2 \mathrm{E}-06$ & $1.2 \mathrm{E}-04$ \\
Diet 2 & $3.7 \mathrm{E}-08$ & $1.5 \mathrm{E}-06$ & $4.7 \mathrm{E}-05$ \\
Diet 3 & $2.2 \mathrm{E}-08$ & $8.0 \mathrm{E}-07$ & $2.6 \mathrm{E}-05$ \\
Diet 4 & $2.7 \mathrm{E}-07$ & $9.7 \mathrm{E}-06$ & $3.6 \mathrm{E}-04$ \\
\hline
\end{tabular}

\section{Relative Risk [ ]}

\begin{tabular}{lccc}
\hline Diet 1 & 1.00014 & 1.003 & 1.072 \\
Diet 2 & 1.000047 & 1.001 & 1.023 \\
Diet 3 & 1.000028 & 1.00054 & 1.012 \\
Diet 4 & 1.00026 & 1.0077 & 1.18 \\
\hline
\end{tabular}

\section{Probability of Causation [\%]}

\begin{tabular}{llcc}
\hline Diet 1 & 0.014 & 0.301 & 6.71 \\
Diet 2 & 0.005 & 0.104 & 2.28 \\
Diet 3 & 0.003 & 0.054 & 1.17 \\
Diet 4 & 0.026 & 0.761 & 15.14 \\
\hline
\end{tabular}

Diet 1 - Backyard cow milk + all other locally produced non-milk exposure pathways

Diet 2 - Locally produced commercial milk + all other locally produced non-milk exposure pathways

Diet 3 - Regionally mixed commercial milk + inhalation (other regionally mixed food items are minor contributors to the total)

Diet 4 - Goat milk (all other exposure pathways are of negligible importance compared to goat milk) 
Location: Harriman

Receptor: Female born in 1930

\begin{tabular}{lccc}
\hline & \multicolumn{3}{c}{ Thyroid Dose [cGy] } \\
Exposure Pathway & $95 \%$ Subjective Confidence Interval \\
\hline Backyard Cow Milk & lower limit & central estimate & upper limit \\
Commercial Milk (locally produced) & 0.18 & 0.91 & 4.9 \\
Commercial Milk (regionally mixed) & 0.036 & 0.21 & 1.3 \\
Goat Milk (locally produced) & 0.042 & 0.25 & 1.5 \\
Beef (locally produced) & 0.31 & 2.3 & 17 \\
Leafy Vegetables (locally produced) & 0.00047 & 0.0076 & 0.14 \\
Eggs (locally produced) & 0.00021 & 0.002 & 0.016 \\
Cottage Cheese (locally produced) & 0.012 & 0.078 & 0.49 \\
Inhalation & 0.00038 & 0.0043 & 0.047 \\
Mother's milk (mother on Diet 1) & 0.0093 & 0.031 & 0.11 \\
Prenatal exposure (mother on Diet 1) & -- & -- & -- \\
Diet 1 & -- & -- & -- \\
Diet 2 & 0.21 & 1.1 & 5.4 \\
Diet 3 & 0.075 & 0.35 & 2 \\
\hline
\end{tabular}

Excess Lifetime Risk [ ]

\begin{tabular}{llll}
\hline Diet 1 & $5.3 \mathrm{E}-07$ & $7.5 \mathrm{E}-06$ & $1.2 \mathrm{E}-04$ \\
Diet 2 & $2.1 \mathrm{E}-07$ & $2.9 \mathrm{E}-06$ & $3.9 \mathrm{E}-05$ \\
Diet 3 & $1.7 \mathrm{E}-07$ & $2.3 \mathrm{E}-06$ & $3.0 \mathrm{E}-05$ \\
Diet 4 & $1.4 \mathrm{E}-06$ & $1.8 \mathrm{E}-05$ & $3.0 \mathrm{E}-04$ \\
\hline
\end{tabular}

\section{Relative Risk [ ]}

\begin{tabular}{lccc}
\hline Diet 1 & 1.00021 & 1.0021 & 1.022 \\
Diet 2 & 1.000089 & 1.00077 & 1.0098 \\
Diet 3 & 1.000077 & 1.00066 & 1.008 \\
Diet 4 & 1.00056 & 1.0049 & 1.069 \\
\hline
\end{tabular}

\begin{tabular}{llcc} 
& \multicolumn{3}{c}{ Probability of Causation [\%] } \\
\hline Diet 1 & 0.021 & 0.212 & 2.18 \\
Diet 2 & 0.009 & 0.077 & 0.97 \\
Diet 3 & 0.008 & 0.066 & 0.79 \\
Diet 4 & 0.056 & 0.492 & 6.42 \\
\hline
\end{tabular}

Diet 1 - Backyard cow milk + all other locally produced non-milk exposure pathways

Diet 2 - Locally produced commercial milk + all other locally produced non-milk exposure pathways

Diet 3 - Regionally mixed commercial milk + inhalation (other regionally mixed food items are minor contributors to the total)

Diet 4 - Goat milk (all other exposure pathways are of negligible importance compared to goat milk) 


\section{Location: Harriman}

\section{Receptor: Male born in 1930}

\begin{tabular}{lccc}
\hline & \multicolumn{3}{c}{ Thyroid Dose [cGy] } \\
& \multicolumn{2}{c}{$95 \%$ Subjective Confidence Interval } \\
Exposure Pathway & lower limit & central estimate & upper limit \\
\hline Backyard Cow Milk & 0.22 & 1.1 & 5.7 \\
Commercial Milk (locally produced) & 0.044 & 0.25 & 1.7 \\
Commercial Milk (regionally mixed) & 0.056 & 0.3 & 2 \\
Goat Milk (locally produced) & 0.43 & 3.2 & 21 \\
Beef (locally produced) & 0.0007 & 0.012 & 0.24 \\
Leafy Vegetables (locally produced) & 0.00022 & 0.002 & 0.015 \\
Eggs (locally produced) & 0.012 & 0.083 & 0.51 \\
Cottage Cheese (locally produced) & 0.00039 & 0.0043 & 0.042 \\
Inhalation & 0.011 & 0.036 & 0.13 \\
Mother's milk (mother on Diet 1) & -- & -- & - \\
Prenatal exposure (mother on Diet 1) & -- & -- & -- \\
Diet 1 & 0.25 & 1.3 & 6.4 \\
Diet 2 & 0.09 & 0.41 & 2.4 \\
Diet 3 & 0.072 & 0.34 & 2 \\
\hline
\end{tabular}

Excess Lifetime Risk [ ]

\begin{tabular}{llll}
\hline Diet 1 & $5.0 \mathrm{E}-08$ & $2.5 \mathrm{E}-06$ & $6.9 \mathrm{E}-05$ \\
Diet 2 & $1.7 \mathrm{E}-08$ & $8.8 \mathrm{E}-07$ & $2.6 \mathrm{E}-05$ \\
Diet 3 & $1.9 \mathrm{E}-08$ & $7.5 \mathrm{E}-07$ & $2.5 \mathrm{E}-05$ \\
Diet 4 & $1.6 \mathrm{E}-07$ & $5.8 \mathrm{E}-06$ & $1.9 \mathrm{E}-04$ \\
\hline
\end{tabular}

\section{Relative Risk [ ]}

\begin{tabular}{lccc}
\hline Diet 1 & 1.000088 & 1.0017 & 1.035 \\
Diet 2 & 1.00003 & 1.00061 & 1.014 \\
Diet 3 & 1.000026 & 1.0005 & 1.011 \\
Diet 4 & 1.00018 & 1.0046 & 1.11 \\
\hline
\end{tabular}

\begin{tabular}{llcc} 
& \multicolumn{3}{c}{ Probability of Causation [\%] } \\
\hline Diet 1 & 0.009 & 0.171 & 3.42 \\
Diet 2 & 0.003 & 0.061 & 1.39 \\
Diet 3 & 0.003 & 0.050 & 1.12 \\
Diet 4 & 0.018 & 0.459 & 10.16 \\
\hline
\end{tabular}

Diet 1 - Backyard cow milk + all other locally produced non-milk exposure pathways

Diet 2 - Locally produced commercial milk + all other locally produced non-milk exposure pathways

Diet 3 - Regionally mixed commercial milk + inhalation (other regionally mixed food items are minor contributors to the total)

Diet 4 - Goat milk (all other exposure pathways are of negligible importance compared to goat milk) 


\section{Location: Cedar Bluff}

Receptor: Female born in 1930

\begin{tabular}{lccc}
\hline & \multicolumn{3}{c}{ Thyroid Dose [cGy] } \\
Exposure Pathway & \multicolumn{2}{c}{$95 \%$ Subjective Confidence Interval } \\
\hline Backyard Cow Milk & lower limit & central estimate & upper limit \\
Commercial Milk (locally produced) & 0.41 & 2.2 & 13 \\
Commercial Milk (regionally mixed) & 0.081 & 0.49 & 3.2 \\
Goat Milk (locally produced) & 0.043 & 0.25 & 1.5 \\
Beef (locally produced) & 0.78 & 5.6 & 38 \\
Leafy Vegetables (locally produced) & 0.0011 & 0.018 & 0.4 \\
Eggs (locally produced) & 0.00048 & 0.0047 & 0.038 \\
Cottage Cheese (locally produced) & 0.026 & 0.18 & 1.1 \\
Inhalation & 0.001 & 0.01 & 0.12 \\
Mother's milk (mother on Diet 1) & 0.023 & 0.078 & 0.27 \\
Prenatal exposure (mother on Diet 1) & -- & -- & -- \\
Diet 1 & -- & -- & -- \\
Diet 2 & 0.5 & 2.5 & 14 \\
Diet 3 & 0.18 & 0.84 & 4.5 \\
\hline
\end{tabular}

Excess Lifetime Risk [ ]

\begin{tabular}{lllc}
\hline Diet 1 & $1.2 \mathrm{E}-06$ & $1.8 \mathrm{E}-05$ & $2.7 \mathrm{E}-04$ \\
Diet 2 & $5.0 \mathrm{E}-07$ & $6.7 \mathrm{E}-06$ & $8.7 \mathrm{E}-05$ \\
Diet 3 & $2.2 \mathrm{E}-07$ & $2.6 \mathrm{E}-06$ & $3.2 \mathrm{E}-05$ \\
Diet 4 & $3.1 \mathrm{E}-06$ & $4.5 \mathrm{E}-05$ & $6.3 \mathrm{E}-04$ \\
\hline
\end{tabular}

Relative Risk [ ]

\begin{tabular}{lccc}
\hline Diet 1 & 1.00053 & 1.0052 & 1.051 \\
Diet 2 & 1.00021 & 1.0019 & 1.021 \\
Diet 3 & 1.000094 & 1.00076 & 1.0085 \\
Diet 4 & 1.0013 & 1.012 & 1.17 \\
\hline
\end{tabular}

\section{Probability of Causation [\%]}

\begin{tabular}{lccc}
\hline Diet 1 & 0.053 & 0.517 & 4.87 \\
Diet 2 & 0.021 & 0.191 & 2.10 \\
Diet 3 & 0.009 & 0.076 & 0.84 \\
Diet 4 & 0.132 & 1.184 & 14.20 \\
\hline
\end{tabular}

Diet 1 - Backyard cow milk + all other locally produced non-milk exposure pathways

Diet 2 - Locally produced commercial milk + all other locally produced non-milk exposure pathways

Diet 3 - Regionally mixed commercial milk + inhalation (other regionally mixed food items are minor contributors to the total)

Diet 4 - Goat milk (all other exposure pathways are of negligible importance compared to goat milk) 


\section{Location: Cedar Bluff}

Receptor: Male born in 1930

\begin{tabular}{lccc}
\hline & \multicolumn{3}{c}{ Thyroid Dose [cGy] } \\
Exposure Pathway & $95 \%$ Subjective Confidence Interval \\
\hline Backyard Cow Milk & lower limit & central estimate & upper limit \\
Commercial Milk (locally produced) & 0.54 & 2.7 & 15 \\
Commercial Milk (regionally mixed) & 0.11 & 0.61 & 3.7 \\
Goat Milk (locally produced) & 0.056 & 0.3 & 2 \\
Beef (locally produced) & 1 & 7.7 & 49 \\
Leafy Vegetables (locally produced) & 0.0016 & 0.028 & 0.61 \\
Eggs (locally produced) & 0.00045 & 0.0046 & 0.04 \\
Cottage Cheese (locally produced) & 0.028 & 0.2 & 1.2 \\
Inhalation & 0.00099 & 0.011 & 0.11 \\
Mother's milk (mother on Diet 1) & 0.027 & 0.095 & 0.32 \\
Prenatal exposure (mother on Diet 1) & -- & -- & - \\
Diet 1 & -- & -- & - \\
Diet 2 & 0.63 & 3.1 & 16 \\
Diet 3 & 0.21 & 1 & 5.7 \\
\hline
\end{tabular}

Excess Lifetime Risk [ ]

\begin{tabular}{llll}
\hline Diet 1 & $1.3 \mathrm{E}-07$ & $6.0 \mathrm{E}-06$ & $1.8 \mathrm{E}-04$ \\
Diet 2 & $5.0 \mathrm{E}-08$ & $2.2 \mathrm{E}-06$ & $6.7 \mathrm{E}-05$ \\
Diet 3 & $2.3 \mathrm{E}-08$ & $8.6 \mathrm{E}-07$ & $2.6 \mathrm{E}-05$ \\
Diet 4 & $4.0 \mathrm{E}-07$ & $1.4 \mathrm{E}-05$ & $4.2 \mathrm{E}-04$ \\
\hline
\end{tabular}

\section{Relative Risk [ ]}

\begin{tabular}{lccc}
\hline Diet 1 & 1.0002 & 1.0041 & 1.1 \\
Diet 2 & 1.000067 & 1.0015 & 1.03 \\
Diet 3 & 1.000031 & 1.00057 & 1.013 \\
Diet 4 & 1.00035 & 1.011 & 1.27 \\
\hline
\end{tabular}

\section{Probability of Causation [\%]}

\begin{tabular}{lccc}
\hline Diet 1 & 0.020 & 0.408 & 9.17 \\
Diet 2 & 0.007 & 0.148 & 2.94 \\
Diet 3 & 0.003 & 0.057 & 1.27 \\
Diet 4 & 0.035 & 1.068 & 21.23 \\
\hline
\end{tabular}

Diet 1 - Backyard cow milk + all other locally produced non-milk exposure pathways

Diet 2 - Locally produced commercial milk + all other locally produced non-milk exposure pathways

Diet 3 - Regionally mixed commercial milk + inhalation (other regionally mixed food items are minor contributors to the total)

Diet 4 - Goat milk (all other exposure pathways are of negligible importance compared to goat milk) 


\section{Location: Oakdale}

Receptor: Female born in 1930

\begin{tabular}{lccc}
\hline & \multicolumn{3}{c}{ Thyroid Dose [cGy] } \\
& \multicolumn{2}{c}{$95 \%$ Subjective Confidence Interval } \\
Exposure Pathway & lower limit & central estimate & upper limit \\
\hline Backyard Cow Milk & 0.09 & 0.51 & 3.1 \\
Commercial Milk (locally produced) & 0.018 & 0.12 & 0.76 \\
Commercial Milk (regionally mixed) & 0.043 & 0.25 & 1.5 \\
Goat Milk (locally produced) & 0.16 & 1.3 & 9.3 \\
Beef (locally produced) & 0.00027 & 0.0042 & 0.087 \\
Leafy Vegetables (locally produced) & 0.0001 & 0.0011 & 0.009 \\
Eggs (locally produced) & 0.0059 & 0.044 & 0.28 \\
Cottage Cheese (locally produced) & 0.00022 & 0.0025 & 0.029 \\
Inhalation & 0.0052 & 0.018 & 0.066 \\
Mother's milk (mother on Diet 1) & -- & -- & -- \\
Prenatal exposure (mother on Diet 1) & -- & -- & -- \\
Diet 1 & 0.11 & 0.6 & 3.5 \\
Diet 2 & 0.038 & 0.2 & 1.1 \\
Diet 3 & 0.054 & 0.27 & 1.6 \\
\hline
\end{tabular}

Excess Lifetime Risk [ ]

\begin{tabular}{llll}
\hline Diet 1 & $2.7 \mathrm{E}-07$ & $4.3 \mathrm{E}-06$ & $6.3 \mathrm{E}-05$ \\
Diet 2 & $1.1 \mathrm{E}-07$ & $1.6 \mathrm{E}-06$ & $2.1 \mathrm{E}-05$ \\
Diet 3 & $1.6 \mathrm{E}-07$ & $2.2 \mathrm{E}-06$ & $3.0 \mathrm{E}-05$ \\
Diet 4 & $6.8 \mathrm{E}-07$ & $1.1 \mathrm{E}-05$ & $1.5 \mathrm{E}-04$ \\
\hline
\end{tabular}

\section{Relative Risk [ ]}

\begin{tabular}{lccc}
\hline Diet 1 & 1.00011 & 1.0012 & 1.013 \\
Diet 2 & 1.000047 & 1.00044 & 1.0053 \\
Diet 3 & 1.000072 & 1.00062 & 1.0077 \\
Diet 4 & 1.00027 & 1.0028 & 1.042 \\
\hline
\end{tabular}

\section{Probability of Causation [\%]}

\begin{tabular}{llll}
\hline Diet 1 & 0.011 & 0.123 & 1.25 \\
Diet 2 & 0.005 & 0.044 & 0.53 \\
Diet 3 & 0.007 & 0.062 & 0.77 \\
Diet 4 & 0.027 & 0.279 & 3.98 \\
\hline
\end{tabular}

Diet 1 - Backyard cow milk + all other locally produced non-milk exposure pathways

Diet 2 - Locally produced commercial milk + all other locally produced non-milk exposure pathways

Diet 3 - Regionally mixed commercial milk + inhalation (other regionally mixed food items are minor contributors to the total)

Diet 4 - Goat milk (all other exposure pathways are of negligible importance compared to goat milk) 


\section{Location: Oakdale}

\section{Receptor: Male born in 1930}

\begin{tabular}{lccc}
\hline & \multicolumn{3}{c}{ Thyroid Dose [cGy] } \\
Exposure Pathway & 95\% Subjective Confidence Interval \\
\hline Backyard Cow Milk & lower limit & central estimate & upper limit \\
Commercial Milk (locally produced) & 0.11 & 0.63 & 3.8 \\
Commercial Milk (regionally mixed) & 0.023 & 0.15 & 0.94 \\
Goat Milk (locally produced) & 0.056 & 0.3 & 2 \\
Beef (locally produced) & 0.22 & 1.8 & 12 \\
Leafy Vegetables (locally produced) & 0.00041 & 0.0066 & 0.14 \\
Eggs (locally produced) & 0.0001 & 0.0011 & 0.0094 \\
Cottage Cheese (locally produced) & 0.0061 & 0.047 & 0.31 \\
Inhalation & 0.00022 & 0.0025 & 0.026 \\
Mother's milk (mother on Diet 1) & 0.0061 & 0.022 & 0.077 \\
Prenatal exposure (mother on Diet 1) & -- & -- & -- \\
Diet 1 & -- & -- & -- \\
Diet 2 & 0.13 & 0.73 & 4.3 \\
Diet 3 & 0.048 & 0.24 & 1.3 \\
\hline
\end{tabular}

Excess Lifetime Risk [ ]

\begin{tabular}{lllc}
\hline Diet 1 & $2.7 \mathrm{E}-08$ & $1.4 \mathrm{E}-06$ & $4.2 \mathrm{E}-05$ \\
Diet 2 & $1.1 \mathrm{E}-08$ & $5.1 \mathrm{E}-07$ & $1.7 \mathrm{E}-05$ \\
Diet 3 & $1.8 \mathrm{E}-08$ & $7.0 \mathrm{E}-07$ & $2.4 \mathrm{E}-05$ \\
Diet 4 & $8.8 \mathrm{E}-08$ & $3.3 \mathrm{E}-06$ & $1.2 \mathrm{E}-04$ \\
\hline
\end{tabular}

\section{Relative Risk [ ]}

\begin{tabular}{lccc}
\hline Diet 1 & 1.00005 & 1.00097 & 1.023 \\
Diet 2 & 1.000016 & 1.00034 & 1.0079 \\
Diet 3 & 1.000023 & 1.00047 & 1.011 \\
Diet 4 & 1.000091 & 1.0026 & 1.067 \\
\hline
\end{tabular}

\begin{tabular}{llcc}
\hline Diet 1 & 0.005 & 0.097 & 2.28 \\
Diet 2 & 0.002 & 0.034 & 0.79 \\
Diet 3 & 0.002 & 0.047 & 1.09 \\
Diet 4 & 0.009 & 0.254 & 6.21 \\
\hline
\end{tabular}

Diet 1 - Backyard cow milk + all other locally produced non-milk exposure pathways

Diet 2 - Locally produced commercial milk + all other locally produced non-milk exposure pathways

Diet 3 - Regionally mixed commercial milk + inhalation (other regionally mixed food items are minor contributors to the total)

Diet 4 - Goat milk (all other exposure pathways are of negligible importance compared to goat milk) 


\section{Location: Claxton}

Receptor: Female born in 1930

\begin{tabular}{lccc}
\hline & \multicolumn{3}{c}{ Thyroid Dose [cGy] } \\
Exposure Pathway & $95 \%$ Subjective Confidence Interval \\
\hline Backyard Cow Milk & lower limit & central estimate & upper limit \\
Commercial Milk (locally produced) & 0.35 & 1.8 & 11 \\
Commercial Milk (regionally mixed) & 0.068 & 0.41 & 2.6 \\
Goat Milk (locally produced) & 0.042 & 0.25 & 1.5 \\
Beef (locally produced) & 0.68 & 4.7 & 32 \\
Leafy Vegetables (locally produced) & 0.00091 & 0.015 & 0.32 \\
Eggs (locally produced) & 0.00039 & 0.0039 & 0.032 \\
Cottage Cheese (locally produced) & 0.022 & 0.15 & 0.94 \\
Inhalation & 0.00085 & 0.0086 & 0.098 \\
Mother's milk (mother on Diet 1) & 0.02 & 0.064 & 0.22 \\
Prenatal exposure (mother on Diet 1) & -- & -- & - \\
Diet 1 & -- & -- & - \\
Diet 2 & 0.42 & 2.1 & 12 \\
Diet 3 & 0.15 & 0.7 & 3.8 \\
\hline
\end{tabular}

Excess Lifetime Risk [ ]

\begin{tabular}{llll}
\hline Diet 1 & $1.1 \mathrm{E}-06$ & $1.5 \mathrm{E}-05$ & $2.2 \mathrm{E}-04$ \\
Diet 2 & $4.3 \mathrm{E}-07$ & $5.7 \mathrm{E}-06$ & $7.2 \mathrm{E}-05$ \\
Diet 3 & $2.1 \mathrm{E}-07$ & $2.6 \mathrm{E}-06$ & $3.2 \mathrm{E}-05$ \\
Diet 4 & $2.7 \mathrm{E}-06$ & $3.8 \mathrm{E}-05$ & $5.1 \mathrm{E}-04$ \\
\hline
\end{tabular}

Relative Risk [ ]

\begin{tabular}{lccc}
\hline Diet 1 & 1.00043 & 1.0043 & 1.043 \\
Diet 2 & 1.00018 & 1.0016 & 1.018 \\
Diet 3 & 1.000089 & 1.00073 & 1.0083 \\
Diet 4 & 1.0011 & 1.0098 & 1.13 \\
\hline
\end{tabular}

\begin{tabular}{lccc} 
& \multicolumn{3}{c}{ Probability of Causation [\%] } \\
\hline Diet 1 & 0.043 & 0.432 & 4.11 \\
Diet 2 & 0.018 & 0.159 & 1.78 \\
Diet 3 & 0.009 & 0.073 & 0.82 \\
Diet 4 & 0.114 & 0.975 & 11.67 \\
\hline
\end{tabular}

Diet 1 - Backyard cow milk + all other locally produced non-milk exposure pathways

Diet 2 - Locally produced commercial milk + all other locally produced non-milk exposure pathways

Diet 3 - Regionally mixed commercial milk + inhalation (other regionally mixed food items are minor contributors to the total)

Diet 4 - Goat milk (all other exposure pathways are of negligible importance compared to goat milk) 


\section{Location: Claxton}

\section{Receptor: Male born in 1930}

\begin{tabular}{lccc}
\hline & \multicolumn{3}{c}{ Thyroid Dose [cGy] } \\
Exposure Pathway & $95 \%$ Subjective Confidence Interval \\
\hline Backyard Cow Milk & lower limit & central estimate & upper limit \\
Commercial Milk (locally produced) & 0.45 & 2.2 & 12 \\
Commercial Milk (regionally mixed) & 0.092 & 0.51 & 3.1 \\
Goat Milk (locally produced) & 0.056 & 0.3 & 2 \\
Beef (locally produced) & 0.9 & 6.4 & 40 \\
Leafy Vegetables (locally produced) & 0.0014 & 0.024 & 0.5 \\
Eggs (locally produced) & 0.00038 & 0.0038 & 0.034 \\
Cottage Cheese (locally produced) & 0.023 & 0.16 & 0.99 \\
Inhalation & 0.00084 & 0.0088 & 0.091 \\
Mother's milk (mother on Diet 1) & 0.023 & 0.079 & 0.26 \\
Prenatal exposure (mother on Diet 1) & -- & -- & - \\
Diet 1 & -- & -- & - \\
Diet 2 & 0.53 & 2.6 & 14 \\
Diet 3 & 0.18 & 0.85 & 4.8 \\
\hline
\end{tabular}

Excess Lifetime Risk [ ]

\begin{tabular}{llll}
\hline Diet 1 & $1.1 \mathrm{E}-07$ & $5.0 \mathrm{E}-06$ & $1.5 \mathrm{E}-04$ \\
Diet 2 & $4.2 \mathrm{E}-08$ & $1.8 \mathrm{E}-06$ & $5.6 \mathrm{E}-05$ \\
Diet 3 & $2.2 \mathrm{E}-08$ & $8.3 \mathrm{E}-07$ & $2.6 \mathrm{E}-05$ \\
Diet 4 & $3.2 \mathrm{E}-07$ & $1.2 \mathrm{E}-05$ & $3.6 \mathrm{E}-04$ \\
\hline
\end{tabular}

\section{Relative Risk [ ]}

\begin{tabular}{lccc}
\hline Diet 1 & 1.00017 & 1.0035 & 1.083 \\
Diet 2 & 1.000056 & 1.0012 & 1.026 \\
Diet 3 & 1.000029 & 1.00055 & 1.012 \\
Diet 4 & 1.0003 & 1.0091 & 1.23 \\
\hline
\end{tabular}

\begin{tabular}{llcc} 
& \multicolumn{3}{c}{ Probability of Causation [\%] } \\
\hline Diet 1 & 0.017 & 0.345 & 7.68 \\
Diet 2 & 0.006 & 0.122 & 2.54 \\
Diet 3 & 0.003 & 0.055 & 1.23 \\
Diet 4 & 0.030 & 0.897 & 18.34 \\
\hline
\end{tabular}

Diet 1 - Backyard cow milk + all other locally produced non-milk exposure pathways

Diet 2 - Locally produced commercial milk + all other locally produced non-milk exposure pathways

Diet 3 - Regionally mixed commercial milk + inhalation (other regionally mixed food items are minor contributors to the total)

Diet 4 - Goat milk (all other exposure pathways are of negligible importance compared to goat milk) 
Location: Dutch Valley

Receptor: Female born in 1930

\begin{tabular}{lccc}
\hline & \multicolumn{3}{c}{ Thyroid Dose [cGy] } \\
Exposure Pathway & $95 \%$ Subjective Confidence Interval \\
\hline Backyard Cow Milk & lower limit & central estimate & upper limit \\
Commercial Milk (locally produced) & 0.16 & 0.89 & 4.6 \\
Commercial Milk (regionally mixed) & 0.033 & 0.2 & 1.3 \\
Goat Milk (locally produced) & 0.043 & 0.25 & 1.5 \\
Beef (locally produced) & 0.28 & 2.2 & 17 \\
Leafy Vegetables (locally produced) & 0.00043 & 0.0073 & 0.13 \\
Eggs (locally produced) & 0.00019 & 0.0019 & 0.015 \\
Cottage Cheese (locally produced) & 0.011 & 0.075 & 0.46 \\
Inhalation & 0.00038 & 0.0042 & 0.044 \\
Mother's milk (mother on Diet 1) & 0.0092 & 0.031 & 0.11 \\
Prenatal exposure (mother on Diet 1) & -- & -- & -- \\
Diet 1 & -- & -- & -- \\
Diet 2 & 0.19 & 1 & 5.2 \\
Diet 3 & 0.071 & 0.34 & 1.8 \\
\hline
\end{tabular}

Excess Lifetime Risk [ ]

\begin{tabular}{llll}
\hline Diet 1 & $5.0 \mathrm{E}-07$ & $7.2 \mathrm{E}-06$ & $1.1 \mathrm{E}-04$ \\
Diet 2 & $2.1 \mathrm{E}-07$ & $2.7 \mathrm{E}-06$ & $3.7 \mathrm{E}-05$ \\
Diet 3 & $1.7 \mathrm{E}-07$ & $2.3 \mathrm{E}-06$ & $3.0 \mathrm{E}-05$ \\
Diet 4 & $1.2 \mathrm{E}-06$ & $1.7 \mathrm{E}-05$ & $2.8 \mathrm{E}-04$ \\
\hline
\end{tabular}

\section{Relative Risk [ ]}

\begin{tabular}{lccc}
\hline Diet 1 & 1.00022 & 1.002 & 1.022 \\
Diet 2 & 1.000087 & 1.00076 & 1.0089 \\
Diet 3 & 1.000078 & 1.00066 & 1.008 \\
Diet 4 & 1.00053 & 1.005 & 1.067 \\
\hline
\end{tabular}

\begin{tabular}{llcc} 
& \multicolumn{3}{c}{ Probability of Causation [\%] } \\
\hline Diet 1 & 0.022 & 0.203 & 2.14 \\
Diet 2 & 0.009 & 0.076 & 0.88 \\
Diet 3 & 0.008 & 0.066 & 0.79 \\
Diet 4 & 0.053 & 0.499 & 6.28 \\
\hline
\end{tabular}

Diet 1 - Backyard cow milk + all other locally produced non-milk exposure pathways

Diet 2 - Locally produced commercial milk + all other locally produced non-milk exposure pathways

Diet 3 - Regionally mixed commercial milk + inhalation (other regionally mixed food items are minor contributors to the total)

Diet 4 - Goat milk (all other exposure pathways are of negligible importance compared to goat milk) 


\section{Location: Dutch Valley}

Receptor: Male born in 1930

\begin{tabular}{lccc}
\hline & \multicolumn{3}{c}{ Thyroid Dose [cGy] } \\
& \multicolumn{2}{c}{$95 \%$ Subjective Confidence Interval } \\
Exposure Pathway & lower limit & central estimate & upper limit \\
\hline Backyard Cow Milk & 0.2 & 1.1 & 5.4 \\
Commercial Milk (locally produced) & 0.043 & 0.24 & 1.5 \\
Commercial Milk (regionally mixed) & 0.056 & 0.3 & 2 \\
Goat Milk (locally produced) & 0.38 & 3.1 & 20 \\
Beef (locally produced) & 0.00065 & 0.011 & 0.22 \\
Leafy Vegetables (locally produced) & 0.00019 & 0.0018 & 0.015 \\
Eggs (locally produced) & 0.012 & 0.079 & 0.5 \\
Cottage Cheese (locally produced) & 0.00038 & 0.0042 & 0.039 \\
Inhalation & 0.011 & 0.037 & 0.13 \\
Mother's milk (mother on Diet 1) & -- & -- & -- \\
Prenatal exposure (mother on Diet 1) & -- & -- & - \\
Diet 1 & 0.24 & 1.2 & 6.2 \\
Diet 2 & 0.084 & 0.4 & 2.2 \\
Diet 3 & 0.071 & 0.34 & 2 \\
\hline
\end{tabular}

Excess Lifetime Risk [ ]

\begin{tabular}{lllc}
\hline Diet 1 & $5.3 \mathrm{E}-08$ & $2.5 \mathrm{E}-06$ & $6.4 \mathrm{E}-05$ \\
Diet 2 & $1.8 \mathrm{E}-08$ & $8.7 \mathrm{E}-07$ & $2.5 \mathrm{E}-05$ \\
Diet 3 & $1.9 \mathrm{E}-08$ & $7.5 \mathrm{E}-07$ & $2.5 \mathrm{E}-05$ \\
Diet 4 & $1.5 \mathrm{E}-07$ & $5.6 \mathrm{E}-06$ & $1.9 \mathrm{E}-04$ \\
\hline
\end{tabular}

\section{Relative Risk [ ]}

\begin{tabular}{lccc}
\hline Diet 1 & 1.000083 & 1.0016 & 1.037 \\
Diet 2 & 1.000028 & 1.00059 & 1.013 \\
Diet 3 & 1.000025 & 1.00048 & 1.011 \\
Diet 4 & 1.00017 & 1.0044 & 1.098 \\
\hline
\end{tabular}

\section{Probability of Causation [\%]}

\begin{tabular}{llcc}
\hline Diet 1 & 0.008 & 0.164 & 3.55 \\
Diet 2 & 0.003 & 0.059 & 1.29 \\
Diet 3 & 0.002 & 0.048 & 1.13 \\
Diet 4 & 0.017 & 0.437 & 8.86 \\
\hline
\end{tabular}

Diet 1 - Backyard cow milk + all other locally produced non-milk exposure pathways

Diet 2 - Locally produced commercial milk + all other locally produced non-milk exposure pathways

Diet 3 - Regionally mixed commercial milk + inhalation (other regionally mixed food items are minor contributors to the total)

Diet 4 - Goat milk (all other exposure pathways are of negligible importance compared to goat milk) 


\section{Location: Clinton}

Receptor: Female born in 1930

\begin{tabular}{lccc}
\hline & \multicolumn{3}{c}{ Thyroid Dose [cGy] } \\
& \multicolumn{2}{c}{$95 \%$ Subjective Confidence Interval } \\
Exposure Pathway & lower limit & central estimate & upper limit \\
\hline Backyard Cow Milk & 0.26 & 1.4 & 8.2 \\
Commercial Milk (locally produced) & 0.054 & 0.32 & 2 \\
Commercial Milk (regionally mixed) & 0.042 & 0.25 & 1.5 \\
Goat Milk (locally produced) & 0.46 & 3.6 & 25 \\
Beef (locally produced) & 0.0007 & 0.011 & 0.24 \\
Leafy Vegetables (locally produced) & 0.0003 & 0.003 & 0.024 \\
Eggs (locally produced) & 0.018 & 0.12 & 0.71 \\
Cottage Cheese (locally produced) & 0.00064 & 0.0069 & 0.075 \\
Inhalation & 0.015 & 0.05 & 0.17 \\
Mother's milk (mother on Diet 1) & -- & -- & -- \\
Prenatal exposure (mother on Diet 1) & -- & -- & -- \\
Diet 1 & 0.32 & 1.7 & 9.2 \\
Diet 2 & 0.11 & 0.55 & 3 \\
Diet 3 & 0.069 & 0.31 & 1.7 \\
\hline
\end{tabular}

Excess Lifetime Risk [ ]

\begin{tabular}{lllc}
\hline Diet 1 & $7.6 \mathrm{E}-07$ & $1.2 \mathrm{E}-05$ & $1.7 \mathrm{E}-04$ \\
Diet 2 & $3.1 \mathrm{E}-07$ & $4.3 \mathrm{E}-06$ & $5.6 \mathrm{E}-05$ \\
Diet 3 & $1.9 \mathrm{E}-07$ & $2.4 \mathrm{E}-06$ & $3.1 \mathrm{E}-05$ \\
Diet 4 & $1.9 \mathrm{E}-06$ & $2.9 \mathrm{E}-05$ & $4.1 \mathrm{E}-04$ \\
\hline
\end{tabular}

Relative Risk [ ]

\begin{tabular}{lccc}
\hline Diet 1 & 1.00033 & 1.0033 & 1.034 \\
Diet 2 & 1.00013 & 1.0012 & 1.013 \\
Diet 3 & 1.000082 & 1.0007 & 1.0081 \\
Diet 4 & 1.00088 & 1.0076 & 1.11 \\
\hline
\end{tabular}

\section{Probability of Causation [\%]}

\begin{tabular}{llcc}
\hline Diet 1 & 0.033 & 0.328 & 3.29 \\
Diet 2 & 0.013 & 0.123 & 1.31 \\
Diet 3 & 0.008 & 0.070 & 0.81 \\
Diet 4 & 0.088 & 0.756 & 10.04 \\
\hline
\end{tabular}

Diet 1 - Backyard cow milk + all other locally produced non-milk exposure pathways

Diet 2 - Locally produced commercial milk + all other locally produced non-milk exposure pathways

Diet 3 - Regionally mixed commercial milk + inhalation (other regionally mixed food items are minor contributors to the total)

Diet 4 - Goat milk (all other exposure pathways are of negligible importance compared to goat milk) 


\section{Location: Clinton}

\section{Receptor: Male born in 1930}

\begin{tabular}{lccc}
\hline & \multicolumn{3}{c}{ Thyroid Dose [cGy] } \\
& \multicolumn{2}{c}{$95 \%$ Subjective Confidence Interval } \\
Exposure Pathway & lower limit & central estimate & upper limit \\
\hline Backyard Cow Milk & 0.34 & 1.7 & 9.6 \\
Commercial Milk (locally produced) & 0.071 & 0.39 & 2.4 \\
Commercial Milk (regionally mixed) & 0.056 & 0.3 & 2 \\
Goat Milk (locally produced) & 0.67 & 4.9 & 32 \\
Beef (locally produced) & 0.0011 & 0.018 & 0.38 \\
Leafy Vegetables (locally produced) & 0.00029 & 0.003 & 0.024 \\
Eggs (locally produced) & 0.019 & 0.13 & 0.78 \\
Cottage Cheese (locally produced) & 0.00064 & 0.0069 & 0.068 \\
Inhalation & 0.018 & 0.059 & 0.2 \\
Mother's milk (mother on Diet 1) & -- & -- & - \\
Prenatal exposure (mother on Diet 1) & -- & -- & - \\
Diet 1 & 0.39 & 2 & 11 \\
Diet 2 & 0.14 & 0.66 & 3.6 \\
Diet 3 & 0.084 & 0.37 & 2.1 \\
\hline
\end{tabular}

Excess Lifetime Risk [ ]

\begin{tabular}{llll}
\hline Diet 1 & $8.5 \mathrm{E}-08$ & $3.9 \mathrm{E}-06$ & $1.1 \mathrm{E}-04$ \\
Diet 2 & $3.1 \mathrm{E}-08$ & $1.4 \mathrm{E}-06$ & $4.5 \mathrm{E}-05$ \\
Diet 3 & $2.1 \mathrm{E}-08$ & $8.0 \mathrm{E}-07$ & $2.5 \mathrm{E}-05$ \\
Diet 4 & $2.4 \mathrm{E}-07$ & $8.9 \mathrm{E}-06$ & $3.1 \mathrm{E}-04$ \\
\hline
\end{tabular}

\section{Relative Risk [ ]}

\begin{tabular}{lccc}
\hline Diet 1 & 1.00013 & 1.0026 & 1.061 \\
Diet 2 & 1.000044 & 1.00092 & 1.02 \\
Diet 3 & 1.000027 & 1.00053 & 1.012 \\
Diet 4 & 1.00022 & 1.0069 & 1.17 \\
\hline
\end{tabular}

\section{Probability of Causation [\%]}

\begin{tabular}{llcc}
\hline Diet 1 & 0.013 & 0.260 & 5.76 \\
Diet 2 & 0.004 & 0.092 & 1.91 \\
Diet 3 & 0.003 & 0.053 & 1.17 \\
Diet 4 & 0.022 & 0.685 & 14.75 \\
\hline
\end{tabular}

Diet 1 - Backyard cow milk + all other locally produced non-milk exposure pathways

Diet 2 - Locally produced commercial milk + all other locally produced non-milk exposure pathways

Diet 3 - Regionally mixed commercial milk + inhalation (other regionally mixed food items are minor contributors to the total)

Diet 4 - Goat milk (all other exposure pathways are of negligible importance compared to goat milk) 
Location: Friendsville

Receptor: Female born in 1930

\begin{tabular}{lccc}
\hline & \multicolumn{3}{c}{ Thyroid Dose [cGy] } \\
Exposure Pathway & \multicolumn{2}{c}{$95 \%$ Subjective Confidence Interval } \\
\hline Backyard Cow Milk & lower limit & central estimate & upper limit \\
Commercial Milk (locally produced) & 0.14 & 0.8 & 4.5 \\
Commercial Milk (regionally mixed) & 0.029 & 0.19 & 1.1 \\
Goat Milk (locally produced) & 0.042 & 0.25 & 1.5 \\
Beef (locally produced) & 0.29 & 2 & 15 \\
Leafy Vegetables (locally produced) & 0.00041 & 0.0064 & 0.14 \\
Eggs (locally produced) & 0.00016 & 0.0017 & 0.015 \\
Cottage Cheese (locally produced) & 0.0095 & 0.065 & 0.45 \\
Inhalation & 0.00035 & 0.0038 & 0.039 \\
Mother's milk (mother on Diet 1) & 0.0096 & 0.034 & 0.13 \\
Prenatal exposure (mother on Diet 1) & -- & -- & -- \\
Diet 1 & -- & -- & -- \\
Diet 2 & 0.17 & 0.94 & 5.1 \\
Diet 3 & 0.063 & 0.32 & 1.6 \\
\hline
\end{tabular}

Excess Lifetime Risk [ ]

\begin{tabular}{llll}
\hline Diet 1 & $5.1 \mathrm{E}-07$ & $6.9 \mathrm{E}-06$ & $1.0 \mathrm{E}-04$ \\
Diet 2 & $1.9 \mathrm{E}-07$ & $2.5 \mathrm{E}-06$ & $3.1 \mathrm{E}-05$ \\
Diet 3 & $1.8 \mathrm{E}-07$ & $2.3 \mathrm{E}-06$ & $3.0 \mathrm{E}-05$ \\
Diet 4 & $1.1 \mathrm{E}-06$ & $1.7 \mathrm{E}-05$ & $2.5 \mathrm{E}-04$ \\
\hline
\end{tabular}

\section{Relative Risk [ ]}

\begin{tabular}{lccc}
\hline Diet 1 & 1.0002 & 1.0019 & 1.02 \\
Diet 2 & 1.00008 & 1.00071 & 1.0082 \\
Diet 3 & 1.000077 & 1.00066 & 1.008 \\
Diet 4 & 1.00051 & 1.0046 & 1.065 \\
\hline
\end{tabular}

\section{Probability of Causation [\%]}

\begin{tabular}{llll}
\hline Diet 1 & 0.020 & 0.189 & 1.95 \\
Diet 2 & 0.008 & 0.071 & 0.81 \\
Diet 3 & 0.008 & 0.066 & 0.79 \\
Diet 4 & 0.051 & 0.459 & 6.12 \\
\hline
\end{tabular}

Diet 1 - Backyard cow milk + all other locally produced non-milk exposure pathways

Diet 2 - Locally produced commercial milk + all other locally produced non-milk exposure pathways

Diet 3 - Regionally mixed commercial milk + inhalation (other regionally mixed food items are minor contributors to the total)

Diet 4 - Goat milk (all other exposure pathways are of negligible importance compared to goat milk) 
Location: Friendsville

Receptor: Male born in 1930

\begin{tabular}{lccc}
\hline & \multicolumn{3}{c}{ Thyroid Dose [cGy] } \\
& \multicolumn{2}{c}{$95 \%$ Subjective Confidence Interval } \\
Exposure Pathway & lower limit & central estimate & upper limit \\
\hline Backyard Cow Milk & 0.18 & 0.96 & 5.3 \\
Commercial Milk (locally produced) & 0.038 & 0.23 & 1.4 \\
Commercial Milk (regionally mixed) & 0.056 & 0.3 & 2 \\
Goat Milk (locally produced) & 0.37 & 2.8 & 20 \\
Beef (locally produced) & 0.00061 & 0.01 & 0.24 \\
Leafy Vegetables (locally produced) & 0.00015 & 0.0017 & 0.014 \\
Eggs (locally produced) & 0.011 & 0.073 & 0.52 \\
Cottage Cheese (locally produced) & 0.00035 & 0.0038 & 0.036 \\
Inhalation & 0.011 & 0.041 & 0.14 \\
Mother's milk (mother on Diet 1) & -- & -- & -- \\
Prenatal exposure (mother on Diet 1) & -- & -- & -- \\
Diet 1 & 0.22 & 1.1 & 5.9 \\
Diet 2 & 0.074 & 0.38 & 2 \\
Diet 3 & 0.074 & 0.35 & 2.1 \\
\hline
\end{tabular}

Excess Lifetime Risk [ ]

\begin{tabular}{lllc}
\hline Diet 1 & $4.9 \mathrm{E}-08$ & $2.3 \mathrm{E}-06$ & $7.1 \mathrm{E}-05$ \\
Diet 2 & $2.0 \mathrm{E}-08$ & $8.0 \mathrm{E}-07$ & $2.5 \mathrm{E}-05$ \\
Diet 3 & $1.9 \mathrm{E}-08$ & $7.6 \mathrm{E}-07$ & $2.5 \mathrm{E}-05$ \\
Diet 4 & $1.3 \mathrm{E}-07$ & $5.3 \mathrm{E}-06$ & $1.9 \mathrm{E}-04$ \\
\hline
\end{tabular}

\section{Relative Risk [ ]}

\begin{tabular}{lccc}
\hline Diet 1 & 1.000076 & 1.0015 & 1.037 \\
Diet 2 & 1.000027 & 1.00056 & 1.012 \\
Diet 3 & 1.000025 & 1.00049 & 1.011 \\
Diet 4 & 1.00016 & 1.004 & 1.094 \\
\hline
\end{tabular}

\begin{tabular}{llcc} 
& \multicolumn{3}{c}{ Probability of Causation [\%] } \\
\hline Diet 1 & 0.008 & 0.154 & 3.56 \\
Diet 2 & 0.003 & 0.056 & 1.20 \\
Diet 3 & 0.002 & 0.049 & 1.13 \\
Diet 4 & 0.016 & 0.399 & 8.56 \\
\hline
\end{tabular}

Diet 1 - Backyard cow milk + all other locally produced non-milk exposure pathways

Diet 2 - Locally produced commercial milk + all other locally produced non-milk exposure pathways

Diet 3 - Regionally mixed commercial milk + inhalation (other regionally mixed food items are minor contributors to the total)

Diet 4 - Goat milk (all other exposure pathways are of negligible importance compared to goat milk) 
${ }^{131}$ I Releases from X-10 Radioactive Lanthanum Processing

TASK 1 REPORT

Appendix 11-C

July 1999

Location: Wartburg

Receptor: Female born in 1930

\begin{tabular}{lccc}
\hline & \multicolumn{3}{c}{ Thyroid Dose [cGy] } \\
Exposure Pathway & \multicolumn{2}{c}{$95 \%$ Subjective Confidence Interval } \\
\hline Backyard Cow Milk & lower limit & central estimate & upper limit \\
Commercial Milk (locally produced) & 0.034 & 0.22 & 1.3 \\
Commercial Milk (regionally mixed) & 0.0076 & 0.051 & 0.34 \\
Goat Milk (locally produced) & 0.043 & 0.25 & 1.5 \\
Beef (locally produced) & 0.075 & 0.59 & 4.2 \\
Leafy Vegetables (locally produced) & 0.00012 & 0.0018 & 0.042 \\
Eggs (locally produced) & 0.000041 & 0.00047 & 0.0046 \\
Cottage Cheese (locally produced) & 0.0023 & 0.018 & 0.12 \\
Inhalation & 0.000091 & 0.0011 & 0.011 \\
Mother's milk (mother on Diet 1) & 0.0022 & 0.008 & 0.033 \\
Prenatal exposure (mother on Diet 1) & -- & -- & - \\
Diet 1 & -- & -- & -- \\
Diet 2 & 0.043 & 0.26 & 1.4 \\
Diet 3 & 0.015 & 0.086 & 0.51 \\
\hline
\end{tabular}

Excess Lifetime Risk [ ]

\begin{tabular}{llll}
\hline Diet 1 & $1.1 \mathrm{E}-07$ & $1.8 \mathrm{E}-06$ & $3.0 \mathrm{E}-05$ \\
Diet 2 & $5.0 \mathrm{E}-08$ & $6.8 \mathrm{E}-07$ & $1.0 \mathrm{E}-05$ \\
Diet 3 & $1.5 \mathrm{E}-07$ & $2.1 \mathrm{E}-06$ & $2.9 \mathrm{E}-05$ \\
Diet 4 & $3.0 \mathrm{E}-07$ & $4.6 \mathrm{E}-06$ & $8.2 \mathrm{E}-05$ \\
\hline
\end{tabular}

Relative Risk [ ]

\begin{tabular}{lccc}
\hline Diet 1 & 1.000052 & 1.00051 & 1.0066 \\
Diet 2 & 1.00002 & 1.00019 & 1.0027 \\
Diet 3 & 1.000066 & 1.00061 & 1.0076 \\
Diet 4 & 1.00012 & 1.0012 & 1.019 \\
\hline
\end{tabular}

Probability of Causation [\%]

\begin{tabular}{llll}
\hline Diet 1 & 0.005 & 0.051 & 0.66 \\
Diet 2 & 0.002 & 0.019 & 0.27 \\
Diet 3 & 0.007 & 0.060 & 0.75 \\
Diet 4 & 0.012 & 0.122 & 1.88 \\
\hline
\end{tabular}

Diet 1 - Backyard cow milk + all other locally produced non-milk exposure pathways

Diet 2 - Locally produced commercial milk + all other locally produced non-milk exposure pathways

Diet 3 - Regionally mixed commercial milk + inhalation (other regionally mixed food items are minor contributors to the total)

Diet 4 - Goat milk (all other exposure pathways are of negligible importance compared to goat milk) 


\section{Location: Wartburg}

\section{Receptor: Male born in 1930}

\begin{tabular}{lccc}
\hline & \multicolumn{3}{c}{ Thyroid Dose [cGy] } \\
Exposure Pathway & $95 \%$ Subjective Confidence Interval \\
\hline Backyard Cow Milk & lower limit & central estimate & upper limit \\
Commercial Milk (locally produced) & 0.044 & 0.27 & 1.5 \\
Commercial Milk (regionally mixed) & 0.0095 & 0.062 & 0.44 \\
Goat Milk (locally produced) & 0.056 & 0.3 & 2 \\
Beef (locally produced) & 0.1 & 0.78 & 5.5 \\
Leafy Vegetables (locally produced) & 0.00018 & 0.0028 & 0.068 \\
Eggs (locally produced) & 0.000042 & 0.00046 & 0.0043 \\
Cottage Cheese (locally produced) & 0.0025 & 0.02 & 0.13 \\
Inhalation & 0.000088 & 0.0011 & 0.011 \\
Mother's milk (mother on Diet 1) & 0.0025 & 0.0097 & 0.038 \\
Prenatal exposure (mother on Diet 1) & -- & -- & -- \\
Diet 1 & -- & -- & -- \\
Diet 2 & 0.052 & 0.31 & 1.7 \\
Diet 3 & 0.018 & 0.1 & 0.65 \\
\hline
\end{tabular}

Excess Lifetime Risk [ ]

\begin{tabular}{llll}
\hline Diet 1 & $1.2 \mathrm{E}-08$ & $6.0 \mathrm{E}-07$ & $1.9 \mathrm{E}-05$ \\
Diet 2 & $4.8 \mathrm{E}-09$ & $2.2 \mathrm{E}-07$ & $6.4 \mathrm{E}-06$ \\
Diet 3 & $1.7 \mathrm{E}-08$ & $6.7 \mathrm{E}-07$ & $2.4 \mathrm{E}-05$ \\
Diet 4 & $3.5 \mathrm{E}-08$ & $1.4 \mathrm{E}-06$ & $5.4 \mathrm{E}-05$ \\
\hline
\end{tabular}

\section{Relative Risk [ ]}

\begin{tabular}{lccc}
\hline Diet 1 & 1.000018 & 1.00042 & 1.0099 \\
Diet 2 & 1.000006 & 1.00015 & 1.0036 \\
Diet 3 & 1.000021 & 1.00046 & 1.011 \\
Diet 4 & 1.000037 & 1.0011 & 1.03 \\
\hline
\end{tabular}

\section{Probability of Causation [\%]}

\begin{tabular}{llll}
\hline Diet 1 & 0.002 & 0.042 & 0.98 \\
Diet 2 & 0.001 & 0.015 & 0.36 \\
Diet 3 & 0.002 & 0.046 & 1.07 \\
Diet 4 & 0.004 & 0.106 & 2.88 \\
\hline
\end{tabular}

Diet 1 - Backyard cow milk + all other locally produced non-milk exposure pathways

Diet 2 - Locally produced commercial milk + all other locally produced non-milk exposure pathways

Diet 3 - Regionally mixed commercial milk + inhalation (other regionally mixed food items are minor contributors to the total)

Diet 4 - Goat milk (all other exposure pathways are of negligible importance compared to goat milk) 
Location: Rockwood

Receptor: Female born in 1930

\begin{tabular}{lccc}
\hline & \multicolumn{3}{c}{ Thyroid Dose [cGy] } \\
Exposure Pathway & \multicolumn{2}{c}{$95 \%$ Subjective Confidence Interval } \\
\hline Backyard Cow Milk & lower limit & central estimate & upper limit \\
Commercial Milk (locally produced) & 0.17 & 0.9 & 5.4 \\
Commercial Milk (regionally mixed) & 0.035 & 0.21 & 1.4 \\
Goat Milk (locally produced) & 0.043 & 0.25 & 1.5 \\
Beef (locally produced) & 0.34 & 2.3 & 17 \\
Leafy Vegetables (locally produced) & 0.00048 & 0.0074 & 0.17 \\
Eggs (locally produced) & 0.00019 & 0.002 & 0.017 \\
Cottage Cheese (locally produced) & 0.011 & 0.077 & 0.47 \\
Inhalation & 0.00041 & 0.0044 & 0.046 \\
Mother's milk (mother on Diet 1) & 0.01 & 0.035 & 0.12 \\
Prenatal exposure (mother on Diet 1) & -- & -- & -- \\
Diet 1 & -- & -- & -- \\
Diet 2 & 0.21 & 1.1 & 6 \\
Diet 3 & 0.075 & 0.36 & 2.1 \\
\hline
\end{tabular}

Excess Lifetime Risk [ ]

\begin{tabular}{llll}
\hline Diet 1 & $5.1 \mathrm{E}-07$ & $7.8 \mathrm{E}-06$ & $1.2 \mathrm{E}-04$ \\
Diet 2 & $2.1 \mathrm{E}-07$ & $2.9 \mathrm{E}-06$ & $3.5 \mathrm{E}-05$ \\
Diet 3 & $1.8 \mathrm{E}-07$ & $2.3 \mathrm{E}-06$ & $3.0 \mathrm{E}-05$ \\
Diet 4 & $1.3 \mathrm{E}-06$ & $1.9 \mathrm{E}-05$ & $2.8 \mathrm{E}-04$ \\
\hline
\end{tabular}

Relative Risk [ ]

\begin{tabular}{lccc}
\hline Diet 1 & 1.00023 & 1.0022 & 1.023 \\
Diet 2 & 1.000096 & 1.00077 & 1.0091 \\
Diet 3 & 1.00008 & 1.00066 & 1.0079 \\
Diet 4 & 1.00054 & 1.0051 & 1.073 \\
\hline
\end{tabular}

\begin{tabular}{lccc} 
& \multicolumn{3}{c}{ Probability of Causation [\%] } \\
\hline Diet 1 & 0.023 & 0.216 & 2.23 \\
Diet 2 & 0.010 & 0.077 & 0.91 \\
Diet 3 & 0.008 & 0.066 & 0.79 \\
Diet 4 & 0.054 & 0.502 & 6.80 \\
\hline
\end{tabular}

Diet 1 - Backyard cow milk + all other locally produced non-milk exposure pathways

Diet 2 - Locally produced commercial milk + all other locally produced non-milk exposure pathways

Diet 3 - Regionally mixed commercial milk + inhalation (other regionally mixed food items are minor contributors to the total)

Diet 4 - Goat milk (all other exposure pathways are of negligible importance compared to goat milk) 


\section{Location: Rockwood}

\section{Receptor: Male born in 1930}

\begin{tabular}{lccc}
\hline & \multicolumn{3}{c}{ Thyroid Dose [cGy] } \\
& \multicolumn{2}{c}{$95 \%$ Subjective Confidence Interval } \\
Exposure Pathway & lower limit & central estimate & upper limit \\
\hline Backyard Cow Milk & 0.21 & 1.1 & 6.4 \\
Commercial Milk (locally produced) & 0.046 & 0.26 & 1.6 \\
Commercial Milk (regionally mixed) & 0.056 & 0.3 & 2 \\
Goat Milk (locally produced) & 0.43 & 3.3 & 21 \\
Beef (locally produced) & 0.00073 & 0.012 & 0.26 \\
Leafy Vegetables (locally produced) & 0.00019 & 0.0019 & 0.017 \\
Eggs (locally produced) & 0.012 & 0.083 & 0.53 \\
Cottage Cheese (locally produced) & 0.0004 & 0.0044 & 0.044 \\
Inhalation & 0.012 & 0.043 & 0.15 \\
Mother's milk (mother on Diet 1) & -- & -- & - \\
Prenatal exposure (mother on Diet 1) & -- & -- & -- \\
Diet 1 & 0.25 & 1.3 & 7.2 \\
Diet 2 & 0.092 & 0.43 & 2.4 \\
Diet 3 & 0.074 & 0.35 & 2 \\
\hline
\end{tabular}

Excess Lifetime Risk [ ]

\begin{tabular}{lllc}
\hline Diet 1 & $5.9 \mathrm{E}-08$ & $2.4 \mathrm{E}-06$ & $7.3 \mathrm{E}-05$ \\
Diet 2 & $2.3 \mathrm{E}-08$ & $8.5 \mathrm{E}-07$ & $2.8 \mathrm{E}-05$ \\
Diet 3 & $2.0 \mathrm{E}-08$ & $7.5 \mathrm{E}-07$ & $2.5 \mathrm{E}-05$ \\
Diet 4 & $1.6 \mathrm{E}-07$ & $5.6 \mathrm{E}-06$ & $2.0 \mathrm{E}-04$ \\
\hline
\end{tabular}

\section{Relative Risk [ ]}

\begin{tabular}{lccc}
\hline Diet 1 & 1.000082 & 1.0017 & 1.042 \\
Diet 2 & 1.00003 & 1.00062 & 1.014 \\
Diet 3 & 1.000025 & 1.0005 & 1.011 \\
Diet 4 & 1.00015 & 1.0045 & 1.11 \\
\hline
\end{tabular}

\begin{tabular}{llcc} 
& \multicolumn{3}{c}{ Probability of Causation [\%] } \\
\hline Diet 1 & 0.008 & 0.172 & 4.02 \\
Diet 2 & 0.003 & 0.062 & 1.41 \\
Diet 3 & 0.002 & 0.050 & 1.13 \\
Diet 4 & 0.015 & 0.444 & 9.94 \\
\hline
\end{tabular}

Diet 1 - Backyard cow milk + all other locally produced non-milk exposure pathways

Diet 2 - Locally produced commercial milk + all other locally produced non-milk exposure pathways

Diet 3 - Regionally mixed commercial milk + inhalation (other regionally mixed food items are minor contributors to the total)

Diet 4 - Goat milk (all other exposure pathways are of negligible importance compared to goat milk) 
Location: Louisville

Receptor: Female born in 1930

\begin{tabular}{lccc}
\hline & \multicolumn{3}{c}{ Thyroid Dose [cGy] } \\
Exposure Pathway & $95 \%$ Subjective Confidence Interval \\
\hline Backyard Cow Milk & lower limit & central estimate & upper limit \\
Commercial Milk (locally produced) & 0.15 & 0.85 & 4.5 \\
Commercial Milk (regionally mixed) & 0.032 & 0.2 & 1.2 \\
Goat Milk (locally produced) & 0.043 & 0.25 & 1.5 \\
Beef (locally produced) & 0.28 & 2.2 & 16 \\
Leafy Vegetables (locally produced) & 0.00044 & 0.0068 & 0.14 \\
Eggs (locally produced) & 0.00019 & 0.0018 & 0.016 \\
Cottage Cheese (locally produced) & 0.01 & 0.071 & 0.45 \\
Inhalation & 0.00037 & 0.0041 & 0.042 \\
Mother's milk (mother on Diet 1) & 0.01 & 0.035 & 0.13 \\
Prenatal exposure (mother on Diet 1) & -- & -- & -- \\
Diet 1 & -- & -- & -- \\
Diet 2 & 0.18 & 0.99 & 5.1 \\
Diet 3 & 0.068 & 0.33 & 1.8 \\
\hline
\end{tabular}

Excess Lifetime Risk [ ]

\begin{tabular}{llll}
\hline Diet 1 & $5.0 \mathrm{E}-07$ & $7.0 \mathrm{E}-06$ & $1.0 \mathrm{E}-04$ \\
Diet 2 & $2.1 \mathrm{E}-07$ & $2.6 \mathrm{E}-06$ & $3.6 \mathrm{E}-05$ \\
Diet 3 & $1.7 \mathrm{E}-07$ & $2.3 \mathrm{E}-06$ & $3.0 \mathrm{E}-05$ \\
Diet 4 & $1.2 \mathrm{E}-06$ & $1.7 \mathrm{E}-05$ & $2.9 \mathrm{E}-04$ \\
\hline
\end{tabular}

Relative Risk [ ]

\begin{tabular}{lccc}
\hline Diet 1 & 1.00022 & 1.002 & 1.022 \\
Diet 2 & 1.000087 & 1.00073 & 1.0089 \\
Diet 3 & 1.000079 & 1.00067 & 1.008 \\
Diet 4 & 1.00051 & 1.0048 & 1.068 \\
\hline
\end{tabular}

\begin{tabular}{llcc} 
& \multicolumn{3}{c}{ Probability of Causation [\%] } \\
\hline Diet 1 & 0.022 & 0.199 & 2.15 \\
Diet 2 & 0.009 & 0.073 & 0.88 \\
Diet 3 & 0.008 & 0.067 & 0.80 \\
Diet 4 & 0.051 & 0.480 & 6.33 \\
\hline
\end{tabular}

Diet 1 - Backyard cow milk + all other locally produced non-milk exposure pathways

Diet 2 - Locally produced commercial milk + all other locally produced non-milk exposure pathways

Diet 3 - Regionally mixed commercial milk + inhalation (other regionally mixed food items are minor contributors to the total)

Diet 4 - Goat milk (all other exposure pathways are of negligible importance compared to goat milk) 


\section{Location: Louisville}

\section{Receptor: Male born in 1930}

\begin{tabular}{lccc}
\hline & \multicolumn{3}{c}{ Thyroid Dose [cGy] } \\
& \multicolumn{2}{c}{$95 \%$ Subjective Confidence Interval } \\
Exposure Pathway & lower limit & central estimate & upper limit \\
\hline Backyard Cow Milk & 0.18 & 1 & 5.2 \\
Commercial Milk (locally produced) & 0.041 & 0.24 & 1.5 \\
Commercial Milk (regionally mixed) & 0.056 & 0.3 & 2 \\
Goat Milk (locally produced) & 0.38 & 2.9 & 20 \\
Beef (locally produced) & 0.00067 & 0.011 & 0.22 \\
Leafy Vegetables (locally produced) & 0.00018 & 0.0018 & 0.015 \\
Eggs (locally produced) & 0.011 & 0.076 & 0.48 \\
Cottage Cheese (locally produced) & 0.00036 & 0.004 & 0.038 \\
Inhalation & 0.012 & 0.042 & 0.15 \\
Mother's milk (mother on Diet 1) & -- & -- & - \\
Prenatal exposure (mother on Diet 1) & -- & -- & -- \\
Diet 1 & 0.22 & 1.2 & 5.9 \\
Diet 2 & 0.083 & 0.4 & 2.2 \\
Diet 3 & 0.073 & 0.35 & 2 \\
\hline
\end{tabular}

Excess Lifetime Risk [ ]

\begin{tabular}{llll}
\hline Diet 1 & $5.2 \mathrm{E}-08$ & $2.3 \mathrm{E}-06$ & $6.5 \mathrm{E}-05$ \\
Diet 2 & $1.9 \mathrm{E}-08$ & $8.5 \mathrm{E}-07$ & $2.4 \mathrm{E}-05$ \\
Diet 3 & $1.9 \mathrm{E}-08$ & $7.5 \mathrm{E}-07$ & $2.5 \mathrm{E}-05$ \\
Diet 4 & $1.4 \mathrm{E}-07$ & $5.5 \mathrm{E}-06$ & $1.9 \mathrm{E}-04$ \\
\hline
\end{tabular}

\section{Relative Risk [ ]}

\begin{tabular}{lccc}
\hline Diet 1 & 1.000072 & 1.0016 & 1.035 \\
Diet 2 & 1.000026 & 1.00058 & 1.013 \\
Diet 3 & 1.000025 & 1.0005 & 1.012 \\
Diet 4 & 1.00015 & 1.0042 & 1.1 \\
\hline
\end{tabular}

\begin{tabular}{llcc} 
& \multicolumn{3}{c}{ Probability of Causation [\%] } \\
\hline Diet 1 & 0.007 & 0.162 & 3.39 \\
Diet 2 & 0.003 & 0.058 & 1.30 \\
Diet 3 & 0.003 & 0.050 & 1.15 \\
Diet 4 & 0.015 & 0.416 & 9.17 \\
\hline
\end{tabular}

Diet 1 - Backyard cow milk + all other locally produced non-milk exposure pathways

Diet 2 - Locally produced commercial milk + all other locally produced non-milk exposure pathways

Diet 3 - Regionally mixed commercial milk + inhalation (other regionally mixed food items are minor contributors to the total)

Diet 4 - Goat milk (all other exposure pathways are of negligible importance compared to goat milk) 
Location: Barnardville

Receptor: Female born in 1930

\begin{tabular}{lccc}
\hline & \multicolumn{3}{c}{ Thyroid Dose [cGy] } \\
Exposure Pathway & \multicolumn{2}{c}{ 95\% } & Subjective Confidence Interval \\
Backyard Cow Milk & lower limit & central estimate & upper limit \\
Commercial Milk (locally produced) & 0.17 & 0.94 & 5.4 \\
Commercial Milk (regionally mixed) & 0.036 & 0.22 & 1.3 \\
Goat Milk (locally produced) & 0.043 & 0.25 & 1.5 \\
Beef (locally produced) & 0.33 & 2.5 & 18 \\
Leafy Vegetables (locally produced) & 0.00051 & 0.0078 & 0.16 \\
Eggs (locally produced) & 0.0002 & 0.002 & 0.018 \\
Cottage Cheese (locally produced) & 0.011 & 0.081 & 0.51 \\
Inhalation & 0.0004 & 0.0046 & 0.05 \\
Mother's milk (mother on Diet 1) & 0.012 & 0.041 & 0.15 \\
Prenatal exposure (mother on Diet 1) & -- & -- & -- \\
Diet 1 & -- & -- & -- \\
Diet 2 & 0.21 & 1.1 & 6.1 \\
Diet 3 & 0.078 & 0.38 & 2.1 \\
\hline
\end{tabular}

Excess Lifetime Risk [ ]

\begin{tabular}{llll}
\hline Diet 1 & $5.7 \mathrm{E}-07$ & $8.4 \mathrm{E}-06$ & $1.2 \mathrm{E}-04$ \\
Diet 2 & $2.2 \mathrm{E}-07$ & $3.0 \mathrm{E}-06$ & $4.0 \mathrm{E}-05$ \\
Diet 3 & $1.8 \mathrm{E}-07$ & $2.4 \mathrm{E}-06$ & $3.0 \mathrm{E}-05$ \\
Diet 4 & $1.5 \mathrm{E}-06$ & $2.0 \mathrm{E}-05$ & $3.1 \mathrm{E}-04$ \\
\hline
\end{tabular}

\section{Relative Risk [ ]}

\begin{tabular}{lccc}
\hline Diet 1 & 1.00024 & 1.0023 & 1.024 \\
Diet 2 & 1.000096 & 1.00082 & 1.0098 \\
Diet 3 & 1.00008 & 1.00068 & 1.0081 \\
Diet 4 & 1.00056 & 1.0054 & 1.081 \\
\hline
\end{tabular}

\begin{tabular}{llcc} 
& \multicolumn{3}{c}{ Probability of Causation [\%] } \\
\hline Diet 1 & 0.024 & 0.225 & 2.33 \\
Diet 2 & 0.010 & 0.082 & 0.97 \\
Diet 3 & 0.008 & 0.068 & 0.80 \\
Diet 4 & 0.056 & 0.536 & 7.50 \\
\hline
\end{tabular}

Diet 1 - Backyard cow milk + all other locally produced non-milk exposure pathways

Diet 2 - Locally produced commercial milk + all other locally produced non-milk exposure pathways

Diet 3 - Regionally mixed commercial milk + inhalation (other regionally mixed food items are minor contributors to the total)

Diet 4 - Goat milk (all other exposure pathways are of negligible importance compared to goat milk) 


\section{Location: Barnardville}

Receptor: Male born in 1930

\begin{tabular}{lccc}
\hline & \multicolumn{3}{c}{ Thyroid Dose [cGy] } \\
Exposure Pathway & \multicolumn{2}{c}{$95 \%$ Subjective Confidence Interval } \\
\hline Backyard Cow Milk & lower limit & central estimate & upper limit \\
Commercial Milk (locally produced) & 0.22 & 1.2 & 6.5 \\
Commercial Milk (regionally mixed) & 0.046 & 0.27 & 1.7 \\
Goat Milk (locally produced) & 0.056 & 0.3 & 2 \\
Beef (locally produced) & 0.45 & 3.4 & 21 \\
Leafy Vegetables (locally produced) & 0.00076 & 0.012 & 0.25 \\
Eggs (locally produced) & 0.00019 & 0.002 & 0.017 \\
Cottage Cheese (locally produced) & 0.012 & 0.088 & 0.55 \\
Inhalation & 0.0004 & 0.0047 & 0.045 \\
Mother's milk (mother on Diet 1) & 0.014 & 0.049 & 0.18 \\
Prenatal exposure (mother on Diet 1) & -- & -- & - \\
Diet 1 & -- & -- & -- \\
Diet 2 & 0.27 & 1.4 & 7.3 \\
Diet 3 & 0.098 & 0.46 & 2.6 \\
\hline
\end{tabular}

Excess Lifetime Risk [ ]

\begin{tabular}{lllc}
\hline Diet 1 & $6.1 \mathrm{E}-08$ & $2.6 \mathrm{E}-06$ & $7.6 \mathrm{E}-05$ \\
Diet 2 & $2.3 \mathrm{E}-08$ & $9.4 \mathrm{E}-07$ & $2.8 \mathrm{E}-05$ \\
Diet 3 & $2.0 \mathrm{E}-08$ & $7.6 \mathrm{E}-07$ & $2.5 \mathrm{E}-05$ \\
Diet 4 & $1.7 \mathrm{E}-07$ & $6.2 \mathrm{E}-06$ & $2.1 \mathrm{E}-04$ \\
\hline
\end{tabular}

\section{Relative Risk [ ]}

\begin{tabular}{lccc}
\hline Diet 1 & 1.000086 & 1.0019 & 1.045 \\
Diet 2 & 1.000031 & 1.00066 & 1.015 \\
Diet 3 & 1.000026 & 1.00052 & 1.012 \\
Diet 4 & 1.00017 & 1.0046 & 1.13 \\
\hline
\end{tabular}

\begin{tabular}{llcc} 
& \multicolumn{3}{c}{ Probability of Causation [\%] } \\
\hline Diet 1 & 0.009 & 0.192 & 4.26 \\
Diet 2 & 0.003 & 0.066 & 1.48 \\
Diet 3 & 0.003 & 0.052 & 1.15 \\
Diet 4 & 0.017 & 0.459 & 11.69 \\
\hline
\end{tabular}

Diet 1 - Backyard cow milk + all other locally produced non-milk exposure pathways

Diet 2 - Locally produced commercial milk + all other locally produced non-milk exposure pathways

Diet 3 - Regionally mixed commercial milk + inhalation (other regionally mixed food items are minor contributors to the total)

Diet 4 - Goat milk (all other exposure pathways are of negligible importance compared to goat milk) 
Location: Greenback

Receptor: Female born in 1930

\begin{tabular}{lccc}
\hline & \multicolumn{3}{c}{ Thyroid Dose [cGy] } \\
& \multicolumn{2}{c}{$95 \%$ Subjective Confidence Interval } \\
Exposure Pathway & lower limit & central estimate & upper limit \\
\hline Backyard Cow Milk & 0.099 & 0.51 & 3.2 \\
Commercial Milk (locally produced) & 0.018 & 0.12 & 0.83 \\
Commercial Milk (regionally mixed) & 0.043 & 0.25 & 1.5 \\
Goat Milk (locally produced) & 0.18 & 1.4 & 10 \\
Beef (locally produced) & 0.00029 & 0.0044 & 0.087 \\
Leafy Vegetables (locally produced) & 0.00011 & 0.0011 & 0.01 \\
Eggs (locally produced) & 0.0064 & 0.044 & 0.3 \\
Cottage Cheese (locally produced) & 0.00026 & 0.0025 & 0.028 \\
Inhalation & 0.0069 & 0.023 & 0.087 \\
Mother's milk (mother on Diet 1) & -- & -- & -- \\
Prenatal exposure (mother on Diet 1) & -- & -- & -- \\
Diet 1 & 0.12 & 0.6 & 3.6 \\
Diet 2 & 0.043 & 0.21 & 1.2 \\
Diet 3 & 0.057 & 0.28 & 1.6 \\
\hline
\end{tabular}

Excess Lifetime Risk [ ]

\begin{tabular}{llll}
\hline Diet 1 & $3.2 \mathrm{E}-07$ & $4.4 \mathrm{E}-06$ & $7.1 \mathrm{E}-05$ \\
Diet 2 & $1.4 \mathrm{E}-07$ & $1.7 \mathrm{E}-06$ & $2.4 \mathrm{E}-05$ \\
Diet 3 & $1.7 \mathrm{E}-07$ & $2.2 \mathrm{E}-06$ & $3.0 \mathrm{E}-05$ \\
Diet 4 & $7.9 \mathrm{E}-07$ & $1.0 \mathrm{E}-05$ & $1.8 \mathrm{E}-04$ \\
\hline
\end{tabular}

Relative Risk [ ]

\begin{tabular}{lccc}
\hline Diet 1 & 1.00013 & 1.0012 & 1.014 \\
Diet 2 & 1.000053 & 1.00045 & 1.0058 \\
Diet 3 & 1.000075 & 1.00064 & 1.0079 \\
Diet 4 & 1.00029 & 1.0029 & 1.046 \\
\hline
\end{tabular}

\begin{tabular}{llcc} 
& \multicolumn{3}{c}{ Probability of Causation [\%] } \\
\hline Diet 1 & 0.013 & 0.122 & 1.42 \\
Diet 2 & 0.005 & 0.045 & 0.58 \\
Diet 3 & 0.007 & 0.064 & 0.78 \\
Diet 4 & 0.029 & 0.288 & 4.35 \\
\hline
\end{tabular}

Diet 1 - Backyard cow milk + all other locally produced non-milk exposure pathways

Diet 2 - Locally produced commercial milk + all other locally produced non-milk exposure pathways

Diet 3 - Regionally mixed commercial milk + inhalation (other regionally mixed food items are minor contributors to the total)

Diet 4 - Goat milk (all other exposure pathways are of negligible importance compared to goat milk) 
Location: Greenback

Receptor: Male born in 1930

\begin{tabular}{lccc}
\hline & \multicolumn{3}{c}{ Thyroid Dose [cGy] } \\
& \multicolumn{2}{c}{$95 \%$ Subjective Confidence Interval } \\
Exposure Pathway & lower limit & central estimate & upper limit \\
\hline Backyard Cow Milk & 0.12 & 0.64 & 3.8 \\
Commercial Milk (locally produced) & 0.024 & 0.15 & 1 \\
Commercial Milk (regionally mixed) & 0.056 & 0.3 & 2 \\
Goat Milk (locally produced) & 0.22 & 1.8 & 13 \\
Beef (locally produced) & 0.00042 & 0.007 & 0.14 \\
Leafy Vegetables (locally produced) & 0.00011 & 0.0011 & 0.011 \\
Eggs (locally produced) & 0.0068 & 0.047 & 0.31 \\
Cottage Cheese (locally produced) & 0.0002 & 0.0025 & 0.027 \\
Inhalation & 0.0085 & 0.028 & 0.11 \\
Mother's milk (mother on Diet 1) & -- & -- & -- \\
Prenatal exposure (mother on Diet 1) & -- & -- & - \\
Diet 1 & 0.15 & 0.75 & 4.3 \\
Diet 2 & 0.052 & 0.25 & 1.5 \\
Diet 3 & 0.069 & 0.33 & 2 \\
\hline
\end{tabular}

Excess Lifetime Risk [ ]

\begin{tabular}{lllc}
\hline Diet 1 & $3.2 \mathrm{E}-08$ & $1.4 \mathrm{E}-06$ & $3.9 \mathrm{E}-05$ \\
Diet 2 & $1.2 \mathrm{E}-08$ & $5.1 \mathrm{E}-07$ & $1.7 \mathrm{E}-05$ \\
Diet 3 & $1.9 \mathrm{E}-08$ & $7.2 \mathrm{E}-07$ & $2.4 \mathrm{E}-05$ \\
Diet 4 & $9.3 \mathrm{E}-08$ & $3.3 \mathrm{E}-06$ & $1.2 \mathrm{E}-04$ \\
\hline
\end{tabular}

\section{Relative Risk [ ]}

\begin{tabular}{lccc}
\hline Diet 1 & 1.000041 & 1.001 & 1.025 \\
Diet 2 & 1.000015 & 1.00039 & 1.0088 \\
Diet 3 & 1.000024 & 1.00048 & 1.011 \\
Diet 4 & 1.00009 & 1.0026 & 1.058 \\
\hline
\end{tabular}

\begin{tabular}{llcc} 
& \multicolumn{3}{c}{ Probability of Causation [\%] } \\
\hline Diet 1 & 0.004 & 0.105 & 2.44 \\
Diet 2 & 0.002 & 0.039 & 0.88 \\
Diet 3 & 0.002 & 0.048 & 1.10 \\
Diet 4 & 0.009 & 0.264 & 5.49 \\
\hline
\end{tabular}

Diet 1 - Backyard cow milk + all other locally produced non-milk exposure pathways

Diet 2 - Locally produced commercial milk + all other locally produced non-milk exposure pathways

Diet 3 - Regionally mixed commercial milk + inhalation (other regionally mixed food items are minor contributors to the total)

Diet 4 - Goat milk (all other exposure pathways are of negligible importance compared to goat milk) 


\section{Location: Rockford}

Receptor: Female born in 1930

\begin{tabular}{lccc}
\hline & \multicolumn{3}{c}{ Thyroid Dose [cGy] } \\
& \multicolumn{2}{c}{$95 \%$ Subjective Confidence Interval } \\
Exposure Pathway & lower limit & central estimate & upper limit \\
\hline Backyard Cow Milk & 0.16 & 0.81 & 4.6 \\
Commercial Milk (locally produced) & 0.03 & 0.19 & 1.2 \\
Commercial Milk (regionally mixed) & 0.043 & 0.25 & 1.5 \\
Goat Milk (locally produced) & 0.29 & 2.1 & 15 \\
Beef (locally produced) & 0.00043 & 0.0068 & 0.14 \\
Leafy Vegetables (locally produced) & 0.00017 & 0.0018 & 0.015 \\
Eggs (locally produced) & 0.01 & 0.069 & 0.42 \\
Cottage Cheese (locally produced) & 0.00038 & 0.004 & 0.043 \\
Inhalation & 0.011 & 0.035 & 0.12 \\
Mother's milk (mother on Diet 1) & -- & -- & -- \\
Prenatal exposure (mother on Diet 1) & -- & -- & -- \\
Diet 1 & 0.2 & 0.95 & 5.2 \\
Diet 2 & 0.068 & 0.33 & 1.7 \\
Diet 3 & 0.062 & 0.29 & 1.6 \\
\hline
\end{tabular}

Excess Lifetime Risk [ ]

\begin{tabular}{llll}
\hline Diet 1 & $5.0 \mathrm{E}-07$ & $7.0 \mathrm{E}-06$ & $9.5 \mathrm{E}-05$ \\
Diet 2 & $2.1 \mathrm{E}-07$ & $2.7 \mathrm{E}-06$ & $3.4 \mathrm{E}-05$ \\
Diet 3 & $1.8 \mathrm{E}-07$ & $2.3 \mathrm{E}-06$ & $3.1 \mathrm{E}-05$ \\
Diet 4 & $1.2 \mathrm{E}-06$ & $1.7 \mathrm{E}-05$ & $2.4 \mathrm{E}-04$ \\
\hline
\end{tabular}

Relative Risk [ ]

\begin{tabular}{lccc}
\hline Diet 1 & 1.0002 & 1.002 & 1.02 \\
Diet 2 & 1.000084 & 1.00072 & 1.0082 \\
Diet 3 & 1.000078 & 1.00067 & 1.008 \\
Diet 4 & 1.00053 & 1.0045 & 1.063 \\
\hline
\end{tabular}

\begin{tabular}{llcc} 
& \multicolumn{3}{c}{ Probability of Causation [\%] } \\
\hline Diet 1 & 0.020 & 0.196 & 1.99 \\
Diet 2 & 0.008 & 0.072 & 0.81 \\
Diet 3 & 0.008 & 0.067 & 0.79 \\
Diet 4 & 0.053 & 0.444 & 5.91 \\
\hline
\end{tabular}

Diet 1 - Backyard cow milk + all other locally produced non-milk exposure pathways

Diet 2 - Locally produced commercial milk + all other locally produced non-milk exposure pathways

Diet 3 - Regionally mixed commercial milk + inhalation (other regionally mixed food items are minor contributors to the total)

Diet 4 - Goat milk (all other exposure pathways are of negligible importance compared to goat milk) 


\section{Location: Rockford}

\section{Receptor: Male born in 1930}

\begin{tabular}{lccc}
\hline & \multicolumn{3}{c}{ Thyroid Dose [cGy] } \\
Exposure Pathway & $95 \%$ Subjective Confidence Interval \\
\hline Backyard Cow Milk & lower limit & central estimate & upper limit \\
Commercial Milk (locally produced) & 0.2 & 1 & 5.5 \\
Commercial Milk (regionally mixed) & 0.04 & 0.23 & 1.4 \\
Goat Milk (locally produced) & 0.056 & 0.3 & 2 \\
Beef (locally produced) & 0.39 & 2.9 & 19 \\
Leafy Vegetables (locally produced) & 0.00066 & 0.011 & 0.22 \\
Eggs (locally produced) & 0.00018 & 0.0017 & 0.015 \\
Cottage Cheese (locally produced) & 0.011 & 0.075 & 0.45 \\
Inhalation & 0.00035 & 0.004 & 0.039 \\
Mother's milk (mother on Diet 1) & 0.013 & 0.042 & 0.15 \\
Prenatal exposure (mother on Diet 1) & -- & -- & -- \\
Diet 1 & -- & -- & -- \\
Diet 2 & 0.24 & 1.2 & 6.3 \\
Diet 3 & 0.089 & 0.4 & 2.1 \\
\hline
\end{tabular}

Excess Lifetime Risk [ ]

\begin{tabular}{llll}
\hline Diet 1 & $5.1 \mathrm{E}-08$ & $2.3 \mathrm{E}-06$ & $6.5 \mathrm{E}-05$ \\
Diet 2 & $1.9 \mathrm{E}-08$ & $8.3 \mathrm{E}-07$ & $2.7 \mathrm{E}-05$ \\
Diet 3 & $2.0 \mathrm{E}-08$ & $7.6 \mathrm{E}-07$ & $2.5 \mathrm{E}-05$ \\
Diet 4 & $1.4 \mathrm{E}-07$ & $5.2 \mathrm{E}-06$ & $1.9 \mathrm{E}-04$ \\
\hline
\end{tabular}

\section{Relative Risk [ ]}

\begin{tabular}{lccc}
\hline Diet 1 & 1.000077 & 1.0016 & 1.037 \\
Diet 2 & 1.000026 & 1.00058 & 1.013 \\
Diet 3 & 1.000026 & 1.0005 & 1.011 \\
Diet 4 & 1.00014 & 1.0041 & 1.1 \\
\hline
\end{tabular}

\section{Probability of Causation [\%]}

\begin{tabular}{llll}
\hline Diet 1 & 0.008 & 0.159 & 3.56 \\
Diet 2 & 0.003 & 0.058 & 1.31 \\
Diet 3 & 0.003 & 0.050 & 1.13 \\
Diet 4 & 0.014 & 0.409 & 9.41 \\
\hline
\end{tabular}

Diet 1 - Backyard cow milk + all other locally produced non-milk exposure pathways

Diet 2 - Locally produced commercial milk + all other locally produced non-milk exposure pathways

Diet 3 - Regionally mixed commercial milk + inhalation (other regionally mixed food items are minor contributors to the total)

Diet 4 - Goat milk (all other exposure pathways are of negligible importance compared to goat milk) 
Location: Lake City

Receptor: Female born in 1930

\begin{tabular}{lccc}
\hline & \multicolumn{3}{c}{ Thyroid Dose [cGy] } \\
Exposure Pathway & \multicolumn{2}{c}{ 95\% } & Subjective Confidence Interval \\
Backyard Cow Milk & lower limit & central estimate & upper limit \\
Commercial Milk (locally produced) & 0.098 & 0.5 & 2.7 \\
Commercial Milk (regionally mixed) & 0.019 & 0.12 & 0.71 \\
Goat Milk (locally produced) & 0.043 & 0.25 & 1.5 \\
Beef (locally produced) & 0.2 & 1.3 & 9.1 \\
Leafy Vegetables (locally produced) & 0.00026 & 0.0042 & 0.096 \\
Eggs (locally produced) & 0.00011 & 0.0011 & 0.0093 \\
Cottage Cheese (locally produced) & 0.0057 & 0.042 & 0.26 \\
Inhalation & 0.00024 & 0.0024 & 0.026 \\
Mother's milk (mother on Diet 1) & 0.0055 & 0.019 & 0.07 \\
Prenatal exposure (mother on Diet 1) & -- & -- & -- \\
Diet 1 & -- & -- & - \\
Diet 2 & 0.12 & 0.58 & 3.1 \\
Diet 3 & 0.041 & 0.2 & 1.1 \\
\hline
\end{tabular}

Excess Lifetime Risk [ ]

\begin{tabular}{llll}
\hline Diet 1 & $3.1 \mathrm{E}-07$ & $4.1 \mathrm{E}-06$ & $6.5 \mathrm{E}-05$ \\
Diet 2 & $1.2 \mathrm{E}-07$ & $1.6 \mathrm{E}-06$ & $2.1 \mathrm{E}-05$ \\
Diet 3 & $1.6 \mathrm{E}-07$ & $2.2 \mathrm{E}-06$ & $3.0 \mathrm{E}-05$ \\
Diet 4 & $7.5 \mathrm{E}-07$ & $1.1 \mathrm{E}-05$ & $1.6 \mathrm{E}-04$ \\
\hline
\end{tabular}

Relative Risk [ ]

\begin{tabular}{lccc}
\hline Diet 1 & 1.00013 & 1.0012 & 1.013 \\
Diet 2 & 1.00005 & 1.00044 & 1.0054 \\
Diet 3 & 1.000072 & 1.00063 & 1.0077 \\
Diet 4 & 1.0003 & 1.0028 & 1.038 \\
\hline
\end{tabular}

Probability of Causation [\%]

\begin{tabular}{llll}
\hline Diet 1 & 0.013 & 0.120 & 1.24 \\
Diet 2 & 0.005 & 0.044 & 0.54 \\
Diet 3 & 0.007 & 0.063 & 0.77 \\
Diet 4 & 0.030 & 0.283 & 3.67 \\
\hline
\end{tabular}

Diet 1 - Backyard cow milk + all other locally produced non-milk exposure pathways

Diet 2 - Locally produced commercial milk + all other locally produced non-milk exposure pathways

Diet 3 - Regionally mixed commercial milk + inhalation (other regionally mixed food items are minor contributors to the total)

Diet 4 - Goat milk (all other exposure pathways are of negligible importance compared to goat milk) 
Location: Lake City

Receptor: Male born in 1930

\begin{tabular}{lccc}
\hline & \multicolumn{3}{c}{ Thyroid Dose [cGy] } \\
& \multicolumn{2}{c}{$95 \%$ Subjective Confidence Interval } \\
Exposure Pathway & lower limit & central estimate & upper limit \\
\hline Backyard Cow Milk & 0.12 & 0.61 & 3.1 \\
Commercial Milk (locally produced) & 0.026 & 0.14 & 0.93 \\
Commercial Milk (regionally mixed) & 0.056 & 0.3 & 2 \\
Goat Milk (locally produced) & 0.25 & 1.8 & 11 \\
Beef (locally produced) & 0.00039 & 0.0067 & 0.15 \\
Leafy Vegetables (locally produced) & 0.0001 & 0.0011 & 0.0093 \\
Eggs (locally produced) & 0.0063 & 0.045 & 0.27 \\
Cottage Cheese (locally produced) & 0.00024 & 0.0024 & 0.025 \\
Inhalation & 0.0067 & 0.023 & 0.081 \\
Mother's milk (mother on Diet 1) & -- & -- & -- \\
Prenatal exposure (mother on Diet 1) & -- & -- & - \\
Diet 1 & 0.14 & 0.7 & 3.5 \\
Diet 2 & 0.051 & 0.23 & 1.3 \\
Diet 3 & 0.066 & 0.33 & 2 \\
\hline
\end{tabular}

Excess Lifetime Risk [ ]

\begin{tabular}{lllc}
\hline Diet 1 & $3.0 \mathrm{E}-08$ & $1.4 \mathrm{E}-06$ & $4.4 \mathrm{E}-05$ \\
Diet 2 & $1.3 \mathrm{E}-08$ & $5.2 \mathrm{E}-07$ & $1.6 \mathrm{E}-05$ \\
Diet 3 & $1.8 \mathrm{E}-08$ & $7.1 \mathrm{E}-07$ & $2.4 \mathrm{E}-05$ \\
Diet 4 & $8.9 \mathrm{E}-08$ & $3.3 \mathrm{E}-06$ & $1.1 \mathrm{E}-04$ \\
\hline
\end{tabular}

\section{Relative Risk [ ]}

\begin{tabular}{lccc}
\hline Diet 1 & 1.000043 & 1.00093 & 1.022 \\
Diet 2 & 1.000015 & 1.00035 & 1.0076 \\
Diet 3 & 1.000022 & 1.00047 & 1.011 \\
Diet 4 & 1.000093 & 1.0025 & 1.061 \\
\hline
\end{tabular}

\begin{tabular}{lccc} 
& \multicolumn{3}{c}{ Probability of Causation [\%] } \\
\hline Diet 1 & 0.004 & 0.093 & 2.11 \\
Diet 2 & 0.001 & 0.035 & 0.76 \\
Diet 3 & 0.002 & 0.047 & 1.10 \\
Diet 4 & 0.009 & 0.248 & 5.74 \\
\hline
\end{tabular}

Diet 1 - Backyard cow milk + all other locally produced non-milk exposure pathways

Diet 2 - Locally produced commercial milk + all other locally produced non-milk exposure pathways

Diet 3 - Regionally mixed commercial milk + inhalation (other regionally mixed food items are minor contributors to the total)

Diet 4 - Goat milk (all other exposure pathways are of negligible importance compared to goat milk) 
Location: Sweetwater

Receptor: Female born in 1930

\begin{tabular}{lccc}
\hline & \multicolumn{3}{c}{ Thyroid Dose [cGy] } \\
Exposure Pathway & \multicolumn{2}{c}{$95 \%$ Subjective Confidence Interval } \\
\hline Backyard Cow Milk & lower limit & central estimate & upper limit \\
Commercial Milk (locally produced) & 0.098 & 0.63 & 3.2 \\
Commercial Milk (regionally mixed) & 0.024 & 0.15 & 0.91 \\
Goat Milk (locally produced) & 0.042 & 0.25 & 1.5 \\
Beef (locally produced) & 0.18 & 1.5 & 12 \\
Leafy Vegetables (locally produced) & 0.00032 & 0.005 & 0.091 \\
Eggs (locally produced) & 0.00014 & 0.0013 & 0.012 \\
Cottage Cheese (locally produced) & 0.0076 & 0.051 & 0.33 \\
Inhalation & 0.00027 & 0.003 & 0.03 \\
Mother's milk (mother on Diet 1) & 0.0079 & 0.027 & 0.098 \\
Prenatal exposure (mother on Diet 1) & -- & -- & -- \\
Diet 1 & -- & -- & - \\
Diet 2 & 0.12 & 0.74 & 3.7 \\
Diet 3 & 0.051 & 0.25 & 1.3 \\
\hline
\end{tabular}

Excess Lifetime Risk [ ]

\begin{tabular}{llll}
\hline Diet 1 & $3.7 \mathrm{E}-07$ & $5.1 \mathrm{E}-06$ & $7.5 \mathrm{E}-05$ \\
Diet 2 & $1.6 \mathrm{E}-07$ & $1.9 \mathrm{E}-06$ & $2.5 \mathrm{E}-05$ \\
Diet 3 & $1.7 \mathrm{E}-07$ & $2.3 \mathrm{E}-06$ & $3.0 \mathrm{E}-05$ \\
Diet 4 & $8.3 \mathrm{E}-07$ & $1.2 \mathrm{E}-05$ & $2.2 \mathrm{E}-04$ \\
\hline
\end{tabular}

Relative Risk [ ]

\begin{tabular}{lccc}
\hline Diet 1 & 1.00016 & 1.0015 & 1.016 \\
Diet 2 & 1.000066 & 1.00054 & 1.0065 \\
Diet 3 & 1.000077 & 1.00066 & 1.0079 \\
Diet 4 & 1.00038 & 1.0036 & 1.048 \\
\hline
\end{tabular}

\section{Probability of Causation [\%]}

\begin{tabular}{llll}
\hline Diet 1 & 0.016 & 0.147 & 1.62 \\
Diet 2 & 0.007 & 0.054 & 0.65 \\
Diet 3 & 0.008 & 0.066 & 0.79 \\
Diet 4 & 0.038 & 0.360 & 4.56 \\
\hline
\end{tabular}

Diet 1 - Backyard cow milk + all other locally produced non-milk exposure pathways

Diet 2 - Locally produced commercial milk + all other locally produced non-milk exposure pathways

Diet 3 - Regionally mixed commercial milk + inhalation (other regionally mixed food items are minor contributors to the total)

Diet 4 - Goat milk (all other exposure pathways are of negligible importance compared to goat milk) 
Location: Sweetwater

Receptor: Male born in 1930

\begin{tabular}{lccc}
\hline & \multicolumn{3}{c}{ Thyroid Dose [cGy] } \\
& \multicolumn{2}{c}{$95 \%$ Subjective Confidence Interval } \\
Exposure Pathway & lower limit & central estimate & upper limit \\
\hline Backyard Cow Milk & 0.13 & 0.77 & 3.8 \\
Commercial Milk (locally produced) & 0.029 & 0.18 & 1.1 \\
Commercial Milk (regionally mixed) & 0.056 & 0.3 & 2 \\
Goat Milk (locally produced) & 0.24 & 2.1 & 15 \\
Beef (locally produced) & 0.00048 & 0.0081 & 0.15 \\
Leafy Vegetables (locally produced) & 0.00014 & 0.0013 & 0.011 \\
Eggs (locally produced) & 0.0083 & 0.056 & 0.37 \\
Cottage Cheese (locally produced) & 0.00026 & 0.003 & 0.027 \\
Inhalation & 0.0093 & 0.034 & 0.12 \\
Mother's milk (mother on Diet 1) & -- & -- & -- \\
Prenatal exposure (mother on Diet 1) & -- & -- & -- \\
Diet 1 & 0.16 & 0.9 & 4.5 \\
Diet 2 & 0.06 & 0.29 & 1.6 \\
Diet 3 & 0.069 & 0.34 & 2 \\
\hline
\end{tabular}

Excess Lifetime Risk [ ]

\begin{tabular}{llll}
\hline Diet 1 & $4.1 \mathrm{E}-08$ & $1.8 \mathrm{E}-06$ & $4.6 \mathrm{E}-05$ \\
Diet 2 & $1.5 \mathrm{E}-08$ & $6.3 \mathrm{E}-07$ & $1.8 \mathrm{E}-05$ \\
Diet 3 & $1.8 \mathrm{E}-08$ & $7.4 \mathrm{E}-07$ & $2.5 \mathrm{E}-05$ \\
Diet 4 & $1.0 \mathrm{E}-07$ & $4.0 \mathrm{E}-06$ & $1.4 \mathrm{E}-04$ \\
\hline
\end{tabular}

\section{Relative Risk [ ]}

\begin{tabular}{lccc}
\hline Diet 1 & 1.000057 & 1.0012 & 1.027 \\
Diet 2 & 1.000021 & 1.00043 & 1.0093 \\
Diet 3 & 1.000024 & 1.00048 & 1.011 \\
Diet 4 & 1.0001 & 1.0032 & 1.065 \\
\hline
\end{tabular}

\begin{tabular}{llcc} 
& \multicolumn{3}{c}{ Probability of Causation [\%] } \\
\hline Diet 1 & 0.006 & 0.122 & 2.62 \\
Diet 2 & 0.002 & 0.043 & 0.92 \\
Diet 3 & 0.002 & 0.048 & 1.12 \\
Diet 4 & 0.010 & 0.314 & 6.07 \\
\hline
\end{tabular}

Diet 1 - Backyard cow milk + all other locally produced non-milk exposure pathways

Diet 2 - Locally produced commercial milk + all other locally produced non-milk exposure pathways

Diet 3 - Regionally mixed commercial milk + inhalation (other regionally mixed food items are minor contributors to the total)

Diet 4 - Goat milk (all other exposure pathways are of negligible importance compared to goat milk) 


\section{Location: Knoxville}

Receptor: Female born in 1930

\begin{tabular}{lccc}
\hline & \multicolumn{3}{c}{ Thyroid Dose [cGy] } \\
& \multicolumn{2}{c}{$95 \%$ Subjective Confidence Interval } \\
Exposure Pathway & lower limit & central estimate & upper limit \\
\hline Backyard Cow Milk & 0.19 & 1 & 5.7 \\
Commercial Milk (locally produced) & 0.04 & 0.24 & 1.5 \\
Commercial Milk (regionally mixed) & 0.043 & 0.25 & 1.5 \\
Goat Milk (locally produced) & 0.38 & 2.7 & 19 \\
Beef (locally produced) & 0.00054 & 0.0085 & 0.17 \\
Leafy Vegetables (locally produced) & 0.00023 & 0.0022 & 0.019 \\
Eggs (locally produced) & 0.013 & 0.088 & 0.55 \\
Cottage Cheese (locally produced) & 0.00049 & 0.0051 & 0.052 \\
Inhalation & 0.013 & 0.043 & 0.16 \\
Mother's milk (mother on Diet 1) & -- & -- & -- \\
Prenatal exposure (mother on Diet 1) & -- & -- & -- \\
Diet 1 & 0.23 & 1.2 & 6.6 \\
Diet 2 & 0.085 & 0.42 & 2.2 \\
Diet 3 & 0.066 & 0.3 & 1.7 \\
\hline
\end{tabular}

Excess Lifetime Risk [ ]

\begin{tabular}{llll}
\hline Diet 1 & $5.9 \mathrm{E}-07$ & $8.7 \mathrm{E}-06$ & $1.2 \mathrm{E}-04$ \\
Diet 2 & $2.4 \mathrm{E}-07$ & $3.3 \mathrm{E}-06$ & $4.3 \mathrm{E}-05$ \\
Diet 3 & $1.9 \mathrm{E}-07$ & $2.4 \mathrm{E}-06$ & $3.1 \mathrm{E}-05$ \\
Diet 4 & $1.4 \mathrm{E}-06$ & $2.2 \mathrm{E}-05$ & $3.1 \mathrm{E}-04$ \\
\hline
\end{tabular}

Relative Risk [ ]

\begin{tabular}{lccc}
\hline Diet 1 & 1.00027 & 1.0025 & 1.025 \\
Diet 2 & 1.00011 & 1.00092 & 1.0099 \\
Diet 3 & 1.000082 & 1.00068 & 1.008 \\
Diet 4 & 1.00067 & 1.0057 & 1.077 \\
\hline
\end{tabular}

\begin{tabular}{llcc} 
& \multicolumn{3}{c}{ Probability of Causation [\%] } \\
\hline Diet 1 & 0.027 & 0.250 & 2.44 \\
Diet 2 & 0.011 & 0.092 & 0.98 \\
Diet 3 & 0.008 & 0.068 & 0.80 \\
Diet 4 & 0.067 & 0.566 & 7.16 \\
\hline
\end{tabular}

Diet 1 - Backyard cow milk + all other locally produced non-milk exposure pathways

Diet 2 - Locally produced commercial milk + all other locally produced non-milk exposure pathways

Diet 3 - Regionally mixed commercial milk + inhalation (other regionally mixed food items are minor contributors to the total)

Diet 4 - Goat milk (all other exposure pathways are of negligible importance compared to goat milk) 


\section{Location: Knoxville}

\section{Receptor: Male born in 1930}

\begin{tabular}{lccc}
\hline & \multicolumn{3}{c}{ Thyroid Dose [cGy] } \\
Exposure Pathway & 95\% & Subjective Confidence Interval \\
Backyard Cow Milk & lower limit & central estimate & upper limit \\
Commercial Milk (locally produced) & 0.24 & 1.3 & 6.9 \\
Commercial Milk (regionally mixed) & 0.052 & 0.3 & 1.8 \\
Goat Milk (locally produced) & 0.056 & 0.3 & 2 \\
Beef (locally produced) & 0.52 & 3.6 & 23 \\
Leafy Vegetables (locally produced) & 0.00083 & 0.013 & 0.28 \\
Eggs (locally produced) & 0.00023 & 0.0022 & 0.019 \\
Cottage Cheese (locally produced) & 0.014 & 0.095 & 0.59 \\
Inhalation & 0.00049 & 0.005 & 0.05 \\
Mother's milk (mother on Diet 1) & 0.015 & 0.053 & 0.18 \\
Prenatal exposure (mother on Diet 1) & -- & -- & -- \\
Diet 1 & -- & -- & -- \\
Diet 2 & 0.29 & 1.5 & 7.9 \\
Diet 3 & 0.11 & 0.51 & 2.7 \\
\hline
\end{tabular}

Excess Lifetime Risk [ ]

\begin{tabular}{llll}
\hline Diet 1 & $6.2 \mathrm{E}-08$ & $3.0 \mathrm{E}-06$ & $8.2 \mathrm{E}-05$ \\
Diet 2 & $2.4 \mathrm{E}-08$ & $1.1 \mathrm{E}-06$ & $3.1 \mathrm{E}-05$ \\
Diet 3 & $2.0 \mathrm{E}-08$ & $7.8 \mathrm{E}-07$ & $2.5 \mathrm{E}-05$ \\
Diet 4 & $1.8 \mathrm{E}-07$ & $6.6 \mathrm{E}-06$ & $2.3 \mathrm{E}-04$ \\
\hline
\end{tabular}

\section{Relative Risk [ ]}

\begin{tabular}{lccc}
\hline Diet 1 & 1.0001 & 1.002 & 1.045 \\
Diet 2 & 1.000033 & 1.00073 & 1.016 \\
Diet 3 & 1.000026 & 1.00051 & 1.012 \\
Diet 4 & 1.00018 & 1.0052 & 1.13 \\
\hline
\end{tabular}

\section{Probability of Causation [\%]}

\begin{tabular}{lccc}
\hline Diet 1 & 0.010 & 0.197 & 4.30 \\
Diet 2 & 0.003 & 0.073 & 1.59 \\
Diet 3 & 0.003 & 0.051 & 1.15 \\
Diet 4 & 0.018 & 0.518 & 11.37 \\
\hline
\end{tabular}

Diet 1 - Backyard cow milk + all other locally produced non-milk exposure pathways

Diet 2 - Locally produced commercial milk + all other locally produced non-milk exposure pathways

Diet 3 - Regionally mixed commercial milk + inhalation (other regionally mixed food items are minor contributors to the total)

Diet 4 - Goat milk (all other exposure pathways are of negligible importance compared to goat milk) 


\section{Location: Maryville}

Receptor: Female born in 1930

\begin{tabular}{lccc}
\hline & \multicolumn{3}{c}{ Thyroid Dose [cGy] } \\
Exposure Pathway & \multicolumn{2}{c}{$95 \%$ Subjective Confidence Interval } \\
\hline Backyard Cow Milk & lower limit & central estimate & upper limit \\
Commercial Milk (locally produced) & 0.096 & 0.51 & 2.9 \\
Commercial Milk (regionally mixed) & 0.016 & 0.12 & 0.73 \\
Goat Milk (locally produced) & 0.042 & 0.25 & 1.5 \\
Beef (locally produced) & 0.17 & 1.3 & 9.6 \\
Leafy Vegetables (locally produced) & 0.00028 & 0.0042 & 0.088 \\
Eggs (locally produced) & 0.0001 & 0.0011 & 0.0092 \\
Cottage Cheese (locally produced) & 0.0061 & 0.044 & 0.26 \\
Inhalation & 0.00021 & 0.0025 & 0.027 \\
Mother's milk (mother on Diet 1) & 0.0067 & 0.023 & 0.092 \\
Prenatal exposure (mother on Diet 1) & -- & -- & -- \\
Diet 1 & -- & -- & -- \\
Diet 2 & 0.12 & 0.6 & 3.3 \\
Diet 3 & 0.041 & 0.21 & 1.1 \\
\hline
\end{tabular}

Excess Lifetime Risk [ ]

\begin{tabular}{llll}
\hline Diet 1 & $3.2 \mathrm{E}-07$ & $4.6 \mathrm{E}-06$ & $7.1 \mathrm{E}-05$ \\
Diet 2 & $1.2 \mathrm{E}-07$ & $1.7 \mathrm{E}-06$ & $2.3 \mathrm{E}-05$ \\
Diet 3 & $1.6 \mathrm{E}-07$ & $2.2 \mathrm{E}-06$ & $3.0 \mathrm{E}-05$ \\
Diet 4 & $7.6 \mathrm{E}-07$ & $1.1 \mathrm{E}-05$ & $1.8 \mathrm{E}-04$ \\
\hline
\end{tabular}

Relative Risk [ ]

\begin{tabular}{lccc}
\hline Diet 1 & 1.00013 & 1.0012 & 1.012 \\
Diet 2 & 1.000047 & 1.00042 & 1.0052 \\
Diet 3 & 1.000074 & 1.00064 & 1.0078 \\
Diet 4 & 1.00027 & 1.0028 & 1.04 \\
\hline
\end{tabular}

\section{Probability of Causation [\%]}

\begin{tabular}{llll}
\hline Diet 1 & 0.013 & 0.117 & 1.19 \\
Diet 2 & 0.005 & 0.042 & 0.52 \\
Diet 3 & 0.007 & 0.064 & 0.78 \\
Diet 4 & 0.027 & 0.277 & 3.89 \\
\hline
\end{tabular}

Diet 1 - Backyard cow milk + all other locally produced non-milk exposure pathways

Diet 2 - Locally produced commercial milk + all other locally produced non-milk exposure pathways

Diet 3 - Regionally mixed commercial milk + inhalation (other regionally mixed food items are minor contributors to the total)

Diet 4 - Goat milk (all other exposure pathways are of negligible importance compared to goat milk) 


\section{Location: Maryville}

\section{Receptor: Male born in 1930}

\begin{tabular}{lccc}
\hline & \multicolumn{3}{c}{ Thyroid Dose [cGy] } \\
Exposure Pathway & \multicolumn{2}{c}{$95 \%$ Subjective Confidence Interval } \\
\hline Backyard Cow Milk & lower limit & central estimate & upper limit \\
Commercial Milk (locally produced) & 0.11 & 0.62 & 3.4 \\
Commercial Milk (regionally mixed) & 0.022 & 0.14 & 0.84 \\
Goat Milk (locally produced) & 0.056 & 0.3 & 2 \\
Beef (locally produced) & 0.23 & 1.8 & 11 \\
Leafy Vegetables (locally produced) & 0.00042 & 0.0067 & 0.14 \\
Eggs (locally produced) & 0.000093 & 0.0011 & 0.009 \\
Cottage Cheese (locally produced) & 0.0065 & 0.046 & 0.27 \\
Inhalation & 0.00021 & 0.0026 & 0.024 \\
Mother's milk (mother on Diet 1) & 0.0079 & 0.028 & 0.11 \\
Prenatal exposure (mother on Diet 1) & -- & -- & - \\
Diet 1 & -- & -- & -- \\
Diet 2 & 0.15 & 0.73 & 3.8 \\
Diet 3 & 0.052 & 0.25 & 1.4 \\
\hline
\end{tabular}

Excess Lifetime Risk [ ]

\begin{tabular}{llll}
\hline Diet 1 & $3.2 \mathrm{E}-08$ & $1.4 \mathrm{E}-06$ & $4.0 \mathrm{E}-05$ \\
Diet 2 & $1.3 \mathrm{E}-08$ & $5.1 \mathrm{E}-07$ & $1.5 \mathrm{E}-05$ \\
Diet 3 & $1.9 \mathrm{E}-08$ & $7.3 \mathrm{E}-07$ & $2.4 \mathrm{E}-05$ \\
Diet 4 & $9.7 \mathrm{E}-08$ & $3.3 \mathrm{E}-06$ & $1.2 \mathrm{E}-04$ \\
\hline
\end{tabular}

Relative Risk [ ]

\begin{tabular}{lccc}
\hline Diet 1 & 1.000046 & 1.0011 & 1.023 \\
Diet 2 & 1.000016 & 1.00036 & 1.0076 \\
Diet 3 & 1.000023 & 1.00048 & 1.011 \\
Diet 4 & 1.000098 & 1.0025 & 1.067 \\
\hline
\end{tabular}

\begin{tabular}{llcc} 
& \multicolumn{3}{c}{ Probability of Causation [\%] } \\
\hline Diet 1 & 0.005 & 0.106 & 2.24 \\
Diet 2 & 0.002 & 0.036 & 0.75 \\
Diet 3 & 0.002 & 0.048 & 1.10 \\
Diet 4 & 0.010 & 0.248 & 6.27 \\
\hline
\end{tabular}

Diet 1 - Backyard cow milk + all other locally produced non-milk exposure pathways

Diet 2 - Locally produced commercial milk + all other locally produced non-milk exposure pathways

Diet 3 - Regionally mixed commercial milk + inhalation (other regionally mixed food items are minor contributors to the total)

Diet 4 - Goat milk (all other exposure pathways are of negligible importance compared to goat milk) 
Location: Cedar Grove

Receptor: Female born in 1930

\begin{tabular}{lccc}
\hline & \multicolumn{3}{c}{ Thyroid Dose [cGy] } \\
Exposure Pathway & $95 \%$ Subjective Confidence Interval \\
\hline Backyard Cow Milk & lower limit & central estimate & upper limit \\
Commercial Milk (locally produced) & 0.18 & 0.94 & 5.2 \\
Commercial Milk (regionally mixed) & 0.036 & 0.22 & 1.3 \\
Goat Milk (locally produced) & 0.043 & 0.25 & 1.5 \\
Beef (locally produced) & 0.36 & 2.4 & 18 \\
Leafy Vegetables (locally produced) & 0.00049 & 0.0077 & 0.16 \\
Eggs (locally produced) & 0.0002 & 0.002 & 0.017 \\
Cottage Cheese (locally produced) & 0.012 & 0.081 & 0.49 \\
Inhalation & 0.00045 & 0.0046 & 0.049 \\
Mother's milk (mother on Diet 1) & 0.012 & 0.038 & 0.13 \\
Prenatal exposure (mother on Diet 1) & -- & -- & -- \\
Diet 1 & -- & -- & -- \\
Diet 2 & 0.22 & 1.1 & 6 \\
Diet 3 & 0.079 & 0.37 & 2 \\
\hline
\end{tabular}

Excess Lifetime Risk [ ]

\begin{tabular}{llll}
\hline Diet 1 & $5.8 \mathrm{E}-07$ & $8.1 \mathrm{E}-06$ & $1.1 \mathrm{E}-04$ \\
Diet 2 & $2.3 \mathrm{E}-07$ & $3.1 \mathrm{E}-06$ & $3.7 \mathrm{E}-05$ \\
Diet 3 & $1.8 \mathrm{E}-07$ & $2.4 \mathrm{E}-06$ & $3.1 \mathrm{E}-05$ \\
Diet 4 & $1.4 \mathrm{E}-06$ & $2.0 \mathrm{E}-05$ & $2.7 \mathrm{E}-04$ \\
\hline
\end{tabular}

Relative Risk [ ]

\begin{tabular}{lccc}
\hline Diet 1 & 1.00022 & 1.0023 & 1.023 \\
Diet 2 & 1.0001 & 1.00084 & 1.0092 \\
Diet 3 & 1.000079 & 1.00067 & 1.008 \\
Diet 4 & 1.00062 & 1.0051 & 1.068 \\
\hline
\end{tabular}

\begin{tabular}{llcc} 
& \multicolumn{3}{c}{ Probability of Causation [\%] } \\
\hline Diet 1 & 0.022 & 0.227 & 2.26 \\
Diet 2 & 0.010 & 0.084 & 0.92 \\
Diet 3 & 0.008 & 0.067 & 0.79 \\
Diet 4 & 0.062 & 0.503 & 6.33 \\
\hline
\end{tabular}

Diet 1 - Backyard cow milk + all other locally produced non-milk exposure pathways

Diet 2 - Locally produced commercial milk + all other locally produced non-milk exposure pathways

Diet 3 - Regionally mixed commercial milk + inhalation (other regionally mixed food items are minor contributors to the total)

Diet 4 - Goat milk (all other exposure pathways are of negligible importance compared to goat milk) 


\section{Location: Cedar Grove}

Receptor: Male born in 1930

\begin{tabular}{lccc}
\hline & \multicolumn{3}{c}{ Thyroid Dose [cGy] } \\
Exposure Pathway & \multicolumn{2}{c}{$95 \%$ Subjective Confidence Interval } \\
\hline Backyard Cow Milk & lower limit & central estimate & upper limit \\
Commercial Milk (locally produced) & 0.24 & 1.2 & 6.2 \\
Commercial Milk (regionally mixed) & 0.048 & 0.27 & 1.6 \\
Goat Milk (locally produced) & 0.056 & 0.3 & 2 \\
Beef (locally produced) & 0.49 & 3.3 & 21 \\
Leafy Vegetables (locally produced) & 0.00075 & 0.012 & 0.26 \\
Eggs (locally produced) & 0.0002 & 0.002 & 0.017 \\
Cottage Cheese (locally produced) & 0.013 & 0.086 & 0.52 \\
Inhalation & 0.00042 & 0.0046 & 0.044 \\
Mother's milk (mother on Diet 1) & 0.014 & 0.046 & 0.16 \\
Prenatal exposure (mother on Diet 1) & -- & -- & - \\
Diet 1 & -- & -- & -- \\
Diet 2 & 0.28 & 1.4 & 7.2 \\
Diet 3 & 0.1 & 0.46 & 2.5 \\
\hline
\end{tabular}

Excess Lifetime Risk [ ]

\begin{tabular}{lllc}
\hline Diet 1 & $5.8 \mathrm{E}-08$ & $2.7 \mathrm{E}-06$ & $7.6 \mathrm{E}-05$ \\
Diet 2 & $2.2 \mathrm{E}-08$ & $9.3 \mathrm{E}-07$ & $3.1 \mathrm{E}-05$ \\
Diet 3 & $2.0 \mathrm{E}-08$ & $7.7 \mathrm{E}-07$ & $2.5 \mathrm{E}-05$ \\
Diet 4 & $1.6 \mathrm{E}-07$ & $6.1 \mathrm{E}-06$ & $2.2 \mathrm{E}-04$ \\
\hline
\end{tabular}

\section{Relative Risk [ ]}

\begin{tabular}{lccc}
\hline Diet 1 & 1.000091 & 1.0018 & 1.041 \\
Diet 2 & 1.00003 & 1.00066 & 1.014 \\
Diet 3 & 1.000026 & 1.00051 & 1.011 \\
Diet 4 & 1.00016 & 1.0048 & 1.12 \\
\hline
\end{tabular}

\section{Probability of Causation [\%]}

\begin{tabular}{lccc}
\hline Diet 1 & 0.009 & 0.183 & 3.96 \\
Diet 2 & 0.003 & 0.066 & 1.43 \\
Diet 3 & 0.003 & 0.051 & 1.13 \\
Diet 4 & 0.016 & 0.480 & 10.60 \\
\hline
\end{tabular}

Diet 1 - Backyard cow milk + all other locally produced non-milk exposure pathways

Diet 2 - Locally produced commercial milk + all other locally produced non-milk exposure pathways

Diet 3 - Regionally mixed commercial milk + inhalation (other regionally mixed food items are minor contributors to the total)

Diet 4 - Goat milk (all other exposure pathways are of negligible importance compared to goat milk) 
Location: Bradbury

Receptor: Female born in 1935

\begin{tabular}{lccc}
\hline & \multicolumn{3}{c}{ Thyroid Dose [cGy] } \\
Exposure Pathway & $95 \%$ Subjective Confidence Interval \\
\hline Backyard Cow Milk & lower limit & central estimate & upper limit \\
Commercial Milk (locally produced) & 2 & 11 & 71 \\
Commercial Milk (regionally mixed) & 0.48 & 3.2 & 21 \\
Goat Milk (locally produced) & 0.071 & 0.43 & 2.6 \\
Beef (locally produced) & 5 & 34 & 220 \\
Leafy Vegetables (locally produced) & 0.0049 & 0.079 & 1.7 \\
Eggs (locally produced) & 0.0016 & 0.02 & 0.17 \\
Cottage Cheese (locally produced) & 0.13 & 0.89 & 5.8 \\
Inhalation & 0.0041 & 0.041 & 0.45 \\
Mother's milk (mother on Diet 1) & 0.089 & 0.29 & 1 \\
Prenatal exposure (mother on Diet 1) & -- & -- & -- \\
Diet 1 & -- & -- & -- \\
Diet 2 & 2.4 & 13 & 79 \\
Diet 3 & 0.87 & 4.7 & 27 \\
\hline
\end{tabular}

Excess Lifetime Risk [ ]

\begin{tabular}{lllc}
\hline Diet 1 & $3.2 \mathrm{E}-05$ & $4.3 \mathrm{E}-04$ & $5.2 \mathrm{E}-03$ \\
Diet 2 & $1.5 \mathrm{E}-05$ & $1.8 \mathrm{E}-04$ & $2.1 \mathrm{E}-03$ \\
Diet 3 & $2.9 \mathrm{E}-06$ & $2.7 \mathrm{E}-05$ & $2.5 \mathrm{E}-04$ \\
Diet 4 & $1.1 \mathrm{E}-04$ & $1.5 \mathrm{E}-03$ & $1.8 \mathrm{E}-02$ \\
\hline
\end{tabular}

\section{Relative Risk [ ]}

\begin{tabular}{lccc}
\hline Diet 1 & 1.012 & 1.1 & 2.1 \\
Diet 2 & 1.005 & 1.046 & 1.5 \\
Diet 3 & 1.00098 & 1.0072 & 1.065 \\
Diet 4 & 1.039 & 1.32 & 5.3 \\
\hline
\end{tabular}

\section{Probability of Causation [\%]}

\begin{tabular}{lccc}
\hline Diet 1 & 1.155 & 9.05 & 51.7 \\
Diet 2 & 0.498 & 4.38 & 33.2 \\
Diet 3 & 0.098 & 0.71 & 6.1 \\
Diet 4 & 3.768 & 24.39 & 81.0 \\
\hline
\end{tabular}

Diet 1 - Backyard cow milk + all other locally produced non-milk exposure pathways

Diet 2 - Locally produced commercial milk + all other locally produced non-milk exposure pathways

Diet 3 - Regionally mixed commercial milk + inhalation (other regionally mixed food items are minor contributors to the total)

Diet 4 - Goat milk (all other exposure pathways are of negligible importance compared to goat milk) 


\section{Location: Bradbury}

Receptor: Male born in 1935

\begin{tabular}{lccc}
\hline & \multicolumn{3}{c}{ Thyroid Dose [cGy] } \\
& \multicolumn{2}{c}{$95 \%$ Subjective Confidence Interval } \\
Exposure Pathway & lower limit & central estimate & upper limit \\
\hline Backyard Cow Milk & 2.4 & 13 & 80 \\
Commercial Milk (locally produced) & 0.55 & 3.5 & 24 \\
Commercial Milk (regionally mixed) & 0.078 & 0.47 & 3.1 \\
Goat Milk (locally produced) & 6.2 & 41 & 260 \\
Beef (locally produced) & 0.0069 & 0.11 & 2.2 \\
Leafy Vegetables (locally produced) & 0.0017 & 0.019 & 0.17 \\
Eggs (locally produced) & 0.14 & 1 & 6.8 \\
Cottage Cheese (locally produced) & 0.004 & 0.043 & 0.48 \\
Inhalation & 0.088 & 0.31 & 1.1 \\
Mother's milk (mother on Diet 1) & -- & -- & -- \\
Prenatal exposure (mother on Diet 1) & -- & -- & - \\
Diet 1 & 2.8 & 15 & 90 \\
Diet 2 & 0.97 & 5.6 & 31 \\
Diet 3 & 0.21 & 0.84 & 4.2 \\
\hline
\end{tabular}

Excess Lifetime Risk [ ]

\begin{tabular}{lllc}
\hline Diet 1 & $3.5 \mathrm{E}-06$ & $1.1 \mathrm{E}-04$ & $2.6 \mathrm{E}-03$ \\
Diet 2 & $1.5 \mathrm{E}-06$ & $4.9 \mathrm{E}-05$ & $1.2 \mathrm{E}-03$ \\
Diet 3 & $2.9 \mathrm{E}-07$ & $7.4 \mathrm{E}-06$ & $1.5 \mathrm{E}-04$ \\
Diet 4 & $9.9 \mathrm{E}-06$ & $3.0 \mathrm{E}-04$ & $8.2 \mathrm{E}-03$ \\
\hline
\end{tabular}

\section{Relative Risk [ ]}

\begin{tabular}{lccc}
\hline Diet 1 & 1.0035 & 1.075 & 2.6 \\
Diet 2 & 1.0017 & 1.032 & 1.66 \\
Diet 3 & 1.00027 & 1.0047 & 1.085 \\
Diet 4 & 1.011 & 1.23 & 5.5 \\
\hline
\end{tabular}

Probability of Causation [\%]

\begin{tabular}{lccc}
\hline Diet 1 & 0.352 & 7.00 & 61.1 \\
Diet 2 & 0.167 & 3.14 & 39.7 \\
Diet 3 & 0.027 & 0.47 & 7.8 \\
Diet 4 & 1.082 & 18.88 & 81.6 \\
\hline
\end{tabular}

Diet 1 - Backyard cow milk + all other locally produced non-milk exposure pathways

Diet 2 - Locally produced commercial milk + all other locally produced non-milk exposure pathways

Diet 3 - Regionally mixed commercial milk + inhalation (other regionally mixed food items are minor contributors to the total)

Diet 4 - Goat milk (all other exposure pathways are of negligible importance compared to goat milk) 
Location: Gallaher Bend

Receptor: Female born in 1935

\begin{tabular}{lccc}
\hline & \multicolumn{3}{c}{ Thyroid Dose [cGy] } \\
Exposure Pathway & $95 \%$ & Subjective Confidence Interval \\
Backyard Cow Milk & lower limit & central estimate & upper limit \\
Commercial Milk (locally produced) & 2.3 & 14 & 82 \\
Commercial Milk (regionally mixed) & 0.65 & 4 & 24 \\
Goat Milk (locally produced) & 0.071 & 0.43 & 2.6 \\
Beef (locally produced) & 5.7 & 44 & 280 \\
Leafy Vegetables (locally produced) & 0.0057 & 0.098 & 2 \\
Eggs (locally produced) & 0.0021 & 0.024 & 0.21 \\
Cottage Cheese (locally produced) & 0.17 & 1.1 & 7.2 \\
Inhalation & 0.0046 & 0.053 & 0.51 \\
Mother's milk (mother on Diet 1) & 0.11 & 0.35 & 1.2 \\
Prenatal exposure (mother on Diet 1) & -- & -- & -- \\
Diet 1 & -- & -- & -- \\
Diet 2 & 2.8 & 16 & 92 \\
Diet 3 & 1.1 & 5.9 & 29 \\
\hline
\end{tabular}

Excess Lifetime Risk [ ]

\begin{tabular}{llll}
\hline Diet 1 & $3.8 \mathrm{E}-05$ & $4.9 \mathrm{E}-04$ & $6.2 \mathrm{E}-03$ \\
Diet 2 & $2.0 \mathrm{E}-05$ & $2.2 \mathrm{E}-04$ & $2.5 \mathrm{E}-03$ \\
Diet 3 & $3.2 \mathrm{E}-06$ & $2.9 \mathrm{E}-05$ & $2.6 \mathrm{E}-04$ \\
Diet 4 & $1.2 \mathrm{E}-04$ & $1.8 \mathrm{E}-03$ & $2.4 \mathrm{E}-02$ \\
\hline
\end{tabular}

\section{Relative Risk [ ]}

\begin{tabular}{lccc}
\hline Diet 1 & 1.014 & 1.12 & 2.4 \\
Diet 2 & 1.006 & 1.058 & 1.59 \\
Diet 3 & 1.0011 & 1.0078 & 1.071 \\
Diet 4 & 1.043 & 1.41 & 6.3 \\
\hline
\end{tabular}

\begin{tabular}{|c|c|c|c|}
\hline Diet 1 & 1.362 & 11.07 & 57.7 \\
\hline Diet 2 & 0.597 & 5.52 & 37.1 \\
\hline Diet 3 & 0.107 & 0.77 & 6.6 \\
\hline Diet 4 & 4.087 & 28.93 & 84.1 \\
\hline
\end{tabular}

Diet 1 - Backyard cow milk + all other locally produced non-milk exposure pathways

Diet 2 - Locally produced commercial milk + all other locally produced non-milk exposure pathways

Diet 3 - Regionally mixed commercial milk + inhalation (other regionally mixed food items are minor contributors to the total)

Diet 4 - Goat milk (all other exposure pathways are of negligible importance compared to goat milk) 
Location: Gallaher Bend

Receptor: Male born in 1935

\begin{tabular}{lccc}
\hline & \multicolumn{3}{c}{ Thyroid Dose [cGy] } \\
& \multicolumn{2}{c}{$95 \%$ Subjective Confidence Interval } \\
Exposure Pathway & lower limit & central estimate & upper limit \\
\hline Backyard Cow Milk & 2.9 & 16 & 89 \\
Commercial Milk (locally produced) & 0.81 & 4.4 & 26 \\
Commercial Milk (regionally mixed) & 0.078 & 0.47 & 3 \\
Goat Milk (locally produced) & 6.8 & 49 & 320 \\
Beef (locally produced) & 0.0075 & 0.13 & 2.8 \\
Leafy Vegetables (locally produced) & 0.002 & 0.024 & 0.2 \\
Eggs (locally produced) & 0.18 & 1.3 & 8.7 \\
Cottage Cheese (locally produced) & 0.0054 & 0.053 & 0.54 \\
Inhalation & 0.11 & 0.39 & 1.3 \\
Mother's milk (mother on Diet 1) & -- & -- & -- \\
Prenatal exposure (mother on Diet 1) & -- & -- & - \\
Diet 1 & 3.5 & 18 & 99 \\
Diet 2 & 1.2 & 6.7 & 35 \\
Diet 3 & 0.22 & 0.92 & 4.4 \\
\hline
\end{tabular}

Excess Lifetime Risk [ ]

\begin{tabular}{llll}
\hline Diet 1 & $4.6 \mathrm{E}-06$ & $1.4 \mathrm{E}-04$ & $3.0 \mathrm{E}-03$ \\
Diet 2 & $1.6 \mathrm{E}-06$ & $5.8 \mathrm{E}-05$ & $1.4 \mathrm{E}-03$ \\
Diet 3 & $3.0 \mathrm{E}-07$ & $8.0 \mathrm{E}-06$ & $1.6 \mathrm{E}-04$ \\
Diet 4 & $1.3 \mathrm{E}-05$ & $3.7 \mathrm{E}-04$ & $9.4 \mathrm{E}-03$ \\
\hline
\end{tabular}

\section{Relative Risk [ ]}

\begin{tabular}{lccc}
\hline Diet 1 & 1.0044 & 1.09 & 2.8 \\
Diet 2 & 1.002 & 1.039 & 1.73 \\
Diet 3 & 1.00028 & 1.005 & 1.088 \\
Diet 4 & 1.012 & 1.29 & 6 \\
\hline
\end{tabular}

Probability of Causation [\%]

\begin{tabular}{lccc}
\hline Diet 1 & 0.439 & 8.29 & 64.4 \\
Diet 2 & 0.200 & 3.72 & 42.2 \\
Diet 3 & 0.028 & 0.50 & 8.1 \\
Diet 4 & 1.205 & 22.47 & 83.2 \\
\hline
\end{tabular}

Diet 1 - Backyard cow milk + all other locally produced non-milk exposure pathways

Diet 2 - Locally produced commercial milk + all other locally produced non-milk exposure pathways

Diet 3 - Regionally mixed commercial milk + inhalation (other regionally mixed food items are minor contributors to the total)

Diet 4 - Goat milk (all other exposure pathways are of negligible importance compared to goat milk) 


\section{Location: EFPC}

Receptor: Female born in 1935

\begin{tabular}{lccc}
\hline & \multicolumn{3}{c}{ Thyroid Dose [cGy] } \\
& \multicolumn{2}{c}{$95 \%$ Subjective Confidence Interval } \\
Exposure Pathway & lower limit & central estimate & upper limit \\
\hline Backyard Cow Milk & 0.5 & 3 & 19 \\
Commercial Milk (locally produced) & 0.12 & 0.84 & 5.4 \\
Commercial Milk (regionally mixed) & 0.071 & 0.43 & 2.6 \\
Goat Milk (locally produced) & -- & -- & -- \\
Beef (locally produced) & 0.0013 & 0.021 & 0.44 \\
Leafy Vegetables (locally produced) & 0.00043 & 0.0051 & 0.047 \\
Eggs (locally produced) & 0.031 & 0.23 & 1.5 \\
Cottage Cheese (locally produced) & 0.0011 & 0.011 & 0.12 \\
Inhalation & 0.023 & 0.078 & 0.27 \\
Mother's milk (mother on Diet 1) & -- & -- & -- \\
Prenatal exposure (mother on Diet 1) & -- & -- & -- \\
Diet 1 & 0.59 & 3.4 & 21 \\
Diet 2 & 0.22 & 1.3 & 7.2 \\
Diet 3 & 0.11 & 0.52 & 2.9 \\
\hline
\end{tabular}

Excess Lifetime Risk [ ]

\begin{tabular}{lccc}
\hline Diet 1 & $8.3 \mathrm{E}-06$ & $1.1 \mathrm{E}-04$ & $1.3 \mathrm{E}-03$ \\
Diet 2 & $3.9 \mathrm{E}-06$ & $4.6 \mathrm{E}-05$ & $5.5 \mathrm{E}-04$ \\
Diet 3 & $1.8 \mathrm{E}-06$ & $2.0 \mathrm{E}-05$ & $2.0 \mathrm{E}-04$ \\
Diet 4 & -- & -- & -- \\
\hline
\end{tabular}

\section{Relative Risk [ ]}

\begin{tabular}{lccc}
\hline Diet 1 & 1.0028 & 1.027 & 1.29 \\
Diet 2 & 1.0013 & 1.012 & 1.13 \\
Diet 3 & 1.00064 & 1.0052 & 1.051 \\
Diet 4 & -- & -- & -- \\
\hline
\end{tabular}

\begin{tabular}{lccc}
\hline Diet 1 & 0.279 & 2.58 & 22.0 \\
Diet 2 & 0.130 & 1.16 & 11.7 \\
Diet 3 & 0.064 & 0.51 & 4.9 \\
Diet 4 & -- & -- & - \\
\hline Diet 1 - Backyard cow milk + all other locally produced non-milk exposure pathways & \\
Diet 2 - Locally produced commercial milk + all other locally produced non-milk exposure pathways \\
Diet 3 - Regionally mixed commercial milk + inhalation (other regionally mixed food items are minor contributors to the total) \\
Diet 4 - Goat milk (all other exposure pathways are of negligible importance compared to goat milk)
\end{tabular}




\section{Location: EFPC}

\section{Receptor: Male born in 1935}

\begin{tabular}{lccc}
\hline & \multicolumn{3}{c}{ Thyroid Dose [cGy] } \\
& \multicolumn{2}{c}{$95 \%$ Subjective Confidence Interval } \\
Exposure Pathway & lower limit & central estimate & upper limit \\
\hline Backyard Cow Milk & 0.63 & 3.4 & 21 \\
Commercial Milk (locally produced) & 0.14 & 0.93 & 6.2 \\
Commercial Milk (regionally mixed) & 0.078 & 0.47 & 3.1 \\
Goat Milk (locally produced) & -- & -- & -- \\
Beef (locally produced) & 0.0018 & 0.029 & 0.62 \\
Leafy Vegetables (locally produced) & 0.00041 & 0.0049 & 0.045 \\
Eggs (locally produced) & 0.035 & 0.27 & 1.8 \\
Cottage Cheese (locally produced) & 0.0011 & 0.011 & 0.12 \\
Inhalation & 0.022 & 0.085 & 0.29 \\
Mother's milk (mother on Diet 1) & -- & -- & -- \\
Prenatal exposure (mother on Diet 1) & -- & -- & -- \\
Diet 1 & 0.73 & 3.8 & 24 \\
Diet 2 & 0.25 & 1.4 & 8.2 \\
Diet 3 & 0.12 & 0.58 & 3.4 \\
\hline
\end{tabular}

Excess Lifetime Risk [ ]

\begin{tabular}{lccc}
\hline Diet 1 & $8.2 \mathrm{E}-07$ & $2.9 \mathrm{E}-05$ & $6.5 \mathrm{E}-04$ \\
Diet 2 & $3.7 \mathrm{E}-07$ & $1.3 \mathrm{E}-05$ & $2.9 \mathrm{E}-04$ \\
Diet 3 & $1.9 \mathrm{E}-07$ & $5.3 \mathrm{E}-06$ & $1.2 \mathrm{E}-04$ \\
Diet 4 & -- & -- & -- \\
\hline
\end{tabular}

\section{Relative Risk [ ]}

\begin{tabular}{lccc}
\hline Diet 1 & 1.00087 & 1.019 & 1.43 \\
Diet 2 & 1.00043 & 1.0082 & 1.18 \\
Diet 3 & 1.00019 & 1.0033 & 1.068 \\
Diet 4 & -- & -- & -- \\
\hline
\end{tabular}

\begin{tabular}{lccc}
\hline Diet 1 & 0.087 & 1.85 & 29.3 \\
Diet 2 & 0.043 & 0.81 & 15.3 \\
Diet 3 & 0.019 & 0.33 & 6.4 \\
Diet 4 & -- & -- & - \\
\hline Diet 1 - Backyard cow milk + all other locally produced non-milk exposure pathways & \\
Diet 2 - Locally produced commercial milk + all other locally produced non-milk exposure pathways \\
Diet 3 - Regionally mixed commercial milk + inhalation (other regionally mixed food items are minor contributors to the total) \\
Diet 4 - Goat milk (all other exposure pathways are of negligible importance compared to goat milk)
\end{tabular}




\section{Location: Hope Creek}

Receptor: Female born in 1935

\begin{tabular}{lccc}
\hline & \multicolumn{3}{c}{ Thyroid Dose [cGy] } \\
& \multicolumn{2}{c}{$95 \%$ Subjective Confidence Interval } \\
Exposure Pathway & lower limit & central estimate & upper limit \\
\hline Backyard Cow Milk & 1.3 & 7.9 & 48 \\
Commercial Milk (locally produced) & 0.35 & 2.2 & 15 \\
Commercial Milk (regionally mixed) & 0.071 & 0.43 & 2.6 \\
Goat Milk (locally produced) & -- & -- & -- \\
Beef (locally produced) & 0.0031 & 0.056 & 1.2 \\
Leafy Vegetables (locally produced) & 0.0013 & 0.014 & 0.12 \\
Eggs (locally produced) & 0.088 & 0.61 & 4.2 \\
Cottage Cheese (locally produced) & 0.0031 & 0.03 & 0.33 \\
Inhalation & 0.062 & 0.21 & 0.71 \\
Mother's milk (mother on Diet 1) & -- & -- & -- \\
Prenatal exposure (mother on Diet 1) & -- & -- & - \\
Diet 1 & 1.6 & 9 & 54 \\
Diet 2 & 0.64 & 3.3 & 19 \\
Diet 3 & 0.17 & 0.65 & 3.2 \\
\hline
\end{tabular}

Excess Lifetime Risk [ ]

\begin{tabular}{lccc}
\hline Diet 1 & $2.1 \mathrm{E}-05$ & $3.0 \mathrm{E}-04$ & $3.7 \mathrm{E}-03$ \\
Diet 2 & $1.1 \mathrm{E}-05$ & $1.3 \mathrm{E}-04$ & $1.5 \mathrm{E}-03$ \\
Diet 3 & $2.5 \mathrm{E}-06$ & $2.4 \mathrm{E}-05$ & $2.3 \mathrm{E}-04$ \\
Diet 4 & -- & -- & -- \\
\hline
\end{tabular}

\section{Relative Risk [ ]}

\begin{tabular}{lccc}
\hline Diet 1 & 1.0085 & 1.073 & 1.81 \\
Diet 2 & 1.0033 & 1.033 & 1.36 \\
Diet 3 & 1.00085 & 1.0064 & 1.057 \\
Diet 4 & -- & -- & -- \\
\hline
\end{tabular}

\section{Probability of Causation [\%]}

\begin{tabular}{lccc}
\hline Diet 1 & 0.838 & 6.80 & 44.5 \\
Diet 2 & 0.330 & 3.23 & 26.3 \\
Diet 3 & 0.085 & 0.64 & 5.4 \\
Diet 4 & -- & -- & - \\
\hline Diet 1 - Backyard cow milk + all other locally produced non-milk exposure pathways & \\
Diet 2 - Locally produced commercial milk + all other locally produced non-milk exposure pathways \\
Diet 3 - Regionally mixed commercial milk + inhalation (other regionally mixed food items are minor contributors to the total) \\
Diet 4 - Goat milk (all other exposure pathways are of negligible importance compared to goat milk)
\end{tabular}




\section{Location: Hope Creek}

\section{Receptor: Male born in 1935}

\begin{tabular}{lccc}
\hline & \multicolumn{3}{c}{ Thyroid Dose [cGy] } \\
& \multicolumn{2}{c}{$95 \%$ Subjective Confidence Interval } \\
Exposure Pathway & lower limit & central estimate & upper limit \\
\hline Backyard Cow Milk & 1.7 & 9.2 & 55 \\
Commercial Milk (locally produced) & 0.39 & 2.5 & 17 \\
Commercial Milk (regionally mixed) & 0.078 & 0.47 & 3 \\
Goat Milk (locally produced) & -- & -- & -- \\
Beef (locally produced) & 0.0046 & 0.077 & 1.7 \\
Leafy Vegetables (locally produced) & 0.0013 & 0.013 & 0.12 \\
Eggs (locally produced) & 0.1 & 0.74 & 4.7 \\
Cottage Cheese (locally produced) & 0.0029 & 0.03 & 0.35 \\
Inhalation & 0.064 & 0.22 & 0.74 \\
Mother's milk (mother on Diet 1) & -- & -- & - \\
Prenatal exposure (mother on Diet 1) & -- & -- & - \\
Diet 1 & 1.9 & 10 & 62 \\
Diet 2 & 0.72 & 3.8 & 23 \\
Diet 3 & 0.18 & 0.75 & 3.8 \\
\hline
\end{tabular}

Excess Lifetime Risk [ ]

\begin{tabular}{lccc}
\hline Diet 1 & $2.4 \mathrm{E}-06$ & $7.9 \mathrm{E}-05$ & $1.9 \mathrm{E}-03$ \\
Diet 2 & $9.9 \mathrm{E}-07$ & $3.5 \mathrm{E}-05$ & $8.1 \mathrm{E}-04$ \\
Diet 3 & $2.5 \mathrm{E}-07$ & $6.7 \mathrm{E}-06$ & $1.4 \mathrm{E}-04$ \\
Diet 4 & -- & -- & -- \\
\hline
\end{tabular}

\section{Relative Risk [ ]}

\begin{tabular}{lccc}
\hline Diet 1 & 1.0024 & 1.053 & 2.1 \\
Diet 2 & 1.0011 & 1.023 & 1.47 \\
Diet 3 & 1.00024 & 1.0042 & 1.079 \\
Diet 4 & -- & -- & -- \\
\hline
\end{tabular}

\begin{tabular}{lccc}
\hline Diet 1 & 0.243 & 5.06 & 52.8 \\
Diet 2 & 0.114 & 2.20 & 31.8 \\
Diet 3 & 0.024 & 0.42 & 7.3 \\
Diet 4 & -- & -- & -- \\
Diet 1 - Backyard cow milk + all other locally produced non-milk exposure pathways & \\
Diet 2 - Locally produced commercial milk + all other locally produced non-milk exposure pathways \\
Diet 3 - Regionally mixed commercial milk + inhalation (other regionally mixed food items are minor contributors to the total) \\
Diet 4 - Goat milk (all other exposure pathways are of negligible importance compared to goat milk)
\end{tabular}


Location: Buttermilk Rd.

Receptor: Female born in 1935

\begin{tabular}{lccc}
\hline & \multicolumn{3}{c}{ Thyroid Dose [cGy] } \\
Exposure Pathway & \multicolumn{2}{c}{$95 \%$ Subjective Confidence Interval } \\
Backyard Cow Milk & lower limit & central estimate & upper limit \\
Commercial Milk (locally produced) & 1.3 & 7.7 & 45 \\
Commercial Milk (regionally mixed) & 0.35 & 2.2 & 14 \\
Goat Milk (locally produced) & 0.071 & 0.43 & 2.6 \\
Beef (locally produced) & 3.5 & 24 & 150 \\
Leafy Vegetables (locally produced) & 0.0031 & 0.055 & 1.1 \\
Eggs (locally produced) & 0.0013 & 0.014 & 0.12 \\
Cottage Cheese (locally produced) & 0.087 & 0.6 & 4.1 \\
Inhalation & 0.003 & 0.03 & 0.31 \\
Mother's milk (mother on Diet 1) & 0.063 & 0.21 & 0.72 \\
Prenatal exposure (mother on Diet 1) & -- & -- & -- \\
Diet 1 & -- & -- & -- \\
Diet 2 & 1.6 & 8.9 & 51 \\
Diet 3 & 0.65 & 3.3 & 19 \\
\hline
\end{tabular}

Excess Lifetime Risk [ ]

\begin{tabular}{llll}
\hline Diet 1 & $2.1 \mathrm{E}-05$ & $3.0 \mathrm{E}-04$ & $3.7 \mathrm{E}-03$ \\
Diet 2 & $1.1 \mathrm{E}-05$ & $1.2 \mathrm{E}-04$ & $1.4 \mathrm{E}-03$ \\
Diet 3 & $2.6 \mathrm{E}-06$ & $2.4 \mathrm{E}-05$ & $2.3 \mathrm{E}-04$ \\
Diet 4 & $6.9 \mathrm{E}-05$ & $9.7 \mathrm{E}-04$ & $1.4 \mathrm{E}-02$ \\
\hline
\end{tabular}

\section{Relative Risk [ ]}

\begin{tabular}{lccc}
\hline Diet 1 & 1.0081 & 1.071 & 1.77 \\
Diet 2 & 1.0033 & 1.033 & 1.34 \\
Diet 3 & 1.00085 & 1.0065 & 1.058 \\
Diet 4 & 1.025 & 1.23 & 3.8 \\
\hline
\end{tabular}

\section{Probability of Causation [\%]}

\begin{tabular}{lccc}
\hline Diet 1 & 0.804 & 6.66 & 43.3 \\
Diet 2 & 0.330 & 3.16 & 25.2 \\
Diet 3 & 0.085 & 0.64 & 5.5 \\
Diet 4 & 2.424 & 18.46 & 73.7 \\
\hline Dien
\end{tabular}

Diet 1 - Backyard cow milk + all other locally produced non-milk exposure pathways

Diet 2 - Locally produced commercial milk + all other locally produced non-milk exposure pathways

Diet 3 - Regionally mixed commercial milk + inhalation (other regionally mixed food items are minor contributors to the total)

Diet 4 - Goat milk (all other exposure pathways are of negligible importance compared to goat milk) 
Location: Buttermilk Rd.

Receptor: Male born in 1935

\begin{tabular}{lccc}
\hline & \multicolumn{3}{c}{ Thyroid Dose [cGy] } \\
& \multicolumn{2}{c}{$95 \%$ Subjective Confidence Interval } \\
Exposure Pathway & lower limit & central estimate & upper limit \\
\hline Backyard Cow Milk & 1.6 & 9.1 & 54 \\
Commercial Milk (locally produced) & 0.4 & 2.4 & 17 \\
Commercial Milk (regionally mixed) & 0.078 & 0.47 & 3 \\
Goat Milk (locally produced) & 4.1 & 28 & 180 \\
Beef (locally produced) & 0.0044 & 0.075 & 1.6 \\
Leafy Vegetables (locally produced) & 0.0013 & 0.013 & 0.11 \\
Eggs (locally produced) & 0.1 & 0.74 & 4.6 \\
Cottage Cheese (locally produced) & 0.0029 & 0.029 & 0.33 \\
Inhalation & 0.064 & 0.22 & 0.76 \\
Mother's milk (mother on Diet 1) & -- & -- & -- \\
Prenatal exposure (mother on Diet 1) & -- & -- & - \\
Diet 1 & 1.9 & 10 & 60 \\
Diet 2 & 0.72 & 3.8 & 22 \\
Diet 3 & 0.18 & 0.75 & 3.8 \\
\hline
\end{tabular}

Excess Lifetime Risk [ ]

\begin{tabular}{llll}
\hline Diet 1 & $2.4 \mathrm{E}-06$ & $7.8 \mathrm{E}-05$ & $1.8 \mathrm{E}-03$ \\
Diet 2 & $9.5 \mathrm{E}-07$ & $3.4 \mathrm{E}-05$ & $7.7 \mathrm{E}-04$ \\
Diet 3 & $2.4 \mathrm{E}-07$ & $6.7 \mathrm{E}-06$ & $1.4 \mathrm{E}-04$ \\
Diet 4 & $6.9 \mathrm{E}-06$ & $2.1 \mathrm{E}-04$ & $5.7 \mathrm{E}-03$ \\
\hline
\end{tabular}

\section{Relative Risk [ ]}

\begin{tabular}{lccc}
\hline Diet 1 & 1.0024 & 1.052 & 2.1 \\
Diet 2 & 1.0012 & 1.022 & 1.45 \\
Diet 3 & 1.00023 & 1.0042 & 1.079 \\
Diet 4 & 1.0077 & 1.16 & 4.1 \\
\hline
\end{tabular}

\section{Probability of Causation [\%]}

\begin{tabular}{lccc}
\hline Diet 1 & 0.240 & 4.90 & 51.2 \\
Diet 2 & 0.118 & 2.16 & 31.0 \\
Diet 3 & 0.023 & 0.42 & 7.4 \\
Diet 4 & 0.763 & 13.87 & 75.1 \\
\hline
\end{tabular}

Diet 1 - Backyard cow milk + all other locally produced non-milk exposure pathways

Diet 2 - Locally produced commercial milk + all other locally produced non-milk exposure pathways

Diet 3 - Regionally mixed commercial milk + inhalation (other regionally mixed food items are minor contributors to the total)

Diet 4 - Goat milk (all other exposure pathways are of negligible importance compared to goat milk) 
Location: Jonesville

Receptor: Female born in 1935

\begin{tabular}{lccc}
\hline & \multicolumn{3}{c}{ Thyroid Dose [cGy] } \\
Exposure Pathway & $95 \%$ Subjective Confidence Interval \\
\hline Backyard Cow Milk & lower limit & central estimate & upper limit \\
Commercial Milk (locally produced) & 0.19 & 1.1 & 7.5 \\
Commercial Milk (regionally mixed) & 0.047 & 0.32 & 2.4 \\
Goat Milk (locally produced) & 0.071 & 0.43 & 2.6 \\
Beef (locally produced) & 0.48 & 3.5 & 26 \\
Leafy Vegetables (locally produced) & 0.00044 & 0.008 & 0.17 \\
Eggs (locally produced) & 0.00017 & 0.0019 & 0.019 \\
Cottage Cheese (locally produced) & 0.013 & 0.085 & 0.64 \\
Inhalation & 0.00037 & 0.0041 & 0.057 \\
Mother's milk (mother on Diet 1) & 0.009 & 0.031 & 0.12 \\
Prenatal exposure (mother on Diet 1) & -- & -- & -- \\
Diet 1 & -- & -- & -- \\
Diet 2 & 0.22 & 1.2 & 8.4 \\
Diet 3 & 0.087 & 0.48 & 3.1 \\
\hline
\end{tabular}

Excess Lifetime Risk [ ]

\begin{tabular}{llcc}
\hline Diet 1 & $3.2 \mathrm{E}-06$ & $4.3 \mathrm{E}-05$ & $6.0 \mathrm{E}-04$ \\
Diet 2 & $1.6 \mathrm{E}-06$ & $1.8 \mathrm{E}-05$ & $2.2 \mathrm{E}-04$ \\
Diet 3 & $1.6 \mathrm{E}-06$ & $1.8 \mathrm{E}-05$ & $1.9 \mathrm{E}-04$ \\
Diet 4 & $9.9 \mathrm{E}-06$ & $1.3 \mathrm{E}-04$ & $1.8 \mathrm{E}-03$ \\
\hline
\end{tabular}

\section{Relative Risk [ ]}

\begin{tabular}{lccc}
\hline Diet 1 & 1.0011 & 1.0098 & 1.13 \\
Diet 2 & 1.00048 & 1.0044 & 1.06 \\
Diet 3 & 1.00056 & 1.0047 & 1.049 \\
Diet 4 & 1.0032 & 1.032 & 1.48 \\
\hline
\end{tabular}

\section{Probability of Causation [\%]}

\begin{tabular}{llcc}
\hline Diet 1 & 0.106 & 0.97 & 11.4 \\
Diet 2 & 0.048 & 0.44 & 5.7 \\
Diet 3 & 0.056 & 0.47 & 4.7 \\
Diet 4 & 0.315 & 3.06 & 32.4 \\
\hline
\end{tabular}

Diet 1 - Backyard cow milk + all other locally produced non-milk exposure pathways

Diet 2 - Locally produced commercial milk + all other locally produced non-milk exposure pathways

Diet 3 - Regionally mixed commercial milk + inhalation (other regionally mixed food items are minor contributors to the total)

Diet 4 - Goat milk (all other exposure pathways are of negligible importance compared to goat milk) 


\section{Location: Jonesville}

\section{Receptor: Male born in 1935}

\begin{tabular}{lccc}
\hline & \multicolumn{3}{c}{ Thyroid Dose [cGy] } \\
& \multicolumn{2}{c}{$95 \%$ Subjective Confidence Interval } \\
Exposure Pathway & lower limit & central estimate & upper limit \\
\hline Backyard Cow Milk & 0.22 & 1.3 & 8.4 \\
Commercial Milk (locally produced) & 0.05 & 0.35 & 2.7 \\
Commercial Milk (regionally mixed) & 0.078 & 0.47 & 3 \\
Goat Milk (locally produced) & 0.54 & 4 & 30 \\
Beef (locally produced) & 0.00061 & 0.011 & 0.23 \\
Leafy Vegetables (locally produced) & 0.00017 & 0.0019 & 0.018 \\
Eggs (locally produced) & 0.014 & 0.1 & 0.71 \\
Cottage Cheese (locally produced) & 0.00038 & 0.0041 & 0.055 \\
Inhalation & 0.0099 & 0.034 & 0.13 \\
Mother's milk (mother on Diet 1) & -- & -- & - \\
Prenatal exposure (mother on Diet 1) & -- & -- & -- \\
Diet 1 & 0.27 & 1.4 & 9.3 \\
Diet 2 & 0.098 & 0.53 & 3.5 \\
Diet 3 & 0.099 & 0.51 & 3.2 \\
\hline
\end{tabular}

Excess Lifetime Risk [ ]

\begin{tabular}{llll}
\hline Diet 1 & $2.9 \mathrm{E}-07$ & $1.0 \mathrm{E}-05$ & $2.7 \mathrm{E}-04$ \\
Diet 2 & $1.5 \mathrm{E}-07$ & $4.8 \mathrm{E}-06$ & $1.2 \mathrm{E}-04$ \\
Diet 3 & $1.8 \mathrm{E}-07$ & $4.8 \mathrm{E}-06$ & $1.2 \mathrm{E}-04$ \\
Diet 4 & $9.9 \mathrm{E}-07$ & $3.0 \mathrm{E}-05$ & $9.0 \mathrm{E}-04$ \\
\hline
\end{tabular}

\section{Relative Risk [ ]}

\begin{tabular}{lccc}
\hline Diet 1 & 1.00032 & 1.0076 & 1.16 \\
Diet 2 & 1.00015 & 1.0033 & 1.065 \\
Diet 3 & 1.00017 & 1.0031 & 1.065 \\
Diet 4 & 1.00097 & 1.023 & 1.46 \\
\hline
\end{tabular}

\section{Probability of Causation [\%]}

\begin{tabular}{llcc}
\hline Diet 1 & 0.032 & 0.75 & 14.0 \\
Diet 2 & 0.015 & 0.33 & 6.1 \\
Diet 3 & 0.017 & 0.31 & 6.1 \\
Diet 4 & 0.097 & 2.25 & 31.5 \\
\hline
\end{tabular}

Diet 1 - Backyard cow milk + all other locally produced non-milk exposure pathways

Diet 2 - Locally produced commercial milk + all other locally produced non-milk exposure pathways

Diet 3 - Regionally mixed commercial milk + inhalation (other regionally mixed food items are minor contributors to the total)

Diet 4 - Goat milk (all other exposure pathways are of negligible importance compared to goat milk) 


\section{Location: OR Scarboro}

Receptor: Female born in 1935

\begin{tabular}{lccc}
\hline & \multicolumn{3}{c}{ Thyroid Dose [cGy] } \\
Exposure Pathway & \multicolumn{2}{c}{ 95\% } & Subjective Confidence Interval \\
Backyard Cow Milk & lower limit & central estimate & upper limit \\
Commercial Milk (locally produced) & -- & -- & -- \\
Commercial Milk (regionally mixed) & -- & -- & -- \\
Goat Milk (locally produced) & 0.071 & 0.43 & 2.6 \\
Beef (locally produced) & -- & -- & -- \\
Leafy Vegetables (locally produced) & -- & -- & -- \\
Eggs (locally produced) & -- & -- & -- \\
Cottage Cheese (locally produced) & -- & -- & -- \\
Inhalation & 0.0019 & 0.022 & 0.22 \\
Mother's milk (mother on Diet 3) & 0.046 & 0.15 & 0.54 \\
Prenatal exposure (mother on Diet 3) & -- & -- & -- \\
Diet 1 & -- & -- & -- \\
Diet 2 & -- & -- & -- \\
Diet 3 & -- & -- & -- \\
\hline
\end{tabular}

Excess Lifetime Risk [ ]

\begin{tabular}{lccc}
\hline Diet 1 & -- & -- & -- \\
Diet 2 & -- & -- & -- \\
Diet 3 & $2.2 \mathrm{E}-06$ & $2.2 \mathrm{E}-05$ & $2.2 \mathrm{E}-04$ \\
Diet 4 & -- & -- & -- \\
\hline
\end{tabular}

\section{Relative Risk [ ]}

\begin{tabular}{lccc}
\hline Diet 1 & -- & -- & -- \\
Diet 2 & -- & -- & -- \\
Diet 3 & 1.00078 & 1.0059 & 1.055 \\
Diet 4 & -- & -- & -- \\
\hline
\end{tabular}

\begin{tabular}{lccc}
\hline Diet 1 & -- & -- & -- \\
Diet 2 & -- & -- & -- \\
Diet 3 & 0.078 & 0.59 & 5.3 \\
Diet 4 & -- & -- & \\
\hline Diet 1 - Backyard cow milk + all other locally produced non-milk exposure pathways & \\
Diet 2 - Locally produced commercial milk + all other locally produced non-milk exposure pathways \\
Diet 3 - Regionally mixed commercial milk + inhalation (other regionally mixed food items are minor contributors to the total) \\
Diet 4 - Goat milk (all other exposure pathways are of negligible importance compared to goat milk)
\end{tabular}




\section{Location: OR Scarboro}

Receptor: Male born in 1935

\begin{tabular}{lccc}
\hline & \multicolumn{3}{c}{ Thyroid Dose [cGy] } \\
Exposure Pathway & \multicolumn{2}{c}{$95 \%$ Subjective Confidence Interval } \\
\hline Backyard Cow Milk & lower limit & central estimate & upper limit \\
Commercial Milk (locally produced) & -- & -- & -- \\
Commercial Milk (regionally mixed) & -- & -- & -- \\
Goat Milk (locally produced) & 0.078 & 0.47 & 3 \\
Beef (locally produced) & -- & -- & -- \\
Leafy Vegetables (locally produced) & -- & -- & -- \\
Eggs (locally produced) & -- & -- & -- \\
Cottage Cheese (locally produced) & -- & -- \\
Inhalation & 0.0021 & 0.022 & 0.23 \\
Mother's milk (mother on Diet 3) & 0.047 & 0.17 & 0.57 \\
Prenatal exposure (mother on Diet 3) & -- & -- & -- \\
Diet 1 & -- & -- & -- \\
Diet 2 & -- & -- & -- \\
Diet 3 & -- & -- & -- \\
\hline
\end{tabular}

Excess Lifetime Risk [ ]

\begin{tabular}{lccc}
\hline Diet 1 & -- & -- & -- \\
Diet 2 & -- & -- & -- \\
Diet 3 & $2.2 \mathrm{E}-07$ & $6.1 \mathrm{E}-06$ & $1.4 \mathrm{E}-04$ \\
Diet 4 & -- & -- & -- \\
\hline
\end{tabular}

\section{Relative Risk [ ]}

\begin{tabular}{lccc}
\hline Diet 1 & -- & -- & -- \\
Diet 2 & -- & -- & -- \\
Diet 3 & 1.00022 & 1.0038 & 1.075 \\
Diet 4 & -- & -- & -- \\
\hline
\end{tabular}

\begin{tabular}{lccc}
\hline Diet 1 & -- & -- & -- \\
Diet 2 & -- & -- & -- \\
Diet 3 & 0.022 & 0.38 & -9 \\
Diet 4 & -- & -- \\
\hline Diet 1 - Backyard cow milk + all other locally produced non-milk exposure pathways & \\
Diet 2 - Locally produced commercial milk + all other locally produced non-milk exposure pathways \\
Diet 3 - Regionally mixed commercial milk + inhalation (other regionally mixed food items are minor contributors to the total) \\
Diet 4 - Goat milk (all other exposure pathways are of negligible importance compared to goat milk)
\end{tabular}




\section{Location: Lawnville/Gallaher}

Receptor: Female born in 1935

\begin{tabular}{lccc}
\hline & \multicolumn{3}{c}{ Thyroid Dose [cGy] } \\
Exposure Pathway & \multicolumn{2}{c}{$95 \%$ Subjective Confidence Interval } \\
\hline Backyard Cow Milk & lower limit & central estimate & upper limit \\
Commercial Milk (locally produced) & 1.3 & 7.3 & 38 \\
Commercial Milk (regionally mixed) & 0.34 & 2.1 & 14 \\
Goat Milk (locally produced) & 0.071 & 0.43 & 2.6 \\
Beef (locally produced) & 3.4 & 23 & 150 \\
Leafy Vegetables (locally produced) & 0.003 & 0.054 & 1.1 \\
Eggs (locally produced) & 0.0013 & 0.013 & 0.1 \\
Cottage Cheese (locally produced) & 0.09 & 0.57 & 3.6 \\
Inhalation & 0.0028 & 0.027 & 0.29 \\
Mother's milk (mother on Diet 1) & 0.064 & 0.2 & 0.71 \\
Prenatal exposure (mother on Diet 1) & -- & -- & -- \\
Diet 1 & -- & -- & -- \\
Diet 2 & 1.6 & 8.5 & 43 \\
Diet 3 & 0.64 & 3.1 & 19 \\
\hline
\end{tabular}

Excess Lifetime Risk [ ]

\begin{tabular}{llll}
\hline Diet 1 & $2.3 \mathrm{E}-05$ & $2.7 \mathrm{E}-04$ & $3.3 \mathrm{E}-03$ \\
Diet 2 & $1.0 \mathrm{E}-05$ & $1.1 \mathrm{E}-04$ & $1.4 \mathrm{E}-03$ \\
Diet 3 & $2.4 \mathrm{E}-06$ & $2.4 \mathrm{E}-05$ & $2.3 \mathrm{E}-04$ \\
Diet 4 & $6.5 \mathrm{E}-05$ & $9.4 \mathrm{E}-04$ & $1.2 \mathrm{E}-02$ \\
\hline
\end{tabular}

\section{Relative Risk [ ]}

\begin{tabular}{lccc}
\hline Diet 1 & 1.0084 & 1.068 & 1.76 \\
Diet 2 & 1.0034 & 1.03 & 1.33 \\
Diet 3 & 1.00085 & 1.0064 & 1.058 \\
Diet 4 & 1.024 & 1.22 & 3.6 \\
\hline
\end{tabular}

\section{Probability of Causation [\%]}

\begin{tabular}{lccc}
\hline Diet 1 & 0.834 & 6.33 & 43.1 \\
Diet 2 & 0.339 & 2.94 & 24.5 \\
Diet 3 & 0.085 & 0.64 & 5.4 \\
Diet 4 & 2.359 & 17.74 & 71.9 \\
\hline Dien
\end{tabular}

Diet 1 - Backyard cow milk + all other locally produced non-milk exposure pathways

Diet 2 - Locally produced commercial milk + all other locally produced non-milk exposure pathways

Diet 3 - Regionally mixed commercial milk + inhalation (other regionally mixed food items are minor contributors to the total)

Diet 4 - Goat milk (all other exposure pathways are of negligible importance compared to goat milk) 


\section{Location: Lawnville/Gallaher}

Receptor: Male born in 1935

\begin{tabular}{lccc}
\hline & \multicolumn{3}{c}{ Thyroid Dose [cGy] } \\
& \multicolumn{2}{c}{$95 \%$ Subjective Confidence Interval } \\
Exposure Pathway & lower limit & central estimate & upper limit \\
\hline Backyard Cow Milk & 1.7 & 8.5 & 46 \\
Commercial Milk (locally produced) & 0.4 & 2.3 & 16 \\
Commercial Milk (regionally mixed) & 0.078 & 0.47 & 3 \\
Goat Milk (locally produced) & 3.9 & 27 & 170 \\
Beef (locally produced) & 0.0042 & 0.074 & 1.4 \\
Leafy Vegetables (locally produced) & 0.0013 & 0.013 & 0.1 \\
Eggs (locally produced) & 0.11 & 0.68 & 4.3 \\
Cottage Cheese (locally produced) & 0.0029 & 0.028 & 0.28 \\
Inhalation & 0.064 & 0.21 & 0.8 \\
Mother's milk (mother on Diet 1) & -- & -- & -- \\
Prenatal exposure (mother on Diet 1) & -- & -- & -- \\
Diet 1 & 1.9 & 9.6 & 52 \\
Diet 2 & 0.7 & 3.5 & 21 \\
Diet 3 & 0.18 & 0.74 & 3.9 \\
\hline
\end{tabular}

Excess Lifetime Risk [ ]

\begin{tabular}{llll}
\hline Diet 1 & $2.2 \mathrm{E}-06$ & $7.2 \mathrm{E}-05$ & $1.7 \mathrm{E}-03$ \\
Diet 2 & $7.9 \mathrm{E}-07$ & $3.1 \mathrm{E}-05$ & $7.1 \mathrm{E}-04$ \\
Diet 3 & $2.3 \mathrm{E}-07$ & $6.7 \mathrm{E}-06$ & $1.4 \mathrm{E}-04$ \\
Diet 4 & $6.5 \mathrm{E}-06$ & $2.0 \mathrm{E}-04$ & $5.3 \mathrm{E}-03$ \\
\hline
\end{tabular}

\section{Relative Risk [ ]}

\begin{tabular}{lccc}
\hline Diet 1 & 1.0021 & 1.047 & 2 \\
Diet 2 & 1.0011 & 1.02 & 1.42 \\
Diet 3 & 1.00023 & 1.0041 & 1.079 \\
Diet 4 & 1.0071 & 1.15 & 4 \\
\hline
\end{tabular}

\begin{tabular}{|c|c|c|c|}
\hline & \\
\hline$\overline{\text { Diet } 1}$ & 0.213 & 4.52 & 50.0 \\
\hline Diet 2 & 0.106 & 1.96 & 29.8 \\
\hline Diet 3 & 0.023 & 0.41 & 7.3 \\
\hline Diet 4 & 0.703 & 13.12 & 75.3 \\
\hline
\end{tabular}

Diet 1 - Backyard cow milk + all other locally produced non-milk exposure pathways

Diet 2 - Locally produced commercial milk + all other locally produced non-milk exposure pathways

Diet 3 - Regionally mixed commercial milk + inhalation (other regionally mixed food items are minor contributors to the total)

Diet 4 - Goat milk (all other exposure pathways are of negligible importance compared to goat milk) 


\section{Location: Dyllis}

Receptor: Female born in 1935

\begin{tabular}{lccc}
\hline & \multicolumn{3}{c}{ Thyroid Dose [cGy] } \\
& \multicolumn{2}{c}{$95 \%$ Subjective Confidence Interval } \\
Exposure Pathway & lower limit & central estimate & upper limit \\
\hline Backyard Cow Milk & 0.24 & 1.5 & 9.7 \\
Commercial Milk (locally produced) & 0.067 & 0.43 & 3 \\
Commercial Milk (regionally mixed) & 0.071 & 0.43 & 2.6 \\
Goat Milk (locally produced) & 0.69 & 4.7 & 32 \\
Beef (locally produced) & 0.00068 & 0.01 & 0.21 \\
Leafy Vegetables (locally produced) & 0.00024 & 0.0026 & 0.024 \\
Eggs (locally produced) & 0.016 & 0.12 & 0.87 \\
Cottage Cheese (locally produced) & 0.00052 & 0.0056 & 0.069 \\
Inhalation & 0.012 & 0.043 & 0.17 \\
Mother's milk (mother on Diet 1) & -- & -- & -- \\
Prenatal exposure (mother on Diet 1) & -- & -- & -- \\
Diet 1 & 0.28 & 1.7 & 11 \\
Diet 2 & 0.13 & 0.65 & 4.3 \\
Diet 3 & 0.097 & 0.47 & 2.8 \\
\hline
\end{tabular}

Excess Lifetime Risk [ ]

\begin{tabular}{lllc}
\hline Diet 1 & $4.0 \mathrm{E}-06$ & $5.7 \mathrm{E}-05$ & $7.8 \mathrm{E}-04$ \\
Diet 2 & $1.8 \mathrm{E}-06$ & $2.4 \mathrm{E}-05$ & $2.8 \mathrm{E}-04$ \\
Diet 3 & $1.6 \mathrm{E}-06$ & $1.9 \mathrm{E}-05$ & $1.9 \mathrm{E}-04$ \\
Diet 4 & $1.2 \mathrm{E}-05$ & $2.0 \mathrm{E}-04$ & $2.7 \mathrm{E}-03$ \\
\hline
\end{tabular}

Relative Risk [ ]

\begin{tabular}{lccc}
\hline Diet 1 & 1.0014 & 1.013 & 1.17 \\
Diet 2 & 1.00074 & 1.006 & 1.089 \\
Diet 3 & 1.00058 & 1.0048 & 1.049 \\
Diet 4 & 1.0054 & 1.043 & 1.6 \\
\hline
\end{tabular}

Probability of Causation [\%]

\begin{tabular}{llcc}
\hline Diet 1 & 0.144 & 1.32 & 14.2 \\
Diet 2 & 0.074 & 0.60 & 8.2 \\
Diet 3 & 0.058 & 0.48 & 4.7 \\
Diet 4 & 0.534 & 4.12 & 37.4 \\
\hline
\end{tabular}

Diet 1 - Backyard cow milk + all other locally produced non-milk exposure pathways

Diet 2 - Locally produced commercial milk + all other locally produced non-milk exposure pathways

Diet 3 - Regionally mixed commercial milk + inhalation (other regionally mixed food items are minor contributors to the total)

Diet 4 - Goat milk (all other exposure pathways are of negligible importance compared to goat milk) 


\section{Location: Dyllis}

\section{Receptor: Male born in 1935}

\begin{tabular}{lccc}
\hline & \multicolumn{3}{c}{ Thyroid Dose [cGy] } \\
& \multicolumn{2}{c}{$95 \%$ Subjective Confidence Interval } \\
Exposure Pathway & lower limit & central estimate & upper limit \\
\hline Backyard Cow Milk & 0.3 & 1.8 & 10 \\
Commercial Milk (locally produced) & 0.073 & 0.49 & 3.6 \\
Commercial Milk (regionally mixed) & 0.078 & 0.47 & 3 \\
Goat Milk (locally produced) & 0.77 & 5.4 & 39 \\
Beef (locally produced) & 0.00098 & 0.015 & 0.33 \\
Leafy Vegetables (locally produced) & 0.00023 & 0.0026 & 0.025 \\
Eggs (locally produced) & 0.019 & 0.14 & 1 \\
Cottage Cheese (locally produced) & 0.00052 & 0.0058 & 0.071 \\
Inhalation & 0.013 & 0.048 & 0.17 \\
Mother's milk (mother on Diet 1) & -- & -- & - \\
Prenatal exposure (mother on Diet 1) & -- & -- & - \\
Diet 1 & 0.35 & 2 & 11 \\
Diet 2 & 0.15 & 0.73 & 4.9 \\
Diet 3 & 0.1 & 0.53 & 3.2 \\
\hline
\end{tabular}

Excess Lifetime Risk [ ]

\begin{tabular}{llll}
\hline Diet 1 & $4.2 \mathrm{E}-07$ & $1.5 \mathrm{E}-05$ & $3.4 \mathrm{E}-04$ \\
Diet 2 & $2.0 \mathrm{E}-07$ & $6.9 \mathrm{E}-06$ & $1.6 \mathrm{E}-04$ \\
Diet 3 & $1.8 \mathrm{E}-07$ & $4.9 \mathrm{E}-06$ & $1.2 \mathrm{E}-04$ \\
Diet 4 & $1.4 \mathrm{E}-06$ & $4.1 \mathrm{E}-05$ & $1.2 \mathrm{E}-03$ \\
\hline
\end{tabular}

\section{Relative Risk [ ]}

\begin{tabular}{lccc}
\hline Diet 1 & 1.00046 & 1.0099 & 1.24 \\
Diet 2 & 1.00022 & 1.0046 & 1.093 \\
Diet 3 & 1.00017 & 1.0032 & 1.066 \\
Diet 4 & 1.0013 & 1.033 & 1.61 \\
\hline
\end{tabular}

\section{Probability of Causation [\%]}

\begin{tabular}{llcc}
\hline Diet 1 & 0.046 & 0.98 & 19.1 \\
Diet 2 & 0.022 & 0.46 & 8.5 \\
Diet 3 & 0.017 & 0.31 & 6.2 \\
Diet 4 & 0.134 & 3.16 & 37.6 \\
\hline
\end{tabular}

Diet 1 - Backyard cow milk + all other locally produced non-milk exposure pathways

Diet 2 - Locally produced commercial milk + all other locally produced non-milk exposure pathways

Diet 3 - Regionally mixed commercial milk + inhalation (other regionally mixed food items are minor contributors to the total)

Diet 4 - Goat milk (all other exposure pathways are of negligible importance compared to goat milk) 
Location: OR High School Area

Receptor: Female born in 1935

\begin{tabular}{lccc}
\hline & \multicolumn{3}{c}{ Thyroid Dose [cGy] } \\
Exposure Pathway & \multicolumn{2}{c}{ 95\% Subjective Confidence Interval } \\
\hline Backyard Cow Milk & lower limit & central estimate & upper limit \\
Commercial Milk (locally produced) & -- & -- & -- \\
Commercial Milk (regionally mixed) & -- & -- & -- \\
Goat Milk (locally produced) & 0.071 & 0.43 & 2.6 \\
Beef (locally produced) & -- & -- & -- \\
Leafy Vegetables (locally produced) & -- & -- & -- \\
Eggs (locally produced) & -- & -- & -- \\
Cottage Cheese (locally produced) & -- & -- & -- \\
Inhalation & 0.0011 & 0.012 & 0.12 \\
Mother's milk (mother on Diet 3) & 0.027 & 0.09 & 0.33 \\
Prenatal exposure (mother on Diet 3) & -- & -- & -- \\
Diet 1 & -- & -- & -- \\
Diet 2 & -- & -- & -- \\
Diet 3 & -- & -- & -- \\
\hline
\end{tabular}

Excess Lifetime Risk [ ]

\begin{tabular}{lccc}
\hline Diet 1 & -- & -- & -- \\
Diet 2 & -- & -- & -- \\
Diet 3 & $1.9 \mathrm{E}-06$ & $2.0 \mathrm{E}-05$ & $2.0 \mathrm{E}-04$ \\
Diet 4 & -- & -- & -- \\
\hline
\end{tabular}

\section{Relative Risk [ ]}

\begin{tabular}{lccc}
\hline Diet 1 & -- & -- & -- \\
Diet 2 & -- & -- & -- \\
Diet 3 & 1.00067 & 1.0054 & 1.051 \\
Diet 4 & -- & -- & -- \\
\hline
\end{tabular}

\begin{tabular}{lccc}
\hline Diet 1 & -- & -- & -- \\
Diet 2 & -- & -- & 4.9 \\
Diet 3 & 0.067 & 0.53 & -- \\
Diet 4 & -- & -- & \\
\hline Diet 1 - Backyard cow milk + all other locally produced non-milk exposure pathways & \\
Diet 2 - Locally produced commercial milk + all other locally produced non-milk exposure pathways \\
Diet 3 - Regionally mixed commercial milk + inhalation (other regionally mixed food items are minor contributors to the total) \\
Diet 4 - Goat milk (all other exposure pathways are of negligible importance compared to goat milk)
\end{tabular}




\section{Location: OR High School Area}

Receptor: Male born in 1935

\begin{tabular}{lccc}
\hline & \multicolumn{3}{c}{ Thyroid Dose [cGy] } \\
Exposure Pathway & \multicolumn{2}{c}{$95 \%$ Subjective Confidence Interval } \\
\hline Backyard Cow Milk & lower limit & central estimate & upper limit \\
Commercial Milk (locally produced) & -- & -- & -- \\
Commercial Milk (regionally mixed) & -- & -- & -- \\
Goat Milk (locally produced) & 0.078 & 0.47 & 3 \\
Beef (locally produced) & -- & -- & -- \\
Leafy Vegetables (locally produced) & -- & -- & -- \\
Eggs (locally produced) & -- & -- & -- \\
Cottage Cheese (locally produced) & -- & -- \\
Inhalation & 0.0014 & 0.013 & 0.13 \\
Mother's milk (mother on Diet 3) & 0.029 & 0.099 & 0.34 \\
Prenatal exposure (mother on Diet 3) & -- & -- & -- \\
Diet 1 & -- & -- & -- \\
Diet 2 & -- & -- & -- \\
Diet 3 & -- & -- & -- \\
\hline
\end{tabular}

Excess Lifetime Risk [ ]

\begin{tabular}{lccc}
\hline Diet 1 & -- & -- & -- \\
Diet 2 & -- & -- & -- \\
Diet 3 & $2.0 \mathrm{E}-07$ & $5.3 \mathrm{E}-06$ & $1.3 \mathrm{E}-04$ \\
Diet 4 & -- & -- & -- \\
\hline
\end{tabular}

\section{Relative Risk [ ]}

\begin{tabular}{lccc}
\hline Diet 1 & -- & -- & -- \\
Diet 2 & -- & -- & -- \\
Diet 3 & 1.0002 & 1.0034 & 1.069 \\
Diet 4 & -- & -- & -- \\
\hline
\end{tabular}

\begin{tabular}{lccc}
\hline Diet 1 & -- & -- & -- \\
Diet 2 & -- & -- & -- \\
Diet 3 & 0.020 & 0.34 & -5 \\
Diet 4 & -- & -- \\
\hline Diet 1 - Backyard cow milk + all other locally produced non-milk exposure pathways & \\
Diet 2 - Locally produced commercial milk + all other locally produced non-milk exposure pathways \\
Diet 3 - Regionally mixed commercial milk + inhalation (other regionally mixed food items are minor contributors to the total) \\
Diet 4 - Goat milk (all other exposure pathways are of negligible importance compared to goat milk)
\end{tabular}




\section{Location: Norwood}

Receptor: Female born in 1935

\begin{tabular}{lccc}
\hline & \multicolumn{3}{c}{ Thyroid Dose [cGy] } \\
& \multicolumn{2}{c}{$95 \%$ Subjective Confidence Interval } \\
Exposure Pathway & lower limit & central estimate & upper limit \\
\hline Backyard Cow Milk & 0.23 & 1.4 & 9.9 \\
Commercial Milk (locally produced) & 0.06 & 0.41 & 2.9 \\
Commercial Milk (regionally mixed) & 0.071 & 0.43 & 2.6 \\
Goat Milk (locally produced) & 0.58 & 4.5 & 32 \\
Beef (locally produced) & 0.00058 & 0.01 & 0.23 \\
Leafy Vegetables (locally produced) & 0.00026 & 0.0025 & 0.023 \\
Eggs (locally produced) & 0.015 & 0.11 & 0.83 \\
Cottage Cheese (locally produced) & 0.00046 & 0.0055 & 0.067 \\
Inhalation & 0.011 & 0.041 & 0.15 \\
Mother's milk (mother on Diet 1) & -- & -- & -- \\
Prenatal exposure (mother on Diet 1) & -- & -- & -- \\
Diet 1 & 0.28 & 1.6 & 11 \\
Diet 2 & 0.11 & 0.61 & 3.7 \\
Diet 3 & 0.094 & 0.47 & 2.8 \\
\hline
\end{tabular}

Excess Lifetime Risk [ ]

\begin{tabular}{lllc}
\hline Diet 1 & $3.7 \mathrm{E}-06$ & $5.5 \mathrm{E}-05$ & $7.7 \mathrm{E}-04$ \\
Diet 2 & $1.9 \mathrm{E}-06$ & $2.4 \mathrm{E}-05$ & $3.1 \mathrm{E}-04$ \\
Diet 3 & $1.6 \mathrm{E}-06$ & $1.9 \mathrm{E}-05$ & $2.0 \mathrm{E}-04$ \\
Diet 4 & $1.4 \mathrm{E}-05$ & $1.8 \mathrm{E}-04$ & $2.6 \mathrm{E}-03$ \\
\hline
\end{tabular}

\section{Relative Risk [ ]}

\begin{tabular}{lccc}
\hline Diet 1 & 1.0014 & 1.013 & 1.14 \\
Diet 2 & 1.00065 & 1.0056 & 1.074 \\
Diet 3 & 1.00057 & 1.0048 & 1.05 \\
Diet 4 & 1.0047 & 1.041 & 1.56 \\
\hline
\end{tabular}

\section{Probability of Causation [\%]}

\begin{tabular}{llcc}
\hline Diet 1 & 0.140 & 1.27 & 12.4 \\
Diet 2 & 0.065 & 0.56 & 6.8 \\
Diet 3 & 0.057 & 0.48 & 4.7 \\
Diet 4 & 0.465 & 3.92 & 35.9 \\
\hline
\end{tabular}

Diet 1 - Backyard cow milk + all other locally produced non-milk exposure pathways

Diet 2 - Locally produced commercial milk + all other locally produced non-milk exposure pathways

Diet 3 - Regionally mixed commercial milk + inhalation (other regionally mixed food items are minor contributors to the total)

Diet 4 - Goat milk (all other exposure pathways are of negligible importance compared to goat milk) 


\section{Location: Norwood}

\section{Receptor: Male born in 1935}

\begin{tabular}{lccc}
\hline & \multicolumn{3}{c}{ Thyroid Dose [cGy] } \\
& \multicolumn{2}{c}{$95 \%$ Subjective Confidence Interval } \\
Exposure Pathway & lower limit & central estimate & upper limit \\
\hline Backyard Cow Milk & 0.28 & 1.6 & 11 \\
Commercial Milk (locally produced) & 0.073 & 0.46 & 3.6 \\
Commercial Milk (regionally mixed) & 0.078 & 0.47 & 3 \\
Goat Milk (locally produced) & 0.68 & 5.2 & 39 \\
Beef (locally produced) & 0.00087 & 0.015 & 0.34 \\
Leafy Vegetables (locally produced) & 0.00025 & 0.0025 & 0.023 \\
Eggs (locally produced) & 0.017 & 0.13 & 0.9 \\
Cottage Cheese (locally produced) & 0.00054 & 0.0055 & 0.065 \\
Inhalation & 0.012 & 0.043 & 0.16 \\
Mother's milk (mother on Diet 1) & -- & -- & -- \\
Prenatal exposure (mother on Diet 1) & -- & -- & -- \\
Diet 1 & 0.32 & 1.8 & 12 \\
Diet 2 & 0.12 & 0.69 & 4.8 \\
Diet 3 & 0.1 & 0.52 & 3.2 \\
\hline
\end{tabular}

Excess Lifetime Risk [ ]

\begin{tabular}{llll}
\hline Diet 1 & $4.3 \mathrm{E}-07$ & $1.4 \mathrm{E}-05$ & $3.2 \mathrm{E}-04$ \\
Diet 2 & $1.8 \mathrm{E}-07$ & $5.9 \mathrm{E}-06$ & $1.4 \mathrm{E}-04$ \\
Diet 3 & $1.8 \mathrm{E}-07$ & $4.9 \mathrm{E}-06$ & $1.2 \mathrm{E}-04$ \\
Diet 4 & $1.3 \mathrm{E}-06$ & $3.9 \mathrm{E}-05$ & $1.2 \mathrm{E}-03$ \\
\hline
\end{tabular}

\section{Relative Risk [ ]}

\begin{tabular}{lccc}
\hline Diet 1 & 1.00041 & 1.0096 & 1.22 \\
Diet 2 & 1.00021 & 1.0042 & 1.096 \\
Diet 3 & 1.00017 & 1.0031 & 1.065 \\
Diet 4 & 1.0012 & 1.03 & 1.71 \\
\hline
\end{tabular}

\section{Probability of Causation [\%]}

\begin{tabular}{llcc}
\hline Diet 1 & 0.041 & 0.95 & 17.8 \\
Diet 2 & 0.021 & 0.42 & 8.7 \\
Diet 3 & 0.017 & 0.31 & 6.1 \\
Diet 4 & 0.116 & 2.87 & 41.4 \\
\hline
\end{tabular}

Diet 1 - Backyard cow milk + all other locally produced non-milk exposure pathways

Diet 2 - Locally produced commercial milk + all other locally produced non-milk exposure pathways

Diet 3 - Regionally mixed commercial milk + inhalation (other regionally mixed food items are minor contributors to the total)

Diet 4 - Goat milk (all other exposure pathways are of negligible importance compared to goat milk) 
Location: Woodland

Receptor: Female born in 1935

\begin{tabular}{lccc}
\hline & \multicolumn{3}{c}{ Thyroid Dose [cGy] } \\
Exposure Pathway & \multicolumn{2}{c}{$95 \%$ Subjective Confidence Interval } \\
\hline Backyard Cow Milk & lower limit & central estimate & upper limit \\
Commercial Milk (locally produced) & -- & -- & -- \\
Commercial Milk (regionally mixed) & -- & -- & -- \\
Goat Milk (locally produced) & 0.071 & 0.43 & 2.6 \\
Beef (locally produced) & -- & -- & -- \\
Leafy Vegetables (locally produced) & -- & -- & -- \\
Eggs (locally produced) & -- & -- & -- \\
Cottage Cheese (locally produced) & -- & -- & -- \\
Inhalation & 0.0018 & 0.018 & 0.19 \\
Mother's milk (mother on Diet 3) & 0.043 & 0.14 & 0.48 \\
Prenatal exposure (mother on Diet 3) & -- & -- & -- \\
Diet 1 & -- & -- & -- \\
Diet 2 & -- & -- & -- \\
Diet 3 & -- & -- & -- \\
\hline
\end{tabular}

Excess Lifetime Risk [ ]

\begin{tabular}{lccc}
\hline Diet 1 & -- & -- & -- \\
Diet 2 & -- & -- & -- \\
Diet 3 & $2.2 \mathrm{E}-06$ & $2.2 \mathrm{E}-05$ & $2.2 \mathrm{E}-04$ \\
Diet 4 & -- & -- & -- \\
\hline
\end{tabular}

\section{Relative Risk [ ]}

\begin{tabular}{lccc}
\hline Diet 1 & -- & -- & -- \\
Diet 2 & -- & -- & -- \\
Diet 3 & 1.00075 & 1.0058 & 1.054 \\
Diet 4 & -- & -- & -- \\
\hline
\end{tabular}

\begin{tabular}{lccc}
\hline Diet 1 & -- & -- & -- \\
Diet 2 & -- & -- & -- \\
Diet 3 & 0.075 & 0.58 & 5.1 \\
Diet 4 & -- & -- \\
\hline Diet 1 - Backyard cow milk + all other locally produced non-milk exposure pathways & \\
Diet 2 - Locally produced commercial milk + all other locally produced non-milk exposure pathways \\
Diet 3 - Regionally mixed commercial milk + inhalation (other regionally mixed food items are minor contributors to the total) \\
Diet 4 - Goat milk (all other exposure pathways are of negligible importance compared to goat milk)
\end{tabular}


Location: Woodland

Receptor: Male born in 1935

\begin{tabular}{lccc}
\hline & \multicolumn{3}{c}{ Thyroid Dose [cGy] } \\
Exposure Pathway & \multicolumn{2}{c}{ 95\% Subjective Confidence Interval } \\
\hline Backyard Cow Milk & lower limit & central estimate & upper limit \\
Commercial Milk (locally produced) & -- & -- & -- \\
Commercial Milk (regionally mixed) & -- & -- & -- \\
Goat Milk (locally produced) & 0.078 & 0.47 & 3 \\
Beef (locally produced) & -- & -- & -- \\
Leafy Vegetables (locally produced) & -- & -- & -- \\
Eggs (locally produced) & -- & -- & -- \\
Cottage Cheese (locally produced) & -- & -- & -- \\
Inhalation & 0.0019 & 0.019 & 0.19 \\
Mother's milk (mother on Diet 3) & 0.043 & 0.14 & 0.52 \\
Prenatal exposure (mother on Diet 3) & -- & -- & -- \\
Diet 1 & -- & -- & -- \\
Diet 2 & -- & -- & -- \\
Diet 3 & -- & -- & -- \\
\hline
\end{tabular}

Excess Lifetime Risk [ ]

\begin{tabular}{lccc}
\hline Diet 1 & -- & -- & -- \\
Diet 2 & -- & -- & -- \\
Diet 3 & $2.2 \mathrm{E}-07$ & $5.9 \mathrm{E}-06$ & $1.3 \mathrm{E}-04$ \\
Diet 4 & -- & -- & -- \\
\hline
\end{tabular}

\section{Relative Risk [ ]}

\begin{tabular}{lccc}
\hline Diet 1 & -- & -- & -- \\
Diet 2 & -- & -- & -- \\
Diet 3 & 1.00021 & 1.0037 & 1.073 \\
Diet 4 & -- & -- & -- \\
\hline
\end{tabular}

\begin{tabular}{lccc}
\hline Diet 1 & -- & -- & -- \\
Diet 2 & -- & -- & -- \\
Diet 3 & 0.021 & 0.37 & -8 \\
Diet 4 & -- & -- & - \\
\hline Diet 1 - Backyard cow milk + all other locally produced non-milk exposure pathways & \\
Diet 2 - Locally produced commercial milk + all other locally produced non-milk exposure pathways \\
Diet 3 - Regionally mixed commercial milk + inhalation (other regionally mixed food items are minor contributors to the total) \\
Diet 4 - Goat milk (all other exposure pathways are of negligible importance compared to goat milk)
\end{tabular}


Location: Hardin Valley

Receptor: Female born in 1935

\begin{tabular}{lccc}
\hline & \multicolumn{3}{c}{ Thyroid Dose [cGy] } \\
Exposure Pathway & $95 \%$ & Subjective Confidence Interval \\
Backyard Cow Milk & lower limit & central estimate & upper limit \\
Commercial Milk (locally produced) & 1.3 & 6.9 & 41 \\
Commercial Milk (regionally mixed) & 0.33 & 2 & 13 \\
Goat Milk (locally produced) & 0.071 & 0.43 & 2.6 \\
Beef (locally produced) & 3.3 & 22 & 130 \\
Leafy Vegetables (locally produced) & 0.0029 & 0.048 & 0.96 \\
Eggs (locally produced) & 0.0012 & 0.013 & 0.11 \\
Cottage Cheese (locally produced) & 0.081 & 0.54 & 3.6 \\
Inhalation & 0.0026 & 0.026 & 0.28 \\
Mother's milk (mother on Diet 1) & 0.06 & 0.2 & 0.72 \\
Prenatal exposure (mother on Diet 1) & -- & -- & -- \\
Diet 1 & -- & -- & -- \\
Diet 2 & 1.5 & 7.9 & 47 \\
Diet 3 & 0.58 & 3 & 18 \\
\hline
\end{tabular}

Excess Lifetime Risk [ ]

\begin{tabular}{llll}
\hline Diet 1 & $1.9 \mathrm{E}-05$ & $2.6 \mathrm{E}-04$ & $3.1 \mathrm{E}-03$ \\
Diet 2 & $9.0 \mathrm{E}-06$ & $1.1 \mathrm{E}-04$ & $1.3 \mathrm{E}-03$ \\
Diet 3 & $2.5 \mathrm{E}-06$ & $2.4 \mathrm{E}-05$ & $2.3 \mathrm{E}-04$ \\
Diet 4 & $6.2 \mathrm{E}-05$ & $9.2 \mathrm{E}-04$ & $1.2 \mathrm{E}-02$ \\
\hline
\end{tabular}

\section{Relative Risk [ ]}

\begin{tabular}{lccc}
\hline Diet 1 & 1.0072 & 1.064 & 1.68 \\
Diet 2 & 1.0033 & 1.029 & 1.3 \\
Diet 3 & 1.00084 & 1.0063 & 1.057 \\
Diet 4 & 1.024 & 1.2 & 3.6 \\
\hline
\end{tabular}

\begin{tabular}{|c|c|c|c|}
\hline Diet 1 & 0.715 & 6.00 & 40.4 \\
\hline Diet 2 & 0.326 & 2.80 & 23.2 \\
\hline Diet 3 & 0.084 & 0.63 & 5.4 \\
\hline Diet 4 & 2.369 & 16.87 & 72.4 \\
\hline
\end{tabular}

Diet 1 - Backyard cow milk + all other locally produced non-milk exposure pathways

Diet 2 - Locally produced commercial milk + all other locally produced non-milk exposure pathways

Diet 3 - Regionally mixed commercial milk + inhalation (other regionally mixed food items are minor contributors to the total)

Diet 4 - Goat milk (all other exposure pathways are of negligible importance compared to goat milk) 
Location: Hardin Valley

Receptor: Male born in 1935

\begin{tabular}{lccc}
\hline & \multicolumn{3}{c}{ Thyroid Dose [cGy] } \\
& \multicolumn{2}{c}{$95 \%$ Subjective Confidence Interval } \\
Exposure Pathway & lower limit & central estimate & upper limit \\
\hline Backyard Cow Milk & 1.4 & 8.1 & 45 \\
Commercial Milk (locally produced) & 0.38 & 2.3 & 15 \\
Commercial Milk (regionally mixed) & 0.078 & 0.47 & 3 \\
Goat Milk (locally produced) & 3.8 & 26 & 160 \\
Beef (locally produced) & 0.0042 & 0.068 & 1.4 \\
Leafy Vegetables (locally produced) & 0.0011 & 0.012 & 0.1 \\
Eggs (locally produced) & 0.09 & 0.64 & 4.4 \\
Cottage Cheese (locally produced) & 0.0026 & 0.027 & 0.31 \\
Inhalation & 0.063 & 0.22 & 0.74 \\
Mother's milk (mother on Diet 1) & -- & -- & - \\
Prenatal exposure (mother on Diet 1) & -- & -- & - \\
Diet 1 & 1.6 & 9.2 & 52 \\
Diet 2 & 0.66 & 3.5 & 21 \\
Diet 3 & 0.18 & 0.74 & 3.8 \\
\hline
\end{tabular}

Excess Lifetime Risk [ ]

\begin{tabular}{llll}
\hline Diet 1 & $2.2 \mathrm{E}-06$ & $6.8 \mathrm{E}-05$ & $1.5 \mathrm{E}-03$ \\
Diet 2 & $9.7 \mathrm{E}-07$ & $3.2 \mathrm{E}-05$ & $7.1 \mathrm{E}-04$ \\
Diet 3 & $2.5 \mathrm{E}-07$ & $6.6 \mathrm{E}-06$ & $1.4 \mathrm{E}-04$ \\
Diet 4 & $6.5 \mathrm{E}-06$ & $1.9 \mathrm{E}-04$ & $4.8 \mathrm{E}-03$ \\
\hline
\end{tabular}

\section{Relative Risk [ ]}

\begin{tabular}{lccc}
\hline Diet 1 & 1.0022 & 1.045 & 2 \\
Diet 2 & 1.0011 & 1.02 & 1.38 \\
Diet 3 & 1.00023 & 1.0041 & 1.077 \\
Diet 4 & 1.0066 & 1.15 & 3.9 \\
\hline
\end{tabular}

\section{Probability of Causation [\%]}

\begin{tabular}{lccc}
\hline Diet 1 & 0.218 & 4.32 & 50.8 \\
Diet 2 & 0.105 & 1.99 & 27.6 \\
Diet 3 & 0.023 & 0.41 & 7.1 \\
Diet 4 & 0.659 & 12.71 & 74.0 \\
\hline
\end{tabular}

Diet 1 - Backyard cow milk + all other locally produced non-milk exposure pathways

Diet 2 - Locally produced commercial milk + all other locally produced non-milk exposure pathways

Diet 3 - Regionally mixed commercial milk + inhalation (other regionally mixed food items are minor contributors to the total)

Diet 4 - Goat milk (all other exposure pathways are of negligible importance compared to goat milk) 


\section{Location: Oliver Springs}

Receptor: Female born in 1935

\begin{tabular}{lccc}
\hline & \multicolumn{3}{c}{ Thyroid Dose [cGy] } \\
Exposure Pathway & \multicolumn{2}{c}{$95 \%$ Subjective Confidence Interval } \\
\hline Backyard Cow Milk & lower limit & central estimate & upper limit \\
Commercial Milk (locally produced) & 0.18 & 1.1 & 6.9 \\
Commercial Milk (regionally mixed) & 0.048 & 0.31 & 2.2 \\
Goat Milk (locally produced) & 0.071 & 0.43 & 2.6 \\
Beef (locally produced) & 0.45 & 3.3 & 23 \\
Leafy Vegetables (locally produced) & 0.00043 & 0.0077 & 0.16 \\
Eggs (locally produced) & 0.00016 & 0.0019 & 0.019 \\
Cottage Cheese (locally produced) & 0.012 & 0.082 & 0.63 \\
Inhalation & 0.00035 & 0.0042 & 0.048 \\
Mother's milk (mother on Diet 1) & 0.0092 & 0.031 & 0.12 \\
Prenatal exposure (mother on Diet 1) & -- & -- & -- \\
Diet 1 & -- & -- & -- \\
Diet 2 & 0.21 & 1.2 & 7.7 \\
Diet 3 & 0.088 & 0.46 & 2.9 \\
\hline
\end{tabular}

Excess Lifetime Risk [ ]

\begin{tabular}{llcc}
\hline Diet 1 & $3.1 \mathrm{E}-06$ & $4.0 \mathrm{E}-05$ & $5.4 \mathrm{E}-04$ \\
Diet 2 & $1.5 \mathrm{E}-06$ & $1.7 \mathrm{E}-05$ & $2.3 \mathrm{E}-04$ \\
Diet 3 & $1.5 \mathrm{E}-06$ & $1.8 \mathrm{E}-05$ & $1.9 \mathrm{E}-04$ \\
Diet 4 & $9.7 \mathrm{E}-06$ & $1.3 \mathrm{E}-04$ & $1.8 \mathrm{E}-03$ \\
\hline
\end{tabular}

Relative Risk [ ]

\begin{tabular}{lccc}
\hline Diet 1 & 1.00099 & 1.01 & 1.12 \\
Diet 2 & 1.00049 & 1.0044 & 1.056 \\
Diet 3 & 1.00056 & 1.0047 & 1.049 \\
Diet 4 & 1.0035 & 1.032 & 1.42 \\
\hline
\end{tabular}

\section{Probability of Causation [\%]}

\begin{tabular}{llcc}
\hline Diet 1 & 0.099 & 0.99 & 10.5 \\
Diet 2 & 0.049 & 0.44 & 5.3 \\
Diet 3 & 0.056 & 0.47 & 4.7 \\
Diet 4 & 0.344 & 3.07 & 29.4 \\
\hline
\end{tabular}

Diet 1 - Backyard cow milk + all other locally produced non-milk exposure pathways

Diet 2 - Locally produced commercial milk + all other locally produced non-milk exposure pathways

Diet 3 - Regionally mixed commercial milk + inhalation (other regionally mixed food items are minor contributors to the total)

Diet 4 - Goat milk (all other exposure pathways are of negligible importance compared to goat milk) 


\section{Location: Oliver Springs}

\section{Receptor: Male born in 1935}

\begin{tabular}{lccc}
\hline & \multicolumn{3}{c}{ Thyroid Dose [cGy] } \\
Exposure Pathway & \multicolumn{2}{c}{$95 \%$ Subjective Confidence Interval } \\
\hline Backyard Cow Milk & lower limit & central estimate & upper limit \\
Commercial Milk (locally produced) & 0.21 & 1.2 & 7.7 \\
Commercial Milk (regionally mixed) & 0.054 & 0.35 & 2.6 \\
Goat Milk (locally produced) & 0.078 & 0.47 & 3 \\
Beef (locally produced) & 0.51 & 3.9 & 28 \\
Leafy Vegetables (locally produced) & 0.00063 & 0.011 & 0.24 \\
Eggs (locally produced) & 0.00016 & 0.0019 & 0.018 \\
Cottage Cheese (locally produced) & 0.013 & 0.097 & 0.67 \\
Inhalation & 0.00038 & 0.0042 & 0.045 \\
Mother's milk (mother on Diet 1) & 0.0096 & 0.034 & 0.13 \\
Prenatal exposure (mother on Diet 1) & -- & -- & -- \\
Diet 1 & -- & -- & -- \\
Diet 2 & 0.25 & 1.4 & 8.5 \\
Diet 3 & 0.097 & 0.53 & 3.5 \\
\hline
\end{tabular}

Excess Lifetime Risk [ ]

\begin{tabular}{llll}
\hline Diet 1 & $2.8 \mathrm{E}-07$ & $1.0 \mathrm{E}-05$ & $2.6 \mathrm{E}-04$ \\
Diet 2 & $1.4 \mathrm{E}-07$ & $4.7 \mathrm{E}-06$ & $1.1 \mathrm{E}-04$ \\
Diet 3 & $1.7 \mathrm{E}-07$ & $4.8 \mathrm{E}-06$ & $1.2 \mathrm{E}-04$ \\
Diet 4 & $9.7 \mathrm{E}-07$ & $3.1 \mathrm{E}-05$ & $8.4 \mathrm{E}-04$ \\
\hline
\end{tabular}

\section{Relative Risk [ ]}

\begin{tabular}{lccc}
\hline Diet 1 & 1.00033 & 1.0074 & 1.16 \\
Diet 2 & 1.00016 & 1.0032 & 1.07 \\
Diet 3 & 1.00017 & 1.0031 & 1.065 \\
Diet 4 & 1.00084 & 1.022 & 1.53 \\
\hline
\end{tabular}

\section{Probability of Causation [\%]}

\begin{tabular}{llcc}
\hline Diet 1 & 0.033 & 0.74 & 13.5 \\
Diet 2 & 0.016 & 0.32 & 6.5 \\
Diet 3 & 0.017 & 0.31 & 6.1 \\
Diet 4 & 0.084 & 2.12 & 34.1 \\
\hline
\end{tabular}

Diet 1 - Backyard cow milk + all other locally produced non-milk exposure pathways

Diet 2 - Locally produced commercial milk + all other locally produced non-milk exposure pathways

Diet 3 - Regionally mixed commercial milk + inhalation (other regionally mixed food items are minor contributors to the total)

Diet 4 - Goat milk (all other exposure pathways are of negligible importance compared to goat milk) 


\section{Location: Solway}

Receptor: Female born in 1935

\begin{tabular}{lccc}
\hline & \multicolumn{3}{c}{ Thyroid Dose [cGy] } \\
& \multicolumn{2}{c}{$95 \%$ Subjective Confidence Interval } \\
Exposure Pathway & lower limit & central estimate & upper limit \\
\hline Backyard Cow Milk & 1.1 & 6.4 & 37 \\
Commercial Milk (locally produced) & 0.29 & 1.8 & 12 \\
Commercial Milk (regionally mixed) & 0.071 & 0.43 & 2.6 \\
Goat Milk (locally produced) & 3 & 20 & 120 \\
Beef (locally produced) & 0.0026 & 0.045 & 0.89 \\
Leafy Vegetables (locally produced) & 0.0011 & 0.011 & 0.1 \\
Eggs (locally produced) & 0.075 & 0.49 & 3.2 \\
Cottage Cheese (locally produced) & 0.0022 & 0.024 & 0.26 \\
Inhalation & 0.057 & 0.18 & 0.65 \\
Mother's milk (mother on Diet 1) & -- & -- & - \\
Prenatal exposure (mother on Diet 1) & -- & -- & - \\
Diet 1 & 1.3 & 7.2 & 43 \\
Diet 2 & 0.53 & 2.7 & 15 \\
Diet 3 & 0.16 & 0.63 & 3.1 \\
\hline
\end{tabular}

Excess Lifetime Risk [ ]

\begin{tabular}{llll}
\hline Diet 1 & $1.8 \mathrm{E}-05$ & $2.4 \mathrm{E}-04$ & $3.1 \mathrm{E}-03$ \\
Diet 2 & $9.1 \mathrm{E}-06$ & $1.0 \mathrm{E}-04$ & $1.3 \mathrm{E}-03$ \\
Diet 3 & $2.5 \mathrm{E}-06$ & $2.3 \mathrm{E}-05$ & $2.3 \mathrm{E}-04$ \\
Diet 4 & $5.9 \mathrm{E}-05$ & $8.0 \mathrm{E}-04$ & $1.1 \mathrm{E}-02$ \\
\hline
\end{tabular}

\section{Relative Risk [ ]}

\begin{tabular}{lccc}
\hline Diet 1 & 1.0067 & 1.058 & 1.63 \\
Diet 2 & 1.003 & 1.026 & 1.29 \\
Diet 3 & 1.00082 & 1.0062 & 1.057 \\
Diet 4 & 1.022 & 1.19 & 3.4 \\
\hline
\end{tabular}

\section{Probability of Causation [\%]}

\begin{tabular}{lccc}
\hline Diet 1 & 0.668 & 5.49 & 38.4 \\
Diet 2 & 0.294 & 2.57 & 22.3 \\
Diet 3 & 0.082 & 0.61 & 5.4 \\
Diet 4 & 2.136 & 15.73 & 70.4 \\
\hline
\end{tabular}

Diet 1 - Backyard cow milk + all other locally produced non-milk exposure pathways

Diet 2 - Locally produced commercial milk + all other locally produced non-milk exposure pathways

Diet 3 - Regionally mixed commercial milk + inhalation (other regionally mixed food items are minor contributors to the total)

Diet 4 - Goat milk (all other exposure pathways are of negligible importance compared to goat milk) 


\section{Location: Solway}

\section{Receptor: Male born in 1935}

\begin{tabular}{lccc}
\hline & \multicolumn{3}{c}{ Thyroid Dose [cGy] } \\
& \multicolumn{2}{c}{$95 \%$ Subjective Confidence Interval } \\
Exposure Pathway & lower limit & central estimate & upper limit \\
\hline Backyard Cow Milk & 1.4 & 7.4 & 43 \\
Commercial Milk (locally produced) & 0.33 & 2 & 13 \\
Commercial Milk (regionally mixed) & 0.078 & 0.47 & 3 \\
Goat Milk (locally produced) & 3.4 & 23 & 150 \\
Beef (locally produced) & 0.0036 & 0.063 & 1.2 \\
Leafy Vegetables (locally produced) & 0.001 & 0.011 & 0.096 \\
Eggs (locally produced) & 0.084 & 0.59 & 3.6 \\
Cottage Cheese (locally produced) & 0.0022 & 0.024 & 0.27 \\
Inhalation & 0.059 & 0.2 & 0.66 \\
Mother's milk (mother on Diet 1) & -- & -- & -- \\
Prenatal exposure (mother on Diet 1) & -- & -- & -- \\
Diet 1 & 1.7 & 8.4 & 49 \\
Diet 2 & 0.62 & 3.1 & 18 \\
Diet 3 & 0.18 & 0.72 & 3.7 \\
\hline
\end{tabular}

Excess Lifetime Risk [ ]

\begin{tabular}{llll}
\hline Diet 1 & $2.0 \mathrm{E}-06$ & $6.1 \mathrm{E}-05$ & $1.4 \mathrm{E}-03$ \\
Diet 2 & $8.8 \mathrm{E}-07$ & $2.7 \mathrm{E}-05$ & $6.4 \mathrm{E}-04$ \\
Diet 3 & $2.4 \mathrm{E}-07$ & $6.5 \mathrm{E}-06$ & $1.4 \mathrm{E}-04$ \\
Diet 4 & $5.9 \mathrm{E}-06$ & $1.7 \mathrm{E}-04$ & $4.5 \mathrm{E}-03$ \\
\hline
\end{tabular}

\section{Relative Risk [ ]}

\begin{tabular}{lccc}
\hline Diet 1 & 1.0017 & 1.042 & 1.9 \\
Diet 2 & 1.00094 & 1.019 & 1.36 \\
Diet 3 & 1.00023 & 1.0041 & 1.076 \\
Diet 4 & 1.0058 & 1.13 & 3.5 \\
\hline
\end{tabular}

\section{Probability of Causation [\%]}

\begin{tabular}{lccc}
\hline Diet 1 & 0.171 & 3.99 & 47.3 \\
Diet 2 & 0.094 & 1.83 & 26.4 \\
Diet 3 & 0.023 & 0.41 & 7.1 \\
Diet 4 & 0.575 & 11.71 & 71.4 \\
\hline
\end{tabular}

Diet 1 - Backyard cow milk + all other locally produced non-milk exposure pathways

Diet 2 - Locally produced commercial milk + all other locally produced non-milk exposure pathways

Diet 3 - Regionally mixed commercial milk + inhalation (other regionally mixed food items are minor contributors to the total)

Diet 4 - Goat milk (all other exposure pathways are of negligible importance compared to goat milk) 
Location: Sugar Grove

Receptor: Female born in 1935

\begin{tabular}{lccc}
\hline & \multicolumn{3}{c}{ Thyroid Dose [cGy] } \\
Exposure Pathway & $95 \%$ Subjective Confidence Interval \\
\hline Backyard Cow Milk & lower limit & central estimate & upper limit \\
Commercial Milk (locally produced) & 0.48 & 2.7 & 16 \\
Commercial Milk (regionally mixed) & 0.12 & 0.77 & 5.1 \\
Goat Milk (locally produced) & 0.071 & 0.43 & 2.6 \\
Beef (locally produced) & 1.3 & 8.3 & 54 \\
Leafy Vegetables (locally produced) & 0.0012 & 0.019 & 0.36 \\
Eggs (locally produced) & 0.00043 & 0.0048 & 0.042 \\
Cottage Cheese (locally produced) & 0.032 & 0.2 & 1.4 \\
Inhalation & 0.00091 & 0.01 & 0.12 \\
Mother's milk (mother on Diet 1) & 0.023 & 0.076 & 0.27 \\
Prenatal exposure (mother on Diet 1) & -- & -- & -- \\
Diet 1 & -- & -- & -- \\
Diet 2 & 0.57 & 3 & 18 \\
Diet 3 & 0.22 & 1.2 & 7.1 \\
\hline
\end{tabular}

Excess Lifetime Risk [ ]

\begin{tabular}{llll}
\hline Diet 1 & $7.6 \mathrm{E}-06$ & $1.0 \mathrm{E}-04$ & $1.3 \mathrm{E}-03$ \\
Diet 2 & $3.7 \mathrm{E}-06$ & $4.3 \mathrm{E}-05$ & $5.3 \mathrm{E}-04$ \\
Diet 3 & $1.9 \mathrm{E}-06$ & $2.0 \mathrm{E}-05$ & $2.0 \mathrm{E}-04$ \\
Diet 4 & $2.5 \mathrm{E}-05$ & $3.4 \mathrm{E}-04$ & $4.3 \mathrm{E}-03$ \\
\hline
\end{tabular}

\section{Relative Risk [ ]}

\begin{tabular}{lccc}
\hline Diet 1 & 1.0028 & 1.025 & 1.28 \\
Diet 2 & 1.0012 & 1.011 & 1.13 \\
Diet 3 & 1.00064 & 1.0051 & 1.051 \\
Diet 4 & 1.0089 & 1.076 & 2.1 \\
\hline
\end{tabular}

\section{Probability of Causation [\%]}

\begin{tabular}{llcc}
\hline Diet 1 & 0.282 & 2.39 & 21.6 \\
Diet 2 & 0.122 & 1.07 & 11.1 \\
Diet 3 & 0.064 & 0.51 & 4.9 \\
Diet 4 & 0.881 & 6.97 & 51.3 \\
\hline
\end{tabular}

Diet 1 - Backyard cow milk + all other locally produced non-milk exposure pathways

Diet 2 - Locally produced commercial milk + all other locally produced non-milk exposure pathways

Diet 3 - Regionally mixed commercial milk + inhalation (other regionally mixed food items are minor contributors to the total)

Diet 4 - Goat milk (all other exposure pathways are of negligible importance compared to goat milk) 
Location: Sugar Grove

Receptor: Male born in 1935

\begin{tabular}{lccc}
\hline & \multicolumn{3}{c}{ Thyroid Dose [cGy] } \\
& \multicolumn{2}{c}{$95 \%$ Subjective Confidence Interval } \\
Exposure Pathway & lower limit & central estimate & upper limit \\
\hline Backyard Cow Milk & 0.58 & 3.1 & 18 \\
Commercial Milk (locally produced) & 0.14 & 0.86 & 6 \\
Commercial Milk (regionally mixed) & 0.078 & 0.47 & 3 \\
Goat Milk (locally produced) & 1.5 & 9.7 & 62 \\
Beef (locally produced) & 0.0016 & 0.026 & 0.55 \\
Leafy Vegetables (locally produced) & 0.00042 & 0.0045 & 0.042 \\
Eggs (locally produced) & 0.035 & 0.25 & 1.7 \\
Cottage Cheese (locally produced) & 0.00094 & 0.01 & 0.12 \\
Inhalation & 0.024 & 0.084 & 0.29 \\
Mother's milk (mother on Diet 1) & -- & -- & - \\
Prenatal exposure (mother on Diet 1) & -- & -- & - \\
Diet 1 & 0.67 & 3.5 & 21 \\
Diet 2 & 0.26 & 1.3 & 8.1 \\
Diet 3 & 0.12 & 0.57 & 3.4 \\
\hline
\end{tabular}

Excess Lifetime Risk [ ]

\begin{tabular}{llll}
\hline Diet 1 & $7.9 \mathrm{E}-07$ & $2.6 \mathrm{E}-05$ & $5.9 \mathrm{E}-04$ \\
Diet 2 & $3.8 \mathrm{E}-07$ & $1.2 \mathrm{E}-05$ & $2.8 \mathrm{E}-04$ \\
Diet 3 & $2.0 \mathrm{E}-07$ & $5.3 \mathrm{E}-06$ & $1.2 \mathrm{E}-04$ \\
Diet 4 & $2.7 \mathrm{E}-06$ & $7.1 \mathrm{E}-05$ & $1.9 \mathrm{E}-03$ \\
\hline
\end{tabular}

\section{Relative Risk [ ]}

\begin{tabular}{lccc}
\hline Diet 1 & 1.00078 & 1.018 & 1.4 \\
Diet 2 & 1.00039 & 1.0079 & 1.15 \\
Diet 3 & 1.00019 & 1.0033 & 1.068 \\
Diet 4 & 1.0025 & 1.055 & 2.1 \\
\hline
\end{tabular}

\section{Probability of Causation [\%]}

\begin{tabular}{lccc}
\hline Diet 1 & 0.078 & 1.73 & 27.7 \\
Diet 2 & 0.039 & 0.78 & 13.1 \\
Diet 3 & 0.019 & 0.33 & 6.4 \\
Diet 4 & 0.249 & 5.16 & 50.5 \\
\hline
\end{tabular}

Diet 1 - Backyard cow milk + all other locally produced non-milk exposure pathways

Diet 2 - Locally produced commercial milk + all other locally produced non-milk exposure pathways

Diet 3 - Regionally mixed commercial milk + inhalation (other regionally mixed food items are minor contributors to the total)

Diet 4 - Goat milk (all other exposure pathways are of negligible importance compared to goat milk) 


\section{Location: OR Townsite}

Receptor: Female born in 1935

\begin{tabular}{lccc}
\hline & \multicolumn{3}{c}{ Thyroid Dose [cGy] } \\
Exposure Pathway & \multicolumn{2}{c}{$9 \%$ Subjective Confidence Interval } \\
\hline Backyard Cow Milk & lower limit & central estimate & upper limit \\
Commercial Milk (locally produced) & -- & -- & -- \\
Commercial Milk (regionally mixed) & -- & -- & -- \\
Goat Milk (locally produced) & 0.071 & 0.43 & 2.6 \\
Beef (locally produced) & -- & -- & -- \\
Leafy Vegetables (locally produced) & -- & -- & -- \\
Eggs (locally produced) & -- & -- & -- \\
Cottage Cheese (locally produced) & -- & -- & -- \\
Inhalation & 0.0011 & 0.011 & 0.12 \\
Mother's milk (mother on Diet 3) & 0.025 & 0.084 & 0.3 \\
Prenatal exposure (mother on Diet 3) & -- & -- & -- \\
Diet 1 & -- & -- & -- \\
Diet 2 & -- & -- & -- \\
Diet 3 & -- & -- & -- \\
\hline
\end{tabular}

Excess Lifetime Risk [ ]

\begin{tabular}{lccc}
\hline Diet 1 & -- & -- & -- \\
Diet 2 & -- & -- & -- \\
Diet 3 & $1.9 \mathrm{E}-06$ & $2.0 \mathrm{E}-05$ & $2.0 \mathrm{E}-04$ \\
Diet 4 & -- & -- & -- \\
\hline
\end{tabular}

\section{Relative Risk [ ]}

\begin{tabular}{lccc}
\hline Diet 1 & -- & -- & -- \\
Diet 2 & -- & -- & -- \\
Diet 3 & 1.00066 & 1.0052 & 1.051 \\
Diet 4 & -- & -- & -- \\
\hline
\end{tabular}

\begin{tabular}{lccc}
\hline Diet 1 & -- & -- & -- \\
Diet 2 & -- & -- & -- \\
Diet 3 & 0.066 & 0.52 & 4.9 \\
Diet 4 & -- & -- & -- \\
\hline Diet 1 - Backyard cow milk + all other locally produced non-milk exposure pathways
\end{tabular}

Diet 1 - Backyard cow milk + all other locally produced non-milk exposure pathways

Diet 2 - Locally produced commercial milk + all other locally produced non-milk exposure pathways

Diet 3 - Regionally mixed commercial milk + inhalation (other regionally mixed food items are minor contributors to the total)

Diet 4 - Goat milk (all other exposure pathways are of negligible importance compared to goat milk) 


\section{Location: OR Townsite}

\section{Receptor: Male born in 1935}

\begin{tabular}{lccc}
\hline & \multicolumn{3}{c}{ Thyroid Dose [cGy] } \\
Exposure Pathway & \multicolumn{2}{c}{$9 \%$ Subjective Confidence Interval } \\
\hline Backyard Cow Milk & lower limit & central estimate & upper limit \\
Commercial Milk (locally produced) & -- & -- & -- \\
Commercial Milk (regionally mixed) & -- & -- & -- \\
Goat Milk (locally produced) & 0.078 & 0.47 & 3 \\
Beef (locally produced) & -- & -- & -- \\
Leafy Vegetables (locally produced) & -- & -- & -- \\
Eggs (locally produced) & -- & -- & -- \\
Cottage Cheese (locally produced) & -- & -- & -- \\
Inhalation & 0.0011 & 0.011 & 0.13 \\
Mother's milk (mother on Diet 3) & 0.026 & 0.091 & 0.32 \\
Prenatal exposure (mother on Diet 3) & -- & -- & -- \\
Diet 1 & -- & -- & -- \\
Diet 2 & -- & -- & -- \\
Diet 3 & -- & -- & -- \\
\hline
\end{tabular}

Excess Lifetime Risk [ ]

\begin{tabular}{lccc}
\hline Diet 1 & -- & -- & -- \\
Diet 2 & -- & -- & -- \\
Diet 3 & $1.9 \mathrm{E}-07$ & $5.3 \mathrm{E}-06$ & $1.3 \mathrm{E}-04$ \\
Diet 4 & -- & -- & -- \\
\hline
\end{tabular}

\section{Relative Risk [ ]}

\begin{tabular}{lccc}
\hline Diet 1 & -- & -- & -- \\
Diet 2 & -- & -- & -- \\
Diet 3 & 1.00019 & 1.0034 & 1.069 \\
Diet 4 & -- & -- & -- \\
\hline
\end{tabular}

\begin{tabular}{lccc}
\hline Diet 1 & -- & -- & -- \\
Diet 2 & -- & -- & -- \\
Diet 3 & 0.019 & 0.34 & 6.4 \\
Diet 4 & -- & -- \\
\hline Diet 1 - Backyard cow milk + all other locally produced non-milk exposure pathways & \\
Diet 2 - Locally produced commercial milk + all other locally produced non-milk exposure pathways \\
Diet 3 - Regionally mixed commercial milk + inhalation (other regionally mixed food items are minor contributors to the total) \\
Diet 4 - Goat milk (all other exposure pathways are of negligible importance compared to goat milk)
\end{tabular}


Location: Hines Valley

Receptor: Female born in 1935

\begin{tabular}{lccc}
\hline & \multicolumn{3}{c}{ Thyroid Dose [cGy] } \\
Exposure Pathway & $95 \%$ Subjective Confidence Interval \\
\hline Backyard Cow Milk & lower limit & central estimate & upper limit \\
Commercial Milk (locally produced) & 0.54 & 3.2 & 20 \\
Commercial Milk (regionally mixed) & 0.15 & 0.9 & 6.1 \\
Goat Milk (locally produced) & 0.071 & 0.43 & 2.6 \\
Beef (locally produced) & 1.5 & 9.8 & 62 \\
Leafy Vegetables (locally produced) & 0.0013 & 0.023 & 0.51 \\
Eggs (locally produced) & 0.00056 & 0.0057 & 0.052 \\
Cottage Cheese (locally produced) & 0.036 & 0.25 & 1.7 \\
Inhalation & 0.0013 & 0.012 & 0.13 \\
Mother's milk (mother on Diet 1) & 0.029 & 0.1 & 0.34 \\
Prenatal exposure (mother on Diet 1) & -- & -- & -- \\
Diet 1 & -- & -- & -- \\
Diet 2 & 0.67 & 3.6 & 23 \\
Diet 3 & 0.27 & 1.4 & 8 \\
\hline
\end{tabular}

Excess Lifetime Risk [ ]

\begin{tabular}{llll}
\hline Diet 1 & $8.8 \mathrm{E}-06$ & $1.2 \mathrm{E}-04$ & $1.5 \mathrm{E}-03$ \\
Diet 2 & $4.8 \mathrm{E}-06$ & $5.3 \mathrm{E}-05$ & $6.5 \mathrm{E}-04$ \\
Diet 3 & $2.0 \mathrm{E}-06$ & $2.1 \mathrm{E}-05$ & $2.1 \mathrm{E}-04$ \\
Diet 4 & $3.0 \mathrm{E}-05$ & $4.0 \mathrm{E}-04$ & $6.1 \mathrm{E}-03$ \\
\hline
\end{tabular}

\section{Relative Risk [ ]}

\begin{tabular}{lccc}
\hline Diet 1 & 1.0035 & 1.03 & 1.3 \\
Diet 2 & 1.0014 & 1.013 & 1.14 \\
Diet 3 & 1.00067 & 1.0054 & 1.052 \\
Diet 4 & 1.011 & 1.093 & 2.2 \\
\hline
\end{tabular}

Probability of Causation [\%]

\begin{tabular}{lccc}
\hline Diet 1 & 0.352 & 2.92 & 23.1 \\
Diet 2 & 0.138 & 1.33 & 12.2 \\
Diet 3 & 0.067 & 0.54 & 4.9 \\
Diet 4 & 1.055 & 8.53 & 53.6 \\
\hline
\end{tabular}

Diet 1 - Backyard cow milk + all other locally produced non-milk exposure pathways

Diet 2 - Locally produced commercial milk + all other locally produced non-milk exposure pathways

Diet 3 - Regionally mixed commercial milk + inhalation (other regionally mixed food items are minor contributors to the total)

Diet 4 - Goat milk (all other exposure pathways are of negligible importance compared to goat milk) 
Location: Hines Valley

Receptor: Male born in 1935

\begin{tabular}{lccc}
\hline & \multicolumn{3}{c}{ Thyroid Dose [cGy] } \\
Exposure Pathway & $95 \%$ Subjective Confidence Interval \\
\hline Backyard Cow Milk & lower limit & central estimate & upper limit \\
Commercial Milk (locally produced) & 0.71 & 3.8 & 22 \\
Commercial Milk (regionally mixed) & 0.16 & 1 & 7.1 \\
Goat Milk (locally produced) & 0.078 & 0.47 & 3.1 \\
Beef (locally produced) & 1.7 & 11 & 73 \\
Leafy Vegetables (locally produced) & 0.0019 & 0.032 & 0.69 \\
Eggs (locally produced) & 0.00055 & 0.0056 & 0.05 \\
Cottage Cheese (locally produced) & 0.04 & 0.29 & 2 \\
Inhalation & 0.0012 & 0.012 & 0.15 \\
Mother's milk (mother on Diet 1) & 0.031 & 0.11 & 0.37 \\
Prenatal exposure (mother on Diet 1) & -- & -- & -- \\
Diet 1 & -- & -- & -- \\
Diet 2 & 0.83 & 4.3 & 24 \\
Diet 3 & 0.3 & 1.6 & 9.2 \\
\hline
\end{tabular}

Excess Lifetime Risk [ ]

\begin{tabular}{llcc}
\hline Diet 1 & $9.7 \mathrm{E}-07$ & $3.1 \mathrm{E}-05$ & $8.1 \mathrm{E}-04$ \\
Diet 2 & $4.4 \mathrm{E}-07$ & $1.4 \mathrm{E}-05$ & $3.5 \mathrm{E}-04$ \\
Diet 3 & $2.0 \mathrm{E}-07$ & $5.5 \mathrm{E}-06$ & $1.3 \mathrm{E}-04$ \\
Diet 4 & $3.2 \mathrm{E}-06$ & $8.8 \mathrm{E}-05$ & $2.5 \mathrm{E}-03$ \\
\hline
\end{tabular}

\section{Relative Risk [ ]}

\begin{tabular}{lccc}
\hline Diet 1 & 1.00096 & 1.022 & 1.45 \\
Diet 2 & 1.00047 & 1.0095 & 1.19 \\
Diet 3 & 1.0002 & 1.0034 & 1.071 \\
Diet 4 & 1.0027 & 1.067 & 2.5 \\
\hline
\end{tabular}

\section{Probability of Causation [\%]}

\begin{tabular}{llcc}
\hline Diet 1 & 0.096 & 2.18 & 31.2 \\
Diet 2 & 0.046 & 0.94 & 15.6 \\
Diet 3 & 0.020 & 0.34 & 6.6 \\
Diet 4 & 0.270 & 6.32 & 59.1 \\
\hline
\end{tabular}

Diet 1 - Backyard cow milk + all other locally produced non-milk exposure pathways

Diet 2 - Locally produced commercial milk + all other locally produced non-milk exposure pathways

Diet 3 - Regionally mixed commercial milk + inhalation (other regionally mixed food items are minor contributors to the total)

Diet 4 - Goat milk (all other exposure pathways are of negligible importance compared to goat milk) 


\section{Location: Farragut}

Receptor: Female born in 1935

\begin{tabular}{lccc}
\hline & \multicolumn{3}{c}{ Thyroid Dose [cGy] } \\
& \multicolumn{2}{c}{$95 \%$ Subjective Confidence Interval } \\
Exposure Pathway & lower limit & central estimate & upper limit \\
\hline Backyard Cow Milk & 0.64 & 3.6 & 20 \\
Commercial Milk (locally produced) & 0.17 & 1.1 & 6.3 \\
Commercial Milk (regionally mixed) & 0.071 & 0.43 & 2.6 \\
Goat Milk (locally produced) & 1.8 & 11 & 76 \\
Beef (locally produced) & 0.0014 & 0.026 & 0.59 \\
Leafy Vegetables (locally produced) & 0.00055 & 0.0065 & 0.059 \\
Eggs (locally produced) & 0.045 & 0.28 & 1.9 \\
Cottage Cheese (locally produced) & 0.0013 & 0.014 & 0.14 \\
Inhalation & 0.034 & 0.11 & 0.41 \\
Mother's milk (mother on Diet 1) & -- & -- & -- \\
Prenatal exposure (mother on Diet 1) & -- & -- & -- \\
Diet 1 & 0.77 & 4.1 & 23 \\
Diet 2 & 0.31 & 1.6 & 8.4 \\
Diet 3 & 0.13 & 0.55 & 2.9 \\
\hline
\end{tabular}

Excess Lifetime Risk [ ]

\begin{tabular}{llll}
\hline Diet 1 & $1.1 \mathrm{E}-05$ & $1.4 \mathrm{E}-04$ & $1.6 \mathrm{E}-03$ \\
Diet 2 & $4.8 \mathrm{E}-06$ & $5.7 \mathrm{E}-05$ & $6.5 \mathrm{E}-04$ \\
Diet 3 & $2.0 \mathrm{E}-06$ & $2.1 \mathrm{E}-05$ & $2.1 \mathrm{E}-04$ \\
Diet 4 & $3.3 \mathrm{E}-05$ & $4.6 \mathrm{E}-04$ & $5.9 \mathrm{E}-03$ \\
\hline
\end{tabular}

\section{Relative Risk [ ]}

\begin{tabular}{lccc}
\hline Diet 1 & 1.0041 & 1.034 & 1.35 \\
Diet 2 & 1.0016 & 1.015 & 1.15 \\
Diet 3 & 1.00072 & 1.0055 & 1.052 \\
Diet 4 & 1.012 & 1.11 & 2.4 \\
\hline
\end{tabular}

\begin{tabular}{llcc} 
& \multicolumn{3}{c}{ Probability of Causation [\%] } \\
\hline Diet 1 & 0.407 & 3.27 & 25.7 \\
Diet 2 & 0.164 & 1.49 & 13.2 \\
Diet 3 & 0.072 & 0.55 & 4.9 \\
Diet 4 & 1.184 & 9.55 & 57.6 \\
\hline
\end{tabular}

Diet 1 - Backyard cow milk + all other locally produced non-milk exposure pathways

Diet 2 - Locally produced commercial milk + all other locally produced non-milk exposure pathways

Diet 3 - Regionally mixed commercial milk + inhalation (other regionally mixed food items are minor contributors to the total)

Diet 4 - Goat milk (all other exposure pathways are of negligible importance compared to goat milk) 


\section{Location: Farragut}

\section{Receptor: Male born in 1935}

\begin{tabular}{lccc}
\hline & \multicolumn{3}{c}{ Thyroid Dose [cGy] } \\
& \multicolumn{2}{c}{$95 \%$ Subjective Confidence Interval } \\
Exposure Pathway & lower limit & central estimate & upper limit \\
\hline Backyard Cow Milk & 0.84 & 4.1 & 23 \\
Commercial Milk (locally produced) & 0.19 & 1.2 & 7.6 \\
Commercial Milk (regionally mixed) & 0.078 & 0.47 & 3.1 \\
Goat Milk (locally produced) & 2.1 & 13 & 86 \\
Beef (locally produced) & 0.0021 & 0.036 & 0.86 \\
Leafy Vegetables (locally produced) & 0.00055 & 0.0063 & 0.058 \\
Eggs (locally produced) & 0.049 & 0.33 & 2.1 \\
Cottage Cheese (locally produced) & 0.0014 & 0.014 & 0.15 \\
Inhalation & 0.033 & 0.12 & 0.41 \\
Mother's milk (mother on Diet 1) & -- & -- & - \\
Prenatal exposure (mother on Diet 1) & -- & -- & - \\
Diet 1 & 0.99 & 4.7 & 26 \\
Diet 2 & 0.35 & 1.8 & 10 \\
Diet 3 & 0.14 & 0.62 & 3.5 \\
\hline
\end{tabular}

Excess Lifetime Risk [ ]

\begin{tabular}{llll}
\hline Diet 1 & $1.1 \mathrm{E}-06$ & $3.4 \mathrm{E}-05$ & $8.7 \mathrm{E}-04$ \\
Diet 2 & $4.9 \mathrm{E}-07$ & $1.6 \mathrm{E}-05$ & $3.8 \mathrm{E}-04$ \\
Diet 3 & $2.1 \mathrm{E}-07$ & $5.6 \mathrm{E}-06$ & $1.3 \mathrm{E}-04$ \\
Diet 4 & $3.6 \mathrm{E}-06$ & $9.7 \mathrm{E}-05$ & $2.8 \mathrm{E}-03$ \\
\hline
\end{tabular}

\section{Relative Risk [ ]}

\begin{tabular}{lccc}
\hline Diet 1 & 1.0011 & 1.023 & 1.52 \\
Diet 2 & 1.00056 & 1.01 & 1.22 \\
Diet 3 & 1.0002 & 1.0035 & 1.071 \\
Diet 4 & 1.0034 & 1.074 & 2.6 \\
\hline
\end{tabular}

\section{Probability of Causation [\%]}

\begin{tabular}{llcc}
\hline Diet 1 & 0.113 & 2.27 & 34.3 \\
Diet 2 & 0.056 & 1.02 & 17.8 \\
Diet 3 & 0.020 & 0.35 & 6.6 \\
Diet 4 & 0.334 & 6.91 & 61.1 \\
\hline
\end{tabular}

Diet 1 - Backyard cow milk + all other locally produced non-milk exposure pathways

Diet 2 - Locally produced commercial milk + all other locally produced non-milk exposure pathways

Diet 3 - Regionally mixed commercial milk + inhalation (other regionally mixed food items are minor contributors to the total)

Diet 4 - Goat milk (all other exposure pathways are of negligible importance compared to goat milk) 
Location: Lenoir City

Receptor: Female born in 1935

\begin{tabular}{lccc}
\hline & \multicolumn{3}{c}{ Thyroid Dose [cGy] } \\
Exposure Pathway & \multicolumn{2}{c}{$95 \%$ Subjective Confidence Interval } \\
\hline Backyard Cow Milk & lower limit & central estimate & upper limit \\
Commercial Milk (locally produced) & 0.42 & 2.2 & 11 \\
Commercial Milk (regionally mixed) & 0.1 & 0.61 & 4.4 \\
Goat Milk (locally produced) & 0.071 & 0.43 & 2.6 \\
Beef (locally produced) & -- & -- & -- \\
Leafy Vegetables (locally produced) & 0.00096 & 0.016 & 0.32 \\
Eggs (locally produced) & 0.00042 & 0.004 & 0.033 \\
Cottage Cheese (locally produced) & 0.027 & 0.17 & 1.1 \\
Inhalation & 0.00084 & 0.0083 & 0.089 \\
Mother's milk (mother on Diet 1) & 0.02 & 0.071 & 0.26 \\
Prenatal exposure (mother on Diet 1) & -- & -- & -- \\
Diet 1 & -- & -- & -- \\
Diet 2 & 0.51 & 2.5 & 13 \\
Diet 3 & 0.19 & 0.93 & 5.4 \\
\hline
\end{tabular}

Excess Lifetime Risk [ ]

\begin{tabular}{lccc}
\hline Diet 1 & $6.7 \mathrm{E}-06$ & $8.1 \mathrm{E}-05$ & $1.1 \mathrm{E}-03$ \\
Diet 2 & $3.1 \mathrm{E}-06$ & $3.5 \mathrm{E}-05$ & $4.2 \mathrm{E}-04$ \\
Diet 3 & $1.8 \mathrm{E}-06$ & $2.0 \mathrm{E}-05$ & $2.0 \mathrm{E}-04$ \\
Diet 4 & -- & -- & -- \\
\hline
\end{tabular}

Relative Risk [ ]

\begin{tabular}{lccc}
\hline Diet 1 & 1.0024 & 1.02 & 1.23 \\
Diet 2 & 1.0011 & 1.0091 & 1.11 \\
Diet 3 & 1.00062 & 1.0051 & 1.051 \\
Diet 4 & -- & -- & -- \\
\hline
\end{tabular}

\section{Probability of Causation [\%]}

\begin{tabular}{lccc}
\hline Diet 1 & 0.244 & 1.93 & 18.5 \\
Diet 2 & 0.105 & 0.91 & 9.9 \\
Diet 3 & 0.062 & 0.51 & 4.8 \\
Diet 4 & -- & -- & -- \\
\hline Diet 1 - Backyard cow milk + all other locally produced non-milk exposure pathways & \\
Diet 2 - Locally produced commercial milk + all other locally produced non-milk exposure pathways \\
Diet 3 - Regionally mixed commercial milk + inhalation (other regionally mixed food items are minor contributors to the total) \\
Diet 4 - Goat milk (all other exposure pathways are of negligible importance compared to goat milk)
\end{tabular}




\section{Location: Lenoir City}

\section{Receptor: Male born in 1935}

\begin{tabular}{lccc}
\hline & \multicolumn{3}{c}{ Thyroid Dose [cGy] } \\
Exposure Pathway & $95 \%$ Subjective Confidence Interval \\
\hline Backyard Cow Milk & lower limit & central estimate & upper limit \\
Commercial Milk (locally produced) & 0.49 & 2.6 & 14 \\
Commercial Milk (regionally mixed) & 0.12 & 0.69 & 5 \\
Goat Milk (locally produced) & 0.078 & 0.47 & 3 \\
Beef (locally produced) & -- & -- & -- \\
Leafy Vegetables (locally produced) & 0.0013 & 0.022 & 0.4 \\
Eggs (locally produced) & 0.00041 & 0.004 & 0.032 \\
Cottage Cheese (locally produced) & 0.032 & 0.2 & 1.3 \\
Inhalation & 0.00087 & 0.0084 & 0.081 \\
Mother's milk (mother on Diet 1) & 0.022 & 0.075 & 0.28 \\
Prenatal exposure (mother on Diet 1) & -- & -- & - \\
Diet 1 & -- & -- & -- \\
Diet 2 & 0.57 & 3 & 15 \\
Diet 3 & 0.23 & 1 & 6.4 \\
\hline
\end{tabular}

Excess Lifetime Risk [ ]

\begin{tabular}{lccc}
\hline Diet 1 & $5.8 \mathrm{E}-07$ & $2.2 \mathrm{E}-05$ & $4.7 \mathrm{E}-04$ \\
Diet 2 & $2.4 \mathrm{E}-07$ & $9.3 \mathrm{E}-06$ & $2.2 \mathrm{E}-04$ \\
Diet 3 & $1.9 \mathrm{E}-07$ & $5.2 \mathrm{E}-06$ & $1.3 \mathrm{E}-04$ \\
Diet 4 & -- & -- & -- \\
\hline
\end{tabular}

\section{Relative Risk [ ]}

\begin{tabular}{lccc}
\hline Diet 1 & 1.00076 & 1.014 & 1.28 \\
Diet 2 & 1.00031 & 1.0065 & 1.13 \\
Diet 3 & 1.00018 & 1.0033 & 1.067 \\
Diet 4 & -- & -- & -- \\
\hline
\end{tabular}

\section{Probability of Causation [\%]}

\begin{tabular}{llcr}
\hline Diet 1 & 0.076 & 1.43 & 21.9 \\
Diet 2 & 0.031 & 0.65 & 11.2 \\
Diet 3 & 0.018 & 0.33 & 6.2
\end{tabular}

Diet 4

Diet 1 - Backyard cow milk + all other locally produced non-milk exposure pathways

Diet 2 - Locally produced commercial milk + all other locally produced non-milk exposure pathways

Diet 3 - Regionally mixed commercial milk + inhalation (other regionally mixed food items are minor contributors to the total)

Diet 4 - Goat milk (all other exposure pathways are of negligible importance compared to goat milk) 


\section{Location: Kingston}

Receptor: Female born in 1935

\begin{tabular}{lccc}
\hline & \multicolumn{3}{c}{ Thyroid Dose [cGy] } \\
& \multicolumn{2}{c}{$95 \%$ Subjective Confidence Interval } \\
Exposure Pathway & lower limit & central estimate & upper limit \\
\hline Backyard Cow Milk & 0.47 & 2.8 & 15 \\
Commercial Milk (locally produced) & 0.13 & 0.82 & 4.9 \\
Commercial Milk (regionally mixed) & 0.071 & 0.43 & 2.6 \\
Goat Milk (locally produced) & 1.3 & 9 & 58 \\
Beef (locally produced) & 0.0012 & 0.02 & 0.41 \\
Leafy Vegetables (locally produced) & 0.00048 & 0.0049 & 0.046 \\
Eggs (locally produced) & 0.034 & 0.21 & 1.4 \\
Cottage Cheese (locally produced) & 0.00089 & 0.011 & 0.11 \\
Inhalation & 0.027 & 0.091 & 0.33 \\
Mother's milk (mother on Diet 1) & -- & -- & -- \\
Prenatal exposure (mother on Diet 1) & -- & -- & -- \\
Diet 1 & 0.57 & 3.2 & 18 \\
Diet 2 & 0.24 & 1.2 & 6.9 \\
Diet 3 & 0.12 & 0.53 & 2.9 \\
\hline
\end{tabular}

Excess Lifetime Risk [ ]

\begin{tabular}{llll}
\hline Diet 1 & $7.7 \mathrm{E}-06$ & $1.1 \mathrm{E}-04$ & $1.3 \mathrm{E}-03$ \\
Diet 2 & $4.1 \mathrm{E}-06$ & $4.7 \mathrm{E}-05$ & $5.4 \mathrm{E}-04$ \\
Diet 3 & $1.9 \mathrm{E}-06$ & $2.0 \mathrm{E}-05$ & $2.1 \mathrm{E}-04$ \\
Diet 4 & $2.7 \mathrm{E}-05$ & $3.4 \mathrm{E}-04$ & $4.5 \mathrm{E}-03$ \\
\hline
\end{tabular}

\section{Relative Risk [ ]}

\begin{tabular}{lccc}
\hline Diet 1 & 1.0031 & 1.026 & 1.28 \\
Diet 2 & 1.0015 & 1.012 & 1.13 \\
Diet 3 & 1.00068 & 1.0053 & 1.052 \\
Diet 4 & 1.01 & 1.084 & 2 \\
\hline
\end{tabular}

\section{Probability of Causation [\%]}

\begin{tabular}{lccc}
\hline Diet 1 & 0.304 & 2.52 & 21.8 \\
Diet 2 & 0.149 & 1.17 & 11.6 \\
Diet 3 & 0.068 & 0.53 & 5.0 \\
Diet 4 & 1.001 & 7.74 & 50.3 \\
\hline
\end{tabular}

Diet 1 - Backyard cow milk + all other locally produced non-milk exposure pathways

Diet 2 - Locally produced commercial milk + all other locally produced non-milk exposure pathways

Diet 3 - Regionally mixed commercial milk + inhalation (other regionally mixed food items are minor contributors to the total)

Diet 4 - Goat milk (all other exposure pathways are of negligible importance compared to goat milk) 


\section{Location: Kingston}

Receptor: Male born in 1935

\begin{tabular}{lccc}
\hline & \multicolumn{3}{c}{ Thyroid Dose [cGy] } \\
& \multicolumn{2}{c}{$95 \%$ Subjective Confidence Interval } \\
Exposure Pathway & lower limit & central estimate & upper limit \\
\hline Backyard Cow Milk & 0.63 & 3.4 & 19 \\
Commercial Milk (locally produced) & 0.15 & 0.89 & 6 \\
Commercial Milk (regionally mixed) & 0.078 & 0.47 & 3 \\
Goat Milk (locally produced) & 1.5 & 10 & 67 \\
Beef (locally produced) & 0.0017 & 0.028 & 0.64 \\
Leafy Vegetables (locally produced) & 0.00047 & 0.0051 & 0.042 \\
Eggs (locally produced) & 0.036 & 0.25 & 1.7 \\
Cottage Cheese (locally produced) & 0.0011 & 0.011 & 0.11 \\
Inhalation & 0.028 & 0.099 & 0.35 \\
Mother's milk (mother on Diet 1) & -- & -- & -- \\
Prenatal exposure (mother on Diet 1) & -- & -- & - \\
Diet 1 & 0.74 & 3.8 & 21 \\
Diet 2 & 0.28 & 1.4 & 8.5 \\
Diet 3 & 0.12 & 0.59 & 3.4 \\
\hline
\end{tabular}

Excess Lifetime Risk [ ]

\begin{tabular}{llll}
\hline Diet 1 & $8.4 \mathrm{E}-07$ & $2.7 \mathrm{E}-05$ & $7.0 \mathrm{E}-04$ \\
Diet 2 & $3.7 \mathrm{E}-07$ & $1.3 \mathrm{E}-05$ & $2.9 \mathrm{E}-04$ \\
Diet 3 & $2.0 \mathrm{E}-07$ & $5.4 \mathrm{E}-06$ & $1.3 \mathrm{E}-04$ \\
Diet 4 & $2.8 \mathrm{E}-06$ & $7.8 \mathrm{E}-05$ & $2.2 \mathrm{E}-03$ \\
\hline
\end{tabular}

\section{Relative Risk [ ]}

\begin{tabular}{lccc}
\hline Diet 1 & 1.00078 & 1.019 & 1.4 \\
Diet 2 & 1.00041 & 1.0083 & 1.17 \\
Diet 3 & 1.0002 & 1.0034 & 1.069 \\
Diet 4 & 1.0025 & 1.054 & 2.3 \\
\hline
\end{tabular}

Probability of Causation [\%]

\begin{tabular}{llcc}
\hline Diet 1 & 0.078 & 1.86 & 28.4 \\
Diet 2 & 0.041 & 0.83 & 14.5 \\
Diet 3 & 0.020 & 0.33 & 6.5 \\
Diet 4 & 0.247 & 5.15 & 56.3 \\
\hline
\end{tabular}

Diet 1 - Backyard cow milk + all other locally produced non-milk exposure pathways

Diet 2 - Locally produced commercial milk + all other locally produced non-milk exposure pathways

Diet 3 - Regionally mixed commercial milk + inhalation (other regionally mixed food items are minor contributors to the total)

Diet 4 - Goat milk (all other exposure pathways are of negligible importance compared to goat milk) 


\section{Location: Karns}

Receptor: Female born in 1935

\begin{tabular}{lccc}
\hline & \multicolumn{3}{c}{ Thyroid Dose [cGy] } \\
Exposure Pathway & \multicolumn{2}{c}{$95 \%$ Subjective Confidence Interval } \\
\hline Backyard Cow Milk & lower limit & central estimate & upper limit \\
Commercial Milk (locally produced) & 0.65 & 3.8 & 21 \\
Commercial Milk (regionally mixed) & 0.18 & 1.1 & 6.2 \\
Goat Milk (locally produced) & 0.071 & 0.43 & 2.6 \\
Beef (locally produced) & 1.8 & 12 & 79 \\
Leafy Vegetables (locally produced) & 0.0015 & 0.027 & 0.56 \\
Eggs (locally produced) & 0.00061 & 0.0068 & 0.059 \\
Cottage Cheese (locally produced) & 0.047 & 0.3 & 2 \\
Inhalation & 0.0013 & 0.014 & 0.14 \\
Mother's milk (mother on Diet 1) & 0.038 & 0.12 & 0.45 \\
Prenatal exposure (mother on Diet 1) & -- & -- & -- \\
Diet 1 & -- & -- & -- \\
Diet 2 & 0.81 & 4.5 & 24 \\
Diet 3 & 0.33 & 1.7 & 8.2 \\
\hline
\end{tabular}

Excess Lifetime Risk [ ]

\begin{tabular}{llll}
\hline Diet 1 & $1.2 \mathrm{E}-05$ & $1.5 \mathrm{E}-04$ & $1.7 \mathrm{E}-03$ \\
Diet 2 & $5.7 \mathrm{E}-06$ & $6.2 \mathrm{E}-05$ & $7.3 \mathrm{E}-04$ \\
Diet 3 & $2.1 \mathrm{E}-06$ & $2.1 \mathrm{E}-05$ & $2.1 \mathrm{E}-04$ \\
Diet 4 & $3.4 \mathrm{E}-05$ & $4.9 \mathrm{E}-04$ & $6.4 \mathrm{E}-03$ \\
\hline
\end{tabular}

\section{Relative Risk [ ]}

\begin{tabular}{lccc}
\hline Diet 1 & 1.0043 & 1.035 & 1.34 \\
Diet 2 & 1.0018 & 1.017 & 1.16 \\
Diet 3 & 1.00075 & 1.0057 & 1.053 \\
Diet 4 & 1.013 & 1.11 & 2.5 \\
\hline
\end{tabular}

\begin{tabular}{lccc} 
& \multicolumn{3}{c}{ Probability of Causation [\%] } \\
\hline Diet 1 & 0.433 & 3.36 & 25.6 \\
Diet 2 & 0.177 & 1.65 & 13.5 \\
Diet 3 & 0.075 & 0.56 & 5.1 \\
Diet 4 & 1.285 & 9.97 & 59.2 \\
\hline
\end{tabular}

Diet 1 - Backyard cow milk + all other locally produced non-milk exposure pathways

Diet 2 - Locally produced commercial milk + all other locally produced non-milk exposure pathways

Diet 3 - Regionally mixed commercial milk + inhalation (other regionally mixed food items are minor contributors to the total)

Diet 4 - Goat milk (all other exposure pathways are of negligible importance compared to goat milk) 


\section{Location: Karns}

\section{Receptor: Male born in 1935}

\begin{tabular}{lccc}
\hline & \multicolumn{3}{c}{ Thyroid Dose [cGy] } \\
& \multicolumn{2}{c}{$95 \%$ Subjective Confidence Interval } \\
Exposure Pathway & lower limit & central estimate & upper limit \\
\hline Backyard Cow Milk & 0.86 & 4.4 & 23 \\
Commercial Milk (locally produced) & 0.21 & 1.2 & 7.5 \\
Commercial Milk (regionally mixed) & 0.078 & 0.47 & 3 \\
Goat Milk (locally produced) & 2 & 14 & 94 \\
Beef (locally produced) & 0.0022 & 0.038 & 0.83 \\
Leafy Vegetables (locally produced) & 0.00056 & 0.0067 & 0.056 \\
Eggs (locally produced) & 0.052 & 0.35 & 2.3 \\
Cottage Cheese (locally produced) & 0.0015 & 0.015 & 0.15 \\
Inhalation & 0.038 & 0.14 & 0.45 \\
Mother's milk (mother on Diet 1) & -- & -- & -- \\
Prenatal exposure (mother on Diet 1) & -- & -- & -- \\
Diet 1 & 1 & 5 & 26 \\
Diet 2 & 0.38 & 1.9 & 9.9 \\
Diet 3 & 0.14 & 0.64 & 3.6 \\
\hline
\end{tabular}

Excess Lifetime Risk [ ]

\begin{tabular}{llll}
\hline Diet 1 & $1.2 \mathrm{E}-06$ & $3.7 \mathrm{E}-05$ & $9.0 \mathrm{E}-04$ \\
Diet 2 & $5.0 \mathrm{E}-07$ & $1.7 \mathrm{E}-05$ & $3.9 \mathrm{E}-04$ \\
Diet 3 & $2.1 \mathrm{E}-07$ & $5.7 \mathrm{E}-06$ & $1.3 \mathrm{E}-04$ \\
Diet 4 & $3.8 \mathrm{E}-06$ & $1.1 \mathrm{E}-04$ & $2.8 \mathrm{E}-03$ \\
\hline
\end{tabular}

\section{Relative Risk [ ]}

\begin{tabular}{lccc}
\hline Diet 1 & 1.0011 & 1.025 & 1.54 \\
Diet 2 & 1.00058 & 1.011 & 1.22 \\
Diet 3 & 1.00021 & 1.0036 & 1.071 \\
Diet 4 & 1.0035 & 1.078 & 2.5 \\
\hline
\end{tabular}

\section{Probability of Causation [\%]}

\begin{tabular}{llcc}
\hline Diet 1 & 0.115 & 2.49 & 34.9 \\
Diet 2 & 0.058 & 1.10 & 17.9 \\
Diet 3 & 0.021 & 0.36 & 6.7 \\
Diet 4 & 0.348 & 7.25 & 60.3 \\
\hline
\end{tabular}

Diet 1 - Backyard cow milk + all other locally produced non-milk exposure pathways

Diet 2 - Locally produced commercial milk + all other locally produced non-milk exposure pathways

Diet 3 - Regionally mixed commercial milk + inhalation (other regionally mixed food items are minor contributors to the total)

Diet 4 - Goat milk (all other exposure pathways are of negligible importance compared to goat milk) 


\section{Location: Loudon}

Receptor: Female born in 1935

\begin{tabular}{lccc}
\hline & \multicolumn{3}{c}{ Thyroid Dose [cGy] } \\
Exposure Pathway & \multicolumn{2}{c}{$95 \%$ Subjective Confidence Interval } \\
\hline Backyard Cow Milk & lower limit & central estimate & upper limit \\
Commercial Milk (locally produced) & 0.38 & 2.1 & 13 \\
Commercial Milk (regionally mixed) & 0.091 & 0.63 & 3.9 \\
Goat Milk (locally produced) & 0.071 & 0.43 & 2.6 \\
Beef (locally produced) & 1 & 6.7 & 44 \\
Leafy Vegetables (locally produced) & 0.00093 & 0.015 & 0.31 \\
Eggs (locally produced) & 0.00035 & 0.0038 & 0.034 \\
Cottage Cheese (locally produced) & 0.025 & 0.17 & 1.1 \\
Inhalation & 0.00079 & 0.0079 & 0.09 \\
Mother's milk (mother on Diet 1) & 0.022 & 0.073 & 0.25 \\
Prenatal exposure (mother on Diet 1) & -- & -- & - \\
Diet 1 & -- & -- & - \\
Diet 2 & 0.46 & 2.5 & 14 \\
Diet 3 & 0.18 & 0.94 & 5.3 \\
\hline
\end{tabular}

Excess Lifetime Risk [ ]

\begin{tabular}{llll}
\hline Diet 1 & $6.4 \mathrm{E}-06$ & $8.2 \mathrm{E}-05$ & $1.0 \mathrm{E}-03$ \\
Diet 2 & $3.2 \mathrm{E}-06$ & $3.6 \mathrm{E}-05$ & $4.2 \mathrm{E}-04$ \\
Diet 3 & $1.8 \mathrm{E}-06$ & $2.0 \mathrm{E}-05$ & $2.0 \mathrm{E}-04$ \\
Diet 4 & $2.0 \mathrm{E}-05$ & $2.7 \mathrm{E}-04$ & $3.5 \mathrm{E}-03$ \\
\hline
\end{tabular}

\section{Relative Risk [ ]}

\begin{tabular}{lccc}
\hline Diet 1 & 1.0024 & 1.02 & 1.2 \\
Diet 2 & 1.001 & 1.0089 & 1.096 \\
Diet 3 & 1.00063 & 1.0051 & 1.051 \\
Diet 4 & 1.0076 & 1.062 & 1.84 \\
\hline
\end{tabular}

\section{Probability of Causation [\%]}

\begin{tabular}{lccc}
\hline Diet 1 & 0.237 & 1.96 & 16.7 \\
Diet 2 & 0.100 & 0.88 & 8.7 \\
Diet 3 & 0.063 & 0.51 & 4.8 \\
Diet 4 & 0.751 & 5.83 & 45.6 \\
\hline
\end{tabular}

Diet 1 - Backyard cow milk + all other locally produced non-milk exposure pathways

Diet 2 - Locally produced commercial milk + all other locally produced non-milk exposure pathways

Diet 3 - Regionally mixed commercial milk + inhalation (other regionally mixed food items are minor contributors to the total)

Diet 4 - Goat milk (all other exposure pathways are of negligible importance compared to goat milk) 


\section{Location: Loudon}

\section{Receptor: Male born in 1935}

\begin{tabular}{lccc}
\hline & \multicolumn{3}{c}{ Thyroid Dose [cGy] } \\
& \multicolumn{2}{c}{$95 \%$ Subjective Confidence Interval } \\
Exposure Pathway & lower limit & central estimate & upper limit \\
\hline Backyard Cow Milk & 0.48 & 2.5 & 15 \\
Commercial Milk (locally produced) & 0.11 & 0.69 & 4.6 \\
Commercial Milk (regionally mixed) & 0.078 & 0.47 & 3 \\
Goat Milk (locally produced) & 1.1 & 7.8 & 50 \\
Beef (locally produced) & 0.0014 & 0.021 & 0.44 \\
Leafy Vegetables (locally produced) & 0.00035 & 0.0037 & 0.033 \\
Eggs (locally produced) & 0.028 & 0.2 & 1.3 \\
Cottage Cheese (locally produced) & 0.00078 & 0.0082 & 0.091 \\
Inhalation & 0.022 & 0.08 & 0.28 \\
Mother's milk (mother on Diet 1) & -- & -- & -- \\
Prenatal exposure (mother on Diet 1) & -- & -- & -- \\
Diet 1 & 0.56 & 2.8 & 17 \\
Diet 2 & 0.2 & 1.1 & 6.4 \\
Diet 3 & 0.12 & 0.57 & 3.3 \\
\hline
\end{tabular}

Excess Lifetime Risk [ ]

\begin{tabular}{llll}
\hline Diet 1 & $6.8 \mathrm{E}-07$ & $2.1 \mathrm{E}-05$ & $5.0 \mathrm{E}-04$ \\
Diet 2 & $3.2 \mathrm{E}-07$ & $9.4 \mathrm{E}-06$ & $2.2 \mathrm{E}-04$ \\
Diet 3 & $1.9 \mathrm{E}-07$ & $5.2 \mathrm{E}-06$ & $1.2 \mathrm{E}-04$ \\
Diet 4 & $2.1 \mathrm{E}-06$ & $5.9 \mathrm{E}-05$ & $1.6 \mathrm{E}-03$ \\
\hline
\end{tabular}

\section{Relative Risk [ ]}

\begin{tabular}{lccc}
\hline Diet 1 & 1.00063 & 1.015 & 1.3 \\
Diet 2 & 1.00031 & 1.0064 & 1.12 \\
Diet 3 & 1.00019 & 1.0033 & 1.068 \\
Diet 4 & 1.002 & 1.044 & 1.85 \\
\hline
\end{tabular}

\section{Probability of Causation [\%]}

\begin{tabular}{llcc}
\hline Diet 1 & 0.063 & 1.44 & 22.8 \\
Diet 2 & 0.031 & 0.63 & 10.6 \\
Diet 3 & 0.019 & 0.33 & 6.4 \\
Diet 4 & 0.196 & 4.23 & 45.9 \\
\hline
\end{tabular}

Diet 1 - Backyard cow milk + all other locally produced non-milk exposure pathways

Diet 2 - Locally produced commercial milk + all other locally produced non-milk exposure pathways

Diet 3 - Regionally mixed commercial milk + inhalation (other regionally mixed food items are minor contributors to the total)

Diet 4 - Goat milk (all other exposure pathways are of negligible importance compared to goat milk) 
Location: Harriman

Receptor: Female born in 1935

\begin{tabular}{lccc}
\hline & \multicolumn{3}{c}{ Thyroid Dose [cGy] } \\
& \multicolumn{2}{c}{$95 \%$ Subjective Confidence Interval } \\
Exposure Pathway & lower limit & central estimate & upper limit \\
\hline Backyard Cow Milk & 0.23 & 1.3 & 6.5 \\
Commercial Milk (locally produced) & 0.06 & 0.36 & 2.4 \\
Commercial Milk (regionally mixed) & 0.071 & 0.43 & 2.6 \\
Goat Milk (locally produced) & 0.6 & 3.9 & 26 \\
Beef (locally produced) & 0.00055 & 0.0092 & 0.19 \\
Leafy Vegetables (locally produced) & 0.00024 & 0.0022 & 0.019 \\
Eggs (locally produced) & 0.016 & 0.098 & 0.61 \\
Cottage Cheese (locally produced) & 0.00049 & 0.0047 & 0.049 \\
Inhalation & 0.012 & 0.04 & 0.15 \\
Mother's milk (mother on Diet 1) & -- & -- & -- \\
Prenatal exposure (mother on Diet 1) & -- & -- & - \\
Diet 1 & 0.29 & 1.4 & 7.4 \\
Diet 2 & 0.11 & 0.54 & 3.2 \\
Diet 3 & 0.098 & 0.47 & 2.7 \\
\hline
\end{tabular}

Excess Lifetime Risk [ ]

\begin{tabular}{lllc}
\hline Diet 1 & $4.0 \mathrm{E}-06$ & $4.8 \mathrm{E}-05$ & $5.9 \mathrm{E}-04$ \\
Diet 2 & $1.8 \mathrm{E}-06$ & $2.0 \mathrm{E}-05$ & $2.5 \mathrm{E}-04$ \\
Diet 3 & $1.6 \mathrm{E}-06$ & $1.8 \mathrm{E}-05$ & $1.9 \mathrm{E}-04$ \\
Diet 4 & $1.2 \mathrm{E}-05$ & $1.6 \mathrm{E}-04$ & $2.1 \mathrm{E}-03$ \\
\hline
\end{tabular}

\section{Relative Risk [ ]}

\begin{tabular}{lccc}
\hline Diet 1 & 1.0014 & 1.011 & 1.13 \\
Diet 2 & 1.0006 & 1.0052 & 1.06 \\
Diet 3 & 1.00055 & 1.0049 & 1.049 \\
Diet 4 & 1.0042 & 1.037 & 1.41 \\
\hline
\end{tabular}

\section{Probability of Causation [\%]}

\begin{tabular}{lccc}
\hline Diet 1 & 0.139 & 1.13 & 11.4 \\
Diet 2 & 0.060 & 0.51 & 5.7 \\
Diet 3 & 0.055 & 0.48 & 4.7 \\
Diet 4 & 0.415 & 3.60 & 29.1 \\
\hline
\end{tabular}

Diet 1 - Backyard cow milk + all other locally produced non-milk exposure pathways

Diet 2 - Locally produced commercial milk + all other locally produced non-milk exposure pathways

Diet 3 - Regionally mixed commercial milk + inhalation (other regionally mixed food items are minor contributors to the total)

Diet 4 - Goat milk (all other exposure pathways are of negligible importance compared to goat milk) 


\section{Location: Harriman}

Receptor: Male born in 1935

\begin{tabular}{lccc}
\hline & \multicolumn{3}{c}{ Thyroid Dose [cGy] } \\
& \multicolumn{2}{c}{$95 \%$ Subjective Confidence Interval } \\
Exposure Pathway & lower limit & central estimate & upper limit \\
\hline Backyard Cow Milk & 0.28 & 1.5 & 8 \\
Commercial Milk (locally produced) & 0.066 & 0.4 & 2.8 \\
Commercial Milk (regionally mixed) & 0.078 & 0.47 & 3 \\
Goat Milk (locally produced) & 0.66 & 4.6 & 31 \\
Beef (locally produced) & 0.00076 & 0.013 & 0.24 \\
Leafy Vegetables (locally produced) & 0.00023 & 0.0023 & 0.019 \\
Eggs (locally produced) & 0.018 & 0.12 & 0.75 \\
Cottage Cheese (locally produced) & 0.0005 & 0.0047 & 0.046 \\
Inhalation & 0.013 & 0.042 & 0.16 \\
Mother's milk (mother on Diet 1) & -- & -- & - \\
Prenatal exposure (mother on Diet 1) & -- & -- & - \\
Diet 1 & 0.33 & 1.7 & 9 \\
Diet 2 & 0.13 & 0.6 & 3.7 \\
Diet 3 & 0.1 & 0.52 & 3.2 \\
\hline
\end{tabular}

Excess Lifetime Risk [ ]

\begin{tabular}{lllc}
\hline Diet 1 & $3.4 \mathrm{E}-07$ & $1.2 \mathrm{E}-05$ & $2.9 \mathrm{E}-04$ \\
Diet 2 & $1.4 \mathrm{E}-07$ & $5.4 \mathrm{E}-06$ & $1.2 \mathrm{E}-04$ \\
Diet 3 & $1.8 \mathrm{E}-07$ & $4.8 \mathrm{E}-06$ & $1.2 \mathrm{E}-04$ \\
Diet 4 & $1.1 \mathrm{E}-06$ & $3.4 \mathrm{E}-05$ & $9.0 \mathrm{E}-04$ \\
\hline
\end{tabular}

\section{Relative Risk [ ]}

\begin{tabular}{lccc}
\hline Diet 1 & 1.00041 & 1.0084 & 1.16 \\
Diet 2 & 1.00018 & 1.0037 & 1.071 \\
Diet 3 & 1.00017 & 1.0031 & 1.065 \\
Diet 4 & 1.0013 & 1.026 & 1.51 \\
\hline
\end{tabular}

\section{Probability of Causation [\%]}

\begin{tabular}{lccc}
\hline Diet 1 & 0.041 & 0.84 & 14.2 \\
Diet 2 & 0.018 & 0.37 & 6.6 \\
Diet 3 & 0.017 & 0.31 & 6.1 \\
Diet 4 & 0.128 & 2.57 & 33.8 \\
\hline
\end{tabular}

Diet 1 - Backyard cow milk + all other locally produced non-milk exposure pathways

Diet 2 - Locally produced commercial milk + all other locally produced non-milk exposure pathways

Diet 3 - Regionally mixed commercial milk + inhalation (other regionally mixed food items are minor contributors to the total)

Diet 4 - Goat milk (all other exposure pathways are of negligible importance compared to goat milk) 


\section{Location: Cedar Bluff}

Receptor: Female born in 1935

\begin{tabular}{lccc}
\hline & \multicolumn{3}{c}{ Thyroid Dose [cGy] } \\
& \multicolumn{2}{c}{$95 \%$ Subjective Confidence Interval } \\
Exposure Pathway & lower limit & central estimate & upper limit \\
\hline Backyard Cow Milk & 0.53 & 3 & 18 \\
Commercial Milk (locally produced) & 0.14 & 0.87 & 5.7 \\
Commercial Milk (regionally mixed) & 0.071 & 0.43 & 2.6 \\
Goat Milk (locally produced) & 1.4 & 9.1 & 59 \\
Beef (locally produced) & 0.0012 & 0.022 & 0.48 \\
Leafy Vegetables (locally produced) & 0.00055 & 0.0053 & 0.045 \\
Eggs (locally produced) & 0.034 & 0.23 & 1.5 \\
Cottage Cheese (locally produced) & 0.0012 & 0.011 & 0.12 \\
Inhalation & 0.03 & 0.1 & 0.35 \\
Mother's milk (mother on Diet 1) & -- & -- & -- \\
Prenatal exposure (mother on Diet 1) & -- & -- & -- \\
Diet 1 & 0.64 & 3.5 & 20 \\
Diet 2 & 0.27 & 1.3 & 7.3 \\
Diet 3 & 0.12 & 0.54 & 2.9 \\
\hline
\end{tabular}

Excess Lifetime Risk [ ]

\begin{tabular}{llll}
\hline Diet 1 & $8.4 \mathrm{E}-06$ & $1.1 \mathrm{E}-04$ & $1.5 \mathrm{E}-03$ \\
Diet 2 & $4.5 \mathrm{E}-06$ & $4.9 \mathrm{E}-05$ & $5.7 \mathrm{E}-04$ \\
Diet 3 & $2.0 \mathrm{E}-06$ & $2.1 \mathrm{E}-05$ & $2.1 \mathrm{E}-04$ \\
Diet 4 & $2.9 \mathrm{E}-05$ & $3.9 \mathrm{E}-04$ & $5.3 \mathrm{E}-03$ \\
\hline
\end{tabular}

Relative Risk [ ]

\begin{tabular}{lccc}
\hline Diet 1 & 1.0035 & 1.029 & 1.26 \\
Diet 2 & 1.0014 & 1.013 & 1.13 \\
Diet 3 & 1.00068 & 1.0054 & 1.052 \\
Diet 4 & 1.01 & 1.087 & 2.1 \\
\hline
\end{tabular}

\section{Probability of Causation [\%]}

\begin{tabular}{lccc}
\hline Diet 1 & 0.347 & 2.79 & 20.8 \\
Diet 2 & 0.136 & 1.27 & 11.1 \\
Diet 3 & 0.068 & 0.54 & 4.9 \\
Diet 4 & 1.016 & 7.97 & 51.7 \\
\hline
\end{tabular}

Diet 1 - Backyard cow milk + all other locally produced non-milk exposure pathways

Diet 2 - Locally produced commercial milk + all other locally produced non-milk exposure pathways

Diet 3 - Regionally mixed commercial milk + inhalation (other regionally mixed food items are minor contributors to the total)

Diet 4 - Goat milk (all other exposure pathways are of negligible importance compared to goat milk) 


\section{Location: Cedar Bluff}

Receptor: Male born in 1935

\begin{tabular}{lccc}
\hline & \multicolumn{3}{c}{ Thyroid Dose [cGy] } \\
Exposure Pathway & $95 \%$ Subjective Confidence Interval \\
\hline Backyard Cow Milk & lower limit & central estimate & upper limit \\
Commercial Milk (locally produced) & 0.68 & 3.5 & 20 \\
Commercial Milk (regionally mixed) & 0.16 & 0.97 & 6.7 \\
Goat Milk (locally produced) & 0.078 & 0.47 & 3 \\
Beef (locally produced) & 1.6 & 11 & 70 \\
Leafy Vegetables (locally produced) & 0.0017 & 0.031 & 0.67 \\
Eggs (locally produced) & 0.00052 & 0.0052 & 0.044 \\
Cottage Cheese (locally produced) & 0.039 & 0.27 & 1.8 \\
Inhalation & 0.0012 & 0.012 & 0.14 \\
Mother's milk (mother on Diet 1) & 0.031 & 0.11 & 0.37 \\
Prenatal exposure (mother on Diet 1) & -- & -- & - \\
Diet 1 & -- & -- & - \\
Diet 2 & 0.8 & 4 & 22 \\
Diet 3 & 0.29 & 1.5 & 8.6 \\
\hline
\end{tabular}

Excess Lifetime Risk [ ]

\begin{tabular}{llll}
\hline Diet 1 & $9.7 \mathrm{E}-07$ & $3.0 \mathrm{E}-05$ & $7.3 \mathrm{E}-04$ \\
Diet 2 & $4.1 \mathrm{E}-07$ & $1.3 \mathrm{E}-05$ & $3.1 \mathrm{E}-04$ \\
Diet 3 & $2.0 \mathrm{E}-07$ & $5.5 \mathrm{E}-06$ & $1.3 \mathrm{E}-04$ \\
Diet 4 & $3.0 \mathrm{E}-06$ & $8.3 \mathrm{E}-05$ & $2.2 \mathrm{E}-03$ \\
\hline
\end{tabular}

\section{Relative Risk [ ]}

\begin{tabular}{lccc}
\hline Diet 1 & 1.0009 & 1.021 & 1.43 \\
Diet 2 & 1.00043 & 1.0089 & 1.17 \\
Diet 3 & 1.0002 & 1.0034 & 1.071 \\
Diet 4 & 1.0027 & 1.06 & 2.3 \\
\hline
\end{tabular}

\section{Probability of Causation [\%]}

\begin{tabular}{llcc}
\hline Diet 1 & 0.090 & 2.01 & 29.9 \\
Diet 2 & 0.043 & 0.88 & 14.8 \\
Diet 3 & 0.020 & 0.34 & 6.6 \\
Diet 4 & 0.270 & 5.68 & 55.9 \\
\hline
\end{tabular}

Diet 1 - Backyard cow milk + all other locally produced non-milk exposure pathways

Diet 2 - Locally produced commercial milk + all other locally produced non-milk exposure pathways

Diet 3 - Regionally mixed commercial milk + inhalation (other regionally mixed food items are minor contributors to the total)

Diet 4 - Goat milk (all other exposure pathways are of negligible importance compared to goat milk) 


\section{Location: Oakdale}

Receptor: Female born in 1935

\begin{tabular}{lccc}
\hline & \multicolumn{3}{c}{ Thyroid Dose [cGy] } \\
& \multicolumn{2}{c}{$95 \%$ Subjective Confidence Interval } \\
Exposure Pathway & lower limit & central estimate & upper limit \\
\hline Backyard Cow Milk & 0.12 & 0.71 & 4.4 \\
Commercial Milk (locally produced) & 0.032 & 0.21 & 1.4 \\
Commercial Milk (regionally mixed) & 0.071 & 0.43 & 2.6 \\
Goat Milk (locally produced) & 0.28 & 2.2 & 15 \\
Beef (locally produced) & 0.00031 & 0.0051 & 0.11 \\
Leafy Vegetables (locally produced) & 0.00012 & 0.0013 & 0.011 \\
Eggs (locally produced) & 0.0074 & 0.055 & 0.38 \\
Cottage Cheese (locally produced) & 0.00028 & 0.0027 & 0.032 \\
Inhalation & 0.0066 & 0.023 & 0.087 \\
Mother's milk (mother on Diet 1) & -- & -- & -- \\
Prenatal exposure (mother on Diet 1) & -- & -- & -- \\
Diet 1 & 0.14 & 0.82 & 5 \\
Diet 2 & 0.058 & 0.31 & 1.8 \\
Diet 3 & 0.087 & 0.45 & 2.7 \\
\hline
\end{tabular}

Excess Lifetime Risk [ ]

\begin{tabular}{llll}
\hline Diet 1 & $1.9 \mathrm{E}-06$ & $2.8 \mathrm{E}-05$ & $3.3 \mathrm{E}-04$ \\
Diet 2 & $9.8 \mathrm{E}-07$ & $1.2 \mathrm{E}-05$ & $1.4 \mathrm{E}-04$ \\
Diet 3 & $1.5 \mathrm{E}-06$ & $1.8 \mathrm{E}-05$ & $1.9 \mathrm{E}-04$ \\
Diet 4 & $6.0 \mathrm{E}-06$ & $9.1 \mathrm{E}-05$ & $1.2 \mathrm{E}-03$ \\
\hline
\end{tabular}

Relative Risk [ ]

\begin{tabular}{lccc}
\hline Diet 1 & 1.00073 & 1.0065 & 1.07 \\
Diet 2 & 1.0003 & 1.003 & 1.032 \\
Diet 3 & 1.00053 & 1.0047 & 1.048 \\
Diet 4 & 1.0021 & 1.021 & 1.25 \\
\hline
\end{tabular}

\section{Probability of Causation [\%]}

\begin{tabular}{llcc}
\hline Diet 1 & 0.073 & 0.65 & 6.5 \\
Diet 2 & 0.030 & 0.30 & 3.1 \\
Diet 3 & 0.053 & 0.47 & 4.6 \\
Diet 4 & 0.210 & 2.04 & 20.1 \\
\hline
\end{tabular}

Diet 1 - Backyard cow milk + all other locally produced non-milk exposure pathways

Diet 2 - Locally produced commercial milk + all other locally produced non-milk exposure pathways

Diet 3 - Regionally mixed commercial milk + inhalation (other regionally mixed food items are minor contributors to the total)

Diet 4 - Goat milk (all other exposure pathways are of negligible importance compared to goat milk) 


\section{Location: Oakdale}

\section{Receptor: Male born in 1935}

\begin{tabular}{lccc}
\hline & \multicolumn{3}{c}{ Thyroid Dose [cGy] } \\
& \multicolumn{2}{c}{$95 \%$ Subjective Confidence Interval } \\
Exposure Pathway & lower limit & central estimate & upper limit \\
\hline Backyard Cow Milk & 0.14 & 0.83 & 5 \\
Commercial Milk (locally produced) & 0.034 & 0.23 & 1.6 \\
Commercial Milk (regionally mixed) & 0.078 & 0.47 & 3 \\
Goat Milk (locally produced) & 0.33 & 2.6 & 17 \\
Beef (locally produced) & 0.00043 & 0.007 & 0.15 \\
Leafy Vegetables (locally produced) & 0.00011 & 0.0013 & 0.011 \\
Eggs (locally produced) & 0.0089 & 0.066 & 0.45 \\
Cottage Cheese (locally produced) & 0.00026 & 0.0027 & 0.03 \\
Inhalation & 0.0068 & 0.025 & 0.091 \\
Mother's milk (mother on Diet 1) & -- & -- & - \\
Prenatal exposure (mother on Diet 1) & -- & -- & -- \\
Diet 1 & 0.17 & 0.95 & 5.6 \\
Diet 2 & 0.067 & 0.36 & 2.1 \\
Diet 3 & 0.096 & 0.5 & 3.1 \\
\hline
\end{tabular}

Excess Lifetime Risk [ ]

\begin{tabular}{llll}
\hline Diet 1 & $1.9 \mathrm{E}-07$ & $7.2 \mathrm{E}-06$ & $1.8 \mathrm{E}-04$ \\
Diet 2 & $8.7 \mathrm{E}-08$ & $3.2 \mathrm{E}-06$ & $7.7 \mathrm{E}-05$ \\
Diet 3 & $1.7 \mathrm{E}-07$ & $4.7 \mathrm{E}-06$ & $1.2 \mathrm{E}-04$ \\
Diet 4 & $6.3 \mathrm{E}-07$ & $2.0 \mathrm{E}-05$ & $5.8 \mathrm{E}-04$ \\
\hline
\end{tabular}

\section{Relative Risk [ ]}

\begin{tabular}{lccc}
\hline Diet 1 & 1.00023 & 1.0049 & 1.094 \\
Diet 2 & 1.00011 & 1.0021 & 1.041 \\
Diet 3 & 1.00016 & 1.0031 & 1.064 \\
Diet 4 & 1.00065 & 1.015 & 1.3 \\
\hline
\end{tabular}

\section{Probability of Causation [\%]}

\begin{tabular}{lccc}
\hline Diet 1 & 0.023 & 0.49 & 8.5 \\
Diet 2 & 0.011 & 0.21 & 3.9 \\
Diet 3 & 0.016 & 0.30 & 6.0 \\
Diet 4 & 0.065 & 1.48 & 22.9 \\
\hline
\end{tabular}

Diet 1 - Backyard cow milk + all other locally produced non-milk exposure pathways

Diet 2 - Locally produced commercial milk + all other locally produced non-milk exposure pathways

Diet 3 - Regionally mixed commercial milk + inhalation (other regionally mixed food items are minor contributors to the total)

Diet 4 - Goat milk (all other exposure pathways are of negligible importance compared to goat milk) 


\section{Location: Claxton}

Receptor: Female born in 1935

\begin{tabular}{lccc}
\hline & \multicolumn{3}{c}{ Thyroid Dose [cGy] } \\
& \multicolumn{2}{c}{$95 \%$ Subjective Confidence Interval } \\
Exposure Pathway & lower limit & central estimate & upper limit \\
\hline Backyard Cow Milk & 0.44 & 2.5 & 15 \\
Commercial Milk (locally produced) & 0.12 & 0.72 & 4.6 \\
Commercial Milk (regionally mixed) & 0.071 & 0.43 & 2.6 \\
Goat Milk (locally produced) & 1.2 & 7.6 & 48 \\
Beef (locally produced) & 0.001 & 0.018 & 0.39 \\
Leafy Vegetables (locally produced) & 0.00045 & 0.0044 & 0.038 \\
Eggs (locally produced) & 0.029 & 0.19 & 1.2 \\
Cottage Cheese (locally produced) & 0.00097 & 0.0095 & 0.098 \\
Inhalation & 0.025 & 0.083 & 0.29 \\
Mother's milk (mother on Diet 1) & -- & -- & - \\
Prenatal exposure (mother on Diet 1) & -- & -- & - \\
Diet 1 & 0.54 & 2.9 & 17 \\
Diet 2 & 0.23 & 1.1 & 6 \\
Diet 3 & 0.11 & 0.52 & 2.9 \\
\hline
\end{tabular}

Excess Lifetime Risk [ ]

\begin{tabular}{llll}
\hline Diet 1 & $7.1 \mathrm{E}-06$ & $9.6 \mathrm{E}-05$ & $1.2 \mathrm{E}-03$ \\
Diet 2 & $3.8 \mathrm{E}-06$ & $4.1 \mathrm{E}-05$ & $5.0 \mathrm{E}-04$ \\
Diet 3 & $1.9 \mathrm{E}-06$ & $2.0 \mathrm{E}-05$ & $2.0 \mathrm{E}-04$ \\
Diet 4 & $2.4 \mathrm{E}-05$ & $3.2 \mathrm{E}-04$ & $4.5 \mathrm{E}-03$ \\
\hline
\end{tabular}

\section{Relative Risk [ ]}

\begin{tabular}{lccc}
\hline Diet 1 & 1.0028 & 1.024 & 1.22 \\
Diet 2 & 1.0012 & 1.011 & 1.11 \\
Diet 3 & 1.00065 & 1.0052 & 1.051 \\
Diet 4 & 1.0088 & 1.073 & 1.9 \\
\hline
\end{tabular}

\section{Probability of Causation [\%]}

\begin{tabular}{lccc}
\hline Diet 1 & 0.283 & 2.30 & 18.2 \\
Diet 2 & 0.117 & 1.08 & 9.6 \\
Diet 3 & 0.065 & 0.52 & 4.9 \\
Diet 4 & 0.876 & 6.82 & 47.3 \\
\hline
\end{tabular}

Diet 1 - Backyard cow milk + all other locally produced non-milk exposure pathways

Diet 2 - Locally produced commercial milk + all other locally produced non-milk exposure pathways

Diet 3 - Regionally mixed commercial milk + inhalation (other regionally mixed food items are minor contributors to the total)

Diet 4 - Goat milk (all other exposure pathways are of negligible importance compared to goat milk) 


\section{Location: Claxton}

\section{Receptor: Male born in 1935}

\begin{tabular}{lccc}
\hline & \multicolumn{3}{c}{ Thyroid Dose [cGy] } \\
& \multicolumn{2}{c}{$95 \%$ Subjective Confidence Interval } \\
Exposure Pathway & lower limit & central estimate & upper limit \\
\hline Backyard Cow Milk & 0.57 & 2.9 & 17 \\
Commercial Milk (locally produced) & 0.13 & 0.8 & 5.6 \\
Commercial Milk (regionally mixed) & 0.078 & 0.47 & 3 \\
Goat Milk (locally produced) & 1.4 & 9.1 & 58 \\
Beef (locally produced) & 0.0015 & 0.025 & 0.55 \\
Leafy Vegetables (locally produced) & 0.00043 & 0.0043 & 0.038 \\
Eggs (locally produced) & 0.032 & 0.23 & 1.5 \\
Cottage Cheese (locally produced) & 0.00097 & 0.0098 & 0.11 \\
Inhalation & 0.026 & 0.091 & 0.3 \\
Mother's milk (mother on Diet 1) & -- & -- & -- \\
Prenatal exposure (mother on Diet 1) & -- & -- & -- \\
Diet 1 & 0.67 & 3.3 & 19 \\
Diet 2 & 0.25 & 1.2 & 7.2 \\
Diet 3 & 0.12 & 0.58 & 3.4 \\
\hline
\end{tabular}

Excess Lifetime Risk [ ]

\begin{tabular}{llll}
\hline Diet 1 & $8.1 \mathrm{E}-07$ & $2.5 \mathrm{E}-05$ & $6.1 \mathrm{E}-04$ \\
Diet 2 & $3.5 \mathrm{E}-07$ & $1.1 \mathrm{E}-05$ & $2.5 \mathrm{E}-04$ \\
Diet 3 & $2.0 \mathrm{E}-07$ & $5.3 \mathrm{E}-06$ & $1.3 \mathrm{E}-04$ \\
Diet 4 & $2.5 \mathrm{E}-06$ & $6.9 \mathrm{E}-05$ & $1.8 \mathrm{E}-03$ \\
\hline
\end{tabular}

\section{Relative Risk [ ]}

\begin{tabular}{lccc}
\hline Diet 1 & 1.00074 & 1.017 & 1.36 \\
Diet 2 & 1.00036 & 1.0074 & 1.14 \\
Diet 3 & 1.00019 & 1.0033 & 1.069 \\
Diet 4 & 1.0022 & 1.052 & 2.1 \\
\hline
\end{tabular}

\section{Probability of Causation [\%]}

\begin{tabular}{lccc}
\hline Diet 1 & 0.074 & 1.68 & 26.2 \\
Diet 2 & 0.036 & 0.74 & 12.4 \\
Diet 3 & 0.019 & 0.33 & 6.4 \\
Diet 4 & 0.224 & 4.93 & 51.2 \\
\hline
\end{tabular}

Diet 1 - Backyard cow milk + all other locally produced non-milk exposure pathways

Diet 2 - Locally produced commercial milk + all other locally produced non-milk exposure pathways

Diet 3 - Regionally mixed commercial milk + inhalation (other regionally mixed food items are minor contributors to the total)

Diet 4 - Goat milk (all other exposure pathways are of negligible importance compared to goat milk) 
Location: Dutch Valley

Receptor: Female born in 1935

\begin{tabular}{lccc}
\hline & \multicolumn{3}{c}{ Thyroid Dose [cGy] } \\
Exposure Pathway & $95 \%$ & Subjective Confidence Interval \\
Backyard Cow Milk & lower limit & central estimate & upper limit \\
Commercial Milk (locally produced) & 0.2 & 1.2 & 6.7 \\
Commercial Milk (regionally mixed) & 0.056 & 0.35 & 2.2 \\
Goat Milk (locally produced) & 0.071 & 0.43 & 2.6 \\
Beef (locally produced) & 0.52 & 3.7 & 26 \\
Leafy Vegetables (locally produced) & 0.0005 & 0.0087 & 0.17 \\
Eggs (locally produced) & 0.00022 & 0.0022 & 0.018 \\
Cottage Cheese (locally produced) & 0.015 & 0.095 & 0.6 \\
Inhalation & 0.00045 & 0.0046 & 0.048 \\
Mother's milk (mother on Diet 1) & 0.012 & 0.04 & 0.14 \\
Prenatal exposure (mother on Diet 1) & -- & -- & -- \\
Diet 1 & -- & -- & -- \\
Diet 2 & 0.26 & 1.4 & 7.6 \\
Diet 3 & 0.1 & 0.53 & 2.8 \\
\hline
\end{tabular}

Excess Lifetime Risk [ ]

\begin{tabular}{llll}
\hline Diet 1 & $3.6 \mathrm{E}-06$ & $4.6 \mathrm{E}-05$ & $5.6 \mathrm{E}-04$ \\
Diet 2 & $1.7 \mathrm{E}-06$ & $2.0 \mathrm{E}-05$ & $2.3 \mathrm{E}-04$ \\
Diet 3 & $1.5 \mathrm{E}-06$ & $1.9 \mathrm{E}-05$ & $1.9 \mathrm{E}-04$ \\
Diet 4 & $1.1 \mathrm{E}-05$ & $1.5 \mathrm{E}-04$ & $2.1 \mathrm{E}-03$ \\
\hline
\end{tabular}

\section{Relative Risk [ ]}

\begin{tabular}{lccc}
\hline Diet 1 & 1.0014 & 1.011 & 1.12 \\
Diet 2 & 1.00057 & 1.0053 & 1.055 \\
Diet 3 & 1.00056 & 1.0048 & 1.049 \\
Diet 4 & 1.0039 & 1.036 & 1.43 \\
\hline
\end{tabular}

\section{Probability of Causation [\%]}

\begin{tabular}{lccc}
\hline Diet 1 & 0.138 & 1.09 & 10.7 \\
Diet 2 & 0.057 & 0.52 & 5.2 \\
Diet 3 & 0.056 & 0.48 & 4.7 \\
Diet 4 & 0.390 & 3.48 & 29.6 \\
\hline
\end{tabular}

Diet 1 - Backyard cow milk + all other locally produced non-milk exposure pathways

Diet 2 - Locally produced commercial milk + all other locally produced non-milk exposure pathways

Diet 3 - Regionally mixed commercial milk + inhalation (other regionally mixed food items are minor contributors to the total)

Diet 4 - Goat milk (all other exposure pathways are of negligible importance compared to goat milk) 


\section{Location: Dutch Valley}

Receptor: Male born in 1935

\begin{tabular}{lccc}
\hline & \multicolumn{3}{c}{ Thyroid Dose [cGy] } \\
Exposure Pathway & \multicolumn{2}{c}{$95 \%$ Subjective Confidence Interval } \\
\hline Backyard Cow Milk & lower limit & central estimate & upper limit \\
Commercial Milk (locally produced) & 0.26 & 1.4 & 7.4 \\
Commercial Milk (regionally mixed) & 0.065 & 0.39 & 2.5 \\
Goat Milk (locally produced) & 0.078 & 0.47 & 3.1 \\
Beef (locally produced) & 0.58 & 4.4 & 30 \\
Leafy Vegetables (locally produced) & 0.00073 & 0.012 & 0.23 \\
Eggs (locally produced) & 0.0002 & 0.0022 & 0.018 \\
Cottage Cheese (locally produced) & 0.017 & 0.11 & 0.72 \\
Inhalation & 0.00048 & 0.0046 & 0.044 \\
Mother's milk (mother on Diet 1) & 0.012 & 0.043 & 0.16 \\
Prenatal exposure (mother on Diet 1) & -- & -- & -- \\
Diet 1 & -- & -- & -- \\
Diet 2 & 0.31 & 1.6 & 8.4 \\
Diet 3 & 0.12 & 0.59 & 3.2 \\
\hline
\end{tabular}

Excess Lifetime Risk [ ]

\begin{tabular}{llll}
\hline Diet 1 & $3.6 \mathrm{E}-07$ & $1.2 \mathrm{E}-05$ & $2.7 \mathrm{E}-04$ \\
Diet 2 & $1.4 \mathrm{E}-07$ & $5.2 \mathrm{E}-06$ & $1.2 \mathrm{E}-04$ \\
Diet 3 & $1.8 \mathrm{E}-07$ & $4.8 \mathrm{E}-06$ & $1.2 \mathrm{E}-04$ \\
Diet 4 & $1.1 \mathrm{E}-06$ & $3.3 \mathrm{E}-05$ & $9.2 \mathrm{E}-04$ \\
\hline
\end{tabular}

Relative Risk [ ]

\begin{tabular}{lccc}
\hline Diet 1 & 1.00037 & 1.0082 & 1.16 \\
Diet 2 & 1.00017 & 1.0035 & 1.069 \\
Diet 3 & 1.00017 & 1.0031 & 1.065 \\
Diet 4 & 1.0011 & 1.025 & 1.47 \\
\hline
\end{tabular}

\section{Probability of Causation [\%]}

\begin{tabular}{llcc}
\hline Diet 1 & 0.037 & 0.82 & 13.9 \\
Diet 2 & 0.017 & 0.35 & 6.4 \\
Diet 3 & 0.017 & 0.31 & 6.1 \\
Diet 4 & 0.114 & 2.44 & 31.5 \\
\hline
\end{tabular}

Diet 1 - Backyard cow milk + all other locally produced non-milk exposure pathways

Diet 2 - Locally produced commercial milk + all other locally produced non-milk exposure pathways

Diet 3 - Regionally mixed commercial milk + inhalation (other regionally mixed food items are minor contributors to the total)

Diet 4 - Goat milk (all other exposure pathways are of negligible importance compared to goat milk) 


\section{Location: Clinton}

Receptor: Female born in 1935

\begin{tabular}{lccc}
\hline & \multicolumn{3}{c}{ Thyroid Dose [cGy] } \\
Exposure Pathway & \multicolumn{2}{c}{$9 \%$ Subjective Confidence Interval } \\
\hline Backyard Cow Milk & lower limit & central estimate & upper limit \\
Commercial Milk (locally produced) & 0.34 & 2 & 11 \\
Commercial Milk (regionally mixed) & 0.089 & 0.56 & 3.5 \\
Goat Milk (locally produced) & 0.071 & 0.43 & 2.6 \\
Beef (locally produced) & 0.89 & 6 & 39 \\
Leafy Vegetables (locally produced) & 0.0008 & 0.014 & 0.28 \\
Eggs (locally produced) & 0.00035 & 0.0035 & 0.028 \\
Cottage Cheese (locally produced) & 0.022 & 0.15 & 0.97 \\
Inhalation & 0.00077 & 0.0073 & 0.079 \\
Mother's milk (mother on Diet 1) & 0.02 & 0.064 & 0.23 \\
Prenatal exposure (mother on Diet 1) & -- & -- & - \\
Diet 1 & -- & -- & - \\
Diet 2 & 0.41 & 2.3 & 13 \\
Diet 3 & 0.17 & 0.84 & 4.6 \\
\hline
\end{tabular}

Excess Lifetime Risk [ ]

\begin{tabular}{llll}
\hline Diet 1 & $5.3 \mathrm{E}-06$ & $7.6 \mathrm{E}-05$ & $9.3 \mathrm{E}-04$ \\
Diet 2 & $2.8 \mathrm{E}-06$ & $3.1 \mathrm{E}-05$ & $3.6 \mathrm{E}-04$ \\
Diet 3 & $1.8 \mathrm{E}-06$ & $1.9 \mathrm{E}-05$ & $2.0 \mathrm{E}-04$ \\
Diet 4 & $1.8 \mathrm{E}-05$ & $2.5 \mathrm{E}-04$ & $3.3 \mathrm{E}-03$ \\
\hline
\end{tabular}

\section{Relative Risk [ ]}

\begin{tabular}{lccc}
\hline Diet 1 & 1.0021 & 1.018 & 1.18 \\
Diet 2 & 1.00088 & 1.0084 & 1.081 \\
Diet 3 & 1.00062 & 1.005 & 1.05 \\
Diet 4 & 1.0064 & 1.057 & 1.67 \\
\hline
\end{tabular}

\section{Probability of Causation [\%]}

\begin{tabular}{lccc}
\hline Diet 1 & 0.214 & 1.81 & 15.0 \\
Diet 2 & 0.088 & 0.84 & 7.5 \\
Diet 3 & 0.062 & 0.50 & 4.8 \\
Diet 4 & 0.639 & 5.40 & 39.6 \\
\hline
\end{tabular}

Diet 1 - Backyard cow milk + all other locally produced non-milk exposure pathways

Diet 2 - Locally produced commercial milk + all other locally produced non-milk exposure pathways

Diet 3 - Regionally mixed commercial milk + inhalation (other regionally mixed food items are minor contributors to the total)

Diet 4 - Goat milk (all other exposure pathways are of negligible importance compared to goat milk) 


\section{Location: Clinton}

\section{Receptor: Male born in 1935}

\begin{tabular}{lccc}
\hline & \multicolumn{3}{c}{ Thyroid Dose [cGy] } \\
& \multicolumn{2}{c}{$95 \%$ Subjective Confidence Interval } \\
Exposure Pathway & lower limit & central estimate & upper limit \\
\hline Backyard Cow Milk & 0.43 & 2.3 & 13 \\
Commercial Milk (locally produced) & 0.1 & 0.62 & 4.1 \\
Commercial Milk (regionally mixed) & 0.078 & 0.47 & 3 \\
Goat Milk (locally produced) & 1 & 7 & 45 \\
Beef (locally produced) & 0.0011 & 0.019 & 0.41 \\
Leafy Vegetables (locally produced) & 0.00034 & 0.0034 & 0.028 \\
Eggs (locally produced) & 0.026 & 0.18 & 1.2 \\
Cottage Cheese (locally produced) & 0.00076 & 0.0074 & 0.081 \\
Inhalation & 0.02 & 0.069 & 0.24 \\
Mother's milk (mother on Diet 1) & -- & -- & - \\
Prenatal exposure (mother on Diet 1) & -- & -- & - \\
Diet 1 & 0.5 & 2.6 & 15 \\
Diet 2 & 0.19 & 0.98 & 5.2 \\
Diet 3 & 0.11 & 0.55 & 3.3 \\
\hline
\end{tabular}

Excess Lifetime Risk [ ]

\begin{tabular}{llll}
\hline Diet 1 & $6.5 \mathrm{E}-07$ & $2.0 \mathrm{E}-05$ & $4.5 \mathrm{E}-04$ \\
Diet 2 & $2.6 \mathrm{E}-07$ & $8.6 \mathrm{E}-06$ & $2.0 \mathrm{E}-04$ \\
Diet 3 & $1.9 \mathrm{E}-07$ & $5.1 \mathrm{E}-06$ & $1.2 \mathrm{E}-04$ \\
Diet 4 & $1.8 \mathrm{E}-06$ & $5.4 \mathrm{E}-05$ & $1.4 \mathrm{E}-03$ \\
\hline
\end{tabular}

\section{Relative Risk [ ]}

\begin{tabular}{lccc}
\hline Diet 1 & 1.00057 & 1.013 & 1.27 \\
Diet 2 & 1.00031 & 1.0058 & 1.11 \\
Diet 3 & 1.00018 & 1.0032 & 1.067 \\
Diet 4 & 1.0018 & 1.041 & 1.79 \\
\hline
\end{tabular}

\section{Probability of Causation [\%]}

\begin{tabular}{lccc}
\hline Diet 1 & 0.057 & 1.28 & 21.0 \\
Diet 2 & 0.031 & 0.58 & 9.7 \\
Diet 3 & 0.018 & 0.32 & 6.3 \\
Diet 4 & 0.183 & 3.90 & 43.6 \\
\hline
\end{tabular}

Diet 1 - Backyard cow milk + all other locally produced non-milk exposure pathways

Diet 2 - Locally produced commercial milk + all other locally produced non-milk exposure pathways

Diet 3 - Regionally mixed commercial milk + inhalation (other regionally mixed food items are minor contributors to the total)

Diet 4 - Goat milk (all other exposure pathways are of negligible importance compared to goat milk) 
Location: Friendsville

Receptor: Female born in 1935

\begin{tabular}{lccc}
\hline & \multicolumn{3}{c}{ Thyroid Dose [cGy] } \\
Exposure Pathway & $95 \%$ & Subjective Confidence Interval \\
Backyard Cow Milk & lower limit & central estimate & upper limit \\
Commercial Milk (locally produced) & 0.17 & 1.1 & 6.6 \\
Commercial Milk (regionally mixed) & 0.052 & 0.33 & 1.9 \\
Goat Milk (locally produced) & 0.071 & 0.43 & 2.6 \\
Beef (locally produced) & 0.51 & 3.4 & 26 \\
Leafy Vegetables (locally produced) & 0.00045 & 0.008 & 0.17 \\
Eggs (locally produced) & 0.00018 & 0.002 & 0.017 \\
Cottage Cheese (locally produced) & 0.013 & 0.086 & 0.61 \\
Inhalation & 0.00042 & 0.0042 & 0.042 \\
Mother's milk (mother on Diet 1) & 0.012 & 0.044 & 0.17 \\
Prenatal exposure (mother on Diet 1) & -- & -- & -- \\
Diet 1 & -- & -- & -- \\
Diet 2 & 0.23 & 1.3 & 7.5 \\
Diet 3 & 0.092 & 0.5 & 2.5 \\
\hline
\end{tabular}

Excess Lifetime Risk [ ]

\begin{tabular}{llll}
\hline Diet 1 & $3.4 \mathrm{E}-06$ & $4.2 \mathrm{E}-05$ & $5.1 \mathrm{E}-04$ \\
Diet 2 & $1.6 \mathrm{E}-06$ & $1.8 \mathrm{E}-05$ & $2.0 \mathrm{E}-04$ \\
Diet 3 & $1.6 \mathrm{E}-06$ & $1.9 \mathrm{E}-05$ & $2.0 \mathrm{E}-04$ \\
Diet 4 & $9.7 \mathrm{E}-06$ & $1.4 \mathrm{E}-04$ & $1.9 \mathrm{E}-03$ \\
\hline
\end{tabular}

\section{Relative Risk [ ]}

\begin{tabular}{lccc}
\hline Diet 1 & 1.0012 & 1.011 & 1.1 \\
Diet 2 & 1.00054 & 1.0048 & 1.047 \\
Diet 3 & 1.00057 & 1.0049 & 1.049 \\
Diet 4 & 1.0035 & 1.034 & 1.41 \\
\hline
\end{tabular}

\section{Probability of Causation [\%]}

\begin{tabular}{llcc}
\hline Diet 1 & 0.118 & 1.04 & 9.2 \\
Diet 2 & 0.054 & 0.47 & 4.5 \\
Diet 3 & 0.057 & 0.49 & 4.7 \\
Diet 4 & 0.347 & 3.25 & 29.2 \\
\hline
\end{tabular}

Diet 1 - Backyard cow milk + all other locally produced non-milk exposure pathways

Diet 2 - Locally produced commercial milk + all other locally produced non-milk exposure pathways

Diet 3 - Regionally mixed commercial milk + inhalation (other regionally mixed food items are minor contributors to the total)

Diet 4 - Goat milk (all other exposure pathways are of negligible importance compared to goat milk) 
Location: Friendsville

Receptor: Male born in 1935

\begin{tabular}{lccc}
\hline & \multicolumn{3}{c}{ Thyroid Dose [cGy] } \\
Exposure Pathway & 95\% & Subjective Confidence Interval \\
Backyard Cow Milk & lower limit & central estimate & upper limit \\
Commercial Milk (locally produced) & 0.24 & 1.3 & 6.8 \\
Commercial Milk (regionally mixed) & 0.059 & 0.36 & 2.1 \\
Goat Milk (locally produced) & 0.078 & 0.47 & 3 \\
Beef (locally produced) & 0.58 & 4 & 28 \\
Leafy Vegetables (locally produced) & 0.00066 & 0.011 & 0.25 \\
Eggs (locally produced) & 0.00017 & 0.002 & 0.016 \\
Cottage Cheese (locally produced) & 0.014 & 0.1 & 0.7 \\
Inhalation & 0.0004 & 0.0043 & 0.044 \\
Mother's milk (mother on Diet 1) & 0.012 & 0.047 & 0.16 \\
Prenatal exposure (mother on Diet 1) & -- & -- & -- \\
Diet 1 & -- & -- & -- \\
Diet 2 & 0.29 & 1.4 & 7.8 \\
Diet 3 & 0.1 & 0.57 & 2.9 \\
\hline
\end{tabular}

Excess Lifetime Risk [ ]

\begin{tabular}{llll}
\hline Diet 1 & $3.2 \mathrm{E}-07$ & $1.1 \mathrm{E}-05$ & $2.8 \mathrm{E}-04$ \\
Diet 2 & $1.6 \mathrm{E}-07$ & $4.7 \mathrm{E}-06$ & $1.2 \mathrm{E}-04$ \\
Diet 3 & $1.8 \mathrm{E}-07$ & $4.9 \mathrm{E}-06$ & $1.2 \mathrm{E}-04$ \\
Diet 4 & $1.1 \mathrm{E}-06$ & $3.0 \mathrm{E}-05$ & $9.0 \mathrm{E}-04$ \\
\hline
\end{tabular}

\section{Relative Risk [ ]}

\begin{tabular}{lccc}
\hline Diet 1 & 1.00036 & 1.0074 & 1.15 \\
Diet 2 & 1.00017 & 1.0033 & 1.064 \\
Diet 3 & 1.00018 & 1.0031 & 1.065 \\
Diet 4 & 1.001 & 1.023 & 1.45 \\
\hline
\end{tabular}

\section{Probability of Causation [\%]}

\begin{tabular}{lccc}
\hline Diet 1 & 0.036 & 0.74 & 13.1 \\
Diet 2 & 0.016 & 0.33 & 6.0 \\
Diet 3 & 0.018 & 0.31 & 6.1 \\
Diet 4 & 0.100 & 2.25 & 31.0 \\
\hline
\end{tabular}

Diet 1 - Backyard cow milk + all other locally produced non-milk exposure pathways

Diet 2 - Locally produced commercial milk + all other locally produced non-milk exposure pathways

Diet 3 - Regionally mixed commercial milk + inhalation (other regionally mixed food items are minor contributors to the total)

Diet 4 - Goat milk (all other exposure pathways are of negligible importance compared to goat milk) 
Location: Wartburg

Receptor: Female born in 1935

\begin{tabular}{lccc}
\hline & \multicolumn{3}{c}{ Thyroid Dose [cGy] } \\
& \multicolumn{2}{c}{$95 \%$ Subjective Confidence Interval } \\
Exposure Pathway & lower limit & central estimate & upper limit \\
\hline Backyard Cow Milk & 0.04 & 0.31 & 1.7 \\
Commercial Milk (locally produced) & 0.012 & 0.086 & 0.57 \\
Commercial Milk (regionally mixed) & 0.071 & 0.43 & 2.6 \\
Goat Milk (locally produced) & 0.13 & 0.98 & 7.1 \\
Beef (locally produced) & 0.00013 & 0.0021 & 0.051 \\
Leafy Vegetables (locally produced) & 0.000048 & 0.00054 & 0.0055 \\
Eggs (locally produced) & 0.0033 & 0.023 & 0.17 \\
Cottage Cheese (locally produced) & 0.00009 & 0.0012 & 0.012 \\
Inhalation & 0.0027 & 0.01 & 0.043 \\
Mother's milk (mother on Diet 1) & -- & -- & -- \\
Prenatal exposure (mother on Diet 1) & -- & -- & -- \\
Diet 1 & 0.053 & 0.36 & 1.9 \\
Diet 2 & 0.023 & 0.13 & 0.79 \\
Diet 3 & 0.077 & 0.44 & 2.6 \\
\hline
\end{tabular}

Excess Lifetime Risk [ ]

\begin{tabular}{llcc}
\hline Diet 1 & $7.7 \mathrm{E}-07$ & $1.1 \mathrm{E}-05$ & $1.4 \mathrm{E}-04$ \\
Diet 2 & $4.3 \mathrm{E}-07$ & $5.0 \mathrm{E}-06$ & $6.5 \mathrm{E}-05$ \\
Diet 3 & $1.4 \mathrm{E}-06$ & $1.7 \mathrm{E}-05$ & $1.9 \mathrm{E}-04$ \\
Diet 4 & $2.6 \mathrm{E}-06$ & $3.7 \mathrm{E}-05$ & $5.1 \mathrm{E}-04$ \\
\hline
\end{tabular}

\section{Relative Risk [ ]}

\begin{tabular}{lccc}
\hline Diet 1 & 1.0003 & 1.0028 & 1.036 \\
Diet 2 & 1.00015 & 1.0013 & 1.018 \\
Diet 3 & 1.00051 & 1.0046 & 1.048 \\
Diet 4 & 1.00098 & 1.0091 & 1.11 \\
\hline
\end{tabular}

\section{Probability of Causation [\%]}

\begin{tabular}{llcc}
\hline Diet 1 & 0.030 & 0.28 & 3.5 \\
Diet 2 & 0.015 & 0.13 & 1.8 \\
Diet 3 & 0.051 & 0.45 & 4.6 \\
Diet 4 & 0.098 & 0.91 & 10.0 \\
\hline
\end{tabular}

Diet 1 - Backyard cow milk + all other locally produced non-milk exposure pathways

Diet 2 - Locally produced commercial milk + all other locally produced non-milk exposure pathways

Diet 3 - Regionally mixed commercial milk + inhalation (other regionally mixed food items are minor contributors to the total)

Diet 4 - Goat milk (all other exposure pathways are of negligible importance compared to goat milk) 


\section{Location: Wartburg}

Receptor: Male born in 1935

\begin{tabular}{lccc}
\hline & \multicolumn{3}{c}{ Thyroid Dose [cGy] } \\
& \multicolumn{2}{c}{$95 \%$ Subjective Confidence Interval } \\
Exposure Pathway & lower limit & central estimate & upper limit \\
\hline Backyard Cow Milk & 0.052 & 0.35 & 2 \\
Commercial Milk (locally produced) & 0.015 & 0.1 & 0.7 \\
Commercial Milk (regionally mixed) & 0.078 & 0.47 & 3 \\
Goat Milk (locally produced) & 0.15 & 1.1 & 8 \\
Beef (locally produced) & 0.0002 & 0.003 & 0.075 \\
Leafy Vegetables (locally produced) & 0.000046 & 0.00054 & 0.005 \\
Eggs (locally produced) & 0.0036 & 0.027 & 0.2 \\
Cottage Cheese (locally produced) & 0.0001 & 0.0012 & 0.012 \\
Inhalation & 0.0029 & 0.011 & 0.045 \\
Mother's milk (mother on Diet 1) & -- & -- & - \\
Prenatal exposure (mother on Diet 1) & -- & -- & -- \\
Diet 1 & 0.062 & 0.4 & 2.2 \\
Diet 2 & 0.026 & 0.15 & 0.98 \\
Diet 3 & 0.084 & 0.49 & 3.1 \\
\hline
\end{tabular}

Excess Lifetime Risk [ ]

\begin{tabular}{llll}
\hline Diet 1 & $7.9 \mathrm{E}-08$ & $3.0 \mathrm{E}-06$ & $8.0 \mathrm{E}-05$ \\
Diet 2 & $4.1 \mathrm{E}-08$ & $1.4 \mathrm{E}-06$ & $3.1 \mathrm{E}-05$ \\
Diet 3 & $1.6 \mathrm{E}-07$ & $4.6 \mathrm{E}-06$ & $1.1 \mathrm{E}-04$ \\
Diet 4 & $2.6 \mathrm{E}-07$ & $8.7 \mathrm{E}-06$ & $2.4 \mathrm{E}-04$ \\
\hline
\end{tabular}

\section{Relative Risk [ ]}

\begin{tabular}{lccc}
\hline Diet 1 & 1.000085 & 1.0021 & 1.043 \\
Diet 2 & 1.000042 & 1.00096 & 1.019 \\
Diet 3 & 1.00015 & 1.003 & 1.063 \\
Diet 4 & 1.00022 & 1.006 & 1.15 \\
\hline
\end{tabular}

\section{Probability of Causation [\%]}

\begin{tabular}{llcc}
\hline Diet 1 & 0.008 & 0.21 & 4.2 \\
Diet 2 & 0.004 & 0.10 & 1.8 \\
Diet 3 & 0.015 & 0.30 & 6.0 \\
Diet 4 & 0.022 & 0.60 & 13.1 \\
\hline
\end{tabular}

Diet 1 - Backyard cow milk + all other locally produced non-milk exposure pathways

Diet 2 - Locally produced commercial milk + all other locally produced non-milk exposure pathways

Diet 3 - Regionally mixed commercial milk + inhalation (other regionally mixed food items are minor contributors to the total)

Diet 4 - Goat milk (all other exposure pathways are of negligible importance compared to goat milk) 
Location: Rockwood

Receptor: Female born in 1935

\begin{tabular}{lccc}
\hline & \multicolumn{3}{c}{ Thyroid Dose [cGy] } \\
Exposure Pathway & $95 \%$ Subjective Confidence Interval \\
\hline Backyard Cow Milk & lower limit & central estimate & upper limit \\
Commercial Milk (locally produced) & 0.23 & 1.3 & 7.4 \\
Commercial Milk (regionally mixed) & 0.058 & 0.38 & 2.3 \\
Goat Milk (locally produced) & 0.071 & 0.43 & 2.6 \\
Beef (locally produced) & 0.61 & 4 & 27 \\
Leafy Vegetables (locally produced) & 0.00054 & 0.0089 & 0.19 \\
Eggs (locally produced) & 0.00021 & 0.0022 & 0.02 \\
Cottage Cheese (locally produced) & 0.015 & 0.099 & 0.62 \\
Inhalation & 0.00045 & 0.0049 & 0.049 \\
Mother's milk (mother on Diet 1) & 0.013 & 0.045 & 0.16 \\
Prenatal exposure (mother on Diet 1) & -- & -- & - \\
Diet 1 & -- & -- & -- \\
Diet 2 & 0.28 & 1.5 & 8.3 \\
Diet 3 & 0.11 & 0.56 & 3.1 \\
\hline
\end{tabular}

Excess Lifetime Risk [ ]

\begin{tabular}{llll}
\hline Diet 1 & $3.8 \mathrm{E}-06$ & $4.8 \mathrm{E}-05$ & $5.9 \mathrm{E}-04$ \\
Diet 2 & $1.8 \mathrm{E}-06$ & $2.1 \mathrm{E}-05$ & $2.4 \mathrm{E}-04$ \\
Diet 3 & $1.6 \mathrm{E}-06$ & $1.9 \mathrm{E}-05$ & $2.0 \mathrm{E}-04$ \\
Diet 4 & $1.2 \mathrm{E}-05$ & $1.6 \mathrm{E}-04$ & $1.8 \mathrm{E}-03$ \\
\hline
\end{tabular}

\section{Relative Risk [ ]}

\begin{tabular}{lccc}
\hline Diet 1 & 1.0014 & 1.012 & 1.12 \\
Diet 2 & 1.00062 & 1.0053 & 1.055 \\
Diet 3 & 1.00058 & 1.0049 & 1.049 \\
Diet 4 & 1.0044 & 1.037 & 1.48 \\
\hline
\end{tabular}

Probability of Causation [\%]

\begin{tabular}{llcc}
\hline Diet 1 & 0.144 & 1.15 & 10.4 \\
Diet 2 & 0.062 & 0.53 & 5.2 \\
Diet 3 & 0.058 & 0.49 & 4.7 \\
Diet 4 & 0.434 & 3.58 & 31.4 \\
\hline
\end{tabular}

Diet 1 - Backyard cow milk + all other locally produced non-milk exposure pathways

Diet 2 - Locally produced commercial milk + all other locally produced non-milk exposure pathways

Diet 3 - Regionally mixed commercial milk + inhalation (other regionally mixed food items are minor contributors to the total)

Diet 4 - Goat milk (all other exposure pathways are of negligible importance compared to goat milk) 


\section{Location: Rockwood}

\section{Receptor: Male born in 1935}

\begin{tabular}{lccc}
\hline & \multicolumn{3}{c}{ Thyroid Dose [cGy] } \\
& \multicolumn{2}{c}{$95 \%$ Subjective Confidence Interval } \\
Exposure Pathway & lower limit & central estimate & upper limit \\
\hline Backyard Cow Milk & 0.28 & 1.5 & 8.5 \\
Commercial Milk (locally produced) & 0.07 & 0.41 & 2.8 \\
Commercial Milk (regionally mixed) & 0.078 & 0.47 & 3 \\
Goat Milk (locally produced) & 0.69 & 4.7 & 29 \\
Beef (locally produced) & 0.00083 & 0.013 & 0.29 \\
Leafy Vegetables (locally produced) & 0.00021 & 0.0022 & 0.02 \\
Eggs (locally produced) & 0.017 & 0.12 & 0.76 \\
Cottage Cheese (locally produced) & 0.00049 & 0.0049 & 0.053 \\
Inhalation & 0.013 & 0.049 & 0.17 \\
Mother's milk (mother on Diet 1) & -- & -- & - \\
Prenatal exposure (mother on Diet 1) & -- & -- & -- \\
Diet 1 & 0.33 & 1.7 & 9.6 \\
Diet 2 & 0.13 & 0.64 & 3.8 \\
Diet 3 & 0.1 & 0.53 & 3.2 \\
\hline
\end{tabular}

Excess Lifetime Risk [ ]

\begin{tabular}{llll}
\hline Diet 1 & $4.0 \mathrm{E}-07$ & $1.2 \mathrm{E}-05$ & $2.9 \mathrm{E}-04$ \\
Diet 2 & $1.9 \mathrm{E}-07$ & $5.5 \mathrm{E}-06$ & $1.3 \mathrm{E}-04$ \\
Diet 3 & $1.8 \mathrm{E}-07$ & $4.9 \mathrm{E}-06$ & $1.2 \mathrm{E}-04$ \\
Diet 4 & $1.3 \mathrm{E}-06$ & $3.3 \mathrm{E}-05$ & $9.7 \mathrm{E}-04$ \\
\hline
\end{tabular}

\section{Relative Risk [ ]}

\begin{tabular}{lccc}
\hline Diet 1 & 1.00039 & 1.0086 & 1.18 \\
Diet 2 & 1.00019 & 1.0038 & 1.071 \\
Diet 3 & 1.00018 & 1.0031 & 1.065 \\
Diet 4 & 1.0011 & 1.026 & 1.56 \\
\hline
\end{tabular}

\section{Probability of Causation [\%]}

\begin{tabular}{llcc}
\hline Diet 1 & 0.039 & 0.85 & 14.9 \\
Diet 2 & 0.019 & 0.38 & 6.6 \\
Diet 3 & 0.018 & 0.31 & 6.1 \\
Diet 4 & 0.112 & 2.50 & 35.1 \\
\hline
\end{tabular}

Diet 1 - Backyard cow milk + all other locally produced non-milk exposure pathways

Diet 2 - Locally produced commercial milk + all other locally produced non-milk exposure pathways

Diet 3 - Regionally mixed commercial milk + inhalation (other regionally mixed food items are minor contributors to the total)

Diet 4 - Goat milk (all other exposure pathways are of negligible importance compared to goat milk) 


\section{Location: Louisville}

Receptor: Female born in 1935

\begin{tabular}{lccc}
\hline & \multicolumn{3}{c}{ Thyroid Dose [cGy] } \\
Exposure Pathway & \multicolumn{2}{c}{$95 \%$ Subjective Confidence Interval } \\
\hline Backyard Cow Milk & lower limit & central estimate & upper limit \\
Commercial Milk (locally produced) & 0.19 & 1.2 & 6.3 \\
Commercial Milk (regionally mixed) & 0.052 & 0.34 & 2.1 \\
Goat Milk (locally produced) & 0.071 & 0.43 & 2.6 \\
Beef (locally produced) & 0.5 & 3.6 & 26 \\
Leafy Vegetables (locally produced) & 0.0005 & 0.0083 & 0.16 \\
Eggs (locally produced) & 0.0002 & 0.0021 & 0.018 \\
Cottage Cheese (locally produced) & 0.014 & 0.088 & 0.61 \\
Inhalation & 0.00041 & 0.0045 & 0.046 \\
Mother's milk (mother on Diet 1) & 0.013 & 0.045 & 0.17 \\
Prenatal exposure (mother on Diet 1) & -- & -- & -- \\
Diet 1 & -- & -- & -- \\
Diet 2 & 0.24 & 1.4 & 7.1 \\
Diet 3 & 0.098 & 0.52 & 2.9 \\
\hline
\end{tabular}

Excess Lifetime Risk [ ]

\begin{tabular}{lllc}
\hline Diet 1 & $3.4 \mathrm{E}-06$ & $4.3 \mathrm{E}-05$ & $5.5 \mathrm{E}-04$ \\
Diet 2 & $1.7 \mathrm{E}-06$ & $1.9 \mathrm{E}-05$ & $2.2 \mathrm{E}-04$ \\
Diet 3 & $1.6 \mathrm{E}-06$ & $1.9 \mathrm{E}-05$ & $2.0 \mathrm{E}-04$ \\
Diet 4 & $1.0 \mathrm{E}-05$ & $1.4 \mathrm{E}-04$ & $2.0 \mathrm{E}-03$ \\
\hline
\end{tabular}

Relative Risk [ ]

\begin{tabular}{lccc}
\hline Diet 1 & 1.0013 & 1.011 & 1.11 \\
Diet 2 & 1.00059 & 1.005 & 1.056 \\
Diet 3 & 1.00057 & 1.0049 & 1.05 \\
Diet 4 & 1.0038 & 1.035 & 1.41 \\
\hline
\end{tabular}

Probability of Causation [\%]

\begin{tabular}{llcc}
\hline Diet 1 & 0.133 & 1.06 & 10.3 \\
Diet 2 & 0.059 & 0.49 & 5.3 \\
Diet 3 & 0.057 & 0.49 & 4.7 \\
Diet 4 & 0.381 & 3.34 & 28.9 \\
\hline
\end{tabular}

Diet 1 - Backyard cow milk + all other locally produced non-milk exposure pathways

Diet 2 - Locally produced commercial milk + all other locally produced non-milk exposure pathways

Diet 3 - Regionally mixed commercial milk + inhalation (other regionally mixed food items are minor contributors to the total)

Diet 4 - Goat milk (all other exposure pathways are of negligible importance compared to goat milk) 


\section{Location: Louisville}

\section{Receptor: Male born in 1935}

\begin{tabular}{lccc}
\hline & \multicolumn{3}{c}{ Thyroid Dose [cGy] } \\
& \multicolumn{2}{c}{$95 \%$ Subjective Confidence Interval } \\
Exposure Pathway & lower limit & central estimate & upper limit \\
\hline Backyard Cow Milk & 0.24 & 1.4 & 7.1 \\
Commercial Milk (locally produced) & 0.061 & 0.38 & 2.5 \\
Commercial Milk (regionally mixed) & 0.078 & 0.47 & 3 \\
Goat Milk (locally produced) & 0.57 & 4.2 & 29 \\
Beef (locally produced) & 0.00075 & 0.012 & 0.24 \\
Leafy Vegetables (locally produced) & 0.0002 & 0.0021 & 0.017 \\
Eggs (locally produced) & 0.016 & 0.11 & 0.73 \\
Cottage Cheese (locally produced) & 0.00045 & 0.0046 & 0.044 \\
Inhalation & 0.014 & 0.049 & 0.18 \\
Mother's milk (mother on Diet 1) & -- & -- & -- \\
Prenatal exposure (mother on Diet 1) & -- & -- & -- \\
Diet 1 & 0.3 & 1.6 & 8.1 \\
Diet 2 & 0.11 & 0.58 & 3.3 \\
Diet 3 & 0.1 & 0.53 & 3.2 \\
\hline
\end{tabular}

Excess Lifetime Risk [ ]

\begin{tabular}{llll}
\hline Diet 1 & $3.5 \mathrm{E}-07$ & $1.2 \mathrm{E}-05$ & $2.7 \mathrm{E}-04$ \\
Diet 2 & $1.6 \mathrm{E}-07$ & $5.2 \mathrm{E}-06$ & $1.2 \mathrm{E}-04$ \\
Diet 3 & $1.8 \mathrm{E}-07$ & $4.9 \mathrm{E}-06$ & $1.2 \mathrm{E}-04$ \\
Diet 4 & $1.1 \mathrm{E}-06$ & $3.2 \mathrm{E}-05$ & $9.7 \mathrm{E}-04$ \\
\hline
\end{tabular}

\section{Relative Risk [ ]}

\begin{tabular}{lccc}
\hline Diet 1 & 1.00034 & 1.0078 & 1.15 \\
Diet 2 & 1.00017 & 1.0036 & 1.067 \\
Diet 3 & 1.00018 & 1.0031 & 1.065 \\
Diet 4 & 1.001 & 1.024 & 1.47 \\
\hline
\end{tabular}

\section{Probability of Causation [\%]}

\begin{tabular}{llcc}
\hline Diet 1 & 0.034 & 0.78 & 13.1 \\
Diet 2 & 0.017 & 0.35 & 6.2 \\
Diet 3 & 0.018 & 0.31 & 6.1 \\
Diet 4 & 0.103 & 2.32 & 31.5 \\
\hline
\end{tabular}

Diet 1 - Backyard cow milk + all other locally produced non-milk exposure pathways

Diet 2 - Locally produced commercial milk + all other locally produced non-milk exposure pathways

Diet 3 - Regionally mixed commercial milk + inhalation (other regionally mixed food items are minor contributors to the total)

Diet 4 - Goat milk (all other exposure pathways are of negligible importance compared to goat milk) 
Location: Barnardville

Receptor: Female born in 1935

\begin{tabular}{lccc}
\hline & \multicolumn{3}{c}{ Thyroid Dose [cGy] } \\
Exposure Pathway & $95 \%$ & Subjective Confidence Interval \\
Backyard Cow Milk & lower limit & central estimate & upper limit \\
Commercial Milk (locally produced) & 0.22 & 1.3 & 7.6 \\
Commercial Milk (regionally mixed) & 0.058 & 0.39 & 2.3 \\
Goat Milk (locally produced) & 0.071 & 0.43 & 2.6 \\
Beef (locally produced) & 0.6 & 4.2 & 27 \\
Leafy Vegetables (locally produced) & 0.00057 & 0.0095 & 0.19 \\
Eggs (locally produced) & 0.00022 & 0.0023 & 0.021 \\
Cottage Cheese (locally produced) & 0.015 & 0.1 & 0.71 \\
Inhalation & 0.00046 & 0.005 & 0.051 \\
Mother's milk (mother on Diet 1) & 0.016 & 0.052 & 0.2 \\
Prenatal exposure (mother on Diet 1) & -- & -- & -- \\
Diet 1 & -- & -- & -- \\
Diet 2 & 0.28 & 1.5 & 8.4 \\
Diet 3 & 0.11 & 0.59 & 3.2 \\
\hline
\end{tabular}

Excess Lifetime Risk [ ]

\begin{tabular}{llcc}
\hline Diet 1 & $3.9 \mathrm{E}-06$ & $5.1 \mathrm{E}-05$ & $6.1 \mathrm{E}-04$ \\
Diet 2 & $1.9 \mathrm{E}-06$ & $2.2 \mathrm{E}-05$ & $2.6 \mathrm{E}-04$ \\
Diet 3 & $1.6 \mathrm{E}-06$ & $1.9 \mathrm{E}-05$ & $2.0 \mathrm{E}-04$ \\
Diet 4 & $1.2 \mathrm{E}-05$ & $1.6 \mathrm{E}-04$ & $2.1 \mathrm{E}-03$ \\
\hline
\end{tabular}

\section{Relative Risk [ ]}

\begin{tabular}{lccc}
\hline Diet 1 & 1.0015 & 1.012 & 1.13 \\
Diet 2 & 1.00067 & 1.0055 & 1.058 \\
Diet 3 & 1.00059 & 1.005 & 1.05 \\
Diet 4 & 1.0045 & 1.039 & 1.48 \\
\hline
\end{tabular}

Probability of Causation [\%]

\begin{tabular}{llcc}
\hline Diet 1 & 0.149 & 1.22 & 11.1 \\
Diet 2 & 0.067 & 0.54 & 5.5 \\
Diet 3 & 0.059 & 0.49 & 4.8 \\
Diet 4 & 0.452 & 3.79 & 32.5 \\
\hline
\end{tabular}

Diet 1 - Backyard cow milk + all other locally produced non-milk exposure pathways

Diet 2 - Locally produced commercial milk + all other locally produced non-milk exposure pathways

Diet 3 - Regionally mixed commercial milk + inhalation (other regionally mixed food items are minor contributors to the total)

Diet 4 - Goat milk (all other exposure pathways are of negligible importance compared to goat milk) 


\section{Location: Barnardville}

Receptor: Male born in 1935

\begin{tabular}{lccc}
\hline & \multicolumn{3}{c}{ Thyroid Dose [cGy] } \\
Exposure Pathway & \multicolumn{2}{c}{$95 \%$ Subjective Confidence Interval } \\
\hline Backyard Cow Milk & lower limit & central estimate & upper limit \\
Commercial Milk (locally produced) & 0.29 & 1.5 & 8.5 \\
Commercial Milk (regionally mixed) & 0.071 & 0.42 & 2.7 \\
Goat Milk (locally produced) & 0.078 & 0.47 & 3.1 \\
Beef (locally produced) & 0.69 & 4.7 & 32 \\
Leafy Vegetables (locally produced) & 0.00084 & 0.013 & 0.28 \\
Eggs (locally produced) & 0.00022 & 0.0023 & 0.02 \\
Cottage Cheese (locally produced) & 0.017 & 0.12 & 0.82 \\
Inhalation & 0.00047 & 0.0052 & 0.052 \\
Mother's milk (mother on Diet 1) & 0.016 & 0.057 & 0.21 \\
Prenatal exposure (mother on Diet 1) & -- & -- & - \\
Diet 1 & -- & -- & -- \\
Diet 2 & 0.35 & 1.8 & 9.5 \\
Diet 3 & 0.13 & 0.67 & 3.7 \\
\hline
\end{tabular}

Excess Lifetime Risk [ ]

\begin{tabular}{lllc}
\hline Diet 1 & $4.3 \mathrm{E}-07$ & $1.3 \mathrm{E}-05$ & $3.3 \mathrm{E}-04$ \\
Diet 2 & $1.9 \mathrm{E}-07$ & $5.9 \mathrm{E}-06$ & $1.4 \mathrm{E}-04$ \\
Diet 3 & $1.8 \mathrm{E}-07$ & $5.0 \mathrm{E}-06$ & $1.2 \mathrm{E}-04$ \\
Diet 4 & $1.4 \mathrm{E}-06$ & $3.6 \mathrm{E}-05$ & $1.1 \mathrm{E}-03$ \\
\hline
\end{tabular}

\section{Relative Risk [ ]}

\begin{tabular}{lccc}
\hline Diet 1 & 1.00041 & 1.0091 & 1.19 \\
Diet 2 & 1.0002 & 1.0041 & 1.075 \\
Diet 3 & 1.00018 & 1.0032 & 1.066 \\
Diet 4 & 1.0012 & 1.026 & 1.58 \\
\hline
\end{tabular}

\section{Probability of Causation [\%]}

\begin{tabular}{lccc}
\hline Diet 1 & 0.041 & 0.90 & 16.2 \\
Diet 2 & 0.020 & 0.41 & 7.0 \\
Diet 3 & 0.018 & 0.31 & 6.2 \\
Diet 4 & 0.121 & 2.54 & 36.5 \\
\hline
\end{tabular}

Diet 1 - Backyard cow milk + all other locally produced non-milk exposure pathways

Diet 2 - Locally produced commercial milk + all other locally produced non-milk exposure pathways

Diet 3 - Regionally mixed commercial milk + inhalation (other regionally mixed food items are minor contributors to the total)

Diet 4 - Goat milk (all other exposure pathways are of negligible importance compared to goat milk) 
Location: Greenback

Receptor: Female born in 1935

\begin{tabular}{lccc}
\hline & \multicolumn{3}{c}{ Thyroid Dose [cGy] } \\
Exposure Pathway & $95 \%$ Subjective Confidence Interval \\
\hline Backyard Cow Milk & lower limit & central estimate & upper limit \\
Commercial Milk (locally produced) & 0.12 & 0.71 & 4.6 \\
Commercial Milk (regionally mixed) & 0.031 & 0.22 & 1.4 \\
Goat Milk (locally produced) & 0.071 & 0.43 & 2.6 \\
Beef (locally produced) & 0.3 & 2.3 & 15 \\
Leafy Vegetables (locally produced) & 0.00033 & 0.0052 & 0.11 \\
Eggs (locally produced) & 0.00012 & 0.0013 & 0.012 \\
Cottage Cheese (locally produced) & 0.0086 & 0.057 & 0.41 \\
Inhalation & 0.00025 & 0.0027 & 0.035 \\
Mother's milk (mother on Diet 1) & 0.0088 & 0.03 & 0.11 \\
Prenatal exposure (mother on Diet 1) & -- & -- & -- \\
Diet 1 & -- & -- & -- \\
Diet 2 & 0.15 & 0.82 & 5.2 \\
Diet 3 & 0.068 & 0.32 & 2 \\
\hline
\end{tabular}

Excess Lifetime Risk [ ]

\begin{tabular}{llll}
\hline Diet 1 & $2.2 \mathrm{E}-06$ & $2.8 \mathrm{E}-05$ & $4.0 \mathrm{E}-04$ \\
Diet 2 & $1.2 \mathrm{E}-06$ & $1.3 \mathrm{E}-05$ & $1.5 \mathrm{E}-04$ \\
Diet 3 & $1.6 \mathrm{E}-06$ & $1.8 \mathrm{E}-05$ & $1.9 \mathrm{E}-04$ \\
Diet 4 & $7.1 \mathrm{E}-06$ & $8.5 \mathrm{E}-05$ & $1.2 \mathrm{E}-03$ \\
\hline
\end{tabular}

\section{Relative Risk [ ]}

\begin{tabular}{lccc}
\hline Diet 1 & 1.00084 & 1.0067 & 1.074 \\
Diet 2 & 1.00039 & 1.003 & 1.032 \\
Diet 3 & 1.00056 & 1.0047 & 1.049 \\
Diet 4 & 1.0025 & 1.021 & 1.28 \\
\hline
\end{tabular}

\section{Probability of Causation [\%]}

\begin{tabular}{llcc}
\hline Diet 1 & 0.084 & 0.67 & 6.9 \\
Diet 2 & 0.039 & 0.30 & 3.1 \\
Diet 3 & 0.056 & 0.47 & 4.7 \\
Diet 4 & 0.246 & 2.08 & 21.7 \\
\hline
\end{tabular}

Diet 1 - Backyard cow milk + all other locally produced non-milk exposure pathways

Diet 2 - Locally produced commercial milk + all other locally produced non-milk exposure pathways

Diet 3 - Regionally mixed commercial milk + inhalation (other regionally mixed food items are minor contributors to the total)

Diet 4 - Goat milk (all other exposure pathways are of negligible importance compared to goat milk) 
Location: Greenback

Receptor: Male born in 1935

\begin{tabular}{lccc}
\hline & \multicolumn{3}{c}{ Thyroid Dose [cGy] } \\
Exposure Pathway & 95\% Subjective Confidence Interval \\
\hline Backyard Cow Milk & lower limit & central estimate & upper limit \\
Commercial Milk (locally produced) & 0.16 & 0.83 & 5.3 \\
Commercial Milk (regionally mixed) & 0.038 & 0.23 & 1.7 \\
Goat Milk (locally produced) & 0.078 & 0.47 & 3 \\
Beef (locally produced) & 0.34 & 2.7 & 18 \\
Leafy Vegetables (locally produced) & 0.00046 & 0.0073 & 0.15 \\
Eggs (locally produced) & 0.00013 & 0.0013 & 0.012 \\
Cottage Cheese (locally produced) & 0.0096 & 0.066 & 0.45 \\
Inhalation & 0.00028 & 0.0027 & 0.035 \\
Mother's milk (mother on Diet 1) & 0.0098 & 0.032 & 0.13 \\
Prenatal exposure (mother on Diet 1) & -- & -- & -- \\
Diet 1 & -- & -- & -- \\
Diet 2 & 0.19 & 0.97 & 6 \\
Diet 3 & 0.075 & 0.37 & 2.3 \\
\hline
\end{tabular}

Excess Lifetime Risk [ ]

\begin{tabular}{llll}
\hline Diet 1 & $2.3 \mathrm{E}-07$ & $6.9 \mathrm{E}-06$ & $1.8 \mathrm{E}-04$ \\
Diet 2 & $1.1 \mathrm{E}-07$ & $3.3 \mathrm{E}-06$ & $7.7 \mathrm{E}-05$ \\
Diet 3 & $1.8 \mathrm{E}-07$ & $4.8 \mathrm{E}-06$ & $1.2 \mathrm{E}-04$ \\
Diet 4 & $7.2 \mathrm{E}-07$ & $1.9 \mathrm{E}-05$ & $5.5 \mathrm{E}-04$ \\
\hline
\end{tabular}

\section{Relative Risk [ ]}

\begin{tabular}{lccc}
\hline Diet 1 & 1.00023 & 1.0051 & 1.11 \\
Diet 2 & 1.0001 & 1.0023 & 1.041 \\
Diet 3 & 1.00017 & 1.0031 & 1.064 \\
Diet 4 & 1.00064 & 1.015 & 1.29 \\
\hline
\end{tabular}

\begin{tabular}{llcc} 
& \multicolumn{3}{c}{ Probability of Causation [\%] } \\
\hline Diet 1 & 0.023 & 0.51 & 9.9 \\
Diet 2 & 0.010 & 0.23 & 4.0 \\
Diet 3 & 0.017 & 0.31 & 6.0 \\
Diet 4 & 0.064 & 1.51 & 22.7 \\
\hline
\end{tabular}

Diet 1 - Backyard cow milk + all other locally produced non-milk exposure pathways

Diet 2 - Locally produced commercial milk + all other locally produced non-milk exposure pathways

Diet 3 - Regionally mixed commercial milk + inhalation (other regionally mixed food items are minor contributors to the total)

Diet 4 - Goat milk (all other exposure pathways are of negligible importance compared to goat milk) 


\section{Location: Rockford}

Receptor: Female born in 1935

\begin{tabular}{lccc}
\hline & \multicolumn{3}{c}{ Thyroid Dose [cGy] } \\
Exposure Pathway & $95 \%$ Subjective Confidence Interval \\
\hline Backyard Cow Milk & lower limit & central estimate & upper limit \\
Commercial Milk (locally produced) & 0.2 & 1.2 & 6.7 \\
Commercial Milk (regionally mixed) & 0.052 & 0.33 & 2 \\
Goat Milk (locally produced) & 0.071 & 0.43 & 2.6 \\
Beef (locally produced) & 0.54 & 3.6 & 22 \\
Leafy Vegetables (locally produced) & 0.00049 & 0.0082 & 0.16 \\
Eggs (locally produced) & 0.00019 & 0.0021 & 0.017 \\
Cottage Cheese (locally produced) & 0.013 & 0.089 & 0.56 \\
Inhalation & 0.00043 & 0.0043 & 0.048 \\
Mother's milk (mother on Diet 1) & 0.014 & 0.045 & 0.16 \\
Prenatal exposure (mother on Diet 1) & -- & -- & -- \\
Diet 1 & -- & -- & -- \\
Diet 2 & 0.24 & 1.3 & 7.7 \\
Diet 3 & 0.11 & 0.51 & 2.7 \\
\hline
\end{tabular}

Excess Lifetime Risk [ ]

\begin{tabular}{llll}
\hline Diet 1 & $3.3 \mathrm{E}-06$ & $4.3 \mathrm{E}-05$ & $5.5 \mathrm{E}-04$ \\
Diet 2 & $1.8 \mathrm{E}-06$ & $1.9 \mathrm{E}-05$ & $2.2 \mathrm{E}-04$ \\
Diet 3 & $1.7 \mathrm{E}-06$ & $1.9 \mathrm{E}-05$ & $2.0 \mathrm{E}-04$ \\
Diet 4 & $1.1 \mathrm{E}-05$ & $1.5 \mathrm{E}-04$ & $2.0 \mathrm{E}-03$ \\
\hline
\end{tabular}

\section{Relative Risk [ ]}

\begin{tabular}{lccc}
\hline Diet 1 & 1.0012 & 1.01 & 1.1 \\
Diet 2 & 1.00059 & 1.0048 & 1.05 \\
Diet 3 & 1.00058 & 1.0049 & 1.05 \\
Diet 4 & 1.0039 & 1.034 & 1.4 \\
\hline
\end{tabular}

\section{Probability of Causation [\%]}

\begin{tabular}{llcc}
\hline Diet 1 & 0.125 & 1.03 & 9.5 \\
Diet 2 & 0.059 & 0.48 & 4.8 \\
Diet 3 & 0.058 & 0.49 & 4.7 \\
Diet 4 & 0.386 & 3.29 & 28.2 \\
\hline
\end{tabular}

Diet 1 - Backyard cow milk + all other locally produced non-milk exposure pathways

Diet 2 - Locally produced commercial milk + all other locally produced non-milk exposure pathways

Diet 3 - Regionally mixed commercial milk + inhalation (other regionally mixed food items are minor contributors to the total)

Diet 4 - Goat milk (all other exposure pathways are of negligible importance compared to goat milk) 


\section{Location: Rockford}

Receptor: Male born in 1935

\begin{tabular}{lccc}
\hline & \multicolumn{3}{c}{ Thyroid Dose [cGy] } \\
& \multicolumn{2}{c}{$95 \%$ Subjective Confidence Interval } \\
Exposure Pathway & lower limit & central estimate & upper limit \\
\hline Backyard Cow Milk & 0.26 & 1.3 & 7.5 \\
Commercial Milk (locally produced) & 0.059 & 0.37 & 2.5 \\
Commercial Milk (regionally mixed) & 0.078 & 0.47 & 3 \\
Goat Milk (locally produced) & 0.6 & 4.2 & 27 \\
Beef (locally produced) & 0.00071 & 0.011 & 0.23 \\
Leafy Vegetables (locally produced) & 0.0002 & 0.002 & 0.017 \\
Eggs (locally produced) & 0.015 & 0.11 & 0.68 \\
Cottage Cheese (locally produced) & 0.00041 & 0.0045 & 0.048 \\
Inhalation & 0.015 & 0.048 & 0.18 \\
Mother's milk (mother on Diet 1) & -- & -- & -- \\
Prenatal exposure (mother on Diet 1) & -- & -- & -- \\
Diet 1 & 0.31 & 1.5 & 8.5 \\
Diet 2 & 0.12 & 0.58 & 3.2 \\
Diet 3 & 0.11 & 0.53 & 3.2 \\
\hline
\end{tabular}

Excess Lifetime Risk [ ]

\begin{tabular}{lllc}
\hline Diet 1 & $3.6 \mathrm{E}-07$ & $1.2 \mathrm{E}-05$ & $2.7 \mathrm{E}-04$ \\
Diet 2 & $1.7 \mathrm{E}-07$ & $5.0 \mathrm{E}-06$ & $1.2 \mathrm{E}-04$ \\
Diet 3 & $1.8 \mathrm{E}-07$ & $4.9 \mathrm{E}-06$ & $1.2 \mathrm{E}-04$ \\
Diet 4 & $1.1 \mathrm{E}-06$ & $3.1 \mathrm{E}-05$ & $8.5 \mathrm{E}-04$ \\
\hline
\end{tabular}

\section{Relative Risk [ ]}

\begin{tabular}{lccc}
\hline Diet 1 & 1.00035 & 1.0079 & 1.16 \\
Diet 2 & 1.00018 & 1.0036 & 1.063 \\
Diet 3 & 1.00017 & 1.0031 & 1.066 \\
Diet 4 & 1.00098 & 1.025 & 1.47 \\
\hline
\end{tabular}

\section{Probability of Causation [\%]}

\begin{tabular}{lccc}
\hline Diet 1 & 0.035 & 0.79 & 13.7 \\
Diet 2 & 0.018 & 0.35 & 6.0 \\
Diet 3 & 0.017 & 0.31 & 6.2 \\
Diet 4 & 0.098 & 2.41 & 31.7 \\
\hline
\end{tabular}

Diet 1 - Backyard cow milk + all other locally produced non-milk exposure pathways

Diet 2 - Locally produced commercial milk + all other locally produced non-milk exposure pathways

Diet 3 - Regionally mixed commercial milk + inhalation (other regionally mixed food items are minor contributors to the total)

Diet 4 - Goat milk (all other exposure pathways are of negligible importance compared to goat milk) 
Location: Lake City

Receptor: Female born in 1935

\begin{tabular}{lccc}
\hline & \multicolumn{3}{c}{ Thyroid Dose [cGy] } \\
Exposure Pathway & \multicolumn{2}{c}{$95 \%$ Subjective Confidence Interval } \\
\hline Backyard Cow Milk & lower limit & central estimate & upper limit \\
Commercial Milk (locally produced) & 0.11 & 0.69 & 3.5 \\
Commercial Milk (regionally mixed) & 0.034 & 0.2 & 1.2 \\
Goat Milk (locally produced) & 0.071 & 0.43 & 2.6 \\
Beef (locally produced) & 0.34 & 2.2 & 14 \\
Leafy Vegetables (locally produced) & 0.00029 & 0.005 & 0.11 \\
Eggs (locally produced) & 0.00012 & 0.0013 & 0.011 \\
Cottage Cheese (locally produced) & 0.008 & 0.052 & 0.32 \\
Inhalation & 0.00026 & 0.0027 & 0.028 \\
Mother's milk (mother on Diet 1) & 0.0071 & 0.025 & 0.092 \\
Prenatal exposure (mother on Diet 1) & -- & -- & -- \\
Diet 1 & -- & -- & -- \\
Diet 2 & 0.14 & 0.8 & 4 \\
Diet 3 & 0.062 & 0.3 & 1.7 \\
\hline
\end{tabular}

Excess Lifetime Risk [ ]

\begin{tabular}{llll}
\hline Diet 1 & $2.0 \mathrm{E}-06$ & $2.5 \mathrm{E}-05$ & $3.2 \mathrm{E}-04$ \\
Diet 2 & $1.1 \mathrm{E}-06$ & $1.2 \mathrm{E}-05$ & $1.3 \mathrm{E}-04$ \\
Diet 3 & $1.5 \mathrm{E}-06$ & $1.8 \mathrm{E}-05$ & $1.9 \mathrm{E}-04$ \\
Diet 4 & $6.5 \mathrm{E}-06$ & $8.7 \mathrm{E}-05$ & $1.3 \mathrm{E}-03$ \\
\hline
\end{tabular}

\section{Relative Risk [ ]}

\begin{tabular}{lccc}
\hline Diet 1 & 1.00074 & 1.0066 & 1.068 \\
Diet 2 & 1.00033 & 1.003 & 1.034 \\
Diet 3 & 1.00054 & 1.0047 & 1.048 \\
Diet 4 & 1.0024 & 1.021 & 1.24 \\
\hline
\end{tabular}

\section{Probability of Causation [\%]}

\begin{tabular}{llcc}
\hline Diet 1 & 0.074 & 0.66 & 6.3 \\
Diet 2 & 0.033 & 0.30 & 3.3 \\
Diet 3 & 0.054 & 0.47 & 4.6 \\
Diet 4 & 0.244 & 2.05 & 19.1 \\
\hline
\end{tabular}

Diet 1 - Backyard cow milk + all other locally produced non-milk exposure pathways

Diet 2 - Locally produced commercial milk + all other locally produced non-milk exposure pathways

Diet 3 - Regionally mixed commercial milk + inhalation (other regionally mixed food items are minor contributors to the total)

Diet 4 - Goat milk (all other exposure pathways are of negligible importance compared to goat milk) 
Location: Lake City

Receptor: Male born in 1935

\begin{tabular}{lccc}
\hline & \multicolumn{3}{c}{ Thyroid Dose [cGy] } \\
& \multicolumn{2}{c}{$95 \%$ Subjective Confidence Interval } \\
Exposure Pathway & lower limit & central estimate & upper limit \\
\hline Backyard Cow Milk & 0.15 & 0.8 & 4.3 \\
Commercial Milk (locally produced) & 0.038 & 0.22 & 1.5 \\
Commercial Milk (regionally mixed) & 0.078 & 0.47 & 3.1 \\
Goat Milk (locally produced) & 0.4 & 2.6 & 17 \\
Beef (locally produced) & 0.00043 & 0.007 & 0.16 \\
Leafy Vegetables (locally produced) & 0.00012 & 0.0013 & 0.01 \\
Eggs (locally produced) & 0.0087 & 0.062 & 0.41 \\
Cottage Cheese (locally produced) & 0.00028 & 0.0027 & 0.029 \\
Inhalation & 0.0076 & 0.027 & 0.097 \\
Mother's milk (mother on Diet 1) & -- & -- & -- \\
Prenatal exposure (mother on Diet 1) & -- & -- & - \\
Diet 1 & 0.18 & 0.92 & 4.8 \\
Diet 2 & 0.069 & 0.34 & 2 \\
Diet 3 & 0.094 & 0.51 & 3.1 \\
\hline
\end{tabular}

Excess Lifetime Risk [ ]

\begin{tabular}{llll}
\hline Diet 1 & $2.1 \mathrm{E}-07$ & $6.8 \mathrm{E}-06$ & $1.7 \mathrm{E}-04$ \\
Diet 2 & $1.0 \mathrm{E}-07$ & $3.1 \mathrm{E}-06$ & $7.3 \mathrm{E}-05$ \\
Diet 3 & $1.7 \mathrm{E}-07$ & $4.7 \mathrm{E}-06$ & $1.2 \mathrm{E}-04$ \\
Diet 4 & $7.1 \mathrm{E}-07$ & $2.0 \mathrm{E}-05$ & $5.3 \mathrm{E}-04$ \\
\hline
\end{tabular}

\section{Relative Risk [ ]}

\begin{tabular}{lccc}
\hline Diet 1 & 1.0002 & 1.0047 & 1.096 \\
Diet 2 & 1.000094 & 1.0021 & 1.039 \\
Diet 3 & 1.00017 & 1.0031 & 1.064 \\
Diet 4 & 1.00056 & 1.014 & 1.3 \\
\hline
\end{tabular}

\section{Probability of Causation [\%]}

\begin{tabular}{lccc}
\hline Diet 1 & 0.020 & 0.47 & 8.7 \\
Diet 2 & 0.009 & 0.21 & 3.7 \\
Diet 3 & 0.016 & 0.31 & 6.0 \\
Diet 4 & 0.056 & 1.40 & 23.2 \\
\hline
\end{tabular}

Diet 1 - Backyard cow milk + all other locally produced non-milk exposure pathways

Diet 2 - Locally produced commercial milk + all other locally produced non-milk exposure pathways

Diet 3 - Regionally mixed commercial milk + inhalation (other regionally mixed food items are minor contributors to the total)

Diet 4 - Goat milk (all other exposure pathways are of negligible importance compared to goat milk) 
Location: Sweetwater

Receptor: Female born in 1935

\begin{tabular}{lccc}
\hline & \multicolumn{3}{c}{ Thyroid Dose [cGy] } \\
Exposure Pathway & $95 \%$ Subjective Confidence Interval \\
\hline Backyard Cow Milk & lower limit & central estimate & upper limit \\
Commercial Milk (locally produced) & 0.13 & 0.88 & 4.9 \\
Commercial Milk (regionally mixed) & 0.038 & 0.26 & 1.6 \\
Goat Milk (locally produced) & 0.071 & 0.43 & 2.6 \\
Beef (locally produced) & 0.34 & 2.5 & 20 \\
Leafy Vegetables (locally produced) & 0.00036 & 0.0061 & 0.11 \\
Eggs (locally produced) & 0.00015 & 0.0015 & 0.013 \\
Cottage Cheese (locally produced) & 0.01 & 0.066 & 0.42 \\
Inhalation & 0.00032 & 0.0034 & 0.034 \\
Mother's milk (mother on Diet 1) & 0.01 & 0.036 & 0.13 \\
Prenatal exposure (mother on Diet 1) & -- & -- & -- \\
Diet 1 & -- & -- & -- \\
Diet 2 & 0.17 & 1 & 5.6 \\
Diet 3 & 0.071 & 0.4 & 2.1 \\
\hline
\end{tabular}

Excess Lifetime Risk [ ]

\begin{tabular}{llcc}
\hline Diet 1 & $2.6 \mathrm{E}-06$ & $3.2 \mathrm{E}-05$ & $4.1 \mathrm{E}-04$ \\
Diet 2 & $1.3 \mathrm{E}-06$ & $1.4 \mathrm{E}-05$ & $1.6 \mathrm{E}-04$ \\
Diet 3 & $1.5 \mathrm{E}-06$ & $1.9 \mathrm{E}-05$ & $1.9 \mathrm{E}-04$ \\
Diet 4 & $7.4 \mathrm{E}-06$ & $1.1 \mathrm{E}-04$ & $1.5 \mathrm{E}-03$ \\
\hline
\end{tabular}

\section{Relative Risk [ ]}

\begin{tabular}{lccc}
\hline Diet 1 & 1.001 & 1.0077 & 1.08 \\
Diet 2 & 1.00046 & 1.0037 & 1.04 \\
Diet 3 & 1.00056 & 1.0048 & 1.049 \\
Diet 4 & 1.0027 & 1.026 & 1.31 \\
\hline
\end{tabular}

\section{Probability of Causation [\%]}

\begin{tabular}{llcc}
\hline Diet 1 & 0.103 & 0.76 & 7.4 \\
Diet 2 & 0.046 & 0.37 & 3.8 \\
Diet 3 & 0.056 & 0.48 & 4.7 \\
Diet 4 & 0.267 & 2.52 & 23.9 \\
\hline
\end{tabular}

Diet 1 - Backyard cow milk + all other locally produced non-milk exposure pathways

Diet 2 - Locally produced commercial milk + all other locally produced non-milk exposure pathways

Diet 3 - Regionally mixed commercial milk + inhalation (other regionally mixed food items are minor contributors to the total)

Diet 4 - Goat milk (all other exposure pathways are of negligible importance compared to goat milk) 
Location: Sweetwater

Receptor: Male born in 1935

\begin{tabular}{lccc}
\hline & \multicolumn{3}{c}{ Thyroid Dose [cGy] } \\
& \multicolumn{2}{c}{$95 \%$ Subjective Confidence Interval } \\
Exposure Pathway & lower limit & central estimate & upper limit \\
\hline Backyard Cow Milk & 0.17 & 1 & 5.1 \\
Commercial Milk (locally produced) & 0.045 & 0.29 & 1.8 \\
Commercial Milk (regionally mixed) & 0.078 & 0.47 & 3.1 \\
Goat Milk (locally produced) & 0.37 & 3.1 & 23 \\
Beef (locally produced) & 0.00053 & 0.0085 & 0.16 \\
Leafy Vegetables (locally produced) & 0.00014 & 0.0016 & 0.013 \\
Eggs (locally produced) & 0.012 & 0.079 & 0.54 \\
Cottage Cheese (locally produced) & 0.00033 & 0.0034 & 0.032 \\
Inhalation & 0.01 & 0.039 & 0.14 \\
Mother's milk (mother on Diet 1) & -- & -- & - \\
Prenatal exposure (mother on Diet 1) & -- & -- & -- \\
Diet 1 & 0.21 & 1.2 & 5.8 \\
Diet 2 & 0.084 & 0.44 & 2.3 \\
Diet 3 & 0.097 & 0.52 & 3.2 \\
\hline
\end{tabular}

Excess Lifetime Risk [ ]

\begin{tabular}{llll}
\hline Diet 1 & $2.7 \mathrm{E}-07$ & $8.7 \mathrm{E}-06$ & $1.9 \mathrm{E}-04$ \\
Diet 2 & $1.1 \mathrm{E}-07$ & $3.8 \mathrm{E}-06$ & $8.7 \mathrm{E}-05$ \\
Diet 3 & $1.8 \mathrm{E}-07$ & $4.8 \mathrm{E}-06$ & $1.2 \mathrm{E}-04$ \\
Diet 4 & $8.3 \mathrm{E}-07$ & $2.3 \mathrm{E}-05$ & $7.3 \mathrm{E}-04$ \\
\hline
\end{tabular}

\section{Relative Risk [ ]}

\begin{tabular}{lccc}
\hline Diet 1 & 1.00025 & 1.006 & 1.11 \\
Diet 2 & 1.00012 & 1.0026 & 1.047 \\
Diet 3 & 1.00017 & 1.0031 & 1.064 \\
Diet 4 & 1.00079 & 1.018 & 1.31 \\
\hline
\end{tabular}

\begin{tabular}{llcc} 
& \multicolumn{3}{c}{ Probability of Causation [\%] } \\
\hline Diet 1 & 0.025 & 0.60 & 9.8 \\
Diet 2 & 0.012 & 0.26 & 4.5 \\
Diet 3 & 0.017 & 0.31 & 6.1 \\
Diet 4 & 0.078 & 1.75 & 23.8 \\
\hline
\end{tabular}

Diet 1 - Backyard cow milk + all other locally produced non-milk exposure pathways

Diet 2 - Locally produced commercial milk + all other locally produced non-milk exposure pathways

Diet 3 - Regionally mixed commercial milk + inhalation (other regionally mixed food items are minor contributors to the total)

Diet 4 - Goat milk (all other exposure pathways are of negligible importance compared to goat milk) 


\section{Location: Knoxville}

Receptor: Female born in 1935

\begin{tabular}{lccc}
\hline & \multicolumn{3}{c}{ Thyroid Dose [cGy] } \\
Exposure Pathway & \multicolumn{2}{c}{$95 \%$ Subjective Confidence Interval } \\
\hline Backyard Cow Milk & lower limit & central estimate & upper limit \\
Commercial Milk (locally produced) & 0.24 & 1.5 & 8.4 \\
Commercial Milk (regionally mixed) & 0.069 & 0.43 & 2.6 \\
Goat Milk (locally produced) & 0.071 & 0.43 & 2.6 \\
Beef (locally produced) & 0.69 & 4.5 & 28 \\
Leafy Vegetables (locally produced) & 0.00061 & 0.01 & 0.2 \\
Eggs (locally produced) & 0.00027 & 0.0026 & 0.022 \\
Cottage Cheese (locally produced) & 0.016 & 0.11 & 0.76 \\
Inhalation & 0.00057 & 0.0055 & 0.059 \\
Mother's milk (mother on Diet 1) & 0.017 & 0.056 & 0.21 \\
Prenatal exposure (mother on Diet 1) & -- & -- & -- \\
Diet 1 & -- & -- & -- \\
Diet 2 & 0.3 & 1.7 & 9.7 \\
Diet 3 & 0.13 & 0.66 & 3.5 \\
\hline
\end{tabular}

Excess Lifetime Risk [ ]

\begin{tabular}{llll}
\hline Diet 1 & $4.1 \mathrm{E}-06$ & $5.5 \mathrm{E}-05$ & $6.7 \mathrm{E}-04$ \\
Diet 2 & $2.0 \mathrm{E}-06$ & $2.4 \mathrm{E}-05$ & $2.8 \mathrm{E}-04$ \\
Diet 3 & $1.7 \mathrm{E}-06$ & $1.9 \mathrm{E}-05$ & $2.0 \mathrm{E}-04$ \\
Diet 4 & $1.3 \mathrm{E}-05$ & $1.8 \mathrm{E}-04$ & $2.5 \mathrm{E}-03$ \\
\hline
\end{tabular}

\section{Relative Risk [ ]}

\begin{tabular}{lccc}
\hline Diet 1 & 1.0016 & 1.014 & 1.13 \\
Diet 2 & 1.00074 & 1.0062 & 1.061 \\
Diet 3 & 1.00061 & 1.005 & 1.05 \\
Diet 4 & 1.0049 & 1.043 & 1.49 \\
\hline
\end{tabular}

\section{Probability of Causation [\%]}

\begin{tabular}{lccc}
\hline Diet 1 & 0.163 & 1.34 & 11.6 \\
Diet 2 & 0.074 & 0.62 & 5.7 \\
Diet 3 & 0.061 & 0.50 & 4.8 \\
Diet 4 & 0.486 & 4.13 & 32.8 \\
\hline
\end{tabular}

Diet 1 - Backyard cow milk + all other locally produced non-milk exposure pathways

Diet 2 - Locally produced commercial milk + all other locally produced non-milk exposure pathways

Diet 3 - Regionally mixed commercial milk + inhalation (other regionally mixed food items are minor contributors to the total)

Diet 4 - Goat milk (all other exposure pathways are of negligible importance compared to goat milk) 


\section{Location: Knoxville}

\section{Receptor: Male born in 1935}

\begin{tabular}{lccc}
\hline & \multicolumn{3}{c}{ Thyroid Dose [cGy] } \\
& \multicolumn{2}{c}{$95 \%$ Subjective Confidence Interval } \\
Exposure Pathway & lower limit & central estimate & upper limit \\
\hline Backyard Cow Milk & 0.31 & 1.7 & 9.1 \\
Commercial Milk (locally produced) & 0.076 & 0.48 & 3.2 \\
Commercial Milk (regionally mixed) & 0.078 & 0.47 & 3.1 \\
Goat Milk (locally produced) & 0.8 & 5.3 & 35 \\
Beef (locally produced) & 0.0009 & 0.014 & 0.3 \\
Leafy Vegetables (locally produced) & 0.00026 & 0.0025 & 0.021 \\
Eggs (locally produced) & 0.019 & 0.13 & 0.9 \\
Cottage Cheese (locally produced) & 0.00058 & 0.0055 & 0.06 \\
Inhalation & 0.017 & 0.061 & 0.22 \\
Mother's milk (mother on Diet 1) & -- & -- & -- \\
Prenatal exposure (mother on Diet 1) & -- & -- & -- \\
Diet 1 & 0.37 & 1.9 & 10 \\
Diet 2 & 0.15 & 0.74 & 4.1 \\
Diet 3 & 0.11 & 0.54 & 3.3 \\
\hline
\end{tabular}

Excess Lifetime Risk [ ]

\begin{tabular}{lllr}
\hline Diet 1 & $4.6 \mathrm{E}-07$ & $1.5 \mathrm{E}-05$ & $3.3 \mathrm{E}-04$ \\
Diet 2 & $2.1 \mathrm{E}-07$ & $6.5 \mathrm{E}-06$ & $1.5 \mathrm{E}-04$ \\
Diet 3 & $1.8 \mathrm{E}-07$ & $5.0 \mathrm{E}-06$ & $1.2 \mathrm{E}-04$ \\
Diet 4 & $1.4 \mathrm{E}-06$ & $4.0 \mathrm{E}-05$ & $1.1 \mathrm{E}-03$ \\
\hline
\end{tabular}

\section{Relative Risk [ ]}

\begin{tabular}{lccc}
\hline Diet 1 & 1.00042 & 1.0096 & 1.2 \\
Diet 2 & 1.00023 & 1.0044 & 1.081 \\
Diet 3 & 1.00018 & 1.0032 & 1.067 \\
Diet 4 & 1.0013 & 1.031 & 1.59 \\
\hline
\end{tabular}

\section{Probability of Causation [\%]}

\begin{tabular}{lccc}
\hline Diet 1 & 0.042 & 0.95 & 16.4 \\
Diet 2 & 0.023 & 0.44 & 7.5 \\
Diet 3 & 0.018 & 0.32 & 6.2 \\
Diet 4 & 0.132 & 2.97 & 37.2 \\
\hline
\end{tabular}

Diet 1 - Backyard cow milk + all other locally produced non-milk exposure pathways

Diet 2 - Locally produced commercial milk + all other locally produced non-milk exposure pathways

Diet 3 - Regionally mixed commercial milk + inhalation (other regionally mixed food items are minor contributors to the total)

Diet 4 - Goat milk (all other exposure pathways are of negligible importance compared to goat milk) 
Location: Maryville

Receptor: Female born in 1935

\begin{tabular}{lccc}
\hline & \multicolumn{3}{c}{ Thyroid Dose [cGy] } \\
Exposure Pathway & \multicolumn{2}{c}{$95 \%$ Subjective Confidence Interval } \\
\hline Backyard Cow Milk & lower limit & central estimate & upper limit \\
Commercial Milk (locally produced) & 0.12 & 0.71 & 4.3 \\
Commercial Milk (regionally mixed) & 0.03 & 0.21 & 1.2 \\
Goat Milk (locally produced) & 0.071 & 0.43 & 2.6 \\
Beef (locally produced) & 0.3 & 2.2 & 14 \\
Leafy Vegetables (locally produced) & 0.00033 & 0.005 & 0.1 \\
Eggs (locally produced) & 0.00011 & 0.0012 & 0.01 \\
Cottage Cheese (locally produced) & 0.0079 & 0.054 & 0.38 \\
Inhalation & 0.00027 & 0.0027 & 0.029 \\
Mother's milk (mother on Diet 1) & 0.0087 & 0.03 & 0.12 \\
Prenatal exposure (mother on Diet 1) & -- & -- & -- \\
Diet 1 & -- & -- & -- \\
Diet 2 & 0.15 & 0.82 & 4.7 \\
Diet 3 & 0.061 & 0.33 & 1.7 \\
\hline
\end{tabular}

Excess Lifetime Risk [ ]

\begin{tabular}{llll}
\hline Diet 1 & $2.2 \mathrm{E}-06$ & $2.8 \mathrm{E}-05$ & $3.2 \mathrm{E}-04$ \\
Diet 2 & $1.1 \mathrm{E}-06$ & $1.2 \mathrm{E}-05$ & $1.4 \mathrm{E}-04$ \\
Diet 3 & $1.5 \mathrm{E}-06$ & $1.8 \mathrm{E}-05$ & $1.9 \mathrm{E}-04$ \\
Diet 4 & $6.4 \mathrm{E}-06$ & $8.8 \mathrm{E}-05$ & $1.2 \mathrm{E}-03$ \\
\hline
\end{tabular}

\section{Relative Risk [ ]}

\begin{tabular}{lccc}
\hline Diet 1 & 1.00074 & 1.0067 & 1.067 \\
Diet 2 & 1.00034 & 1.0029 & 1.031 \\
Diet 3 & 1.00055 & 1.0048 & 1.049 \\
Diet 4 & 1.0025 & 1.022 & 1.26 \\
\hline
\end{tabular}

\section{Probability of Causation [\%]}

\begin{tabular}{llcc}
\hline Diet 1 & 0.074 & 0.67 & 6.2 \\
Diet 2 & 0.034 & 0.29 & 3.0 \\
Diet 3 & 0.055 & 0.47 & 4.7 \\
Diet 4 & 0.247 & 2.11 & 20.9 \\
\hline
\end{tabular}

Diet 1 - Backyard cow milk + all other locally produced non-milk exposure pathways

Diet 2 - Locally produced commercial milk + all other locally produced non-milk exposure pathways

Diet 3 - Regionally mixed commercial milk + inhalation (other regionally mixed food items are minor contributors to the total)

Diet 4 - Goat milk (all other exposure pathways are of negligible importance compared to goat milk) 


\section{Location: Maryville}

\section{Receptor: Male born in 1935}

\begin{tabular}{lccc}
\hline & \multicolumn{3}{c}{ Thyroid Dose [cGy] } \\
& \multicolumn{2}{c}{$95 \%$ Subjective Confidence Interval } \\
Exposure Pathway & lower limit & central estimate & upper limit \\
\hline Backyard Cow Milk & 0.15 & 0.81 & 4.7 \\
Commercial Milk (locally produced) & 0.035 & 0.23 & 1.4 \\
Commercial Milk (regionally mixed) & 0.078 & 0.47 & 3 \\
Goat Milk (locally produced) & 0.36 & 2.6 & 17 \\
Beef (locally produced) & 0.00048 & 0.0071 & 0.15 \\
Leafy Vegetables (locally produced) & 0.00011 & 0.0012 & 0.011 \\
Eggs (locally produced) & 0.0088 & 0.067 & 0.44 \\
Cottage Cheese (locally produced) & 0.00024 & 0.0028 & 0.028 \\
Inhalation & 0.0088 & 0.033 & 0.13 \\
Mother's milk (mother on Diet 1) & -- & -- & -- \\
Prenatal exposure (mother on Diet 1) & -- & -- & -- \\
Diet 1 & 0.19 & 0.93 & 5.1 \\
Diet 2 & 0.07 & 0.36 & 2 \\
Diet 3 & 0.095 & 0.51 & 3.2 \\
\hline
\end{tabular}

\begin{tabular}{llll}
\hline Diet 1 & $2.3 \mathrm{E}-07$ & $6.8 \mathrm{E}-06$ & $1.7 \mathrm{E}-04$ \\
Diet 2 & $1.0 \mathrm{E}-07$ & $3.2 \mathrm{E}-06$ & $6.9 \mathrm{E}-05$ \\
Diet 3 & $1.8 \mathrm{E}-07$ & $4.8 \mathrm{E}-06$ & $1.2 \mathrm{E}-04$ \\
Diet 4 & $7.7 \mathrm{E}-07$ & $2.0 \mathrm{E}-05$ & $5.8 \mathrm{E}-04$ \\
\hline
\end{tabular}

\section{Relative Risk [ ]}

\begin{tabular}{lccc}
\hline Diet 1 & 1.00022 & 1.0048 & 1.1 \\
Diet 2 & 1.00011 & 1.0022 & 1.04 \\
Diet 3 & 1.00017 & 1.0031 & 1.065 \\
Diet 4 & 1.00066 & 1.014 & 1.3 \\
\hline
\end{tabular}

\section{Probability of Causation [\%]}

\begin{tabular}{llcc}
\hline Diet 1 & 0.022 & 0.48 & 9.2 \\
Diet 2 & 0.011 & 0.22 & 3.8 \\
Diet 3 & 0.017 & 0.31 & 6.1 \\
Diet 4 & 0.066 & 1.39 & 23.3 \\
\hline
\end{tabular}

Diet 1 - Backyard cow milk + all other locally produced non-milk exposure pathways

Diet 2 - Locally produced commercial milk + all other locally produced non-milk exposure pathways

Diet 3 - Regionally mixed commercial milk + inhalation (other regionally mixed food items are minor contributors to the total)

Diet 4 - Goat milk (all other exposure pathways are of negligible importance compared to goat milk) 
Location: Cedar Grove

Receptor: Female born in 1935

\begin{tabular}{lccc}
\hline & \multicolumn{3}{c}{ Thyroid Dose [cGy] } \\
Exposure Pathway & $95 \%$ & Subjective Confidence Interval \\
Backyard Cow Milk & lower limit & central estimate & upper limit \\
Commercial Milk (locally produced) & 0.23 & 1.3 & 7.6 \\
Commercial Milk (regionally mixed) & 0.063 & 0.38 & 2.3 \\
Goat Milk (locally produced) & 0.071 & 0.43 & 2.6 \\
Beef (locally produced) & 0.67 & 4.1 & 25 \\
Leafy Vegetables (locally produced) & 0.00055 & 0.0094 & 0.19 \\
Eggs (locally produced) & 0.00023 & 0.0024 & 0.02 \\
Cottage Cheese (locally produced) & 0.016 & 0.1 & 0.64 \\
Inhalation & 0.00049 & 0.005 & 0.051 \\
Mother's milk (mother on Diet 1) & 0.015 & 0.049 & 0.17 \\
Prenatal exposure (mother on Diet 1) & -- & -- & -- \\
Diet 1 & -- & -- & -- \\
Diet 2 & 0.28 & 1.5 & 8.7 \\
Diet 3 & 0.13 & 0.59 & 3.1 \\
\hline
\end{tabular}

Excess Lifetime Risk [ ]

\begin{tabular}{llll}
\hline Diet 1 & $3.9 \mathrm{E}-06$ & $5.0 \mathrm{E}-05$ & $6.1 \mathrm{E}-04$ \\
Diet 2 & $2.1 \mathrm{E}-06$ & $2.2 \mathrm{E}-05$ & $2.6 \mathrm{E}-04$ \\
Diet 3 & $1.7 \mathrm{E}-06$ & $1.9 \mathrm{E}-05$ & $2.0 \mathrm{E}-04$ \\
Diet 4 & $1.2 \mathrm{E}-05$ & $1.7 \mathrm{E}-04$ & $2.4 \mathrm{E}-03$ \\
\hline
\end{tabular}

\section{Relative Risk [ ]}

\begin{tabular}{lccc}
\hline Diet 1 & 1.0014 & 1.012 & 1.12 \\
Diet 2 & 1.00067 & 1.0057 & 1.058 \\
Diet 3 & 1.00059 & 1.0049 & 1.05 \\
Diet 4 & 1.0047 & 1.039 & 1.45 \\
\hline
\end{tabular}

Probability of Causation [\%]

\begin{tabular}{llcc}
\hline Diet 1 & 0.144 & 1.18 & 10.6 \\
Diet 2 & 0.067 & 0.57 & 5.5 \\
Diet 3 & 0.058 & 0.49 & 4.7 \\
Diet 4 & 0.470 & 3.79 & 31.2 \\
\hline
\end{tabular}

Diet 1 - Backyard cow milk + all other locally produced non-milk exposure pathways

Diet 2 - Locally produced commercial milk + all other locally produced non-milk exposure pathways

Diet 3 - Regionally mixed commercial milk + inhalation (other regionally mixed food items are minor contributors to the total)

Diet 4 - Goat milk (all other exposure pathways are of negligible importance compared to goat milk) 


\section{Location: Cedar Grove}

Receptor: Male born in 1935

\begin{tabular}{lccc}
\hline & \multicolumn{3}{c}{ Thyroid Dose [cGy] } \\
Exposure Pathway & \multicolumn{2}{c}{$95 \%$ Subjective Confidence Interval } \\
\hline Backyard Cow Milk & lower limit & central estimate & upper limit \\
Commercial Milk (locally produced) & 0.31 & 1.5 & 8.6 \\
Commercial Milk (regionally mixed) & 0.069 & 0.42 & 2.9 \\
Goat Milk (locally produced) & 0.078 & 0.47 & 3 \\
Beef (locally produced) & 0.74 & 4.9 & 32 \\
Leafy Vegetables (locally produced) & 0.00082 & 0.013 & 0.27 \\
Eggs (locally produced) & 0.00023 & 0.0023 & 0.02 \\
Cottage Cheese (locally produced) & 0.018 & 0.12 & 0.79 \\
Inhalation & 0.0005 & 0.0051 & 0.052 \\
Mother's milk (mother on Diet 1) & 0.016 & 0.053 & 0.19 \\
Prenatal exposure (mother on Diet 1) & -- & -- & - \\
Diet 1 & -- & -- & -- \\
Diet 2 & 0.35 & 1.7 & 9.7 \\
Diet 3 & 0.14 & 0.66 & 3.7 \\
\hline
\end{tabular}

Excess Lifetime Risk [ ]

\begin{tabular}{llll}
\hline Diet 1 & $4.2 \mathrm{E}-07$ & $1.3 \mathrm{E}-05$ & $3.1 \mathrm{E}-04$ \\
Diet 2 & $1.9 \mathrm{E}-07$ & $5.7 \mathrm{E}-06$ & $1.3 \mathrm{E}-04$ \\
Diet 3 & $1.8 \mathrm{E}-07$ & $5.0 \mathrm{E}-06$ & $1.2 \mathrm{E}-04$ \\
Diet 4 & $1.3 \mathrm{E}-06$ & $3.5 \mathrm{E}-05$ & $9.3 \mathrm{E}-04$ \\
\hline
\end{tabular}

\section{Relative Risk [ ]}

\begin{tabular}{lccc}
\hline Diet 1 & 1.00039 & 1.0091 & 1.18 \\
Diet 2 & 1.0002 & 1.004 & 1.071 \\
Diet 3 & 1.00018 & 1.0032 & 1.066 \\
Diet 4 & 1.0012 & 1.028 & 1.55 \\
\hline
\end{tabular}

\section{Probability of Causation [\%]}

\begin{tabular}{lccc}
\hline Diet 1 & 0.039 & 0.90 & 15.3 \\
Diet 2 & 0.020 & 0.40 & 6.6 \\
Diet 3 & 0.018 & 0.32 & 6.2 \\
Diet 4 & 0.118 & 2.73 & 35.3 \\
\hline
\end{tabular}

Diet 1 - Backyard cow milk + all other locally produced non-milk exposure pathways

Diet 2 - Locally produced commercial milk + all other locally produced non-milk exposure pathways

Diet 3 - Regionally mixed commercial milk + inhalation (other regionally mixed food items are minor contributors to the total)

Diet 4 - Goat milk (all other exposure pathways are of negligible importance compared to goat milk) 
Location: Bradbury

Receptor: Female born in 1940

\begin{tabular}{lccc}
\hline & \multicolumn{3}{c}{ Thyroid Dose [cGy] } \\
& \multicolumn{2}{c}{$95 \%$ Subjective Confidence Interval } \\
Exposure Pathway & lower limit & central estimate & upper limit \\
\hline Backyard Cow Milk & 3.1 & 17 & 110 \\
Commercial Milk (locally produced) & 0.82 & 5.8 & 40 \\
Commercial Milk (regionally mixed) & 0.12 & 0.76 & 4.9 \\
Goat Milk (locally produced) & 7 & 57 & 360 \\
Beef (locally produced) & 0.0059 & 0.097 & 2 \\
Leafy Vegetables (locally produced) & 0.0021 & 0.025 & 0.21 \\
Eggs (locally produced) & 0.16 & 1.3 & 8.8 \\
Cottage Cheese (locally produced) & 0.0046 & 0.049 & 0.53 \\
Inhalation & 0.11 & 0.38 & 1.3 \\
Mother's milk (mother on Diet 1) & -- & -- & -- \\
Prenatal exposure (mother on Diet 1) & -- & -- & - \\
Diet 1 & 3.5 & 19 & 120 \\
Diet 2 & 1.4 & 7.9 & 49 \\
Diet 3 & 0.3 & 1.2 & 6.1 \\
\hline
\end{tabular}

Excess Lifetime Risk [ ]

\begin{tabular}{lllc}
\hline Diet 1 & $1.2 \mathrm{E}-04$ & $2.0 \mathrm{E}-03$ & $2.3 \mathrm{E}-02$ \\
Diet 2 & $5.7 \mathrm{E}-05$ & $7.5 \mathrm{E}-04$ & $9.6 \mathrm{E}-03$ \\
Diet 3 & $1.2 \mathrm{E}-05$ & $1.1 \mathrm{E}-04$ & $1.1 \mathrm{E}-03$ \\
Diet 4 & $3.8 \mathrm{E}-04$ & $5.3 \mathrm{E}-03$ & $6.1 \mathrm{E}-02$ \\
\hline
\end{tabular}

\section{Relative Risk [ ]}

\begin{tabular}{lccc}
\hline Diet 1 & 1.048 & 1.44 & 6.1 \\
Diet 2 & 1.02 & 1.19 & 3.4 \\
Diet 3 & 1.0034 & 1.028 & 1.28 \\
Diet 4 & 1.13 & 2.2 & 18 \\
\hline
\end{tabular}

\begin{tabular}{lccc}
\hline Diet 1 & 4.54 & 30.4 & 83 \\
Diet 2 & 2.01 & 15.9 & 71 \\
Diet 3 & 0.34 & 2.8 & 22 \\
Diet 4 & 11.62 & 54.9 & 94 \\
\hline
\end{tabular}

Diet 1 - Backyard cow milk + all other locally produced non-milk exposure pathways

Diet 2 - Locally produced commercial milk + all other locally produced non-milk exposure pathways

Diet 3 - Regionally mixed commercial milk + inhalation (other regionally mixed food items are minor contributors to the total)

Diet 4 - Goat milk (all other exposure pathways are of negligible importance compared to goat milk) 


\section{Location: Bradbury}

Receptor: Male born in 1940

\begin{tabular}{lccc}
\hline & \multicolumn{3}{c}{ Thyroid Dose [cGy] } \\
& \multicolumn{2}{c}{$95 \%$ Subjective Confidence Interval } \\
Exposure Pathway & lower limit & central estimate & upper limit \\
\hline Backyard Cow Milk & 3.1 & 18 & 110 \\
Commercial Milk (locally produced) & 0.88 & 5.9 & 39 \\
Commercial Milk (regionally mixed) & 0.12 & 0.79 & 4.9 \\
Goat Milk (locally produced) & 7.7 & 58 & 380 \\
Beef (locally produced) & 0.0069 & 0.12 & 2.4 \\
Leafy Vegetables (locally produced) & 0.0021 & 0.024 & 0.21 \\
Eggs (locally produced) & 0.2 & 1.5 & 10 \\
Cottage Cheese (locally produced) & 0.0046 & 0.049 & 0.53 \\
Inhalation & 0.11 & 0.39 & 1.4 \\
Mother's milk (mother on Diet 1) & -- & -- & - \\
Prenatal exposure (mother on Diet 1) & -- & -- & - \\
Diet 1 & 3.5 & 20 & 130 \\
Diet 2 & 1.4 & 8.6 & 51 \\
Diet 3 & 0.29 & 1.3 & 6.2 \\
\hline
\end{tabular}

Excess Lifetime Risk [ ]

\begin{tabular}{llll}
\hline Diet 1 & $1.5 \mathrm{E}-05$ & $4.8 \mathrm{E}-04$ & $1.1 \mathrm{E}-02$ \\
Diet 2 & $6.3 \mathrm{E}-06$ & $2.1 \mathrm{E}-04$ & $4.7 \mathrm{E}-03$ \\
Diet 3 & $1.1 \mathrm{E}-06$ & $2.9 \mathrm{E}-05$ & $6.0 \mathrm{E}-04$ \\
Diet 4 & $3.7 \mathrm{E}-05$ & $1.2 \mathrm{E}-03$ & $3.1 \mathrm{E}-02$ \\
\hline
\end{tabular}

\section{Relative Risk [ ]}

\begin{tabular}{lccc}
\hline Diet 1 & 1.015 & 1.31 & 7.8 \\
Diet 2 & 1.0066 & 1.13 & 3.9 \\
Diet 3 & 1.0011 & 1.017 & 1.37 \\
Diet 4 & 1.041 & 1.83 & 20 \\
\hline
\end{tabular}

\section{Probability of Causation [\%]}

\begin{tabular}{lccc}
\hline Diet 1 & 1.46 & 23.9 & 87 \\
Diet 2 & 0.66 & 11.6 & 74 \\
Diet 3 & 0.11 & 1.7 & 27 \\
Diet 4 & 3.92 & 45.3 & 95 \\
\hline
\end{tabular}

Diet 1 - Backyard cow milk + all other locally produced non-milk exposure pathways

Diet 2 - Locally produced commercial milk + all other locally produced non-milk exposure pathways

Diet 3 - Regionally mixed commercial milk + inhalation (other regionally mixed food items are minor contributors to the total)

Diet 4 - Goat milk (all other exposure pathways are of negligible importance compared to goat milk) 
Location: Gallaher Bend

Receptor: Female born in 1940

\begin{tabular}{lccc}
\hline & \multicolumn{3}{c}{ Thyroid Dose [cGy] } \\
Exposure Pathway & \multicolumn{2}{c}{$95 \%$ Subjective Confidence Interval } \\
\hline Backyard Cow Milk & lower limit & central estimate & upper limit \\
Commercial Milk (locally produced) & 3.3 & 21 & 120 \\
Commercial Milk (regionally mixed) & 1.2 & 7.1 & 46 \\
Goat Milk (locally produced) & 0.12 & 0.76 & 4.9 \\
Beef (locally produced) & 8.1 & 68 & 440 \\
Leafy Vegetables (locally produced) & 0.0069 & 0.12 & 2.6 \\
Eggs (locally produced) & 0.0026 & 0.031 & 0.27 \\
Cottage Cheese (locally produced) & 0.22 & 1.6 & 11 \\
Inhalation & 0.0061 & 0.062 & 0.6 \\
Mother's milk (mother on Diet 1) & 0.13 & 0.46 & 1.7 \\
Prenatal exposure (mother on Diet 1) & -- & -- & -- \\
Diet 1 & -- & -- & -- \\
Diet 2 & 4 & 24 & 140 \\
Diet 3 & 1.7 & 9.9 & 55 \\
\hline
\end{tabular}

Excess Lifetime Risk [ ]

\begin{tabular}{lllc}
\hline Diet 1 & $1.5 \mathrm{E}-04$ & $2.4 \mathrm{E}-03$ & $2.8 \mathrm{E}-02$ \\
Diet 2 & $6.6 \mathrm{E}-05$ & $8.9 \mathrm{E}-04$ & $1.2 \mathrm{E}-02$ \\
Diet 3 & $1.3 \mathrm{E}-05$ & $1.2 \mathrm{E}-04$ & $1.1 \mathrm{E}-03$ \\
Diet 4 & $4.2 \mathrm{E}-04$ & $6.4 \mathrm{E}-03$ & $8.1 \mathrm{E}-02$ \\
\hline
\end{tabular}

\section{Relative Risk [ ]}

\begin{tabular}{lccc}
\hline Diet 1 & 1.056 & 1.55 & 7.4 \\
Diet 2 & 1.024 & 1.24 & 3.8 \\
Diet 3 & 1.0038 & 1.03 & 1.3 \\
Diet 4 & 1.16 & 2.6 & 21 \\
\hline
\end{tabular}

\section{Probability of Causation [\%]}

\begin{tabular}{lccc}
\hline Diet 1 & 5.29 & 35.6 & 86 \\
Diet 2 & 2.35 & 19.3 & 74 \\
Diet 3 & 0.38 & 3.0 & 23 \\
Diet 4 & 13.62 & 61.0 & 95 \\
\hline
\end{tabular}

Diet 1 - Backyard cow milk + all other locally produced non-milk exposure pathways

Diet 2 - Locally produced commercial milk + all other locally produced non-milk exposure pathways

Diet 3 - Regionally mixed commercial milk + inhalation (other regionally mixed food items are minor contributors to the total)

Diet 4 - Goat milk (all other exposure pathways are of negligible importance compared to goat milk) 
Location: Gallaher Bend

Receptor: Male born in 1940

\begin{tabular}{lccc}
\hline & \multicolumn{3}{c}{ Thyroid Dose [cGy] } \\
& \multicolumn{2}{c}{$95 \%$ Subjective Confidence Interval } \\
Exposure Pathway & lower limit & central estimate & upper limit \\
\hline Backyard Cow Milk & 3.9 & 22 & 130 \\
Commercial Milk (locally produced) & 1.2 & 7.3 & 43 \\
Commercial Milk (regionally mixed) & 0.12 & 0.79 & 4.9 \\
Goat Milk (locally produced) & 8.5 & 72 & 470 \\
Beef (locally produced) & 0.0081 & 0.14 & 3.1 \\
Leafy Vegetables (locally produced) & 0.0028 & 0.03 & 0.25 \\
Eggs (locally produced) & 0.28 & 1.8 & 12 \\
Cottage Cheese (locally produced) & 0.0066 & 0.061 & 0.62 \\
Inhalation & 0.13 & 0.49 & 1.6 \\
Mother's milk (mother on Diet 1) & -- & -- & - \\
Prenatal exposure (mother on Diet 1) & -- & -- & - \\
Diet 1 & 4.6 & 24 & 150 \\
Diet 2 & 1.8 & 11 & 56 \\
Diet 3 & 0.31 & 1.4 & 6.5 \\
\hline
\end{tabular}

Excess Lifetime Risk [ ]

\begin{tabular}{llll}
\hline Diet 1 & $1.9 \mathrm{E}-05$ & $5.9 \mathrm{E}-04$ & $1.2 \mathrm{E}-02$ \\
Diet 2 & $7.7 \mathrm{E}-06$ & $2.5 \mathrm{E}-04$ & $5.6 \mathrm{E}-03$ \\
Diet 3 & $1.2 \mathrm{E}-06$ & $3.3 \mathrm{E}-05$ & $6.4 \mathrm{E}-04$ \\
Diet 4 & $4.8 \mathrm{E}-05$ & $1.4 \mathrm{E}-03$ & $4.3 \mathrm{E}-02$ \\
\hline
\end{tabular}

\section{Relative Risk [ ]}

\begin{tabular}{lccc}
\hline Diet 1 & 1.018 & 1.38 & 9.9 \\
Diet 2 & 1.0074 & 1.16 & 4.3 \\
Diet 3 & 1.0011 & 1.019 & 1.39 \\
Diet 4 & 1.041 & 2 & 23 \\
\hline
\end{tabular}

\section{Probability of Causation [\%]}

\begin{tabular}{lccc}
\hline Diet 1 & 1.78 & 27.4 & 90 \\
Diet 2 & 0.73 & 13.8 & 77 \\
Diet 3 & 0.11 & 1.9 & 28 \\
Diet 4 & 3.89 & 50.7 & 96 \\
\hline
\end{tabular}

Diet 1 - Backyard cow milk + all other locally produced non-milk exposure pathways

Diet 2 - Locally produced commercial milk + all other locally produced non-milk exposure pathways

Diet 3 - Regionally mixed commercial milk + inhalation (other regionally mixed food items are minor contributors to the total)

Diet 4 - Goat milk (all other exposure pathways are of negligible importance compared to goat milk) 


\section{Location: EFPC}

Receptor: Female born in 1940

\begin{tabular}{lccc}
\hline & \multicolumn{3}{c}{ Thyroid Dose [cGy] } \\
& \multicolumn{2}{c}{$95 \%$ Subjective Confidence Interval } \\
Exposure Pathway & lower limit & central estimate & upper limit \\
\hline Backyard Cow Milk & 0.74 & 4.5 & 27 \\
Commercial Milk (locally produced) & 0.21 & 1.5 & 10 \\
Commercial Milk (regionally mixed) & 0.12 & 0.76 & 4.9 \\
Goat Milk (locally produced) & -- & -- & -- \\
Beef (locally produced) & 0.0015 & 0.025 & 0.54 \\
Leafy Vegetables (locally produced) & 0.00055 & 0.0064 & 0.055 \\
Eggs (locally produced) & 0.042 & 0.32 & 2.4 \\
Cottage Cheese (locally produced) & 0.0012 & 0.013 & 0.14 \\
Inhalation & 0.028 & 0.1 & 0.36 \\
Mother's milk (mother on Diet 1) & -- & -- & -- \\
Prenatal exposure (mother on Diet 1) & -- & -- & -- \\
Diet 1 & 0.86 & 5 & 30 \\
Diet 2 & 0.35 & 2.1 & 13 \\
Diet 3 & 0.18 & 0.88 & 5.1 \\
\hline
\end{tabular}

Excess Lifetime Risk [ ]

\begin{tabular}{lccc}
\hline Diet 1 & $3.3 \mathrm{E}-05$ & $5.0 \mathrm{E}-04$ & $6.1 \mathrm{E}-03$ \\
Diet 2 & $1.6 \mathrm{E}-05$ & $1.9 \mathrm{E}-04$ & $2.4 \mathrm{E}-03$ \\
Diet 3 & $7.7 \mathrm{E}-06$ & $8.4 \mathrm{E}-05$ & $1.0 \mathrm{E}-03$ \\
Diet 4 & -- & -- & -- \\
\hline
\end{tabular}

\section{Relative Risk [ ]}

\begin{tabular}{lccc}
\hline Diet 1 & 1.012 & 1.12 & 2.3 \\
Diet 2 & 1.0052 & 1.05 & 1.62 \\
Diet 3 & 1.0025 & 1.022 & 1.25 \\
Diet 4 & -- & -- & -- \\
\hline
\end{tabular}

\section{Probability of Causation [\%]}

\begin{tabular}{lccc}
\hline Diet 1 & 1.18 & 10.2 & 55 \\
Diet 2 & 0.52 & 4.8 & 37 \\
Diet 3 & 0.25 & 2.1 & 20 \\
Diet 4 & -- & -- & -- \\
\hline
\end{tabular}

Diet 1 - Backyard cow milk + all other locally produced non-milk exposure pathways

Diet 2 - Locally produced commercial milk + all other locally produced non-milk exposure pathways

Diet 3 - Regionally mixed commercial milk + inhalation (other regionally mixed food items are minor contributors to the total)

Diet 4 - Goat milk (all other exposure pathways are of negligible importance compared to goat milk) 


\section{Location: EFPC}

\section{Receptor: Male born in 1940}

\begin{tabular}{lccc}
\hline & \multicolumn{3}{c}{ Thyroid Dose [cGy] } \\
& \multicolumn{2}{c}{$95 \%$ Subjective Confidence Interval } \\
Exposure Pathway & lower limit & central estimate & upper limit \\
\hline Backyard Cow Milk & 0.8 & 4.6 & 30 \\
Commercial Milk (locally produced) & 0.23 & 1.5 & 10 \\
Commercial Milk (regionally mixed) & 0.12 & 0.79 & 4.9 \\
Goat Milk (locally produced) & -- & -- & -- \\
Beef (locally produced) & 0.0018 & 0.03 & 0.67 \\
Leafy Vegetables (locally produced) & 0.00055 & 0.0062 & 0.054 \\
Eggs (locally produced) & 0.052 & 0.38 & 2.7 \\
Cottage Cheese (locally produced) & 0.0012 & 0.013 & 0.15 \\
Inhalation & 0.026 & 0.11 & 0.38 \\
Mother's milk (mother on Diet 1) & -- & -- & - \\
Prenatal exposure (mother on Diet 1) & -- & -- & - \\
Diet 1 & 0.91 & 5.3 & 34 \\
Diet 2 & 0.36 & 2.2 & 13 \\
Diet 3 & 0.18 & 0.93 & 5.1 \\
\hline
\end{tabular}

Excess Lifetime Risk [ ]

\begin{tabular}{lccc}
\hline Diet 1 & $3.6 \mathrm{E}-06$ & $1.3 \mathrm{E}-04$ & $2.7 \mathrm{E}-03$ \\
Diet 2 & $1.7 \mathrm{E}-06$ & $5.5 \mathrm{E}-05$ & $1.2 \mathrm{E}-03$ \\
Diet 3 & $7.8 \mathrm{E}-07$ & $2.1 \mathrm{E}-05$ & $5.0 \mathrm{E}-04$ \\
Diet 4 & -- & -- & -- \\
\hline
\end{tabular}

\section{Relative Risk [ ]}

\begin{tabular}{lccc}
\hline Diet 1 & 1.0036 & 1.08 & 2.9 \\
Diet 2 & 1.0017 & 1.033 & 1.85 \\
Diet 3 & 1.00072 & 1.013 & 1.32 \\
Diet 4 & -- & -- & -- \\
\hline
\end{tabular}

\begin{tabular}{lccc}
\hline Diet 1 & 0.36 & 7.4 & 64 \\
Diet 2 & 0.17 & 3.2 & 45 \\
Diet 3 & 0.07 & 1.3 & 25 \\
Diet 4 & -- & -- & \\
\hline Diet 1 - Backyard cow milk + all other locally produced non-milk exposure pathways & \\
Diet 2 - Locally produced commercial milk + all other locally produced non-milk exposure pathways \\
Diet 3 - Regionally mixed commercial milk + inhalation (other regionally mixed food items are minor contributors to the total) \\
Diet 4 - Goat milk (all other exposure pathways are of negligible importance compared to goat milk)
\end{tabular}




\section{Location: Hope Creek}

Receptor: Female born in 1940

\begin{tabular}{lccc}
\hline & \multicolumn{3}{c}{ Thyroid Dose [cGy] } \\
& \multicolumn{2}{c}{$95 \%$ Subjective Confidence Interval } \\
Exposure Pathway & lower limit & central estimate & upper limit \\
\hline Backyard Cow Milk & 2 & 12 & 71 \\
Commercial Milk (locally produced) & 0.62 & 3.9 & 28 \\
Commercial Milk (regionally mixed) & 0.12 & 0.76 & 4.9 \\
Goat Milk (locally produced) & -- & -- & -- \\
Beef (locally produced) & 0.0038 & 0.069 & 1.5 \\
Leafy Vegetables (locally produced) & 0.0017 & 0.017 & 0.16 \\
Eggs (locally produced) & 0.12 & 0.89 & 6.1 \\
Cottage Cheese (locally produced) & 0.0035 & 0.034 & 0.4 \\
Inhalation & 0.076 & 0.28 & 0.99 \\
Mother's milk (mother on Diet 1) & -- & -- & -- \\
Prenatal exposure (mother on Diet 1) & -- & -- & -- \\
Diet 1 & 2.3 & 14 & 81 \\
Diet 2 & 0.95 & 5.4 & 35 \\
Diet 3 & 0.25 & 1.1 & 5.7 \\
\hline
\end{tabular}

Excess Lifetime Risk [ ]

\begin{tabular}{lccc}
\hline Diet 1 & $8.4 \mathrm{E}-05$ & $1.3 \mathrm{E}-03$ & $1.7 \mathrm{E}-02$ \\
Diet 2 & $4.3 \mathrm{E}-05$ & $5.2 \mathrm{E}-04$ & $6.5 \mathrm{E}-03$ \\
Diet 3 & $1.0 \mathrm{E}-05$ & $1.0 \mathrm{E}-04$ & $1.1 \mathrm{E}-03$ \\
Diet 4 & -- & -- & -- \\
\hline
\end{tabular}

\section{Relative Risk [ ]}

\begin{tabular}{lccc}
\hline Diet 1 & 1.035 & 1.31 & 4.4 \\
Diet 2 & 1.014 & 1.13 & 2.6 \\
Diet 3 & 1.0031 & 1.026 & 1.27 \\
Diet 4 & -- & -- & -- \\
\hline
\end{tabular}

\begin{tabular}{lccc}
\hline Diet 1 & 3.40 & 23.7 & 77 \\
Diet 2 & 1.38 & 11.8 & 62 \\
Diet 3 & 0.31 & 2.5 & 21 \\
Diet 4 & -- & -- & - \\
\hline Diet 1 - Backyard cow milk + all other locally produced non-milk exposure pathways & \\
Diet 2 - Locally produced commercial milk + all other locally produced non-milk exposure pathways \\
Diet 3 - Regionally mixed commercial milk + inhalation (other regionally mixed food items are minor contributors to the total) \\
Diet 4 - Goat milk (all other exposure pathways are of negligible importance compared to goat milk)
\end{tabular}




\section{Location: Hope Creek}

Receptor: Male born in 1940

\begin{tabular}{lccc}
\hline & \multicolumn{3}{c}{ Thyroid Dose [cGy] } \\
& \multicolumn{2}{c}{$95 \%$ Subjective Confidence Interval } \\
Exposure Pathway & lower limit & central estimate & upper limit \\
\hline Backyard Cow Milk & 2.2 & 12 & 81 \\
Commercial Milk (locally produced) & 0.6 & 4.1 & 28 \\
Commercial Milk (regionally mixed) & 0.12 & 0.79 & 4.9 \\
Goat Milk (locally produced) & -- & -- & -- \\
Beef (locally produced) & 0.0046 & 0.081 & 1.9 \\
Leafy Vegetables (locally produced) & 0.0016 & 0.017 & 0.15 \\
Eggs (locally produced) & 0.15 & 1 & 6.8 \\
Cottage Cheese (locally produced) & 0.0033 & 0.034 & 0.4 \\
Inhalation & 0.076 & 0.28 & 0.97 \\
Mother's milk (mother on Diet 1) & -- & -- & - \\
Prenatal exposure (mother on Diet 1) & -- & -- & - \\
Diet 1 & 2.6 & 14 & 91 \\
Diet 2 & 1 & 5.9 & 35 \\
Diet 3 & 0.25 & 1.1 & 5.6 \\
\hline
\end{tabular}

Excess Lifetime Risk [ ]

\begin{tabular}{lccc}
\hline Diet 1 & $9.4 \mathrm{E}-06$ & $3.3 \mathrm{E}-04$ & $7.4 \mathrm{E}-03$ \\
Diet 2 & $4.2 \mathrm{E}-06$ & $1.4 \mathrm{E}-04$ & $3.4 \mathrm{E}-03$ \\
Diet 3 & $1.0 \mathrm{E}-06$ & $2.7 \mathrm{E}-05$ & $5.7 \mathrm{E}-04$ \\
Diet 4 & -- & -- & -- \\
\hline
\end{tabular}

\section{Relative Risk [ ]}

\begin{tabular}{lccc}
\hline Diet 1 & 1.011 & 1.22 & 5.6 \\
Diet 2 & 1.0042 & 1.092 & 3.1 \\
Diet 3 & 1.00092 & 1.016 & 1.35 \\
Diet 4 & -- & -- & -- \\
\hline
\end{tabular}

\section{Probability of Causation [\%]}

\begin{tabular}{lccc}
\hline Diet 1 & 1.09 & 18.1 & 82 \\
Diet 2 & 0.42 & 8.4 & 67 \\
Diet 3 & 0.09 & 1.6 & 26 \\
Diet 4 & -- & -- & -- \\
\hline
\end{tabular}

Diet 1 - Backyard cow milk + all other locally produced non-milk exposure pathways

Diet 2 - Locally produced commercial milk + all other locally produced non-milk exposure pathways

Diet 3 - Regionally mixed commercial milk + inhalation (other regionally mixed food items are minor contributors to the total)

Diet 4 - Goat milk (all other exposure pathways are of negligible importance compared to goat milk) 
Location: Buttermilk Rd.

Receptor: Female born in 1940

\begin{tabular}{lccc}
\hline & \multicolumn{3}{c}{ Thyroid Dose [cGy] } \\
Exposure Pathway & $95 \%$ & Subjective Confidence Interval \\
Backyard Cow Milk & lower limit & central estimate & upper limit \\
Commercial Milk (locally produced) & 1.9 & 12 & 69 \\
Commercial Milk (regionally mixed) & 0.62 & 3.9 & 28 \\
Goat Milk (locally produced) & 0.12 & 0.76 & 4.9 \\
Beef (locally produced) & 4.6 & 38 & 250 \\
Leafy Vegetables (locally produced) & 0.0038 & 0.068 & 1.4 \\
Eggs (locally produced) & 0.0016 & 0.017 & 0.15 \\
Cottage Cheese (locally produced) & 0.12 & 0.89 & 6 \\
Inhalation & 0.0034 & 0.034 & 0.39 \\
Mother's milk (mother on Diet 1) & 0.079 & 0.28 & 1 \\
Prenatal exposure (mother on Diet 1) & -- & -- & -- \\
Diet 1 & -- & -- & -- \\
Diet 2 & 2.2 & 13 & 78 \\
Diet 3 & 0.94 & 5.4 & 35 \\
\hline
\end{tabular}

Excess Lifetime Risk [ ]

\begin{tabular}{llll}
\hline Diet 1 & $7.9 \mathrm{E}-05$ & $1.3 \mathrm{E}-03$ & $1.6 \mathrm{E}-02$ \\
Diet 2 & $4.2 \mathrm{E}-05$ & $5.1 \mathrm{E}-04$ & $6.0 \mathrm{E}-03$ \\
Diet 3 & $1.0 \mathrm{E}-05$ & $1.0 \mathrm{E}-04$ & $1.1 \mathrm{E}-03$ \\
Diet 4 & $2.5 \mathrm{E}-04$ & $3.6 \mathrm{E}-03$ & $4.5 \mathrm{E}-02$ \\
\hline
\end{tabular}

\section{Relative Risk [ ]}

\begin{tabular}{lccc}
\hline Diet 1 & 1.035 & 1.31 & 4.3 \\
Diet 2 & 1.014 & 1.14 & 2.6 \\
Diet 3 & 1.0031 & 1.026 & 1.27 \\
Diet 4 & 1.089 & 1.89 & 12 \\
\hline
\end{tabular}

\section{Probability of Causation [\%]}

\begin{tabular}{lccc}
\hline Diet 1 & 3.34 & 23.6 & 76 \\
Diet 2 & 1.34 & 11.9 & 60 \\
Diet 3 & 0.31 & 2.5 & 21 \\
Diet 4 & 8.17 & 46.8 & 91 \\
\hline
\end{tabular}

Diet 1 - Backyard cow milk + all other locally produced non-milk exposure pathways

Diet 2 - Locally produced commercial milk + all other locally produced non-milk exposure pathways

Diet 3 - Regionally mixed commercial milk + inhalation (other regionally mixed food items are minor contributors to the total)

Diet 4 - Goat milk (all other exposure pathways are of negligible importance compared to goat milk) 
Location: Buttermilk Rd.

Receptor: Male born in 1940

\begin{tabular}{lccc}
\hline & \multicolumn{3}{c}{ Thyroid Dose [cGy] } \\
& \multicolumn{2}{c}{$95 \%$ Subjective Confidence Interval } \\
Exposure Pathway & lower limit & central estimate & upper limit \\
\hline Backyard Cow Milk & 2.2 & 12 & 81 \\
Commercial Milk (locally produced) & 0.6 & 4.1 & 27 \\
Commercial Milk (regionally mixed) & 0.12 & 0.79 & 4.9 \\
Goat Milk (locally produced) & 5.3 & 40 & 280 \\
Beef (locally produced) & 0.0045 & 0.081 & 1.7 \\
Leafy Vegetables (locally produced) & 0.0016 & 0.017 & 0.14 \\
Eggs (locally produced) & 0.15 & 1 & 6.6 \\
Cottage Cheese (locally produced) & 0.0032 & 0.034 & 0.37 \\
Inhalation & 0.076 & 0.27 & 1 \\
Mother's milk (mother on Diet 1) & -- & -- & -- \\
Prenatal exposure (mother on Diet 1) & -- & -- & -- \\
Diet 1 & 2.5 & 14 & 90 \\
Diet 2 & 1 & 5.8 & 34 \\
Diet 3 & 0.25 & 1.1 & 5.7 \\
\hline
\end{tabular}

Excess Lifetime Risk [ ]

\begin{tabular}{llll}
\hline Diet 1 & $9.1 \mathrm{E}-06$ & $3.3 \mathrm{E}-04$ & $6.9 \mathrm{E}-03$ \\
Diet 2 & $4.0 \mathrm{E}-06$ & $1.4 \mathrm{E}-04$ & $3.3 \mathrm{E}-03$ \\
Diet 3 & $1.0 \mathrm{E}-06$ & $2.7 \mathrm{E}-05$ & $5.6 \mathrm{E}-04$ \\
Diet 4 & $2.7 \mathrm{E}-05$ & $8.1 \mathrm{E}-04$ & $2.0 \mathrm{E}-02$ \\
\hline
\end{tabular}

\section{Relative Risk [ ]}

\begin{tabular}{lccc}
\hline Diet 1 & 1.011 & 1.22 & 5.4 \\
Diet 2 & 1.0042 & 1.088 & 3 \\
Diet 3 & 1.00092 & 1.016 & 1.35 \\
Diet 4 & 1.027 & 1.58 & 14 \\
\hline
\end{tabular}

\section{Probability of Causation [\%]}

\begin{tabular}{lccc}
\hline Diet 1 & 1.07 & 17.8 & 81 \\
Diet 2 & 0.42 & 8.1 & 66 \\
Diet 3 & 0.09 & 1.6 & 26 \\
Diet 4 & 2.62 & 36.4 & 93 \\
\hline
\end{tabular}

Diet 1 - Backyard cow milk + all other locally produced non-milk exposure pathways

Diet 2 - Locally produced commercial milk + all other locally produced non-milk exposure pathways

Diet 3 - Regionally mixed commercial milk + inhalation (other regionally mixed food items are minor contributors to the total)

Diet 4 - Goat milk (all other exposure pathways are of negligible importance compared to goat milk) 


\section{Location: Jonesville}

Receptor: Female born in 1940

\begin{tabular}{lccc}
\hline & \multicolumn{3}{c}{ Thyroid Dose [cGy] } \\
Exposure Pathway & \multicolumn{2}{c}{$95 \%$ Subjective Confidence Interval } \\
\hline Backyard Cow Milk & lower limit & central estimate & upper limit \\
Commercial Milk (locally produced) & 0.27 & 1.6 & 11 \\
Commercial Milk (regionally mixed) & 0.078 & 0.57 & 4.3 \\
Goat Milk (locally produced) & 0.12 & 0.76 & 4.9 \\
Beef (locally produced) & 0.66 & 5.4 & 45 \\
Leafy Vegetables (locally produced) & 0.00054 & 0.0098 & 0.21 \\
Eggs (locally produced) & 0.0002 & 0.0025 & 0.023 \\
Cottage Cheese (locally produced) & 0.016 & 0.12 & 0.95 \\
Inhalation & 0.00043 & 0.0048 & 0.064 \\
Mother's milk (mother on Diet 1) & 0.011 & 0.041 & 0.17 \\
Prenatal exposure (mother on Diet 1) & -- & -- & -- \\
Diet 1 & -- & -- & -- \\
Diet 2 & 0.32 & 1.8 & 12 \\
Diet 3 & 0.13 & 0.78 & 5.6 \\
\hline
\end{tabular}

Excess Lifetime Risk [ ]

\begin{tabular}{llll}
\hline Diet 1 & $1.2 \mathrm{E}-05$ & $1.8 \mathrm{E}-04$ & $2.8 \mathrm{E}-03$ \\
Diet 2 & $6.2 \mathrm{E}-06$ & $7.7 \mathrm{E}-05$ & $9.7 \mathrm{E}-04$ \\
Diet 3 & $6.6 \mathrm{E}-06$ & $7.7 \mathrm{E}-05$ & $9.8 \mathrm{E}-04$ \\
Diet 4 & $3.3 \mathrm{E}-05$ & $5.0 \mathrm{E}-04$ & $6.5 \mathrm{E}-03$ \\
\hline
\end{tabular}

Relative Risk [ ]

\begin{tabular}{lccc}
\hline Diet 1 & 1.0043 & 1.044 & 1.59 \\
Diet 2 & 1.0019 & 1.018 & 1.25 \\
Diet 3 & 1.0023 & 1.02 & 1.24 \\
Diet 4 & 1.011 & 1.12 & 3 \\
\hline
\end{tabular}

\section{Probability of Causation [\%]}

\begin{tabular}{lccc}
\hline Diet 1 & 0.43 & 4.2 & 37 \\
Diet 2 & 0.19 & 1.8 & 20 \\
Diet 3 & 0.23 & 2.0 & 19 \\
Diet 4 & 1.10 & 11.1 & 66 \\
\hline
\end{tabular}

Diet 1 - Backyard cow milk + all other locally produced non-milk exposure pathways

Diet 2 - Locally produced commercial milk + all other locally produced non-milk exposure pathways

Diet 3 - Regionally mixed commercial milk + inhalation (other regionally mixed food items are minor contributors to the total)

Diet 4 - Goat milk (all other exposure pathways are of negligible importance compared to goat milk) 


\section{Location: Jonesville}

\section{Receptor: Male born in 1940}

\begin{tabular}{lccc}
\hline & \multicolumn{3}{c}{ Thyroid Dose [cGy] } \\
& \multicolumn{2}{c}{$95 \%$ Subjective Confidence Interval } \\
Exposure Pathway & lower limit & central estimate & upper limit \\
\hline Backyard Cow Milk & 0.28 & 1.7 & 11 \\
Commercial Milk (locally produced) & 0.084 & 0.58 & 4.4 \\
Commercial Milk (regionally mixed) & 0.12 & 0.79 & 4.9 \\
Goat Milk (locally produced) & 0.7 & 5.7 & 48 \\
Beef (locally produced) & 0.00061 & 0.012 & 0.24 \\
Leafy Vegetables (locally produced) & 0.00021 & 0.0025 & 0.023 \\
Eggs (locally produced) & 0.019 & 0.14 & 1.1 \\
Cottage Cheese (locally produced) & 0.00045 & 0.0049 & 0.062 \\
Inhalation & 0.012 & 0.042 & 0.17 \\
Mother's milk (mother on Diet 1) & -- & -- & - \\
Prenatal exposure (mother on Diet 1) & -- & -- & - \\
Diet 1 & 0.34 & 1.9 & 13 \\
Diet 2 & 0.14 & 0.83 & 5.6 \\
Diet 3 & 0.15 & 0.85 & 4.9 \\
\hline
\end{tabular}

Excess Lifetime Risk [ ]

\begin{tabular}{llll}
\hline Diet 1 & $1.2 \mathrm{E}-06$ & $4.6 \mathrm{E}-05$ & $1.1 \mathrm{E}-03$ \\
Diet 2 & $6.8 \mathrm{E}-07$ & $2.0 \mathrm{E}-05$ & $4.9 \mathrm{E}-04$ \\
Diet 3 & $7.1 \mathrm{E}-07$ & $2.0 \mathrm{E}-05$ & $4.8 \mathrm{E}-04$ \\
Diet 4 & $3.6 \mathrm{E}-06$ & $1.2 \mathrm{E}-04$ & $3.3 \mathrm{E}-03$ \\
\hline
\end{tabular}

\section{Relative Risk [ ]}

\begin{tabular}{lccc}
\hline Diet 1 & 1.0012 & 1.032 & 1.7 \\
Diet 2 & 1.0006 & 1.013 & 1.31 \\
Diet 3 & 1.00065 & 1.013 & 1.32 \\
Diet 4 & 1.0034 & 1.086 & 3 \\
\hline
\end{tabular}

\section{Probability of Causation [\%]}

\begin{tabular}{llll}
\hline Diet 1 & 0.12 & 3.1 & 41 \\
Diet 2 & 0.06 & 1.3 & 24 \\
Diet 3 & 0.07 & 1.3 & 24 \\
Diet 4 & 0.34 & 7.9 & 67 \\
\hline
\end{tabular}

Diet 1 - Backyard cow milk + all other locally produced non-milk exposure pathways

Diet 2 - Locally produced commercial milk + all other locally produced non-milk exposure pathways

Diet 3 - Regionally mixed commercial milk + inhalation (other regionally mixed food items are minor contributors to the total)

Diet 4 - Goat milk (all other exposure pathways are of negligible importance compared to goat milk) 


\section{Location: OR Scarboro}

Receptor: Female born in 1940

\begin{tabular}{lccc}
\hline & \multicolumn{3}{c}{ Thyroid Dose [cGy] } \\
Exposure Pathway & \multicolumn{2}{c}{$9 \%$ Subjective Confidence Interval } \\
\hline Backyard Cow Milk & lower limit & central estimate & upper limit \\
Commercial Milk (locally produced) & -- & -- & -- \\
Commercial Milk (regionally mixed) & -- & -- & -- \\
Goat Milk (locally produced) & 0.12 & 0.76 & 4.9 \\
Beef (locally produced) & -- & -- & -- \\
Leafy Vegetables (locally produced) & -- & -- & -- \\
Eggs (locally produced) & -- & -- & -- \\
Cottage Cheese (locally produced) & -- & -- & -- \\
Inhalation & 0.0024 & 0.026 & 0.28 \\
Mother's milk (mother on Diet 3) & 0.057 & 0.2 & 0.75 \\
Prenatal exposure (mother on Diet 3) & -- & -- & -- \\
Diet 1 & -- & -- & -- \\
Diet 2 & -- & -- & -- \\
Diet 3 & -- & -- & -- \\
\hline
\end{tabular}

Excess Lifetime Risk [ ]

\begin{tabular}{lccc}
\hline Diet 1 & -- & -- & -- \\
Diet 2 & -- & -- & -- \\
Diet 3 & $9.3 \mathrm{E}-06$ & $9.3 \mathrm{E}-05$ & $1.1 \mathrm{E}-03$ \\
Diet 4 & -- & -- & -- \\
\hline
\end{tabular}

\section{Relative Risk [ ]}

\begin{tabular}{lccc}
\hline Diet 1 & -- & -- & -- \\
Diet 2 & -- & -- & -- \\
Diet 3 & 1.0029 & 1.024 & 1.26 \\
Diet 4 & -- & -- & -- \\
\hline
\end{tabular}

\begin{tabular}{lccc}
\hline Diet 1 & -- & -- & -- \\
Diet 2 & -- & -- & -- \\
Diet 3 & 0.29 & 2.4 & -- \\
Diet 4 & -- & -- & \\
\hline Diet 1 - Backyard cow milk + all other locally produced non-milk exposure pathways & \\
Diet 2 - Locally produced commercial milk + all other locally produced non-milk exposure pathways \\
Diet 3 - Regionally mixed commercial milk + inhalation (other regionally mixed food items are minor contributors to the total) \\
Diet 4 - Goat milk (all other exposure pathways are of negligible importance compared to goat milk)
\end{tabular}




\section{Location: OR Scarboro}

Receptor: Male born in 1940

\begin{tabular}{lccc}
\hline & \multicolumn{3}{c}{ Thyroid Dose [cGy] } \\
Exposure Pathway & \multicolumn{2}{c}{ 95\% Subjective Confidence Interval } \\
\hline Backyard Cow Milk & lower limit & central estimate & upper limit \\
Commercial Milk (locally produced) & -- & -- & -- \\
Commercial Milk (regionally mixed) & -- & -- & -- \\
Goat Milk (locally produced) & 0.12 & 0.79 & 4.9 \\
Beef (locally produced) & -- & -- & -- \\
Leafy Vegetables (locally produced) & -- & -- & -- \\
Eggs (locally produced) & -- & -- & -- \\
Cottage Cheese (locally produced) & -- & -- & -- \\
Inhalation & 0.0025 & 0.025 & 0.27 \\
Mother's milk (mother on Diet 3) & 0.056 & 0.21 & 0.73 \\
Prenatal exposure (mother on Diet 3) & -- & -- & -- \\
Diet 1 & -- & -- & -- \\
Diet 2 & -- & -- & -- \\
Diet 3 & -- & -- & -- \\
\hline
\end{tabular}

Excess Lifetime Risk [ ]

\begin{tabular}{lccc}
\hline Diet 1 & -- & -- & -- \\
Diet 2 & -- & -- & -- \\
Diet 3 & $9.0 \mathrm{E}-07$ & $2.4 \mathrm{E}-05$ & $5.4 \mathrm{E}-04$ \\
Diet 4 & -- & -- & -- \\
\hline
\end{tabular}

\section{Relative Risk [ ]}

\begin{tabular}{lccc}
\hline Diet 1 & -- & -- & -- \\
Diet 2 & -- & -- & -- \\
Diet 3 & 1.00084 & 1.015 & 1.34 \\
Diet 4 & -- & -- & -- \\
\hline
\end{tabular}

\begin{tabular}{lccc}
\hline Diet 1 & -- & -- & -- \\
Diet 2 & -- & -- & -- \\
Diet 3 & 0.08 & 1.5 & -- \\
Diet 4 & -- & -- & - \\
\hline Diet 1 - Backyard cow milk + all other locally produced non-milk exposure pathways & \\
Diet 2 - Locally produced commercial milk + all other locally produced non-milk exposure pathways \\
Diet 3 - Regionally mixed commercial milk + inhalation (other regionally mixed food items are minor contributors to the total) \\
Diet 4 - Goat milk (all other exposure pathways are of negligible importance compared to goat milk)
\end{tabular}


Location: Lawnville/Gallaher Receptor: Female born in 1940

\begin{tabular}{lccc}
\hline & \multicolumn{3}{c}{ Thyroid Dose [cGy] } \\
Exposure Pathway & \multicolumn{2}{c}{$95 \%$} & Subjective Confidence Interval \\
Backyard Cow Milk & lower limit & central estimate & upper limit \\
Commercial Milk (locally produced) & 1.9 & 11 & 58 \\
Commercial Milk (regionally mixed) & 0.63 & 3.6 & 27 \\
Goat Milk (locally produced) & 0.12 & 0.76 & 4.9 \\
Beef (locally produced) & 4.7 & 36 & 240 \\
Leafy Vegetables (locally produced) & 0.0036 & 0.066 & 1.3 \\
Eggs (locally produced) & 0.0016 & 0.017 & 0.13 \\
Cottage Cheese (locally produced) & 0.12 & 0.83 & 5.7 \\
Inhalation & 0.0032 & 0.032 & 0.33 \\
Mother's milk (mother on Diet 1) & 0.079 & 0.27 & 0.98 \\
Prenatal exposure (mother on Diet 1) & -- & -- & -- \\
Diet 1 & -- & -- & -- \\
Diet 2 & 2.3 & 13 & 65 \\
Diet 3 & 0.97 & 5.2 & 34 \\
\hline
\end{tabular}

Excess Lifetime Risk [ ]

\begin{tabular}{llll}
\hline Diet 1 & $8.1 \mathrm{E}-05$ & $1.2 \mathrm{E}-03$ & $1.7 \mathrm{E}-02$ \\
Diet 2 & $4.2 \mathrm{E}-05$ & $4.8 \mathrm{E}-04$ & $5.8 \mathrm{E}-03$ \\
Diet 3 & $1.0 \mathrm{E}-05$ & $9.8 \mathrm{E}-05$ & $1.1 \mathrm{E}-03$ \\
Diet 4 & $2.5 \mathrm{E}-04$ & $3.4 \mathrm{E}-03$ & $4.4 \mathrm{E}-02$ \\
\hline
\end{tabular}

\section{Relative Risk [ ]}

\begin{tabular}{lccc}
\hline Diet 1 & 1.034 & 1.29 & 4.4 \\
Diet 2 & 1.013 & 1.13 & 2.5 \\
Diet 3 & 1.0031 & 1.026 & 1.27 \\
Diet 4 & 1.087 & 1.84 & 12 \\
\hline
\end{tabular}

\begin{tabular}{lccc}
\hline Diet 1 & 3.31 & 22.7 & 77 \\
Diet 2 & 1.33 & 11.4 & 60 \\
Diet 3 & 0.31 & 2.5 & 21 \\
Diet 4 & 8.04 & 45.5 & 91 \\
\hline
\end{tabular}

Diet 1 - Backyard cow milk + all other locally produced non-milk exposure pathways

Diet 2 - Locally produced commercial milk + all other locally produced non-milk exposure pathways

Diet 3 - Regionally mixed commercial milk + inhalation (other regionally mixed food items are minor contributors to the total)

Diet 4 - Goat milk (all other exposure pathways are of negligible importance compared to goat milk) 


\section{Location: Lawnville/Gallaher}

Receptor: Male born in 1940

\begin{tabular}{lccc}
\hline & \multicolumn{3}{c}{ Thyroid Dose [cGy] } \\
Exposure Pathway & $95 \%$ Subjective Confidence Interval \\
\hline Backyard Cow Milk & lower limit & central estimate & upper limit \\
Commercial Milk (locally produced) & 2.2 & 12 & 66 \\
Commercial Milk (regionally mixed) & 0.61 & 3.8 & 26 \\
Goat Milk (locally produced) & 0.12 & 0.79 & 4.9 \\
Beef (locally produced) & 4.9 & 39 & 240 \\
Leafy Vegetables (locally produced) & 0.0044 & 0.079 & 1.6 \\
Eggs (locally produced) & 0.0017 & 0.016 & 0.13 \\
Cottage Cheese (locally produced) & 0.16 & 0.94 & 6.3 \\
Inhalation & 0.0033 & 0.033 & 0.35 \\
Mother's milk (mother on Diet 1) & 0.075 & 0.26 & 1 \\
Prenatal exposure (mother on Diet 1) & -- & -- & -- \\
Diet 1 & -- & -- & -- \\
Diet 2 & 2.5 & 13 & 75 \\
Diet 3 & 1 & 5.5 & 33 \\
\hline
\end{tabular}

Excess Lifetime Risk [ ]

\begin{tabular}{llll}
\hline Diet 1 & $9.2 \mathrm{E}-06$ & $3.0 \mathrm{E}-04$ & $6.9 \mathrm{E}-03$ \\
Diet 2 & $3.6 \mathrm{E}-06$ & $1.3 \mathrm{E}-04$ & $3.0 \mathrm{E}-03$ \\
Diet 3 & $9.5 \mathrm{E}-07$ & $2.7 \mathrm{E}-05$ & $5.7 \mathrm{E}-04$ \\
Diet 4 & $2.6 \mathrm{E}-05$ & $7.8 \mathrm{E}-04$ & $2.0 \mathrm{E}-02$ \\
\hline
\end{tabular}

\section{Relative Risk [ ]}

\begin{tabular}{lccc}
\hline Diet 1 & 1.0087 & 1.2 & 5.3 \\
Diet 2 & 1.0041 & 1.08 & 2.9 \\
Diet 3 & 1.0009 & 1.016 & 1.35 \\
Diet 4 & 1.027 & 1.56 & 14 \\
\hline
\end{tabular}

\section{Probability of Causation [\%]}

\begin{tabular}{lccc}
\hline Diet 1 & 0.86 & 16.8 & 81 \\
Diet 2 & 0.41 & 7.4 & 66 \\
Diet 3 & 0.09 & 1.6 & 26 \\
Diet 4 & 2.61 & 35.7 & 93 \\
\hline
\end{tabular}

Diet 1 - Backyard cow milk + all other locally produced non-milk exposure pathways

Diet 2 - Locally produced commercial milk + all other locally produced non-milk exposure pathways

Diet 3 - Regionally mixed commercial milk + inhalation (other regionally mixed food items are minor contributors to the total)

Diet 4 - Goat milk (all other exposure pathways are of negligible importance compared to goat milk) 


\section{Location: Dyllis}

Receptor: Female born in 1940

\begin{tabular}{lccc}
\hline & \multicolumn{3}{c}{ Thyroid Dose [cGy] } \\
Exposure Pathway & \multicolumn{2}{c}{ 95\% } & Subjective Confidence Interval \\
Backyard Cow Milk & lower limit & central estimate & upper limit \\
Commercial Milk (locally produced) & 0.39 & 2.2 & 14 \\
Commercial Milk (regionally mixed) & 0.12 & 0.76 & 5.9 \\
Goat Milk (locally produced) & 0.12 & 0.76 & 4.9 \\
Beef (locally produced) & 1 & 7.6 & 55 \\
Leafy Vegetables (locally produced) & 0.00082 & 0.013 & 0.27 \\
Eggs (locally produced) & 0.00029 & 0.0034 & 0.031 \\
Cottage Cheese (locally produced) & 0.022 & 0.17 & 1.4 \\
Inhalation & 0.00064 & 0.0066 & 0.081 \\
Mother's milk (mother on Diet 1) & 0.015 & 0.056 & 0.24 \\
Prenatal exposure (mother on Diet 1) & -- & -- & - \\
Diet 1 & -- & -- & - \\
Diet 2 & 0.44 & 2.5 & 16 \\
Diet 3 & 0.19 & 1.1 & 7.4 \\
\hline
\end{tabular}

Excess Lifetime Risk [ ]

\begin{tabular}{llll}
\hline Diet 1 & $1.6 \mathrm{E}-05$ & $2.6 \mathrm{E}-04$ & $3.3 \mathrm{E}-03$ \\
Diet 2 & $7.3 \mathrm{E}-06$ & $1.1 \mathrm{E}-04$ & $1.3 \mathrm{E}-03$ \\
Diet 3 & $6.7 \mathrm{E}-06$ & $7.8 \mathrm{E}-05$ & $9.9 \mathrm{E}-04$ \\
Diet 4 & $5.1 \mathrm{E}-05$ & $7.5 \mathrm{E}-04$ & $8.7 \mathrm{E}-03$ \\
\hline
\end{tabular}

\section{Relative Risk [ ]}

\begin{tabular}{lccc}
\hline Diet 1 & 1.0062 & 1.058 & 1.78 \\
Diet 2 & 1.003 & 1.025 & 1.4 \\
Diet 3 & 1.0023 & 1.021 & 1.24 \\
Diet 4 & 1.018 & 1.16 & 3.4 \\
\hline
\end{tabular}

\begin{tabular}{lccc}
\hline Diet 1 & 0.62 & 5.4 & 44 \\
Diet 2 & 0.30 & 2.4 & 28 \\
Diet 3 & 0.23 & 2.0 & 19 \\
Diet 4 & 1.80 & 13.9 & 70 \\
\hline
\end{tabular}

Diet 1 - Backyard cow milk + all other locally produced non-milk exposure pathways

Diet 2 - Locally produced commercial milk + all other locally produced non-milk exposure pathways

Diet 3 - Regionally mixed commercial milk + inhalation (other regionally mixed food items are minor contributors to the total)

Diet 4 - Goat milk (all other exposure pathways are of negligible importance compared to goat milk) 


\section{Location: Dyllis}

\section{Receptor: Male born in 1940}

\begin{tabular}{lccc}
\hline & \multicolumn{3}{c}{ Thyroid Dose [cGy] } \\
& \multicolumn{2}{c}{$95 \%$ Subjective Confidence Interval } \\
Exposure Pathway & lower limit & central estimate & upper limit \\
\hline Backyard Cow Milk & 0.38 & 2.3 & 14 \\
Commercial Milk (locally produced) & 0.13 & 0.8 & 5.8 \\
Commercial Milk (regionally mixed) & 0.12 & 0.79 & 4.9 \\
Goat Milk (locally produced) & 0.97 & 7.9 & 63 \\
Beef (locally produced) & 0.00099 & 0.015 & 0.31 \\
Leafy Vegetables (locally produced) & 0.00029 & 0.0033 & 0.031 \\
Eggs (locally produced) & 0.026 & 0.2 & 1.5 \\
Cottage Cheese (locally produced) & 0.00067 & 0.0067 & 0.085 \\
Inhalation & 0.015 & 0.059 & 0.22 \\
Mother's milk (mother on Diet 1) & -- & -- & - \\
Prenatal exposure (mother on Diet 1) & -- & -- & - \\
Diet 1 & 0.45 & 2.6 & 16 \\
Diet 2 & 0.21 & 1.1 & 7.7 \\
Diet 3 & 0.15 & 0.86 & 5 \\
\hline
\end{tabular}

Excess Lifetime Risk [ ]

\begin{tabular}{llll}
\hline Diet 1 & $1.7 \mathrm{E}-06$ & $6.4 \mathrm{E}-05$ & $1.4 \mathrm{E}-03$ \\
Diet 2 & $8.3 \mathrm{E}-07$ & $2.8 \mathrm{E}-05$ & $6.5 \mathrm{E}-04$ \\
Diet 3 & $7.4 \mathrm{E}-07$ & $2.1 \mathrm{E}-05$ & $4.9 \mathrm{E}-04$ \\
Diet 4 & $5.1 \mathrm{E}-06$ & $1.5 \mathrm{E}-04$ & $4.2 \mathrm{E}-03$ \\
\hline
\end{tabular}

\section{Relative Risk [ ]}

\begin{tabular}{lccc}
\hline Diet 1 & 1.0019 & 1.041 & 2.1 \\
Diet 2 & 1.00077 & 1.018 & 1.43 \\
Diet 3 & 1.00066 & 1.013 & 1.32 \\
Diet 4 & 1.005 & 1.12 & 3.6 \\
\hline
\end{tabular}

\section{Probability of Causation [\%]}

\begin{tabular}{lccc}
\hline Diet 1 & 0.19 & 3.9 & 51 \\
Diet 2 & 0.08 & 1.8 & 30 \\
Diet 3 & 0.07 & 1.3 & 24 \\
Diet 4 & 0.50 & 10.5 & 72 \\
\hline
\end{tabular}

Diet 1 - Backyard cow milk + all other locally produced non-milk exposure pathways

Diet 2 - Locally produced commercial milk + all other locally produced non-milk exposure pathways

Diet 3 - Regionally mixed commercial milk + inhalation (other regionally mixed food items are minor contributors to the total)

Diet 4 - Goat milk (all other exposure pathways are of negligible importance compared to goat milk) 
Location: OR High School Area

Receptor: Female born in 1940

\begin{tabular}{lccc}
\hline & \multicolumn{3}{c}{ Thyroid Dose [cGy] } \\
Exposure Pathway & \multicolumn{2}{c}{ 95\% Subjective Confidence Interval } \\
\hline Backyard Cow Milk & lower limit & central estimate & upper limit \\
Commercial Milk (locally produced) & -- & -- & -- \\
Commercial Milk (regionally mixed) & -- & -- & -- \\
Goat Milk (locally produced) & 0.12 & 0.76 & 4.9 \\
Beef (locally produced) & -- & -- & -- \\
Leafy Vegetables (locally produced) & -- & -- & -- \\
Eggs (locally produced) & -- & -- & -- \\
Cottage Cheese (locally produced) & -- & -- & -- \\
Inhalation & 0.0014 & 0.015 & 0.15 \\
Mother's milk (mother on Diet 3) & 0.034 & 0.12 & 0.46 \\
Prenatal exposure (mother on Diet 3) & -- & -- & -- \\
Diet 1 & -- & -- & -- \\
Diet 2 & -- & -- & -- \\
Diet 3 & -- & -- & -- \\
\hline
\end{tabular}

Excess Lifetime Risk [ ]

\begin{tabular}{lccc}
\hline Diet 1 & -- & -- & -- \\
Diet 2 & -- & -- & -- \\
Diet 3 & $8.2 \mathrm{E}-06$ & $8.6 \mathrm{E}-05$ & $1.0 \mathrm{E}-03$ \\
Diet 4 & -- & -- & -- \\
\hline
\end{tabular}

\section{Relative Risk [ ]}

\begin{tabular}{lccc}
\hline Diet 1 & -- & -- & -- \\
Diet 2 & -- & -- & -- \\
Diet 3 & 1.0025 & 1.022 & 1.25 \\
Diet 4 & -- & -- & -- \\
\hline
\end{tabular}

\begin{tabular}{lccc}
\hline Diet 1 & -- & -- & -- \\
Diet 2 & -- & -- & - \\
Diet 3 & 0.25 & 2.2 & -- \\
Diet 4 & -- & -- & \\
\hline Diet 1 - Backyard cow milk + all other locally produced non-milk exposure pathways & \\
Diet 2 - Locally produced commercial milk + all other locally produced non-milk exposure pathways \\
Diet 3 - Regionally mixed commercial milk + inhalation (other regionally mixed food items are minor contributors to the total) \\
Diet 4 - Goat milk (all other exposure pathways are of negligible importance compared to goat milk)
\end{tabular}




\section{Location: OR High School Area}

Receptor: Male born in 1940

\begin{tabular}{lccc}
\hline & \multicolumn{3}{c}{ Thyroid Dose [cGy] } \\
Exposure Pathway & \multicolumn{2}{c}{$9 \%$ Subjective Confidence Interval } \\
\hline Backyard Cow Milk & lower limit & central estimate & upper limit \\
Commercial Milk (locally produced) & -- & -- & -- \\
Commercial Milk (regionally mixed) & -- & -- & -- \\
Goat Milk (locally produced) & 0.12 & 0.79 & 4.9 \\
Beef (locally produced) & -- & -- & -- \\
Leafy Vegetables (locally produced) & -- & -- & -- \\
Eggs (locally produced) & -- & -- & -- \\
Cottage Cheese (locally produced) & -- & -- & -- \\
Inhalation & 0.0016 & 0.014 & 0.15 \\
Mother's milk (mother on Diet 3) & 0.035 & 0.12 & 0.44 \\
Prenatal exposure (mother on Diet 3) & -- & -- & -- \\
Diet 1 & -- & -- & -- \\
Diet 2 & -- & -- & -- \\
Diet 3 & -- & -- & -- \\
\hline
\end{tabular}

Excess Lifetime Risk [ ]

\begin{tabular}{lccc}
\hline Diet 1 & -- & -- & -- \\
Diet 2 & -- & -- & -- \\
Diet 3 & $8.1 \mathrm{E}-07$ & $2.2 \mathrm{E}-05$ & $5.0 \mathrm{E}-04$ \\
Diet 4 & -- & -- & -- \\
\hline
\end{tabular}

\section{Relative Risk [ ]}

\begin{tabular}{lccc}
\hline Diet 1 & -- & -- & -- \\
Diet 2 & -- & -- & -- \\
Diet 3 & 1.00074 & 1.014 & 1.33 \\
Diet 4 & -- & -- & -- \\
\hline
\end{tabular}

\begin{tabular}{lccc}
\hline Diet 1 & -- & -- & -- \\
Diet 2 & -- & -- & -- \\
Diet 3 & 0.07 & 1.4 & 25 \\
Diet 4 & -- & -- \\
\hline Diet 1 - Backyard cow milk + all other locally produced non-milk exposure pathways & \\
Diet 2 - Locally produced commercial milk + all other locally produced non-milk exposure pathways \\
Diet 3 - Regionally mixed commercial milk + inhalation (other regionally mixed food items are minor contributors to the total) \\
Diet 4 - Goat milk (all other exposure pathways are of negligible importance compared to goat milk)
\end{tabular}




\section{Location: Norwood}

Receptor: Female born in 1940

\begin{tabular}{lccc}
\hline & \multicolumn{3}{c}{ Thyroid Dose [cGy] } \\
Exposure Pathway & $95 \%$ Subjective Confidence Interval \\
\hline Backyard Cow Milk & lower limit & central estimate & upper limit \\
Commercial Milk (locally produced) & 0.35 & 2.1 & 15 \\
Commercial Milk (regionally mixed) & 0.11 & 0.73 & 5.6 \\
Goat Milk (locally produced) & 0.12 & 0.76 & 4.9 \\
Beef (locally produced) & 0.88 & 7.1 & 52 \\
Leafy Vegetables (locally produced) & 0.00071 & 0.013 & 0.29 \\
Eggs (locally produced) & 0.00031 & 0.0032 & 0.029 \\
Cottage Cheese (locally produced) & 0.02 & 0.16 & 1.2 \\
Inhalation & 0.00058 & 0.0064 & 0.078 \\
Mother's milk (mother on Diet 1) & 0.014 & 0.054 & 0.21 \\
Prenatal exposure (mother on Diet 1) & -- & -- & -- \\
Diet 1 & -- & -- & -- \\
Diet 2 & 0.41 & 2.3 & 16 \\
Diet 3 & 0.17 & 1 & 7.2 \\
\hline
\end{tabular}

Excess Lifetime Risk [ ]

\begin{tabular}{lllc}
\hline Diet 1 & $1.5 \mathrm{E}-05$ & $2.5 \mathrm{E}-04$ & $3.4 \mathrm{E}-03$ \\
Diet 2 & $7.6 \mathrm{E}-06$ & $9.9 \mathrm{E}-05$ & $1.4 \mathrm{E}-03$ \\
Diet 3 & $6.7 \mathrm{E}-06$ & $7.9 \mathrm{E}-05$ & $9.9 \mathrm{E}-04$ \\
Diet 4 & $4.5 \mathrm{E}-05$ & $6.7 \mathrm{E}-04$ & $8.9 \mathrm{E}-03$ \\
\hline
\end{tabular}

Relative Risk [ ]

\begin{tabular}{lccc}
\hline Diet 1 & 1.0059 & 1.055 & 1.61 \\
Diet 2 & 1.0026 & 1.024 & 1.31 \\
Diet 3 & 1.0022 & 1.02 & 1.24 \\
Diet 4 & 1.017 & 1.15 & 3.1 \\
\hline
\end{tabular}

\section{Probability of Causation [\%]}

\begin{tabular}{lccc}
\hline Diet 1 & 0.59 & 5.2 & 38 \\
Diet 2 & 0.26 & 2.3 & 24 \\
Diet 3 & 0.22 & 2.0 & 19 \\
Diet 4 & 1.70 & 13.3 & 67 \\
\hline
\end{tabular}

Diet 1 - Backyard cow milk + all other locally produced non-milk exposure pathways

Diet 2 - Locally produced commercial milk + all other locally produced non-milk exposure pathways

Diet 3 - Regionally mixed commercial milk + inhalation (other regionally mixed food items are minor contributors to the total)

Diet 4 - Goat milk (all other exposure pathways are of negligible importance compared to goat milk) 


\section{Location: Norwood}

\section{Receptor: Male born in 1940}

\begin{tabular}{lccc}
\hline & \multicolumn{3}{c}{ Thyroid Dose [cGy] } \\
& \multicolumn{2}{c}{$95 \%$ Subjective Confidence Interval } \\
Exposure Pathway & lower limit & central estimate & upper limit \\
\hline Backyard Cow Milk & 0.36 & 2.2 & 16 \\
Commercial Milk (locally produced) & 0.11 & 0.72 & 5.5 \\
Commercial Milk (regionally mixed) & 0.12 & 0.79 & 4.9 \\
Goat Milk (locally produced) & 0.99 & 7.3 & 56 \\
Beef (locally produced) & 0.00084 & 0.015 & 0.35 \\
Leafy Vegetables (locally produced) & 0.00032 & 0.0031 & 0.029 \\
Eggs (locally produced) & 0.024 & 0.18 & 1.4 \\
Cottage Cheese (locally produced) & 0.00057 & 0.0064 & 0.079 \\
Inhalation & 0.014 & 0.054 & 0.21 \\
Mother's milk (mother on Diet 1) & -- & -- & - \\
Prenatal exposure (mother on Diet 1) & -- & -- & - \\
Diet 1 & 0.42 & 2.6 & 18 \\
Diet 2 & 0.18 & 1.1 & 7.2 \\
Diet 3 & 0.16 & 0.86 & 5 \\
\hline
\end{tabular}

Excess Lifetime Risk [ ]

\begin{tabular}{llll}
\hline Diet 1 & $1.6 \mathrm{E}-06$ & $5.8 \mathrm{E}-05$ & $1.3 \mathrm{E}-03$ \\
Diet 2 & $7.9 \mathrm{E}-07$ & $2.5 \mathrm{E}-05$ & $6.4 \mathrm{E}-04$ \\
Diet 3 & $7.2 \mathrm{E}-07$ & $2.0 \mathrm{E}-05$ & $4.8 \mathrm{E}-04$ \\
Diet 4 & $4.9 \mathrm{E}-06$ & $1.5 \mathrm{E}-04$ & $4.0 \mathrm{E}-03$ \\
\hline
\end{tabular}

Relative Risk [ ]

\begin{tabular}{lccc}
\hline Diet 1 & 1.0018 & 1.039 & 1.93 \\
Diet 2 & 1.00077 & 1.017 & 1.41 \\
Diet 3 & 1.00065 & 1.013 & 1.32 \\
Diet 4 & 1.0045 & 1.11 & 4.2 \\
\hline
\end{tabular}

\section{Probability of Causation [\%]}

\begin{tabular}{lccc}
\hline Diet 1 & 0.18 & 3.8 & 48 \\
Diet 2 & 0.08 & 1.6 & 29 \\
Diet 3 & 0.07 & 1.3 & 24 \\
Diet 4 & 0.45 & 10.1 & 76 \\
\hline
\end{tabular}

Diet 1 - Backyard cow milk + all other locally produced non-milk exposure pathways

Diet 2 - Locally produced commercial milk + all other locally produced non-milk exposure pathways

Diet 3 - Regionally mixed commercial milk + inhalation (other regionally mixed food items are minor contributors to the total)

Diet 4 - Goat milk (all other exposure pathways are of negligible importance compared to goat milk) 
Location: Woodland

Receptor: Female born in 1940

\begin{tabular}{lccc}
\hline & \multicolumn{3}{c}{ Thyroid Dose [cGy] } \\
Exposure Pathway & \multicolumn{2}{c}{$95 \%$ Subjective Confidence Interval } \\
\hline Backyard Cow Milk & lower limit & central estimate & upper limit \\
Commercial Milk (locally produced) & -- & -- & -- \\
Commercial Milk (regionally mixed) & -- & -- & -- \\
Goat Milk (locally produced) & 0.12 & 0.76 & 4.9 \\
Beef (locally produced) & -- & -- & -- \\
Leafy Vegetables (locally produced) & -- & -- & -- \\
Eggs (locally produced) & -- & -- & -- \\
Cottage Cheese (locally produced) & -- & -- & -- \\
Inhalation & 0.0021 & 0.022 & 0.22 \\
Mother's milk (mother on Diet 3) & 0.053 & 0.18 & 0.66 \\
Prenatal exposure (mother on Diet 3) & -- & -- & -- \\
Diet 1 & -- & -- & -- \\
Diet 2 & -- & -- & -- \\
Diet 3 & -- & -- & -- \\
\hline
\end{tabular}

Excess Lifetime Risk [ ]

\begin{tabular}{lccc}
\hline Diet 1 & -- & -- & -- \\
Diet 2 & -- & -- & -- \\
Diet 3 & $9.0 \mathrm{E}-06$ & $9.1 \mathrm{E}-05$ & $1.0 \mathrm{E}-03$ \\
Diet 4 & -- & -- & -- \\
\hline
\end{tabular}

\section{Relative Risk [ ]}

\begin{tabular}{lccc}
\hline Diet 1 & -- & -- & -- \\
Diet 2 & -- & -- & -- \\
Diet 3 & 1.0028 & 1.024 & 1.26 \\
Diet 4 & -- & -- & -- \\
\hline
\end{tabular}

\begin{tabular}{lccc}
\hline Diet 1 & -- & -- & -- \\
Diet 2 & -- & -- & -- \\
Diet 3 & 0.28 & 2.3 & -- \\
Diet 4 & -- & -- & \\
\hline Diet 1 - Backyard cow milk + all other locally produced non-milk exposure pathways & \\
Diet 2 - Locally produced commercial milk + all other locally produced non-milk exposure pathways \\
Diet 3 - Regionally mixed commercial milk + inhalation (other regionally mixed food items are minor contributors to the total) \\
Diet 4 - Goat milk (all other exposure pathways are of negligible importance compared to goat milk)
\end{tabular}


Location: Woodland

Receptor: Male born in 1940

\begin{tabular}{lccc}
\hline & \multicolumn{3}{c}{ Thyroid Dose [cGy] } \\
Exposure Pathway & \multicolumn{2}{c}{ 95 } & Subjective Confidence Interval \\
Backyard Cow Milk & lower limit & central estimate & upper limit \\
Commercial Milk (locally produced) & -- & -- & -- \\
Commercial Milk (regionally mixed) & -- & -- & -- \\
Goat Milk (locally produced) & 0.12 & 0.79 & 4.9 \\
Beef (locally produced) & -- & -- & -- \\
Leafy Vegetables (locally produced) & -- & -- & -- \\
Eggs (locally produced) & -- & -- & -- \\
Cottage Cheese (locally produced) & -- & -- & -- \\
Inhalation & 0.0022 & 0.022 & 0.23 \\
Mother's milk (mother on Diet 3) & 0.052 & 0.18 & 0.66 \\
Prenatal exposure (mother on Diet 3) & -- & -- & -- \\
Diet 1 & -- & -- & -- \\
Diet 2 & -- & -- & -- \\
Diet 3 & -- & -- & -- \\
\hline
\end{tabular}

Excess Lifetime Risk [ ]

\begin{tabular}{lccc}
\hline Diet 1 & -- & -- & -- \\
Diet 2 & -- & -- & -- \\
Diet 3 & $8.7 \mathrm{E}-07$ & $2.4 \mathrm{E}-05$ & $5.2 \mathrm{E}-04$ \\
Diet 4 & -- & -- & -- \\
\hline
\end{tabular}

\section{Relative Risk [ ]}

\begin{tabular}{lccc}
\hline Diet 1 & -- & -- & -- \\
Diet 2 & -- & -- & -- \\
Diet 3 & 1.00081 & 1.015 & 1.34 \\
Diet 4 & -- & -- & -- \\
\hline
\end{tabular}

\begin{tabular}{lccc}
\hline Diet 1 & -- & -- & -- \\
Diet 2 & -- & -- & -- \\
Diet 3 & 0.08 & 1.5 & 25 \\
Diet 4 & -- & -- \\
\hline Diet 1 - Backyard cow milk + all other locally produced non-milk exposure pathways & \\
Diet 2 - Locally produced commercial milk + all other locally produced non-milk exposure pathways \\
Diet 3 - Regionally mixed commercial milk + inhalation (other regionally mixed food items are minor contributors to the total) \\
Diet 4 - Goat milk (all other exposure pathways are of negligible importance compared to goat milk)
\end{tabular}


Location: Hardin Valley

Receptor: Female born in 1940

\begin{tabular}{lccc}
\hline & \multicolumn{3}{c}{ Thyroid Dose [cGy] } \\
Exposure Pathway & $95 \%$ Subjective Confidence Interval \\
\hline Backyard Cow Milk & lower limit & central estimate & upper limit \\
Commercial Milk (locally produced) & 1.8 & 10 & 61 \\
Commercial Milk (regionally mixed) & 0.56 & 3.6 & 24 \\
Goat Milk (locally produced) & 0.12 & 0.76 & 4.9 \\
Beef (locally produced) & 4.6 & 35 & 230 \\
Leafy Vegetables (locally produced) & 0.0036 & 0.06 & 1.2 \\
Eggs (locally produced) & 0.0014 & 0.016 & 0.13 \\
Cottage Cheese (locally produced) & 0.11 & 0.77 & 5.7 \\
Inhalation & 0.0031 & 0.031 & 0.32 \\
Mother's milk (mother on Diet 1) & 0.074 & 0.26 & 1 \\
Prenatal exposure (mother on Diet 1) & -- & -- & - \\
Diet 1 & -- & -- & -- \\
Diet 2 & 2.1 & 12 & 70 \\
Diet 3 & 0.95 & 5 & 32 \\
\hline
\end{tabular}

Excess Lifetime Risk [ ]

\begin{tabular}{lllc}
\hline Diet 1 & $7.5 \mathrm{E}-05$ & $1.2 \mathrm{E}-03$ & $1.4 \mathrm{E}-02$ \\
Diet 2 & $3.7 \mathrm{E}-05$ & $4.7 \mathrm{E}-04$ & $5.9 \mathrm{E}-03$ \\
Diet 3 & $1.0 \mathrm{E}-05$ & $9.9 \mathrm{E}-05$ & $1.1 \mathrm{E}-03$ \\
Diet 4 & $2.4 \mathrm{E}-04$ & $3.3 \mathrm{E}-03$ & $3.8 \mathrm{E}-02$ \\
\hline
\end{tabular}

\section{Relative Risk [ ]}

\begin{tabular}{lccc}
\hline Diet 1 & 1.031 & 1.28 & 4.2 \\
Diet 2 & 1.013 & 1.12 & 2.5 \\
Diet 3 & 1.0031 & 1.026 & 1.27 \\
Diet 4 & 1.088 & 1.78 & 12 \\
\hline
\end{tabular}

\section{Probability of Causation [\%]}

\begin{tabular}{lccc}
\hline Diet 1 & 3.04 & 21.8 & 76 \\
Diet 2 & 1.32 & 10.4 & 60 \\
Diet 3 & 0.31 & 2.5 & 21 \\
Diet 4 & 8.10 & 43.7 & 91 \\
\hline
\end{tabular}

Diet 1 - Backyard cow milk + all other locally produced non-milk exposure pathways

Diet 2 - Locally produced commercial milk + all other locally produced non-milk exposure pathways

Diet 3 - Regionally mixed commercial milk + inhalation (other regionally mixed food items are minor contributors to the total)

Diet 4 - Goat milk (all other exposure pathways are of negligible importance compared to goat milk) 
Location: Hardin Valley

Receptor: Male born in 1940

\begin{tabular}{lccc}
\hline & \multicolumn{3}{c}{ Thyroid Dose [cGy] } \\
& \multicolumn{2}{c}{$95 \%$ Subjective Confidence Interval } \\
Exposure Pathway & lower limit & central estimate & upper limit \\
\hline Backyard Cow Milk & 1.8 & 11 & 67 \\
Commercial Milk (locally produced) & 0.59 & 3.7 & 24 \\
Commercial Milk (regionally mixed) & 0.12 & 0.79 & 4.9 \\
Goat Milk (locally produced) & 4.9 & 37 & 250 \\
Beef (locally produced) & 0.0042 & 0.07 & 1.4 \\
Leafy Vegetables (locally produced) & 0.0014 & 0.015 & 0.13 \\
Eggs (locally produced) & 0.13 & 0.9 & 6.5 \\
Cottage Cheese (locally produced) & 0.0032 & 0.031 & 0.34 \\
Inhalation & 0.077 & 0.27 & 0.98 \\
Mother's milk (mother on Diet 1) & -- & -- & - \\
Prenatal exposure (mother on Diet 1) & -- & -- & - \\
Diet 1 & 2.1 & 12 & 76 \\
Diet 2 & 0.96 & 5.4 & 32 \\
Diet 3 & 0.25 & 1.1 & 5.7 \\
\hline
\end{tabular}

Excess Lifetime Risk [ ]

\begin{tabular}{llll}
\hline Diet 1 & $8.0 \mathrm{E}-06$ & $3.0 \mathrm{E}-04$ & $6.0 \mathrm{E}-03$ \\
Diet 2 & $4.0 \mathrm{E}-06$ & $1.2 \mathrm{E}-04$ & $2.9 \mathrm{E}-03$ \\
Diet 3 & $1.0 \mathrm{E}-06$ & $2.6 \mathrm{E}-05$ & $5.7 \mathrm{E}-04$ \\
Diet 4 & $2.3 \mathrm{E}-05$ & $7.0 \mathrm{E}-04$ & $1.7 \mathrm{E}-02$ \\
\hline
\end{tabular}

\section{Relative Risk [ ]}

\begin{tabular}{lccc}
\hline Diet 1 & 1.0091 & 1.19 & 5.2 \\
Diet 2 & 1.0039 & 1.079 & 2.7 \\
Diet 3 & 1.0009 & 1.016 & 1.35 \\
Diet 4 & 1.023 & 1.53 & 13 \\
\hline
\end{tabular}

\begin{tabular}{lccc}
\hline Diet 1 & 0.91 & 15.6 & 80 \\
Diet 2 & 0.39 & 7.4 & 63 \\
Diet 3 & 0.09 & 1.5 & 26 \\
Diet 4 & 2.23 & 34.6 & 92 \\
\hline
\end{tabular}

Diet 1 - Backyard cow milk + all other locally produced non-milk exposure pathways

Diet 2 - Locally produced commercial milk + all other locally produced non-milk exposure pathways

Diet 3 - Regionally mixed commercial milk + inhalation (other regionally mixed food items are minor contributors to the total)

Diet 4 - Goat milk (all other exposure pathways are of negligible importance compared to goat milk) 


\section{Location: Oliver Springs}

Receptor: Female born in 1940

\begin{tabular}{lccc}
\hline & \multicolumn{3}{c}{ Thyroid Dose [cGy] } \\
Exposure Pathway & \multicolumn{2}{c}{$95 \%$ Subjective Confidence Interval } \\
\hline Backyard Cow Milk & lower limit & central estimate & upper limit \\
Commercial Milk (locally produced) & 0.27 & 1.6 & 10 \\
Commercial Milk (regionally mixed) & 0.084 & 0.54 & 4.2 \\
Goat Milk (locally produced) & 0.12 & 0.76 & 4.9 \\
Beef (locally produced) & 0.66 & 5.4 & 41 \\
Leafy Vegetables (locally produced) & 0.00053 & 0.0094 & 0.21 \\
Eggs (locally produced) & 0.0002 & 0.0024 & 0.022 \\
Cottage Cheese (locally produced) & 0.016 & 0.12 & 0.91 \\
Inhalation & 0.00046 & 0.0048 & 0.055 \\
Mother's milk (mother on Diet 1) & 0.011 & 0.041 & 0.17 \\
Prenatal exposure (mother on Diet 1) & -- & -- & -- \\
Diet 1 & -- & -- & -- \\
Diet 2 & 0.32 & 1.8 & 12 \\
Diet 3 & 0.13 & 0.76 & 5.4 \\
\hline
\end{tabular}

Excess Lifetime Risk [ ]

\begin{tabular}{llll}
\hline Diet 1 & $1.2 \mathrm{E}-05$ & $1.7 \mathrm{E}-04$ & $2.6 \mathrm{E}-03$ \\
Diet 2 & $6.0 \mathrm{E}-06$ & $7.3 \mathrm{E}-05$ & $1.0 \mathrm{E}-03$ \\
Diet 3 & $6.6 \mathrm{E}-06$ & $7.7 \mathrm{E}-05$ & $9.8 \mathrm{E}-04$ \\
Diet 4 & $3.3 \mathrm{E}-05$ & $4.9 \mathrm{E}-04$ & $6.7 \mathrm{E}-03$ \\
\hline
\end{tabular}

Relative Risk [ ]

\begin{tabular}{lccc}
\hline Diet 1 & 1.0043 & 1.043 & 1.55 \\
Diet 2 & 1.0019 & 1.018 & 1.25 \\
Diet 3 & 1.0022 & 1.02 & 1.24 \\
Diet 4 & 1.013 & 1.12 & 2.7 \\
\hline
\end{tabular}

\section{Probability of Causation [\%]}

\begin{tabular}{lccc}
\hline Diet 1 & 0.43 & 4.1 & 35 \\
Diet 2 & 0.19 & 1.8 & 20 \\
Diet 3 & 0.22 & 2.0 & 19 \\
Diet 4 & 1.24 & 10.8 & 62 \\
\hline
\end{tabular}

Diet 1 - Backyard cow milk + all other locally produced non-milk exposure pathways

Diet 2 - Locally produced commercial milk + all other locally produced non-milk exposure pathways

Diet 3 - Regionally mixed commercial milk + inhalation (other regionally mixed food items are minor contributors to the total)

Diet 4 - Goat milk (all other exposure pathways are of negligible importance compared to goat milk) 


\section{Location: Oliver Springs}

Receptor: Male born in 1940

\begin{tabular}{lccc}
\hline & \multicolumn{3}{c}{ Thyroid Dose [cGy] } \\
Exposure Pathway & $95 \%$ Subjective Confidence Interval \\
\hline Backyard Cow Milk & lower limit & central estimate & upper limit \\
Commercial Milk (locally produced) & 0.29 & 1.7 & 11 \\
Commercial Milk (regionally mixed) & 0.085 & 0.56 & 4.3 \\
Goat Milk (locally produced) & 0.12 & 0.79 & 4.9 \\
Beef (locally produced) & 0.71 & 5.7 & 41 \\
Leafy Vegetables (locally produced) & 0.00063 & 0.011 & 0.24 \\
Eggs (locally produced) & 0.00021 & 0.0023 & 0.023 \\
Cottage Cheese (locally produced) & 0.02 & 0.14 & 1 \\
Inhalation & 0.00045 & 0.0047 & 0.057 \\
Mother's milk (mother on Diet 1) & 0.012 & 0.042 & 0.16 \\
Prenatal exposure (mother on Diet 1) & -- & -- & - \\
Diet 1 & -- & -- & - \\
Diet 2 & 0.34 & 1.9 & 13 \\
Diet 3 & 0.14 & 0.83 & 5.5 \\
\hline
\end{tabular}

Excess Lifetime Risk [ ]

\begin{tabular}{llll}
\hline Diet 1 & $1.2 \mathrm{E}-06$ & $4.5 \mathrm{E}-05$ & $1.1 \mathrm{E}-03$ \\
Diet 2 & $5.7 \mathrm{E}-07$ & $2.0 \mathrm{E}-05$ & $4.6 \mathrm{E}-04$ \\
Diet 3 & $7.1 \mathrm{E}-07$ & $2.0 \mathrm{E}-05$ & $4.8 \mathrm{E}-04$ \\
Diet 4 & $3.5 \mathrm{E}-06$ & $1.2 \mathrm{E}-04$ & $3.0 \mathrm{E}-03$ \\
\hline
\end{tabular}

\section{Relative Risk [ ]}

\begin{tabular}{lccc}
\hline Diet 1 & 1.0013 & 1.031 & 1.71 \\
Diet 2 & 1.00057 & 1.013 & 1.31 \\
Diet 3 & 1.00064 & 1.013 & 1.32 \\
Diet 4 & 1.003 & 1.085 & 3.1 \\
\hline
\end{tabular}

\section{Probability of Causation [\%]}

\begin{tabular}{llll}
\hline Diet 1 & 0.13 & 3.0 & 41 \\
Diet 2 & 0.06 & 1.3 & 23 \\
Diet 3 & 0.06 & 1.3 & 24 \\
Diet 4 & 0.30 & 7.8 & 67 \\
\hline
\end{tabular}

Diet 1 - Backyard cow milk + all other locally produced non-milk exposure pathways

Diet 2 - Locally produced commercial milk + all other locally produced non-milk exposure pathways

Diet 3 - Regionally mixed commercial milk + inhalation (other regionally mixed food items are minor contributors to the total)

Diet 4 - Goat milk (all other exposure pathways are of negligible importance compared to goat milk) 


\section{Location: Solway}

Receptor: Female born in 1940

\begin{tabular}{lccc}
\hline & \multicolumn{3}{c}{ Thyroid Dose [cGy] } \\
& \multicolumn{2}{c}{$95 \%$ Subjective Confidence Interval } \\
Exposure Pathway & lower limit & central estimate & upper limit \\
\hline Backyard Cow Milk & 1.7 & 9.4 & 55 \\
Commercial Milk (locally produced) & 0.49 & 3.2 & 22 \\
Commercial Milk (regionally mixed) & 0.12 & 0.76 & 4.9 \\
Goat Milk (locally produced) & 4.1 & 31 & 210 \\
Beef (locally produced) & 0.0031 & 0.055 & 1.1 \\
Leafy Vegetables (locally produced) & 0.0014 & 0.014 & 0.12 \\
Eggs (locally produced) & 0.099 & 0.69 & 4.9 \\
Cottage Cheese (locally produced) & 0.0028 & 0.028 & 0.33 \\
Inhalation & 0.072 & 0.24 & 0.88 \\
Mother's milk (mother on Diet 1) & -- & -- & - \\
Prenatal exposure (mother on Diet 1) & -- & -- & - \\
Diet 1 & 2 & 11 & 63 \\
Diet 2 & 0.85 & 4.5 & 29 \\
Diet 3 & 0.25 & 1.1 & 5.7 \\
\hline
\end{tabular}

Excess Lifetime Risk [ ]

\begin{tabular}{llll}
\hline Diet 1 & $6.9 \mathrm{E}-05$ & $1.1 \mathrm{E}-03$ & $1.4 \mathrm{E}-02$ \\
Diet 2 & $3.7 \mathrm{E}-05$ & $4.2 \mathrm{E}-04$ & $5.2 \mathrm{E}-03$ \\
Diet 3 & $1.0 \mathrm{E}-05$ & $9.7 \mathrm{E}-05$ & $1.1 \mathrm{E}-03$ \\
Diet 4 & $2.0 \mathrm{E}-04$ & $2.9 \mathrm{E}-03$ & $3.4 \mathrm{E}-02$ \\
\hline
\end{tabular}

\section{Relative Risk [ ]}

\begin{tabular}{lccc}
\hline Diet 1 & 1.028 & 1.26 & 3.7 \\
Diet 2 & 1.012 & 1.11 & 2.3 \\
Diet 3 & 1.0029 & 1.025 & 1.27 \\
Diet 4 & 1.075 & 1.71 & 11 \\
\hline
\end{tabular}

\section{Probability of Causation [\%]}

\begin{tabular}{lccc}
\hline Diet 1 & 2.76 & 20.4 & 73 \\
Diet 2 & 1.15 & 10.0 & 56 \\
Diet 3 & 0.29 & 2.4 & 21 \\
Diet 4 & 6.94 & 41.3 & 91 \\
\hline
\end{tabular}

Diet 1 - Backyard cow milk + all other locally produced non-milk exposure pathways

Diet 2 - Locally produced commercial milk + all other locally produced non-milk exposure pathways

Diet 3 - Regionally mixed commercial milk + inhalation (other regionally mixed food items are minor contributors to the total)

Diet 4 - Goat milk (all other exposure pathways are of negligible importance compared to goat milk) 


\section{Location: Solway}

\section{Receptor: Male born in 1940}

\begin{tabular}{lccc}
\hline & \multicolumn{3}{c}{ Thyroid Dose [cGy] } \\
& \multicolumn{2}{c}{$95 \%$ Subjective Confidence Interval } \\
Exposure Pathway & lower limit & central estimate & upper limit \\
\hline Backyard Cow Milk & 1.8 & 10 & 63 \\
Commercial Milk (locally produced) & 0.51 & 3.4 & 22 \\
Commercial Milk (regionally mixed) & 0.12 & 0.79 & 4.9 \\
Goat Milk (locally produced) & 4.5 & 33 & 230 \\
Beef (locally produced) & 0.0037 & 0.064 & 1.3 \\
Leafy Vegetables (locally produced) & 0.0014 & 0.014 & 0.12 \\
Eggs (locally produced) & 0.12 & 0.83 & 5.4 \\
Cottage Cheese (locally produced) & 0.0029 & 0.028 & 0.3 \\
Inhalation & 0.072 & 0.25 & 0.88 \\
Mother's milk (mother on Diet 1) & -- & -- & - \\
Prenatal exposure (mother on Diet 1) & -- & -- & - \\
Diet 1 & 2.1 & 11 & 72 \\
Diet 2 & 0.86 & 4.8 & 28 \\
Diet 3 & 0.24 & 1.1 & 5.6 \\
\hline
\end{tabular}

Excess Lifetime Risk [ ]

\begin{tabular}{llll}
\hline Diet 1 & $7.6 \mathrm{E}-06$ & $2.6 \mathrm{E}-04$ & $6.0 \mathrm{E}-03$ \\
Diet 2 & $3.6 \mathrm{E}-06$ & $1.1 \mathrm{E}-04$ & $2.7 \mathrm{E}-03$ \\
Diet 3 & $9.6 \mathrm{E}-07$ & $2.6 \mathrm{E}-05$ & $5.6 \mathrm{E}-04$ \\
Diet 4 & $2.2 \mathrm{E}-05$ & $6.3 \mathrm{E}-04$ & $1.7 \mathrm{E}-02$ \\
\hline
\end{tabular}

\section{Relative Risk [ ]}

\begin{tabular}{lccc}
\hline Diet 1 & 1.0068 & 1.17 & 4.7 \\
Diet 2 & 1.0035 & 1.073 & 2.7 \\
Diet 3 & 1.00091 & 1.016 & 1.35 \\
Diet 4 & 1.022 & 1.49 & 12 \\
\hline
\end{tabular}

\begin{tabular}{lccc}
\hline Diet 1 & 0.68 & 14.8 & 79 \\
Diet 2 & 0.35 & 6.8 & 62 \\
Diet 3 & 0.09 & 1.5 & 26 \\
Diet 4 & 2.11 & 32.6 & 91 \\
\hline
\end{tabular}

Diet 1 - Backyard cow milk + all other locally produced non-milk exposure pathways

Diet 2 - Locally produced commercial milk + all other locally produced non-milk exposure pathways

Diet 3 - Regionally mixed commercial milk + inhalation (other regionally mixed food items are minor contributors to the total)

Diet 4 - Goat milk (all other exposure pathways are of negligible importance compared to goat milk) 
Location: Sugar Grove

Receptor: Female born in 1940

\begin{tabular}{lccc}
\hline & \multicolumn{3}{c}{ Thyroid Dose [cGy] } \\
Exposure Pathway & $95 \%$ & Subjective Confidence Interval \\
Backyard Cow Milk & lower limit & central estimate & upper limit \\
Commercial Milk (locally produced) & 0.73 & 4 & 25 \\
Commercial Milk (regionally mixed) & 0.21 & 1.4 & 9.5 \\
Goat Milk (locally produced) & 0.12 & 0.76 & 4.9 \\
Beef (locally produced) & 1.7 & 13 & 90 \\
Leafy Vegetables (locally produced) & 0.0014 & 0.023 & 0.47 \\
Eggs (locally produced) & 0.00054 & 0.0059 & 0.053 \\
Cottage Cheese (locally produced) & 0.043 & 0.29 & 2.2 \\
Inhalation & 0.0011 & 0.012 & 0.14 \\
Mother's milk (mother on Diet 1) & 0.028 & 0.1 & 0.38 \\
Prenatal exposure (mother on Diet 1) & -- & -- & -- \\
Diet 1 & -- & -- & -- \\
Diet 2 & 0.85 & 4.6 & 27 \\
Diet 3 & 0.36 & 1.9 & 13 \\
\hline
\end{tabular}

Excess Lifetime Risk [ ]

\begin{tabular}{lllc}
\hline Diet 1 & $2.9 \mathrm{E}-05$ & $4.4 \mathrm{E}-04$ & $5.8 \mathrm{E}-03$ \\
Diet 2 & $1.4 \mathrm{E}-05$ & $1.8 \mathrm{E}-04$ & $2.3 \mathrm{E}-03$ \\
Diet 3 & $7.9 \mathrm{E}-06$ & $8.3 \mathrm{E}-05$ & $1.0 \mathrm{E}-03$ \\
Diet 4 & $8.5 \mathrm{E}-05$ & $1.2 \mathrm{E}-03$ & $1.4 \mathrm{E}-02$ \\
\hline
\end{tabular}

\section{Relative Risk [ ]}

\begin{tabular}{lccc}
\hline Diet 1 & 1.012 & 1.11 & 2.3 \\
Diet 2 & 1.0051 & 1.045 & 1.57 \\
Diet 3 & 1.0025 & 1.022 & 1.25 \\
Diet 4 & 1.031 & 1.29 & 5.4 \\
\hline
\end{tabular}

\section{Probability of Causation [\%]}

\begin{tabular}{lccc}
\hline Diet 1 & 1.15 & 9.4 & 55 \\
Diet 2 & 0.51 & 4.3 & 35 \\
Diet 3 & 0.25 & 2.1 & 20 \\
Diet 4 & 3.00 & 22.0 & 80 \\
\hline
\end{tabular}

Diet 1 - Backyard cow milk + all other locally produced non-milk exposure pathways

Diet 2 - Locally produced commercial milk + all other locally produced non-milk exposure pathways

Diet 3 - Regionally mixed commercial milk + inhalation (other regionally mixed food items are minor contributors to the total)

Diet 4 - Goat milk (all other exposure pathways are of negligible importance compared to goat milk) 
Location: Sugar Grove

Receptor: Male born in 1940

\begin{tabular}{lccc}
\hline & \multicolumn{3}{c}{ Thyroid Dose [cGy] } \\
& \multicolumn{2}{c}{$95 \%$ Subjective Confidence Interval } \\
Exposure Pathway & lower limit & central estimate & upper limit \\
\hline Backyard Cow Milk & 0.73 & 4.2 & 27 \\
Commercial Milk (locally produced) & 0.22 & 1.4 & 9.8 \\
Commercial Milk (regionally mixed) & 0.12 & 0.79 & 4.9 \\
Goat Milk (locally produced) & 1.9 & 14 & 100 \\
Beef (locally produced) & 0.0016 & 0.027 & 0.56 \\
Leafy Vegetables (locally produced) & 0.00054 & 0.0059 & 0.053 \\
Eggs (locally produced) & 0.048 & 0.34 & 2.5 \\
Cottage Cheese (locally produced) & 0.0011 & 0.012 & 0.13 \\
Inhalation & 0.028 & 0.1 & 0.38 \\
Mother's milk (mother on Diet 1) & -- & -- & - \\
Prenatal exposure (mother on Diet 1) & -- & -- & - \\
Diet 1 & 0.85 & 4.8 & 30 \\
Diet 2 & 0.36 & 2.1 & 13 \\
Diet 3 & 0.17 & 0.92 & 5.1 \\
\hline
\end{tabular}

Excess Lifetime Risk [ ]

\begin{tabular}{lllc}
\hline Diet 1 & $3.3 \mathrm{E}-06$ & $1.1 \mathrm{E}-04$ & $2.5 \mathrm{E}-03$ \\
Diet 2 & $1.6 \mathrm{E}-06$ & $4.7 \mathrm{E}-05$ & $1.1 \mathrm{E}-03$ \\
Diet 3 & $7.9 \mathrm{E}-07$ & $2.1 \mathrm{E}-05$ & $5.0 \mathrm{E}-04$ \\
Diet 4 & $9.2 \mathrm{E}-06$ & $2.7 \mathrm{E}-04$ & $7.2 \mathrm{E}-03$ \\
\hline
\end{tabular}

\section{Relative Risk [ ]}

\begin{tabular}{lccc}
\hline Diet 1 & 1.003 & 1.072 & 2.7 \\
Diet 2 & 1.0014 & 1.031 & 1.72 \\
Diet 3 & 1.00073 & 1.013 & 1.32 \\
Diet 4 & 1.0089 & 1.2 & 5.7 \\
\hline
\end{tabular}

\section{Probability of Causation [\%]}

\begin{tabular}{lccc}
\hline Diet 1 & 0.30 & 6.7 & 61 \\
Diet 2 & 0.14 & 3.0 & 41 \\
Diet 3 & 0.07 & 1.3 & 24 \\
Diet 4 & 0.88 & 16.2 & 81 \\
\hline
\end{tabular}

Diet 1 - Backyard cow milk + all other locally produced non-milk exposure pathways

Diet 2 - Locally produced commercial milk + all other locally produced non-milk exposure pathways

Diet 3 - Regionally mixed commercial milk + inhalation (other regionally mixed food items are minor contributors to the total)

Diet 4 - Goat milk (all other exposure pathways are of negligible importance compared to goat milk) 


\section{Location: OR Townsite}

Receptor: Female born in 1940

\begin{tabular}{lccc}
\hline & \multicolumn{3}{c}{ Thyroid Dose [cGy] } \\
Exposure Pathway & \multicolumn{2}{c}{$9 \%$ Subjective Confidence Interval } \\
\hline Backyard Cow Milk & lower limit & central estimate & upper limit \\
Commercial Milk (locally produced) & -- & -- & -- \\
Commercial Milk (regionally mixed) & -- & -- & -- \\
Goat Milk (locally produced) & 0.12 & 0.76 & 4.9 \\
Beef (locally produced) & -- & -- & -- \\
Leafy Vegetables (locally produced) & -- & -- & -- \\
Eggs (locally produced) & -- & -- & -- \\
Cottage Cheese (locally produced) & -- & -- & -- \\
Inhalation & 0.0012 & 0.013 & 0.14 \\
Mother's milk (mother on Diet 3) & 0.031 & 0.11 & 0.42 \\
Prenatal exposure (mother on Diet 3) & -- & -- & -- \\
Diet 1 & -- & -- & -- \\
Diet 2 & -- & -- & -- \\
Diet 3 & -- & -- & -- \\
\hline
\end{tabular}

Excess Lifetime Risk [ ]

\begin{tabular}{lccc}
\hline Diet 1 & -- & -- & -- \\
Diet 2 & -- & -- & -- \\
Diet 3 & $7.9 \mathrm{E}-06$ & $8.5 \mathrm{E}-05$ & $1.0 \mathrm{E}-03$ \\
Diet 4 & -- & -- & -- \\
\hline
\end{tabular}

\section{Relative Risk [ ]}

\begin{tabular}{lccc}
\hline Diet 1 & -- & -- & -- \\
Diet 2 & -- & -- & -- \\
Diet 3 & 1.0025 & 1.022 & 1.25 \\
Diet 4 & -- & -- & -- \\
\hline
\end{tabular}

\begin{tabular}{lccc}
\hline Diet 1 & -- & -- & -- \\
Diet 2 & -- & -- & -- \\
Diet 3 & 0.25 & 2.2 & -- \\
Diet 4 & -- & -- & \\
\hline Diet 1 - Backyard cow milk + all other locally produced non-milk exposure pathways & \\
Diet 2 - Locally produced commercial milk + all other locally produced non-milk exposure pathways \\
Diet 3 - Regionally mixed commercial milk + inhalation (other regionally mixed food items are minor contributors to the total) \\
Diet 4 - Goat milk (all other exposure pathways are of negligible importance compared to goat milk)
\end{tabular}




\section{Location: OR Townsite}

\section{Receptor: Male born in 1940}

\begin{tabular}{lccc}
\hline & \multicolumn{3}{c}{ Thyroid Dose [cGy] } \\
Exposure Pathway & \multicolumn{2}{c}{$9 \%$ Subjective Confidence Interval } \\
\hline Backyard Cow Milk & lower limit & central estimate & upper limit \\
Commercial Milk (locally produced) & -- & -- & -- \\
Commercial Milk (regionally mixed) & -- & -- & -- \\
Goat Milk (locally produced) & 0.12 & 0.79 & 4.9 \\
Beef (locally produced) & -- & -- & -- \\
Leafy Vegetables (locally produced) & -- & -- & -- \\
Eggs (locally produced) & -- & -- & -- \\
Cottage Cheese (locally produced) & -- & -- & -- \\
Inhalation & 0.0012 & 0.013 & 0.14 \\
Mother's milk (mother on Diet 3) & 0.031 & 0.11 & 0.41 \\
Prenatal exposure (mother on Diet 3) & -- & -- & -- \\
Diet 1 & -- & -- & -- \\
Diet 2 & -- & -- & -- \\
Diet 3 & -- & -- & -- \\
\hline
\end{tabular}

Excess Lifetime Risk [ ]

\begin{tabular}{lccc}
\hline Diet 1 & -- & -- & -- \\
Diet 2 & -- & -- & -- \\
Diet 3 & $8.0 \mathrm{E}-07$ & $2.2 \mathrm{E}-05$ & $5.0 \mathrm{E}-04$ \\
Diet 4 & -- & -- & -- \\
\hline
\end{tabular}

\section{Relative Risk [ ]}

\begin{tabular}{lccc}
\hline Diet 1 & -- & -- & -- \\
Diet 2 & -- & -- & -- \\
Diet 3 & 1.00074 & 1.014 & 1.33 \\
Diet 4 & -- & -- & -- \\
\hline
\end{tabular}

\begin{tabular}{lccc}
\hline Diet 1 & -- & -- & -- \\
Diet 2 & -- & -- & -- \\
Diet 3 & 0.07 & 1.3 & 25 \\
Diet 4 & -- & -- & \\
\hline Diet 1 - Backyard cow milk + all other locally produced non-milk exposure pathways & \\
Diet 2 - Locally produced commercial milk + all other locally produced non-milk exposure pathways \\
Diet 3 - Regionally mixed commercial milk + inhalation (other regionally mixed food items are minor contributors to the total) \\
Diet 4 - Goat milk (all other exposure pathways are of negligible importance compared to goat milk)
\end{tabular}


Location: Hines Valley

Receptor: Female born in 1940

\begin{tabular}{lccc}
\hline & \multicolumn{3}{c}{ Thyroid Dose [cGy] } \\
Exposure Pathway & $95 \%$ Subjective Confidence Interval \\
\hline Backyard Cow Milk & lower limit & central estimate & upper limit \\
Commercial Milk (locally produced) & 0.82 & 4.9 & 29 \\
Commercial Milk (regionally mixed) & 0.25 & 1.6 & 11 \\
Goat Milk (locally produced) & 0.12 & 0.76 & 4.9 \\
Beef (locally produced) & 1.9 & 16 & 100 \\
Leafy Vegetables (locally produced) & 0.0015 & 0.028 & 0.63 \\
Eggs (locally produced) & 0.00073 & 0.0071 & 0.065 \\
Cottage Cheese (locally produced) & 0.049 & 0.35 & 2.5 \\
Inhalation & 0.0014 & 0.014 & 0.16 \\
Mother's milk (mother on Diet 1) & 0.036 & 0.13 & 0.47 \\
Prenatal exposure (mother on Diet 1) & -- & -- & -- \\
Diet 1 & -- & -- & -- \\
Diet 2 & 0.96 & 5.5 & 34 \\
Diet 3 & 0.41 & 2.2 & 14 \\
\hline
\end{tabular}

Excess Lifetime Risk [ ]

\begin{tabular}{lllc}
\hline Diet 1 & $3.7 \mathrm{E}-05$ & $5.4 \mathrm{E}-04$ & $7.2 \mathrm{E}-03$ \\
Diet 2 & $1.8 \mathrm{E}-05$ & $2.2 \mathrm{E}-04$ & $2.8 \mathrm{E}-03$ \\
Diet 3 & $8.0 \mathrm{E}-06$ & $8.7 \mathrm{E}-05$ & $1.0 \mathrm{E}-03$ \\
Diet 4 & $1.1 \mathrm{E}-04$ & $1.5 \mathrm{E}-03$ & $1.9 \mathrm{E}-02$ \\
\hline
\end{tabular}

\section{Relative Risk [ ]}

\begin{tabular}{lccc}
\hline Diet 1 & 1.015 & 1.13 & 2.3 \\
Diet 2 & 1.006 & 1.056 & 1.65 \\
Diet 3 & 1.0026 & 1.023 & 1.25 \\
Diet 4 & 1.038 & 1.36 & 5.7 \\
\hline
\end{tabular}

\section{Probability of Causation [\%]}

\begin{tabular}{lccc}
\hline Diet 1 & 1.47 & 11.4 & 56 \\
Diet 2 & 0.59 & 5.3 & 39 \\
Diet 3 & 0.26 & 2.2 & 20 \\
Diet 4 & 3.66 & 26.3 & 82 \\
\hline
\end{tabular}

Diet 1 - Backyard cow milk + all other locally produced non-milk exposure pathways

Diet 2 - Locally produced commercial milk + all other locally produced non-milk exposure pathways

Diet 3 - Regionally mixed commercial milk + inhalation (other regionally mixed food items are minor contributors to the total)

Diet 4 - Goat milk (all other exposure pathways are of negligible importance compared to goat milk) 


\section{Location: Hines Valley}

Receptor: Male born in 1940

\begin{tabular}{lccc}
\hline & \multicolumn{3}{c}{ Thyroid Dose [cGy] } \\
& \multicolumn{2}{c}{$95 \%$ Subjective Confidence Interval } \\
Exposure Pathway & lower limit & central estimate & upper limit \\
\hline Backyard Cow Milk & 0.94 & 5.1 & 33 \\
Commercial Milk (locally produced) & 0.25 & 1.7 & 11 \\
Commercial Milk (regionally mixed) & 0.12 & 0.79 & 4.9 \\
Goat Milk (locally produced) & 2.3 & 16 & 120 \\
Beef (locally produced) & 0.0018 & 0.034 & 0.74 \\
Leafy Vegetables (locally produced) & 0.00069 & 0.0072 & 0.064 \\
Eggs (locally produced) & 0.061 & 0.41 & 2.9 \\
Cottage Cheese (locally produced) & 0.0014 & 0.014 & 0.17 \\
Inhalation & 0.037 & 0.14 & 0.48 \\
Mother's milk (mother on Diet 1) & -- & -- & - \\
Prenatal exposure (mother on Diet 1) & -- & -- & - \\
Diet 1 & 1.1 & 5.7 & 37 \\
Diet 2 & 0.42 & 2.4 & 14 \\
Diet 3 & 0.19 & 0.96 & 5.2 \\
\hline
\end{tabular}

Excess Lifetime Risk [ ]

\begin{tabular}{lllc}
\hline Diet 1 & $4.0 \mathrm{E}-06$ & $1.4 \mathrm{E}-04$ & $3.3 \mathrm{E}-03$ \\
Diet 2 & $1.9 \mathrm{E}-06$ & $5.9 \mathrm{E}-05$ & $1.4 \mathrm{E}-03$ \\
Diet 3 & $8.2 \mathrm{E}-07$ & $2.2 \mathrm{E}-05$ & $5.1 \mathrm{E}-04$ \\
Diet 4 & $1.2 \mathrm{E}-05$ & $3.4 \mathrm{E}-04$ & $8.2 \mathrm{E}-03$ \\
\hline
\end{tabular}

\section{Relative Risk [ ]}

\begin{tabular}{lccc}
\hline Diet 1 & 1.0041 & 1.094 & 2.9 \\
Diet 2 & 1.0017 & 1.039 & 1.83 \\
Diet 3 & 1.00076 & 1.014 & 1.33 \\
Diet 4 & 1.01 & 1.24 & 7.1 \\
\hline
\end{tabular}

\section{Probability of Causation [\%]}

\begin{tabular}{lccc}
\hline Diet 1 & 0.41 & 8.6 & 66 \\
Diet 2 & 0.17 & 3.7 & 45 \\
Diet 3 & 0.08 & 1.4 & 25 \\
Diet 4 & 1.02 & 19.2 & 86 \\
\hline
\end{tabular}

Diet 1 - Backyard cow milk + all other locally produced non-milk exposure pathways

Diet 2 - Locally produced commercial milk + all other locally produced non-milk exposure pathways

Diet 3 - Regionally mixed commercial milk + inhalation (other regionally mixed food items are minor contributors to the total)

Diet 4 - Goat milk (all other exposure pathways are of negligible importance compared to goat milk) 


\section{Location: Farragut}

Receptor: Female born in 1940

\begin{tabular}{lccc}
\hline & \multicolumn{3}{c}{ Thyroid Dose [cGy] } \\
& \multicolumn{2}{c}{$95 \%$ Subjective Confidence Interval } \\
Exposure Pathway & lower limit & central estimate & upper limit \\
\hline Backyard Cow Milk & 0.98 & 5.5 & 31 \\
Commercial Milk (locally produced) & 0.29 & 1.8 & 12 \\
Commercial Milk (regionally mixed) & 0.12 & 0.76 & 4.9 \\
Goat Milk (locally produced) & 2.3 & 18 & 130 \\
Beef (locally produced) & 0.0018 & 0.032 & 0.76 \\
Leafy Vegetables (locally produced) & 0.00072 & 0.0082 & 0.072 \\
Eggs (locally produced) & 0.058 & 0.42 & 2.9 \\
Cottage Cheese (locally produced) & 0.0016 & 0.016 & 0.18 \\
Inhalation & 0.041 & 0.15 & 0.56 \\
Mother's milk (mother on Diet 1) & -- & -- & - \\
Prenatal exposure (mother on Diet 1) & -- & -- & - \\
Diet 1 & 1.2 & 6.2 & 35 \\
Diet 2 & 0.49 & 2.5 & 15 \\
Diet 3 & 0.2 & 0.94 & 5.3 \\
\hline
\end{tabular}

Excess Lifetime Risk [ ]

\begin{tabular}{llll}
\hline Diet 1 & $4.1 \mathrm{E}-05$ & $6.3 \mathrm{E}-04$ & $7.1 \mathrm{E}-03$ \\
Diet 2 & $1.9 \mathrm{E}-05$ & $2.5 \mathrm{E}-04$ & $3.0 \mathrm{E}-03$ \\
Diet 3 & $8.6 \mathrm{E}-06$ & $8.8 \mathrm{E}-05$ & $1.0 \mathrm{E}-03$ \\
Diet 4 & $1.2 \mathrm{E}-04$ & $1.8 \mathrm{E}-03$ & $2.0 \mathrm{E}-02$ \\
\hline
\end{tabular}

\section{Relative Risk [ ]}

\begin{tabular}{lccc}
\hline Diet 1 & 1.017 & 1.15 & 2.6 \\
Diet 2 & 1.0066 & 1.061 & 1.74 \\
Diet 3 & 1.0027 & 1.023 & 1.25 \\
Diet 4 & 1.042 & 1.41 & 6.9 \\
\hline
\end{tabular}

\section{Probability of Causation [\%]}

\begin{tabular}{lccc}
\hline Diet 1 & 1.63 & 12.9 & 61 \\
Diet 2 & 0.66 & 5.8 & 42 \\
Diet 3 & 0.27 & 2.2 & 20 \\
Diet 4 & 4.03 & 29.0 & 85 \\
\hline
\end{tabular}

Diet 1 - Backyard cow milk + all other locally produced non-milk exposure pathways

Diet 2 - Locally produced commercial milk + all other locally produced non-milk exposure pathways

Diet 3 - Regionally mixed commercial milk + inhalation (other regionally mixed food items are minor contributors to the total)

Diet 4 - Goat milk (all other exposure pathways are of negligible importance compared to goat milk) 


\section{Location: Farragut}

\section{Receptor: Male born in 1940}

\begin{tabular}{lccc}
\hline & \multicolumn{3}{c}{ Thyroid Dose [cGy] } \\
& \multicolumn{2}{c}{$95 \%$ Subjective Confidence Interval } \\
Exposure Pathway & lower limit & central estimate & upper limit \\
\hline Backyard Cow Milk & 1.1 & 5.6 & 33 \\
Commercial Milk (locally produced) & 0.3 & 1.9 & 12 \\
Commercial Milk (regionally mixed) & 0.12 & 0.79 & 4.9 \\
Goat Milk (locally produced) & 2.6 & 19 & 130 \\
Beef (locally produced) & 0.0021 & 0.038 & 0.93 \\
Leafy Vegetables (locally produced) & 0.00071 & 0.008 & 0.074 \\
Eggs (locally produced) & 0.07 & 0.47 & 3.2 \\
Cottage Cheese (locally produced) & 0.0016 & 0.016 & 0.17 \\
Inhalation & 0.039 & 0.15 & 0.54 \\
Mother's milk (mother on Diet 1) & -- & -- & -- \\
Prenatal exposure (mother on Diet 1) & -- & -- & -- \\
Diet 1 & 1.2 & 6.5 & 38 \\
Diet 2 & 0.5 & 2.7 & 15 \\
Diet 3 & 0.2 & 0.97 & 5.3 \\
\hline
\end{tabular}

Excess Lifetime Risk [ ]

\begin{tabular}{llll}
\hline Diet 1 & $5.1 \mathrm{E}-06$ & $1.5 \mathrm{E}-04$ & $3.3 \mathrm{E}-03$ \\
Diet 2 & $2.2 \mathrm{E}-06$ & $6.3 \mathrm{E}-05$ & $1.6 \mathrm{E}-03$ \\
Diet 3 & $8.3 \mathrm{E}-07$ & $2.3 \mathrm{E}-05$ & $5.1 \mathrm{E}-04$ \\
Diet 4 & $1.3 \mathrm{E}-05$ & $3.7 \mathrm{E}-04$ & $9.8 \mathrm{E}-03$ \\
\hline
\end{tabular}

\section{Relative Risk [ ]}

\begin{tabular}{lccc}
\hline Diet 1 & 1.0049 & 1.1 & 3.3 \\
Diet 2 & 1.0021 & 1.043 & 1.96 \\
Diet 3 & 1.00078 & 1.014 & 1.33 \\
Diet 4 & 1.013 & 1.28 & 7.6 \\
\hline
\end{tabular}

\begin{tabular}{lccc}
\hline Diet 1 & 0.48 & 9.2 & 70 \\
Diet 2 & 0.21 & 4.1 & 49 \\
Diet 3 & 0.08 & 1.4 & 25 \\
Diet 4 & 1.29 & 21.6 & 87 \\
\hline
\end{tabular}

Diet 1 - Backyard cow milk + all other locally produced non-milk exposure pathways

Diet 2 - Locally produced commercial milk + all other locally produced non-milk exposure pathways

Diet 3 - Regionally mixed commercial milk + inhalation (other regionally mixed food items are minor contributors to the total)

Diet 4 - Goat milk (all other exposure pathways are of negligible importance compared to goat milk) 
Location: Lenoir City

Receptor: Female born in 1940

\begin{tabular}{lccc}
\hline & \multicolumn{3}{c}{ Thyroid Dose [cGy] } \\
Exposure Pathway & $95 \%$ Subjective Confidence Interval \\
\hline Backyard Cow Milk & lower limit & central estimate & upper limit \\
Commercial Milk (locally produced) & 0.6 & 3.3 & 16 \\
Commercial Milk (regionally mixed) & 0.19 & 1.1 & 8.1 \\
Goat Milk (locally produced) & 0.12 & 0.76 & 4.9 \\
Beef (locally produced) & -- & -- & -- \\
Leafy Vegetables (locally produced) & 0.0011 & 0.02 & 0.39 \\
Eggs (locally produced) & 0.00054 & 0.005 & 0.041 \\
Cottage Cheese (locally produced) & 0.038 & 0.25 & 1.8 \\
Inhalation & 0.00098 & 0.0099 & 0.11 \\
Mother's milk (mother on Diet 1) & 0.026 & 0.094 & 0.36 \\
Prenatal exposure (mother on Diet 1) & -- & -- & -- \\
Diet 1 & -- & -- & -- \\
Diet 2 & 0.73 & 3.7 & 18 \\
Diet 3 & 0.3 & 1.5 & 9.7 \\
\hline
\end{tabular}

Excess Lifetime Risk [ ]

\begin{tabular}{lccc}
\hline Diet 1 & $2.9 \mathrm{E}-05$ & $3.6 \mathrm{E}-04$ & $5.2 \mathrm{E}-03$ \\
Diet 2 & $1.3 \mathrm{E}-05$ & $1.5 \mathrm{E}-04$ & $2.0 \mathrm{E}-03$ \\
Diet 3 & $7.9 \mathrm{E}-06$ & $8.2 \mathrm{E}-05$ & $1.0 \mathrm{E}-03$ \\
Diet 4 & -- & -- & -- \\
\hline
\end{tabular}

Relative Risk [ ]

\begin{tabular}{lccc}
\hline Diet 1 & 1.01 & 1.09 & 1.96 \\
Diet 2 & 1.0042 & 1.037 & 1.49 \\
Diet 3 & 1.0024 & 1.022 & 1.25 \\
Diet 4 & -- & -- & -- \\
\hline
\end{tabular}

\section{Probability of Causation [\%]}

\begin{tabular}{lccc}
\hline Diet 1 & 1.03 & 8.3 & 49 \\
Diet 2 & 0.42 & 3.5 & 33 \\
Diet 3 & 0.24 & 2.1 & 20 \\
Diet 4 & -- & -- & -- \\
\hline Diet 1 - Backyard cow milk + all other locally produced non-milk exposure pathways
\end{tabular}

Diet 1 - Backyard cow milk + all other locally produced non-milk exposure pathways

Diet 2 - Locally produced commercial milk + all other locally produced non-milk exposure pathways

Diet 3 - Regionally mixed commercial milk + inhalation (other regionally mixed food items are minor contributors to the total)

Diet 4 - Goat milk (all other exposure pathways are of negligible importance compared to goat milk) 


\section{Location: Lenoir City}

\section{Receptor: Male born in 1940}

\begin{tabular}{lccc}
\hline & \multicolumn{3}{c}{ Thyroid Dose [cGy] } \\
& \multicolumn{2}{c}{$95 \%$ Subjective Confidence Interval } \\
Exposure Pathway & lower limit & central estimate & upper limit \\
\hline Backyard Cow Milk & 0.71 & 3.5 & 19 \\
Commercial Milk (locally produced) & 0.18 & 1.1 & 7.8 \\
Commercial Milk (regionally mixed) & 0.12 & 0.79 & 4.9 \\
Goat Milk (locally produced) & -- & -- & -- \\
Beef (locally produced) & 0.0014 & 0.023 & 0.45 \\
Leafy Vegetables (locally produced) & 0.00055 & 0.0049 & 0.039 \\
Eggs (locally produced) & 0.046 & 0.29 & 2 \\
Cottage Cheese (locally produced) & 0.00098 & 0.01 & 0.11 \\
Inhalation & 0.027 & 0.093 & 0.36 \\
Mother's milk (mother on Diet 1) & -- & -- & - \\
Prenatal exposure (mother on Diet 1) & -- & -- & -- \\
Diet 1 & 0.82 & 4 & 22 \\
Diet 2 & 0.32 & 1.6 & 9.8 \\
Diet 3 & 0.17 & 0.92 & 5 \\
\hline
\end{tabular}

Excess Lifetime Risk [ ]

\begin{tabular}{lccc}
\hline Diet 1 & $2.6 \mathrm{E}-06$ & $9.1 \mathrm{E}-05$ & $1.9 \mathrm{E}-03$ \\
Diet 2 & $1.1 \mathrm{E}-06$ & $3.9 \mathrm{E}-05$ & $9.3 \mathrm{E}-04$ \\
Diet 3 & $7.7 \mathrm{E}-07$ & $2.2 \mathrm{E}-05$ & $4.9 \mathrm{E}-04$ \\
Diet 4 & -- & -- & -- \\
\hline
\end{tabular}

\section{Relative Risk [ ]}

\begin{tabular}{lccc}
\hline Diet 1 & 1.0028 & 1.062 & 2.2 \\
Diet 2 & 1.0013 & 1.026 & 1.57 \\
Diet 3 & 1.0007 & 1.013 & 1.32 \\
Diet 4 & -- & -- & -- \\
\hline
\end{tabular}

\begin{tabular}{lccc}
\hline Diet 1 & 0.28 & 5.9 & 55 \\
Diet 2 & 0.13 & 2.5 & 36 \\
Diet 3 & 0.07 & 1.3 & 24 \\
Diet 4 & -- & -- & - \\
\hline Diet 1 - Backyard cow milk + all other locally produced non-milk exposure pathways & \\
Diet 2 - Locally produced commercial milk + all other locally produced non-milk exposure pathways \\
Diet 3 - Regionally mixed commercial milk + inhalation (other regionally mixed food items are minor contributors to the total) \\
Diet 4 - Goat milk (all other exposure pathways are of negligible importance compared to goat milk)
\end{tabular}




\section{Location: Kingston}

Receptor: Female born in 1940

\begin{tabular}{lccc}
\hline & \multicolumn{3}{c}{ Thyroid Dose [cGy] } \\
& \multicolumn{2}{c}{$95 \%$ Subjective Confidence Interval } \\
Exposure Pathway & lower limit & central estimate & upper limit \\
\hline Backyard Cow Milk & 0.7 & 4.3 & 23 \\
Commercial Milk (locally produced) & 0.23 & 1.4 & 9.4 \\
Commercial Milk (regionally mixed) & 0.12 & 0.76 & 4.9 \\
Goat Milk (locally produced) & 1.8 & 14 & 88 \\
Beef (locally produced) & 0.0015 & 0.025 & 0.53 \\
Leafy Vegetables (locally produced) & 0.00057 & 0.0064 & 0.056 \\
Eggs (locally produced) & 0.044 & 0.32 & 2.2 \\
Cottage Cheese (locally produced) & 0.0011 & 0.012 & 0.14 \\
Inhalation & 0.035 & 0.12 & 0.47 \\
Mother's milk (mother on Diet 1) & -- & -- & -- \\
Prenatal exposure (mother on Diet 1) & -- & -- & -- \\
Diet 1 & 0.85 & 4.9 & 27 \\
Diet 2 & 0.39 & 2 & 12 \\
Diet 3 & 0.19 & 0.89 & 5.2 \\
\hline
\end{tabular}

Excess Lifetime Risk [ ]

\begin{tabular}{llll}
\hline Diet 1 & $2.9 \mathrm{E}-05$ & $4.7 \mathrm{E}-04$ & $6.2 \mathrm{E}-03$ \\
Diet 2 & $1.5 \mathrm{E}-05$ & $2.0 \mathrm{E}-04$ & $2.5 \mathrm{E}-03$ \\
Diet 3 & $8.0 \mathrm{E}-06$ & $8.6 \mathrm{E}-05$ & $1.0 \mathrm{E}-03$ \\
Diet 4 & $9.8 \mathrm{E}-05$ & $1.3 \mathrm{E}-03$ & $1.7 \mathrm{E}-02$ \\
\hline
\end{tabular}

\section{Relative Risk [ ]}

\begin{tabular}{lccc}
\hline Diet 1 & 1.013 & 1.11 & 2.3 \\
Diet 2 & 1.0057 & 1.049 & 1.61 \\
Diet 3 & 1.0026 & 1.022 & 1.25 \\
Diet 4 & 1.034 & 1.32 & 5.4 \\
\hline
\end{tabular}

\section{Probability of Causation [\%]}

\begin{tabular}{lccc}
\hline Diet 1 & 1.26 & 10.3 & 57 \\
Diet 2 & 0.56 & 4.7 & 38 \\
Diet 3 & 0.26 & 2.2 & 20 \\
Diet 4 & 3.25 & 24.2 & 81 \\
\hline
\end{tabular}

Diet 1 - Backyard cow milk + all other locally produced non-milk exposure pathways

Diet 2 - Locally produced commercial milk + all other locally produced non-milk exposure pathways

Diet 3 - Regionally mixed commercial milk + inhalation (other regionally mixed food items are minor contributors to the total)

Diet 4 - Goat milk (all other exposure pathways are of negligible importance compared to goat milk) 


\section{Location: Kingston}

\section{Receptor: Male born in 1940}

\begin{tabular}{lccc}
\hline & \multicolumn{3}{c}{ Thyroid Dose [cGy] } \\
& \multicolumn{2}{c}{$95 \%$ Subjective Confidence Interval } \\
Exposure Pathway & lower limit & central estimate & upper limit \\
\hline Backyard Cow Milk & 0.8 & 4.5 & 28 \\
Commercial Milk (locally produced) & 0.24 & 1.5 & 9.7 \\
Commercial Milk (regionally mixed) & 0.12 & 0.79 & 4.9 \\
Goat Milk (locally produced) & 2 & 15 & 99 \\
Beef (locally produced) & 0.0017 & 0.03 & 0.65 \\
Leafy Vegetables (locally produced) & 0.0006 & 0.0061 & 0.054 \\
Eggs (locally produced) & 0.054 & 0.36 & 2.3 \\
Cottage Cheese (locally produced) & 0.0013 & 0.012 & 0.13 \\
Inhalation & 0.034 & 0.12 & 0.45 \\
Mother's milk (mother on Diet 1) & -- & -- & - \\
Prenatal exposure (mother on Diet 1) & -- & -- & - \\
Diet 1 & 0.94 & 5.1 & 31 \\
Diet 2 & 0.4 & 2.1 & 13 \\
Diet 3 & 0.18 & 0.95 & 5.2 \\
\hline
\end{tabular}

Excess Lifetime Risk [ ]

\begin{tabular}{llll}
\hline Diet 1 & $3.5 \mathrm{E}-06$ & $1.2 \mathrm{E}-04$ & $2.8 \mathrm{E}-03$ \\
Diet 2 & $1.7 \mathrm{E}-06$ & $5.3 \mathrm{E}-05$ & $1.2 \mathrm{E}-03$ \\
Diet 3 & $8.0 \mathrm{E}-07$ & $2.2 \mathrm{E}-05$ & $5.0 \mathrm{E}-04$ \\
Diet 4 & $9.8 \mathrm{E}-06$ & $2.9 \mathrm{E}-04$ & $7.8 \mathrm{E}-03$ \\
\hline
\end{tabular}

\section{Relative Risk [ ]}

\begin{tabular}{lccc}
\hline Diet 1 & 1.0033 & 1.078 & 2.7 \\
Diet 2 & 1.0015 & 1.033 & 1.74 \\
Diet 3 & 1.00075 & 1.014 & 1.33 \\
Diet 4 & 1.0091 & 1.21 & 6.7 \\
\hline
\end{tabular}

\section{Probability of Causation [\%]}

\begin{tabular}{lccc}
\hline Diet 1 & 0.33 & 7.2 & 63 \\
Diet 2 & 0.15 & 3.2 & 42 \\
Diet 3 & 0.08 & 1.4 & 25 \\
Diet 4 & 0.91 & 17.3 & 85 \\
\hline
\end{tabular}

Diet 1 - Backyard cow milk + all other locally produced non-milk exposure pathways

Diet 2 - Locally produced commercial milk + all other locally produced non-milk exposure pathways

Diet 3 - Regionally mixed commercial milk + inhalation (other regionally mixed food items are minor contributors to the total)

Diet 4 - Goat milk (all other exposure pathways are of negligible importance compared to goat milk) 


\section{Location: Karns}

Receptor: Female born in 1940

\begin{tabular}{lccc}
\hline & \multicolumn{3}{c}{ Thyroid Dose [cGy] } \\
& \multicolumn{2}{c}{$95 \%$ Subjective Confidence Interval } \\
Exposure Pathway & lower limit & central estimate & upper limit \\
\hline Backyard Cow Milk & 0.94 & 5.8 & 31 \\
Commercial Milk (locally produced) & 0.31 & 2 & 12 \\
Commercial Milk (regionally mixed) & 0.12 & 0.76 & 4.9 \\
Goat Milk (locally produced) & 2.4 & 19 & 130 \\
Beef (locally produced) & 0.0018 & 0.033 & 0.71 \\
Leafy Vegetables (locally produced) & 0.00077 & 0.0088 & 0.073 \\
Eggs (locally produced) & 0.061 & 0.44 & 3 \\
Cottage Cheese (locally produced) & 0.0017 & 0.018 & 0.17 \\
Inhalation & 0.045 & 0.16 & 0.6 \\
Mother's milk (mother on Diet 1) & -- & -- & -- \\
Prenatal exposure (mother on Diet 1) & -- & -- & -- \\
Diet 1 & 1.1 & 6.6 & 36 \\
Diet 2 & 0.52 & 2.8 & 15 \\
Diet 3 & 0.21 & 0.96 & 5.4 \\
\hline
\end{tabular}

Excess Lifetime Risk [ ]

\begin{tabular}{llll}
\hline Diet 1 & $4.5 \mathrm{E}-05$ & $6.7 \mathrm{E}-04$ & $7.5 \mathrm{E}-03$ \\
Diet 2 & $2.1 \mathrm{E}-05$ & $2.6 \mathrm{E}-04$ & $3.2 \mathrm{E}-03$ \\
Diet 3 & $8.8 \mathrm{E}-06$ & $9.0 \mathrm{E}-05$ & $1.0 \mathrm{E}-03$ \\
Diet 4 & $1.3 \mathrm{E}-04$ & $1.8 \mathrm{E}-03$ & $2.3 \mathrm{E}-02$ \\
\hline
\end{tabular}

\section{Relative Risk [ ]}

\begin{tabular}{lccc}
\hline Diet 1 & 1.017 & 1.15 & 2.6 \\
Diet 2 & 1.0071 & 1.068 & 1.74 \\
Diet 3 & 1.0028 & 1.023 & 1.26 \\
Diet 4 & 1.048 & 1.44 & 6.9 \\
\hline
\end{tabular}

\section{Probability of Causation [\%]}

\begin{tabular}{lccc}
\hline Diet 1 & 1.72 & 13.4 & 62 \\
Diet 2 & 0.71 & 6.4 & 42 \\
Diet 3 & 0.28 & 2.3 & 20 \\
Diet 4 & 4.57 & 30.5 & 85 \\
\hline
\end{tabular}

Diet 1 - Backyard cow milk + all other locally produced non-milk exposure pathways

Diet 2 - Locally produced commercial milk + all other locally produced non-milk exposure pathways

Diet 3 - Regionally mixed commercial milk + inhalation (other regionally mixed food items are minor contributors to the total)

Diet 4 - Goat milk (all other exposure pathways are of negligible importance compared to goat milk) 


\section{Location: Karns}

\section{Receptor: Male born in 1940}

\begin{tabular}{lccc}
\hline & \multicolumn{3}{c}{ Thyroid Dose [cGy] } \\
& \multicolumn{2}{c}{$95 \%$ Subjective Confidence Interval } \\
Exposure Pathway & lower limit & central estimate & upper limit \\
\hline Backyard Cow Milk & 1.1 & 6.1 & 34 \\
Commercial Milk (locally produced) & 0.33 & 2 & 12 \\
Commercial Milk (regionally mixed) & 0.12 & 0.79 & 4.9 \\
Goat Milk (locally produced) & 2.7 & 20 & 140 \\
Beef (locally produced) & 0.0022 & 0.038 & 0.85 \\
Leafy Vegetables (locally produced) & 0.00078 & 0.0084 & 0.072 \\
Eggs (locally produced) & 0.077 & 0.5 & 3.4 \\
Cottage Cheese (locally produced) & 0.0018 & 0.017 & 0.17 \\
Inhalation & 0.045 & 0.17 & 0.59 \\
Mother's milk (mother on Diet 1) & -- & -- & - \\
Prenatal exposure (mother on Diet 1) & -- & -- & - \\
Diet 1 & 1.3 & 6.9 & 39 \\
Diet 2 & 0.53 & 2.9 & 15 \\
Diet 3 & 0.2 & 0.99 & 5.4 \\
\hline
\end{tabular}

Excess Lifetime Risk [ ]

\begin{tabular}{llll}
\hline Diet 1 & $5.2 \mathrm{E}-06$ & $1.6 \mathrm{E}-04$ & $3.5 \mathrm{E}-03$ \\
Diet 2 & $2.2 \mathrm{E}-06$ & $6.9 \mathrm{E}-05$ & $1.6 \mathrm{E}-03$ \\
Diet 3 & $8.6 \mathrm{E}-07$ & $2.3 \mathrm{E}-05$ & $5.2 \mathrm{E}-04$ \\
Diet 4 & $1.3 \mathrm{E}-05$ & $3.8 \mathrm{E}-04$ & $1.0 \mathrm{E}-02$ \\
\hline
\end{tabular}

\section{Relative Risk [ ]}

\begin{tabular}{lccc}
\hline Diet 1 & 1.0047 & 1.11 & 3.4 \\
Diet 2 & 1.0021 & 1.045 & 1.94 \\
Diet 3 & 1.0008 & 1.015 & 1.33 \\
Diet 4 & 1.013 & 1.3 & 7.5 \\
\hline
\end{tabular}

\begin{tabular}{lccc}
\hline Diet 1 & 0.47 & 9.7 & 71 \\
Diet 2 & 0.21 & 4.3 & 48 \\
Diet 3 & 0.08 & 1.4 & 25 \\
Diet 4 & 1.26 & 22.8 & 86 \\
\hline
\end{tabular}

Diet 1 - Backyard cow milk + all other locally produced non-milk exposure pathways

Diet 2 - Locally produced commercial milk + all other locally produced non-milk exposure pathways

Diet 3 - Regionally mixed commercial milk + inhalation (other regionally mixed food items are minor contributors to the total)

Diet 4 - Goat milk (all other exposure pathways are of negligible importance compared to goat milk) 


\section{Location: Loudon}

Receptor: Female born in 1940

\begin{tabular}{lccc}
\hline & \multicolumn{3}{c}{ Thyroid Dose [cGy] } \\
Exposure Pathway & \multicolumn{2}{c}{$95 \%$ Subjective Confidence Interval } \\
\hline Backyard Cow Milk & lower limit & central estimate & upper limit \\
Commercial Milk (locally produced) & 0.58 & 3.3 & 20 \\
Commercial Milk (regionally mixed) & 0.16 & 1.1 & 7.5 \\
Goat Milk (locally produced) & 0.12 & 0.76 & 4.9 \\
Beef (locally produced) & 1.3 & 11 & 71 \\
Leafy Vegetables (locally produced) & 0.0011 & 0.019 & 0.39 \\
Eggs (locally produced) & 0.00043 & 0.0048 & 0.042 \\
Cottage Cheese (locally produced) & 0.034 & 0.24 & 1.7 \\
Inhalation & 0.00089 & 0.0096 & 0.11 \\
Mother's milk (mother on Diet 1) & 0.027 & 0.097 & 0.36 \\
Prenatal exposure (mother on Diet 1) & -- & -- & -- \\
Diet 1 & -- & -- & -- \\
Diet 2 & 0.69 & 3.7 & 22 \\
Diet 3 & 0.28 & 1.5 & 9.4 \\
\hline
\end{tabular}

Excess Lifetime Risk [ ]

\begin{tabular}{llcc}
\hline Diet 1 & $2.4 \mathrm{E}-05$ & $3.7 \mathrm{E}-04$ & $4.7 \mathrm{E}-03$ \\
Diet 2 & $1.2 \mathrm{E}-05$ & $1.5 \mathrm{E}-04$ & $1.9 \mathrm{E}-03$ \\
Diet 3 & $7.8 \mathrm{E}-06$ & $8.3 \mathrm{E}-05$ & $1.0 \mathrm{E}-03$ \\
Diet 4 & $6.7 \mathrm{E}-05$ & $1.0 \mathrm{E}-03$ & $1.2 \mathrm{E}-02$ \\
\hline
\end{tabular}

Relative Risk [ ]

\begin{tabular}{lccc}
\hline Diet 1 & 1.0096 & 1.087 & 1.89 \\
Diet 2 & 1.0043 & 1.037 & 1.41 \\
Diet 3 & 1.0024 & 1.022 & 1.24 \\
Diet 4 & 1.024 & 1.24 & 4.2 \\
\hline
\end{tabular}

\begin{tabular}{lccc} 
& \multicolumn{3}{c}{ Probability of Causation [\%] } \\
\hline Diet 1 & 0.95 & 8.0 & 47 \\
Diet 2 & 0.42 & 3.6 & 29 \\
Diet 3 & 0.24 & 2.1 & 20 \\
Diet 4 & 2.31 & 19.0 & 76 \\
\hline
\end{tabular}

Diet 1 - Backyard cow milk + all other locally produced non-milk exposure pathways

Diet 2 - Locally produced commercial milk + all other locally produced non-milk exposure pathways

Diet 3 - Regionally mixed commercial milk + inhalation (other regionally mixed food items are minor contributors to the total)

Diet 4 - Goat milk (all other exposure pathways are of negligible importance compared to goat milk) 


\section{Location: Loudon}

\section{Receptor: Male born in 1940}

\begin{tabular}{lccc}
\hline & \multicolumn{3}{c}{ Thyroid Dose [cGy] } \\
& \multicolumn{2}{c}{$95 \%$ Subjective Confidence Interval } \\
Exposure Pathway & lower limit & central estimate & upper limit \\
\hline Backyard Cow Milk & 0.6 & 3.4 & 21 \\
Commercial Milk (locally produced) & 0.17 & 1.1 & 7.7 \\
Commercial Milk (regionally mixed) & 0.12 & 0.79 & 4.9 \\
Goat Milk (locally produced) & 1.5 & 11 & 78 \\
Beef (locally produced) & 0.0013 & 0.022 & 0.45 \\
Leafy Vegetables (locally produced) & 0.00046 & 0.0047 & 0.041 \\
Eggs (locally produced) & 0.04 & 0.28 & 1.9 \\
Cottage Cheese (locally produced) & 0.0009 & 0.0096 & 0.1 \\
Inhalation & 0.027 & 0.099 & 0.36 \\
Mother's milk (mother on Diet 1) & -- & -- & - \\
Prenatal exposure (mother on Diet 1) & -- & -- & - \\
Diet 1 & 0.7 & 3.9 & 24 \\
Diet 2 & 0.29 & 1.7 & 9.8 \\
Diet 3 & 0.17 & 0.92 & 5.1 \\
\hline
\end{tabular}

Excess Lifetime Risk [ ]

\begin{tabular}{llll}
\hline Diet 1 & $2.9 \mathrm{E}-06$ & $9.0 \mathrm{E}-05$ & $2.1 \mathrm{E}-03$ \\
Diet 2 & $1.3 \mathrm{E}-06$ & $3.9 \mathrm{E}-05$ & $8.7 \mathrm{E}-04$ \\
Diet 3 & $7.8 \mathrm{E}-07$ & $2.1 \mathrm{E}-05$ & $5.0 \mathrm{E}-04$ \\
Diet 4 & $7.6 \mathrm{E}-06$ & $2.2 \mathrm{E}-04$ & $5.7 \mathrm{E}-03$ \\
\hline
\end{tabular}

\section{Relative Risk [ ]}

\begin{tabular}{lccc}
\hline Diet 1 & 1.0025 & 1.061 & 2.3 \\
Diet 2 & 1.0012 & 1.026 & 1.53 \\
Diet 3 & 1.00073 & 1.013 & 1.32 \\
Diet 4 & 1.0074 & 1.16 & 4.7 \\
\hline
\end{tabular}

\section{Probability of Causation [\%]}

\begin{tabular}{lccc}
\hline Diet 1 & 0.25 & 5.8 & 56 \\
Diet 2 & 0.12 & 2.5 & 34 \\
Diet 3 & 0.07 & 1.3 & 24 \\
Diet 4 & 0.73 & 13.8 & 78 \\
\hline
\end{tabular}

Diet 1 - Backyard cow milk + all other locally produced non-milk exposure pathways

Diet 2 - Locally produced commercial milk + all other locally produced non-milk exposure pathways

Diet 3 - Regionally mixed commercial milk + inhalation (other regionally mixed food items are minor contributors to the total)

Diet 4 - Goat milk (all other exposure pathways are of negligible importance compared to goat milk) 
Location: Harriman

Receptor: Female born in 1940

\begin{tabular}{lccc}
\hline & \multicolumn{3}{c}{ Thyroid Dose [cGy] } \\
Exposure Pathway & \multicolumn{2}{c}{$95 \%$ Subjective Confidence Interval } \\
\hline Backyard Cow Milk & lower limit & central estimate & upper limit \\
Commercial Milk (locally produced) & 0.34 & 1.9 & 9.6 \\
Commercial Milk (regionally mixed) & 0.11 & 0.63 & 4.6 \\
Goat Milk (locally produced) & 0.12 & 0.76 & 4.9 \\
Beef (locally produced) & 0.81 & 6.1 & 42 \\
Leafy Vegetables (locally produced) & 0.00065 & 0.011 & 0.23 \\
Eggs (locally produced) & 0.0003 & 0.0029 & 0.024 \\
Cottage Cheese (locally produced) & 0.022 & 0.14 & 1 \\
Inhalation & 0.00056 & 0.0056 & 0.06 \\
Mother's milk (mother on Diet 1) & 0.015 & 0.053 & 0.2 \\
Prenatal exposure (mother on Diet 1) & -- & -- & -- \\
Diet 1 & -- & -- & -- \\
Diet 2 & 0.4 & 2.1 & 11 \\
Diet 3 & 0.18 & 0.87 & 5.7 \\
\hline
\end{tabular}

Excess Lifetime Risk [ ]

\begin{tabular}{llll}
\hline Diet 1 & $1.6 \mathrm{E}-05$ & $2.1 \mathrm{E}-04$ & $3.0 \mathrm{E}-03$ \\
Diet 2 & $7.7 \mathrm{E}-06$ & $8.4 \mathrm{E}-05$ & $1.1 \mathrm{E}-03$ \\
Diet 3 & $6.9 \mathrm{E}-06$ & $7.7 \mathrm{E}-05$ & $9.9 \mathrm{E}-04$ \\
Diet 4 & $4.4 \mathrm{E}-05$ & $5.9 \mathrm{E}-04$ & $7.7 \mathrm{E}-03$ \\
\hline
\end{tabular}

\section{Relative Risk [ ]}

\begin{tabular}{lccc}
\hline Diet 1 & 1.0058 & 1.051 & 1.54 \\
Diet 2 & 1.0024 & 1.021 & 1.28 \\
Diet 3 & 1.0022 & 1.021 & 1.24 \\
Diet 4 & 1.015 & 1.14 & 2.8 \\
\hline
\end{tabular}

\section{Probability of Causation [\%]}

\begin{tabular}{lccc}
\hline Diet 1 & 0.58 & 4.8 & 35 \\
Diet 2 & 0.24 & 2.1 & 22 \\
Diet 3 & 0.22 & 2.0 & 19 \\
Diet 4 & 1.50 & 12.3 & 65 \\
\hline
\end{tabular}

Diet 1 - Backyard cow milk + all other locally produced non-milk exposure pathways

Diet 2 - Locally produced commercial milk + all other locally produced non-milk exposure pathways

Diet 3 - Regionally mixed commercial milk + inhalation (other regionally mixed food items are minor contributors to the total)

Diet 4 - Goat milk (all other exposure pathways are of negligible importance compared to goat milk) 


\section{Location: Harriman}

Receptor: Male born in 1940

\begin{tabular}{lccc}
\hline & \multicolumn{3}{c}{ Thyroid Dose [cGy] } \\
Exposure Pathway & \multicolumn{2}{c}{$95 \%$ Subjective Confidence Interval } \\
\hline Backyard Cow Milk & lower limit & central estimate & upper limit \\
Commercial Milk (locally produced) & 0.4 & 2 & 11 \\
Commercial Milk (regionally mixed) & 0.11 & 0.65 & 4.5 \\
Goat Milk (locally produced) & 0.12 & 0.79 & 4.9 \\
Beef (locally produced) & 0.86 & 6.4 & 45 \\
Leafy Vegetables (locally produced) & 0.00078 & 0.014 & 0.26 \\
Eggs (locally produced) & 0.00031 & 0.0028 & 0.023 \\
Cottage Cheese (locally produced) & 0.026 & 0.16 & 1.2 \\
Inhalation & 0.00056 & 0.0059 & 0.062 \\
Mother's milk (mother on Diet 1) & 0.015 & 0.052 & 0.2 \\
Prenatal exposure (mother on Diet 1) & -- & -- & - \\
Diet 1 & -- & -- & -- \\
Diet 2 & 0.46 & 2.3 & 13 \\
Diet 3 & 0.18 & 0.93 & 5.7 \\
\hline
\end{tabular}

Excess Lifetime Risk [ ]

\begin{tabular}{llll}
\hline Diet 1 & $1.5 \mathrm{E}-06$ & $5.2 \mathrm{E}-05$ & $1.1 \mathrm{E}-03$ \\
Diet 2 & $6.5 \mathrm{E}-07$ & $2.2 \mathrm{E}-05$ & $5.4 \mathrm{E}-04$ \\
Diet 3 & $7.3 \mathrm{E}-07$ & $2.0 \mathrm{E}-05$ & $4.8 \mathrm{E}-04$ \\
Diet 4 & $4.2 \mathrm{E}-06$ & $1.3 \mathrm{E}-04$ & $3.4 \mathrm{E}-03$ \\
\hline
\end{tabular}

\section{Relative Risk [ ]}

\begin{tabular}{lccc}
\hline Diet 1 & 1.0016 & 1.035 & 1.71 \\
Diet 2 & 1.00073 & 1.014 & 1.33 \\
Diet 3 & 1.00065 & 1.013 & 1.32 \\
Diet 4 & 1.0048 & 1.096 & 3.3 \\
\hline
\end{tabular}

\section{Probability of Causation [\%]}

\begin{tabular}{llll}
\hline Diet 1 & 0.16 & 3.4 & 41 \\
Diet 2 & 0.07 & 1.4 & 25 \\
Diet 3 & 0.07 & 1.3 & 24 \\
Diet 4 & 0.48 & 8.8 & 69 \\
\hline
\end{tabular}

Diet 1 - Backyard cow milk + all other locally produced non-milk exposure pathways

Diet 2 - Locally produced commercial milk + all other locally produced non-milk exposure pathways

Diet 3 - Regionally mixed commercial milk + inhalation (other regionally mixed food items are minor contributors to the total)

Diet 4 - Goat milk (all other exposure pathways are of negligible importance compared to goat milk) 


\section{Location: Cedar Bluff}

Receptor: Female born in 1940

\begin{tabular}{lccc}
\hline & \multicolumn{3}{c}{ Thyroid Dose [cGy] } \\
& \multicolumn{2}{c}{$95 \%$ Subjective Confidence Interval } \\
Exposure Pathway & lower limit & central estimate & upper limit \\
\hline Backyard Cow Milk & 0.79 & 4.6 & 27 \\
Commercial Milk (locally produced) & 0.24 & 1.5 & 10 \\
Commercial Milk (regionally mixed) & 0.12 & 0.76 & 4.9 \\
Goat Milk (locally produced) & 1.8 & 15 & 98 \\
Beef (locally produced) & 0.0015 & 0.027 & 0.59 \\
Leafy Vegetables (locally produced) & 0.0007 & 0.0068 & 0.057 \\
Eggs (locally produced) & 0.046 & 0.34 & 2.4 \\
Cottage Cheese (locally produced) & 0.0013 & 0.013 & 0.15 \\
Inhalation & 0.036 & 0.14 & 0.48 \\
Mother's milk (mother on Diet 1) & -- & -- & -- \\
Prenatal exposure (mother on Diet 1) & -- & -- & - \\
Diet 1 & 0.91 & 5.2 & 30 \\
Diet 2 & 0.4 & 2.1 & 12 \\
Diet 3 & 0.2 & 0.93 & 5.2 \\
\hline
\end{tabular}

Excess Lifetime Risk [ ]

\begin{tabular}{llll}
\hline Diet 1 & $3.3 \mathrm{E}-05$ & $5.2 \mathrm{E}-04$ & $6.6 \mathrm{E}-03$ \\
Diet 2 & $1.7 \mathrm{E}-05$ & $2.1 \mathrm{E}-04$ & $2.6 \mathrm{E}-03$ \\
Diet 3 & $8.1 \mathrm{E}-06$ & $8.7 \mathrm{E}-05$ & $1.0 \mathrm{E}-03$ \\
Diet 4 & $1.0 \mathrm{E}-04$ & $1.4 \mathrm{E}-03$ & $1.9 \mathrm{E}-02$ \\
\hline
\end{tabular}

\section{Relative Risk [ ]}

\begin{tabular}{lccc}
\hline Diet 1 & 1.014 & 1.12 & 2.2 \\
Diet 2 & 1.0058 & 1.054 & 1.59 \\
Diet 3 & 1.0026 & 1.023 & 1.25 \\
Diet 4 & 1.036 & 1.34 & 5.4 \\
\hline
\end{tabular}

\section{Probability of Causation [\%]}

\begin{tabular}{lccc}
\hline Diet 1 & 1.42 & 10.9 & 54 \\
Diet 2 & 0.58 & 5.1 & 37 \\
Diet 3 & 0.26 & 2.2 & 20 \\
Diet 4 & 3.48 & 25.1 & 81 \\
\hline
\end{tabular}

Diet 1 - Backyard cow milk + all other locally produced non-milk exposure pathways

Diet 2 - Locally produced commercial milk + all other locally produced non-milk exposure pathways

Diet 3 - Regionally mixed commercial milk + inhalation (other regionally mixed food items are minor contributors to the total)

Diet 4 - Goat milk (all other exposure pathways are of negligible importance compared to goat milk) 


\section{Location: Cedar Bluff}

Receptor: Male born in 1940

\begin{tabular}{lccc}
\hline & \multicolumn{3}{c}{ Thyroid Dose [cGy] } \\
& \multicolumn{2}{c}{$95 \%$ Subjective Confidence Interval } \\
Exposure Pathway & lower limit & central estimate & upper limit \\
\hline Backyard Cow Milk & 0.9 & 4.8 & 30 \\
Commercial Milk (locally produced) & 0.25 & 1.6 & 10 \\
Commercial Milk (regionally mixed) & 0.12 & 0.79 & 4.9 \\
Goat Milk (locally produced) & 2.1 & 16 & 110 \\
Beef (locally produced) & 0.0017 & 0.031 & 0.7 \\
Leafy Vegetables (locally produced) & 0.00068 & 0.0067 & 0.057 \\
Eggs (locally produced) & 0.057 & 0.39 & 2.7 \\
Cottage Cheese (locally produced) & 0.0014 & 0.013 & 0.15 \\
Inhalation & 0.037 & 0.14 & 0.48 \\
Mother's milk (mother on Diet 1) & -- & -- & - \\
Prenatal exposure (mother on Diet 1) & -- & -- & - \\
Diet 1 & 1 & 5.4 & 33 \\
Diet 2 & 0.41 & 2.3 & 13 \\
Diet 3 & 0.19 & 0.96 & 5.2 \\
\hline
\end{tabular}

Excess Lifetime Risk [ ]

\begin{tabular}{lllc}
\hline Diet 1 & $3.7 \mathrm{E}-06$ & $1.3 \mathrm{E}-04$ & $2.9 \mathrm{E}-03$ \\
Diet 2 & $1.7 \mathrm{E}-06$ & $5.5 \mathrm{E}-05$ & $1.3 \mathrm{E}-03$ \\
Diet 3 & $8.2 \mathrm{E}-07$ & $2.2 \mathrm{E}-05$ & $5.1 \mathrm{E}-04$ \\
Diet 4 & $1.1 \mathrm{E}-05$ & $3.2 \mathrm{E}-04$ & $7.3 \mathrm{E}-03$ \\
\hline
\end{tabular}

\section{Relative Risk [ ]}

\begin{tabular}{lccc}
\hline Diet 1 & 1.0039 & 1.087 & 2.8 \\
Diet 2 & 1.0017 & 1.036 & 1.77 \\
Diet 3 & 1.00077 & 1.014 & 1.33 \\
Diet 4 & 1.0099 & 1.23 & 6.4 \\
\hline
\end{tabular}

\section{Probability of Causation [\%]}

\begin{tabular}{lccc}
\hline Diet 1 & 0.39 & 8.0 & 64 \\
Diet 2 & 0.17 & 3.5 & 43 \\
Diet 3 & 0.08 & 1.4 & 25 \\
Diet 4 & 0.98 & 18.5 & 84 \\
\hline
\end{tabular}

Diet 1 - Backyard cow milk + all other locally produced non-milk exposure pathways

Diet 2 - Locally produced commercial milk + all other locally produced non-milk exposure pathways

Diet 3 - Regionally mixed commercial milk + inhalation (other regionally mixed food items are minor contributors to the total)

Diet 4 - Goat milk (all other exposure pathways are of negligible importance compared to goat milk) 


\section{Location: Oakdale}

Receptor: Female born in 1940

\begin{tabular}{lccc}
\hline & \multicolumn{3}{c}{ Thyroid Dose [cGy] } \\
Exposure Pathway & \multicolumn{2}{c}{$95 \%$ Subjective Confidence Interval } \\
\hline Backyard Cow Milk & lower limit & central estimate & upper limit \\
Commercial Milk (locally produced) & 0.17 & 1.1 & 6.5 \\
Commercial Milk (regionally mixed) & 0.054 & 0.37 & 2.6 \\
Goat Milk (locally produced) & 0.12 & 0.76 & 4.9 \\
Beef (locally produced) & 0.4 & 3.5 & 25 \\
Leafy Vegetables (locally produced) & 0.00036 & 0.0063 & 0.13 \\
Eggs (locally produced) & 0.00014 & 0.0016 & 0.014 \\
Cottage Cheese (locally produced) & 0.01 & 0.08 & 0.58 \\
Inhalation & 0.0003 & 0.0032 & 0.037 \\
Mother's milk (mother on Diet 1) & 0.0082 & 0.031 & 0.12 \\
Prenatal exposure (mother on Diet 1) & -- & -- & -- \\
Diet 1 & -- & -- & -- \\
Diet 2 & 0.2 & 1.2 & 7.2 \\
Diet 3 & 0.09 & 0.51 & 3.2 \\
\hline
\end{tabular}

Excess Lifetime Risk [ ]

\begin{tabular}{llll}
\hline Diet 1 & $7.6 \mathrm{E}-06$ & $1.2 \mathrm{E}-04$ & $1.6 \mathrm{E}-03$ \\
Diet 2 & $3.9 \mathrm{E}-06$ & $4.9 \mathrm{E}-05$ & $6.1 \mathrm{E}-04$ \\
Diet 3 & $6.3 \mathrm{E}-06$ & $7.5 \mathrm{E}-05$ & $9.8 \mathrm{E}-04$ \\
Diet 4 & $2.1 \mathrm{E}-05$ & $3.3 \mathrm{E}-04$ & $4.3 \mathrm{E}-03$ \\
\hline
\end{tabular}

Relative Risk [ ]

\begin{tabular}{lccc}
\hline Diet 1 & 1.0029 & 1.029 & 1.31 \\
Diet 2 & 1.0012 & 1.012 & 1.15 \\
Diet 3 & 1.0021 & 1.02 & 1.24 \\
Diet 4 & 1.0073 & 1.08 & 2 \\
\hline
\end{tabular}

\section{Probability of Causation [\%]}

\begin{tabular}{llcc}
\hline Diet 1 & 0.29 & 2.8 & 23 \\
Diet 2 & 0.12 & 1.2 & 13 \\
Diet 3 & 0.21 & 2.0 & 19 \\
Diet 4 & 0.73 & 7.3 & 50 \\
\hline
\end{tabular}

Diet 1 - Backyard cow milk + all other locally produced non-milk exposure pathways

Diet 2 - Locally produced commercial milk + all other locally produced non-milk exposure pathways

Diet 3 - Regionally mixed commercial milk + inhalation (other regionally mixed food items are minor contributors to the total)

Diet 4 - Goat milk (all other exposure pathways are of negligible importance compared to goat milk) 


\section{Location: Oakdale}

\section{Receptor: Male born in 1940}

\begin{tabular}{lccc}
\hline & \multicolumn{3}{c}{ Thyroid Dose [cGy] } \\
& \multicolumn{2}{c}{$95 \%$ Subjective Confidence Interval } \\
Exposure Pathway & lower limit & central estimate & upper limit \\
\hline Backyard Cow Milk & 0.18 & 1.1 & 7.3 \\
Commercial Milk (locally produced) & 0.056 & 0.38 & 2.5 \\
Commercial Milk (regionally mixed) & 0.12 & 0.79 & 4.9 \\
Goat Milk (locally produced) & 0.44 & 3.7 & 26 \\
Beef (locally produced) & 0.00043 & 0.0073 & 0.16 \\
Leafy Vegetables (locally produced) & 0.00015 & 0.0016 & 0.014 \\
Eggs (locally produced) & 0.013 & 0.093 & 0.66 \\
Cottage Cheese (locally produced) & 0.0003 & 0.0032 & 0.037 \\
Inhalation & 0.0083 & 0.031 & 0.12 \\
Mother's milk (mother on Diet 1) & -- & -- & - \\
Prenatal exposure (mother on Diet 1) & -- & -- & -- \\
Diet 1 & 0.21 & 1.3 & 8.2 \\
Diet 2 & 0.093 & 0.54 & 3.2 \\
Diet 3 & 0.14 & 0.84 & 4.9 \\
\hline
\end{tabular}

Excess Lifetime Risk [ ]

\begin{tabular}{llll}
\hline Diet 1 & $8.2 \mathrm{E}-07$ & $3.1 \mathrm{E}-05$ & $7.4 \mathrm{E}-04$ \\
Diet 2 & $3.7 \mathrm{E}-07$ & $1.3 \mathrm{E}-05$ & $3.3 \mathrm{E}-04$ \\
Diet 3 & $7.0 \mathrm{E}-07$ & $2.0 \mathrm{E}-05$ & $4.8 \mathrm{E}-04$ \\
Diet 4 & $2.4 \mathrm{E}-06$ & $7.6 \mathrm{E}-05$ & $1.9 \mathrm{E}-03$ \\
\hline
\end{tabular}

\section{Relative Risk [ ]}

\begin{tabular}{lccc}
\hline Diet 1 & 1.00089 & 1.021 & 1.41 \\
Diet 2 & 1.00039 & 1.0084 & 1.19 \\
Diet 3 & 1.00063 & 1.013 & 1.31 \\
Diet 4 & 1.0023 & 1.055 & 2.3 \\
\hline
\end{tabular}

\section{Probability of Causation [\%]}

\begin{tabular}{llll}
\hline Diet 1 & 0.09 & 2.0 & 29 \\
Diet 2 & 0.04 & 0.8 & 16 \\
Diet 3 & 0.06 & 1.3 & 24 \\
Diet 4 & 0.23 & 5.2 & 55 \\
\hline
\end{tabular}

Diet 1 - Backyard cow milk + all other locally produced non-milk exposure pathways

Diet 2 - Locally produced commercial milk + all other locally produced non-milk exposure pathways

Diet 3 - Regionally mixed commercial milk + inhalation (other regionally mixed food items are minor contributors to the total)

Diet 4 - Goat milk (all other exposure pathways are of negligible importance compared to goat milk) 


\section{Location: Claxton}

Receptor: Female born in 1940

\begin{tabular}{lccc}
\hline & \multicolumn{3}{c}{ Thyroid Dose [cGy] } \\
& \multicolumn{2}{c}{$95 \%$ Subjective Confidence Interval } \\
Exposure Pathway & lower limit & central estimate & upper limit \\
\hline Backyard Cow Milk & 0.68 & 3.8 & 22 \\
Commercial Milk (locally produced) & 0.2 & 1.3 & 8.5 \\
Commercial Milk (regionally mixed) & 0.12 & 0.76 & 4.9 \\
Goat Milk (locally produced) & 1.6 & 12 & 81 \\
Beef (locally produced) & 0.0012 & 0.022 & 0.49 \\
Leafy Vegetables (locally produced) & 0.00057 & 0.0056 & 0.048 \\
Eggs (locally produced) & 0.039 & 0.28 & 1.9 \\
Cottage Cheese (locally produced) & 0.0011 & 0.011 & 0.12 \\
Inhalation & 0.031 & 0.11 & 0.4 \\
Mother's milk (mother on Diet 1) & -- & -- & - \\
Prenatal exposure (mother on Diet 1) & -- & -- & - \\
Diet 1 & 0.79 & 4.4 & 25 \\
Diet 2 & 0.35 & 1.8 & 10 \\
Diet 3 & 0.19 & 0.89 & 5.2 \\
\hline
\end{tabular}

Excess Lifetime Risk [ ]

\begin{tabular}{llcc}
\hline Diet 1 & $2.8 \mathrm{E}-05$ & $4.2 \mathrm{E}-04$ & $5.4 \mathrm{E}-03$ \\
Diet 2 & $1.4 \mathrm{E}-05$ & $1.7 \mathrm{E}-04$ & $2.2 \mathrm{E}-03$ \\
Diet 3 & $7.8 \mathrm{E}-06$ & $8.5 \mathrm{E}-05$ & $1.0 \mathrm{E}-03$ \\
Diet 4 & $8.6 \mathrm{E}-05$ & $1.2 \mathrm{E}-03$ & $1.5 \mathrm{E}-02$ \\
\hline
\end{tabular}

\section{Relative Risk [ ]}

\begin{tabular}{lccc}
\hline Diet 1 & 1.012 & 1.1 & 1.99 \\
Diet 2 & 1.0048 & 1.045 & 1.5 \\
Diet 3 & 1.0025 & 1.022 & 1.25 \\
Diet 4 & 1.031 & 1.28 & 4.6 \\
\hline
\end{tabular}

\section{Probability of Causation [\%]}

\begin{tabular}{lccc}
\hline Diet 1 & 1.18 & 9.2 & 50 \\
Diet 2 & 0.48 & 4.3 & 33 \\
Diet 3 & 0.25 & 2.2 & 20 \\
Diet 4 & 2.98 & 21.6 & 78 \\
\hline
\end{tabular}

Diet 1 - Backyard cow milk + all other locally produced non-milk exposure pathways

Diet 2 - Locally produced commercial milk + all other locally produced non-milk exposure pathways

Diet 3 - Regionally mixed commercial milk + inhalation (other regionally mixed food items are minor contributors to the total)

Diet 4 - Goat milk (all other exposure pathways are of negligible importance compared to goat milk) 


\section{Location: Claxton}

\section{Receptor: Male born in 1940}

\begin{tabular}{lccc}
\hline & \multicolumn{3}{c}{ Thyroid Dose [cGy] } \\
& \multicolumn{2}{c}{$95 \%$ Subjective Confidence Interval } \\
Exposure Pathway & lower limit & central estimate & upper limit \\
\hline Backyard Cow Milk & 0.76 & 4 & 24 \\
Commercial Milk (locally produced) & 0.21 & 1.3 & 8.7 \\
Commercial Milk (regionally mixed) & 0.12 & 0.79 & 4.9 \\
Goat Milk (locally produced) & 1.8 & 13 & 89 \\
Beef (locally produced) & 0.0015 & 0.026 & 0.57 \\
Leafy Vegetables (locally produced) & 0.00054 & 0.0056 & 0.047 \\
Eggs (locally produced) & 0.048 & 0.33 & 2.2 \\
Cottage Cheese (locally produced) & 0.0011 & 0.011 & 0.12 \\
Inhalation & 0.031 & 0.11 & 0.39 \\
Mother's milk (mother on Diet 1) & -- & -- & - \\
Prenatal exposure (mother on Diet 1) & -- & -- & - \\
Diet 1 & 0.87 & 4.5 & 27 \\
Diet 2 & 0.35 & 1.9 & 11 \\
Diet 3 & 0.18 & 0.94 & 5.1 \\
\hline
\end{tabular}

Excess Lifetime Risk [ ]

\begin{tabular}{llll}
\hline Diet 1 & $3.0 \mathrm{E}-06$ & $1.1 \mathrm{E}-04$ & $2.4 \mathrm{E}-03$ \\
Diet 2 & $1.5 \mathrm{E}-06$ & $4.4 \mathrm{E}-05$ & $1.1 \mathrm{E}-03$ \\
Diet 3 & $8.0 \mathrm{E}-07$ & $2.2 \mathrm{E}-05$ & $5.0 \mathrm{E}-04$ \\
Diet 4 & $8.9 \mathrm{E}-06$ & $2.6 \mathrm{E}-04$ & $6.3 \mathrm{E}-03$ \\
\hline
\end{tabular}

\section{Relative Risk [ ]}

\begin{tabular}{lccc}
\hline Diet 1 & 1.0031 & 1.072 & 2.5 \\
Diet 2 & 1.0014 & 1.03 & 1.63 \\
Diet 3 & 1.00074 & 1.014 & 1.33 \\
Diet 4 & 1.0084 & 1.19 & 5.4 \\
\hline
\end{tabular}

\section{Probability of Causation [\%]}

\begin{tabular}{lccc}
\hline Diet 1 & 0.31 & 6.7 & 59 \\
Diet 2 & 0.14 & 2.9 & 39 \\
Diet 3 & 0.07 & 1.3 & 25 \\
Diet 4 & 0.83 & 16.1 & 81 \\
\hline
\end{tabular}

Diet 1 - Backyard cow milk + all other locally produced non-milk exposure pathways

Diet 2 - Locally produced commercial milk + all other locally produced non-milk exposure pathways

Diet 3 - Regionally mixed commercial milk + inhalation (other regionally mixed food items are minor contributors to the total)

Diet 4 - Goat milk (all other exposure pathways are of negligible importance compared to goat milk) 
Location: Dutch Valley

Receptor: Female born in 1940

\begin{tabular}{lccc}
\hline & \multicolumn{3}{c}{ Thyroid Dose [cGy] } \\
Exposure Pathway & \multicolumn{2}{c}{$95 \%$ Subjective Confidence Interval } \\
\hline Backyard Cow Milk & lower limit & central estimate & upper limit \\
Commercial Milk (locally produced) & 0.3 & 1.9 & 9.9 \\
Commercial Milk (regionally mixed) & 0.1 & 0.62 & 4.1 \\
Goat Milk (locally produced) & 0.12 & 0.76 & 4.9 \\
Beef (locally produced) & 0.7 & 5.9 & 41 \\
Leafy Vegetables (locally produced) & 0.0006 & 0.011 & 0.21 \\
Eggs (locally produced) & 0.00027 & 0.0027 & 0.023 \\
Cottage Cheese (locally produced) & 0.02 & 0.14 & 0.96 \\
Inhalation & 0.00053 & 0.0056 & 0.054 \\
Mother's milk (mother on Diet 1) & 0.015 & 0.052 & 0.19 \\
Prenatal exposure (mother on Diet 1) & -- & -- & -- \\
Diet 1 & -- & -- & -- \\
Diet 2 & 0.37 & 2.1 & 11 \\
Diet 3 & 0.16 & 0.86 & 5.1 \\
\hline
\end{tabular}

Excess Lifetime Risk [ ]

\begin{tabular}{llll}
\hline Diet 1 & $1.5 \mathrm{E}-05$ & $2.0 \mathrm{E}-04$ & $2.6 \mathrm{E}-03$ \\
Diet 2 & $7.0 \mathrm{E}-06$ & $8.2 \mathrm{E}-05$ & $1.1 \mathrm{E}-03$ \\
Diet 3 & $6.8 \mathrm{E}-06$ & $7.8 \mathrm{E}-05$ & $9.9 \mathrm{E}-04$ \\
Diet 4 & $3.8 \mathrm{E}-05$ & $5.6 \mathrm{E}-04$ & $7.6 \mathrm{E}-03$ \\
\hline
\end{tabular}

Relative Risk [ ]

\begin{tabular}{lccc}
\hline Diet 1 & 1.0057 & 1.049 & 1.52 \\
Diet 2 & 1.0023 & 1.021 & 1.25 \\
Diet 3 & 1.0022 & 1.021 & 1.24 \\
Diet 4 & 1.015 & 1.14 & 2.9 \\
\hline
\end{tabular}

\section{Probability of Causation [\%]}

\begin{tabular}{lccc}
\hline Diet 1 & 0.57 & 4.7 & 34 \\
Diet 2 & 0.23 & 2.1 & 20 \\
Diet 3 & 0.22 & 2.0 & 19 \\
Diet 4 & 1.43 & 12.2 & 64 \\
\hline
\end{tabular}

Diet 1 - Backyard cow milk + all other locally produced non-milk exposure pathways

Diet 2 - Locally produced commercial milk + all other locally produced non-milk exposure pathways

Diet 3 - Regionally mixed commercial milk + inhalation (other regionally mixed food items are minor contributors to the total)

Diet 4 - Goat milk (all other exposure pathways are of negligible importance compared to goat milk) 


\section{Location: Dutch Valley}

Receptor: Male born in 1940

\begin{tabular}{lccc}
\hline & \multicolumn{3}{c}{ Thyroid Dose [cGy] } \\
& \multicolumn{2}{c}{$95 \%$ Subjective Confidence Interval } \\
Exposure Pathway & lower limit & central estimate & upper limit \\
\hline Backyard Cow Milk & 0.35 & 1.9 & 11 \\
Commercial Milk (locally produced) & 0.1 & 0.64 & 4 \\
Commercial Milk (regionally mixed) & 0.12 & 0.79 & 4.9 \\
Goat Milk (locally produced) & 0.76 & 6.2 & 44 \\
Beef (locally produced) & 0.00071 & 0.012 & 0.25 \\
Leafy Vegetables (locally produced) & 0.00028 & 0.0027 & 0.022 \\
Eggs (locally produced) & 0.025 & 0.16 & 1.1 \\
Cottage Cheese (locally produced) & 0.00057 & 0.0056 & 0.056 \\
Inhalation & 0.015 & 0.054 & 0.2 \\
Mother's milk (mother on Diet 1) & -- & -- & - \\
Prenatal exposure (mother on Diet 1) & -- & -- & - \\
Diet 1 & 0.41 & 2.2 & 12 \\
Diet 2 & 0.17 & 0.92 & 5.1 \\
Diet 3 & 0.15 & 0.87 & 5 \\
\hline
\end{tabular}

Excess Lifetime Risk [ ]

\begin{tabular}{llll}
\hline Diet 1 & $1.6 \mathrm{E}-06$ & $5.1 \mathrm{E}-05$ & $1.1 \mathrm{E}-03$ \\
Diet 2 & $6.4 \mathrm{E}-07$ & $2.2 \mathrm{E}-05$ & $5.1 \mathrm{E}-04$ \\
Diet 3 & $7.3 \mathrm{E}-07$ & $2.0 \mathrm{E}-05$ & $4.8 \mathrm{E}-04$ \\
Diet 4 & $4.2 \mathrm{E}-06$ & $1.2 \mathrm{E}-04$ & $3.6 \mathrm{E}-03$ \\
\hline
\end{tabular}

\section{Relative Risk [ ]}

\begin{tabular}{lccc}
\hline Diet 1 & 1.0015 & 1.034 & 1.72 \\
Diet 2 & 1.00068 & 1.014 & 1.3 \\
Diet 3 & 1.00066 & 1.013 & 1.32 \\
Diet 4 & 1.0041 & 1.093 & 3 \\
\hline
\end{tabular}

\section{Probability of Causation [\%]}

\begin{tabular}{llll}
\hline Diet 1 & 0.15 & 3.3 & 41 \\
Diet 2 & 0.07 & 1.4 & 23 \\
Diet 3 & 0.07 & 1.3 & 24 \\
Diet 4 & 0.41 & 8.5 & 66 \\
\hline
\end{tabular}

Diet 1 - Backyard cow milk + all other locally produced non-milk exposure pathways

Diet 2 - Locally produced commercial milk + all other locally produced non-milk exposure pathways

Diet 3 - Regionally mixed commercial milk + inhalation (other regionally mixed food items are minor contributors to the total)

Diet 4 - Goat milk (all other exposure pathways are of negligible importance compared to goat milk) 


\section{Location: Clinton}

Receptor: Female born in 1940

\begin{tabular}{lccc}
\hline & \multicolumn{3}{c}{ Thyroid Dose [cGy] } \\
Exposure Pathway & \multicolumn{2}{c}{$95 \%$ Subjective Confidence Interval } \\
\hline Backyard Cow Milk & lower limit & central estimate & upper limit \\
Commercial Milk (locally produced) & 0.5 & 3 & 17 \\
Commercial Milk (regionally mixed) & 0.16 & 0.99 & 6.6 \\
Goat Milk (locally produced) & 0.12 & 0.76 & 4.9 \\
Beef (locally produced) & 1.1 & 9.7 & 61 \\
Leafy Vegetables (locally produced) & 0.00094 & 0.017 & 0.36 \\
Eggs (locally produced) & 0.00043 & 0.0044 & 0.037 \\
Cottage Cheese (locally produced) & 0.03 & 0.22 & 1.5 \\
Inhalation & 0.00086 & 0.0087 & 0.097 \\
Mother's milk (mother on Diet 1) & 0.024 & 0.086 & 0.31 \\
Prenatal exposure (mother on Diet 1) & -- & -- & - \\
Diet 1 & -- & -- & -- \\
Diet 2 & 0.59 & 3.4 & 19 \\
Diet 3 & 0.26 & 1.4 & 8.3 \\
\hline
\end{tabular}

Excess Lifetime Risk [ ]

\begin{tabular}{lllc}
\hline Diet 1 & $2.0 \mathrm{E}-05$ & $3.4 \mathrm{E}-04$ & $4.3 \mathrm{E}-03$ \\
Diet 2 & $1.1 \mathrm{E}-05$ & $1.3 \mathrm{E}-04$ & $1.6 \mathrm{E}-03$ \\
Diet 3 & $7.4 \mathrm{E}-06$ & $8.2 \mathrm{E}-05$ & $1.0 \mathrm{E}-03$ \\
Diet 4 & $6.0 \mathrm{E}-05$ & $9.0 \mathrm{E}-04$ & $1.1 \mathrm{E}-02$ \\
\hline
\end{tabular}

\section{Relative Risk [ ]}

\begin{tabular}{lccc}
\hline Diet 1 & 1.009 & 1.08 & 1.77 \\
Diet 2 & 1.0035 & 1.034 & 1.38 \\
Diet 3 & 1.0023 & 1.021 & 1.24 \\
Diet 4 & 1.023 & 1.22 & 3.8 \\
\hline
\end{tabular}

\begin{tabular}{lccc}
\hline Diet 1 & 0.90 & 7.4 & 43 \\
Diet 2 & 0.35 & 3.3 & 27 \\
Diet 3 & 0.23 & 2.1 & 20 \\
Diet 4 & 2.22 & 18.1 & 73 \\
\hline
\end{tabular}

Diet 1 - Backyard cow milk + all other locally produced non-milk exposure pathways

Diet 2 - Locally produced commercial milk + all other locally produced non-milk exposure pathways

Diet 3 - Regionally mixed commercial milk + inhalation (other regionally mixed food items are minor contributors to the total)

Diet 4 - Goat milk (all other exposure pathways are of negligible importance compared to goat milk) 


\section{Location: Clinton}

\section{Receptor: Male born in 1940}

\begin{tabular}{lccc}
\hline & \multicolumn{3}{c}{ Thyroid Dose [cGy] } \\
& \multicolumn{2}{c}{$95 \%$ Subjective Confidence Interval } \\
Exposure Pathway & lower limit & central estimate & upper limit \\
\hline Backyard Cow Milk & 0.57 & 3.1 & 20 \\
Commercial Milk (locally produced) & 0.16 & 1 & 6.4 \\
Commercial Milk (regionally mixed) & 0.12 & 0.79 & 4.9 \\
Goat Milk (locally produced) & 1.3 & 10 & 69 \\
Beef (locally produced) & 0.0011 & 0.02 & 0.43 \\
Leafy Vegetables (locally produced) & 0.00043 & 0.0043 & 0.035 \\
Eggs (locally produced) & 0.037 & 0.26 & 1.7 \\
Cottage Cheese (locally produced) & 0.00088 & 0.0086 & 0.093 \\
Inhalation & 0.024 & 0.085 & 0.32 \\
Mother's milk (mother on Diet 1) & -- & -- & - \\
Prenatal exposure (mother on Diet 1) & -- & -- & - \\
Diet 1 & 0.68 & 3.6 & 22 \\
Diet 2 & 0.26 & 1.5 & 8 \\
Diet 3 & 0.17 & 0.91 & 5 \\
\hline
\end{tabular}

Excess Lifetime Risk [ ]

\begin{tabular}{llll}
\hline Diet 1 & $2.3 \mathrm{E}-06$ & $8.4 \mathrm{E}-05$ & $1.8 \mathrm{E}-03$ \\
Diet 2 & $1.1 \mathrm{E}-06$ & $3.5 \mathrm{E}-05$ & $8.2 \mathrm{E}-04$ \\
Diet 3 & $7.6 \mathrm{E}-07$ & $2.1 \mathrm{E}-05$ & $4.9 \mathrm{E}-04$ \\
Diet 4 & $6.8 \mathrm{E}-06$ & $2.0 \mathrm{E}-04$ & $4.7 \mathrm{E}-03$ \\
\hline
\end{tabular}

\section{Relative Risk [ ]}

\begin{tabular}{lccc}
\hline Diet 1 & 1.0025 & 1.056 & 2.1 \\
Diet 2 & 1.0011 & 1.023 & 1.49 \\
Diet 3 & 1.00071 & 1.013 & 1.32 \\
Diet 4 & 1.0065 & 1.15 & 4.3 \\
\hline
\end{tabular}

\section{Probability of Causation [\%]}

\begin{tabular}{lccc}
\hline Diet 1 & 0.25 & 5.3 & 51 \\
Diet 2 & 0.11 & 2.2 & 33 \\
Diet 3 & 0.07 & 1.3 & 24 \\
Diet 4 & 0.64 & 13.2 & 76 \\
\hline
\end{tabular}

Diet 1 - Backyard cow milk + all other locally produced non-milk exposure pathways

Diet 2 - Locally produced commercial milk + all other locally produced non-milk exposure pathways

Diet 3 - Regionally mixed commercial milk + inhalation (other regionally mixed food items are minor contributors to the total)

Diet 4 - Goat milk (all other exposure pathways are of negligible importance compared to goat milk) 
Location: Friendsville

Receptor: Female born in 1940

\begin{tabular}{lccc}
\hline & \multicolumn{3}{c}{ Thyroid Dose [cGy] } \\
Exposure Pathway & \multicolumn{2}{c}{$95 \%$ Subjective Confidence Interval } \\
\hline Backyard Cow Milk & lower limit & central estimate & upper limit \\
Commercial Milk (locally produced) & 0.28 & 1.7 & 9.6 \\
Commercial Milk (regionally mixed) & 0.089 & 0.57 & 3.7 \\
Goat Milk (locally produced) & 0.12 & 0.76 & 4.9 \\
Beef (locally produced) & 0.65 & 5.4 & 43 \\
Leafy Vegetables (locally produced) & 0.00055 & 0.0095 & 0.22 \\
Eggs (locally produced) & 0.00023 & 0.0025 & 0.023 \\
Cottage Cheese (locally produced) & 0.017 & 0.12 & 0.88 \\
Inhalation & 0.00048 & 0.005 & 0.051 \\
Mother's milk (mother on Diet 1) & 0.015 & 0.059 & 0.24 \\
Prenatal exposure (mother on Diet 1) & -- & -- & -- \\
Diet 1 & -- & -- & -- \\
Diet 2 & 0.34 & 1.9 & 11 \\
Diet 3 & 0.15 & 0.81 & 4.6 \\
\hline
\end{tabular}

Excess Lifetime Risk [ ]

\begin{tabular}{llll}
\hline Diet 1 & $1.4 \mathrm{E}-05$ & $1.9 \mathrm{E}-04$ & $2.3 \mathrm{E}-03$ \\
Diet 2 & $6.0 \mathrm{E}-06$ & $7.8 \mathrm{E}-05$ & $9.2 \mathrm{E}-04$ \\
Diet 3 & $6.7 \mathrm{E}-06$ & $7.9 \mathrm{E}-05$ & $9.9 \mathrm{E}-04$ \\
Diet 4 & $3.7 \mathrm{E}-05$ & $5.4 \mathrm{E}-04$ & $6.7 \mathrm{E}-03$ \\
\hline
\end{tabular}

\section{Relative Risk [ ]}

\begin{tabular}{lccc}
\hline Diet 1 & 1.0051 & 1.045 & 1.5 \\
Diet 2 & 1.0021 & 1.02 & 1.22 \\
Diet 3 & 1.0023 & 1.021 & 1.24 \\
Diet 4 & 1.013 & 1.13 & 2.7 \\
\hline
\end{tabular}

\section{Probability of Causation [\%]}

\begin{tabular}{lccc}
\hline Diet 1 & 0.51 & 4.3 & 33 \\
Diet 2 & 0.21 & 2.0 & 18 \\
Diet 3 & 0.23 & 2.0 & 19 \\
Diet 4 & 1.32 & 11.6 & 63 \\
\hline
\end{tabular}

Diet 1 - Backyard cow milk + all other locally produced non-milk exposure pathways

Diet 2 - Locally produced commercial milk + all other locally produced non-milk exposure pathways

Diet 3 - Regionally mixed commercial milk + inhalation (other regionally mixed food items are minor contributors to the total)

Diet 4 - Goat milk (all other exposure pathways are of negligible importance compared to goat milk) 
Location: Friendsville

Receptor: Male born in 1940

\begin{tabular}{lccc}
\hline & \multicolumn{3}{c}{ Thyroid Dose [cGy] } \\
& \multicolumn{2}{c}{$95 \%$ Subjective Confidence Interval } \\
Exposure Pathway & lower limit & central estimate & upper limit \\
\hline Backyard Cow Milk & 0.3 & 1.7 & 10 \\
Commercial Milk (locally produced) & 0.095 & 0.59 & 3.5 \\
Commercial Milk (regionally mixed) & 0.12 & 0.79 & 4.9 \\
Goat Milk (locally produced) & 0.7 & 5.7 & 45 \\
Beef (locally produced) & 0.00065 & 0.011 & 0.28 \\
Leafy Vegetables (locally produced) & 0.00023 & 0.0025 & 0.021 \\
Eggs (locally produced) & 0.021 & 0.14 & 1.1 \\
Cottage Cheese (locally produced) & 0.00049 & 0.0051 & 0.054 \\
Inhalation & 0.014 & 0.059 & 0.21 \\
Mother's milk (mother on Diet 1) & -- & -- & -- \\
Prenatal exposure (mother on Diet 1) & -- & -- & - \\
Diet 1 & 0.37 & 2 & 11 \\
Diet 2 & 0.15 & 0.86 & 4.5 \\
Diet 3 & 0.16 & 0.88 & 5 \\
\hline
\end{tabular}

Excess Lifetime Risk [ ]

\begin{tabular}{llll}
\hline Diet 1 & $1.5 \mathrm{E}-06$ & $4.7 \mathrm{E}-05$ & $1.1 \mathrm{E}-03$ \\
Diet 2 & $6.8 \mathrm{E}-07$ & $2.0 \mathrm{E}-05$ & $5.0 \mathrm{E}-04$ \\
Diet 3 & $7.3 \mathrm{E}-07$ & $2.0 \mathrm{E}-05$ & $4.9 \mathrm{E}-04$ \\
Diet 4 & $3.9 \mathrm{E}-06$ & $1.1 \mathrm{E}-04$ & $3.1 \mathrm{E}-03$ \\
\hline
\end{tabular}

\section{Relative Risk [ ]}

\begin{tabular}{lccc}
\hline Diet 1 & 1.0014 & 1.031 & 1.73 \\
Diet 2 & 1.00063 & 1.014 & 1.27 \\
Diet 3 & 1.00067 & 1.013 & 1.32 \\
Diet 4 & 1.0037 & 1.087 & 2.9 \\
\hline
\end{tabular}

\section{Probability of Causation [\%]}

\begin{tabular}{llll}
\hline Diet 1 & 0.14 & 3.0 & 42 \\
Diet 2 & 0.06 & 1.3 & 21 \\
Diet 3 & 0.07 & 1.3 & 24 \\
Diet 4 & 0.37 & 8.0 & 65 \\
\hline
\end{tabular}

Diet 1 - Backyard cow milk + all other locally produced non-milk exposure pathways

Diet 2 - Locally produced commercial milk + all other locally produced non-milk exposure pathways

Diet 3 - Regionally mixed commercial milk + inhalation (other regionally mixed food items are minor contributors to the total)

Diet 4 - Goat milk (all other exposure pathways are of negligible importance compared to goat milk) 
Location: Wartburg

Receptor: Female born in 1940

\begin{tabular}{lccc}
\hline & \multicolumn{3}{c}{ Thyroid Dose [cGy] } \\
Exposure Pathway & \multicolumn{2}{c}{$95 \%$ Subjective Confidence Interval } \\
\hline Backyard Cow Milk & lower limit & central estimate & upper limit \\
Commercial Milk (locally produced) & 0.064 & 0.47 & 2.6 \\
Commercial Milk (regionally mixed) & 0.023 & 0.15 & 1.1 \\
Goat Milk (locally produced) & 0.12 & 0.76 & 4.9 \\
Beef (locally produced) & 0.2 & 1.5 & 12 \\
Leafy Vegetables (locally produced) & 0.00017 & 0.0027 & 0.067 \\
Eggs (locally produced) & 0.000061 & 0.00068 & 0.0063 \\
Cottage Cheese (locally produced) & 0.004 & 0.033 & 0.25 \\
Inhalation & 0.0001 & 0.0014 & 0.014 \\
Mother's milk (mother on Diet 1) & 0.0035 & 0.014 & 0.06 \\
Prenatal exposure (mother on Diet 1) & -- & -- & -- \\
Diet 1 & -- & -- & -- \\
Diet 2 & 0.079 & 0.53 & 2.9 \\
Diet 3 & 0.037 & 0.22 & 1.5 \\
\hline
\end{tabular}

Excess Lifetime Risk [ ]

\begin{tabular}{llll}
\hline Diet 1 & $3.1 \mathrm{E}-06$ & $5.0 \mathrm{E}-05$ & $7.6 \mathrm{E}-04$ \\
Diet 2 & $1.7 \mathrm{E}-06$ & $2.1 \mathrm{E}-05$ & $3.0 \mathrm{E}-04$ \\
Diet 3 & $6.1 \mathrm{E}-06$ & $7.3 \mathrm{E}-05$ & $9.7 \mathrm{E}-04$ \\
Diet 4 & $9.2 \mathrm{E}-06$ & $1.4 \mathrm{E}-04$ & $2.0 \mathrm{E}-03$ \\
\hline
\end{tabular}

Relative Risk [ ]

\begin{tabular}{lccc}
\hline Diet 1 & 1.0014 & 1.013 & 1.17 \\
Diet 2 & 1.0006 & 1.0054 & 1.082 \\
Diet 3 & 1.0021 & 1.02 & 1.24 \\
Diet 4 & 1.0034 & 1.035 & 1.46 \\
\hline
\end{tabular}

\section{Probability of Causation [\%]}

\begin{tabular}{lccc}
\hline Diet 1 & 0.14 & 1.2 & 14 \\
Diet 2 & 0.06 & 0.5 & 8 \\
Diet 3 & 0.21 & 1.9 & 19 \\
Diet 4 & 0.34 & 3.4 & 32 \\
\hline
\end{tabular}

Diet 1 - Backyard cow milk + all other locally produced non-milk exposure pathways

Diet 2 - Locally produced commercial milk + all other locally produced non-milk exposure pathways

Diet 3 - Regionally mixed commercial milk + inhalation (other regionally mixed food items are minor contributors to the total)

Diet 4 - Goat milk (all other exposure pathways are of negligible importance compared to goat milk) 


\section{Location: Wartburg}

\section{Receptor: Male born in 1940}

\begin{tabular}{lccc}
\hline & \multicolumn{3}{c}{ Thyroid Dose [cGy] } \\
& \multicolumn{2}{c}{$95 \%$ Subjective Confidence Interval } \\
Exposure Pathway & lower limit & central estimate & upper limit \\
\hline Backyard Cow Milk & 0.071 & 0.49 & 2.9 \\
Commercial Milk (locally produced) & 0.024 & 0.16 & 1.2 \\
Commercial Milk (regionally mixed) & 0.12 & 0.79 & 4.9 \\
Goat Milk (locally produced) & 0.2 & 1.6 & 12 \\
Beef (locally produced) & 0.00019 & 0.0031 & 0.072 \\
Leafy Vegetables (locally produced) & 0.00006 & 0.00066 & 0.0064 \\
Eggs (locally produced) & 0.0052 & 0.039 & 0.3 \\
Cottage Cheese (locally produced) & 0.00012 & 0.0014 & 0.015 \\
Inhalation & 0.0034 & 0.014 & 0.057 \\
Mother's milk (mother on Diet 1) & -- & -- & - \\
Prenatal exposure (mother on Diet 1) & -- & -- & -- \\
Diet 1 & 0.085 & 0.56 & 3.2 \\
Diet 2 & 0.036 & 0.23 & 1.5 \\
Diet 3 & 0.13 & 0.81 & 4.9 \\
\hline
\end{tabular}

Excess Lifetime Risk [ ]

\begin{tabular}{llll}
\hline Diet 1 & $3.5 \mathrm{E}-07$ & $1.3 \mathrm{E}-05$ & $3.0 \mathrm{E}-04$ \\
Diet 2 & $1.7 \mathrm{E}-07$ & $5.9 \mathrm{E}-06$ & $1.3 \mathrm{E}-04$ \\
Diet 3 & $6.6 \mathrm{E}-07$ & $1.9 \mathrm{E}-05$ & $4.7 \mathrm{E}-04$ \\
Diet 4 & $1.0 \mathrm{E}-06$ & $3.3 \mathrm{E}-05$ & $8.6 \mathrm{E}-04$ \\
\hline
\end{tabular}

\section{Relative Risk [ ]}

\begin{tabular}{lccc}
\hline Diet 1 & 1.00035 & 1.0083 & 1.21 \\
Diet 2 & 1.00016 & 1.0039 & 1.084 \\
Diet 3 & 1.0006 & 1.013 & 1.31 \\
Diet 4 & 1.00088 & 1.023 & 1.62 \\
\hline
\end{tabular}

\section{Probability of Causation [\%]}

\begin{tabular}{llcc}
\hline Diet 1 & 0.04 & 0.8 & 17 \\
Diet 2 & 0.02 & 0.4 & 8 \\
Diet 3 & 0.06 & 1.2 & 24 \\
Diet 4 & 0.09 & 2.3 & 38 \\
\hline
\end{tabular}

Diet 1 - Backyard cow milk + all other locally produced non-milk exposure pathways

Diet 2 - Locally produced commercial milk + all other locally produced non-milk exposure pathways

Diet 3 - Regionally mixed commercial milk + inhalation (other regionally mixed food items are minor contributors to the total)

Diet 4 - Goat milk (all other exposure pathways are of negligible importance compared to goat milk) 
Location: Rockwood

Receptor: Female born in 1940

\begin{tabular}{lccc}
\hline & \multicolumn{3}{c}{ Thyroid Dose [cGy] } \\
Exposure Pathway & \multicolumn{2}{c}{$95 \%$ Subjective Confidence Interval } \\
\hline Backyard Cow Milk & lower limit & central estimate & upper limit \\
Commercial Milk (locally produced) & 0.34 & 1.9 & 11 \\
Commercial Milk (regionally mixed) & 0.1 & 0.66 & 4.4 \\
Goat Milk (locally produced) & 0.12 & 0.76 & 4.9 \\
Beef (locally produced) & 0.8 & 6.3 & 42 \\
Leafy Vegetables (locally produced) & 0.00068 & 0.011 & 0.25 \\
Eggs (locally produced) & 0.00026 & 0.0028 & 0.025 \\
Cottage Cheese (locally produced) & 0.02 & 0.14 & 0.97 \\
Inhalation & 0.00054 & 0.0057 & 0.061 \\
Mother's milk (mother on Diet 1) & 0.016 & 0.061 & 0.23 \\
Prenatal exposure (mother on Diet 1) & -- & -- & -- \\
Diet 1 & -- & -- & - \\
Diet 2 & 0.4 & 2.2 & 13 \\
Diet 3 & 0.18 & 0.92 & 5.6 \\
\hline
\end{tabular}

Excess Lifetime Risk [ ]

\begin{tabular}{llll}
\hline Diet 1 & $1.3 \mathrm{E}-05$ & $2.2 \mathrm{E}-04$ & $2.7 \mathrm{E}-03$ \\
Diet 2 & $6.9 \mathrm{E}-06$ & $9.0 \mathrm{E}-05$ & $1.1 \mathrm{E}-03$ \\
Diet 3 & $6.9 \mathrm{E}-06$ & $7.9 \mathrm{E}-05$ & $9.9 \mathrm{E}-04$ \\
Diet 4 & $4.2 \mathrm{E}-05$ & $6.0 \mathrm{E}-04$ & $6.7 \mathrm{E}-03$ \\
\hline
\end{tabular}

Relative Risk [ ]

\begin{tabular}{lccc}
\hline Diet 1 & 1.0059 & 1.052 & 1.56 \\
Diet 2 & 1.0025 & 1.022 & 1.25 \\
Diet 3 & 1.0023 & 1.021 & 1.24 \\
Diet 4 & 1.014 & 1.14 & 2.9 \\
\hline
\end{tabular}

\section{Probability of Causation [\%]}

\begin{tabular}{lccc}
\hline Diet 1 & 0.59 & 4.9 & 35 \\
Diet 2 & 0.25 & 2.2 & 20 \\
Diet 3 & 0.23 & 2.0 & 19 \\
Diet 4 & 1.40 & 12.0 & 64 \\
\hline
\end{tabular}

Diet 1 - Backyard cow milk + all other locally produced non-milk exposure pathways

Diet 2 - Locally produced commercial milk + all other locally produced non-milk exposure pathways

Diet 3 - Regionally mixed commercial milk + inhalation (other regionally mixed food items are minor contributors to the total)

Diet 4 - Goat milk (all other exposure pathways are of negligible importance compared to goat milk) 


\section{Location: Rockwood}

\section{Receptor: Male born in 1940}

\begin{tabular}{lccc}
\hline & \multicolumn{3}{c}{ Thyroid Dose [cGy] } \\
& \multicolumn{2}{c}{$95 \%$ Subjective Confidence Interval } \\
Exposure Pathway & lower limit & central estimate & upper limit \\
\hline Backyard Cow Milk & 0.35 & 2 & 12 \\
Commercial Milk (locally produced) & 0.11 & 0.67 & 4.5 \\
Commercial Milk (regionally mixed) & 0.12 & 0.79 & 4.9 \\
Goat Milk (locally produced) & 0.89 & 6.7 & 45 \\
Beef (locally produced) & 0.00081 & 0.013 & 0.3 \\
Leafy Vegetables (locally produced) & 0.00028 & 0.0028 & 0.025 \\
Eggs (locally produced) & 0.024 & 0.17 & 1.1 \\
Cottage Cheese (locally produced) & 0.00056 & 0.0058 & 0.06 \\
Inhalation & 0.016 & 0.061 & 0.23 \\
Mother's milk (mother on Diet 1) & -- & -- & - \\
Prenatal exposure (mother on Diet 1) & -- & -- & - \\
Diet 1 & 0.42 & 2.3 & 14 \\
Diet 2 & 0.18 & 0.97 & 5.6 \\
Diet 3 & 0.15 & 0.87 & 5 \\
\hline
\end{tabular}

Excess Lifetime Risk [ ]

\begin{tabular}{llll}
\hline Diet 1 & $1.9 \mathrm{E}-06$ & $5.3 \mathrm{E}-05$ & $1.2 \mathrm{E}-03$ \\
Diet 2 & $8.3 \mathrm{E}-07$ & $2.2 \mathrm{E}-05$ & $5.4 \mathrm{E}-04$ \\
Diet 3 & $7.3 \mathrm{E}-07$ & $2.0 \mathrm{E}-05$ & $4.9 \mathrm{E}-04$ \\
Diet 4 & $4.6 \mathrm{E}-06$ & $1.3 \mathrm{E}-04$ & $3.5 \mathrm{E}-03$ \\
\hline
\end{tabular}

\section{Relative Risk [ ]}

\begin{tabular}{lccc}
\hline Diet 1 & 1.0016 & 1.035 & 1.78 \\
Diet 2 & 1.00073 & 1.015 & 1.33 \\
Diet 3 & 1.00067 & 1.013 & 1.32 \\
Diet 4 & 1.0043 & 1.093 & 3.4 \\
\hline
\end{tabular}

\section{Probability of Causation [\%]}

\begin{tabular}{llll}
\hline Diet 1 & 0.16 & 3.4 & 43 \\
Diet 2 & 0.07 & 1.5 & 24 \\
Diet 3 & 0.07 & 1.3 & 24 \\
Diet 4 & 0.43 & 8.5 & 69 \\
\hline
\end{tabular}

Diet 1 - Backyard cow milk + all other locally produced non-milk exposure pathways

Diet 2 - Locally produced commercial milk + all other locally produced non-milk exposure pathways

Diet 3 - Regionally mixed commercial milk + inhalation (other regionally mixed food items are minor contributors to the total)

Diet 4 - Goat milk (all other exposure pathways are of negligible importance compared to goat milk) 


\section{Location: Louisville}

Receptor: Female born in 1940

\begin{tabular}{lccc}
\hline & \multicolumn{3}{c}{ Thyroid Dose [cGy] } \\
Exposure Pathway & $95 \%$ Subjective Confidence Interval \\
\hline Backyard Cow Milk & lower limit & central estimate & upper limit \\
Commercial Milk (locally produced) & 0.28 & 1.8 & 9.5 \\
Commercial Milk (regionally mixed) & 0.098 & 0.6 & 4 \\
Goat Milk (locally produced) & 0.12 & 0.76 & 4.9 \\
Beef (locally produced) & 0.7 & 5.7 & 40 \\
Leafy Vegetables (locally produced) & 0.00061 & 0.01 & 0.21 \\
Eggs (locally produced) & 0.00026 & 0.0026 & 0.023 \\
Cottage Cheese (locally produced) & 0.019 & 0.13 & 0.91 \\
Inhalation & 0.00049 & 0.0053 & 0.053 \\
Mother's milk (mother on Diet 1) & 0.016 & 0.06 & 0.24 \\
Prenatal exposure (mother on Diet 1) & -- & -- & - \\
Diet 1 & -- & -- & -- \\
Diet 2 & 0.35 & 2 & 11 \\
Diet 3 & 0.16 & 0.84 & 5.2 \\
\hline
\end{tabular}

Excess Lifetime Risk [ ]

\begin{tabular}{llll}
\hline Diet 1 & $1.3 \mathrm{E}-05$ & $1.9 \mathrm{E}-04$ & $2.6 \mathrm{E}-03$ \\
Diet 2 & $6.8 \mathrm{E}-06$ & $8.2 \mathrm{E}-05$ & $1.1 \mathrm{E}-03$ \\
Diet 3 & $7.0 \mathrm{E}-06$ & $7.9 \mathrm{E}-05$ & $9.9 \mathrm{E}-04$ \\
Diet 4 & $3.7 \mathrm{E}-05$ & $5.4 \mathrm{E}-04$ & $7.3 \mathrm{E}-03$ \\
\hline
\end{tabular}

Relative Risk [ ]

\begin{tabular}{lccc}
\hline Diet 1 & 1.0056 & 1.047 & 1.53 \\
Diet 2 & 1.0024 & 1.021 & 1.26 \\
Diet 3 & 1.0023 & 1.021 & 1.24 \\
Diet 4 & 1.014 & 1.13 & 2.8 \\
\hline
\end{tabular}

\section{Probability of Causation [\%]}

\begin{tabular}{lccc}
\hline Diet 1 & 0.56 & 4.5 & 34 \\
Diet 2 & 0.24 & 2.0 & 20 \\
Diet 3 & 0.23 & 2.0 & 19 \\
Diet 4 & 1.35 & 11.7 & 63 \\
\hline
\end{tabular}

Diet 1 - Backyard cow milk + all other locally produced non-milk exposure pathways

Diet 2 - Locally produced commercial milk + all other locally produced non-milk exposure pathways

Diet 3 - Regionally mixed commercial milk + inhalation (other regionally mixed food items are minor contributors to the total)

Diet 4 - Goat milk (all other exposure pathways are of negligible importance compared to goat milk) 


\section{Location: Louisville}

\section{Receptor: Male born in 1940}

\begin{tabular}{lccc}
\hline & \multicolumn{3}{c}{ Thyroid Dose [cGy] } \\
& \multicolumn{2}{c}{$95 \%$ Subjective Confidence Interval } \\
Exposure Pathway & lower limit & central estimate & upper limit \\
\hline Backyard Cow Milk & 0.32 & 1.8 & 9.9 \\
Commercial Milk (locally produced) & 0.1 & 0.61 & 4 \\
Commercial Milk (regionally mixed) & 0.12 & 0.79 & 4.9 \\
Goat Milk (locally produced) & 0.76 & 6.1 & 43 \\
Beef (locally produced) & 0.00073 & 0.012 & 0.25 \\
Leafy Vegetables (locally produced) & 0.00027 & 0.0026 & 0.022 \\
Eggs (locally produced) & 0.023 & 0.15 & 1.1 \\
Cottage Cheese (locally produced) & 0.00053 & 0.0053 & 0.054 \\
Inhalation & 0.016 & 0.061 & 0.23 \\
Mother's milk (mother on Diet 1) & -- & -- & - \\
Prenatal exposure (mother on Diet 1) & -- & -- & - \\
Diet 1 & 0.38 & 2.1 & 11 \\
Diet 2 & 0.17 & 0.9 & 5.1 \\
Diet 3 & 0.16 & 0.88 & 5 \\
\hline
\end{tabular}

Excess Lifetime Risk [ ]

\begin{tabular}{llll}
\hline Diet 1 & $1.5 \mathrm{E}-06$ & $5.1 \mathrm{E}-05$ & $1.1 \mathrm{E}-03$ \\
Diet 2 & $6.8 \mathrm{E}-07$ & $2.2 \mathrm{E}-05$ & $5.0 \mathrm{E}-04$ \\
Diet 3 & $7.3 \mathrm{E}-07$ & $2.0 \mathrm{E}-05$ & $4.9 \mathrm{E}-04$ \\
Diet 4 & $4.1 \mathrm{E}-06$ & $1.2 \mathrm{E}-04$ & $3.4 \mathrm{E}-03$ \\
\hline
\end{tabular}

\section{Relative Risk [ ]}

\begin{tabular}{lccc}
\hline Diet 1 & 1.0013 & 1.033 & 1.69 \\
Diet 2 & 1.00064 & 1.014 & 1.29 \\
Diet 3 & 1.00067 & 1.013 & 1.32 \\
Diet 4 & 1.0037 & 1.089 & 3 \\
\hline
\end{tabular}

\section{Probability of Causation [\%]}

\begin{tabular}{llll}
\hline Diet 1 & 0.13 & 3.2 & 40 \\
Diet 2 & 0.06 & 1.4 & 23 \\
Diet 3 & 0.07 & 1.3 & 24 \\
Diet 4 & 0.37 & 8.1 & 66 \\
\hline
\end{tabular}

Diet 1 - Backyard cow milk + all other locally produced non-milk exposure pathways

Diet 2 - Locally produced commercial milk + all other locally produced non-milk exposure pathways

Diet 3 - Regionally mixed commercial milk + inhalation (other regionally mixed food items are minor contributors to the total)

Diet 4 - Goat milk (all other exposure pathways are of negligible importance compared to goat milk) 
Location: Barnardville

Receptor: Female born in 1940

\begin{tabular}{lccc}
\hline & \multicolumn{3}{c}{ Thyroid Dose [cGy] } \\
Exposure Pathway & \multicolumn{2}{c}{$95 \%$ Subjective Confidence Interval } \\
\hline Backyard Cow Milk & lower limit & central estimate & upper limit \\
Commercial Milk (locally produced) & 0.34 & 2 & 12 \\
Commercial Milk (regionally mixed) & 0.11 & 0.68 & 4.4 \\
Goat Milk (locally produced) & 0.12 & 0.76 & 4.9 \\
Beef (locally produced) & 0.86 & 6.5 & 44 \\
Leafy Vegetables (locally produced) & 0.00072 & 0.012 & 0.24 \\
Eggs (locally produced) & 0.00028 & 0.003 & 0.026 \\
Cottage Cheese (locally produced) & 0.02 & 0.15 & 1 \\
Inhalation & 0.00054 & 0.0061 & 0.063 \\
Mother's milk (mother on Diet 1) & 0.019 & 0.07 & 0.28 \\
Prenatal exposure (mother on Diet 1) & -- & -- & -- \\
Diet 1 & -- & -- & -- \\
Diet 2 & 0.41 & 2.3 & 13 \\
Diet 3 & 0.19 & 0.96 & 5.8 \\
\hline
\end{tabular}

Excess Lifetime Risk [ ]

\begin{tabular}{llll}
\hline Diet 1 & $1.4 \mathrm{E}-05$ & $2.3 \mathrm{E}-04$ & $3.0 \mathrm{E}-03$ \\
Diet 2 & $7.4 \mathrm{E}-06$ & $9.4 \mathrm{E}-05$ & $1.2 \mathrm{E}-03$ \\
Diet 3 & $6.9 \mathrm{E}-06$ & $8.0 \mathrm{E}-05$ & $1.0 \mathrm{E}-03$ \\
Diet 4 & $4.4 \mathrm{E}-05$ & $6.3 \mathrm{E}-04$ & $7.9 \mathrm{E}-03$ \\
\hline
\end{tabular}

\section{Relative Risk [ ]}

\begin{tabular}{lccc}
\hline Diet 1 & 1.006 & 1.054 & 1.53 \\
Diet 2 & 1.0027 & 1.023 & 1.26 \\
Diet 3 & 1.0023 & 1.021 & 1.24 \\
Diet 4 & 1.015 & 1.15 & 2.9 \\
\hline
\end{tabular}

\begin{tabular}{llcc} 
& \multicolumn{3}{c}{ Probability of Causation [\%] } \\
\hline Diet 1 & 0.60 & 5.1 & 35 \\
Diet 2 & 0.27 & 2.3 & 20 \\
Diet 3 & 0.23 & 2.0 & 20 \\
Diet 4 & 1.52 & 12.8 & 65 \\
\hline
\end{tabular}

Diet 1 - Backyard cow milk + all other locally produced non-milk exposure pathways

Diet 2 - Locally produced commercial milk + all other locally produced non-milk exposure pathways

Diet 3 - Regionally mixed commercial milk + inhalation (other regionally mixed food items are minor contributors to the total)

Diet 4 - Goat milk (all other exposure pathways are of negligible importance compared to goat milk) 


\section{Location: Barnardville}

Receptor: Male born in 1940

\begin{tabular}{lccc}
\hline & \multicolumn{3}{c}{ Thyroid Dose [cGy] } \\
& \multicolumn{2}{c}{$95 \%$ Subjective Confidence Interval } \\
Exposure Pathway & lower limit & central estimate & upper limit \\
\hline Backyard Cow Milk & 0.35 & 2.1 & 12 \\
Commercial Milk (locally produced) & 0.11 & 0.7 & 4.3 \\
Commercial Milk (regionally mixed) & 0.12 & 0.79 & 4.9 \\
Goat Milk (locally produced) & 0.93 & 7 & 47 \\
Beef (locally produced) & 0.00081 & 0.014 & 0.29 \\
Leafy Vegetables (locally produced) & 0.00028 & 0.0029 & 0.026 \\
Eggs (locally produced) & 0.025 & 0.17 & 1.2 \\
Cottage Cheese (locally produced) & 0.00058 & 0.0061 & 0.06 \\
Inhalation & 0.019 & 0.071 & 0.27 \\
Mother's milk (mother on Diet 1) & -- & -- & - \\
Prenatal exposure (mother on Diet 1) & -- & -- & - \\
Diet 1 & 0.43 & 2.5 & 14 \\
Diet 2 & 0.19 & 1 & 5.9 \\
Diet 3 & 0.16 & 0.89 & 5 \\
\hline
\end{tabular}

Excess Lifetime Risk [ ]

\begin{tabular}{llll}
\hline Diet 1 & $1.9 \mathrm{E}-06$ & $5.6 \mathrm{E}-05$ & $1.3 \mathrm{E}-03$ \\
Diet 2 & $8.1 \mathrm{E}-07$ & $2.4 \mathrm{E}-05$ & $5.8 \mathrm{E}-04$ \\
Diet 3 & $7.4 \mathrm{E}-07$ & $2.1 \mathrm{E}-05$ & $4.9 \mathrm{E}-04$ \\
Diet 4 & $4.7 \mathrm{E}-06$ & $1.4 \mathrm{E}-04$ & $3.5 \mathrm{E}-03$ \\
\hline
\end{tabular}

\section{Relative Risk [ ]}

\begin{tabular}{lccc}
\hline Diet 1 & 1.0016 & 1.038 & 1.82 \\
Diet 2 & 1.00076 & 1.016 & 1.33 \\
Diet 3 & 1.00069 & 1.013 & 1.32 \\
Diet 4 & 1.0043 & 1.099 & 3.5 \\
\hline
\end{tabular}

\section{Probability of Causation [\%]}

\begin{tabular}{llll}
\hline Diet 1 & 0.16 & 3.6 & 45 \\
Diet 2 & 0.08 & 1.6 & 25 \\
Diet 3 & 0.07 & 1.3 & 24 \\
Diet 4 & 0.43 & 9.0 & 71 \\
\hline
\end{tabular}

Diet 1 - Backyard cow milk + all other locally produced non-milk exposure pathways

Diet 2 - Locally produced commercial milk + all other locally produced non-milk exposure pathways

Diet 3 - Regionally mixed commercial milk + inhalation (other regionally mixed food items are minor contributors to the total)

Diet 4 - Goat milk (all other exposure pathways are of negligible importance compared to goat milk) 
Location: Greenback

Receptor: Female born in 1940

\begin{tabular}{lccc}
\hline & \multicolumn{3}{c}{ Thyroid Dose [cGy] } \\
Exposure Pathway & $95 \%$ Subjective Confidence Interval \\
\hline Backyard Cow Milk & lower limit & central estimate & upper limit \\
Commercial Milk (locally produced) & 0.19 & 1.1 & 7 \\
Commercial Milk (regionally mixed) & 0.056 & 0.38 & 2.7 \\
Goat Milk (locally produced) & 0.12 & 0.76 & 4.9 \\
Beef (locally produced) & 0.42 & 3.5 & 28 \\
Leafy Vegetables (locally produced) & 0.00039 & 0.0065 & 0.13 \\
Eggs (locally produced) & 0.00014 & 0.0016 & 0.015 \\
Cottage Cheese (locally produced) & 0.012 & 0.08 & 0.62 \\
Inhalation & 0.0003 & 0.0032 & 0.039 \\
Mother's milk (mother on Diet 1) & 0.011 & 0.04 & 0.16 \\
Prenatal exposure (mother on Diet 1) & -- & -- & -- \\
Diet 1 & -- & -- & -- \\
Diet 2 & 0.23 & 1.3 & 7.8 \\
Diet 3 & 0.1 & 0.53 & 3.4 \\
\hline
\end{tabular}

Excess Lifetime Risk [ ]

\begin{tabular}{llll}
\hline Diet 1 & $8.2 \mathrm{E}-06$ & $1.2 \mathrm{E}-04$ & $1.7 \mathrm{E}-03$ \\
Diet 2 & $4.5 \mathrm{E}-06$ & $5.3 \mathrm{E}-05$ & $6.5 \mathrm{E}-04$ \\
Diet 3 & $6.6 \mathrm{E}-06$ & $7.7 \mathrm{E}-05$ & $9.8 \mathrm{E}-04$ \\
Diet 4 & $2.4 \mathrm{E}-05$ & $3.3 \mathrm{E}-04$ & $4.3 \mathrm{E}-03$ \\
\hline
\end{tabular}

Relative Risk [ ]

\begin{tabular}{lccc}
\hline Diet 1 & 1.0032 & 1.029 & 1.34 \\
Diet 2 & 1.0015 & 1.013 & 1.14 \\
Diet 3 & 1.0023 & 1.02 & 1.24 \\
Diet 4 & 1.0083 & 1.078 & 2.2 \\
\hline
\end{tabular}

\section{Probability of Causation [\%]}

\begin{tabular}{llcc}
\hline Diet 1 & 0.32 & 2.9 & 25 \\
Diet 2 & 0.15 & 1.3 & 12 \\
Diet 3 & 0.23 & 2.0 & 19 \\
Diet 4 & 0.82 & 7.2 & 54 \\
\hline
\end{tabular}

Diet 1 - Backyard cow milk + all other locally produced non-milk exposure pathways

Diet 2 - Locally produced commercial milk + all other locally produced non-milk exposure pathways

Diet 3 - Regionally mixed commercial milk + inhalation (other regionally mixed food items are minor contributors to the total)

Diet 4 - Goat milk (all other exposure pathways are of negligible importance compared to goat milk) 
Location: Greenback

Receptor: Male born in 1940

\begin{tabular}{lccc}
\hline & \multicolumn{3}{c}{ Thyroid Dose [cGy] } \\
& \multicolumn{2}{c}{$95 \%$ Subjective Confidence Interval } \\
Exposure Pathway & lower limit & central estimate & upper limit \\
\hline Backyard Cow Milk & 0.2 & 1.1 & 7.3 \\
Commercial Milk (locally produced) & 0.056 & 0.39 & 2.7 \\
Commercial Milk (regionally mixed) & 0.12 & 0.79 & 4.9 \\
Goat Milk (locally produced) & 0.44 & 3.8 & 29 \\
Beef (locally produced) & 0.00046 & 0.0078 & 0.16 \\
Leafy Vegetables (locally produced) & 0.00016 & 0.0016 & 0.015 \\
Eggs (locally produced) & 0.014 & 0.097 & 0.71 \\
Cottage Cheese (locally produced) & 0.00031 & 0.0033 & 0.039 \\
Inhalation & 0.011 & 0.04 & 0.17 \\
Mother's milk (mother on Diet 1) & -- & -- & -- \\
Prenatal exposure (mother on Diet 1) & -- & -- & -- \\
Diet 1 & 0.24 & 1.3 & 8.4 \\
Diet 2 & 0.11 & 0.57 & 3.6 \\
Diet 3 & 0.15 & 0.85 & 4.9 \\
\hline
\end{tabular}

Excess Lifetime Risk [ ]

\begin{tabular}{lllc}
\hline Diet 1 & $8.9 \mathrm{E}-07$ & $2.9 \mathrm{E}-05$ & $7.1 \mathrm{E}-04$ \\
Diet 2 & $4.6 \mathrm{E}-07$ & $1.3 \mathrm{E}-05$ & $3.1 \mathrm{E}-04$ \\
Diet 3 & $7.1 \mathrm{E}-07$ & $2.0 \mathrm{E}-05$ & $4.8 \mathrm{E}-04$ \\
Diet 4 & $2.6 \mathrm{E}-06$ & $7.5 \mathrm{E}-05$ & $2.1 \mathrm{E}-03$ \\
\hline
\end{tabular}

\section{Relative Risk [ ]}

\begin{tabular}{lccc}
\hline Diet 1 & 1.00084 & 1.02 & 1.46 \\
Diet 2 & 1.00039 & 1.0093 & 1.18 \\
Diet 3 & 1.00065 & 1.013 & 1.31 \\
Diet 4 & 1.0022 & 1.056 & 2.2 \\
\hline
\end{tabular}

\section{Probability of Causation [\%]}

\begin{tabular}{llll}
\hline Diet 1 & 0.08 & 1.9 & 32 \\
Diet 2 & 0.04 & 0.9 & 15 \\
Diet 3 & 0.07 & 1.3 & 24 \\
Diet 4 & 0.22 & 5.3 & 54 \\
\hline
\end{tabular}

Diet 1 - Backyard cow milk + all other locally produced non-milk exposure pathways

Diet 2 - Locally produced commercial milk + all other locally produced non-milk exposure pathways

Diet 3 - Regionally mixed commercial milk + inhalation (other regionally mixed food items are minor contributors to the total)

Diet 4 - Goat milk (all other exposure pathways are of negligible importance compared to goat milk) 


\section{Location: Rockford}

Receptor: Female born in 1940

\begin{tabular}{lccc}
\hline & \multicolumn{3}{c}{ Thyroid Dose [cGy] } \\
Exposure Pathway & \multicolumn{2}{c}{$95 \%$ Subjective Confidence Interval } \\
\hline Backyard Cow Milk & lower limit & central estimate & upper limit \\
Commercial Milk (locally produced) & 0.31 & 1.7 & 10 \\
Commercial Milk (regionally mixed) & 0.093 & 0.59 & 3.9 \\
Goat Milk (locally produced) & 0.12 & 0.76 & 4.9 \\
Beef (locally produced) & 0.72 & 5.5 & 39 \\
Leafy Vegetables (locally produced) & 0.00058 & 0.01 & 0.21 \\
Eggs (locally produced) & 0.00023 & 0.0026 & 0.022 \\
Cottage Cheese (locally produced) & 0.019 & 0.13 & 0.87 \\
Inhalation & 0.0005 & 0.0051 & 0.055 \\
Mother's milk (mother on Diet 1) & 0.017 & 0.06 & 0.23 \\
Prenatal exposure (mother on Diet 1) & -- & -- & -- \\
Diet 1 & -- & -- & -- \\
Diet 2 & 0.37 & 2 & 11 \\
Diet 3 & 0.17 & 0.84 & 4.8 \\
\hline
\end{tabular}

Excess Lifetime Risk [ ]

\begin{tabular}{llll}
\hline Diet 1 & $1.3 \mathrm{E}-05$ & $1.9 \mathrm{E}-04$ & $2.5 \mathrm{E}-03$ \\
Diet 2 & $7.0 \mathrm{E}-06$ & $8.0 \mathrm{E}-05$ & $9.7 \mathrm{E}-04$ \\
Diet 3 & $6.9 \mathrm{E}-06$ & $7.9 \mathrm{E}-05$ & $9.9 \mathrm{E}-04$ \\
Diet 4 & $3.7 \mathrm{E}-05$ & $5.4 \mathrm{E}-04$ & $6.7 \mathrm{E}-03$ \\
\hline
\end{tabular}

\section{Relative Risk [ ]}

\begin{tabular}{lccc}
\hline Diet 1 & 1.0051 & 1.046 & 1.47 \\
Diet 2 & 1.0022 & 1.02 & 1.22 \\
Diet 3 & 1.0023 & 1.021 & 1.24 \\
Diet 4 & 1.014 & 1.13 & 2.7 \\
\hline
\end{tabular}

\section{Probability of Causation [\%]}

\begin{tabular}{lccc}
\hline Diet 1 & 0.51 & 4.4 & 32 \\
Diet 2 & 0.22 & 2.0 & 18 \\
Diet 3 & 0.23 & 2.0 & 19 \\
Diet 4 & 1.35 & 11.2 & 62 \\
\hline
\end{tabular}

Diet 1 - Backyard cow milk + all other locally produced non-milk exposure pathways

Diet 2 - Locally produced commercial milk + all other locally produced non-milk exposure pathways

Diet 3 - Regionally mixed commercial milk + inhalation (other regionally mixed food items are minor contributors to the total)

Diet 4 - Goat milk (all other exposure pathways are of negligible importance compared to goat milk) 


\section{Location: Rockford}

\section{Receptor: Male born in 1940}

\begin{tabular}{lccc}
\hline & \multicolumn{3}{c}{ Thyroid Dose [cGy] } \\
& \multicolumn{2}{c}{$95 \%$ Subjective Confidence Interval } \\
Exposure Pathway & lower limit & central estimate & upper limit \\
\hline Backyard Cow Milk & 0.34 & 1.8 & 11 \\
Commercial Milk (locally produced) & 0.095 & 0.61 & 3.8 \\
Commercial Milk (regionally mixed) & 0.12 & 0.79 & 4.9 \\
Goat Milk (locally produced) & 0.79 & 5.9 & 43 \\
Beef (locally produced) & 0.00069 & 0.012 & 0.24 \\
Leafy Vegetables (locally produced) & 0.00025 & 0.0026 & 0.021 \\
Eggs (locally produced) & 0.021 & 0.15 & 1 \\
Cottage Cheese (locally produced) & 0.00052 & 0.0051 & 0.053 \\
Inhalation & 0.017 & 0.061 & 0.23 \\
Mother's milk (mother on Diet 1) & -- & -- & -- \\
Prenatal exposure (mother on Diet 1) & -- & -- & -- \\
Diet 1 & 0.4 & 2.1 & 12 \\
Diet 2 & 0.17 & 0.89 & 5.1 \\
Diet 3 & 0.16 & 0.87 & 5 \\
\hline
\end{tabular}

Excess Lifetime Risk [ ]

\begin{tabular}{llll}
\hline Diet 1 & $1.3 \mathrm{E}-06$ & $4.9 \mathrm{E}-05$ & $1.1 \mathrm{E}-03$ \\
Diet 2 & $7.2 \mathrm{E}-07$ & $2.0 \mathrm{E}-05$ & $5.0 \mathrm{E}-04$ \\
Diet 3 & $7.4 \mathrm{E}-07$ & $2.0 \mathrm{E}-05$ & $4.8 \mathrm{E}-04$ \\
Diet 4 & $4.0 \mathrm{E}-06$ & $1.2 \mathrm{E}-04$ & $3.0 \mathrm{E}-03$ \\
\hline
\end{tabular}

\section{Relative Risk [ ]}

\begin{tabular}{lccc}
\hline Diet 1 & 1.0013 & 1.033 & 1.67 \\
Diet 2 & 1.00065 & 1.014 & 1.3 \\
Diet 3 & 1.00067 & 1.013 & 1.32 \\
Diet 4 & 1.0038 & 1.092 & 3 \\
\hline
\end{tabular}

\section{Probability of Causation [\%]}

\begin{tabular}{llll}
\hline Diet 1 & 0.13 & 3.2 & 40 \\
Diet 2 & 0.07 & 1.4 & 23 \\
Diet 3 & 0.07 & 1.3 & 24 \\
Diet 4 & 0.38 & 8.4 & 66 \\
\hline
\end{tabular}

Diet 1 - Backyard cow milk + all other locally produced non-milk exposure pathways

Diet 2 - Locally produced commercial milk + all other locally produced non-milk exposure pathways

Diet 3 - Regionally mixed commercial milk + inhalation (other regionally mixed food items are minor contributors to the total)

Diet 4 - Goat milk (all other exposure pathways are of negligible importance compared to goat milk) 
Location: Lake City

Receptor: Female born in 1940

\begin{tabular}{lccc}
\hline & \multicolumn{3}{c}{ Thyroid Dose [cGy] } \\
Exposure Pathway & \multicolumn{2}{c}{$95 \%$ Subjective Confidence Interval } \\
\hline Backyard Cow Milk & lower limit & central estimate & upper limit \\
Commercial Milk (locally produced) & 0.18 & 1.1 & 5.4 \\
Commercial Milk (regionally mixed) & 0.059 & 0.36 & 2.4 \\
Goat Milk (locally produced) & 0.12 & 0.76 & 4.9 \\
Beef (locally produced) & 0.46 & 3.6 & 24 \\
Leafy Vegetables (locally produced) & 0.00035 & 0.0063 & 0.14 \\
Eggs (locally produced) & 0.00015 & 0.0016 & 0.013 \\
Cottage Cheese (locally produced) & 0.01 & 0.077 & 0.53 \\
Inhalation & 0.00029 & 0.0031 & 0.033 \\
Mother's milk (mother on Diet 1) & 0.0088 & 0.032 & 0.13 \\
Prenatal exposure (mother on Diet 1) & -- & -- & -- \\
Diet 1 & -- & -- & -- \\
Diet 2 & 0.21 & 1.2 & 6.2 \\
Diet 3 & 0.098 & 0.5 & 3 \\
\hline
\end{tabular}

Excess Lifetime Risk [ ]

\begin{tabular}{llll}
\hline Diet 1 & $7.9 \mathrm{E}-06$ & $1.1 \mathrm{E}-04$ & $1.5 \mathrm{E}-03$ \\
Diet 2 & $4.0 \mathrm{E}-06$ & $4.9 \mathrm{E}-05$ & $6.2 \mathrm{E}-04$ \\
Diet 3 & $6.4 \mathrm{E}-06$ & $7.6 \mathrm{E}-05$ & $9.8 \mathrm{E}-04$ \\
Diet 4 & $2.3 \mathrm{E}-05$ & $3.3 \mathrm{E}-04$ & $4.5 \mathrm{E}-03$ \\
\hline
\end{tabular}

Relative Risk [ ]

\begin{tabular}{lccc}
\hline Diet 1 & 1.0033 & 1.029 & 1.3 \\
Diet 2 & 1.0014 & 1.012 & 1.16 \\
Diet 3 & 1.0022 & 1.02 & 1.24 \\
Diet 4 & 1.008 & 1.079 & 2 \\
\hline
\end{tabular}

\begin{tabular}{llcc}
\hline Diet 1 & 0.33 & 2.8 & 23 \\
Diet 2 & 0.14 & 1.2 & 14 \\
Diet 3 & 0.22 & 2.0 & 19 \\
Diet 4 & 0.79 & 7.3 & 50 \\
\hline
\end{tabular}

Diet 1 - Backyard cow milk + all other locally produced non-milk exposure pathways

Diet 2 - Locally produced commercial milk + all other locally produced non-milk exposure pathways

Diet 3 - Regionally mixed commercial milk + inhalation (other regionally mixed food items are minor contributors to the total)

Diet 4 - Goat milk (all other exposure pathways are of negligible importance compared to goat milk) 
Location: Lake City

Receptor: Male born in 1940

\begin{tabular}{lccc}
\hline & \multicolumn{3}{c}{ Thyroid Dose [cGy] } \\
& \multicolumn{2}{c}{$95 \%$ Subjective Confidence Interval } \\
Exposure Pathway & lower limit & central estimate & upper limit \\
\hline Backyard Cow Milk & 0.2 & 1.1 & 6.1 \\
Commercial Milk (locally produced) & 0.06 & 0.37 & 2.4 \\
Commercial Milk (regionally mixed) & 0.12 & 0.79 & 4.9 \\
Goat Milk (locally produced) & 0.54 & 3.7 & 24 \\
Beef (locally produced) & 0.00041 & 0.0073 & 0.17 \\
Leafy Vegetables (locally produced) & 0.00016 & 0.0016 & 0.014 \\
Eggs (locally produced) & 0.013 & 0.088 & 0.63 \\
Cottage Cheese (locally produced) & 0.00035 & 0.0032 & 0.033 \\
Inhalation & 0.0092 & 0.033 & 0.12 \\
Mother's milk (mother on Diet 1) & -- & -- & -- \\
Prenatal exposure (mother on Diet 1) & -- & -- & -- \\
Diet 1 & 0.23 & 1.2 & 6.8 \\
Diet 2 & 0.1 & 0.54 & 3.1 \\
Diet 3 & 0.14 & 0.84 & 4.9 \\
\hline
\end{tabular}

Excess Lifetime Risk [ ]

\begin{tabular}{llll}
\hline Diet 1 & $8.8 \mathrm{E}-07$ & $3.0 \mathrm{E}-05$ & $6.7 \mathrm{E}-04$ \\
Diet 2 & $4.4 \mathrm{E}-07$ & $1.3 \mathrm{E}-05$ & $2.9 \mathrm{E}-04$ \\
Diet 3 & $7.0 \mathrm{E}-07$ & $2.0 \mathrm{E}-05$ & $4.8 \mathrm{E}-04$ \\
Diet 4 & $2.7 \mathrm{E}-06$ & $7.5 \mathrm{E}-05$ & $1.9 \mathrm{E}-03$ \\
\hline
\end{tabular}

\section{Relative Risk [ ]}

\begin{tabular}{lccc}
\hline Diet 1 & 1.00082 & 1.02 & 1.41 \\
Diet 2 & 1.00037 & 1.0084 & 1.17 \\
Diet 3 & 1.00063 & 1.013 & 1.32 \\
Diet 4 & 1.0024 & 1.052 & 2.3 \\
\hline
\end{tabular}

\section{Probability of Causation [\%]}

\begin{tabular}{llll}
\hline Diet 1 & 0.08 & 1.9 & 29 \\
Diet 2 & 0.04 & 0.8 & 15 \\
Diet 3 & 0.06 & 1.3 & 24 \\
Diet 4 & 0.24 & 5.0 & 56 \\
\hline
\end{tabular}

Diet 1 - Backyard cow milk + all other locally produced non-milk exposure pathways

Diet 2 - Locally produced commercial milk + all other locally produced non-milk exposure pathways

Diet 3 - Regionally mixed commercial milk + inhalation (other regionally mixed food items are minor contributors to the total)

Diet 4 - Goat milk (all other exposure pathways are of negligible importance compared to goat milk) 
Location: Sweetwater

Receptor: Female born in 1940

\begin{tabular}{lccc}
\hline & \multicolumn{3}{c}{ Thyroid Dose [cGy] } \\
Exposure Pathway & \multicolumn{2}{c}{$95 \%$ Subjective Confidence Interval } \\
\hline Backyard Cow Milk & lower limit & central estimate & upper limit \\
Commercial Milk (locally produced) & 0.2 & 1.3 & 7.1 \\
Commercial Milk (regionally mixed) & 0.07 & 0.44 & 2.9 \\
Goat Milk (locally produced) & 0.12 & 0.76 & 4.9 \\
Beef (locally produced) & 0.45 & 4 & 31 \\
Leafy Vegetables (locally produced) & 0.00043 & 0.0074 & 0.14 \\
Eggs (locally produced) & 0.00019 & 0.0019 & 0.016 \\
Cottage Cheese (locally produced) & 0.014 & 0.092 & 0.66 \\
Inhalation & 0.00039 & 0.0039 & 0.039 \\
Mother's milk (mother on Diet 1) & 0.013 & 0.047 & 0.18 \\
Prenatal exposure (mother on Diet 1) & -- & -- & -- \\
Diet 1 & -- & -- & - \\
Diet 2 & 0.25 & 1.5 & 8.1 \\
Diet 3 & 0.12 & 0.63 & 3.7 \\
\hline
\end{tabular}

Excess Lifetime Risk [ ]

\begin{tabular}{llll}
\hline Diet 1 & $9.9 \mathrm{E}-06$ & $1.4 \mathrm{E}-04$ & $1.8 \mathrm{E}-03$ \\
Diet 2 & $5.1 \mathrm{E}-06$ & $6.0 \mathrm{E}-05$ & $7.9 \mathrm{E}-04$ \\
Diet 3 & $6.6 \mathrm{E}-06$ & $7.8 \mathrm{E}-05$ & $9.8 \mathrm{E}-04$ \\
Diet 4 & $2.7 \mathrm{E}-05$ & $4.0 \mathrm{E}-04$ & $5.5 \mathrm{E}-03$ \\
\hline
\end{tabular}

Relative Risk [ ]

\begin{tabular}{lccc}
\hline Diet 1 & 1.0043 & 1.034 & 1.37 \\
Diet 2 & 1.0018 & 1.016 & 1.18 \\
Diet 3 & 1.0022 & 1.02 & 1.24 \\
Diet 4 & 1.01 & 1.098 & 2.4 \\
\hline
\end{tabular}

\begin{tabular}{llcc} 
& \multicolumn{3}{c}{ Probability of Causation [\%] } \\
\hline Diet 1 & 0.43 & 3.3 & 27 \\
Diet 2 & 0.18 & 1.5 & 15 \\
Diet 3 & 0.22 & 2.0 & 19 \\
Diet 4 & 1.04 & 9.0 & 57 \\
\hline
\end{tabular}

Diet 1 - Backyard cow milk + all other locally produced non-milk exposure pathways

Diet 2 - Locally produced commercial milk + all other locally produced non-milk exposure pathways

Diet 3 - Regionally mixed commercial milk + inhalation (other regionally mixed food items are minor contributors to the total)

Diet 4 - Goat milk (all other exposure pathways are of negligible importance compared to goat milk) 
Location: Sweetwater

Receptor: Male born in 1940

\begin{tabular}{lccc}
\hline & \multicolumn{3}{c}{ Thyroid Dose [cGy] } \\
Exposure Pathway & \multicolumn{2}{c}{$95 \%$ Subjective Confidence Interval } \\
\hline Backyard Cow Milk & lower limit & central estimate & upper limit \\
Commercial Milk (locally produced) & 0.22 & 1.3 & 6.9 \\
Commercial Milk (regionally mixed) & 0.075 & 0.46 & 2.8 \\
Goat Milk (locally produced) & 0.12 & 0.79 & 4.9 \\
Beef (locally produced) & 0.49 & 4.3 & 36 \\
Leafy Vegetables (locally produced) & 0.00052 & 0.009 & 0.17 \\
Eggs (locally produced) & 0.0002 & 0.0019 & 0.016 \\
Cottage Cheese (locally produced) & 0.017 & 0.11 & 0.81 \\
Inhalation & 0.0004 & 0.0039 & 0.04 \\
Mother's milk (mother on Diet 1) & 0.013 & 0.049 & 0.18 \\
Prenatal exposure (mother on Diet 1) & -- & -- & -- \\
Diet 1 & -- & -- & -- \\
Diet 2 & 0.27 & 1.5 & 7.8 \\
Diet 3 & 0.12 & 0.69 & 3.6 \\
\hline
\end{tabular}

Excess Lifetime Risk [ ]

\begin{tabular}{llll}
\hline Diet 1 & $1.2 \mathrm{E}-06$ & $3.8 \mathrm{E}-05$ & $7.4 \mathrm{E}-04$ \\
Diet 2 & $4.9 \mathrm{E}-07$ & $1.6 \mathrm{E}-05$ & $3.8 \mathrm{E}-04$ \\
Diet 3 & $7.2 \mathrm{E}-07$ & $2.0 \mathrm{E}-05$ & $4.8 \mathrm{E}-04$ \\
Diet 4 & $2.9 \mathrm{E}-06$ & $8.6 \mathrm{E}-05$ & $2.6 \mathrm{E}-03$ \\
\hline
\end{tabular}

\section{Relative Risk [ ]}

\begin{tabular}{lccc}
\hline Diet 1 & 1.00097 & 1.025 & 1.51 \\
Diet 2 & 1.00049 & 1.011 & 1.21 \\
Diet 3 & 1.00066 & 1.013 & 1.32 \\
Diet 4 & 1.0026 & 1.068 & 2.4 \\
\hline
\end{tabular}

\section{Probability of Causation [\%]}

\begin{tabular}{llcc}
\hline Diet 1 & 0.10 & 2.4 & 34 \\
Diet 2 & 0.05 & 1.0 & 17 \\
Diet 3 & 0.07 & 1.3 & 24 \\
Diet 4 & 0.26 & 6.4 & 58 \\
\hline
\end{tabular}

Diet 1 - Backyard cow milk + all other locally produced non-milk exposure pathways

Diet 2 - Locally produced commercial milk + all other locally produced non-milk exposure pathways

Diet 3 - Regionally mixed commercial milk + inhalation (other regionally mixed food items are minor contributors to the total)

Diet 4 - Goat milk (all other exposure pathways are of negligible importance compared to goat milk) 


\section{Location: Knoxville}

Receptor: Female born in 1940

\begin{tabular}{lccc}
\hline & \multicolumn{3}{c}{ Thyroid Dose [cGy] } \\
Exposure Pathway & $95 \%$ Subjective Confidence Interval \\
\hline Backyard Cow Milk & lower limit & central estimate & upper limit \\
Commercial Milk (locally produced) & 0.37 & 2.2 & 12 \\
Commercial Milk (regionally mixed) & 0.12 & 0.76 & 5 \\
Goat Milk (locally produced) & 0.12 & 0.76 & 4.9 \\
Beef (locally produced) & 0.93 & 7.1 & 49 \\
Leafy Vegetables (locally produced) & 0.00072 & 0.012 & 0.26 \\
Eggs (locally produced) & 0.00032 & 0.0033 & 0.028 \\
Cottage Cheese (locally produced) & 0.022 & 0.16 & 1.1 \\
Inhalation & 0.00066 & 0.0065 & 0.067 \\
Mother's milk (mother on Diet 1) & 0.02 & 0.075 & 0.29 \\
Prenatal exposure (mother on Diet 1) & -- & -- & -- \\
Diet 1 & -- & -- & -- \\
Diet 2 & 0.44 & 2.5 & 14 \\
Diet 3 & 0.21 & 1.1 & 6.3 \\
\hline
\end{tabular}

Excess Lifetime Risk [ ]

\begin{tabular}{llll}
\hline Diet 1 & $1.6 \mathrm{E}-05$ & $2.5 \mathrm{E}-04$ & $3.0 \mathrm{E}-03$ \\
Diet 2 & $8.3 \mathrm{E}-06$ & $1.0 \mathrm{E}-04$ & $1.2 \mathrm{E}-03$ \\
Diet 3 & $7.1 \mathrm{E}-06$ & $8.1 \mathrm{E}-05$ & $1.0 \mathrm{E}-03$ \\
Diet 4 & $4.6 \mathrm{E}-05$ & $6.9 \mathrm{E}-04$ & $8.5 \mathrm{E}-03$ \\
\hline
\end{tabular}

\section{Relative Risk [ ]}

\begin{tabular}{lccc}
\hline Diet 1 & 1.0068 & 1.06 & 1.6 \\
Diet 2 & 1.0029 & 1.026 & 1.28 \\
Diet 3 & 1.0023 & 1.021 & 1.24 \\
Diet 4 & 1.018 & 1.16 & 3.2 \\
\hline
\end{tabular}

\begin{tabular}{lccc}
\hline Diet 1 & 0.67 & 5.6 & 37 \\
Diet 2 & 0.29 & 2.5 & 22 \\
Diet 3 & 0.23 & 2.1 & 20 \\
Diet 4 & 1.76 & 14.0 & 68 \\
\hline
\end{tabular}

Diet 1 - Backyard cow milk + all other locally produced non-milk exposure pathways

Diet 2 - Locally produced commercial milk + all other locally produced non-milk exposure pathways

Diet 3 - Regionally mixed commercial milk + inhalation (other regionally mixed food items are minor contributors to the total)

Diet 4 - Goat milk (all other exposure pathways are of negligible importance compared to goat milk) 


\section{Location: Knoxville}

\section{Receptor: Male born in 1940}

\begin{tabular}{lccc}
\hline & \multicolumn{3}{c}{ Thyroid Dose [cGy] } \\
& \multicolumn{2}{c}{$95 \%$ Subjective Confidence Interval } \\
Exposure Pathway & lower limit & central estimate & upper limit \\
\hline Backyard Cow Milk & 0.39 & 2.3 & 13 \\
Commercial Milk (locally produced) & 0.12 & 0.78 & 5 \\
Commercial Milk (regionally mixed) & 0.12 & 0.79 & 4.9 \\
Goat Milk (locally produced) & 1 & 7.5 & 52 \\
Beef (locally produced) & 0.00088 & 0.015 & 0.31 \\
Leafy Vegetables (locally produced) & 0.00033 & 0.0032 & 0.026 \\
Eggs (locally produced) & 0.027 & 0.19 & 1.3 \\
Cottage Cheese (locally produced) & 0.00071 & 0.0065 & 0.07 \\
Inhalation & 0.021 & 0.076 & 0.28 \\
Mother's milk (mother on Diet 1) & -- & -- & - \\
Prenatal exposure (mother on Diet 1) & -- & -- & - \\
Diet 1 & 0.47 & 2.6 & 15 \\
Diet 2 & 0.21 & 1.1 & 6.4 \\
Diet 3 & 0.16 & 0.89 & 5 \\
\hline
\end{tabular}

Excess Lifetime Risk [ ]

\begin{tabular}{llll}
\hline Diet 1 & $1.7 \mathrm{E}-06$ & $6.4 \mathrm{E}-05$ & $1.3 \mathrm{E}-03$ \\
Diet 2 & $8.8 \mathrm{E}-07$ & $2.7 \mathrm{E}-05$ & $6.0 \mathrm{E}-04$ \\
Diet 3 & $7.6 \mathrm{E}-07$ & $2.1 \mathrm{E}-05$ & $4.9 \mathrm{E}-04$ \\
Diet 4 & $5.1 \mathrm{E}-06$ & $1.5 \mathrm{E}-04$ & $3.5 \mathrm{E}-03$ \\
\hline
\end{tabular}

\section{Relative Risk [ ]}

\begin{tabular}{lccc}
\hline Diet 1 & 1.0017 & 1.041 & 1.83 \\
Diet 2 & 1.00082 & 1.017 & 1.37 \\
Diet 3 & 1.00069 & 1.013 & 1.32 \\
Diet 4 & 1.0049 & 1.11 & 3.5 \\
\hline
\end{tabular}

\section{Probability of Causation [\%]}

\begin{tabular}{lccc}
\hline Diet 1 & 0.17 & 3.9 & 45 \\
Diet 2 & 0.08 & 1.7 & 27 \\
Diet 3 & 0.07 & 1.3 & 24 \\
Diet 4 & 0.48 & 10.2 & 71 \\
\hline
\end{tabular}

Diet 1 - Backyard cow milk + all other locally produced non-milk exposure pathways

Diet 2 - Locally produced commercial milk + all other locally produced non-milk exposure pathways

Diet 3 - Regionally mixed commercial milk + inhalation (other regionally mixed food items are minor contributors to the total)

Diet 4 - Goat milk (all other exposure pathways are of negligible importance compared to goat milk) 
Location: Maryville

Receptor: Female born in 1940

\begin{tabular}{lccc}
\hline & \multicolumn{3}{c}{ Thyroid Dose [cGy] } \\
& \multicolumn{2}{c}{$95 \%$ Subjective Confidence Interval } \\
Exposure Pathway & lower limit & central estimate & upper limit \\
\hline Backyard Cow Milk & 0.19 & 1.1 & 6.2 \\
Commercial Milk (locally produced) & 0.054 & 0.36 & 2.3 \\
Commercial Milk (regionally mixed) & 0.12 & 0.76 & 4.9 \\
Goat Milk (locally produced) & 0.47 & 3.4 & 23 \\
Beef (locally produced) & 0.0004 & 0.0062 & 0.14 \\
Leafy Vegetables (locally produced) & 0.00014 & 0.0016 & 0.013 \\
Eggs (locally produced) & 0.01 & 0.08 & 0.54 \\
Cottage Cheese (locally produced) & 0.00028 & 0.0033 & 0.033 \\
Inhalation & 0.011 & 0.04 & 0.18 \\
Mother's milk (mother on Diet 1) & -- & -- & -- \\
Prenatal exposure (mother on Diet 1) & -- & -- & -- \\
Diet 1 & 0.23 & 1.2 & 6.9 \\
Diet 2 & 0.095 & 0.52 & 3.1 \\
Diet 3 & 0.14 & 0.81 & 5 \\
\hline
\end{tabular}

Excess Lifetime Risk [ ]

\begin{tabular}{llll}
\hline Diet 1 & $7.7 \mathrm{E}-06$ & $1.2 \mathrm{E}-04$ & $1.6 \mathrm{E}-03$ \\
Diet 2 & $4.2 \mathrm{E}-06$ & $5.0 \mathrm{E}-05$ & $6.5 \mathrm{E}-04$ \\
Diet 3 & $6.4 \mathrm{E}-06$ & $7.7 \mathrm{E}-05$ & $9.8 \mathrm{E}-04$ \\
Diet 4 & $2.2 \mathrm{E}-05$ & $3.5 \mathrm{E}-04$ & $4.4 \mathrm{E}-03$ \\
\hline
\end{tabular}

\section{Relative Risk [ ]}

\begin{tabular}{lccc}
\hline Diet 1 & 1.003 & 1.028 & 1.28 \\
Diet 2 & 1.0014 & 1.012 & 1.14 \\
Diet 3 & 1.0022 & 1.02 & 1.24 \\
Diet 4 & 1.0076 & 1.078 & 1.94 \\
\hline
\end{tabular}

\section{Probability of Causation [\%]}

\begin{tabular}{llcc}
\hline Diet 1 & 0.30 & 2.7 & 22 \\
Diet 2 & 0.14 & 1.2 & 13 \\
Diet 3 & 0.22 & 2.0 & 19 \\
Diet 4 & 0.75 & 7.2 & 48 \\
\hline
\end{tabular}

Diet 1 - Backyard cow milk + all other locally produced non-milk exposure pathways

Diet 2 - Locally produced commercial milk + all other locally produced non-milk exposure pathways

Diet 3 - Regionally mixed commercial milk + inhalation (other regionally mixed food items are minor contributors to the total)

Diet 4 - Goat milk (all other exposure pathways are of negligible importance compared to goat milk) 


\section{Location: Maryville}

\section{Receptor: Male born in 1940}

\begin{tabular}{lccc}
\hline & \multicolumn{3}{c}{ Thyroid Dose [cGy] } \\
Exposure Pathway & 95\% Subjective Confidence Interval \\
\hline Backyard Cow Milk & lower limit & central estimate & upper limit \\
Commercial Milk (locally produced) & 0.2 & 1.1 & 7 \\
Commercial Milk (regionally mixed) & 0.055 & 0.38 & 2.3 \\
Goat Milk (locally produced) & 0.12 & 0.79 & 4.9 \\
Beef (locally produced) & 0.47 & 3.7 & 24 \\
Leafy Vegetables (locally produced) & 0.00046 & 0.0073 & 0.16 \\
Eggs (locally produced) & 0.00015 & 0.0015 & 0.013 \\
Cottage Cheese (locally produced) & 0.013 & 0.093 & 0.65 \\
Inhalation & 0.00032 & 0.0032 & 0.033 \\
Mother's milk (mother on Diet 1) & 0.01 & 0.041 & 0.17 \\
Prenatal exposure (mother on Diet 1) & -- & -- & - \\
Diet 1 & -- & -- & -- \\
Diet 2 & 0.24 & 1.3 & 7.7 \\
Diet 3 & 0.1 & 0.56 & 3.1 \\
\hline
\end{tabular}

Excess Lifetime Risk [ ]

\begin{tabular}{llll}
\hline Diet 1 & $1.0 \mathrm{E}-06$ & $3.0 \mathrm{E}-05$ & $7.3 \mathrm{E}-04$ \\
Diet 2 & $4.4 \mathrm{E}-07$ & $1.3 \mathrm{E}-05$ & $2.8 \mathrm{E}-04$ \\
Diet 3 & $7.1 \mathrm{E}-07$ & $2.0 \mathrm{E}-05$ & $4.8 \mathrm{E}-04$ \\
Diet 4 & $2.7 \mathrm{E}-06$ & $7.7 \mathrm{E}-05$ & $1.8 \mathrm{E}-03$ \\
\hline
\end{tabular}

\section{Relative Risk [ ]}

\begin{tabular}{lccc}
\hline Diet 1 & 1.00096 & 1.02 & 1.4 \\
Diet 2 & 1.00042 & 1.009 & 1.17 \\
Diet 3 & 1.00064 & 1.013 & 1.32 \\
Diet 4 & 1.0027 & 1.053 & 2.5 \\
\hline
\end{tabular}

\section{Probability of Causation [\%]}

\begin{tabular}{llll}
\hline Diet 1 & 0.10 & 2.0 & 28 \\
Diet 2 & 0.04 & 0.9 & 14 \\
Diet 3 & 0.06 & 1.3 & 24 \\
Diet 4 & 0.27 & 5.0 & 60 \\
\hline
\end{tabular}

Diet 1 - Backyard cow milk + all other locally produced non-milk exposure pathways

Diet 2 - Locally produced commercial milk + all other locally produced non-milk exposure pathways

Diet 3 - Regionally mixed commercial milk + inhalation (other regionally mixed food items are minor contributors to the total)

Diet 4 - Goat milk (all other exposure pathways are of negligible importance compared to goat milk) 
Location: Cedar Grove

Receptor: Female born in 1940

\begin{tabular}{lccc}
\hline & \multicolumn{3}{c}{ Thyroid Dose [cGy] } \\
Exposure Pathway & $95 \%$ Subjective Confidence Interval \\
\hline Backyard Cow Milk & lower limit & central estimate & upper limit \\
Commercial Milk (locally produced) & 0.36 & 2 & 11 \\
Commercial Milk (regionally mixed) & 0.11 & 0.68 & 4.4 \\
Goat Milk (locally produced) & 0.12 & 0.76 & 4.9 \\
Beef (locally produced) & 0.87 & 6.3 & 44 \\
Leafy Vegetables (locally produced) & 0.00066 & 0.012 & 0.24 \\
Eggs (locally produced) & 0.00028 & 0.003 & 0.025 \\
Cottage Cheese (locally produced) & 0.022 & 0.15 & 1 \\
Inhalation & 0.0006 & 0.0059 & 0.061 \\
Mother's milk (mother on Diet 1) & 0.019 & 0.065 & 0.24 \\
Prenatal exposure (mother on Diet 1) & -- & -- & -- \\
Diet 1 & -- & -- & -- \\
Diet 2 & 0.43 & 2.2 & 13 \\
Diet 3 & 0.2 & 0.96 & 5.5 \\
\hline
\end{tabular}

Excess Lifetime Risk [ ]

\begin{tabular}{llll}
\hline Diet 1 & $1.5 \mathrm{E}-05$ & $2.2 \mathrm{E}-04$ & $2.8 \mathrm{E}-03$ \\
Diet 2 & $8.1 \mathrm{E}-06$ & $9.2 \mathrm{E}-05$ & $1.1 \mathrm{E}-03$ \\
Diet 3 & $7.0 \mathrm{E}-06$ & $8.0 \mathrm{E}-05$ & $9.9 \mathrm{E}-04$ \\
Diet 4 & $4.4 \mathrm{E}-05$ & $6.3 \mathrm{E}-04$ & $7.8 \mathrm{E}-03$ \\
\hline
\end{tabular}

\section{Relative Risk [ ]}

\begin{tabular}{lccc}
\hline Diet 1 & 1.0059 & 1.053 & 1.54 \\
Diet 2 & 1.0026 & 1.024 & 1.26 \\
Diet 3 & 1.0023 & 1.021 & 1.24 \\
Diet 4 & 1.017 & 1.15 & 2.9 \\
\hline
\end{tabular}

\section{Probability of Causation [\%]}

\begin{tabular}{lccc}
\hline Diet 1 & 0.59 & 5.1 & 35 \\
Diet 2 & 0.26 & 2.3 & 21 \\
Diet 3 & 0.23 & 2.0 & 19 \\
Diet 4 & 1.64 & 12.7 & 66 \\
\hline
\end{tabular}

Diet 1 - Backyard cow milk + all other locally produced non-milk exposure pathways

Diet 2 - Locally produced commercial milk + all other locally produced non-milk exposure pathways

Diet 3 - Regionally mixed commercial milk + inhalation (other regionally mixed food items are minor contributors to the total)

Diet 4 - Goat milk (all other exposure pathways are of negligible importance compared to goat milk) 
Location: Cedar Grove

Receptor: Male born in 1940

\begin{tabular}{lccc}
\hline & \multicolumn{3}{c}{ Thyroid Dose [cGy] } \\
& \multicolumn{2}{c}{$95 \%$ Subjective Confidence Interval } \\
Exposure Pathway & lower limit & central estimate & upper limit \\
\hline Backyard Cow Milk & 0.39 & 2.1 & 12 \\
Commercial Milk (locally produced) & 0.11 & 0.7 & 4.5 \\
Commercial Milk (regionally mixed) & 0.12 & 0.79 & 4.9 \\
Goat Milk (locally produced) & 0.98 & 6.8 & 48 \\
Beef (locally produced) & 0.0008 & 0.014 & 0.28 \\
Leafy Vegetables (locally produced) & 0.00029 & 0.0029 & 0.024 \\
Eggs (locally produced) & 0.025 & 0.17 & 1.1 \\
Cottage Cheese (locally produced) & 0.00061 & 0.0059 & 0.06 \\
Inhalation & 0.019 & 0.066 & 0.24 \\
Mother's milk (mother on Diet 1) & -- & -- & - \\
Prenatal exposure (mother on Diet 1) & -- & -- & - \\
Diet 1 & 0.45 & 2.4 & 14 \\
Diet 2 & 0.2 & 1 & 5.9 \\
Diet 3 & 0.16 & 0.88 & 5 \\
\hline
\end{tabular}

Excess Lifetime Risk [ ]

\begin{tabular}{llll}
\hline Diet 1 & $1.5 \mathrm{E}-06$ & $5.6 \mathrm{E}-05$ & $1.2 \mathrm{E}-03$ \\
Diet 2 & $8.0 \mathrm{E}-07$ & $2.3 \mathrm{E}-05$ & $5.4 \mathrm{E}-04$ \\
Diet 3 & $7.4 \mathrm{E}-07$ & $2.1 \mathrm{E}-05$ & $4.9 \mathrm{E}-04$ \\
Diet 4 & $4.4 \mathrm{E}-06$ & $1.3 \mathrm{E}-04$ & $3.3 \mathrm{E}-03$ \\
\hline
\end{tabular}

Relative Risk [ ]

\begin{tabular}{lccc}
\hline Diet 1 & 1.0015 & 1.038 & 1.77 \\
Diet 2 & 1.00074 & 1.016 & 1.33 \\
Diet 3 & 1.00068 & 1.013 & 1.32 \\
Diet 4 & 1.0045 & 1.11 & 3.3 \\
\hline
\end{tabular}

\section{Probability of Causation [\%]}

\begin{tabular}{llll}
\hline Diet 1 & 0.15 & 3.6 & 43 \\
Diet 2 & 0.07 & 1.6 & 25 \\
Diet 3 & 0.07 & 1.3 & 24 \\
Diet 4 & 0.45 & 9.5 & 69 \\
\hline
\end{tabular}

Diet 1 - Backyard cow milk + all other locally produced non-milk exposure pathways

Diet 2 - Locally produced commercial milk + all other locally produced non-milk exposure pathways

Diet 3 - Regionally mixed commercial milk + inhalation (other regionally mixed food items are minor contributors to the total)

Diet 4 - Goat milk (all other exposure pathways are of negligible importance compared to goat milk) 
Location: Bradbury

Receptor: Female born in 1944

\begin{tabular}{lccc}
\hline & \multicolumn{3}{c}{ Thyroid Dose [cGy] } \\
& \multicolumn{2}{c}{$95 \%$ Subjective Confidence Interval } \\
Exposure Pathway & lower limit & central estimate & upper limit \\
\hline Backyard Cow Milk & 4.6 & 26 & 150 \\
Commercial Milk (locally produced) & 1.3 & 8.7 & 61 \\
Commercial Milk (regionally mixed) & 0.19 & 1.2 & 7.9 \\
Goat Milk (locally produced) & 11 & 83 & 560 \\
Beef (locally produced) & 0.0076 & 0.13 & 2.6 \\
Leafy Vegetables (locally produced) & 0.0022 & 0.026 & 0.24 \\
Eggs (locally produced) & 0.24 & 1.8 & 13 \\
Cottage Cheese (locally produced) & 0.0059 & 0.066 & 0.78 \\
Inhalation & 0.12 & 0.45 & 1.7 \\
Mother's milk (mother on Diet 1) & 0.00083 & 0.029 & 0.79 \\
Prenatal exposure (mother on Diet 1) & -- & -- & -- \\
Diet 1 & 5.2 & 29 & 170 \\
Diet 2 & 1.9 & 12 & 75 \\
Diet 3 & 0.4 & 1.7 & 9.5 \\
\hline
\end{tabular}

Excess Lifetime Risk [ ]

\begin{tabular}{llll}
\hline Diet 1 & $3.0 \mathrm{E}-04$ & $5.0 \mathrm{E}-03$ & $6.4 \mathrm{E}-02$ \\
Diet 2 & $1.5 \mathrm{E}-04$ & $1.9 \mathrm{E}-03$ & $2.6 \mathrm{E}-02$ \\
Diet 3 & $2.6 \mathrm{E}-05$ & $2.6 \mathrm{E}-04$ & $2.8 \mathrm{E}-03$ \\
Diet 4 & $8.6 \mathrm{E}-04$ & $1.4 \mathrm{E}-02$ & $1.6 \mathrm{E}-01$ \\
\hline
\end{tabular}

Relative Risk [ ]

\begin{tabular}{lccc}
\hline Diet 1 & 1.13 & 2.2 & 14 \\
Diet 2 & 1.053 & 1.5 & 7.2 \\
Diet 3 & 1.0082 & 1.071 & 1.73 \\
Diet 4 & 1.34 & 4.1 & 52 \\
\hline
\end{tabular}

\section{Probability of Causation [\%]}

\begin{tabular}{lccc}
\hline Diet 1 & 11.13 & 54.4 & 93 \\
Diet 2 & 5.00 & 33.1 & 86 \\
Diet 3 & 0.82 & 6.6 & 42 \\
Diet 4 & 25.48 & 75.4 & 98 \\
\hline
\end{tabular}

Diet 1 - Backyard cow milk + all other locally produced non-milk exposure pathways

Diet 2 - Locally produced commercial milk + all other locally produced non-milk exposure pathways

Diet 3 - Regionally mixed commercial milk + inhalation (other regionally mixed food items are minor contributors to the total)

Diet 4 - Goat milk (all other exposure pathways are of negligible importance compared to goat milk) 


\section{Location: Bradbury}

Receptor: Male born in 1944

\begin{tabular}{lccc}
\hline & \multicolumn{3}{c}{ Thyroid Dose [cGy] } \\
& \multicolumn{2}{c}{$95 \%$ Subjective Confidence Interval } \\
Exposure Pathway & lower limit & central estimate & upper limit \\
\hline Backyard Cow Milk & 4.6 & 26 & 160 \\
Commercial Milk (locally produced) & 1.2 & 9 & 61 \\
Commercial Milk (regionally mixed) & 0.19 & 1.2 & 7.1 \\
Goat Milk (locally produced) & 10 & 78 & 520 \\
Beef (locally produced) & 0.0077 & 0.13 & 2.7 \\
Leafy Vegetables (locally produced) & 0.0022 & 0.026 & 0.26 \\
Eggs (locally produced) & 0.28 & 2.1 & 14 \\
Cottage Cheese (locally produced) & 0.0055 & 0.066 & 0.83 \\
Inhalation & 0.12 & 0.45 & 1.6 \\
Mother's milk (mother on Diet 1) & 0.00083 & 0.029 & 0.79 \\
Prenatal exposure (mother on Diet 1) & -- & -- & -- \\
Diet 1 & 5.4 & 29 & 190 \\
Diet 2 & 2 & 13 & 77 \\
Diet 3 & 0.36 & 1.7 & 9.4 \\
\hline
\end{tabular}

Excess Lifetime Risk [ ]

\begin{tabular}{lllc}
\hline Diet 1 & $4.1 \mathrm{E}-05$ & $1.3 \mathrm{E}-03$ & $2.5 \mathrm{E}-02$ \\
Diet 2 & $1.9 \mathrm{E}-05$ & $5.2 \mathrm{E}-04$ & $1.1 \mathrm{E}-02$ \\
Diet 3 & $2.9 \mathrm{E}-06$ & $7.4 \mathrm{E}-05$ & $1.4 \mathrm{E}-03$ \\
Diet 4 & $1.2 \mathrm{E}-04$ & $3.1 \mathrm{E}-03$ & $6.8 \mathrm{E}-02$ \\
\hline
\end{tabular}

\section{Relative Risk [ ]}

\begin{tabular}{lccc}
\hline Diet 1 & 1.042 & 1.84 & 20 \\
Diet 2 & 1.017 & 1.34 & 9.2 \\
Diet 3 & 1.0025 & 1.044 & 1.99 \\
Diet 4 & 1.12 & 3.3 & 48 \\
\hline
\end{tabular}

\begin{tabular}{lccc}
\hline Diet 1 & 4.01 & 45.6 & 95 \\
Diet 2 & 1.67 & 25.6 & 89 \\
Diet 3 & 0.25 & 4.2 & 50 \\
Diet 4 & 10.99 & 69.7 & 98 \\
\hline
\end{tabular}

Diet 1 - Backyard cow milk + all other locally produced non-milk exposure pathways

Diet 2 - Locally produced commercial milk + all other locally produced non-milk exposure pathways

Diet 3 - Regionally mixed commercial milk + inhalation (other regionally mixed food items are minor contributors to the total)

Diet 4 - Goat milk (all other exposure pathways are of negligible importance compared to goat milk) 
Location: Gallaher Bend

Receptor: Female born in 1944

\begin{tabular}{lccc}
\hline & \multicolumn{3}{c}{ Thyroid Dose [cGy] } \\
& \multicolumn{2}{c}{$9 \%$ Subjective Confidence Interval } \\
Exposure Pathway & lower limit & central estimate & upper limit \\
\hline Backyard Cow Milk & 5.2 & 33 & 170 \\
Commercial Milk (locally produced) & 1.7 & 11 & 67 \\
Commercial Milk (regionally mixed) & 0.19 & 1.2 & 7.9 \\
Goat Milk (locally produced) & 13 & 100 & 650 \\
Beef (locally produced) & 0.0087 & 0.16 & 3.4 \\
Leafy Vegetables (locally produced) & 0.0029 & 0.032 & 0.29 \\
Eggs (locally produced) & 0.32 & 2.2 & 15 \\
Cottage Cheese (locally produced) & 0.0078 & 0.084 & 0.88 \\
Inhalation & 0.14 & 0.55 & 2 \\
Mother's milk (mother on Diet 1) & 0.0011 & 0.035 & 0.99 \\
Prenatal exposure (mother on Diet 1) & -- & -- & -- \\
Diet 1 & 6.1 & 36 & 190 \\
Diet 2 & 2.4 & 15 & 83 \\
Diet 3 & 0.44 & 1.8 & 9.8 \\
\hline
\end{tabular}

Excess Lifetime Risk [ ]

\begin{tabular}{llll}
\hline Diet 1 & $3.9 \mathrm{E}-04$ & $5.8 \mathrm{E}-03$ & $8.1 \mathrm{E}-02$ \\
Diet 2 & $1.9 \mathrm{E}-04$ & $2.3 \mathrm{E}-03$ & $3.0 \mathrm{E}-02$ \\
Diet 3 & $2.8 \mathrm{E}-05$ & $2.7 \mathrm{E}-04$ & $2.9 \mathrm{E}-03$ \\
Diet 4 & $1.0 \mathrm{E}-03$ & $1.7 \mathrm{E}-02$ & $2.0 \mathrm{E}-01$ \\
\hline
\end{tabular}

\section{Relative Risk [ ]}

\begin{tabular}{lccc}
\hline Diet 1 & 1.15 & 2.5 & 18 \\
Diet 2 & 1.06 & 1.63 & 8.7 \\
Diet 3 & 1.0093 & 1.074 & 1.76 \\
Diet 4 & 1.44 & 4.9 & 58 \\
\hline
\end{tabular}

\begin{tabular}{lccc}
\hline Diet 1 & \multicolumn{3}{c}{ Probability of Causation [\%] } \\
Diet 2 & 12.86 & 60.6 & 94 \\
Diet 3 & 5.63 & 38.8 & 88 \\
Diet 4 & 0.92 & 6.9 & 43 \\
\hline
\end{tabular}

Diet 1 - Backyard cow milk + all other locally produced non-milk exposure pathways

Diet 2 - Locally produced commercial milk + all other locally produced non-milk exposure pathways

Diet 3 - Regionally mixed commercial milk + inhalation (other regionally mixed food items are minor contributors to the total)

Diet 4 - Goat milk (all other exposure pathways are of negligible importance compared to goat milk) 
Location: Gallaher Bend

Receptor: Male born in 1944

\begin{tabular}{lccc}
\hline & \multicolumn{3}{c}{ Thyroid Dose [cGy] } \\
& \multicolumn{2}{c}{$95 \%$ Subjective Confidence Interval } \\
Exposure Pathway & lower limit & central estimate & upper limit \\
\hline Backyard Cow Milk & 5.3 & 33 & 170 \\
Commercial Milk (locally produced) & 1.6 & 11 & 71 \\
Commercial Milk (regionally mixed) & 0.19 & 1.2 & 7.1 \\
Goat Milk (locally produced) & 11 & 98 & 630 \\
Beef (locally produced) & 0.009 & 0.16 & 3.4 \\
Leafy Vegetables (locally produced) & 0.0028 & 0.033 & 0.28 \\
Eggs (locally produced) & 0.37 & 2.6 & 18 \\
Cottage Cheese (locally produced) & 0.0072 & 0.084 & 0.99 \\
Inhalation & 0.15 & 0.57 & 2 \\
Mother's milk (mother on Diet 1) & 0.0011 & 0.035 & 0.99 \\
Prenatal exposure (mother on Diet 1) & -- & -- & - \\
Diet 1 & 6.1 & 37 & 200 \\
Diet 2 & 2.4 & 15 & 87 \\
Diet 3 & 0.4 & 1.9 & 10 \\
\hline
\end{tabular}

Excess Lifetime Risk [ ]

\begin{tabular}{llll}
\hline Diet 1 & $5.4 \mathrm{E}-05$ & $1.5 \mathrm{E}-03$ & $3.0 \mathrm{E}-02$ \\
Diet 2 & $2.1 \mathrm{E}-05$ & $6.1 \mathrm{E}-04$ & $1.2 \mathrm{E}-02$ \\
Diet 3 & $3.0 \mathrm{E}-06$ & $8.1 \mathrm{E}-05$ & $1.5 \mathrm{E}-03$ \\
Diet 4 & $1.4 \mathrm{E}-04$ & $3.7 \mathrm{E}-03$ & $8.4 \mathrm{E}-02$ \\
\hline
\end{tabular}

\section{Relative Risk [ ]}

\begin{tabular}{lccc}
\hline Diet 1 & 1.049 & 2 & 25 \\
Diet 2 & 1.019 & 1.41 & 10.4 \\
Diet 3 & 1.0026 & 1.045 & 2 \\
Diet 4 & 1.14 & 3.8 & 56 \\
\hline
\end{tabular}

\begin{tabular}{lccc}
\hline Diet 1 & 4.63 & 50.1 & 96 \\
Diet 2 & 1.86 & 28.8 & 90 \\
Diet 3 & 0.26 & 4.3 & 51 \\
Diet 4 & 11.97 & 73.8 & 98 \\
\hline
\end{tabular}

Diet 1 - Backyard cow milk + all other locally produced non-milk exposure pathways

Diet 2 - Locally produced commercial milk + all other locally produced non-milk exposure pathways

Diet 3 - Regionally mixed commercial milk + inhalation (other regionally mixed food items are minor contributors to the total)

Diet 4 - Goat milk (all other exposure pathways are of negligible importance compared to goat milk) 


\section{Location: EFPC}

Receptor: Female born in 1944

\begin{tabular}{lccc}
\hline & \multicolumn{3}{c}{ Thyroid Dose [cGy] } \\
& \multicolumn{2}{c}{$95 \%$ Subjective Confidence Interval } \\
Exposure Pathway & lower limit & central estimate & upper limit \\
\hline Backyard Cow Milk & 1.1 & 6.8 & 40 \\
Commercial Milk (locally produced) & 0.32 & 2.3 & 16 \\
Commercial Milk (regionally mixed) & 0.19 & 1.2 & 7.9 \\
Goat Milk (locally produced) & -- & -- & -- \\
Beef (locally produced) & 0.002 & 0.033 & 0.69 \\
Leafy Vegetables (locally produced) & 0.00061 & 0.0069 & 0.065 \\
Eggs (locally produced) & 0.059 & 0.46 & 3.5 \\
Cottage Cheese (locally produced) & 0.0017 & 0.017 & 0.22 \\
Inhalation & 0.032 & 0.12 & 0.44 \\
Mother's milk (mother on Diet 1) & 0.00021 & 0.0074 & 0.2 \\
Prenatal exposure (mother on Diet 1) & -- & -- & -- \\
Diet 1 & 1.3 & 7.6 & 44 \\
Diet 2 & 0.5 & 3.1 & 19 \\
Diet 3 & 0.24 & 1.3 & 8.4 \\
\hline
\end{tabular}

Excess Lifetime Risk [ ]

\begin{tabular}{lccc}
\hline Diet 1 & $8.3 \mathrm{E}-05$ & $1.3 \mathrm{E}-03$ & $1.7 \mathrm{E}-02$ \\
Diet 2 & $3.8 \mathrm{E}-05$ & $4.9 \mathrm{E}-04$ & $6.8 \mathrm{E}-03$ \\
Diet 3 & $1.8 \mathrm{E}-05$ & $2.1 \mathrm{E}-04$ & $2.6 \mathrm{E}-03$ \\
Diet 4 & -- & -- & -- \\
\hline
\end{tabular}

\section{Relative Risk [ ]}

\begin{tabular}{lccc}
\hline Diet 1 & 1.031 & 1.31 & 4.6 \\
Diet 2 & 1.013 & 1.13 & 2.6 \\
Diet 3 & 1.0061 & 1.057 & 1.66 \\
Diet 4 & -- & -- & -- \\
\hline
\end{tabular}

\begin{tabular}{lccc}
\hline Diet 1 & 3.00 & 23.2 & 76 \\
Diet 2 & 1.25 & 11.3 & 59 \\
Diet 3 & 0.60 & 5.4 & 40 \\
Diet 4 & -- & -- & \\
\hline Diet 1 - Backyard cow milk + all other locally produced non-milk exposure pathways & \\
Diet 2 - Locally produced commercial milk + all other locally produced non-milk exposure pathways \\
Diet 3 - Regionally mixed commercial milk + inhalation (other regionally mixed food items are minor contributors to the total) \\
Diet 4 - Goat milk (all other exposure pathways are of negligible importance compared to goat milk)
\end{tabular}




\section{Location: EFPC}

\section{Receptor: Male born in 1944}

\begin{tabular}{lccc}
\hline & \multicolumn{3}{c}{ Thyroid Dose [cGy] } \\
& \multicolumn{2}{c}{$95 \%$ Subjective Confidence Interval } \\
Exposure Pathway & lower limit & central estimate & upper limit \\
\hline Backyard Cow Milk & 1.1 & 6.8 & 44 \\
Commercial Milk (locally produced) & 0.32 & 2.3 & 16 \\
Commercial Milk (regionally mixed) & 0.19 & 1.2 & 7.1 \\
Goat Milk (locally produced) & -- & -- & -- \\
Beef (locally produced) & 0.0021 & 0.035 & 0.75 \\
Leafy Vegetables (locally produced) & 0.00059 & 0.0069 & 0.065 \\
Eggs (locally produced) & 0.07 & 0.53 & 3.7 \\
Cottage Cheese (locally produced) & 0.0015 & 0.017 & 0.22 \\
Inhalation & 0.03 & 0.12 & 0.45 \\
Mother's milk (mother on Diet 1) & 0.00021 & 0.0074 & 0.2 \\
Prenatal exposure (mother on Diet 1) & -- & -- & -- \\
Diet 1 & 1.3 & 7.6 & 50 \\
Diet 2 & 0.5 & 3.2 & 20 \\
Diet 3 & 0.24 & 1.3 & 7.6 \\
\hline
\end{tabular}

Excess Lifetime Risk [ ]

\begin{tabular}{lccc}
\hline Diet 1 & $9.9 \mathrm{E}-06$ & $3.3 \mathrm{E}-04$ & $6.1 \mathrm{E}-03$ \\
Diet 2 & $4.7 \mathrm{E}-06$ & $1.4 \mathrm{E}-04$ & $3.0 \mathrm{E}-03$ \\
Diet 3 & $2.3 \mathrm{E}-06$ & $5.7 \mathrm{E}-05$ & $1.2 \mathrm{E}-03$ \\
Diet 4 & -- & -- & -- \\
\hline
\end{tabular}

\section{Relative Risk [ ]}

\begin{tabular}{lccc}
\hline Diet 1 & 1.0097 & 1.22 & 6.4 \\
Diet 2 & 1.0042 & 1.087 & 3.3 \\
Diet 3 & 1.0018 & 1.036 & 1.91 \\
Diet 4 & -- & -- & -- \\
\hline
\end{tabular}

\begin{tabular}{lccc}
\hline Diet 1 & 0.95 & 17.7 & 84 \\
Diet 2 & 0.42 & 7.9 & 69 \\
Diet 3 & 0.19 & 3.5 & 48 \\
Diet 4 & -- & -- & - \\
\hline Diet 1 - Backyard cow milk + all other locally produced non-milk exposure pathways & \\
Diet 2 - Locally produced commercial milk + all other locally produced non-milk exposure pathways \\
Diet 3 - Regionally mixed commercial milk + inhalation (other regionally mixed food items are minor contributors to the total) \\
Diet 4 - Goat milk (all other exposure pathways are of negligible importance compared to goat milk)
\end{tabular}




\section{Location: Hope Creek}

Receptor: Female born in 1944

\begin{tabular}{lccc}
\hline & \multicolumn{3}{c}{ Thyroid Dose [cGy] } \\
& \multicolumn{2}{c}{$95 \%$ Subjective Confidence Interval } \\
Exposure Pathway & lower limit & central estimate & upper limit \\
\hline Backyard Cow Milk & 3.1 & 19 & 110 \\
Commercial Milk (locally produced) & 0.89 & 6 & 43 \\
Commercial Milk (regionally mixed) & 0.19 & 1.2 & 7.9 \\
Goat Milk (locally produced) & -- & -- & -- \\
Beef (locally produced) & 0.005 & 0.09 & 1.9 \\
Leafy Vegetables (locally produced) & 0.0017 & 0.019 & 0.17 \\
Eggs (locally produced) & 0.17 & 1.2 & 8.9 \\
Cottage Cheese (locally produced) & 0.0044 & 0.046 & 0.58 \\
Inhalation & 0.087 & 0.33 & 1.2 \\
Mother's milk (mother on Diet 1) & 0.00054 & 0.021 & 0.56 \\
Prenatal exposure (mother on Diet 1) & -- & -- & -- \\
Diet 1 & 3.5 & 21 & 120 \\
Diet 2 & 1.4 & 8 & 54 \\
Diet 3 & 0.35 & 1.5 & 9 \\
\hline
\end{tabular}

Excess Lifetime Risk [ ]

\begin{tabular}{lccc}
\hline Diet 1 & $2.2 \mathrm{E}-04$ & $3.5 \mathrm{E}-03$ & $4.6 \mathrm{E}-02$ \\
Diet 2 & $1.1 \mathrm{E}-04$ & $1.3 \mathrm{E}-03$ & $1.8 \mathrm{E}-02$ \\
Diet 3 & $2.3 \mathrm{E}-05$ & $2.4 \mathrm{E}-04$ & $2.7 \mathrm{E}-03$ \\
Diet 4 & -- & -- & -- \\
\hline
\end{tabular}

\section{Relative Risk [ ]}

\begin{tabular}{lccc}
\hline Diet 1 & 1.091 & 1.91 & 10.2 \\
Diet 2 & 1.035 & 1.35 & 5.2 \\
Diet 3 & 1.0074 & 1.066 & 1.7 \\
Diet 4 & -- & -- & -- \\
\hline
\end{tabular}

\section{Probability of Causation [\%]}

\begin{tabular}{lccc}
\hline Diet 1 & 8.32 & 47.4 & 90 \\
Diet 2 & 3.34 & 25.7 & 80 \\
Diet 3 & 0.74 & 6.2 & 41 \\
Diet 4 & -- & -- & - \\
\hline Diet 1 - Backyard cow milk + all other locally produced non-milk exposure pathways & \\
Diet 2 - Locally produced commercial milk + all other locally produced non-milk exposure pathways \\
Diet 3 - Regionally mixed commercial milk + inhalation (other regionally mixed food items are minor contributors to the total) \\
Diet 4 - Goat milk (all other exposure pathways are of negligible importance compared to goat milk)
\end{tabular}




\section{Location: Hope Creek}

\section{Receptor: Male born in 1944}

\begin{tabular}{lccc}
\hline & \multicolumn{3}{c}{ Thyroid Dose [cGy] } \\
& \multicolumn{2}{c}{$95 \%$ Subjective Confidence Interval } \\
Exposure Pathway & lower limit & central estimate & upper limit \\
\hline Backyard Cow Milk & 3.2 & 18 & 110 \\
Commercial Milk (locally produced) & 0.93 & 6.1 & 43 \\
Commercial Milk (regionally mixed) & 0.19 & 1.2 & 7.1 \\
Goat Milk (locally produced) & -- & -- & -- \\
Beef (locally produced) & 0.0052 & 0.093 & 2.1 \\
Leafy Vegetables (locally produced) & 0.0017 & 0.019 & 0.17 \\
Eggs (locally produced) & 0.2 & 1.5 & 10 \\
Cottage Cheese (locally produced) & 0.0041 & 0.045 & 0.6 \\
Inhalation & 0.087 & 0.33 & 1.2 \\
Mother's milk (mother on Diet 1) & 0.00054 & 0.021 & 0.56 \\
Prenatal exposure (mother on Diet 1) & -- & -- & -- \\
Diet 1 & 3.7 & 20 & 130 \\
Diet 2 & 1.4 & 8.5 & 53 \\
Diet 3 & 0.32 & 1.6 & 8.3 \\
\hline
\end{tabular}

Excess Lifetime Risk [ ]

\begin{tabular}{lccc}
\hline Diet 1 & $2.7 \mathrm{E}-05$ & $9.1 \mathrm{E}-04$ & $1.8 \mathrm{E}-02$ \\
Diet 2 & $1.3 \mathrm{E}-05$ & $3.8 \mathrm{E}-04$ & $7.9 \mathrm{E}-03$ \\
Diet 3 & $2.7 \mathrm{E}-06$ & $6.7 \mathrm{E}-05$ & $1.3 \mathrm{E}-03$ \\
Diet 4 & -- & -- & -- \\
\hline
\end{tabular}

\section{Relative Risk [ ]}

\begin{tabular}{lccc}
\hline Diet 1 & 1.029 & 1.6 & 14 \\
Diet 2 & 1.011 & 1.23 & 6.6 \\
Diet 3 & 1.0023 & 1.041 & 1.96 \\
Diet 4 & -- & -- & -- \\
\hline
\end{tabular}

\begin{tabular}{lccc}
\hline Diet 1 & 2.82 & 37.3 & 93 \\
Diet 2 & 1.11 & 18.8 & 85 \\
Diet 3 & 0.23 & 3.9 & 49 \\
Diet 4 & -- & -- & - \\
\hline Diet 1 - Backyard cow milk + all other locally produced non-milk exposure pathways & \\
Diet 2 - Locally produced commercial milk + all other locally produced non-milk exposure pathways \\
Diet 3 - Regionally mixed commercial milk + inhalation (other regionally mixed food items are minor contributors to the total) \\
Diet 4 - Goat milk (all other exposure pathways are of negligible importance compared to goat milk)
\end{tabular}


Location: Buttermilk Rd.

Receptor: Female born in 1944

\begin{tabular}{lccc}
\hline & \multicolumn{3}{c}{ Thyroid Dose [cGy] } \\
Exposure Pathway & \multicolumn{2}{c}{$95 \%$ Subjective Confidence Interval } \\
Backyard Cow Milk & lower limit & central estimate & upper limit \\
Commercial Milk (locally produced) & 3.1 & 18 & 110 \\
Commercial Milk (regionally mixed) & 0.88 & 6 & 42 \\
Goat Milk (locally produced) & 0.19 & 1.2 & 7.9 \\
Beef (locally produced) & 7.6 & 57 & 390 \\
Leafy Vegetables (locally produced) & 0.005 & 0.089 & 1.8 \\
Eggs (locally produced) & 0.0017 & 0.019 & 0.16 \\
Cottage Cheese (locally produced) & 0.17 & 1.2 & 9 \\
Inhalation & 0.0044 & 0.045 & 0.56 \\
Mother's milk (mother on Diet 1) & 0.09 & 0.33 & 1.2 \\
Prenatal exposure (mother on Diet 1) & 0.00051 & 0.02 & 0.53 \\
Diet 1 & -- & -- & -- \\
Diet 2 & 3.4 & 21 & 120 \\
Diet 3 & 1.4 & 8 & 53 \\
\hline
\end{tabular}

Excess Lifetime Risk [ ]

\begin{tabular}{llll}
\hline Diet 1 & $2.1 \mathrm{E}-04$ & $3.5 \mathrm{E}-03$ & $4.4 \mathrm{E}-02$ \\
Diet 2 & $1.1 \mathrm{E}-04$ & $1.3 \mathrm{E}-03$ & $1.7 \mathrm{E}-02$ \\
Diet 3 & $2.3 \mathrm{E}-05$ & $2.4 \mathrm{E}-04$ & $2.7 \mathrm{E}-03$ \\
Diet 4 & $5.5 \mathrm{E}-04$ & $9.5 \mathrm{E}-03$ & $1.2 \mathrm{E}-01$ \\
\hline
\end{tabular}

\section{Relative Risk [ ]}

\begin{tabular}{lccc}
\hline Diet 1 & 1.09 & 1.89 & 10.1 \\
Diet 2 & 1.034 & 1.34 & 5 \\
Diet 3 & 1.0074 & 1.066 & 1.7 \\
Diet 4 & 1.22 & 3.1 & 32 \\
\hline
\end{tabular}

\begin{tabular}{lccc}
\hline Diet 1 & 8.26 & 46.9 & 90 \\
Diet 2 & 3.25 & 25.4 & 80 \\
Diet 3 & 0.73 & 6.2 & 41 \\
Diet 4 & 17.90 & 67.8 & 97 \\
\hline Diet 1 - Backyard cow milk + all other localy produced non-milk expses
\end{tabular}

Diet 1 - Backyard cow milk + all other locally produced non-milk exposure pathways

Diet 2 - Locally produced commercial milk + all other locally produced non-milk exposure pathways

Diet 3 - Regionally mixed commercial milk + inhalation (other regionally mixed food items are minor contributors to the total)

Diet 4 - Goat milk (all other exposure pathways are of negligible importance compared to goat milk) 
Location: Buttermilk Rd.

Receptor: Male born in 1944

\begin{tabular}{lccc}
\hline & \multicolumn{3}{c}{ Thyroid Dose [cGy] } \\
& \multicolumn{2}{c}{$95 \%$ Subjective Confidence Interval } \\
Exposure Pathway & lower limit & central estimate & upper limit \\
\hline Backyard Cow Milk & 3.2 & 18 & 110 \\
Commercial Milk (locally produced) & 0.91 & 6.1 & 41 \\
Commercial Milk (regionally mixed) & 0.19 & 1.2 & 7.1 \\
Goat Milk (locally produced) & 7 & 54 & 350 \\
Beef (locally produced) & 0.0051 & 0.091 & 2 \\
Leafy Vegetables (locally produced) & 0.0017 & 0.018 & 0.17 \\
Eggs (locally produced) & 0.2 & 1.5 & 10 \\
Cottage Cheese (locally produced) & 0.0041 & 0.045 & 0.55 \\
Inhalation & 0.087 & 0.32 & 1.2 \\
Mother's milk (mother on Diet 1) & 0.00051 & 0.02 & 0.53 \\
Prenatal exposure (mother on Diet 1) & -- & -- & -- \\
Diet 1 & 3.7 & 20 & 120 \\
Diet 2 & 1.4 & 8.4 & 52 \\
Diet 3 & 0.32 & 1.6 & 8.3 \\
\hline
\end{tabular}

Excess Lifetime Risk [ ]

\begin{tabular}{llll}
\hline Diet 1 & $2.6 \mathrm{E}-05$ & $9.1 \mathrm{E}-04$ & $1.7 \mathrm{E}-02$ \\
Diet 2 & $1.3 \mathrm{E}-05$ & $3.7 \mathrm{E}-04$ & $7.7 \mathrm{E}-03$ \\
Diet 3 & $2.7 \mathrm{E}-06$ & $6.8 \mathrm{E}-05$ & $1.3 \mathrm{E}-03$ \\
Diet 4 & $7.0 \mathrm{E}-05$ & $2.2 \mathrm{E}-03$ & $4.9 \mathrm{E}-02$ \\
\hline
\end{tabular}

\section{Relative Risk [ ]}

\begin{tabular}{lccc}
\hline Diet 1 & 1.029 & 1.59 & 14 \\
Diet 2 & 1.011 & 1.22 & 6.5 \\
Diet 3 & 1.0022 & 1.041 & 1.97 \\
Diet 4 & 1.083 & 2.5 & 34 \\
\hline
\end{tabular}

\begin{tabular}{lccc}
\hline Diet 1 & 2.77 & 36.8 & 93 \\
Diet 2 & 1.11 & 18.2 & 84 \\
Diet 3 & 0.22 & 3.9 & 49 \\
Diet 4 & 7.66 & 60.2 & 97 \\
\hline
\end{tabular}

Diet 1 - Backyard cow milk + all other locally produced non-milk exposure pathways

Diet 2 - Locally produced commercial milk + all other locally produced non-milk exposure pathways

Diet 3 - Regionally mixed commercial milk + inhalation (other regionally mixed food items are minor contributors to the total)

Diet 4 - Goat milk (all other exposure pathways are of negligible importance compared to goat milk) 
Location: Jonesville

Receptor: Female born in 1944

\begin{tabular}{lccc}
\hline & \multicolumn{3}{c}{ Thyroid Dose [cGy] } \\
Exposure Pathway & $95 \%$ Subjective Confidence Interval \\
\hline Backyard Cow Milk & lower limit & central estimate & upper limit \\
Commercial Milk (locally produced) & 0.42 & 2.5 & 16 \\
Commercial Milk (regionally mixed) & 0.12 & 0.86 & 6.9 \\
Goat Milk (locally produced) & 0.19 & 1.2 & 7.9 \\
Beef (locally produced) & 1 & 7.9 & 66 \\
Leafy Vegetables (locally produced) & 0.00068 & 0.013 & 0.26 \\
Eggs (locally produced) & 0.00022 & 0.0027 & 0.028 \\
Cottage Cheese (locally produced) & 0.023 & 0.18 & 1.4 \\
Inhalation & 0.00054 & 0.0065 & 0.086 \\
Mother's milk (mother on Diet 1) & 0.013 & 0.048 & 0.2 \\
Prenatal exposure (mother on Diet 1) & 0.000072 & 0.0028 & 0.075 \\
Diet 1 & -- & -- & -- \\
Diet 2 & 0.49 & 2.8 & 18 \\
Diet 3 & 0.19 & 1.1 & 8.2 \\
\hline
\end{tabular}

Excess Lifetime Risk [ ]

\begin{tabular}{lllc}
\hline Diet 1 & $3.3 \mathrm{E}-05$ & $4.8 \mathrm{E}-04$ & $7.6 \mathrm{E}-03$ \\
Diet 2 & $1.6 \mathrm{E}-05$ & $2.0 \mathrm{E}-04$ & $2.7 \mathrm{E}-03$ \\
Diet 3 & $1.7 \mathrm{E}-05$ & $1.9 \mathrm{E}-04$ & $2.6 \mathrm{E}-03$ \\
Diet 4 & $9.0 \mathrm{E}-05$ & $1.3 \mathrm{E}-03$ & $1.8 \mathrm{E}-02$ \\
\hline
\end{tabular}

\section{Relative Risk [ ]}

\begin{tabular}{lccc}
\hline Diet 1 & 1.011 & 1.12 & 2.7 \\
Diet 2 & 1.0047 & 1.047 & 1.7 \\
Diet 3 & 1.0057 & 1.054 & 1.65 \\
Diet 4 & 1.029 & 1.3 & 6.3 \\
\hline
\end{tabular}

\section{Probability of Causation [\%]}

\begin{tabular}{lccc}
\hline Diet 1 & 1.12 & 10.4 & 62 \\
Diet 2 & 0.47 & 4.5 & 41 \\
Diet 3 & 0.57 & 5.1 & 39 \\
Diet 4 & 2.83 & 23.2 & 84 \\
\hline
\end{tabular}

Diet 1 - Backyard cow milk + all other locally produced non-milk exposure pathways

Diet 2 - Locally produced commercial milk + all other locally produced non-milk exposure pathways

Diet 3 - Regionally mixed commercial milk + inhalation (other regionally mixed food items are minor contributors to the total)

Diet 4 - Goat milk (all other exposure pathways are of negligible importance compared to goat milk) 


\section{Location: Jonesville}

\section{Receptor: Male born in 1944}

\begin{tabular}{lccc}
\hline & \multicolumn{3}{c}{ Thyroid Dose [cGy] } \\
& \multicolumn{2}{c}{$95 \%$ Subjective Confidence Interval } \\
Exposure Pathway & lower limit & central estimate & upper limit \\
\hline Backyard Cow Milk & 0.44 & 2.5 & 17 \\
Commercial Milk (locally produced) & 0.12 & 0.85 & 6.4 \\
Commercial Milk (regionally mixed) & 0.19 & 1.2 & 7.1 \\
Goat Milk (locally produced) & 0.99 & 7.9 & 63 \\
Beef (locally produced) & 0.00072 & 0.013 & 0.27 \\
Leafy Vegetables (locally produced) & 0.00021 & 0.0027 & 0.025 \\
Eggs (locally produced) & 0.027 & 0.2 & 1.6 \\
Cottage Cheese (locally produced) & 0.00051 & 0.0065 & 0.09 \\
Inhalation & 0.014 & 0.049 & 0.2 \\
Mother's milk (mother on Diet 1) & 0.000072 & 0.0028 & 0.075 \\
Prenatal exposure (mother on Diet 1) & -- & -- & -- \\
Diet 1 & 0.53 & 2.8 & 19 \\
Diet 2 & 0.2 & 1.2 & 8.3 \\
Diet 3 & 0.22 & 1.2 & 7.3 \\
\hline
\end{tabular}

Excess Lifetime Risk [ ]

\begin{tabular}{lllc}
\hline Diet 1 & $3.7 \mathrm{E}-06$ & $1.3 \mathrm{E}-04$ & $2.8 \mathrm{E}-03$ \\
Diet 2 & $1.8 \mathrm{E}-06$ & $5.1 \mathrm{E}-05$ & $1.1 \mathrm{E}-03$ \\
Diet 3 & $2.2 \mathrm{E}-06$ & $5.2 \mathrm{E}-05$ & $1.1 \mathrm{E}-03$ \\
Diet 4 & $1.0 \mathrm{E}-05$ & $3.0 \mathrm{E}-04$ & $8.1 \mathrm{E}-03$ \\
\hline
\end{tabular}

\section{Relative Risk [ ]}

\begin{tabular}{lccc}
\hline Diet 1 & 1.0036 & 1.087 & 2.9 \\
Diet 2 & 1.0015 & 1.032 & 1.85 \\
Diet 3 & 1.0017 & 1.035 & 1.87 \\
Diet 4 & 1.011 & 1.22 & 5.8 \\
\hline
\end{tabular}

\section{Probability of Causation [\%]}

\begin{tabular}{lccc}
\hline Diet 1 & 0.36 & 8.0 & 66 \\
Diet 2 & 0.16 & 3.1 & 46 \\
Diet 3 & 0.17 & 3.3 & 47 \\
Diet 4 & 1.11 & 18.2 & 82 \\
\hline
\end{tabular}

Diet 1 - Backyard cow milk + all other locally produced non-milk exposure pathways

Diet 2 - Locally produced commercial milk + all other locally produced non-milk exposure pathways

Diet 3 - Regionally mixed commercial milk + inhalation (other regionally mixed food items are minor contributors to the total)

Diet 4 - Goat milk (all other exposure pathways are of negligible importance compared to goat milk) 


\section{Location: OR Scarboro}

Receptor: Female born in 1944

\begin{tabular}{lccc}
\hline & \multicolumn{3}{c}{ Thyroid Dose [cGy] } \\
Exposure Pathway & \multicolumn{2}{c}{$9 \%$ Subjective Confidence Interval } \\
\hline Backyard Cow Milk & lower limit & central estimate & upper limit \\
Commercial Milk (locally produced) & -- & -- & -- \\
Commercial Milk (regionally mixed) & -- & -- & -- \\
Goat Milk (locally produced) & 0.19 & 1.2 & 7.9 \\
Beef (locally produced) & -- & -- & -- \\
Leafy Vegetables (locally produced) & -- & -- & -- \\
Eggs (locally produced) & -- & -- & -- \\
Cottage Cheese (locally produced) & -- & -- & -- \\
Inhalation & 0.003 & 0.034 & 0.39 \\
Mother's milk (mother on Diet 3) & 0.064 & 0.24 & 0.92 \\
Prenatal exposure (mother on Diet 3) & 0.000047 & 0.0012 & 0.019 \\
Diet 1 & -- & -- & -- \\
Diet 2 & -- & -- & -- \\
Diet 3 & -- & -- & -- \\
\hline
\end{tabular}

Excess Lifetime Risk [ ]

\begin{tabular}{lccc}
\hline Diet 1 & -- & -- & -- \\
Diet 2 & -- & -- & -- \\
Diet 3 & $2.1 \mathrm{E}-05$ & $2.3 \mathrm{E}-04$ & $2.7 \mathrm{E}-03$ \\
Diet 4 & -- & -- & -- \\
\hline
\end{tabular}

\section{Relative Risk [ ]}

\begin{tabular}{lccc}
\hline Diet 1 & -- & -- & -- \\
Diet 2 & -- & -- & -- \\
Diet 3 & 1.0069 & 1.062 & 1.69 \\
Diet 4 & -- & -- & -- \\
\hline
\end{tabular}

\begin{tabular}{lccc}
\hline Diet 1 & -- & -- & -- \\
Diet 2 & -- & -- & -- \\
Diet 3 & 0.68 & 5.9 & 41 \\
Diet 4 & -- & -- \\
\hline Diet 1 - Backyard cow milk + all other locally produced non-milk exposure pathways & \\
Diet 2 - Locally produced commercial milk + all other locally produced non-milk exposure pathways \\
Diet 3 - Regionally mixed commercial milk + inhalation (other regionally mixed food items are minor contributors to the total) \\
Diet 4 - Goat milk (all other exposure pathways are of negligible importance compared to goat milk)
\end{tabular}




\section{Location: OR Scarboro}

Receptor: Male born in 1944

\begin{tabular}{lccc}
\hline & \multicolumn{3}{c}{ Thyroid Dose [cGy] } \\
Exposure Pathway & \multicolumn{2}{c}{$95 \%$ Subjective Confidence Interval } \\
\hline Backyard Cow Milk & lower limit & central estimate & upper limit \\
Commercial Milk (locally produced) & -- & -- & -- \\
Commercial Milk (regionally mixed) & -- & -- & -- \\
Goat Milk (locally produced) & 0.19 & 1.2 & 7.1 \\
Beef (locally produced) & -- & -- & -- \\
Leafy Vegetables (locally produced) & -- & -- & -- \\
Eggs (locally produced) & -- & -- & -- \\
Cottage Cheese (locally produced) & -- & -- & -- \\
Inhalation & 0.003 & 0.035 & 0.42 \\
Mother's milk (mother on Diet 3) & 0.064 & 0.25 & 0.87 \\
Prenatal exposure (mother on Diet 3) & 0.000047 & 0.0012 & 0.019 \\
Diet 1 & -- & -- & -- \\
Diet 2 & -- & -- & -- \\
Diet 3 & -- & -- & -- \\
\hline
\end{tabular}

Excess Lifetime Risk [ ]

\begin{tabular}{lccc}
\hline Diet 1 & -- & -- & -- \\
Diet 2 & -- & -- & -- \\
Diet 3 & $2.5 \mathrm{E}-06$ & $6.4 \mathrm{E}-05$ & $1.3 \mathrm{E}-03$ \\
Diet 4 & -- & -- & -- \\
\hline
\end{tabular}

\section{Relative Risk [ ]}

\begin{tabular}{lccc}
\hline Diet 1 & -- & -- & -- \\
Diet 2 & -- & -- & -- \\
Diet 3 & 1.0021 & 1.039 & 1.94 \\
Diet 4 & -- & -- & -- \\
\hline
\end{tabular}

\begin{tabular}{lccc}
\hline Diet 1 & -- & -- & -- \\
Diet 2 & -- & -- & -- \\
Diet 3 & 0.21 & 3.8 & -- \\
Diet 4 & -- & -- & \\
\hline Diet 1 - Backyard cow milk + all other locally produced non-milk exposure pathways & \\
Diet 2 - Locally produced commercial milk + all other locally produced non-milk exposure pathways \\
Diet 3 - Regionally mixed commercial milk + inhalation (other regionally mixed food items are minor contributors to the total) \\
Diet 4 - Goat milk (all other exposure pathways are of negligible importance compared to goat milk)
\end{tabular}




\section{Location: Lawnville/Gallaher}

Receptor: Female born in 1944

\begin{tabular}{lccc}
\hline & \multicolumn{3}{c}{ Thyroid Dose [cGy] } \\
Exposure Pathway & $95 \%$ Subjective Confidence Interval \\
\hline Backyard Cow Milk & lower limit & central estimate & upper limit \\
Commercial Milk (locally produced) & 3 & 17 & 88 \\
Commercial Milk (regionally mixed) & 0.92 & 5.6 & 41 \\
Goat Milk (locally produced) & 0.19 & 1.2 & 7.9 \\
Beef (locally produced) & 7.2 & 54 & 350 \\
Leafy Vegetables (locally produced) & 0.0046 & 0.087 & 1.7 \\
Eggs (locally produced) & 0.0017 & 0.018 & 0.14 \\
Cottage Cheese (locally produced) & 0.17 & 1.2 & 8.5 \\
Inhalation & 0.0042 & 0.042 & 0.47 \\
Mother's milk (mother on Diet 1) & 0.087 & 0.31 & 1.2 \\
Prenatal exposure (mother on Diet 1) & 0.00053 & 0.019 & 0.51 \\
Diet 1 & -- & -- & -- \\
Diet 2 & 3.5 & 19 & 97 \\
Diet 3 & 1.4 & 7.5 & 52 \\
\hline
\end{tabular}

Excess Lifetime Risk [ ]

\begin{tabular}{llll}
\hline Diet 1 & $2.2 \mathrm{E}-04$ & $3.1 \mathrm{E}-03$ & $4.2 \mathrm{E}-02$ \\
Diet 2 & $1.1 \mathrm{E}-04$ & $1.2 \mathrm{E}-03$ & $1.7 \mathrm{E}-02$ \\
Diet 3 & $2.4 \mathrm{E}-05$ & $2.4 \mathrm{E}-04$ & $2.7 \mathrm{E}-03$ \\
Diet 4 & $5.4 \mathrm{E}-04$ & $8.8 \mathrm{E}-03$ & $1.1 \mathrm{E}-01$ \\
\hline
\end{tabular}

\section{Relative Risk [ ]}

\begin{tabular}{lccc}
\hline Diet 1 & 1.092 & 1.84 & 10.2 \\
Diet 2 & 1.033 & 1.33 & 5 \\
Diet 3 & 1.0076 & 1.066 & 1.7 \\
Diet 4 & 1.22 & 3 & 31 \\
\hline
\end{tabular}

\begin{tabular}{lccc}
\hline Diet 1 & 8.41 & 45.5 & 90 \\
Diet 2 & 3.21 & 24.8 & 80 \\
Diet 3 & 0.75 & 6.2 & 41 \\
Diet 4 & 17.82 & 66.2 & 97 \\
\hline Dit
\end{tabular}

Diet 1 - Backyard cow milk + all other locally produced non-milk exposure pathways

Diet 2 - Locally produced commercial milk + all other locally produced non-milk exposure pathways

Diet 3 - Regionally mixed commercial milk + inhalation (other regionally mixed food items are minor contributors to the total)

Diet 4 - Goat milk (all other exposure pathways are of negligible importance compared to goat milk) 


\section{Location: Lawnville/Gallaher}

Receptor: Male born in 1944

\begin{tabular}{lccc}
\hline & \multicolumn{3}{c}{ Thyroid Dose [cGy] } \\
& \multicolumn{2}{c}{$95 \%$ Subjective Confidence Interval } \\
Exposure Pathway & lower limit & central estimate & upper limit \\
\hline Backyard Cow Milk & 3.2 & 17 & 93 \\
Commercial Milk (locally produced) & 0.91 & 5.6 & 41 \\
Commercial Milk (regionally mixed) & 0.19 & 1.2 & 7.1 \\
Goat Milk (locally produced) & 6.9 & 53 & 330 \\
Beef (locally produced) & 0.0047 & 0.09 & 1.8 \\
Leafy Vegetables (locally produced) & 0.0018 & 0.018 & 0.15 \\
Eggs (locally produced) & 0.2 & 1.4 & 9.5 \\
Cottage Cheese (locally produced) & 0.0039 & 0.045 & 0.51 \\
Inhalation & 0.085 & 0.31 & 1.2 \\
Mother's milk (mother on Diet 1) & 0.00053 & 0.019 & 0.51 \\
Prenatal exposure (mother on Diet 1) & -- & -- & -- \\
Diet 1 & 3.6 & 19 & 100 \\
Diet 2 & 1.4 & 7.8 & 53 \\
Diet 3 & 0.32 & 1.5 & 8.3 \\
\hline
\end{tabular}

Excess Lifetime Risk [ ]

\begin{tabular}{llll}
\hline Diet 1 & $2.6 \mathrm{E}-05$ & $8.5 \mathrm{E}-04$ & $1.5 \mathrm{E}-02$ \\
Diet 2 & $1.1 \mathrm{E}-05$ & $3.4 \mathrm{E}-04$ & $7.1 \mathrm{E}-03$ \\
Diet 3 & $2.7 \mathrm{E}-06$ & $6.8 \mathrm{E}-05$ & $1.3 \mathrm{E}-03$ \\
Diet 4 & $6.7 \mathrm{E}-05$ & $2.1 \mathrm{E}-03$ & $4.7 \mathrm{E}-02$ \\
\hline
\end{tabular}

\section{Relative Risk [ ]}

\begin{tabular}{lccc}
\hline Diet 1 & 1.024 & 1.56 & 13 \\
Diet 2 & 1.01 & 1.21 & 6.2 \\
Diet 3 & 1.0022 & 1.041 & 1.96 \\
Diet 4 & 1.085 & 2.4 & 31 \\
\hline
\end{tabular}

\begin{tabular}{lccc}
\hline Diet 1 & 2.35 & 36.0 & 92 \\
Diet 2 & 1.03 & 17.3 & 84 \\
Diet 3 & 0.22 & 3.9 & 49 \\
Diet 4 & 7.85 & 58.6 & 97 \\
\hline Diet 1 Backyard cow milk + all
\end{tabular}

Diet 1 - Backyard cow milk + all other locally produced non-milk exposure pathways

Diet 2 - Locally produced commercial milk + all other locally produced non-milk exposure pathways

Diet 3 - Regionally mixed commercial milk + inhalation (other regionally mixed food items are minor contributors to the total)

Diet 4 - Goat milk (all other exposure pathways are of negligible importance compared to goat milk) 


\section{Location: Dyllis}

Receptor: Female born in 1944

\begin{tabular}{lccc}
\hline & \multicolumn{3}{c}{ Thyroid Dose [cGy] } \\
& \multicolumn{2}{c}{$95 \%$ Subjective Confidence Interval } \\
Exposure Pathway & lower limit & central estimate & upper limit \\
\hline Backyard Cow Milk & 0.58 & 3.4 & 23 \\
Commercial Milk (locally produced) & 0.18 & 1.2 & 9.2 \\
Commercial Milk (regionally mixed) & 0.19 & 1.2 & 7.9 \\
Goat Milk (locally produced) & 1.4 & 11 & 78 \\
Beef (locally produced) & 0.0011 & 0.017 & 0.33 \\
Leafy Vegetables (locally produced) & 0.00031 & 0.0035 & 0.037 \\
Eggs (locally produced) & 0.031 & 0.24 & 2 \\
Cottage Cheese (locally produced) & 0.0008 & 0.0087 & 0.12 \\
Inhalation & 0.017 & 0.066 & 0.28 \\
Mother's milk (mother on Diet 1) & 0.00012 & 0.0038 & 0.11 \\
Prenatal exposure (mother on Diet 1) & -- & -- & -- \\
Diet 1 & 0.66 & 3.9 & 25 \\
Diet 2 & 0.28 & 1.6 & 11 \\
Diet 3 & 0.23 & 1.2 & 8.2 \\
\hline
\end{tabular}

Excess Lifetime Risk [ ]

\begin{tabular}{lllc}
\hline Diet 1 & $4.4 \mathrm{E}-05$ & $6.7 \mathrm{E}-04$ & $9.5 \mathrm{E}-03$ \\
Diet 2 & $1.8 \mathrm{E}-05$ & $2.6 \mathrm{E}-04$ & $3.4 \mathrm{E}-03$ \\
Diet 3 & $1.7 \mathrm{E}-05$ & $2.0 \mathrm{E}-04$ & $2.6 \mathrm{E}-03$ \\
Diet 4 & $1.1 \mathrm{E}-04$ & $1.9 \mathrm{E}-03$ & $2.6 \mathrm{E}-02$ \\
\hline
\end{tabular}

\section{Relative Risk [ ]}

\begin{tabular}{lccc}
\hline Diet 1 & 1.016 & 1.16 & 3.2 \\
Diet 2 & 1.0073 & 1.062 & 1.96 \\
Diet 3 & 1.0058 & 1.054 & 1.65 \\
Diet 4 & 1.047 & 1.41 & 7.4 \\
\hline
\end{tabular}

\section{Probability of Causation [\%]}

\begin{tabular}{lccc}
\hline Diet 1 & 1.55 & 14.1 & 69 \\
Diet 2 & 0.72 & 5.9 & 49 \\
Diet 3 & 0.58 & 5.1 & 39 \\
Diet 4 & 4.45 & 28.9 & 86 \\
\hline
\end{tabular}

Diet 1 - Backyard cow milk + all other locally produced non-milk exposure pathways

Diet 2 - Locally produced commercial milk + all other locally produced non-milk exposure pathways

Diet 3 - Regionally mixed commercial milk + inhalation (other regionally mixed food items are minor contributors to the total)

Diet 4 - Goat milk (all other exposure pathways are of negligible importance compared to goat milk) 


\section{Location: Dyllis}

\section{Receptor: Male born in 1944}

\begin{tabular}{lccc}
\hline & \multicolumn{3}{c}{ Thyroid Dose [cGy] } \\
& \multicolumn{2}{c}{$95 \%$ Subjective Confidence Interval } \\
Exposure Pathway & lower limit & central estimate & upper limit \\
\hline Backyard Cow Milk & 0.57 & 3.5 & 23 \\
Commercial Milk (locally produced) & 0.17 & 1.2 & 9 \\
Commercial Milk (regionally mixed) & 0.19 & 1.2 & 7.1 \\
Goat Milk (locally produced) & 1.4 & 11 & 84 \\
Beef (locally produced) & 0.0011 & 0.017 & 0.36 \\
Leafy Vegetables (locally produced) & 0.0003 & 0.0037 & 0.035 \\
Eggs (locally produced) & 0.038 & 0.27 & 2.2 \\
Cottage Cheese (locally produced) & 0.00077 & 0.009 & 0.12 \\
Inhalation & 0.017 & 0.068 & 0.27 \\
Mother's milk (mother on Diet 1) & 0.00012 & 0.0038 & 0.11 \\
Prenatal exposure (mother on Diet 1) & -- & -- & - \\
Diet 1 & 0.69 & 4 & 26 \\
Diet 2 & 0.29 & 1.6 & 12 \\
Diet 3 & 0.22 & 1.3 & 7.4 \\
\hline
\end{tabular}

Excess Lifetime Risk [ ]

\begin{tabular}{llll}
\hline Diet 1 & $4.8 \mathrm{E}-06$ & $1.8 \mathrm{E}-04$ & $3.7 \mathrm{E}-03$ \\
Diet 2 & $2.6 \mathrm{E}-06$ & $7.3 \mathrm{E}-05$ & $1.6 \mathrm{E}-03$ \\
Diet 3 & $2.2 \mathrm{E}-06$ & $5.4 \mathrm{E}-05$ & $1.2 \mathrm{E}-03$ \\
Diet 4 & $1.5 \mathrm{E}-05$ & $4.1 \mathrm{E}-04$ & $1.0 \mathrm{E}-02$ \\
\hline
\end{tabular}

\section{Relative Risk [ ]}

\begin{tabular}{lccc}
\hline Diet 1 & 1.0054 & 1.11 & 3.7 \\
Diet 2 & 1.0021 & 1.047 & 2.1 \\
Diet 3 & 1.0018 & 1.035 & 1.89 \\
Diet 4 & 1.013 & 1.3 & 8.1 \\
\hline
\end{tabular}

\section{Probability of Causation [\%]}

\begin{tabular}{lccc}
\hline Diet 1 & 0.54 & 10.1 & 73 \\
Diet 2 & 0.21 & 4.5 & 53 \\
Diet 3 & 0.18 & 3.4 & 47 \\
Diet 4 & 1.29 & 23.3 & 87 \\
\hline
\end{tabular}

Diet 1 - Backyard cow milk + all other locally produced non-milk exposure pathways

Diet 2 - Locally produced commercial milk + all other locally produced non-milk exposure pathways

Diet 3 - Regionally mixed commercial milk + inhalation (other regionally mixed food items are minor contributors to the total)

Diet 4 - Goat milk (all other exposure pathways are of negligible importance compared to goat milk) 


\section{Location: OR High School Area}

Receptor: Female born in 1944

\begin{tabular}{lccc}
\hline & \multicolumn{3}{c}{ Thyroid Dose [cGy] } \\
Exposure Pathway & \multicolumn{2}{c}{$9 \%$ Subjective Confidence Interval } \\
\hline Backyard Cow Milk & lower limit & central estimate & upper limit \\
Commercial Milk (locally produced) & -- & -- & -- \\
Commercial Milk (regionally mixed) & -- & -- & -- \\
Goat Milk (locally produced) & 0.19 & 1.2 & 7.9 \\
Beef (locally produced) & -- & -- & -- \\
Leafy Vegetables (locally produced) & -- & -- & -- \\
Eggs (locally produced) & -- & -- & -- \\
Cottage Cheese (locally produced) & -- & -- & -- \\
Inhalation & 0.002 & 0.019 & 0.22 \\
Mother's milk (mother on Diet 3) & 0.038 & 0.14 & 0.56 \\
Prenatal exposure (mother on Diet 3) & 0.000038 & 0.001 & 0.017 \\
Diet 1 & -- & -- & -- \\
Diet 2 & -- & -- & -- \\
Diet 3 & -- & -- & -- \\
\hline
\end{tabular}

Excess Lifetime Risk [ ]

\begin{tabular}{lccc}
\hline Diet 1 & -- & -- & -- \\
Diet 2 & -- & -- & -- \\
Diet 3 & $1.9 \mathrm{E}-05$ & $2.1 \mathrm{E}-04$ & $2.6 \mathrm{E}-03$ \\
Diet 4 & -- & -- & -- \\
\hline
\end{tabular}

\section{Relative Risk [ ]}

\begin{tabular}{lccc}
\hline Diet 1 & -- & -- & -- \\
Diet 2 & -- & -- & -- \\
Diet 3 & 1.0062 & 1.058 & 1.66 \\
Diet 4 & -- & -- & -- \\
\hline
\end{tabular}

\begin{tabular}{lccc}
\hline Diet 1 & -- & -- & -- \\
Diet 2 & -- & -- & -- \\
Diet 3 & 0.62 & 5.5 & 40 \\
Diet 4 & -- & -- \\
\hline Diet 1 - Backyard cow milk + all other locally produced non-milk exposure pathways & \\
Diet 2 - Locally produced commercial milk + all other locally produced non-milk exposure pathways \\
Diet 3 - Regionally mixed commercial milk + inhalation (other regionally mixed food items are minor contributors to the total) \\
Diet 4 - Goat milk (all other exposure pathways are of negligible importance compared to goat milk)
\end{tabular}




\section{Location: OR High School Area}

Receptor: Male born in 1944

\begin{tabular}{lccc}
\hline & \multicolumn{3}{c}{ Thyroid Dose [cGy] } \\
Exposure Pathway & \multicolumn{2}{c}{$95 \%$ Subjective Confidence Interval } \\
\hline Backyard Cow Milk & lower limit & central estimate & upper limit \\
Commercial Milk (locally produced) & -- & -- & -- \\
Commercial Milk (regionally mixed) & -- & -- & -- \\
Goat Milk (locally produced) & 0.19 & 1.2 & 7.1 \\
Beef (locally produced) & -- & -- & -- \\
Leafy Vegetables (locally produced) & -- & -- & -- \\
Eggs (locally produced) & -- & -- & -- \\
Cottage Cheese (locally produced) & -- & -- & -- \\
Inhalation & 0.0018 & 0.02 & 0.23 \\
Mother's milk (mother on Diet 3) & 0.04 & 0.14 & 0.52 \\
Prenatal exposure (mother on Diet 3) & 0.000038 & 0.001 & 0.017 \\
Diet 1 & -- & -- & -- \\
Diet 2 & -- & -- & -- \\
Diet 3 & -- & -- & -- \\
\hline
\end{tabular}

Excess Lifetime Risk [ ]

\begin{tabular}{lccc}
\hline Diet 1 & -- & -- & -- \\
Diet 2 & -- & -- & -- \\
Diet 3 & $2.4 \mathrm{E}-06$ & $5.8 \mathrm{E}-05$ & $1.2 \mathrm{E}-03$ \\
Diet 4 & -- & -- & -- \\
\hline
\end{tabular}

\section{Relative Risk [ ]}

\begin{tabular}{lccc}
\hline Diet 1 & -- & -- & -- \\
Diet 2 & -- & -- & -- \\
Diet 3 & 1.0019 & 1.037 & 1.92 \\
Diet 4 & -- & -- & -- \\
\hline
\end{tabular}

\begin{tabular}{lccc}
\hline Diet 1 & -- & -- & -- \\
Diet 2 & -- & -- & -- \\
Diet 3 & 0.19 & 3.6 & -- \\
Diet 4 & -- & -- & - \\
\hline Diet 1 - Backyard cow milk + all other locally produced non-milk exposure pathways & \\
Diet 2 - Locally produced commercial milk + all other locally produced non-milk exposure pathways \\
Diet 3 - Regionally mixed commercial milk + inhalation (other regionally mixed food items are minor contributors to the total) \\
Diet 4 - Goat milk (all other exposure pathways are of negligible importance compared to goat milk)
\end{tabular}




\section{Location: Norwood}

Receptor: Female born in 1944

\begin{tabular}{lccc}
\hline & \multicolumn{3}{c}{ Thyroid Dose [cGy] } \\
Exposure Pathway & \multicolumn{2}{c}{$95 \%$ Subjective Confidence Interval } \\
\hline Backyard Cow Milk & lower limit & central estimate & upper limit \\
Commercial Milk (locally produced) & 0.51 & 3.2 & 21 \\
Commercial Milk (regionally mixed) & 0.17 & 1.1 & 8.6 \\
Goat Milk (locally produced) & 0.19 & 1.2 & 7.9 \\
Beef (locally produced) & 1.3 & 10 & 84 \\
Leafy Vegetables (locally produced) & 0.00089 & 0.016 & 0.38 \\
Eggs (locally produced) & 0.00035 & 0.0035 & 0.031 \\
Cottage Cheese (locally produced) & 0.029 & 0.22 & 1.9 \\
Inhalation & 0.00072 & 0.0086 & 0.11 \\
Mother's milk (mother on Diet 1) & 0.017 & 0.064 & 0.25 \\
Prenatal exposure (mother on Diet 1) & 0.000099 & 0.0038 & 0.097 \\
Diet 1 & -- & -- & -- \\
Diet 2 & 0.58 & 3.5 & 24 \\
Diet 3 & 0.25 & 1.5 & 11 \\
\hline
\end{tabular}

Excess Lifetime Risk [ ]

\begin{tabular}{llll}
\hline Diet 1 & $4.0 \mathrm{E}-05$ & $6.5 \mathrm{E}-04$ & $9.3 \mathrm{E}-03$ \\
Diet 2 & $1.7 \mathrm{E}-05$ & $2.5 \mathrm{E}-04$ & $3.8 \mathrm{E}-03$ \\
Diet 3 & $1.7 \mathrm{E}-05$ & $2.0 \mathrm{E}-04$ & $2.6 \mathrm{E}-03$ \\
Diet 4 & $1.1 \mathrm{E}-04$ & $1.8 \mathrm{E}-03$ & $2.3 \mathrm{E}-02$ \\
\hline
\end{tabular}

\section{Relative Risk [ ]}

\begin{tabular}{lccc}
\hline Diet 1 & 1.015 & 1.15 & 2.7 \\
Diet 2 & 1.0064 & 1.062 & 1.82 \\
Diet 3 & 1.0057 & 1.054 & 1.65 \\
Diet 4 & 1.042 & 1.4 & 7.2 \\
\hline
\end{tabular}

\section{Probability of Causation [\%]}

\begin{tabular}{lccc}
\hline Diet 1 & 1.51 & 13.2 & 63 \\
Diet 2 & 0.64 & 5.8 & 45 \\
Diet 3 & 0.57 & 5.2 & 39 \\
Diet 4 & 4.06 & 28.3 & 86 \\
\hline
\end{tabular}

Diet 1 - Backyard cow milk + all other locally produced non-milk exposure pathways

Diet 2 - Locally produced commercial milk + all other locally produced non-milk exposure pathways

Diet 3 - Regionally mixed commercial milk + inhalation (other regionally mixed food items are minor contributors to the total)

Diet 4 - Goat milk (all other exposure pathways are of negligible importance compared to goat milk) 


\section{Location: Norwood}

\section{Receptor: Male born in 1944}

\begin{tabular}{lccc}
\hline & \multicolumn{3}{c}{ Thyroid Dose [cGy] } \\
& \multicolumn{2}{c}{$95 \%$ Subjective Confidence Interval } \\
Exposure Pathway & lower limit & central estimate & upper limit \\
\hline Backyard Cow Milk & 0.52 & 3.2 & 23 \\
Commercial Milk (locally produced) & 0.16 & 1.1 & 8.7 \\
Commercial Milk (regionally mixed) & 0.19 & 1.2 & 7.1 \\
Goat Milk (locally produced) & 1.3 & 9.9 & 81 \\
Beef (locally produced) & 0.001 & 0.017 & 0.4 \\
Leafy Vegetables (locally produced) & 0.00034 & 0.0033 & 0.033 \\
Eggs (locally produced) & 0.033 & 0.26 & 1.9 \\
Cottage Cheese (locally produced) & 0.00071 & 0.0089 & 0.12 \\
Inhalation & 0.015 & 0.064 & 0.24 \\
Mother's milk (mother on Diet 1) & 0.000099 & 0.0038 & 0.097 \\
Prenatal exposure (mother on Diet 1) & -- & -- & - \\
Diet 1 & 0.61 & 3.6 & 25 \\
Diet 2 & 0.25 & 1.5 & 12 \\
Diet 3 & 0.22 & 1.3 & 7.4 \\
\hline
\end{tabular}

Excess Lifetime Risk [ ]

\begin{tabular}{llll}
\hline Diet 1 & $5.2 \mathrm{E}-06$ & $1.6 \mathrm{E}-04$ & $3.3 \mathrm{E}-03$ \\
Diet 2 & $2.1 \mathrm{E}-06$ & $6.5 \mathrm{E}-05$ & $1.4 \mathrm{E}-03$ \\
Diet 3 & $2.2 \mathrm{E}-06$ & $5.4 \mathrm{E}-05$ & $1.2 \mathrm{E}-03$ \\
Diet 4 & $1.4 \mathrm{E}-05$ & $4.0 \mathrm{E}-04$ & $9.9 \mathrm{E}-03$ \\
\hline
\end{tabular}

\section{Relative Risk [ ]}

\begin{tabular}{lccc}
\hline Diet 1 & 1.0045 & 1.11 & 3.6 \\
Diet 2 & 1.002 & 1.043 & 2.1 \\
Diet 3 & 1.0017 & 1.035 & 1.89 \\
Diet 4 & 1.013 & 1.29 & 8.8 \\
\hline
\end{tabular}

\begin{tabular}{lccc}
\hline Diet 1 & 0.45 & 10.0 & 72 \\
Diet 2 & 0.20 & 4.2 & 53 \\
Diet 3 & 0.17 & 3.4 & 47 \\
Diet 4 & 1.29 & 22.2 & 88 \\
\hline
\end{tabular}

Diet 1 - Backyard cow milk + all other locally produced non-milk exposure pathways

Diet 2 - Locally produced commercial milk + all other locally produced non-milk exposure pathways

Diet 3 - Regionally mixed commercial milk + inhalation (other regionally mixed food items are minor contributors to the total)

Diet 4 - Goat milk (all other exposure pathways are of negligible importance compared to goat milk) 
Location: Woodland

Receptor: Female born in 1944

\begin{tabular}{lccc}
\hline & \multicolumn{3}{c}{ Thyroid Dose [cGy] } \\
Exposure Pathway & \multicolumn{2}{c}{$95 \%$ Subjective Confidence Interval } \\
\hline Backyard Cow Milk & lower limit & central estimate & upper limit \\
Commercial Milk (locally produced) & -- & -- & -- \\
Commercial Milk (regionally mixed) & -- & -- & -- \\
Goat Milk (locally produced) & 0.19 & 1.2 & 7.9 \\
Beef (locally produced) & -- & -- & -- \\
Leafy Vegetables (locally produced) & -- & -- & -- \\
Eggs (locally produced) & -- & -- & -- \\
Cottage Cheese (locally produced) & -- & -- & -- \\
Inhalation & 0.0028 & 0.028 & 0.32 \\
Mother's milk (mother on Diet 3) & 0.059 & 0.21 & 0.8 \\
Prenatal exposure (mother on Diet 3) & 0.000046 & 0.0012 & 0.017 \\
Diet 1 & -- & -- & -- \\
Diet 2 & -- & -- & -- \\
Diet 3 & -- & -- & -- \\
\hline
\end{tabular}

Excess Lifetime Risk [ ]

\begin{tabular}{lccc}
\hline Diet 1 & -- & -- & -- \\
Diet 2 & -- & -- & -- \\
Diet 3 & $2.1 \mathrm{E}-05$ & $2.2 \mathrm{E}-04$ & $2.7 \mathrm{E}-03$ \\
Diet 4 & -- & -- & -- \\
\hline
\end{tabular}

\section{Relative Risk [ ]}

\begin{tabular}{lccc}
\hline Diet 1 & -- & -- & -- \\
Diet 2 & -- & -- & -- \\
Diet 3 & 1.0067 & 1.061 & 1.68 \\
Diet 4 & -- & -- & -- \\
\hline
\end{tabular}

\begin{tabular}{lccc}
\hline Diet 1 & -- & -- & -- \\
Diet 2 & -- & -- & -- \\
Diet 3 & 0.67 & 5.8 & 40 \\
Diet 4 & -- & -- \\
\hline Diet 1 - Backyard cow milk + all other locally produced non-milk exposure pathways & \\
Diet 2 - Locally produced commercial milk + all other locally produced non-milk exposure pathways \\
Diet 3 - Regionally mixed commercial milk + inhalation (other regionally mixed food items are minor contributors to the total) \\
Diet 4 - Goat milk (all other exposure pathways are of negligible importance compared to goat milk)
\end{tabular}


Location: Woodland

Receptor: Male born in 1944

\begin{tabular}{lccc}
\hline & \multicolumn{3}{c}{ Thyroid Dose [cGy] } \\
Exposure Pathway & \multicolumn{2}{c}{$9 \%$ Subjective Confidence Interval } \\
\hline Backyard Cow Milk & lower limit & central estimate & upper limit \\
Commercial Milk (locally produced) & -- & -- & -- \\
Commercial Milk (regionally mixed) & -- & -- & -- \\
Goat Milk (locally produced) & 0.19 & 1.2 & 7.1 \\
Beef (locally produced) & -- & -- & -- \\
Leafy Vegetables (locally produced) & -- & -- & -- \\
Eggs (locally produced) & -- & -- & -- \\
Cottage Cheese (locally produced) & -- & -- & -- \\
Inhalation & 0.0026 & 0.03 & 0.34 \\
Mother's milk (mother on Diet 3) & 0.059 & 0.21 & 0.79 \\
Prenatal exposure (mother on Diet 3) & 0.000046 & 0.0012 & 0.017 \\
Diet 1 & -- & -- & -- \\
Diet 2 & -- & -- & -- \\
Diet 3 & -- & -- & -- \\
\hline
\end{tabular}

Excess Lifetime Risk [ ]

\begin{tabular}{lccc}
\hline Diet 1 & -- & -- & -- \\
Diet 2 & -- & -- & -- \\
Diet 3 & $2.5 \mathrm{E}-06$ & $6.2 \mathrm{E}-05$ & $1.3 \mathrm{E}-03$ \\
Diet 4 & -- & -- & -- \\
\hline
\end{tabular}

\section{Relative Risk [ ]}

\begin{tabular}{lccc}
\hline Diet 1 & -- & -- & -- \\
Diet 2 & -- & -- & -- \\
Diet 3 & 1.002 & 1.038 & 1.93 \\
Diet 4 & -- & -- & -- \\
\hline
\end{tabular}

\begin{tabular}{lccc}
\hline Diet 1 & -- & -- & -- \\
Diet 2 & -- & -- & -- \\
Diet 3 & 0.20 & 3.7 & -- \\
Diet 4 & -- & -- & - \\
\hline Diet 1 - Backyard cow milk + all other locally produced non-milk exposure pathways & \\
Diet 2 - Locally produced commercial milk + all other locally produced non-milk exposure pathways \\
Diet 3 - Regionally mixed commercial milk + inhalation (other regionally mixed food items are minor contributors to the total) \\
Diet 4 - Goat milk (all other exposure pathways are of negligible importance compared to goat milk)
\end{tabular}


Location: Hardin Valley

Receptor: Female born in 1944

\begin{tabular}{lccc}
\hline & \multicolumn{3}{c}{ Thyroid Dose [cGy] } \\
Exposure Pathway & \multicolumn{2}{c}{$95 \%$ Subjective Confidence Interval } \\
Backyard Cow Milk & lower limit & central estimate & upper limit \\
Commercial Milk (locally produced) & 2.8 & 16 & 93 \\
Commercial Milk (regionally mixed) & 0.86 & 5.5 & 38 \\
Goat Milk (locally produced) & 0.19 & 1.2 & 7.9 \\
Beef (locally produced) & 7 & 52 & 340 \\
Leafy Vegetables (locally produced) & 0.0044 & 0.078 & 1.5 \\
Eggs (locally produced) & 0.0015 & 0.017 & 0.15 \\
Cottage Cheese (locally produced) & 0.15 & 1.1 & 8.4 \\
Inhalation & 0.004 & 0.042 & 0.49 \\
Mother's milk (mother on Diet 1) & 0.084 & 0.31 & 1.2 \\
Prenatal exposure (mother on Diet 1) & 0.00053 & 0.018 & 0.51 \\
Diet 1 & -- & -- & -- \\
Diet 2 & 3.2 & 18 & 100 \\
Diet 3 & 1.3 & 7.3 & 49 \\
\hline
\end{tabular}

Excess Lifetime Risk [ ]

\begin{tabular}{llll}
\hline Diet 1 & $2.0 \mathrm{E}-04$ & $3.0 \mathrm{E}-03$ & $3.8 \mathrm{E}-02$ \\
Diet 2 & $8.7 \mathrm{E}-05$ & $1.2 \mathrm{E}-03$ & $1.6 \mathrm{E}-02$ \\
Diet 3 & $2.3 \mathrm{E}-05$ & $2.4 \mathrm{E}-04$ & $2.7 \mathrm{E}-03$ \\
Diet 4 & $5.5 \mathrm{E}-04$ & $9.0 \mathrm{E}-03$ & $1.0 \mathrm{E}-01$ \\
\hline
\end{tabular}

\section{Relative Risk [ ]}

\begin{tabular}{lccc}
\hline Diet 1 & 1.084 & 1.77 & 9.3 \\
Diet 2 & 1.033 & 1.3 & 4.9 \\
Diet 3 & 1.0074 & 1.065 & 1.7 \\
Diet 4 & 1.23 & 2.9 & 31 \\
\hline
\end{tabular}

\section{Probability of Causation [\%]}

\begin{tabular}{lccc}
\hline Diet 1 & 7.76 & 43.5 & 89 \\
Diet 2 & 3.19 & 23.1 & 79 \\
Diet 3 & 0.74 & 6.1 & 41 \\
Diet 4 & 18.31 & 65.9 & 97 \\
\hline
\end{tabular}

Diet 1 - Backyard cow milk + all other locally produced non-milk exposure pathways

Diet 2 - Locally produced commercial milk + all other locally produced non-milk exposure pathways

Diet 3 - Regionally mixed commercial milk + inhalation (other regionally mixed food items are minor contributors to the total)

Diet 4 - Goat milk (all other exposure pathways are of negligible importance compared to goat milk) 
Location: Hardin Valley

Receptor: Male born in 1944

\begin{tabular}{lccc}
\hline & \multicolumn{3}{c}{ Thyroid Dose [cGy] } \\
& \multicolumn{2}{c}{$95 \%$ Subjective Confidence Interval } \\
Exposure Pathway & lower limit & central estimate & upper limit \\
\hline Backyard Cow Milk & 2.8 & 16 & 95 \\
Commercial Milk (locally produced) & 0.84 & 5.6 & 37 \\
Commercial Milk (regionally mixed) & 0.19 & 1.2 & 7.1 \\
Goat Milk (locally produced) & 6.9 & 50 & 340 \\
Beef (locally produced) & 0.0047 & 0.082 & 1.6 \\
Leafy Vegetables (locally produced) & 0.0015 & 0.016 & 0.15 \\
Eggs (locally produced) & 0.19 & 1.3 & 9.2 \\
Cottage Cheese (locally produced) & 0.0037 & 0.043 & 0.49 \\
Inhalation & 0.086 & 0.31 & 1.2 \\
Mother's milk (mother on Diet 1) & 0.00053 & 0.018 & 0.51 \\
Prenatal exposure (mother on Diet 1) & -- & -- & -- \\
Diet 1 & 3.3 & 18 & 110 \\
Diet 2 & 1.3 & 7.7 & 50 \\
Diet 3 & 0.32 & 1.6 & 8.4 \\
\hline
\end{tabular}

Excess Lifetime Risk [ ]

\begin{tabular}{llll}
\hline Diet 1 & $2.5 \mathrm{E}-05$ & $8.1 \mathrm{E}-04$ & $1.4 \mathrm{E}-02$ \\
Diet 2 & $1.2 \mathrm{E}-05$ & $3.3 \mathrm{E}-04$ & $6.7 \mathrm{E}-03$ \\
Diet 3 & $2.7 \mathrm{E}-06$ & $6.8 \mathrm{E}-05$ & $1.3 \mathrm{E}-03$ \\
Diet 4 & $6.9 \mathrm{E}-05$ & $1.9 \mathrm{E}-03$ & $4.2 \mathrm{E}-02$ \\
\hline
\end{tabular}

\section{Relative Risk [ ]}

\begin{tabular}{lccc}
\hline Diet 1 & 1.026 & 1.52 & 14 \\
Diet 2 & 1.011 & 1.2 & 5.8 \\
Diet 3 & 1.0022 & 1.041 & 1.95 \\
Diet 4 & 1.067 & 2.4 & 32 \\
\hline
\end{tabular}

\begin{tabular}{|c|c|c|c|}
\hline Diet 1 & 2.52 & 34.1 & 93 \\
\hline Diet 2 & 1.05 & 16.9 & 83 \\
\hline Diet 3 & 0.22 & 3.9 & 49 \\
\hline Diet 4 & 6.31 & 57.7 & 97 \\
\hline
\end{tabular}

Diet 1 - Backyard cow milk + all other locally produced non-milk exposure pathways

Diet 2 - Locally produced commercial milk + all other locally produced non-milk exposure pathways

Diet 3 - Regionally mixed commercial milk + inhalation (other regionally mixed food items are minor contributors to the total)

Diet 4 - Goat milk (all other exposure pathways are of negligible importance compared to goat milk) 


\section{Location: Oliver Springs}

Receptor: Female born in 1944

\begin{tabular}{lccc}
\hline & \multicolumn{3}{c}{ Thyroid Dose [cGy] } \\
Exposure Pathway & \multicolumn{2}{c}{$95 \%$ Subjective Confidence Interval } \\
\hline Backyard Cow Milk & lower limit & central estimate & upper limit \\
Commercial Milk (locally produced) & 0.4 & 2.5 & 15 \\
Commercial Milk (regionally mixed) & 0.13 & 0.82 & 6.5 \\
Goat Milk (locally produced) & 0.19 & 1.2 & 7.9 \\
Beef (locally produced) & 1 & 7.9 & 63 \\
Leafy Vegetables (locally produced) & 0.00068 & 0.012 & 0.27 \\
Eggs (locally produced) & 0.00022 & 0.0026 & 0.026 \\
Cottage Cheese (locally produced) & 0.023 & 0.17 & 1.4 \\
Inhalation & 0.00057 & 0.0066 & 0.077 \\
Mother's milk (mother on Diet 1) & 0.013 & 0.048 & 0.2 \\
Prenatal exposure (mother on Diet 1) & 0.000072 & 0.0028 & 0.077 \\
Diet 1 & -- & -- & -- \\
Diet 2 & 0.46 & 2.8 & 17 \\
Diet 3 & 0.2 & 1.1 & 8.3 \\
\hline
\end{tabular}

Excess Lifetime Risk [ ]

\begin{tabular}{llcc}
\hline Diet 1 & $3.3 \mathrm{E}-05$ & $4.7 \mathrm{E}-04$ & $7.1 \mathrm{E}-03$ \\
Diet 2 & $1.5 \mathrm{E}-05$ & $1.8 \mathrm{E}-04$ & $2.8 \mathrm{E}-03$ \\
Diet 3 & $1.7 \mathrm{E}-05$ & $1.9 \mathrm{E}-04$ & $2.6 \mathrm{E}-03$ \\
Diet 4 & $7.8 \mathrm{E}-05$ & $1.3 \mathrm{E}-03$ & $1.7 \mathrm{E}-02$ \\
\hline
\end{tabular}

\section{Relative Risk [ ]}

\begin{tabular}{lccc}
\hline Diet 1 & 1.011 & 1.12 & 2.5 \\
Diet 2 & 1.0045 & 1.047 & 1.68 \\
Diet 3 & 1.0056 & 1.054 & 1.65 \\
Diet 4 & 1.031 & 1.31 & 5.9 \\
\hline
\end{tabular}

\section{Probability of Causation [\%]}

\begin{tabular}{lccc}
\hline Diet 1 & 1.08 & 10.7 & 60 \\
Diet 2 & 0.45 & 4.5 & 40 \\
Diet 3 & 0.56 & 5.1 & 39 \\
Diet 4 & 3.01 & 23.7 & 82 \\
\hline
\end{tabular}

Diet 1 - Backyard cow milk + all other locally produced non-milk exposure pathways

Diet 2 - Locally produced commercial milk + all other locally produced non-milk exposure pathways

Diet 3 - Regionally mixed commercial milk + inhalation (other regionally mixed food items are minor contributors to the total)

Diet 4 - Goat milk (all other exposure pathways are of negligible importance compared to goat milk) 


\section{Location: Oliver Springs}

\section{Receptor: Male born in 1944}

\begin{tabular}{lccc}
\hline & \multicolumn{3}{c}{ Thyroid Dose [cGy] } \\
& \multicolumn{2}{c}{$95 \%$ Subjective Confidence Interval } \\
Exposure Pathway & lower limit & central estimate & upper limit \\
\hline Backyard Cow Milk & 0.4 & 2.4 & 17 \\
Commercial Milk (locally produced) & 0.13 & 0.84 & 6.6 \\
Commercial Milk (regionally mixed) & 0.19 & 1.2 & 7.1 \\
Goat Milk (locally produced) & 0.96 & 7.9 & 60 \\
Beef (locally produced) & 0.00074 & 0.013 & 0.28 \\
Leafy Vegetables (locally produced) & 0.00022 & 0.0025 & 0.026 \\
Eggs (locally produced) & 0.028 & 0.2 & 1.5 \\
Cottage Cheese (locally produced) & 0.00054 & 0.0065 & 0.083 \\
Inhalation & 0.013 & 0.05 & 0.2 \\
Mother's milk (mother on Diet 1) & 0.000072 & 0.0028 & 0.077 \\
Prenatal exposure (mother on Diet 1) & -- & -- & -- \\
Diet 1 & 0.47 & 2.7 & 18 \\
Diet 2 & 0.2 & 1.2 & 8.5 \\
Diet 3 & 0.22 & 1.2 & 7.3 \\
\hline
\end{tabular}

Excess Lifetime Risk [ ]

\begin{tabular}{llll}
\hline Diet 1 & $3.6 \mathrm{E}-06$ & $1.2 \mathrm{E}-04$ & $2.6 \mathrm{E}-03$ \\
Diet 2 & $1.6 \mathrm{E}-06$ & $5.0 \mathrm{E}-05$ & $1.1 \mathrm{E}-03$ \\
Diet 3 & $2.2 \mathrm{E}-06$ & $5.3 \mathrm{E}-05$ & $1.1 \mathrm{E}-03$ \\
Diet 4 & $1.1 \mathrm{E}-05$ & $3.2 \mathrm{E}-04$ & $7.0 \mathrm{E}-03$ \\
\hline
\end{tabular}

\section{Relative Risk [ ]}

\begin{tabular}{lccc}
\hline Diet 1 & 1.0034 & 1.084 & 2.9 \\
Diet 2 & 1.0014 & 1.033 & 1.88 \\
Diet 3 & 1.0017 & 1.035 & 1.88 \\
Diet 4 & 1.0091 & 1.22 & 6.4 \\
\hline
\end{tabular}

\section{Probability of Causation [\%]}

\begin{tabular}{lccc}
\hline Diet 1 & 0.34 & 7.7 & 65 \\
Diet 2 & 0.14 & 3.2 & 46 \\
Diet 3 & 0.17 & 3.3 & 47 \\
Diet 4 & 0.91 & 18.1 & 84 \\
\hline
\end{tabular}

Diet 1 - Backyard cow milk + all other locally produced non-milk exposure pathways

Diet 2 - Locally produced commercial milk + all other locally produced non-milk exposure pathways

Diet 3 - Regionally mixed commercial milk + inhalation (other regionally mixed food items are minor contributors to the total)

Diet 4 - Goat milk (all other exposure pathways are of negligible importance compared to goat milk) 


\section{Location: Solway}

Receptor: Female born in 1944

\begin{tabular}{lccc}
\hline & \multicolumn{3}{c}{ Thyroid Dose [cGy] } \\
& \multicolumn{2}{c}{$95 \%$ Subjective Confidence Interval } \\
Exposure Pathway & lower limit & central estimate & upper limit \\
\hline Backyard Cow Milk & 2.7 & 15 & 85 \\
Commercial Milk (locally produced) & 0.76 & 4.9 & 34 \\
Commercial Milk (regionally mixed) & 0.19 & 1.2 & 7.9 \\
Goat Milk (locally produced) & 6.3 & 47 & 290 \\
Beef (locally produced) & 0.004 & 0.072 & 1.4 \\
Leafy Vegetables (locally produced) & 0.0014 & 0.016 & 0.14 \\
Eggs (locally produced) & 0.14 & 1 & 7.3 \\
Cottage Cheese (locally produced) & 0.0035 & 0.038 & 0.48 \\
Inhalation & 0.08 & 0.29 & 1.1 \\
Mother's milk (mother on Diet 1) & 0.00048 & 0.016 & 0.43 \\
Prenatal exposure (mother on Diet 1) & -- & -- & -- \\
Diet 1 & 3.1 & 16 & 96 \\
Diet 2 & 1.2 & 6.5 & 43 \\
Diet 3 & 0.34 & 1.5 & 9 \\
\hline
\end{tabular}

Excess Lifetime Risk [ ]

\begin{tabular}{lllc}
\hline Diet 1 & $1.8 \mathrm{E}-04$ & $2.8 \mathrm{E}-03$ & $3.7 \mathrm{E}-02$ \\
Diet 2 & $9.3 \mathrm{E}-05$ & $1.1 \mathrm{E}-03$ & $1.5 \mathrm{E}-02$ \\
Diet 3 & $2.3 \mathrm{E}-05$ & $2.4 \mathrm{E}-04$ & $2.7 \mathrm{E}-03$ \\
Diet 4 & $4.7 \mathrm{E}-04$ & $7.8 \mathrm{E}-03$ & $9.2 \mathrm{E}-02$ \\
\hline
\end{tabular}

\section{Relative Risk [ ]}

\begin{tabular}{lccc}
\hline Diet 1 & 1.072 & 1.71 & 8.6 \\
Diet 2 & 1.028 & 1.28 & 4.4 \\
Diet 3 & 1.007 & 1.064 & 1.7 \\
Diet 4 & 1.19 & 2.7 & 28 \\
\hline
\end{tabular}

\section{Probability of Causation [\%]}

\begin{tabular}{lccc}
\hline Diet 1 & 6.72 & 41.2 & 88 \\
Diet 2 & 2.74 & 22.1 & 77 \\
Diet 3 & 0.69 & 6.0 & 41 \\
Diet 4 & 15.89 & 63.0 & 96 \\
\hline
\end{tabular}

Diet 1 - Backyard cow milk + all other locally produced non-milk exposure pathways

Diet 2 - Locally produced commercial milk + all other locally produced non-milk exposure pathways

Diet 3 - Regionally mixed commercial milk + inhalation (other regionally mixed food items are minor contributors to the total)

Diet 4 - Goat milk (all other exposure pathways are of negligible importance compared to goat milk) 


\section{Location: Solway}

\section{Receptor: Male born in 1944}

\begin{tabular}{lccc}
\hline & \multicolumn{3}{c}{ Thyroid Dose [cGy] } \\
& \multicolumn{2}{c}{$95 \%$ Subjective Confidence Interval } \\
Exposure Pathway & lower limit & central estimate & upper limit \\
\hline Backyard Cow Milk & 2.8 & 15 & 89 \\
Commercial Milk (locally produced) & 0.76 & 5 & 34 \\
Commercial Milk (regionally mixed) & 0.19 & 1.2 & 7.1 \\
Goat Milk (locally produced) & 6.2 & 45 & 300 \\
Beef (locally produced) & 0.0041 & 0.077 & 1.4 \\
Leafy Vegetables (locally produced) & 0.0014 & 0.015 & 0.13 \\
Eggs (locally produced) & 0.17 & 1.2 & 7.8 \\
Cottage Cheese (locally produced) & 0.0031 & 0.038 & 0.43 \\
Inhalation & 0.08 & 0.29 & 1 \\
Mother's milk (mother on Diet 1) & 0.00048 & 0.016 & 0.43 \\
Prenatal exposure (mother on Diet 1) & -- & -- & -- \\
Diet 1 & 3.3 & 16 & 100 \\
Diet 2 & 1.2 & 6.9 & 44 \\
Diet 3 & 0.32 & 1.5 & 8.2 \\
\hline
\end{tabular}

Excess Lifetime Risk [ ]

\begin{tabular}{llll}
\hline Diet 1 & $2.4 \mathrm{E}-05$ & $7.2 \mathrm{E}-04$ & $1.4 \mathrm{E}-02$ \\
Diet 2 & $1.1 \mathrm{E}-05$ & $2.9 \mathrm{E}-04$ & $6.2 \mathrm{E}-03$ \\
Diet 3 & $2.7 \mathrm{E}-06$ & $6.7 \mathrm{E}-05$ & $1.3 \mathrm{E}-03$ \\
Diet 4 & $6.2 \mathrm{E}-05$ & $1.7 \mathrm{E}-03$ & $4.0 \mathrm{E}-02$ \\
\hline
\end{tabular}

\section{Relative Risk [ ]}

\begin{tabular}{lccc}
\hline Diet 1 & 1.02 & 1.49 & 12 \\
Diet 2 & 1.0087 & 1.18 & 5.4 \\
Diet 3 & 1.0022 & 1.04 & 1.95 \\
Diet 4 & 1.071 & 2.3 & 28 \\
\hline
\end{tabular}

\section{Probability of Causation [\%]}

\begin{tabular}{lccc}
\hline Diet 1 & 1.97 & 32.6 & 91 \\
Diet 2 & 0.86 & 15.5 & 81 \\
Diet 3 & 0.22 & 3.8 & 49 \\
Diet 4 & 6.59 & 56.2 & 96 \\
\hline
\end{tabular}

Diet 1 - Backyard cow milk + all other locally produced non-milk exposure pathways

Diet 2 - Locally produced commercial milk + all other locally produced non-milk exposure pathways

Diet 3 - Regionally mixed commercial milk + inhalation (other regionally mixed food items are minor contributors to the total)

Diet 4 - Goat milk (all other exposure pathways are of negligible importance compared to goat milk) 
Location: Sugar Grove

Receptor: Female born in 1944

\begin{tabular}{lccc}
\hline & \multicolumn{3}{c}{ Thyroid Dose [cGy] } \\
Exposure Pathway & \multicolumn{2}{c}{$95 \%$ Subjective Confidence Interval } \\
Backyard Cow Milk & lower limit & central estimate & upper limit \\
Commercial Milk (locally produced) & 1.1 & 6.2 & 38 \\
Commercial Milk (regionally mixed) & 0.32 & 2.1 & 15 \\
Goat Milk (locally produced) & 0.19 & 1.2 & 7.9 \\
Beef (locally produced) & 2.7 & 20 & 140 \\
Leafy Vegetables (locally produced) & 0.0017 & 0.031 & 0.57 \\
Eggs (locally produced) & 0.00058 & 0.0065 & 0.062 \\
Cottage Cheese (locally produced) & 0.059 & 0.43 & 3.2 \\
Inhalation & 0.0014 & 0.016 & 0.2 \\
Mother's milk (mother on Diet 1) & 0.032 & 0.12 & 0.47 \\
Prenatal exposure (mother on Diet 1) & 0.0002 & 0.0068 & 0.2 \\
Diet 1 & -- & -- & -- \\
Diet 2 & 1.3 & 7 & 42 \\
Diet 3 & 0.5 & 2.8 & 20 \\
\hline
\end{tabular}

Excess Lifetime Risk [ ]

\begin{tabular}{llll}
\hline Diet 1 & $7.9 \mathrm{E}-05$ & $1.2 \mathrm{E}-03$ & $1.6 \mathrm{E}-02$ \\
Diet 2 & $3.6 \mathrm{E}-05$ & $4.6 \mathrm{E}-04$ & $6.5 \mathrm{E}-03$ \\
Diet 3 & $1.9 \mathrm{E}-05$ & $2.1 \mathrm{E}-04$ & $2.6 \mathrm{E}-03$ \\
Diet 4 & $2.1 \mathrm{E}-04$ & $3.3 \mathrm{E}-03$ & $3.9 \mathrm{E}-02$ \\
\hline
\end{tabular}

\section{Relative Risk [ ]}

\begin{tabular}{lccc}
\hline Diet 1 & 1.03 & 1.29 & 4.5 \\
Diet 2 & 1.013 & 1.12 & 2.5 \\
Diet 3 & 1.0061 & 1.057 & 1.66 \\
Diet 4 & 1.082 & 1.73 & 12 \\
\hline
\end{tabular}

\begin{tabular}{lccc}
\hline Diet 1 & \multicolumn{3}{c}{ Probability of Causation [\%] } \\
Diet 2 & 1.23 & 21.7 & 76 \\
Diet 3 & 0.61 & 10.3 & 57 \\
Diet 4 & 7.47 & 5.4 & 40 \\
\hline
\end{tabular}

Diet 1 - Backyard cow milk + all other locally produced non-milk exposure pathways

Diet 2 - Locally produced commercial milk + all other locally produced non-milk exposure pathways

Diet 3 - Regionally mixed commercial milk + inhalation (other regionally mixed food items are minor contributors to the total)

Diet 4 - Goat milk (all other exposure pathways are of negligible importance compared to goat milk) 
Location: Sugar Grove

Receptor: Male born in 1944

\begin{tabular}{lccc}
\hline & \multicolumn{3}{c}{ Thyroid Dose [cGy] } \\
& \multicolumn{2}{c}{$95 \%$ Subjective Confidence Interval } \\
Exposure Pathway & 1.1 & 6.1 & 38 \\
\hline Backyard Cow Milk & 0.32 & 2.1 & 15 \\
Commercial Milk (locally produced) & 0.19 & 1.2 & 7.1 \\
Commercial Milk (regionally mixed) & 2.6 & 19 & 130 \\
Goat Milk (locally produced) & 0.0018 & 0.032 & 0.64 \\
Beef (locally produced) & 0.00056 & 0.0063 & 0.061 \\
Leafy Vegetables (locally produced) & 0.068 & 0.49 & 3.6 \\
Eggs (locally produced) & 0.0013 & 0.017 & 0.2 \\
Cottage Cheese (locally produced) & 0.033 & 0.12 & 0.45 \\
Inhalation & 0.0002 & 0.0068 & 0.2 \\
Mother's milk (mother on Diet 1) & -- & -- & - \\
Prenatal exposure (mother on Diet 1) & 1.4 & 6.9 & 43 \\
Diet 1 & 0.5 & 2.9 & 20 \\
Diet 2 & 0.24 & 1.3 & 7.5 \\
Diet 3 & & upper limit \\
\hline
\end{tabular}

Excess Lifetime Risk [ ]

\begin{tabular}{llcc}
\hline Diet 1 & $9.6 \mathrm{E}-06$ & $3.0 \mathrm{E}-04$ & $6.1 \mathrm{E}-03$ \\
Diet 2 & $4.8 \mathrm{E}-06$ & $1.2 \mathrm{E}-04$ & $2.6 \mathrm{E}-03$ \\
Diet 3 & $2.3 \mathrm{E}-06$ & $5.6 \mathrm{E}-05$ & $1.2 \mathrm{E}-03$ \\
Diet 4 & $2.7 \mathrm{E}-05$ & $7.0 \mathrm{E}-04$ & $1.7 \mathrm{E}-02$ \\
\hline
\end{tabular}

\section{Relative Risk [ ]}

\begin{tabular}{lccc}
\hline Diet 1 & 1.0089 & 1.2 & 5.8 \\
Diet 2 & 1.0037 & 1.079 & 3 \\
Diet 3 & 1.0019 & 1.036 & 1.91 \\
Diet 4 & 1.026 & 1.52 & 12 \\
\hline
\end{tabular}

\section{Probability of Causation [\%]}

\begin{tabular}{lccc}
\hline Diet 1 & 0.88 & 16.3 & 81 \\
Diet 2 & 0.37 & 7.2 & 65 \\
Diet 3 & 0.19 & 3.5 & 48 \\
Diet 4 & 2.51 & 33.2 & 91 \\
\hline
\end{tabular}

Diet 1 - Backyard cow milk + all other locally produced non-milk exposure pathways

Diet 2 - Locally produced commercial milk + all other locally produced non-milk exposure pathways

Diet 3 - Regionally mixed commercial milk + inhalation (other regionally mixed food items are minor contributors to the total)

Diet 4 - Goat milk (all other exposure pathways are of negligible importance compared to goat milk) 


\section{Location: OR Townsite}

Receptor: Female born in 1944

\begin{tabular}{lccc}
\hline & \multicolumn{3}{c}{ Thyroid Dose [cGy] } \\
Exposure Pathway & \multicolumn{2}{c}{$9 \%$ Subjective Confidence Interval } \\
\hline Backyard Cow Milk & lower limit & central estimate & upper limit \\
Commercial Milk (locally produced) & -- & -- & -- \\
Commercial Milk (regionally mixed) & -- & -- & -- \\
Goat Milk (locally produced) & 0.19 & 1.2 & 7.9 \\
Beef (locally produced) & -- & -- & -- \\
Leafy Vegetables (locally produced) & -- & -- & -- \\
Eggs (locally produced) & -- & -- & -- \\
Cottage Cheese (locally produced) & -- & -- & -- \\
Inhalation & 0.0017 & 0.017 & 0.21 \\
Mother's milk (mother on Diet 3) & 0.036 & 0.13 & 0.51 \\
Prenatal exposure (mother on Diet 3) & 0.000038 & 0.001 & 0.017 \\
Diet 1 & -- & -- & -- \\
Diet 2 & -- & -- & -- \\
Diet 3 & -- & -- & -- \\
\hline
\end{tabular}

Excess Lifetime Risk [ ]

\begin{tabular}{lccc}
\hline Diet 1 & -- & -- & -- \\
Diet 2 & -- & -- & -- \\
Diet 3 & $1.9 \mathrm{E}-05$ & $2.1 \mathrm{E}-04$ & $2.6 \mathrm{E}-03$ \\
Diet 4 & -- & -- & -- \\
\hline
\end{tabular}

\section{Relative Risk [ ]}

\begin{tabular}{lccc}
\hline Diet 1 & -- & -- & -- \\
Diet 2 & -- & -- & -- \\
Diet 3 & 1.0061 & 1.058 & 1.66 \\
Diet 4 & -- & -- & -- \\
\hline
\end{tabular}

\begin{tabular}{lccc}
\hline Diet 1 & -- & -- & -- \\
Diet 2 & -- & -- & -- \\
Diet 3 & 0.61 & 5.4 & 40 \\
Diet 4 & -- & -- \\
\hline Diet 1 - Backyard cow milk + all other locally produced non-milk exposure pathways & \\
Diet 2 - Locally produced commercial milk + all other locally produced non-milk exposure pathways \\
Diet 3 - Regionally mixed commercial milk + inhalation (other regionally mixed food items are minor contributors to the total) \\
Diet 4 - Goat milk (all other exposure pathways are of negligible importance compared to goat milk)
\end{tabular}




\section{Location: OR Townsite}

\section{Receptor: Male born in 1944}

\begin{tabular}{lccc}
\hline & \multicolumn{3}{c}{ Thyroid Dose [cGy] } \\
Exposure Pathway & \multicolumn{2}{c}{$9 \%$ Subjective Confidence Interval } \\
\hline Backyard Cow Milk & lower limit & central estimate & upper limit \\
Commercial Milk (locally produced) & -- & -- & -- \\
Commercial Milk (regionally mixed) & -- & -- & -- \\
Goat Milk (locally produced) & 0.19 & 1.2 & 7.1 \\
Beef (locally produced) & -- & -- & -- \\
Leafy Vegetables (locally produced) & -- & -- & -- \\
Eggs (locally produced) & -- & -- & -- \\
Cottage Cheese (locally produced) & -- & -- & -- \\
Inhalation & 0.0015 & 0.017 & 0.2 \\
Mother's milk (mother on Diet 3) & 0.035 & 0.13 & 0.48 \\
Prenatal exposure (mother on Diet 3) & 0.000038 & 0.001 & 0.017 \\
Diet 1 & -- & -- & -- \\
Diet 2 & -- & -- & -- \\
Diet 3 & -- & -- & -- \\
\hline
\end{tabular}

Excess Lifetime Risk [ ]

\begin{tabular}{lccc}
\hline Diet 1 & -- & -- & -- \\
Diet 2 & -- & -- & -- \\
Diet 3 & $2.3 \mathrm{E}-06$ & $5.7 \mathrm{E}-05$ & $1.2 \mathrm{E}-03$ \\
Diet 4 & -- & -- & -- \\
\hline
\end{tabular}

\section{Relative Risk [ ]}

\begin{tabular}{lccc}
\hline Diet 1 & -- & -- & -- \\
Diet 2 & -- & -- & -- \\
Diet 3 & 1.0019 & 1.036 & 1.92 \\
Diet 4 & -- & -- & -- \\
\hline
\end{tabular}

\begin{tabular}{lccc}
\hline Diet 1 & -- & -- & -- \\
Diet 2 & -- & -- & -- \\
Diet 3 & 0.19 & 3.5 & 48 \\
Diet 4 & -- & -- & - \\
\hline Diet 1 - Backyard cow milk + all other locally produced non-milk exposure pathways & \\
Diet 2 - Locally produced commercial milk + all other locally produced non-milk exposure pathways \\
Diet 3 - Regionally mixed commercial milk + inhalation (other regionally mixed food items are minor contributors to the total) \\
Diet 4 - Goat milk (all other exposure pathways are of negligible importance compared to goat milk)
\end{tabular}


Location: Hines Valley

Receptor: Female born in 1944

\begin{tabular}{lccc}
\hline & \multicolumn{3}{c}{ Thyroid Dose [cGy] } \\
Exposure Pathway & $95 \%$ Subjective Confidence Interval \\
\hline Backyard Cow Milk & lower limit & central estimate & upper limit \\
Commercial Milk (locally produced) & 1.3 & 7.6 & 43 \\
Commercial Milk (regionally mixed) & 0.38 & 2.5 & 18 \\
Goat Milk (locally produced) & 0.19 & 1.2 & 7.9 \\
Beef (locally produced) & 3.3 & 23 & 160 \\
Leafy Vegetables (locally produced) & 0.002 & 0.037 & 0.81 \\
Eggs (locally produced) & 0.00078 & 0.0076 & 0.069 \\
Cottage Cheese (locally produced) & 0.068 & 0.51 & 3.5 \\
Inhalation & 0.0018 & 0.019 & 0.22 \\
Mother's milk (mother on Diet 1) & 0.041 & 0.16 & 0.57 \\
Prenatal exposure (mother on Diet 1) & 0.00023 & 0.0084 & 0.24 \\
Diet 1 & -- & -- & -- \\
Diet 2 & 1.4 & 8.6 & 47 \\
Diet 3 & 0.6 & 3.3 & 21 \\
\hline
\end{tabular}

Excess Lifetime Risk [ ]

\begin{tabular}{lllc}
\hline Diet 1 & $8.9 \mathrm{E}-05$ & $1.4 \mathrm{E}-03$ & $2.0 \mathrm{E}-02$ \\
Diet 2 & $4.6 \mathrm{E}-05$ & $5.6 \mathrm{E}-04$ & $7.9 \mathrm{E}-03$ \\
Diet 3 & $1.9 \mathrm{E}-05$ & $2.1 \mathrm{E}-04$ & $2.6 \mathrm{E}-03$ \\
Diet 4 & $2.6 \mathrm{E}-04$ & $3.9 \mathrm{E}-03$ & $5.1 \mathrm{E}-02$ \\
\hline
\end{tabular}

\section{Relative Risk [ ]}

\begin{tabular}{lccc}
\hline Diet 1 & 1.039 & 1.36 & 4.5 \\
Diet 2 & 1.015 & 1.14 & 2.7 \\
Diet 3 & 1.0063 & 1.059 & 1.67 \\
Diet 4 & 1.093 & 1.88 & 14 \\
\hline
\end{tabular}

\begin{tabular}{lccc}
\hline Diet 1 & 3.75 & 26.6 & 78 \\
Diet 2 & 1.45 & 12.5 & 63 \\
Diet 3 & 0.63 & 5.5 & 40 \\
Diet 4 & 8.50 & 46.9 & 93 \\
\hline
\end{tabular}

Diet 1 - Backyard cow milk + all other locally produced non-milk exposure pathways

Diet 2 - Locally produced commercial milk + all other locally produced non-milk exposure pathways

Diet 3 - Regionally mixed commercial milk + inhalation (other regionally mixed food items are minor contributors to the total)

Diet 4 - Goat milk (all other exposure pathways are of negligible importance compared to goat milk) 


\section{Location: Hines Valley}

Receptor: Male born in 1944

\begin{tabular}{lccc}
\hline & \multicolumn{3}{c}{ Thyroid Dose [cGy] } \\
& \multicolumn{2}{c}{$95 \%$ Subjective Confidence Interval } \\
Exposure Pathway & lower limit & central estimate & upper limit \\
\hline Backyard Cow Milk & 1.3 & 7.4 & 46 \\
Commercial Milk (locally produced) & 0.4 & 2.5 & 17 \\
Commercial Milk (regionally mixed) & 0.19 & 1.2 & 7.1 \\
Goat Milk (locally produced) & 2.9 & 22 & 150 \\
Beef (locally produced) & 0.0021 & 0.039 & 0.86 \\
Leafy Vegetables (locally produced) & 0.00072 & 0.0078 & 0.07 \\
Eggs (locally produced) & 0.078 & 0.59 & 4.2 \\
Cottage Cheese (locally produced) & 0.0017 & 0.019 & 0.24 \\
Inhalation & 0.042 & 0.16 & 0.57 \\
Mother's milk (mother on Diet 1) & 0.00023 & 0.0084 & 0.24 \\
Prenatal exposure (mother on Diet 1) & -- & -- & - \\
Diet 1 & 1.5 & 8.3 & 51 \\
Diet 2 & 0.62 & 3.4 & 21 \\
Diet 3 & 0.25 & 1.4 & 7.7 \\
\hline
\end{tabular}

Excess Lifetime Risk [ ]

\begin{tabular}{llll}
\hline Diet 1 & $1.1 \mathrm{E}-05$ & $3.8 \mathrm{E}-04$ & $7.9 \mathrm{E}-03$ \\
Diet 2 & $5.6 \mathrm{E}-06$ & $1.5 \mathrm{E}-04$ & $3.4 \mathrm{E}-03$ \\
Diet 3 & $2.4 \mathrm{E}-06$ & $5.9 \mathrm{E}-05$ & $1.2 \mathrm{E}-03$ \\
Diet 4 & $3.4 \mathrm{E}-05$ & $8.9 \mathrm{E}-04$ & $2.0 \mathrm{E}-02$ \\
\hline
\end{tabular}

\section{Relative Risk [ ]}

\begin{tabular}{lccc}
\hline Diet 1 & 1.011 & 1.25 & 6.3 \\
Diet 2 & 1.0047 & 1.098 & 3.3 \\
Diet 3 & 1.002 & 1.037 & 1.92 \\
Diet 4 & 1.031 & 1.63 & 17 \\
\hline
\end{tabular}

\section{Probability of Causation [\%]}

\begin{tabular}{lccc}
\hline Diet 1 & 1.12 & 20.3 & 84 \\
Diet 2 & 0.46 & 8.9 & 69 \\
Diet 3 & 0.20 & 3.6 & 48 \\
Diet 4 & 3.05 & 38.5 & 94 \\
\hline
\end{tabular}

Diet 1 - Backyard cow milk + all other locally produced non-milk exposure pathways

Diet 2 - Locally produced commercial milk + all other locally produced non-milk exposure pathways

Diet 3 - Regionally mixed commercial milk + inhalation (other regionally mixed food items are minor contributors to the total)

Diet 4 - Goat milk (all other exposure pathways are of negligible importance compared to goat milk) 


\section{Location: Farragut}

Receptor: Female born in 1944

\begin{tabular}{lccc}
\hline & \multicolumn{3}{c}{ Thyroid Dose [cGy] } \\
Exposure Pathway & \multicolumn{2}{c}{$95 \%$ Subjective Confidence Interval } \\
\hline Backyard Cow Milk & 1.4 & 8.4 & 46 \\
Commercial Milk (locally produced) & 0.44 & 2.8 & 19 \\
Commercial Milk (regionally mixed) & 0.19 & 1.2 & 7.9 \\
Goat Milk (locally produced) & 3.6 & 27 & 190 \\
Beef (locally produced) & 0.0022 & 0.041 & 0.95 \\
Leafy Vegetables (locally produced) & 0.00078 & 0.0088 & 0.081 \\
Eggs (locally produced) & 0.076 & 0.58 & 4.1 \\
Cottage Cheese (locally produced) & 0.0021 & 0.021 & 0.26 \\
Inhalation & 0.047 & 0.18 & 0.67 \\
Mother's milk (mother on Diet 1) & 0.00028 & 0.009 & 0.25 \\
Prenatal exposure (mother on Diet 1) & -- & -- & - \\
Diet 1 & 1.7 & 9.3 & 51 \\
Diet 2 & 0.69 & 3.8 & 24 \\
Diet 3 & 0.28 & 1.3 & 8.6 \\
\hline
\end{tabular}

Excess Lifetime Risk [ ]

\begin{tabular}{llll}
\hline Diet 1 & $1.0 \mathrm{E}-04$ & $1.6 \mathrm{E}-03$ & $2.0 \mathrm{E}-02$ \\
Diet 2 & $4.8 \mathrm{E}-05$ & $6.2 \mathrm{E}-04$ & $8.0 \mathrm{E}-03$ \\
Diet 3 & $2.0 \mathrm{E}-05$ & $2.2 \mathrm{E}-04$ & $2.6 \mathrm{E}-03$ \\
Diet 4 & $2.9 \mathrm{E}-04$ & $4.6 \mathrm{E}-03$ & $5.3 \mathrm{E}-02$ \\
\hline
\end{tabular}

\section{Relative Risk [ ]}

\begin{tabular}{lccc}
\hline Diet 1 & 1.043 & 1.42 & 5.2 \\
Diet 2 & 1.016 & 1.16 & 3 \\
Diet 3 & 1.0064 & 1.06 & 1.67 \\
Diet 4 & 1.11 & 2 & 16 \\
\hline
\end{tabular}

\section{Probability of Causation [\%]}

\begin{tabular}{lccc}
\hline Diet 1 & 4.16 & 29.3 & 81 \\
Diet 2 & 1.61 & 13.9 & 66 \\
Diet 3 & 0.64 & 5.6 & 40 \\
Diet 4 & 9.72 & 50.2 & 94 \\
\hline
\end{tabular}

Diet 1 - Backyard cow milk + all other locally produced non-milk exposure pathways

Diet 2 - Locally produced commercial milk + all other locally produced non-milk exposure pathways

Diet 3 - Regionally mixed commercial milk + inhalation (other regionally mixed food items are minor contributors to the total)

Diet 4 - Goat milk (all other exposure pathways are of negligible importance compared to goat milk) 


\section{Location: Farragut}

\section{Receptor: Male born in 1944}

\begin{tabular}{lccc}
\hline & \multicolumn{3}{c}{ Thyroid Dose [cGy] } \\
& \multicolumn{2}{c}{$95 \%$ Subjective Confidence Interval } \\
Exposure Pathway & lower limit & central estimate & upper limit \\
\hline Backyard Cow Milk & 1.5 & 8.3 & 49 \\
Commercial Milk (locally produced) & 0.43 & 2.9 & 18 \\
Commercial Milk (regionally mixed) & 0.19 & 1.2 & 7.1 \\
Goat Milk (locally produced) & 3.5 & 26 & 180 \\
Beef (locally produced) & 0.0024 & 0.044 & 1.1 \\
Leafy Vegetables (locally produced) & 0.00074 & 0.0086 & 0.081 \\
Eggs (locally produced) & 0.093 & 0.66 & 4.7 \\
Cottage Cheese (locally produced) & 0.0019 & 0.022 & 0.26 \\
Inhalation & 0.044 & 0.18 & 0.63 \\
Mother's milk (mother on Diet 1) & 0.00028 & 0.009 & 0.25 \\
Prenatal exposure (mother on Diet 1) & -- & -- & - \\
Diet 1 & 1.7 & 9.4 & 53 \\
Diet 2 & 0.69 & 3.9 & 24 \\
Diet 3 & 0.27 & 1.4 & 7.8 \\
\hline
\end{tabular}

Excess Lifetime Risk [ ]

\begin{tabular}{llll}
\hline Diet 1 & $1.4 \mathrm{E}-05$ & $4.0 \mathrm{E}-04$ & $8.0 \mathrm{E}-03$ \\
Diet 2 & $6.0 \mathrm{E}-06$ & $1.7 \mathrm{E}-04$ & $3.6 \mathrm{E}-03$ \\
Diet 3 & $2.4 \mathrm{E}-06$ & $6.0 \mathrm{E}-05$ & $1.2 \mathrm{E}-03$ \\
Diet 4 & $4.0 \mathrm{E}-05$ & $9.8 \mathrm{E}-04$ & $2.4 \mathrm{E}-02$ \\
\hline
\end{tabular}

\section{Relative Risk [ ]}

\begin{tabular}{lccc}
\hline Diet 1 & 1.014 & 1.27 & 7.3 \\
Diet 2 & 1.0054 & 1.11 & 3.6 \\
Diet 3 & 1.002 & 1.038 & 1.93 \\
Diet 4 & 1.04 & 1.73 & 18 \\
\hline
\end{tabular}

\section{Probability of Causation [\%]}

\begin{tabular}{lccc}
\hline Diet 1 & 1.34 & 21.0 & 86 \\
Diet 2 & 0.54 & 9.6 & 72 \\
Diet 3 & 0.20 & 3.6 & 48 \\
Diet 4 & 3.84 & 42.0 & 94 \\
\hline
\end{tabular}

Diet 1 - Backyard cow milk + all other locally produced non-milk exposure pathways

Diet 2 - Locally produced commercial milk + all other locally produced non-milk exposure pathways

Diet 3 - Regionally mixed commercial milk + inhalation (other regionally mixed food items are minor contributors to the total)

Diet 4 - Goat milk (all other exposure pathways are of negligible importance compared to goat milk) 
Location: Lenoir City

Receptor: Female born in 1944

\begin{tabular}{lccc}
\hline & \multicolumn{3}{c}{ Thyroid Dose [cGy] } \\
Exposure Pathway & \multicolumn{2}{c}{$95 \%$ Subjective Confidence Interval } \\
Backyard Cow Milk & lower limit & central estimate & upper limit \\
Commercial Milk (locally produced) & 0.91 & 5 & 26 \\
Commercial Milk (regionally mixed) & 0.27 & 1.6 & 13 \\
Goat Milk (locally produced) & 0.19 & 1.2 & 7.9 \\
Beef (locally produced) & -- & -- & -- \\
Leafy Vegetables (locally produced) & 0.0014 & 0.026 & 0.51 \\
Eggs (locally produced) & 0.00056 & 0.0055 & 0.044 \\
Cottage Cheese (locally produced) & 0.051 & 0.36 & 2.5 \\
Inhalation & 0.0012 & 0.013 & 0.14 \\
Mother's milk (mother on Diet 1) & 0.03 & 0.11 & 0.43 \\
Prenatal exposure (mother on Diet 1) & 0.00016 & 0.0057 & 0.16 \\
Diet 1 & -- & -- & -- \\
Diet 2 & 1 & 5.5 & 29 \\
Diet 3 & 0.42 & 2.3 & 15 \\
\hline
\end{tabular}

Excess Lifetime Risk [ ]

\begin{tabular}{lccc}
\hline Diet 1 & $7.3 \mathrm{E}-05$ & $9.6 \mathrm{E}-04$ & $1.4 \mathrm{E}-02$ \\
Diet 2 & $3.4 \mathrm{E}-05$ & $3.6 \mathrm{E}-04$ & $5.6 \mathrm{E}-03$ \\
Diet 3 & $1.9 \mathrm{E}-05$ & $2.0 \mathrm{E}-04$ & $2.6 \mathrm{E}-03$ \\
Diet 4 & -- & -- & -- \\
\hline
\end{tabular}

\section{Relative Risk [ ]}

\begin{tabular}{lccc}
\hline Diet 1 & 1.028 & 1.24 & 3.7 \\
Diet 2 & 1.01 & 1.098 & 2.4 \\
Diet 3 & 1.0061 & 1.056 & 1.66 \\
Diet 4 & -- & -- & -- \\
\hline
\end{tabular}

\begin{tabular}{lccc}
\hline Diet 1 & 2.70 & 19.1 & 73 \\
Diet 2 & 1.02 & 9.0 & 58 \\
Diet 3 & 0.61 & 5.3 & 40 \\
Diet 4 & -- & -- & -- \\
\hline Diet 1 - Backyard cow milk + all other locally produced non-milk exposure pathways & \\
Diet 2 - Locally produced commercial milk + all other locally produced non-milk exposure pathways \\
Diet 3 - Regionally mixed commercial milk + inhalation (other regionally mixed food items are minor contributors to the total) \\
Diet 4 - Goat milk (all other exposure pathways are of negligible importance compared to goat milk)
\end{tabular}




\section{Location: Lenoir City}

\section{Receptor: Male born in 1944}

\begin{tabular}{lccc}
\hline & \multicolumn{3}{c}{ Thyroid Dose [cGy] } \\
& \multicolumn{2}{c}{$95 \%$ Subjective Confidence Interval } \\
Exposure Pathway & lower limit & central estimate & upper limit \\
\hline Backyard Cow Milk & 0.93 & 5 & 27 \\
Commercial Milk (locally produced) & 0.28 & 1.7 & 13 \\
Commercial Milk (regionally mixed) & 0.19 & 1.2 & 7.1 \\
Goat Milk (locally produced) & -- & -- & -- \\
Beef (locally produced) & 0.0016 & 0.026 & 0.51 \\
Leafy Vegetables (locally produced) & 0.00057 & 0.0054 & 0.047 \\
Eggs (locally produced) & 0.062 & 0.41 & 2.8 \\
Cottage Cheese (locally produced) & 0.0012 & 0.014 & 0.15 \\
Inhalation & 0.03 & 0.11 & 0.42 \\
Mother's milk (mother on Diet 1) & 0.00016 & 0.0057 & 0.16 \\
Prenatal exposure (mother on Diet 1) & -- & -- & -- \\
Diet 1 & 1.1 & 5.6 & 31 \\
Diet 2 & 0.45 & 2.3 & 15 \\
Diet 3 & 0.24 & 1.3 & 7.5 \\
\hline
\end{tabular}

Excess Lifetime Risk [ ]

\begin{tabular}{lccc}
\hline Diet 1 & $7.0 \mathrm{E}-06$ & $2.6 \mathrm{E}-04$ & $4.6 \mathrm{E}-03$ \\
Diet 2 & $3.5 \mathrm{E}-06$ & $9.9 \mathrm{E}-05$ & $2.2 \mathrm{E}-03$ \\
Diet 3 & $2.3 \mathrm{E}-06$ & $5.6 \mathrm{E}-05$ & $1.2 \mathrm{E}-03$ \\
Diet 4 & -- & -- & -- \\
\hline
\end{tabular}

\section{Relative Risk [ ]}

\begin{tabular}{lccc}
\hline Diet 1 & 1.0082 & 1.17 & 4.5 \\
Diet 2 & 1.0034 & 1.066 & 2.6 \\
Diet 3 & 1.0019 & 1.035 & 1.92 \\
Diet 4 & -- & -- & -- \\
\hline
\end{tabular}

\begin{tabular}{lccc}
\hline Diet 1 & 0.82 & 14.5 & 78 \\
Diet 2 & 0.34 & 6.2 & 61 \\
Diet 3 & 0.19 & 3.4 & 48 \\
Diet 4 & -- & -- & \\
\hline Diet 1 - Backyard cow milk + all other locally produced non-milk exposure pathways & \\
Diet 2 - Locally produced commercial milk + all other locally produced non-milk exposure pathways \\
Diet 3 - Regionally mixed commercial milk + inhalation (other regionally mixed food items are minor contributors to the total) \\
Diet 4 - Goat milk (all other exposure pathways are of negligible importance compared to goat milk)
\end{tabular}




\section{Location: Kingston}

Receptor: Female born in 1944

\begin{tabular}{lccc}
\hline & \multicolumn{3}{c}{ Thyroid Dose [cGy] } \\
Exposure Pathway & \multicolumn{2}{c}{$95 \%$ Subjective Confidence Interval } \\
\hline Backyard Cow Milk & 1.1 & 6.6 & 35 \\
Commercial Milk (locally produced) & 0.34 & 2.2 & 15 \\
Commercial Milk (regionally mixed) & 0.19 & 1.2 & 7.9 \\
Goat Milk (locally produced) & 2.9 & 21 & 140 \\
Beef (locally produced) & 0.0019 & 0.033 & 0.66 \\
Leafy Vegetables (locally produced) & 0.00062 & 0.007 & 0.063 \\
Eggs (locally produced) & 0.063 & 0.44 & 3.4 \\
Cottage Cheese (locally produced) & 0.0016 & 0.017 & 0.19 \\
Inhalation & 0.04 & 0.14 & 0.56 \\
Mother's milk (mother on Diet 1) & 0.00022 & 0.0072 & 0.22 \\
Prenatal exposure (mother on Diet 1) & -- & -- & -- \\
Diet 1 & 1.2 & 7.4 & 39 \\
Diet 2 & 0.57 & 3 & 20 \\
Diet 3 & 0.26 & 1.3 & 8.4 \\
\hline
\end{tabular}

Excess Lifetime Risk [ ]

\begin{tabular}{llll}
\hline Diet 1 & $8.1 \mathrm{E}-05$ & $1.2 \mathrm{E}-03$ & $1.6 \mathrm{E}-02$ \\
Diet 2 & $4.3 \mathrm{E}-05$ & $4.9 \mathrm{E}-04$ & $6.9 \mathrm{E}-03$ \\
Diet 3 & $1.9 \mathrm{E}-05$ & $2.1 \mathrm{E}-04$ & $2.6 \mathrm{E}-03$ \\
Diet 4 & $2.1 \mathrm{E}-04$ & $3.4 \mathrm{E}-03$ & $4.0 \mathrm{E}-02$ \\
\hline
\end{tabular}

\section{Relative Risk [ ]}

\begin{tabular}{lccc}
\hline Diet 1 & 1.034 & 1.32 & 4.6 \\
Diet 2 & 1.014 & 1.13 & 2.6 \\
Diet 3 & 1.0062 & 1.058 & 1.67 \\
Diet 4 & 1.095 & 1.79 & 12 \\
\hline
\end{tabular}

\section{Probability of Causation [\%]}

\begin{tabular}{lccc}
\hline Diet 1 & 3.30 & 24.0 & 78 \\
Diet 2 & 1.36 & 11.3 & 62 \\
Diet 3 & 0.62 & 5.5 & 40 \\
Diet 4 & 8.70 & 43.9 & 92 \\
\hline
\end{tabular}

Diet 1 - Backyard cow milk + all other locally produced non-milk exposure pathways

Diet 2 - Locally produced commercial milk + all other locally produced non-milk exposure pathways

Diet 3 - Regionally mixed commercial milk + inhalation (other regionally mixed food items are minor contributors to the total)

Diet 4 - Goat milk (all other exposure pathways are of negligible importance compared to goat milk) 


\section{Location: Kingston}

Receptor: Male born in 1944

\begin{tabular}{lccc}
\hline & \multicolumn{3}{c}{ Thyroid Dose [cGy] } \\
& \multicolumn{2}{c}{$95 \%$ Subjective Confidence Interval } \\
Exposure Pathway & lower limit & central estimate & upper limit \\
\hline Backyard Cow Milk & 1.1 & 6.6 & 37 \\
Commercial Milk (locally produced) & 0.35 & 2.2 & 14 \\
Commercial Milk (regionally mixed) & 0.19 & 1.2 & 7.1 \\
Goat Milk (locally produced) & 2.7 & 20 & 140 \\
Beef (locally produced) & 0.0019 & 0.034 & 0.74 \\
Leafy Vegetables (locally produced) & 0.00061 & 0.0066 & 0.064 \\
Eggs (locally produced) & 0.07 & 0.51 & 3.5 \\
Cottage Cheese (locally produced) & 0.0015 & 0.017 & 0.2 \\
Inhalation & 0.038 & 0.14 & 0.54 \\
Mother's milk (mother on Diet 1) & 0.00022 & 0.0072 & 0.22 \\
Prenatal exposure (mother on Diet 1) & -- & -- & - \\
Diet 1 & 1.3 & 7.4 & 41 \\
Diet 2 & 0.54 & 3.1 & 20 \\
Diet 3 & 0.25 & 1.4 & 7.6 \\
\hline
\end{tabular}

Excess Lifetime Risk [ ]

\begin{tabular}{llll}
\hline Diet 1 & $1.0 \mathrm{E}-05$ & $3.2 \mathrm{E}-04$ & $6.2 \mathrm{E}-03$ \\
Diet 2 & $4.5 \mathrm{E}-06$ & $1.3 \mathrm{E}-04$ & $2.9 \mathrm{E}-03$ \\
Diet 3 & $2.4 \mathrm{E}-06$ & $5.9 \mathrm{E}-05$ & $1.2 \mathrm{E}-03$ \\
Diet 4 & $2.8 \mathrm{E}-05$ & $7.8 \mathrm{E}-04$ & $1.9 \mathrm{E}-02$ \\
\hline
\end{tabular}

\section{Relative Risk [ ]}

\begin{tabular}{lccc}
\hline Diet 1 & 1.009 & 1.22 & 5.8 \\
Diet 2 & 1.004 & 1.086 & 3.1 \\
Diet 3 & 1.0019 & 1.037 & 1.93 \\
Diet 4 & 1.027 & 1.56 & 14 \\
\hline
\end{tabular}

\section{Probability of Causation [\%]}

\begin{tabular}{lccc}
\hline Diet 1 & 0.89 & 17.9 & 82 \\
Diet 2 & 0.40 & 7.9 & 68 \\
Diet 3 & 0.19 & 3.6 & 48 \\
Diet 4 & 2.66 & 35.8 & 93 \\
\hline
\end{tabular}

Diet 1 - Backyard cow milk + all other locally produced non-milk exposure pathways

Diet 2 - Locally produced commercial milk + all other locally produced non-milk exposure pathways

Diet 3 - Regionally mixed commercial milk + inhalation (other regionally mixed food items are minor contributors to the total)

Diet 4 - Goat milk (all other exposure pathways are of negligible importance compared to goat milk) 


\section{Location: Karns}

Receptor: Female born in 1944

\begin{tabular}{lccc}
\hline & \multicolumn{3}{c}{ Thyroid Dose [cGy] } \\
& \multicolumn{2}{c}{$95 \%$ Subjective Confidence Interval } \\
Exposure Pathway & lower limit & central estimate & upper limit \\
\hline Backyard Cow Milk & 1.5 & 9.1 & 46 \\
Commercial Milk (locally produced) & 0.47 & 3 & 20 \\
Commercial Milk (regionally mixed) & 0.19 & 1.2 & 7.9 \\
Goat Milk (locally produced) & 3.8 & 28 & 190 \\
Beef (locally produced) & 0.0024 & 0.043 & 0.91 \\
Leafy Vegetables (locally produced) & 0.00084 & 0.0094 & 0.08 \\
Eggs (locally produced) & 0.082 & 0.61 & 4.2 \\
Cottage Cheese (locally produced) & 0.0022 & 0.023 & 0.26 \\
Inhalation & 0.05 & 0.19 & 0.74 \\
Mother's milk (mother on Diet 1) & 0.00031 & 0.0096 & 0.29 \\
Prenatal exposure (mother on Diet 1) & -- & -- & -- \\
Diet 1 & 1.8 & 10 & 51 \\
Diet 2 & 0.73 & 4.1 & 24 \\
Diet 3 & 0.28 & 1.4 & 8.7 \\
\hline
\end{tabular}

Excess Lifetime Risk [ ]

\begin{tabular}{lllc}
\hline Diet 1 & $1.1 \mathrm{E}-04$ & $1.7 \mathrm{E}-03$ & $2.1 \mathrm{E}-02$ \\
Diet 2 & $5.4 \mathrm{E}-05$ & $6.5 \mathrm{E}-04$ & $8.7 \mathrm{E}-03$ \\
Diet 3 & $2.0 \mathrm{E}-05$ & $2.2 \mathrm{E}-04$ & $2.6 \mathrm{E}-03$ \\
Diet 4 & $3.1 \mathrm{E}-04$ & $4.9 \mathrm{E}-03$ & $5.8 \mathrm{E}-02$ \\
\hline
\end{tabular}

\section{Relative Risk [ ]}

\begin{tabular}{lccc}
\hline Diet 1 & 1.046 & 1.44 & 5.4 \\
Diet 2 & 1.018 & 1.18 & 3 \\
Diet 3 & 1.0065 & 1.06 & 1.68 \\
Diet 4 & 1.12 & 2.1 & 17 \\
\hline
\end{tabular}

\section{Probability of Causation [\%]}

\begin{tabular}{lccc}
\hline Diet 1 & 4.39 & 30.3 & 81 \\
Diet 2 & 1.74 & 15.0 & 67 \\
Diet 3 & 0.65 & 5.7 & 40 \\
Diet 4 & 10.62 & 51.7 & 94 \\
\hline
\end{tabular}

Diet 1 - Backyard cow milk + all other locally produced non-milk exposure pathways

Diet 2 - Locally produced commercial milk + all other locally produced non-milk exposure pathways

Diet 3 - Regionally mixed commercial milk + inhalation (other regionally mixed food items are minor contributors to the total)

Diet 4 - Goat milk (all other exposure pathways are of negligible importance compared to goat milk) 


\section{Location: Karns}

\section{Receptor: Male born in 1944}

\begin{tabular}{lccc}
\hline & \multicolumn{3}{c}{ Thyroid Dose [cGy] } \\
Exposure Pathway & $95 \%$ Subjective Confidence Interval \\
\hline Backyard Cow Milk & lower limit & central estimate & upper limit \\
Commercial Milk (locally produced) & 1.6 & 8.9 & 46 \\
Commercial Milk (regionally mixed) & 0.48 & 3 & 18 \\
Goat Milk (locally produced) & 0.19 & 1.2 & 7.1 \\
Beef (locally produced) & 3.5 & 27 & 190 \\
Leafy Vegetables (locally produced) & 0.0026 & 0.045 & 0.98 \\
Eggs (locally produced) & 0.00083 & 0.0091 & 0.078 \\
Cottage Cheese (locally produced) & 0.099 & 0.69 & 4.9 \\
Inhalation & 0.0021 & 0.023 & 0.27 \\
Mother's milk (mother on Diet 1) & 0.051 & 0.2 & 0.71 \\
Prenatal exposure (mother on Diet 1) & 0.00031 & 0.0096 & 0.29 \\
Diet 1 & -- & -- & -- \\
Diet 2 & 1.8 & 10 & 53 \\
Diet 3 & 0.74 & 4.3 & 23 \\
\hline
\end{tabular}

Excess Lifetime Risk [ ]

\begin{tabular}{llll}
\hline Diet 1 & $1.5 \mathrm{E}-05$ & $4.3 \mathrm{E}-04$ & $8.4 \mathrm{E}-03$ \\
Diet 2 & $6.4 \mathrm{E}-06$ & $1.8 \mathrm{E}-04$ & $3.8 \mathrm{E}-03$ \\
Diet 3 & $2.4 \mathrm{E}-06$ & $6.1 \mathrm{E}-05$ & $1.3 \mathrm{E}-03$ \\
Diet 4 & $4.0 \mathrm{E}-05$ & $1.0 \mathrm{E}-03$ & $2.4 \mathrm{E}-02$ \\
\hline
\end{tabular}

\section{Relative Risk [ ]}

\begin{tabular}{lccc}
\hline Diet 1 & 1.014 & 1.29 & 7.6 \\
Diet 2 & 1.0057 & 1.11 & 3.6 \\
Diet 3 & 1.002 & 1.038 & 1.93 \\
Diet 4 & 1.041 & 1.78 & 17 \\
\hline
\end{tabular}

\section{Probability of Causation [\%]}

\begin{tabular}{lccc}
\hline Diet 1 & 1.33 & 22.2 & 87 \\
Diet 2 & 0.57 & 10.2 & 72 \\
Diet 3 & 0.20 & 3.7 & 48 \\
Diet 4 & 3.94 & 43.8 & 94 \\
\hline
\end{tabular}

Diet 1 - Backyard cow milk + all other locally produced non-milk exposure pathways

Diet 2 - Locally produced commercial milk + all other locally produced non-milk exposure pathways

Diet 3 - Regionally mixed commercial milk + inhalation (other regionally mixed food items are minor contributors to the total)

Diet 4 - Goat milk (all other exposure pathways are of negligible importance compared to goat milk) 


\section{Location: Loudon}

Receptor: Female born in 1944

\begin{tabular}{lccc}
\hline & \multicolumn{3}{c}{ Thyroid Dose [cGy] } \\
& \multicolumn{2}{c}{$95 \%$ Subjective Confidence Interval } \\
Exposure Pathway & lower limit & central estimate & upper limit \\
\hline Backyard Cow Milk & 0.88 & 5 & 29 \\
Commercial Milk (locally produced) & 0.25 & 1.7 & 11 \\
Commercial Milk (regionally mixed) & 0.19 & 1.2 & 7.9 \\
Goat Milk (locally produced) & 2.1 & 16 & 110 \\
Beef (locally produced) & 0.0014 & 0.025 & 0.48 \\
Leafy Vegetables (locally produced) & 0.00047 & 0.0053 & 0.048 \\
Eggs (locally produced) & 0.046 & 0.35 & 2.5 \\
Cottage Cheese (locally produced) & 0.0011 & 0.013 & 0.15 \\
Inhalation & 0.031 & 0.11 & 0.44 \\
Mother's milk (mother on Diet 1) & 0.00017 & 0.0057 & 0.15 \\
Prenatal exposure (mother on Diet 1) & -- & -- & -- \\
Diet 1 & 1 & 5.6 & 33 \\
Diet 2 & 0.4 & 2.3 & 14 \\
Diet 3 & 0.25 & 1.3 & 8.4 \\
\hline
\end{tabular}

Excess Lifetime Risk [ ]

\begin{tabular}{llll}
\hline Diet 1 & $6.3 \mathrm{E}-05$ & $9.5 \mathrm{E}-04$ & $1.3 \mathrm{E}-02$ \\
Diet 2 & $3.1 \mathrm{E}-05$ & $3.8 \mathrm{E}-04$ & $5.0 \mathrm{E}-03$ \\
Diet 3 & $1.9 \mathrm{E}-05$ & $2.1 \mathrm{E}-04$ & $2.6 \mathrm{E}-03$ \\
Diet 4 & $1.6 \mathrm{E}-04$ & $2.6 \mathrm{E}-03$ & $3.2 \mathrm{E}-02$ \\
\hline
\end{tabular}

\section{Relative Risk [ ]}

\begin{tabular}{lccc}
\hline Diet 1 & 1.025 & 1.24 & 3.5 \\
Diet 2 & 1.01 & 1.097 & 2.1 \\
Diet 3 & 1.006 & 1.057 & 1.66 \\
Diet 4 & 1.062 & 1.61 & 9.8 \\
\hline
\end{tabular}

\section{Probability of Causation [\%]}

\begin{tabular}{lccc}
\hline Diet 1 & 2.44 & 19.0 & 71 \\
Diet 2 & 1.03 & 8.9 & 52 \\
Diet 3 & 0.60 & 5.4 & 40 \\
Diet 4 & 5.85 & 38.0 & 90 \\
\hline
\end{tabular}

Diet 1 - Backyard cow milk + all other locally produced non-milk exposure pathways

Diet 2 - Locally produced commercial milk + all other locally produced non-milk exposure pathways

Diet 3 - Regionally mixed commercial milk + inhalation (other regionally mixed food items are minor contributors to the total)

Diet 4 - Goat milk (all other exposure pathways are of negligible importance compared to goat milk) 


\section{Location: Loudon}

\section{Receptor: Male born in 1944}

\begin{tabular}{lccc}
\hline & \multicolumn{3}{c}{ Thyroid Dose [cGy] } \\
& \multicolumn{2}{c}{$95 \%$ Subjective Confidence Interval } \\
Exposure Pathway & lower limit & central estimate & upper limit \\
\hline Backyard Cow Milk & 0.92 & 5 & 30 \\
Commercial Milk (locally produced) & 0.25 & 1.7 & 11 \\
Commercial Milk (regionally mixed) & 0.19 & 1.2 & 7.1 \\
Goat Milk (locally produced) & 2 & 15 & 100 \\
Beef (locally produced) & 0.0015 & 0.026 & 0.52 \\
Leafy Vegetables (locally produced) & 0.00046 & 0.0051 & 0.047 \\
Eggs (locally produced) & 0.055 & 0.39 & 2.7 \\
Cottage Cheese (locally produced) & 0.0011 & 0.013 & 0.15 \\
Inhalation & 0.03 & 0.12 & 0.43 \\
Mother's milk (mother on Diet 1) & 0.00017 & 0.0057 & 0.15 \\
Prenatal exposure (mother on Diet 1) & -- & -- & - \\
Diet 1 & 1.1 & 5.6 & 34 \\
Diet 2 & 0.4 & 2.4 & 15 \\
Diet 3 & 0.24 & 1.3 & 7.5 \\
\hline
\end{tabular}

Excess Lifetime Risk [ ]

\begin{tabular}{llll}
\hline Diet 1 & $8.0 \mathrm{E}-06$ & $2.4 \mathrm{E}-04$ & $5.1 \mathrm{E}-03$ \\
Diet 2 & $3.8 \mathrm{E}-06$ & $1.0 \mathrm{E}-04$ & $2.0 \mathrm{E}-03$ \\
Diet 3 & $2.3 \mathrm{E}-06$ & $5.6 \mathrm{E}-05$ & $1.2 \mathrm{E}-03$ \\
Diet 4 & $2.3 \mathrm{E}-05$ & $5.7 \mathrm{E}-04$ & $1.4 \mathrm{E}-02$ \\
\hline
\end{tabular}

\section{Relative Risk [ ]}

\begin{tabular}{lccc}
\hline Diet 1 & 1.0074 & 1.16 & 4.6 \\
Diet 2 & 1.003 & 1.066 & 2.5 \\
Diet 3 & 1.0019 & 1.036 & 1.91 \\
Diet 4 & 1.023 & 1.43 & 9.9 \\
\hline
\end{tabular}

\begin{tabular}{lccc} 
& \multicolumn{3}{c}{ Probability of Causation [\%] } \\
\hline Diet 1 & 0.73 & 14.0 & 78 \\
Diet 2 & 0.30 & 6.2 & 60 \\
Diet 3 & 0.19 & 3.5 & 48 \\
Diet 4 & 2.20 & 30.0 & 90 \\
\hline
\end{tabular}

Diet 1 - Backyard cow milk + all other locally produced non-milk exposure pathways

Diet 2 - Locally produced commercial milk + all other locally produced non-milk exposure pathways

Diet 3 - Regionally mixed commercial milk + inhalation (other regionally mixed food items are minor contributors to the total)

Diet 4 - Goat milk (all other exposure pathways are of negligible importance compared to goat milk) 
Location: Harriman

Receptor: Female born in 1944

\begin{tabular}{lccc}
\hline & \multicolumn{3}{c}{ Thyroid Dose [cGy] } \\
Exposure Pathway & \multicolumn{2}{c}{ 95\% Subjective Confidence Interval } \\
\hline Backyard Cow Milk & lower limit & central estimate & upper limit \\
Commercial Milk (locally produced) & 0.52 & 2.9 & 15 \\
Commercial Milk (regionally mixed) & 0.16 & 0.96 & 7.2 \\
Goat Milk (locally produced) & 0.19 & 1.2 & 7.9 \\
Beef (locally produced) & 1.2 & 9.2 & 61 \\
Leafy Vegetables (locally produced) & 0.00082 & 0.015 & 0.3 \\
Eggs (locally produced) & 0.00031 & 0.0032 & 0.025 \\
Cottage Cheese (locally produced) & 0.029 & 0.2 & 1.4 \\
Inhalation & 0.00072 & 0.0075 & 0.079 \\
Mother's milk (mother on Diet 1) & 0.017 & 0.062 & 0.24 \\
Prenatal exposure (mother on Diet 1) & 0.000092 & 0.0032 & 0.088 \\
Diet 1 & -- & -- & -- \\
Diet 2 & 0.6 & 3.3 & 17 \\
Diet 3 & 0.25 & 1.3 & 8.9 \\
\hline
\end{tabular}

Excess Lifetime Risk [ ]

\begin{tabular}{llll}
\hline Diet 1 & $4.1 \mathrm{E}-05$ & $5.6 \mathrm{E}-04$ & $7.5 \mathrm{E}-03$ \\
Diet 2 & $1.9 \mathrm{E}-05$ & $2.1 \mathrm{E}-04$ & $3.1 \mathrm{E}-03$ \\
Diet 3 & $1.7 \mathrm{E}-05$ & $2.0 \mathrm{E}-04$ & $2.6 \mathrm{E}-03$ \\
Diet 4 & $1.0 \mathrm{E}-04$ & $1.5 \mathrm{E}-03$ & $1.9 \mathrm{E}-02$ \\
\hline
\end{tabular}

\section{Relative Risk [ ]}

\begin{tabular}{lccc}
\hline Diet 1 & 1.015 & 1.14 & 2.6 \\
Diet 2 & 1.0059 & 1.056 & 1.75 \\
Diet 3 & 1.0057 & 1.055 & 1.65 \\
Diet 4 & 1.036 & 1.35 & 6.1 \\
\hline
\end{tabular}

\section{Probability of Causation [\%]}

\begin{tabular}{lccc}
\hline Diet 1 & 1.51 & 12.1 & 61 \\
Diet 2 & 0.59 & 5.3 & 43 \\
Diet 3 & 0.57 & 5.2 & 39 \\
Diet 4 & 3.44 & 26.0 & 83 \\
\hline
\end{tabular}

Diet 1 - Backyard cow milk + all other locally produced non-milk exposure pathways

Diet 2 - Locally produced commercial milk + all other locally produced non-milk exposure pathways

Diet 3 - Regionally mixed commercial milk + inhalation (other regionally mixed food items are minor contributors to the total)

Diet 4 - Goat milk (all other exposure pathways are of negligible importance compared to goat milk) 


\section{Location: Harriman}

\section{Receptor: Male born in 1944}

\begin{tabular}{lccc}
\hline & \multicolumn{3}{c}{ Thyroid Dose [cGy] } \\
& \multicolumn{2}{c}{$95 \%$ Subjective Confidence Interval } \\
Exposure Pathway & lower limit & central estimate & upper limit \\
\hline Backyard Cow Milk & 0.55 & 2.8 & 16 \\
Commercial Milk (locally produced) & 0.16 & 0.97 & 7.1 \\
Commercial Milk (regionally mixed) & 0.19 & 1.2 & 7.1 \\
Goat Milk (locally produced) & 1.2 & 9 & 58 \\
Beef (locally produced) & 0.00088 & 0.015 & 0.3 \\
Leafy Vegetables (locally produced) & 0.00032 & 0.0031 & 0.027 \\
Eggs (locally produced) & 0.035 & 0.23 & 1.6 \\
Cottage Cheese (locally produced) & 0.00066 & 0.0077 & 0.083 \\
Inhalation & 0.017 & 0.061 & 0.24 \\
Mother's milk (mother on Diet 1) & 0.000092 & 0.0032 & 0.088 \\
Prenatal exposure (mother on Diet 1) & -- & -- & -- \\
Diet 1 & 0.63 & 3.2 & 18 \\
Diet 2 & 0.26 & 1.3 & 8.9 \\
Diet 3 & 0.22 & 1.3 & 7.3 \\
\hline
\end{tabular}

Excess Lifetime Risk [ ]

\begin{tabular}{llll}
\hline Diet 1 & $3.9 \mathrm{E}-06$ & $1.5 \mathrm{E}-04$ & $2.6 \mathrm{E}-03$ \\
Diet 2 & $2.0 \mathrm{E}-06$ & $5.7 \mathrm{E}-05$ & $1.2 \mathrm{E}-03$ \\
Diet 3 & $2.2 \mathrm{E}-06$ & $5.3 \mathrm{E}-05$ & $1.2 \mathrm{E}-03$ \\
Diet 4 & $1.1 \mathrm{E}-05$ & $3.6 \mathrm{E}-04$ & $8.2 \mathrm{E}-03$ \\
\hline
\end{tabular}

\section{Relative Risk [ ]}

\begin{tabular}{lccc}
\hline Diet 1 & 1.0044 & 1.097 & 3 \\
Diet 2 & 1.0019 & 1.038 & 1.91 \\
Diet 3 & 1.0017 & 1.035 & 1.89 \\
Diet 4 & 1.015 & 1.26 & 6.3 \\
\hline
\end{tabular}

\section{Probability of Causation [\%]}

\begin{tabular}{lccc}
\hline Diet 1 & 0.44 & 8.9 & 66 \\
Diet 2 & 0.19 & 3.6 & 48 \\
Diet 3 & 0.17 & 3.4 & 47 \\
Diet 4 & 1.48 & 20.6 & 84 \\
\hline
\end{tabular}

Diet 1 - Backyard cow milk + all other locally produced non-milk exposure pathways

Diet 2 - Locally produced commercial milk + all other locally produced non-milk exposure pathways

Diet 3 - Regionally mixed commercial milk + inhalation (other regionally mixed food items are minor contributors to the total)

Diet 4 - Goat milk (all other exposure pathways are of negligible importance compared to goat milk) 


\section{Location: Cedar Bluff}

Receptor: Female born in 1944

\begin{tabular}{lccc}
\hline & \multicolumn{3}{c}{ Thyroid Dose [cGy] } \\
& \multicolumn{2}{c}{$95 \%$ Subjective Confidence Interval } \\
Exposure Pathway & 1.2 & 7.2 & 39 \\
\hline Backyard Cow Milk & 0.35 & 2.4 & 16 \\
Commercial Milk (locally produced) & 0.19 & 1.2 & 7.9 \\
Commercial Milk (regionally mixed) & 3.1 & 22 & 150 \\
Goat Milk (locally produced) & 0.0019 & 0.035 & 0.73 \\
Beef (locally produced) & 0.00073 & 0.0074 & 0.065 \\
Leafy Vegetables (locally produced) & 0.067 & 0.47 & 3.3 \\
Eggs (locally produced) & 0.0018 & 0.018 & 0.21 \\
Cottage Cheese (locally produced) & 0.042 & 0.16 & 0.59 \\
Inhalation & 0.00021 & 0.0078 & 0.22 \\
Mother's milk (mother on Diet 1) & -- & -- & - \\
Prenatal exposure (mother on Diet 1) & 1.4 & 8.1 & 43 \\
Diet 1 & 0.58 & 3.2 & 19 \\
Diet 2 & 0.26 & 1.3 & 8.5 \\
Diet 3 & & upper limit \\
\hline
\end{tabular}

Excess Lifetime Risk [ ]

\begin{tabular}{llll}
\hline Diet 1 & $8.4 \mathrm{E}-05$ & $1.3 \mathrm{E}-03$ & $1.9 \mathrm{E}-02$ \\
Diet 2 & $4.4 \mathrm{E}-05$ & $5.2 \mathrm{E}-04$ & $7.2 \mathrm{E}-03$ \\
Diet 3 & $1.9 \mathrm{E}-05$ & $2.1 \mathrm{E}-04$ & $2.6 \mathrm{E}-03$ \\
Diet 4 & $2.4 \mathrm{E}-04$ & $3.7 \mathrm{E}-03$ & $4.8 \mathrm{E}-02$ \\
\hline
\end{tabular}

\section{Relative Risk [ ]}

\begin{tabular}{lccc}
\hline Diet 1 & 1.038 & 1.35 & 4.2 \\
Diet 2 & 1.014 & 1.14 & 2.5 \\
Diet 3 & 1.0063 & 1.059 & 1.67 \\
Diet 4 & 1.09 & 1.84 & 13 \\
\hline
\end{tabular}

\begin{tabular}{llcc} 
& \multicolumn{3}{c}{ Probability of Causation [\%] } \\
\hline Diet 1 & 3.67 & 25.8 & 76 \\
Diet 2 & 1.42 & 12.1 & 60 \\
Diet 3 & 0.62 & 5.5 & 40 \\
Diet 4 & 8.27 & 45.5 & 92 \\
\hline
\end{tabular}

Diet 1 - Backyard cow milk + all other locally produced non-milk exposure pathways

Diet 2 - Locally produced commercial milk + all other locally produced non-milk exposure pathways

Diet 3 - Regionally mixed commercial milk + inhalation (other regionally mixed food items are minor contributors to the total)

Diet 4 - Goat milk (all other exposure pathways are of negligible importance compared to goat milk) 


\section{Location: Cedar Bluff}

Receptor: Male born in 1944

\begin{tabular}{lccc}
\hline & \multicolumn{3}{c}{ Thyroid Dose [cGy] } \\
Exposure Pathway & \multicolumn{2}{c}{$9 \%$ Subjective Confidence Interval } \\
\hline Backyard Cow Milk & 1.3 & 7 & 40 \\
Commercial Milk (locally produced) & 0.39 & 2.4 & 15 \\
Commercial Milk (regionally mixed) & 0.19 & 1.2 & 7.1 \\
Goat Milk (locally produced) & 2.8 & 21 & 140 \\
Beef (locally produced) & 0.002 & 0.037 & 0.81 \\
Leafy Vegetables (locally produced) & 0.0007 & 0.0072 & 0.065 \\
Eggs (locally produced) & 0.074 & 0.55 & 3.9 \\
Cottage Cheese (locally produced) & 0.0016 & 0.018 & 0.22 \\
Inhalation & 0.042 & 0.16 & 0.58 \\
Mother's milk (mother on Diet 1) & 0.00021 & 0.0078 & 0.22 \\
Prenatal exposure (mother on Diet 1) & -- & -- & - \\
Diet 1 & 1.5 & 7.8 & 45 \\
Diet 2 & 0.59 & 3.2 & 19 \\
Diet 3 & 0.25 & 1.4 & 7.7 \\
\hline
\end{tabular}

Excess Lifetime Risk [ ]

\begin{tabular}{llcc}
\hline Diet 1 & $1.1 \mathrm{E}-05$ & $3.6 \mathrm{E}-04$ & $6.9 \mathrm{E}-03$ \\
Diet 2 & $5.2 \mathrm{E}-06$ & $1.5 \mathrm{E}-04$ & $3.1 \mathrm{E}-03$ \\
Diet 3 & $2.4 \mathrm{E}-06$ & $5.9 \mathrm{E}-05$ & $1.2 \mathrm{E}-03$ \\
Diet 4 & $3.1 \mathrm{E}-05$ & $8.4 \mathrm{E}-04$ & $1.8 \mathrm{E}-02$ \\
\hline
\end{tabular}

\section{Relative Risk [ ]}

\begin{tabular}{lccc}
\hline Diet 1 & 1.011 & 1.24 & 6.3 \\
Diet 2 & 1.0045 & 1.093 & 3.1 \\
Diet 3 & 1.002 & 1.037 & 1.93 \\
Diet 4 & 1.03 & 1.58 & 15 \\
\hline
\end{tabular}

\section{Probability of Causation [\%]}

\begin{tabular}{lccc}
\hline Diet 1 & 1.07 & 19.3 & 84 \\
Diet 2 & 0.45 & 8.5 & 68 \\
Diet 3 & 0.20 & 3.6 & 48 \\
Diet 4 & 2.93 & 36.6 & 93 \\
\hline
\end{tabular}

Diet 1 - Backyard cow milk + all other locally produced non-milk exposure pathways

Diet 2 - Locally produced commercial milk + all other locally produced non-milk exposure pathways

Diet 3 - Regionally mixed commercial milk + inhalation (other regionally mixed food items are minor contributors to the total)

Diet 4 - Goat milk (all other exposure pathways are of negligible importance compared to goat milk) 


\section{Location: Oakdale}

Receptor: Female born in 1944

\begin{tabular}{lccc}
\hline & \multicolumn{3}{c}{ Thyroid Dose [cGy] } \\
Exposure Pathway & $95 \%$ Subjective Confidence Interval \\
\hline Backyard Cow Milk & lower limit & central estimate & upper limit \\
Commercial Milk (locally produced) & 0.26 & 1.7 & 10 \\
Commercial Milk (regionally mixed) & 0.082 & 0.56 & 4 \\
Goat Milk (locally produced) & 0.19 & 1.2 & 7.9 \\
Beef (locally produced) & 0.63 & 5.1 & 37 \\
Leafy Vegetables (locally produced) & 0.00048 & 0.0082 & 0.17 \\
Eggs (locally produced) & 0.00016 & 0.0018 & 0.015 \\
Cottage Cheese (locally produced) & 0.014 & 0.12 & 0.84 \\
Inhalation & 0.00039 & 0.0042 & 0.051 \\
Mother's milk (mother on Diet 1) & 0.0095 & 0.036 & 0.14 \\
Prenatal exposure (mother on Diet 1) & 0.000046 & 0.0018 & 0.05 \\
Diet 1 & -- & -- & -- \\
Diet 2 & 0.3 & 1.9 & 11 \\
Diet 3 & 0.13 & 0.76 & 4.9 \\
\hline
\end{tabular}

Excess Lifetime Risk [ ]

\begin{tabular}{llcc}
\hline Diet 1 & $2.0 \mathrm{E}-05$ & $3.2 \mathrm{E}-04$ & $4.3 \mathrm{E}-03$ \\
Diet 2 & $9.9 \mathrm{E}-06$ & $1.2 \mathrm{E}-04$ & $1.7 \mathrm{E}-03$ \\
Diet 3 & $1.7 \mathrm{E}-05$ & $1.9 \mathrm{E}-04$ & $2.6 \mathrm{E}-03$ \\
Diet 4 & $5.2 \mathrm{E}-05$ & $8.7 \mathrm{E}-04$ & $1.1 \mathrm{E}-02$ \\
\hline
\end{tabular}

\section{Relative Risk [ ]}

\begin{tabular}{lccc}
\hline Diet 1 & 1.0081 & 1.081 & 1.85 \\
Diet 2 & 1.003 & 1.032 & 1.4 \\
Diet 3 & 1.0055 & 1.053 & 1.64 \\
Diet 4 & 1.019 & 1.2 & 3.9 \\
\hline
\end{tabular}

\begin{tabular}{lccc}
\hline Diet 1 & 0.80 & 7.5 & 45 \\
Diet 2 & 0.30 & 3.1 & 28 \\
Diet 3 & 0.55 & 5.1 & 39 \\
Diet 4 & 1.84 & 16.6 & 74 \\
\hline
\end{tabular}

Diet 1 - Backyard cow milk + all other locally produced non-milk exposure pathways

Diet 2 - Locally produced commercial milk + all other locally produced non-milk exposure pathways

Diet 3 - Regionally mixed commercial milk + inhalation (other regionally mixed food items are minor contributors to the total)

Diet 4 - Goat milk (all other exposure pathways are of negligible importance compared to goat milk) 


\section{Location: Oakdale}

Receptor: Male born in 1944

\begin{tabular}{lccc}
\hline & \multicolumn{3}{c}{ Thyroid Dose [cGy] } \\
& \multicolumn{2}{c}{$95 \%$ Subjective Confidence Interval } \\
Exposure Pathway & lower limit & central estimate & upper limit \\
\hline Backyard Cow Milk & 0.28 & 1.6 & 10 \\
Commercial Milk (locally produced) & 0.081 & 0.56 & 4.1 \\
Commercial Milk (regionally mixed) & 0.19 & 1.2 & 7.1 \\
Goat Milk (locally produced) & 0.64 & 5.1 & 35 \\
Beef (locally produced) & 0.0005 & 0.0084 & 0.18 \\
Leafy Vegetables (locally produced) & 0.00015 & 0.0018 & 0.016 \\
Eggs (locally produced) & 0.017 & 0.13 & 0.95 \\
Cottage Cheese (locally produced) & 0.00037 & 0.0043 & 0.052 \\
Inhalation & 0.0092 & 0.037 & 0.14 \\
Mother's milk (mother on Diet 1) & 0.000046 & 0.0018 & 0.05 \\
Prenatal exposure (mother on Diet 1) & -- & -- & -- \\
Diet 1 & 0.31 & 1.8 & 11 \\
Diet 2 & 0.13 & 0.79 & 5.1 \\
Diet 3 & 0.21 & 1.2 & 7.2 \\
\hline
\end{tabular}

Excess Lifetime Risk [ ]

\begin{tabular}{llll}
\hline Diet 1 & $2.2 \mathrm{E}-06$ & $8.5 \mathrm{E}-05$ & $1.8 \mathrm{E}-03$ \\
Diet 2 & $1.1 \mathrm{E}-06$ & $3.4 \mathrm{E}-05$ & $7.5 \mathrm{E}-04$ \\
Diet 3 & $2.2 \mathrm{E}-06$ & $5.1 \mathrm{E}-05$ & $1.1 \mathrm{E}-03$ \\
Diet 4 & $6.8 \mathrm{E}-06$ & $2.0 \mathrm{E}-04$ & $4.7 \mathrm{E}-03$ \\
\hline
\end{tabular}

\section{Relative Risk [ ]}

\begin{tabular}{lccc}
\hline Diet 1 & 1.0025 & 1.055 & 2.2 \\
Diet 2 & 1.0011 & 1.021 & 1.52 \\
Diet 3 & 1.0017 & 1.034 & 1.87 \\
Diet 4 & 1.0073 & 1.15 & 4.1 \\
\hline
\end{tabular}

\section{Probability of Causation [\%]}

\begin{tabular}{lccc}
\hline Diet 1 & 0.25 & 5.2 & 53 \\
Diet 2 & 0.11 & 2.1 & 34 \\
Diet 3 & 0.17 & 3.3 & 47 \\
Diet 4 & 0.72 & 12.5 & 74 \\
\hline
\end{tabular}

Diet 1 - Backyard cow milk + all other locally produced non-milk exposure pathways

Diet 2 - Locally produced commercial milk + all other locally produced non-milk exposure pathways

Diet 3 - Regionally mixed commercial milk + inhalation (other regionally mixed food items are minor contributors to the total)

Diet 4 - Goat milk (all other exposure pathways are of negligible importance compared to goat milk) 


\section{Location: Claxton}

Receptor: Female born in 1944

\begin{tabular}{lccc}
\hline & \multicolumn{3}{c}{ Thyroid Dose [cGy] } \\
& \multicolumn{2}{c}{$95 \%$ Subjective Confidence Interval } \\
Exposure Pathway & lower limit & central estimate & upper limit \\
\hline Backyard Cow Milk & 1.1 & 6 & 33 \\
Commercial Milk (locally produced) & 0.31 & 2 & 13 \\
Commercial Milk (regionally mixed) & 0.19 & 1.2 & 7.9 \\
Goat Milk (locally produced) & 2.7 & 18 & 130 \\
Beef (locally produced) & 0.0016 & 0.029 & 0.59 \\
Leafy Vegetables (locally produced) & 0.00059 & 0.0062 & 0.055 \\
Eggs (locally produced) & 0.054 & 0.4 & 2.7 \\
Cottage Cheese (locally produced) & 0.0015 & 0.015 & 0.17 \\
Inhalation & 0.035 & 0.13 & 0.49 \\
Mother's milk (mother on Diet 1) & 0.00019 & 0.0064 & 0.18 \\
Prenatal exposure (mother on Diet 1) & -- & -- & - \\
Diet 1 & 1.2 & 6.7 & 36 \\
Diet 2 & 0.49 & 2.6 & 16 \\
Diet 3 & 0.25 & 1.3 & 8.4 \\
\hline
\end{tabular}

Excess Lifetime Risk [ ]

\begin{tabular}{llll}
\hline Diet 1 & $7.2 \mathrm{E}-05$ & $1.1 \mathrm{E}-03$ & $1.5 \mathrm{E}-02$ \\
Diet 2 & $3.8 \mathrm{E}-05$ & $4.3 \mathrm{E}-04$ & $6.0 \mathrm{E}-03$ \\
Diet 3 & $1.8 \mathrm{E}-05$ & $2.1 \mathrm{E}-04$ & $2.6 \mathrm{E}-03$ \\
Diet 4 & $2.0 \mathrm{E}-04$ & $3.2 \mathrm{E}-03$ & $4.0 \mathrm{E}-02$ \\
\hline
\end{tabular}

\section{Relative Risk [ ]}

\begin{tabular}{lccc}
\hline Diet 1 & 1.031 & 1.29 & 3.7 \\
Diet 2 & 1.012 & 1.11 & 2.3 \\
Diet 3 & 1.0061 & 1.058 & 1.66 \\
Diet 4 & 1.078 & 1.69 & 10.8 \\
\hline
\end{tabular}

\begin{tabular}{|c|c|c|c|}
\hline Diet 1 & 2.99 & 22.3 & 73 \\
\hline Diet 2 & 1.18 & 10.3 & 56 \\
\hline Diet 3 & 0.61 & 5.4 & 40 \\
\hline Diet 4 & 7.25 & 40.8 & 91 \\
\hline
\end{tabular}

Diet 1 - Backyard cow milk + all other locally produced non-milk exposure pathways

Diet 2 - Locally produced commercial milk + all other locally produced non-milk exposure pathways

Diet 3 - Regionally mixed commercial milk + inhalation (other regionally mixed food items are minor contributors to the total)

Diet 4 - Goat milk (all other exposure pathways are of negligible importance compared to goat milk) 


\section{Location: Claxton}

Receptor: Male born in 1944

\begin{tabular}{lccc}
\hline & \multicolumn{3}{c}{ Thyroid Dose [cGy] } \\
& \multicolumn{2}{c}{$95 \%$ Subjective Confidence Interval } \\
Exposure Pathway & lower limit & central estimate & upper limit \\
\hline Backyard Cow Milk & 1.1 & 5.9 & 34 \\
Commercial Milk (locally produced) & 0.32 & 2 & 13 \\
Commercial Milk (regionally mixed) & 0.19 & 1.2 & 7.1 \\
Goat Milk (locally produced) & 2.4 & 18 & 120 \\
Beef (locally produced) & 0.0017 & 0.031 & 0.65 \\
Leafy Vegetables (locally produced) & 0.00058 & 0.0061 & 0.054 \\
Eggs (locally produced) & 0.063 & 0.46 & 3.2 \\
Cottage Cheese (locally produced) & 0.0014 & 0.015 & 0.18 \\
Inhalation & 0.035 & 0.13 & 0.47 \\
Mother's milk (mother on Diet 1) & 0.00019 & 0.0064 & 0.18 \\
Prenatal exposure (mother on Diet 1) & -- & -- & -- \\
Diet 1 & 1.3 & 6.5 & 38 \\
Diet 2 & 0.5 & 2.8 & 16 \\
Diet 3 & 0.24 & 1.3 & 7.6 \\
\hline
\end{tabular}

Excess Lifetime Risk [ ]

\begin{tabular}{lllc}
\hline Diet 1 & $9.1 \mathrm{E}-06$ & $3.0 \mathrm{E}-04$ & $5.7 \mathrm{E}-03$ \\
Diet 2 & $4.4 \mathrm{E}-06$ & $1.2 \mathrm{E}-04$ & $2.5 \mathrm{E}-03$ \\
Diet 3 & $2.3 \mathrm{E}-06$ & $5.7 \mathrm{E}-05$ & $1.2 \mathrm{E}-03$ \\
Diet 4 & $2.6 \mathrm{E}-05$ & $6.9 \mathrm{E}-04$ & $1.5 \mathrm{E}-02$ \\
\hline
\end{tabular}

\section{Relative Risk [ ]}

\begin{tabular}{lccc}
\hline Diet 1 & 1.0087 & 1.2 & 5.3 \\
Diet 2 & 1.0037 & 1.077 & 2.7 \\
Diet 3 & 1.0019 & 1.036 & 1.91 \\
Diet 4 & 1.026 & 1.5 & 13 \\
\hline
\end{tabular}

\section{Probability of Causation [\%]}

\begin{tabular}{lccc}
\hline Diet 1 & 0.87 & 16.5 & 81 \\
Diet 2 & 0.37 & 7.2 & 63 \\
Diet 3 & 0.19 & 3.5 & 48 \\
Diet 4 & 2.50 & 33.1 & 92 \\
\hline
\end{tabular}

Diet 1 - Backyard cow milk + all other locally produced non-milk exposure pathways

Diet 2 - Locally produced commercial milk + all other locally produced non-milk exposure pathways

Diet 3 - Regionally mixed commercial milk + inhalation (other regionally mixed food items are minor contributors to the total)

Diet 4 - Goat milk (all other exposure pathways are of negligible importance compared to goat milk) 
Location: Dutch Valley

Receptor: Female born in 1944

\begin{tabular}{lccc}
\hline & \multicolumn{3}{c}{ Thyroid Dose [cGy] } \\
Exposure Pathway & \multicolumn{2}{c}{$95 \%$ Subjective Confidence Interval } \\
Backyard Cow Milk & lower limit & central estimate & upper limit \\
Commercial Milk (locally produced) & 0.48 & 2.8 & 15 \\
Commercial Milk (regionally mixed) & 0.15 & 0.94 & 6.4 \\
Goat Milk (locally produced) & 0.19 & 1.2 & 7.9 \\
Beef (locally produced) & 1.1 & 8.9 & 58 \\
Leafy Vegetables (locally produced) & 0.00076 & 0.014 & 0.27 \\
Eggs (locally produced) & 0.0003 & 0.003 & 0.025 \\
Cottage Cheese (locally produced) & 0.029 & 0.2 & 1.4 \\
Inhalation & 0.0007 & 0.0073 & 0.08 \\
Mother's milk (mother on Diet 1) & 0.016 & 0.062 & 0.24 \\
Prenatal exposure (mother on Diet 1) & 0.000091 & 0.0031 & 0.088 \\
Diet 1 & -- & -- & -- \\
Diet 2 & 0.56 & 3.2 & 17 \\
Diet 3 & 0.23 & 1.3 & 7.7 \\
\hline
\end{tabular}

Excess Lifetime Risk [ ]

\begin{tabular}{llll}
\hline Diet 1 & $3.6 \mathrm{E}-05$ & $5.2 \mathrm{E}-04$ & $7.3 \mathrm{E}-03$ \\
Diet 2 & $1.8 \mathrm{E}-05$ & $2.0 \mathrm{E}-04$ & $3.0 \mathrm{E}-03$ \\
Diet 3 & $1.7 \mathrm{E}-05$ & $2.0 \mathrm{E}-04$ & $2.6 \mathrm{E}-03$ \\
Diet 4 & $9.8 \mathrm{E}-05$ & $1.5 \mathrm{E}-03$ & $1.9 \mathrm{E}-02$ \\
\hline
\end{tabular}

\section{Relative Risk [ ]}

\begin{tabular}{lccc}
\hline Diet 1 & 1.015 & 1.13 & 2.4 \\
Diet 2 & 1.0056 & 1.055 & 1.71 \\
Diet 3 & 1.0057 & 1.055 & 1.65 \\
Diet 4 & 1.036 & 1.34 & 6.2 \\
\hline
\end{tabular}

\section{Probability of Causation [\%]}

\begin{tabular}{lccc}
\hline Diet 1 & 1.49 & 11.8 & 59 \\
Diet 2 & 0.56 & 5.2 & 41 \\
Diet 3 & 0.57 & 5.2 & 39 \\
Diet 4 & 3.51 & 25.1 & 83 \\
\hline
\end{tabular}

Diet 1 - Backyard cow milk + all other locally produced non-milk exposure pathways

Diet 2 - Locally produced commercial milk + all other locally produced non-milk exposure pathways

Diet 3 - Regionally mixed commercial milk + inhalation (other regionally mixed food items are minor contributors to the total)

Diet 4 - Goat milk (all other exposure pathways are of negligible importance compared to goat milk) 


\section{Location: Dutch Valley}

Receptor: Male born in 1944

\begin{tabular}{lccc}
\hline & \multicolumn{3}{c}{ Thyroid Dose [cGy] } \\
Exposure Pathway & \multicolumn{2}{c}{$95 \%$ Subjective Confidence Interval } \\
\hline Backyard Cow Milk & lower limit & central estimate & upper limit \\
Commercial Milk (locally produced) & 0.5 & 2.8 & 15 \\
Commercial Milk (regionally mixed) & 0.15 & 0.96 & 6.3 \\
Goat Milk (locally produced) & 0.19 & 1.2 & 7.1 \\
Beef (locally produced) & 1.1 & 8.7 & 60 \\
Leafy Vegetables (locally produced) & 0.00083 & 0.014 & 0.28 \\
Eggs (locally produced) & 0.00028 & 0.0029 & 0.026 \\
Cottage Cheese (locally produced) & 0.033 & 0.23 & 1.5 \\
Inhalation & 0.00064 & 0.0075 & 0.079 \\
Mother's milk (mother on Diet 1) & 0.016 & 0.062 & 0.24 \\
Prenatal exposure (mother on Diet 1) & 0.000091 & 0.0031 & 0.088 \\
Diet 1 & -- & -- & -- \\
Diet 2 & 0.57 & 3.1 & 18 \\
Diet 3 & 0.24 & 1.3 & 7.8 \\
\hline
\end{tabular}

Excess Lifetime Risk [ ]

\begin{tabular}{llll}
\hline Diet 1 & $4.5 \mathrm{E}-06$ & $1.4 \mathrm{E}-04$ & $2.6 \mathrm{E}-03$ \\
Diet 2 & $1.9 \mathrm{E}-06$ & $5.6 \mathrm{E}-05$ & $1.2 \mathrm{E}-03$ \\
Diet 3 & $2.2 \mathrm{E}-06$ & $5.3 \mathrm{E}-05$ & $1.2 \mathrm{E}-03$ \\
Diet 4 & $1.2 \mathrm{E}-05$ & $3.4 \mathrm{E}-04$ & $7.6 \mathrm{E}-03$ \\
\hline
\end{tabular}

\section{Relative Risk [ ]}

\begin{tabular}{lccc}
\hline Diet 1 & 1.004 & 1.093 & 2.9 \\
Diet 2 & 1.0018 & 1.037 & 1.83 \\
Diet 3 & 1.0017 & 1.035 & 1.89 \\
Diet 4 & 1.013 & 1.25 & 5.9 \\
\hline
\end{tabular}

\section{Probability of Causation [\%]}

\begin{tabular}{lccc}
\hline Diet 1 & 0.40 & 8.5 & 65 \\
Diet 2 & 0.18 & 3.5 & 45 \\
Diet 3 & 0.17 & 3.4 & 47 \\
Diet 4 & 1.29 & 19.8 & 82 \\
\hline
\end{tabular}

Diet 1 - Backyard cow milk + all other locally produced non-milk exposure pathways

Diet 2 - Locally produced commercial milk + all other locally produced non-milk exposure pathways

Diet 3 - Regionally mixed commercial milk + inhalation (other regionally mixed food items are minor contributors to the total)

Diet 4 - Goat milk (all other exposure pathways are of negligible importance compared to goat milk) 


\section{Location: Clinton}

Receptor: Female born in 1944

\begin{tabular}{lccc}
\hline & \multicolumn{3}{c}{ Thyroid Dose [cGy] } \\
& \multicolumn{2}{c}{$95 \%$ Subjective Confidence Interval } \\
Exposure Pathway & lower limit & central estimate & upper limit \\
\hline Backyard Cow Milk & 0.78 & 4.7 & 25 \\
Commercial Milk (locally produced) & 0.23 & 1.5 & 10 \\
Commercial Milk (regionally mixed) & 0.19 & 1.2 & 7.9 \\
Goat Milk (locally produced) & 1.9 & 14 & 94 \\
Beef (locally produced) & 0.0012 & 0.022 & 0.45 \\
Leafy Vegetables (locally produced) & 0.00047 & 0.0049 & 0.042 \\
Eggs (locally produced) & 0.045 & 0.31 & 2.1 \\
Cottage Cheese (locally produced) & 0.0012 & 0.012 & 0.14 \\
Inhalation & 0.028 & 0.1 & 0.38 \\
Mother's milk (mother on Diet 1) & 0.00013 & 0.005 & 0.14 \\
Prenatal exposure (mother on Diet 1) & -- & -- & -- \\
Diet 1 & 0.91 & 5.2 & 28 \\
Diet 2 & 0.37 & 2.1 & 12 \\
Diet 3 & 0.24 & 1.3 & 8.3 \\
\hline
\end{tabular}

Excess Lifetime Risk [ ]

\begin{tabular}{lllc}
\hline Diet 1 & $5.3 \mathrm{E}-05$ & $8.6 \mathrm{E}-04$ & $1.2 \mathrm{E}-02$ \\
Diet 2 & $2.8 \mathrm{E}-05$ & $3.3 \mathrm{E}-04$ & $4.4 \mathrm{E}-03$ \\
Diet 3 & $1.8 \mathrm{E}-05$ & $2.0 \mathrm{E}-04$ & $2.6 \mathrm{E}-03$ \\
Diet 4 & $1.4 \mathrm{E}-04$ & $2.4 \mathrm{E}-03$ & $2.9 \mathrm{E}-02$ \\
\hline
\end{tabular}

\section{Relative Risk [ ]}

\begin{tabular}{lccc}
\hline Diet 1 & 1.023 & 1.23 & 3.1 \\
Diet 2 & 1.0088 & 1.089 & 1.99 \\
Diet 3 & 1.0059 & 1.057 & 1.66 \\
Diet 4 & 1.056 & 1.54 & 9 \\
\hline
\end{tabular}

\begin{tabular}{lccc}
\hline Diet 1 & 2.27 & 18.3 & 67 \\
Diet 2 & 0.87 & 8.2 & 49 \\
Diet 3 & 0.59 & 5.4 & 40 \\
Diet 4 & 5.27 & 34.6 & 88 \\
\hline
\end{tabular}

Diet 1 - Backyard cow milk + all other locally produced non-milk exposure pathways

Diet 2 - Locally produced commercial milk + all other locally produced non-milk exposure pathways

Diet 3 - Regionally mixed commercial milk + inhalation (other regionally mixed food items are minor contributors to the total)

Diet 4 - Goat milk (all other exposure pathways are of negligible importance compared to goat milk) 


\section{Location: Clinton}

\section{Receptor: Male born in 1944}

\begin{tabular}{lccc}
\hline & \multicolumn{3}{c}{ Thyroid Dose [cGy] } \\
& \multicolumn{2}{c}{$95 \%$ Subjective Confidence Interval } \\
Exposure Pathway & lower limit & central estimate & upper limit \\
\hline Backyard Cow Milk & 0.83 & 4.6 & 26 \\
Commercial Milk (locally produced) & 0.24 & 1.5 & 10 \\
Commercial Milk (regionally mixed) & 0.19 & 1.2 & 7.1 \\
Goat Milk (locally produced) & 1.9 & 14 & 93 \\
Beef (locally produced) & 0.0013 & 0.023 & 0.49 \\
Leafy Vegetables (locally produced) & 0.00047 & 0.0046 & 0.041 \\
Eggs (locally produced) & 0.049 & 0.36 & 2.5 \\
Cottage Cheese (locally produced) & 0.0011 & 0.012 & 0.13 \\
Inhalation & 0.028 & 0.1 & 0.37 \\
Mother's milk (mother on Diet 1) & 0.00013 & 0.005 & 0.14 \\
Prenatal exposure (mother on Diet 1) & -- & -- & -- \\
Diet 1 & 0.96 & 5.1 & 29 \\
Diet 2 & 0.37 & 2.1 & 12 \\
Diet 3 & 0.23 & 1.3 & 7.5 \\
\hline
\end{tabular}

Excess Lifetime Risk [ ]

\begin{tabular}{llll}
\hline Diet 1 & $7.2 \mathrm{E}-06$ & $2.4 \mathrm{E}-04$ & $4.3 \mathrm{E}-03$ \\
Diet 2 & $3.2 \mathrm{E}-06$ & $9.3 \mathrm{E}-05$ & $2.0 \mathrm{E}-03$ \\
Diet 3 & $2.3 \mathrm{E}-06$ & $5.5 \mathrm{E}-05$ & $1.2 \mathrm{E}-03$ \\
Diet 4 & $1.9 \mathrm{E}-05$ & $5.5 \mathrm{E}-04$ & $1.2 \mathrm{E}-02$ \\
\hline
\end{tabular}

\section{Relative Risk [ ]}

\begin{tabular}{lccc}
\hline Diet 1 & 1.007 & 1.15 & 4.2 \\
Diet 2 & 1.0029 & 1.058 & 2.3 \\
Diet 3 & 1.0018 & 1.036 & 1.91 \\
Diet 4 & 1.021 & 1.39 & 9.3 \\
\hline
\end{tabular}

\begin{tabular}{lccc}
\hline Diet 1 & 0.69 & 13.2 & 75 \\
Diet 2 & 0.29 & 5.5 & 56 \\
Diet 3 & 0.18 & 3.4 & 48 \\
Diet 4 & 2.02 & 27.6 & 89 \\
\hline
\end{tabular}

Diet 1 - Backyard cow milk + all other locally produced non-milk exposure pathways

Diet 2 - Locally produced commercial milk + all other locally produced non-milk exposure pathways

Diet 3 - Regionally mixed commercial milk + inhalation (other regionally mixed food items are minor contributors to the total)

Diet 4 - Goat milk (all other exposure pathways are of negligible importance compared to goat milk) 
Location: Friendsville

Receptor: Female born in 1944

\begin{tabular}{lccc}
\hline & \multicolumn{3}{c}{ Thyroid Dose [cGy] } \\
Exposure Pathway & $95 \%$ Subjective Confidence Interval \\
\hline Backyard Cow Milk & lower limit & central estimate & upper limit \\
Commercial Milk (locally produced) & 0.42 & 2.6 & 14 \\
Commercial Milk (regionally mixed) & 0.13 & 0.88 & 5.3 \\
Goat Milk (locally produced) & 0.19 & 1.2 & 7.9 \\
Beef (locally produced) & 1.1 & 8.2 & 61 \\
Leafy Vegetables (locally produced) & 0.00073 & 0.013 & 0.29 \\
Eggs (locally produced) & 0.00025 & 0.0027 & 0.024 \\
Cottage Cheese (locally produced) & 0.023 & 0.17 & 1.4 \\
Inhalation & 0.00059 & 0.0065 & 0.076 \\
Mother's milk (mother on Diet 1) & 0.017 & 0.07 & 0.29 \\
Prenatal exposure (mother on Diet 1) & 0.000095 & 0.0028 & 0.087 \\
Diet 1 & -- & -- & -- \\
Diet 2 & 0.49 & 2.9 & 16 \\
Diet 3 & 0.21 & 1.2 & 6.7 \\
\hline
\end{tabular}

Excess Lifetime Risk [ ]

\begin{tabular}{llll}
\hline Diet 1 & $3.5 \mathrm{E}-05$ & $4.8 \mathrm{E}-04$ & $6.9 \mathrm{E}-03$ \\
Diet 2 & $1.4 \mathrm{E}-05$ & $2.0 \mathrm{E}-04$ & $2.5 \mathrm{E}-03$ \\
Diet 3 & $1.7 \mathrm{E}-05$ & $2.0 \mathrm{E}-04$ & $2.6 \mathrm{E}-03$ \\
Diet 4 & $9.2 \mathrm{E}-05$ & $1.4 \mathrm{E}-03$ & $1.8 \mathrm{E}-02$ \\
\hline
\end{tabular}

\section{Relative Risk [ ]}

\begin{tabular}{lccc}
\hline Diet 1 & 1.014 & 1.12 & 2.4 \\
Diet 2 & 1.0053 & 1.051 & 1.58 \\
Diet 3 & 1.0057 & 1.055 & 1.65 \\
Diet 4 & 1.035 & 1.31 & 5.8 \\
\hline
\end{tabular}

\section{Probability of Causation [\%]}

\begin{tabular}{lccc}
\hline Diet 1 & 1.35 & 10.9 & 59 \\
Diet 2 & 0.53 & 4.8 & 37 \\
Diet 3 & 0.57 & 5.2 & 39 \\
Diet 4 & 3.34 & 23.9 & 83 \\
\hline
\end{tabular}

Diet 1 - Backyard cow milk + all other locally produced non-milk exposure pathways

Diet 2 - Locally produced commercial milk + all other locally produced non-milk exposure pathways

Diet 3 - Regionally mixed commercial milk + inhalation (other regionally mixed food items are minor contributors to the total)

Diet 4 - Goat milk (all other exposure pathways are of negligible importance compared to goat milk) 
Location: Friendsville

Receptor: Male born in 1944

\begin{tabular}{lccc}
\hline & \multicolumn{3}{c}{ Thyroid Dose [cGy] } \\
Exposure Pathway & \multicolumn{2}{c}{$95 \%$ Subjective Confidence Interval } \\
\hline Backyard Cow Milk & lower limit & central estimate & upper limit \\
Commercial Milk (locally produced) & 0.44 & 2.5 & 16 \\
Commercial Milk (regionally mixed) & 0.13 & 0.89 & 5.4 \\
Goat Milk (locally produced) & 0.19 & 1.2 & 7.1 \\
Beef (locally produced) & 1 & 7.8 & 60 \\
Leafy Vegetables (locally produced) & 0.0008 & 0.013 & 0.32 \\
Eggs (locally produced) & 0.00024 & 0.0027 & 0.025 \\
Cottage Cheese (locally produced) & 0.027 & 0.2 & 1.4 \\
Inhalation & 0.00057 & 0.0066 & 0.075 \\
Mother's milk (mother on Diet 1) & 0.016 & 0.069 & 0.25 \\
Prenatal exposure (mother on Diet 1) & 0.000095 & 0.0028 & 0.087 \\
Diet 1 & -- & -- & -- \\
Diet 2 & 0.51 & 2.9 & 17 \\
Diet 3 & 0.2 & 1.2 & 6.9 \\
\hline
\end{tabular}

Excess Lifetime Risk [ ]

\begin{tabular}{llll}
\hline Diet 1 & $4.0 \mathrm{E}-06$ & $1.3 \mathrm{E}-04$ & $2.6 \mathrm{E}-03$ \\
Diet 2 & $1.8 \mathrm{E}-06$ & $5.1 \mathrm{E}-05$ & $1.1 \mathrm{E}-03$ \\
Diet 3 & $2.2 \mathrm{E}-06$ & $5.4 \mathrm{E}-05$ & $1.2 \mathrm{E}-03$ \\
Diet 4 & $1.1 \mathrm{E}-05$ & $3.0 \mathrm{E}-04$ & $7.3 \mathrm{E}-03$ \\
\hline
\end{tabular}

\section{Relative Risk [ ]}

\begin{tabular}{lccc}
\hline Diet 1 & 1.004 & 1.085 & 2.9 \\
Diet 2 & 1.0017 & 1.034 & 1.71 \\
Diet 3 & 1.0018 & 1.035 & 1.89 \\
Diet 4 & 1.012 & 1.23 & 5.8 \\
\hline
\end{tabular}

\section{Probability of Causation [\%]}

\begin{tabular}{lccc}
\hline Diet 1 & 0.40 & 7.8 & 65 \\
Diet 2 & 0.17 & 3.3 & 42 \\
Diet 3 & 0.18 & 3.4 & 47 \\
Diet 4 & 1.17 & 18.5 & 82 \\
\hline
\end{tabular}

Diet 1 - Backyard cow milk + all other locally produced non-milk exposure pathways

Diet 2 - Locally produced commercial milk + all other locally produced non-milk exposure pathways

Diet 3 - Regionally mixed commercial milk + inhalation (other regionally mixed food items are minor contributors to the total)

Diet 4 - Goat milk (all other exposure pathways are of negligible importance compared to goat milk) 
Location: Wartburg

Receptor: Female born in 1944

\begin{tabular}{lccc}
\hline & \multicolumn{3}{c}{ Thyroid Dose [cGy] } \\
Exposure Pathway & \multicolumn{2}{c}{$95 \%$ Subjective Confidence Interval } \\
\hline Backyard Cow Milk & lower limit & central estimate & upper limit \\
Commercial Milk (locally produced) & 0.09 & 0.71 & 4.1 \\
Commercial Milk (regionally mixed) & 0.033 & 0.24 & 1.8 \\
Goat Milk (locally produced) & 0.19 & 1.2 & 7.9 \\
Beef (locally produced) & 0.29 & 2.4 & 17 \\
Leafy Vegetables (locally produced) & 0.00021 & 0.0034 & 0.084 \\
Eggs (locally produced) & 0.000068 & 0.00074 & 0.007 \\
Cottage Cheese (locally produced) & 0.0058 & 0.048 & 0.36 \\
Inhalation & 0.00016 & 0.0018 & 0.02 \\
Mother's milk (mother on Diet 1) & 0.004 & 0.016 & 0.073 \\
Prenatal exposure (mother on Diet 1) & 0.00002 & 0.00076 & 0.024 \\
Diet 1 & -- & -- & -- \\
Diet 2 & 0.11 & 0.78 & 4.6 \\
Diet 3 & 0.051 & 0.32 & 2.3 \\
\hline
\end{tabular}

Excess Lifetime Risk [ ]

\begin{tabular}{llll}
\hline Diet 1 & $8.0 \mathrm{E}-06$ & $1.4 \mathrm{E}-04$ & $1.9 \mathrm{E}-03$ \\
Diet 2 & $4.0 \mathrm{E}-06$ & $5.1 \mathrm{E}-05$ & $7.9 \mathrm{E}-04$ \\
Diet 3 & $1.6 \mathrm{E}-05$ & $1.9 \mathrm{E}-04$ & $2.6 \mathrm{E}-03$ \\
Diet 4 & $2.1 \mathrm{E}-05$ & $3.4 \mathrm{E}-04$ & $5.2 \mathrm{E}-03$ \\
\hline
\end{tabular}

\section{Relative Risk [ ]}

\begin{tabular}{llcc}
\hline Diet 1 & 1.0034 & 1.034 & 1.49 \\
Diet 2 & 1.0015 & 1.014 & 1.23 \\
Diet 3 & 1.0054 & 1.052 & 1.64 \\
Diet 4 & 1.0081 & 1.089 & 2.3 \\
\hline
\end{tabular}

\section{Probability of Causation [\%]}

\begin{tabular}{llll}
\hline Diet 1 & 0.34 & 3.3 & 33 \\
Diet 2 & 0.15 & 1.4 & 18 \\
Diet 3 & 0.54 & 4.9 & 39 \\
Diet 4 & 0.81 & 8.2 & 56 \\
\hline
\end{tabular}

Diet 1 - Backyard cow milk + all other locally produced non-milk exposure pathways

Diet 2 - Locally produced commercial milk + all other locally produced non-milk exposure pathways

Diet 3 - Regionally mixed commercial milk + inhalation (other regionally mixed food items are minor contributors to the total)

Diet 4 - Goat milk (all other exposure pathways are of negligible importance compared to goat milk) 


\section{Location: Wartburg}

Receptor: Male born in 1944

\begin{tabular}{lccc}
\hline & \multicolumn{3}{c}{ Thyroid Dose [cGy] } \\
Exposure Pathway & \multicolumn{2}{c}{$95 \%$ Subjective Confidence Interval } \\
\hline Backyard Cow Milk & lower limit & central estimate & upper limit \\
Commercial Milk (locally produced) & 0.097 & 0.69 & 4.5 \\
Commercial Milk (regionally mixed) & 0.034 & 0.24 & 1.9 \\
Goat Milk (locally produced) & 0.19 & 1.2 & 7.1 \\
Beef (locally produced) & 0.28 & 2.2 & 16 \\
Leafy Vegetables (locally produced) & 0.00022 & 0.0036 & 0.086 \\
Eggs (locally produced) & 0.000068 & 0.00074 & 0.0074 \\
Cottage Cheese (locally produced) & 0.0066 & 0.055 & 0.38 \\
Inhalation & 0.00014 & 0.0019 & 0.02 \\
Mother's milk (mother on Diet 1) & 0.0039 & 0.016 & 0.068 \\
Prenatal exposure (mother on Diet 1) & 0.00002 & 0.00076 & 0.024 \\
Diet 1 & -- & -- & -- \\
Diet 2 & 0.11 & 0.78 & 4.9 \\
Diet 3 & 0.052 & 0.33 & 2.4 \\
\hline
\end{tabular}

Excess Lifetime Risk [ ]

\begin{tabular}{lllc}
\hline Diet 1 & $1.0 \mathrm{E}-06$ & $3.5 \mathrm{E}-05$ & $7.2 \mathrm{E}-04$ \\
Diet 2 & $4.8 \mathrm{E}-07$ & $1.5 \mathrm{E}-05$ & $3.2 \mathrm{E}-04$ \\
Diet 3 & $2.1 \mathrm{E}-06$ & $5.1 \mathrm{E}-05$ & $1.1 \mathrm{E}-03$ \\
Diet 4 & $3.1 \mathrm{E}-06$ & $8.8 \mathrm{E}-05$ & $2.1 \mathrm{E}-03$ \\
\hline
\end{tabular}

\section{Relative Risk [ ]}

\begin{tabular}{lccc}
\hline Diet 1 & 1.00093 & 1.024 & 1.55 \\
Diet 2 & 1.00044 & 1.0097 & 1.24 \\
Diet 3 & 1.0016 & 1.034 & 1.86 \\
Diet 4 & 1.0024 & 1.062 & 2.6 \\
\hline
\end{tabular}

\section{Probability of Causation [\%]}

\begin{tabular}{llll}
\hline Diet 1 & 0.09 & 2.3 & 35 \\
Diet 2 & 0.04 & 1.0 & 19 \\
Diet 3 & 0.16 & 3.3 & 46 \\
Diet 4 & 0.24 & 5.8 & 62 \\
\hline
\end{tabular}

Diet 1 - Backyard cow milk + all other locally produced non-milk exposure pathways

Diet 2 - Locally produced commercial milk + all other locally produced non-milk exposure pathways

Diet 3 - Regionally mixed commercial milk + inhalation (other regionally mixed food items are minor contributors to the total)

Diet 4 - Goat milk (all other exposure pathways are of negligible importance compared to goat milk) 
Location: Rockwood

Receptor: Female born in 1944

\begin{tabular}{lccc}
\hline & \multicolumn{3}{c}{ Thyroid Dose [cGy] } \\
Exposure Pathway & \multicolumn{2}{c}{$95 \%$ Subjective Confidence Interval } \\
\hline Backyard Cow Milk & 0.5 & 3 & 17 \\
Commercial Milk (locally produced) & 0.16 & 1 & 6.9 \\
Commercial Milk (regionally mixed) & 0.19 & 1.2 & 7.9 \\
Goat Milk (locally produced) & 1.3 & 9.4 & 67 \\
Beef (locally produced) & 0.00086 & 0.014 & 0.31 \\
Leafy Vegetables (locally produced) & 0.0003 & 0.0031 & 0.029 \\
Eggs (locally produced) & 0.028 & 0.2 & 1.4 \\
Cottage Cheese (locally produced) & 0.0007 & 0.0076 & 0.088 \\
Inhalation & 0.019 & 0.071 & 0.27 \\
Mother's milk (mother on Diet 1) & 0.000097 & 0.0032 & 0.089 \\
Prenatal exposure (mother on Diet 1) & -- & -- & - \\
Diet 1 & 0.59 & 3.3 & 19 \\
Diet 2 & 0.25 & 1.4 & 8.5 \\
Diet 3 & 0.23 & 1.2 & 8.2 \\
\hline
\end{tabular}

Excess Lifetime Risk [ ]

\begin{tabular}{llll}
\hline Diet 1 & $3.6 \mathrm{E}-05$ & $5.7 \mathrm{E}-04$ & $7.5 \mathrm{E}-03$ \\
Diet 2 & $1.8 \mathrm{E}-05$ & $2.2 \mathrm{E}-04$ & $3.0 \mathrm{E}-03$ \\
Diet 3 & $1.7 \mathrm{E}-05$ & $2.0 \mathrm{E}-04$ & $2.6 \mathrm{E}-03$ \\
Diet 4 & $1.0 \mathrm{E}-04$ & $1.6 \mathrm{E}-03$ & $1.8 \mathrm{E}-02$ \\
\hline
\end{tabular}

\section{Relative Risk [ ]}

\begin{tabular}{lccc}
\hline Diet 1 & 1.015 & 1.14 & 2.4 \\
Diet 2 & 1.0061 & 1.058 & 1.66 \\
Diet 3 & 1.0058 & 1.055 & 1.65 \\
Diet 4 & 1.037 & 1.36 & 5.9 \\
\hline
\end{tabular}

\begin{tabular}{|c|c|c|c|}
\hline Diet 1 & 1.52 & 12.2 & 58 \\
\hline Diet 2 & 0.61 & 5.4 & 39 \\
\hline Diet 3 & 0.58 & 5.2 & 39 \\
\hline Diet 4 & 3.59 & 25.7 & 82 \\
\hline
\end{tabular}

Diet 1 - Backyard cow milk + all other locally produced non-milk exposure pathways

Diet 2 - Locally produced commercial milk + all other locally produced non-milk exposure pathways

Diet 3 - Regionally mixed commercial milk + inhalation (other regionally mixed food items are minor contributors to the total)

Diet 4 - Goat milk (all other exposure pathways are of negligible importance compared to goat milk) 


\section{Location: Rockwood}

\section{Receptor: Male born in 1944}

\begin{tabular}{lccc}
\hline & \multicolumn{3}{c}{ Thyroid Dose [cGy] } \\
& \multicolumn{2}{c}{$95 \%$ Subjective Confidence Interval } \\
Exposure Pathway & lower limit & central estimate & upper limit \\
\hline Backyard Cow Milk & 0.52 & 3 & 18 \\
Commercial Milk (locally produced) & 0.16 & 0.99 & 6.7 \\
Commercial Milk (regionally mixed) & 0.19 & 1.2 & 7.1 \\
Goat Milk (locally produced) & 1.2 & 9 & 62 \\
Beef (locally produced) & 0.00092 & 0.015 & 0.34 \\
Leafy Vegetables (locally produced) & 0.00028 & 0.003 & 0.029 \\
Eggs (locally produced) & 0.031 & 0.23 & 1.6 \\
Cottage Cheese (locally produced) & 0.00064 & 0.0078 & 0.089 \\
Inhalation & 0.018 & 0.071 & 0.27 \\
Mother's milk (mother on Diet 1) & 0.000097 & 0.0032 & 0.089 \\
Prenatal exposure (mother on Diet 1) & -- & -- & -- \\
Diet 1 & 0.62 & 3.3 & 20 \\
Diet 2 & 0.25 & 1.4 & 8.7 \\
Diet 3 & 0.22 & 1.3 & 7.4 \\
\hline
\end{tabular}

Excess Lifetime Risk [ ]

\begin{tabular}{llll}
\hline Diet 1 & $4.9 \mathrm{E}-06$ & $1.5 \mathrm{E}-04$ & $2.8 \mathrm{E}-03$ \\
Diet 2 & $2.3 \mathrm{E}-06$ & $5.9 \mathrm{E}-05$ & $1.2 \mathrm{E}-03$ \\
Diet 3 & $2.2 \mathrm{E}-06$ & $5.4 \mathrm{E}-05$ & $1.2 \mathrm{E}-03$ \\
Diet 4 & $1.4 \mathrm{E}-05$ & $3.3 \mathrm{E}-04$ & $8.0 \mathrm{E}-03$ \\
\hline
\end{tabular}

\section{Relative Risk [ ]}

\begin{tabular}{lccc}
\hline Diet 1 & 1.0044 & 1.096 & 3.1 \\
Diet 2 & 1.0018 & 1.04 & 1.92 \\
Diet 3 & 1.0018 & 1.035 & 1.89 \\
Diet 4 & 1.013 & 1.25 & 6.6 \\
\hline
\end{tabular}

\section{Probability of Causation [\%]}

\begin{tabular}{lccc}
\hline Diet 1 & 0.44 & 8.7 & 67 \\
Diet 2 & 0.18 & 3.8 & 47 \\
Diet 3 & 0.18 & 3.4 & 47 \\
Diet 4 & 1.30 & 19.8 & 84 \\
\hline
\end{tabular}

Diet 1 - Backyard cow milk + all other locally produced non-milk exposure pathways

Diet 2 - Locally produced commercial milk + all other locally produced non-milk exposure pathways

Diet 3 - Regionally mixed commercial milk + inhalation (other regionally mixed food items are minor contributors to the total)

Diet 4 - Goat milk (all other exposure pathways are of negligible importance compared to goat milk) 


\section{Location: Louisville}

Receptor: Female born in 1944

\begin{tabular}{lccc}
\hline & \multicolumn{3}{c}{ Thyroid Dose [cGy] } \\
Exposure Pathway & $95 \%$ Subjective Confidence Interval \\
\hline Backyard Cow Milk & lower limit & central estimate & upper limit \\
Commercial Milk (locally produced) & 0.42 & 2.7 & 15 \\
Commercial Milk (regionally mixed) & 0.14 & 0.92 & 6.1 \\
Goat Milk (locally produced) & 0.19 & 1.2 & 7.9 \\
Beef (locally produced) & 1.1 & 8.6 & 64 \\
Leafy Vegetables (locally produced) & 0.00077 & 0.013 & 0.27 \\
Eggs (locally produced) & 0.00029 & 0.0029 & 0.026 \\
Cottage Cheese (locally produced) & 0.026 & 0.19 & 1.3 \\
Inhalation & 0.00068 & 0.0071 & 0.075 \\
Mother's milk (mother on Diet 1) & 0.019 & 0.07 & 0.29 \\
Prenatal exposure (mother on Diet 1) & 0.000091 & 0.003 & 0.087 \\
Diet 1 & -- & -- & -- \\
Diet 2 & 0.5 & 3 & 16 \\
Diet 3 & 0.23 & 1.3 & 7.8 \\
\hline
\end{tabular}

Excess Lifetime Risk [ ]

\begin{tabular}{llll}
\hline Diet 1 & $3.5 \mathrm{E}-05$ & $5.1 \mathrm{E}-04$ & $7.0 \mathrm{E}-03$ \\
Diet 2 & $1.8 \mathrm{E}-05$ & $2.0 \mathrm{E}-04$ & $2.9 \mathrm{E}-03$ \\
Diet 3 & $1.7 \mathrm{E}-05$ & $2.0 \mathrm{E}-04$ & $2.6 \mathrm{E}-03$ \\
Diet 4 & $9.3 \mathrm{E}-05$ & $1.4 \mathrm{E}-03$ & $1.9 \mathrm{E}-02$ \\
\hline
\end{tabular}

\section{Relative Risk [ ]}

\begin{tabular}{lccc}
\hline Diet 1 & 1.014 & 1.13 & 2.5 \\
Diet 2 & 1.0057 & 1.053 & 1.68 \\
Diet 3 & 1.0058 & 1.055 & 1.65 \\
Diet 4 & 1.036 & 1.34 & 5.8 \\
\hline
\end{tabular}

\section{Probability of Causation [\%]}

\begin{tabular}{lccc}
\hline Diet 1 & 1.43 & 11.4 & 59 \\
Diet 2 & 0.57 & 5.0 & 40 \\
Diet 3 & 0.57 & 5.2 & 39 \\
Diet 4 & 3.47 & 25.0 & 83 \\
\hline
\end{tabular}

Diet 1 - Backyard cow milk + all other locally produced non-milk exposure pathways

Diet 2 - Locally produced commercial milk + all other locally produced non-milk exposure pathways

Diet 3 - Regionally mixed commercial milk + inhalation (other regionally mixed food items are minor contributors to the total)

Diet 4 - Goat milk (all other exposure pathways are of negligible importance compared to goat milk) 


\section{Location: Louisville}

\section{Receptor: Male born in 1944}

\begin{tabular}{lccc}
\hline & \multicolumn{3}{c}{ Thyroid Dose [cGy] } \\
& \multicolumn{2}{c}{$95 \%$ Subjective Confidence Interval } \\
Exposure Pathway & lower limit & central estimate & upper limit \\
\hline Backyard Cow Milk & 0.44 & 2.7 & 15 \\
Commercial Milk (locally produced) & 0.14 & 0.93 & 6.1 \\
Commercial Milk (regionally mixed) & 0.19 & 1.2 & 7.1 \\
Goat Milk (locally produced) & 1.1 & 8.4 & 61 \\
Beef (locally produced) & 0.00085 & 0.014 & 0.28 \\
Leafy Vegetables (locally produced) & 0.00026 & 0.0028 & 0.025 \\
Eggs (locally produced) & 0.03 & 0.21 & 1.4 \\
Cottage Cheese (locally produced) & 0.00062 & 0.0072 & 0.075 \\
Inhalation & 0.018 & 0.071 & 0.28 \\
Mother's milk (mother on Diet 1) & 0.000091 & 0.003 & 0.087 \\
Prenatal exposure (mother on Diet 1) & -- & -- & -- \\
Diet 1 & 0.53 & 3 & 17 \\
Diet 2 & 0.23 & 1.3 & 7.9 \\
Diet 3 & 0.22 & 1.3 & 7.4 \\
\hline
\end{tabular}

Excess Lifetime Risk [ ]

\begin{tabular}{llll}
\hline Diet 1 & $4.4 \mathrm{E}-06$ & $1.4 \mathrm{E}-04$ & $2.5 \mathrm{E}-03$ \\
Diet 2 & $1.9 \mathrm{E}-06$ & $5.5 \mathrm{E}-05$ & $1.2 \mathrm{E}-03$ \\
Diet 3 & $2.2 \mathrm{E}-06$ & $5.4 \mathrm{E}-05$ & $1.2 \mathrm{E}-03$ \\
Diet 4 & $1.1 \mathrm{E}-05$ & $3.2 \mathrm{E}-04$ & $7.9 \mathrm{E}-03$ \\
\hline
\end{tabular}

\section{Relative Risk [ ]}

\begin{tabular}{lccc}
\hline Diet 1 & 1.0036 & 1.09 & 2.9 \\
Diet 2 & 1.0017 & 1.036 & 1.82 \\
Diet 3 & 1.0018 & 1.035 & 1.9 \\
Diet 4 & 1.011 & 1.24 & 6 \\
\hline
\end{tabular}

\section{Probability of Causation [\%]}

\begin{tabular}{lccc}
\hline Diet 1 & 0.36 & 8.2 & 65 \\
Diet 2 & 0.17 & 3.5 & 45 \\
Diet 3 & 0.18 & 3.4 & 47 \\
Diet 4 & 1.11 & 19.6 & 82 \\
\hline
\end{tabular}

Diet 1 - Backyard cow milk + all other locally produced non-milk exposure pathways

Diet 2 - Locally produced commercial milk + all other locally produced non-milk exposure pathways

Diet 3 - Regionally mixed commercial milk + inhalation (other regionally mixed food items are minor contributors to the total)

Diet 4 - Goat milk (all other exposure pathways are of negligible importance compared to goat milk) 
Location: Barnardville

Receptor: Female born in 1944

\begin{tabular}{lccc}
\hline & \multicolumn{3}{c}{ Thyroid Dose [cGy] } \\
Exposure Pathway & $95 \%$ Subjective Confidence Interval \\
\hline Backyard Cow Milk & lower limit & central estimate & upper limit \\
Commercial Milk (locally produced) & 0.5 & 3.1 & 17 \\
Commercial Milk (regionally mixed) & 0.16 & 1 & 6.8 \\
Goat Milk (locally produced) & 0.19 & 1.2 & 7.9 \\
Beef (locally produced) & 1.3 & 9.8 & 72 \\
Leafy Vegetables (locally produced) & 0.00092 & 0.015 & 0.31 \\
Eggs (locally produced) & 0.0003 & 0.0032 & 0.03 \\
Cottage Cheese (locally produced) & 0.028 & 0.21 & 1.5 \\
Inhalation & 0.00069 & 0.0081 & 0.089 \\
Mother's milk (mother on Diet 1) & 0.022 & 0.082 & 0.34 \\
Prenatal exposure (mother on Diet 1) & 0.00011 & 0.0034 & 0.095 \\
Diet 1 & -- & -- & -- \\
Diet 2 & 0.59 & 3.5 & 19 \\
Diet 3 & 0.25 & 1.4 & 8.6 \\
\hline
\end{tabular}

Excess Lifetime Risk [ ]

\begin{tabular}{lllc}
\hline Diet 1 & $3.7 \mathrm{E}-05$ & $6.1 \mathrm{E}-04$ & $8.3 \mathrm{E}-03$ \\
Diet 2 & $2.0 \mathrm{E}-05$ & $2.4 \mathrm{E}-04$ & $3.2 \mathrm{E}-03$ \\
Diet 3 & $1.7 \mathrm{E}-05$ & $2.0 \mathrm{E}-04$ & $2.6 \mathrm{E}-03$ \\
Diet 4 & $9.8 \mathrm{E}-05$ & $1.7 \mathrm{E}-03$ & $2.1 \mathrm{E}-02$ \\
\hline
\end{tabular}

\section{Relative Risk [ ]}

\begin{tabular}{lccc}
\hline Diet 1 & 1.016 & 1.15 & 2.5 \\
Diet 2 & 1.0067 & 1.059 & 1.66 \\
Diet 3 & 1.0058 & 1.055 & 1.66 \\
Diet 4 & 1.041 & 1.37 & 6.2 \\
\hline
\end{tabular}

\section{Probability of Causation [\%]}

\begin{tabular}{lccc}
\hline Diet 1 & 1.55 & 12.7 & 61 \\
Diet 2 & 0.67 & 5.6 & 40 \\
Diet 3 & 0.58 & 5.3 & 40 \\
Diet 4 & 3.92 & 27.0 & 84 \\
\hline
\end{tabular}

Diet 1 - Backyard cow milk + all other locally produced non-milk exposure pathways

Diet 2 - Locally produced commercial milk + all other locally produced non-milk exposure pathways

Diet 3 - Regionally mixed commercial milk + inhalation (other regionally mixed food items are minor contributors to the total)

Diet 4 - Goat milk (all other exposure pathways are of negligible importance compared to goat milk) 


\section{Location: Barnardville}

Receptor: Male born in 1944

\begin{tabular}{lccc}
\hline & \multicolumn{3}{c}{ Thyroid Dose [cGy] } \\
& \multicolumn{2}{c}{$95 \%$ Subjective Confidence Interval } \\
Exposure Pathway & lower limit & central estimate & upper limit \\
\hline Backyard Cow Milk & 0.54 & 3 & 18 \\
Commercial Milk (locally produced) & 0.15 & 1 & 6.9 \\
Commercial Milk (regionally mixed) & 0.19 & 1.2 & 7.1 \\
Goat Milk (locally produced) & 1.3 & 9.4 & 63 \\
Beef (locally produced) & 0.00096 & 0.016 & 0.33 \\
Leafy Vegetables (locally produced) & 0.00029 & 0.0032 & 0.029 \\
Eggs (locally produced) & 0.033 & 0.24 & 1.7 \\
Cottage Cheese (locally produced) & 0.00067 & 0.0079 & 0.089 \\
Inhalation & 0.021 & 0.083 & 0.32 \\
Mother's milk (mother on Diet 1) & 0.00011 & 0.0034 & 0.095 \\
Prenatal exposure (mother on Diet 1) & -- & -- & -- \\
Diet 1 & 0.65 & 3.4 & 20 \\
Diet 2 & 0.25 & 1.5 & 9 \\
Diet 3 & 0.22 & 1.3 & 7.4 \\
\hline
\end{tabular}

Excess Lifetime Risk [ ]

\begin{tabular}{lllc}
\hline Diet 1 & $5.1 \mathrm{E}-06$ & $1.5 \mathrm{E}-04$ & $3.1 \mathrm{E}-03$ \\
Diet 2 & $2.3 \mathrm{E}-06$ & $6.3 \mathrm{E}-05$ & $1.3 \mathrm{E}-03$ \\
Diet 3 & $2.2 \mathrm{E}-06$ & $5.5 \mathrm{E}-05$ & $1.2 \mathrm{E}-03$ \\
Diet 4 & $1.4 \mathrm{E}-05$ & $3.7 \mathrm{E}-04$ & $8.5 \mathrm{E}-03$ \\
\hline
\end{tabular}

\section{Relative Risk [ ]}

\begin{tabular}{lccc}
\hline Diet 1 & 1.0046 & 1.1 & 3.3 \\
Diet 2 & 1.0019 & 1.042 & 1.92 \\
Diet 3 & 1.0018 & 1.035 & 1.9 \\
Diet 4 & 1.013 & 1.26 & 7.3 \\
\hline
\end{tabular}

\section{Probability of Causation [\%]}

\begin{tabular}{lccc}
\hline Diet 1 & 0.45 & 9.4 & 69 \\
Diet 2 & 0.19 & 4.0 & 48 \\
Diet 3 & 0.18 & 3.4 & 47 \\
Diet 4 & 1.31 & 20.9 & 86 \\
\hline
\end{tabular}

Diet 1 - Backyard cow milk + all other locally produced non-milk exposure pathways

Diet 2 - Locally produced commercial milk + all other locally produced non-milk exposure pathways

Diet 3 - Regionally mixed commercial milk + inhalation (other regionally mixed food items are minor contributors to the total)

Diet 4 - Goat milk (all other exposure pathways are of negligible importance compared to goat milk) 
Location: Greenback

Receptor: Female born in 1944

\begin{tabular}{lccc}
\hline & \multicolumn{3}{c}{ Thyroid Dose [cGy] } \\
Exposure Pathway & $95 \%$ & Subjective Confidence Interval \\
Backyard Cow Milk & lower limit & central estimate & upper limit \\
Commercial Milk (locally produced) & 0.27 & 1.7 & 11 \\
Commercial Milk (regionally mixed) & 0.086 & 0.58 & 4.2 \\
Goat Milk (locally produced) & 0.19 & 1.2 & 7.9 \\
Beef (locally produced) & 0.68 & 5.2 & 42 \\
Leafy Vegetables (locally produced) & 0.00051 & 0.0084 & 0.18 \\
Eggs (locally produced) & 0.00016 & 0.0018 & 0.018 \\
Cottage Cheese (locally produced) & 0.016 & 0.12 & 0.92 \\
Inhalation & 0.0004 & 0.0043 & 0.055 \\
Mother's milk (mother on Diet 1) & 0.012 & 0.047 & 0.19 \\
Prenatal exposure (mother on Diet 1) & 0.000056 & 0.0019 & 0.053 \\
Diet 1 & -- & -- & -- \\
Diet 2 & 0.33 & 1.9 & 12 \\
Diet 3 & 0.15 & 0.79 & 5.3 \\
\hline
\end{tabular}

Excess Lifetime Risk [ ]

\begin{tabular}{llcc}
\hline Diet 1 & $2.3 \mathrm{E}-05$ & $3.3 \mathrm{E}-04$ & $4.8 \mathrm{E}-03$ \\
Diet 2 & $1.1 \mathrm{E}-05$ & $1.3 \mathrm{E}-04$ & $1.8 \mathrm{E}-03$ \\
Diet 3 & $1.7 \mathrm{E}-05$ & $1.9 \mathrm{E}-04$ & $2.6 \mathrm{E}-03$ \\
Diet 4 & $5.9 \mathrm{E}-05$ & $8.7 \mathrm{E}-04$ & $1.1 \mathrm{E}-02$ \\
\hline
\end{tabular}

Relative Risk [ ]

\begin{tabular}{lccc}
\hline Diet 1 & 1.0085 & 1.08 & 1.93 \\
Diet 2 & 1.0038 & 1.033 & 1.38 \\
Diet 3 & 1.0057 & 1.053 & 1.65 \\
Diet 4 & 1.021 & 1.2 & 4.2 \\
\hline
\end{tabular}

\begin{tabular}{lccc} 
& \multicolumn{3}{c}{ Probability of Causation [\%] } \\
\hline Diet 1 & 0.84 & 7.4 & 48 \\
Diet 2 & 0.38 & 3.2 & 27 \\
Diet 3 & 0.57 & 5.1 & 39 \\
Diet 4 & 2.06 & 16.9 & 76 \\
\hline
\end{tabular}

Diet 1 - Backyard cow milk + all other locally produced non-milk exposure pathways

Diet 2 - Locally produced commercial milk + all other locally produced non-milk exposure pathways

Diet 3 - Regionally mixed commercial milk + inhalation (other regionally mixed food items are minor contributors to the total)

Diet 4 - Goat milk (all other exposure pathways are of negligible importance compared to goat milk) 
Location: Greenback

Receptor: Male born in 1944

\begin{tabular}{lccc}
\hline & \multicolumn{3}{c}{ Thyroid Dose [cGy] } \\
Exposure Pathway & $95 \%$ Subjective Confidence Interval \\
\hline Backyard Cow Milk & lower limit & central estimate & upper limit \\
Commercial Milk (locally produced) & 0.29 & 1.7 & 11 \\
Commercial Milk (regionally mixed) & 0.085 & 0.57 & 3.9 \\
Goat Milk (locally produced) & 0.19 & 1.2 & 7.1 \\
Beef (locally produced) & 0.64 & 5.2 & 40 \\
Leafy Vegetables (locally produced) & 0.00052 & 0.009 & 0.18 \\
Eggs (locally produced) & 0.00016 & 0.0017 & 0.017 \\
Cottage Cheese (locally produced) & 0.019 & 0.13 & 0.92 \\
Inhalation & 0.00034 & 0.0045 & 0.053 \\
Mother's milk (mother on Diet 1) & 0.013 & 0.048 & 0.2 \\
Prenatal exposure (mother on Diet 1) & 0.000056 & 0.0019 & 0.053 \\
Diet 1 & -- & -- & -- \\
Diet 2 & 0.35 & 1.9 & 12 \\
Diet 3 & 0.15 & 0.8 & 5.3 \\
\hline
\end{tabular}

Excess Lifetime Risk [ ]

\begin{tabular}{lllc}
\hline Diet 1 & $2.7 \mathrm{E}-06$ & $7.8 \mathrm{E}-05$ & $1.7 \mathrm{E}-03$ \\
Diet 2 & $1.3 \mathrm{E}-06$ & $3.4 \mathrm{E}-05$ & $7.5 \mathrm{E}-04$ \\
Diet 3 & $2.2 \mathrm{E}-06$ & $5.3 \mathrm{E}-05$ & $1.1 \mathrm{E}-03$ \\
Diet 4 & $7.5 \mathrm{E}-06$ & $1.9 \mathrm{E}-04$ & $4.7 \mathrm{E}-03$ \\
\hline
\end{tabular}

\section{Relative Risk [ ]}

\begin{tabular}{lccc}
\hline Diet 1 & 1.0024 & 1.057 & 2.3 \\
Diet 2 & 1.00098 & 1.023 & 1.54 \\
Diet 3 & 1.0017 & 1.035 & 1.87 \\
Diet 4 & 1.0066 & 1.15 & 3.8 \\
\hline
\end{tabular}

\begin{tabular}{lccc} 
& \multicolumn{3}{c}{ Probability of Causation [\%] } \\
\hline Diet 1 & 0.24 & 5.4 & 55 \\
Diet 2 & 0.10 & 2.3 & 35 \\
Diet 3 & 0.17 & 3.3 & 47 \\
Diet 4 & 0.65 & 13.0 & 74 \\
\hline
\end{tabular}

Diet 1 - Backyard cow milk + all other locally produced non-milk exposure pathways

Diet 2 - Locally produced commercial milk + all other locally produced non-milk exposure pathways

Diet 3 - Regionally mixed commercial milk + inhalation (other regionally mixed food items are minor contributors to the total)

Diet 4 - Goat milk (all other exposure pathways are of negligible importance compared to goat milk) 


\section{Location: Rockford}

Receptor: Female born in 1944

\begin{tabular}{lccc}
\hline & \multicolumn{3}{c}{ Thyroid Dose [cGy] } \\
Exposure Pathway & \multicolumn{2}{c}{$95 \%$ Subjective Confidence Interval } \\
\hline Backyard Cow Milk & lower limit & central estimate & upper limit \\
Commercial Milk (locally produced) & 0.48 & 2.7 & 16 \\
Commercial Milk (regionally mixed) & 0.14 & 0.9 & 5.9 \\
Goat Milk (locally produced) & 0.19 & 1.2 & 7.9 \\
Beef (locally produced) & 1.2 & 8.3 & 57 \\
Leafy Vegetables (locally produced) & 0.00077 & 0.013 & 0.26 \\
Eggs (locally produced) & 0.00026 & 0.0028 & 0.025 \\
Cottage Cheese (locally produced) & 0.025 & 0.19 & 1.3 \\
Inhalation & 0.00062 & 0.0067 & 0.078 \\
Mother's milk (mother on Diet 1) & 0.019 & 0.07 & 0.27 \\
Prenatal exposure (mother on Diet 1) & 0.00009 & 0.003 & 0.081 \\
Diet 1 & -- & -- & -- \\
Diet 2 & 0.55 & 3.1 & 17 \\
Diet 3 & 0.24 & 1.2 & 7.4 \\
\hline
\end{tabular}

Excess Lifetime Risk [ ]

\begin{tabular}{llll}
\hline Diet 1 & $3.4 \mathrm{E}-05$ & $5.2 \mathrm{E}-04$ & $6.6 \mathrm{E}-03$ \\
Diet 2 & $1.8 \mathrm{E}-05$ & $2.0 \mathrm{E}-04$ & $2.8 \mathrm{E}-03$ \\
Diet 3 & $1.8 \mathrm{E}-05$ & $2.0 \mathrm{E}-04$ & $2.6 \mathrm{E}-03$ \\
Diet 4 & $8.9 \mathrm{E}-05$ & $1.4 \mathrm{E}-03$ & $1.7 \mathrm{E}-02$ \\
\hline
\end{tabular}

\section{Relative Risk [ ]}

\begin{tabular}{lccc}
\hline Diet 1 & 1.013 & 1.13 & 2.3 \\
Diet 2 & 1.0054 & 1.053 & 1.58 \\
Diet 3 & 1.0058 & 1.055 & 1.65 \\
Diet 4 & 1.035 & 1.32 & 5.7 \\
\hline
\end{tabular}

\begin{tabular}{|c|c|c|c|}
\hline Diet 1 & 1.32 & 11.2 & 56 \\
\hline Diet 2 & 0.54 & 5.0 & 36 \\
\hline Diet 3 & 0.58 & 5.2 & 39 \\
\hline Diet 4 & 3.40 & 24.1 & 82 \\
\hline
\end{tabular}

Diet 1 - Backyard cow milk + all other locally produced non-milk exposure pathways

Diet 2 - Locally produced commercial milk + all other locally produced non-milk exposure pathways

Diet 3 - Regionally mixed commercial milk + inhalation (other regionally mixed food items are minor contributors to the total)

Diet 4 - Goat milk (all other exposure pathways are of negligible importance compared to goat milk) 


\section{Location: Rockford}

Receptor: Male born in 1944

\begin{tabular}{lccc}
\hline & \multicolumn{3}{c}{ Thyroid Dose [cGy] } \\
& \multicolumn{2}{c}{$95 \%$ Subjective Confidence Interval } \\
Exposure Pathway & lower limit & central estimate & upper limit \\
\hline Backyard Cow Milk & 0.51 & 2.7 & 15 \\
Commercial Milk (locally produced) & 0.14 & 0.91 & 5.9 \\
Commercial Milk (regionally mixed) & 0.19 & 1.2 & 7.1 \\
Goat Milk (locally produced) & 1.1 & 8.2 & 56 \\
Beef (locally produced) & 0.00078 & 0.014 & 0.27 \\
Leafy Vegetables (locally produced) & 0.00026 & 0.0027 & 0.025 \\
Eggs (locally produced) & 0.03 & 0.21 & 1.4 \\
Cottage Cheese (locally produced) & 0.00059 & 0.0071 & 0.077 \\
Inhalation & 0.02 & 0.071 & 0.27 \\
Mother's milk (mother on Diet 1) & 0.00009 & 0.003 & 0.081 \\
Prenatal exposure (mother on Diet 1) & -- & -- & - \\
Diet 1 & 0.6 & 3 & 18 \\
Diet 2 & 0.24 & 1.3 & 7.8 \\
Diet 3 & 0.23 & 1.3 & 7.4 \\
\hline
\end{tabular}

Excess Lifetime Risk [ ]

\begin{tabular}{llll}
\hline Diet 1 & $4.3 \mathrm{E}-06$ & $1.3 \mathrm{E}-04$ & $2.6 \mathrm{E}-03$ \\
Diet 2 & $2.0 \mathrm{E}-06$ & $5.2 \mathrm{E}-05$ & $1.2 \mathrm{E}-03$ \\
Diet 3 & $2.2 \mathrm{E}-06$ & $5.4 \mathrm{E}-05$ & $1.2 \mathrm{E}-03$ \\
Diet 4 & $1.2 \mathrm{E}-05$ & $3.0 \mathrm{E}-04$ & $7.0 \mathrm{E}-03$ \\
\hline
\end{tabular}

\section{Relative Risk [ ]}

\begin{tabular}{lccc}
\hline Diet 1 & 1.0039 & 1.09 & 2.8 \\
Diet 2 & 1.0017 & 1.035 & 1.81 \\
Diet 3 & 1.0018 & 1.035 & 1.89 \\
Diet 4 & 1.011 & 1.24 & 5.8 \\
\hline
\end{tabular}

\section{Probability of Causation [\%]}

\begin{tabular}{lccc}
\hline Diet 1 & 0.39 & 8.3 & 64 \\
Diet 2 & 0.17 & 3.4 & 45 \\
Diet 3 & 0.18 & 3.4 & 47 \\
Diet 4 & 1.12 & 19.2 & 82 \\
\hline
\end{tabular}

Diet 1 - Backyard cow milk + all other locally produced non-milk exposure pathways

Diet 2 - Locally produced commercial milk + all other locally produced non-milk exposure pathways

Diet 3 - Regionally mixed commercial milk + inhalation (other regionally mixed food items are minor contributors to the total)

Diet 4 - Goat milk (all other exposure pathways are of negligible importance compared to goat milk) 
Location: Lake City

Receptor: Female born in 1944

\begin{tabular}{lccc}
\hline & \multicolumn{3}{c}{ Thyroid Dose [cGy] } \\
Exposure Pathway & \multicolumn{2}{c}{$95 \%$ Subjective Confidence Interval } \\
\hline Backyard Cow Milk & lower limit & central estimate & upper limit \\
Commercial Milk (locally produced) & 0.26 & 1.6 & 8.3 \\
Commercial Milk (regionally mixed) & 0.084 & 0.55 & 3.6 \\
Goat Milk (locally produced) & 0.19 & 1.2 & 7.9 \\
Beef (locally produced) & 0.78 & 5.2 & 36 \\
Leafy Vegetables (locally produced) & 0.00045 & 0.0081 & 0.17 \\
Eggs (locally produced) & 0.00017 & 0.0017 & 0.015 \\
Cottage Cheese (locally produced) & 0.014 & 0.11 & 0.71 \\
Inhalation & 0.00045 & 0.0041 & 0.045 \\
Mother's milk (mother on Diet 1) & 0.01 & 0.038 & 0.15 \\
Prenatal exposure (mother on Diet 1) & 0.000055 & 0.0018 & 0.056 \\
Diet 1 & -- & -- & -- \\
Diet 2 & 0.31 & 1.8 & 9.1 \\
Diet 3 & 0.14 & 0.74 & 4.8 \\
\hline
\end{tabular}

Excess Lifetime Risk [ ]

\begin{tabular}{lllc}
\hline Diet 1 & $2.1 \mathrm{E}-05$ & $3.1 \mathrm{E}-04$ & $4.2 \mathrm{E}-03$ \\
Diet 2 & $1.1 \mathrm{E}-05$ & $1.2 \mathrm{E}-04$ & $1.7 \mathrm{E}-03$ \\
Diet 3 & $1.7 \mathrm{E}-05$ & $1.9 \mathrm{E}-04$ & $2.6 \mathrm{E}-03$ \\
Diet 4 & $5.5 \mathrm{E}-05$ & $8.3 \mathrm{E}-04$ & $1.2 \mathrm{E}-02$ \\
\hline
\end{tabular}

Relative Risk [ ]

\begin{tabular}{lccc}
\hline Diet 1 & 1.0087 & 1.078 & 1.84 \\
Diet 2 & 1.0035 & 1.032 & 1.43 \\
Diet 3 & 1.0055 & 1.053 & 1.64 \\
Diet 4 & 1.022 & 1.2 & 3.7 \\
\hline
\end{tabular}

\begin{tabular}{lccc}
\hline Diet 1 & 0.86 & 7.3 & 45 \\
Diet 2 & 0.35 & 3.1 & 30 \\
Diet 3 & 0.55 & 5.0 & 39 \\
Diet 4 & 2.12 & 16.5 & 73 \\
\hline
\end{tabular}

Diet 1 - Backyard cow milk + all other locally produced non-milk exposure pathways

Diet 2 - Locally produced commercial milk + all other locally produced non-milk exposure pathways

Diet 3 - Regionally mixed commercial milk + inhalation (other regionally mixed food items are minor contributors to the total)

Diet 4 - Goat milk (all other exposure pathways are of negligible importance compared to goat milk) 
Location: Lake City

Receptor: Male born in 1944

\begin{tabular}{lccc}
\hline & \multicolumn{3}{c}{ Thyroid Dose [cGy] } \\
Exposure Pathway & \multicolumn{2}{c}{$95 \%$ Subjective Confidence Interval } \\
\hline Backyard Cow Milk & lower limit & central estimate & upper limit \\
Commercial Milk (locally produced) & 0.27 & 1.6 & 8.6 \\
Commercial Milk (regionally mixed) & 0.087 & 0.56 & 3.6 \\
Goat Milk (locally produced) & 0.19 & 1.2 & 7.1 \\
Beef (locally produced) & 0.68 & 5.1 & 33 \\
Leafy Vegetables (locally produced) & 0.00049 & 0.0086 & 0.2 \\
Eggs (locally produced) & 0.00016 & 0.0017 & 0.015 \\
Cottage Cheese (locally produced) & 0.017 & 0.13 & 0.84 \\
Inhalation & 0.00041 & 0.0044 & 0.046 \\
Mother's milk (mother on Diet 1) & 0.011 & 0.039 & 0.15 \\
Prenatal exposure (mother on Diet 1) & 0.000055 & 0.0018 & 0.056 \\
Diet 1 & -- & -- & -- \\
Diet 2 & 0.33 & 1.8 & 9.7 \\
Diet 3 & 0.14 & 0.77 & 4.7 \\
\hline
\end{tabular}

Excess Lifetime Risk [ ]

\begin{tabular}{llll}
\hline Diet 1 & $2.5 \mathrm{E}-06$ & $8.3 \mathrm{E}-05$ & $1.6 \mathrm{E}-03$ \\
Diet 2 & $1.2 \mathrm{E}-06$ & $3.4 \mathrm{E}-05$ & $7.2 \mathrm{E}-04$ \\
Diet 3 & $2.2 \mathrm{E}-06$ & $5.2 \mathrm{E}-05$ & $1.1 \mathrm{E}-03$ \\
Diet 4 & $7.9 \mathrm{E}-06$ & $2.0 \mathrm{E}-04$ & $4.7 \mathrm{E}-03$ \\
\hline
\end{tabular}

\section{Relative Risk [ ]}

\begin{tabular}{lccc}
\hline Diet 1 & 1.0023 & 1.054 & 2.2 \\
Diet 2 & 1.001 & 1.022 & 1.49 \\
Diet 3 & 1.0017 & 1.034 & 1.87 \\
Diet 4 & 1.0066 & 1.14 & 4.2 \\
\hline
\end{tabular}

\section{Probability of Causation [\%]}

\begin{tabular}{lccc}
\hline Diet 1 & 0.23 & 5.1 & 53 \\
Diet 2 & 0.10 & 2.1 & 33 \\
Diet 3 & 0.17 & 3.3 & 47 \\
Diet 4 & 0.66 & 12.4 & 76 \\
\hline
\end{tabular}

Diet 1 - Backyard cow milk + all other locally produced non-milk exposure pathways

Diet 2 - Locally produced commercial milk + all other locally produced non-milk exposure pathways

Diet 3 - Regionally mixed commercial milk + inhalation (other regionally mixed food items are minor contributors to the total)

Diet 4 - Goat milk (all other exposure pathways are of negligible importance compared to goat milk) 
Location: Sweetwater

Receptor: Female born in 1944

\begin{tabular}{lccc}
\hline & \multicolumn{3}{c}{ Thyroid Dose [cGy] } \\
Exposure Pathway & \multicolumn{2}{c}{$95 \%$ Subjective Confidence Interval } \\
\hline Backyard Cow Milk & lower limit & central estimate & upper limit \\
Commercial Milk (locally produced) & 0.3 & 2 & 11 \\
Commercial Milk (regionally mixed) & 0.1 & 0.7 & 4.4 \\
Goat Milk (locally produced) & 0.19 & 1.2 & 7.9 \\
Beef (locally produced) & 0.7 & 6.1 & 48 \\
Leafy Vegetables (locally produced) & 0.00055 & 0.0097 & 0.19 \\
Eggs (locally produced) & 0.00022 & 0.0021 & 0.019 \\
Cottage Cheese (locally produced) & 0.02 & 0.13 & 1.1 \\
Inhalation & 0.00049 & 0.0052 & 0.055 \\
Mother's milk (mother on Diet 1) & 0.014 & 0.056 & 0.22 \\
Prenatal exposure (mother on Diet 1) & 0.00007 & 0.0022 & 0.063 \\
Diet 1 & -- & -- & - \\
Diet 2 & 0.36 & 2.2 & 12 \\
Diet 3 & 0.17 & 0.95 & 5.3 \\
\hline
\end{tabular}

Excess Lifetime Risk [ ]

\begin{tabular}{llcc}
\hline Diet 1 & $2.5 \mathrm{E}-05$ & $3.6 \mathrm{E}-04$ & $5.2 \mathrm{E}-03$ \\
Diet 2 & $1.3 \mathrm{E}-05$ & $1.5 \mathrm{E}-04$ & $2.2 \mathrm{E}-03$ \\
Diet 3 & $1.7 \mathrm{E}-05$ & $1.9 \mathrm{E}-04$ & $2.6 \mathrm{E}-03$ \\
Diet 4 & $7.4 \mathrm{E}-05$ & $1.1 \mathrm{E}-03$ & $1.5 \mathrm{E}-02$ \\
\hline
\end{tabular}

\section{Relative Risk [ ]}

\begin{tabular}{lccc}
\hline Diet 1 & 1.011 & 1.093 & 2 \\
Diet 2 & 1.0042 & 1.04 & 1.49 \\
Diet 3 & 1.0057 & 1.054 & 1.65 \\
Diet 4 & 1.026 & 1.25 & 4.7 \\
\hline
\end{tabular}

\section{Probability of Causation [\%]}

\begin{tabular}{lccc}
\hline Diet 1 & 1.11 & 8.5 & 51 \\
Diet 2 & 0.42 & 3.9 & 33 \\
Diet 3 & 0.57 & 5.1 & 39 \\
Diet 4 & 2.49 & 19.9 & 79 \\
\hline
\end{tabular}

Diet 1 - Backyard cow milk + all other locally produced non-milk exposure pathways

Diet 2 - Locally produced commercial milk + all other locally produced non-milk exposure pathways

Diet 3 - Regionally mixed commercial milk + inhalation (other regionally mixed food items are minor contributors to the total)

Diet 4 - Goat milk (all other exposure pathways are of negligible importance compared to goat milk) 
Location: Sweetwater

Receptor: Male born in 1944

\begin{tabular}{lccc}
\hline & \multicolumn{3}{c}{ Thyroid Dose [cGy] } \\
& \multicolumn{2}{c}{$95 \%$ Subjective Confidence Interval } \\
Exposure Pathway & lower limit & central estimate & upper limit \\
\hline Backyard Cow Milk & 0.32 & 1.9 & 11 \\
Commercial Milk (locally produced) & 0.1 & 0.69 & 4.4 \\
Commercial Milk (regionally mixed) & 0.19 & 1.2 & 7.1 \\
Goat Milk (locally produced) & 0.7 & 6 & 50 \\
Beef (locally produced) & 0.0006 & 0.01 & 0.2 \\
Leafy Vegetables (locally produced) & 0.00019 & 0.0021 & 0.018 \\
Eggs (locally produced) & 0.022 & 0.16 & 1 \\
Cottage Cheese (locally produced) & 0.00046 & 0.0053 & 0.054 \\
Inhalation & 0.014 & 0.057 & 0.22 \\
Mother's milk (mother on Diet 1) & 0.00007 & 0.0022 & 0.063 \\
Prenatal exposure (mother on Diet 1) & -- & -- & -- \\
Diet 1 & 0.38 & 2.2 & 13 \\
Diet 2 & 0.16 & 0.97 & 5.4 \\
Diet 3 & 0.21 & 1.3 & 7.4 \\
\hline
\end{tabular}

Excess Lifetime Risk [ ]

\begin{tabular}{llll}
\hline Diet 1 & $3.5 \mathrm{E}-06$ & $1.0 \mathrm{E}-04$ & $1.9 \mathrm{E}-03$ \\
Diet 2 & $1.4 \mathrm{E}-06$ & $3.9 \mathrm{E}-05$ & $8.4 \mathrm{E}-04$ \\
Diet 3 & $2.2 \mathrm{E}-06$ & $5.3 \mathrm{E}-05$ & $1.2 \mathrm{E}-03$ \\
Diet 4 & $8.2 \mathrm{E}-06$ & $2.3 \mathrm{E}-04$ & $5.6 \mathrm{E}-03$ \\
\hline
\end{tabular}

Relative Risk [ ]

\begin{tabular}{lccc}
\hline Diet 1 & 1.0027 & 1.065 & 2.4 \\
Diet 2 & 1.0012 & 1.027 & 1.56 \\
Diet 3 & 1.0017 & 1.035 & 1.88 \\
Diet 4 & 1.0084 & 1.19 & 4.3 \\
\hline
\end{tabular}

\section{Probability of Causation [\%]}

\begin{tabular}{lccc}
\hline Diet 1 & 0.27 & 6.1 & 57 \\
Diet 2 & 0.12 & 2.6 & 36 \\
Diet 3 & 0.17 & 3.4 & 47 \\
Diet 4 & 0.83 & 15.8 & 77 \\
\hline
\end{tabular}

Diet 1 - Backyard cow milk + all other locally produced non-milk exposure pathways

Diet 2 - Locally produced commercial milk + all other locally produced non-milk exposure pathways

Diet 3 - Regionally mixed commercial milk + inhalation (other regionally mixed food items are minor contributors to the total)

Diet 4 - Goat milk (all other exposure pathways are of negligible importance compared to goat milk) 
Location: Knoxville

Receptor: Female born in 1944

\begin{tabular}{lccc}
\hline & \multicolumn{3}{c}{ Thyroid Dose [cGy] } \\
Exposure Pathway & \multicolumn{2}{c}{$95 \%$ Subjective Confidence Interval } \\
\hline Backyard Cow Milk & lower limit & central estimate & upper limit \\
Commercial Milk (locally produced) & 0.57 & 3.4 & 18 \\
Commercial Milk (regionally mixed) & 0.18 & 1.2 & 7.9 \\
Goat Milk (locally produced) & 0.19 & 1.2 & 7.9 \\
Beef (locally produced) & 1.5 & 10 & 71 \\
Leafy Vegetables (locally produced) & 0.00096 & 0.016 & 0.32 \\
Eggs (locally produced) & 0.00036 & 0.0036 & 0.033 \\
Cottage Cheese (locally produced) & 0.031 & 0.23 & 1.6 \\
Inhalation & 0.00084 & 0.0086 & 0.098 \\
Mother's milk (mother on Diet 1) & 0.023 & 0.088 & 0.35 \\
Prenatal exposure (mother on Diet 1) & 0.00011 & 0.0037 & 0.1 \\
Diet 1 & -- & -- & -- \\
Diet 2 & 0.66 & 3.9 & 20 \\
Diet 3 & 0.28 & 1.6 & 9.6 \\
\hline
\end{tabular}

Excess Lifetime Risk [ ]

\begin{tabular}{lllc}
\hline Diet 1 & $4.1 \mathrm{E}-05$ & $6.4 \mathrm{E}-04$ & $8.5 \mathrm{E}-03$ \\
Diet 2 & $2.0 \mathrm{E}-05$ & $2.5 \mathrm{E}-04$ & $3.5 \mathrm{E}-03$ \\
Diet 3 & $1.8 \mathrm{E}-05$ & $2.0 \mathrm{E}-04$ & $2.6 \mathrm{E}-03$ \\
Diet 4 & $1.1 \mathrm{E}-04$ & $1.9 \mathrm{E}-03$ & $2.2 \mathrm{E}-02$ \\
\hline
\end{tabular}

\section{Relative Risk [ ]}

\begin{tabular}{lccc}
\hline Diet 1 & 1.018 & 1.17 & 2.6 \\
Diet 2 & 1.0074 & 1.065 & 1.73 \\
Diet 3 & 1.0059 & 1.056 & 1.65 \\
Diet 4 & 1.045 & 1.41 & 6.8 \\
\hline
\end{tabular}

\begin{tabular}{lccc} 
& \multicolumn{3}{c}{ Probability of Causation [\%] } \\
\hline Diet 1 & 1.77 & 14.4 & 61 \\
Diet 2 & 0.73 & 6.1 & 42 \\
Diet 3 & 0.58 & 5.3 & 40 \\
Diet 4 & 4.32 & 28.8 & 85 \\
\hline
\end{tabular}

Diet 1 - Backyard cow milk + all other locally produced non-milk exposure pathways

Diet 2 - Locally produced commercial milk + all other locally produced non-milk exposure pathways

Diet 3 - Regionally mixed commercial milk + inhalation (other regionally mixed food items are minor contributors to the total)

Diet 4 - Goat milk (all other exposure pathways are of negligible importance compared to goat milk) 


\section{Location: Knoxville}

\section{Receptor: Male born in 1944}

\begin{tabular}{lccc}
\hline & \multicolumn{3}{c}{ Thyroid Dose [cGy] } \\
& \multicolumn{2}{c}{$95 \%$ Subjective Confidence Interval } \\
Exposure Pathway & lower limit & central estimate & upper limit \\
\hline Backyard Cow Milk & 0.61 & 3.4 & 19 \\
Commercial Milk (locally produced) & 0.18 & 1.2 & 7.7 \\
Commercial Milk (regionally mixed) & 0.19 & 1.2 & 7.1 \\
Goat Milk (locally produced) & 1.5 & 10 & 70 \\
Beef (locally produced) & 0.001 & 0.017 & 0.35 \\
Leafy Vegetables (locally produced) & 0.00035 & 0.0035 & 0.032 \\
Eggs (locally produced) & 0.037 & 0.27 & 1.9 \\
Cottage Cheese (locally produced) & 0.00081 & 0.0088 & 0.099 \\
Inhalation & 0.024 & 0.088 & 0.34 \\
Mother's milk (mother on Diet 1) & 0.00011 & 0.0037 & 0.1 \\
Prenatal exposure (mother on Diet 1) & -- & -- & -- \\
Diet 1 & 0.71 & 3.8 & 21 \\
Diet 2 & 0.29 & 1.6 & 9.8 \\
Diet 3 & 0.23 & 1.3 & 7.4 \\
\hline
\end{tabular}

Excess Lifetime Risk [ ]

\begin{tabular}{llll}
\hline Diet 1 & $5.3 \mathrm{E}-06$ & $1.7 \mathrm{E}-04$ & $3.2 \mathrm{E}-03$ \\
Diet 2 & $2.6 \mathrm{E}-06$ & $7.0 \mathrm{E}-05$ & $1.5 \mathrm{E}-03$ \\
Diet 3 & $2.3 \mathrm{E}-06$ & $5.5 \mathrm{E}-05$ & $1.2 \mathrm{E}-03$ \\
Diet 4 & $1.5 \mathrm{E}-05$ & $3.9 \mathrm{E}-04$ & $8.8 \mathrm{E}-03$ \\
\hline
\end{tabular}

\section{Relative Risk [ ]}

\begin{tabular}{lccc}
\hline Diet 1 & 1.005 & 1.11 & 3.3 \\
Diet 2 & 1.0022 & 1.045 & 2 \\
Diet 3 & 1.0018 & 1.035 & 1.9 \\
Diet 4 & 1.015 & 1.29 & 7 \\
\hline
\end{tabular}

\section{Probability of Causation [\%]}

\begin{tabular}{lccc}
\hline Diet 1 & 0.50 & 10.1 & 70 \\
Diet 2 & 0.22 & 4.3 & 51 \\
Diet 3 & 0.18 & 3.4 & 47 \\
Diet 4 & 1.45 & 22.6 & 86 \\
\hline
\end{tabular}

Diet 1 - Backyard cow milk + all other locally produced non-milk exposure pathways

Diet 2 - Locally produced commercial milk + all other locally produced non-milk exposure pathways

Diet 3 - Regionally mixed commercial milk + inhalation (other regionally mixed food items are minor contributors to the total)

Diet 4 - Goat milk (all other exposure pathways are of negligible importance compared to goat milk) 
Location: Maryville

Receptor: Female born in 1944

\begin{tabular}{lccc}
\hline & \multicolumn{3}{c}{ Thyroid Dose [cGy] } \\
Exposure Pathway & $95 \%$ & Subjective Confidence Interval \\
Backyard Cow Milk & lower limit & central estimate & upper limit \\
Commercial Milk (locally produced) & 0.27 & 1.7 & 8.9 \\
Commercial Milk (regionally mixed) & 0.077 & 0.54 & 3.6 \\
Goat Milk (locally produced) & 0.19 & 1.2 & 7.9 \\
Beef (locally produced) & 0.67 & 5.1 & 38 \\
Leafy Vegetables (locally produced) & 0.0005 & 0.0081 & 0.17 \\
Eggs (locally produced) & 0.00015 & 0.0018 & 0.015 \\
Cottage Cheese (locally produced) & 0.015 & 0.11 & 0.74 \\
Inhalation & 0.00037 & 0.0043 & 0.05 \\
Mother's milk (mother on Diet 1) & 0.012 & 0.046 & 0.21 \\
Prenatal exposure (mother on Diet 1) & 0.000057 & 0.002 & 0.051 \\
Diet 1 & -- & -- & - \\
Diet 2 & 0.33 & 1.9 & 9.7 \\
Diet 3 & 0.13 & 0.76 & 4.5 \\
\hline
\end{tabular}

Excess Lifetime Risk [ ]

\begin{tabular}{llll}
\hline Diet 1 & $1.9 \mathrm{E}-05$ & $3.3 \mathrm{E}-04$ & $4.8 \mathrm{E}-03$ \\
Diet 2 & $1.0 \mathrm{E}-05$ & $1.3 \mathrm{E}-04$ & $1.7 \mathrm{E}-03$ \\
Diet 3 & $1.7 \mathrm{E}-05$ & $1.9 \mathrm{E}-04$ & $2.6 \mathrm{E}-03$ \\
Diet 4 & $5.2 \mathrm{E}-05$ & $9.1 \mathrm{E}-04$ & $1.2 \mathrm{E}-02$ \\
\hline
\end{tabular}

\section{Relative Risk [ ]}

\begin{tabular}{lccc}
\hline Diet 1 & 1.0081 & 1.078 & 1.83 \\
Diet 2 & 1.0036 & 1.031 & 1.36 \\
Diet 3 & 1.0056 & 1.054 & 1.65 \\
Diet 4 & 1.02 & 1.2 & 3.7 \\
\hline
\end{tabular}

\begin{tabular}{lccc}
\hline Diet 1 & 0.81 & 7.2 & 45 \\
Diet 2 & 0.36 & 3.0 & 26 \\
Diet 3 & 0.56 & 5.1 & 39 \\
Diet 4 & 1.98 & 16.6 & 73 \\
\hline
\end{tabular}

Diet 1 - Backyard cow milk + all other locally produced non-milk exposure pathways

Diet 2 - Locally produced commercial milk + all other locally produced non-milk exposure pathways

Diet 3 - Regionally mixed commercial milk + inhalation (other regionally mixed food items are minor contributors to the total)

Diet 4 - Goat milk (all other exposure pathways are of negligible importance compared to goat milk) 


\section{Location: Maryville}

\section{Receptor: Male born in 1944}

\begin{tabular}{lccc}
\hline & \multicolumn{3}{c}{ Thyroid Dose [cGy] } \\
Exposure Pathway & 95\% Subjective Confidence Interval \\
\hline Backyard Cow Milk & lower limit & central estimate & upper limit \\
Commercial Milk (locally produced) & 0.29 & 1.6 & 9.6 \\
Commercial Milk (regionally mixed) & 0.076 & 0.55 & 3.6 \\
Goat Milk (locally produced) & 0.19 & 1.2 & 7.1 \\
Beef (locally produced) & 0.65 & 5.1 & 34 \\
Leafy Vegetables (locally produced) & 0.00054 & 0.0084 & 0.18 \\
Eggs (locally produced) & 0.00015 & 0.0017 & 0.015 \\
Cottage Cheese (locally produced) & 0.018 & 0.13 & 0.96 \\
Inhalation & 0.00034 & 0.0042 & 0.046 \\
Mother's milk (mother on Diet 1) & 0.012 & 0.048 & 0.2 \\
Prenatal exposure (mother on Diet 1) & 0.000057 & 0.002 & 0.051 \\
Diet 1 & -- & -- & -- \\
Diet 2 & 0.36 & 1.8 & 11 \\
Diet 3 & 0.14 & 0.81 & 4.4 \\
\hline
\end{tabular}

Excess Lifetime Risk [ ]

\begin{tabular}{llll}
\hline Diet 1 & $2.6 \mathrm{E}-06$ & $8.0 \mathrm{E}-05$ & $1.6 \mathrm{E}-03$ \\
Diet 2 & $1.3 \mathrm{E}-06$ & $3.4 \mathrm{E}-05$ & $6.6 \mathrm{E}-04$ \\
Diet 3 & $2.2 \mathrm{E}-06$ & $5.3 \mathrm{E}-05$ & $1.1 \mathrm{E}-03$ \\
Diet 4 & $7.6 \mathrm{E}-06$ & $2.1 \mathrm{E}-04$ & $4.3 \mathrm{E}-03$ \\
\hline
\end{tabular}

\section{Relative Risk [ ]}

\begin{tabular}{lccc}
\hline Diet 1 & 1.0027 & 1.057 & 2.2 \\
Diet 2 & 1.001 & 1.023 & 1.47 \\
Diet 3 & 1.0017 & 1.035 & 1.88 \\
Diet 4 & 1.0077 & 1.14 & 4.3 \\
\hline
\end{tabular}

\begin{tabular}{lccc}
\hline Diet 1 & 0.27 & 5.4 & 54 \\
Diet 2 & 0.10 & 2.2 & 32 \\
Diet 3 & 0.17 & 3.3 & 47 \\
Diet 4 & 0.77 & 12.1 & 77 \\
\hline
\end{tabular}

Diet 1 - Backyard cow milk + all other locally produced non-milk exposure pathways

Diet 2 - Locally produced commercial milk + all other locally produced non-milk exposure pathways

Diet 3 - Regionally mixed commercial milk + inhalation (other regionally mixed food items are minor contributors to the total)

Diet 4 - Goat milk (all other exposure pathways are of negligible importance compared to goat milk) 
Location: Cedar Grove

Receptor: Female born in 1944

\begin{tabular}{lccc}
\hline & \multicolumn{3}{c}{ Thyroid Dose [cGy] } \\
Exposure Pathway & $95 \%$ Subjective Confidence Interval \\
\hline Backyard Cow Milk & lower limit & central estimate & upper limit \\
Commercial Milk (locally produced) & 0.57 & 3.1 & 17 \\
Commercial Milk (regionally mixed) & 0.17 & 1 & 6.8 \\
Goat Milk (locally produced) & 0.19 & 1.2 & 7.9 \\
Beef (locally produced) & 1.5 & 9.7 & 65 \\
Leafy Vegetables (locally produced) & 0.00086 & 0.015 & 0.29 \\
Eggs (locally produced) & 0.00031 & 0.0033 & 0.029 \\
Cottage Cheese (locally produced) & 0.029 & 0.21 & 1.5 \\
Inhalation & 0.00074 & 0.0079 & 0.088 \\
Mother's milk (mother on Diet 1) & 0.021 & 0.077 & 0.29 \\
Prenatal exposure (mother on Diet 1) & 0.00011 & 0.0034 & 0.096 \\
Diet 1 & -- & -- & -- \\
Diet 2 & 0.65 & 3.5 & 19 \\
Diet 3 & 0.27 & 1.4 & 8.6 \\
\hline
\end{tabular}

Excess Lifetime Risk [ ]

\begin{tabular}{llll}
\hline Diet 1 & $4.0 \mathrm{E}-05$ & $5.9 \mathrm{E}-04$ & $7.5 \mathrm{E}-03$ \\
Diet 2 & $2.0 \mathrm{E}-05$ & $2.3 \mathrm{E}-04$ & $3.1 \mathrm{E}-03$ \\
Diet 3 & $1.8 \mathrm{E}-05$ & $2.0 \mathrm{E}-04$ & $2.6 \mathrm{E}-03$ \\
Diet 4 & $1.0 \mathrm{E}-04$ & $1.7 \mathrm{E}-03$ & $2.0 \mathrm{E}-02$ \\
\hline
\end{tabular}

\section{Relative Risk [ ]}

\begin{tabular}{lccc}
\hline Diet 1 & 1.015 & 1.15 & 2.5 \\
Diet 2 & 1.0064 & 1.061 & 1.68 \\
Diet 3 & 1.0058 & 1.055 & 1.65 \\
Diet 4 & 1.042 & 1.37 & 6.2 \\
\hline
\end{tabular}

\section{Probability of Causation [\%]}

\begin{tabular}{lccc}
\hline Diet 1 & 1.49 & 12.9 & 59 \\
Diet 2 & 0.63 & 5.7 & 41 \\
Diet 3 & 0.58 & 5.2 & 39 \\
Diet 4 & 4.08 & 26.8 & 84 \\
\hline
\end{tabular}

Diet 1 - Backyard cow milk + all other locally produced non-milk exposure pathways

Diet 2 - Locally produced commercial milk + all other locally produced non-milk exposure pathways

Diet 3 - Regionally mixed commercial milk + inhalation (other regionally mixed food items are minor contributors to the total)

Diet 4 - Goat milk (all other exposure pathways are of negligible importance compared to goat milk) 


\section{Location: Cedar Grove}

Receptor: Male born in 1944

\begin{tabular}{lccc}
\hline & \multicolumn{3}{c}{ Thyroid Dose [cGy] } \\
Exposure Pathway & \multicolumn{2}{c}{ 95\% } & Subjective Confidence Interval \\
Backyard Cow Milk & lower limit & central estimate & upper limit \\
Commercial Milk (locally produced) & 0.59 & 3.1 & 18 \\
Commercial Milk (regionally mixed) & 0.17 & 1 & 6.6 \\
Goat Milk (locally produced) & 0.19 & 1.2 & 7.1 \\
Beef (locally produced) & 1.4 & 9.3 & 64 \\
Leafy Vegetables (locally produced) & 0.00091 & 0.016 & 0.32 \\
Eggs (locally produced) & 0.00031 & 0.0032 & 0.028 \\
Cottage Cheese (locally produced) & 0.035 & 0.24 & 1.7 \\
Inhalation & 0.00069 & 0.0081 & 0.086 \\
Mother's milk (mother on Diet 1) & 0.021 & 0.077 & 0.29 \\
Prenatal exposure (mother on Diet 1) & 0.00011 & 0.0034 & 0.096 \\
Diet 1 & -- & -- & -- \\
Diet 2 & 0.7 & 3.5 & 20 \\
Diet 3 & 0.28 & 1.5 & 8.9 \\
\hline
\end{tabular}

Excess Lifetime Risk [ ]

\begin{tabular}{llll}
\hline Diet 1 & $4.8 \mathrm{E}-06$ & $1.5 \mathrm{E}-04$ & $2.9 \mathrm{E}-03$ \\
Diet 2 & $2.3 \mathrm{E}-06$ & $6.0 \mathrm{E}-05$ & $1.3 \mathrm{E}-03$ \\
Diet 3 & $2.2 \mathrm{E}-06$ & $5.4 \mathrm{E}-05$ & $1.2 \mathrm{E}-03$ \\
Diet 4 & $1.4 \mathrm{E}-05$ & $3.5 \mathrm{E}-04$ & $8.0 \mathrm{E}-03$ \\
\hline
\end{tabular}

\section{Relative Risk [ ]}

\begin{tabular}{lccc}
\hline Diet 1 & 1.0045 & 1.1 & 3.2 \\
Diet 2 & 1.002 & 1.04 & 1.9 \\
Diet 3 & 1.0018 & 1.035 & 1.89 \\
Diet 4 & 1.013 & 1.28 & 6.8 \\
\hline
\end{tabular}

\section{Probability of Causation [\%]}

\begin{tabular}{lccc}
\hline Diet 1 & 0.44 & 9.4 & 68 \\
Diet 2 & 0.20 & 3.9 & 47 \\
Diet 3 & 0.18 & 3.4 & 47 \\
Diet 4 & 1.33 & 21.6 & 85 \\
\hline
\end{tabular}

Diet 1 - Backyard cow milk + all other locally produced non-milk exposure pathways

Diet 2 - Locally produced commercial milk + all other locally produced non-milk exposure pathways

Diet 3 - Regionally mixed commercial milk + inhalation (other regionally mixed food items are minor contributors to the total)

Diet 4 - Goat milk (all other exposure pathways are of negligible importance compared to goat milk) 
Location: Bradbury

Receptor: Female born in 1950

\begin{tabular}{lccc}
\hline & \multicolumn{3}{c}{ Thyroid Dose [cGy] } \\
& \multicolumn{2}{c}{$95 \%$ Subjective Confidence Interval } \\
Exposure Pathway & lower limit & central estimate & upper limit \\
\hline Backyard Cow Milk & 4.4 & 26 & 170 \\
Commercial Milk (locally produced) & 1.1 & 8.7 & 61 \\
Commercial Milk (regionally mixed) & 0.17 & 1.2 & 7.9 \\
Goat Milk (locally produced) & 8.3 & 70 & 630 \\
Beef (locally produced) & 0.0062 & 0.11 & 2.7 \\
Leafy Vegetables (locally produced) & 0.0016 & 0.018 & 0.17 \\
Eggs (locally produced) & 0.21 & 1.7 & 15 \\
Cottage Cheese (locally produced) & 0.0056 & 0.063 & 0.78 \\
Inhalation & 0.075 & 0.3 & 1.2 \\
Mother's milk (mother on Diet 1) & 0.0017 & 0.045 & 1.3 \\
Prenatal exposure (mother on Diet 1) & 0.012 & 0.13 & 1.4 \\
Diet 1 & 4.8 & 28 & 190 \\
Diet 2 & 1.8 & 11 & 77 \\
Diet 3 & 0.31 & 1.5 & 9.1 \\
\hline
\end{tabular}

Excess Lifetime Risk [ ]

\begin{tabular}{llll}
\hline Diet 1 & $4.6 \mathrm{E}-04$ & $7.8 \mathrm{E}-03$ & $1.1 \mathrm{E}-01$ \\
Diet 2 & $2.3 \mathrm{E}-04$ & $2.8 \mathrm{E}-03$ & $3.8 \mathrm{E}-02$ \\
Diet 3 & $3.6 \mathrm{E}-05$ & $3.8 \mathrm{E}-04$ & $4.3 \mathrm{E}-03$ \\
Diet 4 & $1.2 \mathrm{E}-03$ & $1.9 \mathrm{E}-02$ & $2.6 \mathrm{E}-01$ \\
\hline
\end{tabular}

\section{Relative Risk [ ]}

\begin{tabular}{lccc}
\hline Diet 1 & 1.18 & 2.9 & 23 \\
Diet 2 & 1.069 & 1.72 & 11 \\
Diet 3 & 1.011 & 1.094 & 2.2 \\
Diet 4 & 1.4 & 5.1 & 81 \\
\hline
\end{tabular}

\section{Probability of Causation [\%]}

\begin{tabular}{lccc}
\hline Diet 1 & 15.21 & 64.9 & 96 \\
Diet 2 & 6.43 & 41.7 & 91 \\
Diet 3 & 1.10 & 8.6 & 54 \\
Diet 4 & 28.39 & 80.2 & 99 \\
\hline
\end{tabular}

Diet 1 - Backyard cow milk + all other locally produced non-milk exposure pathways

Diet 2 - Locally produced commercial milk + all other locally produced non-milk exposure pathways

Diet 3 - Regionally mixed commercial milk + inhalation (other regionally mixed food items are minor contributors to the total)

Diet 4 - Goat milk (all other exposure pathways are of negligible importance compared to goat milk) 


\section{Location: Bradbury}

Receptor: Male born in 1950

\begin{tabular}{lccc}
\hline & \multicolumn{3}{c}{ Thyroid Dose [cGy] } \\
& \multicolumn{2}{c}{$95 \%$ Subjective Confidence Interval } \\
Exposure Pathway & lower limit & central estimate & upper limit \\
\hline Backyard Cow Milk & 4.4 & 25 & 170 \\
Commercial Milk (locally produced) & 1.2 & 8.3 & 64 \\
Commercial Milk (regionally mixed) & 0.17 & 1.1 & 8.9 \\
Goat Milk (locally produced) & 7.9 & 73 & 560 \\
Beef (locally produced) & 0.0065 & 0.11 & 2.5 \\
Leafy Vegetables (locally produced) & 0.0016 & 0.018 & 0.17 \\
Eggs (locally produced) & 0.24 & 1.7 & 14 \\
Cottage Cheese (locally produced) & 0.0053 & 0.063 & 0.68 \\
Inhalation & 0.07 & 0.3 & 1.1 \\
Mother's milk (mother on Diet 1) & 0.0017 & 0.045 & 1.3 \\
Prenatal exposure (mother on Diet 1) & 0.012 & 0.13 & 1.4 \\
Diet 1 & 5 & 28 & 190 \\
Diet 2 & 1.7 & 11 & 78 \\
Diet 3 & 0.29 & 1.5 & 9.9 \\
\hline
\end{tabular}

Excess Lifetime Risk [ ]

\begin{tabular}{lllc}
\hline Diet 1 & $5.4 \mathrm{E}-05$ & $2.1 \mathrm{E}-03$ & $3.6 \mathrm{E}-02$ \\
Diet 2 & $3.1 \mathrm{E}-05$ & $7.5 \mathrm{E}-04$ & $1.7 \mathrm{E}-02$ \\
Diet 3 & $4.4 \mathrm{E}-06$ & $9.8 \mathrm{E}-05$ & $2.1 \mathrm{E}-03$ \\
Diet 4 & $1.4 \mathrm{E}-04$ & $4.7 \mathrm{E}-03$ & $1.1 \mathrm{E}-01$ \\
\hline
\end{tabular}

\section{Relative Risk [ ]}

\begin{tabular}{lccc}
\hline Diet 1 & 1.071 & 2.3 & 30 \\
Diet 2 & 1.026 & 1.52 & 14 \\
Diet 3 & 1.0039 & 1.067 & 2.5 \\
Diet 4 & 1.15 & 4.4 & 82 \\
\hline
\end{tabular}

\begin{tabular}{lccc} 
& \multicolumn{3}{c}{ Probability of Causation [\%] } \\
\hline Diet 1 & 6.64 & 57.0 & 97 \\
Diet 2 & 2.57 & 34.0 & 93 \\
Diet 3 & 0.39 & 6.2 & 60 \\
Diet 4 & 13.21 & 76.9 & 99 \\
\hline
\end{tabular}

Diet 1 - Backyard cow milk + all other locally produced non-milk exposure pathways

Diet 2 - Locally produced commercial milk + all other locally produced non-milk exposure pathways

Diet 3 - Regionally mixed commercial milk + inhalation (other regionally mixed food items are minor contributors to the total)

Diet 4 - Goat milk (all other exposure pathways are of negligible importance compared to goat milk) 
Location: Gallaher Bend

Receptor: Female born in 1950

\begin{tabular}{lccc}
\hline & \multicolumn{3}{c}{ Thyroid Dose [cGy] } \\
& \multicolumn{2}{c}{$95 \%$ Subjective Confidence Interval } \\
Exposure Pathway & lower limit & central estimate & upper limit \\
\hline Backyard Cow Milk & 5.7 & 31 & 190 \\
Commercial Milk (locally produced) & 1.5 & 11 & 76 \\
Commercial Milk (regionally mixed) & 0.17 & 1.2 & 7.9 \\
Goat Milk (locally produced) & 10 & 87 & 720 \\
Beef (locally produced) & 0.0068 & 0.13 & 3.2 \\
Leafy Vegetables (locally produced) & 0.0021 & 0.022 & 0.2 \\
Eggs (locally produced) & 0.27 & 2 & 17 \\
Cottage Cheese (locally produced) & 0.0068 & 0.077 & 0.86 \\
Inhalation & 0.088 & 0.36 & 1.4 \\
Mother's milk (mother on Diet 1) & 0.0019 & 0.057 & 1.4 \\
Prenatal exposure (mother on Diet 1) & 0.016 & 0.16 & 1.7 \\
Diet 1 & 6.4 & 35 & 210 \\
Diet 2 & 2.1 & 14 & 95 \\
Diet 3 & 0.34 & 1.6 & 9.2 \\
\hline
\end{tabular}

Excess Lifetime Risk [ ]

\begin{tabular}{llll}
\hline Diet 1 & $5.9 \mathrm{E}-04$ & $9.1 \mathrm{E}-03$ & $1.4 \mathrm{E}-01$ \\
Diet 2 & $2.9 \mathrm{E}-04$ & $3.4 \mathrm{E}-03$ & $4.8 \mathrm{E}-02$ \\
Diet 3 & $3.9 \mathrm{E}-05$ & $4.0 \mathrm{E}-04$ & $4.5 \mathrm{E}-03$ \\
Diet 4 & $1.4 \mathrm{E}-03$ & $2.3 \mathrm{E}-02$ & $3.0 \mathrm{E}-01$ \\
\hline
\end{tabular}

\section{Relative Risk [ ]}

\begin{tabular}{lccc}
\hline Diet 1 & 1.23 & 3.4 & 25 \\
Diet 2 & 1.088 & 1.9 & 13 \\
Diet 3 & 1.012 & 1.1 & 2.2 \\
Diet 4 & 1.54 & 6.3 & 94 \\
\hline
\end{tabular}

Probability of Causation [\%]

\begin{tabular}{lccc}
\hline Diet 1 & 18.73 & 70.9 & 96 \\
Diet 2 & 8.07 & 47.4 & 92 \\
Diet 3 & 1.21 & 9.1 & 54 \\
Diet 4 & 35.03 & 84.1 & 99 \\
\hline
\end{tabular}

Diet 1 - Backyard cow milk + all other locally produced non-milk exposure pathways

Diet 2 - Locally produced commercial milk + all other locally produced non-milk exposure pathways

Diet 3 - Regionally mixed commercial milk + inhalation (other regionally mixed food items are minor contributors to the total)

Diet 4 - Goat milk (all other exposure pathways are of negligible importance compared to goat milk) 


\section{Location: Gallaher Bend}

Receptor: Male born in 1950

\begin{tabular}{lccc}
\hline & \multicolumn{3}{c}{ Thyroid Dose [cGy] } \\
& \multicolumn{2}{c}{$95 \%$ Subjective Confidence Interval } \\
Exposure Pathway & lower limit & central estimate & upper limit \\
\hline Backyard Cow Milk & 5.4 & 33 & 180 \\
Commercial Milk (locally produced) & 1.5 & 11 & 75 \\
Commercial Milk (regionally mixed) & 0.17 & 1.1 & 8.9 \\
Goat Milk (locally produced) & 9.3 & 87 & 650 \\
Beef (locally produced) & 0.0073 & 0.14 & 3 \\
Leafy Vegetables (locally produced) & 0.0019 & 0.022 & 0.2 \\
Eggs (locally produced) & 0.28 & 2.1 & 16 \\
Cottage Cheese (locally produced) & 0.0062 & 0.077 & 0.8 \\
Inhalation & 0.094 & 0.38 & 1.4 \\
Mother's milk (mother on Diet 1) & 0.0019 & 0.057 & 1.4 \\
Prenatal exposure (mother on Diet 1) & 0.016 & 0.16 & 1.7 \\
Diet 1 & 6.2 & 36 & 200 \\
Diet 2 & 2.2 & 14 & 93 \\
Diet 3 & 0.31 & 1.6 & 10 \\
\hline
\end{tabular}

Excess Lifetime Risk [ ]

\begin{tabular}{lllc}
\hline Diet 1 & $6.9 \mathrm{E}-05$ & $2.5 \mathrm{E}-03$ & $4.1 \mathrm{E}-02$ \\
Diet 2 & $3.3 \mathrm{E}-05$ & $9.5 \mathrm{E}-04$ & $2.0 \mathrm{E}-02$ \\
Diet 3 & $4.6 \mathrm{E}-06$ & $1.1 \mathrm{E}-04$ & $2.1 \mathrm{E}-03$ \\
Diet 4 & $1.6 \mathrm{E}-04$ & $5.7 \mathrm{E}-03$ & $1.2 \mathrm{E}-01$ \\
\hline
\end{tabular}

\section{Relative Risk [ ]}

\begin{tabular}{lccc}
\hline Diet 1 & 1.088 & 2.5 & 36 \\
Diet 2 & 1.032 & 1.64 & 14 \\
Diet 3 & 1.0042 & 1.07 & 2.5 \\
Diet 4 & 1.19 & 5.1 & 91 \\
\hline
\end{tabular}

\section{Probability of Causation [\%]}

\begin{tabular}{lccc}
\hline Diet 1 & 8.05 & 60.3 & 97 \\
Diet 2 & 3.09 & 39.1 & 93 \\
Diet 3 & 0.42 & 6.5 & 60 \\
Diet 4 & 16.10 & 80.5 & 99 \\
\hline
\end{tabular}

Diet 1 - Backyard cow milk + all other locally produced non-milk exposure pathways

Diet 2 - Locally produced commercial milk + all other locally produced non-milk exposure pathways

Diet 3 - Regionally mixed commercial milk + inhalation (other regionally mixed food items are minor contributors to the total)

Diet 4 - Goat milk (all other exposure pathways are of negligible importance compared to goat milk) 


\section{Location: EFPC}

Receptor: Female born in 1950

\begin{tabular}{lccc}
\hline & \multicolumn{3}{c}{ Thyroid Dose [cGy] } \\
Exposure Pathway & \multicolumn{2}{c}{$95 \%$ Subjective Confidence Interval } \\
\hline Backyard Cow Milk & 1.1 & 6.7 & 43 \\
Commercial Milk (locally produced) & 0.29 & 2.3 & 16 \\
Commercial Milk (regionally mixed) & 0.17 & 1.2 & 7.9 \\
Goat Milk (locally produced) & -- & -- & -- \\
Beef (locally produced) & 0.0017 & 0.028 & 0.72 \\
Leafy Vegetables (locally produced) & 0.00039 & 0.0048 & 0.045 \\
Eggs (locally produced) & 0.052 & 0.44 & 3.6 \\
Cottage Cheese (locally produced) & 0.0015 & 0.016 & 0.2 \\
Inhalation & 0.02 & 0.08 & 0.31 \\
Mother's milk (mother on Diet 1) & 0.00042 & 0.012 & 0.32 \\
Prenatal exposure (mother on Diet 1) & 0.0032 & 0.035 & 0.37 \\
Diet 1 & 1.2 & 7.4 & 47 \\
Diet 2 & 0.44 & 3 & 20 \\
Diet 3 & 0.21 & 1.3 & 8.2 \\
\hline
\end{tabular}

Excess Lifetime Risk [ ]

\begin{tabular}{lccc}
\hline Diet 1 & $1.3 \mathrm{E}-04$ & $2.0 \mathrm{E}-03$ & $2.8 \mathrm{E}-02$ \\
Diet 2 & $6.0 \mathrm{E}-05$ & $7.4 \mathrm{E}-04$ & $1.0 \mathrm{E}-02$ \\
Diet 3 & $2.9 \mathrm{E}-05$ & $3.1 \mathrm{E}-04$ & $4.1 \mathrm{E}-03$ \\
Diet 4 & -- & -- & -- \\
\hline
\end{tabular}

\section{Relative Risk [ ]}

\begin{tabular}{lccc}
\hline Diet 1 & 1.045 & 1.5 & 6.7 \\
Diet 2 & 1.017 & 1.19 & 3.6 \\
Diet 3 & 1.0087 & 1.082 & 2.1 \\
Diet 4 & -- & -- & -- \\
\hline
\end{tabular}

\begin{tabular}{lccc}
\hline Diet 1 & 4.27 & 32.4 & 84 \\
Diet 2 & 1.68 & 15.6 & 70 \\
Diet 3 & 0.86 & 7.6 & 52 \\
Diet 4 & -- & -- & -- \\
\hline Diet 1 - Backyard cow milk + all other locally produced non-milk exposure pathways \\
Diet 2 - Locally produced commercial milk + all other locally produced non-milk exposure pathways \\
Diet 3 - Regionally mixed commercial milk + inhalation (other regionally mixed food items are minor contributors to the total) \\
Diet 4 - Goat milk (all other exposure pathways are of negligible importance compared to goat milk)
\end{tabular}




\section{Location: EFPC}

\section{Receptor: Male born in 1950}

\begin{tabular}{lccc}
\hline & \multicolumn{3}{c}{ Thyroid Dose [cGy] } \\
& \multicolumn{2}{c}{$95 \%$ Subjective Confidence Interval } \\
Exposure Pathway & lower limit & central estimate & upper limit \\
\hline Backyard Cow Milk & 1.1 & 6.8 & 44 \\
Commercial Milk (locally produced) & 0.29 & 2.3 & 17 \\
Commercial Milk (regionally mixed) & 0.17 & 1.1 & 8.9 \\
Goat Milk (locally produced) & -- & -- & -- \\
Beef (locally produced) & 0.0017 & 0.028 & 0.72 \\
Leafy Vegetables (locally produced) & 0.00039 & 0.0045 & 0.045 \\
Eggs (locally produced) & 0.058 & 0.42 & 3.5 \\
Cottage Cheese (locally produced) & 0.0014 & 0.017 & 0.19 \\
Inhalation & 0.018 & 0.082 & 0.32 \\
Mother's milk (mother on Diet 1) & 0.00042 & 0.012 & 0.32 \\
Prenatal exposure (mother on Diet 1) & 0.0032 & 0.035 & 0.37 \\
Diet 1 & 1.2 & 7.6 & 48 \\
Diet 2 & 0.44 & 3 & 21 \\
Diet 3 & 0.21 & 1.2 & 9.1 \\
\hline
\end{tabular}

Excess Lifetime Risk [ ]

\begin{tabular}{lccc}
\hline Diet 1 & $1.4 \mathrm{E}-05$ & $5.4 \mathrm{E}-04$ & $9.0 \mathrm{E}-03$ \\
Diet 2 & $7.5 \mathrm{E}-06$ & $2.0 \mathrm{E}-04$ & $4.5 \mathrm{E}-03$ \\
Diet 3 & $3.7 \mathrm{E}-06$ & $8.4 \mathrm{E}-05$ & $1.9 \mathrm{E}-03$ \\
Diet 4 & -- & -- & -- \\
\hline
\end{tabular}

\section{Relative Risk [ ]}

\begin{tabular}{lccc}
\hline Diet 1 & 1.018 & 1.34 & 9.1 \\
Diet 2 & 1.0067 & 1.13 & 4.5 \\
Diet 3 & 1.0029 & 1.057 & 2.4 \\
Diet 4 & -- & -- & -- \\
\hline
\end{tabular}

\section{Probability of Causation [\%]}

\begin{tabular}{lccc}
\hline Diet 1 & 1.74 & 24.7 & 88 \\
Diet 2 & 0.67 & 11.3 & 77 \\
Diet 3 & 0.29 & 5.4 & 58 \\
Diet 4 & -- & -- \\
\hline Diet 1 - Backyard cow milk + all other locally produced non-milk exposure pathways & \\
Diet 2 - Locally produced commercial milk + all other locally produced non-milk exposure pathways \\
Diet 3 - Regionally mixed commercial milk + inhalation (other regionally mixed food items are minor contributors to the total) \\
Diet 4 - Goat milk (all other exposure pathways are of negligible importance compared to goat milk)
\end{tabular}




\section{Location: Hope Creek}

Receptor: Female born in 1950

\begin{tabular}{lccc}
\hline & \multicolumn{3}{c}{ Thyroid Dose [cGy] } \\
& \multicolumn{2}{c}{$95 \%$ Subjective Confidence Interval } \\
Exposure Pathway & lower limit & central estimate & upper limit \\
\hline Backyard Cow Milk & 3 & 18 & 120 \\
Commercial Milk (locally produced) & 0.82 & 6.1 & 45 \\
Commercial Milk (regionally mixed) & 0.17 & 1.2 & 7.9 \\
Goat Milk (locally produced) & -- & -- & -- \\
Beef (locally produced) & 0.004 & 0.078 & 2 \\
Leafy Vegetables (locally produced) & 0.0012 & 0.013 & 0.12 \\
Eggs (locally produced) & 0.15 & 1.2 & 9.2 \\
Cottage Cheese (locally produced) & 0.0039 & 0.044 & 0.53 \\
Inhalation & 0.054 & 0.21 & 0.86 \\
Mother's milk (mother on Diet 1) & 0.0011 & 0.032 & 0.85 \\
Prenatal exposure (mother on Diet 1) & 0.0096 & 0.09 & 1 \\
Diet 1 & 3.3 & 19 & 130 \\
Diet 2 & 1.2 & 8 & 57 \\
Diet 3 & 0.28 & 1.4 & 8.7 \\
\hline
\end{tabular}

Excess Lifetime Risk [ ]

\begin{tabular}{lccc}
\hline Diet 1 & $3.4 \mathrm{E}-04$ & $5.3 \mathrm{E}-03$ & $7.7 \mathrm{E}-02$ \\
Diet 2 & $1.7 \mathrm{E}-04$ & $2.0 \mathrm{E}-03$ & $2.7 \mathrm{E}-02$ \\
Diet 3 & $3.3 \mathrm{E}-05$ & $3.5 \mathrm{E}-04$ & $4.2 \mathrm{E}-03$ \\
Diet 4 & -- & -- & -- \\
\hline
\end{tabular}

\section{Relative Risk [ ]}

\begin{tabular}{lccc}
\hline Diet 1 & 1.12 & 2.4 & 16 \\
Diet 2 & 1.049 & 1.51 & 7.9 \\
Diet 3 & 1.01 & 1.089 & 2.1 \\
Diet 4 & -- & -- & -- \\
\hline
\end{tabular}

\section{Probability of Causation [\%]}

\begin{tabular}{lccc}
\hline Diet 1 & 10.96 & 57.4 & 93 \\
Diet 2 & 4.65 & 33.6 & 87 \\
Diet 3 & 1.02 & 8.1 & 53 \\
Diet 4 & -- & -- & -- \\
\hline
\end{tabular}

Diet 1 - Backyard cow milk + all other locally produced non-milk exposure pathways

Diet 2 - Locally produced commercial milk + all other locally produced non-milk exposure pathways

Diet 3 - Regionally mixed commercial milk + inhalation (other regionally mixed food items are minor contributors to the total)

Diet 4 - Goat milk (all other exposure pathways are of negligible importance compared to goat milk) 


\section{Location: Hope Creek}

Receptor: Male born in 1950

\begin{tabular}{lccc}
\hline & \multicolumn{3}{c}{ Thyroid Dose [cGy] } \\
& \multicolumn{2}{c}{$95 \%$ Subjective Confidence Interval } \\
Exposure Pathway & lower limit & central estimate & upper limit \\
\hline Backyard Cow Milk & 3.2 & 18 & 120 \\
Commercial Milk (locally produced) & 0.84 & 6 & 48 \\
Commercial Milk (regionally mixed) & 0.17 & 1.1 & 8.9 \\
Goat Milk (locally produced) & -- & -- & -- \\
Beef (locally produced) & 0.0044 & 0.074 & 2 \\
Leafy Vegetables (locally produced) & 0.0012 & 0.013 & 0.12 \\
Eggs (locally produced) & 0.16 & 1.1 & 9.1 \\
Cottage Cheese (locally produced) & 0.0039 & 0.045 & 0.5 \\
Inhalation & 0.054 & 0.22 & 0.83 \\
Mother's milk (mother on Diet 1) & 0.0011 & 0.032 & 0.85 \\
Prenatal exposure (mother on Diet 1) & 0.0096 & 0.09 & 1 \\
Diet 1 & 3.5 & 20 & 130 \\
Diet 2 & 1.3 & 7.7 & 57 \\
Diet 3 & 0.27 & 1.4 & 9.6 \\
\hline
\end{tabular}

Excess Lifetime Risk [ ]

\begin{tabular}{lccc}
\hline Diet 1 & $3.8 \mathrm{E}-05$ & $1.4 \mathrm{E}-03$ & $2.6 \mathrm{E}-02$ \\
Diet 2 & $1.9 \mathrm{E}-05$ & $5.6 \mathrm{E}-04$ & $1.2 \mathrm{E}-02$ \\
Diet 3 & $4.2 \mathrm{E}-06$ & $9.4 \mathrm{E}-05$ & $2.0 \mathrm{E}-03$ \\
Diet 4 & -- & -- & -- \\
\hline
\end{tabular}

\section{Relative Risk [ ]}

\begin{tabular}{lccc}
\hline Diet 1 & 1.052 & 1.89 & 21 \\
Diet 2 & 1.018 & 1.36 & 9.5 \\
Diet 3 & 1.0035 & 1.062 & 2.5 \\
Diet 4 & -- & -- & -- \\
\hline
\end{tabular}

\section{Probability of Causation [\%]}

\begin{tabular}{lccc}
\hline Diet 1 & 4.89 & 46.9 & 95 \\
Diet 2 & 1.76 & 26.4 & 89 \\
Diet 3 & 0.34 & 5.9 & 59 \\
Diet 4 & -- & -- & -- \\
\hline
\end{tabular}

Diet 1 - Backyard cow milk + all other locally produced non-milk exposure pathways

Diet 2 - Locally produced commercial milk + all other locally produced non-milk exposure pathways

Diet 3 - Regionally mixed commercial milk + inhalation (other regionally mixed food items are minor contributors to the total)

Diet 4 - Goat milk (all other exposure pathways are of negligible importance compared to goat milk) 
Location: Buttermilk Rd.

Receptor: Female born in 1950

\begin{tabular}{lccc}
\hline & \multicolumn{3}{c}{ Thyroid Dose [cGy] } \\
Exposure Pathway & $95 \%$ Subjective Confidence Interval \\
\hline Backyard Cow Milk & lower limit & central estimate & upper limit \\
Commercial Milk (locally produced) & 2.9 & 17 & 120 \\
Commercial Milk (regionally mixed) & 0.81 & 6 & 44 \\
Goat Milk (locally produced) & 0.17 & 1.2 & 7.9 \\
Beef (locally produced) & 5.3 & 48 & 420 \\
Leafy Vegetables (locally produced) & 0.0039 & 0.077 & 1.8 \\
Eggs (locally produced) & 0.0012 & 0.013 & 0.12 \\
Cottage Cheese (locally produced) & 0.15 & 1.2 & 9 \\
Inhalation & 0.0037 & 0.042 & 0.53 \\
Mother's milk (mother on Diet 1) & 0.055 & 0.22 & 0.88 \\
Prenatal exposure (mother on Diet 1) & 0.001 & 0.031 & 0.85 \\
Diet 1 & 0.0096 & 0.09 & 0.96 \\
Diet 2 & 3.3 & 19 & 130 \\
Diet 3 & 1.2 & 7.9 & 57 \\
\hline
\end{tabular}

Excess Lifetime Risk [ ]

\begin{tabular}{llll}
\hline Diet 1 & $3.3 \mathrm{E}-04$ & $5.2 \mathrm{E}-03$ & $7.5 \mathrm{E}-02$ \\
Diet 2 & $1.7 \mathrm{E}-04$ & $1.9 \mathrm{E}-03$ & $2.6 \mathrm{E}-02$ \\
Diet 3 & $3.4 \mathrm{E}-05$ & $3.5 \mathrm{E}-04$ & $4.2 \mathrm{E}-03$ \\
Diet 4 & $8.1 \mathrm{E}-04$ & $1.3 \mathrm{E}-02$ & $2.0 \mathrm{E}-01$ \\
\hline
\end{tabular}

\section{Relative Risk [ ]}

\begin{tabular}{lccc}
\hline Diet 1 & 1.12 & 2.3 & 15 \\
Diet 2 & 1.047 & 1.51 & 7.5 \\
Diet 3 & 1.01 & 1.089 & 2.1 \\
Diet 4 & 1.28 & 4 & 53 \\
\hline
\end{tabular}

\section{Probability of Causation [\%]}

\begin{tabular}{lccc}
\hline Diet 1 & 10.93 & 56.9 & 93 \\
Diet 2 & 4.47 & 33.5 & 86 \\
Diet 3 & 1.01 & 8.2 & 53 \\
Diet 4 & 21.71 & 74.5 & 98 \\
\hline
\end{tabular}

Diet 1 - Backyard cow milk + all other locally produced non-milk exposure pathways

Diet 2 - Locally produced commercial milk + all other locally produced non-milk exposure pathways

Diet 3 - Regionally mixed commercial milk + inhalation (other regionally mixed food items are minor contributors to the total)

Diet 4 - Goat milk (all other exposure pathways are of negligible importance compared to goat milk) 
Location: Buttermilk Rd.

Receptor: Male born in 1950

\begin{tabular}{lccc}
\hline & \multicolumn{3}{c}{ Thyroid Dose [cGy] } \\
& \multicolumn{2}{c}{$95 \%$ Subjective Confidence Interval } \\
Exposure Pathway & lower limit & central estimate & upper limit \\
\hline Backyard Cow Milk & 3.3 & 18 & 110 \\
Commercial Milk (locally produced) & 0.82 & 5.9 & 46 \\
Commercial Milk (regionally mixed) & 0.17 & 1.1 & 8.9 \\
Goat Milk (locally produced) & 5.1 & 48 & 370 \\
Beef (locally produced) & 0.0043 & 0.074 & 1.8 \\
Leafy Vegetables (locally produced) & 0.0011 & 0.012 & 0.11 \\
Eggs (locally produced) & 0.16 & 1.1 & 8.9 \\
Cottage Cheese (locally produced) & 0.0037 & 0.043 & 0.47 \\
Inhalation & 0.053 & 0.22 & 0.84 \\
Mother's milk (mother on Diet 1) & 0.001 & 0.031 & 0.85 \\
Prenatal exposure (mother on Diet 1) & 0.0096 & 0.09 & 0.96 \\
Diet 1 & 3.6 & 20 & 120 \\
Diet 2 & 1.2 & 7.7 & 56 \\
Diet 3 & 0.27 & 1.4 & 9.6 \\
\hline
\end{tabular}

Excess Lifetime Risk [ ]

\begin{tabular}{lllc}
\hline Diet 1 & $3.5 \mathrm{E}-05$ & $1.4 \mathrm{E}-03$ & $2.6 \mathrm{E}-02$ \\
Diet 2 & $1.9 \mathrm{E}-05$ & $5.5 \mathrm{E}-04$ & $1.2 \mathrm{E}-02$ \\
Diet 3 & $4.1 \mathrm{E}-06$ & $9.4 \mathrm{E}-05$ & $2.0 \mathrm{E}-03$ \\
Diet 4 & $9.7 \mathrm{E}-05$ & $3.3 \mathrm{E}-03$ & $7.6 \mathrm{E}-02$ \\
\hline
\end{tabular}

\section{Relative Risk [ ]}

\begin{tabular}{lccc}
\hline Diet 1 & 1.051 & 1.89 & 20 \\
Diet 2 & 1.018 & 1.35 & 9.3 \\
Diet 3 & 1.0035 & 1.062 & 2.5 \\
Diet 4 & 1.11 & 3.2 & 51 \\
\hline
\end{tabular}

\section{Probability of Causation [\%]}

\begin{tabular}{lccc}
\hline Diet 1 & 4.89 & 46.7 & 95 \\
Diet 2 & 1.73 & 26.1 & 89 \\
Diet 3 & 0.35 & 5.9 & 60 \\
Diet 4 & 10.21 & 68.8 & 98 \\
\hline
\end{tabular}

Diet 1 - Backyard cow milk + all other locally produced non-milk exposure pathways

Diet 2 - Locally produced commercial milk + all other locally produced non-milk exposure pathways

Diet 3 - Regionally mixed commercial milk + inhalation (other regionally mixed food items are minor contributors to the total)

Diet 4 - Goat milk (all other exposure pathways are of negligible importance compared to goat milk) 


\section{Location: Jonesville}

Receptor: Female born in 1950

\begin{tabular}{lccc}
\hline & \multicolumn{3}{c}{ Thyroid Dose [cGy] } \\
Exposure Pathway & \multicolumn{2}{c}{$95 \%$ Subjective Confidence Interval } \\
\hline Backyard Cow Milk & lower limit & central estimate & upper limit \\
Commercial Milk (locally produced) & 0.43 & 2.5 & 20 \\
Commercial Milk (regionally mixed) & 0.1 & 0.86 & 7.3 \\
Goat Milk (locally produced) & 0.17 & 1.2 & 7.9 \\
Beef (locally produced) & 0.75 & 6.6 & 68 \\
Leafy Vegetables (locally produced) & 0.00055 & 0.011 & 0.25 \\
Eggs (locally produced) & 0.00015 & 0.0018 & 0.019 \\
Cottage Cheese (locally produced) & 0.02 & 0.17 & 1.4 \\
Inhalation & 0.00051 & 0.0064 & 0.096 \\
Mother's milk (mother on Diet 1) & 0.008 & 0.032 & 0.14 \\
Prenatal exposure (mother on Diet 1) & 0.00017 & 0.0045 & 0.13 \\
Diet 1 & 0.0014 & 0.013 & 0.15 \\
Diet 2 & 0.48 & 2.7 & 21 \\
Diet 3 & 0.17 & 1.1 & 8.8 \\
\hline
\end{tabular}

Excess Lifetime Risk [ ]

\begin{tabular}{llll}
\hline Diet 1 & $4.4 \mathrm{E}-05$ & $7.5 \mathrm{E}-04$ & $1.2 \mathrm{E}-02$ \\
Diet 2 & $2.4 \mathrm{E}-05$ & $2.8 \mathrm{E}-04$ & $4.2 \mathrm{E}-03$ \\
Diet 3 & $2.7 \mathrm{E}-05$ & $3.0 \mathrm{E}-04$ & $4.1 \mathrm{E}-03$ \\
Diet 4 & $1.1 \mathrm{E}-04$ & $1.8 \mathrm{E}-03$ & $3.3 \mathrm{E}-02$ \\
\hline
\end{tabular}

\section{Relative Risk [ ]}

\begin{tabular}{lccc}
\hline Diet 1 & 1.017 & 1.19 & 3.4 \\
Diet 2 & 1.0064 & 1.071 & 2 \\
Diet 3 & 1.0078 & 1.079 & 2.1 \\
Diet 4 & 1.038 & 1.42 & 9.2 \\
\hline
\end{tabular}

\section{Probability of Causation [\%]}

\begin{tabular}{lccc}
\hline Diet 1 & 1.63 & 15.9 & 71 \\
Diet 2 & 0.63 & 6.6 & 50 \\
Diet 3 & 0.77 & 7.3 & 52 \\
Diet 4 & 3.64 & 29.5 & 89 \\
\hline
\end{tabular}

Diet 1 - Backyard cow milk + all other locally produced non-milk exposure pathways

Diet 2 - Locally produced commercial milk + all other locally produced non-milk exposure pathways

Diet 3 - Regionally mixed commercial milk + inhalation (other regionally mixed food items are minor contributors to the total)

Diet 4 - Goat milk (all other exposure pathways are of negligible importance compared to goat milk) 


\section{Location: Jonesville}

\section{Receptor: Male born in 1950}

\begin{tabular}{lccc}
\hline & \multicolumn{3}{c}{ Thyroid Dose [cGy] } \\
& \multicolumn{2}{c}{$95 \%$ Subjective Confidence Interval } \\
Exposure Pathway & lower limit & central estimate & upper limit \\
\hline Backyard Cow Milk & 0.44 & 2.6 & 19 \\
Commercial Milk (locally produced) & 0.11 & 0.82 & 6.7 \\
Commercial Milk (regionally mixed) & 0.17 & 1.1 & 8.9 \\
Goat Milk (locally produced) & 0.76 & 7 & 61 \\
Beef (locally produced) & 0.00058 & 0.011 & 0.25 \\
Leafy Vegetables (locally produced) & 0.00015 & 0.0018 & 0.018 \\
Eggs (locally produced) & 0.023 & 0.17 & 1.2 \\
Cottage Cheese (locally produced) & 0.00046 & 0.0061 & 0.078 \\
Inhalation & 0.0083 & 0.032 & 0.14 \\
Mother's milk (mother on Diet 1) & 0.00017 & 0.0045 & 0.13 \\
Prenatal exposure (mother on Diet 1) & 0.0014 & 0.013 & 0.15 \\
Diet 1 & 0.49 & 2.8 & 21 \\
Diet 2 & 0.17 & 1.1 & 8.4 \\
Diet 3 & 0.19 & 1.2 & 9 \\
\hline
\end{tabular}

Excess Lifetime Risk [ ]

\begin{tabular}{llll}
\hline Diet 1 & $5.3 \mathrm{E}-06$ & $1.9 \mathrm{E}-04$ & $4.0 \mathrm{E}-03$ \\
Diet 2 & $2.7 \mathrm{E}-06$ & $7.6 \mathrm{E}-05$ & $1.7 \mathrm{E}-03$ \\
Diet 3 & $3.3 \mathrm{E}-06$ & $8.0 \mathrm{E}-05$ & $1.9 \mathrm{E}-03$ \\
Diet 4 & $1.4 \mathrm{E}-05$ & $4.6 \mathrm{E}-04$ & $1.2 \mathrm{E}-02$ \\
\hline
\end{tabular}

\section{Relative Risk [ ]}

\begin{tabular}{lccc}
\hline Diet 1 & 1.0063 & 1.14 & 3.9 \\
Diet 2 & 1.0023 & 1.051 & 2.4 \\
Diet 3 & 1.0028 & 1.055 & 2.3 \\
Diet 4 & 1.014 & 1.33 & 8.7 \\
\hline
\end{tabular}

\begin{tabular}{lccc} 
& \multicolumn{3}{c}{ Probability of Causation [\%] } \\
\hline Diet 1 & 0.62 & 11.9 & 74 \\
Diet 2 & 0.23 & 4.8 & 57 \\
Diet 3 & 0.28 & 5.2 & 57 \\
Diet 4 & 1.35 & 24.6 & 88 \\
\hline
\end{tabular}

Diet 1 - Backyard cow milk + all other locally produced non-milk exposure pathways

Diet 2 - Locally produced commercial milk + all other locally produced non-milk exposure pathways

Diet 3 - Regionally mixed commercial milk + inhalation (other regionally mixed food items are minor contributors to the total)

Diet 4 - Goat milk (all other exposure pathways are of negligible importance compared to goat milk) 


\section{Location: OR Scarboro}

Receptor: Female born in 1950

\begin{tabular}{lccc}
\hline & \multicolumn{3}{c}{ Thyroid Dose [cGy] } \\
Exposure Pathway & \multicolumn{2}{c}{$9 \%$ Subjective Confidence Interval } \\
\hline Backyard Cow Milk & lower limit & central estimate & upper limit \\
Commercial Milk (locally produced) & -- & -- & -- \\
Commercial Milk (regionally mixed) & -- & -- & -- \\
Goat Milk (locally produced) & 0.17 & 1.2 & 7.9 \\
Beef (locally produced) & -- & -- & -- \\
Leafy Vegetables (locally produced) & -- & -- & -- \\
Eggs (locally produced) & -- & -- & -- \\
Cottage Cheese (locally produced) & -- & -- & -- \\
Inhalation & 0.0028 & 0.032 & 0.38 \\
Mother's milk (mother on Diet 3) & 0.039 & 0.16 & 0.66 \\
Prenatal exposure (mother on Diet 3) & 0.00013 & 0.0023 & 0.027 \\
Diet 1 & 0.0011 & 0.0065 & 0.051 \\
Diet 2 & -- & -- & -- \\
Diet 3 & -- & -- & -- \\
\hline
\end{tabular}

Excess Lifetime Risk [ ]

\begin{tabular}{lccc}
\hline Diet 1 & -- & -- & -- \\
Diet 2 & -- & -- & -- \\
Diet 3 & $3.2 \mathrm{E}-05$ & $3.3 \mathrm{E}-04$ & $4.2 \mathrm{E}-03$ \\
Diet 4 & -- & -- & -- \\
\hline
\end{tabular}

\section{Relative Risk [ ]}

\begin{tabular}{lccc}
\hline Diet 1 & -- & -- & -- \\
Diet 2 & -- & -- & -- \\
Diet 3 & 1.0097 & 1.086 & 2.1 \\
Diet 4 & -- & -- & -- \\
\hline
\end{tabular}

\begin{tabular}{lccc}
\hline Diet 1 & -- & -- & -- \\
Diet 2 & -- & -- & -- \\
Diet 3 & 0.97 & 7.9 & 53 \\
Diet 4 & -- & -- \\
\hline Diet 1 - Backyard cow milk + all other locally produced non-milk exposure pathways & \\
Diet 2 - Locally produced commercial milk + all other locally produced non-milk exposure pathways \\
Diet 3 - Regionally mixed commercial milk + inhalation (other regionally mixed food items are minor contributors to the total) \\
Diet 4 - Goat milk (all other exposure pathways are of negligible importance compared to goat milk)
\end{tabular}




\section{Location: OR Scarboro}

Receptor: Male born in 1950

\begin{tabular}{lccc}
\hline & \multicolumn{3}{c}{ Thyroid Dose [cGy] } \\
Exposure Pathway & \multicolumn{2}{c}{$95 \%$ Subjective Confidence Interval } \\
\hline Backyard Cow Milk & lower limit & central estimate & upper limit \\
Commercial Milk (locally produced) & -- & -- & -- \\
Commercial Milk (regionally mixed) & -- & -- & -- \\
Goat Milk (locally produced) & 0.17 & 1.1 & 8.9 \\
Beef (locally produced) & -- & -- & -- \\
Leafy Vegetables (locally produced) & -- & -- & -- \\
Eggs (locally produced) & -- & -- & -- \\
Cottage Cheese (locally produced) & -- & -- & -- \\
Inhalation & 0.0026 & 0.033 & 0.34 \\
Mother's milk (mother on Diet 3) & 0.039 & 0.17 & 0.62 \\
Prenatal exposure (mother on Diet 3) & 0.00013 & 0.0023 & 0.027 \\
Diet 1 & 0.0011 & 0.0065 & 0.051 \\
Diet 2 & -- & -- & -- \\
Diet 3 & -- & -- & -- \\
\hline
\end{tabular}

Excess Lifetime Risk [ ]

\begin{tabular}{lccc}
\hline Diet 1 & -- & -- & -- \\
Diet 2 & -- & -- & -- \\
Diet 3 & $4.0 \mathrm{E}-06$ & $8.9 \mathrm{E}-05$ & $2.0 \mathrm{E}-03$ \\
Diet 4 & -- & -- & -- \\
\hline
\end{tabular}

\section{Relative Risk [ ]}

\begin{tabular}{lccc}
\hline Diet 1 & -- & -- & -- \\
Diet 2 & -- & -- & -- \\
Diet 3 & 1.0032 & 1.06 & 2.4 \\
Diet 4 & -- & -- & -- \\
\hline
\end{tabular}

\begin{tabular}{lccc}
\hline Diet 1 & -- & -- & -- \\
Diet 2 & -- & -- & 59 \\
Diet 3 & 0.32 & 5.7 & -- \\
Diet 4 & -- & -- & \\
\hline Diet 1 - Backyard cow milk + all other locally produced non-milk exposure pathways & \\
Diet 2 - Locally produced commercial milk + all other locally produced non-milk exposure pathways \\
Diet 3 - Regionally mixed commercial milk + inhalation (other regionally mixed food items are minor contributors to the total) \\
Diet 4 - Goat milk (all other exposure pathways are of negligible importance compared to goat milk)
\end{tabular}


Location: Lawnville/Gallaher Receptor: Female born in 1950

\begin{tabular}{lccc}
\hline & \multicolumn{3}{c}{ Thyroid Dose [cGy] } \\
Exposure Pathway & $95 \%$ Subjective Confidence Interval \\
\hline Backyard Cow Milk & lower limit & central estimate & upper limit \\
Commercial Milk (locally produced) & 2.9 & 17 & 100 \\
Commercial Milk (regionally mixed) & 0.82 & 5.5 & 45 \\
Goat Milk (locally produced) & 0.17 & 1.2 & 7.9 \\
Beef (locally produced) & 5.4 & 45 & 380 \\
Leafy Vegetables (locally produced) & 0.0034 & 0.076 & 1.8 \\
Eggs (locally produced) & 0.0011 & 0.012 & 0.11 \\
Cottage Cheese (locally produced) & 0.14 & 1.1 & 8.2 \\
Inhalation & 0.0036 & 0.042 & 0.52 \\
Mother's milk (mother on Diet 1) & 0.053 & 0.21 & 0.85 \\
Prenatal exposure (mother on Diet 1) & 0.001 & 0.029 & 0.79 \\
Diet 1 & 0.0093 & 0.088 & 0.88 \\
Diet 2 & 3.3 & 19 & 110 \\
Diet 3 & 1.2 & 7.4 & 57 \\
\hline
\end{tabular}

Excess Lifetime Risk [ ]

\begin{tabular}{llll}
\hline Diet 1 & $3.4 \mathrm{E}-04$ & $4.9 \mathrm{E}-03$ & $7.4 \mathrm{E}-02$ \\
Diet 2 & $1.6 \mathrm{E}-04$ & $1.7 \mathrm{E}-03$ & $2.6 \mathrm{E}-02$ \\
Diet 3 & $3.3 \mathrm{E}-05$ & $3.5 \mathrm{E}-04$ & $4.3 \mathrm{E}-03$ \\
Diet 4 & $8.3 \mathrm{E}-04$ & $1.3 \mathrm{E}-02$ & $2.0 \mathrm{E}-01$ \\
\hline
\end{tabular}

\section{Relative Risk [ ]}

\begin{tabular}{lccc}
\hline Diet 1 & 1.13 & 2.3 & 14 \\
Diet 2 & 1.043 & 1.49 & 7.4 \\
Diet 3 & 1.01 & 1.089 & 2.1 \\
Diet 4 & 1.3 & 3.8 & 46 \\
\hline
\end{tabular}

\section{Probability of Causation [\%]}

\begin{tabular}{lccc}
\hline Diet 1 & 11.55 & 56.3 & 93 \\
Diet 2 & 4.16 & 32.7 & 87 \\
Diet 3 & 1.02 & 8.1 & 53 \\
Diet 4 & 22.86 & 73.7 & 98 \\
\hline
\end{tabular}

Diet 1 - Backyard cow milk + all other locally produced non-milk exposure pathways

Diet 2 - Locally produced commercial milk + all other locally produced non-milk exposure pathways

Diet 3 - Regionally mixed commercial milk + inhalation (other regionally mixed food items are minor contributors to the total)

Diet 4 - Goat milk (all other exposure pathways are of negligible importance compared to goat milk) 


\section{Location: Lawnville/Gallaher}

Receptor: Male born in 1950

\begin{tabular}{|c|c|c|c|}
\hline \multirow[b]{2}{*}{ Exposure Pathway } & \multicolumn{3}{|c|}{$\begin{array}{c}\text { Thyroid Dose [cGy] } \\
\text { 95\% Subjective Confidence Interval }\end{array}$} \\
\hline & lower limit & central estimate & upper limit \\
\hline Backyard Cow Milk & 3.1 & 17 & 96 \\
\hline Commercial Milk (locally produced) & 0.82 & 5.4 & 46 \\
\hline Commercial Milk (regionally mixed) & 0.17 & 1.1 & 8.9 \\
\hline Goat Milk (locally produced) & 5.1 & 47 & 350 \\
\hline Beef (locally produced) & 0.0037 & 0.073 & 1.7 \\
\hline Leafy Vegetables (locally produced) & 0.0012 & 0.012 & 0.11 \\
\hline Eggs (locally produced) & 0.16 & 1.1 & 8.2 \\
\hline Cottage Cheese (locally produced) & 0.0033 & 0.042 & 0.42 \\
\hline Inhalation & 0.054 & 0.21 & 0.86 \\
\hline Mother's milk (mother on Diet 1) & 0.001 & 0.029 & 0.79 \\
\hline Prenatal exposure (mother on Diet 1) & 0.0093 & 0.088 & 0.88 \\
\hline Diet 1 & 3.4 & 19 & 100 \\
\hline Diet 2 & 1.2 & 7.3 & 54 \\
\hline Diet 3 & 0.27 & 1.4 & 9.6 \\
\hline
\end{tabular}

Excess Lifetime Risk [ ]

\begin{tabular}{lllc}
\hline Diet 1 & $3.7 \mathrm{E}-05$ & $1.4 \mathrm{E}-03$ & $2.2 \mathrm{E}-02$ \\
Diet 2 & $1.8 \mathrm{E}-05$ & $4.9 \mathrm{E}-04$ & $1.1 \mathrm{E}-02$ \\
Diet 3 & $4.2 \mathrm{E}-06$ & $9.4 \mathrm{E}-05$ & $2.0 \mathrm{E}-03$ \\
Diet 4 & $8.7 \mathrm{E}-05$ & $3.0 \mathrm{E}-03$ & $6.3 \mathrm{E}-02$ \\
\hline
\end{tabular}

\section{Relative Risk [ ]}

\begin{tabular}{lccc}
\hline Diet 1 & 1.047 & 1.84 & 20 \\
Diet 2 & 1.018 & 1.33 & 9.3 \\
Diet 3 & 1.0036 & 1.061 & 2.5 \\
Diet 4 & 1.1 & 3.2 & 45 \\
\hline
\end{tabular}

\begin{tabular}{lccc}
\hline Diet 1 & 4.52 & 45.5 & 95 \\
Diet 2 & 1.74 & 24.8 & 89 \\
Diet 3 & 0.36 & 5.8 & 60 \\
Diet 4 & 9.43 & 68.6 & 98 \\
\hline
\end{tabular}

Diet 1 - Backyard cow milk + all other locally produced non-milk exposure pathways

Diet 2 - Locally produced commercial milk + all other locally produced non-milk exposure pathways

Diet 3 - Regionally mixed commercial milk + inhalation (other regionally mixed food items are minor contributors to the total)

Diet 4 - Goat milk (all other exposure pathways are of negligible importance compared to goat milk) 


\section{Location: Dyllis}

Receptor: Female born in 1950

\begin{tabular}{lccc}
\hline & \multicolumn{3}{c}{ Thyroid Dose [cGy] } \\
& \multicolumn{2}{c}{$95 \%$ Subjective Confidence Interval } \\
Exposure Pathway & lower limit & central estimate & upper limit \\
\hline Backyard Cow Milk & 0.56 & 3.4 & 25 \\
Commercial Milk (locally produced) & 0.15 & 1.1 & 9.8 \\
Commercial Milk (regionally mixed) & 0.17 & 1.2 & 7.9 \\
Goat Milk (locally produced) & 1.2 & 9.1 & 87 \\
Beef (locally produced) & 0.00079 & 0.015 & 0.33 \\
Leafy Vegetables (locally produced) & 0.00021 & 0.0025 & 0.025 \\
Eggs (locally produced) & 0.026 & 0.23 & 1.9 \\
Cottage Cheese (locally produced) & 0.00076 & 0.0085 & 0.12 \\
Inhalation & 0.01 & 0.044 & 0.19 \\
Mother's milk (mother on Diet 1) & 0.00019 & 0.0061 & 0.17 \\
Prenatal exposure (mother on Diet 1) & 0.0019 & 0.018 & 0.23 \\
Diet 1 & 0.62 & 3.7 & 28 \\
Diet 2 & 0.23 & 1.5 & 12 \\
Diet 3 & 0.2 & 1.2 & 8.1 \\
\hline
\end{tabular}

Excess Lifetime Risk [ ]

\begin{tabular}{llll}
\hline Diet 1 & $6.6 \mathrm{E}-05$ & $1.0 \mathrm{E}-03$ & $1.7 \mathrm{E}-02$ \\
Diet 2 & $2.6 \mathrm{E}-05$ & $3.9 \mathrm{E}-04$ & $5.3 \mathrm{E}-03$ \\
Diet 3 & $2.7 \mathrm{E}-05$ & $3.1 \mathrm{E}-04$ & $4.1 \mathrm{E}-03$ \\
Diet 4 & $1.5 \mathrm{E}-04$ & $2.6 \mathrm{E}-03$ & $4.1 \mathrm{E}-02$ \\
\hline
\end{tabular}

\section{Relative Risk [ ]}

\begin{tabular}{lccc}
\hline Diet 1 & 1.026 & 1.26 & 3.9 \\
Diet 2 & 1.0099 & 1.096 & 2.5 \\
Diet 3 & 1.0081 & 1.08 & 2.1 \\
Diet 4 & 1.053 & 1.57 & 12 \\
\hline
\end{tabular}

\begin{tabular}{lccc} 
& \multicolumn{3}{c}{ Probability of Causation [\%] } \\
\hline Diet 1 & 2.51 & 20.4 & 74 \\
Diet 2 & 0.98 & 8.8 & 59 \\
Diet 3 & 0.81 & 7.4 & 52 \\
Diet 4 & 5.01 & 36.1 & 91 \\
\hline
\end{tabular}

Diet 1 - Backyard cow milk + all other locally produced non-milk exposure pathways

Diet 2 - Locally produced commercial milk + all other locally produced non-milk exposure pathways

Diet 3 - Regionally mixed commercial milk + inhalation (other regionally mixed food items are minor contributors to the total)

Diet 4 - Goat milk (all other exposure pathways are of negligible importance compared to goat milk) 


\section{Location: Dyllis}

\section{Receptor: Male born in 1950}

\begin{tabular}{lccc}
\hline & \multicolumn{3}{c}{ Thyroid Dose [cGy] } \\
& \multicolumn{2}{c}{$95 \%$ Subjective Confidence Interval } \\
Exposure Pathway & lower limit & central estimate & upper limit \\
\hline Backyard Cow Milk & 0.55 & 3.5 & 24 \\
Commercial Milk (locally produced) & 0.16 & 1.1 & 9.6 \\
Commercial Milk (regionally mixed) & 0.17 & 1.1 & 8.9 \\
Goat Milk (locally produced) & 1.1 & 9.7 & 88 \\
Beef (locally produced) & 0.00084 & 0.014 & 0.32 \\
Leafy Vegetables (locally produced) & 0.00022 & 0.0025 & 0.024 \\
Eggs (locally produced) & 0.031 & 0.23 & 1.8 \\
Cottage Cheese (locally produced) & 0.00074 & 0.0086 & 0.1 \\
Inhalation & 0.011 & 0.045 & 0.2 \\
Mother's milk (mother on Diet 1) & 0.00019 & 0.0061 & 0.17 \\
Prenatal exposure (mother on Diet 1) & 0.0019 & 0.018 & 0.23 \\
Diet 1 & 0.61 & 3.9 & 26 \\
Diet 2 & 0.24 & 1.5 & 12 \\
Diet 3 & 0.2 & 1.2 & 9.1 \\
\hline
\end{tabular}

Excess Lifetime Risk [ ]

\begin{tabular}{llll}
\hline Diet 1 & $7.0 \mathrm{E}-06$ & $2.7 \mathrm{E}-04$ & $5.0 \mathrm{E}-03$ \\
Diet 2 & $3.8 \mathrm{E}-06$ & $1.0 \mathrm{E}-04$ & $2.3 \mathrm{E}-03$ \\
Diet 3 & $3.5 \mathrm{E}-06$ & $8.1 \mathrm{E}-05$ & $1.9 \mathrm{E}-03$ \\
Diet 4 & $1.9 \mathrm{E}-05$ & $6.4 \mathrm{E}-04$ & $1.5 \mathrm{E}-02$ \\
\hline
\end{tabular}

\section{Relative Risk [ ]}

\begin{tabular}{lccc}
\hline Diet 1 & 1.0083 & 1.18 & 5.1 \\
Diet 2 & 1.003 & 1.069 & 2.8 \\
Diet 3 & 1.0028 & 1.056 & 2.3 \\
Diet 4 & 1.018 & 1.46 & 10.1 \\
\hline
\end{tabular}

\section{Probability of Causation [\%]}

\begin{tabular}{lccc}
\hline Diet 1 & 0.82 & 15.0 & 80 \\
Diet 2 & 0.30 & 6.4 & 64 \\
Diet 3 & 0.28 & 5.3 & 57 \\
Diet 4 & 1.80 & 31.5 & 90 \\
\hline
\end{tabular}

Diet 1 - Backyard cow milk + all other locally produced non-milk exposure pathways

Diet 2 - Locally produced commercial milk + all other locally produced non-milk exposure pathways

Diet 3 - Regionally mixed commercial milk + inhalation (other regionally mixed food items are minor contributors to the total)

Diet 4 - Goat milk (all other exposure pathways are of negligible importance compared to goat milk) 


\section{Location: OR High School Area}

Receptor: Female born in 1950

\begin{tabular}{lccc}
\hline & \multicolumn{3}{c}{ Thyroid Dose [cGy] } \\
Exposure Pathway & \multicolumn{2}{c}{$9 \%$ Subjective Confidence Interval } \\
\hline Backyard Cow Milk & lower limit & central estimate & upper limit \\
Commercial Milk (locally produced) & -- & -- & -- \\
Commercial Milk (regionally mixed) & -- & -- & -- \\
Goat Milk (locally produced) & 0.17 & 1.2 & 7.9 \\
Beef (locally produced) & -- & -- & -- \\
Leafy Vegetables (locally produced) & -- & -- & -- \\
Eggs (locally produced) & -- & -- & -- \\
Cottage Cheese (locally produced) & -- & -- & -- \\
Inhalation & 0.0016 & 0.019 & 0.21 \\
Mother's milk (mother on Diet 3) & 0.023 & 0.093 & 0.4 \\
Prenatal exposure (mother on Diet 3) & 0.00011 & 0.0019 & 0.024 \\
Diet 1 & 0.00087 & 0.0055 & 0.044 \\
Diet 2 & -- & -- & -- \\
Diet 3 & -- & -- & -- \\
\hline
\end{tabular}

Excess Lifetime Risk [ ]

\begin{tabular}{lccc}
\hline Diet 1 & -- & -- & -- \\
Diet 2 & -- & -- & -- \\
Diet 3 & $2.9 \mathrm{E}-05$ & $3.2 \mathrm{E}-04$ & $4.1 \mathrm{E}-03$ \\
Diet 4 & -- & -- & -- \\
\hline
\end{tabular}

\section{Relative Risk [ ]}

\begin{tabular}{lccc}
\hline Diet 1 & -- & -- & -- \\
Diet 2 & -- & -- & -- \\
Diet 3 & 1.009 & 1.082 & 2.1 \\
Diet 4 & -- & -- & -- \\
\hline
\end{tabular}

\begin{tabular}{lccc}
\hline Diet 1 & -- & -- & -- \\
Diet 2 & -- & -- & -- \\
Diet 3 & 0.89 & 7.6 & 52 \\
Diet 4 & -- & -- \\
\hline Diet 1 - Backyard cow milk + all other locally produced non-milk exposure pathways & \\
Diet 2 - Locally produced commercial milk + all other locally produced non-milk exposure pathways \\
Diet 3 - Regionally mixed commercial milk + inhalation (other regionally mixed food items are minor contributors to the total) \\
Diet 4 - Goat milk (all other exposure pathways are of negligible importance compared to goat milk)
\end{tabular}




\section{Location: OR High School Area}

Receptor: Male born in 1950

\begin{tabular}{lccc}
\hline & \multicolumn{3}{c}{ Thyroid Dose [cGy] } \\
Exposure Pathway & \multicolumn{2}{c}{$95 \%$ Subjective Confidence Interval } \\
\hline Backyard Cow Milk & lower limit & central estimate & upper limit \\
Commercial Milk (locally produced) & -- & -- & -- \\
Commercial Milk (regionally mixed) & -- & -- & -- \\
Goat Milk (locally produced) & 0.17 & 1.1 & 8.9 \\
Beef (locally produced) & -- & -- & -- \\
Leafy Vegetables (locally produced) & -- & -- & -- \\
Eggs (locally produced) & -- & -- & -- \\
Cottage Cheese (locally produced) & -- & -- & -- \\
Inhalation & 0.0015 & 0.019 & 0.19 \\
Mother's milk (mother on Diet 3) & 0.025 & 0.094 & 0.38 \\
Prenatal exposure (mother on Diet 3) & 0.00011 & 0.0019 & 0.024 \\
Diet 1 & 0.00087 & 0.0055 & 0.044 \\
Diet 2 & -- & -- & -- \\
Diet 3 & -- & -- & -- \\
\hline
\end{tabular}

Excess Lifetime Risk [ ]

\begin{tabular}{lccc}
\hline Diet 1 & -- & -- & -- \\
Diet 2 & -- & -- & -- \\
Diet 3 & $3.8 \mathrm{E}-06$ & $8.5 \mathrm{E}-05$ & $1.9 \mathrm{E}-03$ \\
Diet 4 & -- & -- & -- \\
\hline
\end{tabular}

\section{Relative Risk [ ]}

\begin{tabular}{lccc}
\hline Diet 1 & -- & -- & -- \\
Diet 2 & -- & -- & -- \\
Diet 3 & 1.003 & 1.057 & 2.4 \\
Diet 4 & -- & -- & -- \\
\hline
\end{tabular}

\begin{tabular}{lccc}
\hline Diet 1 & -- & -- & -- \\
Diet 2 & -- & -- & -- \\
Diet 3 & 0.29 & 5.4 & 58 \\
Diet 4 & -- & -- & - \\
\hline Diet 1 - Backyard cow milk + all other locally produced non-milk exposure pathways & \\
Diet 2 - Locally produced commercial milk + all other locally produced non-milk exposure pathways \\
Diet 3 - Regionally mixed commercial milk + inhalation (other regionally mixed food items are minor contributors to the total) \\
Diet 4 - Goat milk (all other exposure pathways are of negligible importance compared to goat milk)
\end{tabular}




\section{Location: Norwood}

Receptor: Female born in 1950

\begin{tabular}{lccc}
\hline & \multicolumn{3}{c}{ Thyroid Dose [cGy] } \\
Exposure Pathway & \multicolumn{2}{c}{$95 \%$ Subjective Confidence Interval } \\
\hline Backyard Cow Milk & lower limit & central estimate & upper limit \\
Commercial Milk (locally produced) & 0.51 & 3.3 & 24 \\
Commercial Milk (regionally mixed) & 0.14 & 1.1 & 10 \\
Goat Milk (locally produced) & 0.17 & 1.2 & 7.9 \\
Beef (locally produced) & 0.96 & 8.8 & 84 \\
Leafy Vegetables (locally produced) & 0.00078 & 0.014 & 0.37 \\
Eggs (locally produced) & 0.00021 & 0.0022 & 0.022 \\
Cottage Cheese (locally produced) & 0.025 & 0.21 & 1.9 \\
Inhalation & 0.00067 & 0.0081 & 0.11 \\
Mother's milk (mother on Diet 1) & 0.011 & 0.042 & 0.18 \\
Prenatal exposure (mother on Diet 1) & 0.00022 & 0.0058 & 0.16 \\
Diet 1 & 0.0019 & 0.017 & 0.2 \\
Diet 2 & 0.57 & 3.7 & 27 \\
Diet 3 & 0.22 & 1.4 & 12 \\
\hline
\end{tabular}

\section{Excess Lifetime Risk [ ]}

\begin{tabular}{llll}
\hline Diet 1 & $5.9 \mathrm{E}-05$ & $1.0 \mathrm{E}-03$ & $1.5 \mathrm{E}-02$ \\
Diet 2 & $2.8 \mathrm{E}-05$ & $3.6 \mathrm{E}-04$ & $5.5 \mathrm{E}-03$ \\
Diet 3 & $2.7 \mathrm{E}-05$ & $3.1 \mathrm{E}-04$ & $4.1 \mathrm{E}-03$ \\
Diet 4 & $1.4 \mathrm{E}-04$ & $2.4 \mathrm{E}-03$ & $4.2 \mathrm{E}-02$ \\
\hline
\end{tabular}

\section{Relative Risk [ ]}

\begin{tabular}{lccc}
\hline Diet 1 & 1.021 & 1.24 & 3.8 \\
Diet 2 & 1.0084 & 1.091 & 2.4 \\
Diet 3 & 1.008 & 1.08 & 2.1 \\
Diet 4 & 1.054 & 1.55 & 11 \\
\hline
\end{tabular}

\section{Probability of Causation [\%]}

\begin{tabular}{lccc}
\hline Diet 1 & 2.03 & 19.4 & 74 \\
Diet 2 & 0.83 & 8.3 & 58 \\
Diet 3 & 0.79 & 7.4 & 52 \\
Diet 4 & 5.08 & 35.3 & 91 \\
\hline
\end{tabular}

Diet 1 - Backyard cow milk + all other locally produced non-milk exposure pathways

Diet 2 - Locally produced commercial milk + all other locally produced non-milk exposure pathways

Diet 3 - Regionally mixed commercial milk + inhalation (other regionally mixed food items are minor contributors to the total)

Diet 4 - Goat milk (all other exposure pathways are of negligible importance compared to goat milk) 


\section{Location: Norwood}

\section{Receptor: Male born in 1950}

\begin{tabular}{lccc}
\hline & \multicolumn{3}{c}{ Thyroid Dose [cGy] } \\
& \multicolumn{2}{c}{$95 \%$ Subjective Confidence Interval } \\
Exposure Pathway & lower limit & central estimate & upper limit \\
\hline Backyard Cow Milk & 0.55 & 3.2 & 24 \\
Commercial Milk (locally produced) & 0.15 & 1.1 & 9.7 \\
Commercial Milk (regionally mixed) & 0.17 & 1.1 & 8.9 \\
Goat Milk (locally produced) & 0.94 & 9.2 & 92 \\
Beef (locally produced) & 0.00083 & 0.014 & 0.34 \\
Leafy Vegetables (locally produced) & 0.00021 & 0.0023 & 0.024 \\
Eggs (locally produced) & 0.028 & 0.21 & 1.9 \\
Cottage Cheese (locally produced) & 0.00065 & 0.0081 & 0.11 \\
Inhalation & 0.0091 & 0.042 & 0.17 \\
Mother's milk (mother on Diet 1) & 0.00022 & 0.0058 & 0.16 \\
Prenatal exposure (mother on Diet 1) & 0.0019 & 0.017 & 0.2 \\
Diet 1 & 0.6 & 3.6 & 26 \\
Diet 2 & 0.22 & 1.4 & 12 \\
Diet 3 & 0.2 & 1.2 & 9 \\
\hline
\end{tabular}

Excess Lifetime Risk [ ]

\begin{tabular}{llll}
\hline Diet 1 & $6.8 \mathrm{E}-06$ & $2.5 \mathrm{E}-04$ & $4.7 \mathrm{E}-03$ \\
Diet 2 & $3.4 \mathrm{E}-06$ & $9.6 \mathrm{E}-05$ & $2.0 \mathrm{E}-03$ \\
Diet 3 & $3.5 \mathrm{E}-06$ & $8.1 \mathrm{E}-05$ & $1.9 \mathrm{E}-03$ \\
Diet 4 & $1.7 \mathrm{E}-05$ & $6.0 \mathrm{E}-04$ & $1.3 \mathrm{E}-02$ \\
\hline
\end{tabular}

\section{Relative Risk [ ]}

\begin{tabular}{lccc}
\hline Diet 1 & 1.0089 & 1.17 & 4.9 \\
Diet 2 & 1.0034 & 1.062 & 2.8 \\
Diet 3 & 1.0028 & 1.055 & 2.3 \\
Diet 4 & 1.019 & 1.42 & 12 \\
\hline
\end{tabular}

\section{Probability of Causation [\%]}

\begin{tabular}{lccc}
\hline Diet 1 & 0.88 & 14.4 & 80 \\
Diet 2 & 0.34 & 5.9 & 64 \\
Diet 3 & 0.28 & 5.2 & 57 \\
Diet 4 & 1.91 & 29.3 & 92 \\
\hline
\end{tabular}

Diet 1 - Backyard cow milk + all other locally produced non-milk exposure pathways

Diet 2 - Locally produced commercial milk + all other locally produced non-milk exposure pathways

Diet 3 - Regionally mixed commercial milk + inhalation (other regionally mixed food items are minor contributors to the total)

Diet 4 - Goat milk (all other exposure pathways are of negligible importance compared to goat milk) 
Location: Woodland

Receptor: Female born in 1950

\begin{tabular}{lccc}
\hline & \multicolumn{3}{c}{ Thyroid Dose [cGy] } \\
Exposure Pathway & \multicolumn{2}{c}{$95 \%$ Subjective Confidence Interval } \\
\hline Backyard Cow Milk & lower limit & central estimate & upper limit \\
Commercial Milk (locally produced) & -- & -- & -- \\
Commercial Milk (regionally mixed) & -- & -- & -- \\
Goat Milk (locally produced) & 0.17 & 1.2 & 7.9 \\
Beef (locally produced) & -- & -- & -- \\
Leafy Vegetables (locally produced) & -- & -- & -- \\
Eggs (locally produced) & -- & -- & -- \\
Cottage Cheese (locally produced) & -- & -- & -- \\
Inhalation & 0.0024 & 0.027 & 0.33 \\
Mother's milk (mother on Diet 3) & 0.036 & 0.14 & 0.58 \\
Prenatal exposure (mother on Diet 3) & 0.00012 & 0.0022 & 0.026 \\
Diet 1 & 0.0011 & 0.0064 & 0.047 \\
Diet 2 & -- & -- & -- \\
Diet 3 & -- & -- & -- \\
\hline
\end{tabular}

Excess Lifetime Risk [ ]

\begin{tabular}{lccc}
\hline Diet 1 & -- & -- & -- \\
Diet 2 & -- & -- & -- \\
Diet 3 & $3.1 \mathrm{E}-05$ & $3.3 \mathrm{E}-04$ & $4.2 \mathrm{E}-03$ \\
Diet 4 & -- & -- & -- \\
\hline
\end{tabular}

\section{Relative Risk [ ]}

\begin{tabular}{lccc}
\hline Diet 1 & -- & -- & -- \\
Diet 2 & -- & -- & -- \\
Diet 3 & 1.0095 & 1.085 & 2.1 \\
Diet 4 & -- & -- & -- \\
\hline
\end{tabular}

\begin{tabular}{lccc}
\hline Diet 1 & -- & -- & -- \\
Diet 2 & -- & -- & -- \\
Diet 3 & 0.95 & 7.8 & 52 \\
Diet 4 & -- & -- \\
\hline Diet 1 - Backyard cow milk + all other locally produced non-milk exposure pathways & \\
Diet 2 - Locally produced commercial milk + all other locally produced non-milk exposure pathways \\
Diet 3 - Regionally mixed commercial milk + inhalation (other regionally mixed food items are minor contributors to the total) \\
Diet 4 - Goat milk (all other exposure pathways are of negligible importance compared to goat milk)
\end{tabular}


Location: Woodland

Receptor: Male born in 1950

\begin{tabular}{lccc}
\hline & \multicolumn{3}{c}{ Thyroid Dose [cGy] } \\
Exposure Pathway & \multicolumn{2}{c}{$9 \%$ Subjective Confidence Interval } \\
\hline Backyard Cow Milk & lower limit & central estimate & upper limit \\
Commercial Milk (locally produced) & -- & -- & -- \\
Commercial Milk (regionally mixed) & -- & -- & -- \\
Goat Milk (locally produced) & 0.17 & 1.1 & 8.9 \\
Beef (locally produced) & -- & -- & -- \\
Leafy Vegetables (locally produced) & -- & -- & -- \\
Eggs (locally produced) & -- & -- & -- \\
Cottage Cheese (locally produced) & -- & -- & -- \\
Inhalation & 0.0022 & 0.028 & 0.28 \\
Mother's milk (mother on Diet 3) & 0.036 & 0.14 & 0.57 \\
Prenatal exposure (mother on Diet 3) & 0.00012 & 0.0022 & 0.026 \\
Diet 1 & 0.0011 & 0.0064 & 0.047 \\
Diet 2 & -- & -- & -- \\
Diet 3 & -- & -- & -- \\
\hline
\end{tabular}

Excess Lifetime Risk [ ]

\begin{tabular}{lccc}
\hline Diet 1 & -- & -- & -- \\
Diet 2 & -- & -- & -- \\
Diet 3 & $4.0 \mathrm{E}-06$ & $8.8 \mathrm{E}-05$ & $2.0 \mathrm{E}-03$ \\
Diet 4 & -- & -- & -- \\
\hline
\end{tabular}

\section{Relative Risk [ ]}

\begin{tabular}{lccc}
\hline Diet 1 & -- & -- & -- \\
Diet 2 & -- & -- & -- \\
Diet 3 & 1.0032 & 1.059 & 2.4 \\
Diet 4 & -- & -- & -- \\
\hline
\end{tabular}

\begin{tabular}{lccc}
\hline Diet 1 & -- & -- & -- \\
Diet 2 & -- & -- & -- \\
Diet 3 & 0.32 & 5.6 & 59 \\
Diet 4 & -- & -- \\
\hline Diet 1 - Backyard cow milk + all other locally produced non-milk exposure pathways & \\
Diet 2 - Locally produced commercial milk + all other locally produced non-milk exposure pathways \\
Diet 3 - Regionally mixed commercial milk + inhalation (other regionally mixed food items are minor contributors to the total) \\
Diet 4 - Goat milk (all other exposure pathways are of negligible importance compared to goat milk)
\end{tabular}


Location: Hardin Valley

Receptor: Female born in 1950

\begin{tabular}{lccc}
\hline & \multicolumn{3}{c}{ Thyroid Dose [cGy] } \\
& \multicolumn{2}{c}{$95 \%$ Subjective Confidence Interval } \\
Exposure Pathway & lower limit & central estimate & upper limit \\
\hline Backyard Cow Milk & 2.7 & 15 & 100 \\
Commercial Milk (locally produced) & 0.75 & 5.5 & 40 \\
Commercial Milk (regionally mixed) & 0.17 & 1.2 & 7.9 \\
Goat Milk (locally produced) & 5.4 & 43 & 380 \\
Beef (locally produced) & 0.0037 & 0.068 & 1.5 \\
Leafy Vegetables (locally produced) & 0.0011 & 0.012 & 0.11 \\
Eggs (locally produced) & 0.13 & 1 & 8.2 \\
Cottage Cheese (locally produced) & 0.0036 & 0.039 & 0.5 \\
Inhalation & 0.05 & 0.2 & 0.87 \\
Mother's milk (mother on Diet 1) & 0.00099 & 0.029 & 0.73 \\
Prenatal exposure (mother on Diet 1) & 0.0087 & 0.084 & 0.89 \\
Diet 1 & 3 & 17 & 110 \\
Diet 2 & 1.2 & 7.3 & 51 \\
Diet 3 & 0.28 & 1.4 & 8.8 \\
\hline
\end{tabular}

Excess Lifetime Risk [ ]

\begin{tabular}{llll}
\hline Diet 1 & $3.1 \mathrm{E}-04$ & $4.7 \mathrm{E}-03$ & $6.8 \mathrm{E}-02$ \\
Diet 2 & $1.4 \mathrm{E}-04$ & $1.8 \mathrm{E}-03$ & $2.3 \mathrm{E}-02$ \\
Diet 3 & $3.3 \mathrm{E}-05$ & $3.5 \mathrm{E}-04$ & $4.3 \mathrm{E}-03$ \\
Diet 4 & $7.3 \mathrm{E}-04$ & $1.2 \mathrm{E}-02$ & $1.7 \mathrm{E}-01$ \\
\hline
\end{tabular}

\section{Relative Risk [ ]}

\begin{tabular}{lccc}
\hline Diet 1 & 1.11 & 2.2 & 13 \\
Diet 2 & 1.045 & 1.44 & 6.9 \\
Diet 3 & 1.01 & 1.089 & 2.1 \\
Diet 4 & 1.24 & 3.7 & 47 \\
\hline
\end{tabular}

\begin{tabular}{lccc}
\hline Diet 1 & \multicolumn{3}{c}{ Probability of Causation [\%] } \\
Diet 2 & 10.25 & 54.7 & 92 \\
Diet 3 & 4.35 & 30.5 & 85 \\
Diet 4 & 1.01 & 8.2 & 53 \\
\hline
\end{tabular}

Diet 1 - Backyard cow milk + all other locally produced non-milk exposure pathways

Diet 2 - Locally produced commercial milk + all other locally produced non-milk exposure pathways

Diet 3 - Regionally mixed commercial milk + inhalation (other regionally mixed food items are minor contributors to the total)

Diet 4 - Goat milk (all other exposure pathways are of negligible importance compared to goat milk) 
Location: Hardin Valley

Receptor: Male born in 1950

\begin{tabular}{lccc}
\hline & \multicolumn{3}{c}{ Thyroid Dose [cGy] } \\
& \multicolumn{2}{c}{$95 \%$ Subjective Confidence Interval } \\
Exposure Pathway & lower limit & central estimate & upper limit \\
\hline Backyard Cow Milk & 2.7 & 16 & 98 \\
Commercial Milk (locally produced) & 0.78 & 5.3 & 42 \\
Commercial Milk (regionally mixed) & 0.17 & 1.1 & 8.9 \\
Goat Milk (locally produced) & 5.2 & 45 & 350 \\
Beef (locally produced) & 0.0038 & 0.068 & 1.5 \\
Leafy Vegetables (locally produced) & 0.0011 & 0.011 & 0.11 \\
Eggs (locally produced) & 0.15 & 1 & 7.8 \\
Cottage Cheese (locally produced) & 0.0035 & 0.04 & 0.41 \\
Inhalation & 0.053 & 0.21 & 0.81 \\
Mother's milk (mother on Diet 1) & 0.00099 & 0.029 & 0.73 \\
Prenatal exposure (mother on Diet 1) & 0.0087 & 0.084 & 0.89 \\
Diet 1 & 3.1 & 18 & 110 \\
Diet 2 & 1.2 & 7 & 53 \\
Diet 3 & 0.27 & 1.4 & 9.6 \\
\hline
\end{tabular}

Excess Lifetime Risk [ ]

\begin{tabular}{lllc}
\hline Diet 1 & $3.4 \mathrm{E}-05$ & $1.2 \mathrm{E}-03$ & $2.1 \mathrm{E}-02$ \\
Diet 2 & $1.8 \mathrm{E}-05$ & $4.8 \mathrm{E}-04$ & $1.0 \mathrm{E}-02$ \\
Diet 3 & $4.2 \mathrm{E}-06$ & $9.2 \mathrm{E}-05$ & $2.0 \mathrm{E}-03$ \\
Diet 4 & $8.8 \mathrm{E}-05$ & $2.9 \mathrm{E}-03$ & $5.8 \mathrm{E}-02$ \\
\hline
\end{tabular}

\section{Relative Risk [ ]}

\begin{tabular}{lccc}
\hline Diet 1 & 1.043 & 1.77 & 19 \\
Diet 2 & 1.016 & 1.32 & 9.3 \\
Diet 3 & 1.0034 & 1.062 & 2.5 \\
Diet 4 & 1.086 & 3.1 & 44 \\
\hline
\end{tabular}

\section{Probability of Causation [\%]}

\begin{tabular}{lccc}
\hline Diet 1 & 4.14 & 43.5 & 95 \\
Diet 2 & 1.58 & 24.3 & 89 \\
Diet 3 & 0.34 & 5.8 & 59 \\
Diet 4 & 7.93 & 67.6 & 98 \\
\hline
\end{tabular}

Diet 1 - Backyard cow milk + all other locally produced non-milk exposure pathways

Diet 2 - Locally produced commercial milk + all other locally produced non-milk exposure pathways

Diet 3 - Regionally mixed commercial milk + inhalation (other regionally mixed food items are minor contributors to the total)

Diet 4 - Goat milk (all other exposure pathways are of negligible importance compared to goat milk) 


\section{Location: Oliver Springs}

Receptor: Female born in 1950

\begin{tabular}{lccc}
\hline & \multicolumn{3}{c}{ Thyroid Dose [cGy] } \\
& \multicolumn{2}{c}{$95 \%$ Subjective Confidence Interval } \\
Exposure Pathway & lower limit & central estimate & upper limit \\
\hline Backyard Cow Milk & 0.41 & 2.5 & 18 \\
Commercial Milk (locally produced) & 0.11 & 0.82 & 7.3 \\
Commercial Milk (regionally mixed) & 0.17 & 1.2 & 7.9 \\
Goat Milk (locally produced) & 0.76 & 6.8 & 64 \\
Beef (locally produced) & 0.00055 & 0.011 & 0.27 \\
Leafy Vegetables (locally produced) & 0.00014 & 0.0017 & 0.018 \\
Eggs (locally produced) & 0.02 & 0.16 & 1.4 \\
Cottage Cheese (locally produced) & 0.0005 & 0.0061 & 0.074 \\
Inhalation & 0.0079 & 0.032 & 0.15 \\
Mother's milk (mother on Diet 1) & 0.00016 & 0.0044 & 0.13 \\
Prenatal exposure (mother on Diet 1) & 0.0013 & 0.013 & 0.15 \\
Diet 1 & 0.46 & 2.7 & 20 \\
Diet 2 & 0.18 & 1.1 & 9 \\
Diet 3 & 0.19 & 1.2 & 8 \\
\hline
\end{tabular}

Excess Lifetime Risk [ ]

\begin{tabular}{llll}
\hline Diet 1 & $4.4 \mathrm{E}-05$ & $7.5 \mathrm{E}-04$ & $1.3 \mathrm{E}-02$ \\
Diet 2 & $2.3 \mathrm{E}-05$ & $2.7 \mathrm{E}-04$ & $4.2 \mathrm{E}-03$ \\
Diet 3 & $2.7 \mathrm{E}-05$ & $3.0 \mathrm{E}-04$ & $4.1 \mathrm{E}-03$ \\
Diet 4 & $1.1 \mathrm{E}-04$ & $1.8 \mathrm{E}-03$ & $3.1 \mathrm{E}-02$ \\
\hline
\end{tabular}

\section{Relative Risk [ ]}

\begin{tabular}{lccc}
\hline Diet 1 & 1.016 & 1.19 & 3.2 \\
Diet 2 & 1.0057 & 1.071 & 2 \\
Diet 3 & 1.0078 & 1.079 & 2.1 \\
Diet 4 & 1.036 & 1.43 & 8.5 \\
\hline
\end{tabular}

\section{Probability of Causation [\%]}

\begin{tabular}{lccc}
\hline Diet 1 & 1.62 & 15.7 & 68 \\
Diet 2 & 0.57 & 6.6 & 51 \\
Diet 3 & 0.77 & 7.3 & 52 \\
Diet 4 & 3.52 & 29.8 & 88 \\
\hline
\end{tabular}

Diet 1 - Backyard cow milk + all other locally produced non-milk exposure pathways

Diet 2 - Locally produced commercial milk + all other locally produced non-milk exposure pathways

Diet 3 - Regionally mixed commercial milk + inhalation (other regionally mixed food items are minor contributors to the total)

Diet 4 - Goat milk (all other exposure pathways are of negligible importance compared to goat milk) 


\section{Location: Oliver Springs}

Receptor: Male born in 1950

\begin{tabular}{lccc}
\hline & \multicolumn{3}{c}{ Thyroid Dose [cGy] } \\
& \multicolumn{2}{c}{$95 \%$ Subjective Confidence Interval } \\
Exposure Pathway & lower limit & central estimate & upper limit \\
\hline Backyard Cow Milk & 0.39 & 2.5 & 18 \\
Commercial Milk (locally produced) & 0.12 & 0.82 & 7.2 \\
Commercial Milk (regionally mixed) & 0.17 & 1.1 & 8.9 \\
Goat Milk (locally produced) & 0.71 & 7 & 60 \\
Beef (locally produced) & 0.00056 & 0.011 & 0.26 \\
Leafy Vegetables (locally produced) & 0.00014 & 0.0017 & 0.018 \\
Eggs (locally produced) & 0.023 & 0.16 & 1.4 \\
Cottage Cheese (locally produced) & 0.00049 & 0.0062 & 0.071 \\
Inhalation & 0.0079 & 0.033 & 0.14 \\
Mother's milk (mother on Diet 1) & 0.00016 & 0.0044 & 0.13 \\
Prenatal exposure (mother on Diet 1) & 0.0013 & 0.013 & 0.15 \\
Diet 1 & 0.45 & 2.7 & 19 \\
Diet 2 & 0.18 & 1.1 & 9 \\
Diet 3 & 0.19 & 1.2 & 9 \\
\hline
\end{tabular}

Excess Lifetime Risk [ ]

\begin{tabular}{llcc}
\hline Diet 1 & $4.9 \mathrm{E}-06$ & $1.9 \mathrm{E}-04$ & $3.5 \mathrm{E}-03$ \\
Diet 2 & $2.5 \mathrm{E}-06$ & $7.5 \mathrm{E}-05$ & $1.5 \mathrm{E}-03$ \\
Diet 3 & $3.3 \mathrm{E}-06$ & $8.0 \mathrm{E}-05$ & $1.9 \mathrm{E}-03$ \\
Diet 4 & $1.3 \mathrm{E}-05$ & $4.7 \mathrm{E}-04$ & $1.1 \mathrm{E}-02$ \\
\hline
\end{tabular}

\section{Relative Risk [ ]}

\begin{tabular}{lccc}
\hline Diet 1 & 1.0063 & 1.13 & 4.1 \\
Diet 2 & 1.0023 & 1.049 & 2.3 \\
Diet 3 & 1.0028 & 1.055 & 2.3 \\
Diet 4 & 1.012 & 1.32 & 8.8 \\
\hline
\end{tabular}

\section{Probability of Causation [\%]}

\begin{tabular}{lccc}
\hline Diet 1 & 0.63 & 11.4 & 75 \\
Diet 2 & 0.23 & 4.7 & 56 \\
Diet 3 & 0.28 & 5.2 & 57 \\
Diet 4 & 1.19 & 24.3 & 88 \\
\hline
\end{tabular}

Diet 1 - Backyard cow milk + all other locally produced non-milk exposure pathways

Diet 2 - Locally produced commercial milk + all other locally produced non-milk exposure pathways

Diet 3 - Regionally mixed commercial milk + inhalation (other regionally mixed food items are minor contributors to the total)

Diet 4 - Goat milk (all other exposure pathways are of negligible importance compared to goat milk) 


\section{Location: Solway}

Receptor: Female born in 1950

\begin{tabular}{lccc}
\hline & \multicolumn{3}{c}{ Thyroid Dose [cGy] } \\
& \multicolumn{2}{c}{$95 \%$ Subjective Confidence Interval } \\
Exposure Pathway & lower limit & central estimate & upper limit \\
\hline Backyard Cow Milk & 2.5 & 14 & 94 \\
Commercial Milk (locally produced) & 0.68 & 5 & 36 \\
Commercial Milk (regionally mixed) & 0.17 & 1.2 & 7.9 \\
Goat Milk (locally produced) & 4.7 & 39 & 340 \\
Beef (locally produced) & 0.0031 & 0.062 & 1.5 \\
Leafy Vegetables (locally produced) & 0.00095 & 0.01 & 0.099 \\
Eggs (locally produced) & 0.12 & 0.96 & 7 \\
Cottage Cheese (locally produced) & 0.0032 & 0.035 & 0.43 \\
Inhalation & 0.049 & 0.19 & 0.79 \\
Mother's milk (mother on Diet 1) & 0.0009 & 0.026 & 0.68 \\
Prenatal exposure (mother on Diet 1) & 0.0082 & 0.074 & 0.76 \\
Diet 1 & 2.8 & 16 & 100 \\
Diet 2 & 1 & 6.5 & 46 \\
Diet 3 & 0.27 & 1.4 & 8.7 \\
\hline
\end{tabular}

Excess Lifetime Risk [ ]

\begin{tabular}{llll}
\hline Diet 1 & $2.8 \mathrm{E}-04$ & $4.2 \mathrm{E}-03$ & $6.1 \mathrm{E}-02$ \\
Diet 2 & $1.5 \mathrm{E}-04$ & $1.6 \mathrm{E}-03$ & $2.0 \mathrm{E}-02$ \\
Diet 3 & $3.4 \mathrm{E}-05$ & $3.4 \mathrm{E}-04$ & $4.2 \mathrm{E}-03$ \\
Diet 4 & $6.9 \mathrm{E}-04$ & $1.1 \mathrm{E}-02$ & $1.5 \mathrm{E}-01$ \\
\hline
\end{tabular}

\section{Relative Risk [ ]}

\begin{tabular}{lccc}
\hline Diet 1 & 1.097 & 2.1 & 12 \\
Diet 2 & 1.04 & 1.42 & 6.1 \\
Diet 3 & 1.01 & 1.088 & 2.1 \\
Diet 4 & 1.23 & 3.4 & 45 \\
\hline
\end{tabular}

\section{Probability of Causation [\%]}

\begin{tabular}{lccc}
\hline Diet 1 & 8.84 & 52.0 & 92 \\
Diet 2 & 3.82 & 29.6 & 84 \\
Diet 3 & 1.00 & 8.1 & 53 \\
Diet 4 & 18.69 & 70.6 & 98 \\
\hline
\end{tabular}

Diet 1 - Backyard cow milk + all other locally produced non-milk exposure pathways

Diet 2 - Locally produced commercial milk + all other locally produced non-milk exposure pathways

Diet 3 - Regionally mixed commercial milk + inhalation (other regionally mixed food items are minor contributors to the total)

Diet 4 - Goat milk (all other exposure pathways are of negligible importance compared to goat milk) 


\section{Location: Solway}

\section{Receptor: Male born in 1950}

\begin{tabular}{lccc}
\hline & \multicolumn{3}{c}{ Thyroid Dose [cGy] } \\
& \multicolumn{2}{c}{$95 \%$ Subjective Confidence Interval } \\
Exposure Pathway & lower limit & central estimate & upper limit \\
\hline Backyard Cow Milk & 2.9 & 15 & 91 \\
Commercial Milk (locally produced) & 0.7 & 4.9 & 37 \\
Commercial Milk (regionally mixed) & 0.17 & 1.1 & 8.9 \\
Goat Milk (locally produced) & 4.6 & 41 & 300 \\
Beef (locally produced) & 0.0033 & 0.062 & 1.4 \\
Leafy Vegetables (locally produced) & 0.00094 & 0.01 & 0.095 \\
Eggs (locally produced) & 0.14 & 0.95 & 6.8 \\
Cottage Cheese (locally produced) & 0.0029 & 0.035 & 0.4 \\
Inhalation & 0.049 & 0.19 & 0.75 \\
Mother's milk (mother on Diet 1) & 0.0009 & 0.026 & 0.68 \\
Prenatal exposure (mother on Diet 1) & 0.0082 & 0.074 & 0.76 \\
Diet 1 & 3.2 & 16 & 99 \\
Diet 2 & 1 & 6.4 & 47 \\
Diet 3 & 0.27 & 1.4 & 9.6 \\
\hline
\end{tabular}

Excess Lifetime Risk [ ]

\begin{tabular}{llll}
\hline Diet 1 & $3.1 \mathrm{E}-05$ & $1.1 \mathrm{E}-03$ & $2.1 \mathrm{E}-02$ \\
Diet 2 & $1.7 \mathrm{E}-05$ & $4.3 \mathrm{E}-04$ & $9.7 \mathrm{E}-03$ \\
Diet 3 & $4.2 \mathrm{E}-06$ & $9.1 \mathrm{E}-05$ & $2.0 \mathrm{E}-03$ \\
Diet 4 & $8.2 \mathrm{E}-05$ & $2.7 \mathrm{E}-03$ & $5.6 \mathrm{E}-02$ \\
\hline
\end{tabular}

\section{Relative Risk [ ]}

\begin{tabular}{lccc}
\hline Diet 1 & 1.038 & 1.73 & 17 \\
Diet 2 & 1.014 & 1.29 & 8.1 \\
Diet 3 & 1.0035 & 1.061 & 2.5 \\
Diet 4 & 1.083 & 2.9 & 40 \\
\hline
\end{tabular}

\begin{tabular}{lccc}
\hline Diet 1 & 3.63 & 42.1 & 94 \\
Diet 2 & 1.43 & 22.7 & 87 \\
Diet 3 & 0.35 & 5.8 & 59 \\
Diet 4 & 7.67 & 65.0 & 97 \\
\hline
\end{tabular}

Diet 1 - Backyard cow milk + all other locally produced non-milk exposure pathways

Diet 2 - Locally produced commercial milk + all other locally produced non-milk exposure pathways

Diet 3 - Regionally mixed commercial milk + inhalation (other regionally mixed food items are minor contributors to the total)

Diet 4 - Goat milk (all other exposure pathways are of negligible importance compared to goat milk) 
Location: Sugar Grove

Receptor: Female born in 1950

\begin{tabular}{lccc}
\hline & \multicolumn{3}{c}{ Thyroid Dose [cGy] } \\
Exposure Pathway & \multicolumn{2}{c}{$95 \%$ Subjective Confidence Interval } \\
\hline Backyard Cow Milk & 1 & 6.1 & 42 \\
Commercial Milk (locally produced) & 0.28 & 2.1 & 16 \\
Commercial Milk (regionally mixed) & 0.17 & 1.2 & 7.9 \\
Goat Milk (locally produced) & 2.1 & 16 & 150 \\
Beef (locally produced) & 0.0015 & 0.026 & 0.58 \\
Leafy Vegetables (locally produced) & 0.0004 & 0.0044 & 0.044 \\
Eggs (locally produced) & 0.051 & 0.39 & 3.2 \\
Cottage Cheese (locally produced) & 0.0013 & 0.015 & 0.2 \\
Inhalation & 0.019 & 0.079 & 0.34 \\
Mother's milk (mother on Diet 1) & 0.0004 & 0.011 & 0.29 \\
Prenatal exposure (mother on Diet 1) & 0.0032 & 0.032 & 0.34 \\
Diet 1 & 1.1 & 6.8 & 46 \\
Diet 2 & 0.43 & 2.8 & 20 \\
Diet 3 & 0.22 & 1.3 & 8.2 \\
\hline
\end{tabular}

Excess Lifetime Risk [ ]

\begin{tabular}{llll}
\hline Diet 1 & $1.2 \mathrm{E}-04$ & $1.8 \mathrm{E}-03$ & $2.6 \mathrm{E}-02$ \\
Diet 2 & $5.8 \mathrm{E}-05$ & $6.8 \mathrm{E}-04$ & $8.9 \mathrm{E}-03$ \\
Diet 3 & $2.9 \mathrm{E}-05$ & $3.1 \mathrm{E}-04$ & $4.1 \mathrm{E}-03$ \\
Diet 4 & $2.8 \mathrm{E}-04$ & $4.6 \mathrm{E}-03$ & $6.9 \mathrm{E}-02$ \\
\hline
\end{tabular}

\section{Relative Risk [ ]}

\begin{tabular}{lccc}
\hline Diet 1 & 1.042 & 1.45 & 5.9 \\
Diet 2 & 1.017 & 1.17 & 3.2 \\
Diet 3 & 1.0086 & 1.082 & 2.1 \\
Diet 4 & 1.093 & 2 & 19 \\
\hline
\end{tabular}

\begin{tabular}{lccc} 
& \multicolumn{3}{c}{ Probability of Causation [\%] } \\
\hline Diet 1 & 3.98 & 30.3 & 82 \\
Diet 2 & 1.68 & 14.3 & 67 \\
Diet 3 & 0.86 & 7.6 & 52 \\
Diet 4 & 8.43 & 48.9 & 94 \\
\hline
\end{tabular}

Diet 1 - Backyard cow milk + all other locally produced non-milk exposure pathways

Diet 2 - Locally produced commercial milk + all other locally produced non-milk exposure pathways

Diet 3 - Regionally mixed commercial milk + inhalation (other regionally mixed food items are minor contributors to the total)

Diet 4 - Goat milk (all other exposure pathways are of negligible importance compared to goat milk) 
Location: Sugar Grove

Receptor: Male born in 1950

\begin{tabular}{lccc}
\hline & \multicolumn{3}{c}{ Thyroid Dose [cGy] } \\
& \multicolumn{2}{c}{$95 \%$ Subjective Confidence Interval } \\
Exposure Pathway & lower limit & central estimate & upper limit \\
\hline Backyard Cow Milk & 1.1 & 6.3 & 40 \\
Commercial Milk (locally produced) & 0.3 & 2.1 & 16 \\
Commercial Milk (regionally mixed) & 0.17 & 1.1 & 8.9 \\
Goat Milk (locally produced) & 1.9 & 17 & 140 \\
Beef (locally produced) & 0.0015 & 0.026 & 0.57 \\
Leafy Vegetables (locally produced) & 0.00041 & 0.0043 & 0.043 \\
Eggs (locally produced) & 0.058 & 0.39 & 3 \\
Cottage Cheese (locally produced) & 0.0012 & 0.015 & 0.18 \\
Inhalation & 0.02 & 0.08 & 0.31 \\
Mother's milk (mother on Diet 1) & 0.0004 & 0.011 & 0.29 \\
Prenatal exposure (mother on Diet 1) & 0.0032 & 0.032 & 0.34 \\
Diet 1 & 1.2 & 6.8 & 45 \\
Diet 2 & 0.44 & 2.7 & 21 \\
Diet 3 & 0.21 & 1.2 & 9.2 \\
\hline
\end{tabular}

Excess Lifetime Risk [ ]

\begin{tabular}{llll}
\hline Diet 1 & $1.4 \mathrm{E}-05$ & $4.6 \mathrm{E}-04$ & $8.9 \mathrm{E}-03$ \\
Diet 2 & $6.9 \mathrm{E}-06$ & $1.8 \mathrm{E}-04$ & $3.9 \mathrm{E}-03$ \\
Diet 3 & $3.7 \mathrm{E}-06$ & $8.3 \mathrm{E}-05$ & $1.9 \mathrm{E}-03$ \\
Diet 4 & $3.6 \mathrm{E}-05$ & $1.1 \mathrm{E}-03$ & $2.5 \mathrm{E}-02$ \\
\hline
\end{tabular}

\section{Relative Risk [ ]}

\begin{tabular}{lccc}
\hline Diet 1 & 1.016 & 1.31 & 7.8 \\
Diet 2 & 1.0058 & 1.12 & 4.2 \\
Diet 3 & 1.0029 & 1.057 & 2.4 \\
Diet 4 & 1.033 & 1.8 & 18 \\
\hline
\end{tabular}

\section{Probability of Causation [\%]}

\begin{tabular}{lccc}
\hline Diet 1 & 1.57 & 23.0 & 86 \\
Diet 2 & 0.58 & 10.6 & 75 \\
Diet 3 & 0.29 & 5.4 & 58 \\
Diet 4 & 3.15 & 43.0 & 94 \\
\hline
\end{tabular}

Diet 1 - Backyard cow milk + all other locally produced non-milk exposure pathways

Diet 2 - Locally produced commercial milk + all other locally produced non-milk exposure pathways

Diet 3 - Regionally mixed commercial milk + inhalation (other regionally mixed food items are minor contributors to the total)

Diet 4 - Goat milk (all other exposure pathways are of negligible importance compared to goat milk) 


\section{Location: OR Townsite}

Receptor: Female born in 1950

\begin{tabular}{lccc}
\hline & \multicolumn{3}{c}{ Thyroid Dose [cGy] } \\
Exposure Pathway & \multicolumn{2}{c}{$9 \%$ Subjective Confidence Interval } \\
\hline Backyard Cow Milk & lower limit & central estimate & upper limit \\
Commercial Milk (locally produced) & -- & -- & -- \\
Commercial Milk (regionally mixed) & -- & -- & -- \\
Goat Milk (locally produced) & 0.17 & 1.2 & 7.9 \\
Beef (locally produced) & -- & -- & -- \\
Leafy Vegetables (locally produced) & -- & -- & -- \\
Eggs (locally produced) & -- & -- & -- \\
Cottage Cheese (locally produced) & -- & -- & -- \\
Inhalation & 0.0015 & 0.016 & 0.2 \\
Mother's milk (mother on Diet 3) & 0.022 & 0.087 & 0.36 \\
Prenatal exposure (mother on Diet 3) & 0.00011 & 0.0019 & 0.024 \\
Diet 1 & 0.00085 & 0.0053 & 0.045 \\
Diet 2 & -- & -- & -- \\
Diet 3 & -- & -- & -- \\
\hline
\end{tabular}

Excess Lifetime Risk [ ]

\begin{tabular}{lccc}
\hline Diet 1 & -- & -- & -- \\
Diet 2 & -- & -- & -- \\
Diet 3 & $2.9 \mathrm{E}-05$ & $3.2 \mathrm{E}-04$ & $4.1 \mathrm{E}-03$ \\
Diet 4 & -- & -- & -- \\
\hline
\end{tabular}

\section{Relative Risk [ ]}

\begin{tabular}{lccc}
\hline Diet 1 & -- & -- & -- \\
Diet 2 & -- & -- & -- \\
Diet 3 & 1.0088 & 1.082 & 2.1 \\
Diet 4 & -- & -- & -- \\
\hline
\end{tabular}

\begin{tabular}{lccc}
\hline Diet 1 & -- & -- & -- \\
Diet 2 & -- & -- & 52 \\
Diet 3 & 0.87 & 7.6 & -- \\
Diet 4 & -- & -- & \\
\hline Diet 1 - Backyard cow milk + all other locally produced non-milk exposure pathways & \\
Diet 2 - Locally produced commercial milk + all other locally produced non-milk exposure pathways \\
Diet 3 - Regionally mixed commercial milk + inhalation (other regionally mixed food items are minor contributors to the total) \\
Diet 4 - Goat milk (all other exposure pathways are of negligible importance compared to goat milk)
\end{tabular}




\section{Location: OR Townsite}

\section{Receptor: Male born in 1950}

\begin{tabular}{lccc}
\hline & \multicolumn{3}{c}{ Thyroid Dose [cGy] } \\
Exposure Pathway & \multicolumn{2}{c}{$9 \%$ Subjective Confidence Interval } \\
\hline Backyard Cow Milk & lower limit & central estimate & upper limit \\
Commercial Milk (locally produced) & -- & -- & -- \\
Commercial Milk (regionally mixed) & -- & -- & -- \\
Goat Milk (locally produced) & 0.17 & 1.1 & 8.9 \\
Beef (locally produced) & -- & -- & -- \\
Leafy Vegetables (locally produced) & -- & -- & -- \\
Eggs (locally produced) & -- & -- & -- \\
Cottage Cheese (locally produced) & -- & -- & -- \\
Inhalation & 0.0015 & 0.016 & 0.18 \\
Mother's milk (mother on Diet 3) & 0.022 & 0.088 & 0.34 \\
Prenatal exposure (mother on Diet 3) & 0.00011 & 0.0019 & 0.024 \\
Diet 1 & 0.00085 & 0.0053 & 0.045 \\
Diet 2 & -- & -- & -- \\
Diet 3 & -- & -- & -- \\
\hline
\end{tabular}

Excess Lifetime Risk [ ]

\begin{tabular}{lccc}
\hline Diet 1 & -- & -- & -- \\
Diet 2 & -- & -- & -- \\
Diet 3 & $3.7 \mathrm{E}-06$ & $8.4 \mathrm{E}-05$ & $1.9 \mathrm{E}-03$ \\
Diet 4 & -- & -- & -- \\
\hline
\end{tabular}

\section{Relative Risk [ ]}

\begin{tabular}{lccc}
\hline Diet 1 & -- & -- & -- \\
Diet 2 & -- & -- & -- \\
Diet 3 & 1.0029 & 1.057 & 2.4 \\
Diet 4 & -- & -- & -- \\
\hline
\end{tabular}

\begin{tabular}{lccc}
\hline Diet 1 & -- & -- & -- \\
Diet 2 & -- & -- & -- \\
Diet 3 & 0.29 & 5.4 & 58 \\
Diet 4 & -- & -- & - \\
\hline Diet 1 - Backyard cow milk + all other locally produced non-milk exposure pathways & \\
Diet 2 - Locally produced commercial milk + all other locally produced non-milk exposure pathways \\
Diet 3 - Regionally mixed commercial milk + inhalation (other regionally mixed food items are minor contributors to the total) \\
Diet 4 - Goat milk (all other exposure pathways are of negligible importance compared to goat milk)
\end{tabular}


Location: Hines Valley

Receptor: Female born in 1950

\begin{tabular}{lccc}
\hline & \multicolumn{3}{c}{ Thyroid Dose [cGy] } \\
& \multicolumn{2}{c}{$95 \%$ Subjective Confidence Interval } \\
Exposure Pathway & lower limit & central estimate & upper limit \\
\hline Backyard Cow Milk & 1.2 & 7.2 & 48 \\
Commercial Milk (locally produced) & 0.34 & 2.5 & 19 \\
Commercial Milk (regionally mixed) & 0.17 & 1.2 & 7.9 \\
Goat Milk (locally produced) & 2.4 & 20 & 160 \\
Beef (locally produced) & 0.0016 & 0.032 & 0.86 \\
Leafy Vegetables (locally produced) & 0.0005 & 0.0054 & 0.049 \\
Eggs (locally produced) & 0.061 & 0.49 & 3.6 \\
Cottage Cheese (locally produced) & 0.0016 & 0.018 & 0.22 \\
Inhalation & 0.025 & 0.11 & 0.41 \\
Mother's milk (mother on Diet 1) & 0.00047 & 0.013 & 0.35 \\
Prenatal exposure (mother on Diet 1) & 0.0042 & 0.038 & 0.43 \\
Diet 1 & 1.4 & 8 & 52 \\
Diet 2 & 0.51 & 3.2 & 22 \\
Diet 3 & 0.23 & 1.3 & 8.3 \\
\hline
\end{tabular}

Excess Lifetime Risk [ ]

\begin{tabular}{lllc}
\hline Diet 1 & $1.4 \mathrm{E}-04$ & $2.2 \mathrm{E}-03$ & $3.4 \mathrm{E}-02$ \\
Diet 2 & $6.9 \mathrm{E}-05$ & $8.4 \mathrm{E}-04$ & $1.1 \mathrm{E}-02$ \\
Diet 3 & $2.9 \mathrm{E}-05$ & $3.2 \mathrm{E}-04$ & $4.1 \mathrm{E}-03$ \\
Diet 4 & $3.4 \mathrm{E}-04$ & $5.4 \mathrm{E}-03$ & $8.1 \mathrm{E}-02$ \\
\hline
\end{tabular}

\section{Relative Risk [ ]}

\begin{tabular}{lccc}
\hline Diet 1 & 1.052 & 1.57 & 6.6 \\
Diet 2 & 1.02 & 1.21 & 3.7 \\
Diet 3 & 1.009 & 1.083 & 2.1 \\
Diet 4 & 1.11 & 2.2 & 24 \\
\hline
\end{tabular}

\begin{tabular}{lccc}
\hline Diet 1 & 4.90 & 36.3 & 85 \\
Diet 2 & 1.97 & 17.5 & 73 \\
Diet 3 & 0.89 & 7.7 & 52 \\
Diet 4 & 10.15 & 55.3 & 96 \\
\hline
\end{tabular}

Diet 1 - Backyard cow milk + all other locally produced non-milk exposure pathways

Diet 2 - Locally produced commercial milk + all other locally produced non-milk exposure pathways

Diet 3 - Regionally mixed commercial milk + inhalation (other regionally mixed food items are minor contributors to the total)

Diet 4 - Goat milk (all other exposure pathways are of negligible importance compared to goat milk) 


\section{Location: Hines Valley}

Receptor: Male born in 1950

\begin{tabular}{lccc}
\hline & \multicolumn{3}{c}{ Thyroid Dose [cGy] } \\
& \multicolumn{2}{c}{$95 \%$ Subjective Confidence Interval } \\
Exposure Pathway & lower limit & central estimate & upper limit \\
\hline Backyard Cow Milk & 1.3 & 7.6 & 48 \\
Commercial Milk (locally produced) & 0.35 & 2.5 & 20 \\
Commercial Milk (regionally mixed) & 0.17 & 1.1 & 8.9 \\
Goat Milk (locally produced) & 2.2 & 20 & 150 \\
Beef (locally produced) & 0.0018 & 0.031 & 0.82 \\
Leafy Vegetables (locally produced) & 0.00051 & 0.0053 & 0.045 \\
Eggs (locally produced) & 0.069 & 0.47 & 3.7 \\
Cottage Cheese (locally produced) & 0.0017 & 0.019 & 0.21 \\
Inhalation & 0.026 & 0.1 & 0.41 \\
Mother's milk (mother on Diet 1) & 0.00047 & 0.013 & 0.35 \\
Prenatal exposure (mother on Diet 1) & 0.0042 & 0.038 & 0.43 \\
Diet 1 & 1.5 & 8.3 & 53 \\
Diet 2 & 0.52 & 3.2 & 23 \\
Diet 3 & 0.22 & 1.2 & 9.3 \\
\hline
\end{tabular}

Excess Lifetime Risk [ ]

\begin{tabular}{llll}
\hline Diet 1 & $1.6 \mathrm{E}-05$ & $5.9 \mathrm{E}-04$ & $1.1 \mathrm{E}-02$ \\
Diet 2 & $8.4 \mathrm{E}-06$ & $2.3 \mathrm{E}-04$ & $5.0 \mathrm{E}-03$ \\
Diet 3 & $3.8 \mathrm{E}-06$ & $8.4 \mathrm{E}-05$ & $1.9 \mathrm{E}-03$ \\
Diet 4 & $4.1 \mathrm{E}-05$ & $1.4 \mathrm{E}-03$ & $3.0 \mathrm{E}-02$ \\
\hline
\end{tabular}

\section{Relative Risk [ ]}

\begin{tabular}{lccc}
\hline Diet 1 & 1.02 & 1.37 & 9.4 \\
Diet 2 & 1.0076 & 1.15 & 4.3 \\
Diet 3 & 1.003 & 1.057 & 2.4 \\
Diet 4 & 1.042 & 1.93 & 23 \\
\hline
\end{tabular}

\section{Probability of Causation [\%]}

\begin{tabular}{lccc}
\hline Diet 1 & 1.99 & 26.9 & 89 \\
Diet 2 & 0.76 & 13.2 & 77 \\
Diet 3 & 0.30 & 5.4 & 58 \\
Diet 4 & 4.07 & 48.2 & 96 \\
\hline
\end{tabular}

Diet 1 - Backyard cow milk + all other locally produced non-milk exposure pathways

Diet 2 - Locally produced commercial milk + all other locally produced non-milk exposure pathways

Diet 3 - Regionally mixed commercial milk + inhalation (other regionally mixed food items are minor contributors to the total)

Diet 4 - Goat milk (all other exposure pathways are of negligible importance compared to goat milk) 


\section{Location: Farragut}

Receptor: Female born in 1950

\begin{tabular}{lccc}
\hline & \multicolumn{3}{c}{ Thyroid Dose [cGy] } \\
& \multicolumn{2}{c}{$95 \%$ Subjective Confidence Interval } \\
Exposure Pathway & lower limit & central estimate & upper limit \\
\hline Backyard Cow Milk & 1.4 & 8.1 & 53 \\
Commercial Milk (locally produced) & 0.39 & 2.8 & 20 \\
Commercial Milk (regionally mixed) & 0.17 & 1.2 & 7.9 \\
Goat Milk (locally produced) & 2.6 & 23 & 200 \\
Beef (locally produced) & 0.0019 & 0.035 & 1 \\
Leafy Vegetables (locally produced) & 0.0005 & 0.006 & 0.056 \\
Eggs (locally produced) & 0.068 & 0.56 & 4 \\
Cottage Cheese (locally produced) & 0.0018 & 0.021 & 0.23 \\
Inhalation & 0.029 & 0.12 & 0.48 \\
Mother's milk (mother on Diet 1) & 0.00059 & 0.015 & 0.41 \\
Prenatal exposure (mother on Diet 1) & 0.0045 & 0.044 & 0.45 \\
Diet 1 & 1.6 & 8.8 & 58 \\
Diet 2 & 0.59 & 3.7 & 25 \\
Diet 3 & 0.24 & 1.3 & 8.4 \\
\hline
\end{tabular}

Excess Lifetime Risk [ ]

\begin{tabular}{llll}
\hline Diet 1 & $1.6 \mathrm{E}-04$ & $2.5 \mathrm{E}-03$ & $3.8 \mathrm{E}-02$ \\
Diet 2 & $8.1 \mathrm{E}-05$ & $9.4 \mathrm{E}-04$ & $1.2 \mathrm{E}-02$ \\
Diet 3 & $3.0 \mathrm{E}-05$ & $3.2 \mathrm{E}-04$ & $4.2 \mathrm{E}-03$ \\
Diet 4 & $3.8 \mathrm{E}-04$ & $6.4 \mathrm{E}-03$ & $8.8 \mathrm{E}-02$ \\
\hline
\end{tabular}

\section{Relative Risk [ ]}

\begin{tabular}{lccc}
\hline Diet 1 & 1.057 & 1.63 & 7.3 \\
Diet 2 & 1.023 & 1.24 & 4.3 \\
Diet 3 & 1.0092 & 1.084 & 2.1 \\
Diet 4 & 1.12 & 2.4 & 26 \\
\hline
\end{tabular}

\section{Probability of Causation [\%]}

\begin{tabular}{lccc}
\hline Diet 1 & 5.36 & 38.6 & 86 \\
Diet 2 & 2.24 & 19.2 & 77 \\
Diet 3 & 0.91 & 7.7 & 52 \\
Diet 4 & 10.76 & 58.3 & 96 \\
\hline
\end{tabular}

Diet 1 - Backyard cow milk + all other locally produced non-milk exposure pathways

Diet 2 - Locally produced commercial milk + all other locally produced non-milk exposure pathways

Diet 3 - Regionally mixed commercial milk + inhalation (other regionally mixed food items are minor contributors to the total)

Diet 4 - Goat milk (all other exposure pathways are of negligible importance compared to goat milk) 


\section{Location: Farragut}

\section{Receptor: Male born in 1950}

\begin{tabular}{lccc}
\hline & \multicolumn{3}{c}{ Thyroid Dose [cGy] } \\
& \multicolumn{2}{c}{$95 \%$ Subjective Confidence Interval } \\
Exposure Pathway & lower limit & central estimate & upper limit \\
\hline Backyard Cow Milk & 1.4 & 8.2 & 53 \\
Commercial Milk (locally produced) & 0.41 & 2.7 & 21 \\
Commercial Milk (regionally mixed) & 0.17 & 1.1 & 8.9 \\
Goat Milk (locally produced) & 2.5 & 23 & 180 \\
Beef (locally produced) & 0.002 & 0.036 & 0.95 \\
Leafy Vegetables (locally produced) & 0.00052 & 0.006 & 0.055 \\
Eggs (locally produced) & 0.077 & 0.53 & 4 \\
Cottage Cheese (locally produced) & 0.0018 & 0.021 & 0.21 \\
Inhalation & 0.027 & 0.12 & 0.45 \\
Mother's milk (mother on Diet 1) & 0.00059 & 0.015 & 0.41 \\
Prenatal exposure (mother on Diet 1) & 0.0045 & 0.044 & 0.45 \\
Diet 1 & 1.6 & 9.1 & 58 \\
Diet 2 & 0.61 & 3.6 & 25 \\
Diet 3 & 0.23 & 1.3 & 9.3 \\
\hline
\end{tabular}

Excess Lifetime Risk [ ]

\begin{tabular}{llll}
\hline Diet 1 & $2.0 \mathrm{E}-05$ & $6.5 \mathrm{E}-04$ & $1.1 \mathrm{E}-02$ \\
Diet 2 & $9.5 \mathrm{E}-06$ & $2.4 \mathrm{E}-04$ & $5.6 \mathrm{E}-03$ \\
Diet 3 & $3.9 \mathrm{E}-06$ & $8.5 \mathrm{E}-05$ & $2.0 \mathrm{E}-03$ \\
Diet 4 & $4.6 \mathrm{E}-05$ & $1.6 \mathrm{E}-03$ & $3.3 \mathrm{E}-02$ \\
\hline
\end{tabular}

\section{Relative Risk [ ]}

\begin{tabular}{lccc}
\hline Diet 1 & 1.023 & 1.42 & 10.7 \\
Diet 2 & 1.0088 & 1.17 & 5 \\
Diet 3 & 1.003 & 1.058 & 2.4 \\
Diet 4 & 1.048 & 2.1 & 26 \\
\hline
\end{tabular}

\begin{tabular}{lccc}
\hline Diet 1 & 2.28 & 29.4 & 91 \\
Diet 2 & 0.88 & 14.2 & 80 \\
Diet 3 & 0.30 & 5.5 & 59 \\
Diet 4 & 4.54 & 52.0 & 96 \\
\hline
\end{tabular}

Diet 1 - Backyard cow milk + all other locally produced non-milk exposure pathways

Diet 2 - Locally produced commercial milk + all other locally produced non-milk exposure pathways

Diet 3 - Regionally mixed commercial milk + inhalation (other regionally mixed food items are minor contributors to the total)

Diet 4 - Goat milk (all other exposure pathways are of negligible importance compared to goat milk) 
Location: Lenoir City

Receptor: Female born in 1950

\begin{tabular}{lccc}
\hline & \multicolumn{3}{c}{ Thyroid Dose [cGy] } \\
Exposure Pathway & \multicolumn{2}{c}{$95 \%$ Subjective Confidence Interval } \\
\hline Backyard Cow Milk & lower limit & central estimate & upper limit \\
Commercial Milk (locally produced) & 0.95 & 5.1 & 30 \\
Commercial Milk (regionally mixed) & 0.25 & 1.7 & 13 \\
Goat Milk (locally produced) & 0.17 & 1.2 & 7.9 \\
Beef (locally produced) & -- & -- & -- \\
Leafy Vegetables (locally produced) & 0.0011 & 0.022 & 0.53 \\
Eggs (locally produced) & 0.00035 & 0.0037 & 0.034 \\
Cottage Cheese (locally produced) & 0.045 & 0.33 & 2.4 \\
Inhalation & 0.001 & 0.013 & 0.15 \\
Mother's milk (mother on Diet 1) & 0.017 & 0.073 & 0.3 \\
Prenatal exposure (mother on Diet 1) & 0.00031 & 0.009 & 0.24 \\
Diet 1 & 0.003 & 0.028 & 0.28 \\
Diet 2 & 1 & 5.6 & 33 \\
Diet 3 & 0.38 & 2.2 & 16 \\
\hline
\end{tabular}

Excess Lifetime Risk [ ]

\begin{tabular}{lccc}
\hline Diet 1 & $1.1 \mathrm{E}-04$ & $1.5 \mathrm{E}-03$ & $2.5 \mathrm{E}-02$ \\
Diet 2 & $5.4 \mathrm{E}-05$ & $5.3 \mathrm{E}-04$ & $8.5 \mathrm{E}-03$ \\
Diet 3 & $2.8 \mathrm{E}-05$ & $3.1 \mathrm{E}-04$ & $4.1 \mathrm{E}-03$ \\
Diet 4 & -- & -- & -- \\
\hline
\end{tabular}

Relative Risk [ ]

\begin{tabular}{lccc}
\hline Diet 1 & 1.04 & 1.38 & 5.3 \\
Diet 2 & 1.013 & 1.15 & 3 \\
Diet 3 & 1.0087 & 1.082 & 2.1 \\
Diet 4 & -- & -- & -- \\
\hline
\end{tabular}

Probability of Causation [\%]

\begin{tabular}{lccc}
\hline Diet 1 & 3.88 & 27.6 & 81 \\
Diet 2 & 1.31 & 12.8 & 67 \\
Diet 3 & 0.86 & 7.5 & 52 \\
Diet 4 & -- & -- & -- \\
\hline Diet 1 - Backyard cow milk + all other locally produced non-milk exposure pathways & \\
Diet 2 - Locally produced commercial milk + all other locally produced non-milk exposure pathways \\
Diet 3 - Regionally mixed commercial milk + inhalation (other regionally mixed food items are minor contributors to the total) \\
Diet 4 - Goat milk (all other exposure pathways are of negligible importance compared to goat milk)
\end{tabular}




\section{Location: Lenoir City}

\section{Receptor: Male born in 1950}

\begin{tabular}{lccc}
\hline & \multicolumn{3}{c}{ Thyroid Dose [cGy] } \\
& \multicolumn{2}{c}{$95 \%$ Subjective Confidence Interval } \\
Exposure Pathway & lower limit & central estimate & upper limit \\
\hline Backyard Cow Milk & 0.92 & 5.2 & 31 \\
Commercial Milk (locally produced) & 0.25 & 1.6 & 13 \\
Commercial Milk (regionally mixed) & 0.17 & 1.1 & 8.9 \\
Goat Milk (locally produced) & -- & -- & -- \\
Beef (locally produced) & 0.0011 & 0.021 & 0.5 \\
Leafy Vegetables (locally produced) & 0.00037 & 0.0037 & 0.032 \\
Eggs (locally produced) & 0.05 & 0.34 & 2.4 \\
Cottage Cheese (locally produced) & 0.001 & 0.013 & 0.12 \\
Inhalation & 0.019 & 0.071 & 0.31 \\
Mother's milk (mother on Diet 1) & 0.00031 & 0.009 & 0.24 \\
Prenatal exposure (mother on Diet 1) & 0.003 & 0.028 & 0.28 \\
Diet 1 & 1 & 5.6 & 33 \\
Diet 2 & 0.39 & 2.1 & 16 \\
Diet 3 & 0.21 & 1.2 & 9.2 \\
\hline
\end{tabular}

Excess Lifetime Risk [ ]

\begin{tabular}{lccc}
\hline Diet 1 & $1.1 \mathrm{E}-05$ & $4.1 \mathrm{E}-04$ & $6.5 \mathrm{E}-03$ \\
Diet 2 & $5.4 \mathrm{E}-06$ & $1.5 \mathrm{E}-04$ & $3.1 \mathrm{E}-03$ \\
Diet 3 & $3.7 \mathrm{E}-06$ & $8.3 \mathrm{E}-05$ & $1.9 \mathrm{E}-03$ \\
Diet 4 & -- & -- & -- \\
\hline
\end{tabular}

\section{Relative Risk [ ]}

\begin{tabular}{lccc}
\hline Diet 1 & 1.014 & 1.25 & 6.7 \\
Diet 2 & 1.0054 & 1.093 & 3.4 \\
Diet 3 & 1.0029 & 1.057 & 2.4 \\
Diet 4 & -- & -- & -- \\
\hline
\end{tabular}

\section{Probability of Causation [\%]}

\begin{tabular}{lccc}
\hline Diet 1 & 1.36 & 20.1 & 85 \\
Diet 2 & 0.54 & 8.5 & 71 \\
Diet 3 & 0.29 & 5.4 & 58 \\
Diet 4 & -- & -- \\
\hline Diet 1 - Backyard cow milk + all other locally produced non-milk exposure pathways & \\
Diet 2 - Locally produced commercial milk + all other locally produced non-milk exposure pathways \\
Diet 3 - Regionally mixed commercial milk + inhalation (other regionally mixed food items are minor contributors to the total) \\
Diet 4 - Goat milk (all other exposure pathways are of negligible importance compared to goat milk)
\end{tabular}




\section{Location: Kingston}

Receptor: Female born in 1950

\begin{tabular}{lccc}
\hline & \multicolumn{3}{c}{ Thyroid Dose [cGy] } \\
& \multicolumn{2}{c}{$95 \%$ Subjective Confidence Interval } \\
Exposure Pathway & lower limit & central estimate & upper limit \\
\hline Backyard Cow Milk & 1.1 & 6.5 & 41 \\
Commercial Milk (locally produced) & 0.32 & 2.2 & 15 \\
Commercial Milk (regionally mixed) & 0.17 & 1.2 & 7.9 \\
Goat Milk (locally produced) & 2 & 18 & 160 \\
Beef (locally produced) & 0.0015 & 0.028 & 0.67 \\
Leafy Vegetables (locally produced) & 0.00044 & 0.0046 & 0.042 \\
Eggs (locally produced) & 0.051 & 0.43 & 3.5 \\
Cottage Cheese (locally produced) & 0.0014 & 0.016 & 0.17 \\
Inhalation & 0.023 & 0.094 & 0.4 \\
Mother's milk (mother on Diet 1) & 0.00044 & 0.012 & 0.3 \\
Prenatal exposure (mother on Diet 1) & 0.0037 & 0.033 & 0.35 \\
Diet 1 & 1.2 & 7.2 & 45 \\
Diet 2 & 0.49 & 3 & 19 \\
Diet 3 & 0.22 & 1.3 & 8.3 \\
\hline
\end{tabular}

Excess Lifetime Risk [ ]

\begin{tabular}{lllc}
\hline Diet 1 & $1.2 \mathrm{E}-04$ & $1.9 \mathrm{E}-03$ & $2.6 \mathrm{E}-02$ \\
Diet 2 & $7.1 \mathrm{E}-05$ & $7.2 \mathrm{E}-04$ & $9.5 \mathrm{E}-03$ \\
Diet 3 & $2.9 \mathrm{E}-05$ & $3.2 \mathrm{E}-04$ & $4.1 \mathrm{E}-03$ \\
Diet 4 & $3.0 \mathrm{E}-04$ & $4.8 \mathrm{E}-03$ & $6.1 \mathrm{E}-02$ \\
\hline
\end{tabular}

\section{Relative Risk [ ]}

\begin{tabular}{lccc}
\hline Diet 1 & 1.048 & 1.5 & 6.1 \\
Diet 2 & 1.017 & 1.19 & 3.3 \\
Diet 3 & 1.009 & 1.082 & 2.1 \\
Diet 4 & 1.11 & 2.1 & 18 \\
\hline
\end{tabular}

\begin{tabular}{lccc}
\hline Diet 1 & 4.58 & 33.1 & 83 \\
Diet 2 & 1.67 & 15.7 & 70 \\
Diet 3 & 0.89 & 7.6 & 52 \\
Diet 4 & 9.63 & 52.3 & 94 \\
\hline
\end{tabular}

Diet 1 - Backyard cow milk + all other locally produced non-milk exposure pathways

Diet 2 - Locally produced commercial milk + all other locally produced non-milk exposure pathways

Diet 3 - Regionally mixed commercial milk + inhalation (other regionally mixed food items are minor contributors to the total)

Diet 4 - Goat milk (all other exposure pathways are of negligible importance compared to goat milk) 


\section{Location: Kingston}

\section{Receptor: Male born in 1950}

\begin{tabular}{lccc}
\hline & \multicolumn{3}{c}{ Thyroid Dose [cGy] } \\
& \multicolumn{2}{c}{$95 \%$ Subjective Confidence Interval } \\
Exposure Pathway & lower limit & central estimate & upper limit \\
\hline Backyard Cow Milk & 1.1 & 6.7 & 39 \\
Commercial Milk (locally produced) & 0.33 & 2.1 & 17 \\
Commercial Milk (regionally mixed) & 0.17 & 1.1 & 8.9 \\
Goat Milk (locally produced) & 2 & 18 & 140 \\
Beef (locally produced) & 0.0015 & 0.027 & 0.66 \\
Leafy Vegetables (locally produced) & 0.00041 & 0.0045 & 0.041 \\
Eggs (locally produced) & 0.06 & 0.42 & 3.3 \\
Cottage Cheese (locally produced) & 0.0013 & 0.016 & 0.16 \\
Inhalation & 0.023 & 0.096 & 0.39 \\
Mother's milk (mother on Diet 1) & 0.00044 & 0.012 & 0.3 \\
Prenatal exposure (mother on Diet 1) & 0.0037 & 0.033 & 0.35 \\
Diet 1 & 1.2 & 7.3 & 43 \\
Diet 2 & 0.49 & 2.9 & 21 \\
Diet 3 & 0.22 & 1.2 & 9.3 \\
\hline
\end{tabular}

Excess Lifetime Risk [ ]

\begin{tabular}{llll}
\hline Diet 1 & $1.4 \mathrm{E}-05$ & $5.1 \mathrm{E}-04$ & $9.0 \mathrm{E}-03$ \\
Diet 2 & $6.7 \mathrm{E}-06$ & $2.0 \mathrm{E}-04$ & $3.9 \mathrm{E}-03$ \\
Diet 3 & $3.8 \mathrm{E}-06$ & $8.4 \mathrm{E}-05$ & $1.9 \mathrm{E}-03$ \\
Diet 4 & $3.9 \mathrm{E}-05$ & $1.2 \mathrm{E}-03$ & $2.5 \mathrm{E}-02$ \\
\hline
\end{tabular}

\section{Relative Risk [ ]}

\begin{tabular}{lccc}
\hline Diet 1 & 1.017 & 1.33 & 8 \\
Diet 2 & 1.007 & 1.13 & 4.4 \\
Diet 3 & 1.0029 & 1.057 & 2.4 \\
Diet 4 & 1.034 & 1.84 & 21 \\
\hline
\end{tabular}

\section{Probability of Causation [\%]}

\begin{tabular}{lccc}
\hline Diet 1 & 1.65 & 24.6 & 87 \\
Diet 2 & 0.70 & 11.5 & 77 \\
Diet 3 & 0.29 & 5.4 & 59 \\
Diet 4 & 3.29 & 45.6 & 95 \\
\hline
\end{tabular}

Diet 1 - Backyard cow milk + all other locally produced non-milk exposure pathways

Diet 2 - Locally produced commercial milk + all other locally produced non-milk exposure pathways

Diet 3 - Regionally mixed commercial milk + inhalation (other regionally mixed food items are minor contributors to the total)

Diet 4 - Goat milk (all other exposure pathways are of negligible importance compared to goat milk) 


\section{Location: Karns}

Receptor: Female born in 1950

\begin{tabular}{|c|c|c|c|}
\hline \multirow[b]{2}{*}{ Exposure Pathway } & \multicolumn{3}{|c|}{$\begin{array}{c}\text { Thyroid Dose [cGy] } \\
\text { 95\% Subjective Confidence Interval }\end{array}$} \\
\hline & lower limit & central estimate & upper limit \\
\hline Backyard Cow Milk & 1.5 & 8.6 & 53 \\
\hline Commercial Milk (locally produced) & 0.42 & 3 & 20 \\
\hline Commercial Milk (regionally mixed) & 0.17 & 1.2 & 7.9 \\
\hline Goat Milk (locally produced) & 2.9 & 24 & 210 \\
\hline Beef (locally produced) & 0.002 & 0.038 & 0.93 \\
\hline Leafy Vegetables (locally produced) & 0.00058 & 0.0064 & 0.058 \\
\hline Eggs (locally produced) & 0.071 & 0.58 & 4.4 \\
\hline Cottage Cheese (locally produced) & 0.0019 & 0.022 & 0.23 \\
\hline Inhalation & 0.031 & 0.13 & 0.53 \\
\hline Mother's milk (mother on Diet 1) & 0.00061 & 0.016 & 0.41 \\
\hline Prenatal exposure (mother on Diet 1) & 0.0047 & 0.046 & 0.46 \\
\hline Diet 1 & 1.7 & 9.5 & 58 \\
\hline Diet 2 & 0.63 & 3.9 & 25 \\
\hline Diet 3 & 0.24 & 1.3 & 8.4 \\
\hline
\end{tabular}

Excess Lifetime Risk [ ]

\begin{tabular}{llll}
\hline Diet 1 & $1.8 \mathrm{E}-04$ & $2.6 \mathrm{E}-03$ & $4.1 \mathrm{E}-02$ \\
Diet 2 & $8.6 \mathrm{E}-05$ & $9.9 \mathrm{E}-04$ & $1.3 \mathrm{E}-02$ \\
Diet 3 & $3.1 \mathrm{E}-05$ & $3.3 \mathrm{E}-04$ & $4.2 \mathrm{E}-03$ \\
Diet 4 & $4.4 \mathrm{E}-04$ & $6.7 \mathrm{E}-03$ & $8.5 \mathrm{E}-02$ \\
\hline
\end{tabular}

\section{Relative Risk [ ]}

\begin{tabular}{lccc}
\hline Diet 1 & 1.065 & 1.67 & 7.4 \\
Diet 2 & 1.025 & 1.26 & 4.3 \\
Diet 3 & 1.0094 & 1.085 & 2.1 \\
Diet 4 & 1.14 & 2.5 & 26 \\
\hline
\end{tabular}

\begin{tabular}{lccc}
\hline Diet 1 & 6.06 & 40.2 & 86 \\
Diet 2 & 2.45 & 20.5 & 77 \\
Diet 3 & 0.93 & 7.8 & 52 \\
Diet 4 & 12.65 & 60.1 & 96 \\
\hline
\end{tabular}

Diet 1 - Backyard cow milk + all other locally produced non-milk exposure pathways

Diet 2 - Locally produced commercial milk + all other locally produced non-milk exposure pathways

Diet 3 - Regionally mixed commercial milk + inhalation (other regionally mixed food items are minor contributors to the total)

Diet 4 - Goat milk (all other exposure pathways are of negligible importance compared to goat milk) 


\section{Location: Karns}

\section{Receptor: Male born in 1950}

\begin{tabular}{lccc}
\hline & \multicolumn{3}{c}{ Thyroid Dose [cGy] } \\
& \multicolumn{2}{c}{$95 \%$ Subjective Confidence Interval } \\
Exposure Pathway & lower limit & central estimate & upper limit \\
\hline Backyard Cow Milk & 1.5 & 8.9 & 51 \\
Commercial Milk (locally produced) & 0.44 & 2.9 & 21 \\
Commercial Milk (regionally mixed) & 0.17 & 1.1 & 8.9 \\
Goat Milk (locally produced) & 2.7 & 24 & 190 \\
Beef (locally produced) & 0.0021 & 0.038 & 0.87 \\
Leafy Vegetables (locally produced) & 0.00056 & 0.0063 & 0.055 \\
Eggs (locally produced) & 0.083 & 0.56 & 4.2 \\
Cottage Cheese (locally produced) & 0.0018 & 0.022 & 0.22 \\
Inhalation & 0.032 & 0.13 & 0.5 \\
Mother's milk (mother on Diet 1) & 0.00061 & 0.016 & 0.41 \\
Prenatal exposure (mother on Diet 1) & 0.0047 & 0.046 & 0.46 \\
Diet 1 & 1.7 & 9.8 & 55 \\
Diet 2 & 0.65 & 3.9 & 26 \\
Diet 3 & 0.24 & 1.3 & 9.3 \\
\hline
\end{tabular}

Excess Lifetime Risk [ ]

\begin{tabular}{llll}
\hline Diet 1 & $2.0 \mathrm{E}-05$ & $7.1 \mathrm{E}-04$ & $1.2 \mathrm{E}-02$ \\
Diet 2 & $1.0 \mathrm{E}-05$ & $2.6 \mathrm{E}-04$ & $5.6 \mathrm{E}-03$ \\
Diet 3 & $4.0 \mathrm{E}-06$ & $8.6 \mathrm{E}-05$ & $2.0 \mathrm{E}-03$ \\
Diet 4 & $4.9 \mathrm{E}-05$ & $1.6 \mathrm{E}-03$ & $3.4 \mathrm{E}-02$ \\
\hline
\end{tabular}

\section{Relative Risk [ ]}

\begin{tabular}{lccc}
\hline Diet 1 & 1.025 & 1.45 & 10.6 \\
Diet 2 & 1.0095 & 1.18 & 4.9 \\
Diet 3 & 1.003 & 1.059 & 2.4 \\
Diet 4 & 1.049 & 2.2 & 27 \\
\hline
\end{tabular}

\begin{tabular}{lccc}
\hline Diet 1 & 2.48 & 31.0 & 91 \\
Diet 2 & 0.94 & 15.4 & 80 \\
Diet 3 & 0.30 & 5.5 & 59 \\
Diet 4 & 4.63 & 53.8 & 96 \\
\hline
\end{tabular}

Diet 1 - Backyard cow milk + all other locally produced non-milk exposure pathways

Diet 2 - Locally produced commercial milk + all other locally produced non-milk exposure pathways

Diet 3 - Regionally mixed commercial milk + inhalation (other regionally mixed food items are minor contributors to the total)

Diet 4 - Goat milk (all other exposure pathways are of negligible importance compared to goat milk) 


\section{Location: Loudon}

Receptor: Female born in 1950

\begin{tabular}{lccc}
\hline & \multicolumn{3}{c}{ Thyroid Dose [cGy] } \\
& \multicolumn{2}{c}{$95 \%$ Subjective Confidence Interval } \\
Exposure Pathway & lower limit & central estimate & upper limit \\
\hline Backyard Cow Milk & 0.86 & 4.9 & 34 \\
Commercial Milk (locally produced) & 0.22 & 1.7 & 12 \\
Commercial Milk (regionally mixed) & 0.17 & 1.2 & 7.9 \\
Goat Milk (locally produced) & 1.6 & 13 & 120 \\
Beef (locally produced) & 0.0012 & 0.021 & 0.47 \\
Leafy Vegetables (locally produced) & 0.00034 & 0.0035 & 0.034 \\
Eggs (locally produced) & 0.041 & 0.32 & 2.5 \\
Cottage Cheese (locally produced) & 0.0011 & 0.012 & 0.15 \\
Inhalation & 0.019 & 0.075 & 0.31 \\
Mother's milk (mother on Diet 1) & 0.00034 & 0.0085 & 0.24 \\
Prenatal exposure (mother on Diet 1) & 0.0027 & 0.026 & 0.26 \\
Diet 1 & 0.97 & 5.4 & 37 \\
Diet 2 & 0.35 & 2.3 & 15 \\
Diet 3 & 0.21 & 1.3 & 8.2 \\
\hline
\end{tabular}

Excess Lifetime Risk [ ]

\begin{tabular}{lllc}
\hline Diet 1 & $8.6 \mathrm{E}-05$ & $1.5 \mathrm{E}-03$ & $2.2 \mathrm{E}-02$ \\
Diet 2 & $4.7 \mathrm{E}-05$ & $5.6 \mathrm{E}-04$ & $7.4 \mathrm{E}-03$ \\
Diet 3 & $2.9 \mathrm{E}-05$ & $3.1 \mathrm{E}-04$ & $4.1 \mathrm{E}-03$ \\
Diet 4 & $2.2 \mathrm{E}-04$ & $3.6 \mathrm{E}-03$ & $5.4 \mathrm{E}-02$ \\
\hline
\end{tabular}

\section{Relative Risk [ ]}

\begin{tabular}{lccc}
\hline Diet 1 & 1.035 & 1.37 & 4.8 \\
Diet 2 & 1.014 & 1.14 & 2.7 \\
Diet 3 & 1.0086 & 1.082 & 2.1 \\
Diet 4 & 1.075 & 1.8 & 15 \\
\hline
\end{tabular}

\section{Probability of Causation [\%]}

\begin{tabular}{lccc}
\hline Diet 1 & 3.34 & 26.7 & 79 \\
Diet 2 & 1.37 & 12.3 & 62 \\
Diet 3 & 0.85 & 7.6 & 52 \\
Diet 4 & 6.97 & 44.5 & 93 \\
\hline
\end{tabular}

Diet 1 - Backyard cow milk + all other locally produced non-milk exposure pathways

Diet 2 - Locally produced commercial milk + all other locally produced non-milk exposure pathways

Diet 3 - Regionally mixed commercial milk + inhalation (other regionally mixed food items are minor contributors to the total)

Diet 4 - Goat milk (all other exposure pathways are of negligible importance compared to goat milk) 


\section{Location: Loudon}

\section{Receptor: Male born in 1950}

\begin{tabular}{lccc}
\hline & \multicolumn{3}{c}{ Thyroid Dose [cGy] } \\
& \multicolumn{2}{c}{$95 \%$ Subjective Confidence Interval } \\
Exposure Pathway & lower limit & central estimate & upper limit \\
\hline Backyard Cow Milk & 0.86 & 5 & 32 \\
Commercial Milk (locally produced) & 0.23 & 1.6 & 12 \\
Commercial Milk (regionally mixed) & 0.17 & 1.1 & 8.9 \\
Goat Milk (locally produced) & 1.5 & 14 & 110 \\
Beef (locally produced) & 0.0012 & 0.021 & 0.47 \\
Leafy Vegetables (locally produced) & 0.00033 & 0.0034 & 0.032 \\
Eggs (locally produced) & 0.045 & 0.32 & 2.4 \\
Cottage Cheese (locally produced) & 0.001 & 0.012 & 0.13 \\
Inhalation & 0.018 & 0.077 & 0.3 \\
Mother's milk (mother on Diet 1) & 0.00034 & 0.0085 & 0.24 \\
Prenatal exposure (mother on Diet 1) & 0.0027 & 0.026 & 0.26 \\
Diet 1 & 0.99 & 5.5 & 36 \\
Diet 2 & 0.35 & 2.2 & 15 \\
Diet 3 & 0.21 & 1.2 & 9.2 \\
\hline
\end{tabular}

Excess Lifetime Risk [ ]

\begin{tabular}{lllc}
\hline Diet 1 & $1.1 \mathrm{E}-05$ & $3.8 \mathrm{E}-04$ & $7.1 \mathrm{E}-03$ \\
Diet 2 & $5.8 \mathrm{E}-06$ & $1.5 \mathrm{E}-04$ & $3.1 \mathrm{E}-03$ \\
Diet 3 & $3.7 \mathrm{E}-06$ & $8.3 \mathrm{E}-05$ & $1.9 \mathrm{E}-03$ \\
Diet 4 & $2.8 \mathrm{E}-05$ & $8.9 \mathrm{E}-04$ & $2.1 \mathrm{E}-02$ \\
\hline
\end{tabular}

\section{Relative Risk [ ]}

\begin{tabular}{lccc}
\hline Diet 1 & 1.013 & 1.26 & 6.1 \\
Diet 2 & 1.0049 & 1.098 & 3.4 \\
Diet 3 & 1.0029 & 1.056 & 2.4 \\
Diet 4 & 1.027 & 1.65 & 16 \\
\hline
\end{tabular}

\section{Probability of Causation [\%]}

\begin{tabular}{lccc}
\hline Diet 1 & 1.29 & 20.5 & 84 \\
Diet 2 & 0.49 & 8.9 & 71 \\
Diet 3 & 0.29 & 5.3 & 58 \\
Diet 4 & 2.66 & 39.3 & 94 \\
\hline
\end{tabular}

Diet 1 - Backyard cow milk + all other locally produced non-milk exposure pathways

Diet 2 - Locally produced commercial milk + all other locally produced non-milk exposure pathways

Diet 3 - Regionally mixed commercial milk + inhalation (other regionally mixed food items are minor contributors to the total)

Diet 4 - Goat milk (all other exposure pathways are of negligible importance compared to goat milk) 
Location: Harriman

Receptor: Female born in 1950

\begin{tabular}{lccc}
\hline & \multicolumn{3}{c}{ Thyroid Dose [cGy] } \\
Exposure Pathway & \multicolumn{2}{c}{$95 \%$ Subjective Confidence Interval } \\
\hline Backyard Cow Milk & lower limit & central estimate & upper limit \\
Commercial Milk (locally produced) & 0.52 & 2.9 & 18 \\
Commercial Milk (regionally mixed) & 0.14 & 0.97 & 7.7 \\
Goat Milk (locally produced) & 0.17 & 1.2 & 7.9 \\
Beef (locally produced) & 0.98 & 7.5 & 65 \\
Leafy Vegetables (locally produced) & 0.00065 & 0.013 & 0.31 \\
Eggs (locally produced) & 0.00019 & 0.0021 & 0.019 \\
Cottage Cheese (locally produced) & 0.025 & 0.19 & 1.4 \\
Inhalation & 0.0006 & 0.0074 & 0.086 \\
Mother's milk (mother on Diet 1) & 0.01 & 0.04 & 0.17 \\
Prenatal exposure (mother on Diet 1) & 0.00017 & 0.0051 & 0.14 \\
Diet 1 & 0.0017 & 0.016 & 0.16 \\
Diet 2 & 0.58 & 3.2 & 19 \\
Diet 3 & 0.21 & 1.3 & 9.1 \\
\hline
\end{tabular}

Excess Lifetime Risk [ ]

\begin{tabular}{llll}
\hline Diet 1 & $6.2 \mathrm{E}-05$ & $8.7 \mathrm{E}-04$ & $1.4 \mathrm{E}-02$ \\
Diet 2 & $3.1 \mathrm{E}-05$ & $3.1 \mathrm{E}-04$ & $4.8 \mathrm{E}-03$ \\
Diet 3 & $2.7 \mathrm{E}-05$ & $3.1 \mathrm{E}-04$ & $4.1 \mathrm{E}-03$ \\
Diet 4 & $1.4 \mathrm{E}-04$ & $2.2 \mathrm{E}-03$ & $3.4 \mathrm{E}-02$ \\
\hline
\end{tabular}

\section{Relative Risk [ ]}

\begin{tabular}{lccc}
\hline Diet 1 & 1.022 & 1.22 & 3.4 \\
Diet 2 & 1.0076 & 1.085 & 2.1 \\
Diet 3 & 1.008 & 1.08 & 2.1 \\
Diet 4 & 1.049 & 1.48 & 8.5 \\
\hline
\end{tabular}

\section{Probability of Causation [\%]}

\begin{tabular}{lccc}
\hline Diet 1 & 2.15 & 18.2 & 70 \\
Diet 2 & 0.75 & 7.8 & 53 \\
Diet 3 & 0.80 & 7.4 & 52 \\
Diet 4 & 4.69 & 32.6 & 88 \\
\hline
\end{tabular}

Diet 1 - Backyard cow milk + all other locally produced non-milk exposure pathways

Diet 2 - Locally produced commercial milk + all other locally produced non-milk exposure pathways

Diet 3 - Regionally mixed commercial milk + inhalation (other regionally mixed food items are minor contributors to the total)

Diet 4 - Goat milk (all other exposure pathways are of negligible importance compared to goat milk) 


\section{Location: Harriman}

\section{Receptor: Male born in 1950}

\begin{tabular}{lccc}
\hline & \multicolumn{3}{c}{ Thyroid Dose [cGy] } \\
& \multicolumn{2}{c}{$95 \%$ Subjective Confidence Interval } \\
Exposure Pathway & lower limit & central estimate & upper limit \\
\hline Backyard Cow Milk & 0.52 & 3 & 18 \\
Commercial Milk (locally produced) & 0.14 & 0.91 & 7.5 \\
Commercial Milk (regionally mixed) & 0.17 & 1.1 & 8.9 \\
Goat Milk (locally produced) & 0.9 & 8 & 62 \\
Beef (locally produced) & 0.00065 & 0.013 & 0.29 \\
Leafy Vegetables (locally produced) & 0.0002 & 0.0021 & 0.018 \\
Eggs (locally produced) & 0.029 & 0.19 & 1.4 \\
Cottage Cheese (locally produced) & 0.00056 & 0.0073 & 0.071 \\
Inhalation & 0.01 & 0.04 & 0.17 \\
Mother's milk (mother on Diet 1) & 0.00017 & 0.0051 & 0.14 \\
Prenatal exposure (mother on Diet 1) & 0.0017 & 0.016 & 0.16 \\
Diet 1 & 0.58 & 3.2 & 19 \\
Diet 2 & 0.22 & 1.2 & 9.3 \\
Diet 3 & 0.19 & 1.2 & 9 \\
\hline
\end{tabular}

Excess Lifetime Risk [ ]

\begin{tabular}{llll}
\hline Diet 1 & $6.6 \mathrm{E}-06$ & $2.3 \mathrm{E}-04$ & $3.7 \mathrm{E}-03$ \\
Diet 2 & $3.1 \mathrm{E}-06$ & $8.7 \mathrm{E}-05$ & $1.7 \mathrm{E}-03$ \\
Diet 3 & $3.4 \mathrm{E}-06$ & $8.0 \mathrm{E}-05$ & $1.9 \mathrm{E}-03$ \\
Diet 4 & $1.5 \mathrm{E}-05$ & $5.2 \mathrm{E}-04$ & $1.1 \mathrm{E}-02$ \\
\hline
\end{tabular}

\section{Relative Risk [ ]}

\begin{tabular}{lccc}
\hline Diet 1 & 1.0078 & 1.14 & 4.2 \\
Diet 2 & 1.003 & 1.055 & 2.4 \\
Diet 3 & 1.0028 & 1.055 & 2.3 \\
Diet 4 & 1.019 & 1.38 & 8.4 \\
\hline
\end{tabular}

\begin{tabular}{lccc}
\hline Diet 1 & 0.78 & 12.5 & 76 \\
Diet 2 & 0.30 & 5.2 & 58 \\
Diet 3 & 0.28 & 5.2 & 57 \\
Diet 4 & 1.87 & 27.7 & 88 \\
\hline
\end{tabular}

Diet 1 - Backyard cow milk + all other locally produced non-milk exposure pathways

Diet 2 - Locally produced commercial milk + all other locally produced non-milk exposure pathways

Diet 3 - Regionally mixed commercial milk + inhalation (other regionally mixed food items are minor contributors to the total)

Diet 4 - Goat milk (all other exposure pathways are of negligible importance compared to goat milk) 


\section{Location: Cedar Bluff}

Receptor: Female born in 1950

\begin{tabular}{lccc}
\hline & \multicolumn{3}{c}{ Thyroid Dose [cGy] } \\
& \multicolumn{2}{c}{$95 \%$ Subjective Confidence Interval } \\
Exposure Pathway & lower limit & central estimate & upper limit \\
\hline Backyard Cow Milk & 1.2 & 6.8 & 44 \\
Commercial Milk (locally produced) & 0.33 & 2.4 & 16 \\
Commercial Milk (regionally mixed) & 0.17 & 1.2 & 7.9 \\
Goat Milk (locally produced) & 2.2 & 18 & 160 \\
Beef (locally produced) & 0.0015 & 0.03 & 0.78 \\
Leafy Vegetables (locally produced) & 0.00049 & 0.005 & 0.046 \\
Eggs (locally produced) & 0.058 & 0.46 & 3.4 \\
Cottage Cheese (locally produced) & 0.0015 & 0.017 & 0.2 \\
Inhalation & 0.026 & 0.11 & 0.41 \\
Mother's milk (mother on Diet 1) & 0.00045 & 0.012 & 0.32 \\
Prenatal exposure (mother on Diet 1) & 0.0041 & 0.036 & 0.39 \\
Diet 1 & 1.4 & 7.5 & 48 \\
Diet 2 & 0.51 & 3.1 & 20 \\
Diet 3 & 0.23 & 1.3 & 8.3 \\
\hline
\end{tabular}

Excess Lifetime Risk [ ]

\begin{tabular}{lllc}
\hline Diet 1 & $1.3 \mathrm{E}-04$ & $2.0 \mathrm{E}-03$ & $3.1 \mathrm{E}-02$ \\
Diet 2 & $6.6 \mathrm{E}-05$ & $7.8 \mathrm{E}-04$ & $1.0 \mathrm{E}-02$ \\
Diet 3 & $2.9 \mathrm{E}-05$ & $3.2 \mathrm{E}-04$ & $4.1 \mathrm{E}-03$ \\
Diet 4 & $3.4 \mathrm{E}-04$ & $5.1 \mathrm{E}-03$ & $7.1 \mathrm{E}-02$ \\
\hline
\end{tabular}

\section{Relative Risk [ ]}

\begin{tabular}{lccc}
\hline Diet 1 & 1.048 & 1.53 & 6 \\
Diet 2 & 1.019 & 1.2 & 3.4 \\
Diet 3 & 1.009 & 1.083 & 2.1 \\
Diet 4 & 1.11 & 2.2 & 22 \\
\hline
\end{tabular}

\begin{tabular}{lccc} 
& \multicolumn{3}{c}{ Probability of Causation [\%] } \\
\hline Diet 1 & 4.61 & 34.7 & 83 \\
Diet 2 & 1.90 & 16.7 & 71 \\
Diet 3 & 0.89 & 7.7 & 52 \\
Diet 4 & 9.76 & 53.9 & 95 \\
\hline
\end{tabular}

Diet 1 - Backyard cow milk + all other locally produced non-milk exposure pathways

Diet 2 - Locally produced commercial milk + all other locally produced non-milk exposure pathways

Diet 3 - Regionally mixed commercial milk + inhalation (other regionally mixed food items are minor contributors to the total)

Diet 4 - Goat milk (all other exposure pathways are of negligible importance compared to goat milk) 


\section{Location: Cedar Bluff}

Receptor: Male born in 1950

\begin{tabular}{lccc}
\hline & \multicolumn{3}{c}{ Thyroid Dose [cGy] } \\
& \multicolumn{2}{c}{$95 \%$ Subjective Confidence Interval } \\
Exposure Pathway & lower limit & central estimate & upper limit \\
\hline Backyard Cow Milk & 1.3 & 7 & 43 \\
Commercial Milk (locally produced) & 0.35 & 2.3 & 18 \\
Commercial Milk (regionally mixed) & 0.17 & 1.1 & 8.9 \\
Goat Milk (locally produced) & 2.1 & 19 & 140 \\
Beef (locally produced) & 0.0017 & 0.029 & 0.74 \\
Leafy Vegetables (locally produced) & 0.0005 & 0.0049 & 0.042 \\
Eggs (locally produced) & 0.064 & 0.44 & 3.5 \\
Cottage Cheese (locally produced) & 0.0016 & 0.017 & 0.18 \\
Inhalation & 0.026 & 0.11 & 0.42 \\
Mother's milk (mother on Diet 1) & 0.00045 & 0.012 & 0.32 \\
Prenatal exposure (mother on Diet 1) & 0.0041 & 0.036 & 0.39 \\
Diet 1 & 1.4 & 7.7 & 47 \\
Diet 2 & 0.51 & 3.1 & 21 \\
Diet 3 & 0.22 & 1.3 & 9.3 \\
\hline
\end{tabular}

Excess Lifetime Risk [ ]

\begin{tabular}{lllc}
\hline Diet 1 & $1.4 \mathrm{E}-05$ & $5.7 \mathrm{E}-04$ & $9.9 \mathrm{E}-03$ \\
Diet 2 & $7.7 \mathrm{E}-06$ & $2.1 \mathrm{E}-04$ & $4.7 \mathrm{E}-03$ \\
Diet 3 & $3.8 \mathrm{E}-06$ & $8.5 \mathrm{E}-05$ & $1.9 \mathrm{E}-03$ \\
Diet 4 & $3.9 \mathrm{E}-05$ & $1.3 \mathrm{E}-03$ & $2.7 \mathrm{E}-02$ \\
\hline
\end{tabular}

\section{Relative Risk [ ]}

\begin{tabular}{lccc}
\hline Diet 1 & 1.019 & 1.34 & 8.6 \\
Diet 2 & 1.0075 & 1.14 & 4.1 \\
Diet 3 & 1.003 & 1.057 & 2.4 \\
Diet 4 & 1.04 & 1.88 & 21 \\
\hline
\end{tabular}

\section{Probability of Causation [\%]}

\begin{tabular}{lccc}
\hline Diet 1 & 1.91 & 25.6 & 88 \\
Diet 2 & 0.75 & 12.5 & 75 \\
Diet 3 & 0.30 & 5.4 & 58 \\
Diet 4 & 3.86 & 46.7 & 95 \\
\hline
\end{tabular}

Diet 1 - Backyard cow milk + all other locally produced non-milk exposure pathways

Diet 2 - Locally produced commercial milk + all other locally produced non-milk exposure pathways

Diet 3 - Regionally mixed commercial milk + inhalation (other regionally mixed food items are minor contributors to the total)

Diet 4 - Goat milk (all other exposure pathways are of negligible importance compared to goat milk) 


\section{Location: Oakdale}

Receptor: Female born in 1950

\begin{tabular}{lccc}
\hline & \multicolumn{3}{c}{ Thyroid Dose [cGy] } \\
Exposure Pathway & \multicolumn{2}{c}{$95 \%$ Subjective Confidence Interval } \\
\hline Backyard Cow Milk & lower limit & central estimate & upper limit \\
Commercial Milk (locally produced) & 0.26 & 1.6 & 11 \\
Commercial Milk (regionally mixed) & 0.073 & 0.56 & 4.4 \\
Goat Milk (locally produced) & 0.17 & 1.2 & 7.9 \\
Beef (locally produced) & 0.46 & 4.2 & 40 \\
Leafy Vegetables (locally produced) & 0.00038 & 0.0072 & 0.17 \\
Eggs (locally produced) & 0.0001 & 0.0012 & 0.011 \\
Cottage Cheese (locally produced) & 0.012 & 0.11 & 0.86 \\
Inhalation & 0.00034 & 0.0041 & 0.05 \\
Mother's milk (mother on Diet 1) & 0.0058 & 0.024 & 0.1 \\
Prenatal exposure (mother on Diet 1) & 0.000093 & 0.0029 & 0.082 \\
Diet 1 & 0.0009 & 0.0087 & 0.096 \\
Diet 2 & 0.29 & 1.8 & 12 \\
Diet 3 & 0.11 & 0.74 & 5.1 \\
\hline
\end{tabular}

Excess Lifetime Risk [ ]

\begin{tabular}{llll}
\hline Diet 1 & $3.0 \mathrm{E}-05$ & $4.8 \mathrm{E}-04$ & $7.4 \mathrm{E}-03$ \\
Diet 2 & $1.5 \mathrm{E}-05$ & $1.8 \mathrm{E}-04$ & $2.6 \mathrm{E}-03$ \\
Diet 3 & $2.6 \mathrm{E}-05$ & $3.0 \mathrm{E}-04$ & $4.0 \mathrm{E}-03$ \\
Diet 4 & $6.7 \mathrm{E}-05$ & $1.2 \mathrm{E}-03$ & $1.8 \mathrm{E}-02$ \\
\hline
\end{tabular}

\section{Relative Risk [ ]}

\begin{tabular}{lccc}
\hline Diet 1 & 1.01 & 1.12 & 2.3 \\
Diet 2 & 1.0041 & 1.048 & 1.62 \\
Diet 3 & 1.0077 & 1.079 & 2.1 \\
Diet 4 & 1.024 & 1.28 & 5.4 \\
\hline
\end{tabular}

\section{Probability of Causation [\%]}

\begin{tabular}{lccc}
\hline Diet 1 & 1.04 & 10.8 & 56 \\
Diet 2 & 0.41 & 4.6 & 37 \\
Diet 3 & 0.76 & 7.3 & 51 \\
Diet 4 & 2.29 & 21.4 & 81 \\
\hline
\end{tabular}

Diet 1 - Backyard cow milk + all other locally produced non-milk exposure pathways

Diet 2 - Locally produced commercial milk + all other locally produced non-milk exposure pathways

Diet 3 - Regionally mixed commercial milk + inhalation (other regionally mixed food items are minor contributors to the total)

Diet 4 - Goat milk (all other exposure pathways are of negligible importance compared to goat milk) 


\section{Location: Oakdale}

\section{Receptor: Male born in 1950}

\begin{tabular}{lccc}
\hline & \multicolumn{3}{c}{ Thyroid Dose [cGy] } \\
& \multicolumn{2}{c}{$95 \%$ Subjective Confidence Interval } \\
Exposure Pathway & lower limit & central estimate & upper limit \\
\hline Backyard Cow Milk & 0.28 & 1.7 & 11 \\
Commercial Milk (locally produced) & 0.075 & 0.55 & 4.4 \\
Commercial Milk (regionally mixed) & 0.17 & 1.1 & 8.9 \\
Goat Milk (locally produced) & 0.44 & 4.5 & 35 \\
Beef (locally produced) & 0.00041 & 0.007 & 0.17 \\
Leafy Vegetables (locally produced) & 0.0001 & 0.0011 & 0.011 \\
Eggs (locally produced) & 0.013 & 0.11 & 0.84 \\
Cottage Cheese (locally produced) & 0.00035 & 0.0041 & 0.047 \\
Inhalation & 0.0057 & 0.024 & 0.1 \\
Mother's milk (mother on Diet 1) & 0.000093 & 0.0029 & 0.082 \\
Prenatal exposure (mother on Diet 1) & 0.0009 & 0.0087 & 0.096 \\
Diet 1 & 0.31 & 1.8 & 12 \\
Diet 2 & 0.11 & 0.72 & 5.4 \\
Diet 3 & 0.18 & 1.2 & 9 \\
\hline
\end{tabular}

Excess Lifetime Risk [ ]

\begin{tabular}{llll}
\hline Diet 1 & $3.2 \mathrm{E}-06$ & $1.3 \mathrm{E}-04$ & $2.5 \mathrm{E}-03$ \\
Diet 2 & $1.8 \mathrm{E}-06$ & $5.1 \mathrm{E}-05$ & $1.1 \mathrm{E}-03$ \\
Diet 3 & $3.2 \mathrm{E}-06$ & $7.9 \mathrm{E}-05$ & $1.9 \mathrm{E}-03$ \\
Diet 4 & $9.1 \mathrm{E}-06$ & $3.0 \mathrm{E}-04$ & $6.6 \mathrm{E}-03$ \\
\hline
\end{tabular}

\section{Relative Risk [ ]}

\begin{tabular}{lccc}
\hline Diet 1 & 1.0044 & 1.082 & 2.8 \\
Diet 2 & 1.0016 & 1.033 & 1.78 \\
Diet 3 & 1.0027 & 1.054 & 2.3 \\
Diet 4 & 1.01 & 1.21 & 5.6 \\
\hline
\end{tabular}

\section{Probability of Causation [\%]}

\begin{tabular}{lccc}
\hline Diet 1 & 0.44 & 7.5 & 63 \\
Diet 2 & 0.16 & 3.2 & 43 \\
Diet 3 & 0.27 & 5.2 & 57 \\
Diet 4 & 1.03 & 17.3 & 81 \\
\hline
\end{tabular}

Diet 1 - Backyard cow milk + all other locally produced non-milk exposure pathways

Diet 2 - Locally produced commercial milk + all other locally produced non-milk exposure pathways

Diet 3 - Regionally mixed commercial milk + inhalation (other regionally mixed food items are minor contributors to the total)

Diet 4 - Goat milk (all other exposure pathways are of negligible importance compared to goat milk) 


\section{Location: Claxton}

Receptor: Female born in 1950

\begin{tabular}{lccc}
\hline & \multicolumn{3}{c}{ Thyroid Dose [cGy] } \\
Exposure Pathway & \multicolumn{2}{c}{$95 \%$ Subjective Confidence Interval } \\
\hline Backyard Cow Milk & 1 & 5.7 & 36 \\
Commercial Milk (locally produced) & 0.28 & 2 & 14 \\
Commercial Milk (regionally mixed) & 0.17 & 1.2 & 7.9 \\
Goat Milk (locally produced) & 2 & 15 & 130 \\
Beef (locally produced) & 0.0013 & 0.025 & 0.65 \\
Leafy Vegetables (locally produced) & 0.0004 & 0.0042 & 0.038 \\
Eggs (locally produced) & 0.048 & 0.38 & 2.8 \\
Cottage Cheese (locally produced) & 0.0013 & 0.014 & 0.16 \\
Inhalation & 0.022 & 0.088 & 0.34 \\
Mother's milk (mother on Diet 1) & 0.00037 & 0.011 & 0.27 \\
Prenatal exposure (mother on Diet 1) & 0.0035 & 0.03 & 0.32 \\
Diet 1 & 1.2 & 6.3 & 39 \\
Diet 2 & 0.43 & 2.6 & 17 \\
Diet 3 & 0.22 & 1.3 & 8.2 \\
\hline
\end{tabular}

Excess Lifetime Risk [ ]

\begin{tabular}{llll}
\hline Diet 1 & $1.1 \mathrm{E}-04$ & $1.7 \mathrm{E}-03$ & $2.6 \mathrm{E}-02$ \\
Diet 2 & $5.8 \mathrm{E}-05$ & $6.6 \mathrm{E}-04$ & $8.2 \mathrm{E}-03$ \\
Diet 3 & $2.9 \mathrm{E}-05$ & $3.2 \mathrm{E}-04$ & $4.1 \mathrm{E}-03$ \\
Diet 4 & $2.8 \mathrm{E}-04$ & $4.4 \mathrm{E}-03$ & $5.9 \mathrm{E}-02$ \\
\hline
\end{tabular}

\section{Relative Risk [ ]}

\begin{tabular}{lccc}
\hline Diet 1 & 1.041 & 1.45 & 5.2 \\
Diet 2 & 1.016 & 1.17 & 3.1 \\
Diet 3 & 1.0088 & 1.082 & 2.1 \\
Diet 4 & 1.092 & 1.97 & 18 \\
\hline
\end{tabular}

\begin{tabular}{lccc} 
& \multicolumn{3}{c}{ Probability of Causation [\%] } \\
\hline Diet 1 & 3.90 & 30.8 & 81 \\
Diet 2 & 1.61 & 14.3 & 67 \\
Diet 3 & 0.88 & 7.6 & 52 \\
Diet 4 & 8.38 & 49.1 & 94 \\
\hline
\end{tabular}

Diet 1 - Backyard cow milk + all other locally produced non-milk exposure pathways

Diet 2 - Locally produced commercial milk + all other locally produced non-milk exposure pathways

Diet 3 - Regionally mixed commercial milk + inhalation (other regionally mixed food items are minor contributors to the total)

Diet 4 - Goat milk (all other exposure pathways are of negligible importance compared to goat milk) 


\section{Location: Claxton}

\section{Receptor: Male born in 1950}

\begin{tabular}{lccc}
\hline & \multicolumn{3}{c}{ Thyroid Dose [cGy] } \\
& \multicolumn{2}{c}{$95 \%$ Subjective Confidence Interval } \\
Exposure Pathway & lower limit & central estimate & upper limit \\
\hline Backyard Cow Milk & 1.1 & 5.9 & 35 \\
Commercial Milk (locally produced) & 0.29 & 1.9 & 15 \\
Commercial Milk (regionally mixed) & 0.17 & 1.1 & 8.9 \\
Goat Milk (locally produced) & 1.8 & 16 & 120 \\
Beef (locally produced) & 0.0014 & 0.025 & 0.61 \\
Leafy Vegetables (locally produced) & 0.00041 & 0.0042 & 0.035 \\
Eggs (locally produced) & 0.055 & 0.38 & 2.9 \\
Cottage Cheese (locally produced) & 0.0013 & 0.015 & 0.15 \\
Inhalation & 0.022 & 0.088 & 0.34 \\
Mother's milk (mother on Diet 1) & 0.00037 & 0.011 & 0.27 \\
Prenatal exposure (mother on Diet 1) & 0.0035 & 0.03 & 0.32 \\
Diet 1 & 1.2 & 6.4 & 39 \\
Diet 2 & 0.44 & 2.5 & 18 \\
Diet 3 & 0.22 & 1.2 & 9.2 \\
\hline
\end{tabular}

Excess Lifetime Risk [ ]

\begin{tabular}{llll}
\hline Diet 1 & $1.2 \mathrm{E}-05$ & $4.7 \mathrm{E}-04$ & $8.2 \mathrm{E}-03$ \\
Diet 2 & $6.5 \mathrm{E}-06$ & $1.7 \mathrm{E}-04$ & $3.9 \mathrm{E}-03$ \\
Diet 3 & $3.7 \mathrm{E}-06$ & $8.4 \mathrm{E}-05$ & $1.9 \mathrm{E}-03$ \\
Diet 4 & $3.3 \mathrm{E}-05$ & $1.1 \mathrm{E}-03$ & $2.3 \mathrm{E}-02$ \\
\hline
\end{tabular}

\section{Relative Risk [ ]}

\begin{tabular}{lccc}
\hline Diet 1 & 1.016 & 1.29 & 7.2 \\
Diet 2 & 1.0061 & 1.12 & 3.6 \\
Diet 3 & 1.0029 & 1.057 & 2.4 \\
Diet 4 & 1.033 & 1.75 & 17 \\
\hline
\end{tabular}

\section{Probability of Causation [\%]}

\begin{tabular}{lccc}
\hline Diet 1 & 1.54 & 22.3 & 86 \\
Diet 2 & 0.61 & 10.7 & 72 \\
Diet 3 & 0.29 & 5.4 & 58 \\
Diet 4 & 3.17 & 42.6 & 94 \\
\hline
\end{tabular}

Diet 1 - Backyard cow milk + all other locally produced non-milk exposure pathways

Diet 2 - Locally produced commercial milk + all other locally produced non-milk exposure pathways

Diet 3 - Regionally mixed commercial milk + inhalation (other regionally mixed food items are minor contributors to the total)

Diet 4 - Goat milk (all other exposure pathways are of negligible importance compared to goat milk) 
Location: Dutch Valley

Receptor: Female born in 1950

\begin{tabular}{lccc}
\hline & \multicolumn{3}{c}{ Thyroid Dose [cGy] } \\
Exposure Pathway & $95 \%$ Subjective Confidence Interval \\
\hline Backyard Cow Milk & lower limit & central estimate & upper limit \\
Commercial Milk (locally produced) & 0.49 & 2.8 & 17 \\
Commercial Milk (regionally mixed) & 0.13 & 0.95 & 7 \\
Goat Milk (locally produced) & 0.17 & 1.2 & 7.9 \\
Beef (locally produced) & 0.89 & 7.5 & 65 \\
Leafy Vegetables (locally produced) & 0.00062 & 0.012 & 0.28 \\
Eggs (locally produced) & 0.00019 & 0.002 & 0.019 \\
Cottage Cheese (locally produced) & 0.023 & 0.18 & 1.4 \\
Inhalation & 0.00058 & 0.0071 & 0.077 \\
Mother's milk (mother on Diet 1) & 0.0098 & 0.041 & 0.17 \\
Prenatal exposure (mother on Diet 1) & 0.00017 & 0.0049 & 0.13 \\
Diet 1 & 0.0015 & 0.015 & 0.15 \\
Diet 2 & 0.56 & 3.1 & 18 \\
Diet 3 & 0.19 & 1.2 & 8.4 \\
\hline
\end{tabular}

Excess Lifetime Risk [ ]

\begin{tabular}{llll}
\hline Diet 1 & $5.7 \mathrm{E}-05$ & $8.2 \mathrm{E}-04$ & $1.4 \mathrm{E}-02$ \\
Diet 2 & $2.8 \mathrm{E}-05$ & $3.0 \mathrm{E}-04$ & $4.6 \mathrm{E}-03$ \\
Diet 3 & $2.7 \mathrm{E}-05$ & $3.1 \mathrm{E}-04$ & $4.1 \mathrm{E}-03$ \\
Diet 4 & $1.3 \mathrm{E}-04$ & $2.1 \mathrm{E}-03$ & $3.0 \mathrm{E}-02$ \\
\hline
\end{tabular}

\section{Relative Risk [ ]}

\begin{tabular}{lccc}
\hline Diet 1 & 1.023 & 1.22 & 3.2 \\
Diet 2 & 1.0077 & 1.082 & 2.1 \\
Diet 3 & 1.008 & 1.08 & 2.1 \\
Diet 4 & 1.046 & 1.47 & 8.5 \\
\hline
\end{tabular}

\begin{tabular}{lccc} 
& \multicolumn{3}{c}{ Probability of Causation [\%] } \\
\hline Diet 1 & 2.22 & 17.6 & 68 \\
Diet 2 & 0.76 & 7.5 & 51 \\
Diet 3 & 0.79 & 7.4 & 52 \\
Diet 4 & 4.38 & 31.7 & 88 \\
\hline
\end{tabular}

Diet 1 - Backyard cow milk + all other locally produced non-milk exposure pathways

Diet 2 - Locally produced commercial milk + all other locally produced non-milk exposure pathways

Diet 3 - Regionally mixed commercial milk + inhalation (other regionally mixed food items are minor contributors to the total)

Diet 4 - Goat milk (all other exposure pathways are of negligible importance compared to goat milk) 


\section{Location: Dutch Valley}

Receptor: Male born in 1950

\begin{tabular}{lccc}
\hline & \multicolumn{3}{c}{ Thyroid Dose [cGy] } \\
& \multicolumn{2}{c}{$95 \%$ Subjective Confidence Interval } \\
Exposure Pathway & lower limit & central estimate & upper limit \\
\hline Backyard Cow Milk & 0.49 & 2.9 & 16 \\
Commercial Milk (locally produced) & 0.14 & 0.91 & 6.6 \\
Commercial Milk (regionally mixed) & 0.17 & 1.1 & 8.9 \\
Goat Milk (locally produced) & 0.82 & 7.8 & 58 \\
Beef (locally produced) & 0.00063 & 0.012 & 0.27 \\
Leafy Vegetables (locally produced) & 0.00019 & 0.002 & 0.018 \\
Eggs (locally produced) & 0.026 & 0.18 & 1.3 \\
Cottage Cheese (locally produced) & 0.00058 & 0.0071 & 0.069 \\
Inhalation & 0.01 & 0.042 & 0.17 \\
Mother's milk (mother on Diet 1) & 0.00017 & 0.0049 & 0.13 \\
Prenatal exposure (mother on Diet 1) & 0.0015 & 0.015 & 0.15 \\
Diet 1 & 0.55 & 3.1 & 18 \\
Diet 2 & 0.2 & 1.2 & 8.3 \\
Diet 3 & 0.19 & 1.2 & 9 \\
\hline
\end{tabular}

Excess Lifetime Risk [ ]

\begin{tabular}{llll}
\hline Diet 1 & $6.4 \mathrm{E}-06$ & $2.3 \mathrm{E}-04$ & $3.6 \mathrm{E}-03$ \\
Diet 2 & $3.0 \mathrm{E}-06$ & $8.3 \mathrm{E}-05$ & $1.7 \mathrm{E}-03$ \\
Diet 3 & $3.4 \mathrm{E}-06$ & $8.1 \mathrm{E}-05$ & $1.9 \mathrm{E}-03$ \\
Diet 4 & $1.5 \mathrm{E}-05$ & $5.0 \mathrm{E}-04$ & $1.0 \mathrm{E}-02$ \\
\hline
\end{tabular}

\section{Relative Risk [ ]}

\begin{tabular}{lccc}
\hline Diet 1 & 1.0075 & 1.14 & 4.1 \\
Diet 2 & 1.0029 & 1.055 & 2.2 \\
Diet 3 & 1.0028 & 1.055 & 2.3 \\
Diet 4 & 1.017 & 1.37 & 8.7 \\
\hline
\end{tabular}

\begin{tabular}{lccc} 
& \multicolumn{3}{c}{ Probability of Causation [\%] } \\
\hline Diet 1 & 0.74 & 12.2 & 75 \\
Diet 2 & 0.29 & 5.2 & 55 \\
Diet 3 & 0.28 & 5.2 & 57 \\
Diet 4 & 1.66 & 26.5 & 88 \\
\hline
\end{tabular}

Diet 1 - Backyard cow milk + all other locally produced non-milk exposure pathways

Diet 2 - Locally produced commercial milk + all other locally produced non-milk exposure pathways

Diet 3 - Regionally mixed commercial milk + inhalation (other regionally mixed food items are minor contributors to the total)

Diet 4 - Goat milk (all other exposure pathways are of negligible importance compared to goat milk) 


\section{Location: Clinton}

Receptor: Female born in 1950

\begin{tabular}{lccc}
\hline & \multicolumn{3}{c}{ Thyroid Dose [cGy] } \\
Exposure Pathway & \multicolumn{2}{c}{$95 \%$ Subjective Confidence Interval } \\
\hline Backyard Cow Milk & lower limit & central estimate & upper limit \\
Commercial Milk (locally produced) & 0.79 & 4.4 & 29 \\
Commercial Milk (regionally mixed) & 0.21 & 1.5 & 11 \\
Goat Milk (locally produced) & 0.17 & 1.2 & 7.9 \\
Beef (locally produced) & 1.4 & 12 & 100 \\
Leafy Vegetables (locally produced) & 0.00099 & 0.019 & 0.46 \\
Eggs (locally produced) & 0.00031 & 0.0032 & 0.029 \\
Cottage Cheese (locally produced) & 0.037 & 0.29 & 2.1 \\
Inhalation & 0.00097 & 0.011 & 0.13 \\
Mother's milk (mother on Diet 1) & 0.017 & 0.067 & 0.27 \\
Prenatal exposure (mother on Diet 1) & 0.00027 & 0.0078 & 0.21 \\
Diet 1 & 0.0026 & 0.023 & 0.25 \\
Diet 2 & 0.89 & 4.8 & 32 \\
Diet 3 & 0.33 & 2.1 & 13 \\
\hline
\end{tabular}

Excess Lifetime Risk [ ]

\begin{tabular}{llll}
\hline Diet 1 & $8.6 \mathrm{E}-05$ & $1.3 \mathrm{E}-03$ & $1.9 \mathrm{E}-02$ \\
Diet 2 & $4.4 \mathrm{E}-05$ & $5.1 \mathrm{E}-04$ & $6.3 \mathrm{E}-03$ \\
Diet 3 & $2.8 \mathrm{E}-05$ & $3.1 \mathrm{E}-04$ & $4.1 \mathrm{E}-03$ \\
Diet 4 & $2.0 \mathrm{E}-04$ & $3.3 \mathrm{E}-03$ & $4.5 \mathrm{E}-02$ \\
\hline
\end{tabular}

\section{Relative Risk [ ]}

\begin{tabular}{lccc}
\hline Diet 1 & 1.031 & 1.34 & 4.3 \\
Diet 2 & 1.012 & 1.13 & 2.5 \\
Diet 3 & 1.0084 & 1.081 & 2.1 \\
Diet 4 & 1.069 & 1.75 & 14 \\
\hline
\end{tabular}

\begin{tabular}{lccc}
\hline Diet 1 & 3.03 & 25.0 & 76 \\
Diet 2 & 1.22 & 11.4 & 59 \\
Diet 3 & 0.84 & 7.5 & 52 \\
Diet 4 & 6.41 & 42.3 & 92 \\
\hline
\end{tabular}

Diet 1 - Backyard cow milk + all other locally produced non-milk exposure pathways

Diet 2 - Locally produced commercial milk + all other locally produced non-milk exposure pathways

Diet 3 - Regionally mixed commercial milk + inhalation (other regionally mixed food items are minor contributors to the total)

Diet 4 - Goat milk (all other exposure pathways are of negligible importance compared to goat milk) 


\section{Location: Clinton}

\section{Receptor: Male born in 1950}

\begin{tabular}{lccc}
\hline & \multicolumn{3}{c}{ Thyroid Dose [cGy] } \\
& \multicolumn{2}{c}{$95 \%$ Subjective Confidence Interval } \\
Exposure Pathway & lower limit & central estimate & upper limit \\
\hline Backyard Cow Milk & 0.84 & 4.5 & 28 \\
Commercial Milk (locally produced) & 0.22 & 1.5 & 11 \\
Commercial Milk (regionally mixed) & 0.17 & 1.1 & 8.9 \\
Goat Milk (locally produced) & 1.3 & 12 & 90 \\
Beef (locally produced) & 0.0011 & 0.019 & 0.46 \\
Leafy Vegetables (locally produced) & 0.0003 & 0.0032 & 0.027 \\
Eggs (locally produced) & 0.04 & 0.29 & 2.2 \\
Cottage Cheese (locally produced) & 0.00099 & 0.011 & 0.12 \\
Inhalation & 0.016 & 0.067 & 0.27 \\
Mother's milk (mother on Diet 1) & 0.00027 & 0.0078 & 0.21 \\
Prenatal exposure (mother on Diet 1) & 0.0026 & 0.023 & 0.25 \\
Diet 1 & 0.95 & 5 & 30 \\
Diet 2 & 0.32 & 2 & 14 \\
Diet 3 & 0.21 & 1.2 & 9.1 \\
\hline
\end{tabular}

Excess Lifetime Risk [ ]

\begin{tabular}{llll}
\hline Diet 1 & $8.9 \mathrm{E}-06$ & $3.7 \mathrm{E}-04$ & $6.4 \mathrm{E}-03$ \\
Diet 2 & $4.9 \mathrm{E}-06$ & $1.4 \mathrm{E}-04$ & $3.1 \mathrm{E}-03$ \\
Diet 3 & $3.6 \mathrm{E}-06$ & $8.3 \mathrm{E}-05$ & $1.9 \mathrm{E}-03$ \\
Diet 4 & $2.5 \mathrm{E}-05$ & $8.1 \mathrm{E}-04$ & $1.7 \mathrm{E}-02$ \\
\hline
\end{tabular}

\section{Relative Risk [ ]}

\begin{tabular}{lccc}
\hline Diet 1 & 1.013 & 1.22 & 5.8 \\
Diet 2 & 1.0045 & 1.091 & 3 \\
Diet 3 & 1.0029 & 1.056 & 2.4 \\
Diet 4 & 1.028 & 1.56 & 13 \\
\hline
\end{tabular}

\section{Probability of Causation [\%]}

\begin{tabular}{lccc}
\hline Diet 1 & 1.27 & 18.1 & 82 \\
Diet 2 & 0.45 & 8.3 & 66 \\
Diet 3 & 0.29 & 5.3 & 58 \\
Diet 4 & 2.69 & 35.6 & 92 \\
\hline
\end{tabular}

Diet 1 - Backyard cow milk + all other locally produced non-milk exposure pathways

Diet 2 - Locally produced commercial milk + all other locally produced non-milk exposure pathways

Diet 3 - Regionally mixed commercial milk + inhalation (other regionally mixed food items are minor contributors to the total)

Diet 4 - Goat milk (all other exposure pathways are of negligible importance compared to goat milk) 
Location: Friendsville

Receptor: Female born in 1950

\begin{tabular}{lccc}
\hline & \multicolumn{3}{c}{ Thyroid Dose [cGy] } \\
Exposure Pathway & \multicolumn{2}{c}{$95 \%$ Subjective Confidence Interval } \\
\hline Backyard Cow Milk & lower limit & central estimate & upper limit \\
Commercial Milk (locally produced) & 0.44 & 2.5 & 16 \\
Commercial Milk (regionally mixed) & 0.11 & 0.88 & 5.6 \\
Goat Milk (locally produced) & 0.17 & 1.2 & 7.9 \\
Beef (locally produced) & 0.77 & 6.9 & 64 \\
Leafy Vegetables (locally produced) & 0.00065 & 0.011 & 0.28 \\
Eggs (locally produced) & 0.00017 & 0.0018 & 0.018 \\
Cottage Cheese (locally produced) & 0.021 & 0.17 & 1.4 \\
Inhalation & 0.0005 & 0.0065 & 0.073 \\
Mother's milk (mother on Diet 1) & 0.01 & 0.046 & 0.21 \\
Prenatal exposure (mother on Diet 1) & 0.00018 & 0.0047 & 0.12 \\
Diet 1 & 0.0014 & 0.014 & 0.14 \\
Diet 2 & 0.48 & 2.8 & 18 \\
Diet 3 & 0.17 & 1.2 & 7.1 \\
\hline
\end{tabular}

Excess Lifetime Risk [ ]

\begin{tabular}{llll}
\hline Diet 1 & $5.6 \mathrm{E}-05$ & $7.6 \mathrm{E}-04$ & $1.3 \mathrm{E}-02$ \\
Diet 2 & $2.4 \mathrm{E}-05$ & $3.0 \mathrm{E}-04$ & $3.8 \mathrm{E}-03$ \\
Diet 3 & $2.7 \mathrm{E}-05$ & $3.1 \mathrm{E}-04$ & $4.1 \mathrm{E}-03$ \\
Diet 4 & $1.2 \mathrm{E}-04$ & $1.9 \mathrm{E}-03$ & $2.7 \mathrm{E}-02$ \\
\hline
\end{tabular}

\section{Relative Risk [ ]}

\begin{tabular}{lccc}
\hline Diet 1 & 1.019 & 1.2 & 3.1 \\
Diet 2 & 1.0073 & 1.075 & 1.94 \\
Diet 3 & 1.0081 & 1.08 & 2.1 \\
Diet 4 & 1.039 & 1.44 & 8 \\
\hline
\end{tabular}

\section{Probability of Causation [\%]}

\begin{tabular}{lccc}
\hline Diet 1 & 1.86 & 16.6 & 67 \\
Diet 2 & 0.72 & 7.0 & 48 \\
Diet 3 & 0.81 & 7.4 & 52 \\
Diet 4 & 3.72 & 30.4 & 87 \\
\hline
\end{tabular}

Diet 1 - Backyard cow milk + all other locally produced non-milk exposure pathways

Diet 2 - Locally produced commercial milk + all other locally produced non-milk exposure pathways

Diet 3 - Regionally mixed commercial milk + inhalation (other regionally mixed food items are minor contributors to the total)

Diet 4 - Goat milk (all other exposure pathways are of negligible importance compared to goat milk) 
Location: Friendsville

Receptor: Male born in 1950

\begin{tabular}{lccc}
\hline & \multicolumn{3}{c}{ Thyroid Dose [cGy] } \\
& \multicolumn{2}{c}{$95 \%$ Subjective Confidence Interval } \\
Exposure Pathway & lower limit & central estimate & upper limit \\
\hline Backyard Cow Milk & 0.42 & 2.6 & 17 \\
Commercial Milk (locally produced) & 0.12 & 0.87 & 5.8 \\
Commercial Milk (regionally mixed) & 0.17 & 1.1 & 8.9 \\
Goat Milk (locally produced) & 0.76 & 7.2 & 62 \\
Beef (locally produced) & 0.00063 & 0.011 & 0.27 \\
Leafy Vegetables (locally produced) & 0.00016 & 0.0019 & 0.018 \\
Eggs (locally produced) & 0.023 & 0.17 & 1.2 \\
Cottage Cheese (locally produced) & 0.00054 & 0.0066 & 0.065 \\
Inhalation & 0.01 & 0.047 & 0.18 \\
Mother's milk (mother on Diet 1) & 0.00018 & 0.0047 & 0.12 \\
Prenatal exposure (mother on Diet 1) & 0.0014 & 0.014 & 0.14 \\
Diet 1 & 0.48 & 2.9 & 18 \\
Diet 2 & 0.18 & 1.1 & 7.5 \\
Diet 3 & 0.2 & 1.2 & 9 \\
\hline
\end{tabular}

Excess Lifetime Risk [ ]

\begin{tabular}{lllc}
\hline Diet 1 & $6.0 \mathrm{E}-06$ & $2.0 \mathrm{E}-04$ & $3.6 \mathrm{E}-03$ \\
Diet 2 & $2.8 \mathrm{E}-06$ & $7.6 \mathrm{E}-05$ & $1.7 \mathrm{E}-03$ \\
Diet 3 & $3.4 \mathrm{E}-06$ & $8.1 \mathrm{E}-05$ & $1.9 \mathrm{E}-03$ \\
Diet 4 & $1.5 \mathrm{E}-05$ & $4.7 \mathrm{E}-04$ & $8.7 \mathrm{E}-03$ \\
\hline
\end{tabular}

\section{Relative Risk [ ]}

\begin{tabular}{lccc}
\hline Diet 1 & 1.0075 & 1.14 & 4 \\
Diet 2 & 1.0027 & 1.053 & 2.2 \\
Diet 3 & 1.0028 & 1.056 & 2.3 \\
Diet 4 & 1.014 & 1.34 & 8.5 \\
\hline
\end{tabular}

\begin{tabular}{lccc} 
& \multicolumn{3}{c}{ Probability of Causation [\%] } \\
\hline Diet 1 & 0.74 & 12.3 & 75 \\
Diet 2 & 0.27 & 5.1 & 54 \\
Diet 3 & 0.28 & 5.3 & 57 \\
Diet 4 & 1.34 & 25.3 & 88 \\
\hline
\end{tabular}

Diet 1 - Backyard cow milk + all other locally produced non-milk exposure pathways

Diet 2 - Locally produced commercial milk + all other locally produced non-milk exposure pathways

Diet 3 - Regionally mixed commercial milk + inhalation (other regionally mixed food items are minor contributors to the total)

Diet 4 - Goat milk (all other exposure pathways are of negligible importance compared to goat milk) 
Location: Wartburg

Receptor: Female born in 1950

\begin{tabular}{lccc}
\hline & \multicolumn{3}{c}{ Thyroid Dose [cGy] } \\
Exposure Pathway & \multicolumn{2}{c}{$95 \%$ Subjective Confidence Interval } \\
\hline Backyard Cow Milk & lower limit & central estimate & upper limit \\
Commercial Milk (locally produced) & 0.11 & 0.73 & 4.6 \\
Commercial Milk (regionally mixed) & 0.032 & 0.24 & 1.9 \\
Goat Milk (locally produced) & 0.17 & 1.2 & 7.9 \\
Beef (locally produced) & 0.23 & 1.9 & 18 \\
Leafy Vegetables (locally produced) & 0.00017 & 0.0029 & 0.078 \\
Eggs (locally produced) & 0.000042 & 0.00049 & 0.0053 \\
Cottage Cheese (locally produced) & 0.0052 & 0.046 & 0.37 \\
Inhalation & 0.00015 & 0.0018 & 0.021 \\
Mother's milk (mother on Diet 1) & 0.0024 & 0.011 & 0.051 \\
Prenatal exposure (mother on Diet 1) & 0.000042 & 0.0012 & 0.038 \\
Diet 1 & 0.00037 & 0.0035 & 0.04 \\
Diet 2 & 0.13 & 0.79 & 5 \\
Diet 3 & 0.05 & 0.31 & 2.3 \\
\hline
\end{tabular}

Excess Lifetime Risk [ ]

\begin{tabular}{lllc}
\hline Diet 1 & $1.1 \mathrm{E}-05$ & $2.1 \mathrm{E}-04$ & $3.4 \mathrm{E}-03$ \\
Diet 2 & $6.5 \mathrm{E}-06$ & $7.9 \mathrm{E}-05$ & $1.1 \mathrm{E}-03$ \\
Diet 3 & $2.6 \mathrm{E}-05$ & $2.9 \mathrm{E}-04$ & $4.0 \mathrm{E}-03$ \\
Diet 4 & $3.0 \mathrm{E}-05$ & $5.0 \mathrm{E}-04$ & $7.7 \mathrm{E}-03$ \\
\hline
\end{tabular}

Relative Risk [ ]

\begin{tabular}{lccc}
\hline Diet 1 & 1.0052 & 1.051 & 1.61 \\
Diet 2 & 1.0019 & 1.02 & 1.31 \\
Diet 3 & 1.0074 & 1.078 & 2.1 \\
Diet 4 & 1.011 & 1.13 & 3.2 \\
\hline
\end{tabular}

\section{Probability of Causation [\%]}

\begin{tabular}{lccc}
\hline Diet 1 & 0.52 & 4.9 & 38 \\
Diet 2 & 0.19 & 1.9 & 24 \\
Diet 3 & 0.74 & 7.2 & 51 \\
Diet 4 & 1.07 & 11.2 & 68 \\
\hline
\end{tabular}

Diet 1 - Backyard cow milk + all other locally produced non-milk exposure pathways

Diet 2 - Locally produced commercial milk + all other locally produced non-milk exposure pathways

Diet 3 - Regionally mixed commercial milk + inhalation (other regionally mixed food items are minor contributors to the total)

Diet 4 - Goat milk (all other exposure pathways are of negligible importance compared to goat milk) 


\section{Location: Wartburg}

\section{Receptor: Male born in 1950}

\begin{tabular}{lccc}
\hline & \multicolumn{3}{c}{ Thyroid Dose [cGy] } \\
& \multicolumn{2}{c}{$95 \%$ Subjective Confidence Interval } \\
Exposure Pathway & lower limit & central estimate & upper limit \\
\hline Backyard Cow Milk & 0.11 & 0.7 & 4.7 \\
Commercial Milk (locally produced) & 0.035 & 0.23 & 1.9 \\
Commercial Milk (regionally mixed) & 0.17 & 1.1 & 8.9 \\
Goat Milk (locally produced) & 0.2 & 1.9 & 17 \\
Beef (locally produced) & 0.00017 & 0.0029 & 0.077 \\
Leafy Vegetables (locally produced) & 0.000042 & 0.0005 & 0.0051 \\
Eggs (locally produced) & 0.0055 & 0.046 & 0.34 \\
Cottage Cheese (locally produced) & 0.00013 & 0.0018 & 0.018 \\
Inhalation & 0.0025 & 0.011 & 0.046 \\
Mother's milk (mother on Diet 1) & 0.000042 & 0.0012 & 0.038 \\
Prenatal exposure (mother on Diet 1) & 0.00037 & 0.0035 & 0.04 \\
Diet 1 & 0.12 & 0.78 & 5.1 \\
Diet 2 & 0.052 & 0.31 & 2.4 \\
Diet 3 & 0.18 & 1.1 & 8.9 \\
\hline
\end{tabular}

Excess Lifetime Risk [ ]

\begin{tabular}{llll}
\hline Diet 1 & $1.3 \mathrm{E}-06$ & $5.6 \mathrm{E}-05$ & $1.1 \mathrm{E}-03$ \\
Diet 2 & $7.2 \mathrm{E}-07$ & $2.2 \mathrm{E}-05$ & $4.2 \mathrm{E}-04$ \\
Diet 3 & $3.1 \mathrm{E}-06$ & $7.8 \mathrm{E}-05$ & $1.9 \mathrm{E}-03$ \\
Diet 4 & $4.4 \mathrm{E}-06$ & $1.3 \mathrm{E}-04$ & $3.4 \mathrm{E}-03$ \\
\hline
\end{tabular}

\section{Relative Risk [ ]}

\begin{tabular}{lccc}
\hline Diet 1 & 1.0015 & 1.036 & 1.77 \\
Diet 2 & 1.00063 & 1.014 & 1.35 \\
Diet 3 & 1.0027 & 1.054 & 2.3 \\
Diet 4 & 1.0031 & 1.09 & 3.2 \\
\hline
\end{tabular}

\section{Probability of Causation [\%]}

\begin{tabular}{llll}
\hline Diet 1 & 0.15 & 3.5 & 43 \\
Diet 2 & 0.06 & 1.3 & 26 \\
Diet 3 & 0.27 & 5.1 & 56 \\
Diet 4 & 0.31 & 8.2 & 68 \\
\hline
\end{tabular}

Diet 1 - Backyard cow milk + all other locally produced non-milk exposure pathways

Diet 2 - Locally produced commercial milk + all other locally produced non-milk exposure pathways

Diet 3 - Regionally mixed commercial milk + inhalation (other regionally mixed food items are minor contributors to the total)

Diet 4 - Goat milk (all other exposure pathways are of negligible importance compared to goat milk) 
Location: Rockwood

Receptor: Female born in 1950

\begin{tabular}{lccc}
\hline & \multicolumn{3}{c}{ Thyroid Dose [cGy] } \\
Exposure Pathway & \multicolumn{2}{c}{$95 \%$ Subjective Confidence Interval } \\
\hline Backyard Cow Milk & lower limit & central estimate & upper limit \\
Commercial Milk (locally produced) & 0.48 & 2.9 & 19 \\
Commercial Milk (regionally mixed) & 0.15 & 1 & 7.2 \\
Goat Milk (locally produced) & 0.17 & 1.2 & 7.9 \\
Beef (locally produced) & 0.95 & 7.8 & 72 \\
Leafy Vegetables (locally produced) & 0.00072 & 0.013 & 0.29 \\
Eggs (locally produced) & 0.0002 & 0.002 & 0.02 \\
Cottage Cheese (locally produced) & 0.023 & 0.19 & 1.4 \\
Inhalation & 0.00064 & 0.0073 & 0.084 \\
Mother's milk (mother on Diet 1) & 0.012 & 0.048 & 0.2 \\
Prenatal exposure (mother on Diet 1) & 0.0002 & 0.0053 & 0.14 \\
Diet 1 & 0.0016 & 0.016 & 0.16 \\
Diet 2 & 0.54 & 3.2 & 20 \\
Diet 3 & 0.22 & 1.3 & 9.2 \\
\hline
\end{tabular}

Excess Lifetime Risk [ ]

\begin{tabular}{llll}
\hline Diet 1 & $5.2 \mathrm{E}-05$ & $8.7 \mathrm{E}-04$ & $1.3 \mathrm{E}-02$ \\
Diet 2 & $2.9 \mathrm{E}-05$ & $3.4 \mathrm{E}-04$ & $4.5 \mathrm{E}-03$ \\
Diet 3 & $2.8 \mathrm{E}-05$ & $3.1 \mathrm{E}-04$ & $4.1 \mathrm{E}-03$ \\
Diet 4 & $1.3 \mathrm{E}-04$ & $2.2 \mathrm{E}-03$ & $3.0 \mathrm{E}-02$ \\
\hline
\end{tabular}

\section{Relative Risk [ ]}

\begin{tabular}{lccc}
\hline Diet 1 & 1.021 & 1.22 & 3.2 \\
Diet 2 & 1.0081 & 1.084 & 2 \\
Diet 3 & 1.0081 & 1.08 & 2.1 \\
Diet 4 & 1.044 & 1.49 & 9.3 \\
\hline
\end{tabular}

\begin{tabular}{lccc} 
& \multicolumn{3}{c}{ Probability of Causation [\%] } \\
\hline Diet 1 & 2.07 & 17.7 & 67 \\
Diet 2 & 0.80 & 7.7 & 49 \\
Diet 3 & 0.81 & 7.4 & 52 \\
Diet 4 & 4.21 & 32.1 & 88 \\
\hline
\end{tabular}

Diet 1 - Backyard cow milk + all other locally produced non-milk exposure pathways

Diet 2 - Locally produced commercial milk + all other locally produced non-milk exposure pathways

Diet 3 - Regionally mixed commercial milk + inhalation (other regionally mixed food items are minor contributors to the total)

Diet 4 - Goat milk (all other exposure pathways are of negligible importance compared to goat milk) 


\section{Location: Rockwood}

\section{Receptor: Male born in 1950}

\begin{tabular}{lccc}
\hline & \multicolumn{3}{c}{ Thyroid Dose [cGy] } \\
& \multicolumn{2}{c}{$95 \%$ Subjective Confidence Interval } \\
Exposure Pathway & lower limit & central estimate & upper limit \\
\hline Backyard Cow Milk & 0.5 & 2.9 & 18 \\
Commercial Milk (locally produced) & 0.15 & 0.97 & 7.4 \\
Commercial Milk (regionally mixed) & 0.17 & 1.1 & 8.9 \\
Goat Milk (locally produced) & 0.91 & 8.1 & 61 \\
Beef (locally produced) & 0.00073 & 0.012 & 0.32 \\
Leafy Vegetables (locally produced) & 0.00019 & 0.0021 & 0.019 \\
Eggs (locally produced) & 0.026 & 0.19 & 1.4 \\
Cottage Cheese (locally produced) & 0.00064 & 0.0072 & 0.074 \\
Inhalation & 0.011 & 0.047 & 0.19 \\
Mother's milk (mother on Diet 1) & 0.0002 & 0.0053 & 0.14 \\
Prenatal exposure (mother on Diet 1) & 0.0016 & 0.016 & 0.16 \\
Diet 1 & 0.57 & 3.3 & 20 \\
Diet 2 & 0.22 & 1.3 & 9 \\
Diet 3 & 0.2 & 1.2 & 9 \\
\hline
\end{tabular}

Excess Lifetime Risk [ ]

\begin{tabular}{llcc}
\hline Diet 1 & $6.8 \mathrm{E}-06$ & $2.2 \mathrm{E}-04$ & $4.1 \mathrm{E}-03$ \\
Diet 2 & $3.4 \mathrm{E}-06$ & $8.6 \mathrm{E}-05$ & $1.8 \mathrm{E}-03$ \\
Diet 3 & $3.5 \mathrm{E}-06$ & $8.1 \mathrm{E}-05$ & $1.9 \mathrm{E}-03$ \\
Diet 4 & $1.8 \mathrm{E}-05$ & $5.3 \mathrm{E}-04$ & $1.2 \mathrm{E}-02$ \\
\hline
\end{tabular}

\section{Relative Risk [ ]}

\begin{tabular}{lccc}
\hline Diet 1 & 1.0077 & 1.15 & 4.3 \\
Diet 2 & 1.003 & 1.058 & 2.4 \\
Diet 3 & 1.0028 & 1.056 & 2.3 \\
Diet 4 & 1.016 & 1.38 & 10 \\
\hline
\end{tabular}

\begin{tabular}{lccc}
\hline Diet 1 & 0.76 & 12.7 & 75 \\
Diet 2 & 0.30 & 5.4 & 57 \\
Diet 3 & 0.28 & 5.3 & 57 \\
Diet 4 & 1.59 & 27.1 & 90 \\
\hline
\end{tabular}

Diet 1 - Backyard cow milk + all other locally produced non-milk exposure pathways

Diet 2 - Locally produced commercial milk + all other locally produced non-milk exposure pathways

Diet 3 - Regionally mixed commercial milk + inhalation (other regionally mixed food items are minor contributors to the total)

Diet 4 - Goat milk (all other exposure pathways are of negligible importance compared to goat milk) 


\section{Location: Louisville}

Receptor: Female born in 1950

\begin{tabular}{lccc}
\hline & \multicolumn{3}{c}{ Thyroid Dose [cGy] } \\
Exposure Pathway & \multicolumn{2}{c}{$95 \%$ Subjective Confidence Interval } \\
\hline Backyard Cow Milk & lower limit & central estimate & upper limit \\
Commercial Milk (locally produced) & 0.47 & 2.7 & 16 \\
Commercial Milk (regionally mixed) & 0.13 & 0.93 & 6.4 \\
Goat Milk (locally produced) & 0.17 & 1.2 & 7.9 \\
Beef (locally produced) & 0.84 & 7.2 & 64 \\
Leafy Vegetables (locally produced) & 0.00065 & 0.011 & 0.26 \\
Eggs (locally produced) & 0.00019 & 0.0019 & 0.018 \\
Cottage Cheese (locally produced) & 0.022 & 0.17 & 1.4 \\
Inhalation & 0.00057 & 0.0069 & 0.073 \\
Mother's milk (mother on Diet 1) & 0.011 & 0.047 & 0.2 \\
Prenatal exposure (mother on Diet 1) & 0.00018 & 0.0049 & 0.13 \\
Diet 1 & 0.0015 & 0.014 & 0.15 \\
Diet 2 & 0.52 & 3 & 17 \\
Diet 3 & 0.2 & 1.2 & 7.9 \\
\hline
\end{tabular}

Excess Lifetime Risk [ ]

\begin{tabular}{llll}
\hline Diet 1 & $5.2 \mathrm{E}-05$ & $7.9 \mathrm{E}-04$ & $1.2 \mathrm{E}-02$ \\
Diet 2 & $2.7 \mathrm{E}-05$ & $3.1 \mathrm{E}-04$ & $4.4 \mathrm{E}-03$ \\
Diet 3 & $2.7 \mathrm{E}-05$ & $3.1 \mathrm{E}-04$ & $4.1 \mathrm{E}-03$ \\
Diet 4 & $1.2 \mathrm{E}-04$ & $2.0 \mathrm{E}-03$ & $2.9 \mathrm{E}-02$ \\
\hline
\end{tabular}

\section{Relative Risk [ ]}

\begin{tabular}{lccc}
\hline Diet 1 & 1.022 & 1.2 & 3.2 \\
Diet 2 & 1.0074 & 1.078 & 1.98 \\
Diet 3 & 1.0082 & 1.08 & 2.1 \\
Diet 4 & 1.042 & 1.46 & 8.1 \\
\hline
\end{tabular}

\section{Probability of Causation [\%]}

\begin{tabular}{lccc}
\hline Diet 1 & 2.11 & 16.9 & 68 \\
Diet 2 & 0.74 & 7.3 & 49 \\
Diet 3 & 0.81 & 7.4 & 52 \\
Diet 4 & 4.06 & 31.5 & 87 \\
\hline
\end{tabular}

Diet 1 - Backyard cow milk + all other locally produced non-milk exposure pathways

Diet 2 - Locally produced commercial milk + all other locally produced non-milk exposure pathways

Diet 3 - Regionally mixed commercial milk + inhalation (other regionally mixed food items are minor contributors to the total)

Diet 4 - Goat milk (all other exposure pathways are of negligible importance compared to goat milk) 


\section{Location: Louisville}

\section{Receptor: Male born in 1950}

\begin{tabular}{lccc}
\hline & \multicolumn{3}{c}{ Thyroid Dose [cGy] } \\
& \multicolumn{2}{c}{$95 \%$ Subjective Confidence Interval } \\
Exposure Pathway & lower limit & central estimate & upper limit \\
\hline Backyard Cow Milk & 0.47 & 2.8 & 16 \\
Commercial Milk (locally produced) & 0.14 & 0.9 & 6.4 \\
Commercial Milk (regionally mixed) & 0.17 & 1.1 & 8.9 \\
Goat Milk (locally produced) & 0.79 & 7.5 & 59 \\
Beef (locally produced) & 0.00063 & 0.011 & 0.27 \\
Leafy Vegetables (locally produced) & 0.00018 & 0.0019 & 0.018 \\
Eggs (locally produced) & 0.025 & 0.17 & 1.2 \\
Cottage Cheese (locally produced) & 0.00056 & 0.007 & 0.064 \\
Inhalation & 0.011 & 0.047 & 0.2 \\
Mother's milk (mother on Diet 1) & 0.00018 & 0.0049 & 0.13 \\
Prenatal exposure (mother on Diet 1) & 0.0015 & 0.014 & 0.15 \\
Diet 1 & 0.53 & 3.1 & 18 \\
Diet 2 & 0.21 & 1.2 & 8.2 \\
Diet 3 & 0.2 & 1.2 & 9 \\
\hline
\end{tabular}

Excess Lifetime Risk [ ]

\begin{tabular}{llll}
\hline Diet 1 & $5.9 \mathrm{E}-06$ & $2.2 \mathrm{E}-04$ & $3.5 \mathrm{E}-03$ \\
Diet 2 & $2.8 \mathrm{E}-06$ & $8.1 \mathrm{E}-05$ & $1.6 \mathrm{E}-03$ \\
Diet 3 & $3.5 \mathrm{E}-06$ & $8.1 \mathrm{E}-05$ & $1.9 \mathrm{E}-03$ \\
Diet 4 & $1.5 \mathrm{E}-05$ & $4.9 \mathrm{E}-04$ & $9.9 \mathrm{E}-03$ \\
\hline
\end{tabular}

\section{Relative Risk [ ]}

\begin{tabular}{lccc}
\hline Diet 1 & 1.0069 & 1.14 & 3.8 \\
Diet 2 & 1.0028 & 1.053 & 2.2 \\
Diet 3 & 1.0028 & 1.056 & 2.3 \\
Diet 4 & 1.014 & 1.35 & 8.5 \\
\hline
\end{tabular}

\begin{tabular}{lccc} 
& \multicolumn{3}{c}{ Probability of Causation [\%] } \\
\hline Diet 1 & 0.69 & 12.2 & 74 \\
Diet 2 & 0.28 & 5.0 & 55 \\
Diet 3 & 0.28 & 5.3 & 57 \\
Diet 4 & 1.41 & 25.9 & 88 \\
\hline
\end{tabular}

Diet 1 - Backyard cow milk + all other locally produced non-milk exposure pathways

Diet 2 - Locally produced commercial milk + all other locally produced non-milk exposure pathways

Diet 3 - Regionally mixed commercial milk + inhalation (other regionally mixed food items are minor contributors to the total)

Diet 4 - Goat milk (all other exposure pathways are of negligible importance compared to goat milk) 
Location: Barnardville

Receptor: Female born in 1950

\begin{tabular}{lccc}
\hline & \multicolumn{3}{c}{ Thyroid Dose [cGy] } \\
Exposure Pathway & \multicolumn{2}{c}{$95 \%$ Subjective Confidence Interval } \\
\hline Backyard Cow Milk & lower limit & central estimate & upper limit \\
Commercial Milk (locally produced) & 0.51 & 3 & 19 \\
Commercial Milk (regionally mixed) & 0.14 & 1 & 7.6 \\
Goat Milk (locally produced) & 0.17 & 1.2 & 7.9 \\
Beef (locally produced) & 0.95 & 8.3 & 71 \\
Leafy Vegetables (locally produced) & 0.00076 & 0.013 & 0.3 \\
Eggs (locally produced) & 0.00021 & 0.0022 & 0.02 \\
Cottage Cheese (locally produced) & 0.024 & 0.2 & 1.6 \\
Inhalation & 0.00062 & 0.0077 & 0.082 \\
Mother's milk (mother on Diet 1) & 0.013 & 0.055 & 0.24 \\
Prenatal exposure (mother on Diet 1) & 0.00022 & 0.0056 & 0.15 \\
Diet 1 & 0.0017 & 0.016 & 0.17 \\
Diet 2 & 0.57 & 3.4 & 21 \\
Diet 3 & 0.23 & 1.4 & 9.2 \\
\hline
\end{tabular}

Excess Lifetime Risk [ ]

\begin{tabular}{llll}
\hline Diet 1 & $5.6 \mathrm{E}-05$ & $9.3 \mathrm{E}-04$ & $1.4 \mathrm{E}-02$ \\
Diet 2 & $3.0 \mathrm{E}-05$ & $3.6 \mathrm{E}-04$ & $4.5 \mathrm{E}-03$ \\
Diet 3 & $2.8 \mathrm{E}-05$ & $3.1 \mathrm{E}-04$ & $4.1 \mathrm{E}-03$ \\
Diet 4 & $1.4 \mathrm{E}-04$ & $2.3 \mathrm{E}-03$ & $3.0 \mathrm{E}-02$ \\
\hline
\end{tabular}

\section{Relative Risk [ ]}

\begin{tabular}{lccc}
\hline Diet 1 & 1.022 & 1.23 & 3.2 \\
Diet 2 & 1.0083 & 1.089 & 1.98 \\
Diet 3 & 1.0083 & 1.08 & 2.1 \\
Diet 4 & 1.048 & 1.52 & 9.3 \\
\hline
\end{tabular}

\section{Probability of Causation [\%]}

\begin{tabular}{lccc}
\hline Diet 1 & 2.18 & 18.8 & 69 \\
Diet 2 & 0.82 & 8.1 & 49 \\
Diet 3 & 0.82 & 7.4 & 52 \\
Diet 4 & 4.61 & 34.4 & 89 \\
\hline
\end{tabular}

Diet 1 - Backyard cow milk + all other locally produced non-milk exposure pathways

Diet 2 - Locally produced commercial milk + all other locally produced non-milk exposure pathways

Diet 3 - Regionally mixed commercial milk + inhalation (other regionally mixed food items are minor contributors to the total)

Diet 4 - Goat milk (all other exposure pathways are of negligible importance compared to goat milk) 


\section{Location: Barnardville}

Receptor: Male born in 1950

\begin{tabular}{lccc}
\hline & \multicolumn{3}{c}{ Thyroid Dose [cGy] } \\
Exposure Pathway & \multicolumn{2}{c}{$95 \%$ Subjective Confidence Interval } \\
\hline Backyard Cow Milk & lower limit & central estimate & upper limit \\
Commercial Milk (locally produced) & 0.5 & 3.1 & 19 \\
Commercial Milk (regionally mixed) & 0.15 & 1 & 7.6 \\
Goat Milk (locally produced) & 0.17 & 1.1 & 8.9 \\
Beef (locally produced) & 0.93 & 8.4 & 68 \\
Leafy Vegetables (locally produced) & 0.00077 & 0.013 & 0.3 \\
Eggs (locally produced) & 0.0002 & 0.0021 & 0.02 \\
Cottage Cheese (locally produced) & 0.028 & 0.2 & 1.5 \\
Inhalation & 0.00062 & 0.0077 & 0.076 \\
Mother's milk (mother on Diet 1) & 0.013 & 0.056 & 0.23 \\
Prenatal exposure (mother on Diet 1) & 0.00022 & 0.0056 & 0.15 \\
Diet 1 & 0.0017 & 0.016 & 0.17 \\
Diet 2 & 0.57 & 3.4 & 20 \\
Diet 3 & 0.23 & 1.4 & 9.2 \\
\hline
\end{tabular}

Excess Lifetime Risk [ ]

\begin{tabular}{llll}
\hline Diet 1 & $6.9 \mathrm{E}-06$ & $2.5 \mathrm{E}-04$ & $4.2 \mathrm{E}-03$ \\
Diet 2 & $3.4 \mathrm{E}-06$ & $9.1 \mathrm{E}-05$ & $1.8 \mathrm{E}-03$ \\
Diet 3 & $3.6 \mathrm{E}-06$ & $8.2 \mathrm{E}-05$ & $1.9 \mathrm{E}-03$ \\
Diet 4 & $1.9 \mathrm{E}-05$ & $5.6 \mathrm{E}-04$ & $1.2 \mathrm{E}-02$ \\
\hline
\end{tabular}

\section{Relative Risk [ ]}

\begin{tabular}{lccc}
\hline Diet 1 & 1.0079 & 1.16 & 4.4 \\
Diet 2 & 1.0031 & 1.062 & 2.5 \\
Diet 3 & 1.0028 & 1.056 & 2.4 \\
Diet 4 & 1.017 & 1.4 & 12 \\
\hline
\end{tabular}

\section{Probability of Causation [\%]}

\begin{tabular}{lccc}
\hline Diet 1 & 0.78 & 13.7 & 77 \\
Diet 2 & 0.31 & 5.9 & 59 \\
Diet 3 & 0.28 & 5.3 & 57 \\
Diet 4 & 1.68 & 28.4 & 91 \\
\hline
\end{tabular}

Diet 1 - Backyard cow milk + all other locally produced non-milk exposure pathways

Diet 2 - Locally produced commercial milk + all other locally produced non-milk exposure pathways

Diet 3 - Regionally mixed commercial milk + inhalation (other regionally mixed food items are minor contributors to the total)

Diet 4 - Goat milk (all other exposure pathways are of negligible importance compared to goat milk) 
Location: Greenback

Receptor: Female born in 1950

\begin{tabular}{lccc}
\hline & \multicolumn{3}{c}{ Thyroid Dose [cGy] } \\
Exposure Pathway & \multicolumn{2}{c}{$95 \%$ Subjective Confidence Interval } \\
\hline Backyard Cow Milk & 0.3 & 1.7 & 13 \\
Commercial Milk (locally produced) & 0.069 & 0.56 & 4.4 \\
Commercial Milk (regionally mixed) & 0.17 & 1.2 & 7.9 \\
Goat Milk (locally produced) & 0.48 & 4.3 & 42 \\
Beef (locally produced) & 0.0004 & 0.0072 & 0.18 \\
Leafy Vegetables (locally produced) & 0.00011 & 0.0012 & 0.011 \\
Eggs (locally produced) & 0.014 & 0.11 & 0.88 \\
Cottage Cheese (locally produced) & 0.00034 & 0.0041 & 0.061 \\
Inhalation & 0.0074 & 0.032 & 0.13 \\
Mother's milk (mother on Diet 1) & 0.00013 & 0.003 & 0.09 \\
Prenatal exposure (mother on Diet 1) & 0.001 & 0.0092 & 0.096 \\
Diet 1 & 0.34 & 1.9 & 14 \\
Diet 2 & 0.12 & 0.77 & 5.2 \\
Diet 3 & 0.19 & 1.2 & 8 \\
\hline
\end{tabular}

Excess Lifetime Risk [ ]

\begin{tabular}{llll}
\hline Diet 1 & $2.8 \mathrm{E}-05$ & $4.8 \mathrm{E}-04$ & $7.1 \mathrm{E}-03$ \\
Diet 2 & $1.5 \mathrm{E}-05$ & $1.9 \mathrm{E}-04$ & $2.8 \mathrm{E}-03$ \\
Diet 3 & $2.7 \mathrm{E}-05$ & $3.0 \mathrm{E}-04$ & $4.1 \mathrm{E}-03$ \\
Diet 4 & $7.9 \mathrm{E}-05$ & $1.2 \mathrm{E}-03$ & $2.3 \mathrm{E}-02$ \\
\hline
\end{tabular}

\section{Relative Risk [ ]}

\begin{tabular}{lccc}
\hline Diet 1 & 1.012 & 1.12 & 2.4 \\
Diet 2 & 1.0048 & 1.048 & 1.62 \\
Diet 3 & 1.0078 & 1.079 & 2.1 \\
Diet 4 & 1.027 & 1.28 & 6 \\
\hline
\end{tabular}

\section{Probability of Causation [\%]}

\begin{tabular}{lccc}
\hline Diet 1 & 1.14 & 11.1 & 59 \\
Diet 2 & 0.48 & 4.6 & 38 \\
Diet 3 & 0.77 & 7.3 & 52 \\
Diet 4 & 2.61 & 22.0 & 83 \\
\hline
\end{tabular}

Diet 1 - Backyard cow milk + all other locally produced non-milk exposure pathways

Diet 2 - Locally produced commercial milk + all other locally produced non-milk exposure pathways

Diet 3 - Regionally mixed commercial milk + inhalation (other regionally mixed food items are minor contributors to the total)

Diet 4 - Goat milk (all other exposure pathways are of negligible importance compared to goat milk) 
Location: Greenback

Receptor: Male born in 1950

\begin{tabular}{lccc}
\hline & \multicolumn{3}{c}{ Thyroid Dose [cGy] } \\
Exposure Pathway & 95\% Subjective Confidence Interval \\
\hline Backyard Cow Milk & lower limit & central estimate & upper limit \\
Commercial Milk (locally produced) & 0.29 & 1.7 & 13 \\
Commercial Milk (regionally mixed) & 0.074 & 0.54 & 4.3 \\
Goat Milk (locally produced) & 0.17 & 1.1 & 8.9 \\
Beef (locally produced) & 0.46 & 4.7 & 40 \\
Leafy Vegetables (locally produced) & 0.00041 & 0.0075 & 0.17 \\
Eggs (locally produced) & 0.00012 & 0.0012 & 0.011 \\
Cottage Cheese (locally produced) & 0.015 & 0.11 & 0.84 \\
Inhalation & 0.00034 & 0.0041 & 0.051 \\
Mother's milk (mother on Diet 1) & 0.0079 & 0.031 & 0.14 \\
Prenatal exposure (mother on Diet 1) & 0.00013 & 0.003 & 0.09 \\
Diet 1 & 0.001 & 0.0092 & 0.096 \\
Diet 2 & 0.35 & 1.9 & 14 \\
Diet 3 & 0.13 & 0.75 & 5 \\
\hline
\end{tabular}

Excess Lifetime Risk [ ]

\begin{tabular}{llll}
\hline Diet 1 & $4.1 \mathrm{E}-06$ & $1.3 \mathrm{E}-04$ & $2.5 \mathrm{E}-03$ \\
Diet 2 & $1.8 \mathrm{E}-06$ & $4.8 \mathrm{E}-05$ & $9.9 \mathrm{E}-04$ \\
Diet 3 & $3.4 \mathrm{E}-06$ & $8.0 \mathrm{E}-05$ & $1.9 \mathrm{E}-03$ \\
Diet 4 & $1.0 \mathrm{E}-05$ & $2.9 \mathrm{E}-04$ & $7.3 \mathrm{E}-03$ \\
\hline
\end{tabular}

\section{Relative Risk [ ]}

\begin{tabular}{lccc}
\hline Diet 1 & 1.0044 & 1.086 & 2.9 \\
Diet 2 & 1.0016 & 1.034 & 1.83 \\
Diet 3 & 1.0028 & 1.055 & 2.3 \\
Diet 4 & 1.0088 & 1.23 & 5.8 \\
\hline
\end{tabular}

\section{Probability of Causation [\%]}

\begin{tabular}{lccc}
\hline Diet 1 & 0.44 & 8.0 & 65 \\
Diet 2 & 0.16 & 3.3 & 45 \\
Diet 3 & 0.28 & 5.2 & 57 \\
Diet 4 & 0.87 & 18.4 & 82 \\
\hline
\end{tabular}

Diet 1 - Backyard cow milk + all other locally produced non-milk exposure pathways

Diet 2 - Locally produced commercial milk + all other locally produced non-milk exposure pathways

Diet 3 - Regionally mixed commercial milk + inhalation (other regionally mixed food items are minor contributors to the total)

Diet 4 - Goat milk (all other exposure pathways are of negligible importance compared to goat milk) 


\section{Location: Rockford}

Receptor: Female born in 1950

\begin{tabular}{lccc}
\hline & \multicolumn{3}{c}{ Thyroid Dose [cGy] } \\
Exposure Pathway & \multicolumn{2}{c}{$95 \%$ Subjective Confidence Interval } \\
\hline Backyard Cow Milk & lower limit & central estimate & upper limit \\
Commercial Milk (locally produced) & 0.48 & 2.6 & 17 \\
Commercial Milk (regionally mixed) & 0.12 & 0.9 & 6.1 \\
Goat Milk (locally produced) & 0.17 & 1.2 & 7.9 \\
Beef (locally produced) & 0.86 & 6.8 & 60 \\
Leafy Vegetables (locally produced) & 0.00061 & 0.011 & 0.27 \\
Eggs (locally produced) & 0.00018 & 0.0019 & 0.017 \\
Cottage Cheese (locally produced) & 0.021 & 0.17 & 1.3 \\
Inhalation & 0.00058 & 0.0064 & 0.082 \\
Mother's milk (mother on Diet 1) & 0.012 & 0.047 & 0.2 \\
Prenatal exposure (mother on Diet 1) & 0.00017 & 0.0048 & 0.13 \\
Diet 1 & 0.0016 & 0.014 & 0.15 \\
Diet 2 & 0.53 & 2.9 & 19 \\
Diet 3 & 0.2 & 1.2 & 7.7 \\
\hline
\end{tabular}

Excess Lifetime Risk [ ]

\begin{tabular}{llll}
\hline Diet 1 & $5.0 \mathrm{E}-05$ & $7.9 \mathrm{E}-04$ & $1.1 \mathrm{E}-02$ \\
Diet 2 & $2.7 \mathrm{E}-05$ & $3.0 \mathrm{E}-04$ & $3.8 \mathrm{E}-03$ \\
Diet 3 & $2.8 \mathrm{E}-05$ & $3.1 \mathrm{E}-04$ & $4.1 \mathrm{E}-03$ \\
Diet 4 & $1.2 \mathrm{E}-04$ & $2.0 \mathrm{E}-03$ & $2.9 \mathrm{E}-02$ \\
\hline
\end{tabular}

\section{Relative Risk [ ]}

\begin{tabular}{lccc}
\hline Diet 1 & 1.018 & 1.19 & 3 \\
Diet 2 & 1.0072 & 1.077 & 1.89 \\
Diet 3 & 1.0081 & 1.08 & 2.1 \\
Diet 4 & 1.04 & 1.44 & 8.1 \\
\hline
\end{tabular}

\section{Probability of Causation [\%]}

\begin{tabular}{lccc}
\hline Diet 1 & 1.78 & 16.2 & 67 \\
Diet 2 & 0.71 & 7.2 & 47 \\
Diet 3 & 0.80 & 7.4 & 52 \\
Diet 4 & 3.85 & 30.4 & 88 \\
\hline
\end{tabular}

Diet 1 - Backyard cow milk + all other locally produced non-milk exposure pathways

Diet 2 - Locally produced commercial milk + all other locally produced non-milk exposure pathways

Diet 3 - Regionally mixed commercial milk + inhalation (other regionally mixed food items are minor contributors to the total)

Diet 4 - Goat milk (all other exposure pathways are of negligible importance compared to goat milk) 


\section{Location: Rockford}

\section{Receptor: Male born in 1950}

\begin{tabular}{lccc}
\hline & \multicolumn{3}{c}{ Thyroid Dose [cGy] } \\
& \multicolumn{2}{c}{$95 \%$ Subjective Confidence Interval } \\
Exposure Pathway & lower limit & central estimate & upper limit \\
\hline Backyard Cow Milk & 0.48 & 2.7 & 17 \\
Commercial Milk (locally produced) & 0.13 & 0.88 & 6.5 \\
Commercial Milk (regionally mixed) & 0.17 & 1.1 & 8.9 \\
Goat Milk (locally produced) & 0.78 & 7.3 & 57 \\
Beef (locally produced) & 0.00063 & 0.011 & 0.27 \\
Leafy Vegetables (locally produced) & 0.00017 & 0.0019 & 0.016 \\
Eggs (locally produced) & 0.024 & 0.17 & 1.2 \\
Cottage Cheese (locally produced) & 0.00056 & 0.0065 & 0.072 \\
Inhalation & 0.012 & 0.047 & 0.19 \\
Mother's milk (mother on Diet 1) & 0.00017 & 0.0048 & 0.13 \\
Prenatal exposure (mother on Diet 1) & 0.0016 & 0.014 & 0.15 \\
Diet 1 & 0.55 & 2.9 & 19 \\
Diet 2 & 0.21 & 1.2 & 8 \\
Diet 3 & 0.2 & 1.2 & 9.1 \\
\hline
\end{tabular}

Excess Lifetime Risk [ ]

\begin{tabular}{llll}
\hline Diet 1 & $5.9 \mathrm{E}-06$ & $2.1 \mathrm{E}-04$ & $3.8 \mathrm{E}-03$ \\
Diet 2 & $3.1 \mathrm{E}-06$ & $7.8 \mathrm{E}-05$ & $1.7 \mathrm{E}-03$ \\
Diet 3 & $3.5 \mathrm{E}-06$ & $8.1 \mathrm{E}-05$ & $1.9 \mathrm{E}-03$ \\
Diet 4 & $1.6 \mathrm{E}-05$ & $4.7 \mathrm{E}-04$ & $1.0 \mathrm{E}-02$ \\
\hline
\end{tabular}

\section{Relative Risk [ ]}

\begin{tabular}{lccc}
\hline Diet 1 & 1.007 & 1.14 & 3.8 \\
Diet 2 & 1.0025 & 1.054 & 2.3 \\
Diet 3 & 1.0028 & 1.056 & 2.3 \\
Diet 4 & 1.014 & 1.35 & 8.1 \\
\hline
\end{tabular}

\section{Probability of Causation [\%]}

\begin{tabular}{lccc}
\hline Diet 1 & 0.69 & 12.0 & 73 \\
Diet 2 & 0.25 & 5.1 & 55 \\
Diet 3 & 0.28 & 5.3 & 57 \\
Diet 4 & 1.42 & 25.7 & 87 \\
\hline
\end{tabular}

Diet 1 - Backyard cow milk + all other locally produced non-milk exposure pathways

Diet 2 - Locally produced commercial milk + all other locally produced non-milk exposure pathways

Diet 3 - Regionally mixed commercial milk + inhalation (other regionally mixed food items are minor contributors to the total)

Diet 4 - Goat milk (all other exposure pathways are of negligible importance compared to goat milk) 
Location: Lake City

Receptor: Female born in 1950

\begin{tabular}{lccc}
\hline & \multicolumn{3}{c}{ Thyroid Dose [cGy] } \\
Exposure Pathway & $95 \%$ Subjective Confidence Interval \\
\hline Backyard Cow Milk & lower limit & central estimate & upper limit \\
Commercial Milk (locally produced) & 0.29 & 1.6 & 9.3 \\
Commercial Milk (regionally mixed) & 0.082 & 0.56 & 3.9 \\
Goat Milk (locally produced) & 0.17 & 1.2 & 7.9 \\
Beef (locally produced) & 0.55 & 4.4 & 36 \\
Leafy Vegetables (locally produced) & 0.00038 & 0.007 & 0.18 \\
Eggs (locally produced) & 0.00011 & 0.0012 & 0.011 \\
Cottage Cheese (locally produced) & 0.013 & 0.1 & 0.78 \\
Inhalation & 0.00038 & 0.004 & 0.045 \\
Mother's milk (mother on Diet 1) & 0.0061 & 0.026 & 0.11 \\
Prenatal exposure (mother on Diet 1) & 0.00011 & 0.0029 & 0.079 \\
Diet 1 & 0.0009 & 0.0084 & 0.085 \\
Diet 2 & 0.33 & 1.8 & 10 \\
Diet 3 & 0.13 & 0.73 & 4.9 \\
\hline
\end{tabular}

Excess Lifetime Risk [ ]

\begin{tabular}{llll}
\hline Diet 1 & $3.0 \mathrm{E}-05$ & $4.8 \mathrm{E}-04$ & $7.3 \mathrm{E}-03$ \\
Diet 2 & $1.6 \mathrm{E}-05$ & $1.8 \mathrm{E}-04$ & $2.5 \mathrm{E}-03$ \\
Diet 3 & $2.6 \mathrm{E}-05$ & $3.0 \mathrm{E}-04$ & $4.0 \mathrm{E}-03$ \\
Diet 4 & $7.8 \mathrm{E}-05$ & $1.2 \mathrm{E}-03$ & $1.6 \mathrm{E}-02$ \\
\hline
\end{tabular}

\section{Relative Risk [ ]}

\begin{tabular}{lccc}
\hline Diet 1 & 1.012 & 1.12 & 2.2 \\
Diet 2 & 1.0045 & 1.047 & 1.62 \\
Diet 3 & 1.0077 & 1.079 & 2.1 \\
Diet 4 & 1.026 & 1.28 & 5.6 \\
\hline
\end{tabular}

\section{Probability of Causation [\%]}

\begin{tabular}{lccc}
\hline Diet 1 & 1.22 & 10.8 & 54 \\
Diet 2 & 0.45 & 4.5 & 38 \\
Diet 3 & 0.77 & 7.3 & 51 \\
Diet 4 & 2.50 & 21.6 & 82 \\
\hline
\end{tabular}

Diet 1 - Backyard cow milk + all other locally produced non-milk exposure pathways

Diet 2 - Locally produced commercial milk + all other locally produced non-milk exposure pathways

Diet 3 - Regionally mixed commercial milk + inhalation (other regionally mixed food items are minor contributors to the total)

Diet 4 - Goat milk (all other exposure pathways are of negligible importance compared to goat milk) 


\section{Location: Lake City}

Receptor: Male born in 1950

\begin{tabular}{lccc}
\hline & \multicolumn{3}{c}{ Thyroid Dose [cGy] } \\
& \multicolumn{2}{c}{$95 \%$ Subjective Confidence Interval } \\
Exposure Pathway & lower limit & central estimate & upper limit \\
\hline Backyard Cow Milk & 0.3 & 1.6 & 9.1 \\
Commercial Milk (locally produced) & 0.087 & 0.55 & 4.1 \\
Commercial Milk (regionally mixed) & 0.17 & 1.1 & 8.9 \\
Goat Milk (locally produced) & 0.5 & 4.5 & 36 \\
Beef (locally produced) & 0.00038 & 0.0068 & 0.17 \\
Leafy Vegetables (locally produced) & 0.00011 & 0.0012 & 0.011 \\
Eggs (locally produced) & 0.015 & 0.1 & 0.73 \\
Cottage Cheese (locally produced) & 0.00035 & 0.0041 & 0.04 \\
Inhalation & 0.0065 & 0.026 & 0.1 \\
Mother's milk (mother on Diet 1) & 0.00011 & 0.0029 & 0.079 \\
Prenatal exposure (mother on Diet 1) & 0.0009 & 0.0084 & 0.085 \\
Diet 1 & 0.34 & 1.8 & 10 \\
Diet 2 & 0.13 & 0.73 & 5.2 \\
Diet 3 & 0.18 & 1.2 & 9 \\
\hline
\end{tabular}

Excess Lifetime Risk [ ]

\begin{tabular}{llll}
\hline Diet 1 & $3.4 \mathrm{E}-06$ & $1.3 \mathrm{E}-04$ & $2.3 \mathrm{E}-03$ \\
Diet 2 & $1.9 \mathrm{E}-06$ & $5.1 \mathrm{E}-05$ & $1.0 \mathrm{E}-03$ \\
Diet 3 & $3.3 \mathrm{E}-06$ & $8.0 \mathrm{E}-05$ & $1.9 \mathrm{E}-03$ \\
Diet 4 & $9.9 \mathrm{E}-06$ & $3.0 \mathrm{E}-04$ & $6.2 \mathrm{E}-03$ \\
\hline
\end{tabular}

\section{Relative Risk [ ]}

\begin{tabular}{lccc}
\hline Diet 1 & 1.0041 & 1.083 & 2.6 \\
Diet 2 & 1.0017 & 1.032 & 1.7 \\
Diet 3 & 1.0027 & 1.055 & 2.3 \\
Diet 4 & 1.0085 & 1.21 & 5.4 \\
\hline
\end{tabular}

\section{Probability of Causation [\%]}

\begin{tabular}{lccc}
\hline Diet 1 & 0.41 & 7.6 & 61 \\
Diet 2 & 0.17 & 3.1 & 41 \\
Diet 3 & 0.27 & 5.2 & 57 \\
Diet 4 & 0.84 & 17.0 & 81 \\
\hline
\end{tabular}

Diet 1 - Backyard cow milk + all other locally produced non-milk exposure pathways

Diet 2 - Locally produced commercial milk + all other locally produced non-milk exposure pathways

Diet 3 - Regionally mixed commercial milk + inhalation (other regionally mixed food items are minor contributors to the total)

Diet 4 - Goat milk (all other exposure pathways are of negligible importance compared to goat milk) 
Location: Sweetwater

Receptor: Female born in 1950

\begin{tabular}{lccc}
\hline & \multicolumn{3}{c}{ Thyroid Dose [cGy] } \\
Exposure Pathway & $95 \%$ & Subjective Confidence Interval \\
Backyard Cow Milk & lower limit & central estimate & upper limit \\
Commercial Milk (locally produced) & 0.33 & 2 & 11 \\
Commercial Milk (regionally mixed) & 0.091 & 0.69 & 4.6 \\
Goat Milk (locally produced) & 0.17 & 1.2 & 7.9 \\
Beef (locally produced) & 0.56 & 5.3 & 49 \\
Leafy Vegetables (locally produced) & 0.00048 & 0.0082 & 0.19 \\
Eggs (locally produced) & 0.00015 & 0.0014 & 0.014 \\
Cottage Cheese (locally produced) & 0.016 & 0.12 & 0.97 \\
Inhalation & 0.00039 & 0.0053 & 0.055 \\
Mother's milk (mother on Diet 1) & 0.0084 & 0.037 & 0.15 \\
Prenatal exposure (mother on Diet 1) & 0.00013 & 0.0035 & 0.095 \\
Diet 1 & 0.001 & 0.011 & 0.11 \\
Diet 2 & 0.38 & 2.2 & 12 \\
Diet 3 & 0.13 & 0.91 & 5.6 \\
\hline
\end{tabular}

Excess Lifetime Risk [ ]

\begin{tabular}{llll}
\hline Diet 1 & $4.0 \mathrm{E}-05$ & $5.8 \mathrm{E}-04$ & $9.7 \mathrm{E}-03$ \\
Diet 2 & $1.8 \mathrm{E}-05$ & $2.3 \mathrm{E}-04$ & $3.3 \mathrm{E}-03$ \\
Diet 3 & $2.7 \mathrm{E}-05$ & $3.0 \mathrm{E}-04$ & $4.1 \mathrm{E}-03$ \\
Diet 4 & $9.1 \mathrm{E}-05$ & $1.5 \mathrm{E}-03$ & $2.3 \mathrm{E}-02$ \\
\hline
\end{tabular}

\section{Relative Risk [ ]}

\begin{tabular}{lccc}
\hline Diet 1 & 1.017 & 1.15 & 2.5 \\
Diet 2 & 1.0057 & 1.06 & 1.72 \\
Diet 3 & 1.0079 & 1.08 & 2.1 \\
Diet 4 & 1.03 & 1.34 & 6.2 \\
\hline
\end{tabular}

\section{Probability of Causation [\%]}

\begin{tabular}{lccc}
\hline Diet 1 & 1.70 & 13.1 & 61 \\
Diet 2 & 0.57 & 5.7 & 42 \\
Diet 3 & 0.79 & 7.4 & 52 \\
Diet 4 & 2.92 & 25.3 & 84 \\
\hline
\end{tabular}

Diet 1 - Backyard cow milk + all other locally produced non-milk exposure pathways

Diet 2 - Locally produced commercial milk + all other locally produced non-milk exposure pathways

Diet 3 - Regionally mixed commercial milk + inhalation (other regionally mixed food items are minor contributors to the total)

Diet 4 - Goat milk (all other exposure pathways are of negligible importance compared to goat milk) 
Location: Sweetwater

Receptor: Male born in 1950

\begin{tabular}{lccc}
\hline & \multicolumn{3}{c}{ Thyroid Dose [cGy] } \\
& \multicolumn{2}{c}{$95 \%$ Subjective Confidence Interval } \\
Exposure Pathway & lower limit & central estimate & upper limit \\
\hline Backyard Cow Milk & 0.33 & 2 & 12 \\
Commercial Milk (locally produced) & 0.1 & 0.68 & 4.4 \\
Commercial Milk (regionally mixed) & 0.17 & 1.1 & 8.9 \\
Goat Milk (locally produced) & 0.56 & 5.5 & 44 \\
Beef (locally produced) & 0.00048 & 0.0084 & 0.18 \\
Leafy Vegetables (locally produced) & 0.00014 & 0.0014 & 0.014 \\
Eggs (locally produced) & 0.018 & 0.12 & 0.9 \\
Cottage Cheese (locally produced) & 0.00043 & 0.0052 & 0.05 \\
Inhalation & 0.0086 & 0.038 & 0.16 \\
Mother's milk (mother on Diet 1) & 0.00013 & 0.0035 & 0.095 \\
Prenatal exposure (mother on Diet 1) & 0.001 & 0.011 & 0.11 \\
Diet 1 & 0.38 & 2.3 & 12 \\
Diet 2 & 0.15 & 0.9 & 5.8 \\
Diet 3 & 0.19 & 1.2 & 9 \\
\hline
\end{tabular}

Excess Lifetime Risk [ ]

\begin{tabular}{llll}
\hline Diet 1 & $4.8 \mathrm{E}-06$ & $1.6 \mathrm{E}-04$ & $2.6 \mathrm{E}-03$ \\
Diet 2 & $2.1 \mathrm{E}-06$ & $5.9 \mathrm{E}-05$ & $1.2 \mathrm{E}-03$ \\
Diet 3 & $3.4 \mathrm{E}-06$ & $8.1 \mathrm{E}-05$ & $1.9 \mathrm{E}-03$ \\
Diet 4 & $1.0 \mathrm{E}-05$ & $3.6 \mathrm{E}-04$ & $6.8 \mathrm{E}-03$ \\
\hline
\end{tabular}

\section{Relative Risk [ ]}

\begin{tabular}{lccc}
\hline Diet 1 & 1.005 & 1.11 & 3.2 \\
Diet 2 & 1.002 & 1.041 & 1.89 \\
Diet 3 & 1.0028 & 1.055 & 2.3 \\
Diet 4 & 1.0098 & 1.26 & 6.5 \\
\hline
\end{tabular}

\section{Probability of Causation [\%]}

\begin{tabular}{lccc}
\hline Diet 1 & 0.50 & 9.6 & 68 \\
Diet 2 & 0.20 & 3.9 & 47 \\
Diet 3 & 0.28 & 5.2 & 57 \\
Diet 4 & 0.97 & 20.9 & 85 \\
\hline
\end{tabular}

Diet 1 - Backyard cow milk + all other locally produced non-milk exposure pathways

Diet 2 - Locally produced commercial milk + all other locally produced non-milk exposure pathways

Diet 3 - Regionally mixed commercial milk + inhalation (other regionally mixed food items are minor contributors to the total)

Diet 4 - Goat milk (all other exposure pathways are of negligible importance compared to goat milk) 


\section{Location: Knoxville}

Receptor: Female born in 1950

\begin{tabular}{lccc}
\hline & \multicolumn{3}{c}{ Thyroid Dose [cGy] } \\
Exposure Pathway & \multicolumn{2}{c}{$95 \%$ Subjective Confidence Interval } \\
\hline Backyard Cow Milk & lower limit & central estimate & upper limit \\
Commercial Milk (locally produced) & 0.57 & 3.3 & 20 \\
Commercial Milk (regionally mixed) & 0.16 & 1.2 & 7.9 \\
Goat Milk (locally produced) & 0.17 & 1.2 & 7.9 \\
Beef (locally produced) & 1.1 & 8.8 & 79 \\
Leafy Vegetables (locally produced) & 0.00077 & 0.014 & 0.33 \\
Eggs (locally produced) & 0.00024 & 0.0024 & 0.022 \\
Cottage Cheese (locally produced) & 0.026 & 0.21 & 1.6 \\
Inhalation & 0.00074 & 0.008 & 0.1 \\
Mother's milk (mother on Diet 1) & 0.015 & 0.059 & 0.25 \\
Prenatal exposure (mother on Diet 1) & 0.0002 & 0.006 & 0.15 \\
Diet 1 & 0.002 & 0.018 & 0.19 \\
Diet 2 & 0.64 & 3.7 & 23 \\
Diet 3 & 0.24 & 1.5 & 10 \\
\hline
\end{tabular}

Excess Lifetime Risk [ ]

\begin{tabular}{llll}
\hline Diet 1 & $6.4 \mathrm{E}-05$ & $9.8 \mathrm{E}-04$ & $1.4 \mathrm{E}-02$ \\
Diet 2 & $3.1 \mathrm{E}-05$ & $3.9 \mathrm{E}-04$ & $4.8 \mathrm{E}-03$ \\
Diet 3 & $2.8 \mathrm{E}-05$ & $3.1 \mathrm{E}-04$ & $4.1 \mathrm{E}-03$ \\
Diet 4 & $1.6 \mathrm{E}-04$ & $2.5 \mathrm{E}-03$ & $3.4 \mathrm{E}-02$ \\
\hline
\end{tabular}

\section{Relative Risk [ ]}

\begin{tabular}{lccc}
\hline Diet 1 & 1.025 & 1.25 & 3.5 \\
Diet 2 & 1.0096 & 1.097 & 2.1 \\
Diet 3 & 1.0083 & 1.081 & 2.1 \\
Diet 4 & 1.051 & 1.57 & 9.8 \\
\hline
\end{tabular}

\begin{tabular}{lccc} 
& \multicolumn{3}{c}{ Probability of Causation [\%] } \\
\hline Diet 1 & 2.45 & 20.2 & 71 \\
Diet 2 & 0.96 & 8.8 & 53 \\
Diet 3 & 0.83 & 7.5 & 52 \\
Diet 4 & 4.85 & 36.1 & 90 \\
\hline
\end{tabular}

Diet 1 - Backyard cow milk + all other locally produced non-milk exposure pathways

Diet 2 - Locally produced commercial milk + all other locally produced non-milk exposure pathways

Diet 3 - Regionally mixed commercial milk + inhalation (other regionally mixed food items are minor contributors to the total)

Diet 4 - Goat milk (all other exposure pathways are of negligible importance compared to goat milk) 


\section{Location: Knoxville}

\section{Receptor: Male born in 1950}

\begin{tabular}{lccc}
\hline & \multicolumn{3}{c}{ Thyroid Dose [cGy] } \\
& \multicolumn{2}{c}{$95 \%$ Subjective Confidence Interval } \\
Exposure Pathway & lower limit & central estimate & upper limit \\
\hline Backyard Cow Milk & 0.58 & 3.4 & 20 \\
Commercial Milk (locally produced) & 0.17 & 1.1 & 8.4 \\
Commercial Milk (regionally mixed) & 0.17 & 1.1 & 8.9 \\
Goat Milk (locally produced) & 1.1 & 9.2 & 72 \\
Beef (locally produced) & 0.00082 & 0.014 & 0.32 \\
Leafy Vegetables (locally produced) & 0.00023 & 0.0024 & 0.022 \\
Eggs (locally produced) & 0.029 & 0.22 & 1.5 \\
Cottage Cheese (locally produced) & 0.00077 & 0.0083 & 0.085 \\
Inhalation & 0.015 & 0.059 & 0.24 \\
Mother's milk (mother on Diet 1) & 0.0002 & 0.006 & 0.15 \\
Prenatal exposure (mother on Diet 1) & 0.002 & 0.018 & 0.19 \\
Diet 1 & 0.65 & 3.7 & 22 \\
Diet 2 & 0.25 & 1.5 & 10 \\
Diet 3 & 0.21 & 1.2 & 9.1 \\
\hline
\end{tabular}

Excess Lifetime Risk [ ]

\begin{tabular}{llcc}
\hline Diet 1 & $6.8 \mathrm{E}-06$ & $2.7 \mathrm{E}-04$ & $4.5 \mathrm{E}-03$ \\
Diet 2 & $3.9 \mathrm{E}-06$ & $1.0 \mathrm{E}-04$ & $2.2 \mathrm{E}-03$ \\
Diet 3 & $3.6 \mathrm{E}-06$ & $8.2 \mathrm{E}-05$ & $1.9 \mathrm{E}-03$ \\
Diet 4 & $1.9 \mathrm{E}-05$ & $6.1 \mathrm{E}-04$ & $1.2 \mathrm{E}-02$ \\
\hline
\end{tabular}

\section{Relative Risk [ ]}

\begin{tabular}{lccc}
\hline Diet 1 & 1.0095 & 1.17 & 4.4 \\
Diet 2 & 1.0033 & 1.068 & 2.6 \\
Diet 3 & 1.0028 & 1.056 & 2.4 \\
Diet 4 & 1.018 & 1.43 & 10.1 \\
\hline
\end{tabular}

\section{Probability of Causation [\%]}

\begin{tabular}{lccc}
\hline Diet 1 & 0.94 & 14.6 & 77 \\
Diet 2 & 0.33 & 6.3 & 61 \\
Diet 3 & 0.28 & 5.3 & 58 \\
Diet 4 & 1.78 & 30.3 & 90 \\
\hline
\end{tabular}

Diet 1 - Backyard cow milk + all other locally produced non-milk exposure pathways

Diet 2 - Locally produced commercial milk + all other locally produced non-milk exposure pathways

Diet 3 - Regionally mixed commercial milk + inhalation (other regionally mixed food items are minor contributors to the total)

Diet 4 - Goat milk (all other exposure pathways are of negligible importance compared to goat milk) 


\section{Location: Maryville}

Receptor: Female born in 1950

\begin{tabular}{lccc}
\hline & \multicolumn{3}{c}{ Thyroid Dose [cGy] } \\
Exposure Pathway & \multicolumn{2}{c}{$95 \%$ Subjective Confidence Interval } \\
\hline Backyard Cow Milk & lower limit & central estimate & upper limit \\
Commercial Milk (locally produced) & 0.28 & 1.6 & 11 \\
Commercial Milk (regionally mixed) & 0.072 & 0.54 & 4.3 \\
Goat Milk (locally produced) & 0.17 & 1.2 & 7.9 \\
Beef (locally produced) & 0.5 & 4.4 & 38 \\
Leafy Vegetables (locally produced) & 0.00043 & 0.0072 & 0.16 \\
Eggs (locally produced) & 0.00011 & 0.0012 & 0.01 \\
Cottage Cheese (locally produced) & 0.013 & 0.11 & 0.84 \\
Inhalation & 0.00032 & 0.004 & 0.045 \\
Mother's milk (mother on Diet 1) & 0.0074 & 0.031 & 0.15 \\
Prenatal exposure (mother on Diet 1) & 0.00012 & 0.0031 & 0.084 \\
Diet 1 & 0.0009 & 0.0088 & 0.091 \\
Diet 2 & 0.31 & 1.8 & 12 \\
Diet 3 & 0.12 & 0.74 & 5 \\
\hline
\end{tabular}

Excess Lifetime Risk [ ]

\begin{tabular}{llll}
\hline Diet 1 & $2.8 \mathrm{E}-05$ & $5.0 \mathrm{E}-04$ & $8.8 \mathrm{E}-03$ \\
Diet 2 & $1.5 \mathrm{E}-05$ & $1.9 \mathrm{E}-04$ & $2.6 \mathrm{E}-03$ \\
Diet 3 & $2.7 \mathrm{E}-05$ & $3.0 \mathrm{E}-04$ & $4.0 \mathrm{E}-03$ \\
Diet 4 & $7.0 \mathrm{E}-05$ & $1.2 \mathrm{E}-03$ & $1.7 \mathrm{E}-02$ \\
\hline
\end{tabular}

\section{Relative Risk [ ]}

\begin{tabular}{lccc}
\hline Diet 1 & 1.012 & 1.12 & 2.1 \\
Diet 2 & 1.0046 & 1.048 & 1.56 \\
Diet 3 & 1.0078 & 1.079 & 2.1 \\
Diet 4 & 1.024 & 1.28 & 5.2 \\
\hline
\end{tabular}

\section{Probability of Causation [\%]}

\begin{tabular}{lccc}
\hline Diet 1 & 1.18 & 10.5 & 52 \\
Diet 2 & 0.46 & 4.6 & 36 \\
Diet 3 & 0.77 & 7.3 & 52 \\
Diet 4 & 2.37 & 21.6 & 80 \\
\hline
\end{tabular}

Diet 1 - Backyard cow milk + all other locally produced non-milk exposure pathways

Diet 2 - Locally produced commercial milk + all other locally produced non-milk exposure pathways

Diet 3 - Regionally mixed commercial milk + inhalation (other regionally mixed food items are minor contributors to the total)

Diet 4 - Goat milk (all other exposure pathways are of negligible importance compared to goat milk) 


\section{Location: Maryville}

\section{Receptor: Male born in 1950}

\begin{tabular}{lccc}
\hline & \multicolumn{3}{c}{ Thyroid Dose [cGy] } \\
& \multicolumn{2}{c}{$95 \%$ Subjective Confidence Interval } \\
Exposure Pathway & lower limit & central estimate & upper limit \\
\hline Backyard Cow Milk & 0.28 & 1.6 & 11 \\
Commercial Milk (locally produced) & 0.081 & 0.54 & 4.2 \\
Commercial Milk (regionally mixed) & 0.17 & 1.1 & 8.9 \\
Goat Milk (locally produced) & 0.48 & 4.4 & 38 \\
Beef (locally produced) & 0.00043 & 0.007 & 0.16 \\
Leafy Vegetables (locally produced) & 0.00011 & 0.0011 & 0.01 \\
Eggs (locally produced) & 0.015 & 0.11 & 0.75 \\
Cottage Cheese (locally produced) & 0.00032 & 0.004 & 0.041 \\
Inhalation & 0.0074 & 0.032 & 0.14 \\
Mother's milk (mother on Diet 1) & 0.00012 & 0.0031 & 0.084 \\
Prenatal exposure (mother on Diet 1) & 0.0009 & 0.0088 & 0.091 \\
Diet 1 & 0.31 & 1.8 & 12 \\
Diet 2 & 0.12 & 0.73 & 5 \\
Diet 3 & 0.19 & 1.2 & 9 \\
\hline
\end{tabular}

Excess Lifetime Risk [ ]

\begin{tabular}{llll}
\hline Diet 1 & $3.7 \mathrm{E}-06$ & $1.3 \mathrm{E}-04$ & $2.3 \mathrm{E}-03$ \\
Diet 2 & $2.0 \mathrm{E}-06$ & $4.8 \mathrm{E}-05$ & $1.1 \mathrm{E}-03$ \\
Diet 3 & $3.3 \mathrm{E}-06$ & $7.9 \mathrm{E}-05$ & $1.9 \mathrm{E}-03$ \\
Diet 4 & $9.8 \mathrm{E}-06$ & $3.0 \mathrm{E}-04$ & $7.2 \mathrm{E}-03$ \\
\hline
\end{tabular}

Relative Risk [ ]

\begin{tabular}{lccc}
\hline Diet 1 & 1.0042 & 1.086 & 2.7 \\
Diet 2 & 1.0016 & 1.034 & 1.74 \\
Diet 3 & 1.0028 & 1.055 & 2.3 \\
Diet 4 & 1.0098 & 1.21 & 7.5 \\
\hline
\end{tabular}

\section{Probability of Causation [\%]}

\begin{tabular}{lccc}
\hline Diet 1 & 0.41 & 7.9 & 63 \\
Diet 2 & 0.16 & 3.2 & 43 \\
Diet 3 & 0.28 & 5.2 & 57 \\
Diet 4 & 0.97 & 17.4 & 86 \\
\hline
\end{tabular}

Diet 1 - Backyard cow milk + all other locally produced non-milk exposure pathways

Diet 2 - Locally produced commercial milk + all other locally produced non-milk exposure pathways

Diet 3 - Regionally mixed commercial milk + inhalation (other regionally mixed food items are minor contributors to the total)

Diet 4 - Goat milk (all other exposure pathways are of negligible importance compared to goat milk) 
Location: Cedar Grove

Receptor: Female born in 1950

\begin{tabular}{lccc}
\hline & \multicolumn{3}{c}{ Thyroid Dose [cGy] } \\
Exposure Pathway & 95\% Subjective Confidence Interval \\
\hline Backyard Cow Milk & lower limit & central estimate & upper limit \\
Commercial Milk (locally produced) & 0.54 & 3 & 19 \\
Commercial Milk (regionally mixed) & 0.15 & 1 & 6.9 \\
Goat Milk (locally produced) & 0.17 & 1.2 & 7.9 \\
Beef (locally produced) & 1 & 8 & 69 \\
Leafy Vegetables (locally produced) & 0.00071 & 0.013 & 0.33 \\
Eggs (locally produced) & 0.00021 & 0.0022 & 0.019 \\
Cottage Cheese (locally produced) & 0.024 & 0.2 & 1.5 \\
Inhalation & 0.00068 & 0.0073 & 0.09 \\
Mother's milk (mother on Diet 1) & 0.013 & 0.051 & 0.21 \\
Prenatal exposure (mother on Diet 1) & 0.00019 & 0.0055 & 0.14 \\
Diet 1 & 0.0019 & 0.016 & 0.17 \\
Diet 2 & 0.61 & 3.3 & 21 \\
Diet 3 & 0.23 & 1.4 & 9 \\
\hline
\end{tabular}

Excess Lifetime Risk [ ]

\begin{tabular}{llll}
\hline Diet 1 & $6.0 \mathrm{E}-05$ & $9.1 \mathrm{E}-04$ & $1.3 \mathrm{E}-02$ \\
Diet 2 & $3.2 \mathrm{E}-05$ & $3.5 \mathrm{E}-04$ & $4.2 \mathrm{E}-03$ \\
Diet 3 & $2.8 \mathrm{E}-05$ & $3.1 \mathrm{E}-04$ & $4.1 \mathrm{E}-03$ \\
Diet 4 & $1.4 \mathrm{E}-04$ & $2.3 \mathrm{E}-03$ & $3.2 \mathrm{E}-02$ \\
\hline
\end{tabular}

\section{Relative Risk [ ]}

\begin{tabular}{lccc}
\hline Diet 1 & 1.021 & 1.23 & 3.3 \\
Diet 2 & 1.0087 & 1.089 & 2 \\
Diet 3 & 1.0082 & 1.08 & 2.1 \\
Diet 4 & 1.048 & 1.5 & 9.2 \\
\hline
\end{tabular}

Probability of Causation [\%]

\begin{tabular}{lccc}
\hline Diet 1 & 2.03 & 18.6 & 69 \\
Diet 2 & 0.86 & 8.2 & 51 \\
Diet 3 & 0.82 & 7.4 & 52 \\
Diet 4 & 4.54 & 33.3 & 89 \\
\hline
\end{tabular}

Diet 1 - Backyard cow milk + all other locally produced non-milk exposure pathways

Diet 2 - Locally produced commercial milk + all other locally produced non-milk exposure pathways

Diet 3 - Regionally mixed commercial milk + inhalation (other regionally mixed food items are minor contributors to the total)

Diet 4 - Goat milk (all other exposure pathways are of negligible importance compared to goat milk) 
Location: Cedar Grove

Receptor: Male born in 1950

\begin{tabular}{lccc}
\hline & \multicolumn{3}{c}{ Thyroid Dose [cGy] } \\
Exposure Pathway & 95\% Subjective Confidence Interval \\
\hline Backyard Cow Milk & lower limit & central estimate & upper limit \\
Commercial Milk (locally produced) & 0.54 & 3.1 & 19 \\
Commercial Milk (regionally mixed) & 0.16 & 1 & 7.6 \\
Goat Milk (locally produced) & 0.17 & 1.1 & 8.9 \\
Beef (locally produced) & 0.97 & 8.4 & 67 \\
Leafy Vegetables (locally produced) & 0.00075 & 0.013 & 0.31 \\
Eggs (locally produced) & 0.00021 & 0.0022 & 0.019 \\
Cottage Cheese (locally produced) & 0.029 & 0.2 & 1.4 \\
Inhalation & 0.00066 & 0.0075 & 0.079 \\
Mother's milk (mother on Diet 1) & 0.013 & 0.051 & 0.2 \\
Prenatal exposure (mother on Diet 1) & 0.00019 & 0.0055 & 0.14 \\
Diet 1 & 0.0019 & 0.016 & 0.17 \\
Diet 2 & 0.62 & 3.4 & 21 \\
Diet 3 & 0.24 & 1.4 & 9.5 \\
\hline
\end{tabular}

Excess Lifetime Risk [ ]

\begin{tabular}{llll}
\hline Diet 1 & $6.8 \mathrm{E}-06$ & $2.4 \mathrm{E}-04$ & $4.3 \mathrm{E}-03$ \\
Diet 2 & $3.6 \mathrm{E}-06$ & $9.0 \mathrm{E}-05$ & $2.0 \mathrm{E}-03$ \\
Diet 3 & $3.6 \mathrm{E}-06$ & $8.2 \mathrm{E}-05$ & $1.9 \mathrm{E}-03$ \\
Diet 4 & $1.8 \mathrm{E}-05$ & $5.5 \mathrm{E}-04$ & $1.2 \mathrm{E}-02$ \\
\hline
\end{tabular}

\section{Relative Risk [ ]}

\begin{tabular}{lccc}
\hline Diet 1 & 1.0079 & 1.16 & 4.2 \\
Diet 2 & 1.003 & 1.063 & 2.4 \\
Diet 3 & 1.0028 & 1.056 & 2.3 \\
Diet 4 & 1.016 & 1.4 & 9 \\
\hline
\end{tabular}

\begin{tabular}{lccc}
\hline Diet 1 & 0.78 & 13.5 & 76 \\
Diet 2 & 0.30 & 5.9 & 58 \\
Diet 3 & 0.28 & 5.3 & 57 \\
Diet 4 & 1.62 & 28.5 & 89 \\
\hline
\end{tabular}

Diet 1 - Backyard cow milk + all other locally produced non-milk exposure pathways

Diet 2 - Locally produced commercial milk + all other locally produced non-milk exposure pathways

Diet 3 - Regionally mixed commercial milk + inhalation (other regionally mixed food items are minor contributors to the total)

Diet 4 - Goat milk (all other exposure pathways are of negligible importance compared to goat milk) 
Location: Bradbury

Receptor: Female born in 1952

\begin{tabular}{lccc}
\hline & \multicolumn{3}{c}{ Thyroid Dose [cGy] } \\
Exposure Pathway & $95 \%$ & Subjective Confidence Interval \\
Backyard Cow Milk & lower limit & central estimate & upper limit \\
Commercial Milk (locally produced) & 4.4 & 28 & 200 \\
Commercial Milk (regionally mixed) & 1.2 & 9.2 & 65 \\
Goat Milk (locally produced) & 0.18 & 1.3 & 8 \\
Beef (locally produced) & 8.9 & 75 & 650 \\
Leafy Vegetables (locally produced) & 0.0061 & 0.1 & 2.5 \\
Eggs (locally produced) & 0.001 & 0.013 & 0.13 \\
Cottage Cheese (locally produced) & 0.2 & 1.7 & 13 \\
Inhalation & 0.0051 & 0.071 & 0.82 \\
Mother's milk (mother on Diet 1) & 0.058 & 0.24 & 0.96 \\
Prenatal exposure (mother on Diet 1) & 0.0011 & 0.026 & 0.54 \\
Diet 1 & 0.011 & 0.075 & 0.82 \\
Diet 2 & 4.9 & 30 & 220 \\
Diet 3 & 1.8 & 12 & 78 \\
\hline
\end{tabular}

Excess Lifetime Risk [ ]

\begin{tabular}{llll}
\hline Diet 1 & $6.0 \mathrm{E}-04$ & $9.3 \mathrm{E}-03$ & $1.4 \mathrm{E}-01$ \\
Diet 2 & $2.6 \mathrm{E}-04$ & $3.5 \mathrm{E}-03$ & $4.5 \mathrm{E}-02$ \\
Diet 3 & $4.2 \mathrm{E}-05$ & $4.5 \mathrm{E}-04$ & $5.0 \mathrm{E}-03$ \\
Diet 4 & $1.3 \mathrm{E}-03$ & $2.5 \mathrm{E}-02$ & $3.6 \mathrm{E}-01$ \\
\hline
\end{tabular}

\section{Relative Risk [ ]}

\begin{tabular}{lccc}
\hline Diet 1 & 1.24 & 3.2 & 29 \\
Diet 2 & 1.086 & 1.87 & 13 \\
Diet 3 & 1.013 & 1.11 & 2.3 \\
Diet 4 & 1.52 & 6.4 & 93 \\
\hline
\end{tabular}

\begin{tabular}{lccc} 
& \multicolumn{3}{c}{ Probability of Causation [\%] } \\
\hline Diet 1 & 19.01 & 68.8 & 97 \\
Diet 2 & 7.89 & 46.2 & 92 \\
Diet 3 & 1.30 & 9.9 & 57 \\
Diet 4 & 34.35 & 84.2 & 99 \\
\hline
\end{tabular}

Diet 1 - Backyard cow milk + all other locally produced non-milk exposure pathways

Diet 2 - Locally produced commercial milk + all other locally produced non-milk exposure pathways

Diet 3 - Regionally mixed commercial milk + inhalation (other regionally mixed food items are minor contributors to the total)

Diet 4 - Goat milk (all other exposure pathways are of negligible importance compared to goat milk) 


\section{Location: Bradbury}

Receptor: Male born in 1952

\begin{tabular}{lccc}
\hline & \multicolumn{3}{c}{ Thyroid Dose [cGy] } \\
& \multicolumn{2}{c}{$95 \%$ Subjective Confidence Interval } \\
Exposure Pathway & lower limit & central estimate & upper limit \\
\hline Backyard Cow Milk & 4.4 & 27 & 190 \\
Commercial Milk (locally produced) & 1.2 & 9 & 68 \\
Commercial Milk (regionally mixed) & 0.18 & 1.3 & 9 \\
Goat Milk (locally produced) & 9.4 & 73 & 650 \\
Beef (locally produced) & 0.006 & 0.1 & 2.3 \\
Leafy Vegetables (locally produced) & 0.0011 & 0.013 & 0.12 \\
Eggs (locally produced) & 0.24 & 1.7 & 13 \\
Cottage Cheese (locally produced) & 0.0053 & 0.069 & 0.85 \\
Inhalation & 0.057 & 0.24 & 0.93 \\
Mother's milk (mother on Diet 1) & 0.0011 & 0.026 & 0.54 \\
Prenatal exposure (mother on Diet 1) & 0.011 & 0.075 & 0.82 \\
Diet 1 & 4.8 & 29 & 210 \\
Diet 2 & 1.7 & 12 & 82 \\
Diet 3 & 0.28 & 1.5 & 9.7 \\
\hline
\end{tabular}

Excess Lifetime Risk [ ]

\begin{tabular}{llll}
\hline Diet 1 & $7.9 \mathrm{E}-05$ & $2.4 \mathrm{E}-03$ & $4.7 \mathrm{E}-02$ \\
Diet 2 & $3.7 \mathrm{E}-05$ & $8.9 \mathrm{E}-04$ & $2.0 \mathrm{E}-02$ \\
Diet 3 & $5.4 \mathrm{E}-06$ & $1.2 \mathrm{E}-04$ & $2.2 \mathrm{E}-03$ \\
Diet 4 & $1.8 \mathrm{E}-04$ & $5.8 \mathrm{E}-03$ & $1.3 \mathrm{E}-01$ \\
\hline
\end{tabular}

\section{Relative Risk [ ]}

\begin{tabular}{lccc}
\hline Diet 1 & 1.1 & 2.6 & 41 \\
Diet 2 & 1.033 & 1.62 & 18 \\
Diet 3 & 1.0048 & 1.079 & 2.9 \\
Diet 4 & 1.2 & 5.5 & 101 \\
\hline
\end{tabular}

\section{Probability of Causation [\%]}

\begin{tabular}{lccc}
\hline Diet 1 & 9.12 & 61.2 & 98 \\
Diet 2 & 3.16 & 38.2 & 94 \\
Diet 3 & 0.47 & 7.3 & 66 \\
Diet 4 & 16.70 & 81.6 & 99 \\
\hline
\end{tabular}

Diet 1 - Backyard cow milk + all other locally produced non-milk exposure pathways

Diet 2 - Locally produced commercial milk + all other locally produced non-milk exposure pathways

Diet 3 - Regionally mixed commercial milk + inhalation (other regionally mixed food items are minor contributors to the total)

Diet 4 - Goat milk (all other exposure pathways are of negligible importance compared to goat milk) 
Location: Gallaher Bend

Receptor: Female born in 1952

\begin{tabular}{lccc}
\hline & \multicolumn{3}{c}{ Thyroid Dose [cGy] } \\
Exposure Pathway & \multicolumn{2}{c}{$95 \%$ Subjective Confidence Interval } \\
Backyard Cow Milk & lower limit & central estimate & upper limit \\
Commercial Milk (locally produced) & 5.5 & 35 & 230 \\
Commercial Milk (regionally mixed) & 1.6 & 11 & 84 \\
Goat Milk (locally produced) & 0.17 & 1.3 & 8 \\
Beef (locally produced) & 10 & 88 & 760 \\
Leafy Vegetables (locally produced) & 0.007 & 0.13 & 2.9 \\
Eggs (locally produced) & 0.0013 & 0.016 & 0.15 \\
Cottage Cheese (locally produced) & 0.25 & 2 & 16 \\
Inhalation & 0.0062 & 0.088 & 0.97 \\
Mother's milk (mother on Diet 1) & 0.065 & 0.29 & 1.2 \\
Prenatal exposure (mother on Diet 1) & 0.0012 & 0.03 & 0.67 \\
Diet 1 & 0.014 & 0.092 & 0.89 \\
Diet 2 & 6.1 & 39 & 250 \\
Diet 3 & 2.2 & 15 & 97 \\
\hline
\end{tabular}

Excess Lifetime Risk [ ]

\begin{tabular}{llll}
\hline Diet 1 & $7.1 \mathrm{E}-04$ & $1.1 \mathrm{E}-02$ & $1.7 \mathrm{E}-01$ \\
Diet 2 & $3.2 \mathrm{E}-04$ & $4.3 \mathrm{E}-03$ & $5.9 \mathrm{E}-02$ \\
Diet 3 & $4.4 \mathrm{E}-05$ & $4.7 \mathrm{E}-04$ & $5.1 \mathrm{E}-03$ \\
Diet 4 & $1.8 \mathrm{E}-03$ & $3.1 \mathrm{E}-02$ & $4.3 \mathrm{E}-01$ \\
\hline
\end{tabular}

\section{Relative Risk [ ]}

\begin{tabular}{lccc}
\hline Diet 1 & 1.29 & 3.8 & 33 \\
Diet 2 & 1.11 & 2.1 & 15 \\
Diet 3 & 1.014 & 1.12 & 2.4 \\
Diet 4 & 1.68 & 7.9 & 111 \\
\hline
\end{tabular}

\begin{tabular}{lccc} 
& \multicolumn{3}{c}{ Probability of Causation [\%] } \\
\hline Diet 1 & 22.50 & 73.6 & 97 \\
Diet 2 & 9.54 & 52.0 & 93 \\
Diet 3 & 1.41 & 10.3 & 58 \\
Diet 4 & 40.56 & 87.3 & 99 \\
\hline
\end{tabular}

Diet 1 - Backyard cow milk + all other locally produced non-milk exposure pathways

Diet 2 - Locally produced commercial milk + all other locally produced non-milk exposure pathways

Diet 3 - Regionally mixed commercial milk + inhalation (other regionally mixed food items are minor contributors to the total)

Diet 4 - Goat milk (all other exposure pathways are of negligible importance compared to goat milk) 


\section{Location: Gallaher Bend}

Receptor: Male born in 1952

\begin{tabular}{lccc}
\hline & \multicolumn{3}{c}{ Thyroid Dose [cGy] } \\
& \multicolumn{2}{c}{$95 \%$ Subjective Confidence Interval } \\
Exposure Pathway & lower limit & central estimate & upper limit \\
\hline Backyard Cow Milk & 5 & 35 & 210 \\
Commercial Milk (locally produced) & 1.5 & 11 & 83 \\
Commercial Milk (regionally mixed) & 0.18 & 1.3 & 9 \\
Goat Milk (locally produced) & 10 & 90 & 800 \\
Beef (locally produced) & 0.007 & 0.13 & 3 \\
Leafy Vegetables (locally produced) & 0.0014 & 0.016 & 0.14 \\
Eggs (locally produced) & 0.28 & 2.1 & 14 \\
Cottage Cheese (locally produced) & 0.0068 & 0.092 & 0.95 \\
Inhalation & 0.072 & 0.3 & 1.1 \\
Mother's milk (mother on Diet 1) & 0.0012 & 0.03 & 0.67 \\
Prenatal exposure (mother on Diet 1) & 0.014 & 0.092 & 0.89 \\
Diet 1 & 5.6 & 38 & 230 \\
Diet 2 & 2.2 & 15 & 94 \\
Diet 3 & 0.31 & 1.6 & 10 \\
\hline
\end{tabular}

Excess Lifetime Risk [ ]

\begin{tabular}{lllc}
\hline Diet 1 & $1.0 \mathrm{E}-04$ & $3.2 \mathrm{E}-03$ & $5.4 \mathrm{E}-02$ \\
Diet 2 & $4.0 \mathrm{E}-05$ & $1.2 \mathrm{E}-03$ & $2.3 \mathrm{E}-02$ \\
Diet 3 & $5.8 \mathrm{E}-06$ & $1.2 \mathrm{E}-04$ & $2.3 \mathrm{E}-03$ \\
Diet 4 & $2.3 \mathrm{E}-04$ & $7.3 \mathrm{E}-03$ & $1.3 \mathrm{E}-01$ \\
\hline
\end{tabular}

\section{Relative Risk [ ]}

\begin{tabular}{lccc}
\hline Diet 1 & 1.11 & 2.8 & 50 \\
Diet 2 & 1.039 & 1.75 & 19 \\
Diet 3 & 1.0051 & 1.084 & 2.9 \\
Diet 4 & 1.27 & 6.4 & 121 \\
\hline
\end{tabular}

\section{Probability of Causation [\%]}

\begin{tabular}{lccc}
\hline Diet 1 & 9.89 & 64.8 & 98 \\
Diet 2 & 3.80 & 42.8 & 95 \\
Diet 3 & 0.50 & 7.7 & 66 \\
Diet 4 & 21.19 & 84.4 & 99 \\
\hline
\end{tabular}

Diet 1 - Backyard cow milk + all other locally produced non-milk exposure pathways

Diet 2 - Locally produced commercial milk + all other locally produced non-milk exposure pathways

Diet 3 - Regionally mixed commercial milk + inhalation (other regionally mixed food items are minor contributors to the total)

Diet 4 - Goat milk (all other exposure pathways are of negligible importance compared to goat milk) 


\section{Location: EFPC}

Receptor: Female born in 1952

\begin{tabular}{lccc}
\hline & \multicolumn{3}{c}{ Thyroid Dose [cGy] } \\
& \multicolumn{2}{c}{$95 \%$ Subjective Confidence Interval } \\
Exposure Pathway & lower limit & central estimate & upper limit \\
\hline Backyard Cow Milk & 1.1 & 7.3 & 50 \\
Commercial Milk (locally produced) & 0.3 & 2.4 & 17 \\
Commercial Milk (regionally mixed) & 0.18 & 1.3 & 8 \\
Goat Milk (locally produced) & -- & -- & -- \\
Beef (locally produced) & 0.0016 & 0.027 & 0.65 \\
Leafy Vegetables (locally produced) & 0.00029 & 0.0034 & 0.033 \\
Eggs (locally produced) & 0.051 & 0.43 & 3.5 \\
Cottage Cheese (locally produced) & 0.0014 & 0.019 & 0.21 \\
Inhalation & 0.015 & 0.064 & 0.25 \\
Mother's milk (mother on Diet 1) & 0.00029 & 0.0065 & 0.14 \\
Prenatal exposure (mother on Diet 1) & 0.0027 & 0.02 & 0.2 \\
Diet 1 & 1.2 & 8 & 54 \\
Diet 2 & 0.46 & 3.2 & 21 \\
Diet 3 & 0.21 & 1.3 & 8.1 \\
\hline
\end{tabular}

Excess Lifetime Risk [ ]

\begin{tabular}{lccc}
\hline Diet 1 & $1.5 \mathrm{E}-04$ & $2.4 \mathrm{E}-03$ & $3.6 \mathrm{E}-02$ \\
Diet 2 & $6.9 \mathrm{E}-05$ & $9.2 \mathrm{E}-04$ & $1.2 \mathrm{E}-02$ \\
Diet 3 & $3.5 \mathrm{E}-05$ & $3.8 \mathrm{E}-04$ & $4.8 \mathrm{E}-03$ \\
Diet 4 & -- & -- & -- \\
\hline
\end{tabular}

\section{Relative Risk [ ]}

\begin{tabular}{lccc}
\hline Diet 1 & 1.058 & 1.6 & 8.4 \\
Diet 2 & 1.022 & 1.22 & 4.1 \\
Diet 3 & 1.01 & 1.098 & 2.2 \\
Diet 4 & -- & -- & -- \\
\hline
\end{tabular}

\section{Probability of Causation [\%]}

\begin{tabular}{lccc}
\hline Diet 1 & 5.40 & 36.4 & 87 \\
Diet 2 & 2.13 & 17.9 & 74 \\
Diet 3 & 1.03 & 8.9 & 55 \\
Diet 4 & -- & -- \\
\hline Diet 1 - Backyard cow milk + all other locally produced non-milk exposure pathways & \\
Diet 2 - Locally produced commercial milk + all other locally produced non-milk exposure pathways \\
Diet 3 - Regionally mixed commercial milk + inhalation (other regionally mixed food items are minor contributors to the total) \\
Diet 4 - Goat milk (all other exposure pathways are of negligible importance compared to goat milk)
\end{tabular}




\section{Location: EFPC}

\section{Receptor: Male born in 1952}

\begin{tabular}{lccc}
\hline & \multicolumn{3}{c}{ Thyroid Dose [cGy] } \\
& \multicolumn{2}{c}{$95 \%$ Subjective Confidence Interval } \\
Exposure Pathway & lower limit & central estimate & upper limit \\
\hline Backyard Cow Milk & 1.1 & 7.2 & 49 \\
Commercial Milk (locally produced) & 0.29 & 2.4 & 18 \\
Commercial Milk (regionally mixed) & 0.18 & 1.3 & 9 \\
Goat Milk (locally produced) & -- & -- & -- \\
Beef (locally produced) & 0.0015 & 0.026 & 0.66 \\
Leafy Vegetables (locally produced) & 0.00028 & 0.0033 & 0.033 \\
Eggs (locally produced) & 0.058 & 0.44 & 3.3 \\
Cottage Cheese (locally produced) & 0.0014 & 0.018 & 0.23 \\
Inhalation & 0.015 & 0.064 & 0.25 \\
Mother's milk (mother on Diet 1) & 0.00029 & 0.0065 & 0.14 \\
Prenatal exposure (mother on Diet 1) & 0.0027 & 0.02 & 0.2 \\
Diet 1 & 1.2 & 7.9 & 53 \\
Diet 2 & 0.43 & 3.1 & 21 \\
Diet 3 & 0.2 & 1.3 & 9.2 \\
\hline
\end{tabular}

Excess Lifetime Risk [ ]

\begin{tabular}{lccc}
\hline Diet 1 & $2.0 \mathrm{E}-05$ & $6.5 \mathrm{E}-04$ & $1.1 \mathrm{E}-02$ \\
Diet 2 & $9.3 \mathrm{E}-06$ & $2.4 \mathrm{E}-04$ & $5.2 \mathrm{E}-03$ \\
Diet 3 & $4.3 \mathrm{E}-06$ & $9.7 \mathrm{E}-05$ & $2.0 \mathrm{E}-03$ \\
Diet 4 & -- & -- & -- \\
\hline
\end{tabular}

\section{Relative Risk [ ]}

\begin{tabular}{lccc}
\hline Diet 1 & 1.024 & 1.41 & 12 \\
Diet 2 & 1.0084 & 1.16 & 5.5 \\
Diet 3 & 1.0037 & 1.067 & 2.8 \\
Diet 4 & -- & -- & -- \\
\hline
\end{tabular}

\begin{tabular}{lccc}
\hline Diet 1 & 2.36 & 28.4 & 91 \\
Diet 2 & 0.83 & 13.2 & 81 \\
Diet 3 & 0.37 & 6.3 & 64 \\
Diet 4 & -- & -- & - \\
Diet 1 - Backyard cow milk + all other locally produced non-milk exposure pathways & \\
Diet 2 - Locally produced commercial milk + all other locally produced non-milk exposure pathways \\
Diet 3 - Regionally mixed commercial milk + inhalation (other regionally mixed food items are minor contributors to the total) \\
Diet 4 - Goat milk (all other exposure pathways are of negligible importance compared to goat milk)
\end{tabular}




\section{Location: Hope Creek}

Receptor: Female born in 1952

\begin{tabular}{lccc}
\hline & \multicolumn{3}{c}{ Thyroid Dose [cGy] } \\
Exposure Pathway & \multicolumn{2}{c}{$95 \%$ Subjective Confidence Interval } \\
\hline Backyard Cow Milk & lower limit & central estimate & upper limit \\
Commercial Milk (locally produced) & 3.2 & 20 & 140 \\
Commercial Milk (regionally mixed) & 0.88 & 6.3 & 49 \\
Goat Milk (locally produced) & 0.17 & 1.3 & 8 \\
Beef (locally produced) & -- & -- & -- \\
Leafy Vegetables (locally produced) & 0.004 & 0.073 & 1.8 \\
Eggs (locally produced) & 0.00082 & 0.0092 & 0.089 \\
Cottage Cheese (locally produced) & 0.15 & 1.1 & 9.4 \\
Inhalation & 0.0035 & 0.05 & 0.56 \\
Mother's milk (mother on Diet 1) & 0.041 & 0.17 & 0.69 \\
Prenatal exposure (mother on Diet 1) & 0.00074 & 0.018 & 0.34 \\
Diet 1 & 0.0078 & 0.057 & 0.49 \\
Diet 2 & 3.5 & 22 & 150 \\
Diet 3 & 1.2 & 8.2 & 58 \\
\hline
\end{tabular}

Excess Lifetime Risk [ ]

\begin{tabular}{lccc}
\hline Diet 1 & $4.2 \mathrm{E}-04$ & $6.6 \mathrm{E}-03$ & $9.7 \mathrm{E}-02$ \\
Diet 2 & $1.9 \mathrm{E}-04$ & $2.5 \mathrm{E}-03$ & $3.3 \mathrm{E}-02$ \\
Diet 3 & $3.9 \mathrm{E}-05$ & $4.2 \mathrm{E}-04$ & $4.9 \mathrm{E}-03$ \\
Diet 4 & -- & -- & -- \\
\hline
\end{tabular}

\section{Relative Risk [ ]}

\begin{tabular}{lccc}
\hline Diet 1 & 1.16 & 2.6 & 20 \\
Diet 2 & 1.062 & 1.62 & 9.4 \\
Diet 3 & 1.012 & 1.1 & 2.3 \\
Diet 4 & -- & -- & -- \\
\hline
\end{tabular}

\begin{tabular}{lccc}
\hline Diet 1 & 13.63 & 61.2 & 95 \\
Diet 2 & 5.86 & 38.1 & 89 \\
Diet 3 & 1.19 & 9.5 & 57 \\
Diet 4 & -- & -- & -- \\
\hline Diet 1 - Backyard cow milk + all other locally produced non-milk exposure pathways & \\
Diet 2 - Locally produced commercial milk + all other locally produced non-milk exposure pathways \\
Diet 3 - Regionally mixed commercial milk + inhalation (other regionally mixed food items are minor contributors to the total) \\
Diet 4 - Goat milk (all other exposure pathways are of negligible importance compared to goat milk)
\end{tabular}




\section{Location: Hope Creek}

Receptor: Male born in 1952

\begin{tabular}{lccc}
\hline & \multicolumn{3}{c}{ Thyroid Dose [cGy] } \\
& \multicolumn{2}{c}{$95 \%$ Subjective Confidence Interval } \\
Exposure Pathway & lower limit & central estimate & upper limit \\
\hline Backyard Cow Milk & 3 & 19 & 130 \\
Commercial Milk (locally produced) & 0.86 & 6.4 & 54 \\
Commercial Milk (regionally mixed) & 0.18 & 1.3 & 9 \\
Goat Milk (locally produced) & -- & -- & -- \\
Beef (locally produced) & 0.004 & 0.073 & 1.8 \\
Leafy Vegetables (locally produced) & 0.00078 & 0.009 & 0.09 \\
Eggs (locally produced) & 0.17 & 1.2 & 8.9 \\
Cottage Cheese (locally produced) & 0.0038 & 0.051 & 0.59 \\
Inhalation & 0.043 & 0.17 & 0.66 \\
Mother's milk (mother on Diet 1) & 0.00074 & 0.018 & 0.34 \\
Prenatal exposure (mother on Diet 1) & 0.0078 & 0.057 & 0.49 \\
Diet 1 & 3.3 & 21 & 140 \\
Diet 2 & 1.3 & 8.4 & 62 \\
Diet 3 & 0.25 & 1.4 & 9.6 \\
\hline
\end{tabular}

Excess Lifetime Risk [ ]

\begin{tabular}{lccc}
\hline Diet 1 & $5.1 \mathrm{E}-05$ & $1.7 \mathrm{E}-03$ & $3.3 \mathrm{E}-02$ \\
Diet 2 & $2.4 \mathrm{E}-05$ & $6.4 \mathrm{E}-04$ & $1.4 \mathrm{E}-02$ \\
Diet 3 & $4.9 \mathrm{E}-06$ & $1.1 \mathrm{E}-04$ & $2.1 \mathrm{E}-03$ \\
Diet 4 & -- & -- & -- \\
\hline
\end{tabular}

\section{Relative Risk [ ]}

\begin{tabular}{lccc}
\hline Diet 1 & 1.069 & 2.1 & 26 \\
Diet 2 & 1.022 & 1.44 & 12 \\
Diet 3 & 1.0043 & 1.075 & 2.9 \\
Diet 4 & -- & -- & -- \\
\hline
\end{tabular}

\section{Probability of Causation [\%]}

\begin{tabular}{lccc}
\hline Diet 1 & 6.41 & 52.7 & 96 \\
Diet 2 & 2.15 & 30.2 & 91 \\
Diet 3 & 0.43 & 7.0 & 65 \\
Diet 4 & -- & -- & - \\
\hline Diet 1 - Backyard cow milk + all other locally produced non-milk exposure pathways & \\
Diet 2 - Locally produced commercial milk + all other locally produced non-milk exposure pathways \\
Diet 3 - Regionally mixed commercial milk + inhalation (other regionally mixed food items are minor contributors to the total) \\
Diet 4 - Goat milk (all other exposure pathways are of negligible importance compared to goat milk)
\end{tabular}


Location: Buttermilk Rd.

Receptor: Female born in 1952

\begin{tabular}{lccc}
\hline & \multicolumn{3}{c}{ Thyroid Dose [cGy] } \\
Exposure Pathway & \multicolumn{2}{c}{$95 \%$ Subjective Confidence Interval } \\
Backyard Cow Milk & lower limit & central estimate & upper limit \\
Commercial Milk (locally produced) & 3.1 & 20 & 130 \\
Commercial Milk (regionally mixed) & 0.88 & 6.4 & 48 \\
Goat Milk (locally produced) & 0.17 & 1.3 & 8 \\
Beef (locally produced) & 5.8 & 51 & 440 \\
Leafy Vegetables (locally produced) & 0.0039 & 0.073 & 1.6 \\
Eggs (locally produced) & 0.00078 & 0.0092 & 0.088 \\
Cottage Cheese (locally produced) & 0.14 & 1.1 & 9.2 \\
Inhalation & 0.0035 & 0.05 & 0.54 \\
Mother's milk (mother on Diet 1) & 0.043 & 0.17 & 0.7 \\
Prenatal exposure (mother on Diet 1) & 0.00072 & 0.018 & 0.33 \\
Diet 1 & 0.0074 & 0.056 & 0.46 \\
Diet 2 & 3.4 & 22 & 150 \\
Diet 3 & 1.3 & 8.1 & 56 \\
\hline
\end{tabular}

Excess Lifetime Risk [ ]

\begin{tabular}{llll}
\hline Diet 1 & $4.0 \mathrm{E}-04$ & $6.5 \mathrm{E}-03$ & $9.2 \mathrm{E}-02$ \\
Diet 2 & $1.9 \mathrm{E}-04$ & $2.4 \mathrm{E}-03$ & $3.2 \mathrm{E}-02$ \\
Diet 3 & $3.9 \mathrm{E}-05$ & $4.2 \mathrm{E}-04$ & $4.9 \mathrm{E}-03$ \\
Diet 4 & $9.4 \mathrm{E}-04$ & $1.7 \mathrm{E}-02$ & $2.5 \mathrm{E}-01$ \\
\hline
\end{tabular}

\section{Relative Risk [ ]}

\begin{tabular}{lccc}
\hline Diet 1 & 1.15 & 2.6 & 20 \\
Diet 2 & 1.059 & 1.61 & 9.3 \\
Diet 3 & 1.012 & 1.1 & 2.3 \\
Diet 4 & 1.36 & 4.8 & 58 \\
\hline
\end{tabular}

\begin{tabular}{lccc} 
& \multicolumn{3}{c}{ Probability of Causation [\%] } \\
\hline Diet 1 & 13.28 & 61.0 & 95 \\
Diet 2 & 5.53 & 37.7 & 89 \\
Diet 3 & 1.19 & 9.5 & 57 \\
Diet 4 & 26.11 & 79.0 & 98 \\
\hline
\end{tabular}

Diet 1 - Backyard cow milk + all other locally produced non-milk exposure pathways

Diet 2 - Locally produced commercial milk + all other locally produced non-milk exposure pathways

Diet 3 - Regionally mixed commercial milk + inhalation (other regionally mixed food items are minor contributors to the total)

Diet 4 - Goat milk (all other exposure pathways are of negligible importance compared to goat milk) 
Location: Buttermilk Rd.

Receptor: Male born in 1952

\begin{tabular}{lccc}
\hline & \multicolumn{3}{c}{ Thyroid Dose [cGy] } \\
& \multicolumn{2}{c}{$95 \%$ Subjective Confidence Interval } \\
Exposure Pathway & lower limit & central estimate & upper limit \\
\hline Backyard Cow Milk & 3 & 19 & 120 \\
Commercial Milk (locally produced) & 0.82 & 6.3 & 51 \\
Commercial Milk (regionally mixed) & 0.18 & 1.3 & 9 \\
Goat Milk (locally produced) & 5.8 & 50 & 470 \\
Beef (locally produced) & 0.0039 & 0.071 & 1.6 \\
Leafy Vegetables (locally produced) & 0.0008 & 0.009 & 0.086 \\
Eggs (locally produced) & 0.17 & 1.2 & 8.6 \\
Cottage Cheese (locally produced) & 0.0037 & 0.05 & 0.57 \\
Inhalation & 0.044 & 0.17 & 0.67 \\
Mother's milk (mother on Diet 1) & 0.00072 & 0.018 & 0.33 \\
Prenatal exposure (mother on Diet 1) & 0.0074 & 0.056 & 0.46 \\
Diet 1 & 3.3 & 21 & 130 \\
Diet 2 & 1.3 & 8.3 & 60 \\
Diet 3 & 0.25 & 1.4 & 9.6 \\
\hline
\end{tabular}

Excess Lifetime Risk [ ]

\begin{tabular}{llcc}
\hline Diet 1 & $4.9 \mathrm{E}-05$ & $1.7 \mathrm{E}-03$ & $3.1 \mathrm{E}-02$ \\
Diet 2 & $2.3 \mathrm{E}-05$ & $6.3 \mathrm{E}-04$ & $1.4 \mathrm{E}-02$ \\
Diet 3 & $4.9 \mathrm{E}-06$ & $1.1 \mathrm{E}-04$ & $2.1 \mathrm{E}-03$ \\
Diet 4 & $1.3 \mathrm{E}-04$ & $4.3 \mathrm{E}-03$ & $8.8 \mathrm{E}-02$ \\
\hline
\end{tabular}

\section{Relative Risk [ ]}

\begin{tabular}{lccc}
\hline Diet 1 & 1.07 & 2.1 & 26 \\
Diet 2 & 1.022 & 1.42 & 12 \\
Diet 3 & 1.0044 & 1.075 & 2.9 \\
Diet 4 & 1.15 & 4 & 69 \\
\hline
\end{tabular}

\section{Probability of Causation [\%]}

\begin{tabular}{lccc}
\hline Diet 1 & 6.51 & 52.1 & 96 \\
Diet 2 & 2.14 & 29.6 & 91 \\
Diet 3 & 0.44 & 6.9 & 65 \\
Diet 4 & 12.77 & 74.9 & 98 \\
\hline
\end{tabular}

Diet 1 - Backyard cow milk + all other locally produced non-milk exposure pathways

Diet 2 - Locally produced commercial milk + all other locally produced non-milk exposure pathways

Diet 3 - Regionally mixed commercial milk + inhalation (other regionally mixed food items are minor contributors to the total)

Diet 4 - Goat milk (all other exposure pathways are of negligible importance compared to goat milk) 


\section{Location: Jonesville}

Receptor: Female born in 1952

\begin{tabular}{lccc}
\hline & \multicolumn{3}{c}{ Thyroid Dose [cGy] } \\
Exposure Pathway & \multicolumn{2}{c}{$95 \%$ Subjective Confidence Interval } \\
\hline Backyard Cow Milk & 0.4 & 2.8 & 21 \\
Commercial Milk (locally produced) & 0.11 & 0.89 & 7.3 \\
Commercial Milk (regionally mixed) & 0.17 & 1.3 & 8 \\
Goat Milk (locally produced) & 0.84 & 7.3 & 73 \\
Beef (locally produced) & 0.00053 & 0.01 & 0.24 \\
Leafy Vegetables (locally produced) & 0.0001 & 0.0013 & 0.014 \\
Eggs (locally produced) & 0.019 & 0.16 & 1.4 \\
Cottage Cheese (locally produced) & 0.00048 & 0.007 & 0.094 \\
Inhalation & 0.0063 & 0.026 & 0.11 \\
Mother's milk (mother on Diet 1) & 0.00012 & 0.0026 & 0.057 \\
Prenatal exposure (mother on Diet 1) & 0.0011 & 0.0077 & 0.082 \\
Diet 1 & 0.45 & 3 & 23 \\
Diet 2 & 0.17 & 1.2 & 9.1 \\
Diet 3 & 0.19 & 1.3 & 8 \\
\hline
\end{tabular}

Excess Lifetime Risk [ ]

\begin{tabular}{llll}
\hline Diet 1 & $5.6 \mathrm{E}-05$ & $9.0 \mathrm{E}-04$ & $1.4 \mathrm{E}-02$ \\
Diet 2 & $2.6 \mathrm{E}-05$ & $3.4 \mathrm{E}-04$ & $4.8 \mathrm{E}-03$ \\
Diet 3 & $3.3 \mathrm{E}-05$ & $3.7 \mathrm{E}-04$ & $4.7 \mathrm{E}-03$ \\
Diet 4 & $1.4 \mathrm{E}-04$ & $2.3 \mathrm{E}-03$ & $4.4 \mathrm{E}-02$ \\
\hline
\end{tabular}

\section{Relative Risk [ ]}

\begin{tabular}{lccc}
\hline Diet 1 & 1.022 & 1.23 & 4.2 \\
Diet 2 & 1.0079 & 1.084 & 2.1 \\
Diet 3 & 1.0095 & 1.095 & 2.2 \\
Diet 4 & 1.049 & 1.52 & 10.8 \\
\hline
\end{tabular}

\section{Probability of Causation [\%]}

\begin{tabular}{lccc}
\hline Diet 1 & 2.18 & 18.5 & 76 \\
Diet 2 & 0.78 & 7.7 & 53 \\
Diet 3 & 0.94 & 8.6 & 55 \\
Diet 4 & 4.67 & 34.2 & 91 \\
\hline
\end{tabular}

Diet 1 - Backyard cow milk + all other locally produced non-milk exposure pathways

Diet 2 - Locally produced commercial milk + all other locally produced non-milk exposure pathways

Diet 3 - Regionally mixed commercial milk + inhalation (other regionally mixed food items are minor contributors to the total)

Diet 4 - Goat milk (all other exposure pathways are of negligible importance compared to goat milk) 


\section{Location: Jonesville}

\section{Receptor: Male born in 1952}

\begin{tabular}{lccc}
\hline & \multicolumn{3}{c}{ Thyroid Dose [cGy] } \\
& \multicolumn{2}{c}{$95 \%$ Subjective Confidence Interval } \\
Exposure Pathway & lower limit & central estimate & upper limit \\
\hline Backyard Cow Milk & 0.42 & 2.6 & 20 \\
Commercial Milk (locally produced) & 0.11 & 0.87 & 6.9 \\
Commercial Milk (regionally mixed) & 0.18 & 1.3 & 9 \\
Goat Milk (locally produced) & 0.8 & 7.3 & 73 \\
Beef (locally produced) & 0.00052 & 0.01 & 0.23 \\
Leafy Vegetables (locally produced) & 0.0001 & 0.0013 & 0.013 \\
Eggs (locally produced) & 0.021 & 0.17 & 1.3 \\
Cottage Cheese (locally produced) & 0.00053 & 0.0069 & 0.094 \\
Inhalation & 0.0067 & 0.025 & 0.12 \\
Mother's milk (mother on Diet 1) & 0.00012 & 0.0026 & 0.057 \\
Prenatal exposure (mother on Diet 1) & 0.0011 & 0.0077 & 0.082 \\
Diet 1 & 0.47 & 2.9 & 22 \\
Diet 2 & 0.17 & 1.2 & 8.3 \\
Diet 3 & 0.2 & 1.3 & 9.1 \\
\hline
\end{tabular}

Excess Lifetime Risk [ ]

\begin{tabular}{llll}
\hline Diet 1 & $6.8 \mathrm{E}-06$ & $2.4 \mathrm{E}-04$ & $5.2 \mathrm{E}-03$ \\
Diet 2 & $3.8 \mathrm{E}-06$ & $9.0 \mathrm{E}-05$ & $2.0 \mathrm{E}-03$ \\
Diet 3 & $4.0 \mathrm{E}-06$ & $9.3 \mathrm{E}-05$ & $2.0 \mathrm{E}-03$ \\
Diet 4 & $2.1 \mathrm{E}-05$ & $6.0 \mathrm{E}-04$ & $1.4 \mathrm{E}-02$ \\
\hline
\end{tabular}

\section{Relative Risk [ ]}

\begin{tabular}{lccc}
\hline Diet 1 & 1.0086 & 1.16 & 4.6 \\
Diet 2 & 1.0031 & 1.059 & 2.7 \\
Diet 3 & 1.0036 & 1.065 & 2.7 \\
Diet 4 & 1.02 & 1.43 & 10.9 \\
\hline
\end{tabular}

\section{Probability of Causation [\%]}

\begin{tabular}{lccc}
\hline Diet 1 & 0.85 & 14.1 & 78 \\
Diet 2 & 0.31 & 5.6 & 62 \\
Diet 3 & 0.36 & 6.1 & 63 \\
Diet 4 & 1.91 & 29.8 & 91 \\
\hline
\end{tabular}

Diet 1 - Backyard cow milk + all other locally produced non-milk exposure pathways

Diet 2 - Locally produced commercial milk + all other locally produced non-milk exposure pathways

Diet 3 - Regionally mixed commercial milk + inhalation (other regionally mixed food items are minor contributors to the total)

Diet 4 - Goat milk (all other exposure pathways are of negligible importance compared to goat milk) 


\section{Location: OR Scarboro}

Receptor: Female born in 1952

\begin{tabular}{lccc}
\hline & \multicolumn{3}{c}{ Thyroid Dose [cGy] } \\
Exposure Pathway & \multicolumn{2}{c}{$9 \%$ Subjective Confidence Interval } \\
\hline Backyard Cow Milk & lower limit & central estimate & upper limit \\
Commercial Milk (locally produced) & -- & -- & -- \\
Commercial Milk (regionally mixed) & -- & -- & -- \\
Goat Milk (locally produced) & 0.17 & 1.3 & 8 \\
Beef (locally produced) & -- & -- & -- \\
Leafy Vegetables (locally produced) & -- & -- & -- \\
Eggs (locally produced) & -- & -- & -- \\
Cottage Cheese (locally produced) & -- & -- & -- \\
Inhalation & 0.0025 & 0.037 & 0.41 \\
Mother's milk (mother on Diet 3) & 0.03 & 0.13 & 0.53 \\
Prenatal exposure (mother on Diet 3) & 0.00046 & 0.0083 & 0.13 \\
Diet 1 & 0.0046 & 0.026 & 0.2 \\
Diet 2 & -- & -- & -- \\
Diet 3 & -- & -- & -- \\
\hline
\end{tabular}

Excess Lifetime Risk [ ]

\begin{tabular}{lccc}
\hline Diet 1 & -- & -- & -- \\
Diet 2 & -- & -- & -- \\
Diet 3 & $3.7 \mathrm{E}-05$ & $4.0 \mathrm{E}-04$ & $4.8 \mathrm{E}-03$ \\
Diet 4 & -- & -- & -- \\
\hline
\end{tabular}

\section{Relative Risk [ ]}

\begin{tabular}{lccc}
\hline Diet 1 & -- & -- & -- \\
Diet 2 & -- & -- & -- \\
Diet 3 & 1.011 & 1.1 & 2.3 \\
Diet 4 & -- & -- & -- \\
\hline
\end{tabular}

\begin{tabular}{lccc}
\hline Diet 1 & -- & -- & -- \\
Diet 2 & -- & -- & -- \\
Diet 3 & 1.13 & 9.3 & 56 \\
Diet 4 & -- & -- & \\
\hline Diet 1 - Backyard cow milk + all other locally produced non-milk exposure pathways & \\
Diet 2 - Locally produced commercial milk + all other locally produced non-milk exposure pathways \\
Diet 3 - Regionally mixed commercial milk + inhalation (other regionally mixed food items are minor contributors to the total) \\
Diet 4 - Goat milk (all other exposure pathways are of negligible importance compared to goat milk)
\end{tabular}




\section{Location: OR Scarboro}

Receptor: Male born in 1952

\begin{tabular}{lccc}
\hline & \multicolumn{3}{c}{ Thyroid Dose [cGy] } \\
Exposure Pathway & \multicolumn{2}{c}{ 95\% Subjective Confidence Interval } \\
\hline Backyard Cow Milk & lower limit & central estimate & upper limit \\
Commercial Milk (locally produced) & -- & -- & -- \\
Commercial Milk (regionally mixed) & -- & -- & -- \\
Goat Milk (locally produced) & 0.18 & 1.3 & 9 \\
Beef (locally produced) & -- & -- & -- \\
Leafy Vegetables (locally produced) & -- & -- & -- \\
Eggs (locally produced) & -- & -- & -- \\
Cottage Cheese (locally produced) & -- & -- & -- \\
Inhalation & 0.0028 & 0.037 & 0.41 \\
Mother's milk (mother on Diet 3) & 0.031 & 0.13 & 0.5 \\
Prenatal exposure (mother on Diet 3) & 0.00046 & 0.0083 & 0.13 \\
Diet 1 & 0.0046 & 0.026 & 0.2 \\
Diet 2 & -- & -- & -- \\
Diet 3 & -- & -- & -- \\
\hline
\end{tabular}

Excess Lifetime Risk [ ]

\begin{tabular}{lccc}
\hline Diet 1 & -- & -- & -- \\
Diet 2 & -- & -- & -- \\
Diet 3 & $4.7 \mathrm{E}-06$ & $1.0 \mathrm{E}-04$ & $2.1 \mathrm{E}-03$ \\
Diet 4 & -- & -- & -- \\
\hline
\end{tabular}

\section{Relative Risk [ ]}

\begin{tabular}{lccc}
\hline Diet 1 & -- & -- & -- \\
Diet 2 & -- & -- & -- \\
Diet 3 & 1.0041 & 1.072 & 2.8 \\
Diet 4 & -- & -- & -- \\
\hline
\end{tabular}

\begin{tabular}{lccc}
\hline Diet 1 & -- & -- & -- \\
Diet 2 & -- & -- & -- \\
Diet 3 & 0.41 & 6.7 & -- \\
Diet 4 & -- & -- & \\
\hline Diet 1 - Backyard cow milk + all other locally produced non-milk exposure pathways & \\
Diet 2 - Locally produced commercial milk + all other locally produced non-milk exposure pathways \\
Diet 3 - Regionally mixed commercial milk + inhalation (other regionally mixed food items are minor contributors to the total) \\
Diet 4 - Goat milk (all other exposure pathways are of negligible importance compared to goat milk)
\end{tabular}


Location: Lawnville/Gallaher

Receptor: Female born in 1952

\begin{tabular}{lccc}
\hline & \multicolumn{3}{c}{ Thyroid Dose [cGy] } \\
Exposure Pathway & $95 \%$ & Subjective Confidence Interval \\
Backyard Cow Milk & lower limit & central estimate & upper limit \\
Commercial Milk (locally produced) & 3.3 & 19 & 120 \\
Commercial Milk (regionally mixed) & 0.86 & 5.9 & 46 \\
Goat Milk (locally produced) & 0.17 & 1.2 & 8 \\
Beef (locally produced) & 5.9 & 49 & 400 \\
Leafy Vegetables (locally produced) & 0.0035 & 0.072 & 1.6 \\
Eggs (locally produced) & 0.0008 & 0.0087 & 0.08 \\
Cottage Cheese (locally produced) & 0.15 & 1.1 & 8.5 \\
Inhalation & 0.0035 & 0.047 & 0.51 \\
Mother's milk (mother on Diet 1) & 0.04 & 0.16 & 0.68 \\
Prenatal exposure (mother on Diet 1) & 0.00071 & 0.017 & 0.34 \\
Diet 1 & 0.0073 & 0.051 & 0.44 \\
Diet 2 & 3.6 & 20 & 130 \\
Diet 3 & 1.2 & 7.5 & 57 \\
\hline
\end{tabular}

Excess Lifetime Risk [ ]

\begin{tabular}{llll}
\hline Diet 1 & $3.9 \mathrm{E}-04$ & $6.0 \mathrm{E}-03$ & $9.1 \mathrm{E}-02$ \\
Diet 2 & $2.0 \mathrm{E}-04$ & $2.3 \mathrm{E}-03$ & $3.3 \mathrm{E}-02$ \\
Diet 3 & $4.0 \mathrm{E}-05$ & $4.2 \mathrm{E}-04$ & $4.9 \mathrm{E}-03$ \\
Diet 4 & $1.0 \mathrm{E}-03$ & $1.6 \mathrm{E}-02$ & $2.7 \mathrm{E}-01$ \\
\hline
\end{tabular}

\section{Relative Risk [ ]}

\begin{tabular}{lccc}
\hline Diet 1 & 1.15 & 2.5 & 18 \\
Diet 2 & 1.054 & 1.57 & 9.3 \\
Diet 3 & 1.012 & 1.1 & 2.3 \\
Diet 4 & 1.37 & 4.7 & 54 \\
\hline
\end{tabular}

\begin{tabular}{lccc}
\hline Diet 1 & 13.29 & 59.8 & 95 \\
Diet 2 & 5.13 & 36.3 & 89 \\
Diet 3 & 1.17 & 9.5 & 57 \\
Diet 4 & 26.86 & 78.5 & 98 \\
\hline
\end{tabular}

Diet 1 - Backyard cow milk + all other locally produced non-milk exposure pathways

Diet 2 - Locally produced commercial milk + all other locally produced non-milk exposure pathways

Diet 3 - Regionally mixed commercial milk + inhalation (other regionally mixed food items are minor contributors to the total)

Diet 4 - Goat milk (all other exposure pathways are of negligible importance compared to goat milk) 


\section{Location: Lawnville/Gallaher}

Receptor: Male born in 1952

\begin{tabular}{lccc}
\hline & \multicolumn{3}{c}{ Thyroid Dose [cGy] } \\
& \multicolumn{2}{c}{$95 \%$ Subjective Confidence Interval } \\
Exposure Pathway & lower limit & central estimate & upper limit \\
\hline Backyard Cow Milk & 3.2 & 19 & 100 \\
Commercial Milk (locally produced) & 0.77 & 5.8 & 45 \\
Commercial Milk (regionally mixed) & 0.18 & 1.3 & 9 \\
Goat Milk (locally produced) & 5.8 & 48 & 410 \\
Beef (locally produced) & 0.0035 & 0.069 & 1.6 \\
Leafy Vegetables (locally produced) & 0.00089 & 0.0087 & 0.078 \\
Eggs (locally produced) & 0.17 & 1.1 & 8.4 \\
Cottage Cheese (locally produced) & 0.0035 & 0.048 & 0.54 \\
Inhalation & 0.043 & 0.16 & 0.69 \\
Mother's milk (mother on Diet 1) & 0.00071 & 0.017 & 0.34 \\
Prenatal exposure (mother on Diet 1) & 0.0073 & 0.051 & 0.44 \\
Diet 1 & 3.5 & 21 & 110 \\
Diet 2 & 1.2 & 7.6 & 56 \\
Diet 3 & 0.25 & 1.4 & 9.5 \\
\hline
\end{tabular}

Excess Lifetime Risk [ ]

\begin{tabular}{llll}
\hline Diet 1 & $4.7 \mathrm{E}-05$ & $1.6 \mathrm{E}-03$ & $2.9 \mathrm{E}-02$ \\
Diet 2 & $2.2 \mathrm{E}-05$ & $6.0 \mathrm{E}-04$ & $1.3 \mathrm{E}-02$ \\
Diet 3 & $4.9 \mathrm{E}-06$ & $1.1 \mathrm{E}-04$ & $2.2 \mathrm{E}-03$ \\
Diet 4 & $1.3 \mathrm{E}-04$ & $3.8 \mathrm{E}-03$ & $8.0 \mathrm{E}-02$ \\
\hline
\end{tabular}

\begin{tabular}{lccc}
\hline Diet 1 & 1.065 & 2 & 24 \\
Diet 2 & 1.024 & 1.4 & 12 \\
Diet 3 & 1.0045 & 1.072 & 2.9 \\
Diet 4 & 1.15 & 3.9 & 65 \\
\hline
\end{tabular}

\section{Probability of Causation [\%]}

\begin{tabular}{lccc}
\hline Diet 1 & 6.13 & 50.9 & 96 \\
Diet 2 & 2.31 & 28.7 & 91 \\
Diet 3 & 0.45 & 6.7 & 65 \\
Diet 4 & 12.67 & 74.4 & 98 \\
\hline
\end{tabular}

Diet 1 - Backyard cow milk + all other locally produced non-milk exposure pathways

Diet 2 - Locally produced commercial milk + all other locally produced non-milk exposure pathways

Diet 3 - Regionally mixed commercial milk + inhalation (other regionally mixed food items are minor contributors to the total)

Diet 4 - Goat milk (all other exposure pathways are of negligible importance compared to goat milk) 


\section{Location: Dyllis}

Receptor: Female born in 1952

\begin{tabular}{lccc}
\hline & \multicolumn{3}{c}{ Thyroid Dose [cGy] } \\
& \multicolumn{2}{c}{$95 \%$ Subjective Confidence Interval } \\
Exposure Pathway & lower limit & central estimate & upper limit \\
\hline Backyard Cow Milk & 0.54 & 3.7 & 28 \\
Commercial Milk (locally produced) & 0.17 & 1.2 & 10 \\
Commercial Milk (regionally mixed) & 0.17 & 1.2 & 8 \\
Goat Milk (locally produced) & 1.1 & 9.8 & 93 \\
Beef (locally produced) & 0.00079 & 0.014 & 0.31 \\
Leafy Vegetables (locally produced) & 0.00014 & 0.0018 & 0.018 \\
Eggs (locally produced) & 0.027 & 0.21 & 2 \\
Cottage Cheese (locally produced) & 0.00074 & 0.0093 & 0.13 \\
Inhalation & 0.0083 & 0.036 & 0.16 \\
Mother's milk (mother on Diet 1) & 0.00017 & 0.0037 & 0.075 \\
Prenatal exposure (mother on Diet 1) & 0.0017 & 0.011 & 0.11 \\
Diet 1 & 0.6 & 4.1 & 30 \\
Diet 2 & 0.23 & 1.6 & 13 \\
Diet 3 & 0.2 & 1.3 & 8.1 \\
\hline
\end{tabular}

Excess Lifetime Risk [ ]

\begin{tabular}{llll}
\hline Diet 1 & $8.2 \mathrm{E}-05$ & $1.2 \mathrm{E}-03$ & $2.0 \mathrm{E}-02$ \\
Diet 2 & $2.8 \mathrm{E}-05$ & $4.7 \mathrm{E}-04$ & $6.4 \mathrm{E}-03$ \\
Diet 3 & $3.4 \mathrm{E}-05$ & $3.7 \mathrm{E}-04$ & $4.7 \mathrm{E}-03$ \\
Diet 4 & $1.7 \mathrm{E}-04$ & $3.4 \mathrm{E}-03$ & $5.3 \mathrm{E}-02$ \\
\hline
\end{tabular}

\section{Relative Risk [ ]}

\begin{tabular}{lccc}
\hline Diet 1 & 1.035 & 1.29 & 4.6 \\
Diet 2 & 1.012 & 1.11 & 2.6 \\
Diet 3 & 1.0099 & 1.096 & 2.2 \\
Diet 4 & 1.076 & 1.75 & 13 \\
\hline
\end{tabular}

\section{Probability of Causation [\%]}

\begin{tabular}{lccc}
\hline Diet 1 & 3.34 & 22.5 & 78 \\
Diet 2 & 1.16 & 10.2 & 61 \\
Diet 3 & 0.98 & 8.7 & 55 \\
Diet 4 & 7.07 & 42.6 & 92 \\
\hline
\end{tabular}

Diet 1 - Backyard cow milk + all other locally produced non-milk exposure pathways

Diet 2 - Locally produced commercial milk + all other locally produced non-milk exposure pathways

Diet 3 - Regionally mixed commercial milk + inhalation (other regionally mixed food items are minor contributors to the total)

Diet 4 - Goat milk (all other exposure pathways are of negligible importance compared to goat milk) 


\section{Location: Dyllis}

Receptor: Male born in 1952

\begin{tabular}{lccc}
\hline & \multicolumn{3}{c}{ Thyroid Dose [cGy] } \\
& \multicolumn{2}{c}{$95 \%$ Subjective Confidence Interval } \\
Exposure Pathway & lower limit & central estimate & upper limit \\
\hline Backyard Cow Milk & 0.52 & 3.8 & 28 \\
Commercial Milk (locally produced) & 0.17 & 1.2 & 9.8 \\
Commercial Milk (regionally mixed) & 0.18 & 1.3 & 9 \\
Goat Milk (locally produced) & 1.2 & 9.9 & 98 \\
Beef (locally produced) & 0.00077 & 0.013 & 0.31 \\
Leafy Vegetables (locally produced) & 0.00014 & 0.0017 & 0.019 \\
Eggs (locally produced) & 0.028 & 0.23 & 1.8 \\
Cottage Cheese (locally produced) & 0.00075 & 0.0099 & 0.12 \\
Inhalation & 0.0086 & 0.036 & 0.15 \\
Mother's milk (mother on Diet 1) & 0.00017 & 0.0037 & 0.075 \\
Prenatal exposure (mother on Diet 1) & 0.0017 & 0.011 & 0.11 \\
Diet 1 & 0.57 & 4.1 & 29 \\
Diet 2 & 0.24 & 1.6 & 12 \\
Diet 3 & 0.19 & 1.3 & 9.1 \\
\hline
\end{tabular}

Excess Lifetime Risk [ ]

\begin{tabular}{lllc}
\hline Diet 1 & $9.6 \mathrm{E}-06$ & $3.3 \mathrm{E}-04$ & $6.4 \mathrm{E}-03$ \\
Diet 2 & $5.0 \mathrm{E}-06$ & $1.3 \mathrm{E}-04$ & $2.7 \mathrm{E}-03$ \\
Diet 3 & $4.1 \mathrm{E}-06$ & $9.4 \mathrm{E}-05$ & $2.0 \mathrm{E}-03$ \\
Diet 4 & $2.8 \mathrm{E}-05$ & $8.0 \mathrm{E}-04$ & $1.6 \mathrm{E}-02$ \\
\hline
\end{tabular}

\section{Relative Risk [ ]}

\begin{tabular}{lccc}
\hline Diet 1 & 1.012 & 1.21 & 6.1 \\
Diet 2 & 1.004 & 1.084 & 3.2 \\
Diet 3 & 1.0036 & 1.066 & 2.7 \\
Diet 4 & 1.026 & 1.57 & 14 \\
\hline
\end{tabular}

\section{Probability of Causation [\%]}

\begin{tabular}{lccc}
\hline Diet 1 & 1.14 & 17.3 & 83 \\
Diet 2 & 0.40 & 7.7 & 69 \\
Diet 3 & 0.36 & 6.2 & 63 \\
Diet 4 & 2.54 & 36.4 & 93 \\
\hline
\end{tabular}

Diet 1 - Backyard cow milk + all other locally produced non-milk exposure pathways

Diet 2 - Locally produced commercial milk + all other locally produced non-milk exposure pathways

Diet 3 - Regionally mixed commercial milk + inhalation (other regionally mixed food items are minor contributors to the total)

Diet 4 - Goat milk (all other exposure pathways are of negligible importance compared to goat milk) 


\section{Location: OR High School Area}

Receptor: Female born in 1952

\begin{tabular}{lccc}
\hline & \multicolumn{3}{c}{ Thyroid Dose [cGy] } \\
Exposure Pathway & \multicolumn{2}{c}{ 95\% } & Subjective Confidence Interval \\
Backyard Cow Milk & lower limit & central estimate & upper limit \\
Commercial Milk (locally produced) & -- & -- & -- \\
Commercial Milk (regionally mixed) & -- & -- & -- \\
Goat Milk (locally produced) & 0.17 & 1.3 & 8 \\
Beef (locally produced) & -- & -- & -- \\
Leafy Vegetables (locally produced) & -- & -- & -- \\
Eggs (locally produced) & -- & -- & -- \\
Cottage Cheese (locally produced) & -- & -- & -- \\
Inhalation & 0.0014 & 0.021 & 0.23 \\
Mother's milk (mother on Diet 3) & 0.018 & 0.073 & 0.32 \\
Prenatal exposure (mother on Diet 3) & 0.00037 & 0.0069 & 0.12 \\
Diet 1 & 0.0037 & 0.022 & 0.19 \\
Diet 2 & -- & -- & -- \\
Diet 3 & -- & -- & -- \\
\hline
\end{tabular}

Excess Lifetime Risk [ ]

\begin{tabular}{lccc}
\hline Diet 1 & -- & -- & -- \\
Diet 2 & -- & -- & -- \\
Diet 3 & $3.5 \mathrm{E}-05$ & $3.9 \mathrm{E}-04$ & $4.8 \mathrm{E}-03$ \\
Diet 4 & -- & -- & -- \\
\hline
\end{tabular}

\section{Relative Risk [ ]}

\begin{tabular}{lccc}
\hline Diet 1 & -- & -- & -- \\
Diet 2 & -- & -- & -- \\
Diet 3 & 1.011 & 1.098 & 2.2 \\
Diet 4 & -- & -- & -- \\
\hline
\end{tabular}

\begin{tabular}{|c|c|c|c|}
\hline Diet 1 & -- & -- & -- \\
\hline Diet 2 & -- & -- & -- \\
\hline Diet 3 & 1.05 & 9.0 & 55 \\
\hline Diet 4 & -- & -- & -- \\
\hline
\end{tabular}




\section{Location: OR High School Area}

Receptor: Male born in 1952

\begin{tabular}{lccc}
\hline & \multicolumn{3}{c}{ Thyroid Dose [cGy] } \\
Exposure Pathway & \multicolumn{2}{c}{ 95\% } & Subjective Confidence Interval \\
Backyard Cow Milk & lower limit & central estimate & upper limit \\
Commercial Milk (locally produced) & -- & -- & -- \\
Commercial Milk (regionally mixed) & -- & -- & -- \\
Goat Milk (locally produced) & 0.18 & 1.3 & 9 \\
Beef (locally produced) & -- & -- & -- \\
Leafy Vegetables (locally produced) & -- & -- & -- \\
Eggs (locally produced) & -- & -- & -- \\
Cottage Cheese (locally produced) & -- & -- & -- \\
Inhalation & 0.0016 & 0.022 & 0.23 \\
Mother's milk (mother on Diet 3) & 0.019 & 0.075 & 0.3 \\
Prenatal exposure (mother on Diet 3) & 0.00037 & 0.0069 & 0.12 \\
Diet 1 & 0.0037 & 0.022 & 0.19 \\
Diet 2 & -- & -- & -- \\
Diet 3 & -- & -- & -- \\
\hline
\end{tabular}

Excess Lifetime Risk [ ]

\begin{tabular}{lccc}
\hline Diet 1 & -- & -- & -- \\
Diet 2 & -- & -- & -- \\
Diet 3 & $4.3 \mathrm{E}-06$ & $9.9 \mathrm{E}-05$ & $2.0 \mathrm{E}-03$ \\
Diet 4 & -- & -- & -- \\
\hline
\end{tabular}

\section{Relative Risk [ ]}

\begin{tabular}{lccc}
\hline Diet 1 & -- & -- & -- \\
Diet 2 & -- & -- & -- \\
Diet 3 & 1.0038 & 1.068 & 2.8 \\
Diet 4 & -- & -- & -- \\
\hline
\end{tabular}

\begin{tabular}{lccc}
\hline Diet 1 & -- & -- & -- \\
Diet 2 & -- & -- & -- \\
Diet 3 & 0.38 & 6.3 & -- \\
Diet 4 & -- & -- & \\
\hline Diet 1 - Backyard cow milk + all other locally produced non-milk exposure pathways & \\
Diet 2 - Locally produced commercial milk + all other locally produced non-milk exposure pathways \\
Diet 3 - Regionally mixed commercial milk + inhalation (other regionally mixed food items are minor contributors to the total) \\
Diet 4 - Goat milk (all other exposure pathways are of negligible importance compared to goat milk)
\end{tabular}




\section{Location: Norwood}

Receptor: Female born in 1952

\begin{tabular}{lccc}
\hline & \multicolumn{3}{c}{ Thyroid Dose [cGy] } \\
& \multicolumn{2}{c}{$95 \%$ Subjective Confidence Interval } \\
Exposure Pathway & lower limit & central estimate & upper limit \\
\hline Backyard Cow Milk & 0.51 & 3.5 & 29 \\
Commercial Milk (locally produced) & 0.16 & 1.1 & 10 \\
Commercial Milk (regionally mixed) & 0.17 & 1.3 & 8 \\
Goat Milk (locally produced) & 1 & 9.7 & 96 \\
Beef (locally produced) & 0.00074 & 0.013 & 0.34 \\
Leafy Vegetables (locally produced) & 0.00015 & 0.0017 & 0.016 \\
Eggs (locally produced) & 0.026 & 0.2 & 1.9 \\
Cottage Cheese (locally produced) & 0.00068 & 0.0091 & 0.12 \\
Inhalation & 0.0082 & 0.033 & 0.15 \\
Mother's milk (mother on Diet 1) & 0.00014 & 0.0034 & 0.068 \\
Prenatal exposure (mother on Diet 1) & 0.0013 & 0.01 & 0.11 \\
Diet 1 & 0.56 & 3.9 & 31 \\
Diet 2 & 0.23 & 1.5 & 12 \\
Diet 3 & 0.2 & 1.3 & 8 \\
\hline
\end{tabular}

Excess Lifetime Risk [ ]

\begin{tabular}{llll}
\hline Diet 1 & $7.3 \mathrm{E}-05$ & $1.3 \mathrm{E}-03$ & $1.9 \mathrm{E}-02$ \\
Diet 2 & $3.5 \mathrm{E}-05$ & $4.5 \mathrm{E}-04$ & $7.1 \mathrm{E}-03$ \\
Diet 3 & $3.3 \mathrm{E}-05$ & $3.7 \mathrm{E}-04$ & $4.7 \mathrm{E}-03$ \\
Diet 4 & $1.8 \mathrm{E}-04$ & $3.2 \mathrm{E}-03$ & $5.6 \mathrm{E}-02$ \\
\hline
\end{tabular}

\section{Relative Risk [ ]}

\begin{tabular}{lccc}
\hline Diet 1 & 1.029 & 1.3 & 4.9 \\
Diet 2 & 1.01 & 1.11 & 2.6 \\
Diet 3 & 1.0097 & 1.096 & 2.2 \\
Diet 4 & 1.066 & 1.74 & 12 \\
\hline
\end{tabular}

\section{Probability of Causation [\%]}

\begin{tabular}{lccc}
\hline Diet 1 & 2.78 & 22.7 & 79 \\
Diet 2 & 1.02 & 9.8 & 62 \\
Diet 3 & 0.96 & 8.7 & 55 \\
Diet 4 & 6.21 & 42.4 & 92 \\
\hline
\end{tabular}

Diet 1 - Backyard cow milk + all other locally produced non-milk exposure pathways

Diet 2 - Locally produced commercial milk + all other locally produced non-milk exposure pathways

Diet 3 - Regionally mixed commercial milk + inhalation (other regionally mixed food items are minor contributors to the total)

Diet 4 - Goat milk (all other exposure pathways are of negligible importance compared to goat milk) 


\section{Location: Norwood}

\section{Receptor: Male born in 1952}

\begin{tabular}{lccc}
\hline & \multicolumn{3}{c}{ Thyroid Dose [cGy] } \\
& \multicolumn{2}{c}{$95 \%$ Subjective Confidence Interval } \\
Exposure Pathway & lower limit & central estimate & upper limit \\
\hline Backyard Cow Milk & 0.54 & 3.5 & 27 \\
Commercial Milk (locally produced) & 0.16 & 1.1 & 10 \\
Commercial Milk (regionally mixed) & 0.18 & 1.3 & 9 \\
Goat Milk (locally produced) & 1.1 & 9.5 & 110 \\
Beef (locally produced) & 0.00078 & 0.013 & 0.31 \\
Leafy Vegetables (locally produced) & 0.00016 & 0.0016 & 0.017 \\
Eggs (locally produced) & 0.028 & 0.21 & 1.8 \\
Cottage Cheese (locally produced) & 0.00069 & 0.0091 & 0.12 \\
Inhalation & 0.0074 & 0.034 & 0.14 \\
Mother's milk (mother on Diet 1) & 0.00014 & 0.0034 & 0.068 \\
Prenatal exposure (mother on Diet 1) & 0.0013 & 0.01 & 0.11 \\
Diet 1 & 0.6 & 3.8 & 30 \\
Diet 2 & 0.22 & 1.5 & 12 \\
Diet 3 & 0.19 & 1.3 & 9.1 \\
\hline
\end{tabular}

Excess Lifetime Risk [ ]

\begin{tabular}{llll}
\hline Diet 1 & $9.8 \mathrm{E}-06$ & $3.0 \mathrm{E}-04$ & $6.3 \mathrm{E}-03$ \\
Diet 2 & $4.3 \mathrm{E}-06$ & $1.1 \mathrm{E}-04$ & $2.6 \mathrm{E}-03$ \\
Diet 3 & $4.0 \mathrm{E}-06$ & $9.3 \mathrm{E}-05$ & $2.0 \mathrm{E}-03$ \\
Diet 4 & $2.2 \mathrm{E}-05$ & $7.4 \mathrm{E}-04$ & $1.7 \mathrm{E}-02$ \\
\hline
\end{tabular}

\section{Relative Risk [ ]}

\begin{tabular}{lccc}
\hline Diet 1 & 1.012 & 1.21 & 6.2 \\
Diet 2 & 1.0042 & 1.077 & 3.3 \\
Diet 3 & 1.0036 & 1.066 & 2.7 \\
Diet 4 & 1.024 & 1.56 & 17 \\
\hline
\end{tabular}

\section{Probability of Causation [\%]}

\begin{tabular}{lccc}
\hline Diet 1 & 1.18 & 17.2 & 84 \\
Diet 2 & 0.42 & 7.2 & 70 \\
Diet 3 & 0.36 & 6.2 & 63 \\
Diet 4 & 2.36 & 35.8 & 94 \\
\hline
\end{tabular}

Diet 1 - Backyard cow milk + all other locally produced non-milk exposure pathways

Diet 2 - Locally produced commercial milk + all other locally produced non-milk exposure pathways

Diet 3 - Regionally mixed commercial milk + inhalation (other regionally mixed food items are minor contributors to the total)

Diet 4 - Goat milk (all other exposure pathways are of negligible importance compared to goat milk) 
Location: Woodland

Receptor: Female born in 1952

\begin{tabular}{lccc}
\hline & \multicolumn{3}{c}{ Thyroid Dose [cGy] } \\
Exposure Pathway & \multicolumn{2}{c}{$95 \%$ Subjective Confidence Interval } \\
\hline Backyard Cow Milk & lower limit & central estimate & upper limit \\
Commercial Milk (locally produced) & -- & -- & -- \\
Commercial Milk (regionally mixed) & -- & -- & -- \\
Goat Milk (locally produced) & 0.17 & 1.3 & 8 \\
Beef (locally produced) & -- & -- & -- \\
Leafy Vegetables (locally produced) & -- & -- & -- \\
Eggs (locally produced) & -- & -- & -- \\
Cottage Cheese (locally produced) & -- & -- & -- \\
Inhalation & 0.0022 & 0.031 & 0.34 \\
Mother's milk (mother on Diet 3) & 0.028 & 0.11 & 0.46 \\
Prenatal exposure (mother on Diet 3) & 0.00043 & 0.0079 & 0.12 \\
Diet 1 & 0.0043 & 0.025 & 0.2 \\
Diet 2 & -- & -- & -- \\
Diet 3 & -- & -- & -- \\
\hline
\end{tabular}

Excess Lifetime Risk [ ]

\begin{tabular}{lccc}
\hline Diet 1 & -- & -- & -- \\
Diet 2 & -- & -- & -- \\
Diet 3 & $3.7 \mathrm{E}-05$ & $4.0 \mathrm{E}-04$ & $4.8 \mathrm{E}-03$ \\
Diet 4 & -- & -- & -- \\
\hline
\end{tabular}

Relative Risk [ ]

\begin{tabular}{lccc}
\hline Diet 1 & -- & -- & -- \\
Diet 2 & -- & -- & -- \\
Diet 3 & 1.011 & 1.1 & 2.3 \\
Diet 4 & -- & -- & -- \\
\hline
\end{tabular}

\begin{tabular}{lccc}
\hline Diet 1 & -- & -- & -- \\
Diet 2 & -- & -- & -- \\
Diet 3 & 1.11 & 9.2 & 56 \\
Diet 4 & -- & -- \\
\hline Diet 1 - Backyard cow milk + all other locally produced non-milk exposure pathways & \\
Diet 2 - Locally produced commercial milk + all other locally produced non-milk exposure pathways \\
Diet 3 - Regionally mixed commercial milk + inhalation (other regionally mixed food items are minor contributors to the total) \\
Diet 4 - Goat milk (all other exposure pathways are of negligible importance compared to goat milk)
\end{tabular}


Location: Woodland

Receptor: Male born in 1952

\begin{tabular}{lccc}
\hline & \multicolumn{3}{c}{ Thyroid Dose [cGy] } \\
Exposure Pathway & \multicolumn{2}{c}{$9 \%$ Subjective Confidence Interval } \\
\hline Backyard Cow Milk & lower limit & central estimate & upper limit \\
Commercial Milk (locally produced) & -- & -- & -- \\
Commercial Milk (regionally mixed) & -- & -- & -- \\
Goat Milk (locally produced) & 0.18 & 1.3 & 9 \\
Beef (locally produced) & -- & -- & -- \\
Leafy Vegetables (locally produced) & -- & -- & -- \\
Eggs (locally produced) & -- & -- & -- \\
Cottage Cheese (locally produced) & -- & -- & -- \\
Inhalation & 0.0023 & 0.032 & 0.35 \\
Mother's milk (mother on Diet 3) & 0.029 & 0.11 & 0.45 \\
Prenatal exposure (mother on Diet 3) & 0.00043 & 0.0079 & 0.12 \\
Diet 1 & 0.0043 & 0.025 & 0.2 \\
Diet 2 & -- & -- & -- \\
Diet 3 & -- & -- & -- \\
\hline
\end{tabular}

Excess Lifetime Risk [ ]

\begin{tabular}{lccc}
\hline Diet 1 & -- & -- & -- \\
Diet 2 & -- & -- & -- \\
Diet 3 & $4.6 \mathrm{E}-06$ & $1.0 \mathrm{E}-04$ & $2.1 \mathrm{E}-03$ \\
Diet 4 & -- & -- & -- \\
\hline
\end{tabular}

\section{Relative Risk [ ]}

\begin{tabular}{lccc}
\hline Diet 1 & -- & -- & -- \\
Diet 2 & -- & -- & -- \\
Diet 3 & 1.0041 & 1.07 & 2.8 \\
Diet 4 & -- & -- & -- \\
\hline
\end{tabular}

\begin{tabular}{lccc}
\hline Diet 1 & -- & -- & -- \\
Diet 2 & -- & -- & -- \\
Diet 3 & 0.41 & 6.5 & -- \\
Diet 4 & -- & -- & - \\
\hline Diet 1 - Backyard cow milk + all other locally produced non-milk exposure pathways & \\
Diet 2 - Locally produced commercial milk + all other locally produced non-milk exposure pathways \\
Diet 3 - Regionally mixed commercial milk + inhalation (other regionally mixed food items are minor contributors to the total) \\
Diet 4 - Goat milk (all other exposure pathways are of negligible importance compared to goat milk)
\end{tabular}


Location: Hardin Valley

Receptor: Female born in 1952

\begin{tabular}{lccc}
\hline & \multicolumn{3}{c}{ Thyroid Dose [cGy] } \\
& \multicolumn{2}{c}{$95 \%$ Subjective Confidence Interval } \\
Exposure Pathway & lower limit & central estimate & upper limit \\
\hline Backyard Cow Milk & 2.7 & 17 & 110 \\
Commercial Milk (locally produced) & 0.8 & 5.7 & 42 \\
Commercial Milk (regionally mixed) & 0.17 & 1.3 & 8 \\
Goat Milk (locally produced) & 5.5 & 46 & 390 \\
Beef (locally produced) & 0.0036 & 0.064 & 1.4 \\
Leafy Vegetables (locally produced) & 0.0007 & 0.0083 & 0.077 \\
Eggs (locally produced) & 0.13 & 1 & 8.3 \\
Cottage Cheese (locally produced) & 0.0033 & 0.044 & 0.49 \\
Inhalation & 0.04 & 0.16 & 0.7 \\
Mother's milk (mother on Diet 1) & 0.00068 & 0.016 & 0.31 \\
Prenatal exposure (mother on Diet 1) & 0.0073 & 0.05 & 0.43 \\
Diet 1 & 3 & 19 & 130 \\
Diet 2 & 1.2 & 7.5 & 50 \\
Diet 3 & 0.26 & 1.4 & 8.5 \\
\hline
\end{tabular}

Excess Lifetime Risk [ ]

\begin{tabular}{llll}
\hline Diet 1 & $3.7 \mathrm{E}-04$ & $5.7 \mathrm{E}-03$ & $8.3 \mathrm{E}-02$ \\
Diet 2 & $1.5 \mathrm{E}-04$ & $2.2 \mathrm{E}-03$ & $2.9 \mathrm{E}-02$ \\
Diet 3 & $3.9 \mathrm{E}-05$ & $4.2 \mathrm{E}-04$ & $4.9 \mathrm{E}-03$ \\
Diet 4 & $8.6 \mathrm{E}-04$ & $1.6 \mathrm{E}-02$ & $2.3 \mathrm{E}-01$ \\
\hline
\end{tabular}

\section{Relative Risk [ ]}

\begin{tabular}{lccc}
\hline Diet 1 & 1.15 & 2.4 & 17 \\
Diet 2 & 1.056 & 1.52 & 7.5 \\
Diet 3 & 1.012 & 1.1 & 2.3 \\
Diet 4 & 1.35 & 4.4 & 54 \\
\hline
\end{tabular}

\begin{tabular}{lccc}
\hline Diet 1 & \multicolumn{3}{c}{ Probability of Causation [\%] } \\
Diet 2 & 5.32 & 58.4 & 94 \\
Diet 3 & 1.20 & 34.2 & 87 \\
Diet 4 & 25.70 & 9.4 & 56 \\
\hline
\end{tabular}

Diet 1 - Backyard cow milk + all other locally produced non-milk exposure pathways

Diet 2 - Locally produced commercial milk + all other locally produced non-milk exposure pathways

Diet 3 - Regionally mixed commercial milk + inhalation (other regionally mixed food items are minor contributors to the total)

Diet 4 - Goat milk (all other exposure pathways are of negligible importance compared to goat milk) 
Location: Hardin Valley

Receptor: Male born in 1952

\begin{tabular}{lccc}
\hline & \multicolumn{3}{c}{ Thyroid Dose [cGy] } \\
& \multicolumn{2}{c}{$95 \%$ Subjective Confidence Interval } \\
Exposure Pathway & lower limit & central estimate & upper limit \\
\hline Backyard Cow Milk & 2.7 & 18 & 100 \\
Commercial Milk (locally produced) & 0.8 & 5.7 & 44 \\
Commercial Milk (regionally mixed) & 0.18 & 1.3 & 9 \\
Goat Milk (locally produced) & 5.6 & 44 & 410 \\
Beef (locally produced) & 0.0037 & 0.063 & 1.4 \\
Leafy Vegetables (locally produced) & 0.00074 & 0.008 & 0.08 \\
Eggs (locally produced) & 0.15 & 1 & 7.7 \\
Cottage Cheese (locally produced) & 0.0033 & 0.045 & 0.5 \\
Inhalation & 0.042 & 0.17 & 0.65 \\
Mother's milk (mother on Diet 1) & 0.00068 & 0.016 & 0.31 \\
Prenatal exposure (mother on Diet 1) & 0.0073 & 0.05 & 0.43 \\
Diet 1 & 3 & 19 & 110 \\
Diet 2 & 1.2 & 7.5 & 52 \\
Diet 3 & 0.25 & 1.4 & 9.5 \\
\hline
\end{tabular}

Excess Lifetime Risk [ ]

\begin{tabular}{lllc}
\hline Diet 1 & $4.5 \mathrm{E}-05$ & $1.5 \mathrm{E}-03$ & $2.8 \mathrm{E}-02$ \\
Diet 2 & $2.3 \mathrm{E}-05$ & $5.6 \mathrm{E}-04$ & $1.2 \mathrm{E}-02$ \\
Diet 3 & $4.8 \mathrm{E}-06$ & $1.1 \mathrm{E}-04$ & $2.1 \mathrm{E}-03$ \\
Diet 4 & $1.2 \mathrm{E}-04$ & $3.8 \mathrm{E}-03$ & $7.3 \mathrm{E}-02$ \\
\hline
\end{tabular}

\section{Relative Risk [ ]}

\begin{tabular}{lccc}
\hline Diet 1 & 1.062 & 1.98 & 24 \\
Diet 2 & 1.021 & 1.39 & 10.9 \\
Diet 3 & 1.0043 & 1.074 & 2.9 \\
Diet 4 & 1.13 & 3.7 & 60 \\
\hline
\end{tabular}

\section{Probability of Causation [\%]}

\begin{tabular}{lccc}
\hline Diet 1 & 5.80 & 49.3 & 96 \\
Diet 2 & 2.02 & 27.9 & 91 \\
Diet 3 & 0.43 & 6.9 & 65 \\
Diet 4 & 11.85 & 73.0 & 98 \\
\hline
\end{tabular}

Diet 1 - Backyard cow milk + all other locally produced non-milk exposure pathways

Diet 2 - Locally produced commercial milk + all other locally produced non-milk exposure pathways

Diet 3 - Regionally mixed commercial milk + inhalation (other regionally mixed food items are minor contributors to the total)

Diet 4 - Goat milk (all other exposure pathways are of negligible importance compared to goat milk) 


\section{Location: Oliver Springs}

Receptor: Female born in 1952

\begin{tabular}{lccc}
\hline & \multicolumn{3}{c}{ Thyroid Dose [cGy] } \\
Exposure Pathway & $95 \%$ Subjective Confidence Interval \\
\hline Backyard Cow Milk & lower limit & central estimate & upper limit \\
Commercial Milk (locally produced) & 0.42 & 2.7 & 20 \\
Commercial Milk (regionally mixed) & 0.12 & 0.88 & 7.4 \\
Goat Milk (locally produced) & 0.17 & 1.3 & 8 \\
Beef (locally produced) & 0.83 & 7.1 & 68 \\
Leafy Vegetables (locally produced) & 0.00052 & 0.01 & 0.24 \\
Eggs (locally produced) & 0.0001 & 0.0012 & 0.013 \\
Cottage Cheese (locally produced) & 0.021 & 0.16 & 1.4 \\
Inhalation & 0.00047 & 0.0069 & 0.083 \\
Mother's milk (mother on Diet 1) & 0.0063 & 0.025 & 0.12 \\
Prenatal exposure (mother on Diet 1) & 0.00012 & 0.0026 & 0.051 \\
Diet 1 & 0.0011 & 0.0079 & 0.081 \\
Diet 2 & 0.46 & 2.9 & 22 \\
Diet 3 & 0.18 & 1.1 & 9.1 \\
\hline
\end{tabular}

Excess Lifetime Risk [ ]

\begin{tabular}{llll}
\hline Diet 1 & $5.2 \mathrm{E}-05$ & $9.1 \mathrm{E}-04$ & $1.6 \mathrm{E}-02$ \\
Diet 2 & $2.7 \mathrm{E}-05$ & $3.3 \mathrm{E}-04$ & $5.0 \mathrm{E}-03$ \\
Diet 3 & $3.3 \mathrm{E}-05$ & $3.7 \mathrm{E}-04$ & $4.7 \mathrm{E}-03$ \\
Diet 4 & $1.4 \mathrm{E}-04$ & $2.3 \mathrm{E}-03$ & $4.0 \mathrm{E}-02$ \\
\hline
\end{tabular}

\section{Relative Risk [ ]}

\begin{tabular}{lccc}
\hline Diet 1 & 1.02 & 1.23 & 3.7 \\
Diet 2 & 1.007 & 1.085 & 2.2 \\
Diet 3 & 1.0095 & 1.095 & 2.2 \\
Diet 4 & 1.046 & 1.57 & 10.5 \\
\hline
\end{tabular}

\section{Probability of Causation [\%]}

\begin{tabular}{lccc}
\hline Diet 1 & 1.95 & 18.4 & 73 \\
Diet 2 & 0.69 & 7.8 & 55 \\
Diet 3 & 0.95 & 8.7 & 55 \\
Diet 4 & 4.41 & 35.9 & 90 \\
\hline
\end{tabular}

Diet 1 - Backyard cow milk + all other locally produced non-milk exposure pathways

Diet 2 - Locally produced commercial milk + all other locally produced non-milk exposure pathways

Diet 3 - Regionally mixed commercial milk + inhalation (other regionally mixed food items are minor contributors to the total)

Diet 4 - Goat milk (all other exposure pathways are of negligible importance compared to goat milk) 


\section{Location: Oliver Springs}

Receptor: Male born in 1952

\begin{tabular}{lccc}
\hline & \multicolumn{3}{c}{ Thyroid Dose [cGy] } \\
Exposure Pathway & 95\% Subjective Confidence Interval \\
\hline Backyard Cow Milk & lower limit & central estimate & upper limit \\
Commercial Milk (locally produced) & 0.42 & 2.6 & 19 \\
Commercial Milk (regionally mixed) & 0.12 & 0.86 & 7.1 \\
Goat Milk (locally produced) & 0.18 & 1.3 & 9 \\
Beef (locally produced) & 0.78 & 7.2 & 76 \\
Leafy Vegetables (locally produced) & 0.00054 & 0.0099 & 0.25 \\
Eggs (locally produced) & 0.0001 & 0.0012 & 0.014 \\
Cottage Cheese (locally produced) & 0.022 & 0.17 & 1.3 \\
Inhalation & 0.00052 & 0.0068 & 0.084 \\
Mother's milk (mother on Diet 1) & 0.0064 & 0.026 & 0.11 \\
Prenatal exposure (mother on Diet 1) & 0.00012 & 0.0026 & 0.051 \\
Diet 1 & 0.0011 & 0.0079 & 0.081 \\
Diet 2 & 0.46 & 2.9 & 21 \\
Diet 3 & 0.17 & 1.1 & 8.9 \\
\hline
\end{tabular}

Excess Lifetime Risk [ ]

\begin{tabular}{llll}
\hline Diet 1 & $6.5 \mathrm{E}-06$ & $2.4 \mathrm{E}-04$ & $4.6 \mathrm{E}-03$ \\
Diet 2 & $3.1 \mathrm{E}-06$ & $8.8 \mathrm{E}-05$ & $1.8 \mathrm{E}-03$ \\
Diet 3 & $4.0 \mathrm{E}-06$ & $9.3 \mathrm{E}-05$ & $2.0 \mathrm{E}-03$ \\
Diet 4 & $1.9 \mathrm{E}-05$ & $5.8 \mathrm{E}-04$ & $1.3 \mathrm{E}-02$ \\
\hline
\end{tabular}

\section{Relative Risk [ ]}

\begin{tabular}{lccc}
\hline Diet 1 & 1.0083 & 1.16 & 4.7 \\
Diet 2 & 1.003 & 1.059 & 2.6 \\
Diet 3 & 1.0036 & 1.065 & 2.7 \\
Diet 4 & 1.018 & 1.44 & 12 \\
\hline
\end{tabular}

\section{Probability of Causation [\%]}

\begin{tabular}{lccc}
\hline Diet 1 & 0.82 & 13.6 & 78 \\
Diet 2 & 0.30 & 5.5 & 61 \\
Diet 3 & 0.36 & 6.1 & 63 \\
Diet 4 & 1.81 & 30.5 & 91 \\
\hline
\end{tabular}

Diet 1 - Backyard cow milk + all other locally produced non-milk exposure pathways

Diet 2 - Locally produced commercial milk + all other locally produced non-milk exposure pathways

Diet 3 - Regionally mixed commercial milk + inhalation (other regionally mixed food items are minor contributors to the total)

Diet 4 - Goat milk (all other exposure pathways are of negligible importance compared to goat milk) 


\section{Location: Solway}

Receptor: Female born in 1952

\begin{tabular}{lccc}
\hline & \multicolumn{3}{c}{ Thyroid Dose [cGy] } \\
& \multicolumn{2}{c}{$95 \%$ Subjective Confidence Interval } \\
Exposure Pathway & lower limit & central estimate & upper limit \\
\hline Backyard Cow Milk & 2.6 & 16 & 110 \\
Commercial Milk (locally produced) & 0.72 & 5.1 & 37 \\
Commercial Milk (regionally mixed) & 0.17 & 1.3 & 8 \\
Goat Milk (locally produced) & 5.2 & 42 & 360 \\
Beef (locally produced) & 0.0032 & 0.06 & 1.3 \\
Leafy Vegetables (locally produced) & 0.00063 & 0.0075 & 0.07 \\
Eggs (locally produced) & 0.12 & 0.89 & 7.2 \\
Cottage Cheese (locally produced) & 0.003 & 0.041 & 0.44 \\
Inhalation & 0.039 & 0.15 & 0.62 \\
Mother's milk (mother on Diet 1) & 0.00064 & 0.015 & 0.29 \\
Prenatal exposure (mother on Diet 1) & 0.0065 & 0.046 & 0.4 \\
Diet 1 & 2.9 & 18 & 120 \\
Diet 2 & 1.1 & 6.7 & 46 \\
Diet 3 & 0.26 & 1.4 & 8.4 \\
\hline
\end{tabular}

Excess Lifetime Risk [ ]

\begin{tabular}{lllc}
\hline Diet 1 & $3.2 \mathrm{E}-04$ & $5.2 \mathrm{E}-03$ & $7.4 \mathrm{E}-02$ \\
Diet 2 & $1.5 \mathrm{E}-04$ & $2.0 \mathrm{E}-03$ & $2.5 \mathrm{E}-02$ \\
Diet 3 & $3.9 \mathrm{E}-05$ & $4.1 \mathrm{E}-04$ & $4.9 \mathrm{E}-03$ \\
Diet 4 & $8.0 \mathrm{E}-04$ & $1.4 \mathrm{E}-02$ & $2.1 \mathrm{E}-01$ \\
\hline
\end{tabular}

Relative Risk [ ]

\begin{tabular}{lccc}
\hline Diet 1 & 1.13 & 2.3 & 16 \\
Diet 2 & 1.05 & 1.5 & 7.4 \\
Diet 3 & 1.012 & 1.1 & 2.3 \\
Diet 4 & 1.31 & 4.1 & 52 \\
\hline
\end{tabular}

\section{Probability of Causation [\%]}

\begin{tabular}{lccc}
\hline Diet 1 & 11.88 & 56.1 & 94 \\
Diet 2 & 4.79 & 33.2 & 86 \\
Diet 3 & 1.17 & 9.4 & 56 \\
Diet 4 & 23.51 & 75.7 & 98 \\
\hline
\end{tabular}

Diet 1 - Backyard cow milk + all other locally produced non-milk exposure pathways

Diet 2 - Locally produced commercial milk + all other locally produced non-milk exposure pathways

Diet 3 - Regionally mixed commercial milk + inhalation (other regionally mixed food items are minor contributors to the total)

Diet 4 - Goat milk (all other exposure pathways are of negligible importance compared to goat milk) 


\section{Location: Solway}

Receptor: Male born in 1952

\begin{tabular}{lccc}
\hline & \multicolumn{3}{c}{ Thyroid Dose [cGy] } \\
& \multicolumn{2}{c}{$95 \%$ Subjective Confidence Interval } \\
Exposure Pathway & lower limit & central estimate & upper limit \\
\hline Backyard Cow Milk & 2.6 & 16 & 97 \\
Commercial Milk (locally produced) & 0.69 & 5.2 & 39 \\
Commercial Milk (regionally mixed) & 0.18 & 1.3 & 9 \\
Goat Milk (locally produced) & 5 & 41 & 390 \\
Beef (locally produced) & 0.0031 & 0.059 & 1.3 \\
Leafy Vegetables (locally produced) & 0.00066 & 0.0074 & 0.068 \\
Eggs (locally produced) & 0.14 & 0.98 & 6.7 \\
Cottage Cheese (locally produced) & 0.0031 & 0.04 & 0.49 \\
Inhalation & 0.039 & 0.15 & 0.6 \\
Mother's milk (mother on Diet 1) & 0.00064 & 0.015 & 0.29 \\
Prenatal exposure (mother on Diet 1) & 0.0065 & 0.046 & 0.4 \\
Diet 1 & 2.8 & 17 & 110 \\
Diet 2 & 1.1 & 6.8 & 47 \\
Diet 3 & 0.24 & 1.4 & 9.5 \\
\hline
\end{tabular}

Excess Lifetime Risk [ ]

\begin{tabular}{llcc}
\hline Diet 1 & $4.5 \mathrm{E}-05$ & $1.4 \mathrm{E}-03$ & $2.6 \mathrm{E}-02$ \\
Diet 2 & $2.2 \mathrm{E}-05$ & $5.1 \mathrm{E}-04$ & $1.1 \mathrm{E}-02$ \\
Diet 3 & $4.8 \mathrm{E}-06$ & $1.1 \mathrm{E}-04$ & $2.1 \mathrm{E}-03$ \\
Diet 4 & $1.2 \mathrm{E}-04$ & $3.5 \mathrm{E}-03$ & $6.4 \mathrm{E}-02$ \\
\hline
\end{tabular}

\section{Relative Risk [ ]}

\begin{tabular}{lccc}
\hline Diet 1 & 1.054 & 1.9 & 22 \\
Diet 2 & 1.019 & 1.34 & 9.7 \\
Diet 3 & 1.0043 & 1.072 & 2.8 \\
Diet 4 & 1.12 & 3.5 & 52 \\
\hline
\end{tabular}

\section{Probability of Causation [\%]}

\begin{tabular}{lccc}
\hline Diet 1 & 5.09 & 47.1 & 95 \\
Diet 2 & 1.88 & 25.4 & 90 \\
Diet 3 & 0.43 & 6.8 & 65 \\
Diet 4 & 10.54 & 71.1 & 98 \\
\hline
\end{tabular}

Diet 1 - Backyard cow milk + all other locally produced non-milk exposure pathways

Diet 2 - Locally produced commercial milk + all other locally produced non-milk exposure pathways

Diet 3 - Regionally mixed commercial milk + inhalation (other regionally mixed food items are minor contributors to the total)

Diet 4 - Goat milk (all other exposure pathways are of negligible importance compared to goat milk) 
Location: Sugar Grove

Receptor: Female born in 1952

\begin{tabular}{lccc}
\hline & \multicolumn{3}{c}{ Thyroid Dose [cGy] } \\
& \multicolumn{2}{c}{$95 \%$ Subjective Confidence Interval } \\
Exposure Pathway & 1 & 6.8 & 47 \\
\hline Backyard Cow Milk & 0.3 & 2.2 & 17 \\
Commercial Milk (locally produced) & 0.17 & 1.3 & 8 \\
Commercial Milk (regionally mixed) & 2.2 & 18 & 160 \\
Goat Milk (locally produced) & 0.0014 & 0.025 & 0.57 \\
Beef (locally produced) & 0.00027 & 0.0032 & 0.032 \\
Leafy Vegetables (locally produced) & 0.052 & 0.38 & 3.2 \\
Eggs (locally produced) & 0.0013 & 0.017 & 0.2 \\
Cottage Cheese (locally produced) & 0.015 & 0.063 & 0.27 \\
Inhalation & 0.00028 & 0.0064 & 0.12 \\
Mother's milk (mother on Diet 1) & 0.0027 & 0.019 & 0.17 \\
Prenatal exposure (mother on Diet 1) & 1.1 & 7.4 & 51 \\
Diet 1 & 0.45 & 2.9 & 20 \\
Diet 2 & 0.21 & 1.3 & 8.1 \\
Diet 3 & & & upper limit \\
\hline
\end{tabular}

Excess Lifetime Risk [ ]

\begin{tabular}{lllc}
\hline Diet 1 & $1.4 \mathrm{E}-04$ & $2.2 \mathrm{E}-03$ & $3.3 \mathrm{E}-02$ \\
Diet 2 & $6.3 \mathrm{E}-05$ & $8.4 \mathrm{E}-04$ & $1.1 \mathrm{E}-02$ \\
Diet 3 & $3.5 \mathrm{E}-05$ & $3.8 \mathrm{E}-04$ & $4.7 \mathrm{E}-03$ \\
Diet 4 & $3.3 \mathrm{E}-04$ & $5.9 \mathrm{E}-03$ & $9.1 \mathrm{E}-02$ \\
\hline
\end{tabular}

\section{Relative Risk [ ]}

\begin{tabular}{lccc}
\hline Diet 1 & 1.056 & 1.55 & 7.6 \\
Diet 2 & 1.022 & 1.21 & 3.6 \\
Diet 3 & 1.01 & 1.098 & 2.2 \\
Diet 4 & 1.12 & 2.3 & 22 \\
\hline
\end{tabular}

\section{Probability of Causation [\%]}

\begin{tabular}{lccc}
\hline Diet 1 & 5.22 & 34.4 & 86 \\
Diet 2 & 2.11 & 16.9 & 70 \\
Diet 3 & 1.03 & 8.9 & 55 \\
Diet 4 & 10.75 & 54.4 & 95 \\
\hline
\end{tabular}

Diet 1 - Backyard cow milk + all other locally produced non-milk exposure pathways

Diet 2 - Locally produced commercial milk + all other locally produced non-milk exposure pathways

Diet 3 - Regionally mixed commercial milk + inhalation (other regionally mixed food items are minor contributors to the total)

Diet 4 - Goat milk (all other exposure pathways are of negligible importance compared to goat milk) 
Location: Sugar Grove

Receptor: Male born in 1952

\begin{tabular}{lccc}
\hline & \multicolumn{3}{c}{ Thyroid Dose [cGy] } \\
& \multicolumn{2}{c}{$95 \%$ Subjective Confidence Interval } \\
Exposure Pathway & lower limit & central estimate & upper limit \\
\hline Backyard Cow Milk & 1.1 & 6.6 & 45 \\
Commercial Milk (locally produced) & 0.3 & 2.2 & 17 \\
Commercial Milk (regionally mixed) & 0.18 & 1.3 & 9 \\
Goat Milk (locally produced) & 2.1 & 17 & 160 \\
Beef (locally produced) & 0.0013 & 0.025 & 0.55 \\
Leafy Vegetables (locally produced) & 0.00027 & 0.003 & 0.031 \\
Eggs (locally produced) & 0.056 & 0.4 & 3 \\
Cottage Cheese (locally produced) & 0.0013 & 0.017 & 0.21 \\
Inhalation & 0.016 & 0.064 & 0.25 \\
Mother's milk (mother on Diet 1) & 0.00028 & 0.0064 & 0.12 \\
Prenatal exposure (mother on Diet 1) & 0.0027 & 0.019 & 0.17 \\
Diet 1 & 1.2 & 7.2 & 49 \\
Diet 2 & 0.45 & 2.9 & 20 \\
Diet 3 & 0.2 & 1.3 & 9.2 \\
\hline
\end{tabular}

Excess Lifetime Risk [ ]

\begin{tabular}{lllc}
\hline Diet 1 & $1.9 \mathrm{E}-05$ & $5.9 \mathrm{E}-04$ & $1.1 \mathrm{E}-02$ \\
Diet 2 & $8.8 \mathrm{E}-06$ & $2.1 \mathrm{E}-04$ & $4.6 \mathrm{E}-03$ \\
Diet 3 & $4.3 \mathrm{E}-06$ & $9.7 \mathrm{E}-05$ & $2.0 \mathrm{E}-03$ \\
Diet 4 & $5.2 \mathrm{E}-05$ & $1.4 \mathrm{E}-03$ & $2.9 \mathrm{E}-02$ \\
\hline
\end{tabular}

\section{Relative Risk [ ]}

\begin{tabular}{lccc}
\hline Diet 1 & 1.022 & 1.39 & 9.6 \\
Diet 2 & 1.0075 & 1.14 & 4.9 \\
Diet 3 & 1.0038 & 1.067 & 2.8 \\
Diet 4 & 1.048 & 2.1 & 25 \\
\hline
\end{tabular}

\section{Probability of Causation [\%]}

\begin{tabular}{lccc}
\hline Diet 1 & 2.16 & 27.4 & 88 \\
Diet 2 & 0.75 & 12.1 & 78 \\
Diet 3 & 0.38 & 6.3 & 64 \\
Diet 4 & 4.52 & 49.7 & 96 \\
\hline
\end{tabular}

Diet 1 - Backyard cow milk + all other locally produced non-milk exposure pathways

Diet 2 - Locally produced commercial milk + all other locally produced non-milk exposure pathways

Diet 3 - Regionally mixed commercial milk + inhalation (other regionally mixed food items are minor contributors to the total)

Diet 4 - Goat milk (all other exposure pathways are of negligible importance compared to goat milk) 


\section{Location: OR Townsite}

Receptor: Female born in 1952

\begin{tabular}{lccc}
\hline & \multicolumn{3}{c}{ Thyroid Dose [cGy] } \\
Exposure Pathway & \multicolumn{2}{c}{ 95\% } & Subjective Confidence Interval \\
Backyard Cow Milk & lower limit & central estimate & upper limit \\
Commercial Milk (locally produced) & -- & -- & -- \\
Commercial Milk (regionally mixed) & -- & -- & -- \\
Goat Milk (locally produced) & 0.17 & 1.2 & 8 \\
Beef (locally produced) & -- & -- & -- \\
Leafy Vegetables (locally produced) & -- & -- & -- \\
Eggs (locally produced) & -- & -- & -- \\
Cottage Cheese (locally produced) & -- & -- & -- \\
Inhalation & 0.0013 & 0.019 & 0.2 \\
Mother's milk (mother on Diet 3) & 0.017 & 0.069 & 0.29 \\
Prenatal exposure (mother on Diet 3) & 0.00037 & 0.0067 & 0.11 \\
Diet 1 & 0.0035 & 0.022 & 0.19 \\
Diet 2 & -- & -- & -- \\
Diet 3 & -- & -- & -- \\
\hline
\end{tabular}

Excess Lifetime Risk [ ]

\begin{tabular}{lccc}
\hline Diet 1 & -- & -- & -- \\
Diet 2 & -- & -- & -- \\
Diet 3 & $3.5 \mathrm{E}-05$ & $3.8 \mathrm{E}-04$ & $4.7 \mathrm{E}-03$ \\
Diet 4 & -- & -- & -- \\
\hline
\end{tabular}

\section{Relative Risk [ ]}

\begin{tabular}{lccc}
\hline Diet 1 & -- & -- & -- \\
Diet 2 & -- & -- & -- \\
Diet 3 & 1.01 & 1.098 & 2.2 \\
Diet 4 & -- & -- & -- \\
\hline
\end{tabular}

\begin{tabular}{lccc}
\hline Diet 1 & -- & -- & -- \\
Diet 2 & -- & -- & -- \\
Diet 3 & 1.03 & 8.9 & 55 \\
Diet 4 & -- & -- \\
\hline Diet 1 - Backyard cow milk + all other locally produced non-milk exposure pathways & \\
Diet 2 - Locally produced commercial milk + all other locally produced non-milk exposure pathways \\
Diet 3 - Regionally mixed commercial milk + inhalation (other regionally mixed food items are minor contributors to the total) \\
Diet 4 - Goat milk (all other exposure pathways are of negligible importance compared to goat milk)
\end{tabular}




\section{Location: OR Townsite}

\section{Receptor: Male born in 1952}

\begin{tabular}{lccc}
\hline & \multicolumn{3}{c}{ Thyroid Dose [cGy] } \\
Exposure Pathway & \multicolumn{2}{c}{$95 \%$ Subjective Confidence Interval } \\
\hline Backyard Cow Milk & lower limit & central estimate & upper limit \\
Commercial Milk (locally produced) & -- & -- & -- \\
Commercial Milk (regionally mixed) & -- & -- & -- \\
Goat Milk (locally produced) & 0.18 & 1.3 & 9 \\
Beef (locally produced) & -- & -- & -- \\
Leafy Vegetables (locally produced) & -- & -- & -- \\
Eggs (locally produced) & -- & -- & -- \\
Cottage Cheese (locally produced) & -- & -- & -- \\
Inhalation & 0.0014 & 0.018 & 0.21 \\
Mother's milk (mother on Diet 3) & 0.018 & 0.07 & 0.28 \\
Prenatal exposure (mother on Diet 3) & 0.00037 & 0.0067 & 0.11 \\
Diet 1 & 0.0035 & 0.022 & 0.19 \\
Diet 2 & -- & -- & -- \\
Diet 3 & -- & -- & -- \\
\hline
\end{tabular}

Excess Lifetime Risk [ ]

\begin{tabular}{lccc}
\hline Diet 1 & -- & -- & -- \\
Diet 2 & -- & -- & -- \\
Diet 3 & $4.3 \mathrm{E}-06$ & $9.8 \mathrm{E}-05$ & $2.0 \mathrm{E}-03$ \\
Diet 4 & -- & -- & -- \\
\hline
\end{tabular}

\section{Relative Risk [ ]}

\begin{tabular}{lccc}
\hline Diet 1 & -- & -- & -- \\
Diet 2 & -- & -- & -- \\
Diet 3 & 1.0038 & 1.068 & 2.8 \\
Diet 4 & -- & -- & -- \\
\hline
\end{tabular}

\begin{tabular}{lccc}
\hline Diet 1 & -- & -- & -- \\
Diet 2 & -- & -- & -- \\
Diet 3 & 0.38 & 6.3 & 64 \\
Diet 4 & -- & -- & - \\
\hline Diet 1 - Backyard cow milk + all other locally produced non-milk exposure pathways & \\
Diet 2 - Locally produced commercial milk + all other locally produced non-milk exposure pathways \\
Diet 3 - Regionally mixed commercial milk + inhalation (other regionally mixed food items are minor contributors to the total) \\
Diet 4 - Goat milk (all other exposure pathways are of negligible importance compared to goat milk)
\end{tabular}


Location: Hines Valley

Receptor: Female born in 1952

\begin{tabular}{lccc}
\hline & \multicolumn{3}{c}{ Thyroid Dose [cGy] } \\
Exposure Pathway & \multicolumn{2}{c}{$95 \%$} & Subjective Confidence Interval \\
Backyard Cow Milk & lower limit & central estimate & upper limit \\
\hline Commercial Milk (locally produced) & 1.3 & 8 & 55 \\
Commercial Milk (regionally mixed) & 0.37 & 2.6 & 19 \\
Goat Milk (locally produced) & 0.17 & 1.3 & 8 \\
Beef (locally produced) & 2.7 & 21 & 180 \\
Leafy Vegetables (locally produced) & 0.0016 & 0.03 & 0.78 \\
Eggs (locally produced) & 0.00037 & 0.0038 & 0.037 \\
Cottage Cheese (locally produced) & 0.06 & 0.47 & 3.7 \\
Inhalation & 0.0014 & 0.021 & 0.22 \\
Mother's milk (mother on Diet 1) & 0.019 & 0.083 & 0.33 \\
Prenatal exposure (mother on Diet 1) & 0.00034 & 0.008 & 0.14 \\
Diet 1 & 0.0035 & 0.025 & 0.2 \\
Diet 2 & 1.4 & 8.7 & 61 \\
Diet 3 & 0.54 & 3.4 & 23 \\
\hline
\end{tabular}

Excess Lifetime Risk [ ]

\begin{tabular}{llll}
\hline Diet 1 & $1.7 \mathrm{E}-04$ & $2.7 \mathrm{E}-03$ & $4.2 \mathrm{E}-02$ \\
Diet 2 & $7.9 \mathrm{E}-05$ & $1.0 \mathrm{E}-03$ & $1.5 \mathrm{E}-02$ \\
Diet 3 & $3.6 \mathrm{E}-05$ & $3.9 \mathrm{E}-04$ & $4.8 \mathrm{E}-03$ \\
Diet 4 & $3.8 \mathrm{E}-04$ & $7.2 \mathrm{E}-03$ & $1.0 \mathrm{E}-01$ \\
\hline
\end{tabular}

\section{Relative Risk [ ]}

\begin{tabular}{lccc}
\hline Diet 1 & 1.064 & 1.65 & 8.7 \\
Diet 2 & 1.025 & 1.25 & 4.2 \\
Diet 3 & 1.011 & 1.099 & 2.2 \\
Diet 4 & 1.14 & 2.6 & 28 \\
\hline
\end{tabular}

\begin{tabular}{lccc} 
& \multicolumn{3}{c}{ Probability of Causation [\%] } \\
\hline Diet 1 & 6.05 & 39.4 & 88 \\
Diet 2 & 2.45 & 20.0 & 76 \\
Diet 3 & 1.05 & 9.0 & 55 \\
Diet 4 & 12.41 & 60.9 & 96 \\
\hline Dien
\end{tabular}

Diet 1 - Backyard cow milk + all other locally produced non-milk exposure pathways

Diet 2 - Locally produced commercial milk + all other locally produced non-milk exposure pathways

Diet 3 - Regionally mixed commercial milk + inhalation (other regionally mixed food items are minor contributors to the total)

Diet 4 - Goat milk (all other exposure pathways are of negligible importance compared to goat milk) 


\section{Location: Hines Valley}

Receptor: Male born in 1952

\begin{tabular}{lccc}
\hline & \multicolumn{3}{c}{ Thyroid Dose [cGy] } \\
& \multicolumn{2}{c}{$95 \%$ Subjective Confidence Interval } \\
Exposure Pathway & lower limit & central estimate & upper limit \\
\hline Backyard Cow Milk & 1.3 & 8 & 51 \\
Commercial Milk (locally produced) & 0.37 & 2.6 & 22 \\
Commercial Milk (regionally mixed) & 0.18 & 1.3 & 9 \\
Goat Milk (locally produced) & 2.5 & 20 & 180 \\
Beef (locally produced) & 0.0017 & 0.03 & 0.74 \\
Leafy Vegetables (locally produced) & 0.00034 & 0.0037 & 0.036 \\
Eggs (locally produced) & 0.067 & 0.5 & 3.7 \\
Cottage Cheese (locally produced) & 0.0016 & 0.02 & 0.25 \\
Inhalation & 0.021 & 0.084 & 0.34 \\
Mother's milk (mother on Diet 1) & 0.00034 & 0.008 & 0.14 \\
Prenatal exposure (mother on Diet 1) & 0.0035 & 0.025 & 0.2 \\
Diet 1 & 1.5 & 8.8 & 54 \\
Diet 2 & 0.54 & 3.4 & 25 \\
Diet 3 & 0.21 & 1.3 & 9.3 \\
\hline
\end{tabular}

Excess Lifetime Risk [ ]

\begin{tabular}{lllc}
\hline Diet 1 & $2.2 \mathrm{E}-05$ & $7.2 \mathrm{E}-04$ & $1.3 \mathrm{E}-02$ \\
Diet 2 & $1.0 \mathrm{E}-05$ & $2.7 \mathrm{E}-04$ & $5.8 \mathrm{E}-03$ \\
Diet 3 & $4.4 \mathrm{E}-06$ & $9.9 \mathrm{E}-05$ & $2.1 \mathrm{E}-03$ \\
Diet 4 & $5.4 \mathrm{E}-05$ & $1.7 \mathrm{E}-03$ & $3.9 \mathrm{E}-02$ \\
\hline
\end{tabular}

\section{Relative Risk [ ]}

\begin{tabular}{lccc}
\hline Diet 1 & 1.026 & 1.46 & 11 \\
Diet 2 & 1.0094 & 1.18 & 5.1 \\
Diet 3 & 1.0039 & 1.069 & 2.8 \\
Diet 4 & 1.056 & 2.2 & 31 \\
\hline
\end{tabular}

\section{Probability of Causation [\%]}

\begin{tabular}{lccc}
\hline Diet 1 & 2.55 & 31.5 & 91 \\
Diet 2 & 0.94 & 15.4 & 81 \\
Diet 3 & 0.39 & 6.4 & 64 \\
Diet 4 & 5.34 & 55.5 & 97 \\
\hline
\end{tabular}

Diet 1 - Backyard cow milk + all other locally produced non-milk exposure pathways

Diet 2 - Locally produced commercial milk + all other locally produced non-milk exposure pathways

Diet 3 - Regionally mixed commercial milk + inhalation (other regionally mixed food items are minor contributors to the total)

Diet 4 - Goat milk (all other exposure pathways are of negligible importance compared to goat milk) 


\section{Location: Farragut}

Receptor: Female born in 1952

\begin{tabular}{lccc}
\hline & \multicolumn{3}{c}{ Thyroid Dose [cGy] } \\
Exposure Pathway & \multicolumn{2}{c}{$95 \%$ Subjective Confidence Interval } \\
\hline Backyard Cow Milk & 1.4 & 9 & 63 \\
Commercial Milk (locally produced) & 0.42 & 2.9 & 20 \\
Commercial Milk (regionally mixed) & 0.18 & 1.3 & 8 \\
Goat Milk (locally produced) & 2.9 & 24 & 210 \\
Beef (locally produced) & 0.002 & 0.034 & 0.92 \\
Leafy Vegetables (locally produced) & 0.00035 & 0.0043 & 0.042 \\
Eggs (locally produced) & 0.074 & 0.53 & 4.4 \\
Cottage Cheese (locally produced) & 0.0017 & 0.023 & 0.24 \\
Inhalation & 0.022 & 0.095 & 0.4 \\
Mother's milk (mother on Diet 1) & 0.00043 & 0.0087 & 0.17 \\
Prenatal exposure (mother on Diet 1) & 0.0038 & 0.026 & 0.24 \\
Diet 1 & 1.6 & 9.9 & 70 \\
Diet 2 & 0.61 & 3.8 & 24 \\
Diet 3 & 0.23 & 1.3 & 8.2 \\
\hline
\end{tabular}

Excess Lifetime Risk [ ]

\begin{tabular}{llll}
\hline Diet 1 & $1.9 \mathrm{E}-04$ & $3.1 \mathrm{E}-03$ & $4.9 \mathrm{E}-02$ \\
Diet 2 & $9.5 \mathrm{E}-05$ & $1.2 \mathrm{E}-03$ & $1.5 \mathrm{E}-02$ \\
Diet 3 & $3.6 \mathrm{E}-05$ & $3.9 \mathrm{E}-04$ & $4.8 \mathrm{E}-03$ \\
Diet 4 & $4.7 \mathrm{E}-04$ & $8.2 \mathrm{E}-03$ & $1.2 \mathrm{E}-01$ \\
\hline
\end{tabular}

\section{Relative Risk [ ]}

\begin{tabular}{lccc}
\hline Diet 1 & 1.076 & 1.75 & 9.2 \\
Diet 2 & 1.027 & 1.29 & 4.9 \\
Diet 3 & 1.011 & 1.1 & 2.2 \\
Diet 4 & 1.17 & 2.8 & 29 \\
\hline
\end{tabular}

\section{Probability of Causation [\%]}

\begin{tabular}{lccc}
\hline Diet 1 & 7.06 & 42.7 & 89 \\
Diet 2 & 2.67 & 22.5 & 79 \\
Diet 3 & 1.07 & 9.1 & 56 \\
Diet 4 & 14.29 & 63.8 & 97 \\
\hline
\end{tabular}

Diet 1 - Backyard cow milk + all other locally produced non-milk exposure pathways

Diet 2 - Locally produced commercial milk + all other locally produced non-milk exposure pathways

Diet 3 - Regionally mixed commercial milk + inhalation (other regionally mixed food items are minor contributors to the total)

Diet 4 - Goat milk (all other exposure pathways are of negligible importance compared to goat milk) 


\section{Location: Farragut}

\section{Receptor: Male born in 1952}

\begin{tabular}{lccc}
\hline & \multicolumn{3}{c}{ Thyroid Dose [cGy] } \\
& \multicolumn{2}{c}{$95 \%$ Subjective Confidence Interval } \\
Exposure Pathway & lower limit & central estimate & upper limit \\
\hline Backyard Cow Milk & 1.4 & 8.8 & 56 \\
Commercial Milk (locally produced) & 0.41 & 3 & 23 \\
Commercial Milk (regionally mixed) & 0.18 & 1.3 & 9 \\
Goat Milk (locally produced) & 2.7 & 23 & 230 \\
Beef (locally produced) & 0.002 & 0.034 & 0.87 \\
Leafy Vegetables (locally produced) & 0.00035 & 0.0043 & 0.04 \\
Eggs (locally produced) & 0.077 & 0.56 & 4.3 \\
Cottage Cheese (locally produced) & 0.0019 & 0.023 & 0.26 \\
Inhalation & 0.022 & 0.095 & 0.36 \\
Mother's milk (mother on Diet 1) & 0.00043 & 0.0087 & 0.17 \\
Prenatal exposure (mother on Diet 1) & 0.0038 & 0.026 & 0.24 \\
Diet 1 & 1.6 & 9.6 & 61 \\
Diet 2 & 0.61 & 3.8 & 27 \\
Diet 3 & 0.22 & 1.4 & 9.3 \\
\hline
\end{tabular}

\section{Excess Lifetime Risk [ ]}

\begin{tabular}{llll}
\hline Diet 1 & $3.0 \mathrm{E}-05$ & $7.9 \mathrm{E}-04$ & $1.6 \mathrm{E}-02$ \\
Diet 2 & $1.2 \mathrm{E}-05$ & $3.0 \mathrm{E}-04$ & $6.4 \mathrm{E}-03$ \\
Diet 3 & $4.5 \mathrm{E}-06$ & $1.0 \mathrm{E}-04$ & $2.1 \mathrm{E}-03$ \\
Diet 4 & $6.2 \mathrm{E}-05$ & $1.9 \mathrm{E}-03$ & $3.9 \mathrm{E}-02$ \\
\hline
\end{tabular}

\section{Relative Risk [ ]}

\begin{tabular}{lccc}
\hline Diet 1 & 1.033 & 1.51 & 13 \\
Diet 2 & 1.011 & 1.2 & 5.9 \\
Diet 3 & 1.0039 & 1.069 & 2.8 \\
Diet 4 & 1.074 & 2.4 & 33 \\
\hline
\end{tabular}

\section{Probability of Causation [\%]}

\begin{tabular}{lccc}
\hline Diet 1 & 3.20 & 33.7 & 92 \\
Diet 2 & 1.13 & 16.6 & 83 \\
Diet 3 & 0.39 & 6.4 & 64 \\
Diet 4 & 6.87 & 58.4 & 97 \\
\hline
\end{tabular}

Diet 1 - Backyard cow milk + all other locally produced non-milk exposure pathways

Diet 2 - Locally produced commercial milk + all other locally produced non-milk exposure pathways

Diet 3 - Regionally mixed commercial milk + inhalation (other regionally mixed food items are minor contributors to the total)

Diet 4 - Goat milk (all other exposure pathways are of negligible importance compared to goat milk) 
Location: Lenoir City

Receptor: Female born in 1952

\begin{tabular}{lccc}
\hline & \multicolumn{3}{c}{ Thyroid Dose [cGy] } \\
& \multicolumn{2}{c}{$95 \%$ Subjective Confidence Interval } \\
Exposure Pathway & lower limit & central estimate & upper limit \\
\hline Backyard Cow Milk & 0.95 & 5.6 & 34 \\
Commercial Milk (locally produced) & 0.26 & 1.7 & 14 \\
Commercial Milk (regionally mixed) & 0.17 & 1.3 & 8 \\
Goat Milk (locally produced) & -- & -- & -- \\
Beef (locally produced) & 0.001 & 0.021 & 0.47 \\
Leafy Vegetables (locally produced) & 0.00025 & 0.0027 & 0.025 \\
Eggs (locally produced) & 0.045 & 0.32 & 2.5 \\
Cottage Cheese (locally produced) & 0.00098 & 0.014 & 0.15 \\
Inhalation & 0.014 & 0.059 & 0.25 \\
Mother's milk (mother on Diet 1) & 0.00021 & 0.0053 & 0.097 \\
Prenatal exposure (mother on Diet 1) & 0.0024 & 0.016 & 0.13 \\
Diet 1 & 1 & 6.2 & 37 \\
Diet 2 & 0.38 & 2.3 & 16 \\
Diet 3 & 0.21 & 1.3 & 8.1 \\
\hline
\end{tabular}

Excess Lifetime Risk [ ]

\begin{tabular}{lccc}
\hline Diet 1 & $1.1 \mathrm{E}-04$ & $1.8 \mathrm{E}-03$ & $3.0 \mathrm{E}-02$ \\
Diet 2 & $6.2 \mathrm{E}-05$ & $6.9 \mathrm{E}-04$ & $1.0 \mathrm{E}-02$ \\
Diet 3 & $3.5 \mathrm{E}-05$ & $3.8 \mathrm{E}-04$ & $4.8 \mathrm{E}-03$ \\
Diet 4 & -- & -- & -- \\
\hline
\end{tabular}

\section{Relative Risk [ ]}

\begin{tabular}{lccc}
\hline Diet 1 & 1.045 & 1.45 & 6.2 \\
Diet 2 & 1.017 & 1.17 & 3.6 \\
Diet 3 & 1.01 & 1.097 & 2.2 \\
Diet 4 & -- & -- & -- \\
\hline
\end{tabular}

\section{Probability of Causation [\%]}

\begin{tabular}{lccc}
\hline Diet 1 & 4.30 & 31.1 & 84 \\
Diet 2 & 1.64 & 14.4 & 72 \\
Diet 3 & 1.01 & 8.9 & 55 \\
Diet 4 & -- & -- \\
\hline Diet 1 - Backyard cow milk + all other locally produced non-milk exposure pathways & \\
Diet 2 - Locally produced commercial milk + all other locally produced non-milk exposure pathways \\
Diet 3 - Regionally mixed commercial milk + inhalation (other regionally mixed food items are minor contributors to the total) \\
Diet 4 - Goat milk (all other exposure pathways are of negligible importance compared to goat milk)
\end{tabular}




\section{Location: Lenoir City}

Receptor: Male born in 1952

\begin{tabular}{lccc}
\hline & \multicolumn{3}{c}{ Thyroid Dose [cGy] } \\
& \multicolumn{2}{c}{$95 \%$ Subjective Confidence Interval } \\
Exposure Pathway & lower limit & central estimate & upper limit \\
\hline Backyard Cow Milk & 1 & 5.7 & 34 \\
Commercial Milk (locally produced) & 0.25 & 1.7 & 13 \\
Commercial Milk (regionally mixed) & 0.18 & 1.3 & 9 \\
Goat Milk (locally produced) & -- & -- & -- \\
Beef (locally produced) & 0.001 & 0.021 & 0.45 \\
Leafy Vegetables (locally produced) & 0.00026 & 0.0026 & 0.025 \\
Eggs (locally produced) & 0.048 & 0.34 & 2.5 \\
Cottage Cheese (locally produced) & 0.0011 & 0.015 & 0.15 \\
Inhalation & 0.014 & 0.057 & 0.25 \\
Mother's milk (mother on Diet 1) & 0.00021 & 0.0053 & 0.097 \\
Prenatal exposure (mother on Diet 1) & 0.0024 & 0.016 & 0.13 \\
Diet 1 & 1.1 & 6.2 & 36 \\
Diet 2 & 0.39 & 2.2 & 15 \\
Diet 3 & 0.2 & 1.3 & 9.2 \\
\hline
\end{tabular}

Excess Lifetime Risk [ ]

\begin{tabular}{lccc}
\hline Diet 1 & $1.4 \mathrm{E}-05$ & $4.8 \mathrm{E}-04$ & $8.2 \mathrm{E}-03$ \\
Diet 2 & $6.3 \mathrm{E}-06$ & $1.7 \mathrm{E}-04$ & $3.9 \mathrm{E}-03$ \\
Diet 3 & $4.2 \mathrm{E}-06$ & $9.6 \mathrm{E}-05$ & $2.0 \mathrm{E}-03$ \\
Diet 4 & -- & -- & -- \\
\hline
\end{tabular}

\section{Relative Risk [ ]}

\begin{tabular}{lccc}
\hline Diet 1 & 1.018 & 1.32 & 7.7 \\
Diet 2 & 1.0066 & 1.12 & 4.1 \\
Diet 3 & 1.0038 & 1.066 & 2.8 \\
Diet 4 & -- & -- & -- \\
\hline
\end{tabular}

\section{Probability of Causation [\%]}

\begin{tabular}{lccc}
\hline Diet 1 & 1.81 & 24.3 & 87 \\
Diet 2 & 0.66 & 10.5 & 75 \\
Diet 3 & 0.38 & 6.2 & 64 \\
Diet 4 & -- & -- \\
\hline Diet 1 - Backyard cow milk + all other locally produced non-milk exposure pathways & \\
Diet 2 - Locally produced commercial milk + all other locally produced non-milk exposure pathways \\
Diet 3 - Regionally mixed commercial milk + inhalation (other regionally mixed food items are minor contributors to the total) \\
Diet 4 - Goat milk (all other exposure pathways are of negligible importance compared to goat milk)
\end{tabular}




\section{Location: Kingston}

Receptor: Female born in 1952

\begin{tabular}{lccc}
\hline & \multicolumn{3}{c}{ Thyroid Dose [cGy] } \\
& \multicolumn{2}{c}{$95 \%$ Subjective Confidence Interval } \\
Exposure Pathway & lower limit & central estimate & upper limit \\
\hline Backyard Cow Milk & 1.1 & 7.3 & 47 \\
Commercial Milk (locally produced) & 0.34 & 2.3 & 16 \\
Commercial Milk (regionally mixed) & 0.17 & 1.2 & 8 \\
Goat Milk (locally produced) & 2.4 & 19 & 170 \\
Beef (locally produced) & 0.0015 & 0.026 & 0.59 \\
Leafy Vegetables (locally produced) & 0.0003 & 0.0033 & 0.032 \\
Eggs (locally produced) & 0.054 & 0.41 & 3.6 \\
Cottage Cheese (locally produced) & 0.0012 & 0.018 & 0.19 \\
Inhalation & 0.019 & 0.075 & 0.32 \\
Mother's milk (mother on Diet 1) & 0.00032 & 0.0069 & 0.12 \\
Prenatal exposure (mother on Diet 1) & 0.0031 & 0.021 & 0.18 \\
Diet 1 & 1.3 & 7.8 & 51 \\
Diet 2 & 0.51 & 3.1 & 19 \\
Diet 3 & 0.22 & 1.3 & 8.2 \\
\hline
\end{tabular}

Excess Lifetime Risk [ ]

\begin{tabular}{llll}
\hline Diet 1 & $1.4 \mathrm{E}-04$ & $2.4 \mathrm{E}-03$ & $3.5 \mathrm{E}-02$ \\
Diet 2 & $7.4 \mathrm{E}-05$ & $8.9 \mathrm{E}-04$ & $1.2 \mathrm{E}-02$ \\
Diet 3 & $3.5 \mathrm{E}-05$ & $3.8 \mathrm{E}-04$ & $4.8 \mathrm{E}-03$ \\
Diet 4 & $3.6 \mathrm{E}-04$ & $6.2 \mathrm{E}-03$ & $9.2 \mathrm{E}-02$ \\
\hline
\end{tabular}

\section{Relative Risk [ ]}

\begin{tabular}{lccc}
\hline Diet 1 & 1.063 & 1.61 & 7.5 \\
Diet 2 & 1.022 & 1.22 & 3.7 \\
Diet 3 & 1.011 & 1.098 & 2.3 \\
Diet 4 & 1.14 & 2.4 & 21 \\
\hline
\end{tabular}

\section{Probability of Causation [\%]}

\begin{tabular}{lccc}
\hline Diet 1 & 5.95 & 37.7 & 86 \\
Diet 2 & 2.12 & 18.2 & 73 \\
Diet 3 & 1.06 & 9.0 & 56 \\
Diet 4 & 11.93 & 58.6 & 95 \\
\hline
\end{tabular}

Diet 1 - Backyard cow milk + all other locally produced non-milk exposure pathways

Diet 2 - Locally produced commercial milk + all other locally produced non-milk exposure pathways

Diet 3 - Regionally mixed commercial milk + inhalation (other regionally mixed food items are minor contributors to the total)

Diet 4 - Goat milk (all other exposure pathways are of negligible importance compared to goat milk) 


\section{Location: Kingston}

Receptor: Male born in 1952

\begin{tabular}{lccc}
\hline & \multicolumn{3}{c}{ Thyroid Dose [cGy] } \\
& \multicolumn{2}{c}{$95 \%$ Subjective Confidence Interval } \\
Exposure Pathway & lower limit & central estimate & upper limit \\
\hline Backyard Cow Milk & 1.1 & 6.9 & 42 \\
Commercial Milk (locally produced) & 0.33 & 2.3 & 17 \\
Commercial Milk (regionally mixed) & 0.18 & 1.3 & 9 \\
Goat Milk (locally produced) & 2.3 & 19 & 170 \\
Beef (locally produced) & 0.0015 & 0.026 & 0.61 \\
Leafy Vegetables (locally produced) & 0.00031 & 0.0032 & 0.033 \\
Eggs (locally produced) & 0.055 & 0.43 & 2.9 \\
Cottage Cheese (locally produced) & 0.0014 & 0.018 & 0.2 \\
Inhalation & 0.019 & 0.077 & 0.31 \\
Mother's milk (mother on Diet 1) & 0.00032 & 0.0069 & 0.12 \\
Prenatal exposure (mother on Diet 1) & 0.0031 & 0.021 & 0.18 \\
Diet 1 & 1.2 & 7.6 & 46 \\
Diet 2 & 0.49 & 3.1 & 20 \\
Diet 3 & 0.21 & 1.3 & 9.2 \\
\hline
\end{tabular}

Excess Lifetime Risk [ ]

\begin{tabular}{lllc}
\hline Diet 1 & $1.9 \mathrm{E}-05$ & $6.3 \mathrm{E}-04$ & $1.1 \mathrm{E}-02$ \\
Diet 2 & $8.8 \mathrm{E}-06$ & $2.4 \mathrm{E}-04$ & $4.8 \mathrm{E}-03$ \\
Diet 3 & $4.4 \mathrm{E}-06$ & $9.9 \mathrm{E}-05$ & $2.0 \mathrm{E}-03$ \\
Diet 4 & $5.0 \mathrm{E}-05$ & $1.5 \mathrm{E}-03$ & $3.2 \mathrm{E}-02$ \\
\hline
\end{tabular}

\section{Relative Risk [ ]}

\begin{tabular}{lccc}
\hline Diet 1 & 1.022 & 1.39 & 10.1 \\
Diet 2 & 1.0086 & 1.15 & 5.2 \\
Diet 3 & 1.0038 & 1.068 & 2.8 \\
Diet 4 & 1.056 & 2.1 & 28 \\
\hline
\end{tabular}

\section{Probability of Causation [\%]}

\begin{tabular}{lccc}
\hline Diet 1 & 2.15 & 28.0 & 90 \\
Diet 2 & 0.85 & 13.0 & 81 \\
Diet 3 & 0.38 & 6.4 & 64 \\
Diet 4 & 5.29 & 52.5 & 96 \\
\hline
\end{tabular}

Diet 1 - Backyard cow milk + all other locally produced non-milk exposure pathways

Diet 2 - Locally produced commercial milk + all other locally produced non-milk exposure pathways

Diet 3 - Regionally mixed commercial milk + inhalation (other regionally mixed food items are minor contributors to the total)

Diet 4 - Goat milk (all other exposure pathways are of negligible importance compared to goat milk) 


\section{Location: Karns}

Receptor: Female born in 1952

\begin{tabular}{lccc}
\hline & \multicolumn{3}{c}{ Thyroid Dose [cGy] } \\
Exposure Pathway & \multicolumn{2}{c}{$95 \%$ Subjective Confidence Interval } \\
\hline Backyard Cow Milk & 1.5 & 9.7 & 62 \\
Commercial Milk (locally produced) & 0.45 & 3.1 & 21 \\
Commercial Milk (regionally mixed) & 0.17 & 1.2 & 8 \\
Goat Milk (locally produced) & 3 & 25 & 220 \\
Beef (locally produced) & 0.002 & 0.035 & 0.85 \\
Leafy Vegetables (locally produced) & 0.00039 & 0.0046 & 0.042 \\
Eggs (locally produced) & 0.074 & 0.55 & 4.4 \\
Cottage Cheese (locally produced) & 0.0016 & 0.025 & 0.26 \\
Inhalation & 0.024 & 0.1 & 0.43 \\
Mother's milk (mother on Diet 1) & 0.00043 & 0.0093 & 0.19 \\
Prenatal exposure (mother on Diet 1) & 0.0043 & 0.029 & 0.25 \\
Diet 1 & 1.7 & 11 & 67 \\
Diet 2 & 0.66 & 4 & 25 \\
Diet 3 & 0.23 & 1.3 & 8.2 \\
\hline
\end{tabular}

Excess Lifetime Risk [ ]

\begin{tabular}{llll}
\hline Diet 1 & $2.0 \mathrm{E}-04$ & $3.2 \mathrm{E}-03$ & $4.9 \mathrm{E}-02$ \\
Diet 2 & $1.0 \mathrm{E}-04$ & $1.2 \mathrm{E}-03$ & $1.6 \mathrm{E}-02$ \\
Diet 3 & $3.6 \mathrm{E}-05$ & $4.0 \mathrm{E}-04$ & $4.8 \mathrm{E}-03$ \\
Diet 4 & $5.1 \mathrm{E}-04$ & $8.7 \mathrm{E}-03$ & $1.2 \mathrm{E}-01$ \\
\hline
\end{tabular}

\section{Relative Risk [ ]}

\begin{tabular}{lccc}
\hline Diet 1 & 1.082 & 1.81 & 9.7 \\
Diet 2 & 1.031 & 1.31 & 4.7 \\
Diet 3 & 1.011 & 1.1 & 2.3 \\
Diet 4 & 1.19 & 2.9 & 31 \\
\hline
\end{tabular}

\section{Probability of Causation [\%]}

\begin{tabular}{lccc}
\hline Diet 1 & 7.56 & 44.6 & 90 \\
Diet 2 & 2.98 & 23.9 & 79 \\
Diet 3 & 1.09 & 9.1 & 56 \\
Diet 4 & 15.91 & 65.6 & 97 \\
\hline
\end{tabular}

Diet 1 - Backyard cow milk + all other locally produced non-milk exposure pathways

Diet 2 - Locally produced commercial milk + all other locally produced non-milk exposure pathways

Diet 3 - Regionally mixed commercial milk + inhalation (other regionally mixed food items are minor contributors to the total)

Diet 4 - Goat milk (all other exposure pathways are of negligible importance compared to goat milk) 


\section{Location: Karns}

\section{Receptor: Male born in 1952}

\begin{tabular}{lccc}
\hline & \multicolumn{3}{c}{ Thyroid Dose [cGy] } \\
& \multicolumn{2}{c}{$95 \%$ Subjective Confidence Interval } \\
Exposure Pathway & lower limit & central estimate & upper limit \\
\hline Backyard Cow Milk & 1.5 & 9.5 & 55 \\
Commercial Milk (locally produced) & 0.45 & 3.2 & 22 \\
Commercial Milk (regionally mixed) & 0.18 & 1.3 & 9 \\
Goat Milk (locally produced) & 2.9 & 25 & 230 \\
Beef (locally produced) & 0.0021 & 0.035 & 0.82 \\
Leafy Vegetables (locally produced) & 0.00038 & 0.0044 & 0.039 \\
Eggs (locally produced) & 0.084 & 0.59 & 4.2 \\
Cottage Cheese (locally produced) & 0.0021 & 0.025 & 0.27 \\
Inhalation & 0.025 & 0.11 & 0.4 \\
Mother's milk (mother on Diet 1) & 0.00043 & 0.0093 & 0.19 \\
Prenatal exposure (mother on Diet 1) & 0.0043 & 0.029 & 0.25 \\
Diet 1 & 1.7 & 10 & 60 \\
Diet 2 & 0.64 & 4.1 & 26 \\
Diet 3 & 0.22 & 1.4 & 9.3 \\
\hline
\end{tabular}

Excess Lifetime Risk [ ]

\begin{tabular}{llll}
\hline Diet 1 & $2.8 \mathrm{E}-05$ & $8.4 \mathrm{E}-04$ & $1.5 \mathrm{E}-02$ \\
Diet 2 & $1.2 \mathrm{E}-05$ & $3.1 \mathrm{E}-04$ & $6.5 \mathrm{E}-03$ \\
Diet 3 & $4.5 \mathrm{E}-06$ & $1.0 \mathrm{E}-04$ & $2.1 \mathrm{E}-03$ \\
Diet 4 & $6.7 \mathrm{E}-05$ & $2.0 \mathrm{E}-03$ & $4.0 \mathrm{E}-02$ \\
\hline
\end{tabular}

\section{Relative Risk [ ]}

\begin{tabular}{lccc}
\hline Diet 1 & 1.035 & 1.54 & 14 \\
Diet 2 & 1.012 & 1.21 & 5.9 \\
Diet 3 & 1.004 & 1.07 & 2.8 \\
Diet 4 & 1.077 & 2.5 & 34 \\
\hline
\end{tabular}

\section{Probability of Causation [\%]}

\begin{tabular}{lccc}
\hline Diet 1 & 3.34 & 35.1 & 93 \\
Diet 2 & 1.14 & 17.4 & 83 \\
Diet 3 & 0.40 & 6.5 & 64 \\
Diet 4 & 7.16 & 59.7 & 97 \\
\hline
\end{tabular}

Diet 1 - Backyard cow milk + all other locally produced non-milk exposure pathways

Diet 2 - Locally produced commercial milk + all other locally produced non-milk exposure pathways

Diet 3 - Regionally mixed commercial milk + inhalation (other regionally mixed food items are minor contributors to the total)

Diet 4 - Goat milk (all other exposure pathways are of negligible importance compared to goat milk) 


\section{Location: Loudon}

Receptor: Female born in 1952

\begin{tabular}{lccc}
\hline & \multicolumn{3}{c}{ Thyroid Dose [cGy] } \\
& \multicolumn{2}{c}{$95 \%$ Subjective Confidence Interval } \\
Exposure Pathway & lower limit & central estimate & upper limit \\
\hline Backyard Cow Milk & 0.88 & 5.3 & 37 \\
Commercial Milk (locally produced) & 0.23 & 1.8 & 13 \\
Commercial Milk (regionally mixed) & 0.17 & 1.3 & 8 \\
Goat Milk (locally produced) & 1.7 & 14 & 120 \\
Beef (locally produced) & 0.0012 & 0.02 & 0.46 \\
Leafy Vegetables (locally produced) & 0.00022 & 0.0025 & 0.025 \\
Eggs (locally produced) & 0.039 & 0.3 & 2.5 \\
Cottage Cheese (locally produced) & 0.001 & 0.014 & 0.16 \\
Inhalation & 0.015 & 0.06 & 0.25 \\
Mother's milk (mother on Diet 1) & 0.00027 & 0.0055 & 0.1 \\
Prenatal exposure (mother on Diet 1) & 0.0024 & 0.016 & 0.15 \\
Diet 1 & 0.98 & 5.8 & 41 \\
Diet 2 & 0.35 & 2.3 & 16 \\
Diet 3 & 0.21 & 1.3 & 8.1 \\
\hline
\end{tabular}

Excess Lifetime Risk [ ]

\begin{tabular}{lllc}
\hline Diet 1 & $1.1 \mathrm{E}-04$ & $1.8 \mathrm{E}-03$ & $2.9 \mathrm{E}-02$ \\
Diet 2 & $5.5 \mathrm{E}-05$ & $6.8 \mathrm{E}-04$ & $8.8 \mathrm{E}-03$ \\
Diet 3 & $3.5 \mathrm{E}-05$ & $3.8 \mathrm{E}-04$ & $4.7 \mathrm{E}-03$ \\
Diet 4 & $2.7 \mathrm{E}-04$ & $4.8 \mathrm{E}-03$ & $7.5 \mathrm{E}-02$ \\
\hline
\end{tabular}

\section{Relative Risk [ ]}

\begin{tabular}{lccc}
\hline Diet 1 & 1.045 & 1.44 & 6.1 \\
Diet 2 & 1.017 & 1.17 & 3 \\
Diet 3 & 1.01 & 1.098 & 2.2 \\
Diet 4 & 1.1 & 2 & 18 \\
\hline
\end{tabular}

\begin{tabular}{lccc}
\hline Diet 1 & 4.26 & 30.5 & 83 \\
Diet 2 & 1.68 & 14.4 & 67 \\
Diet 3 & 1.02 & 8.9 & 55 \\
Diet 4 & 9.04 & 51.0 & 94 \\
\hline
\end{tabular}

Diet 1 - Backyard cow milk + all other locally produced non-milk exposure pathways

Diet 2 - Locally produced commercial milk + all other locally produced non-milk exposure pathways

Diet 3 - Regionally mixed commercial milk + inhalation (other regionally mixed food items are minor contributors to the total)

Diet 4 - Goat milk (all other exposure pathways are of negligible importance compared to goat milk) 


\section{Location: Loudon}

\section{Receptor: Male born in 1952}

\begin{tabular}{lccc}
\hline & \multicolumn{3}{c}{ Thyroid Dose [cGy] } \\
& \multicolumn{2}{c}{$95 \%$ Subjective Confidence Interval } \\
Exposure Pathway & lower limit & central estimate & upper limit \\
\hline Backyard Cow Milk & 0.88 & 5.3 & 35 \\
Commercial Milk (locally produced) & 0.24 & 1.8 & 13 \\
Commercial Milk (regionally mixed) & 0.18 & 1.3 & 9 \\
Goat Milk (locally produced) & 1.7 & 14 & 130 \\
Beef (locally produced) & 0.0011 & 0.02 & 0.44 \\
Leafy Vegetables (locally produced) & 0.00022 & 0.0025 & 0.024 \\
Eggs (locally produced) & 0.045 & 0.32 & 2.4 \\
Cottage Cheese (locally produced) & 0.0011 & 0.014 & 0.16 \\
Inhalation & 0.015 & 0.061 & 0.24 \\
Mother's milk (mother on Diet 1) & 0.00027 & 0.0055 & 0.1 \\
Prenatal exposure (mother on Diet 1) & 0.0024 & 0.016 & 0.15 \\
Diet 1 & 0.97 & 5.8 & 38 \\
Diet 2 & 0.36 & 2.3 & 15 \\
Diet 3 & 0.2 & 1.3 & 9.2 \\
\hline
\end{tabular}

Excess Lifetime Risk [ ]

\begin{tabular}{llll}
\hline Diet 1 & $1.6 \mathrm{E}-05$ & $4.6 \mathrm{E}-04$ & $8.7 \mathrm{E}-03$ \\
Diet 2 & $7.3 \mathrm{E}-06$ & $1.7 \mathrm{E}-04$ & $3.8 \mathrm{E}-03$ \\
Diet 3 & $4.3 \mathrm{E}-06$ & $9.7 \mathrm{E}-05$ & $2.0 \mathrm{E}-03$ \\
Diet 4 & $4.0 \mathrm{E}-05$ & $1.1 \mathrm{E}-03$ & $2.4 \mathrm{E}-02$ \\
\hline
\end{tabular}

\section{Relative Risk [ ]}

\begin{tabular}{lccc}
\hline Diet 1 & 1.017 & 1.32 & 7.8 \\
Diet 2 & 1.006 & 1.12 & 3.9 \\
Diet 3 & 1.0038 & 1.067 & 2.8 \\
Diet 4 & 1.039 & 1.87 & 20 \\
\hline
\end{tabular}

\section{Probability of Causation [\%]}

\begin{tabular}{lccc}
\hline Diet 1 & 1.70 & 24.2 & 87 \\
Diet 2 & 0.60 & 10.5 & 74 \\
Diet 3 & 0.38 & 6.3 & 64 \\
Diet 4 & 3.76 & 46.5 & 95 \\
\hline
\end{tabular}

Diet 1 - Backyard cow milk + all other locally produced non-milk exposure pathways

Diet 2 - Locally produced commercial milk + all other locally produced non-milk exposure pathways

Diet 3 - Regionally mixed commercial milk + inhalation (other regionally mixed food items are minor contributors to the total)

Diet 4 - Goat milk (all other exposure pathways are of negligible importance compared to goat milk) 
Location: Harriman

Receptor: Female born in 1952

\begin{tabular}{lccc}
\hline & \multicolumn{3}{c}{ Thyroid Dose [cGy] } \\
Exposure Pathway & \multicolumn{2}{c}{$95 \%$ Subjective Confidence Interval } \\
\hline Backyard Cow Milk & lower limit & central estimate & upper limit \\
Commercial Milk (locally produced) & 0.54 & 3.2 & 20 \\
Commercial Milk (regionally mixed) & 0.15 & 1 & 7.8 \\
Goat Milk (locally produced) & 0.17 & 1.3 & 8 \\
Beef (locally produced) & 0.99 & 8.3 & 69 \\
Leafy Vegetables (locally produced) & 0.00062 & 0.012 & 0.27 \\
Eggs (locally produced) & 0.00014 & 0.0015 & 0.014 \\
Cottage Cheese (locally produced) & 0.026 & 0.18 & 1.4 \\
Inhalation & 0.00057 & 0.0081 & 0.089 \\
Mother's milk (mother on Diet 1) & 0.0079 & 0.033 & 0.14 \\
Prenatal exposure (mother on Diet 1) & 0.00012 & 0.003 & 0.057 \\
Diet 1 & 0.0014 & 0.0092 & 0.078 \\
Diet 2 & 0.59 & 3.5 & 22 \\
Diet 3 & 0.22 & 1.3 & 9.5 \\
\hline
\end{tabular}

Excess Lifetime Risk [ ]

\begin{tabular}{llll}
\hline Diet 1 & $6.7 \mathrm{E}-05$ & $1.1 \mathrm{E}-03$ & $1.7 \mathrm{E}-02$ \\
Diet 2 & $3.6 \mathrm{E}-05$ & $4.0 \mathrm{E}-04$ & $5.8 \mathrm{E}-03$ \\
Diet 3 & $3.4 \mathrm{E}-05$ & $3.7 \mathrm{E}-04$ & $4.7 \mathrm{E}-03$ \\
Diet 4 & $1.8 \mathrm{E}-04$ & $2.7 \mathrm{E}-03$ & $4.8 \mathrm{E}-02$ \\
\hline
\end{tabular}

\section{Relative Risk [ ]}

\begin{tabular}{lccc}
\hline Diet 1 & 1.025 & 1.26 & 4 \\
Diet 2 & 1.0097 & 1.097 & 2.4 \\
Diet 3 & 1.0098 & 1.095 & 2.2 \\
Diet 4 & 1.063 & 1.64 & 9.8 \\
\hline
\end{tabular}

\begin{tabular}{lccc} 
& \multicolumn{3}{c}{ Probability of Causation [\%] } \\
\hline Diet 1 & 2.47 & 20.5 & 75 \\
Diet 2 & 0.96 & 8.9 & 59 \\
Diet 3 & 0.97 & 8.7 & 55 \\
Diet 4 & 5.90 & 38.9 & 90 \\
\hline
\end{tabular}

Diet 1 - Backyard cow milk + all other locally produced non-milk exposure pathways

Diet 2 - Locally produced commercial milk + all other locally produced non-milk exposure pathways

Diet 3 - Regionally mixed commercial milk + inhalation (other regionally mixed food items are minor contributors to the total)

Diet 4 - Goat milk (all other exposure pathways are of negligible importance compared to goat milk) 


\section{Location: Harriman}

Receptor: Male born in 1952

\begin{tabular}{lccc}
\hline & \multicolumn{3}{c}{ Thyroid Dose [cGy] } \\
Exposure Pathway & 95\% Subjective Confidence Interval \\
\hline Backyard Cow Milk & lower limit & central estimate & upper limit \\
Commercial Milk (locally produced) & 0.57 & 3.2 & 19 \\
Commercial Milk (regionally mixed) & 0.14 & 0.99 & 7.5 \\
Goat Milk (locally produced) & 0.18 & 1.3 & 9 \\
Beef (locally produced) & 0.99 & 8.2 & 71 \\
Leafy Vegetables (locally produced) & 0.00059 & 0.012 & 0.26 \\
Eggs (locally produced) & 0.00015 & 0.0015 & 0.014 \\
Cottage Cheese (locally produced) & 0.028 & 0.2 & 1.4 \\
Inhalation & 0.00062 & 0.0082 & 0.084 \\
Mother's milk (mother on Diet 1) & 0.008 & 0.032 & 0.14 \\
Prenatal exposure (mother on Diet 1) & 0.00012 & 0.003 & 0.057 \\
Diet 1 & 0.0014 & 0.0092 & 0.078 \\
Diet 2 & 0.62 & 3.5 & 21 \\
Diet 3 & 0.22 & 1.3 & 9 \\
\hline
\end{tabular}

Excess Lifetime Risk [ ]

\begin{tabular}{llll}
\hline Diet 1 & $7.8 \mathrm{E}-06$ & $2.8 \mathrm{E}-04$ & $4.8 \mathrm{E}-03$ \\
Diet 2 & $3.7 \mathrm{E}-06$ & $1.0 \mathrm{E}-04$ & $2.2 \mathrm{E}-03$ \\
Diet 3 & $4.0 \mathrm{E}-06$ & $9.3 \mathrm{E}-05$ & $2.0 \mathrm{E}-03$ \\
Diet 4 & $2.1 \mathrm{E}-05$ & $6.7 \mathrm{E}-04$ & $1.5 \mathrm{E}-02$ \\
\hline
\end{tabular}

\section{Relative Risk [ ]}

\begin{tabular}{lccc}
\hline Diet 1 & 1.011 & 1.19 & 4.8 \\
Diet 2 & 1.0038 & 1.069 & 2.7 \\
Diet 3 & 1.0036 & 1.065 & 2.7 \\
Diet 4 & 1.026 & 1.5 & 12 \\
\hline
\end{tabular}

\section{Probability of Causation [\%]}

\begin{tabular}{lccc}
\hline Diet 1 & 1.04 & 15.7 & 79 \\
Diet 2 & 0.38 & 6.5 & 63 \\
Diet 3 & 0.36 & 6.1 & 64 \\
Diet 4 & 2.49 & 33.2 & 92 \\
\hline
\end{tabular}

Diet 1 - Backyard cow milk + all other locally produced non-milk exposure pathways

Diet 2 - Locally produced commercial milk + all other locally produced non-milk exposure pathways

Diet 3 - Regionally mixed commercial milk + inhalation (other regionally mixed food items are minor contributors to the total)

Diet 4 - Goat milk (all other exposure pathways are of negligible importance compared to goat milk) 


\section{Location: Cedar Bluff}

Receptor: Female born in 1952

\begin{tabular}{lccc}
\hline & \multicolumn{3}{c}{ Thyroid Dose [cGy] } \\
Exposure Pathway & \multicolumn{2}{c}{$95 \%$ Subjective Confidence Interval } \\
\hline Backyard Cow Milk & 1.2 & 7.5 & 49 \\
Commercial Milk (locally produced) & 0.36 & 2.5 & 18 \\
Commercial Milk (regionally mixed) & 0.17 & 1.3 & 8 \\
Goat Milk (locally produced) & 2.6 & 20 & 160 \\
Beef (locally produced) & 0.0015 & 0.028 & 0.72 \\
Leafy Vegetables (locally produced) & 0.00033 & 0.0036 & 0.034 \\
Eggs (locally produced) & 0.057 & 0.43 & 3.4 \\
Cottage Cheese (locally produced) & 0.0013 & 0.019 & 0.2 \\
Inhalation & 0.02 & 0.085 & 0.33 \\
Mother's milk (mother on Diet 1) & 0.00033 & 0.0076 & 0.13 \\
Prenatal exposure (mother on Diet 1) & 0.0034 & 0.024 & 0.19 \\
Diet 1 & 1.3 & 8.2 & 54 \\
Diet 2 & 0.53 & 3.2 & 21 \\
Diet 3 & 0.23 & 1.3 & 8.2 \\
\hline
\end{tabular}

Excess Lifetime Risk [ ]

\begin{tabular}{llll}
\hline Diet 1 & $1.6 \mathrm{E}-04$ & $2.5 \mathrm{E}-03$ & $4.0 \mathrm{E}-02$ \\
Diet 2 & $7.5 \mathrm{E}-05$ & $9.7 \mathrm{E}-04$ & $1.3 \mathrm{E}-02$ \\
Diet 3 & $3.6 \mathrm{E}-05$ & $3.9 \mathrm{E}-04$ & $4.8 \mathrm{E}-03$ \\
Diet 4 & $3.7 \mathrm{E}-04$ & $6.8 \mathrm{E}-03$ & $9.6 \mathrm{E}-02$ \\
\hline
\end{tabular}

\section{Relative Risk [ ]}

\begin{tabular}{lccc}
\hline Diet 1 & 1.063 & 1.62 & 8 \\
Diet 2 & 1.025 & 1.25 & 3.9 \\
Diet 3 & 1.011 & 1.099 & 2.2 \\
Diet 4 & 1.13 & 2.5 & 25 \\
\hline
\end{tabular}

\begin{tabular}{lccc} 
& \multicolumn{3}{c}{ Probability of Causation [\%] } \\
\hline Diet 1 & 5.90 & 38.0 & 87 \\
Diet 2 & 2.41 & 19.7 & 74 \\
Diet 3 & 1.05 & 9.0 & 56 \\
Diet 4 & 11.85 & 59.5 & 96 \\
\hline
\end{tabular}

Diet 1 - Backyard cow milk + all other locally produced non-milk exposure pathways

Diet 2 - Locally produced commercial milk + all other locally produced non-milk exposure pathways

Diet 3 - Regionally mixed commercial milk + inhalation (other regionally mixed food items are minor contributors to the total)

Diet 4 - Goat milk (all other exposure pathways are of negligible importance compared to goat milk) 


\section{Location: Cedar Bluff}

Receptor: Male born in 1952

\begin{tabular}{lccc}
\hline & \multicolumn{3}{c}{ Thyroid Dose [cGy] } \\
& \multicolumn{2}{c}{$95 \%$ Subjective Confidence Interval } \\
Exposure Pathway & lower limit & central estimate & upper limit \\
\hline Backyard Cow Milk & 1.2 & 7.4 & 45 \\
Commercial Milk (locally produced) & 0.36 & 2.5 & 20 \\
Commercial Milk (regionally mixed) & 0.18 & 1.3 & 9 \\
Goat Milk (locally produced) & 2.4 & 19 & 170 \\
Beef (locally produced) & 0.0016 & 0.029 & 0.66 \\
Leafy Vegetables (locally produced) & 0.00032 & 0.0035 & 0.034 \\
Eggs (locally produced) & 0.065 & 0.47 & 3.5 \\
Cottage Cheese (locally produced) & 0.0016 & 0.019 & 0.22 \\
Inhalation & 0.021 & 0.085 & 0.34 \\
Mother's milk (mother on Diet 1) & 0.00033 & 0.0076 & 0.13 \\
Prenatal exposure (mother on Diet 1) & 0.0034 & 0.024 & 0.19 \\
Diet 1 & 1.4 & 8.1 & 48 \\
Diet 2 & 0.53 & 3.2 & 23 \\
Diet 3 & 0.21 & 1.3 & 9.3 \\
\hline
\end{tabular}

Excess Lifetime Risk [ ]

\begin{tabular}{lllc}
\hline Diet 1 & $2.0 \mathrm{E}-05$ & $6.7 \mathrm{E}-04$ & $1.2 \mathrm{E}-02$ \\
Diet 2 & $9.4 \mathrm{E}-06$ & $2.5 \mathrm{E}-04$ & $5.6 \mathrm{E}-03$ \\
Diet 3 & $4.4 \mathrm{E}-06$ & $9.9 \mathrm{E}-05$ & $2.1 \mathrm{E}-03$ \\
Diet 4 & $5.2 \mathrm{E}-05$ & $1.6 \mathrm{E}-03$ & $3.3 \mathrm{E}-02$ \\
\hline
\end{tabular}

\section{Relative Risk [ ]}

\begin{tabular}{lccc}
\hline Diet 1 & 1.025 & 1.45 & 10.9 \\
Diet 2 & 1.0092 & 1.17 & 5 \\
Diet 3 & 1.0039 & 1.069 & 2.8 \\
Diet 4 & 1.056 & 2.2 & 29 \\
\hline
\end{tabular}

\begin{tabular}{lccc}
\hline Diet 1 & 2.48 & 31.0 & 91 \\
Diet 2 & 0.91 & 14.5 & 80 \\
Diet 3 & 0.39 & 6.4 & 64 \\
Diet 4 & 5.33 & 54.0 & 97 \\
\hline
\end{tabular}

Diet 1 - Backyard cow milk + all other locally produced non-milk exposure pathways

Diet 2 - Locally produced commercial milk + all other locally produced non-milk exposure pathways

Diet 3 - Regionally mixed commercial milk + inhalation (other regionally mixed food items are minor contributors to the total)

Diet 4 - Goat milk (all other exposure pathways are of negligible importance compared to goat milk) 


\section{Location: Oakdale}

Receptor: Female born in 1952

\begin{tabular}{lccc}
\hline & \multicolumn{3}{c}{ Thyroid Dose [cGy] } \\
& \multicolumn{2}{c}{$95 \%$ Subjective Confidence Interval } \\
Exposure Pathway & lower limit & central estimate & upper limit \\
\hline Backyard Cow Milk & 0.26 & 1.8 & 13 \\
Commercial Milk (locally produced) & 0.077 & 0.59 & 4.4 \\
Commercial Milk (regionally mixed) & 0.17 & 1.3 & 8 \\
Goat Milk (locally produced) & 0.49 & 4.7 & 42 \\
Beef (locally produced) & 0.00034 & 0.0068 & 0.15 \\
Leafy Vegetables (locally produced) & 0.000072 & 0.00087 & 0.0084 \\
Eggs (locally produced) & 0.012 & 0.1 & 0.85 \\
Cottage Cheese (locally produced) & 0.00031 & 0.0046 & 0.051 \\
Inhalation & 0.0045 & 0.019 & 0.084 \\
Mother's milk (mother on Diet 1) & 0.00007 & 0.0018 & 0.033 \\
Prenatal exposure (mother on Diet 1) & 0.00073 & 0.0055 & 0.046 \\
Diet 1 & 0.29 & 2 & 14 \\
Diet 2 & 0.11 & 0.77 & 5.2 \\
Diet 3 & 0.19 & 1.3 & 8 \\
\hline
\end{tabular}

Excess Lifetime Risk [ ]

\begin{tabular}{llll}
\hline Diet 1 & $3.3 \mathrm{E}-05$ & $6.0 \mathrm{E}-04$ & $9.1 \mathrm{E}-03$ \\
Diet 2 & $1.7 \mathrm{E}-05$ & $2.3 \mathrm{E}-04$ & $3.3 \mathrm{E}-03$ \\
Diet 3 & $3.3 \mathrm{E}-05$ & $3.7 \mathrm{E}-04$ & $4.7 \mathrm{E}-03$ \\
Diet 4 & $8.4 \mathrm{E}-05$ & $1.5 \mathrm{E}-03$ & $2.4 \mathrm{E}-02$ \\
\hline
\end{tabular}

\section{Relative Risk [ ]}

\begin{tabular}{lccc}
\hline Diet 1 & 1.013 & 1.15 & 2.7 \\
Diet 2 & 1.005 & 1.056 & 1.74 \\
Diet 3 & 1.0094 & 1.095 & 2.2 \\
Diet 4 & 1.032 & 1.36 & 6.4 \\
\hline
\end{tabular}

\section{Probability of Causation [\%]}

\begin{tabular}{lccc}
\hline Diet 1 & 1.27 & 12.6 & 62 \\
Diet 2 & 0.50 & 5.3 & 41 \\
Diet 3 & 0.93 & 8.6 & 55 \\
Diet 4 & 3.04 & 25.9 & 84 \\
\hline
\end{tabular}

Diet 1 - Backyard cow milk + all other locally produced non-milk exposure pathways

Diet 2 - Locally produced commercial milk + all other locally produced non-milk exposure pathways

Diet 3 - Regionally mixed commercial milk + inhalation (other regionally mixed food items are minor contributors to the total)

Diet 4 - Goat milk (all other exposure pathways are of negligible importance compared to goat milk) 


\section{Location: Oakdale}

Receptor: Male born in 1952

\begin{tabular}{lccc}
\hline & \multicolumn{3}{c}{ Thyroid Dose [cGy] } \\
& \multicolumn{2}{c}{$95 \%$ Subjective Confidence Interval } \\
Exposure Pathway & lower limit & central estimate & upper limit \\
\hline Backyard Cow Milk & 0.28 & 1.8 & 12 \\
Commercial Milk (locally produced) & 0.073 & 0.59 & 4.3 \\
Commercial Milk (regionally mixed) & 0.18 & 1.3 & 9 \\
Goat Milk (locally produced) & 0.49 & 4.7 & 44 \\
Beef (locally produced) & 0.00036 & 0.0067 & 0.15 \\
Leafy Vegetables (locally produced) & 0.000071 & 0.00084 & 0.0087 \\
Eggs (locally produced) & 0.014 & 0.11 & 0.83 \\
Cottage Cheese (locally produced) & 0.00034 & 0.0046 & 0.054 \\
Inhalation & 0.0045 & 0.019 & 0.083 \\
Mother's milk (mother on Diet 1) & 0.00007 & 0.0018 & 0.033 \\
Prenatal exposure (mother on Diet 1) & 0.00073 & 0.0055 & 0.046 \\
Diet 1 & 0.31 & 2 & 13 \\
Diet 2 & 0.11 & 0.75 & 5.2 \\
Diet 3 & 0.19 & 1.3 & 9 \\
\hline
\end{tabular}

Excess Lifetime Risk [ ]

\begin{tabular}{llll}
\hline Diet 1 & $4.4 \mathrm{E}-06$ & $1.6 \mathrm{E}-04$ & $3.0 \mathrm{E}-03$ \\
Diet 2 & $2.2 \mathrm{E}-06$ & $6.0 \mathrm{E}-05$ & $1.3 \mathrm{E}-03$ \\
Diet 3 & $3.9 \mathrm{E}-06$ & $9.2 \mathrm{E}-05$ & $2.0 \mathrm{E}-03$ \\
Diet 4 & $1.2 \mathrm{E}-05$ & $3.8 \mathrm{E}-04$ & $8.4 \mathrm{E}-03$ \\
\hline
\end{tabular}

\section{Relative Risk [ ]}

\begin{tabular}{lccc}
\hline Diet 1 & 1.0057 & 1.1 & 3.2 \\
Diet 2 & 1.002 & 1.04 & 1.96 \\
Diet 3 & 1.0036 & 1.065 & 2.7 \\
Diet 4 & 1.014 & 1.28 & 7.1 \\
\hline
\end{tabular}

\section{Probability of Causation [\%]}

\begin{tabular}{lccc}
\hline Diet 1 & 0.57 & 9.4 & 68 \\
Diet 2 & 0.20 & 3.8 & 48 \\
Diet 3 & 0.36 & 6.1 & 63 \\
Diet 4 & 1.37 & 21.6 & 85 \\
\hline
\end{tabular}

Diet 1 - Backyard cow milk + all other locally produced non-milk exposure pathways

Diet 2 - Locally produced commercial milk + all other locally produced non-milk exposure pathways

Diet 3 - Regionally mixed commercial milk + inhalation (other regionally mixed food items are minor contributors to the total)

Diet 4 - Goat milk (all other exposure pathways are of negligible importance compared to goat milk) 


\section{Location: Claxton}

Receptor: Female born in 1952

\begin{tabular}{lccc}
\hline & \multicolumn{3}{c}{ Thyroid Dose [cGy] } \\
& \multicolumn{2}{c}{$95 \%$ Subjective Confidence Interval } \\
Exposure Pathway & 1 & 6.3 & 40 \\
\hline Backyard Cow Milk & 0.31 & 2.1 & 15 \\
Commercial Milk (locally produced) & 0.17 & 1.3 & 8 \\
Commercial Milk (regionally mixed) & 2.1 & 16 & 140 \\
Goat Milk (locally produced) & 0.0013 & 0.023 & 0.59 \\
Beef (locally produced) & 0.00027 & 0.003 & 0.028 \\
Leafy Vegetables (locally produced) & 0.048 & 0.36 & 2.9 \\
Eggs (locally produced) & 0.0011 & 0.016 & 0.17 \\
Cottage Cheese (locally produced) & 0.017 & 0.07 & 0.28 \\
Inhalation & 0.00028 & 0.0063 & 0.11 \\
Mother's milk (mother on Diet 1) & 0.0029 & 0.019 & 0.15 \\
Prenatal exposure (mother on Diet 1) & 1.1 & 6.9 & 44 \\
Diet 1 & 0.45 & 2.7 & 18 \\
Diet 2 & 0.22 & 1.3 & 8.1 \\
Diet 3 & & upper limit \\
\hline
\end{tabular}

Excess Lifetime Risk [ ]

\begin{tabular}{lllc}
\hline Diet 1 & $1.4 \mathrm{E}-04$ & $2.1 \mathrm{E}-03$ & $3.2 \mathrm{E}-02$ \\
Diet 2 & $6.4 \mathrm{E}-05$ & $8.0 \mathrm{E}-04$ & $1.1 \mathrm{E}-02$ \\
Diet 3 & $3.5 \mathrm{E}-05$ & $3.8 \mathrm{E}-04$ & $4.8 \mathrm{E}-03$ \\
Diet 4 & $3.2 \mathrm{E}-04$ & $5.8 \mathrm{E}-03$ & $8.1 \mathrm{E}-02$ \\
\hline
\end{tabular}

\section{Relative Risk [ ]}

\begin{tabular}{lccc}
\hline Diet 1 & 1.054 & 1.51 & 6.8 \\
Diet 2 & 1.021 & 1.2 & 3.4 \\
Diet 3 & 1.011 & 1.098 & 2.2 \\
Diet 4 & 1.12 & 2.2 & 20 \\
\hline
\end{tabular}

\section{Probability of Causation [\%]}

\begin{tabular}{lccc}
\hline Diet 1 & 5.09 & 33.9 & 85 \\
Diet 2 & 2.03 & 16.6 & 71 \\
Diet 3 & 1.04 & 8.9 & 55 \\
Diet 4 & 10.50 & 55.0 & 95 \\
\hline
\end{tabular}

Diet 1 - Backyard cow milk + all other locally produced non-milk exposure pathways

Diet 2 - Locally produced commercial milk + all other locally produced non-milk exposure pathways

Diet 3 - Regionally mixed commercial milk + inhalation (other regionally mixed food items are minor contributors to the total)

Diet 4 - Goat milk (all other exposure pathways are of negligible importance compared to goat milk) 


\section{Location: Claxton}

Receptor: Male born in 1952

\begin{tabular}{lccc}
\hline & \multicolumn{3}{c}{ Thyroid Dose [cGy] } \\
& \multicolumn{2}{c}{$95 \%$ Subjective Confidence Interval } \\
Exposure Pathway & 1 & 6.2 & 38 \\
\hline Backyard Cow Milk & 0.3 & 2.1 & 16 \\
Commercial Milk (locally produced) & 0.18 & 1.3 & 9 \\
Commercial Milk (regionally mixed) & 2 & 16 & 140 \\
Goat Milk (locally produced) & 0.0013 & 0.024 & 0.55 \\
Beef (locally produced) & 0.00027 & 0.0029 & 0.028 \\
Leafy Vegetables (locally produced) & 0.056 & 0.39 & 2.8 \\
Eggs (locally produced) & 0.0013 & 0.016 & 0.18 \\
Cottage Cheese (locally produced) & 0.017 & 0.071 & 0.28 \\
Inhalation & 0.00028 & 0.0063 & 0.11 \\
Mother's milk (mother on Diet 1) & 0.0029 & 0.019 & 0.15 \\
Prenatal exposure (mother on Diet 1) & 1.1 & 6.8 & 41 \\
Diet 1 & 0.45 & 2.7 & 19 \\
Diet 2 & 0.2 & 1.3 & 9.2 \\
Diet 3 & & upper limit \\
\hline
\end{tabular}

Excess Lifetime Risk [ ]

\begin{tabular}{llll}
\hline Diet 1 & $1.7 \mathrm{E}-05$ & $5.6 \mathrm{E}-04$ & $1.0 \mathrm{E}-02$ \\
Diet 2 & $8.3 \mathrm{E}-06$ & $2.1 \mathrm{E}-04$ & $4.6 \mathrm{E}-03$ \\
Diet 3 & $4.3 \mathrm{E}-06$ & $9.8 \mathrm{E}-05$ & $2.0 \mathrm{E}-03$ \\
Diet 4 & $4.6 \mathrm{E}-05$ & $1.4 \mathrm{E}-03$ & $2.7 \mathrm{E}-02$ \\
\hline
\end{tabular}

\section{Relative Risk [ ]}

\begin{tabular}{lccc}
\hline Diet 1 & 1.021 & 1.37 & 9 \\
Diet 2 & 1.0076 & 1.14 & 4.3 \\
Diet 3 & 1.0038 & 1.068 & 2.8 \\
Diet 4 & 1.047 & 1.98 & 24 \\
\hline
\end{tabular}

\section{Probability of Causation [\%]}

\begin{tabular}{lccc}
\hline Diet 1 & 2.03 & 26.8 & 89 \\
Diet 2 & 0.76 & 12.4 & 77 \\
Diet 3 & 0.38 & 6.3 & 64 \\
Diet 4 & 4.44 & 49.4 & 96 \\
\hline
\end{tabular}

Diet 1 - Backyard cow milk + all other locally produced non-milk exposure pathways

Diet 2 - Locally produced commercial milk + all other locally produced non-milk exposure pathways

Diet 3 - Regionally mixed commercial milk + inhalation (other regionally mixed food items are minor contributors to the total)

Diet 4 - Goat milk (all other exposure pathways are of negligible importance compared to goat milk) 
Location: Dutch Valley

Receptor: Female born in 1952

\begin{tabular}{lccc}
\hline & \multicolumn{3}{c}{ Thyroid Dose [cGy] } \\
& \multicolumn{2}{c}{$95 \%$ Subjective Confidence Interval } \\
Exposure Pathway & lower limit & central estimate & upper limit \\
\hline Backyard Cow Milk & 0.49 & 3.1 & 19 \\
Commercial Milk (locally produced) & 0.14 & 0.99 & 7.5 \\
Commercial Milk (regionally mixed) & 0.18 & 1.3 & 8 \\
Goat Milk (locally produced) & 0.97 & 8 & 67 \\
Beef (locally produced) & 0.00058 & 0.011 & 0.25 \\
Leafy Vegetables (locally produced) & 0.00013 & 0.0014 & 0.014 \\
Eggs (locally produced) & 0.023 & 0.18 & 1.4 \\
Cottage Cheese (locally produced) & 0.00054 & 0.008 & 0.081 \\
Inhalation & 0.0076 & 0.033 & 0.14 \\
Mother's milk (mother on Diet 1) & 0.00012 & 0.0029 & 0.061 \\
Prenatal exposure (mother on Diet 1) & 0.0014 & 0.0091 & 0.078 \\
Diet 1 & 0.53 & 3.4 & 21 \\
Diet 2 & 0.2 & 1.3 & 8.6 \\
Diet 3 & 0.2 & 1.3 & 8 \\
\hline
\end{tabular}

Excess Lifetime Risk [ ]

\begin{tabular}{llll}
\hline Diet 1 & $6.1 \mathrm{E}-05$ & $1.0 \mathrm{E}-03$ & $1.6 \mathrm{E}-02$ \\
Diet 2 & $3.2 \mathrm{E}-05$ & $3.8 \mathrm{E}-04$ & $5.8 \mathrm{E}-03$ \\
Diet 3 & $3.4 \mathrm{E}-05$ & $3.7 \mathrm{E}-04$ & $4.7 \mathrm{E}-03$ \\
Diet 4 & $1.6 \mathrm{E}-04$ & $2.7 \mathrm{E}-03$ & $4.3 \mathrm{E}-02$ \\
\hline
\end{tabular}

\section{Relative Risk [ ]}

\begin{tabular}{lccc}
\hline Diet 1 & 1.026 & 1.25 & 3.7 \\
Diet 2 & 1.0093 & 1.096 & 2.3 \\
Diet 3 & 1.0098 & 1.096 & 2.2 \\
Diet 4 & 1.06 & 1.62 & 10.4 \\
\hline
\end{tabular}

\section{Probability of Causation [\%]}

\begin{tabular}{lccc}
\hline Diet 1 & 2.55 & 20.1 & 72 \\
Diet 2 & 0.92 & 8.7 & 56 \\
Diet 3 & 0.97 & 8.7 & 55 \\
Diet 4 & 5.62 & 37.8 & 90 \\
\hline
\end{tabular}

Diet 1 - Backyard cow milk + all other locally produced non-milk exposure pathways

Diet 2 - Locally produced commercial milk + all other locally produced non-milk exposure pathways

Diet 3 - Regionally mixed commercial milk + inhalation (other regionally mixed food items are minor contributors to the total)

Diet 4 - Goat milk (all other exposure pathways are of negligible importance compared to goat milk) 
Location: Dutch Valley

Receptor: Male born in 1952

\begin{tabular}{lccc}
\hline & \multicolumn{3}{c}{ Thyroid Dose [cGy] } \\
Exposure Pathway & \multicolumn{2}{c}{$95 \%$ Subjective Confidence Interval } \\
\hline Backyard Cow Milk & lower limit & central estimate & upper limit \\
Commercial Milk (locally produced) & 0.5 & 3.1 & 18 \\
Commercial Milk (regionally mixed) & 0.13 & 0.99 & 6.8 \\
Goat Milk (locally produced) & 0.18 & 1.3 & 9 \\
Beef (locally produced) & 0.9 & 7.8 & 71 \\
Leafy Vegetables (locally produced) & 0.00059 & 0.011 & 0.25 \\
Eggs (locally produced) & 0.00013 & 0.0014 & 0.013 \\
Cottage Cheese (locally produced) & 0.026 & 0.19 & 1.3 \\
Inhalation & 0.0006 & 0.008 & 0.083 \\
Mother's milk (mother on Diet 1) & 0.0079 & 0.033 & 0.14 \\
Prenatal exposure (mother on Diet 1) & 0.00012 & 0.0029 & 0.061 \\
Diet 1 & 0.0014 & 0.0091 & 0.078 \\
Diet 2 & 0.55 & 3.4 & 20 \\
Diet 3 & 0.2 & 1.3 & 8.1 \\
\hline
\end{tabular}

Excess Lifetime Risk [ ]

\begin{tabular}{llll}
\hline Diet 1 & $8.2 \mathrm{E}-06$ & $2.7 \mathrm{E}-04$ & $4.6 \mathrm{E}-03$ \\
Diet 2 & $3.5 \mathrm{E}-06$ & $9.9 \mathrm{E}-05$ & $2.1 \mathrm{E}-03$ \\
Diet 3 & $4.0 \mathrm{E}-06$ & $9.3 \mathrm{E}-05$ & $2.0 \mathrm{E}-03$ \\
Diet 4 & $2.0 \mathrm{E}-05$ & $6.4 \mathrm{E}-04$ & $1.3 \mathrm{E}-02$ \\
\hline
\end{tabular}

\section{Relative Risk [ ]}

\begin{tabular}{lccc}
\hline Diet 1 & 1.01 & 1.17 & 4.8 \\
Diet 2 & 1.0036 & 1.065 & 2.6 \\
Diet 3 & 1.0036 & 1.065 & 2.7 \\
Diet 4 & 1.024 & 1.48 & 12 \\
\hline
\end{tabular}

\section{Probability of Causation [\%]}

\begin{tabular}{lccc}
\hline Diet 1 & 0.99 & 14.6 & 79 \\
Diet 2 & 0.36 & 6.1 & 60 \\
Diet 3 & 0.36 & 6.1 & 63 \\
Diet 4 & 2.38 & 32.0 & 91 \\
\hline
\end{tabular}

Diet 1 - Backyard cow milk + all other locally produced non-milk exposure pathways

Diet 2 - Locally produced commercial milk + all other locally produced non-milk exposure pathways

Diet 3 - Regionally mixed commercial milk + inhalation (other regionally mixed food items are minor contributors to the total)

Diet 4 - Goat milk (all other exposure pathways are of negligible importance compared to goat milk) 


\section{Location: Clinton}

Receptor: Female born in 1952

\begin{tabular}{lccc}
\hline & \multicolumn{3}{c}{ Thyroid Dose [cGy] } \\
& \multicolumn{2}{c}{$95 \%$ Subjective Confidence Interval } \\
Exposure Pathway & lower limit & central estimate & upper limit \\
\hline Backyard Cow Milk & 0.78 & 4.9 & 33 \\
Commercial Milk (locally produced) & 0.23 & 1.6 & 11 \\
Commercial Milk (regionally mixed) & 0.17 & 1.2 & 8 \\
Goat Milk (locally produced) & 1.6 & 13 & 110 \\
Beef (locally produced) & 0.00096 & 0.018 & 0.41 \\
Leafy Vegetables (locally produced) & 0.00021 & 0.0024 & 0.021 \\
Eggs (locally produced) & 0.037 & 0.28 & 2.2 \\
Cottage Cheese (locally produced) & 0.00089 & 0.013 & 0.13 \\
Inhalation & 0.013 & 0.053 & 0.22 \\
Mother's milk (mother on Diet 1) & 0.00019 & 0.0048 & 0.083 \\
Prenatal exposure (mother on Diet 1) & 0.002 & 0.015 & 0.11 \\
Diet 1 & 0.86 & 5.5 & 36 \\
Diet 2 & 0.34 & 2.1 & 14 \\
Diet 3 & 0.21 & 1.3 & 8.1 \\
\hline
\end{tabular}

Excess Lifetime Risk [ ]

\begin{tabular}{llll}
\hline Diet 1 & $1.0 \mathrm{E}-04$ & $1.6 \mathrm{E}-03$ & $2.4 \mathrm{E}-02$ \\
Diet 2 & $4.8 \mathrm{E}-05$ & $6.3 \mathrm{E}-04$ & $8.0 \mathrm{E}-03$ \\
Diet 3 & $3.4 \mathrm{E}-05$ & $3.8 \mathrm{E}-04$ & $4.7 \mathrm{E}-03$ \\
Diet 4 & $2.3 \mathrm{E}-04$ & $4.3 \mathrm{E}-03$ & $6.0 \mathrm{E}-02$ \\
\hline
\end{tabular}

\section{Relative Risk [ ]}

\begin{tabular}{lccc}
\hline Diet 1 & 1.04 & 1.39 & 5.3 \\
Diet 2 & 1.015 & 1.16 & 2.9 \\
Diet 3 & 1.01 & 1.097 & 2.2 \\
Diet 4 & 1.089 & 1.98 & 15 \\
\hline
\end{tabular}

\section{Probability of Causation [\%]}

\begin{tabular}{lccc}
\hline Diet 1 & 3.81 & 27.9 & 80 \\
Diet 2 & 1.52 & 13.6 & 65 \\
Diet 3 & 1.00 & 8.9 & 55 \\
Diet 4 & 8.14 & 48.9 & 93 \\
\hline
\end{tabular}

Diet 1 - Backyard cow milk + all other locally produced non-milk exposure pathways

Diet 2 - Locally produced commercial milk + all other locally produced non-milk exposure pathways

Diet 3 - Regionally mixed commercial milk + inhalation (other regionally mixed food items are minor contributors to the total)

Diet 4 - Goat milk (all other exposure pathways are of negligible importance compared to goat milk) 


\section{Location: Clinton}

\section{Receptor: Male born in 1952}

\begin{tabular}{lccc}
\hline & \multicolumn{3}{c}{ Thyroid Dose [cGy] } \\
& \multicolumn{2}{c}{$95 \%$ Subjective Confidence Interval } \\
Exposure Pathway & lower limit & central estimate & upper limit \\
\hline Backyard Cow Milk & 0.79 & 4.8 & 28 \\
Commercial Milk (locally produced) & 0.23 & 1.6 & 12 \\
Commercial Milk (regionally mixed) & 0.18 & 1.3 & 9 \\
Goat Milk (locally produced) & 1.5 & 12 & 120 \\
Beef (locally produced) & 0.001 & 0.018 & 0.42 \\
Leafy Vegetables (locally produced) & 0.0002 & 0.0023 & 0.021 \\
Eggs (locally produced) & 0.042 & 0.3 & 2.1 \\
Cottage Cheese (locally produced) & 0.00096 & 0.012 & 0.15 \\
Inhalation & 0.014 & 0.054 & 0.22 \\
Mother's milk (mother on Diet 1) & 0.00019 & 0.0048 & 0.083 \\
Prenatal exposure (mother on Diet 1) & 0.002 & 0.015 & 0.11 \\
Diet 1 & 0.87 & 5.3 & 31 \\
Diet 2 & 0.34 & 2.1 & 14 \\
Diet 3 & 0.2 & 1.3 & 9.2 \\
\hline
\end{tabular}

Excess Lifetime Risk [ ]

\begin{tabular}{llll}
\hline Diet 1 & $1.2 \mathrm{E}-05$ & $4.3 \mathrm{E}-04$ & $7.8 \mathrm{E}-03$ \\
Diet 2 & $6.3 \mathrm{E}-06$ & $1.7 \mathrm{E}-04$ & $3.6 \mathrm{E}-03$ \\
Diet 3 & $4.1 \mathrm{E}-06$ & $9.6 \mathrm{E}-05$ & $2.0 \mathrm{E}-03$ \\
Diet 4 & $3.3 \mathrm{E}-05$ & $1.1 \mathrm{E}-03$ & $2.0 \mathrm{E}-02$ \\
\hline
\end{tabular}

\section{Relative Risk [ ]}

\begin{tabular}{lccc}
\hline Diet 1 & 1.017 & 1.29 & 7.4 \\
Diet 2 & 1.0058 & 1.1 & 3.5 \\
Diet 3 & 1.0037 & 1.066 & 2.8 \\
Diet 4 & 1.04 & 1.78 & 18 \\
\hline
\end{tabular}

\section{Probability of Causation [\%]}

\begin{tabular}{lccc}
\hline Diet 1 & 1.71 & 22.0 & 85 \\
Diet 2 & 0.57 & 9.4 & 71 \\
Diet 3 & 0.37 & 6.2 & 64 \\
Diet 4 & 3.85 & 43.1 & 94 \\
\hline
\end{tabular}

Diet 1 - Backyard cow milk + all other locally produced non-milk exposure pathways

Diet 2 - Locally produced commercial milk + all other locally produced non-milk exposure pathways

Diet 3 - Regionally mixed commercial milk + inhalation (other regionally mixed food items are minor contributors to the total)

Diet 4 - Goat milk (all other exposure pathways are of negligible importance compared to goat milk) 
Location: Friendsville

Receptor: Female born in 1952

\begin{tabular}{lccc}
\hline & \multicolumn{3}{c}{ Thyroid Dose [cGy] } \\
Exposure Pathway & \multicolumn{2}{c}{$95 \%$ Subjective Confidence Interval } \\
\hline Backyard Cow Milk & lower limit & central estimate & upper limit \\
Commercial Milk (locally produced) & 0.41 & 2.8 & 19 \\
Commercial Milk (regionally mixed) & 0.12 & 0.92 & 6.2 \\
Goat Milk (locally produced) & 0.17 & 1.2 & 8 \\
Beef (locally produced) & 0.9 & 7.4 & 68 \\
Leafy Vegetables (locally produced) & 0.00058 & 0.01 & 0.26 \\
Eggs (locally produced) & 0.00011 & 0.0013 & 0.012 \\
Cottage Cheese (locally produced) & 0.022 & 0.16 & 1.3 \\
Inhalation & 0.00048 & 0.0073 & 0.071 \\
Mother's milk (mother on Diet 1) & 0.0081 & 0.037 & 0.16 \\
Prenatal exposure (mother on Diet 1) & 0.00014 & 0.003 & 0.059 \\
Diet 1 & 0.0013 & 0.0089 & 0.076 \\
Diet 2 & 0.46 & 3.1 & 20 \\
Diet 3 & 0.18 & 1.2 & 7.5 \\
\hline
\end{tabular}

Excess Lifetime Risk [ ]

\begin{tabular}{llll}
\hline Diet 1 & $6.1 \mathrm{E}-05$ & $9.2 \mathrm{E}-04$ & $1.5 \mathrm{E}-02$ \\
Diet 2 & $3.0 \mathrm{E}-05$ & $3.6 \mathrm{E}-04$ & $4.6 \mathrm{E}-03$ \\
Diet 3 & $3.4 \mathrm{E}-05$ & $3.7 \mathrm{E}-04$ & $4.7 \mathrm{E}-03$ \\
Diet 4 & $1.7 \mathrm{E}-04$ & $2.5 \mathrm{E}-03$ & $3.8 \mathrm{E}-02$ \\
\hline
\end{tabular}

\section{Relative Risk [ ]}

\begin{tabular}{lccc}
\hline Diet 1 & 1.023 & 1.24 & 3.5 \\
Diet 2 & 1.0084 & 1.093 & 2.1 \\
Diet 3 & 1.0098 & 1.096 & 2.2 \\
Diet 4 & 1.052 & 1.58 & 10.6 \\
\hline
\end{tabular}

\begin{tabular}{lccc} 
& \multicolumn{3}{c}{ Probability of Causation [\%] } \\
\hline Diet 1 & 2.27 & 19.2 & 72 \\
Diet 2 & 0.84 & 8.5 & 52 \\
Diet 3 & 0.98 & 8.8 & 55 \\
Diet 4 & 4.93 & 36.6 & 91 \\
\hline
\end{tabular}

Diet 1 - Backyard cow milk + all other locally produced non-milk exposure pathways

Diet 2 - Locally produced commercial milk + all other locally produced non-milk exposure pathways

Diet 3 - Regionally mixed commercial milk + inhalation (other regionally mixed food items are minor contributors to the total)

Diet 4 - Goat milk (all other exposure pathways are of negligible importance compared to goat milk) 
Location: Friendsville

Receptor: Male born in 1952

\begin{tabular}{lccc}
\hline & \multicolumn{3}{c}{ Thyroid Dose [cGy] } \\
Exposure Pathway & 95\% Subjective Confidence Interval \\
\hline Backyard Cow Milk & lower limit & central estimate & upper limit \\
Commercial Milk (locally produced) & 0.4 & 2.8 & 17 \\
Commercial Milk (regionally mixed) & 0.12 & 0.93 & 5.9 \\
Goat Milk (locally produced) & 0.18 & 1.3 & 9 \\
Beef (locally produced) & 0.83 & 7.1 & 76 \\
Leafy Vegetables (locally produced) & 0.00058 & 0.01 & 0.23 \\
Eggs (locally produced) & 0.00011 & 0.0013 & 0.012 \\
Cottage Cheese (locally produced) & 0.023 & 0.17 & 1.3 \\
Inhalation & 0.00055 & 0.0075 & 0.082 \\
Mother's milk (mother on Diet 1) & 0.0082 & 0.037 & 0.15 \\
Prenatal exposure (mother on Diet 1) & 0.00014 & 0.003 & 0.059 \\
Diet 1 & 0.0013 & 0.0089 & 0.076 \\
Diet 2 & 0.46 & 3 & 19 \\
Diet 3 & 0.18 & 1.2 & 7.3 \\
\hline
\end{tabular}

Excess Lifetime Risk [ ]

\begin{tabular}{llll}
\hline Diet 1 & $8.4 \mathrm{E}-06$ & $2.4 \mathrm{E}-04$ & $4.6 \mathrm{E}-03$ \\
Diet 2 & $3.6 \mathrm{E}-06$ & $8.9 \mathrm{E}-05$ & $1.9 \mathrm{E}-03$ \\
Diet 3 & $4.1 \mathrm{E}-06$ & $9.4 \mathrm{E}-05$ & $2.0 \mathrm{E}-03$ \\
Diet 4 & $1.9 \mathrm{E}-05$ & $5.8 \mathrm{E}-04$ & $1.2 \mathrm{E}-02$ \\
\hline
\end{tabular}

\section{Relative Risk [ ]}

\begin{tabular}{lccc}
\hline Diet 1 & 1.0092 & 1.16 & 4.8 \\
Diet 2 & 1.0033 & 1.062 & 2.3 \\
Diet 3 & 1.0036 & 1.065 & 2.7 \\
Diet 4 & 1.024 & 1.44 & 11 \\
\hline
\end{tabular}

\section{Probability of Causation [\%]}

\begin{tabular}{lccc}
\hline Diet 1 & 0.92 & 14.1 & 79 \\
Diet 2 & 0.33 & 5.9 & 57 \\
Diet 3 & 0.36 & 6.1 & 63 \\
Diet 4 & 2.32 & 30.6 & 91 \\
\hline
\end{tabular}

Diet 1 - Backyard cow milk + all other locally produced non-milk exposure pathways

Diet 2 - Locally produced commercial milk + all other locally produced non-milk exposure pathways

Diet 3 - Regionally mixed commercial milk + inhalation (other regionally mixed food items are minor contributors to the total)

Diet 4 - Goat milk (all other exposure pathways are of negligible importance compared to goat milk) 
Location: Wartburg

Receptor: Female born in 1952

\begin{tabular}{lccc}
\hline & \multicolumn{3}{c}{ Thyroid Dose [cGy] } \\
Exposure Pathway & \multicolumn{2}{c}{$95 \%$ Subjective Confidence Interval } \\
\hline Backyard Cow Milk & lower limit & central estimate & upper limit \\
Commercial Milk (locally produced) & 0.11 & 0.79 & 5.4 \\
Commercial Milk (regionally mixed) & 0.032 & 0.25 & 2 \\
Goat Milk (locally produced) & 0.17 & 1.3 & 8 \\
Beef (locally produced) & 0.25 & 2.1 & 19 \\
Leafy Vegetables (locally produced) & 0.00016 & 0.0028 & 0.07 \\
Eggs (locally produced) & 0.00003 & 0.00037 & 0.0037 \\
Cottage Cheese (locally produced) & 0.005 & 0.045 & 0.38 \\
Inhalation & 0.00013 & 0.0019 & 0.023 \\
Mother's milk (mother on Diet 1) & 0.0019 & 0.0085 & 0.042 \\
Prenatal exposure (mother on Diet 1) & 0.000036 & 0.00079 & 0.016 \\
Diet 1 & 0.00033 & 0.0023 & 0.024 \\
Diet 2 & 0.12 & 0.86 & 5.7 \\
Diet 3 & 0.048 & 0.33 & 2.4 \\
\hline
\end{tabular}

Excess Lifetime Risk [ ]

\begin{tabular}{lllc}
\hline Diet 1 & $1.3 \mathrm{E}-05$ & $2.6 \mathrm{E}-04$ & $4.8 \mathrm{E}-03$ \\
Diet 2 & $7.6 \mathrm{E}-06$ & $9.5 \mathrm{E}-05$ & $1.4 \mathrm{E}-03$ \\
Diet 3 & $3.3 \mathrm{E}-05$ & $3.6 \mathrm{E}-04$ & $4.7 \mathrm{E}-03$ \\
Diet 4 & $3.4 \mathrm{E}-05$ & $6.2 \mathrm{E}-04$ & $1.1 \mathrm{E}-02$ \\
\hline
\end{tabular}

Relative Risk [ ]

\begin{tabular}{lccc}
\hline Diet 1 & 1.006 & 1.063 & 1.76 \\
Diet 2 & 1.0022 & 1.024 & 1.36 \\
Diet 3 & 1.0092 & 1.093 & 2.2 \\
Diet 4 & 1.014 & 1.16 & 3.7 \\
\hline
\end{tabular}

\begin{tabular}{lccc}
\hline Diet 1 & 0.60 & 5.9 & 43 \\
Diet 2 & 0.22 & 2.3 & 26 \\
Diet 3 & 0.91 & 8.5 & 55 \\
Diet 4 & 1.35 & 13.7 & 73 \\
\hline
\end{tabular}

Diet 1 - Backyard cow milk + all other locally produced non-milk exposure pathways

Diet 2 - Locally produced commercial milk + all other locally produced non-milk exposure pathways

Diet 3 - Regionally mixed commercial milk + inhalation (other regionally mixed food items are minor contributors to the total)

Diet 4 - Goat milk (all other exposure pathways are of negligible importance compared to goat milk) 


\section{Location: Wartburg}

Receptor: Male born in 1952

\begin{tabular}{lccc}
\hline & \multicolumn{3}{c}{ Thyroid Dose [cGy] } \\
Exposure Pathway & \multicolumn{2}{c}{$95 \%$ Subjective Confidence Interval } \\
\hline Backyard Cow Milk & lower limit & central estimate & upper limit \\
Commercial Milk (locally produced) & 0.11 & 0.75 & 5.2 \\
Commercial Milk (regionally mixed) & 0.035 & 0.25 & 1.9 \\
Goat Milk (locally produced) & 0.18 & 1.3 & 9 \\
Beef (locally produced) & 0.22 & 2.1 & 20 \\
Leafy Vegetables (locally produced) & 0.00016 & 0.0028 & 0.072 \\
Eggs (locally produced) & 0.00003 & 0.00035 & 0.004 \\
Cottage Cheese (locally produced) & 0.0053 & 0.047 & 0.36 \\
Inhalation & 0.00014 & 0.0021 & 0.021 \\
Mother's milk (mother on Diet 1) & 0.002 & 0.0086 & 0.037 \\
Prenatal exposure (mother on Diet 1) & 0.000036 & 0.00079 & 0.016 \\
Diet 1 & 0.00033 & 0.0023 & 0.024 \\
Diet 2 & 0.13 & 0.82 & 5.7 \\
Diet 3 & 0.05 & 0.32 & 2.5 \\
\hline
\end{tabular}

\section{Excess Lifetime Risk [ ]}

\begin{tabular}{llll}
\hline Diet 1 & $1.8 \mathrm{E}-06$ & $7.0 \mathrm{E}-05$ & $1.3 \mathrm{E}-03$ \\
Diet 2 & $9.4 \mathrm{E}-07$ & $2.5 \mathrm{E}-05$ & $5.3 \mathrm{E}-04$ \\
Diet 3 & $3.9 \mathrm{E}-06$ & $9.1 \mathrm{E}-05$ & $2.0 \mathrm{E}-03$ \\
Diet 4 & $5.0 \mathrm{E}-06$ & $1.6 \mathrm{E}-04$ & $4.2 \mathrm{E}-03$ \\
\hline
\end{tabular}

\section{Relative Risk [ ]}

\begin{tabular}{lccc}
\hline Diet 1 & 1.002 & 1.043 & 1.94 \\
Diet 2 & 1.0008 & 1.016 & 1.45 \\
Diet 3 & 1.0035 & 1.064 & 2.7 \\
Diet 4 & 1.0048 & 1.12 & 4.2 \\
\hline
\end{tabular}

\begin{tabular}{lccc}
\hline Diet 1 & 0.20 & 4.1 & 48 \\
Diet 2 & 0.08 & 1.6 & 31 \\
Diet 3 & 0.35 & 6.0 & 63 \\
Diet 4 & 0.48 & 10.6 & 76 \\
\hline
\end{tabular}

Diet 1 - Backyard cow milk + all other locally produced non-milk exposure pathways

Diet 2 - Locally produced commercial milk + all other locally produced non-milk exposure pathways

Diet 3 - Regionally mixed commercial milk + inhalation (other regionally mixed food items are minor contributors to the total)

Diet 4 - Goat milk (all other exposure pathways are of negligible importance compared to goat milk) 
Location: Rockwood

Receptor: Female born in 1952

\begin{tabular}{lccc}
\hline & \multicolumn{3}{c}{ Thyroid Dose [cGy] } \\
Exposure Pathway & \multicolumn{2}{c}{$95 \%$ Subjective Confidence Interval } \\
Backyard Cow Milk & lower limit & central estimate & upper limit \\
Commercial Milk (locally produced) & 0.5 & 3.2 & 22 \\
Commercial Milk (regionally mixed) & 0.15 & 1.1 & 7.4 \\
Goat Milk (locally produced) & 0.18 & 1.3 & 8 \\
Beef (locally produced) & 1.1 & 8.4 & 72 \\
Leafy Vegetables (locally produced) & 0.0007 & 0.012 & 0.28 \\
Eggs (locally produced) & 0.00013 & 0.0015 & 0.015 \\
Cottage Cheese (locally produced) & 0.024 & 0.19 & 1.5 \\
Inhalation & 0.00063 & 0.0083 & 0.087 \\
Mother's milk (mother on Diet 1) & 0.009 & 0.037 & 0.16 \\
Prenatal exposure (mother on Diet 1) & 0.00016 & 0.0033 & 0.054 \\
Diet 1 & 0.0015 & 0.0097 & 0.083 \\
Diet 2 & 0.57 & 3.5 & 24 \\
Diet 3 & 0.22 & 1.4 & 9 \\
\hline
\end{tabular}

Excess Lifetime Risk [ ]

\begin{tabular}{llll}
\hline Diet 1 & $6.6 \mathrm{E}-05$ & $1.1 \mathrm{E}-03$ & $1.7 \mathrm{E}-02$ \\
Diet 2 & $3.2 \mathrm{E}-05$ & $4.1 \mathrm{E}-04$ & $5.5 \mathrm{E}-03$ \\
Diet 3 & $3.4 \mathrm{E}-05$ & $3.7 \mathrm{E}-04$ & $4.7 \mathrm{E}-03$ \\
Diet 4 & $1.5 \mathrm{E}-04$ & $2.9 \mathrm{E}-03$ & $4.0 \mathrm{E}-02$ \\
\hline
\end{tabular}

\section{Relative Risk [ ]}

\begin{tabular}{lccc}
\hline Diet 1 & 1.028 & 1.26 & 3.7 \\
Diet 2 & 1.0099 & 1.1 & 2.2 \\
Diet 3 & 1.0099 & 1.096 & 2.2 \\
Diet 4 & 1.057 & 1.62 & 10.6 \\
\hline
\end{tabular}

\section{Probability of Causation [\%]}

\begin{tabular}{lccc}
\hline Diet 1 & 2.69 & 20.3 & 72 \\
Diet 2 & 0.98 & 9.1 & 53 \\
Diet 3 & 0.98 & 8.8 & 55 \\
Diet 4 & 5.36 & 37.3 & 90 \\
\hline
\end{tabular}

Diet 1 - Backyard cow milk + all other locally produced non-milk exposure pathways

Diet 2 - Locally produced commercial milk + all other locally produced non-milk exposure pathways

Diet 3 - Regionally mixed commercial milk + inhalation (other regionally mixed food items are minor contributors to the total)

Diet 4 - Goat milk (all other exposure pathways are of negligible importance compared to goat milk) 


\section{Location: Rockwood}

Receptor: Male born in 1952

\begin{tabular}{lccc}
\hline & \multicolumn{3}{c}{ Thyroid Dose [cGy] } \\
& \multicolumn{2}{c}{$95 \%$ Subjective Confidence Interval } \\
Exposure Pathway & lower limit & central estimate & upper limit \\
\hline Backyard Cow Milk & 0.5 & 3.1 & 20 \\
Commercial Milk (locally produced) & 0.15 & 1 & 8.1 \\
Commercial Milk (regionally mixed) & 0.18 & 1.3 & 9 \\
Goat Milk (locally produced) & 0.99 & 8.3 & 77 \\
Beef (locally produced) & 0.00068 & 0.012 & 0.28 \\
Leafy Vegetables (locally produced) & 0.00014 & 0.0015 & 0.015 \\
Eggs (locally produced) & 0.026 & 0.19 & 1.4 \\
Cottage Cheese (locally produced) & 0.00062 & 0.0084 & 0.091 \\
Inhalation & 0.0089 & 0.038 & 0.15 \\
Mother's milk (mother on Diet 1) & 0.00016 & 0.0033 & 0.054 \\
Prenatal exposure (mother on Diet 1) & 0.0015 & 0.0097 & 0.083 \\
Diet 1 & 0.56 & 3.4 & 22 \\
Diet 2 & 0.22 & 1.4 & 9.3 \\
Diet 3 & 0.19 & 1.3 & 9.1 \\
\hline
\end{tabular}

Excess Lifetime Risk [ ]

\begin{tabular}{lllc}
\hline Diet 1 & $9.4 \mathrm{E}-06$ & $2.8 \mathrm{E}-04$ & $5.1 \mathrm{E}-03$ \\
Diet 2 & $4.3 \mathrm{E}-06$ & $1.0 \mathrm{E}-04$ & $2.2 \mathrm{E}-03$ \\
Diet 3 & $4.1 \mathrm{E}-06$ & $9.4 \mathrm{E}-05$ & $2.0 \mathrm{E}-03$ \\
Diet 4 & $2.4 \mathrm{E}-05$ & $6.7 \mathrm{E}-04$ & $1.4 \mathrm{E}-02$ \\
\hline
\end{tabular}

\section{Relative Risk [ ]}

\begin{tabular}{lccc}
\hline Diet 1 & 1.0099 & 1.18 & 5.2 \\
Diet 2 & 1.0037 & 1.069 & 2.7 \\
Diet 3 & 1.0036 & 1.066 & 2.7 \\
Diet 4 & 1.025 & 1.51 & 13 \\
\hline
\end{tabular}

\section{Probability of Causation [\%]}

\begin{tabular}{lccc}
\hline Diet 1 & 0.98 & 15.2 & 80 \\
Diet 2 & 0.37 & 6.4 & 62 \\
Diet 3 & 0.36 & 6.2 & 63 \\
Diet 4 & 2.45 & 32.9 & 92 \\
\hline
\end{tabular}

Diet 1 - Backyard cow milk + all other locally produced non-milk exposure pathways

Diet 2 - Locally produced commercial milk + all other locally produced non-milk exposure pathways

Diet 3 - Regionally mixed commercial milk + inhalation (other regionally mixed food items are minor contributors to the total)

Diet 4 - Goat milk (all other exposure pathways are of negligible importance compared to goat milk) 


\section{Location: Louisville}

Receptor: Female born in 1952

\begin{tabular}{lccc}
\hline & \multicolumn{3}{c}{ Thyroid Dose [cGy] } \\
& \multicolumn{2}{c}{$95 \%$ Subjective Confidence Interval } \\
Exposure Pathway & lower limit & central estimate & upper limit \\
\hline Backyard Cow Milk & 0.46 & 3 & 18 \\
Commercial Milk (locally produced) & 0.13 & 0.96 & 6.8 \\
Commercial Milk (regionally mixed) & 0.18 & 1.3 & 8 \\
Goat Milk (locally produced) & 0.95 & 7.9 & 70 \\
Beef (locally produced) & 0.0006 & 0.011 & 0.24 \\
Leafy Vegetables (locally produced) & 0.00012 & 0.0014 & 0.013 \\
Eggs (locally produced) & 0.022 & 0.17 & 1.4 \\
Cottage Cheese (locally produced) & 0.00051 & 0.0075 & 0.077 \\
Inhalation & 0.0087 & 0.037 & 0.16 \\
Mother's milk (mother on Diet 1) & 0.00014 & 0.0031 & 0.059 \\
Prenatal exposure (mother on Diet 1) & 0.0015 & 0.0094 & 0.08 \\
Diet 1 & 0.52 & 3.3 & 20 \\
Diet 2 & 0.2 & 1.2 & 8.3 \\
Diet 3 & 0.2 & 1.3 & 8.1 \\
\hline
\end{tabular}

Excess Lifetime Risk [ ]

\begin{tabular}{llll}
\hline Diet 1 & $5.6 \mathrm{E}-05$ & $9.6 \mathrm{E}-04$ & $1.6 \mathrm{E}-02$ \\
Diet 2 & $3.1 \mathrm{E}-05$ & $3.7 \mathrm{E}-04$ & $5.3 \mathrm{E}-03$ \\
Diet 3 & $3.4 \mathrm{E}-05$ & $3.7 \mathrm{E}-04$ & $4.7 \mathrm{E}-03$ \\
Diet 4 & $1.6 \mathrm{E}-04$ & $2.5 \mathrm{E}-03$ & $4.2 \mathrm{E}-02$ \\
\hline
\end{tabular}

\section{Relative Risk [ ]}

\begin{tabular}{lccc}
\hline Diet 1 & 1.025 & 1.25 & 3.6 \\
Diet 2 & 1.0088 & 1.094 & 2.2 \\
Diet 3 & 1.0099 & 1.096 & 2.2 \\
Diet 4 & 1.056 & 1.6 & 9.8 \\
\hline
\end{tabular}

\begin{tabular}{lccc}
\hline Diet 1 & 2.47 & 19.8 & 72 \\
Diet 2 & 0.87 & 8.6 & 54 \\
Diet 3 & 0.98 & 8.8 & 55 \\
Diet 4 & 5.27 & 37.3 & 89 \\
\hline
\end{tabular}

Diet 1 - Backyard cow milk + all other locally produced non-milk exposure pathways

Diet 2 - Locally produced commercial milk + all other locally produced non-milk exposure pathways

Diet 3 - Regionally mixed commercial milk + inhalation (other regionally mixed food items are minor contributors to the total)

Diet 4 - Goat milk (all other exposure pathways are of negligible importance compared to goat milk) 


\section{Location: Louisville}

\section{Receptor: Male born in 1952}

\begin{tabular}{lccc}
\hline & \multicolumn{3}{c}{ Thyroid Dose [cGy] } \\
& \multicolumn{2}{c}{$95 \%$ Subjective Confidence Interval } \\
Exposure Pathway & lower limit & central estimate & upper limit \\
\hline Backyard Cow Milk & 0.47 & 3 & 17 \\
Commercial Milk (locally produced) & 0.14 & 0.97 & 6.4 \\
Commercial Milk (regionally mixed) & 0.18 & 1.3 & 9 \\
Goat Milk (locally produced) & 0.9 & 7.6 & 74 \\
Beef (locally produced) & 0.00058 & 0.01 & 0.24 \\
Leafy Vegetables (locally produced) & 0.00013 & 0.0014 & 0.014 \\
Eggs (locally produced) & 0.023 & 0.18 & 1.2 \\
Cottage Cheese (locally produced) & 0.00058 & 0.0079 & 0.08 \\
Inhalation & 0.009 & 0.038 & 0.16 \\
Mother's milk (mother on Diet 1) & 0.00014 & 0.0031 & 0.059 \\
Prenatal exposure (mother on Diet 1) & 0.0015 & 0.0094 & 0.08 \\
Diet 1 & 0.52 & 3.2 & 19 \\
Diet 2 & 0.2 & 1.3 & 7.9 \\
Diet 3 & 0.19 & 1.3 & 9.1 \\
\hline
\end{tabular}

Excess Lifetime Risk [ ]

\begin{tabular}{llll}
\hline Diet 1 & $7.8 \mathrm{E}-06$ & $2.6 \mathrm{E}-04$ & $4.4 \mathrm{E}-03$ \\
Diet 2 & $3.6 \mathrm{E}-06$ & $9.7 \mathrm{E}-05$ & $2.0 \mathrm{E}-03$ \\
Diet 3 & $4.1 \mathrm{E}-06$ & $9.4 \mathrm{E}-05$ & $2.0 \mathrm{E}-03$ \\
Diet 4 & $2.0 \mathrm{E}-05$ & $6.2 \mathrm{E}-04$ & $1.3 \mathrm{E}-02$ \\
\hline
\end{tabular}

\section{Relative Risk [ ]}

\begin{tabular}{lccc}
\hline Diet 1 & 1.0088 & 1.17 & 4.5 \\
Diet 2 & 1.0034 & 1.063 & 2.5 \\
Diet 3 & 1.0036 & 1.065 & 2.7 \\
Diet 4 & 1.023 & 1.46 & 12 \\
\hline
\end{tabular}

\begin{tabular}{llcc} 
& \multicolumn{3}{c}{ Probability of Causation [\%] } \\
\hline Diet 1 & 0.87 & 14.3 & 77 \\
Diet 2 & 0.34 & 5.9 & 60 \\
Diet 3 & 0.36 & 6.1 & 64 \\
Diet 4 & 2.27 & 31.4 & 91 \\
\hline
\end{tabular}

Diet 1 - Backyard cow milk + all other locally produced non-milk exposure pathways

Diet 2 - Locally produced commercial milk + all other locally produced non-milk exposure pathways

Diet 3 - Regionally mixed commercial milk + inhalation (other regionally mixed food items are minor contributors to the total)

Diet 4 - Goat milk (all other exposure pathways are of negligible importance compared to goat milk) 
Location: Barnardville

Receptor: Female born in 1952

\begin{tabular}{lccc}
\hline & \multicolumn{3}{c}{ Thyroid Dose [cGy] } \\
Exposure Pathway & \multicolumn{2}{c}{$95 \%$ Subjective Confidence Interval } \\
Backyard Cow Milk & lower limit & central estimate & upper limit \\
Commercial Milk (locally produced) & 0.52 & 3.3 & 22 \\
Commercial Milk (regionally mixed) & 0.16 & 1.1 & 8.1 \\
Goat Milk (locally produced) & 0.18 & 1.3 & 8 \\
Beef (locally produced) & 1.1 & 8.7 & 77 \\
Leafy Vegetables (locally produced) & 0.00073 & 0.012 & 0.28 \\
Eggs (locally produced) & 0.00013 & 0.0016 & 0.015 \\
Cottage Cheese (locally produced) & 0.025 & 0.2 & 1.6 \\
Inhalation & 0.00057 & 0.0087 & 0.096 \\
Mother's milk (mother on Diet 1) & 0.01 & 0.043 & 0.19 \\
Prenatal exposure (mother on Diet 1) & 0.00017 & 0.0036 & 0.064 \\
Diet 1 & 0.0017 & 0.011 & 0.087 \\
Diet 2 & 0.57 & 3.6 & 24 \\
Diet 3 & 0.23 & 1.4 & 9.7 \\
\hline
\end{tabular}

Excess Lifetime Risk [ ]

\begin{tabular}{lllc}
\hline Diet 1 & $6.7 \mathrm{E}-05$ & $1.1 \mathrm{E}-03$ & $2.1 \mathrm{E}-02$ \\
Diet 2 & $3.5 \mathrm{E}-05$ & $4.4 \mathrm{E}-04$ & $5.6 \mathrm{E}-03$ \\
Diet 3 & $3.4 \mathrm{E}-05$ & $3.7 \mathrm{E}-04$ & $4.7 \mathrm{E}-03$ \\
Diet 4 & $1.7 \mathrm{E}-04$ & $3.0 \mathrm{E}-03$ & $4.2 \mathrm{E}-02$ \\
\hline
\end{tabular}

\section{Relative Risk [ ]}

\begin{tabular}{lccc}
\hline Diet 1 & 1.028 & 1.27 & 3.8 \\
Diet 2 & 1.01 & 1.1 & 2.1 \\
Diet 3 & 1.01 & 1.096 & 2.2 \\
Diet 4 & 1.063 & 1.68 & 11 \\
\hline
\end{tabular}

\begin{tabular}{lccc} 
& \multicolumn{3}{c}{ Probability of Causation [\%] } \\
\hline Diet 1 & 2.75 & 21.5 & 74 \\
Diet 2 & 1.03 & 9.5 & 52 \\
Diet 3 & 1.00 & 8.8 & 55 \\
Diet 4 & 5.95 & 40.3 & 91 \\
\hline
\end{tabular}

Diet 1 - Backyard cow milk + all other locally produced non-milk exposure pathways

Diet 2 - Locally produced commercial milk + all other locally produced non-milk exposure pathways

Diet 3 - Regionally mixed commercial milk + inhalation (other regionally mixed food items are minor contributors to the total)

Diet 4 - Goat milk (all other exposure pathways are of negligible importance compared to goat milk) 


\section{Location: Barnardville}

Receptor: Male born in 1952

\begin{tabular}{lccc}
\hline & \multicolumn{3}{c}{ Thyroid Dose [cGy] } \\
& \multicolumn{2}{c}{$95 \%$ Subjective Confidence Interval } \\
Exposure Pathway & lower limit & central estimate & upper limit \\
\hline Backyard Cow Milk & 0.52 & 3.3 & 20 \\
Commercial Milk (locally produced) & 0.15 & 1.1 & 7.6 \\
Commercial Milk (regionally mixed) & 0.18 & 1.3 & 9 \\
Goat Milk (locally produced) & 1.1 & 8.8 & 81 \\
Beef (locally produced) & 0.00071 & 0.012 & 0.28 \\
Leafy Vegetables (locally produced) & 0.00014 & 0.0015 & 0.015 \\
Eggs (locally produced) & 0.027 & 0.2 & 1.4 \\
Cottage Cheese (locally produced) & 0.00064 & 0.0087 & 0.098 \\
Inhalation & 0.01 & 0.044 & 0.19 \\
Mother's milk (mother on Diet 1) & 0.00017 & 0.0036 & 0.064 \\
Prenatal exposure (mother on Diet 1) & 0.0017 & 0.011 & 0.087 \\
Diet 1 & 0.57 & 3.6 & 22 \\
Diet 2 & 0.23 & 1.5 & 9.2 \\
Diet 3 & 0.19 & 1.3 & 9.1 \\
\hline
\end{tabular}

Excess Lifetime Risk [ ]

\begin{tabular}{llll}
\hline Diet 1 & $9.7 \mathrm{E}-06$ & $3.0 \mathrm{E}-04$ & $5.0 \mathrm{E}-03$ \\
Diet 2 & $4.4 \mathrm{E}-06$ & $1.1 \mathrm{E}-04$ & $2.2 \mathrm{E}-03$ \\
Diet 3 & $4.1 \mathrm{E}-06$ & $9.5 \mathrm{E}-05$ & $2.0 \mathrm{E}-03$ \\
Diet 4 & $2.2 \mathrm{E}-05$ & $7.1 \mathrm{E}-04$ & $1.5 \mathrm{E}-02$ \\
\hline
\end{tabular}

\section{Relative Risk [ ]}

\begin{tabular}{lccc}
\hline Diet 1 & 1.011 & 1.19 & 5.5 \\
Diet 2 & 1.0039 & 1.074 & 2.8 \\
Diet 3 & 1.0036 & 1.066 & 2.7 \\
Diet 4 & 1.026 & 1.52 & 15 \\
\hline
\end{tabular}

\section{Probability of Causation [\%]}

\begin{tabular}{lccc}
\hline Diet 1 & 1.05 & 16.2 & 82 \\
Diet 2 & 0.39 & 6.9 & 64 \\
Diet 3 & 0.36 & 6.2 & 64 \\
Diet 4 & 2.52 & 34.4 & 93 \\
\hline
\end{tabular}

Diet 1 - Backyard cow milk + all other locally produced non-milk exposure pathways

Diet 2 - Locally produced commercial milk + all other locally produced non-milk exposure pathways

Diet 3 - Regionally mixed commercial milk + inhalation (other regionally mixed food items are minor contributors to the total)

Diet 4 - Goat milk (all other exposure pathways are of negligible importance compared to goat milk) 
Location: Greenback

Receptor: Female born in 1952

\begin{tabular}{lccc}
\hline & \multicolumn{3}{c}{ Thyroid Dose [cGy] } \\
Exposure Pathway & $95 \%$ Subjective Confidence Interval \\
\hline Backyard Cow Milk & lower limit & central estimate & upper limit \\
Commercial Milk (locally produced) & 0.29 & 1.8 & 14 \\
Commercial Milk (regionally mixed) & 0.079 & 0.58 & 4.5 \\
Goat Milk (locally produced) & 0.17 & 1.3 & 8 \\
Beef (locally produced) & 0.61 & 5 & 44 \\
Leafy Vegetables (locally produced) & 0.00038 & 0.007 & 0.17 \\
Eggs (locally produced) & 0.000079 & 0.00084 & 0.0085 \\
Cottage Cheese (locally produced) & 0.014 & 0.1 & 0.83 \\
Inhalation & 0.00035 & 0.0047 & 0.057 \\
Mother's milk (mother on Diet 1) & 0.006 & 0.025 & 0.11 \\
Prenatal exposure (mother on Diet 1) & 0.00011 & 0.002 & 0.036 \\
Diet 1 & 0.00092 & 0.0061 & 0.054 \\
Diet 2 & 0.32 & 2 & 15 \\
Diet 3 & 0.12 & 0.78 & 5.6 \\
\hline
\end{tabular}

Excess Lifetime Risk [ ]

\begin{tabular}{llll}
\hline Diet 1 & $3.8 \mathrm{E}-05$ & $6.1 \mathrm{E}-04$ & $1.0 \mathrm{E}-02$ \\
Diet 2 & $1.9 \mathrm{E}-05$ & $2.3 \mathrm{E}-04$ & $3.2 \mathrm{E}-03$ \\
Diet 3 & $3.3 \mathrm{E}-05$ & $3.7 \mathrm{E}-04$ & $4.7 \mathrm{E}-03$ \\
Diet 4 & $9.9 \mathrm{E}-05$ & $1.5 \mathrm{E}-03$ & $3.0 \mathrm{E}-02$ \\
\hline
\end{tabular}

\section{Relative Risk [ ]}

\begin{tabular}{lccc}
\hline Diet 1 & 1.016 & 1.15 & 2.9 \\
Diet 2 & 1.0058 & 1.059 & 1.74 \\
Diet 3 & 1.0095 & 1.095 & 2.2 \\
Diet 4 & 1.032 & 1.35 & 7.7 \\
\hline
\end{tabular}

\section{Probability of Causation [\%]}

\begin{tabular}{lccc}
\hline Diet 1 & 1.58 & 12.8 & 65 \\
Diet 2 & 0.58 & 5.6 & 42 \\
Diet 3 & 0.94 & 8.7 & 55 \\
Diet 4 & 3.12 & 25.7 & 87 \\
\hline
\end{tabular}

Diet 1 - Backyard cow milk + all other locally produced non-milk exposure pathways

Diet 2 - Locally produced commercial milk + all other locally produced non-milk exposure pathways

Diet 3 - Regionally mixed commercial milk + inhalation (other regionally mixed food items are minor contributors to the total)

Diet 4 - Goat milk (all other exposure pathways are of negligible importance compared to goat milk) 
Location: Greenback

Receptor: Male born in 1952

\begin{tabular}{lccc}
\hline & \multicolumn{3}{c}{ Thyroid Dose [cGy] } \\
Exposure Pathway & 95\% Subjective Confidence Interval \\
\hline Backyard Cow Milk & lower limit & central estimate & upper limit \\
Commercial Milk (locally produced) & 0.3 & 1.8 & 13 \\
Commercial Milk (regionally mixed) & 0.081 & 0.58 & 4.2 \\
Goat Milk (locally produced) & 0.18 & 1.3 & 9 \\
Beef (locally produced) & 0.55 & 4.7 & 49 \\
Leafy Vegetables (locally produced) & 0.00038 & 0.0069 & 0.16 \\
Eggs (locally produced) & 0.000081 & 0.00081 & 0.0093 \\
Cottage Cheese (locally produced) & 0.015 & 0.11 & 0.87 \\
Inhalation & 0.00037 & 0.0045 & 0.059 \\
Mother's milk (mother on Diet 1) & 0.0062 & 0.025 & 0.11 \\
Prenatal exposure (mother on Diet 1) & 0.00011 & 0.002 & 0.036 \\
Diet 1 & 0.00092 & 0.0061 & 0.054 \\
Diet 2 & 0.35 & 2 & 14 \\
Diet 3 & 0.12 & 0.78 & 5.4 \\
\hline
\end{tabular}

Excess Lifetime Risk [ ]

\begin{tabular}{lllc}
\hline Diet 1 & $5.0 \mathrm{E}-06$ & $1.5 \mathrm{E}-04$ & $3.0 \mathrm{E}-03$ \\
Diet 2 & $2.3 \mathrm{E}-06$ & $5.8 \mathrm{E}-05$ & $1.2 \mathrm{E}-03$ \\
Diet 3 & $4.0 \mathrm{E}-06$ & $9.2 \mathrm{E}-05$ & $2.0 \mathrm{E}-03$ \\
Diet 4 & $1.4 \mathrm{E}-05$ & $4.0 \mathrm{E}-04$ & $8.5 \mathrm{E}-03$ \\
\hline
\end{tabular}

\section{Relative Risk [ ]}

\begin{tabular}{lccc}
\hline Diet 1 & 1.0055 & 1.11 & 3.5 \\
Diet 2 & 1.002 & 1.04 & 1.99 \\
Diet 3 & 1.0036 & 1.065 & 2.7 \\
Diet 4 & 1.013 & 1.28 & 7.2 \\
\hline
\end{tabular}

\section{Probability of Causation [\%]}

\begin{tabular}{lccc}
\hline Diet 1 & 0.54 & 9.8 & 71 \\
Diet 2 & 0.20 & 3.8 & 50 \\
Diet 3 & 0.36 & 6.1 & 63 \\
Diet 4 & 1.25 & 22.1 & 86 \\
\hline
\end{tabular}

Diet 1 - Backyard cow milk + all other locally produced non-milk exposure pathways

Diet 2 - Locally produced commercial milk + all other locally produced non-milk exposure pathways

Diet 3 - Regionally mixed commercial milk + inhalation (other regionally mixed food items are minor contributors to the total)

Diet 4 - Goat milk (all other exposure pathways are of negligible importance compared to goat milk) 


\section{Location: Rockford}

Receptor: Female born in 1952

\begin{tabular}{lccc}
\hline & \multicolumn{3}{c}{ Thyroid Dose [cGy] } \\
Exposure Pathway & \multicolumn{2}{c}{$95 \%$ Subjective Confidence Interval } \\
\hline Backyard Cow Milk & lower limit & central estimate & upper limit \\
Commercial Milk (locally produced) & 0.46 & 2.9 & 19 \\
Commercial Milk (regionally mixed) & 0.13 & 0.93 & 6.5 \\
Goat Milk (locally produced) & 0.18 & 1.3 & 8 \\
Beef (locally produced) & 0.93 & 7.5 & 65 \\
Leafy Vegetables (locally produced) & 0.00059 & 0.011 & 0.23 \\
Eggs (locally produced) & 0.00011 & 0.0014 & 0.013 \\
Cottage Cheese (locally produced) & 0.022 & 0.16 & 1.2 \\
Inhalation & 0.00052 & 0.0073 & 0.081 \\
Mother's milk (mother on Diet 1) & 0.0094 & 0.037 & 0.16 \\
Prenatal exposure (mother on Diet 1) & 0.00014 & 0.0031 & 0.054 \\
Diet 1 & 0.0014 & 0.0093 & 0.075 \\
Diet 2 & 0.52 & 3.2 & 21 \\
Diet 3 & 0.2 & 1.2 & 8.3 \\
\hline
\end{tabular}

Excess Lifetime Risk [ ]

\begin{tabular}{llll}
\hline Diet 1 & $5.9 \mathrm{E}-05$ & $9.6 \mathrm{E}-04$ & $1.4 \mathrm{E}-02$ \\
Diet 2 & $3.0 \mathrm{E}-05$ & $3.7 \mathrm{E}-04$ & $4.8 \mathrm{E}-03$ \\
Diet 3 & $3.4 \mathrm{E}-05$ & $3.7 \mathrm{E}-04$ & $4.7 \mathrm{E}-03$ \\
Diet 4 & $1.5 \mathrm{E}-04$ & $2.5 \mathrm{E}-03$ & $3.9 \mathrm{E}-02$ \\
\hline
\end{tabular}

\section{Relative Risk [ ]}

\begin{tabular}{lccc}
\hline Diet 1 & 1.024 & 1.24 & 3.7 \\
Diet 2 & 1.009 & 1.092 & 2.1 \\
Diet 3 & 1.0098 & 1.096 & 2.2 \\
Diet 4 & 1.054 & 1.56 & 10 \\
\hline
\end{tabular}

\section{Probability of Causation [\%]}

\begin{tabular}{lccc}
\hline Diet 1 & 2.36 & 19.2 & 72 \\
Diet 2 & 0.89 & 8.4 & 51 \\
Diet 3 & 0.97 & 8.8 & 55 \\
Diet 4 & 5.09 & 35.5 & 90 \\
\hline
\end{tabular}

Diet 1 - Backyard cow milk + all other locally produced non-milk exposure pathways

Diet 2 - Locally produced commercial milk + all other locally produced non-milk exposure pathways

Diet 3 - Regionally mixed commercial milk + inhalation (other regionally mixed food items are minor contributors to the total)

Diet 4 - Goat milk (all other exposure pathways are of negligible importance compared to goat milk) 


\section{Location: Rockford}

Receptor: Male born in 1952

\begin{tabular}{lccc}
\hline & \multicolumn{3}{c}{ Thyroid Dose [cGy] } \\
& \multicolumn{2}{c}{$95 \%$ Subjective Confidence Interval } \\
Exposure Pathway & lower limit & central estimate & upper limit \\
\hline Backyard Cow Milk & 0.47 & 2.8 & 18 \\
Commercial Milk (locally produced) & 0.14 & 0.95 & 6.5 \\
Commercial Milk (regionally mixed) & 0.18 & 1.3 & 9 \\
Goat Milk (locally produced) & 0.87 & 7.6 & 68 \\
Beef (locally produced) & 0.0006 & 0.011 & 0.24 \\
Leafy Vegetables (locally produced) & 0.00011 & 0.0013 & 0.013 \\
Eggs (locally produced) & 0.024 & 0.17 & 1.2 \\
Cottage Cheese (locally produced) & 0.00057 & 0.0073 & 0.088 \\
Inhalation & 0.0098 & 0.037 & 0.15 \\
Mother's milk (mother on Diet 1) & 0.00014 & 0.0031 & 0.054 \\
Prenatal exposure (mother on Diet 1) & 0.0014 & 0.0093 & 0.075 \\
Diet 1 & 0.53 & 3.1 & 20 \\
Diet 2 & 0.21 & 1.2 & 8 \\
Diet 3 & 0.2 & 1.3 & 9.1 \\
\hline
\end{tabular}

Excess Lifetime Risk [ ]

\begin{tabular}{llll}
\hline Diet 1 & $8.1 \mathrm{E}-06$ & $2.5 \mathrm{E}-04$ & $4.8 \mathrm{E}-03$ \\
Diet 2 & $4.1 \mathrm{E}-06$ & $9.4 \mathrm{E}-05$ & $2.0 \mathrm{E}-03$ \\
Diet 3 & $4.1 \mathrm{E}-06$ & $9.4 \mathrm{E}-05$ & $2.0 \mathrm{E}-03$ \\
Diet 4 & $2.3 \mathrm{E}-05$ & $6.2 \mathrm{E}-04$ & $1.2 \mathrm{E}-02$ \\
\hline
\end{tabular}

\section{Relative Risk [ ]}

\begin{tabular}{lccc}
\hline Diet 1 & 1.0091 & 1.17 & 4.6 \\
Diet 2 & 1.0032 & 1.063 & 2.5 \\
Diet 3 & 1.0037 & 1.066 & 2.7 \\
Diet 4 & 1.022 & 1.45 & 10.3 \\
\hline
\end{tabular}

\begin{tabular}{lccc} 
& \multicolumn{3}{c}{ Probability of Causation [\%] } \\
\hline Diet 1 & 0.90 & 14.3 & 78 \\
Diet 2 & 0.32 & 5.9 & 59 \\
Diet 3 & 0.37 & 6.2 & 63 \\
Diet 4 & 2.13 & 31.0 & 90 \\
\hline
\end{tabular}

Diet 1 - Backyard cow milk + all other locally produced non-milk exposure pathways

Diet 2 - Locally produced commercial milk + all other locally produced non-milk exposure pathways

Diet 3 - Regionally mixed commercial milk + inhalation (other regionally mixed food items are minor contributors to the total)

Diet 4 - Goat milk (all other exposure pathways are of negligible importance compared to goat milk) 
Location: Lake City

Receptor: Female born in 1952

\begin{tabular}{lccc}
\hline & \multicolumn{3}{c}{ Thyroid Dose [cGy] } \\
& \multicolumn{2}{c}{$95 \%$ Subjective Confidence Interval } \\
Exposure Pathway & lower limit & central estimate & upper limit \\
\hline Backyard Cow Milk & 0.29 & 1.8 & 11 \\
Commercial Milk (locally produced) & 0.086 & 0.58 & 4.1 \\
Commercial Milk (regionally mixed) & 0.18 & 1.3 & 8 \\
Goat Milk (locally produced) & 0.63 & 4.7 & 40 \\
Beef (locally produced) & 0.00036 & 0.0066 & 0.16 \\
Leafy Vegetables (locally produced) & 0.000079 & 0.00084 & 0.0078 \\
Eggs (locally produced) & 0.013 & 0.1 & 0.83 \\
Cottage Cheese (locally produced) & 0.00031 & 0.0043 & 0.048 \\
Inhalation & 0.0047 & 0.02 & 0.089 \\
Mother's milk (mother on Diet 1) & 0.000083 & 0.0019 & 0.035 \\
Prenatal exposure (mother on Diet 1) & 0.00084 & 0.0055 & 0.049 \\
Diet 1 & 0.34 & 1.9 & 12 \\
Diet 2 & 0.13 & 0.75 & 5 \\
Diet 3 & 0.19 & 1.3 & 8 \\
\hline
\end{tabular}

Excess Lifetime Risk [ ]

\begin{tabular}{llll}
\hline Diet 1 & $3.5 \mathrm{E}-05$ & $5.9 \mathrm{E}-04$ & $8.7 \mathrm{E}-03$ \\
Diet 2 & $1.9 \mathrm{E}-05$ & $2.2 \mathrm{E}-04$ & $3.0 \mathrm{E}-03$ \\
Diet 3 & $3.3 \mathrm{E}-05$ & $3.7 \mathrm{E}-04$ & $4.7 \mathrm{E}-03$ \\
Diet 4 & $8.8 \mathrm{E}-05$ & $1.6 \mathrm{E}-03$ & $2.4 \mathrm{E}-02$ \\
\hline
\end{tabular}

\section{Relative Risk [ ]}

\begin{tabular}{lccc}
\hline Diet 1 & 1.015 & 1.15 & 2.6 \\
Diet 2 & 1.0056 & 1.055 & 1.74 \\
Diet 3 & 1.0095 & 1.094 & 2.2 \\
Diet 4 & 1.032 & 1.35 & 6.5 \\
\hline
\end{tabular}

\section{Probability of Causation [\%]}

\begin{tabular}{lccc}
\hline Diet 1 & 1.44 & 12.7 & 61 \\
Diet 2 & 0.56 & 5.2 & 42 \\
Diet 3 & 0.94 & 8.6 & 55 \\
Diet 4 & 3.13 & 25.8 & 84 \\
\hline
\end{tabular}

Diet 1 - Backyard cow milk + all other locally produced non-milk exposure pathways

Diet 2 - Locally produced commercial milk + all other locally produced non-milk exposure pathways

Diet 3 - Regionally mixed commercial milk + inhalation (other regionally mixed food items are minor contributors to the total)

Diet 4 - Goat milk (all other exposure pathways are of negligible importance compared to goat milk) 
Location: Lake City

Receptor: Male born in 1952

\begin{tabular}{lccc}
\hline & \multicolumn{3}{c}{ Thyroid Dose [cGy] } \\
Exposure Pathway & 95\% Subjective Confidence Interval \\
\hline Backyard Cow Milk & lower limit & central estimate & upper limit \\
Commercial Milk (locally produced) & 0.3 & 1.7 & 10 \\
Commercial Milk (regionally mixed) & 0.086 & 0.57 & 4.2 \\
Goat Milk (locally produced) & 0.18 & 1.3 & 9 \\
Beef (locally produced) & 0.58 & 4.6 & 42 \\
Leafy Vegetables (locally produced) & 0.00036 & 0.0065 & 0.15 \\
Eggs (locally produced) & 0.00008 & 0.00082 & 0.0079 \\
Cottage Cheese (locally produced) & 0.014 & 0.11 & 0.75 \\
Inhalation & 0.00038 & 0.0047 & 0.049 \\
Mother's milk (mother on Diet 1) & 0.0051 & 0.021 & 0.083 \\
Prenatal exposure (mother on Diet 1) & 0.000083 & 0.0019 & 0.035 \\
Diet 1 & 0.00084 & 0.0055 & 0.049 \\
Diet 2 & 0.34 & 1.9 & 11 \\
Diet 3 & 0.13 & 0.74 & 5.1 \\
\hline
\end{tabular}

Excess Lifetime Risk [ ]

\begin{tabular}{llll}
\hline Diet 1 & $4.6 \mathrm{E}-06$ & $1.5 \mathrm{E}-04$ & $2.7 \mathrm{E}-03$ \\
Diet 2 & $2.6 \mathrm{E}-06$ & $5.9 \mathrm{E}-05$ & $1.3 \mathrm{E}-03$ \\
Diet 3 & $4.0 \mathrm{E}-06$ & $9.2 \mathrm{E}-05$ & $2.0 \mathrm{E}-03$ \\
Diet 4 & $1.3 \mathrm{E}-05$ & $3.7 \mathrm{E}-04$ & $8.2 \mathrm{E}-03$ \\
\hline
\end{tabular}

\section{Relative Risk [ ]}

\begin{tabular}{lccc}
\hline Diet 1 & 1.0054 & 1.1 & 3.1 \\
Diet 2 & 1.0021 & 1.039 & 1.93 \\
Diet 3 & 1.0036 & 1.065 & 2.7 \\
Diet 4 & 1.013 & 1.27 & 7.5 \\
\hline
\end{tabular}

\section{Probability of Causation [\%]}

\begin{tabular}{lccc}
\hline Diet 1 & 0.54 & 9.1 & 67 \\
Diet 2 & 0.21 & 3.8 & 48 \\
Diet 3 & 0.35 & 6.1 & 63 \\
Diet 4 & 1.29 & 21.4 & 86 \\
\hline
\end{tabular}

Diet 1 - Backyard cow milk + all other locally produced non-milk exposure pathways

Diet 2 - Locally produced commercial milk + all other locally produced non-milk exposure pathways

Diet 3 - Regionally mixed commercial milk + inhalation (other regionally mixed food items are minor contributors to the total)

Diet 4 - Goat milk (all other exposure pathways are of negligible importance compared to goat milk) 
Location: Sweetwater

Receptor: Female born in 1952

\begin{tabular}{lccc}
\hline & \multicolumn{3}{c}{ Thyroid Dose [cGy] } \\
Exposure Pathway & $95 \%$ Subjective Confidence Interval \\
\hline Backyard Cow Milk & lower limit & central estimate & upper limit \\
Commercial Milk (locally produced) & 0.33 & 2.2 & 13 \\
Commercial Milk (regionally mixed) & 0.092 & 0.72 & 5.1 \\
Goat Milk (locally produced) & 0.18 & 1.3 & 8 \\
Beef (locally produced) & 0.64 & 5.7 & 55 \\
Leafy Vegetables (locally produced) & 0.00042 & 0.008 & 0.17 \\
Eggs (locally produced) & 0.000093 & 0.001 & 0.0098 \\
Cottage Cheese (locally produced) & 0.016 & 0.13 & 0.97 \\
Inhalation & 0.0004 & 0.0057 & 0.056 \\
Mother's milk (mother on Diet 1) & 0.0066 & 0.029 & 0.13 \\
Prenatal exposure (mother on Diet 1) & 0.000097 & 0.0023 & 0.049 \\
Diet 1 & 0.0011 & 0.0072 & 0.061 \\
Diet 2 & 0.37 & 2.4 & 14 \\
Diet 3 & 0.13 & 0.94 & 6.1 \\
\hline
\end{tabular}

Excess Lifetime Risk [ ]

\begin{tabular}{llll}
\hline Diet 1 & $4.2 \mathrm{E}-05$ & $7.0 \mathrm{E}-04$ & $1.2 \mathrm{E}-02$ \\
Diet 2 & $2.1 \mathrm{E}-05$ & $2.8 \mathrm{E}-04$ & $3.9 \mathrm{E}-03$ \\
Diet 3 & $3.3 \mathrm{E}-05$ & $3.7 \mathrm{E}-04$ & $4.7 \mathrm{E}-03$ \\
Diet 4 & $1.2 \mathrm{E}-04$ & $1.9 \mathrm{E}-03$ & $3.2 \mathrm{E}-02$ \\
\hline
\end{tabular}

\section{Relative Risk [ ]}

\begin{tabular}{lccc}
\hline Diet 1 & 1.02 & 1.18 & 2.9 \\
Diet 2 & 1.0066 & 1.071 & 1.86 \\
Diet 3 & 1.0097 & 1.096 & 2.2 \\
Diet 4 & 1.042 & 1.45 & 8 \\
\hline
\end{tabular}

\begin{tabular}{lccc}
\hline Diet 1 & 1.92 & 15.3 & 65 \\
Diet 2 & 0.66 & 6.6 & 46 \\
Diet 3 & 0.96 & 8.7 & 55 \\
Diet 4 & 4.01 & 31.0 & 87 \\
\hline
\end{tabular}

Diet 1 - Backyard cow milk + all other locally produced non-milk exposure pathways

Diet 2 - Locally produced commercial milk + all other locally produced non-milk exposure pathways

Diet 3 - Regionally mixed commercial milk + inhalation (other regionally mixed food items are minor contributors to the total)

Diet 4 - Goat milk (all other exposure pathways are of negligible importance compared to goat milk) 
Location: Sweetwater

Receptor: Male born in 1952

\begin{tabular}{lccc}
\hline & \multicolumn{3}{c}{ Thyroid Dose [cGy] } \\
Exposure Pathway & 95\% Subjective Confidence Interval \\
\hline Backyard Cow Milk & lower limit & central estimate & upper limit \\
Commercial Milk (locally produced) & 0.31 & 2.2 & 13 \\
Commercial Milk (regionally mixed) & 0.098 & 0.72 & 4.4 \\
Goat Milk (locally produced) & 0.18 & 1.3 & 9 \\
Beef (locally produced) & 0.61 & 5.6 & 57 \\
Leafy Vegetables (locally produced) & 0.00043 & 0.0077 & 0.17 \\
Eggs (locally produced) & 0.000091 & 0.001 & 0.0099 \\
Cottage Cheese (locally produced) & 0.018 & 0.13 & 0.91 \\
Inhalation & 0.00044 & 0.0058 & 0.057 \\
Mother's milk (mother on Diet 1) & 0.0068 & 0.03 & 0.13 \\
Prenatal exposure (mother on Diet 1) & 0.000097 & 0.0023 & 0.049 \\
Diet 1 & 0.0011 & 0.0072 & 0.061 \\
Diet 2 & 0.36 & 2.4 & 13 \\
Diet 3 & 0.14 & 0.94 & 5.5 \\
\hline
\end{tabular}

Excess Lifetime Risk [ ]

\begin{tabular}{llll}
\hline Diet 1 & $6.0 \mathrm{E}-06$ & $1.9 \mathrm{E}-04$ & $3.3 \mathrm{E}-03$ \\
Diet 2 & $2.5 \mathrm{E}-06$ & $7.0 \mathrm{E}-05$ & $1.5 \mathrm{E}-03$ \\
Diet 3 & $4.0 \mathrm{E}-06$ & $9.3 \mathrm{E}-05$ & $2.0 \mathrm{E}-03$ \\
Diet 4 & $1.4 \mathrm{E}-05$ & $4.4 \mathrm{E}-04$ & $9.4 \mathrm{E}-03$ \\
\hline
\end{tabular}

\section{Relative Risk [ ]}

\begin{tabular}{lccc}
\hline Diet 1 & 1.0067 & 1.13 & 3.4 \\
Diet 2 & 1.0025 & 1.048 & 2 \\
Diet 3 & 1.0036 & 1.065 & 2.7 \\
Diet 4 & 1.018 & 1.34 & 8.6 \\
\hline
\end{tabular}

\section{Probability of Causation [\%]}

\begin{tabular}{lccc}
\hline Diet 1 & 0.66 & 11.2 & 71 \\
Diet 2 & 0.25 & 4.6 & 50 \\
Diet 3 & 0.36 & 6.1 & 63 \\
Diet 4 & 1.75 & 25.5 & 88 \\
\hline
\end{tabular}

Diet 1 - Backyard cow milk + all other locally produced non-milk exposure pathways

Diet 2 - Locally produced commercial milk + all other locally produced non-milk exposure pathways

Diet 3 - Regionally mixed commercial milk + inhalation (other regionally mixed food items are minor contributors to the total)

Diet 4 - Goat milk (all other exposure pathways are of negligible importance compared to goat milk) 


\section{Location: Knoxville}

Receptor: Female born in 1952

\begin{tabular}{lccc}
\hline & \multicolumn{3}{c}{ Thyroid Dose [cGy] } \\
Exposure Pathway & \multicolumn{2}{c}{$95 \%$ Subjective Confidence Interval } \\
\hline Backyard Cow Milk & lower limit & central estimate & upper limit \\
Commercial Milk (locally produced) & 0.56 & 3.6 & 24 \\
Commercial Milk (regionally mixed) & 0.17 & 1.2 & 8.2 \\
Goat Milk (locally produced) & 0.17 & 1.3 & 8 \\
Beef (locally produced) & 1.2 & 9.5 & 80 \\
Leafy Vegetables (locally produced) & 0.00074 & 0.013 & 0.3 \\
Eggs (locally produced) & 0.00015 & 0.0017 & 0.017 \\
Cottage Cheese (locally produced) & 0.027 & 0.21 & 1.6 \\
Inhalation & 0.00066 & 0.0089 & 0.099 \\
Mother's milk (mother on Diet 1) & 0.012 & 0.047 & 0.2 \\
Prenatal exposure (mother on Diet 1) & 0.00017 & 0.0039 & 0.066 \\
Diet 1 & 0.0018 & 0.012 & 0.093 \\
Diet 2 & 0.64 & 3.9 & 27 \\
Diet 3 & 0.25 & 1.6 & 10 \\
\hline
\end{tabular}

Excess Lifetime Risk [ ]

\begin{tabular}{llll}
\hline Diet 1 & $7.3 \mathrm{E}-05$ & $1.2 \mathrm{E}-03$ & $1.8 \mathrm{E}-02$ \\
Diet 2 & $3.5 \mathrm{E}-05$ & $4.7 \mathrm{E}-04$ & $6.3 \mathrm{E}-03$ \\
Diet 3 & $3.4 \mathrm{E}-05$ & $3.7 \mathrm{E}-04$ & $4.7 \mathrm{E}-03$ \\
Diet 4 & $1.8 \mathrm{E}-04$ & $3.3 \mathrm{E}-03$ & $4.7 \mathrm{E}-02$ \\
\hline
\end{tabular}

\section{Relative Risk [ ]}

\begin{tabular}{lccc}
\hline Diet 1 & 1.033 & 1.3 & 4.2 \\
Diet 2 & 1.012 & 1.11 & 2.3 \\
Diet 3 & 1.01 & 1.097 & 2.2 \\
Diet 4 & 1.067 & 1.71 & 12 \\
\hline
\end{tabular}

\section{Probability of Causation [\%]}

\begin{tabular}{lccc}
\hline Diet 1 & 3.17 & 23.0 & 76 \\
Diet 2 & 1.19 & 10.3 & 57 \\
Diet 3 & 0.99 & 8.8 & 55 \\
Diet 4 & 6.25 & 41.3 & 92 \\
\hline
\end{tabular}

Diet 1 - Backyard cow milk + all other locally produced non-milk exposure pathways

Diet 2 - Locally produced commercial milk + all other locally produced non-milk exposure pathways

Diet 3 - Regionally mixed commercial milk + inhalation (other regionally mixed food items are minor contributors to the total)

Diet 4 - Goat milk (all other exposure pathways are of negligible importance compared to goat milk) 


\section{Location: Knoxville}

\section{Receptor: Male born in 1952}

\begin{tabular}{lccc}
\hline & \multicolumn{3}{c}{ Thyroid Dose [cGy] } \\
& \multicolumn{2}{c}{$95 \%$ Subjective Confidence Interval } \\
Exposure Pathway & lower limit & central estimate & upper limit \\
\hline Backyard Cow Milk & 0.57 & 3.6 & 21 \\
Commercial Milk (locally produced) & 0.17 & 1.2 & 8.6 \\
Commercial Milk (regionally mixed) & 0.18 & 1.3 & 9 \\
Goat Milk (locally produced) & 1.2 & 9.5 & 86 \\
Beef (locally produced) & 0.00078 & 0.013 & 0.3 \\
Leafy Vegetables (locally produced) & 0.00016 & 0.0017 & 0.017 \\
Eggs (locally produced) & 0.031 & 0.22 & 1.5 \\
Cottage Cheese (locally produced) & 0.00072 & 0.0094 & 0.11 \\
Inhalation & 0.012 & 0.047 & 0.19 \\
Mother's milk (mother on Diet 1) & 0.00017 & 0.0039 & 0.066 \\
Prenatal exposure (mother on Diet 1) & 0.0018 & 0.012 & 0.093 \\
Diet 1 & 0.63 & 3.9 & 24 \\
Diet 2 & 0.25 & 1.6 & 10 \\
Diet 3 & 0.2 & 1.3 & 9.1 \\
\hline
\end{tabular}

Excess Lifetime Risk [ ]

\begin{tabular}{lllc}
\hline Diet 1 & $9.7 \mathrm{E}-06$ & $3.2 \mathrm{E}-04$ & $5.6 \mathrm{E}-03$ \\
Diet 2 & $4.9 \mathrm{E}-06$ & $1.2 \mathrm{E}-04$ & $2.5 \mathrm{E}-03$ \\
Diet 3 & $4.1 \mathrm{E}-06$ & $9.5 \mathrm{E}-05$ & $2.0 \mathrm{E}-03$ \\
Diet 4 & $2.8 \mathrm{E}-05$ & $8.1 \mathrm{E}-04$ & $1.5 \mathrm{E}-02$ \\
\hline
\end{tabular}

\section{Relative Risk [ ]}

\begin{tabular}{lccc}
\hline Diet 1 & 1.013 & 1.21 & 5.5 \\
Diet 2 & 1.0042 & 1.08 & 2.9 \\
Diet 3 & 1.0037 & 1.066 & 2.8 \\
Diet 4 & 1.029 & 1.57 & 13 \\
\hline
\end{tabular}

\section{Probability of Causation [\%]}

\begin{tabular}{lccc}
\hline Diet 1 & 1.27 & 17.6 & 81 \\
Diet 2 & 0.42 & 7.4 & 66 \\
Diet 3 & 0.37 & 6.2 & 64 \\
Diet 4 & 2.78 & 36.3 & 92 \\
\hline
\end{tabular}

Diet 1 - Backyard cow milk + all other locally produced non-milk exposure pathways

Diet 2 - Locally produced commercial milk + all other locally produced non-milk exposure pathways

Diet 3 - Regionally mixed commercial milk + inhalation (other regionally mixed food items are minor contributors to the total)

Diet 4 - Goat milk (all other exposure pathways are of negligible importance compared to goat milk) 
Location: Maryville

Receptor: Female born in 1952

\begin{tabular}{lccc}
\hline & \multicolumn{3}{c}{ Thyroid Dose [cGy] } \\
Exposure Pathway & $95 \%$ Subjective Confidence Interval \\
\hline Backyard Cow Milk & lower limit & central estimate & upper limit \\
Commercial Milk (locally produced) & 0.28 & 1.8 & 12 \\
Commercial Milk (regionally mixed) & 0.075 & 0.58 & 4.5 \\
Goat Milk (locally produced) & 0.17 & 1.2 & 8 \\
Beef (locally produced) & 0.55 & 4.7 & 42 \\
Leafy Vegetables (locally produced) & 0.00039 & 0.0067 & 0.15 \\
Eggs (locally produced) & 0.000068 & 0.00083 & 0.0074 \\
Cottage Cheese (locally produced) & 0.013 & 0.1 & 0.87 \\
Inhalation & 0.00031 & 0.0046 & 0.052 \\
Mother's milk (mother on Diet 1) & 0.0057 & 0.025 & 0.11 \\
Prenatal exposure (mother on Diet 1) & 0.0001 & 0.002 & 0.038 \\
Diet 1 & 0.00087 & 0.0059 & 0.05 \\
Diet 2 & 0.32 & 1.9 & 13 \\
Diet 3 & 0.12 & 0.74 & 5.4 \\
\hline
\end{tabular}

Excess Lifetime Risk [ ]

\begin{tabular}{llll}
\hline Diet 1 & $3.9 \mathrm{E}-05$ & $6.1 \mathrm{E}-04$ & $1.2 \mathrm{E}-02$ \\
Diet 2 & $1.8 \mathrm{E}-05$ & $2.3 \mathrm{E}-04$ & $3.3 \mathrm{E}-03$ \\
Diet 3 & $3.3 \mathrm{E}-05$ & $3.7 \mathrm{E}-04$ & $4.7 \mathrm{E}-03$ \\
Diet 4 & $8.1 \mathrm{E}-05$ & $1.6 \mathrm{E}-03$ & $2.4 \mathrm{E}-02$ \\
\hline
\end{tabular}

\section{Relative Risk [ ]}

\begin{tabular}{lccc}
\hline Diet 1 & 1.014 & 1.14 & 2.5 \\
Diet 2 & 1.0056 & 1.055 & 1.67 \\
Diet 3 & 1.0096 & 1.094 & 2.2 \\
Diet 4 & 1.032 & 1.35 & 6.1 \\
\hline
\end{tabular}

\section{Probability of Causation [\%]}

\begin{tabular}{lccc}
\hline Diet 1 & 1.41 & 12.1 & 60 \\
Diet 2 & 0.56 & 5.2 & 40 \\
Diet 3 & 0.95 & 8.6 & 55 \\
Diet 4 & 3.09 & 26.0 & 84 \\
\hline
\end{tabular}

Diet 1 - Backyard cow milk + all other locally produced non-milk exposure pathways

Diet 2 - Locally produced commercial milk + all other locally produced non-milk exposure pathways

Diet 3 - Regionally mixed commercial milk + inhalation (other regionally mixed food items are minor contributors to the total)

Diet 4 - Goat milk (all other exposure pathways are of negligible importance compared to goat milk) 


\section{Location: Maryville}

\section{Receptor: Male born in 1952}

\begin{tabular}{lccc}
\hline & \multicolumn{3}{c}{ Thyroid Dose [cGy] } \\
& \multicolumn{2}{c}{$95 \%$ Subjective Confidence Interval } \\
Exposure Pathway & lower limit & central estimate & upper limit \\
\hline Backyard Cow Milk & 0.28 & 1.7 & 12 \\
Commercial Milk (locally produced) & 0.083 & 0.58 & 4 \\
Commercial Milk (regionally mixed) & 0.18 & 1.3 & 9 \\
Goat Milk (locally produced) & 0.57 & 4.8 & 43 \\
Beef (locally produced) & 0.00038 & 0.0066 & 0.15 \\
Leafy Vegetables (locally produced) & 0.000068 & 0.00083 & 0.0079 \\
Eggs (locally produced) & 0.014 & 0.1 & 0.77 \\
Cottage Cheese (locally produced) & 0.00034 & 0.0045 & 0.051 \\
Inhalation & 0.006 & 0.025 & 0.11 \\
Mother's milk (mother on Diet 1) & 0.0001 & 0.002 & 0.038 \\
Prenatal exposure (mother on Diet 1) & 0.00087 & 0.0059 & 0.05 \\
Diet 1 & 0.31 & 1.9 & 12 \\
Diet 2 & 0.12 & 0.76 & 5.2 \\
Diet 3 & 0.19 & 1.3 & 9 \\
\hline
\end{tabular}

Excess Lifetime Risk [ ]

\begin{tabular}{llll}
\hline Diet 1 & $5.1 \mathrm{E}-06$ & $1.5 \mathrm{E}-04$ & $2.6 \mathrm{E}-03$ \\
Diet 2 & $2.6 \mathrm{E}-06$ & $5.8 \mathrm{E}-05$ & $1.2 \mathrm{E}-03$ \\
Diet 3 & $4.0 \mathrm{E}-06$ & $9.3 \mathrm{E}-05$ & $2.0 \mathrm{E}-03$ \\
Diet 4 & $1.2 \mathrm{E}-05$ & $3.9 \mathrm{E}-04$ & $7.8 \mathrm{E}-03$ \\
\hline
\end{tabular}

\section{Relative Risk [ ]}

\begin{tabular}{lccc}
\hline Diet 1 & 1.0058 & 1.11 & 3.2 \\
Diet 2 & 1.002 & 1.04 & 1.92 \\
Diet 3 & 1.0036 & 1.065 & 2.7 \\
Diet 4 & 1.013 & 1.29 & 9 \\
\hline
\end{tabular}

\section{Probability of Causation [\%]}

\begin{tabular}{lccc}
\hline Diet 1 & 0.58 & 9.6 & 69 \\
Diet 2 & 0.20 & 3.9 & 48 \\
Diet 3 & 0.36 & 6.1 & 63 \\
Diet 4 & 1.27 & 22.3 & 89 \\
\hline
\end{tabular}

Diet 1 - Backyard cow milk + all other locally produced non-milk exposure pathways

Diet 2 - Locally produced commercial milk + all other locally produced non-milk exposure pathways

Diet 3 - Regionally mixed commercial milk + inhalation (other regionally mixed food items are minor contributors to the total)

Diet 4 - Goat milk (all other exposure pathways are of negligible importance compared to goat milk) 
Location: Cedar Grove

Receptor: Female born in 1952

\begin{tabular}{lccc}
\hline & \multicolumn{3}{c}{ Thyroid Dose [cGy] } \\
Exposure Pathway & $95 \%$ Subjective Confidence Interval \\
\hline Backyard Cow Milk & lower limit & central estimate & upper limit \\
Commercial Milk (locally produced) & 0.53 & 3.3 & 22 \\
Commercial Milk (regionally mixed) & 0.16 & 1.1 & 7.6 \\
Goat Milk (locally produced) & 0.17 & 1.3 & 8 \\
Beef (locally produced) & 1.1 & 8.6 & 74 \\
Leafy Vegetables (locally produced) & 0.00069 & 0.012 & 0.28 \\
Eggs (locally produced) & 0.00013 & 0.0016 & 0.014 \\
Cottage Cheese (locally produced) & 0.025 & 0.19 & 1.5 \\
Inhalation & 0.00059 & 0.0083 & 0.093 \\
Mother's milk (mother on Diet 1) & 0.011 & 0.04 & 0.17 \\
Prenatal exposure (mother on Diet 1) & 0.00016 & 0.0035 & 0.061 \\
Diet 1 & 0.0016 & 0.011 & 0.085 \\
Diet 2 & 0.6 & 3.6 & 23 \\
Diet 3 & 0.24 & 1.4 & 9.5 \\
\hline
\end{tabular}

Excess Lifetime Risk [ ]

\begin{tabular}{llll}
\hline Diet 1 & $7.0 \mathrm{E}-05$ & $1.1 \mathrm{E}-03$ & $1.6 \mathrm{E}-02$ \\
Diet 2 & $3.5 \mathrm{E}-05$ & $4.3 \mathrm{E}-04$ & $5.6 \mathrm{E}-03$ \\
Diet 3 & $3.4 \mathrm{E}-05$ & $3.7 \mathrm{E}-04$ & $4.7 \mathrm{E}-03$ \\
Diet 4 & $1.7 \mathrm{E}-04$ & $3.0 \mathrm{E}-03$ & $4.3 \mathrm{E}-02$ \\
\hline
\end{tabular}

\section{Relative Risk [ ]}

\begin{tabular}{lccc}
\hline Diet 1 & 1.029 & 1.27 & 4 \\
Diet 2 & 1.011 & 1.1 & 2.2 \\
Diet 3 & 1.01 & 1.096 & 2.2 \\
Diet 4 & 1.064 & 1.65 & 10.7 \\
\hline
\end{tabular}

\section{Probability of Causation [\%]}

\begin{tabular}{lccc}
\hline Diet 1 & 2.79 & 21.4 & 75 \\
Diet 2 & 1.07 & 9.5 & 55 \\
Diet 3 & 0.99 & 8.8 & 55 \\
Diet 4 & 5.99 & 39.3 & 90 \\
\hline
\end{tabular}

Diet 1 - Backyard cow milk + all other locally produced non-milk exposure pathways

Diet 2 - Locally produced commercial milk + all other locally produced non-milk exposure pathways

Diet 3 - Regionally mixed commercial milk + inhalation (other regionally mixed food items are minor contributors to the total)

Diet 4 - Goat milk (all other exposure pathways are of negligible importance compared to goat milk) 
Location: Cedar Grove

Receptor: Male born in 1952

\begin{tabular}{lccc}
\hline & \multicolumn{3}{c}{ Thyroid Dose [cGy] } \\
& \multicolumn{2}{c}{$95 \%$ Subjective Confidence Interval } \\
Exposure Pathway & lower limit & central estimate & upper limit \\
\hline Backyard Cow Milk & 0.53 & 3.2 & 20 \\
Commercial Milk (locally produced) & 0.16 & 1.1 & 7.7 \\
Commercial Milk (regionally mixed) & 0.18 & 1.3 & 9 \\
Goat Milk (locally produced) & 1.1 & 8.8 & 81 \\
Beef (locally produced) & 0.00069 & 0.012 & 0.28 \\
Leafy Vegetables (locally produced) & 0.00014 & 0.0015 & 0.015 \\
Eggs (locally produced) & 0.029 & 0.2 & 1.4 \\
Cottage Cheese (locally produced) & 0.00066 & 0.0085 & 0.096 \\
Inhalation & 0.011 & 0.041 & 0.16 \\
Mother's milk (mother on Diet 1) & 0.00016 & 0.0035 & 0.061 \\
Prenatal exposure (mother on Diet 1) & 0.0016 & 0.011 & 0.085 \\
Diet 1 & 0.6 & 3.6 & 22 \\
Diet 2 & 0.25 & 1.4 & 9.4 \\
Diet 3 & 0.2 & 1.3 & 9.1 \\
\hline
\end{tabular}

Excess Lifetime Risk [ ]

\begin{tabular}{llll}
\hline Diet 1 & $9.3 \mathrm{E}-06$ & $2.9 \mathrm{E}-04$ & $5.6 \mathrm{E}-03$ \\
Diet 2 & $4.8 \mathrm{E}-06$ & $1.1 \mathrm{E}-04$ & $2.3 \mathrm{E}-03$ \\
Diet 3 & $4.1 \mathrm{E}-06$ & $9.5 \mathrm{E}-05$ & $2.0 \mathrm{E}-03$ \\
Diet 4 & $2.6 \mathrm{E}-05$ & $7.3 \mathrm{E}-04$ & $1.4 \mathrm{E}-02$ \\
\hline
\end{tabular}

\section{Relative Risk [ ]}

\begin{tabular}{lccc}
\hline Diet 1 & 1.011 & 1.19 & 5.1 \\
Diet 2 & 1.0038 & 1.074 & 2.7 \\
Diet 3 & 1.0037 & 1.066 & 2.7 \\
Diet 4 & 1.025 & 1.52 & 12 \\
\hline
\end{tabular}

\section{Probability of Causation [\%]}

\begin{tabular}{lccc}
\hline Diet 1 & 1.05 & 16.0 & 80 \\
Diet 2 & 0.38 & 6.9 & 62 \\
Diet 3 & 0.37 & 6.2 & 63 \\
Diet 4 & 2.47 & 34.0 & 92 \\
\hline
\end{tabular}

Diet 1 - Backyard cow milk + all other locally produced non-milk exposure pathways

Diet 2 - Locally produced commercial milk + all other locally produced non-milk exposure pathways

Diet 3 - Regionally mixed commercial milk + inhalation (other regionally mixed food items are minor contributors to the total)

Diet 4 - Goat milk (all other exposure pathways are of negligible importance compared to goat milk) 
Location: Bradbury

Receptor: Female born in 1954

\begin{tabular}{lccc}
\hline & \multicolumn{3}{c}{ Thyroid Dose [cGy] } \\
Exposure Pathway & \multicolumn{2}{c}{$95 \%$ Subjective Confidence Interval } \\
Backyard Cow Milk & lower limit & central estimate & upper limit \\
Commercial Milk (locally produced) & 2.5 & 18 & 150 \\
Commercial Milk (regionally mixed) & 0.69 & 5.8 & 47 \\
Goat Milk (locally produced) & 0.094 & 0.82 & 5.7 \\
Beef (locally produced) & 4.7 & 51 & 540 \\
Leafy Vegetables (locally produced) & 0.0025 & 0.051 & 1.2 \\
Eggs (locally produced) & 0.00079 & 0.0087 & 0.089 \\
Cottage Cheese (locally produced) & 0.11 & 0.97 & 8.1 \\
Inhalation & 0.0028 & 0.048 & 0.62 \\
Mother's milk (mother on Diet 1) & 0.029 & 0.12 & 0.54 \\
Prenatal exposure (mother on Diet 1) & 0.011 & 0.37 & 7.7 \\
Diet 1 & 0.099 & 1.2 & 8.9 \\
Diet 2 & 2.8 & 19 & 160 \\
Diet 3 & 1 & 7.6 & 57 \\
\hline
\end{tabular}

Excess Lifetime Risk [ ]

\begin{tabular}{llll}
\hline Diet 1 & $3.7 \mathrm{E}-04$ & $6.4 \mathrm{E}-03$ & $9.8 \mathrm{E}-02$ \\
Diet 2 & $1.4 \mathrm{E}-04$ & $2.2 \mathrm{E}-03$ & $3.0 \mathrm{E}-02$ \\
Diet 3 & $2.3 \mathrm{E}-05$ & $2.8 \mathrm{E}-04$ & $3.6 \mathrm{E}-03$ \\
Diet 4 & $7.7 \mathrm{E}-04$ & $1.7 \mathrm{E}-02$ & $3.0 \mathrm{E}-01$ \\
\hline
\end{tabular}

\section{Relative Risk [ ]}

\begin{tabular}{lccc}
\hline Diet 1 & 1.13 & 2.4 & 22 \\
Diet 2 & 1.046 & 1.54 & 10.5 \\
Diet 3 & 1.0071 & 1.069 & 2 \\
Diet 4 & 1.3 & 5 & 67 \\
\hline
\end{tabular}

\section{Probability of Causation [\%]}

\begin{tabular}{lccc}
\hline Diet 1 & 11.59 & 58.6 & 95 \\
Diet 2 & 4.38 & 35.0 & 90 \\
Diet 3 & 0.71 & 6.5 & 50 \\
Diet 4 & 23.18 & 79.7 & 98 \\
\hline
\end{tabular}

Diet 1 - Backyard cow milk + all other locally produced non-milk exposure pathways

Diet 2 - Locally produced commercial milk + all other locally produced non-milk exposure pathways

Diet 3 - Regionally mixed commercial milk + inhalation (other regionally mixed food items are minor contributors to the total)

Diet 4 - Goat milk (all other exposure pathways are of negligible importance compared to goat milk) 


\section{Location: Bradbury}

Receptor: Male born in 1954

\begin{tabular}{lccc}
\hline & \multicolumn{3}{c}{ Thyroid Dose [cGy] } \\
& \multicolumn{2}{c}{$95 \%$ Subjective Confidence Interval } \\
Exposure Pathway & lower limit & central estimate & upper limit \\
\hline Backyard Cow Milk & 2.8 & 18 & 130 \\
Commercial Milk (locally produced) & 0.66 & 5.8 & 41 \\
Commercial Milk (regionally mixed) & 0.1 & 0.8 & 5 \\
Goat Milk (locally produced) & 5.6 & 51 & 500 \\
Beef (locally produced) & 0.0025 & 0.05 & 1.1 \\
Leafy Vegetables (locally produced) & 0.00064 & 0.0086 & 0.098 \\
Eggs (locally produced) & 0.12 & 0.96 & 8 \\
Cottage Cheese (locally produced) & 0.0031 & 0.045 & 0.59 \\
Inhalation & 0.029 & 0.13 & 0.54 \\
Mother's milk (mother on Diet 1) & 0.011 & 0.37 & 7.7 \\
Prenatal exposure (mother on Diet 1) & 0.099 & 1.2 & 8.9 \\
Diet 1 & 3 & 19 & 140 \\
Diet 2 & 0.96 & 7.5 & 50 \\
Diet 3 & 0.15 & 0.94 & 5.2 \\
\hline
\end{tabular}

Excess Lifetime Risk [ ]

\begin{tabular}{llll}
\hline Diet 1 & $5.1 \mathrm{E}-05$ & $1.6 \mathrm{E}-03$ & $3.0 \mathrm{E}-02$ \\
Diet 2 & $2.1 \mathrm{E}-05$ & $5.7 \mathrm{E}-04$ & $1.3 \mathrm{E}-02$ \\
Diet 3 & $3.0 \mathrm{E}-06$ & $7.4 \mathrm{E}-05$ & $1.6 \mathrm{E}-03$ \\
Diet 4 & $1.1 \mathrm{E}-04$ & $4.3 \mathrm{E}-03$ & $1.1 \mathrm{E}-01$ \\
\hline
\end{tabular}

\section{Relative Risk [ ]}

\begin{tabular}{lccc}
\hline Diet 1 & 1.061 & 2 & 26 \\
Diet 2 & 1.02 & 1.4 & 10.5 \\
Diet 3 & 1.0027 & 1.05 & 2 \\
Diet 4 & 1.12 & 4 & 88 \\
\hline
\end{tabular}

\section{Probability of Causation [\%]}

\begin{tabular}{lccc}
\hline Diet 1 & 5.74 & 50.7 & 96 \\
Diet 2 & 1.92 & 28.6 & 90 \\
Diet 3 & 0.27 & 4.8 & 50 \\
Diet 4 & 10.43 & 74.6 & 99 \\
\hline
\end{tabular}

Diet 1 - Backyard cow milk + all other locally produced non-milk exposure pathways

Diet 2 - Locally produced commercial milk + all other locally produced non-milk exposure pathways

Diet 3 - Regionally mixed commercial milk + inhalation (other regionally mixed food items are minor contributors to the total)

Diet 4 - Goat milk (all other exposure pathways are of negligible importance compared to goat milk) 
Location: Gallaher Bend

Receptor: Female born in 1954

\begin{tabular}{lccc}
\hline & \multicolumn{3}{c}{ Thyroid Dose [cGy] } \\
& \multicolumn{2}{c}{$9 \%$ Subjective Confidence Interval } \\
Exposure Pathway & lower limit & central estimate & upper limit \\
\hline Backyard Cow Milk & 3.2 & 23 & 160 \\
Commercial Milk (locally produced) & 0.89 & 7.7 & 51 \\
Commercial Milk (regionally mixed) & 0.094 & 0.81 & 5.7 \\
Goat Milk (locally produced) & 6 & 65 & 620 \\
Beef (locally produced) & 0.0031 & 0.062 & 1.4 \\
Leafy Vegetables (locally produced) & 0.00094 & 0.011 & 0.11 \\
Eggs (locally produced) & 0.14 & 1.1 & 9 \\
Cottage Cheese (locally produced) & 0.0035 & 0.059 & 0.66 \\
Inhalation & 0.031 & 0.15 & 0.67 \\
Mother's milk (mother on Diet 1) & 0.014 & 0.44 & 9.7 \\
Prenatal exposure (mother on Diet 1) & 0.13 & 1.5 & 10 \\
Diet 1 & 3.5 & 25 & 180 \\
Diet 2 & 1.3 & 9.5 & 60 \\
Diet 3 & 0.16 & 0.99 & 6.2 \\
\hline
\end{tabular}

Excess Lifetime Risk [ ]

\begin{tabular}{llll}
\hline Diet 1 & $4.2 \mathrm{E}-04$ & $7.5 \mathrm{E}-03$ & $1.1 \mathrm{E}-01$ \\
Diet 2 & $1.9 \mathrm{E}-04$ & $2.7 \mathrm{E}-03$ & $4.1 \mathrm{E}-02$ \\
Diet 3 & $2.4 \mathrm{E}-05$ & $2.9 \mathrm{E}-04$ & $3.8 \mathrm{E}-03$ \\
Diet 4 & $9.2 \mathrm{E}-04$ & $2.1 \mathrm{E}-02$ & $3.7 \mathrm{E}-01$ \\
\hline
\end{tabular}

\section{Relative Risk [ ]}

\begin{tabular}{lccc}
\hline Diet 1 & 1.17 & 2.8 & 26 \\
Diet 2 & 1.057 & 1.68 & 12 \\
Diet 3 & 1.0078 & 1.072 & 2 \\
Diet 4 & 1.34 & 6 & 81 \\
\hline
\end{tabular}

\section{Probability of Causation [\%]}

\begin{tabular}{lccc}
\hline Diet 1 & 14.61 & 64.0 & 96 \\
Diet 2 & 5.41 & 40.4 & 91 \\
Diet 3 & 0.77 & 6.7 & 51 \\
Diet 4 & 25.33 & 83.2 & 99 \\
\hline
\end{tabular}

Diet 1 - Backyard cow milk + all other locally produced non-milk exposure pathways

Diet 2 - Locally produced commercial milk + all other locally produced non-milk exposure pathways

Diet 3 - Regionally mixed commercial milk + inhalation (other regionally mixed food items are minor contributors to the total)

Diet 4 - Goat milk (all other exposure pathways are of negligible importance compared to goat milk) 


\section{Location: Gallaher Bend}

Receptor: Male born in 1954

\begin{tabular}{lccc}
\hline & \multicolumn{3}{c}{ Thyroid Dose [cGy] } \\
& \multicolumn{2}{c}{$95 \%$ Subjective Confidence Interval } \\
Exposure Pathway & lower limit & central estimate & upper limit \\
\hline Backyard Cow Milk & 3.5 & 22 & 160 \\
Commercial Milk (locally produced) & 0.83 & 7.5 & 51 \\
Commercial Milk (regionally mixed) & 0.1 & 0.8 & 5 \\
Goat Milk (locally produced) & 6.9 & 67 & 630 \\
Beef (locally produced) & 0.0033 & 0.059 & 1.5 \\
Leafy Vegetables (locally produced) & 0.00077 & 0.01 & 0.11 \\
Eggs (locally produced) & 0.15 & 1.2 & 10 \\
Cottage Cheese (locally produced) & 0.0038 & 0.059 & 0.74 \\
Inhalation & 0.036 & 0.16 & 0.65 \\
Mother's milk (mother on Diet 1) & 0.014 & 0.44 & 9.7 \\
Prenatal exposure (mother on Diet 1) & 0.13 & 1.5 & 10 \\
Diet 1 & 3.8 & 23 & 170 \\
Diet 2 & 1.2 & 9.4 & 59 \\
Diet 3 & 0.16 & 0.97 & 5.2 \\
\hline
\end{tabular}

Excess Lifetime Risk [ ]

\begin{tabular}{llll}
\hline Diet 1 & $6.4 \mathrm{E}-05$ & $2.0 \mathrm{E}-03$ & $3.6 \mathrm{E}-02$ \\
Diet 2 & $2.6 \mathrm{E}-05$ & $7.4 \mathrm{E}-04$ & $1.5 \mathrm{E}-02$ \\
Diet 3 & $3.2 \mathrm{E}-06$ & $7.8 \mathrm{E}-05$ & $1.6 \mathrm{E}-03$ \\
Diet 4 & $1.5 \mathrm{E}-04$ & $5.4 \mathrm{E}-03$ & $1.3 \mathrm{E}-01$ \\
\hline
\end{tabular}

\section{Relative Risk [ ]}

\begin{tabular}{lccc}
\hline Diet 1 & 1.072 & 2.2 & 30 \\
Diet 2 & 1.024 & 1.5 & 11 \\
Diet 3 & 1.0029 & 1.053 & 2 \\
Diet 4 & 1.14 & 4.5 & 111 \\
\hline
\end{tabular}

\section{Probability of Causation [\%]}

\begin{tabular}{lccc}
\hline Diet 1 & 6.72 & 55.2 & 97 \\
Diet 2 & 2.35 & 33.2 & 91 \\
Diet 3 & 0.29 & 5.1 & 51 \\
Diet 4 & 11.92 & 77.8 & 99 \\
\hline
\end{tabular}

Diet 1 - Backyard cow milk + all other locally produced non-milk exposure pathways

Diet 2 - Locally produced commercial milk + all other locally produced non-milk exposure pathways

Diet 3 - Regionally mixed commercial milk + inhalation (other regionally mixed food items are minor contributors to the total)

Diet 4 - Goat milk (all other exposure pathways are of negligible importance compared to goat milk) 


\section{Location: EFPC}

Receptor: Female born in 1954

\begin{tabular}{lccc}
\hline & \multicolumn{3}{c}{ Thyroid Dose [cGy] } \\
Exposure Pathway & $95 \%$ Subjective Confidence Interval \\
\hline Backyard Cow Milk & lower limit & central estimate & upper limit \\
Commercial Milk (locally produced) & 0.63 & 4.8 & 36 \\
Commercial Milk (regionally mixed) & 0.17 & 1.6 & 12 \\
Goat Milk (locally produced) & 0.095 & 0.82 & 5.7 \\
Beef (locally produced) & -- & -- & -- \\
Leafy Vegetables (locally produced) & 0.00066 & 0.013 & 0.31 \\
Eggs (locally produced) & 0.00019 & 0.0023 & 0.024 \\
Cottage Cheese (locally produced) & 0.028 & 0.25 & 2.2 \\
Inhalation & 0.00073 & 0.012 & 0.16 \\
Mother's milk (mother on Diet 1) & 0.0076 & 0.034 & 0.14 \\
Prenatal exposure (mother on Diet 1) & 0.0028 & 0.095 & 2.1 \\
Diet 1 & 0.027 & 0.31 & 2.4 \\
Diet 2 & 0.7 & 5.3 & 39 \\
Diet 3 & 0.25 & 2 & 14 \\
\hline
\end{tabular}

Excess Lifetime Risk [ ]

\begin{tabular}{lccc}
\hline Diet 1 & $9.7 \mathrm{E}-05$ & $1.6 \mathrm{E}-03$ & $2.4 \mathrm{E}-02$ \\
Diet 2 & $3.7 \mathrm{E}-05$ & $5.7 \mathrm{E}-04$ & $8.3 \mathrm{E}-03$ \\
Diet 3 & $1.6 \mathrm{E}-05$ & $2.4 \mathrm{E}-04$ & $3.3 \mathrm{E}-03$ \\
Diet 4 & -- & -- & -- \\
\hline
\end{tabular}

Relative Risk [ ]

\begin{tabular}{lccc}
\hline Diet 1 & 1.031 & 1.37 & 6.5 \\
Diet 2 & 1.012 & 1.14 & 3.3 \\
Diet 3 & 1.0055 & 1.061 & 1.98 \\
Diet 4 & -- & -- & -- \\
\hline
\end{tabular}

\begin{tabular}{lccc}
\hline Diet 1 & 3.02 & 26.3 & 83 \\
Diet 2 & 1.16 & 12.1 & 68 \\
Diet 3 & 0.55 & 5.7 & 49 \\
Diet 4 & -- & -- & - \\
\hline Diet 1 - Backyard cow milk + all other locally produced non-milk exposure pathways & \\
Diet 2 - Locally produced commercial milk + all other locally produced non-milk exposure pathways \\
Diet 3 - Regionally mixed commercial milk + inhalation (other regionally mixed food items are minor contributors to the total) \\
Diet 4 - Goat milk (all other exposure pathways are of negligible importance compared to goat milk)
\end{tabular}




\section{Location: EFPC}

\section{Receptor: Male born in 1954}

\begin{tabular}{lccc}
\hline & \multicolumn{3}{c}{ Thyroid Dose [cGy] } \\
& \multicolumn{2}{c}{$95 \%$ Subjective Confidence Interval } \\
Exposure Pathway & lower limit & central estimate & upper limit \\
\hline Backyard Cow Milk & 0.65 & 4.6 & 32 \\
Commercial Milk (locally produced) & 0.17 & 1.5 & 11 \\
Commercial Milk (regionally mixed) & 0.1 & 0.8 & 5 \\
Goat Milk (locally produced) & -- & -- & -- \\
Beef (locally produced) & 0.00065 & 0.013 & 0.31 \\
Leafy Vegetables (locally produced) & 0.00017 & 0.0022 & 0.025 \\
Eggs (locally produced) & 0.031 & 0.25 & 2.1 \\
Cottage Cheese (locally produced) & 0.00084 & 0.012 & 0.16 \\
Inhalation & 0.0074 & 0.034 & 0.14 \\
Mother's milk (mother on Diet 1) & 0.0028 & 0.095 & 2.1 \\
Prenatal exposure (mother on Diet 1) & 0.027 & 0.31 & 2.4 \\
Diet 1 & 0.71 & 5 & 34 \\
Diet 2 & 0.25 & 2 & 13 \\
Diet 3 & 0.11 & 0.84 & 5 \\
\hline
\end{tabular}

Excess Lifetime Risk [ ]

\begin{tabular}{lccc}
\hline Diet 1 & $1.2 \mathrm{E}-05$ & $4.1 \mathrm{E}-04$ & $7.6 \mathrm{E}-03$ \\
Diet 2 & $5.4 \mathrm{E}-06$ & $1.5 \mathrm{E}-04$ & $3.4 \mathrm{E}-03$ \\
Diet 3 & $2.3 \mathrm{E}-06$ & $6.3 \mathrm{E}-05$ & $1.5 \mathrm{E}-03$ \\
Diet 4 & -- & -- & -- \\
\hline
\end{tabular}

\section{Relative Risk [ ]}

\begin{tabular}{lccc}
\hline Diet 1 & 1.015 & 1.26 & 7.3 \\
Diet 2 & 1.0052 & 1.1 & 3.5 \\
Diet 3 & 1.0023 & 1.045 & 1.96 \\
Diet 4 & -- & -- & -- \\
\hline
\end{tabular}

\section{Probability of Causation [\%]}

\begin{tabular}{lccc}
\hline Diet 1 & 1.51 & 20.4 & 85 \\
Diet 2 & 0.52 & 9.1 & 70 \\
Diet 3 & 0.23 & 4.3 & 49 \\
Diet 4 & -- & -- \\
\hline Diet 1 - Backyard cow milk + all other locally produced non-milk exposure pathways & \\
Diet 2 - Locally produced commercial milk + all other locally produced non-milk exposure pathways \\
Diet 3 - Regionally mixed commercial milk + inhalation (other regionally mixed food items are minor contributors to the total) \\
Diet 4 - Goat milk (all other exposure pathways are of negligible importance compared to goat milk)
\end{tabular}




\section{Location: Hope Creek}

Receptor: Female born in 1954

\begin{tabular}{lccc}
\hline & \multicolumn{3}{c}{ Thyroid Dose [cGy] } \\
& \multicolumn{2}{c}{$95 \%$ Subjective Confidence Interval } \\
Exposure Pathway & lower limit & central estimate & upper limit \\
\hline Backyard Cow Milk & 1.7 & 13 & 100 \\
Commercial Milk (locally produced) & 0.49 & 4.2 & 32 \\
Commercial Milk (regionally mixed) & 0.094 & 0.81 & 5.7 \\
Goat Milk (locally produced) & -- & -- & -- \\
Beef (locally produced) & 0.0018 & 0.036 & 0.79 \\
Leafy Vegetables (locally produced) & 0.00051 & 0.0062 & 0.065 \\
Eggs (locally produced) & 0.084 & 0.66 & 5.5 \\
Cottage Cheese (locally produced) & 0.0019 & 0.032 & 0.43 \\
Inhalation & 0.02 & 0.09 & 0.39 \\
Mother's milk (mother on Diet 1) & 0.0072 & 0.26 & 4.9 \\
Prenatal exposure (mother on Diet 1) & 0.074 & 0.83 & 6.4 \\
Diet 1 & 1.9 & 14 & 110 \\
Diet 2 & 0.69 & 5.3 & 37 \\
Diet 3 & 0.13 & 0.91 & 6 \\
\hline
\end{tabular}

Excess Lifetime Risk [ ]

\begin{tabular}{lccc}
\hline Diet 1 & $2.6 \mathrm{E}-04$ & $4.2 \mathrm{E}-03$ & $6.8 \mathrm{E}-02$ \\
Diet 2 & $9.4 \mathrm{E}-05$ & $1.6 \mathrm{E}-03$ & $2.2 \mathrm{E}-02$ \\
Diet 3 & $2.0 \mathrm{E}-05$ & $2.7 \mathrm{E}-04$ & $3.5 \mathrm{E}-03$ \\
Diet 4 & -- & -- & -- \\
\hline
\end{tabular}

\section{Relative Risk [ ]}

\begin{tabular}{lccc}
\hline Diet 1 & 1.096 & 2 & 15 \\
Diet 2 & 1.033 & 1.38 & 6.9 \\
Diet 3 & 1.0066 & 1.066 & 2 \\
Diet 4 & -- & -- & -- \\
\hline
\end{tabular}

\section{Probability of Causation [\%]}

\begin{tabular}{lccc}
\hline Diet 1 & 8.75 & 50.0 & 93 \\
Diet 2 & 3.17 & 27.4 & 85 \\
Diet 3 & 0.65 & 6.2 & 50 \\
Diet 4 & -- & -- & \\
\hline Diet 1 - Backyard cow milk + all other locally produced non-milk exposure pathways & \\
Diet 2 - Locally produced commercial milk + all other locally produced non-milk exposure pathways \\
Diet 3 - Regionally mixed commercial milk + inhalation (other regionally mixed food items are minor contributors to the total) \\
Diet 4 - Goat milk (all other exposure pathways are of negligible importance compared to goat milk)
\end{tabular}




\section{Location: Hope Creek}

\section{Receptor: Male born in 1954}

\begin{tabular}{lccc}
\hline & \multicolumn{3}{c}{ Thyroid Dose [cGy] } \\
& \multicolumn{2}{c}{$95 \%$ Subjective Confidence Interval } \\
Exposure Pathway & 1.9 & 12 & 87 \\
\hline Backyard Cow Milk & 0.48 & 4.2 & 29 \\
Commercial Milk (locally produced) & 0.1 & 0.8 & 5 \\
Commercial Milk (regionally mixed) & -- & -- & -- \\
Goat Milk (locally produced) & 0.0018 & 0.036 & 0.8 \\
Beef (locally produced) & 0.0005 & 0.0061 & 0.069 \\
Leafy Vegetables (locally produced) & 0.088 & 0.66 & 5.8 \\
Eggs (locally produced) & 0.0022 & 0.032 & 0.46 \\
Cottage Cheese (locally produced) & 0.02 & 0.091 & 0.39 \\
Inhalation & 0.0072 & 0.26 & 4.9 \\
Mother's milk (mother on Diet 1) & 0.074 & 0.83 & 6.4 \\
Prenatal exposure (mother on Diet 1) & 2 & 13 & 92 \\
Diet 1 & 0.71 & 5.3 & 34 \\
Diet 2 & 0.14 & 0.9 & 5.1 \\
Diet 3 & & upper limit \\
\hline
\end{tabular}

Excess Lifetime Risk [ ]

\begin{tabular}{lccc}
\hline Diet 1 & $3.7 \mathrm{E}-05$ & $1.1 \mathrm{E}-03$ & $2.3 \mathrm{E}-02$ \\
Diet 2 & $1.5 \mathrm{E}-05$ & $4.2 \mathrm{E}-04$ & $9.2 \mathrm{E}-03$ \\
Diet 3 & $2.8 \mathrm{E}-06$ & $7.0 \mathrm{E}-05$ & $1.6 \mathrm{E}-03$ \\
Diet 4 & -- & -- & -- \\
\hline
\end{tabular}

\section{Relative Risk [ ]}

\begin{tabular}{lccc}
\hline Diet 1 & 1.041 & 1.74 & 16 \\
Diet 2 & 1.014 & 1.28 & 7.4 \\
Diet 3 & 1.0025 & 1.048 & 1.99 \\
Diet 4 & -- & -- & -- \\
\hline
\end{tabular}

\begin{tabular}{lccc}
\hline Diet 1 & 3.96 & 42.4 & 94 \\
Diet 2 & 1.41 & 21.8 & 86 \\
Diet 3 & 0.25 & 4.6 & 50 \\
Diet 4 & -- & -- & - \\
\hline Diet 1 - Backyard cow milk + all other locally produced non-milk exposure pathways & \\
Diet 2 - Locally produced commercial milk + all other locally produced non-milk exposure pathways \\
Diet 3 - Regionally mixed commercial milk + inhalation (other regionally mixed food items are minor contributors to the total) \\
Diet 4 - Goat milk (all other exposure pathways are of negligible importance compared to goat milk)
\end{tabular}


Location: Buttermilk Rd.

Receptor: Female born in 1954

\begin{tabular}{lccc}
\hline & \multicolumn{3}{c}{ Thyroid Dose [cGy] } \\
Exposure Pathway & \multicolumn{2}{c}{$95 \%$ Subjective Confidence Interval } \\
Backyard Cow Milk & lower limit & central estimate & upper limit \\
Commercial Milk (locally produced) & 1.7 & 13 & 98 \\
Commercial Milk (regionally mixed) & 0.46 & 4.1 & 30 \\
Goat Milk (locally produced) & 0.094 & 0.81 & 5.7 \\
Beef (locally produced) & 3.1 & 36 & 330 \\
Leafy Vegetables (locally produced) & 0.0017 & 0.035 & 0.74 \\
Eggs (locally produced) & 0.0005 & 0.0062 & 0.063 \\
Cottage Cheese (locally produced) & 0.082 & 0.64 & 5.4 \\
Inhalation & 0.0019 & 0.031 & 0.41 \\
Mother's milk (mother on Diet 1) & 0.021 & 0.09 & 0.39 \\
Prenatal exposure (mother on Diet 1) & 0.0073 & 0.25 & 5 \\
Diet 1 & 0.074 & 0.82 & 6.1 \\
Diet 2 & 1.9 & 14 & 110 \\
Diet 3 & 0.68 & 5.2 & 36 \\
\hline
\end{tabular}

Excess Lifetime Risk [ ]

\begin{tabular}{llll}
\hline Diet 1 & $2.5 \mathrm{E}-04$ & $4.3 \mathrm{E}-03$ & $6.7 \mathrm{E}-02$ \\
Diet 2 & $8.9 \mathrm{E}-05$ & $1.5 \mathrm{E}-03$ & $2.2 \mathrm{E}-02$ \\
Diet 3 & $2.0 \mathrm{E}-05$ & $2.7 \mathrm{E}-04$ & $3.5 \mathrm{E}-03$ \\
Diet 4 & $5.0 \mathrm{E}-04$ & $1.2 \mathrm{E}-02$ & $2.0 \mathrm{E}-01$ \\
\hline
\end{tabular}

\section{Relative Risk [ ]}

\begin{tabular}{lccc}
\hline Diet 1 & 1.093 & 1.97 & 15 \\
Diet 2 & 1.032 & 1.38 & 6.8 \\
Diet 3 & 1.0066 & 1.066 & 2 \\
Diet 4 & 1.22 & 3.8 & 45 \\
\hline
\end{tabular}

\begin{tabular}{|c|c|c|c|}
\hline \\
\hline Diet 1 & 8.52 & 49.0 & 93 \\
\hline Diet 2 & 3.11 & 27.2 & 85 \\
\hline Diet 3 & 0.66 & 6.2 & 50 \\
\hline Diet 4 & 17.95 & 73.0 & 98 \\
\hline
\end{tabular}

Diet 1 - Backyard cow milk + all other locally produced non-milk exposure pathways

Diet 2 - Locally produced commercial milk + all other locally produced non-milk exposure pathways

Diet 3 - Regionally mixed commercial milk + inhalation (other regionally mixed food items are minor contributors to the total)

Diet 4 - Goat milk (all other exposure pathways are of negligible importance compared to goat milk) 
Location: Buttermilk Rd.

Receptor: Male born in 1954

\begin{tabular}{lccc}
\hline & \multicolumn{3}{c}{ Thyroid Dose [cGy] } \\
& \multicolumn{2}{c}{$95 \%$ Subjective Confidence Interval } \\
Exposure Pathway & lower limit & central estimate & upper limit \\
\hline Backyard Cow Milk & 1.8 & 12 & 84 \\
Commercial Milk (locally produced) & 0.46 & 4.1 & 27 \\
Commercial Milk (regionally mixed) & 0.1 & 0.8 & 5 \\
Goat Milk (locally produced) & 3.8 & 37 & 350 \\
Beef (locally produced) & 0.0018 & 0.035 & 0.76 \\
Leafy Vegetables (locally produced) & 0.00048 & 0.006 & 0.066 \\
Eggs (locally produced) & 0.083 & 0.64 & 5.8 \\
Cottage Cheese (locally produced) & 0.0021 & 0.032 & 0.44 \\
Inhalation & 0.02 & 0.091 & 0.4 \\
Mother's milk (mother on Diet 1) & 0.0073 & 0.25 & 5 \\
Prenatal exposure (mother on Diet 1) & 0.074 & 0.82 & 6.1 \\
Diet 1 & 2 & 13 & 89 \\
Diet 2 & 0.69 & 5.3 & 33 \\
Diet 3 & 0.14 & 0.9 & 5.1 \\
\hline
\end{tabular}

Excess Lifetime Risk [ ]

\begin{tabular}{lllc}
\hline Diet 1 & $3.3 \mathrm{E}-05$ & $1.1 \mathrm{E}-03$ & $2.2 \mathrm{E}-02$ \\
Diet 2 & $1.4 \mathrm{E}-05$ & $4.2 \mathrm{E}-04$ & $9.1 \mathrm{E}-03$ \\
Diet 3 & $2.7 \mathrm{E}-06$ & $7.0 \mathrm{E}-05$ & $1.6 \mathrm{E}-03$ \\
Diet 4 & $7.8 \mathrm{E}-05$ & $3.0 \mathrm{E}-03$ & $7.4 \mathrm{E}-02$ \\
\hline
\end{tabular}

\section{Relative Risk [ ]}

\begin{tabular}{lccc}
\hline Diet 1 & 1.042 & 1.72 & 16 \\
Diet 2 & 1.014 & 1.27 & 7.3 \\
Diet 3 & 1.0025 & 1.048 & 1.99 \\
Diet 4 & 1.082 & 3 & 62 \\
\hline
\end{tabular}

\begin{tabular}{lccc}
\hline Diet 1 & 4.02 & 41.5 & 93 \\
Diet 2 & 1.43 & 21.4 & 86 \\
Diet 3 & 0.25 & 4.6 & 50 \\
Diet 4 & 7.54 & 66.6 & 98 \\
\hline
\end{tabular}

Diet 1 - Backyard cow milk + all other locally produced non-milk exposure pathways

Diet 2 - Locally produced commercial milk + all other locally produced non-milk exposure pathways

Diet 3 - Regionally mixed commercial milk + inhalation (other regionally mixed food items are minor contributors to the total)

Diet 4 - Goat milk (all other exposure pathways are of negligible importance compared to goat milk) 


\section{Location: Jonesville}

Receptor: Female born in 1954

\begin{tabular}{lccc}
\hline & \multicolumn{3}{c}{ Thyroid Dose [cGy] } \\
Exposure Pathway & \multicolumn{2}{c}{$95 \%$ Subjective Confidence Interval } \\
\hline Backyard Cow Milk & lower limit & central estimate & upper limit \\
Commercial Milk (locally produced) & 0.24 & 1.7 & 14 \\
Commercial Milk (regionally mixed) & 0.062 & 0.58 & 5.2 \\
Goat Milk (locally produced) & 0.094 & 0.81 & 5.7 \\
Beef (locally produced) & 0.48 & 5.2 & 56 \\
Leafy Vegetables (locally produced) & 0.00024 & 0.0052 & 0.12 \\
Eggs (locally produced) & 0.000069 & 0.00091 & 0.0098 \\
Cottage Cheese (locally produced) & 0.01 & 0.09 & 0.83 \\
Inhalation & 0.00029 & 0.0047 & 0.074 \\
Mother's milk (mother on Diet 1) & 0.0031 & 0.013 & 0.067 \\
Prenatal exposure (mother on Diet 1) & 0.0011 & 0.037 & 0.8 \\
Diet 1 & 0.011 & 0.11 & 1.1 \\
Diet 2 & 0.26 & 1.9 & 15 \\
Diet 3 & 0.091 & 0.72 & 5.9 \\
\hline
\end{tabular}

Excess Lifetime Risk [ ]

\begin{tabular}{llll}
\hline Diet 1 & $3.4 \mathrm{E}-05$ & $6.1 \mathrm{E}-04$ & $9.2 \mathrm{E}-03$ \\
Diet 2 & $1.4 \mathrm{E}-05$ & $2.1 \mathrm{E}-04$ & $3.0 \mathrm{E}-03$ \\
Diet 3 & $1.4 \mathrm{E}-05$ & $2.3 \mathrm{E}-04$ & $3.2 \mathrm{E}-03$ \\
Diet 4 & $7.5 \mathrm{E}-05$ & $1.7 \mathrm{E}-03$ & $3.3 \mathrm{E}-02$ \\
\hline
\end{tabular}

\section{Relative Risk [ ]}

\begin{tabular}{lccc}
\hline Diet 1 & 1.013 & 1.14 & 3.2 \\
Diet 2 & 1.0048 & 1.052 & 1.84 \\
Diet 3 & 1.0051 & 1.059 & 1.96 \\
Diet 4 & 1.03 & 1.39 & 8 \\
\hline
\end{tabular}

\begin{tabular}{|c|c|c|c|}
\hline Diet 1 & 1.29 & 12.2 & 68 \\
\hline Diet 2 & 0.48 & 4.9 & 45 \\
\hline Diet 3 & 0.51 & 5.6 & 49 \\
\hline Diet 4 & 2.92 & 28.1 & 87 \\
\hline
\end{tabular}

Diet 1 - Backyard cow milk + all other locally produced non-milk exposure pathways

Diet 2 - Locally produced commercial milk + all other locally produced non-milk exposure pathways

Diet 3 - Regionally mixed commercial milk + inhalation (other regionally mixed food items are minor contributors to the total)

Diet 4 - Goat milk (all other exposure pathways are of negligible importance compared to goat milk) 


\section{Location: Jonesville}

\section{Receptor: Male born in 1954}

\begin{tabular}{lccc}
\hline & \multicolumn{3}{c}{ Thyroid Dose [cGy] } \\
& \multicolumn{2}{c}{$95 \%$ Subjective Confidence Interval } \\
Exposure Pathway & lower limit & central estimate & upper limit \\
\hline Backyard Cow Milk & 0.24 & 1.7 & 13 \\
Commercial Milk (locally produced) & 0.061 & 0.58 & 4.3 \\
Commercial Milk (regionally mixed) & 0.1 & 0.8 & 5 \\
Goat Milk (locally produced) & 0.5 & 5.2 & 56 \\
Beef (locally produced) & 0.00023 & 0.0051 & 0.12 \\
Leafy Vegetables (locally produced) & 0.000066 & 0.00083 & 0.01 \\
Eggs (locally produced) & 0.011 & 0.095 & 0.89 \\
Cottage Cheese (locally produced) & 0.0003 & 0.0046 & 0.069 \\
Inhalation & 0.0032 & 0.013 & 0.066 \\
Mother's milk (mother on Diet 1) & 0.0011 & 0.037 & 0.8 \\
Prenatal exposure (mother on Diet 1) & 0.011 & 0.11 & 1.1 \\
Diet 1 & 0.27 & 1.9 & 14 \\
Diet 2 & 0.09 & 0.73 & 5.4 \\
Diet 3 & 0.11 & 0.82 & 5 \\
\hline
\end{tabular}

Excess Lifetime Risk [ ]

\begin{tabular}{llll}
\hline Diet 1 & $4.3 \mathrm{E}-06$ & $1.5 \mathrm{E}-04$ & $3.3 \mathrm{E}-03$ \\
Diet 2 & $2.0 \mathrm{E}-06$ & $6.0 \mathrm{E}-05$ & $1.3 \mathrm{E}-03$ \\
Diet 3 & $2.2 \mathrm{E}-06$ & $6.1 \mathrm{E}-05$ & $1.5 \mathrm{E}-03$ \\
Diet 4 & $9.8 \mathrm{E}-06$ & $4.5 \mathrm{E}-04$ & $1.2 \mathrm{E}-02$ \\
\hline
\end{tabular}

\section{Relative Risk [ ]}

\begin{tabular}{lccc}
\hline Diet 1 & 1.0056 & 1.11 & 3.4 \\
Diet 2 & 1.0019 & 1.04 & 1.94 \\
Diet 3 & 1.0023 & 1.044 & 1.95 \\
Diet 4 & 1.011 & 1.29 & 10.1 \\
\hline
\end{tabular}

\section{Probability of Causation [\%]}

\begin{tabular}{lccc}
\hline Diet 1 & 0.56 & 9.6 & 70 \\
Diet 2 & 0.19 & 3.8 & 48 \\
Diet 3 & 0.23 & 4.2 & 49 \\
Diet 4 & 1.04 & 22.4 & 90 \\
\hline
\end{tabular}

Diet 1 - Backyard cow milk + all other locally produced non-milk exposure pathways

Diet 2 - Locally produced commercial milk + all other locally produced non-milk exposure pathways

Diet 3 - Regionally mixed commercial milk + inhalation (other regionally mixed food items are minor contributors to the total)

Diet 4 - Goat milk (all other exposure pathways are of negligible importance compared to goat milk) 


\section{Location: OR Scarboro}

Receptor: Female born in 1954

\begin{tabular}{lccc}
\hline & \multicolumn{3}{c}{ Thyroid Dose [cGy] } \\
Exposure Pathway & \multicolumn{2}{c}{$9 \%$ Subjective Confidence Interval } \\
\hline Backyard Cow Milk & lower limit & central estimate & upper limit \\
Commercial Milk (locally produced) & -- & -- & -- \\
Commercial Milk (regionally mixed) & -- & -- & -- \\
Goat Milk (locally produced) & 0.094 & 0.81 & 5.7 \\
Beef (locally produced) & -- & -- & -- \\
Leafy Vegetables (locally produced) & -- & -- & -- \\
Eggs (locally produced) & -- & -- & -- \\
Cottage Cheese (locally produced) & -- & -- & -- \\
Inhalation & 0.0015 & 0.024 & 0.3 \\
Mother's milk (mother on Diet 3) & 0.015 & 0.067 & 0.3 \\
Prenatal exposure (mother on Diet 3) & 0.00084 & 0.017 & 0.26 \\
Diet 1 & 0.0086 & 0.051 & 0.39 \\
Diet 2 & -- & -- & -- \\
Diet 3 & -- & -- & -- \\
\hline
\end{tabular}

Excess Lifetime Risk [ ]

\begin{tabular}{lccc}
\hline Diet 1 & -- & -- & -- \\
Diet 2 & -- & -- & -- \\
Diet 3 & $1.8 \mathrm{E}-05$ & $2.6 \mathrm{E}-04$ & $3.4 \mathrm{E}-03$ \\
Diet 4 & -- & -- & -- \\
\hline
\end{tabular}

\section{Relative Risk [ ]}

\begin{tabular}{lccc}
\hline Diet 1 & -- & -- & -- \\
Diet 2 & -- & -- & -- \\
Diet 3 & 1.0061 & 1.064 & 1.99 \\
Diet 4 & -- & -- & -- \\
\hline
\end{tabular}

\begin{tabular}{lccc}
\hline Diet 1 & -- & -- & -- \\
Diet 2 & -- & -- & -- \\
Diet 3 & 0.61 & 6.0 & 50 \\
Diet 4 & -- & -- \\
\hline Diet 1 - Backyard cow milk + all other locally produced non-milk exposure pathways & \\
Diet 2 - Locally produced commercial milk + all other locally produced non-milk exposure pathways \\
Diet 3 - Regionally mixed commercial milk + inhalation (other regionally mixed food items are minor contributors to the total) \\
Diet 4 - Goat milk (all other exposure pathways are of negligible importance compared to goat milk)
\end{tabular}




\section{Location: OR Scarboro}

Receptor: Male born in 1954

\begin{tabular}{lccc}
\hline & \multicolumn{3}{c}{ Thyroid Dose [cGy] } \\
Exposure Pathway & \multicolumn{2}{c}{$95 \%$ Subjective Confidence Interval } \\
\hline Backyard Cow Milk & lower limit & central estimate & upper limit \\
Commercial Milk (locally produced) & -- & -- & -- \\
Commercial Milk (regionally mixed) & -- & -- & -- \\
Goat Milk (locally produced) & 0.1 & 0.8 & 5 \\
Beef (locally produced) & -- & -- & -- \\
Leafy Vegetables (locally produced) & -- & -- & -- \\
Eggs (locally produced) & -- & -- & -- \\
Cottage Cheese (locally produced) & -- & -- & 0.32 \\
Inhalation & 0.0015 & 0.025 & 0.29 \\
Mother's milk (mother on Diet 3) & 0.016 & 0.069 & 0.26 \\
Prenatal exposure (mother on Diet 3) & 0.00084 & 0.017 & 0.39 \\
Diet 1 & 0.0086 & 0.051 & -- \\
Diet 2 & -- & -- & -- \\
Diet 3 & -- & -- & 5.1 \\
\hline
\end{tabular}

Excess Lifetime Risk [ ]

\begin{tabular}{lccc}
\hline Diet 1 & -- & -- & -- \\
Diet 2 & -- & -- & -- \\
Diet 3 & $2.6 \mathrm{E}-06$ & $6.8 \mathrm{E}-05$ & $1.6 \mathrm{E}-03$ \\
Diet 4 & -- & -- & -- \\
\hline
\end{tabular}

\section{Relative Risk [ ]}

\begin{tabular}{lccc}
\hline Diet 1 & -- & -- & -- \\
Diet 2 & -- & -- & -- \\
Diet 3 & 1.0024 & 1.047 & 1.98 \\
Diet 4 & -- & -- & -- \\
\hline
\end{tabular}

\begin{tabular}{lccc}
\hline Diet 1 & -- & -- & -- \\
Diet 2 & -- & -- & -- \\
Diet 3 & 0.24 & 4.5 & 49 \\
Diet 4 & -- & -- \\
\hline Diet 1 - Backyard cow milk + all other locally produced non-milk exposure pathways & \\
Diet 2 - Locally produced commercial milk + all other locally produced non-milk exposure pathways \\
Diet 3 - Regionally mixed commercial milk + inhalation (other regionally mixed food items are minor contributors to the total) \\
Diet 4 - Goat milk (all other exposure pathways are of negligible importance compared to goat milk)
\end{tabular}




\section{Location: Lawnville/Gallaher}

Receptor: Female born in 1954

\begin{tabular}{lccc}
\hline & \multicolumn{3}{c}{ Thyroid Dose [cGy] } \\
Exposure Pathway & \multicolumn{2}{c}{$95 \%$} & Subjective Confidence Interval \\
Backyard Cow Milk & lower limit & central estimate & upper limit \\
Commercial Milk (locally produced) & 1.8 & 12 & 84 \\
Commercial Milk (regionally mixed) & 0.49 & 3.8 & 30 \\
Goat Milk (locally produced) & 0.094 & 0.81 & 5.7 \\
Beef (locally produced) & 3 & 35 & 330 \\
Leafy Vegetables (locally produced) & 0.0016 & 0.034 & 0.79 \\
Eggs (locally produced) & 0.00054 & 0.0059 & 0.061 \\
Cottage Cheese (locally produced) & 0.076 & 0.6 & 5 \\
Inhalation & 0.0019 & 0.031 & 0.37 \\
Mother's milk (mother on Diet 1) & 0.019 & 0.088 & 0.38 \\
Prenatal exposure (mother on Diet 1) & 0.0074 & 0.23 & 5.3 \\
Diet 1 & 0.074 & 0.78 & 5.8 \\
Diet 2 & 2 & 13 & 91 \\
Diet 3 & 0.69 & 4.8 & 36 \\
\hline
\end{tabular}

Excess Lifetime Risk [ ]

\begin{tabular}{llll}
\hline Diet 1 & $2.5 \mathrm{E}-04$ & $4.1 \mathrm{E}-03$ & $6.1 \mathrm{E}-02$ \\
Diet 2 & $9.1 \mathrm{E}-05$ & $1.4 \mathrm{E}-03$ & $2.1 \mathrm{E}-02$ \\
Diet 3 & $2.1 \mathrm{E}-05$ & $2.7 \mathrm{E}-04$ & $3.5 \mathrm{E}-03$ \\
Diet 4 & $4.9 \mathrm{E}-04$ & $1.1 \mathrm{E}-02$ & $2.2 \mathrm{E}-01$ \\
\hline
\end{tabular}

\section{Relative Risk [ ]}

\begin{tabular}{lccc}
\hline Diet 1 & 1.085 & 1.95 & 15 \\
Diet 2 & 1.033 & 1.35 & 6.6 \\
Diet 3 & 1.0065 & 1.066 & 2 \\
Diet 4 & 1.2 & 3.7 & 45 \\
\hline
\end{tabular}

\begin{tabular}{|c|c|c|c|}
\hline Diet 1 & $\begin{array}{l}7.81 \\
\end{array}$ & 48.7 & 93 \\
\hline Diet 2 & 3.16 & 25.8 & 85 \\
\hline Diet 3 & 0.65 & 6.2 & 50 \\
\hline Diet 4 & 16.81 & 72.7 & 98 \\
\hline
\end{tabular}

Diet 1 - Backyard cow milk + all other locally produced non-milk exposure pathways

Diet 2 - Locally produced commercial milk + all other locally produced non-milk exposure pathways

Diet 3 - Regionally mixed commercial milk + inhalation (other regionally mixed food items are minor contributors to the total)

Diet 4 - Goat milk (all other exposure pathways are of negligible importance compared to goat milk) 


\section{Location: Lawnville/Gallaher}

Receptor: Male born in 1954

\begin{tabular}{lccc}
\hline & \multicolumn{3}{c}{ Thyroid Dose [cGy] } \\
& \multicolumn{2}{c}{$95 \%$ Subjective Confidence Interval } \\
Exposure Pathway & lower limit & central estimate & upper limit \\
\hline Backyard Cow Milk & 1.9 & 12 & 79 \\
Commercial Milk (locally produced) & 0.44 & 3.8 & 25 \\
Commercial Milk (regionally mixed) & 0.1 & 0.8 & 5 \\
Goat Milk (locally produced) & 3.4 & 34 & 320 \\
Beef (locally produced) & 0.0018 & 0.034 & 0.71 \\
Leafy Vegetables (locally produced) & 0.00047 & 0.0057 & 0.056 \\
Eggs (locally produced) & 0.079 & 0.64 & 5.1 \\
Cottage Cheese (locally produced) & 0.0021 & 0.031 & 0.39 \\
Inhalation & 0.021 & 0.089 & 0.38 \\
Mother's milk (mother on Diet 1) & 0.0074 & 0.23 & 5.3 \\
Prenatal exposure (mother on Diet 1) & 0.074 & 0.78 & 5.8 \\
Diet 1 & 2.1 & 12 & 84 \\
Diet 2 & 0.65 & 5 & 32 \\
Diet 3 & 0.13 & 0.89 & 5.1 \\
\hline
\end{tabular}

Excess Lifetime Risk [ ]

\begin{tabular}{llll}
\hline Diet 1 & $3.2 \mathrm{E}-05$ & $1.0 \mathrm{E}-03$ & $1.9 \mathrm{E}-02$ \\
Diet 2 & $1.3 \mathrm{E}-05$ & $3.9 \mathrm{E}-04$ & $9.4 \mathrm{E}-03$ \\
Diet 3 & $2.7 \mathrm{E}-06$ & $7.0 \mathrm{E}-05$ & $1.6 \mathrm{E}-03$ \\
Diet 4 & $7.4 \mathrm{E}-05$ & $2.8 \mathrm{E}-03$ & $7.4 \mathrm{E}-02$ \\
\hline
\end{tabular}

\section{Relative Risk [ ]}

\begin{tabular}{lccc}
\hline Diet 1 & 1.039 & 1.66 & 15 \\
Diet 2 & 1.014 & 1.25 & 6.9 \\
Diet 3 & 1.0025 & 1.049 & 1.99 \\
Diet 4 & 1.073 & 2.9 & 59 \\
\hline
\end{tabular}

\begin{tabular}{lccc}
\hline Diet 1 & 3.80 & 39.7 & 93 \\
Diet 2 & 1.40 & 20.1 & 85 \\
Diet 3 & 0.25 & 4.6 & 50 \\
Diet 4 & 6.83 & 65.7 & 98 \\
\hline Diet 1 Backand
\end{tabular}

Diet 1 - Backyard cow milk + all other locally produced non-milk exposure pathways

Diet 2 - Locally produced commercial milk + all other locally produced non-milk exposure pathways

Diet 3 - Regionally mixed commercial milk + inhalation (other regionally mixed food items are minor contributors to the total)

Diet 4 - Goat milk (all other exposure pathways are of negligible importance compared to goat milk) 


\section{Location: Dyllis}

Receptor: Female born in 1954

\begin{tabular}{lccc}
\hline & \multicolumn{3}{c}{ Thyroid Dose [cGy] } \\
Exposure Pathway & \multicolumn{2}{c}{$95 \%$ Subjective Confidence Interval } \\
\hline Backyard Cow Milk & lower limit & central estimate & upper limit \\
Commercial Milk (locally produced) & 0.3 & 2.4 & 20 \\
Commercial Milk (regionally mixed) & 0.088 & 0.79 & 7.1 \\
Goat Milk (locally produced) & 0.094 & 0.81 & 5.7 \\
Beef (locally produced) & 0.65 & 7 & 75 \\
Leafy Vegetables (locally produced) & 0.00038 & 0.0065 & 0.15 \\
Eggs (locally produced) & 0.000096 & 0.0012 & 0.012 \\
Cottage Cheese (locally produced) & 0.013 & 0.12 & 1.2 \\
Inhalation & 0.00042 & 0.0059 & 0.09 \\
Mother's milk (mother on Diet 1) & 0.0041 & 0.018 & 0.095 \\
Prenatal exposure (mother on Diet 1) & 0.0013 & 0.049 & 1.1 \\
Diet 1 & 0.013 & 0.16 & 1.3 \\
Diet 2 & 0.34 & 2.5 & 22 \\
Diet 3 & 0.12 & 0.98 & 8.4 \\
\hline
\end{tabular}

Excess Lifetime Risk [ ]

\begin{tabular}{llll}
\hline Diet 1 & $4.9 \mathrm{E}-05$ & $8.2 \mathrm{E}-04$ & $1.3 \mathrm{E}-02$ \\
Diet 2 & $1.6 \mathrm{E}-05$ & $3.1 \mathrm{E}-04$ & $4.4 \mathrm{E}-03$ \\
Diet 3 & $1.4 \mathrm{E}-05$ & $2.4 \mathrm{E}-04$ & $3.3 \mathrm{E}-03$ \\
Diet 4 & $1.1 \mathrm{E}-04$ & $2.4 \mathrm{E}-03$ & $4.5 \mathrm{E}-02$ \\
\hline
\end{tabular}

\section{Relative Risk [ ]}

\begin{tabular}{lccc}
\hline Diet 1 & 1.02 & 1.19 & 4.2 \\
Diet 2 & 1.0069 & 1.071 & 2.2 \\
Diet 3 & 1.0053 & 1.059 & 1.97 \\
Diet 4 & 1.041 & 1.51 & 9.8 \\
\hline
\end{tabular}

\section{Probability of Causation [\%]}

\begin{tabular}{lccc}
\hline Diet 1 & 1.93 & 15.7 & 75 \\
Diet 2 & 0.68 & 6.6 & 54 \\
Diet 3 & 0.53 & 5.6 & 49 \\
Diet 4 & 3.97 & 33.9 & 90 \\
\hline
\end{tabular}

Diet 1 - Backyard cow milk + all other locally produced non-milk exposure pathways

Diet 2 - Locally produced commercial milk + all other locally produced non-milk exposure pathways

Diet 3 - Regionally mixed commercial milk + inhalation (other regionally mixed food items are minor contributors to the total)

Diet 4 - Goat milk (all other exposure pathways are of negligible importance compared to goat milk) 


\section{Location: Dyllis}

\section{Receptor: Male born in 1954}

\begin{tabular}{lccc}
\hline & \multicolumn{3}{c}{ Thyroid Dose [cGy] } \\
& \multicolumn{2}{c}{$95 \%$ Subjective Confidence Interval } \\
Exposure Pathway & lower limit & central estimate & upper limit \\
\hline Backyard Cow Milk & 0.34 & 2.3 & 18 \\
Commercial Milk (locally produced) & 0.087 & 0.79 & 6.5 \\
Commercial Milk (regionally mixed) & 0.1 & 0.8 & 5 \\
Goat Milk (locally produced) & 0.73 & 6.9 & 77 \\
Beef (locally produced) & 0.00038 & 0.0064 & 0.15 \\
Leafy Vegetables (locally produced) & 0.000089 & 0.0011 & 0.014 \\
Eggs (locally produced) & 0.015 & 0.13 & 1.1 \\
Cottage Cheese (locally produced) & 0.00042 & 0.0063 & 0.093 \\
Inhalation & 0.0044 & 0.018 & 0.086 \\
Mother's milk (mother on Diet 1) & 0.0013 & 0.049 & 1.1 \\
Prenatal exposure (mother on Diet 1) & 0.013 & 0.16 & 1.3 \\
Diet 1 & 0.37 & 2.5 & 19 \\
Diet 2 & 0.12 & 0.99 & 8 \\
Diet 3 & 0.11 & 0.82 & 5 \\
\hline
\end{tabular}

Excess Lifetime Risk [ ]

\begin{tabular}{lllc}
\hline Diet 1 & $6.3 \mathrm{E}-06$ & $2.1 \mathrm{E}-04$ & $4.1 \mathrm{E}-03$ \\
Diet 2 & $2.7 \mathrm{E}-06$ & $8.1 \mathrm{E}-05$ & $1.7 \mathrm{E}-03$ \\
Diet 3 & $2.2 \mathrm{E}-06$ & $6.2 \mathrm{E}-05$ & $1.5 \mathrm{E}-03$ \\
Diet 4 & $1.5 \mathrm{E}-05$ & $6.0 \mathrm{E}-04$ & $1.6 \mathrm{E}-02$ \\
\hline
\end{tabular}

\section{Relative Risk [ ]}

\begin{tabular}{lccc}
\hline Diet 1 & 1.0077 & 1.15 & 4.5 \\
Diet 2 & 1.0027 & 1.055 & 2.3 \\
Diet 3 & 1.0023 & 1.044 & 1.95 \\
Diet 4 & 1.016 & 1.39 & 13 \\
\hline
\end{tabular}

\begin{tabular}{lccc}
\hline Diet 1 & 0.76 & 12.7 & 77 \\
Diet 2 & 0.27 & 5.2 & 55 \\
Diet 3 & 0.23 & 4.2 & 49 \\
Diet 4 & 1.56 & 28.1 & 92 \\
\hline
\end{tabular}

Diet 1 - Backyard cow milk + all other locally produced non-milk exposure pathways

Diet 2 - Locally produced commercial milk + all other locally produced non-milk exposure pathways

Diet 3 - Regionally mixed commercial milk + inhalation (other regionally mixed food items are minor contributors to the total)

Diet 4 - Goat milk (all other exposure pathways are of negligible importance compared to goat milk) 


\section{Location: OR High School Area}

Receptor: Female born in 1954

\begin{tabular}{lccc}
\hline & \multicolumn{3}{c}{ Thyroid Dose [cGy] } \\
Exposure Pathway & \multicolumn{2}{c}{$9 \%$ Subjective Confidence Interval } \\
\hline Backyard Cow Milk & lower limit & central estimate & upper limit \\
Commercial Milk (locally produced) & -- & -- & -- \\
Commercial Milk (regionally mixed) & -- & -- & -- \\
Goat Milk (locally produced) & 0.095 & 0.81 & 5.7 \\
Beef (locally produced) & -- & -- & -- \\
Leafy Vegetables (locally produced) & -- & -- & -- \\
Eggs (locally produced) & -- & -- & -- \\
Cottage Cheese (locally produced) & -- & -- & -- \\
Inhalation & 0.00073 & 0.014 & 0.18 \\
Mother's milk (mother on Diet 3) & 0.0084 & 0.039 & 0.19 \\
Prenatal exposure (mother on Diet 3) & 0.00064 & 0.014 & 0.25 \\
Diet 1 & 0.0069 & 0.043 & 0.35 \\
Diet 2 & -- & -- & -- \\
Diet 3 & -- & -- & -- \\
\hline
\end{tabular}

Excess Lifetime Risk [ ]

\begin{tabular}{lccc}
\hline Diet 1 & -- & -- & -- \\
Diet 2 & -- & -- & -- \\
Diet 3 & $1.6 \mathrm{E}-05$ & $2.5 \mathrm{E}-04$ & $3.3 \mathrm{E}-03$ \\
Diet 4 & -- & -- & -- \\
\hline
\end{tabular}

\section{Relative Risk [ ]}

\begin{tabular}{lccc}
\hline Diet 1 & -- & -- & -- \\
Diet 2 & -- & -- & -- \\
Diet 3 & 1.0056 & 1.061 & 1.98 \\
Diet 4 & -- & -- & -- \\
\hline
\end{tabular}

\begin{tabular}{lccc}
\hline Diet 1 & -- & -- & -- \\
Diet 2 & -- & -- & -- \\
Diet 3 & 0.56 & 5.8 & -- \\
Diet 4 & -- & -- & - \\
\hline Diet 1 - Backyard cow milk + all other locally produced non-milk exposure pathways & \\
Diet 2 - Locally produced commercial milk + all other locally produced non-milk exposure pathways \\
Diet 3 - Regionally mixed commercial milk + inhalation (other regionally mixed food items are minor contributors to the total) \\
Diet 4 - Goat milk (all other exposure pathways are of negligible importance compared to goat milk)
\end{tabular}




\section{Location: OR High School Area}

Receptor: Male born in 1954

\begin{tabular}{lccc}
\hline & \multicolumn{3}{c}{ Thyroid Dose [cGy] } \\
Exposure Pathway & \multicolumn{2}{c}{$95 \%$ Subjective Confidence Interval } \\
\hline Backyard Cow Milk & lower limit & central estimate & upper limit \\
Commercial Milk (locally produced) & -- & -- & -- \\
Commercial Milk (regionally mixed) & -- & -- & -- \\
Goat Milk (locally produced) & 0.1 & 0.8 & 5 \\
Beef (locally produced) & -- & -- & -- \\
Leafy Vegetables (locally produced) & -- & -- & -- \\
Eggs (locally produced) & -- & -- & -- \\
Cottage Cheese (locally produced) & -- & -- & -- \\
Inhalation & 0.00092 & 0.014 & 0.16 \\
Mother's milk (mother on Diet 3) & 0.0095 & 0.039 & 0.17 \\
Prenatal exposure (mother on Diet 3) & 0.00064 & 0.014 & 0.25 \\
Diet 1 & 0.0069 & 0.043 & 0.35 \\
Diet 2 & -- & -- & -- \\
Diet 3 & -- & -- & -- \\
\hline
\end{tabular}

Excess Lifetime Risk [ ]

\begin{tabular}{lccc}
\hline Diet 1 & -- & -- & -- \\
Diet 2 & -- & -- & -- \\
Diet 3 & $2.4 \mathrm{E}-06$ & $6.5 \mathrm{E}-05$ & $1.5 \mathrm{E}-03$ \\
Diet 4 & -- & -- & -- \\
\hline
\end{tabular}

\section{Relative Risk [ ]}

\begin{tabular}{lccc}
\hline Diet 1 & -- & -- & -- \\
Diet 2 & -- & -- & -- \\
Diet 3 & 1.0024 & 1.045 & 1.96 \\
Diet 4 & -- & -- & -- \\
\hline
\end{tabular}

\begin{tabular}{lccc}
\hline Diet 1 & -- & -- & -- \\
Diet 2 & -- & -- & -- \\
Diet 3 & 0.24 & 4.3 & 49 \\
Diet 4 & -- & -- \\
\hline Diet 1 - Backyard cow milk + all other locally produced non-milk exposure pathways & \\
Diet 2 - Locally produced commercial milk + all other locally produced non-milk exposure pathways \\
Diet 3 - Regionally mixed commercial milk + inhalation (other regionally mixed food items are minor contributors to the total) \\
Diet 4 - Goat milk (all other exposure pathways are of negligible importance compared to goat milk)
\end{tabular}




\section{Location: Norwood}

Receptor: Female born in 1954

\begin{tabular}{lccc}
\hline & \multicolumn{3}{c}{ Thyroid Dose [cGy] } \\
Exposure Pathway & \multicolumn{2}{c}{$95 \%$ Subjective Confidence Interval } \\
\hline Backyard Cow Milk & lower limit & central estimate & upper limit \\
Commercial Milk (locally produced) & 0.3 & 2.3 & 20 \\
Commercial Milk (regionally mixed) & 0.089 & 0.75 & 7 \\
Goat Milk (locally produced) & 0.094 & 0.81 & 5.7 \\
Beef (locally produced) & 0.58 & 6.9 & 72 \\
Leafy Vegetables (locally produced) & 0.00031 & 0.0063 & 0.18 \\
Eggs (locally produced) & 0.0001 & 0.0011 & 0.012 \\
Cottage Cheese (locally produced) & 0.013 & 0.12 & 1.1 \\
Inhalation & 0.00038 & 0.0059 & 0.097 \\
Mother's milk (mother on Diet 1) & 0.0039 & 0.018 & 0.085 \\
Prenatal exposure (mother on Diet 1) & 0.0013 & 0.048 & 0.94 \\
Diet 1 & 0.013 & 0.15 & 1.2 \\
Diet 2 & 0.33 & 2.5 & 23 \\
Diet 3 & 0.13 & 0.92 & 8.2 \\
\hline
\end{tabular}

Excess Lifetime Risk [ ]

\begin{tabular}{llll}
\hline Diet 1 & $4.0 \mathrm{E}-05$ & $7.7 \mathrm{E}-04$ & $1.4 \mathrm{E}-02$ \\
Diet 2 & $1.9 \mathrm{E}-05$ & $2.9 \mathrm{E}-04$ & $4.6 \mathrm{E}-03$ \\
Diet 3 & $1.4 \mathrm{E}-05$ & $2.4 \mathrm{E}-04$ & $3.2 \mathrm{E}-03$ \\
Diet 4 & $1.0 \mathrm{E}-04$ & $2.3 \mathrm{E}-03$ & $4.2 \mathrm{E}-02$ \\
\hline
\end{tabular}

\section{Relative Risk [ ]}

\begin{tabular}{lccc}
\hline Diet 1 & 1.016 & 1.19 & 3.6 \\
Diet 2 & 1.0059 & 1.069 & 2.2 \\
Diet 3 & 1.0053 & 1.059 & 1.97 \\
Diet 4 & 1.04 & 1.5 & 10.2 \\
\hline
\end{tabular}

\section{Probability of Causation [\%]}

\begin{tabular}{lccc}
\hline Diet 1 & 1.62 & 16.3 & 72 \\
Diet 2 & 0.58 & 6.4 & 54 \\
Diet 3 & 0.52 & 5.6 & 49 \\
Diet 4 & 3.88 & 33.4 & 90 \\
\hline
\end{tabular}

Diet 1 - Backyard cow milk + all other locally produced non-milk exposure pathways

Diet 2 - Locally produced commercial milk + all other locally produced non-milk exposure pathways

Diet 3 - Regionally mixed commercial milk + inhalation (other regionally mixed food items are minor contributors to the total)

Diet 4 - Goat milk (all other exposure pathways are of negligible importance compared to goat milk) 


\section{Location: Norwood}

\section{Receptor: Male born in 1954}

\begin{tabular}{lccc}
\hline & \multicolumn{3}{c}{ Thyroid Dose [cGy] } \\
& \multicolumn{2}{c}{$95 \%$ Subjective Confidence Interval } \\
Exposure Pathway & lower limit & central estimate & upper limit \\
\hline Backyard Cow Milk & 0.33 & 2.2 & 18 \\
Commercial Milk (locally produced) & 0.087 & 0.72 & 7.2 \\
Commercial Milk (regionally mixed) & 0.1 & 0.8 & 5 \\
Goat Milk (locally produced) & 0.67 & 6.8 & 73 \\
Beef (locally produced) & 0.00034 & 0.0062 & 0.16 \\
Leafy Vegetables (locally produced) & 0.000094 & 0.0011 & 0.012 \\
Eggs (locally produced) & 0.014 & 0.12 & 1.3 \\
Cottage Cheese (locally produced) & 0.0004 & 0.006 & 0.083 \\
Inhalation & 0.0037 & 0.018 & 0.077 \\
Mother's milk (mother on Diet 1) & 0.0013 & 0.048 & 0.94 \\
Prenatal exposure (mother on Diet 1) & 0.013 & 0.15 & 1.2 \\
Diet 1 & 0.36 & 2.4 & 19 \\
Diet 2 & 0.13 & 0.94 & 8.1 \\
Diet 3 & 0.11 & 0.82 & 5 \\
\hline
\end{tabular}

Excess Lifetime Risk [ ]

\begin{tabular}{llll}
\hline Diet 1 & $6.3 \mathrm{E}-06$ & $1.8 \mathrm{E}-04$ & $3.9 \mathrm{E}-03$ \\
Diet 2 & $2.4 \mathrm{E}-06$ & $7.1 \mathrm{E}-05$ & $1.8 \mathrm{E}-03$ \\
Diet 3 & $2.2 \mathrm{E}-06$ & $6.1 \mathrm{E}-05$ & $1.5 \mathrm{E}-03$ \\
Diet 4 & $1.3 \mathrm{E}-05$ & $5.7 \mathrm{E}-04$ & $1.4 \mathrm{E}-02$ \\
\hline
\end{tabular}

\section{Relative Risk [ ]}

\begin{tabular}{lccc}
\hline Diet 1 & 1.0066 & 1.13 & 4.4 \\
Diet 2 & 1.0027 & 1.051 & 2.5 \\
Diet 3 & 1.0022 & 1.044 & 1.95 \\
Diet 4 & 1.015 & 1.36 & 12 \\
\hline
\end{tabular}

\section{Probability of Causation [\%]}

\begin{tabular}{lccc}
\hline Diet 1 & 0.65 & 11.7 & 77 \\
Diet 2 & 0.27 & 4.8 & 59 \\
Diet 3 & 0.22 & 4.2 & 49 \\
Diet 4 & 1.45 & 26.6 & 92 \\
\hline
\end{tabular}

Diet 1 - Backyard cow milk + all other locally produced non-milk exposure pathways

Diet 2 - Locally produced commercial milk + all other locally produced non-milk exposure pathways

Diet 3 - Regionally mixed commercial milk + inhalation (other regionally mixed food items are minor contributors to the total)

Diet 4 - Goat milk (all other exposure pathways are of negligible importance compared to goat milk) 
Location: Woodland

Receptor: Female born in 1954

\begin{tabular}{lccc}
\hline & \multicolumn{3}{c}{ Thyroid Dose [cGy] } \\
Exposure Pathway & \multicolumn{2}{c}{$95 \%$ Subjective Confidence Interval } \\
\hline Backyard Cow Milk & lower limit & central estimate & upper limit \\
Commercial Milk (locally produced) & -- & -- & -- \\
Commercial Milk (regionally mixed) & -- & -- & -- \\
Goat Milk (locally produced) & 0.094 & 0.81 & 5.7 \\
Beef (locally produced) & -- & -- & -- \\
Leafy Vegetables (locally produced) & -- & -- & -- \\
Eggs (locally produced) & -- & -- & -- \\
Cottage Cheese (locally produced) & -- & -- & -- \\
Inhalation & 0.0012 & 0.02 & 0.26 \\
Mother's milk (mother on Diet 3) & 0.013 & 0.06 & 0.26 \\
Prenatal exposure (mother on Diet 3) & 0.00079 & 0.017 & 0.26 \\
Diet 1 & 0.0087 & 0.049 & 0.37 \\
Diet 2 & -- & -- & -- \\
Diet 3 & -- & -- & -- \\
\hline
\end{tabular}

Excess Lifetime Risk [ ]

\begin{tabular}{lccc}
\hline Diet 1 & -- & -- & -- \\
Diet 2 & -- & -- & -- \\
Diet 3 & $1.8 \mathrm{E}-05$ & $2.6 \mathrm{E}-04$ & $3.4 \mathrm{E}-03$ \\
Diet 4 & -- & -- & -- \\
\hline
\end{tabular}

\section{Relative Risk [ ]}

\begin{tabular}{lccc}
\hline Diet 1 & -- & -- & -- \\
Diet 2 & -- & -- & -- \\
Diet 3 & 1.006 & 1.063 & 1.99 \\
Diet 4 & -- & -- & -- \\
\hline
\end{tabular}

\begin{tabular}{lccc}
\hline Diet 1 & -- & -- & -- \\
Diet 2 & -- & -- & -- \\
Diet 3 & 0.60 & 5.9 & 50 \\
Diet 4 & -- & -- \\
\hline Diet 1 - Backyard cow milk + all other locally produced non-milk exposure pathways & \\
Diet 2 - Locally produced commercial milk + all other locally produced non-milk exposure pathways \\
Diet 3 - Regionally mixed commercial milk + inhalation (other regionally mixed food items are minor contributors to the total) \\
Diet 4 - Goat milk (all other exposure pathways are of negligible importance compared to goat milk)
\end{tabular}


Location: Woodland

Receptor: Male born in 1954

\begin{tabular}{lccc}
\hline & \multicolumn{3}{c}{ Thyroid Dose [cGy] } \\
Exposure Pathway & \multicolumn{2}{c}{$9 \%$ Subjective Confidence Interval } \\
\hline Backyard Cow Milk & lower limit & central estimate & upper limit \\
Commercial Milk (locally produced) & -- & -- & -- \\
Commercial Milk (regionally mixed) & -- & -- & -- \\
Goat Milk (locally produced) & 0.1 & 0.8 & 5 \\
Beef (locally produced) & -- & -- & -- \\
Leafy Vegetables (locally produced) & -- & -- & -- \\
Eggs (locally produced) & -- & -- & -- \\
Cottage Cheese (locally produced) & -- & -- & -- \\
Inhalation & 0.0014 & 0.021 & 0.25 \\
Mother's milk (mother on Diet 3) & 0.014 & 0.06 & 0.25 \\
Prenatal exposure (mother on Diet 3) & 0.00079 & 0.017 & 0.26 \\
Diet 1 & 0.0087 & 0.049 & 0.37 \\
Diet 2 & -- & -- & -- \\
Diet 3 & -- & -- & -- \\
\hline
\end{tabular}

Excess Lifetime Risk [ ]

\begin{tabular}{lccc}
\hline Diet 1 & -- & -- & -- \\
Diet 2 & -- & -- & -- \\
Diet 3 & $2.5 \mathrm{E}-06$ & $6.7 \mathrm{E}-05$ & $1.5 \mathrm{E}-03$ \\
Diet 4 & -- & -- & -- \\
\hline
\end{tabular}

\section{Relative Risk [ ]}

\begin{tabular}{lccc}
\hline Diet 1 & -- & -- & -- \\
Diet 2 & -- & -- & -- \\
Diet 3 & 1.0024 & 1.047 & 1.97 \\
Diet 4 & -- & -- & -- \\
\hline
\end{tabular}

\begin{tabular}{lccc}
\hline Diet 1 & -- & -- & -- \\
Diet 2 & -- & -- & -- \\
Diet 3 & 0.24 & 4.5 & -- \\
Diet 4 & -- & -- & \\
\hline Diet 1 - Backyard cow milk + all other locally produced non-milk exposure pathways & \\
Diet 2 - Locally produced commercial milk + all other locally produced non-milk exposure pathways \\
Diet 3 - Regionally mixed commercial milk + inhalation (other regionally mixed food items are minor contributors to the total) \\
Diet 4 - Goat milk (all other exposure pathways are of negligible importance compared to goat milk)
\end{tabular}


Location: Hardin Valley

Receptor: Female born in 1954

\begin{tabular}{lccc}
\hline & \multicolumn{3}{c}{ Thyroid Dose [cGy] } \\
Exposure Pathway & \multicolumn{2}{c}{$95 \%$ Subjective Confidence Interval } \\
Backyard Cow Milk & lower limit & central estimate & upper limit \\
Commercial Milk (locally produced) & 1.6 & 11 & 87 \\
Commercial Milk (regionally mixed) & 0.42 & 3.7 & 28 \\
Goat Milk (locally produced) & 0.094 & 0.81 & 5.7 \\
Beef (locally produced) & 2.9 & 32 & 300 \\
Leafy Vegetables (locally produced) & 0.0017 & 0.032 & 0.66 \\
Eggs (locally produced) & 0.00048 & 0.0055 & 0.056 \\
Cottage Cheese (locally produced) & 0.071 & 0.58 & 4.8 \\
Inhalation & 0.002 & 0.028 & 0.35 \\
Mother's milk (mother on Diet 1) & 0.019 & 0.085 & 0.41 \\
Prenatal exposure (mother on Diet 1) & 0.0064 & 0.22 & 4.8 \\
Diet 1 & 0.065 & 0.75 & 5.7 \\
Diet 2 & 1.7 & 12 & 94 \\
Diet 3 & 0.62 & 4.8 & 33 \\
\hline
\end{tabular}

Excess Lifetime Risk [ ]

\begin{tabular}{llll}
\hline Diet 1 & $2.4 \mathrm{E}-04$ & $3.7 \mathrm{E}-03$ & $5.7 \mathrm{E}-02$ \\
Diet 2 & $8.3 \mathrm{E}-05$ & $1.4 \mathrm{E}-03$ & $2.0 \mathrm{E}-02$ \\
Diet 3 & $2.0 \mathrm{E}-05$ & $2.7 \mathrm{E}-04$ & $3.5 \mathrm{E}-03$ \\
Diet 4 & $5.1 \mathrm{E}-04$ & $1.1 \mathrm{E}-02$ & $1.9 \mathrm{E}-01$ \\
\hline
\end{tabular}

\section{Relative Risk [ ]}

\begin{tabular}{lccc}
\hline Diet 1 & 1.085 & 1.87 & 14 \\
Diet 2 & 1.032 & 1.33 & 5.9 \\
Diet 3 & 1.0066 & 1.066 & 2 \\
Diet 4 & 1.2 & 3.4 & 38 \\
\hline
\end{tabular}

\begin{tabular}{|c|c|c|c|}
\hline Diet 1 & 7.86 & 46.4 & 93 \\
\hline Diet 2 & 3.12 & 24.9 & 83 \\
\hline Diet 3 & 0.66 & 6.2 & 50 \\
\hline Diet 4 & 16.59 & 70.9 & 97 \\
\hline
\end{tabular}

Diet 1 - Backyard cow milk + all other locally produced non-milk exposure pathways

Diet 2 - Locally produced commercial milk + all other locally produced non-milk exposure pathways

Diet 3 - Regionally mixed commercial milk + inhalation (other regionally mixed food items are minor contributors to the total)

Diet 4 - Goat milk (all other exposure pathways are of negligible importance compared to goat milk) 
Location: Hardin Valley

Receptor: Male born in 1954

\begin{tabular}{lccc}
\hline & \multicolumn{3}{c}{ Thyroid Dose [cGy] } \\
& \multicolumn{2}{c}{$95 \%$ Subjective Confidence Interval } \\
Exposure Pathway & lower limit & central estimate & upper limit \\
\hline Backyard Cow Milk & 1.7 & 11 & 80 \\
Commercial Milk (locally produced) & 0.42 & 3.6 & 25 \\
Commercial Milk (regionally mixed) & 0.1 & 0.8 & 5 \\
Goat Milk (locally produced) & 3.5 & 33 & 300 \\
Beef (locally produced) & 0.0017 & 0.031 & 0.71 \\
Leafy Vegetables (locally produced) & 0.00044 & 0.0053 & 0.059 \\
Eggs (locally produced) & 0.078 & 0.59 & 5 \\
Cottage Cheese (locally produced) & 0.0019 & 0.029 & 0.4 \\
Inhalation & 0.021 & 0.086 & 0.38 \\
Mother's milk (mother on Diet 1) & 0.0064 & 0.22 & 4.8 \\
Prenatal exposure (mother on Diet 1) & 0.065 & 0.75 & 5.7 \\
Diet 1 & 1.8 & 12 & 86 \\
Diet 2 & 0.62 & 4.6 & 31 \\
Diet 3 & 0.13 & 0.89 & 5.1 \\
\hline
\end{tabular}

Excess Lifetime Risk [ ]

\begin{tabular}{llll}
\hline Diet 1 & $3.0 \mathrm{E}-05$ & $9.9 \mathrm{E}-04$ & $1.8 \mathrm{E}-02$ \\
Diet 2 & $1.3 \mathrm{E}-05$ & $3.7 \mathrm{E}-04$ & $8.2 \mathrm{E}-03$ \\
Diet 3 & $2.7 \mathrm{E}-06$ & $7.0 \mathrm{E}-05$ & $1.6 \mathrm{E}-03$ \\
Diet 4 & $7.0 \mathrm{E}-05$ & $2.8 \mathrm{E}-03$ & $7.0 \mathrm{E}-02$ \\
\hline
\end{tabular}

\section{Relative Risk [ ]}

\begin{tabular}{lccc}
\hline Diet 1 & 1.038 & 1.64 & 15 \\
Diet 2 & 1.013 & 1.25 & 6.5 \\
Diet 3 & 1.0025 & 1.049 & 1.98 \\
Diet 4 & 1.071 & 2.7 & 55 \\
\hline
\end{tabular}

\section{Probability of Causation [\%]}

\begin{tabular}{lccc}
\hline Diet 1 & 3.70 & 39.0 & 93 \\
Diet 2 & 1.30 & 20.2 & 84 \\
Diet 3 & 0.25 & 4.6 & 50 \\
Diet 4 & 6.64 & 63.4 & 98 \\
\hline
\end{tabular}

Diet 1 - Backyard cow milk + all other locally produced non-milk exposure pathways

Diet 2 - Locally produced commercial milk + all other locally produced non-milk exposure pathways

Diet 3 - Regionally mixed commercial milk + inhalation (other regionally mixed food items are minor contributors to the total)

Diet 4 - Goat milk (all other exposure pathways are of negligible importance compared to goat milk) 


\section{Location: Oliver Springs}

Receptor: Female born in 1954

\begin{tabular}{lccc}
\hline & \multicolumn{3}{c}{ Thyroid Dose [cGy] } \\
Exposure Pathway & $95 \%$ Subjective Confidence Interval \\
\hline Backyard Cow Milk & lower limit & central estimate & upper limit \\
Commercial Milk (locally produced) & 0.23 & 1.7 & 14 \\
Commercial Milk (regionally mixed) & 0.067 & 0.57 & 5.1 \\
Goat Milk (locally produced) & 0.094 & 0.81 & 5.7 \\
Beef (locally produced) & 0.46 & 5.1 & 55 \\
Leafy Vegetables (locally produced) & 0.00023 & 0.0051 & 0.12 \\
Eggs (locally produced) & 0.000068 & 0.00085 & 0.0094 \\
Cottage Cheese (locally produced) & 0.01 & 0.093 & 0.91 \\
Inhalation & 0.00027 & 0.0046 & 0.069 \\
Mother's milk (mother on Diet 1) & 0.0031 & 0.013 & 0.068 \\
Prenatal exposure (mother on Diet 1) & 0.00098 & 0.035 & 0.75 \\
Diet 1 & 0.0099 & 0.12 & 0.95 \\
Diet 2 & 0.25 & 1.8 & 15 \\
Diet 3 & 0.097 & 0.72 & 5.9 \\
\hline
\end{tabular}

Excess Lifetime Risk [ ]

\begin{tabular}{lllc}
\hline Diet 1 & $3.3 \mathrm{E}-05$ & $5.8 \mathrm{E}-04$ & $1.1 \mathrm{E}-02$ \\
Diet 2 & $1.4 \mathrm{E}-05$ & $2.1 \mathrm{E}-04$ & $3.4 \mathrm{E}-03$ \\
Diet 3 & $1.4 \mathrm{E}-05$ & $2.3 \mathrm{E}-04$ & $3.2 \mathrm{E}-03$ \\
Diet 4 & $7.1 \mathrm{E}-05$ & $1.7 \mathrm{E}-03$ & $2.9 \mathrm{E}-02$ \\
\hline
\end{tabular}

\section{Relative Risk [ ]}

\begin{tabular}{lccc}
\hline Diet 1 & 1.012 & 1.14 & 3 \\
Diet 2 & 1.0042 & 1.054 & 1.85 \\
Diet 3 & 1.0052 & 1.059 & 1.97 \\
Diet 4 & 1.027 & 1.4 & 7.3 \\
\hline
\end{tabular}

\section{Probability of Causation [\%]}

\begin{tabular}{lccc}
\hline Diet 1 & 1.14 & 12.6 & 66 \\
Diet 2 & 0.42 & 5.1 & 46 \\
Diet 3 & 0.51 & 5.6 & 49 \\
Diet 4 & 2.61 & 28.2 & 86 \\
\hline
\end{tabular}

Diet 1 - Backyard cow milk + all other locally produced non-milk exposure pathways

Diet 2 - Locally produced commercial milk + all other locally produced non-milk exposure pathways

Diet 3 - Regionally mixed commercial milk + inhalation (other regionally mixed food items are minor contributors to the total)

Diet 4 - Goat milk (all other exposure pathways are of negligible importance compared to goat milk) 


\section{Location: Oliver Springs}

\section{Receptor: Male born in 1954}

\begin{tabular}{lccc}
\hline & \multicolumn{3}{c}{ Thyroid Dose [cGy] } \\
& \multicolumn{2}{c}{$95 \%$ Subjective Confidence Interval } \\
Exposure Pathway & lower limit & central estimate & upper limit \\
\hline Backyard Cow Milk & 0.25 & 1.7 & 13 \\
Commercial Milk (locally produced) & 0.066 & 0.56 & 4.6 \\
Commercial Milk (regionally mixed) & 0.1 & 0.8 & 5 \\
Goat Milk (locally produced) & 0.47 & 5.3 & 55 \\
Beef (locally produced) & 0.00025 & 0.005 & 0.13 \\
Leafy Vegetables (locally produced) & 0.00006 & 0.00082 & 0.0097 \\
Eggs (locally produced) & 0.011 & 0.095 & 0.92 \\
Cottage Cheese (locally produced) & 0.00031 & 0.0047 & 0.06 \\
Inhalation & 0.003 & 0.014 & 0.065 \\
Mother's milk (mother on Diet 1) & 0.00098 & 0.035 & 0.75 \\
Prenatal exposure (mother on Diet 1) & 0.0099 & 0.12 & 0.95 \\
Diet 1 & 0.27 & 1.8 & 14 \\
Diet 2 & 0.097 & 0.73 & 5.8 \\
Diet 3 & 0.11 & 0.82 & 5 \\
\hline
\end{tabular}

Excess Lifetime Risk [ ]

\begin{tabular}{llll}
\hline Diet 1 & $4.0 \mathrm{E}-06$ & $1.5 \mathrm{E}-04$ & $3.0 \mathrm{E}-03$ \\
Diet 2 & $1.8 \mathrm{E}-06$ & $5.5 \mathrm{E}-05$ & $1.2 \mathrm{E}-03$ \\
Diet 3 & $2.2 \mathrm{E}-06$ & $6.0 \mathrm{E}-05$ & $1.5 \mathrm{E}-03$ \\
Diet 4 & $1.1 \mathrm{E}-05$ & $4.2 \mathrm{E}-04$ & $1.1 \mathrm{E}-02$ \\
\hline
\end{tabular}

\section{Relative Risk [ ]}

\begin{tabular}{lccc}
\hline Diet 1 & 1.0049 & 1.099 & 3.3 \\
Diet 2 & 1.0019 & 1.038 & 1.99 \\
Diet 3 & 1.0022 & 1.043 & 1.95 \\
Diet 4 & 1.0098 & 1.28 & 9.6 \\
\hline
\end{tabular}

\section{Probability of Causation [\%]}

\begin{tabular}{lccc}
\hline Diet 1 & 0.48 & 9.0 & 69 \\
Diet 2 & 0.19 & 3.6 & 49 \\
Diet 3 & 0.22 & 4.2 & 49 \\
Diet 4 & 0.97 & 21.8 & 89 \\
\hline
\end{tabular}

Diet 1 - Backyard cow milk + all other locally produced non-milk exposure pathways

Diet 2 - Locally produced commercial milk + all other locally produced non-milk exposure pathways

Diet 3 - Regionally mixed commercial milk + inhalation (other regionally mixed food items are minor contributors to the total)

Diet 4 - Goat milk (all other exposure pathways are of negligible importance compared to goat milk) 


\section{Location: Solway}

Receptor: Female born in 1954

\begin{tabular}{lccc}
\hline & \multicolumn{3}{c}{ Thyroid Dose [cGy] } \\
& \multicolumn{2}{c}{$95 \%$ Subjective Confidence Interval } \\
Exposure Pathway & lower limit & central estimate & upper limit \\
\hline Backyard Cow Milk & 1.5 & 10 & 81 \\
Commercial Milk (locally produced) & 0.41 & 3.3 & 26 \\
Commercial Milk (regionally mixed) & 0.094 & 0.81 & 5.7 \\
Goat Milk (locally produced) & 2.8 & 29 & 280 \\
Beef (locally produced) & 0.0015 & 0.03 & 0.62 \\
Leafy Vegetables (locally produced) & 0.00042 & 0.0051 & 0.05 \\
Eggs (locally produced) & 0.069 & 0.52 & 4.5 \\
Cottage Cheese (locally produced) & 0.0018 & 0.026 & 0.33 \\
Inhalation & 0.019 & 0.079 & 0.35 \\
Mother's milk (mother on Diet 1) & 0.0062 & 0.21 & 4.3 \\
Prenatal exposure (mother on Diet 1) & 0.062 & 0.67 & 5.3 \\
Diet 1 & 1.6 & 11 & 86 \\
Diet 2 & 0.59 & 4.2 & 30 \\
Diet 3 & 0.13 & 0.89 & 6 \\
\hline
\end{tabular}

Excess Lifetime Risk [ ]

\begin{tabular}{llll}
\hline Diet 1 & $2.1 \mathrm{E}-04$ & $3.5 \mathrm{E}-03$ & $4.9 \mathrm{E}-02$ \\
Diet 2 & $7.7 \mathrm{E}-05$ & $1.2 \mathrm{E}-03$ & $1.8 \mathrm{E}-02$ \\
Diet 3 & $2.0 \mathrm{E}-05$ & $2.7 \mathrm{E}-04$ & $3.5 \mathrm{E}-03$ \\
Diet 4 & $4.3 \mathrm{E}-04$ & $9.8 \mathrm{E}-03$ & $1.6 \mathrm{E}-01$ \\
\hline
\end{tabular}

\section{Relative Risk [ ]}

\begin{tabular}{lccc}
\hline Diet 1 & 1.079 & 1.8 & 12 \\
Diet 2 & 1.028 & 1.32 & 5.6 \\
Diet 3 & 1.0065 & 1.065 & 2 \\
Diet 4 & 1.18 & 3.3 & 36 \\
\hline
\end{tabular}

\section{Probability of Causation [\%]}

\begin{tabular}{lccc}
\hline Diet 1 & 7.32 & 44.3 & 92 \\
Diet 2 & 2.71 & 24.0 & 82 \\
Diet 3 & 0.64 & 6.1 & 50 \\
Diet 4 & 15.09 & 69.4 & 97 \\
\hline
\end{tabular}

Diet 1 - Backyard cow milk + all other locally produced non-milk exposure pathways

Diet 2 - Locally produced commercial milk + all other locally produced non-milk exposure pathways

Diet 3 - Regionally mixed commercial milk + inhalation (other regionally mixed food items are minor contributors to the total)

Diet 4 - Goat milk (all other exposure pathways are of negligible importance compared to goat milk) 


\section{Location: Solway}

\section{Receptor: Male born in 1954}

\begin{tabular}{lccc}
\hline & \multicolumn{3}{c}{ Thyroid Dose [cGy] } \\
& \multicolumn{2}{c}{$95 \%$ Subjective Confidence Interval } \\
Exposure Pathway & lower limit & central estimate & upper limit \\
\hline Backyard Cow Milk & 1.5 & 10 & 70 \\
Commercial Milk (locally produced) & 0.41 & 3.3 & 22 \\
Commercial Milk (regionally mixed) & 0.1 & 0.8 & 5 \\
Goat Milk (locally produced) & 3.1 & 29 & 270 \\
Beef (locally produced) & 0.0015 & 0.029 & 0.65 \\
Leafy Vegetables (locally produced) & 0.00039 & 0.0049 & 0.053 \\
Eggs (locally produced) & 0.068 & 0.52 & 4.4 \\
Cottage Cheese (locally produced) & 0.0017 & 0.027 & 0.36 \\
Inhalation & 0.019 & 0.079 & 0.35 \\
Mother's milk (mother on Diet 1) & 0.0062 & 0.21 & 4.3 \\
Prenatal exposure (mother on Diet 1) & 0.062 & 0.67 & 5.3 \\
Diet 1 & 1.7 & 11 & 74 \\
Diet 2 & 0.6 & 4.2 & 27 \\
Diet 3 & 0.13 & 0.88 & 5.1 \\
\hline
\end{tabular}

Excess Lifetime Risk [ ]

\begin{tabular}{llll}
\hline Diet 1 & $2.8 \mathrm{E}-05$ & $8.8 \mathrm{E}-04$ & $1.6 \mathrm{E}-02$ \\
Diet 2 & $1.2 \mathrm{E}-05$ & $3.3 \mathrm{E}-04$ & $7.4 \mathrm{E}-03$ \\
Diet 3 & $2.7 \mathrm{E}-06$ & $6.9 \mathrm{E}-05$ & $1.6 \mathrm{E}-03$ \\
Diet 4 & $6.2 \mathrm{E}-05$ & $2.5 \mathrm{E}-03$ & $6.1 \mathrm{E}-02$ \\
\hline
\end{tabular}

\section{Relative Risk [ ]}

\begin{tabular}{lccc}
\hline Diet 1 & 1.035 & 1.61 & 14 \\
Diet 2 & 1.012 & 1.23 & 6.1 \\
Diet 3 & 1.0025 & 1.048 & 1.98 \\
Diet 4 & 1.068 & 2.7 & 52 \\
\hline
\end{tabular}

\section{Probability of Causation [\%]}

\begin{tabular}{lccc}
\hline Diet 1 & 3.38 & 37.8 & 93 \\
Diet 2 & 1.16 & 18.7 & 84 \\
Diet 3 & 0.25 & 4.6 & 49 \\
Diet 4 & 6.31 & 62.1 & 98 \\
\hline
\end{tabular}

Diet 1 - Backyard cow milk + all other locally produced non-milk exposure pathways

Diet 2 - Locally produced commercial milk + all other locally produced non-milk exposure pathways

Diet 3 - Regionally mixed commercial milk + inhalation (other regionally mixed food items are minor contributors to the total)

Diet 4 - Goat milk (all other exposure pathways are of negligible importance compared to goat milk) 
Location: Sugar Grove

Receptor: Female born in 1954

\begin{tabular}{lccc}
\hline & \multicolumn{3}{c}{ Thyroid Dose [cGy] } \\
Exposure Pathway & \multicolumn{2}{c}{$95 \%$ Subjective Confidence Interval } \\
Backyard Cow Milk & lower limit & central estimate & upper limit \\
Commercial Milk (locally produced) & 0.59 & 4.3 & 33 \\
Commercial Milk (regionally mixed) & 0.17 & 1.4 & 11 \\
Goat Milk (locally produced) & 0.094 & 0.81 & 5.7 \\
Beef (locally produced) & 1.1 & 12 & 120 \\
Leafy Vegetables (locally produced) & 0.00063 & 0.012 & 0.26 \\
Eggs (locally produced) & 0.00018 & 0.0021 & 0.022 \\
Cottage Cheese (locally produced) & 0.027 & 0.22 & 1.9 \\
Inhalation & 0.00073 & 0.011 & 0.15 \\
Mother's milk (mother on Diet 1) & 0.0071 & 0.033 & 0.16 \\
Prenatal exposure (mother on Diet 1) & 0.0026 & 0.088 & 1.9 \\
Diet 1 & 0.025 & 0.28 & 2.3 \\
Diet 2 & 0.65 & 4.6 & 36 \\
Diet 3 & 0.25 & 1.8 & 13 \\
\hline
\end{tabular}

Excess Lifetime Risk [ ]

\begin{tabular}{llll}
\hline Diet 1 & $8.7 \mathrm{E}-05$ & $1.4 \mathrm{E}-03$ & $2.3 \mathrm{E}-02$ \\
Diet 2 & $3.4 \mathrm{E}-05$ & $5.2 \mathrm{E}-04$ & $7.8 \mathrm{E}-03$ \\
Diet 3 & $1.6 \mathrm{E}-05$ & $2.5 \mathrm{E}-04$ & $3.3 \mathrm{E}-03$ \\
Diet 4 & $1.9 \mathrm{E}-04$ & $4.3 \mathrm{E}-03$ & $7.0 \mathrm{E}-02$ \\
\hline
\end{tabular}

\section{Relative Risk [ ]}

\begin{tabular}{lccc}
\hline Diet 1 & 1.034 & 1.34 & 5.8 \\
Diet 2 & 1.012 & 1.13 & 2.9 \\
Diet 3 & 1.0056 & 1.06 & 1.98 \\
Diet 4 & 1.073 & 1.96 & 15 \\
\hline
\end{tabular}

\section{Probability of Causation [\%]}

\begin{tabular}{lccc}
\hline Diet 1 & 3.25 & 24.6 & 81 \\
Diet 2 & 1.22 & 11.3 & 64 \\
Diet 3 & 0.55 & 5.7 & 49 \\
Diet 4 & 6.74 & 47.4 & 93 \\
\hline
\end{tabular}

Diet 1 - Backyard cow milk + all other locally produced non-milk exposure pathways

Diet 2 - Locally produced commercial milk + all other locally produced non-milk exposure pathways

Diet 3 - Regionally mixed commercial milk + inhalation (other regionally mixed food items are minor contributors to the total)

Diet 4 - Goat milk (all other exposure pathways are of negligible importance compared to goat milk) 
Location: Sugar Grove

Receptor: Male born in 1954

\begin{tabular}{lccc}
\hline & \multicolumn{3}{c}{ Thyroid Dose [cGy] } \\
Exposure Pathway & $95 \%$ Subjective Confidence Interval \\
\hline Backyard Cow Milk & lower limit & central estimate & upper limit \\
Commercial Milk (locally produced) & 0.65 & 4.2 & 30 \\
Commercial Milk (regionally mixed) & 0.17 & 1.4 & 9.8 \\
Goat Milk (locally produced) & 0.1 & 0.8 & 5 \\
Beef (locally produced) & 1.4 & 12 & 120 \\
Leafy Vegetables (locally produced) & 0.00062 & 0.012 & 0.28 \\
Eggs (locally produced) & 0.00017 & 0.0021 & 0.024 \\
Cottage Cheese (locally produced) & 0.029 & 0.23 & 2 \\
Inhalation & 0.00073 & 0.011 & 0.15 \\
Mother's milk (mother on Diet 1) & 0.0081 & 0.034 & 0.15 \\
Prenatal exposure (mother on Diet 1) & 0.0026 & 0.088 & 1.9 \\
Diet 1 & 0.025 & 0.28 & 2.3 \\
Diet 2 & 0.72 & 4.5 & 32 \\
Diet 3 & 0.25 & 1.8 & 12 \\
\hline
\end{tabular}

Excess Lifetime Risk [ ]

\begin{tabular}{llll}
\hline Diet 1 & $1.2 \mathrm{E}-05$ & $3.8 \mathrm{E}-04$ & $6.9 \mathrm{E}-03$ \\
Diet 2 & $5.0 \mathrm{E}-06$ & $1.4 \mathrm{E}-04$ & $3.0 \mathrm{E}-03$ \\
Diet 3 & $2.3 \mathrm{E}-06$ & $6.4 \mathrm{E}-05$ & $1.5 \mathrm{E}-03$ \\
Diet 4 & $2.6 \mathrm{E}-05$ & $1.1 \mathrm{E}-03$ & $2.8 \mathrm{E}-02$ \\
\hline
\end{tabular}

\section{Relative Risk [ ]}

\begin{tabular}{lccc}
\hline Diet 1 & 1.014 & 1.26 & 6.1 \\
Diet 2 & 1.0049 & 1.097 & 3.2 \\
Diet 3 & 1.0023 & 1.045 & 1.96 \\
Diet 4 & 1.027 & 1.69 & 23 \\
\hline
\end{tabular}

\section{Probability of Causation [\%]}

\begin{tabular}{lccc}
\hline Diet 1 & 1.34 & 20.0 & 82 \\
Diet 2 & 0.49 & 8.7 & 67 \\
Diet 3 & 0.23 & 4.3 & 49 \\
Diet 4 & 2.65 & 39.7 & 95 \\
\hline
\end{tabular}

Diet 1 - Backyard cow milk + all other locally produced non-milk exposure pathways

Diet 2 - Locally produced commercial milk + all other locally produced non-milk exposure pathways

Diet 3 - Regionally mixed commercial milk + inhalation (other regionally mixed food items are minor contributors to the total)

Diet 4 - Goat milk (all other exposure pathways are of negligible importance compared to goat milk) 


\section{Location: OR Townsite}

Receptor: Female born in 1954

\begin{tabular}{lccc}
\hline & \multicolumn{3}{c}{ Thyroid Dose [cGy] } \\
Exposure Pathway & \multicolumn{2}{c}{$9 \%$ Subjective Confidence Interval } \\
\hline Backyard Cow Milk & lower limit & central estimate & upper limit \\
Commercial Milk (locally produced) & -- & -- & -- \\
Commercial Milk (regionally mixed) & -- & -- & -- \\
Goat Milk (locally produced) & 0.094 & 0.81 & 5.7 \\
Beef (locally produced) & -- & -- & -- \\
Leafy Vegetables (locally produced) & -- & -- & -- \\
Eggs (locally produced) & -- & -- & -- \\
Cottage Cheese (locally produced) & -- & -- & -- \\
Inhalation & 0.00077 & 0.012 & 0.15 \\
Mother's milk (mother on Diet 3) & 0.0082 & 0.037 & 0.17 \\
Prenatal exposure (mother on Diet 3) & 0.00062 & 0.014 & 0.24 \\
Diet 1 & 0.0066 & 0.042 & 0.35 \\
Diet 2 & -- & -- & -- \\
Diet 3 & -- & -- & -- \\
\hline
\end{tabular}

Excess Lifetime Risk [ ]

\begin{tabular}{lccc}
\hline Diet 1 & -- & -- & -- \\
Diet 2 & -- & -- & -- \\
Diet 3 & $1.6 \mathrm{E}-05$ & $2.5 \mathrm{E}-04$ & $3.3 \mathrm{E}-03$ \\
Diet 4 & -- & -- & -- \\
\hline
\end{tabular}

\section{Relative Risk [ ]}

\begin{tabular}{lccc}
\hline Diet 1 & -- & -- & -- \\
Diet 2 & -- & -- & -- \\
Diet 3 & 1.0056 & 1.061 & 1.98 \\
Diet 4 & -- & -- & -- \\
\hline
\end{tabular}

\begin{tabular}{lccc}
\hline Diet 1 & -- & -- & -- \\
Diet 2 & -- & -- & -- \\
Diet 3 & 0.56 & 5.7 & -- \\
Diet 4 & -- & -- & - \\
\hline Diet 1 - Backyard cow milk + all other locally produced non-milk exposure pathways & \\
Diet 2 - Locally produced commercial milk + all other locally produced non-milk exposure pathways \\
Diet 3 - Regionally mixed commercial milk + inhalation (other regionally mixed food items are minor contributors to the total) \\
Diet 4 - Goat milk (all other exposure pathways are of negligible importance compared to goat milk)
\end{tabular}




\section{Location: OR Townsite}

\section{Receptor: Male born in 1954}

\begin{tabular}{lccc}
\hline & \multicolumn{3}{c}{ Thyroid Dose [cGy] } \\
Exposure Pathway & \multicolumn{2}{c}{$95 \%$ Subjective Confidence Interval } \\
\hline Backyard Cow Milk & lower limit & central estimate & upper limit \\
Commercial Milk (locally produced) & -- & -- & -- \\
Commercial Milk (regionally mixed) & -- & -- & -- \\
Goat Milk (locally produced) & 0.1 & 0.8 & 5 \\
Beef (locally produced) & -- & -- & -- \\
Leafy Vegetables (locally produced) & -- & -- & -- \\
Eggs (locally produced) & -- & -- & -- \\
Cottage Cheese (locally produced) & -- & -- & -- \\
Inhalation & 0.0008 & 0.012 & 0.17 \\
Mother's milk (mother on Diet 3) & 0.0086 & 0.037 & 0.16 \\
Prenatal exposure (mother on Diet 3) & 0.00062 & 0.014 & 0.24 \\
Diet 1 & 0.0066 & 0.042 & 0.35 \\
Diet 2 & -- & -- & -- \\
Diet 3 & -- & -- & -- \\
\hline
\end{tabular}

Excess Lifetime Risk [ ]

\begin{tabular}{lccc}
\hline Diet 1 & -- & -- & -- \\
Diet 2 & -- & -- & -- \\
Diet 3 & $2.3 \mathrm{E}-06$ & $6.4 \mathrm{E}-05$ & $1.5 \mathrm{E}-03$ \\
Diet 4 & -- & -- & -- \\
\hline
\end{tabular}

\section{Relative Risk [ ]}

\begin{tabular}{lccc}
\hline Diet 1 & -- & -- & -- \\
Diet 2 & -- & -- & -- \\
Diet 3 & 1.0023 & 1.045 & 1.96 \\
Diet 4 & -- & -- & -- \\
\hline
\end{tabular}

\begin{tabular}{lccc}
\hline Diet 1 & -- & -- & -- \\
Diet 2 & -- & -- & -- \\
Diet 3 & 0.23 & 4.3 & 49 \\
Diet 4 & -- & -- & - \\
\hline Diet 1 - Backyard cow milk + all other locally produced non-milk exposure pathways & \\
Diet 2 - Locally produced commercial milk + all other locally produced non-milk exposure pathways \\
Diet 3 - Regionally mixed commercial milk + inhalation (other regionally mixed food items are minor contributors to the total) \\
Diet 4 - Goat milk (all other exposure pathways are of negligible importance compared to goat milk)
\end{tabular}


Location: Hines Valley

Receptor: Female born in 1954

\begin{tabular}{lccc}
\hline & \multicolumn{3}{c}{ Thyroid Dose [cGy] } \\
Exposure Pathway & \multicolumn{2}{c}{$95 \%$ Subjective Confidence Interval } \\
Backyard Cow Milk & lower limit & central estimate & upper limit \\
Commercial Milk (locally produced) & 0.71 & 5.3 & 39 \\
Commercial Milk (regionally mixed) & 0.21 & 1.7 & 13 \\
Goat Milk (locally produced) & 0.094 & 0.81 & 5.7 \\
Beef (locally produced) & 1.4 & 15 & 150 \\
Leafy Vegetables (locally produced) & 0.00071 & 0.015 & 0.32 \\
Eggs (locally produced) & 0.00023 & 0.0026 & 0.027 \\
Cottage Cheese (locally produced) & 0.034 & 0.28 & 2.1 \\
Inhalation & 0.00078 & 0.013 & 0.17 \\
Mother's milk (mother on Diet 1) & 0.009 & 0.044 & 0.19 \\
Prenatal exposure (mother on Diet 1) & 0.0031 & 0.1 & 2.1 \\
Diet 1 & 0.03 & 0.34 & 2.6 \\
Diet 2 & 0.79 & 5.6 & 42 \\
Diet 3 & 0.28 & 2.2 & 15 \\
\hline
\end{tabular}

Excess Lifetime Risk [ ]

\begin{tabular}{lllc}
\hline Diet 1 & $1.1 \mathrm{E}-04$ & $1.7 \mathrm{E}-03$ & $2.9 \mathrm{E}-02$ \\
Diet 2 & $3.9 \mathrm{E}-05$ & $6.4 \mathrm{E}-04$ & $9.3 \mathrm{E}-03$ \\
Diet 3 & $1.7 \mathrm{E}-05$ & $2.5 \mathrm{E}-04$ & $3.3 \mathrm{E}-03$ \\
Diet 4 & $2.2 \mathrm{E}-04$ & $5.1 \mathrm{E}-03$ & $8.9 \mathrm{E}-02$ \\
\hline
\end{tabular}

\section{Relative Risk [ ]}

\begin{tabular}{lccc}
\hline Diet 1 & 1.039 & 1.43 & 6.6 \\
Diet 2 & 1.014 & 1.16 & 3.3 \\
Diet 3 & 1.0057 & 1.061 & 1.99 \\
Diet 4 & 1.086 & 2.1 & 18 \\
\hline
\end{tabular}

\begin{tabular}{lccc}
\hline Diet 1 & 3.76 & 29.9 & 85 \\
Diet 2 & 1.36 & 13.8 & 69 \\
Diet 3 & 0.57 & 5.8 & 50 \\
Diet 4 & 7.92 & 52.9 & 95 \\
\hline
\end{tabular}

Diet 1 - Backyard cow milk + all other locally produced non-milk exposure pathways

Diet 2 - Locally produced commercial milk + all other locally produced non-milk exposure pathways

Diet 3 - Regionally mixed commercial milk + inhalation (other regionally mixed food items are minor contributors to the total)

Diet 4 - Goat milk (all other exposure pathways are of negligible importance compared to goat milk) 


\section{Location: Hines Valley}

Receptor: Male born in 1954

\begin{tabular}{lccc}
\hline & \multicolumn{3}{c}{ Thyroid Dose [cGy] } \\
& \multicolumn{2}{c}{$95 \%$ Subjective Confidence Interval } \\
Exposure Pathway & lower limit & central estimate & upper limit \\
\hline Backyard Cow Milk & 0.77 & 5 & 35 \\
Commercial Milk (locally produced) & 0.21 & 1.7 & 11 \\
Commercial Milk (regionally mixed) & 0.1 & 0.8 & 5 \\
Goat Milk (locally produced) & 1.7 & 15 & 150 \\
Beef (locally produced) & 0.00074 & 0.015 & 0.33 \\
Leafy Vegetables (locally produced) & 0.00022 & 0.0025 & 0.028 \\
Eggs (locally produced) & 0.038 & 0.28 & 2.3 \\
Cottage Cheese (locally produced) & 0.00093 & 0.013 & 0.2 \\
Inhalation & 0.0094 & 0.044 & 0.19 \\
Mother's milk (mother on Diet 1) & 0.0031 & 0.1 & 2.1 \\
Prenatal exposure (mother on Diet 1) & 0.03 & 0.34 & 2.6 \\
Diet 1 & 0.85 & 5.4 & 37 \\
Diet 2 & 0.3 & 2.2 & 13 \\
Diet 3 & 0.12 & 0.85 & 5.1 \\
\hline
\end{tabular}

Excess Lifetime Risk [ ]

\begin{tabular}{llll}
\hline Diet 1 & $1.6 \mathrm{E}-05$ & $4.5 \mathrm{E}-04$ & $9.6 \mathrm{E}-03$ \\
Diet 2 & $6.2 \mathrm{E}-06$ & $1.8 \mathrm{E}-04$ & $3.8 \mathrm{E}-03$ \\
Diet 3 & $2.4 \mathrm{E}-06$ & $6.5 \mathrm{E}-05$ & $1.5 \mathrm{E}-03$ \\
Diet 4 & $3.1 \mathrm{E}-05$ & $1.3 \mathrm{E}-03$ & $3.2 \mathrm{E}-02$ \\
\hline
\end{tabular}

\section{Relative Risk [ ]}

\begin{tabular}{lccc}
\hline Diet 1 & 1.016 & 1.31 & 7.2 \\
Diet 2 & 1.0058 & 1.12 & 3.6 \\
Diet 3 & 1.0023 & 1.045 & 1.97 \\
Diet 4 & 1.036 & 1.83 & 25 \\
\hline
\end{tabular}

\section{Probability of Causation [\%]}

\begin{tabular}{lccc}
\hline Diet 1 & 1.62 & 23.4 & 86 \\
Diet 2 & 0.58 & 10.6 & 72 \\
Diet 3 & 0.23 & 4.4 & 49 \\
Diet 4 & 3.45 & 45.4 & 96 \\
\hline
\end{tabular}

Diet 1 - Backyard cow milk + all other locally produced non-milk exposure pathways

Diet 2 - Locally produced commercial milk + all other locally produced non-milk exposure pathways

Diet 3 - Regionally mixed commercial milk + inhalation (other regionally mixed food items are minor contributors to the total)

Diet 4 - Goat milk (all other exposure pathways are of negligible importance compared to goat milk) 


\section{Location: Farragut}

Receptor: Female born in 1954

\begin{tabular}{lccc}
\hline & \multicolumn{3}{c}{ Thyroid Dose [cGy] } \\
Exposure Pathway & \multicolumn{2}{c}{$95 \%$ Subjective Confidence Interval } \\
\hline Backyard Cow Milk & lower limit & central estimate & upper limit \\
Commercial Milk (locally produced) & 0.79 & 5.6 & 42 \\
Commercial Milk (regionally mixed) & 0.23 & 1.9 & 14 \\
Goat Milk (locally produced) & 0.095 & 0.82 & 5.7 \\
Beef (locally produced) & 1.6 & 17 & 180 \\
Leafy Vegetables (locally produced) & 0.00085 & 0.017 & 0.38 \\
Eggs (locally produced) & 0.00024 & 0.0029 & 0.03 \\
Cottage Cheese (locally produced) & 0.039 & 0.31 & 2.5 \\
Inhalation & 0.00098 & 0.015 & 0.2 \\
Mother's milk (mother on Diet 1) & 0.011 & 0.05 & 0.22 \\
Prenatal exposure (mother on Diet 1) & 0.0033 & 0.12 & 2.5 \\
Diet 1 & 0.034 & 0.38 & 2.7 \\
Diet 2 & 0.88 & 6.2 & 45 \\
Diet 3 & 0.33 & 2.4 & 16 \\
\hline
\end{tabular}

Excess Lifetime Risk [ ]

\begin{tabular}{llll}
\hline Diet 1 & $1.2 \mathrm{E}-04$ & $2.0 \mathrm{E}-03$ & $3.2 \mathrm{E}-02$ \\
Diet 2 & $4.5 \mathrm{E}-05$ & $7.2 \mathrm{E}-04$ & $9.6 \mathrm{E}-03$ \\
Diet 3 & $1.7 \mathrm{E}-05$ & $2.5 \mathrm{E}-04$ & $3.4 \mathrm{E}-03$ \\
Diet 4 & $2.5 \mathrm{E}-04$ & $5.8 \mathrm{E}-03$ & $1.0 \mathrm{E}-01$ \\
\hline
\end{tabular}

\section{Relative Risk [ ]}

\begin{tabular}{lccc}
\hline Diet 1 & 1.046 & 1.48 & 7 \\
Diet 2 & 1.015 & 1.18 & 3.9 \\
Diet 3 & 1.0059 & 1.062 & 1.99 \\
Diet 4 & 1.094 & 2.3 & 22 \\
\hline
\end{tabular}

\begin{tabular}{lccc}
\hline Diet 1 & 4.42 & 32.1 & 85 \\
Diet 2 & 1.50 & 15.0 & 74 \\
Diet 3 & 0.58 & 5.8 & 50 \\
Diet 4 & 8.57 & 55.7 & 95 \\
\hline
\end{tabular}

Diet 1 - Backyard cow milk + all other locally produced non-milk exposure pathways

Diet 2 - Locally produced commercial milk + all other locally produced non-milk exposure pathways

Diet 3 - Regionally mixed commercial milk + inhalation (other regionally mixed food items are minor contributors to the total)

Diet 4 - Goat milk (all other exposure pathways are of negligible importance compared to goat milk) 


\section{Location: Farragut}

\section{Receptor: Male born in 1954}

\begin{tabular}{lccc}
\hline & \multicolumn{3}{c}{ Thyroid Dose [cGy] } \\
& \multicolumn{2}{c}{$95 \%$ Subjective Confidence Interval } \\
Exposure Pathway & lower limit & central estimate & upper limit \\
\hline Backyard Cow Milk & 0.9 & 5.6 & 43 \\
Commercial Milk (locally produced) & 0.21 & 1.9 & 12 \\
Commercial Milk (regionally mixed) & 0.1 & 0.8 & 5 \\
Goat Milk (locally produced) & 1.8 & 18 & 180 \\
Beef (locally produced) & 0.00086 & 0.016 & 0.4 \\
Leafy Vegetables (locally produced) & 0.00022 & 0.0028 & 0.03 \\
Eggs (locally produced) & 0.041 & 0.31 & 2.6 \\
Cottage Cheese (locally produced) & 0.001 & 0.016 & 0.19 \\
Inhalation & 0.011 & 0.051 & 0.21 \\
Mother's milk (mother on Diet 1) & 0.0033 & 0.12 & 2.5 \\
Prenatal exposure (mother on Diet 1) & 0.034 & 0.38 & 2.7 \\
Diet 1 & 0.98 & 6.1 & 46 \\
Diet 2 & 0.32 & 2.4 & 15 \\
Diet 3 & 0.12 & 0.86 & 5 \\
\hline
\end{tabular}

Excess Lifetime Risk [ ]

\begin{tabular}{llll}
\hline Diet 1 & $1.5 \mathrm{E}-05$ & $4.8 \mathrm{E}-04$ & $9.3 \mathrm{E}-03$ \\
Diet 2 & $6.6 \mathrm{E}-06$ & $1.9 \mathrm{E}-04$ & $4.6 \mathrm{E}-03$ \\
Diet 3 & $2.4 \mathrm{E}-06$ & $6.6 \mathrm{E}-05$ & $1.5 \mathrm{E}-03$ \\
Diet 4 & $3.4 \mathrm{E}-05$ & $1.4 \mathrm{E}-03$ & $3.7 \mathrm{E}-02$ \\
\hline
\end{tabular}

\section{Relative Risk [ ]}

\begin{tabular}{lccc}
\hline Diet 1 & 1.02 & 1.34 & 8.1 \\
Diet 2 & 1.0063 & 1.13 & 4 \\
Diet 3 & 1.0024 & 1.046 & 1.97 \\
Diet 4 & 1.039 & 1.98 & 29 \\
\hline
\end{tabular}

\section{Probability of Causation [\%]}

\begin{tabular}{lccc}
\hline Diet 1 & 1.95 & 25.2 & 88 \\
Diet 2 & 0.63 & 11.3 & 75 \\
Diet 3 & 0.24 & 4.4 & 49 \\
Diet 4 & 3.78 & 49.4 & 96 \\
\hline
\end{tabular}

Diet 1 - Backyard cow milk + all other locally produced non-milk exposure pathways

Diet 2 - Locally produced commercial milk + all other locally produced non-milk exposure pathways

Diet 3 - Regionally mixed commercial milk + inhalation (other regionally mixed food items are minor contributors to the total)

Diet 4 - Goat milk (all other exposure pathways are of negligible importance compared to goat milk) 
Location: Lenoir City

Receptor: Female born in 1954

\begin{tabular}{lccc}
\hline & \multicolumn{3}{c}{ Thyroid Dose [cGy] } \\
Exposure Pathway & \multicolumn{2}{c}{$95 \%$ Subjective Confidence Interval } \\
\hline Backyard Cow Milk & lower limit & central estimate & upper limit \\
Commercial Milk (locally produced) & 0.54 & 3.6 & 26 \\
Commercial Milk (regionally mixed) & 0.14 & 1.1 & 9.5 \\
Goat Milk (locally produced) & 0.094 & 0.81 & 5.7 \\
Beef (locally produced) & -- & -- & -- \\
Leafy Vegetables (locally produced) & 0.00051 & 0.01 & 0.23 \\
Eggs (locally produced) & 0.00017 & 0.0018 & 0.018 \\
Cottage Cheese (locally produced) & 0.023 & 0.18 & 1.6 \\
Inhalation & 0.00051 & 0.0092 & 0.12 \\
Mother's milk (mother on Diet 1) & 0.0068 & 0.031 & 0.14 \\
Prenatal exposure (mother on Diet 1) & 0.0022 & 0.064 & 1.6 \\
Diet 1 & 0.024 & 0.23 & 1.8 \\
Diet 2 & 0.61 & 3.9 & 28 \\
Diet 3 & 0.21 & 1.4 & 11 \\
\hline
\end{tabular}

Excess Lifetime Risk [ ]

\begin{tabular}{lccc}
\hline Diet 1 & $7.2 \mathrm{E}-05$ & $1.2 \mathrm{E}-03$ & $1.9 \mathrm{E}-02$ \\
Diet 2 & $2.8 \mathrm{E}-05$ & $4.2 \mathrm{E}-04$ & $6.4 \mathrm{E}-03$ \\
Diet 3 & $1.5 \mathrm{E}-05$ & $2.5 \mathrm{E}-04$ & $3.3 \mathrm{E}-03$ \\
Diet 4 & -- & -- & -- \\
\hline
\end{tabular}

\section{Relative Risk [ ]}

\begin{tabular}{lccc}
\hline Diet 1 & 1.027 & 1.28 & 5.2 \\
Diet 2 & 1.01 & 1.1 & 2.7 \\
Diet 3 & 1.0055 & 1.06 & 1.98 \\
Diet 4 & -- & -- & -- \\
\hline
\end{tabular}

\begin{tabular}{lccc}
\hline Diet 1 & 2.62 & 21.6 & 81 \\
Diet 2 & 0.99 & 9.5 & 62 \\
Diet 3 & 0.55 & 5.7 & 49 \\
Diet 4 & -- & -- & - \\
\hline Diet 1 - Backyard cow milk + all other locally produced non-milk exposure pathways & \\
Diet 2 - Locally produced commercial milk + all other locally produced non-milk exposure pathways \\
Diet 3 - Regionally mixed commercial milk + inhalation (other regionally mixed food items are minor contributors to the total) \\
Diet 4 - Goat milk (all other exposure pathways are of negligible importance compared to goat milk)
\end{tabular}




\section{Location: Lenoir City}

\section{Receptor: Male born in 1954}

\begin{tabular}{lccc}
\hline & \multicolumn{3}{c}{ Thyroid Dose [cGy] } \\
& \multicolumn{2}{c}{$95 \%$ Subjective Confidence Interval } \\
Exposure Pathway & lower limit & central estimate & upper limit \\
\hline Backyard Cow Milk & 0.52 & 3.5 & 24 \\
Commercial Milk (locally produced) & 0.14 & 1.1 & 8 \\
Commercial Milk (regionally mixed) & 0.1 & 0.8 & 5 \\
Goat Milk (locally produced) & -- & -- & -- \\
Beef (locally produced) & 0.00056 & 0.01 & 0.24 \\
Leafy Vegetables (locally produced) & 0.00015 & 0.0017 & 0.017 \\
Eggs (locally produced) & 0.026 & 0.19 & 1.5 \\
Cottage Cheese (locally produced) & 0.00065 & 0.0091 & 0.12 \\
Inhalation & 0.0073 & 0.03 & 0.14 \\
Mother's milk (mother on Diet 1) & 0.0022 & 0.064 & 1.6 \\
Prenatal exposure (mother on Diet 1) & 0.024 & 0.23 & 1.8 \\
Diet 1 & 0.59 & 3.7 & 25 \\
Diet 2 & 0.2 & 1.4 & 9.3 \\
Diet 3 & 0.11 & 0.84 & 5 \\
\hline
\end{tabular}

Excess Lifetime Risk [ ]

\begin{tabular}{lccc}
\hline Diet 1 & $8.8 \mathrm{E}-06$ & $2.9 \mathrm{E}-04$ & $5.6 \mathrm{E}-03$ \\
Diet 2 & $3.9 \mathrm{E}-06$ & $1.2 \mathrm{E}-04$ & $2.9 \mathrm{E}-03$ \\
Diet 3 & $2.3 \mathrm{E}-06$ & $6.3 \mathrm{E}-05$ & $1.5 \mathrm{E}-03$ \\
Diet 4 & -- & -- & -- \\
\hline
\end{tabular}

\section{Relative Risk [ ]}

\begin{tabular}{lccc}
\hline Diet 1 & 1.011 & 1.21 & 5.1 \\
Diet 2 & 1.0041 & 1.075 & 2.8 \\
Diet 3 & 1.0023 & 1.045 & 1.96 \\
Diet 4 & -- & -- & -- \\
\hline
\end{tabular}

\section{Probability of Causation [\%]}

\begin{tabular}{lccc}
\hline Diet 1 & 1.09 & 17.2 & 80 \\
Diet 2 & 0.41 & 7.0 & 64 \\
Diet 3 & 0.23 & 4.3 & 49 \\
Diet 4 & -- & -- \\
\hline Diet 1 - Backyard cow milk + all other locally produced non-milk exposure pathways & \\
Diet 2 - Locally produced commercial milk + all other locally produced non-milk exposure pathways \\
Diet 3 - Regionally mixed commercial milk + inhalation (other regionally mixed food items are minor contributors to the total) \\
Diet 4 - Goat milk (all other exposure pathways are of negligible importance compared to goat milk)
\end{tabular}




\section{Location: Kingston}

Receptor: Female born in 1954

\begin{tabular}{lccc}
\hline & \multicolumn{3}{c}{ Thyroid Dose [cGy] } \\
Exposure Pathway & \multicolumn{2}{c}{$95 \%$ Subjective Confidence Interval } \\
\hline Backyard Cow Milk & lower limit & central estimate & upper limit \\
Commercial Milk (locally produced) & 0.64 & 4.5 & 37 \\
Commercial Milk (regionally mixed) & 0.19 & 1.5 & 11 \\
Goat Milk (locally produced) & 0.094 & 0.81 & 5.7 \\
Beef (locally produced) & 1.3 & 14 & 130 \\
Leafy Vegetables (locally produced) & 0.00062 & 0.013 & 0.29 \\
Eggs (locally produced) & 0.00019 & 0.0023 & 0.023 \\
Cottage Cheese (locally produced) & 0.03 & 0.24 & 2 \\
Inhalation & 0.00077 & 0.012 & 0.15 \\
Mother's milk (mother on Diet 1) & 0.0089 & 0.039 & 0.19 \\
Prenatal exposure (mother on Diet 1) & 0.0028 & 0.096 & 1.8 \\
Diet 1 & 0.028 & 0.3 & 2.2 \\
Diet 2 & 0.71 & 4.9 & 39 \\
Diet 3 & 0.26 & 1.9 & 13 \\
\hline
\end{tabular}

Excess Lifetime Risk [ ]

\begin{tabular}{lllc}
\hline Diet 1 & $9.5 \mathrm{E}-05$ & $1.5 \mathrm{E}-03$ & $2.5 \mathrm{E}-02$ \\
Diet 2 & $3.7 \mathrm{E}-05$ & $5.5 \mathrm{E}-04$ & $7.7 \mathrm{E}-03$ \\
Diet 3 & $1.6 \mathrm{E}-05$ & $2.5 \mathrm{E}-04$ & $3.3 \mathrm{E}-03$ \\
Diet 4 & $1.8 \mathrm{E}-04$ & $4.4 \mathrm{E}-03$ & $8.3 \mathrm{E}-02$ \\
\hline
\end{tabular}

\section{Relative Risk [ ]}

\begin{tabular}{lccc}
\hline Diet 1 & 1.035 & 1.38 & 5.9 \\
Diet 2 & 1.012 & 1.14 & 3.3 \\
Diet 3 & 1.0056 & 1.061 & 1.98 \\
Diet 4 & 1.081 & 1.97 & 18 \\
\hline
\end{tabular}

\begin{tabular}{lccc}
\hline Diet 1 & 3.41 & 27.7 & 83 \\
Diet 2 & 1.23 & 12.5 & 69 \\
Diet 3 & 0.56 & 5.7 & 50 \\
Diet 4 & 7.46 & 49.1 & 94 \\
\hline
\end{tabular}

Diet 1 - Backyard cow milk + all other locally produced non-milk exposure pathways

Diet 2 - Locally produced commercial milk + all other locally produced non-milk exposure pathways

Diet 3 - Regionally mixed commercial milk + inhalation (other regionally mixed food items are minor contributors to the total)

Diet 4 - Goat milk (all other exposure pathways are of negligible importance compared to goat milk) 


\section{Location: Kingston}

\section{Receptor: Male born in 1954}

\begin{tabular}{lccc}
\hline & \multicolumn{3}{c}{ Thyroid Dose [cGy] } \\
& \multicolumn{2}{c}{$95 \%$ Subjective Confidence Interval } \\
Exposure Pathway & lower limit & central estimate & upper limit \\
\hline Backyard Cow Milk & 0.7 & 4.4 & 33 \\
Commercial Milk (locally produced) & 0.19 & 1.5 & 10 \\
Commercial Milk (regionally mixed) & 0.1 & 0.8 & 5 \\
Goat Milk (locally produced) & 1.4 & 13 & 120 \\
Beef (locally produced) & 0.00071 & 0.013 & 0.31 \\
Leafy Vegetables (locally produced) & 0.00017 & 0.0022 & 0.024 \\
Eggs (locally produced) & 0.03 & 0.24 & 2.1 \\
Cottage Cheese (locally produced) & 0.00075 & 0.013 & 0.15 \\
Inhalation & 0.0096 & 0.04 & 0.17 \\
Mother's milk (mother on Diet 1) & 0.0028 & 0.096 & 1.8 \\
Prenatal exposure (mother on Diet 1) & 0.028 & 0.3 & 2.2 \\
Diet 1 & 0.76 & 4.8 & 35 \\
Diet 2 & 0.27 & 2 & 12 \\
Diet 3 & 0.11 & 0.85 & 5 \\
\hline
\end{tabular}

Excess Lifetime Risk [ ]

\begin{tabular}{llll}
\hline Diet 1 & $1.3 \mathrm{E}-05$ & $3.9 \mathrm{E}-04$ & $7.0 \mathrm{E}-03$ \\
Diet 2 & $5.4 \mathrm{E}-06$ & $1.5 \mathrm{E}-04$ & $3.3 \mathrm{E}-03$ \\
Diet 3 & $2.4 \mathrm{E}-06$ & $6.5 \mathrm{E}-05$ & $1.5 \mathrm{E}-03$ \\
Diet 4 & $2.9 \mathrm{E}-05$ & $1.2 \mathrm{E}-03$ & $2.7 \mathrm{E}-02$ \\
\hline
\end{tabular}

\section{Relative Risk [ ]}

\begin{tabular}{lccc}
\hline Diet 1 & 1.015 & 1.26 & 6.6 \\
Diet 2 & 1.0051 & 1.1 & 3.3 \\
Diet 3 & 1.0023 & 1.045 & 1.96 \\
Diet 4 & 1.031 & 1.73 & 24 \\
\hline
\end{tabular}

\section{Probability of Causation [\%]}

\begin{tabular}{lccc}
\hline Diet 1 & 1.45 & 20.8 & 85 \\
Diet 2 & 0.50 & 9.3 & 69 \\
Diet 3 & 0.23 & 4.3 & 49 \\
Diet 4 & 3.05 & 41.9 & 96 \\
\hline
\end{tabular}

Diet 1 - Backyard cow milk + all other locally produced non-milk exposure pathways

Diet 2 - Locally produced commercial milk + all other locally produced non-milk exposure pathways

Diet 3 - Regionally mixed commercial milk + inhalation (other regionally mixed food items are minor contributors to the total)

Diet 4 - Goat milk (all other exposure pathways are of negligible importance compared to goat milk) 


\section{Location: Karns}

Receptor: Female born in 1954

\begin{tabular}{lccc}
\hline & \multicolumn{3}{c}{ Thyroid Dose [cGy] } \\
Exposure Pathway & \multicolumn{2}{c}{$95 \%$ Subjective Confidence Interval } \\
\hline Backyard Cow Milk & lower limit & central estimate & upper limit \\
Commercial Milk (locally produced) & 0.86 & 6.1 & 42 \\
Commercial Milk (regionally mixed) & 0.25 & 2.1 & 14 \\
Goat Milk (locally produced) & 0.094 & 0.81 & 5.7 \\
Beef (locally produced) & 1.8 & 18 & 180 \\
Leafy Vegetables (locally produced) & 0.00088 & 0.017 & 0.39 \\
Eggs (locally produced) & 0.00027 & 0.003 & 0.03 \\
Cottage Cheese (locally produced) & 0.042 & 0.33 & 2.7 \\
Inhalation & 0.00095 & 0.016 & 0.19 \\
Mother's milk (mother on Diet 1) & 0.011 & 0.055 & 0.24 \\
Prenatal exposure (mother on Diet 1) & 0.0038 & 0.13 & 2.5 \\
Diet 1 & 0.038 & 0.41 & 2.8 \\
Diet 2 & 0.96 & 6.6 & 45 \\
Diet 3 & 0.36 & 2.6 & 16 \\
\hline
\end{tabular}

Excess Lifetime Risk [ ]

\begin{tabular}{lllr}
\hline Diet 1 & $1.3 \mathrm{E}-04$ & $2.1 \mathrm{E}-03$ & $3.3 \mathrm{E}-02$ \\
Diet 2 & $4.9 \mathrm{E}-05$ & $7.4 \mathrm{E}-04$ & $1.0 \mathrm{E}-02$ \\
Diet 3 & $1.7 \mathrm{E}-05$ & $2.6 \mathrm{E}-04$ & $3.4 \mathrm{E}-03$ \\
Diet 4 & $2.6 \mathrm{E}-04$ & $6.0 \mathrm{E}-03$ & $1.0 \mathrm{E}-01$ \\
\hline
\end{tabular}

\section{Relative Risk [ ]}

\begin{tabular}{lccc}
\hline Diet 1 & 1.049 & 1.51 & 7 \\
Diet 2 & 1.017 & 1.19 & 3.9 \\
Diet 3 & 1.0059 & 1.062 & 1.99 \\
Diet 4 & 1.1 & 2.4 & 23 \\
\hline
\end{tabular}

\begin{tabular}{lccc}
\hline Diet 1 & 4.71 & 33.9 & 86 \\
Diet 2 & 1.64 & 16.3 & 74 \\
Diet 3 & 0.59 & 5.9 & 50 \\
Diet 4 & 9.30 & 57.8 & 96 \\
\hline
\end{tabular}

Diet 1 - Backyard cow milk + all other locally produced non-milk exposure pathways

Diet 2 - Locally produced commercial milk + all other locally produced non-milk exposure pathways

Diet 3 - Regionally mixed commercial milk + inhalation (other regionally mixed food items are minor contributors to the total)

Diet 4 - Goat milk (all other exposure pathways are of negligible importance compared to goat milk) 


\section{Location: Karns}

\section{Receptor: Male born in 1954}

\begin{tabular}{lccc}
\hline & \multicolumn{3}{c}{ Thyroid Dose [cGy] } \\
& \multicolumn{2}{c}{$95 \%$ Subjective Confidence Interval } \\
Exposure Pathway & lower limit & central estimate & upper limit \\
\hline Backyard Cow Milk & 0.96 & 6.1 & 41 \\
Commercial Milk (locally produced) & 0.23 & 2.1 & 13 \\
Commercial Milk (regionally mixed) & 0.1 & 0.8 & 5 \\
Goat Milk (locally produced) & 2 & 19 & 170 \\
Beef (locally produced) & 0.00094 & 0.017 & 0.4 \\
Leafy Vegetables (locally produced) & 0.00022 & 0.003 & 0.03 \\
Eggs (locally produced) & 0.043 & 0.33 & 2.8 \\
Cottage Cheese (locally produced) & 0.0011 & 0.017 & 0.2 \\
Inhalation & 0.012 & 0.056 & 0.22 \\
Mother's milk (mother on Diet 1) & 0.0038 & 0.13 & 2.5 \\
Prenatal exposure (mother on Diet 1) & 0.038 & 0.41 & 2.8 \\
Diet 1 & 1 & 6.6 & 45 \\
Diet 2 & 0.35 & 2.6 & 15 \\
Diet 3 & 0.12 & 0.86 & 5.1 \\
\hline
\end{tabular}

Excess Lifetime Risk [ ]

\begin{tabular}{lllc}
\hline Diet 1 & $1.7 \mathrm{E}-05$ & $5.2 \mathrm{E}-04$ & $9.6 \mathrm{E}-03$ \\
Diet 2 & $7.1 \mathrm{E}-06$ & $2.0 \mathrm{E}-04$ & $4.5 \mathrm{E}-03$ \\
Diet 3 & $2.5 \mathrm{E}-06$ & $6.7 \mathrm{E}-05$ & $1.5 \mathrm{E}-03$ \\
Diet 4 & $3.9 \mathrm{E}-05$ & $1.5 \mathrm{E}-03$ & $3.8 \mathrm{E}-02$ \\
\hline
\end{tabular}

\section{Relative Risk [ ]}

\begin{tabular}{lccc}
\hline Diet 1 & 1.021 & 1.36 & 8.7 \\
Diet 2 & 1.0069 & 1.14 & 4 \\
Diet 3 & 1.0024 & 1.046 & 1.97 \\
Diet 4 & 1.042 & 2 & 30 \\
\hline
\end{tabular}

\begin{tabular}{lccc}
\hline Diet 1 & 2.08 & 26.5 & 88 \\
Diet 2 & 0.69 & 12.1 & 75 \\
Diet 3 & 0.24 & 4.4 & 49 \\
Diet 4 & 4.03 & 50.1 & 97 \\
\hline
\end{tabular}

Diet 1 - Backyard cow milk + all other locally produced non-milk exposure pathways

Diet 2 - Locally produced commercial milk + all other locally produced non-milk exposure pathways

Diet 3 - Regionally mixed commercial milk + inhalation (other regionally mixed food items are minor contributors to the total)

Diet 4 - Goat milk (all other exposure pathways are of negligible importance compared to goat milk) 


\section{Location: Loudon}

Receptor: Female born in 1954

\begin{tabular}{lccc}
\hline & \multicolumn{3}{c}{ Thyroid Dose [cGy] } \\
& \multicolumn{2}{c}{$95 \%$ Subjective Confidence Interval } \\
Exposure Pathway & lower limit & central estimate & upper limit \\
\hline Backyard Cow Milk & 0.49 & 3.4 & 27 \\
Commercial Milk (locally produced) & 0.14 & 1.1 & 8.7 \\
Commercial Milk (regionally mixed) & 0.094 & 0.81 & 5.7 \\
Goat Milk (locally produced) & 0.93 & 9.9 & 99 \\
Beef (locally produced) & 0.00049 & 0.01 & 0.22 \\
Leafy Vegetables (locally produced) & 0.00016 & 0.0017 & 0.017 \\
Eggs (locally produced) & 0.022 & 0.18 & 1.5 \\
Cottage Cheese (locally produced) & 0.00053 & 0.009 & 0.12 \\
Inhalation & 0.0071 & 0.031 & 0.15 \\
Mother's milk (mother on Diet 1) & 0.0021 & 0.072 & 1.4 \\
Prenatal exposure (mother on Diet 1) & 0.02 & 0.23 & 1.7 \\
Diet 1 & 0.55 & 3.7 & 28 \\
Diet 2 & 0.2 & 1.5 & 11 \\
Diet 3 & 0.11 & 0.86 & 5.8 \\
\hline
\end{tabular}

Excess Lifetime Risk [ ]

\begin{tabular}{llll}
\hline Diet 1 & $7.4 \mathrm{E}-05$ & $1.2 \mathrm{E}-03$ & $1.9 \mathrm{E}-02$ \\
Diet 2 & $2.9 \mathrm{E}-05$ & $4.2 \mathrm{E}-04$ & $6.0 \mathrm{E}-03$ \\
Diet 3 & $1.5 \mathrm{E}-05$ & $2.4 \mathrm{E}-04$ & $3.3 \mathrm{E}-03$ \\
Diet 4 & $1.5 \mathrm{E}-04$ & $3.4 \mathrm{E}-03$ & $5.7 \mathrm{E}-02$ \\
\hline
\end{tabular}

\section{Relative Risk [ ]}

\begin{tabular}{lccc}
\hline Diet 1 & 1.027 & 1.28 & 4.7 \\
Diet 2 & 1.0092 & 1.11 & 2.6 \\
Diet 3 & 1.0055 & 1.06 & 1.98 \\
Diet 4 & 1.057 & 1.77 & 13 \\
\hline
\end{tabular}

\begin{tabular}{lccc} 
& \multicolumn{3}{c}{ Probability of Causation [\%] } \\
\hline Diet 1 & 2.63 & 22.0 & 78 \\
Diet 2 & 0.91 & 9.6 & 61 \\
Diet 3 & 0.55 & 5.7 & 49 \\
Diet 4 & 5.40 & 43.3 & 92 \\
\hline
\end{tabular}

Diet 1 - Backyard cow milk + all other locally produced non-milk exposure pathways

Diet 2 - Locally produced commercial milk + all other locally produced non-milk exposure pathways

Diet 3 - Regionally mixed commercial milk + inhalation (other regionally mixed food items are minor contributors to the total)

Diet 4 - Goat milk (all other exposure pathways are of negligible importance compared to goat milk) 


\section{Location: Loudon}

\section{Receptor: Male born in 1954}

\begin{tabular}{lccc}
\hline & \multicolumn{3}{c}{ Thyroid Dose [cGy] } \\
& \multicolumn{2}{c}{$95 \%$ Subjective Confidence Interval } \\
Exposure Pathway & lower limit & central estimate & upper limit \\
\hline Backyard Cow Milk & 0.53 & 3.4 & 23 \\
Commercial Milk (locally produced) & 0.14 & 1.1 & 7.5 \\
Commercial Milk (regionally mixed) & 0.1 & 0.8 & 5 \\
Goat Milk (locally produced) & 1.1 & 10 & 100 \\
Beef (locally produced) & 0.00051 & 0.0099 & 0.22 \\
Leafy Vegetables (locally produced) & 0.00014 & 0.0017 & 0.018 \\
Eggs (locally produced) & 0.024 & 0.18 & 1.6 \\
Cottage Cheese (locally produced) & 0.00059 & 0.009 & 0.11 \\
Inhalation & 0.0071 & 0.032 & 0.14 \\
Mother's milk (mother on Diet 1) & 0.0021 & 0.072 & 1.4 \\
Prenatal exposure (mother on Diet 1) & 0.02 & 0.23 & 1.7 \\
Diet 1 & 0.59 & 3.7 & 25 \\
Diet 2 & 0.21 & 1.5 & 9.4 \\
Diet 3 & 0.11 & 0.84 & 5 \\
\hline
\end{tabular}

Excess Lifetime Risk [ ]

\begin{tabular}{lllc}
\hline Diet 1 & $9.8 \mathrm{E}-06$ & $2.9 \mathrm{E}-04$ & $5.4 \mathrm{E}-03$ \\
Diet 2 & $4.1 \mathrm{E}-06$ & $1.1 \mathrm{E}-04$ & $2.4 \mathrm{E}-03$ \\
Diet 3 & $2.3 \mathrm{E}-06$ & $6.3 \mathrm{E}-05$ & $1.5 \mathrm{E}-03$ \\
Diet 4 & $2.0 \mathrm{E}-05$ & $8.4 \mathrm{E}-04$ & $2.2 \mathrm{E}-02$ \\
\hline
\end{tabular}

\section{Relative Risk [ ]}

\begin{tabular}{lccc}
\hline Diet 1 & 1.011 & 1.21 & 5.1 \\
Diet 2 & 1.0039 & 1.08 & 2.7 \\
Diet 3 & 1.0023 & 1.045 & 1.96 \\
Diet 4 & 1.023 & 1.56 & 17 \\
\hline
\end{tabular}

\section{Probability of Causation [\%]}

\begin{tabular}{lccc}
\hline Diet 1 & 1.08 & 17.3 & 80 \\
Diet 2 & 0.39 & 7.4 & 63 \\
Diet 3 & 0.23 & 4.3 & 49 \\
Diet 4 & 2.21 & 35.9 & 94 \\
\hline
\end{tabular}

Diet 1 - Backyard cow milk + all other locally produced non-milk exposure pathways

Diet 2 - Locally produced commercial milk + all other locally produced non-milk exposure pathways

Diet 3 - Regionally mixed commercial milk + inhalation (other regionally mixed food items are minor contributors to the total)

Diet 4 - Goat milk (all other exposure pathways are of negligible importance compared to goat milk) 
Location: Harriman

Receptor: Female born in 1954

\begin{tabular}{lccc}
\hline & \multicolumn{3}{c}{ Thyroid Dose [cGy] } \\
Exposure Pathway & \multicolumn{2}{c}{$95 \%$ Subjective Confidence Interval } \\
Backyard Cow Milk & lower limit & central estimate & upper limit \\
Commercial Milk (locally produced) & 0.3 & 2.1 & 15 \\
Commercial Milk (regionally mixed) & 0.082 & 0.65 & 5.5 \\
Goat Milk (locally produced) & 0.094 & 0.81 & 5.7 \\
Beef (locally produced) & 0.57 & 5.9 & 58 \\
Leafy Vegetables (locally produced) & 0.00029 & 0.0058 & 0.13 \\
Eggs (locally produced) & 0.000097 & 0.001 & 0.011 \\
Cottage Cheese (locally produced) & 0.013 & 0.11 & 0.91 \\
Inhalation & 0.0003 & 0.0053 & 0.069 \\
Mother's milk (mother on Diet 1) & 0.0039 & 0.017 & 0.075 \\
Prenatal exposure (mother on Diet 1) & 0.0012 & 0.038 & 0.93 \\
Diet 1 & 0.014 & 0.13 & 1.1 \\
Diet 2 & 0.34 & 2.2 & 16 \\
Diet 3 & 0.12 & 0.82 & 6.1 \\
\hline
\end{tabular}

Excess Lifetime Risk [ ]

\begin{tabular}{llll}
\hline Diet 1 & $4.3 \mathrm{E}-05$ & $7.1 \mathrm{E}-04$ & $1.1 \mathrm{E}-02$ \\
Diet 2 & $1.6 \mathrm{E}-05$ & $2.5 \mathrm{E}-04$ & $3.6 \mathrm{E}-03$ \\
Diet 3 & $1.4 \mathrm{E}-05$ & $2.4 \mathrm{E}-04$ & $3.2 \mathrm{E}-03$ \\
Diet 4 & $9.5 \mathrm{E}-05$ & $2.1 \mathrm{E}-03$ & $4.0 \mathrm{E}-02$ \\
\hline
\end{tabular}

\section{Relative Risk [ ]}

\begin{tabular}{lccc}
\hline Diet 1 & 1.015 & 1.16 & 3.4 \\
Diet 2 & 1.0058 & 1.061 & 1.95 \\
Diet 3 & 1.0052 & 1.059 & 1.97 \\
Diet 4 & 1.035 & 1.46 & 8.4 \\
\hline
\end{tabular}

\section{Probability of Causation [\%]}

\begin{tabular}{lccc}
\hline Diet 1 & 1.51 & 13.5 & 70 \\
Diet 2 & 0.57 & 5.8 & 49 \\
Diet 3 & 0.52 & 5.6 & 49 \\
Diet 4 & 3.36 & 31.4 & 88 \\
\hline
\end{tabular}

Diet 1 - Backyard cow milk + all other locally produced non-milk exposure pathways

Diet 2 - Locally produced commercial milk + all other locally produced non-milk exposure pathways

Diet 3 - Regionally mixed commercial milk + inhalation (other regionally mixed food items are minor contributors to the total)

Diet 4 - Goat milk (all other exposure pathways are of negligible importance compared to goat milk) 


\section{Location: Harriman}

\section{Receptor: Male born in 1954}

\begin{tabular}{lccc}
\hline & \multicolumn{3}{c}{ Thyroid Dose [cGy] } \\
Exposure Pathway & 95\% Subjective Confidence Interval \\
\hline Backyard Cow Milk & lower limit & central estimate & upper limit \\
Commercial Milk (locally produced) & 0.3 & 2 & 14 \\
Commercial Milk (regionally mixed) & 0.079 & 0.63 & 4.5 \\
Goat Milk (locally produced) & 0.1 & 0.8 & 5 \\
Beef (locally produced) & 0.62 & 5.8 & 54 \\
Leafy Vegetables (locally produced) & 0.00032 & 0.0059 & 0.13 \\
Eggs (locally produced) & 0.000086 & 0.00099 & 0.0095 \\
Cottage Cheese (locally produced) & 0.015 & 0.11 & 0.89 \\
Inhalation & 0.00037 & 0.0054 & 0.069 \\
Mother's milk (mother on Diet 1) & 0.0041 & 0.017 & 0.074 \\
Prenatal exposure (mother on Diet 1) & 0.0012 & 0.038 & 0.93 \\
Diet 1 & 0.014 & 0.13 & 1.1 \\
Diet 2 & 0.34 & 2.2 & 15 \\
Diet 3 & 0.12 & 0.84 & 5.4 \\
\hline
\end{tabular}

Excess Lifetime Risk [ ]

\begin{tabular}{llll}
\hline Diet 1 & $5.1 \mathrm{E}-06$ & $1.7 \mathrm{E}-04$ & $3.2 \mathrm{E}-03$ \\
Diet 2 & $2.3 \mathrm{E}-06$ & $6.9 \mathrm{E}-05$ & $1.6 \mathrm{E}-03$ \\
Diet 3 & $2.2 \mathrm{E}-06$ & $6.1 \mathrm{E}-05$ & $1.5 \mathrm{E}-03$ \\
Diet 4 & $1.2 \mathrm{E}-05$ & $5.0 \mathrm{E}-04$ & $1.3 \mathrm{E}-02$ \\
\hline
\end{tabular}

\section{Relative Risk [ ]}

\begin{tabular}{lccc}
\hline Diet 1 & 1.0065 & 1.12 & 3.4 \\
Diet 2 & 1.0023 & 1.043 & 2 \\
Diet 3 & 1.0022 & 1.044 & 1.95 \\
Diet 4 & 1.014 & 1.34 & 10.9 \\
\hline
\end{tabular}

\section{Probability of Causation [\%]}

\begin{tabular}{lccc}
\hline Diet 1 & 0.64 & 10.7 & 70 \\
Diet 2 & 0.23 & 4.1 & 51 \\
Diet 3 & 0.22 & 4.2 & 49 \\
Diet 4 & 1.40 & 25.1 & 91 \\
\hline
\end{tabular}

Diet 1 - Backyard cow milk + all other locally produced non-milk exposure pathways

Diet 2 - Locally produced commercial milk + all other locally produced non-milk exposure pathways

Diet 3 - Regionally mixed commercial milk + inhalation (other regionally mixed food items are minor contributors to the total)

Diet 4 - Goat milk (all other exposure pathways are of negligible importance compared to goat milk) 


\section{Location: Cedar Bluff}

Receptor: Female born in 1954

\begin{tabular}{lccc}
\hline & \multicolumn{3}{c}{ Thyroid Dose [cGy] } \\
Exposure Pathway & \multicolumn{2}{c}{$95 \%$ Subjective Confidence Interval } \\
\hline Backyard Cow Milk & lower limit & central estimate & upper limit \\
Commercial Milk (locally produced) & 0.68 & 4.9 & 36 \\
Commercial Milk (regionally mixed) & 0.19 & 1.6 & 12 \\
Goat Milk (locally produced) & 0.095 & 0.82 & 5.7 \\
Beef (locally produced) & 1.4 & 14 & 130 \\
Leafy Vegetables (locally produced) & 0.00068 & 0.014 & 0.29 \\
Eggs (locally produced) & 0.00021 & 0.0024 & 0.025 \\
Cottage Cheese (locally produced) & 0.033 & 0.26 & 2 \\
Inhalation & 0.00072 & 0.012 & 0.15 \\
Mother's milk (mother on Diet 1) & 0.0096 & 0.044 & 0.19 \\
Prenatal exposure (mother on Diet 1) & 0.0031 & 0.1 & 1.9 \\
Diet 1 & 0.03 & 0.33 & 2.3 \\
Diet 2 & 0.77 & 5.3 & 39 \\
Diet 3 & 0.27 & 2.1 & 13 \\
\hline
\end{tabular}

Excess Lifetime Risk [ ]

\begin{tabular}{lllc}
\hline Diet 1 & $1.0 \mathrm{E}-04$ & $1.6 \mathrm{E}-03$ & $2.8 \mathrm{E}-02$ \\
Diet 2 & $3.6 \mathrm{E}-05$ & $6.0 \mathrm{E}-04$ & $8.6 \mathrm{E}-03$ \\
Diet 3 & $1.7 \mathrm{E}-05$ & $2.5 \mathrm{E}-04$ & $3.3 \mathrm{E}-03$ \\
Diet 4 & $2.1 \mathrm{E}-04$ & $4.8 \mathrm{E}-03$ & $8.2 \mathrm{E}-02$ \\
\hline
\end{tabular}

\section{Relative Risk [ ]}

\begin{tabular}{lccc}
\hline Diet 1 & 1.036 & 1.4 & 6.3 \\
Diet 2 & 1.013 & 1.15 & 3.2 \\
Diet 3 & 1.0057 & 1.061 & 1.99 \\
Diet 4 & 1.082 & 2.1 & 17 \\
\hline
\end{tabular}

\begin{tabular}{lccc}
\hline Diet 1 & 3.50 & 28.7 & 84 \\
Diet 2 & 1.30 & 13.3 & 69 \\
Diet 3 & 0.57 & 5.8 & 50 \\
Diet 4 & 7.57 & 51.4 & 94 \\
\hline
\end{tabular}

Diet 1 - Backyard cow milk + all other locally produced non-milk exposure pathways

Diet 2 - Locally produced commercial milk + all other locally produced non-milk exposure pathways

Diet 3 - Regionally mixed commercial milk + inhalation (other regionally mixed food items are minor contributors to the total)

Diet 4 - Goat milk (all other exposure pathways are of negligible importance compared to goat milk) 


\section{Location: Cedar Bluff}

Receptor: Male born in 1954

\begin{tabular}{lccc}
\hline & \multicolumn{3}{c}{ Thyroid Dose [cGy] } \\
& \multicolumn{2}{c}{$95 \%$ Subjective Confidence Interval } \\
Exposure Pathway & lower limit & central estimate & upper limit \\
\hline Backyard Cow Milk & 0.74 & 4.7 & 31 \\
Commercial Milk (locally produced) & 0.19 & 1.6 & 10 \\
Commercial Milk (regionally mixed) & 0.1 & 0.8 & 5 \\
Goat Milk (locally produced) & 1.6 & 14 & 130 \\
Beef (locally produced) & 0.0007 & 0.014 & 0.3 \\
Leafy Vegetables (locally produced) & 0.0002 & 0.0024 & 0.026 \\
Eggs (locally produced) & 0.036 & 0.26 & 2.2 \\
Cottage Cheese (locally produced) & 0.00084 & 0.013 & 0.18 \\
Inhalation & 0.0095 & 0.045 & 0.2 \\
Mother's milk (mother on Diet 1) & 0.0031 & 0.1 & 1.9 \\
Prenatal exposure (mother on Diet 1) & 0.03 & 0.33 & 2.3 \\
Diet 1 & 0.83 & 5.2 & 34 \\
Diet 2 & 0.29 & 2.1 & 13 \\
Diet 3 & 0.12 & 0.85 & 5.1 \\
\hline
\end{tabular}

Excess Lifetime Risk [ ]

\begin{tabular}{llll}
\hline Diet 1 & $1.4 \mathrm{E}-05$ & $4.3 \mathrm{E}-04$ & $8.6 \mathrm{E}-03$ \\
Diet 2 & $5.6 \mathrm{E}-06$ & $1.7 \mathrm{E}-04$ & $3.5 \mathrm{E}-03$ \\
Diet 3 & $2.4 \mathrm{E}-06$ & $6.5 \mathrm{E}-05$ & $1.5 \mathrm{E}-03$ \\
Diet 4 & $2.9 \mathrm{E}-05$ & $1.2 \mathrm{E}-03$ & $2.9 \mathrm{E}-02$ \\
\hline
\end{tabular}

\section{Relative Risk [ ]}

\begin{tabular}{lccc}
\hline Diet 1 & 1.016 & 1.28 & 6.7 \\
Diet 2 & 1.0056 & 1.11 & 3.4 \\
Diet 3 & 1.0023 & 1.045 & 1.97 \\
Diet 4 & 1.035 & 1.78 & 23 \\
\hline
\end{tabular}

\section{Probability of Causation [\%]}

\begin{tabular}{lccc}
\hline Diet 1 & 1.57 & 22.1 & 85 \\
Diet 2 & 0.56 & 9.9 & 70 \\
Diet 3 & 0.23 & 4.4 & 49 \\
Diet 4 & 3.38 & 43.7 & 96 \\
\hline
\end{tabular}

Diet 1 - Backyard cow milk + all other locally produced non-milk exposure pathways

Diet 2 - Locally produced commercial milk + all other locally produced non-milk exposure pathways

Diet 3 - Regionally mixed commercial milk + inhalation (other regionally mixed food items are minor contributors to the total)

Diet 4 - Goat milk (all other exposure pathways are of negligible importance compared to goat milk) 


\section{Location: Oakdale}

Receptor: Female born in 1954

\begin{tabular}{lccc}
\hline & \multicolumn{3}{c}{ Thyroid Dose [cGy] } \\
Exposure Pathway & \multicolumn{2}{c}{$95 \%$ Subjective Confidence Interval } \\
\hline Backyard Cow Milk & lower limit & central estimate & upper limit \\
Commercial Milk (locally produced) & 0.16 & 1.2 & 8.9 \\
Commercial Milk (regionally mixed) & 0.04 & 0.37 & 3.1 \\
Goat Milk (locally produced) & 0.095 & 0.82 & 5.7 \\
Beef (locally produced) & 0.29 & 3.3 & 33 \\
Leafy Vegetables (locally produced) & 0.00016 & 0.0032 & 0.067 \\
Eggs (locally produced) & 0.000047 & 0.00058 & 0.006 \\
Cottage Cheese (locally produced) & 0.0067 & 0.06 & 0.51 \\
Inhalation & 0.00018 & 0.0029 & 0.039 \\
Mother's milk (mother on Diet 1) & 0.0022 & 0.01 & 0.048 \\
Prenatal exposure (mother on Diet 1) & 0.00067 & 0.023 & 0.5 \\
Diet 1 & 0.0073 & 0.076 & 0.59 \\
Diet 2 & 0.17 & 1.3 & 9.5 \\
Diet 3 & 0.061 & 0.48 & 3.6 \\
\hline
\end{tabular}

Excess Lifetime Risk [ ]

\begin{tabular}{llll}
\hline Diet 1 & $2.2 \mathrm{E}-05$ & $4.0 \mathrm{E}-04$ & $6.3 \mathrm{E}-03$ \\
Diet 2 & $7.8 \mathrm{E}-06$ & $1.4 \mathrm{E}-04$ & $2.0 \mathrm{E}-03$ \\
Diet 3 & $1.4 \mathrm{E}-05$ & $2.3 \mathrm{E}-04$ & $3.2 \mathrm{E}-03$ \\
Diet 4 & $4.7 \mathrm{E}-05$ & $1.1 \mathrm{E}-03$ & $2.0 \mathrm{E}-02$ \\
\hline
\end{tabular}

\section{Relative Risk [ ]}

\begin{tabular}{lccc}
\hline Diet 1 & 1.0076 & 1.09 & 2.3 \\
Diet 2 & 1.0028 & 1.035 & 1.57 \\
Diet 3 & 1.0051 & 1.059 & 1.96 \\
Diet 4 & 1.018 & 1.26 & 5.3 \\
\hline
\end{tabular}

\section{Probability of Causation [\%]}

\begin{tabular}{lccc}
\hline Diet 1 & 0.75 & 8.2 & 56 \\
Diet 2 & 0.28 & 3.4 & 36 \\
Diet 3 & 0.51 & 5.6 & 49 \\
Diet 4 & 1.80 & 20.1 & 80 \\
\hline
\end{tabular}

Diet 1 - Backyard cow milk + all other locally produced non-milk exposure pathways

Diet 2 - Locally produced commercial milk + all other locally produced non-milk exposure pathways

Diet 3 - Regionally mixed commercial milk + inhalation (other regionally mixed food items are minor contributors to the total)

Diet 4 - Goat milk (all other exposure pathways are of negligible importance compared to goat milk) 


\section{Location: Oakdale}

\section{Receptor: Male born in 1954}

\begin{tabular}{lccc}
\hline & \multicolumn{3}{c}{ Thyroid Dose [cGy] } \\
& \multicolumn{2}{c}{$95 \%$ Subjective Confidence Interval } \\
Exposure Pathway & lower limit & central estimate & upper limit \\
\hline Backyard Cow Milk & 0.16 & 1.1 & 8.1 \\
Commercial Milk (locally produced) & 0.04 & 0.38 & 2.6 \\
Commercial Milk (regionally mixed) & 0.1 & 0.8 & 5 \\
Goat Milk (locally produced) & 0.35 & 3.3 & 33 \\
Beef (locally produced) & 0.00016 & 0.0033 & 0.07 \\
Leafy Vegetables (locally produced) & 0.000044 & 0.00056 & 0.0059 \\
Eggs (locally produced) & 0.0073 & 0.06 & 0.52 \\
Cottage Cheese (locally produced) & 0.0002 & 0.003 & 0.044 \\
Inhalation & 0.0022 & 0.01 & 0.046 \\
Mother's milk (mother on Diet 1) & 0.00067 & 0.023 & 0.5 \\
Prenatal exposure (mother on Diet 1) & 0.0073 & 0.076 & 0.59 \\
Diet 1 & 0.18 & 1.2 & 8.8 \\
Diet 2 & 0.061 & 0.48 & 3.1 \\
Diet 3 & 0.11 & 0.81 & 5 \\
\hline
\end{tabular}

Excess Lifetime Risk [ ]

\begin{tabular}{lllc}
\hline Diet 1 & $2.9 \mathrm{E}-06$ & $9.7 \mathrm{E}-05$ & $2.1 \mathrm{E}-03$ \\
Diet 2 & $1.3 \mathrm{E}-06$ & $4.0 \mathrm{E}-05$ & $8.4 \mathrm{E}-04$ \\
Diet 3 & $2.1 \mathrm{E}-06$ & $6.0 \mathrm{E}-05$ & $1.5 \mathrm{E}-03$ \\
Diet 4 & $6.7 \mathrm{E}-06$ & $2.9 \mathrm{E}-04$ & $7.2 \mathrm{E}-03$ \\
\hline
\end{tabular}

Relative Risk [ ]

\begin{tabular}{lccc}
\hline Diet 1 & 1.0036 & 1.066 & 2.5 \\
Diet 2 & 1.0013 & 1.026 & 1.57 \\
Diet 3 & 1.0022 & 1.043 & 1.95 \\
Diet 4 & 1.0073 & 1.19 & 6.5 \\
\hline
\end{tabular}

\section{Probability of Causation [\%]}

\begin{tabular}{lccc}
\hline Diet 1 & 0.36 & 6.2 & 58 \\
Diet 2 & 0.13 & 2.5 & 36 \\
Diet 3 & 0.22 & 4.1 & 49 \\
Diet 4 & 0.73 & 16.0 & 84 \\
\hline
\end{tabular}

Diet 1 - Backyard cow milk + all other locally produced non-milk exposure pathways

Diet 2 - Locally produced commercial milk + all other locally produced non-milk exposure pathways

Diet 3 - Regionally mixed commercial milk + inhalation (other regionally mixed food items are minor contributors to the total)

Diet 4 - Goat milk (all other exposure pathways are of negligible importance compared to goat milk) 


\section{Location: Claxton}

Receptor: Female born in 1954

\begin{tabular}{lccc}
\hline & \multicolumn{3}{c}{ Thyroid Dose [cGy] } \\
& \multicolumn{2}{c}{$95 \%$ Subjective Confidence Interval } \\
Exposure Pathway & lower limit & central estimate & upper limit \\
\hline Backyard Cow Milk & 0.58 & 4.1 & 31 \\
Commercial Milk (locally produced) & 0.17 & 1.4 & 9.5 \\
Commercial Milk (regionally mixed) & 0.094 & 0.81 & 5.7 \\
Goat Milk (locally produced) & 1.2 & 12 & 110 \\
Beef (locally produced) & 0.00058 & 0.012 & 0.24 \\
Leafy Vegetables (locally produced) & 0.00018 & 0.002 & 0.02 \\
Eggs (locally produced) & 0.028 & 0.21 & 1.7 \\
Cottage Cheese (locally produced) & 0.00063 & 0.01 & 0.13 \\
Inhalation & 0.008 & 0.037 & 0.16 \\
Mother's milk (mother on Diet 1) & 0.0025 & 0.082 & 1.7 \\
Prenatal exposure (mother on Diet 1) & 0.025 & 0.27 & 1.9 \\
Diet 1 & 0.64 & 4.5 & 33 \\
Diet 2 & 0.23 & 1.7 & 11 \\
Diet 3 & 0.11 & 0.86 & 5.8 \\
\hline
\end{tabular}

Excess Lifetime Risk [ ]

\begin{tabular}{llll}
\hline Diet 1 & $8.7 \mathrm{E}-05$ & $1.4 \mathrm{E}-03$ & $2.2 \mathrm{E}-02$ \\
Diet 2 & $3.1 \mathrm{E}-05$ & $5.0 \mathrm{E}-04$ & $7.1 \mathrm{E}-03$ \\
Diet 3 & $1.6 \mathrm{E}-05$ & $2.5 \mathrm{E}-04$ & $3.3 \mathrm{E}-03$ \\
Diet 4 & $1.7 \mathrm{E}-04$ & $4.0 \mathrm{E}-03$ & $6.7 \mathrm{E}-02$ \\
\hline
\end{tabular}

\section{Relative Risk [ ]}

\begin{tabular}{lccc}
\hline Diet 1 & 1.032 & 1.33 & 5.6 \\
Diet 2 & 1.011 & 1.13 & 2.8 \\
Diet 3 & 1.0056 & 1.061 & 1.98 \\
Diet 4 & 1.071 & 1.89 & 14 \\
\hline
\end{tabular}

\section{Probability of Causation [\%]}

\begin{tabular}{lccc}
\hline Diet 1 & 3.14 & 25.0 & 82 \\
Diet 2 & 1.10 & 11.3 & 64 \\
Diet 3 & 0.56 & 5.7 & 49 \\
Diet 4 & 6.65 & 47.0 & 93 \\
\hline
\end{tabular}

Diet 1 - Backyard cow milk + all other locally produced non-milk exposure pathways

Diet 2 - Locally produced commercial milk + all other locally produced non-milk exposure pathways

Diet 3 - Regionally mixed commercial milk + inhalation (other regionally mixed food items are minor contributors to the total)

Diet 4 - Goat milk (all other exposure pathways are of negligible importance compared to goat milk) 


\section{Location: Claxton}

\section{Receptor: Male born in 1954}

\begin{tabular}{lccc}
\hline & \multicolumn{3}{c}{ Thyroid Dose [cGy] } \\
& \multicolumn{2}{c}{$95 \%$ Subjective Confidence Interval } \\
Exposure Pathway & lower limit & central estimate & upper limit \\
\hline Backyard Cow Milk & 0.63 & 3.9 & 27 \\
Commercial Milk (locally produced) & 0.16 & 1.3 & 8.8 \\
Commercial Milk (regionally mixed) & 0.1 & 0.8 & 5 \\
Goat Milk (locally produced) & 1.4 & 12 & 110 \\
Beef (locally produced) & 0.00061 & 0.012 & 0.26 \\
Leafy Vegetables (locally produced) & 0.00016 & 0.002 & 0.021 \\
Eggs (locally produced) & 0.03 & 0.22 & 1.7 \\
Cottage Cheese (locally produced) & 0.00073 & 0.011 & 0.14 \\
Inhalation & 0.0081 & 0.037 & 0.16 \\
Mother's milk (mother on Diet 1) & 0.0025 & 0.082 & 1.7 \\
Prenatal exposure (mother on Diet 1) & 0.025 & 0.27 & 1.9 \\
Diet 1 & 0.69 & 4.3 & 29 \\
Diet 2 & 0.25 & 1.7 & 11 \\
Diet 3 & 0.11 & 0.84 & 5 \\
\hline
\end{tabular}

Excess Lifetime Risk [ ]

\begin{tabular}{lllc}
\hline Diet 1 & $1.2 \mathrm{E}-05$ & $3.5 \mathrm{E}-04$ & $7.1 \mathrm{E}-03$ \\
Diet 2 & $4.9 \mathrm{E}-06$ & $1.4 \mathrm{E}-04$ & $3.0 \mathrm{E}-03$ \\
Diet 3 & $2.4 \mathrm{E}-06$ & $6.4 \mathrm{E}-05$ & $1.5 \mathrm{E}-03$ \\
Diet 4 & $2.5 \mathrm{E}-05$ & $9.8 \mathrm{E}-04$ & $2.5 \mathrm{E}-02$ \\
\hline
\end{tabular}

\section{Relative Risk [ ]}

\begin{tabular}{lccc}
\hline Diet 1 & 1.013 & 1.24 & 5.8 \\
Diet 2 & 1.0047 & 1.092 & 3 \\
Diet 3 & 1.0023 & 1.045 & 1.96 \\
Diet 4 & 1.029 & 1.65 & 20 \\
\hline
\end{tabular}

\section{Probability of Causation [\%]}

\begin{tabular}{lccc}
\hline Diet 1 & 1.30 & 19.4 & 83 \\
Diet 2 & 0.47 & 8.4 & 67 \\
Diet 3 & 0.23 & 4.3 & 49 \\
Diet 4 & 2.77 & 39.4 & 95 \\
\hline
\end{tabular}

Diet 1 - Backyard cow milk + all other locally produced non-milk exposure pathways

Diet 2 - Locally produced commercial milk + all other locally produced non-milk exposure pathways

Diet 3 - Regionally mixed commercial milk + inhalation (other regionally mixed food items are minor contributors to the total)

Diet 4 - Goat milk (all other exposure pathways are of negligible importance compared to goat milk) 
Location: Dutch Valley

Receptor: Female born in 1954

\begin{tabular}{lccc}
\hline & \multicolumn{3}{c}{ Thyroid Dose [cGy] } \\
Exposure Pathway & $95 \%$ Subjective Confidence Interval \\
\hline Backyard Cow Milk & lower limit & central estimate & upper limit \\
Commercial Milk (locally produced) & 0.28 & 2 & 13 \\
Commercial Milk (regionally mixed) & 0.079 & 0.65 & 5 \\
Goat Milk (locally produced) & 0.095 & 0.82 & 5.7 \\
Beef (locally produced) & 0.55 & 5.7 & 57 \\
Leafy Vegetables (locally produced) & 0.00027 & 0.0055 & 0.13 \\
Eggs (locally produced) & 0.00009 & 0.00097 & 0.0097 \\
Cottage Cheese (locally produced) & 0.012 & 0.1 & 0.86 \\
Inhalation & 0.00029 & 0.0052 & 0.062 \\
Mother's milk (mother on Diet 1) & 0.0037 & 0.017 & 0.073 \\
Prenatal exposure (mother on Diet 1) & 0.0012 & 0.039 & 0.83 \\
Diet 1 & 0.013 & 0.13 & 0.96 \\
Diet 2 & 0.32 & 2.1 & 15 \\
Diet 3 & 0.11 & 0.82 & 5.6 \\
\hline
\end{tabular}

Excess Lifetime Risk [ ]

\begin{tabular}{llll}
\hline Diet 1 & $4.0 \mathrm{E}-05$ & $6.7 \mathrm{E}-04$ & $1.1 \mathrm{E}-02$ \\
Diet 2 & $1.5 \mathrm{E}-05$ & $2.4 \mathrm{E}-04$ & $3.5 \mathrm{E}-03$ \\
Diet 3 & $1.4 \mathrm{E}-05$ & $2.4 \mathrm{E}-04$ & $3.2 \mathrm{E}-03$ \\
Diet 4 & $8.9 \mathrm{E}-05$ & $2.0 \mathrm{E}-03$ & $3.6 \mathrm{E}-02$ \\
\hline
\end{tabular}

\section{Relative Risk [ ]}

\begin{tabular}{lccc}
\hline Diet 1 & 1.015 & 1.16 & 3.1 \\
Diet 2 & 1.0051 & 1.061 & 1.94 \\
Diet 3 & 1.0052 & 1.059 & 1.97 \\
Diet 4 & 1.032 & 1.44 & 8.2 \\
\hline
\end{tabular}

\section{Probability of Causation [\%]}

\begin{tabular}{lccc}
\hline Diet 1 & 1.45 & 13.5 & 67 \\
Diet 2 & 0.51 & 5.7 & 48 \\
Diet 3 & 0.52 & 5.6 & 49 \\
Diet 4 & 3.12 & 30.3 & 87 \\
\hline
\end{tabular}

Diet 1 - Backyard cow milk + all other locally produced non-milk exposure pathways

Diet 2 - Locally produced commercial milk + all other locally produced non-milk exposure pathways

Diet 3 - Regionally mixed commercial milk + inhalation (other regionally mixed food items are minor contributors to the total)

Diet 4 - Goat milk (all other exposure pathways are of negligible importance compared to goat milk) 
Location: Dutch Valley

Receptor: Male born in 1954

\begin{tabular}{lccc}
\hline & \multicolumn{3}{c}{ Thyroid Dose [cGy] } \\
Exposure Pathway & $95 \%$ Subjective Confidence Interval \\
\hline Backyard Cow Milk & lower limit & central estimate & upper limit \\
Commercial Milk (locally produced) & 0.3 & 2 & 13 \\
Commercial Milk (regionally mixed) & 0.076 & 0.63 & 4.4 \\
Goat Milk (locally produced) & 0.1 & 0.8 & 5 \\
Beef (locally produced) & 0.59 & 5.7 & 54 \\
Leafy Vegetables (locally produced) & 0.0003 & 0.0056 & 0.12 \\
Eggs (locally produced) & 0.000079 & 0.00094 & 0.0095 \\
Cottage Cheese (locally produced) & 0.013 & 0.1 & 0.88 \\
Inhalation & 0.00035 & 0.0052 & 0.066 \\
Mother's milk (mother on Diet 1) & 0.004 & 0.018 & 0.076 \\
Prenatal exposure (mother on Diet 1) & 0.0012 & 0.039 & 0.83 \\
Diet 1 & 0.013 & 0.13 & 0.96 \\
Diet 2 & 0.33 & 2.1 & 14 \\
Diet 3 & 0.11 & 0.82 & 5.2 \\
\hline
\end{tabular}

Excess Lifetime Risk [ ]

\begin{tabular}{lllc}
\hline Diet 1 & $5.4 \mathrm{E}-06$ & $1.7 \mathrm{E}-04$ & $3.1 \mathrm{E}-03$ \\
Diet 2 & $2.2 \mathrm{E}-06$ & $6.5 \mathrm{E}-05$ & $1.5 \mathrm{E}-03$ \\
Diet 3 & $2.2 \mathrm{E}-06$ & $6.1 \mathrm{E}-05$ & $1.5 \mathrm{E}-03$ \\
Diet 4 & $1.3 \mathrm{E}-05$ & $4.8 \mathrm{E}-04$ & $1.2 \mathrm{E}-02$ \\
\hline
\end{tabular}

\section{Relative Risk [ ]}

\begin{tabular}{lccc}
\hline Diet 1 & 1.006 & 1.12 & 3.3 \\
Diet 2 & 1.0021 & 1.043 & 1.93 \\
Diet 3 & 1.0022 & 1.044 & 1.95 \\
Diet 4 & 1.013 & 1.32 & 10.3 \\
\hline
\end{tabular}

\section{Probability of Causation [\%]}

\begin{tabular}{lccc}
\hline Diet 1 & 0.60 & 10.3 & 69 \\
Diet 2 & 0.21 & 4.1 & 48 \\
Diet 3 & 0.22 & 4.2 & 49 \\
Diet 4 & 1.31 & 24.0 & 90 \\
\hline
\end{tabular}

Diet 1 - Backyard cow milk + all other locally produced non-milk exposure pathways

Diet 2 - Locally produced commercial milk + all other locally produced non-milk exposure pathways

Diet 3 - Regionally mixed commercial milk + inhalation (other regionally mixed food items are minor contributors to the total)

Diet 4 - Goat milk (all other exposure pathways are of negligible importance compared to goat milk) 


\section{Location: Clinton}

Receptor: Female born in 1954

\begin{tabular}{lccc}
\hline & \multicolumn{3}{c}{ Thyroid Dose [cGy] } \\
Exposure Pathway & \multicolumn{2}{c}{$95 \%$ Subjective Confidence Interval } \\
\hline Backyard Cow Milk & lower limit & central estimate & upper limit \\
Commercial Milk (locally produced) & 0.44 & 3.2 & 24 \\
Commercial Milk (regionally mixed) & 0.12 & 1 & 7.6 \\
Goat Milk (locally produced) & 0.094 & 0.81 & 5.7 \\
Beef (locally produced) & 0.85 & 8.8 & 85 \\
Leafy Vegetables (locally produced) & 0.00044 & 0.009 & 0.18 \\
Eggs (locally produced) & 0.00013 & 0.0016 & 0.016 \\
Cottage Cheese (locally produced) & 0.021 & 0.16 & 1.3 \\
Inhalation & 0.00051 & 0.0078 & 0.099 \\
Mother's milk (mother on Diet 1) & 0.0065 & 0.028 & 0.12 \\
Prenatal exposure (mother on Diet 1) & 0.0019 & 0.065 & 1.2 \\
Diet 1 & 0.02 & 0.21 & 1.5 \\
Diet 2 & 0.48 & 3.5 & 26 \\
Diet 3 & 0.17 & 1.3 & 8.8 \\
\hline
\end{tabular}

Excess Lifetime Risk [ ]

\begin{tabular}{llll}
\hline Diet 1 & $6.3 \mathrm{E}-05$ & $1.1 \mathrm{E}-03$ & $1.7 \mathrm{E}-02$ \\
Diet 2 & $2.2 \mathrm{E}-05$ & $3.9 \mathrm{E}-04$ & $5.2 \mathrm{E}-03$ \\
Diet 3 & $1.5 \mathrm{E}-05$ & $2.4 \mathrm{E}-04$ & $3.3 \mathrm{E}-03$ \\
Diet 4 & $1.3 \mathrm{E}-04$ & $3.1 \mathrm{E}-03$ & $5.0 \mathrm{E}-02$ \\
\hline
\end{tabular}

\section{Relative Risk [ ]}

\begin{tabular}{lccc}
\hline Diet 1 & 1.023 & 1.25 & 4.5 \\
Diet 2 & 1.0081 & 1.099 & 2.4 \\
Diet 3 & 1.0055 & 1.06 & 1.98 \\
Diet 4 & 1.052 & 1.69 & 11 \\
\hline
\end{tabular}

\begin{tabular}{lccc} 
& \multicolumn{3}{c}{ Probability of Causation [\%] } \\
\hline Diet 1 & 2.27 & 19.9 & 77 \\
Diet 2 & 0.81 & 9.0 & 57 \\
Diet 3 & 0.54 & 5.7 & 49 \\
Diet 4 & 4.92 & 40.5 & 91 \\
\hline
\end{tabular}

Diet 1 - Backyard cow milk + all other locally produced non-milk exposure pathways

Diet 2 - Locally produced commercial milk + all other locally produced non-milk exposure pathways

Diet 3 - Regionally mixed commercial milk + inhalation (other regionally mixed food items are minor contributors to the total)

Diet 4 - Goat milk (all other exposure pathways are of negligible importance compared to goat milk) 


\section{Location: Clinton}

\section{Receptor: Male born in 1954}

\begin{tabular}{lccc}
\hline & \multicolumn{3}{c}{ Thyroid Dose [cGy] } \\
& \multicolumn{2}{c}{$95 \%$ Subjective Confidence Interval } \\
Exposure Pathway & lower limit & central estimate & upper limit \\
\hline Backyard Cow Milk & 0.47 & 3.1 & 20 \\
Commercial Milk (locally produced) & 0.12 & 1.1 & 6.7 \\
Commercial Milk (regionally mixed) & 0.1 & 0.8 & 5 \\
Goat Milk (locally produced) & 0.99 & 9.1 & 84 \\
Beef (locally produced) & 0.00047 & 0.0089 & 0.19 \\
Leafy Vegetables (locally produced) & 0.00012 & 0.0015 & 0.016 \\
Eggs (locally produced) & 0.022 & 0.16 & 1.4 \\
Cottage Cheese (locally produced) & 0.00053 & 0.0082 & 0.11 \\
Inhalation & 0.0064 & 0.028 & 0.13 \\
Mother's milk (mother on Diet 1) & 0.0019 & 0.065 & 1.2 \\
Prenatal exposure (mother on Diet 1) & 0.02 & 0.21 & 1.5 \\
Diet 1 & 0.52 & 3.3 & 22 \\
Diet 2 & 0.18 & 1.3 & 8.4 \\
Diet 3 & 0.11 & 0.83 & 5 \\
\hline
\end{tabular}

Excess Lifetime Risk [ ]

\begin{tabular}{llll}
\hline Diet 1 & $8.4 \mathrm{E}-06$ & $2.7 \mathrm{E}-04$ & $5.3 \mathrm{E}-03$ \\
Diet 2 & $3.7 \mathrm{E}-06$ & $1.1 \mathrm{E}-04$ & $2.3 \mathrm{E}-03$ \\
Diet 3 & $2.3 \mathrm{E}-06$ & $6.2 \mathrm{E}-05$ & $1.5 \mathrm{E}-03$ \\
Diet 4 & $1.9 \mathrm{E}-05$ & $7.7 \mathrm{E}-04$ & $1.8 \mathrm{E}-02$ \\
\hline
\end{tabular}

\section{Relative Risk [ ]}

\begin{tabular}{lccc}
\hline Diet 1 & 1.011 & 1.18 & 4.8 \\
Diet 2 & 1.0037 & 1.07 & 2.5 \\
Diet 3 & 1.0023 & 1.044 & 1.96 \\
Diet 4 & 1.022 & 1.51 & 16 \\
\hline
\end{tabular}

\section{Probability of Causation [\%]}

\begin{tabular}{lccc}
\hline Diet 1 & 1.08 & 15.5 & 78 \\
Diet 2 & 0.37 & 6.6 & 60 \\
Diet 3 & 0.23 & 4.3 & 49 \\
Diet 4 & 2.17 & 33.4 & 93 \\
\hline
\end{tabular}

Diet 1 - Backyard cow milk + all other locally produced non-milk exposure pathways

Diet 2 - Locally produced commercial milk + all other locally produced non-milk exposure pathways

Diet 3 - Regionally mixed commercial milk + inhalation (other regionally mixed food items are minor contributors to the total)

Diet 4 - Goat milk (all other exposure pathways are of negligible importance compared to goat milk) 
Location: Friendsville

Receptor: Female born in 1954

\begin{tabular}{lccc}
\hline & \multicolumn{3}{c}{ Thyroid Dose [cGy] } \\
Exposure Pathway & $95 \%$ Subjective Confidence Interval \\
\hline Backyard Cow Milk & lower limit & central estimate & upper limit \\
Commercial Milk (locally produced) & 0.23 & 1.7 & 12 \\
Commercial Milk (regionally mixed) & 0.067 & 0.59 & 4 \\
Goat Milk (locally produced) & 0.094 & 0.81 & 5.7 \\
Beef (locally produced) & 0.55 & 5.2 & 53 \\
Leafy Vegetables (locally produced) & 0.00025 & 0.005 & 0.11 \\
Eggs (locally produced) & 0.000076 & 0.00086 & 0.0084 \\
Cottage Cheese (locally produced) & 0.011 & 0.096 & 0.75 \\
Inhalation & 0.00027 & 0.0048 & 0.056 \\
Mother's milk (mother on Diet 1) & 0.0038 & 0.019 & 0.088 \\
Prenatal exposure (mother on Diet 1) & 0.0011 & 0.036 & 0.76 \\
Diet 1 & 0.011 & 0.12 & 0.9 \\
Diet 2 & 0.27 & 1.9 & 13 \\
Diet 3 & 0.1 & 0.76 & 4.8 \\
\hline
\end{tabular}

Excess Lifetime Risk [ ]

\begin{tabular}{lllc}
\hline Diet 1 & $3.5 \mathrm{E}-05$ & $6.1 \mathrm{E}-04$ & $9.9 \mathrm{E}-03$ \\
Diet 2 & $1.4 \mathrm{E}-05$ & $2.2 \mathrm{E}-04$ & $2.9 \mathrm{E}-03$ \\
Diet 3 & $1.4 \mathrm{E}-05$ & $2.4 \mathrm{E}-04$ & $3.2 \mathrm{E}-03$ \\
Diet 4 & $7.3 \mathrm{E}-05$ & $1.8 \mathrm{E}-03$ & $3.1 \mathrm{E}-02$ \\
\hline
\end{tabular}

\section{Relative Risk [ ]}

\begin{tabular}{lccc}
\hline Diet 1 & 1.014 & 1.15 & 2.6 \\
Diet 2 & 1.0046 & 1.057 & 1.78 \\
Diet 3 & 1.0053 & 1.059 & 1.97 \\
Diet 4 & 1.028 & 1.4 & 7.9 \\
\hline
\end{tabular}

\section{Probability of Causation [\%]}

\begin{tabular}{lccc}
\hline Diet 1 & 1.35 & 13.3 & 61 \\
Diet 2 & 0.45 & 5.4 & 44 \\
Diet 3 & 0.53 & 5.6 & 49 \\
Diet 4 & 2.73 & 28.7 & 87 \\
\hline
\end{tabular}

Diet 1 - Backyard cow milk + all other locally produced non-milk exposure pathways

Diet 2 - Locally produced commercial milk + all other locally produced non-milk exposure pathways

Diet 3 - Regionally mixed commercial milk + inhalation (other regionally mixed food items are minor contributors to the total)

Diet 4 - Goat milk (all other exposure pathways are of negligible importance compared to goat milk) 
Location: Friendsville

Receptor: Male born in 1954

\begin{tabular}{lccc}
\hline & \multicolumn{3}{c}{ Thyroid Dose [cGy] } \\
Exposure Pathway & 95\% Subjective Confidence Interval \\
\hline Backyard Cow Milk & lower limit & central estimate & upper limit \\
Commercial Milk (locally produced) & 0.26 & 1.8 & 12 \\
Commercial Milk (regionally mixed) & 0.063 & 0.61 & 4 \\
Goat Milk (locally produced) & 0.1 & 0.8 & 5 \\
Beef (locally produced) & 0.52 & 5.3 & 52 \\
Leafy Vegetables (locally produced) & 0.00026 & 0.0049 & 0.11 \\
Eggs (locally produced) & 0.000072 & 0.00086 & 0.0083 \\
Cottage Cheese (locally produced) & 0.012 & 0.094 & 0.89 \\
Inhalation & 0.00032 & 0.0051 & 0.062 \\
Mother's milk (mother on Diet 1) & 0.0041 & 0.02 & 0.082 \\
Prenatal exposure (mother on Diet 1) & 0.0011 & 0.036 & 0.76 \\
Diet 1 & 0.011 & 0.12 & 0.9 \\
Diet 2 & 0.29 & 2 & 12 \\
Diet 3 & 0.099 & 0.75 & 4.8 \\
\hline
\end{tabular}

Excess Lifetime Risk [ ]

\begin{tabular}{lllr}
\hline Diet 1 & $5.1 \mathrm{E}-06$ & $1.5 \mathrm{E}-04$ & $3.0 \mathrm{E}-03$ \\
Diet 2 & $1.9 \mathrm{E}-06$ & $5.8 \mathrm{E}-05$ & $1.3 \mathrm{E}-03$ \\
Diet 3 & $2.2 \mathrm{E}-06$ & $6.2 \mathrm{E}-05$ & $1.5 \mathrm{E}-03$ \\
Diet 4 & $1.0 \mathrm{E}-05$ & $4.3 \mathrm{E}-04$ & $1.2 \mathrm{E}-02$ \\
\hline
\end{tabular}

\section{Relative Risk [ ]}

\begin{tabular}{lccc}
\hline Diet 1 & 1.0056 & 1.11 & 3.5 \\
Diet 2 & 1.0018 & 1.04 & 1.87 \\
Diet 3 & 1.0022 & 1.044 & 1.95 \\
Diet 4 & 1.011 & 1.31 & 9.5 \\
\hline
\end{tabular}

\section{Probability of Causation [\%]}

\begin{tabular}{lccc}
\hline Diet 1 & 0.56 & 9.7 & 71 \\
Diet 2 & 0.18 & 3.8 & 47 \\
Diet 3 & 0.22 & 4.2 & 49 \\
Diet 4 & 1.08 & 23.5 & 89 \\
\hline
\end{tabular}

Diet 1 - Backyard cow milk + all other locally produced non-milk exposure pathways

Diet 2 - Locally produced commercial milk + all other locally produced non-milk exposure pathways

Diet 3 - Regionally mixed commercial milk + inhalation (other regionally mixed food items are minor contributors to the total)

Diet 4 - Goat milk (all other exposure pathways are of negligible importance compared to goat milk) 
Location: Wartburg

Receptor: Female born in 1954

\begin{tabular}{lccc}
\hline & \multicolumn{3}{c}{ Thyroid Dose [cGy] } \\
Exposure Pathway & \multicolumn{2}{c}{$95 \%$ Subjective Confidence Interval } \\
\hline Backyard Cow Milk & lower limit & central estimate & upper limit \\
Commercial Milk (locally produced) & 0.062 & 0.5 & 4.2 \\
Commercial Milk (regionally mixed) & 0.018 & 0.16 & 1.4 \\
Goat Milk (locally produced) & 0.094 & 0.81 & 5.7 \\
Beef (locally produced) & 0.14 & 1.5 & 15 \\
Leafy Vegetables (locally produced) & 0.00007 & 0.0014 & 0.038 \\
Eggs (locally produced) & 0.000019 & 0.00025 & 0.0026 \\
Cottage Cheese (locally produced) & 0.0024 & 0.026 & 0.24 \\
Inhalation & 0.000077 & 0.0013 & 0.018 \\
Mother's milk (mother on Diet 1) & 0.00089 & 0.0043 & 0.024 \\
Prenatal exposure (mother on Diet 1) & 0.00028 & 0.01 & 0.22 \\
Diet 1 & 0.003 & 0.032 & 0.26 \\
Diet 2 & 0.072 & 0.54 & 4.4 \\
Diet 3 & 0.026 & 0.2 & 1.7 \\
\hline
\end{tabular}

Excess Lifetime Risk [ ]

\begin{tabular}{lllc}
\hline Diet 1 & $8.3 \mathrm{E}-06$ & $1.6 \mathrm{E}-04$ & $3.0 \mathrm{E}-03$ \\
Diet 2 & $3.8 \mathrm{E}-06$ & $6.0 \mathrm{E}-05$ & $9.4 \mathrm{E}-04$ \\
Diet 3 & $1.3 \mathrm{E}-05$ & $2.3 \mathrm{E}-04$ & $3.2 \mathrm{E}-03$ \\
Diet 4 & $1.7 \mathrm{E}-05$ & $4.6 \mathrm{E}-04$ & $9.8 \mathrm{E}-03$ \\
\hline
\end{tabular}

\section{Relative Risk [ ]}

\begin{tabular}{lccc}
\hline Diet 1 & 1.0038 & 1.04 & 1.54 \\
Diet 2 & 1.0014 & 1.015 & 1.25 \\
Diet 3 & 1.005 & 1.058 & 1.96 \\
Diet 4 & 1.0082 & 1.11 & 3.1 \\
\hline
\end{tabular}

\section{Probability of Causation [\%]}

\begin{tabular}{llll}
\hline Diet 1 & 0.38 & 3.9 & 35 \\
Diet 2 & 0.14 & 1.5 & 20 \\
Diet 3 & 0.49 & 5.5 & 49 \\
Diet 4 & 0.81 & 9.7 & 68 \\
\hline
\end{tabular}

Diet 1 - Backyard cow milk + all other locally produced non-milk exposure pathways

Diet 2 - Locally produced commercial milk + all other locally produced non-milk exposure pathways

Diet 3 - Regionally mixed commercial milk + inhalation (other regionally mixed food items are minor contributors to the total)

Diet 4 - Goat milk (all other exposure pathways are of negligible importance compared to goat milk) 


\section{Location: Wartburg}

\section{Receptor: Male born in 1954}

\begin{tabular}{lccc}
\hline & \multicolumn{3}{c}{ Thyroid Dose [cGy] } \\
& \multicolumn{2}{c}{$95 \%$ Subjective Confidence Interval } \\
Exposure Pathway & lower limit & central estimate & upper limit \\
\hline Backyard Cow Milk & 0.064 & 0.48 & 4 \\
Commercial Milk (locally produced) & 0.018 & 0.16 & 1.2 \\
Commercial Milk (regionally mixed) & 0.1 & 0.8 & 5 \\
Goat Milk (locally produced) & 0.14 & 1.5 & 15 \\
Beef (locally produced) & 0.000075 & 0.0014 & 0.039 \\
Leafy Vegetables (locally produced) & 0.000018 & 0.00024 & 0.0027 \\
Eggs (locally produced) & 0.0027 & 0.028 & 0.26 \\
Cottage Cheese (locally produced) & 0.000081 & 0.0014 & 0.017 \\
Inhalation & 0.00096 & 0.0046 & 0.023 \\
Mother's milk (mother on Diet 1) & 0.00028 & 0.01 & 0.22 \\
Prenatal exposure (mother on Diet 1) & 0.003 & 0.032 & 0.26 \\
Diet 1 & 0.072 & 0.52 & 4.3 \\
Diet 2 & 0.028 & 0.21 & 1.5 \\
Diet 3 & 0.1 & 0.81 & 5 \\
\hline
\end{tabular}

Excess Lifetime Risk [ ]

\begin{tabular}{llll}
\hline Diet 1 & $1.2 \mathrm{E}-06$ & $4.1 \mathrm{E}-05$ & $8.0 \mathrm{E}-04$ \\
Diet 2 & $5.5 \mathrm{E}-07$ & $1.6 \mathrm{E}-05$ & $3.5 \mathrm{E}-04$ \\
Diet 3 & $2.1 \mathrm{E}-06$ & $5.9 \mathrm{E}-05$ & $1.5 \mathrm{E}-03$ \\
Diet 4 & $3.1 \mathrm{E}-06$ & $1.2 \mathrm{E}-04$ & $3.1 \mathrm{E}-03$ \\
\hline
\end{tabular}

\section{Relative Risk [ ]}

\begin{tabular}{lccc}
\hline Diet 1 & 1.0013 & 1.029 & 1.65 \\
Diet 2 & 1.0005 & 1.01 & 1.28 \\
Diet 3 & 1.0022 & 1.043 & 1.94 \\
Diet 4 & 1.0028 & 1.079 & 3.3 \\
\hline
\end{tabular}

\section{Probability of Causation [\%]}

\begin{tabular}{llll}
\hline Diet 1 & 0.13 & 2.8 & 39 \\
Diet 2 & 0.05 & 1.0 & 22 \\
Diet 3 & 0.22 & 4.1 & 49 \\
Diet 4 & 0.28 & 7.3 & 70 \\
\hline
\end{tabular}

Diet 1 - Backyard cow milk + all other locally produced non-milk exposure pathways

Diet 2 - Locally produced commercial milk + all other locally produced non-milk exposure pathways

Diet 3 - Regionally mixed commercial milk + inhalation (other regionally mixed food items are minor contributors to the total)

Diet 4 - Goat milk (all other exposure pathways are of negligible importance compared to goat milk) 
Location: Rockwood

Receptor: Female born in 1954

\begin{tabular}{lccc}
\hline & \multicolumn{3}{c}{ Thyroid Dose [cGy] } \\
Exposure Pathway & $95 \%$ Subjective Confidence Interval \\
Backyard Cow Milk & lower limit & central estimate & upper limit \\
Commercial Milk (locally produced) & 0.26 & 2 & 16 \\
Commercial Milk (regionally mixed) & 0.086 & 0.68 & 5.2 \\
Goat Milk (locally produced) & 0.095 & 0.82 & 5.7 \\
Beef (locally produced) & 0.58 & 6 & 58 \\
Leafy Vegetables (locally produced) & 0.00029 & 0.0059 & 0.13 \\
Eggs (locally produced) & 0.000088 & 0.001 & 0.011 \\
Cottage Cheese (locally produced) & 0.013 & 0.11 & 0.9 \\
Inhalation & 0.00035 & 0.0052 & 0.071 \\
Mother's milk (mother on Diet 1) & 0.0042 & 0.02 & 0.093 \\
Prenatal exposure (mother on Diet 1) & 0.0012 & 0.043 & 0.86 \\
Diet 1 & 0.012 & 0.13 & 1 \\
Diet 2 & 0.29 & 2.2 & 17 \\
Diet 3 & 0.12 & 0.87 & 6.3 \\
\hline
\end{tabular}

Excess Lifetime Risk [ ]

\begin{tabular}{llll}
\hline Diet 1 & $4.2 \mathrm{E}-05$ & $6.9 \mathrm{E}-04$ & $1.2 \mathrm{E}-02$ \\
Diet 2 & $1.7 \mathrm{E}-05$ & $2.6 \mathrm{E}-04$ & $3.7 \mathrm{E}-03$ \\
Diet 3 & $1.4 \mathrm{E}-05$ & $2.4 \mathrm{E}-04$ & $3.3 \mathrm{E}-03$ \\
Diet 4 & $8.3 \mathrm{E}-05$ & $2.0 \mathrm{E}-03$ & $3.5 \mathrm{E}-02$ \\
\hline
\end{tabular}

\section{Relative Risk [ ]}

\begin{tabular}{lccc}
\hline Diet 1 & 1.016 & 1.17 & 3.1 \\
Diet 2 & 1.0056 & 1.063 & 1.96 \\
Diet 3 & 1.0053 & 1.059 & 1.97 \\
Diet 4 & 1.033 & 1.45 & 7.9 \\
\hline
\end{tabular}

\section{Probability of Causation [\%]}

\begin{tabular}{lccc}
\hline Diet 1 & 1.55 & 14.2 & 67 \\
Diet 2 & 0.56 & 5.9 & 48 \\
Diet 3 & 0.53 & 5.6 & 49 \\
Diet 4 & 3.22 & 30.2 & 87 \\
\hline
\end{tabular}

Diet 1 - Backyard cow milk + all other locally produced non-milk exposure pathways

Diet 2 - Locally produced commercial milk + all other locally produced non-milk exposure pathways

Diet 3 - Regionally mixed commercial milk + inhalation (other regionally mixed food items are minor contributors to the total)

Diet 4 - Goat milk (all other exposure pathways are of negligible importance compared to goat milk) 


\section{Location: Rockwood}

\section{Receptor: Male born in 1954}

\begin{tabular}{lccc}
\hline & \multicolumn{3}{c}{ Thyroid Dose [cGy] } \\
& \multicolumn{2}{c}{$95 \%$ Subjective Confidence Interval } \\
Exposure Pathway & lower limit & central estimate & upper limit \\
\hline Backyard Cow Milk & 0.31 & 2 & 14 \\
Commercial Milk (locally produced) & 0.082 & 0.67 & 4.6 \\
Commercial Milk (regionally mixed) & 0.1 & 0.8 & 5 \\
Goat Milk (locally produced) & 0.68 & 6 & 62 \\
Beef (locally produced) & 0.0003 & 0.0059 & 0.14 \\
Leafy Vegetables (locally produced) & 0.000081 & 0.00098 & 0.011 \\
Eggs (locally produced) & 0.014 & 0.11 & 0.96 \\
Cottage Cheese (locally produced) & 0.00034 & 0.0055 & 0.065 \\
Inhalation & 0.0044 & 0.02 & 0.086 \\
Mother's milk (mother on Diet 1) & 0.0012 & 0.043 & 0.86 \\
Prenatal exposure (mother on Diet 1) & 0.012 & 0.13 & 1 \\
Diet 1 & 0.34 & 2.2 & 15 \\
Diet 2 & 0.12 & 0.86 & 5.5 \\
Diet 3 & 0.11 & 0.82 & 5 \\
\hline
\end{tabular}

Excess Lifetime Risk [ ]

\begin{tabular}{lllc}
\hline Diet 1 & $5.8 \mathrm{E}-06$ & $1.8 \mathrm{E}-04$ & $3.2 \mathrm{E}-03$ \\
Diet 2 & $2.5 \mathrm{E}-06$ & $6.7 \mathrm{E}-05$ & $1.5 \mathrm{E}-03$ \\
Diet 3 & $2.2 \mathrm{E}-06$ & $6.1 \mathrm{E}-05$ & $1.5 \mathrm{E}-03$ \\
Diet 4 & $1.4 \mathrm{E}-05$ & $5.0 \mathrm{E}-04$ & $1.3 \mathrm{E}-02$ \\
\hline
\end{tabular}

\section{Relative Risk [ ]}

\begin{tabular}{lccc}
\hline Diet 1 & 1.0062 & 1.12 & 3.3 \\
Diet 2 & 1.0023 & 1.045 & 2.1 \\
Diet 3 & 1.0023 & 1.044 & 1.95 \\
Diet 4 & 1.014 & 1.33 & 10.6 \\
\hline
\end{tabular}

Probability of Causation [\%]

\begin{tabular}{lccc}
\hline Diet 1 & 0.62 & 10.6 & 69 \\
Diet 2 & 0.23 & 4.3 & 51 \\
Diet 3 & 0.23 & 4.2 & 49 \\
Diet 4 & 1.40 & 24.2 & 90 \\
\hline
\end{tabular}

Diet 1 - Backyard cow milk + all other locally produced non-milk exposure pathways

Diet 2 - Locally produced commercial milk + all other locally produced non-milk exposure pathways

Diet 3 - Regionally mixed commercial milk + inhalation (other regionally mixed food items are minor contributors to the total)

Diet 4 - Goat milk (all other exposure pathways are of negligible importance compared to goat milk) 


\section{Location: Louisville}

Receptor: Female born in 1954

\begin{tabular}{lccc}
\hline & \multicolumn{3}{c}{ Thyroid Dose [cGy] } \\
Exposure Pathway & \multicolumn{2}{c}{$95 \%$ Subjective Confidence Interval } \\
\hline Backyard Cow Milk & lower limit & central estimate & upper limit \\
Commercial Milk (locally produced) & 0.26 & 1.9 & 14 \\
Commercial Milk (regionally mixed) & 0.074 & 0.62 & 4.7 \\
Goat Milk (locally produced) & 0.094 & 0.82 & 5.7 \\
Beef (locally produced) & 0.53 & 5.5 & 54 \\
Leafy Vegetables (locally produced) & 0.00027 & 0.0053 & 0.12 \\
Eggs (locally produced) & 0.000085 & 0.00093 & 0.0092 \\
Cottage Cheese (locally produced) & 0.011 & 0.1 & 0.8 \\
Inhalation & 0.0003 & 0.0049 & 0.063 \\
Mother's milk (mother on Diet 1) & 0.0042 & 0.019 & 0.092 \\
Prenatal exposure (mother on Diet 1) & 0.0012 & 0.038 & 0.79 \\
Diet 1 & 0.012 & 0.12 & 0.93 \\
Diet 2 & 0.29 & 2 & 15 \\
Diet 3 & 0.11 & 0.8 & 5.4 \\
\hline
\end{tabular}

Excess Lifetime Risk [ ]

\begin{tabular}{llll}
\hline Diet 1 & $3.7 \mathrm{E}-05$ & $6.2 \mathrm{E}-04$ & $1.1 \mathrm{E}-02$ \\
Diet 2 & $1.5 \mathrm{E}-05$ & $2.3 \mathrm{E}-04$ & $3.5 \mathrm{E}-03$ \\
Diet 3 & $1.4 \mathrm{E}-05$ & $2.4 \mathrm{E}-04$ & $3.2 \mathrm{E}-03$ \\
Diet 4 & $7.8 \mathrm{E}-05$ & $1.9 \mathrm{E}-03$ & $3.6 \mathrm{E}-02$ \\
\hline
\end{tabular}

\section{Relative Risk [ ]}

\begin{tabular}{lccc}
\hline Diet 1 & 1.015 & 1.15 & 2.8 \\
Diet 2 & 1.0052 & 1.059 & 1.92 \\
Diet 3 & 1.0053 & 1.06 & 1.97 \\
Diet 4 & 1.032 & 1.42 & 8.1 \\
\hline
\end{tabular}

\section{Probability of Causation [\%]}

\begin{tabular}{lccc}
\hline Diet 1 & 1.44 & 13.1 & 64 \\
Diet 2 & 0.52 & 5.6 & 48 \\
Diet 3 & 0.53 & 5.6 & 49 \\
Diet 4 & 3.11 & 29.3 & 87 \\
\hline
\end{tabular}

Diet 1 - Backyard cow milk + all other locally produced non-milk exposure pathways

Diet 2 - Locally produced commercial milk + all other locally produced non-milk exposure pathways

Diet 3 - Regionally mixed commercial milk + inhalation (other regionally mixed food items are minor contributors to the total)

Diet 4 - Goat milk (all other exposure pathways are of negligible importance compared to goat milk) 


\section{Location: Louisville}

\section{Receptor: Male born in 1954}

\begin{tabular}{lccc}
\hline & \multicolumn{3}{c}{ Thyroid Dose [cGy] } \\
& \multicolumn{2}{c}{$95 \%$ Subjective Confidence Interval } \\
Exposure Pathway & lower limit & central estimate & upper limit \\
\hline Backyard Cow Milk & 0.28 & 1.8 & 12 \\
Commercial Milk (locally produced) & 0.076 & 0.61 & 4.3 \\
Commercial Milk (regionally mixed) & 0.1 & 0.8 & 5 \\
Goat Milk (locally produced) & 0.54 & 5.5 & 53 \\
Beef (locally produced) & 0.00028 & 0.0053 & 0.12 \\
Leafy Vegetables (locally produced) & 0.000076 & 0.00089 & 0.0095 \\
Eggs (locally produced) & 0.012 & 0.1 & 0.9 \\
Cottage Cheese (locally produced) & 0.00032 & 0.0051 & 0.063 \\
Inhalation & 0.0045 & 0.02 & 0.09 \\
Mother's milk (mother on Diet 1) & 0.0012 & 0.038 & 0.79 \\
Prenatal exposure (mother on Diet 1) & 0.012 & 0.12 & 0.93 \\
Diet 1 & 0.31 & 2 & 13 \\
Diet 2 & 0.11 & 0.8 & 5 \\
Diet 3 & 0.11 & 0.82 & 5 \\
\hline
\end{tabular}

Excess Lifetime Risk [ ]

\begin{tabular}{llll}
\hline Diet 1 & $5.3 \mathrm{E}-06$ & $1.6 \mathrm{E}-04$ & $2.8 \mathrm{E}-03$ \\
Diet 2 & $2.1 \mathrm{E}-06$ & $6.1 \mathrm{E}-05$ & $1.4 \mathrm{E}-03$ \\
Diet 3 & $2.2 \mathrm{E}-06$ & $6.1 \mathrm{E}-05$ & $1.5 \mathrm{E}-03$ \\
Diet 4 & $1.2 \mathrm{E}-05$ & $4.6 \mathrm{E}-04$ & $1.2 \mathrm{E}-02$ \\
\hline
\end{tabular}

\section{Relative Risk [ ]}

\begin{tabular}{lccc}
\hline Diet 1 & 1.0054 & 1.11 & 3.2 \\
Diet 2 & 1.002 & 1.042 & 1.93 \\
Diet 3 & 1.0023 & 1.044 & 1.95 \\
Diet 4 & 1.013 & 1.3 & 9.8 \\
\hline
\end{tabular}

\section{Probability of Causation [\%]}

\begin{tabular}{lccc}
\hline Diet 1 & 0.54 & 10.0 & 68 \\
Diet 2 & 0.20 & 4.0 & 48 \\
Diet 3 & 0.23 & 4.2 & 49 \\
Diet 4 & 1.24 & 23.1 & 89 \\
\hline
\end{tabular}

Diet 1 - Backyard cow milk + all other locally produced non-milk exposure pathways

Diet 2 - Locally produced commercial milk + all other locally produced non-milk exposure pathways

Diet 3 - Regionally mixed commercial milk + inhalation (other regionally mixed food items are minor contributors to the total)

Diet 4 - Goat milk (all other exposure pathways are of negligible importance compared to goat milk) 
Location: Barnardville

Receptor: Female born in 1954

\begin{tabular}{lccc}
\hline & \multicolumn{3}{c}{ Thyroid Dose [cGy] } \\
Exposure Pathway & \multicolumn{2}{c}{$95 \%$ Subjective Confidence Interval } \\
Backyard Cow Milk & lower limit & central estimate & upper limit \\
Commercial Milk (locally produced) & 0.3 & 2.2 & 17 \\
Commercial Milk (regionally mixed) & 0.087 & 0.71 & 5.2 \\
Goat Milk (locally produced) & 0.095 & 0.81 & 5.7 \\
Beef (locally produced) & 0.59 & 6.3 & 59 \\
Leafy Vegetables (locally produced) & 0.00031 & 0.0061 & 0.14 \\
Eggs (locally produced) & 0.000097 & 0.001 & 0.011 \\
Cottage Cheese (locally produced) & 0.013 & 0.12 & 0.97 \\
Inhalation & 0.00035 & 0.0056 & 0.07 \\
Mother's milk (mother on Diet 1) & 0.0051 & 0.023 & 0.11 \\
Prenatal exposure (mother on Diet 1) & 0.0013 & 0.044 & 0.83 \\
Diet 1 & 0.013 & 0.14 & 1 \\
Diet 2 & 0.34 & 2.3 & 18 \\
Diet 3 & 0.12 & 0.91 & 6.2 \\
\hline
\end{tabular}

Excess Lifetime Risk [ ]

\begin{tabular}{llll}
\hline Diet 1 & $4.5 \mathrm{E}-05$ & $7.3 \mathrm{E}-04$ & $1.3 \mathrm{E}-02$ \\
Diet 2 & $1.7 \mathrm{E}-05$ & $2.7 \mathrm{E}-04$ & $3.6 \mathrm{E}-03$ \\
Diet 3 & $1.5 \mathrm{E}-05$ & $2.4 \mathrm{E}-04$ & $3.3 \mathrm{E}-03$ \\
Diet 4 & $9.0 \mathrm{E}-05$ & $2.1 \mathrm{E}-03$ & $3.8 \mathrm{E}-02$ \\
\hline
\end{tabular}

\section{Relative Risk [ ]}

\begin{tabular}{lccc}
\hline Diet 1 & 1.016 & 1.18 & 3.1 \\
Diet 2 & 1.0058 & 1.067 & 1.9 \\
Diet 3 & 1.0053 & 1.06 & 1.98 \\
Diet 4 & 1.037 & 1.47 & 8.6 \\
\hline
\end{tabular}

\section{Probability of Causation [\%]}

\begin{tabular}{lccc}
\hline Diet 1 & 1.56 & 15.1 & 68 \\
Diet 2 & 0.58 & 6.3 & 47 \\
Diet 3 & 0.53 & 5.6 & 49 \\
Diet 4 & 3.53 & 32.0 & 88 \\
\hline
\end{tabular}

Diet 1 - Backyard cow milk + all other locally produced non-milk exposure pathways

Diet 2 - Locally produced commercial milk + all other locally produced non-milk exposure pathways

Diet 3 - Regionally mixed commercial milk + inhalation (other regionally mixed food items are minor contributors to the total)

Diet 4 - Goat milk (all other exposure pathways are of negligible importance compared to goat milk) 


\section{Location: Barnardville}

Receptor: Male born in 1954

\begin{tabular}{lccc}
\hline & \multicolumn{3}{c}{ Thyroid Dose [cGy] } \\
& \multicolumn{2}{c}{$95 \%$ Subjective Confidence Interval } \\
Exposure Pathway & lower limit & central estimate & upper limit \\
\hline Backyard Cow Milk & 0.32 & 2.1 & 15 \\
Commercial Milk (locally produced) & 0.084 & 0.7 & 4.7 \\
Commercial Milk (regionally mixed) & 0.1 & 0.8 & 5 \\
Goat Milk (locally produced) & 0.68 & 6.4 & 59 \\
Beef (locally produced) & 0.00032 & 0.006 & 0.15 \\
Leafy Vegetables (locally produced) & 0.000082 & 0.001 & 0.011 \\
Eggs (locally produced) & 0.014 & 0.11 & 1 \\
Cottage Cheese (locally produced) & 0.00035 & 0.0058 & 0.071 \\
Inhalation & 0.0052 & 0.023 & 0.1 \\
Mother's milk (mother on Diet 1) & 0.0013 & 0.044 & 0.83 \\
Prenatal exposure (mother on Diet 1) & 0.013 & 0.14 & 1 \\
Diet 1 & 0.36 & 2.3 & 16 \\
Diet 2 & 0.13 & 0.91 & 5.4 \\
Diet 3 & 0.11 & 0.83 & 5 \\
\hline
\end{tabular}

Excess Lifetime Risk [ ]

\begin{tabular}{llll}
\hline Diet 1 & $6.4 \mathrm{E}-06$ & $1.8 \mathrm{E}-04$ & $3.2 \mathrm{E}-03$ \\
Diet 2 & $2.6 \mathrm{E}-06$ & $6.8 \mathrm{E}-05$ & $1.5 \mathrm{E}-03$ \\
Diet 3 & $2.2 \mathrm{E}-06$ & $6.2 \mathrm{E}-05$ & $1.5 \mathrm{E}-03$ \\
Diet 4 & $1.3 \mathrm{E}-05$ & $5.1 \mathrm{E}-04$ & $1.3 \mathrm{E}-02$ \\
\hline
\end{tabular}

\section{Relative Risk [ ]}

\begin{tabular}{lccc}
\hline Diet 1 & 1.0067 & 1.13 & 3.7 \\
Diet 2 & 1.0024 & 1.049 & 2.1 \\
Diet 3 & 1.0023 & 1.044 & 1.95 \\
Diet 4 & 1.014 & 1.35 & 11 \\
\hline
\end{tabular}

\section{Probability of Causation [\%]}

\begin{tabular}{lccc}
\hline Diet 1 & 0.67 & 11.3 & 73 \\
Diet 2 & 0.24 & 4.7 & 52 \\
Diet 3 & 0.23 & 4.2 & 49 \\
Diet 4 & 1.43 & 26.0 & 91 \\
\hline
\end{tabular}

Diet 1 - Backyard cow milk + all other locally produced non-milk exposure pathways

Diet 2 - Locally produced commercial milk + all other locally produced non-milk exposure pathways

Diet 3 - Regionally mixed commercial milk + inhalation (other regionally mixed food items are minor contributors to the total)

Diet 4 - Goat milk (all other exposure pathways are of negligible importance compared to goat milk) 
Location: Greenback

Receptor: Female born in 1954

\begin{tabular}{lccc}
\hline & \multicolumn{3}{c}{ Thyroid Dose [cGy] } \\
Exposure Pathway & 95\% Subjective Confidence Interval \\
\hline Backyard Cow Milk & lower limit & central estimate & upper limit \\
Commercial Milk (locally produced) & 0.16 & 1.1 & 9 \\
Commercial Milk (regionally mixed) & 0.043 & 0.39 & 3.4 \\
Goat Milk (locally produced) & 0.094 & 0.81 & 5.7 \\
Beef (locally produced) & 0.36 & 3.5 & 34 \\
Leafy Vegetables (locally produced) & 0.00018 & 0.0034 & 0.074 \\
Eggs (locally produced) & 0.00005 & 0.00057 & 0.0059 \\
Cottage Cheese (locally produced) & 0.007 & 0.059 & 0.57 \\
Inhalation & 0.0002 & 0.0032 & 0.048 \\
Mother's milk (mother on Diet 1) & 0.0029 & 0.013 & 0.066 \\
Prenatal exposure (mother on Diet 1) & 0.00088 & 0.024 & 0.47 \\
Diet 1 & 0.0068 & 0.075 & 0.65 \\
Diet 2 & 0.18 & 1.3 & 9.6 \\
Diet 3 & 0.067 & 0.5 & 3.9 \\
\hline
\end{tabular}

Excess Lifetime Risk [ ]

\begin{tabular}{lllc}
\hline Diet 1 & $2.5 \mathrm{E}-05$ & $3.8 \mathrm{E}-04$ & $6.3 \mathrm{E}-03$ \\
Diet 2 & $1.1 \mathrm{E}-05$ & $1.4 \mathrm{E}-04$ & $2.1 \mathrm{E}-03$ \\
Diet 3 & $1.4 \mathrm{E}-05$ & $2.3 \mathrm{E}-04$ & $3.2 \mathrm{E}-03$ \\
Diet 4 & $4.7 \mathrm{E}-05$ & $1.2 \mathrm{E}-03$ & $2.1 \mathrm{E}-02$ \\
\hline
\end{tabular}

\section{Relative Risk [ ]}

\begin{tabular}{lccc}
\hline Diet 1 & 1.0095 & 1.091 & 2.2 \\
Diet 2 & 1.0034 & 1.035 & 1.53 \\
Diet 3 & 1.0052 & 1.059 & 1.96 \\
Diet 4 & 1.019 & 1.26 & 5.3 \\
\hline
\end{tabular}

\section{Probability of Causation [\%]}

\begin{tabular}{lccc}
\hline Diet 1 & 0.94 & 8.3 & 54 \\
Diet 2 & 0.34 & 3.4 & 34 \\
Diet 3 & 0.51 & 5.6 & 49 \\
Diet 4 & 1.87 & 20.5 & 81 \\
\hline
\end{tabular}

Diet 1 - Backyard cow milk + all other locally produced non-milk exposure pathways

Diet 2 - Locally produced commercial milk + all other locally produced non-milk exposure pathways

Diet 3 - Regionally mixed commercial milk + inhalation (other regionally mixed food items are minor contributors to the total)

Diet 4 - Goat milk (all other exposure pathways are of negligible importance compared to goat milk) 


\section{Location: Greenback}

\section{Receptor: Male born in 1954}

\begin{tabular}{lccc}
\hline & \multicolumn{3}{c}{ Thyroid Dose [cGy] } \\
& \multicolumn{2}{c}{$95 \%$ Subjective Confidence Interval } \\
Exposure Pathway & lower limit & central estimate & upper limit \\
\hline Backyard Cow Milk & 0.16 & 1.1 & 8.1 \\
Commercial Milk (locally produced) & 0.046 & 0.39 & 2.6 \\
Commercial Milk (regionally mixed) & 0.1 & 0.8 & 5 \\
Goat Milk (locally produced) & 0.36 & 3.4 & 35 \\
Beef (locally produced) & 0.00018 & 0.0034 & 0.08 \\
Leafy Vegetables (locally produced) & 0.000049 & 0.00054 & 0.0067 \\
Eggs (locally produced) & 0.0079 & 0.062 & 0.59 \\
Cottage Cheese (locally produced) & 0.00019 & 0.003 & 0.041 \\
Inhalation & 0.0031 & 0.013 & 0.065 \\
Mother's milk (mother on Diet 1) & 0.00088 & 0.024 & 0.47 \\
Prenatal exposure (mother on Diet 1) & 0.0068 & 0.075 & 0.65 \\
Diet 1 & 0.18 & 1.2 & 8.6 \\
Diet 2 & 0.07 & 0.5 & 3.3 \\
Diet 3 & 0.11 & 0.82 & 5 \\
\hline
\end{tabular}

Excess Lifetime Risk [ ]

\begin{tabular}{llll}
\hline Diet 1 & $3.0 \mathrm{E}-06$ & $1.0 \mathrm{E}-04$ & $1.8 \mathrm{E}-03$ \\
Diet 2 & $1.4 \mathrm{E}-06$ & $3.8 \mathrm{E}-05$ & $8.4 \mathrm{E}-04$ \\
Diet 3 & $2.2 \mathrm{E}-06$ & $6.1 \mathrm{E}-05$ & $1.5 \mathrm{E}-03$ \\
Diet 4 & $6.7 \mathrm{E}-06$ & $2.9 \mathrm{E}-04$ & $7.1 \mathrm{E}-03$ \\
\hline
\end{tabular}

\section{Relative Risk [ ]}

\begin{tabular}{lccc}
\hline Diet 1 & 1.0036 & 1.072 & 2.4 \\
Diet 2 & 1.0013 & 1.027 & 1.55 \\
Diet 3 & 1.0023 & 1.044 & 1.95 \\
Diet 4 & 1.0078 & 1.2 & 6.5 \\
\hline
\end{tabular}

\section{Probability of Causation [\%]}

\begin{tabular}{lccc}
\hline Diet 1 & 0.36 & 6.8 & 58 \\
Diet 2 & 0.13 & 2.6 & 36 \\
Diet 3 & 0.23 & 4.2 & 49 \\
Diet 4 & 0.78 & 16.3 & 85 \\
\hline
\end{tabular}

Diet 1 - Backyard cow milk + all other locally produced non-milk exposure pathways

Diet 2 - Locally produced commercial milk + all other locally produced non-milk exposure pathways

Diet 3 - Regionally mixed commercial milk + inhalation (other regionally mixed food items are minor contributors to the total)

Diet 4 - Goat milk (all other exposure pathways are of negligible importance compared to goat milk) 


\section{Location: Rockford}

Receptor: Female born in 1954

\begin{tabular}{lccc}
\hline & \multicolumn{3}{c}{ Thyroid Dose [cGy] } \\
Exposure Pathway & \multicolumn{2}{c}{$95 \%$ Subjective Confidence Interval } \\
\hline Backyard Cow Milk & lower limit & central estimate & upper limit \\
Commercial Milk (locally produced) & 0.27 & 1.9 & 14 \\
Commercial Milk (regionally mixed) & 0.074 & 0.6 & 4.8 \\
Goat Milk (locally produced) & 0.095 & 0.81 & 5.7 \\
Beef (locally produced) & 0.56 & 5.4 & 50 \\
Leafy Vegetables (locally produced) & 0.00027 & 0.0053 & 0.11 \\
Eggs (locally produced) & 0.00008 & 0.00093 & 0.009 \\
Cottage Cheese (locally produced) & 0.012 & 0.094 & 0.82 \\
Inhalation & 0.00031 & 0.0047 & 0.065 \\
Mother's milk (mother on Diet 1) & 0.0045 & 0.019 & 0.092 \\
Prenatal exposure (mother on Diet 1) & 0.0012 & 0.036 & 0.77 \\
Diet 1 & 0.012 & 0.12 & 0.93 \\
Diet 2 & 0.3 & 2 & 15 \\
Diet 3 & 0.11 & 0.78 & 5.5 \\
\hline
\end{tabular}

Excess Lifetime Risk [ ]

\begin{tabular}{llll}
\hline Diet 1 & $4.0 \mathrm{E}-05$ & $6.2 \mathrm{E}-04$ & $8.9 \mathrm{E}-03$ \\
Diet 2 & $1.5 \mathrm{E}-05$ & $2.3 \mathrm{E}-04$ & $3.0 \mathrm{E}-03$ \\
Diet 3 & $1.4 \mathrm{E}-05$ & $2.4 \mathrm{E}-04$ & $3.3 \mathrm{E}-03$ \\
Diet 4 & $7.7 \mathrm{E}-05$ & $1.9 \mathrm{E}-03$ & $3.0 \mathrm{E}-02$ \\
\hline
\end{tabular}

\section{Relative Risk [ ]}

\begin{tabular}{lccc}
\hline Diet 1 & 1.015 & 1.15 & 2.9 \\
Diet 2 & 1.005 & 1.058 & 1.8 \\
Diet 3 & 1.0053 & 1.06 & 1.97 \\
Diet 4 & 1.031 & 1.4 & 7.2 \\
\hline
\end{tabular}

\section{Probability of Causation [\%]}

\begin{tabular}{lccc}
\hline Diet 1 & 1.44 & 12.7 & 65 \\
Diet 2 & 0.50 & 5.5 & 44 \\
Diet 3 & 0.53 & 5.6 & 49 \\
Diet 4 & 3.00 & 28.5 & 86 \\
\hline
\end{tabular}

Diet 1 - Backyard cow milk + all other locally produced non-milk exposure pathways

Diet 2 - Locally produced commercial milk + all other locally produced non-milk exposure pathways

Diet 3 - Regionally mixed commercial milk + inhalation (other regionally mixed food items are minor contributors to the total)

Diet 4 - Goat milk (all other exposure pathways are of negligible importance compared to goat milk) 


\section{Location: Rockford}

\section{Receptor: Male born in 1954}

\begin{tabular}{lccc}
\hline & \multicolumn{3}{c}{ Thyroid Dose [cGy] } \\
Exposure Pathway & 95\% Subjective Confidence Interval \\
\hline Backyard Cow Milk & lower limit & central estimate & upper limit \\
Commercial Milk (locally produced) & 0.28 & 1.8 & 12 \\
Commercial Milk (regionally mixed) & 0.07 & 0.62 & 3.9 \\
Goat Milk (locally produced) & 0.1 & 0.8 & 5 \\
Beef (locally produced) & 0.58 & 5.3 & 50 \\
Leafy Vegetables (locally produced) & 0.00028 & 0.0052 & 0.11 \\
Eggs (locally produced) & 0.000069 & 0.00088 & 0.0093 \\
Cottage Cheese (locally produced) & 0.013 & 0.098 & 0.8 \\
Inhalation & 0.00032 & 0.0048 & 0.062 \\
Mother's milk (mother on Diet 1) & 0.0048 & 0.02 & 0.087 \\
Prenatal exposure (mother on Diet 1) & 0.0012 & 0.036 & 0.77 \\
Diet 1 & 0.012 & 0.12 & 0.93 \\
Diet 2 & 0.31 & 2 & 13 \\
Diet 3 & 0.11 & 0.77 & 4.8 \\
\hline
\end{tabular}

Excess Lifetime Risk [ ]

\begin{tabular}{llll}
\hline Diet 1 & $4.8 \mathrm{E}-06$ & $1.6 \mathrm{E}-04$ & $3.0 \mathrm{E}-03$ \\
Diet 2 & $2.3 \mathrm{E}-06$ & $6.2 \mathrm{E}-05$ & $1.3 \mathrm{E}-03$ \\
Diet 3 & $2.2 \mathrm{E}-06$ & $6.1 \mathrm{E}-05$ & $1.5 \mathrm{E}-03$ \\
Diet 4 & $1.1 \mathrm{E}-05$ & $4.4 \mathrm{E}-04$ & $1.1 \mathrm{E}-02$ \\
\hline
\end{tabular}

\section{Relative Risk [ ]}

\begin{tabular}{lccc}
\hline Diet 1 & 1.0059 & 1.11 & 3.3 \\
Diet 2 & 1.0021 & 1.042 & 1.9 \\
Diet 3 & 1.0023 & 1.044 & 1.95 \\
Diet 4 & 1.012 & 1.31 & 9.8 \\
\hline
\end{tabular}

\section{Probability of Causation [\%]}

\begin{tabular}{lccc}
\hline Diet 1 & 0.58 & 10.1 & 69 \\
Diet 2 & 0.21 & 4.1 & 47 \\
Diet 3 & 0.23 & 4.2 & 49 \\
Diet 4 & 1.19 & 23.3 & 89 \\
\hline
\end{tabular}

Diet 1 - Backyard cow milk + all other locally produced non-milk exposure pathways

Diet 2 - Locally produced commercial milk + all other locally produced non-milk exposure pathways

Diet 3 - Regionally mixed commercial milk + inhalation (other regionally mixed food items are minor contributors to the total)

Diet 4 - Goat milk (all other exposure pathways are of negligible importance compared to goat milk) 
Location: Lake City

Receptor: Female born in 1954

\begin{tabular}{lccc}
\hline & \multicolumn{3}{c}{ Thyroid Dose [cGy] } \\
Exposure Pathway & \multicolumn{2}{c}{$95 \%$ Subjective Confidence Interval } \\
\hline Backyard Cow Milk & lower limit & central estimate & upper limit \\
Commercial Milk (locally produced) & 0.17 & 1.1 & 8.2 \\
Commercial Milk (regionally mixed) & 0.047 & 0.38 & 2.8 \\
Goat Milk (locally produced) & 0.095 & 0.82 & 5.7 \\
Beef (locally produced) & 0.34 & 3.4 & 30 \\
Leafy Vegetables (locally produced) & 0.00017 & 0.0033 & 0.075 \\
Eggs (locally produced) & 0.000049 & 0.00059 & 0.0057 \\
Cottage Cheese (locally produced) & 0.0071 & 0.059 & 0.45 \\
Inhalation & 0.00017 & 0.0029 & 0.038 \\
Mother's milk (mother on Diet 1) & 0.0022 & 0.011 & 0.05 \\
Prenatal exposure (mother on Diet 1) & 0.00069 & 0.023 & 0.48 \\
Diet 1 & 0.0072 & 0.075 & 0.53 \\
Diet 2 & 0.18 & 1.2 & 8.7 \\
Diet 3 & 0.065 & 0.48 & 3.2 \\
\hline
\end{tabular}

Excess Lifetime Risk [ ]

\begin{tabular}{llll}
\hline Diet 1 & $2.4 \mathrm{E}-05$ & $3.8 \mathrm{E}-04$ & $6.2 \mathrm{E}-03$ \\
Diet 2 & $8.7 \mathrm{E}-06$ & $1.4 \mathrm{E}-04$ & $2.1 \mathrm{E}-03$ \\
Diet 3 & $1.4 \mathrm{E}-05$ & $2.3 \mathrm{E}-04$ & $3.2 \mathrm{E}-03$ \\
Diet 4 & $4.4 \mathrm{E}-05$ & $1.1 \mathrm{E}-03$ & $2.0 \mathrm{E}-02$ \\
\hline
\end{tabular}

\section{Relative Risk [ ]}

\begin{tabular}{lccc}
\hline Diet 1 & 1.0092 & 1.091 & 2.1 \\
Diet 2 & 1.0033 & 1.036 & 1.57 \\
Diet 3 & 1.0051 & 1.059 & 1.96 \\
Diet 4 & 1.021 & 1.25 & 5.1 \\
\hline
\end{tabular}

\begin{tabular}{lccc}
\hline Diet 1 & 0.91 & 8.3 & 52 \\
Diet 2 & 0.33 & 3.4 & 36 \\
Diet 3 & 0.51 & 5.6 & 49 \\
Diet 4 & 2.05 & 19.9 & 80 \\
\hline
\end{tabular}

Diet 1 - Backyard cow milk + all other locally produced non-milk exposure pathways

Diet 2 - Locally produced commercial milk + all other locally produced non-milk exposure pathways

Diet 3 - Regionally mixed commercial milk + inhalation (other regionally mixed food items are minor contributors to the total)

Diet 4 - Goat milk (all other exposure pathways are of negligible importance compared to goat milk) 
Location: Lake City

Receptor: Male born in 1954

\begin{tabular}{lccc}
\hline & \multicolumn{3}{c}{ Thyroid Dose [cGy] } \\
& \multicolumn{2}{c}{$95 \%$ Subjective Confidence Interval } \\
Exposure Pathway & lower limit & central estimate & upper limit \\
\hline Backyard Cow Milk & 0.17 & 1.1 & 7.2 \\
Commercial Milk (locally produced) & 0.049 & 0.37 & 2.5 \\
Commercial Milk (regionally mixed) & 0.1 & 0.8 & 5 \\
Goat Milk (locally produced) & 0.37 & 3.4 & 30 \\
Beef (locally produced) & 0.00019 & 0.0032 & 0.076 \\
Leafy Vegetables (locally produced) & 0.000047 & 0.00055 & 0.0059 \\
Eggs (locally produced) & 0.008 & 0.062 & 0.51 \\
Cottage Cheese (locally produced) & 0.0002 & 0.003 & 0.041 \\
Inhalation & 0.0024 & 0.011 & 0.048 \\
Mother's milk (mother on Diet 1) & 0.00069 & 0.023 & 0.48 \\
Prenatal exposure (mother on Diet 1) & 0.0072 & 0.075 & 0.53 \\
Diet 1 & 0.19 & 1.2 & 7.8 \\
Diet 2 & 0.07 & 0.47 & 3 \\
Diet 3 & 0.11 & 0.81 & 5 \\
\hline
\end{tabular}

Excess Lifetime Risk [ ]

\begin{tabular}{llll}
\hline Diet 1 & $3.2 \mathrm{E}-06$ & $1.0 \mathrm{E}-04$ & $1.8 \mathrm{E}-03$ \\
Diet 2 & $1.4 \mathrm{E}-06$ & $3.8 \mathrm{E}-05$ & $8.4 \mathrm{E}-04$ \\
Diet 3 & $2.2 \mathrm{E}-06$ & $6.0 \mathrm{E}-05$ & $1.5 \mathrm{E}-03$ \\
Diet 4 & $7.6 \mathrm{E}-06$ & $2.7 \mathrm{E}-04$ & $6.9 \mathrm{E}-03$ \\
\hline
\end{tabular}

Relative Risk [ ]

\begin{tabular}{lccc}
\hline Diet 1 & 1.0035 & 1.068 & 2.3 \\
Diet 2 & 1.0012 & 1.025 & 1.56 \\
Diet 3 & 1.0022 & 1.043 & 1.95 \\
Diet 4 & 1.008 & 1.18 & 6.5 \\
\hline
\end{tabular}

\section{Probability of Causation [\%]}

\begin{tabular}{lccc}
\hline Diet 1 & 0.35 & 6.4 & 57 \\
Diet 2 & 0.12 & 2.4 & 35 \\
Diet 3 & 0.22 & 4.1 & 49 \\
Diet 4 & 0.80 & 15.3 & 84 \\
\hline
\end{tabular}

Diet 1 - Backyard cow milk + all other locally produced non-milk exposure pathways

Diet 2 - Locally produced commercial milk + all other locally produced non-milk exposure pathways

Diet 3 - Regionally mixed commercial milk + inhalation (other regionally mixed food items are minor contributors to the total)

Diet 4 - Goat milk (all other exposure pathways are of negligible importance compared to goat milk) 
Location: Sweetwater

Receptor: Female born in 1954

\begin{tabular}{lccc}
\hline & \multicolumn{3}{c}{ Thyroid Dose [cGy] } \\
Exposure Pathway & $95 \%$ Subjective Confidence Interval \\
\hline Backyard Cow Milk & lower limit & central estimate & upper limit \\
Commercial Milk (locally produced) & 0.18 & 1.4 & 9.7 \\
Commercial Milk (regionally mixed) & 0.053 & 0.46 & 3.3 \\
Goat Milk (locally produced) & 0.094 & 0.82 & 5.7 \\
Beef (locally produced) & 0.37 & 4.1 & 42 \\
Leafy Vegetables (locally produced) & 0.00019 & 0.0038 & 0.082 \\
Eggs (locally produced) & 0.000063 & 0.00066 & 0.0066 \\
Cottage Cheese (locally produced) & 0.0081 & 0.075 & 0.59 \\
Inhalation & 0.00021 & 0.0037 & 0.045 \\
Mother's milk (mother on Diet 1) & 0.0032 & 0.015 & 0.064 \\
Prenatal exposure (mother on Diet 1) & 0.00088 & 0.028 & 0.6 \\
Diet 1 & 0.0085 & 0.094 & 0.7 \\
Diet 2 & 0.2 & 1.5 & 10 \\
Diet 3 & 0.077 & 0.59 & 3.9 \\
\hline
\end{tabular}

Excess Lifetime Risk [ ]

\begin{tabular}{llll}
\hline Diet 1 & $2.6 \mathrm{E}-05$ & $4.6 \mathrm{E}-04$ & $7.9 \mathrm{E}-03$ \\
Diet 2 & $1.1 \mathrm{E}-05$ & $1.7 \mathrm{E}-04$ & $2.7 \mathrm{E}-03$ \\
Diet 3 & $1.4 \mathrm{E}-05$ & $2.4 \mathrm{E}-04$ & $3.2 \mathrm{E}-03$ \\
Diet 4 & $5.8 \mathrm{E}-05$ & $1.4 \mathrm{E}-03$ & $2.5 \mathrm{E}-02$ \\
\hline
\end{tabular}

\section{Relative Risk [ ]}

\begin{tabular}{lccc}
\hline Diet 1 & 1.011 & 1.11 & 2.2 \\
Diet 2 & 1.0036 & 1.045 & 1.69 \\
Diet 3 & 1.0052 & 1.059 & 1.97 \\
Diet 4 & 1.021 & 1.32 & 5.9 \\
\hline
\end{tabular}

\section{Probability of Causation [\%]}

\begin{tabular}{lccc}
\hline Diet 1 & 1.06 & 10.0 & 54 \\
Diet 2 & 0.36 & 4.3 & 41 \\
Diet 3 & 0.52 & 5.6 & 49 \\
Diet 4 & 2.09 & 24.1 & 83 \\
\hline
\end{tabular}

Diet 1 - Backyard cow milk + all other locally produced non-milk exposure pathways

Diet 2 - Locally produced commercial milk + all other locally produced non-milk exposure pathways

Diet 3 - Regionally mixed commercial milk + inhalation (other regionally mixed food items are minor contributors to the total)

Diet 4 - Goat milk (all other exposure pathways are of negligible importance compared to goat milk) 
Location: Sweetwater

Receptor: Male born in 1954

\begin{tabular}{lccc}
\hline & \multicolumn{3}{c}{ Thyroid Dose [cGy] } \\
& \multicolumn{2}{c}{$95 \%$ Subjective Confidence Interval } \\
Exposure Pathway & lower limit & central estimate & upper limit \\
\hline Backyard Cow Milk & 0.21 & 1.4 & 8.7 \\
Commercial Milk (locally produced) & 0.052 & 0.45 & 3.2 \\
Commercial Milk (regionally mixed) & 0.1 & 0.8 & 5 \\
Goat Milk (locally produced) & 0.38 & 4.1 & 41 \\
Beef (locally produced) & 0.0002 & 0.0039 & 0.079 \\
Leafy Vegetables (locally produced) & 0.000058 & 0.00064 & 0.0068 \\
Eggs (locally produced) & 0.0085 & 0.07 & 0.65 \\
Cottage Cheese (locally produced) & 0.00024 & 0.0036 & 0.046 \\
Inhalation & 0.0035 & 0.016 & 0.071 \\
Mother's milk (mother on Diet 1) & 0.00088 & 0.028 & 0.6 \\
Prenatal exposure (mother on Diet 1) & 0.0085 & 0.094 & 0.7 \\
Diet 1 & 0.23 & 1.5 & 9.2 \\
Diet 2 & 0.08 & 0.57 & 3.7 \\
Diet 3 & 0.11 & 0.82 & 5 \\
\hline
\end{tabular}

Excess Lifetime Risk [ ]

\begin{tabular}{lllc}
\hline Diet 1 & $4.1 \mathrm{E}-06$ & $1.2 \mathrm{E}-04$ & $2.2 \mathrm{E}-03$ \\
Diet 2 & $1.6 \mathrm{E}-06$ & $4.4 \mathrm{E}-05$ & $9.9 \mathrm{E}-04$ \\
Diet 3 & $2.2 \mathrm{E}-06$ & $6.0 \mathrm{E}-05$ & $1.5 \mathrm{E}-03$ \\
Diet 4 & $9.4 \mathrm{E}-06$ & $3.4 \mathrm{E}-04$ & $8.8 \mathrm{E}-03$ \\
\hline
\end{tabular}

\section{Relative Risk [ ]}

\begin{tabular}{lccc}
\hline Diet 1 & 1.0038 & 1.084 & 2.6 \\
Diet 2 & 1.0014 & 1.031 & 1.64 \\
Diet 3 & 1.0022 & 1.043 & 1.95 \\
Diet 4 & 1.0087 & 1.23 & 7.1 \\
\hline
\end{tabular}

\section{Probability of Causation [\%]}

\begin{tabular}{lccc}
\hline Diet 1 & 0.38 & 7.7 & 61 \\
Diet 2 & 0.14 & 3.0 & 39 \\
Diet 3 & 0.22 & 4.2 & 49 \\
Diet 4 & 0.86 & 18.4 & 86 \\
\hline
\end{tabular}

Diet 1 - Backyard cow milk + all other locally produced non-milk exposure pathways

Diet 2 - Locally produced commercial milk + all other locally produced non-milk exposure pathways

Diet 3 - Regionally mixed commercial milk + inhalation (other regionally mixed food items are minor contributors to the total)

Diet 4 - Goat milk (all other exposure pathways are of negligible importance compared to goat milk) 


\section{Location: Knoxville}

Receptor: Female born in 1954

\begin{tabular}{lccc}
\hline & \multicolumn{3}{c}{ Thyroid Dose [cGy] } \\
Exposure Pathway & \multicolumn{2}{c}{$95 \%$ Subjective Confidence Interval } \\
\hline Backyard Cow Milk & lower limit & central estimate & upper limit \\
Commercial Milk (locally produced) & 0.33 & 2.4 & 17 \\
Commercial Milk (regionally mixed) & 0.088 & 0.77 & 5.9 \\
Goat Milk (locally produced) & 0.094 & 0.82 & 5.7 \\
Beef (locally produced) & 0.68 & 6.8 & 63 \\
Leafy Vegetables (locally produced) & 0.00034 & 0.0066 & 0.14 \\
Eggs (locally produced) & 0.0001 & 0.0012 & 0.011 \\
Cottage Cheese (locally produced) & 0.015 & 0.12 & 1 \\
Inhalation & 0.00039 & 0.0059 & 0.077 \\
Mother's milk (mother on Diet 1) & 0.0056 & 0.024 & 0.11 \\
Prenatal exposure (mother on Diet 1) & 0.0014 & 0.047 & 0.95 \\
Diet 1 & 0.015 & 0.16 & 1.2 \\
Diet 2 & 0.37 & 2.6 & 18 \\
Diet 3 & 0.13 & 0.98 & 7 \\
\hline
\end{tabular}

Excess Lifetime Risk [ ]

\begin{tabular}{llll}
\hline Diet 1 & $4.8 \mathrm{E}-05$ & $7.8 \mathrm{E}-04$ & $1.2 \mathrm{E}-02$ \\
Diet 2 & $1.7 \mathrm{E}-05$ & $2.9 \mathrm{E}-04$ & $3.9 \mathrm{E}-03$ \\
Diet 3 & $1.5 \mathrm{E}-05$ & $2.4 \mathrm{E}-04$ & $3.3 \mathrm{E}-03$ \\
Diet 4 & $1.0 \mathrm{E}-04$ & $2.4 \mathrm{E}-03$ & $4.0 \mathrm{E}-02$ \\
\hline
\end{tabular}

\section{Relative Risk [ ]}

\begin{tabular}{lccc}
\hline Diet 1 & 1.018 & 1.19 & 3.4 \\
Diet 2 & 1.0067 & 1.073 & 1.97 \\
Diet 3 & 1.0054 & 1.06 & 1.97 \\
Diet 4 & 1.041 & 1.52 & 8.4 \\
\hline
\end{tabular}

\section{Probability of Causation [\%]}

\begin{tabular}{lccc}
\hline Diet 1 & 1.79 & 16.0 & 70 \\
Diet 2 & 0.67 & 6.8 & 49 \\
Diet 3 & 0.54 & 5.6 & 49 \\
Diet 4 & 3.90 & 34.1 & 88 \\
\hline
\end{tabular}

Diet 1 - Backyard cow milk + all other locally produced non-milk exposure pathways

Diet 2 - Locally produced commercial milk + all other locally produced non-milk exposure pathways

Diet 3 - Regionally mixed commercial milk + inhalation (other regionally mixed food items are minor contributors to the total)

Diet 4 - Goat milk (all other exposure pathways are of negligible importance compared to goat milk) 


\section{Location: Knoxville}

\section{Receptor: Male born in 1954}

\begin{tabular}{lccc}
\hline & \multicolumn{3}{c}{ Thyroid Dose [cGy] } \\
& \multicolumn{2}{c}{$95 \%$ Subjective Confidence Interval } \\
Exposure Pathway & lower limit & central estimate & upper limit \\
\hline Backyard Cow Milk & 0.34 & 2.3 & 15 \\
Commercial Milk (locally produced) & 0.087 & 0.77 & 5.1 \\
Commercial Milk (regionally mixed) & 0.1 & 0.8 & 5 \\
Goat Milk (locally produced) & 0.75 & 6.8 & 63 \\
Beef (locally produced) & 0.00036 & 0.0065 & 0.14 \\
Leafy Vegetables (locally produced) & 0.000094 & 0.0011 & 0.012 \\
Eggs (locally produced) & 0.016 & 0.12 & 1 \\
Cottage Cheese (locally produced) & 0.00041 & 0.0062 & 0.08 \\
Inhalation & 0.0058 & 0.025 & 0.11 \\
Mother's milk (mother on Diet 1) & 0.0014 & 0.047 & 0.95 \\
Prenatal exposure (mother on Diet 1) & 0.015 & 0.16 & 1.2 \\
Diet 1 & 0.38 & 2.6 & 16 \\
Diet 2 & 0.13 & 0.99 & 6 \\
Diet 3 & 0.11 & 0.83 & 5 \\
\hline
\end{tabular}

Excess Lifetime Risk [ ]

\begin{tabular}{lllc}
\hline Diet 1 & $6.2 \mathrm{E}-06$ & $2.0 \mathrm{E}-04$ & $3.7 \mathrm{E}-03$ \\
Diet 2 & $2.8 \mathrm{E}-06$ & $7.9 \mathrm{E}-05$ & $1.6 \mathrm{E}-03$ \\
Diet 3 & $2.3 \mathrm{E}-06$ & $6.2 \mathrm{E}-05$ & $1.5 \mathrm{E}-03$ \\
Diet 4 & $1.5 \mathrm{E}-05$ & $5.6 \mathrm{E}-04$ & $1.5 \mathrm{E}-02$ \\
\hline
\end{tabular}

\section{Relative Risk [ ]}

\begin{tabular}{lccc}
\hline Diet 1 & 1.0077 & 1.14 & 3.8 \\
Diet 2 & 1.0028 & 1.054 & 2.1 \\
Diet 3 & 1.0023 & 1.044 & 1.95 \\
Diet 4 & 1.016 & 1.38 & 12 \\
\hline
\end{tabular}

\begin{tabular}{lccc}
\hline Diet 1 & 0.76 & 12.4 & 74 \\
Diet 2 & 0.28 & 5.1 & 52 \\
Diet 3 & 0.23 & 4.2 & 49 \\
Diet 4 & 1.57 & 27.4 & 92 \\
\hline
\end{tabular}

Diet 1 - Backyard cow milk + all other locally produced non-milk exposure pathways

Diet 2 - Locally produced commercial milk + all other locally produced non-milk exposure pathways

Diet 3 - Regionally mixed commercial milk + inhalation (other regionally mixed food items are minor contributors to the total)

Diet 4 - Goat milk (all other exposure pathways are of negligible importance compared to goat milk) 


\section{Location: Maryville}

Receptor: Female born in 1954

\begin{tabular}{lccc}
\hline & \multicolumn{3}{c}{ Thyroid Dose [cGy] } \\
Exposure Pathway & \multicolumn{2}{c}{$95 \%$ Subjective Confidence Interval } \\
\hline Backyard Cow Milk & lower limit & central estimate & upper limit \\
Commercial Milk (locally produced) & 0.16 & 1.2 & 8.3 \\
Commercial Milk (regionally mixed) & 0.044 & 0.37 & 2.8 \\
Goat Milk (locally produced) & 0.094 & 0.81 & 5.7 \\
Beef (locally produced) & 0.31 & 3.2 & 33 \\
Leafy Vegetables (locally produced) & 0.00017 & 0.0032 & 0.071 \\
Eggs (locally produced) & 0.000052 & 0.00058 & 0.0056 \\
Cottage Cheese (locally produced) & 0.0066 & 0.063 & 0.52 \\
Inhalation & 0.00016 & 0.003 & 0.04 \\
Mother's milk (mother on Diet 1) & 0.0028 & 0.013 & 0.066 \\
Prenatal exposure (mother on Diet 1) & 0.00071 & 0.024 & 0.48 \\
Diet 1 & 0.0068 & 0.079 & 0.56 \\
Diet 2 & 0.18 & 1.3 & 8.8 \\
Diet 3 & 0.066 & 0.48 & 3.3 \\
\hline
\end{tabular}

Excess Lifetime Risk [ ]

\begin{tabular}{llll}
\hline Diet 1 & $2.5 \mathrm{E}-05$ & $4.0 \mathrm{E}-04$ & $7.5 \mathrm{E}-03$ \\
Diet 2 & $8.8 \mathrm{E}-06$ & $1.5 \mathrm{E}-04$ & $2.0 \mathrm{E}-03$ \\
Diet 3 & $1.4 \mathrm{E}-05$ & $2.3 \mathrm{E}-04$ & $3.2 \mathrm{E}-03$ \\
Diet 4 & $5.0 \mathrm{E}-05$ & $1.1 \mathrm{E}-03$ & $1.9 \mathrm{E}-02$ \\
\hline
\end{tabular}

\section{Relative Risk [ ]}

\begin{tabular}{lccc}
\hline Diet 1 & 1.0084 & 1.093 & 2.2 \\
Diet 2 & 1.003 & 1.035 & 1.47 \\
Diet 3 & 1.0052 & 1.059 & 1.96 \\
Diet 4 & 1.018 & 1.25 & 4.9 \\
\hline
\end{tabular}

\section{Probability of Causation [\%]}

\begin{tabular}{lccc}
\hline Diet 1 & 0.83 & 8.5 & 54 \\
Diet 2 & 0.30 & 3.4 & 32 \\
Diet 3 & 0.51 & 5.6 & 49 \\
Diet 4 & 1.81 & 19.9 & 79 \\
\hline
\end{tabular}

Diet 1 - Backyard cow milk + all other locally produced non-milk exposure pathways

Diet 2 - Locally produced commercial milk + all other locally produced non-milk exposure pathways

Diet 3 - Regionally mixed commercial milk + inhalation (other regionally mixed food items are minor contributors to the total)

Diet 4 - Goat milk (all other exposure pathways are of negligible importance compared to goat milk) 


\section{Location: Maryville}

\section{Receptor: Male born in 1954}

\begin{tabular}{lccc}
\hline & \multicolumn{3}{c}{ Thyroid Dose [cGy] } \\
& \multicolumn{2}{c}{$95 \%$ Subjective Confidence Interval } \\
Exposure Pathway & lower limit & central estimate & upper limit \\
\hline Backyard Cow Milk & 0.16 & 1.2 & 7.6 \\
Commercial Milk (locally produced) & 0.045 & 0.38 & 2.6 \\
Commercial Milk (regionally mixed) & 0.1 & 0.8 & 5 \\
Goat Milk (locally produced) & 0.39 & 3.4 & 33 \\
Beef (locally produced) & 0.00018 & 0.0031 & 0.07 \\
Leafy Vegetables (locally produced) & 0.000045 & 0.00055 & 0.0052 \\
Eggs (locally produced) & 0.0078 & 0.062 & 0.55 \\
Cottage Cheese (locally produced) & 0.00019 & 0.0029 & 0.034 \\
Inhalation & 0.0029 & 0.014 & 0.058 \\
Mother's milk (mother on Diet 1) & 0.00071 & 0.024 & 0.48 \\
Prenatal exposure (mother on Diet 1) & 0.0068 & 0.079 & 0.56 \\
Diet 1 & 0.18 & 1.3 & 8.3 \\
Diet 2 & 0.066 & 0.49 & 3 \\
Diet 3 & 0.11 & 0.82 & 5 \\
\hline
\end{tabular}

Excess Lifetime Risk [ ]

\begin{tabular}{llll}
\hline Diet 1 & $3.5 \mathrm{E}-06$ & $9.3 \mathrm{E}-05$ & $1.8 \mathrm{E}-03$ \\
Diet 2 & $1.4 \mathrm{E}-06$ & $3.8 \mathrm{E}-05$ & $7.3 \mathrm{E}-04$ \\
Diet 3 & $2.2 \mathrm{E}-06$ & $6.0 \mathrm{E}-05$ & $1.5 \mathrm{E}-03$ \\
Diet 4 & $7.2 \mathrm{E}-06$ & $2.7 \mathrm{E}-04$ & $8.2 \mathrm{E}-03$ \\
\hline
\end{tabular}

\section{Relative Risk [ ]}

\begin{tabular}{lccc}
\hline Diet 1 & 1.0036 & 1.07 & 2.5 \\
Diet 2 & 1.0013 & 1.027 & 1.57 \\
Diet 3 & 1.0022 & 1.043 & 1.95 \\
Diet 4 & 1.0083 & 1.19 & 6.8 \\
\hline
\end{tabular}

\section{Probability of Causation [\%]}

\begin{tabular}{lccc}
\hline Diet 1 & 0.36 & 6.5 & 60 \\
Diet 2 & 0.13 & 2.6 & 36 \\
Diet 3 & 0.22 & 4.2 & 49 \\
Diet 4 & 0.83 & 15.7 & 85 \\
\hline
\end{tabular}

Diet 1 - Backyard cow milk + all other locally produced non-milk exposure pathways

Diet 2 - Locally produced commercial milk + all other locally produced non-milk exposure pathways

Diet 3 - Regionally mixed commercial milk + inhalation (other regionally mixed food items are minor contributors to the total)

Diet 4 - Goat milk (all other exposure pathways are of negligible importance compared to goat milk) 
Location: Cedar Grove

Receptor: Female born in 1954

\begin{tabular}{lccc}
\hline & \multicolumn{3}{c}{ Thyroid Dose [cGy] } \\
Exposure Pathway & \multicolumn{2}{c}{$95 \%$ Subjective Confidence Interval } \\
\hline Backyard Cow Milk & lower limit & central estimate & upper limit \\
Commercial Milk (locally produced) & 0.31 & 2.2 & 16 \\
Commercial Milk (regionally mixed) & 0.089 & 0.69 & 5.1 \\
Goat Milk (locally produced) & 0.094 & 0.81 & 5.7 \\
Beef (locally produced) & 0.65 & 6.2 & 59 \\
Leafy Vegetables (locally produced) & 0.00032 & 0.0062 & 0.12 \\
Eggs (locally produced) & 0.000093 & 0.0011 & 0.01 \\
Cottage Cheese (locally produced) & 0.014 & 0.11 & 0.93 \\
Inhalation & 0.00035 & 0.0055 & 0.073 \\
Mother's milk (mother on Diet 1) & 0.0049 & 0.021 & 0.096 \\
Prenatal exposure (mother on Diet 1) & 0.0013 & 0.041 & 0.95 \\
Diet 1 & 0.013 & 0.14 & 1 \\
Diet 2 & 0.34 & 2.3 & 17 \\
Diet 3 & 0.12 & 0.89 & 6.2 \\
\hline
\end{tabular}

Excess Lifetime Risk [ ]

\begin{tabular}{llll}
\hline Diet 1 & $4.7 \mathrm{E}-05$ & $7.3 \mathrm{E}-04$ & $1.0 \mathrm{E}-02$ \\
Diet 2 & $1.7 \mathrm{E}-05$ & $2.7 \mathrm{E}-04$ & $3.5 \mathrm{E}-03$ \\
Diet 3 & $1.4 \mathrm{E}-05$ & $2.4 \mathrm{E}-04$ & $3.3 \mathrm{E}-03$ \\
Diet 4 & $9.2 \mathrm{E}-05$ & $2.1 \mathrm{E}-03$ & $3.4 \mathrm{E}-02$ \\
\hline
\end{tabular}

\section{Relative Risk [ ]}

\begin{tabular}{lccc}
\hline Diet 1 & 1.018 & 1.17 & 3.3 \\
Diet 2 & 1.0059 & 1.066 & 1.93 \\
Diet 3 & 1.0053 & 1.06 & 1.97 \\
Diet 4 & 1.037 & 1.47 & 7.9 \\
\hline
\end{tabular}

\begin{tabular}{lccc} 
& \multicolumn{3}{c}{ Probability of Causation [\%] } \\
\hline Diet 1 & 1.77 & 14.4 & 70 \\
Diet 2 & 0.59 & 6.2 & 48 \\
Diet 3 & 0.53 & 5.6 & 49 \\
Diet 4 & 3.60 & 31.7 & 87 \\
\hline
\end{tabular}

Diet 1 - Backyard cow milk + all other locally produced non-milk exposure pathways

Diet 2 - Locally produced commercial milk + all other locally produced non-milk exposure pathways

Diet 3 - Regionally mixed commercial milk + inhalation (other regionally mixed food items are minor contributors to the total)

Diet 4 - Goat milk (all other exposure pathways are of negligible importance compared to goat milk) 
Location: Cedar Grove

Receptor: Male born in 1954

\begin{tabular}{lccc}
\hline & \multicolumn{3}{c}{ Thyroid Dose [cGy] } \\
& \multicolumn{2}{c}{$95 \%$ Subjective Confidence Interval } \\
Exposure Pathway & lower limit & central estimate & upper limit \\
\hline Backyard Cow Milk & 0.33 & 2.1 & 14 \\
Commercial Milk (locally produced) & 0.082 & 0.7 & 4.7 \\
Commercial Milk (regionally mixed) & 0.1 & 0.8 & 5 \\
Goat Milk (locally produced) & 0.72 & 6.2 & 57 \\
Beef (locally produced) & 0.00034 & 0.006 & 0.13 \\
Leafy Vegetables (locally produced) & 0.00008 & 0.001 & 0.011 \\
Eggs (locally produced) & 0.015 & 0.11 & 0.93 \\
Cottage Cheese (locally produced) & 0.00038 & 0.0056 & 0.067 \\
Inhalation & 0.0052 & 0.021 & 0.091 \\
Mother's milk (mother on Diet 1) & 0.0013 & 0.041 & 0.95 \\
Prenatal exposure (mother on Diet 1) & 0.013 & 0.14 & 1 \\
Diet 1 & 0.36 & 2.3 & 15 \\
Diet 2 & 0.13 & 0.9 & 5.7 \\
Diet 3 & 0.11 & 0.82 & 5 \\
\hline
\end{tabular}

Excess Lifetime Risk [ ]

\begin{tabular}{lllc}
\hline Diet 1 & $5.6 \mathrm{E}-06$ & $1.8 \mathrm{E}-04$ & $3.5 \mathrm{E}-03$ \\
Diet 2 & $2.7 \mathrm{E}-06$ & $7.0 \mathrm{E}-05$ & $1.5 \mathrm{E}-03$ \\
Diet 3 & $2.3 \mathrm{E}-06$ & $6.2 \mathrm{E}-05$ & $1.5 \mathrm{E}-03$ \\
Diet 4 & $1.3 \mathrm{E}-05$ & $5.0 \mathrm{E}-04$ & $1.4 \mathrm{E}-02$ \\
\hline
\end{tabular}

\section{Relative Risk [ ]}

\begin{tabular}{lccc}
\hline Diet 1 & 1.0068 & 1.13 & 3.6 \\
Diet 2 & 1.0024 & 1.049 & 2.1 \\
Diet 3 & 1.0023 & 1.044 & 1.95 \\
Diet 4 & 1.014 & 1.35 & 11 \\
\hline
\end{tabular}

\begin{tabular}{lccc}
\hline Diet 1 & 0.68 & 11.7 & 72 \\
Diet 2 & 0.24 & 4.7 & 52 \\
Diet 3 & 0.23 & 4.2 & 49 \\
Diet 4 & 1.42 & 26.0 & 91 \\
\hline
\end{tabular}

Diet 1 - Backyard cow milk + all other locally produced non-milk exposure pathways

Diet 2 - Locally produced commercial milk + all other locally produced non-milk exposure pathways

Diet 3 - Regionally mixed commercial milk + inhalation (other regionally mixed food items are minor contributors to the total)

Diet 4 - Goat milk (all other exposure pathways are of negligible importance compared to goat milk) 
Location: Bradbury

Receptor: Female born in 1956

\begin{tabular}{lccc}
\hline & \multicolumn{3}{c}{ Thyroid Dose [cGy] } \\
Exposure Pathway & \multicolumn{2}{c}{$95 \%$ Subjective Confidence Interval } \\
\hline Backyard Cow Milk & lower limit & central estimate & upper limit \\
Commercial Milk (locally produced) & 0.31 & 4.5 & 58 \\
Commercial Milk (regionally mixed) & 0.12 & 1.7 & 21 \\
Goat Milk (locally produced) & 0.018 & 0.22 & 2.4 \\
Beef (locally produced) & 0.93 & 18 & 260 \\
Leafy Vegetables (locally produced) & 0.00018 & 0.0039 & 0.089 \\
Eggs (locally produced) & 0.00024 & 0.003 & 0.034 \\
Cottage Cheese (locally produced) & 0.012 & 0.19 & 2.6 \\
Inhalation & 0.0006 & 0.015 & 0.35 \\
Mother's milk (mother on Diet 1) & 0.0059 & 0.037 & 0.2 \\
Prenatal exposure (mother on Diet 1) & 0.0085 & 0.26 & 9.1 \\
Diet 1 & 0.079 & 0.89 & 11 \\
Diet 2 & 0.36 & 5 & 60 \\
Diet 3 & 0.21 & 2.1 & 24 \\
\hline
\end{tabular}

Excess Lifetime Risk [ ]

\begin{tabular}{llll}
\hline Diet 1 & $6.2 \mathrm{E}-05$ & $1.4 \mathrm{E}-03$ & $3.1 \mathrm{E}-02$ \\
Diet 2 & $3.4 \mathrm{E}-05$ & $6.4 \mathrm{E}-04$ & $1.1 \mathrm{E}-02$ \\
Diet 3 & $5.6 \mathrm{E}-06$ & $8.8 \mathrm{E}-05$ & $1.3 \mathrm{E}-03$ \\
Diet 4 & $1.8 \mathrm{E}-04$ & $5.7 \mathrm{E}-03$ & $1.6 \mathrm{E}-01$ \\
\hline
\end{tabular}

\section{Relative Risk [ ]}

\begin{tabular}{lccc}
\hline Diet 1 & 1.021 & 1.36 & 9.2 \\
Diet 2 & 1.0099 & 1.15 & 4.7 \\
Diet 3 & 1.0015 & 1.02 & 1.39 \\
Diet 4 & 1.056 & 2.3 & 33 \\
\hline
\end{tabular}

\section{Probability of Causation [\%]}

\begin{tabular}{lccc}
\hline Diet 1 & 2.10 & 26.2 & 89 \\
Diet 2 & 0.99 & 12.8 & 78 \\
Diet 3 & 0.15 & 2.0 & 28 \\
Diet 4 & 5.28 & 56.0 & 97 \\
\hline
\end{tabular}

Diet 1 - Backyard cow milk + all other locally produced non-milk exposure pathways

Diet 2 - Locally produced commercial milk + all other locally produced non-milk exposure pathways

Diet 3 - Regionally mixed commercial milk + inhalation (other regionally mixed food items are minor contributors to the total)

Diet 4 - Goat milk (all other exposure pathways are of negligible importance compared to goat milk) 


\section{Location: Bradbury}

Receptor: Male born in 1956

\begin{tabular}{lccc}
\hline & \multicolumn{3}{c}{ Thyroid Dose [cGy] } \\
& \multicolumn{2}{c}{$95 \%$ Subjective Confidence Interval } \\
Exposure Pathway & lower limit & central estimate & upper limit \\
\hline Backyard Cow Milk & 0.33 & 4.7 & 61 \\
Commercial Milk (locally produced) & 0.12 & 1.7 & 16 \\
Commercial Milk (regionally mixed) & 0.017 & 0.23 & 2.2 \\
Goat Milk (locally produced) & 1.3 & 18 & 320 \\
Beef (locally produced) & 0.00018 & 0.0036 & 0.09 \\
Leafy Vegetables (locally produced) & 0.00018 & 0.003 & 0.037 \\
Eggs (locally produced) & 0.013 & 0.19 & 2.8 \\
Cottage Cheese (locally produced) & 0.00064 & 0.015 & 0.27 \\
Inhalation & 0.0068 & 0.037 & 0.2 \\
Mother's milk (mother on Diet 1) & 0.0085 & 0.26 & 9.1 \\
Prenatal exposure (mother on Diet 1) & 0.079 & 0.89 & 11 \\
Diet 1 & 0.37 & 5 & 66 \\
Diet 2 & 0.2 & 2.1 & 18 \\
Diet 3 & 0.035 & 0.28 & 2.3 \\
\hline
\end{tabular}

Excess Lifetime Risk [ ]

\begin{tabular}{llll}
\hline Diet 1 & $1.1 \mathrm{E}-05$ & $3.6 \mathrm{E}-04$ & $1.3 \mathrm{E}-02$ \\
Diet 2 & $4.7 \mathrm{E}-06$ & $1.5 \mathrm{E}-04$ & $4.3 \mathrm{E}-03$ \\
Diet 3 & $6.9 \mathrm{E}-07$ & $2.0 \mathrm{E}-05$ & $5.9 \mathrm{E}-04$ \\
Diet 4 & $3.4 \mathrm{E}-05$ & $1.3 \mathrm{E}-03$ & $5.5 \mathrm{E}-02$ \\
\hline
\end{tabular}

\section{Relative Risk [ ]}

\begin{tabular}{lccc}
\hline Diet 1 & 1.011 & 1.27 & 9.7 \\
Diet 2 & 1.005 & 1.11 & 4.2 \\
Diet 3 & 1.00076 & 1.014 & 1.38 \\
Diet 4 & 1.03 & 2 & 41 \\
\hline
\end{tabular}

\section{Probability of Causation [\%]}

\begin{tabular}{lccc}
\hline Diet 1 & 1.10 & 21.1 & 89 \\
Diet 2 & 0.50 & 9.7 & 76 \\
Diet 3 & 0.08 & 1.4 & 27 \\
Diet 4 & 2.90 & 49.9 & 98 \\
\hline
\end{tabular}

Diet 1 - Backyard cow milk + all other locally produced non-milk exposure pathways

Diet 2 - Locally produced commercial milk + all other locally produced non-milk exposure pathways

Diet 3 - Regionally mixed commercial milk + inhalation (other regionally mixed food items are minor contributors to the total)

Diet 4 - Goat milk (all other exposure pathways are of negligible importance compared to goat milk) 
Location: Gallaher Bend

Receptor: Female born in 1956

\begin{tabular}{lccc}
\hline & \multicolumn{3}{c}{ Thyroid Dose [cGy] } \\
Exposure Pathway & \multicolumn{2}{c}{$95 \%$} & Subjective Confidence Interval \\
Backyard Cow Milk & lower limit & central estimate & upper limit \\
Commercial Milk (locally produced) & 0.4 & 5.7 & 66 \\
Commercial Milk (regionally mixed) & 0.15 & 2.1 & 22 \\
Goat Milk (locally produced) & 0.018 & 0.22 & 2.4 \\
Beef (locally produced) & 1.2 & 21 & 300 \\
Leafy Vegetables (locally produced) & 0.00024 & 0.0046 & 0.11 \\
Eggs (locally produced) & 0.00032 & 0.0037 & 0.041 \\
Cottage Cheese (locally produced) & 0.015 & 0.23 & 3 \\
Inhalation & 0.00075 & 0.018 & 0.37 \\
Mother's milk (mother on Diet 1) & 0.0075 & 0.046 & 0.23 \\
Prenatal exposure (mother on Diet 1) & 0.011 & 0.32 & 10 \\
Diet 1 & 0.1 & 1.1 & 13 \\
Diet 2 & 0.46 & 6.1 & 70 \\
Diet 3 & 0.24 & 2.6 & 25 \\
\hline
\end{tabular}

Excess Lifetime Risk [ ]

\begin{tabular}{llll}
\hline Diet 1 & $7.2 \mathrm{E}-05$ & $1.8 \mathrm{E}-03$ & $4.0 \mathrm{E}-02$ \\
Diet 2 & $4.7 \mathrm{E}-05$ & $8.2 \mathrm{E}-04$ & $1.5 \mathrm{E}-02$ \\
Diet 3 & $6.2 \mathrm{E}-06$ & $9.2 \mathrm{E}-05$ & $1.3 \mathrm{E}-03$ \\
Diet 4 & $2.0 \mathrm{E}-04$ & $6.8 \mathrm{E}-03$ & $1.9 \mathrm{E}-01$ \\
\hline
\end{tabular}

\section{Relative Risk [ ]}

\begin{tabular}{lccc}
\hline Diet 1 & 1.031 & 1.44 & 10.8 \\
Diet 2 & 1.011 & 1.18 & 5 \\
Diet 3 & 1.0016 & 1.021 & 1.4 \\
Diet 4 & 1.071 & 2.7 & 40 \\
\hline
\end{tabular}

\section{Probability of Causation [\%]}

\begin{tabular}{lccc}
\hline Diet 1 & 3.05 & 30.5 & 91 \\
Diet 2 & 1.09 & 15.4 & 80 \\
Diet 3 & 0.16 & 2.1 & 28 \\
Diet 4 & 6.59 & 62.8 & 97 \\
\hline
\end{tabular}

Diet 1 - Backyard cow milk + all other locally produced non-milk exposure pathways

Diet 2 - Locally produced commercial milk + all other locally produced non-milk exposure pathways

Diet 3 - Regionally mixed commercial milk + inhalation (other regionally mixed food items are minor contributors to the total)

Diet 4 - Goat milk (all other exposure pathways are of negligible importance compared to goat milk) 


\section{Location: Gallaher Bend}

Receptor: Male born in 1956

\begin{tabular}{lccc}
\hline & \multicolumn{3}{c}{ Thyroid Dose [cGy] } \\
& \multicolumn{2}{c}{$95 \%$ Subjective Confidence Interval } \\
Exposure Pathway & lower limit & central estimate & upper limit \\
\hline Backyard Cow Milk & 0.41 & 5.8 & 66 \\
Commercial Milk (locally produced) & 0.15 & 2.1 & 19 \\
Commercial Milk (regionally mixed) & 0.017 & 0.23 & 2.2 \\
Goat Milk (locally produced) & 1.5 & 22 & 370 \\
Beef (locally produced) & 0.0002 & 0.0044 & 0.11 \\
Leafy Vegetables (locally produced) & 0.00022 & 0.0037 & 0.043 \\
Eggs (locally produced) & 0.017 & 0.24 & 3.2 \\
Cottage Cheese (locally produced) & 0.00075 & 0.018 & 0.32 \\
Inhalation & 0.009 & 0.045 & 0.23 \\
Mother's milk (mother on Diet 1) & 0.011 & 0.32 & 10 \\
Prenatal exposure (mother on Diet 1) & 0.1 & 1.1 & 13 \\
Diet 1 & 0.49 & 6.2 & 69 \\
Diet 2 & 0.26 & 2.7 & 22 \\
Diet 3 & 0.038 & 0.28 & 2.3 \\
\hline
\end{tabular}

Excess Lifetime Risk [ ]

\begin{tabular}{llll}
\hline Diet 1 & $1.5 \mathrm{E}-05$ & $4.4 \mathrm{E}-04$ & $1.7 \mathrm{E}-02$ \\
Diet 2 & $6.7 \mathrm{E}-06$ & $1.9 \mathrm{E}-04$ & $5.3 \mathrm{E}-03$ \\
Diet 3 & $7.3 \mathrm{E}-07$ & $2.0 \mathrm{E}-05$ & $6.0 \mathrm{E}-04$ \\
Diet 4 & $4.5 \mathrm{E}-05$ & $1.6 \mathrm{E}-03$ & $6.5 \mathrm{E}-02$ \\
\hline
\end{tabular}

\section{Relative Risk [ ]}

\begin{tabular}{lccc}
\hline Diet 1 & 1.014 & 1.34 & 11 \\
Diet 2 & 1.0059 & 1.13 & 4.7 \\
Diet 3 & 1.0008 & 1.014 & 1.38 \\
Diet 4 & 1.038 & 2.2 & 46 \\
\hline
\end{tabular}

\section{Probability of Causation [\%]}

\begin{tabular}{lccc}
\hline Diet 1 & 1.35 & 25.3 & 91 \\
Diet 2 & 0.59 & 11.6 & 79 \\
Diet 3 & 0.08 & 1.4 & 27 \\
Diet 4 & 3.63 & 55.4 & 98 \\
\hline
\end{tabular}

Diet 1 - Backyard cow milk + all other locally produced non-milk exposure pathways

Diet 2 - Locally produced commercial milk + all other locally produced non-milk exposure pathways

Diet 3 - Regionally mixed commercial milk + inhalation (other regionally mixed food items are minor contributors to the total)

Diet 4 - Goat milk (all other exposure pathways are of negligible importance compared to goat milk) 


\section{Location: EFPC}

Receptor: Female born in 1956

\begin{tabular}{lccc}
\hline & \multicolumn{3}{c}{ Thyroid Dose [cGy] } \\
Exposure Pathway & \multicolumn{2}{c}{$95 \%$ Subjective Confidence Interval } \\
\hline Backyard Cow Milk & lower limit & central estimate & upper limit \\
Commercial Milk (locally produced) & 0.081 & 1.2 & 15 \\
Commercial Milk (regionally mixed) & 0.031 & 0.43 & 5.5 \\
Goat Milk (locally produced) & 0.018 & 0.22 & 2.4 \\
Beef (locally produced) & -- & -- & -- \\
Leafy Vegetables (locally produced) & 0.000043 & 0.00096 & 0.023 \\
Eggs (locally produced) & 0.000063 & 0.00078 & 0.0086 \\
Cottage Cheese (locally produced) & 0.003 & 0.049 & 0.68 \\
Inhalation & 0.00017 & 0.0038 & 0.084 \\
Mother's milk (mother on Diet 1) & 0.0016 & 0.0099 & 0.051 \\
Prenatal exposure (mother on Diet 1) & 0.0022 & 0.072 & 2.3 \\
Diet 1 & 0.022 & 0.23 & 2.9 \\
Diet 2 & 0.092 & 1.3 & 15 \\
Diet 3 & 0.054 & 0.53 & 6.1 \\
\hline
\end{tabular}

Excess Lifetime Risk [ ]

\begin{tabular}{lccc}
\hline Diet 1 & $1.6 \mathrm{E}-05$ & $3.9 \mathrm{E}-04$ & $7.9 \mathrm{E}-03$ \\
Diet 2 & $9.0 \mathrm{E}-06$ & $1.7 \mathrm{E}-04$ & $3.1 \mathrm{E}-03$ \\
Diet 3 & $4.4 \mathrm{E}-06$ & $7.4 \mathrm{E}-05$ & $1.2 \mathrm{E}-03$ \\
Diet 4 & -- & -- & -- \\
\hline
\end{tabular}

Relative Risk [ ]

\begin{tabular}{lccc}
\hline Diet 1 & 1.0056 & 1.093 & 3.1 \\
Diet 2 & 1.0025 & 1.039 & 1.88 \\
Diet 3 & 1.001 & 1.018 & 1.38 \\
Diet 4 & -- & -- & -- \\
\hline
\end{tabular}

\section{Probability of Causation [\%]}

\begin{tabular}{lccc}
\hline Diet 1 & 0.56 & 8.4 & 65 \\
Diet 2 & 0.25 & 3.7 & 45 \\
Diet 3 & 0.10 & 1.7 & 27 \\
Diet 4 & -- & -- & \\
\hline Diet 1 - Backyard cow milk + all other locally produced non-milk exposure pathways & \\
Diet 2 - Locally produced commercial milk + all other locally produced non-milk exposure pathways \\
Diet 3 - Regionally mixed commercial milk + inhalation (other regionally mixed food items are minor contributors to the total) \\
Diet 4 - Goat milk (all other exposure pathways are of negligible importance compared to goat milk)
\end{tabular}




\section{Location: EFPC}

\section{Receptor: Male born in 1956}

\begin{tabular}{lccc}
\hline & \multicolumn{3}{c}{ Thyroid Dose [cGy] } \\
& \multicolumn{2}{c}{$95 \%$ Subjective Confidence Interval } \\
Exposure Pathway & lower limit & central estimate & upper limit \\
\hline Backyard Cow Milk & 0.086 & 1.2 & 15 \\
Commercial Milk (locally produced) & 0.031 & 0.43 & 4.7 \\
Commercial Milk (regionally mixed) & 0.017 & 0.23 & 2.2 \\
Goat Milk (locally produced) & -- & -- & -- \\
Beef (locally produced) & 0.000046 & 0.00094 & 0.025 \\
Leafy Vegetables (locally produced) & 0.000047 & 0.00076 & 0.0096 \\
Eggs (locally produced) & 0.0035 & 0.052 & 0.66 \\
Cottage Cheese (locally produced) & 0.00018 & 0.0039 & 0.073 \\
Inhalation & 0.0019 & 0.01 & 0.052 \\
Mother's milk (mother on Diet 1) & 0.0022 & 0.072 & 2.3 \\
Prenatal exposure (mother on Diet 1) & 0.022 & 0.23 & 2.9 \\
Diet 1 & 0.099 & 1.3 & 16 \\
Diet 2 & 0.051 & 0.54 & 5.2 \\
Diet 3 & 0.023 & 0.24 & 2.2 \\
\hline
\end{tabular}

Excess Lifetime Risk [ ]

\begin{tabular}{lccc}
\hline Diet 1 & $2.9 \mathrm{E}-06$ & $9.7 \mathrm{E}-05$ & $3.4 \mathrm{E}-03$ \\
Diet 2 & $1.3 \mathrm{E}-06$ & $4.0 \mathrm{E}-05$ & $1.1 \mathrm{E}-03$ \\
Diet 3 & $6.1 \mathrm{E}-07$ & $1.8 \mathrm{E}-05$ & $5.5 \mathrm{E}-04$ \\
Diet 4 & -- & -- & -- \\
\hline
\end{tabular}

\section{Relative Risk [ ]}

\begin{tabular}{lccc}
\hline Diet 1 & 1.003 & 1.07 & 3.2 \\
Diet 2 & 1.0013 & 1.028 & 1.93 \\
Diet 3 & 1.00056 & 1.012 & 1.36 \\
Diet 4 & -- & -- & -- \\
\hline
\end{tabular}

\section{Probability of Causation [\%]}

\begin{tabular}{lccc}
\hline Diet 1 & 0.30 & 6.5 & 67 \\
Diet 2 & 0.13 & 2.7 & 47 \\
Diet 3 & 0.06 & 1.2 & 26 \\
Diet 4 & -- & -- & -- \\
\hline
\end{tabular}

Diet 1 - Backyard cow milk + all other locally produced non-milk exposure pathways

Diet 2 - Locally produced commercial milk + all other locally produced non-milk exposure pathways

Diet 3 - Regionally mixed commercial milk + inhalation (other regionally mixed food items are minor contributors to the total)

Diet 4 - Goat milk (all other exposure pathways are of negligible importance compared to goat milk) 


\section{Location: Hope Creek}

Receptor: Female born in 1956

\begin{tabular}{lccc}
\hline & \multicolumn{3}{c}{ Thyroid Dose [cGy] } \\
Exposure Pathway & \multicolumn{2}{c}{$95 \%$ Subjective Confidence Interval } \\
\hline Backyard Cow Milk & lower limit & central estimate & upper limit \\
Commercial Milk (locally produced) & 0.21 & 3.2 & 38 \\
Commercial Milk (regionally mixed) & 0.086 & 1.2 & 13 \\
Goat Milk (locally produced) & 0.018 & 0.22 & 2.4 \\
Beef (locally produced) & -- & -- & -- \\
Leafy Vegetables (locally produced) & 0.00013 & 0.0027 & 0.062 \\
Eggs (locally produced) & 0.00018 & 0.0022 & 0.024 \\
Cottage Cheese (locally produced) & 0.0084 & 0.13 & 1.7 \\
Inhalation & 0.00043 & 0.01 & 0.24 \\
Mother's milk (mother on Diet 1) & 0.0044 & 0.026 & 0.14 \\
Prenatal exposure (mother on Diet 1) & 0.0063 & 0.19 & 6.1 \\
Diet 1 & 0.058 & 0.62 & 7.8 \\
Diet 2 & 0.24 & 3.5 & 40 \\
Diet 3 & 0.15 & 1.5 & 14 \\
\hline
\end{tabular}

Excess Lifetime Risk [ ]

\begin{tabular}{lccc}
\hline Diet 1 & $4.0 \mathrm{E}-05$ & $1.0 \mathrm{E}-03$ & $2.2 \mathrm{E}-02$ \\
Diet 2 & $2.5 \mathrm{E}-05$ & $4.6 \mathrm{E}-04$ & $7.8 \mathrm{E}-03$ \\
Diet 3 & $5.2 \mathrm{E}-06$ & $8.2 \mathrm{E}-05$ & $1.3 \mathrm{E}-03$ \\
Diet 4 & -- & -- & -- \\
\hline
\end{tabular}

Relative Risk [ ]

\begin{tabular}{lccc}
\hline Diet 1 & 1.016 & 1.25 & 6.6 \\
Diet 2 & 1.0069 & 1.11 & 3.2 \\
Diet 3 & 1.0013 & 1.019 & 1.38 \\
Diet 4 & -- & -- & -- \\
\hline
\end{tabular}

\section{Probability of Causation [\%]}

\begin{tabular}{lccc}
\hline Diet 1 & 1.54 & 19.9 & 85 \\
Diet 2 & 0.68 & 9.8 & 68 \\
Diet 3 & 0.13 & 1.9 & 28 \\
Diet 4 & -- & -- & - \\
\hline Diet 1 - Backyard cow milk + all other locally produced non-milk exposure pathways & \\
Diet 2 - Locally produced commercial milk + all other locally produced non-milk exposure pathways \\
Diet 3 - Regionally mixed commercial milk + inhalation (other regionally mixed food items are minor contributors to the total) \\
Diet 4 - Goat milk (all other exposure pathways are of negligible importance compared to goat milk)
\end{tabular}




\section{Location: Hope Creek}

\section{Receptor: Male born in 1956}

\begin{tabular}{lccc}
\hline & \multicolumn{3}{c}{ Thyroid Dose [cGy] } \\
Exposure Pathway & 95\% Subjective Confidence Interval \\
\hline Backyard Cow Milk & lower limit & central estimate & upper limit \\
Commercial Milk (locally produced) & 0.22 & 3.4 & 40 \\
Commercial Milk (regionally mixed) & 0.088 & 1.2 & 12 \\
Goat Milk (locally produced) & 0.017 & 0.23 & 2.2 \\
Beef (locally produced) & -- & -- & -- \\
Leafy Vegetables (locally produced) & 0.00011 & 0.0026 & 0.065 \\
Eggs (locally produced) & 0.00012 & 0.0021 & 0.026 \\
Cottage Cheese (locally produced) & 0.0089 & 0.14 & 1.9 \\
Inhalation & 0.00048 & 0.01 & 0.2 \\
Mother's milk (mother on Diet 1) & 0.0051 & 0.027 & 0.14 \\
Prenatal exposure (mother on Diet 1) & 0.0063 & 0.19 & 6.1 \\
Diet 1 & 0.058 & 0.62 & 7.8 \\
Diet 2 & 0.25 & 3.5 & 43 \\
Diet 3 & 0.14 & 1.5 & 13 \\
\hline
\end{tabular}

Excess Lifetime Risk [ ]

\begin{tabular}{lccc}
\hline Diet 1 & $7.6 \mathrm{E}-06$ & $2.4 \mathrm{E}-04$ & $9.4 \mathrm{E}-03$ \\
Diet 2 & $3.4 \mathrm{E}-06$ & $1.1 \mathrm{E}-04$ & $2.9 \mathrm{E}-03$ \\
Diet 3 & $6.6 \mathrm{E}-07$ & $1.9 \mathrm{E}-05$ & $5.7 \mathrm{E}-04$ \\
Diet 4 & -- & -- & -- \\
\hline
\end{tabular}

\section{Relative Risk [ ]}

\begin{tabular}{lccc}
\hline Diet 1 & 1.0076 & 1.2 & 7.4 \\
Diet 2 & 1.0034 & 1.074 & 3.5 \\
Diet 3 & 1.0007 & 1.013 & 1.37 \\
Diet 4 & -- & -- & -- \\
\hline
\end{tabular}

\begin{tabular}{lccc}
\hline Diet 1 & 0.76 & 16.9 & 86 \\
Diet 2 & 0.34 & 6.9 & 71 \\
Diet 3 & 0.07 & 1.3 & 27 \\
Diet 4 & -- & -- & - \\
\hline Diet 1 - Backyard cow milk + all other locally produced non-milk exposure pathways & \\
Diet 2 - Locally produced commercial milk + all other locally produced non-milk exposure pathways \\
Diet 3 - Regionally mixed commercial milk + inhalation (other regionally mixed food items are minor contributors to the total) \\
Diet 4 - Goat milk (all other exposure pathways are of negligible importance compared to goat milk)
\end{tabular}


Location: Buttermilk Rd.

Receptor: Female born in 1956

\begin{tabular}{lccc}
\hline & \multicolumn{3}{c}{ Thyroid Dose [cGy] } \\
Exposure Pathway & $95 \%$ Subjective Confidence Interval \\
\hline Backyard Cow Milk & lower limit & central estimate & upper limit \\
Commercial Milk (locally produced) & 0.21 & 3.2 & 37 \\
Commercial Milk (regionally mixed) & 0.084 & 1.2 & 12 \\
Goat Milk (locally produced) & 0.018 & 0.22 & 2.4 \\
Beef (locally produced) & 0.63 & 12 & 190 \\
Leafy Vegetables (locally produced) & 0.00013 & 0.0027 & 0.06 \\
Eggs (locally produced) & 0.00017 & 0.0021 & 0.023 \\
Cottage Cheese (locally produced) & 0.0084 & 0.13 & 1.6 \\
Inhalation & 0.00041 & 0.01 & 0.23 \\
Mother's milk (mother on Diet 1) & 0.0044 & 0.027 & 0.14 \\
Prenatal exposure (mother on Diet 1) & 0.0061 & 0.19 & 5.9 \\
Diet 1 & 0.059 & 0.61 & 7.6 \\
Diet 2 & 0.24 & 3.4 & 39 \\
Diet 3 & 0.14 & 1.5 & 14 \\
\hline
\end{tabular}

Excess Lifetime Risk [ ]

\begin{tabular}{lllc}
\hline Diet 1 & $4.0 \mathrm{E}-05$ & $9.9 \mathrm{E}-04$ & $2.1 \mathrm{E}-02$ \\
Diet 2 & $2.6 \mathrm{E}-05$ & $4.5 \mathrm{E}-04$ & $7.8 \mathrm{E}-03$ \\
Diet 3 & $5.2 \mathrm{E}-06$ & $8.2 \mathrm{E}-05$ & $1.3 \mathrm{E}-03$ \\
Diet 4 & $1.2 \mathrm{E}-04$ & $4.0 \mathrm{E}-03$ & $1.1 \mathrm{E}-01$ \\
\hline
\end{tabular}

\section{Relative Risk [ ]}

\begin{tabular}{lccc}
\hline Diet 1 & 1.016 & 1.25 & 6.7 \\
Diet 2 & 1.0071 & 1.11 & 3.1 \\
Diet 3 & 1.0013 & 1.019 & 1.38 \\
Diet 4 & 1.042 & 1.92 & 24 \\
\hline
\end{tabular}

\section{Probability of Causation [\%]}

\begin{tabular}{lccc}
\hline Diet 1 & 1.56 & 19.9 & 85 \\
Diet 2 & 0.71 & 9.6 & 68 \\
Diet 3 & 0.13 & 1.9 & 28 \\
Diet 4 & 3.99 & 47.6 & 96 \\
\hline
\end{tabular}

Diet 1 - Backyard cow milk + all other locally produced non-milk exposure pathways

Diet 2 - Locally produced commercial milk + all other locally produced non-milk exposure pathways

Diet 3 - Regionally mixed commercial milk + inhalation (other regionally mixed food items are minor contributors to the total)

Diet 4 - Goat milk (all other exposure pathways are of negligible importance compared to goat milk) 
Location: Buttermilk Rd.

Receptor: Male born in 1956

\begin{tabular}{lccc}
\hline & \multicolumn{3}{c}{ Thyroid Dose [cGy] } \\
& \multicolumn{2}{c}{$95 \%$ Subjective Confidence Interval } \\
Exposure Pathway & lower limit & central estimate & upper limit \\
\hline Backyard Cow Milk & 0.21 & 3.3 & 38 \\
Commercial Milk (locally produced) & 0.084 & 1.2 & 11 \\
Commercial Milk (regionally mixed) & 0.017 & 0.23 & 2.2 \\
Goat Milk (locally produced) & 0.89 & 12 & 210 \\
Beef (locally produced) & 0.00011 & 0.0025 & 0.065 \\
Leafy Vegetables (locally produced) & 0.00012 & 0.0021 & 0.024 \\
Eggs (locally produced) & 0.0088 & 0.14 & 1.8 \\
Cottage Cheese (locally produced) & 0.00046 & 0.01 & 0.19 \\
Inhalation & 0.0051 & 0.027 & 0.14 \\
Mother's milk (mother on Diet 1) & 0.0061 & 0.19 & 5.9 \\
Prenatal exposure (mother on Diet 1) & 0.059 & 0.61 & 7.6 \\
Diet 1 & 0.24 & 3.5 & 41 \\
Diet 2 & 0.14 & 1.4 & 13 \\
Diet 3 & 0.031 & 0.26 & 2.3 \\
\hline
\end{tabular}

Excess Lifetime Risk [ ]

\begin{tabular}{llll}
\hline Diet 1 & $7.0 \mathrm{E}-06$ & $2.4 \mathrm{E}-04$ & $8.9 \mathrm{E}-03$ \\
Diet 2 & $3.3 \mathrm{E}-06$ & $1.1 \mathrm{E}-04$ & $2.9 \mathrm{E}-03$ \\
Diet 3 & $6.6 \mathrm{E}-07$ & $1.9 \mathrm{E}-05$ & $5.7 \mathrm{E}-04$ \\
Diet 4 & $2.3 \mathrm{E}-05$ & $9.0 \mathrm{E}-04$ & $3.7 \mathrm{E}-02$ \\
\hline
\end{tabular}

\section{Relative Risk [ ]}

\begin{tabular}{lccc}
\hline Diet 1 & 1.0072 & 1.2 & 7.1 \\
Diet 2 & 1.0034 & 1.072 & 3.5 \\
Diet 3 & 1.0007 & 1.013 & 1.37 \\
Diet 4 & 1.021 & 1.71 & 31 \\
\hline
\end{tabular}

\section{Probability of Causation [\%]}

\begin{tabular}{lccc}
\hline Diet 1 & 0.71 & 16.4 & 85 \\
Diet 2 & 0.34 & 6.7 & 71 \\
Diet 3 & 0.07 & 1.3 & 27 \\
Diet 4 & 2.04 & 41.4 & 97 \\
\hline
\end{tabular}

Diet 1 - Backyard cow milk + all other locally produced non-milk exposure pathways

Diet 2 - Locally produced commercial milk + all other locally produced non-milk exposure pathways

Diet 3 - Regionally mixed commercial milk + inhalation (other regionally mixed food items are minor contributors to the total)

Diet 4 - Goat milk (all other exposure pathways are of negligible importance compared to goat milk) 


\section{Location: Jonesville}

Receptor: Female born in 1956

\begin{tabular}{lccc}
\hline & \multicolumn{3}{c}{ Thyroid Dose [cGy] } \\
Exposure Pathway & \multicolumn{2}{c}{$95 \%$ Subjective Confidence Interval } \\
\hline Backyard Cow Milk & lower limit & central estimate & upper limit \\
Commercial Milk (locally produced) & 0.028 & 0.45 & 6.1 \\
Commercial Milk (regionally mixed) & 0.012 & 0.17 & 1.9 \\
Goat Milk (locally produced) & 0.018 & 0.22 & 2.4 \\
Beef (locally produced) & 0.091 & 1.8 & 30 \\
Leafy Vegetables (locally produced) & 0.000016 & 0.00036 & 0.0093 \\
Eggs (locally produced) & 0.000022 & 0.00031 & 0.0039 \\
Cottage Cheese (locally produced) & 0.0012 & 0.019 & 0.27 \\
Inhalation & 0.000057 & 0.0015 & 0.035 \\
Mother's milk (mother on Diet 1) & 0.00071 & 0.004 & 0.025 \\
Prenatal exposure (mother on Diet 1) & 0.00087 & 0.026 & 0.86 \\
Diet 1 & 0.0085 & 0.09 & 1.1 \\
Diet 2 & 0.032 & 0.49 & 6.4 \\
Diet 3 & 0.02 & 0.21 & 2.2 \\
\hline
\end{tabular}

Excess Lifetime Risk [ ]

\begin{tabular}{lllc}
\hline Diet 1 & $5.9 \mathrm{E}-06$ & $1.4 \mathrm{E}-04$ & $3.4 \mathrm{E}-03$ \\
Diet 2 & $3.1 \mathrm{E}-06$ & $6.5 \mathrm{E}-05$ & $1.2 \mathrm{E}-03$ \\
Diet 3 & $4.2 \mathrm{E}-06$ & $7.1 \mathrm{E}-05$ & $1.2 \mathrm{E}-03$ \\
Diet 4 & $1.6 \mathrm{E}-05$ & $6.2 \mathrm{E}-04$ & $1.7 \mathrm{E}-02$ \\
\hline
\end{tabular}

Relative Risk [ ]

\begin{tabular}{lccc}
\hline Diet 1 & 1.0025 & 1.036 & 1.94 \\
Diet 2 & 1.0011 & 1.015 & 1.3 \\
Diet 3 & 1.00094 & 1.017 & 1.37 \\
Diet 4 & 1.005 & 1.13 & 4.5 \\
\hline
\end{tabular}

\begin{tabular}{lccc} 
& \multicolumn{3}{c}{ Probability of Causation [\%] } \\
\hline Diet 1 & 0.25 & 3.5 & 48 \\
Diet 2 & 0.11 & 1.5 & 23 \\
Diet 3 & 0.09 & 1.7 & 27 \\
Diet 4 & 0.50 & 11.4 & 77 \\
\hline
\end{tabular}

Diet 1 - Backyard cow milk + all other locally produced non-milk exposure pathways

Diet 2 - Locally produced commercial milk + all other locally produced non-milk exposure pathways

Diet 3 - Regionally mixed commercial milk + inhalation (other regionally mixed food items are minor contributors to the total)

Diet 4 - Goat milk (all other exposure pathways are of negligible importance compared to goat milk) 


\section{Location: Jonesville}

\section{Receptor: Male born in 1956}

\begin{tabular}{lccc}
\hline & \multicolumn{3}{c}{ Thyroid Dose [cGy] } \\
& \multicolumn{2}{c}{$95 \%$ Subjective Confidence Interval } \\
Exposure Pathway & lower limit & central estimate & upper limit \\
\hline Backyard Cow Milk & 0.029 & 0.45 & 6.2 \\
Commercial Milk (locally produced) & 0.01 & 0.17 & 1.7 \\
Commercial Milk (regionally mixed) & 0.017 & 0.23 & 2.2 \\
Goat Milk (locally produced) & 0.12 & 1.8 & 33 \\
Beef (locally produced) & 0.000017 & 0.00037 & 0.0092 \\
Leafy Vegetables (locally produced) & 0.000017 & 0.00029 & 0.0039 \\
Eggs (locally produced) & 0.0014 & 0.02 & 0.25 \\
Cottage Cheese (locally produced) & 0.000075 & 0.0015 & 0.026 \\
Inhalation & 0.00076 & 0.0039 & 0.023 \\
Mother's milk (mother on Diet 1) & 0.00087 & 0.026 & 0.86 \\
Prenatal exposure (mother on Diet 1) & 0.0085 & 0.09 & 1.1 \\
Diet 1 & 0.035 & 0.49 & 6.5 \\
Diet 2 & 0.019 & 0.21 & 2 \\
Diet 3 & 0.019 & 0.24 & 2.2 \\
\hline
\end{tabular}

Excess Lifetime Risk [ ]

\begin{tabular}{llll}
\hline Diet 1 & $9.7 \mathrm{E}-07$ & $3.5 \mathrm{E}-05$ & $1.4 \mathrm{E}-03$ \\
Diet 2 & $4.6 \mathrm{E}-07$ & $1.5 \mathrm{E}-05$ & $4.9 \mathrm{E}-04$ \\
Diet 3 & $5.7 \mathrm{E}-07$ & $1.7 \mathrm{E}-05$ & $5.4 \mathrm{E}-04$ \\
Diet 4 & $3.0 \mathrm{E}-06$ & $1.3 \mathrm{E}-04$ & $5.8 \mathrm{E}-03$ \\
\hline
\end{tabular}

\section{Relative Risk [ ]}

\begin{tabular}{lccc}
\hline Diet 1 & 1.0011 & 1.029 & 1.94 \\
Diet 2 & 1.00052 & 1.012 & 1.33 \\
Diet 3 & 1.00053 & 1.012 & 1.36 \\
Diet 4 & 1.0029 & 1.11 & 5.6 \\
\hline
\end{tabular}

\section{Probability of Causation [\%]}

\begin{tabular}{llll}
\hline Diet 1 & 0.11 & 2.8 & 48 \\
Diet 2 & 0.05 & 1.2 & 25 \\
Diet 3 & 0.05 & 1.2 & 26 \\
Diet 4 & 0.29 & 9.6 & 82 \\
\hline
\end{tabular}

Diet 1 - Backyard cow milk + all other locally produced non-milk exposure pathways

Diet 2 - Locally produced commercial milk + all other locally produced non-milk exposure pathways

Diet 3 - Regionally mixed commercial milk + inhalation (other regionally mixed food items are minor contributors to the total)

Diet 4 - Goat milk (all other exposure pathways are of negligible importance compared to goat milk) 


\section{Location: OR Scarboro}

Receptor: Female born in 1956

\begin{tabular}{lccc}
\hline & \multicolumn{3}{c}{ Thyroid Dose [cGy] } \\
Exposure Pathway & \multicolumn{2}{c}{$9 \%$ Subjective Confidence Interval } \\
\hline Backyard Cow Milk & lower limit & central estimate & upper limit \\
Commercial Milk (locally produced) & -- & -- & -- \\
Commercial Milk (regionally mixed) & -- & -- & -- \\
Goat Milk (locally produced) & 0.018 & 0.22 & 2.4 \\
Beef (locally produced) & -- & -- & -- \\
Leafy Vegetables (locally produced) & -- & -- & -- \\
Eggs (locally produced) & -- & -- & -- \\
Cottage Cheese (locally produced) & -- & -- & -- \\
Inhalation & 0.0003 & 0.0077 & 0.17 \\
Mother's milk (mother on Diet 3) & 0.0033 & 0.02 & 0.11 \\
Prenatal exposure (mother on Diet 3) & 0.00083 & 0.014 & 0.21 \\
Diet 1 & 0.0068 & 0.043 & 0.31 \\
Diet 2 & -- & -- & -- \\
Diet 3 & -- & -- & -- \\
\hline
\end{tabular}

Excess Lifetime Risk [ ]

\begin{tabular}{lccc}
\hline Diet 1 & -- & -- & -- \\
Diet 2 & -- & -- & -- \\
Diet 3 & $4.8 \mathrm{E}-06$ & $7.8 \mathrm{E}-05$ & $1.2 \mathrm{E}-03$ \\
Diet 4 & -- & -- & -- \\
\hline
\end{tabular}

\section{Relative Risk [ ]}

\begin{tabular}{lccc}
\hline Diet 1 & -- & -- & -- \\
Diet 2 & -- & -- & -- \\
Diet 3 & 1.0012 & 1.019 & 1.38 \\
Diet 4 & -- & -- & -- \\
\hline
\end{tabular}

\begin{tabular}{lccc}
\hline Diet 1 & -- & -- & -- \\
Diet 2 & -- & -- & -- \\
Diet 3 & 0.12 & 1.8 & - \\
Diet 4 & -- & -- \\
\hline Diet 1 - Backyard cow milk + all other locally produced non-milk exposure pathways & \\
Diet 2 - Locally produced commercial milk + all other locally produced non-milk exposure pathways \\
Diet 3 - Regionally mixed commercial milk + inhalation (other regionally mixed food items are minor contributors to the total) \\
Diet 4 - Goat milk (all other exposure pathways are of negligible importance compared to goat milk)
\end{tabular}




\section{Location: OR Scarboro}

Receptor: Male born in 1956

\begin{tabular}{lccc}
\hline & \multicolumn{3}{c}{ Thyroid Dose [cGy] } \\
Exposure Pathway & \multicolumn{2}{c}{$95 \%$ Subjective Confidence Interval } \\
\hline Backyard Cow Milk & lower limit & central estimate & upper limit \\
Commercial Milk (locally produced) & -- & -- & -- \\
Commercial Milk (regionally mixed) & -- & -- & -- \\
Goat Milk (locally produced) & 0.017 & 0.23 & 2.2 \\
Beef (locally produced) & -- & -- & -- \\
Leafy Vegetables (locally produced) & -- & -- & -- \\
Eggs (locally produced) & -- & -- & -- \\
Cottage Cheese (locally produced) & -- & -- & -- \\
Inhalation & 0.00033 & 0.0077 & 0.14 \\
Mother's milk (mother on Diet 3) & 0.0038 & 0.02 & 0.11 \\
Prenatal exposure (mother on Diet 3) & 0.00083 & 0.014 & 0.21 \\
Diet 1 & 0.0068 & 0.043 & 0.31 \\
Diet 2 & -- & -- & -- \\
Diet 3 & -- & -- & -- \\
\hline
\end{tabular}

Excess Lifetime Risk [ ]

\begin{tabular}{lccc}
\hline Diet 1 & -- & -- & -- \\
Diet 2 & -- & -- & -- \\
Diet 3 & $6.4 \mathrm{E}-07$ & $1.8 \mathrm{E}-05$ & $5.6 \mathrm{E}-04$ \\
Diet 4 & -- & -- & -- \\
\hline
\end{tabular}

\section{Relative Risk [ ]}

\begin{tabular}{lccc}
\hline Diet 1 & -- & -- & -- \\
Diet 2 & -- & -- & -- \\
Diet 3 & 1.00065 & 1.013 & 1.37 \\
Diet 4 & -- & -- & -- \\
\hline
\end{tabular}

\begin{tabular}{lccc}
\hline Diet 1 & -- & -- & -- \\
Diet 2 & -- & -- & -- \\
Diet 3 & 0.07 & 1.3 & -- \\
Diet 4 & -- & -- & \\
\hline Diet 1 - Backyard cow milk + all other locally produced non-milk exposure pathways & \\
Diet 2 - Locally produced commercial milk + all other locally produced non-milk exposure pathways \\
Diet 3 - Regionally mixed commercial milk + inhalation (other regionally mixed food items are minor contributors to the total) \\
Diet 4 - Goat milk (all other exposure pathways are of negligible importance compared to goat milk)
\end{tabular}


Location: Lawnville/Gallaher Receptor: Female born in 1956

\begin{tabular}{lccc}
\hline & \multicolumn{3}{c}{ Thyroid Dose [cGy] } \\
Exposure Pathway & \multicolumn{2}{c}{$95 \%$ Subjective Confidence Interval } \\
\hline Backyard Cow Milk & lower limit & central estimate & upper limit \\
Commercial Milk (locally produced) & 0.23 & 3.1 & 34 \\
Commercial Milk (regionally mixed) & 0.088 & 1.1 & 12 \\
Goat Milk (locally produced) & 0.018 & 0.22 & 2.4 \\
Beef (locally produced) & 0.68 & 12 & 170 \\
Leafy Vegetables (locally produced) & 0.00012 & 0.0026 & 0.062 \\
Eggs (locally produced) & 0.00015 & 0.002 & 0.021 \\
Cottage Cheese (locally produced) & 0.008 & 0.13 & 1.4 \\
Inhalation & 0.00038 & 0.0096 & 0.22 \\
Mother's milk (mother on Diet 1) & 0.0044 & 0.026 & 0.14 \\
Prenatal exposure (mother on Diet 1) & 0.006 & 0.18 & 6 \\
Diet 1 & 0.063 & 0.6 & 7.3 \\
Diet 2 & 0.27 & 3.3 & 36 \\
Diet 3 & 0.14 & 1.4 & 14 \\
\hline
\end{tabular}

Excess Lifetime Risk [ ]

\begin{tabular}{lllc}
\hline Diet 1 & $4.4 \mathrm{E}-05$ & $9.6 \mathrm{E}-04$ & $1.9 \mathrm{E}-02$ \\
Diet 2 & $2.7 \mathrm{E}-05$ & $4.2 \mathrm{E}-04$ & $8.1 \mathrm{E}-03$ \\
Diet 3 & $5.2 \mathrm{E}-06$ & $8.2 \mathrm{E}-05$ & $1.3 \mathrm{E}-03$ \\
Diet 4 & $1.2 \mathrm{E}-04$ & $3.8 \mathrm{E}-03$ & $1.1 \mathrm{E}-01$ \\
\hline
\end{tabular}

\section{Relative Risk [ ]}

\begin{tabular}{lccc}
\hline Diet 1 & 1.015 & 1.24 & 6.4 \\
Diet 2 & 1.0071 & 1.1 & 3 \\
Diet 3 & 1.0013 & 1.019 & 1.38 \\
Diet 4 & 1.042 & 1.88 & 22 \\
\hline
\end{tabular}

\section{Probability of Causation [\%]}

\begin{tabular}{lccc}
\hline Diet 1 & 1.49 & 19.6 & 84 \\
Diet 2 & 0.71 & 9.1 & 67 \\
Diet 3 & 0.13 & 1.9 & 28 \\
Diet 4 & 4.00 & 46.7 & 96 \\
\hline
\end{tabular}

Diet 1 - Backyard cow milk + all other locally produced non-milk exposure pathways

Diet 2 - Locally produced commercial milk + all other locally produced non-milk exposure pathways

Diet 3 - Regionally mixed commercial milk + inhalation (other regionally mixed food items are minor contributors to the total)

Diet 4 - Goat milk (all other exposure pathways are of negligible importance compared to goat milk) 


\section{Location: Lawnville/Gallaher} Receptor: Male born in 1956

\begin{tabular}{lccc}
\hline & \multicolumn{3}{c}{ Thyroid Dose [cGy] } \\
& \multicolumn{2}{c}{$95 \%$ Subjective Confidence Interval } \\
Exposure Pathway & lower limit & central estimate & upper limit \\
\hline Backyard Cow Milk & 0.21 & 3.1 & 37 \\
Commercial Milk (locally produced) & 0.087 & 1.1 & 11 \\
Commercial Milk (regionally mixed) & 0.017 & 0.23 & 2.2 \\
Goat Milk (locally produced) & 0.88 & 12 & 210 \\
Beef (locally produced) & 0.00011 & 0.0023 & 0.062 \\
Leafy Vegetables (locally produced) & 0.00012 & 0.002 & 0.023 \\
Eggs (locally produced) & 0.0094 & 0.12 & 1.6 \\
Cottage Cheese (locally produced) & 0.00044 & 0.0098 & 0.17 \\
Inhalation & 0.005 & 0.026 & 0.15 \\
Mother's milk (mother on Diet 1) & 0.006 & 0.18 & 6 \\
Prenatal exposure (mother on Diet 1) & 0.063 & 0.6 & 7.3 \\
Diet 1 & 0.24 & 3.3 & 39 \\
Diet 2 & 0.14 & 1.4 & 14 \\
Diet 3 & 0.032 & 0.26 & 2.3 \\
\hline
\end{tabular}

Excess Lifetime Risk [ ]

\begin{tabular}{lllc}
\hline Diet 1 & $7.5 \mathrm{E}-06$ & $2.5 \mathrm{E}-04$ & $7.8 \mathrm{E}-03$ \\
Diet 2 & $3.4 \mathrm{E}-06$ & $1.0 \mathrm{E}-04$ & $3.1 \mathrm{E}-03$ \\
Diet 3 & $6.7 \mathrm{E}-07$ & $1.9 \mathrm{E}-05$ & $5.8 \mathrm{E}-04$ \\
Diet 4 & $2.3 \mathrm{E}-05$ & $8.6 \mathrm{E}-04$ & $4.0 \mathrm{E}-02$ \\
\hline
\end{tabular}

\section{Relative Risk [ ]}

\begin{tabular}{lccc}
\hline Diet 1 & 1.0071 & 1.19 & 7.2 \\
Diet 2 & 1.0033 & 1.071 & 3 \\
Diet 3 & 1.0007 & 1.013 & 1.37 \\
Diet 4 & 1.021 & 1.64 & 27 \\
\hline
\end{tabular}

\begin{tabular}{lccc}
\hline Diet 1 & 0.71 & 15.6 & 86 \\
Diet 2 & 0.33 & 6.6 & 67 \\
Diet 3 & 0.07 & 1.3 & 27 \\
Diet 4 & 2.01 & 39.1 & 96 \\
\hline
\end{tabular}

Diet 1 - Backyard cow milk + all other locally produced non-milk exposure pathways

Diet 2 - Locally produced commercial milk + all other locally produced non-milk exposure pathways

Diet 3 - Regionally mixed commercial milk + inhalation (other regionally mixed food items are minor contributors to the total)

Diet 4 - Goat milk (all other exposure pathways are of negligible importance compared to goat milk) 


\section{Location: Dyllis}

Receptor: Female born in 1956

\begin{tabular}{lccc}
\hline & \multicolumn{3}{c}{ Thyroid Dose [cGy] } \\
Exposure Pathway & \multicolumn{2}{c}{$95 \%$ Subjective Confidence Interval } \\
\hline Backyard Cow Milk & lower limit & central estimate & upper limit \\
Commercial Milk (locally produced) & 0.045 & 0.65 & 8.9 \\
Commercial Milk (regionally mixed) & 0.016 & 0.22 & 2.9 \\
Goat Milk (locally produced) & 0.018 & 0.22 & 2.4 \\
Beef (locally produced) & 0.13 & 2.4 & 37 \\
Leafy Vegetables (locally produced) & 0.000023 & 0.0005 & 0.013 \\
Eggs (locally produced) & 0.000032 & 0.00043 & 0.0052 \\
Cottage Cheese (locally produced) & 0.0014 & 0.026 & 0.38 \\
Inhalation & 0.000085 & 0.0019 & 0.046 \\
Mother's milk (mother on Diet 1) & 0.00094 & 0.0055 & 0.031 \\
Prenatal exposure (mother on Diet 1) & 0.0013 & 0.035 & 1.4 \\
Diet 1 & 0.011 & 0.12 & 1.5 \\
Diet 2 & 0.051 & 0.7 & 9.3 \\
Diet 3 & 0.026 & 0.28 & 3.2 \\
\hline
\end{tabular}

Excess Lifetime Risk [ ]

\begin{tabular}{llll}
\hline Diet 1 & $8.4 \mathrm{E}-06$ & $2.0 \mathrm{E}-04$ & $4.3 \mathrm{E}-03$ \\
Diet 2 & $4.0 \mathrm{E}-06$ & $8.9 \mathrm{E}-05$ & $1.8 \mathrm{E}-03$ \\
Diet 3 & $4.2 \mathrm{E}-06$ & $7.1 \mathrm{E}-05$ & $1.2 \mathrm{E}-03$ \\
Diet 4 & $2.0 \mathrm{E}-05$ & $7.7 \mathrm{E}-04$ & $2.2 \mathrm{E}-02$ \\
\hline
\end{tabular}

\section{Relative Risk [ ]}

\begin{tabular}{lccc}
\hline Diet 1 & 1.0034 & 1.047 & 2.3 \\
Diet 2 & 1.0014 & 1.021 & 1.42 \\
Diet 3 & 1.00098 & 1.017 & 1.37 \\
Diet 4 & 1.0063 & 1.18 & 5 \\
\hline
\end{tabular}

\section{Probability of Causation [\%]}

\begin{tabular}{lccc}
\hline Diet 1 & 0.34 & 4.5 & 57 \\
Diet 2 & 0.14 & 2.0 & 29 \\
Diet 3 & 0.10 & 1.7 & 27 \\
Diet 4 & 0.63 & 14.9 & 80 \\
\hline
\end{tabular}

Diet 1 - Backyard cow milk + all other locally produced non-milk exposure pathways

Diet 2 - Locally produced commercial milk + all other locally produced non-milk exposure pathways

Diet 3 - Regionally mixed commercial milk + inhalation (other regionally mixed food items are minor contributors to the total)

Diet 4 - Goat milk (all other exposure pathways are of negligible importance compared to goat milk) 


\section{Location: Dyllis}

\section{Receptor: Male born in 1956}

\begin{tabular}{lccc}
\hline & \multicolumn{3}{c}{ Thyroid Dose [cGy] } \\
& \multicolumn{2}{c}{$95 \%$ Subjective Confidence Interval } \\
Exposure Pathway & lower limit & central estimate & upper limit \\
\hline Backyard Cow Milk & 0.038 & 0.61 & 7.9 \\
Commercial Milk (locally produced) & 0.016 & 0.23 & 2.4 \\
Commercial Milk (regionally mixed) & 0.017 & 0.23 & 2.2 \\
Goat Milk (locally produced) & 0.15 & 2.5 & 39 \\
Beef (locally produced) & 0.000023 & 0.00049 & 0.013 \\
Leafy Vegetables (locally produced) & 0.000023 & 0.00039 & 0.0053 \\
Eggs (locally produced) & 0.0016 & 0.024 & 0.34 \\
Cottage Cheese (locally produced) & 0.000088 & 0.0021 & 0.04 \\
Inhalation & 0.0011 & 0.0054 & 0.031 \\
Mother's milk (mother on Diet 1) & 0.0013 & 0.035 & 1.4 \\
Prenatal exposure (mother on Diet 1) & 0.011 & 0.12 & 1.5 \\
Diet 1 & 0.044 & 0.66 & 8.2 \\
Diet 2 & 0.026 & 0.28 & 2.6 \\
Diet 3 & 0.02 & 0.24 & 2.2 \\
\hline
\end{tabular}

Excess Lifetime Risk [ ]

\begin{tabular}{llll}
\hline Diet 1 & $1.6 \mathrm{E}-06$ & $4.7 \mathrm{E}-05$ & $1.8 \mathrm{E}-03$ \\
Diet 2 & $6.2 \mathrm{E}-07$ & $2.1 \mathrm{E}-05$ & $5.7 \mathrm{E}-04$ \\
Diet 3 & $5.9 \mathrm{E}-07$ & $1.7 \mathrm{E}-05$ & $5.4 \mathrm{E}-04$ \\
Diet 4 & $5.0 \mathrm{E}-06$ & $1.8 \mathrm{E}-04$ & $8.8 \mathrm{E}-03$ \\
\hline
\end{tabular}

\section{Relative Risk [ ]}

\begin{tabular}{lccc}
\hline Diet 1 & 1.0015 & 1.038 & 2.2 \\
Diet 2 & 1.00071 & 1.015 & 1.39 \\
Diet 3 & 1.00054 & 1.012 & 1.36 \\
Diet 4 & 1.0037 & 1.14 & 7.1 \\
\hline
\end{tabular}

\section{Probability of Causation [\%]}

\begin{tabular}{lccc}
\hline Diet 1 & 0.15 & 3.7 & 54 \\
Diet 2 & 0.07 & 1.5 & 28 \\
Diet 3 & 0.05 & 1.2 & 26 \\
Diet 4 & 0.37 & 12.2 & 86 \\
\hline
\end{tabular}

Diet 1 - Backyard cow milk + all other locally produced non-milk exposure pathways

Diet 2 - Locally produced commercial milk + all other locally produced non-milk exposure pathways

Diet 3 - Regionally mixed commercial milk + inhalation (other regionally mixed food items are minor contributors to the total)

Diet 4 - Goat milk (all other exposure pathways are of negligible importance compared to goat milk) 


\section{Location: OR High School Area}

Receptor: Female born in 1956

\begin{tabular}{lccc}
\hline & \multicolumn{3}{c}{ Thyroid Dose [cGy] } \\
Exposure Pathway & \multicolumn{2}{c}{$9 \%$ Subjective Confidence Interval } \\
\hline Backyard Cow Milk & lower limit & central estimate & upper limit \\
Commercial Milk (locally produced) & -- & -- & -- \\
Commercial Milk (regionally mixed) & -- & -- & -- \\
Goat Milk (locally produced) & 0.018 & 0.22 & 2.4 \\
Beef (locally produced) & -- & -- & -- \\
Leafy Vegetables (locally produced) & -- & -- & -- \\
Eggs (locally produced) & -- & -- & -- \\
Cottage Cheese (locally produced) & -- & -- & -- \\
Inhalation & 0.00019 & 0.0043 & 0.093 \\
Mother's milk (mother on Diet 3) & 0.0019 & 0.012 & 0.063 \\
Prenatal exposure (mother on Diet 3) & 0.00071 & 0.012 & 0.19 \\
Diet 1 & 0.0054 & 0.035 & 0.28 \\
Diet 2 & -- & -- & -- \\
Diet 3 & -- & -- & -- \\
\hline
\end{tabular}

Excess Lifetime Risk [ ]

\begin{tabular}{lccc}
\hline Diet 1 & -- & -- & -- \\
Diet 2 & -- & -- & -- \\
Diet 3 & $4.4 \mathrm{E}-06$ & $7.4 \mathrm{E}-05$ & $1.2 \mathrm{E}-03$ \\
Diet 4 & -- & -- & -- \\
\hline
\end{tabular}

\section{Relative Risk [ ]}

\begin{tabular}{lccc}
\hline Diet 1 & -- & -- & -- \\
Diet 2 & -- & -- & -- \\
Diet 3 & 1.0011 & 1.018 & 1.38 \\
Diet 4 & -- & -- & -- \\
\hline
\end{tabular}

\begin{tabular}{lccc}
\hline Diet 1 & -- & -- & -- \\
Diet 2 & -- & -- & -- \\
Diet 3 & 0.11 & 1.8 & -- \\
Diet 4 & -- & -- & \\
\hline Diet 1 - Backyard cow milk + all other locally produced non-milk exposure pathways & \\
Diet 2 - Locally produced commercial milk + all other locally produced non-milk exposure pathways \\
Diet 3 - Regionally mixed commercial milk + inhalation (other regionally mixed food items are minor contributors to the total) \\
Diet 4 - Goat milk (all other exposure pathways are of negligible importance compared to goat milk)
\end{tabular}




\section{Location: OR High School Area}

Receptor: Male born in 1956

\begin{tabular}{lccc}
\hline & \multicolumn{3}{c}{ Thyroid Dose [cGy] } \\
Exposure Pathway & \multicolumn{2}{c}{$95 \%$ Subjective Confidence Interval } \\
\hline Backyard Cow Milk & lower limit & central estimate & upper limit \\
Commercial Milk (locally produced) & -- & -- & -- \\
Commercial Milk (regionally mixed) & -- & -- & -- \\
Goat Milk (locally produced) & 0.017 & 0.23 & 2.2 \\
Beef (locally produced) & -- & -- & -- \\
Leafy Vegetables (locally produced) & -- & -- & -- \\
Eggs (locally produced) & -- & -- & -- \\
Cottage Cheese (locally produced) & -- & -- & -- \\
Inhalation & 0.00022 & 0.0043 & 0.075 \\
Mother's milk (mother on Diet 3) & 0.0023 & 0.012 & 0.063 \\
Prenatal exposure (mother on Diet 3) & 0.00071 & 0.012 & 0.19 \\
Diet 1 & 0.0054 & 0.035 & 0.28 \\
Diet 2 & -- & -- & -- \\
Diet 3 & -- & -- & -- \\
\hline
\end{tabular}

Excess Lifetime Risk [ ]

\begin{tabular}{lccc}
\hline Diet 1 & -- & -- & -- \\
Diet 2 & -- & -- & -- \\
Diet 3 & $6.2 \mathrm{E}-07$ & $1.8 \mathrm{E}-05$ & $5.5 \mathrm{E}-04$ \\
Diet 4 & -- & -- & -- \\
\hline
\end{tabular}

\section{Relative Risk [ ]}

\begin{tabular}{lccc}
\hline Diet 1 & -- & -- & -- \\
Diet 2 & -- & -- & -- \\
Diet 3 & 1.0006 & 1.012 & 1.36 \\
Diet 4 & -- & -- & -- \\
\hline
\end{tabular}

\begin{tabular}{lccc}
\hline Diet 1 & -- & -- & -- \\
Diet 2 & -- & -- & -- \\
Diet 3 & 0.06 & 1.2 & -- \\
Diet 4 & -- & -- & \\
\hline Diet 1 - Backyard cow milk + all other locally produced non-milk exposure pathways & \\
Diet 2 - Locally produced commercial milk + all other locally produced non-milk exposure pathways \\
Diet 3 - Regionally mixed commercial milk + inhalation (other regionally mixed food items are minor contributors to the total) \\
Diet 4 - Goat milk (all other exposure pathways are of negligible importance compared to goat milk)
\end{tabular}




\section{Location: Norwood}

Receptor: Female born in 1956

\begin{tabular}{lccc}
\hline & \multicolumn{3}{c}{ Thyroid Dose [cGy] } \\
Exposure Pathway & \multicolumn{2}{c}{$95 \%$ Subjective Confidence Interval } \\
\hline Backyard Cow Milk & lower limit & central estimate & upper limit \\
Commercial Milk (locally produced) & 0.037 & 0.58 & 7.9 \\
Commercial Milk (regionally mixed) & 0.015 & 0.21 & 3.4 \\
Goat Milk (locally produced) & 0.018 & 0.22 & 2.4 \\
Beef (locally produced) & 0.13 & 2.3 & 38 \\
Leafy Vegetables (locally produced) & 0.000021 & 0.00046 & 0.012 \\
Eggs (locally produced) & 0.000026 & 0.00039 & 0.0042 \\
Cottage Cheese (locally produced) & 0.0015 & 0.024 & 0.36 \\
Inhalation & 0.00008 & 0.0019 & 0.049 \\
Mother's milk (mother on Diet 1) & 0.00085 & 0.0052 & 0.03 \\
Prenatal exposure (mother on Diet 1) & 0.0011 & 0.034 & 1.3 \\
Diet 1 & 0.01 & 0.12 & 1.5 \\
Diet 2 & 0.043 & 0.62 & 8.6 \\
Diet 3 & 0.025 & 0.26 & 3.7 \\
\hline
\end{tabular}

Excess Lifetime Risk [ ]

\begin{tabular}{llll}
\hline Diet 1 & $7.8 \mathrm{E}-06$ & $1.9 \mathrm{E}-04$ & $4.7 \mathrm{E}-03$ \\
Diet 2 & $3.8 \mathrm{E}-06$ & $8.3 \mathrm{E}-05$ & $1.8 \mathrm{E}-03$ \\
Diet 3 & $4.2 \mathrm{E}-06$ & $7.2 \mathrm{E}-05$ & $1.2 \mathrm{E}-03$ \\
Diet 4 & $2.1 \mathrm{E}-05$ & $7.6 \mathrm{E}-04$ & $2.2 \mathrm{E}-02$ \\
\hline
\end{tabular}

\section{Relative Risk [ ]}

\begin{tabular}{lccc}
\hline Diet 1 & 1.003 & 1.048 & 2.1 \\
Diet 2 & 1.0012 & 1.019 & 1.49 \\
Diet 3 & 1.00096 & 1.017 & 1.37 \\
Diet 4 & 1.0069 & 1.16 & 5.1 \\
\hline
\end{tabular}

\section{Probability of Causation [\%]}

\begin{tabular}{lccc}
\hline Diet 1 & 0.30 & 4.6 & 51 \\
Diet 2 & 0.12 & 1.8 & 33 \\
Diet 3 & 0.10 & 1.7 & 27 \\
Diet 4 & 0.69 & 13.7 & 80 \\
\hline
\end{tabular}

Diet 1 - Backyard cow milk + all other locally produced non-milk exposure pathways

Diet 2 - Locally produced commercial milk + all other locally produced non-milk exposure pathways

Diet 3 - Regionally mixed commercial milk + inhalation (other regionally mixed food items are minor contributors to the total)

Diet 4 - Goat milk (all other exposure pathways are of negligible importance compared to goat milk) 


\section{Location: Norwood}

\section{Receptor: Male born in 1956}

\begin{tabular}{lccc}
\hline & \multicolumn{3}{c}{ Thyroid Dose [cGy] } \\
& \multicolumn{2}{c}{$95 \%$ Subjective Confidence Interval } \\
Exposure Pathway & lower limit & central estimate & upper limit \\
\hline Backyard Cow Milk & 0.035 & 0.6 & 8.2 \\
Commercial Milk (locally produced) & 0.014 & 0.22 & 2.4 \\
Commercial Milk (regionally mixed) & 0.017 & 0.23 & 2.2 \\
Goat Milk (locally produced) & 0.13 & 2.2 & 45 \\
Beef (locally produced) & 0.000022 & 0.00044 & 0.013 \\
Leafy Vegetables (locally produced) & 0.000022 & 0.00039 & 0.0051 \\
Eggs (locally produced) & 0.0016 & 0.026 & 0.34 \\
Cottage Cheese (locally produced) & 0.000078 & 0.0019 & 0.036 \\
Inhalation & 0.00095 & 0.0051 & 0.027 \\
Mother's milk (mother on Diet 1) & 0.0011 & 0.034 & 1.3 \\
Prenatal exposure (mother on Diet 1) & 0.01 & 0.12 & 1.5 \\
Diet 1 & 0.04 & 0.65 & 8.7 \\
Diet 2 & 0.024 & 0.27 & 2.7 \\
Diet 3 & 0.02 & 0.24 & 2.2 \\
\hline
\end{tabular}

Excess Lifetime Risk [ ]

\begin{tabular}{llll}
\hline Diet 1 & $1.4 \mathrm{E}-06$ & $4.4 \mathrm{E}-05$ & $1.8 \mathrm{E}-03$ \\
Diet 2 & $6.1 \mathrm{E}-07$ & $1.9 \mathrm{E}-05$ & $6.7 \mathrm{E}-04$ \\
Diet 3 & $5.8 \mathrm{E}-07$ & $1.7 \mathrm{E}-05$ & $5.4 \mathrm{E}-04$ \\
Diet 4 & $4.3 \mathrm{E}-06$ & $1.7 \mathrm{E}-04$ & $7.1 \mathrm{E}-03$ \\
\hline
\end{tabular}

\section{Relative Risk [ ]}

\begin{tabular}{lccc}
\hline Diet 1 & 1.0013 & 1.036 & 2.2 \\
Diet 2 & 1.00067 & 1.013 & 1.39 \\
Diet 3 & 1.00054 & 1.012 & 1.36 \\
Diet 4 & 1.0037 & 1.13 & 6.3 \\
\hline
\end{tabular}

\section{Probability of Causation [\%]}

\begin{tabular}{lccc}
\hline Diet 1 & 0.13 & 3.5 & 54 \\
Diet 2 & 0.07 & 1.3 & 28 \\
Diet 3 & 0.05 & 1.2 & 26 \\
Diet 4 & 0.37 & 11.3 & 84 \\
\hline
\end{tabular}

Diet 1 - Backyard cow milk + all other locally produced non-milk exposure pathways

Diet 2 - Locally produced commercial milk + all other locally produced non-milk exposure pathways

Diet 3 - Regionally mixed commercial milk + inhalation (other regionally mixed food items are minor contributors to the total)

Diet 4 - Goat milk (all other exposure pathways are of negligible importance compared to goat milk) 
Location: Woodland

Receptor: Female born in 1956

\begin{tabular}{lccc}
\hline & \multicolumn{3}{c}{ Thyroid Dose [cGy] } \\
Exposure Pathway & \multicolumn{2}{c}{$95 \%$ Subjective Confidence Interval } \\
\hline Backyard Cow Milk & lower limit & central estimate & upper limit \\
Commercial Milk (locally produced) & -- & -- & -- \\
Commercial Milk (regionally mixed) & -- & -- & -- \\
Goat Milk (locally produced) & 0.018 & 0.22 & 2.4 \\
Beef (locally produced) & -- & -- & -- \\
Leafy Vegetables (locally produced) & -- & -- & -- \\
Eggs (locally produced) & -- & -- & -- \\
Cottage Cheese (locally produced) & -- & -- & -- \\
Inhalation & 0.00027 & 0.0064 & 0.14 \\
Mother's milk (mother on Diet 3) & 0.0029 & 0.017 & 0.093 \\
Prenatal exposure (mother on Diet 3) & 0.00078 & 0.014 & 0.2 \\
Diet 1 & 0.0065 & 0.041 & 0.3 \\
Diet 2 & -- & -- & -- \\
Diet 3 & -- & -- & -- \\
\hline
\end{tabular}

Excess Lifetime Risk [ ]

\begin{tabular}{lccc}
\hline Diet 1 & -- & -- & -- \\
Diet 2 & -- & -- & -- \\
Diet 3 & $4.8 \mathrm{E}-06$ & $7.7 \mathrm{E}-05$ & $1.2 \mathrm{E}-03$ \\
Diet 4 & -- & -- & -- \\
\hline
\end{tabular}

\section{Relative Risk [ ]}

\begin{tabular}{lccc}
\hline Diet 1 & -- & -- & -- \\
Diet 2 & -- & -- & -- \\
Diet 3 & 1.0012 & 1.018 & 1.38 \\
Diet 4 & -- & -- & -- \\
\hline
\end{tabular}

\begin{tabular}{lccc}
\hline Diet 1 & -- & -- & -- \\
Diet 2 & -- & -- & - \\
Diet 3 & 0.12 & 1.8 & - \\
Diet 4 & -- & -- & - \\
\hline Diet 1 - Backyard cow milk + all other locally produced non-milk exposure pathways & \\
Diet 2 - Locally produced commercial milk + all other locally produced non-milk exposure pathways \\
Diet 3 - Regionally mixed commercial milk + inhalation (other regionally mixed food items are minor contributors to the total) \\
Diet 4 - Goat milk (all other exposure pathways are of negligible importance compared to goat milk)
\end{tabular}


Location: Woodland

Receptor: Male born in 1956

\begin{tabular}{lccc}
\hline & \multicolumn{3}{c}{ Thyroid Dose [cGy] } \\
Exposure Pathway & \multicolumn{2}{c}{ 95\% Subjective Confidence Interval } \\
\hline Backyard Cow Milk & lower limit & central estimate & upper limit \\
Commercial Milk (locally produced) & -- & -- & -- \\
Commercial Milk (regionally mixed) & -- & -- & -- \\
Goat Milk (locally produced) & 0.017 & 0.23 & 2.2 \\
Beef (locally produced) & -- & -- & -- \\
Leafy Vegetables (locally produced) & -- & -- & -- \\
Eggs (locally produced) & -- & -- & -- \\
Cottage Cheese (locally produced) & -- & -- & -- \\
Inhalation & 0.00032 & 0.0065 & 0.11 \\
Mother's milk (mother on Diet 3) & 0.0034 & 0.017 & 0.094 \\
Prenatal exposure (mother on Diet 3) & 0.00078 & 0.014 & 0.2 \\
Diet 1 & 0.0065 & 0.041 & 0.3 \\
Diet 2 & -- & -- & -- \\
Diet 3 & -- & -- & -- \\
\hline
\end{tabular}

Excess Lifetime Risk [ ]

\begin{tabular}{lccc}
\hline Diet 1 & -- & -- & -- \\
Diet 2 & -- & -- & -- \\
Diet 3 & $6.4 \mathrm{E}-07$ & $1.8 \mathrm{E}-05$ & $5.6 \mathrm{E}-04$ \\
Diet 4 & -- & -- & -- \\
\hline
\end{tabular}

\section{Relative Risk [ ]}

\begin{tabular}{lccc}
\hline Diet 1 & -- & -- & -- \\
Diet 2 & -- & -- & -- \\
Diet 3 & 1.00065 & 1.013 & 1.37 \\
Diet 4 & -- & -- & -- \\
\hline
\end{tabular}

\begin{tabular}{lccc}
\hline Diet 1 & -- & -- & -- \\
Diet 2 & -- & -- & -- \\
Diet 3 & 0.07 & 1.3 & -- \\
Diet 4 & -- & -- & \\
\hline Diet 1 - Backyard cow milk + all other locally produced non-milk exposure pathways & \\
Diet 2 - Locally produced commercial milk + all other locally produced non-milk exposure pathways \\
Diet 3 - Regionally mixed commercial milk + inhalation (other regionally mixed food items are minor contributors to the total) \\
Diet 4 - Goat milk (all other exposure pathways are of negligible importance compared to goat milk)
\end{tabular}


Location: Hardin Valley

Receptor: Female born in 1956

\begin{tabular}{lccc}
\hline & \multicolumn{3}{c}{ Thyroid Dose [cGy] } \\
Exposure Pathway & $95 \%$ Subjective Confidence Interval \\
\hline Backyard Cow Milk & lower limit & central estimate & upper limit \\
Commercial Milk (locally produced) & 0.2 & 2.9 & 33 \\
Commercial Milk (regionally mixed) & 0.079 & 1 & 11 \\
Goat Milk (locally produced) & 0.018 & 0.22 & 2.4 \\
Beef (locally produced) & 0.6 & 11 & 160 \\
Leafy Vegetables (locally produced) & 0.00012 & 0.0024 & 0.058 \\
Eggs (locally produced) & 0.00015 & 0.0019 & 0.021 \\
Cottage Cheese (locally produced) & 0.0071 & 0.12 & 1.5 \\
Inhalation & 0.00035 & 0.0093 & 0.2 \\
Mother's milk (mother on Diet 1) & 0.0044 & 0.026 & 0.14 \\
Prenatal exposure (mother on Diet 1) & 0.006 & 0.16 & 5.8 \\
Diet 1 & 0.051 & 0.55 & 6.9 \\
Diet 2 & 0.23 & 3.2 & 35 \\
Diet 3 & 0.13 & 1.3 & 13 \\
\hline
\end{tabular}

Excess Lifetime Risk [ ]

\begin{tabular}{llll}
\hline Diet 1 & $3.6 \mathrm{E}-05$ & $8.9 \mathrm{E}-04$ & $1.9 \mathrm{E}-02$ \\
Diet 2 & $2.0 \mathrm{E}-05$ & $4.0 \mathrm{E}-04$ & $7.8 \mathrm{E}-03$ \\
Diet 3 & $5.1 \mathrm{E}-06$ & $8.2 \mathrm{E}-05$ & $1.3 \mathrm{E}-03$ \\
Diet 4 & $1.1 \mathrm{E}-04$ & $3.6 \mathrm{E}-03$ & $9.9 \mathrm{E}-02$ \\
\hline
\end{tabular}

\section{Relative Risk [ ]}

\begin{tabular}{lccc}
\hline Diet 1 & 1.015 & 1.23 & 6.1 \\
Diet 2 & 1.0067 & 1.094 & 2.8 \\
Diet 3 & 1.0013 & 1.019 & 1.38 \\
Diet 4 & 1.034 & 1.85 & 19 \\
\hline
\end{tabular}

\section{Probability of Causation [\%]}

\begin{tabular}{lccc}
\hline Diet 1 & 1.49 & 18.7 & 83 \\
Diet 2 & 0.66 & 8.5 & 64 \\
Diet 3 & 0.13 & 1.9 & 28 \\
Diet 4 & 3.32 & 46.0 & 95 \\
\hline
\end{tabular}

Diet 1 - Backyard cow milk + all other locally produced non-milk exposure pathways

Diet 2 - Locally produced commercial milk + all other locally produced non-milk exposure pathways

Diet 3 - Regionally mixed commercial milk + inhalation (other regionally mixed food items are minor contributors to the total)

Diet 4 - Goat milk (all other exposure pathways are of negligible importance compared to goat milk) 
Location: Hardin Valley

Receptor: Male born in 1956

\begin{tabular}{lccc}
\hline & \multicolumn{3}{c}{ Thyroid Dose [cGy] } \\
Exposure Pathway & \multicolumn{2}{c}{$95 \%$ Subjective Confidence Interval } \\
\hline Backyard Cow Milk & lower limit & central estimate & upper limit \\
Commercial Milk (locally produced) & 0.18 & 2.9 & 34 \\
Commercial Milk (regionally mixed) & 0.071 & 1.1 & 9.9 \\
Goat Milk (locally produced) & 0.017 & 0.23 & 2.2 \\
Beef (locally produced) & 0.79 & 11 & 190 \\
Leafy Vegetables (locally produced) & 0.00011 & 0.0023 & 0.058 \\
Eggs (locally produced) & 0.00011 & 0.0018 & 0.022 \\
Cottage Cheese (locally produced) & 0.0081 & 0.12 & 1.5 \\
Inhalation & 0.00044 & 0.0095 & 0.17 \\
Mother's milk (mother on Diet 1) & 0.0049 & 0.026 & 0.14 \\
Prenatal exposure (mother on Diet 1) & 0.006 & 0.16 & 5.8 \\
Diet 1 & 0.051 & 0.55 & 6.9 \\
Diet 2 & 0.2 & 3.1 & 36 \\
Diet 3 & 0.13 & 1.3 & 11 \\
\hline
\end{tabular}

Excess Lifetime Risk [ ]

\begin{tabular}{llll}
\hline Diet 1 & $7.5 \mathrm{E}-06$ & $2.2 \mathrm{E}-04$ & $7.6 \mathrm{E}-03$ \\
Diet 2 & $3.1 \mathrm{E}-06$ & $9.6 \mathrm{E}-05$ & $2.5 \mathrm{E}-03$ \\
Diet 3 & $6.6 \mathrm{E}-07$ & $1.9 \mathrm{E}-05$ & $5.7 \mathrm{E}-04$ \\
Diet 4 & $2.3 \mathrm{E}-05$ & $8.6 \mathrm{E}-04$ & $3.6 \mathrm{E}-02$ \\
\hline
\end{tabular}

\section{Relative Risk [ ]}

\begin{tabular}{lccc}
\hline Diet 1 & 1.0074 & 1.18 & 6.5 \\
Diet 2 & 1.0034 & 1.069 & 2.9 \\
Diet 3 & 1.00072 & 1.013 & 1.37 \\
Diet 4 & 1.018 & 1.63 & 28 \\
\hline
\end{tabular}

Probability of Causation [\%]

\begin{tabular}{lccc}
\hline Diet 1 & 0.73 & 15.0 & 84 \\
Diet 2 & 0.34 & 6.4 & 66 \\
Diet 3 & 0.07 & 1.3 & 27 \\
Diet 4 & 1.79 & 38.5 & 96 \\
\hline
\end{tabular}

Diet 1 - Backyard cow milk + all other locally produced non-milk exposure pathways

Diet 2 - Locally produced commercial milk + all other locally produced non-milk exposure pathways

Diet 3 - Regionally mixed commercial milk + inhalation (other regionally mixed food items are minor contributors to the total)

Diet 4 - Goat milk (all other exposure pathways are of negligible importance compared to goat milk) 


\section{Location: Oliver Springs}

Receptor: Female born in 1956

\begin{tabular}{lccc}
\hline & \multicolumn{3}{c}{ Thyroid Dose [cGy] } \\
& \multicolumn{2}{c}{$95 \%$ Subjective Confidence Interval } \\
Exposure Pathway & lower limit & central estimate & upper limit \\
\hline Backyard Cow Milk & 0.03 & 0.44 & 5.5 \\
Commercial Milk (locally produced) & 0.012 & 0.16 & 2.1 \\
Commercial Milk (regionally mixed) & 0.018 & 0.22 & 2.4 \\
Goat Milk (locally produced) & 0.1 & 1.8 & 29 \\
Beef (locally produced) & 0.000015 & 0.00036 & 0.0096 \\
Leafy Vegetables (locally produced) & 0.000021 & 0.00029 & 0.0033 \\
Eggs (locally produced) & 0.0011 & 0.018 & 0.27 \\
Cottage Cheese (locally produced) & 0.000068 & 0.0015 & 0.031 \\
Inhalation & 0.00065 & 0.004 & 0.024 \\
Mother's milk (mother on Diet 1) & 0.00085 & 0.025 & 0.96 \\
Prenatal exposure (mother on Diet 1) & 0.0085 & 0.088 & 1.3 \\
Diet 1 & 0.035 & 0.47 & 5.9 \\
Diet 2 & 0.02 & 0.2 & 2.4 \\
Diet 3 & 0.021 & 0.23 & 2.4 \\
\hline
\end{tabular}

Excess Lifetime Risk [ ]

\begin{tabular}{llll}
\hline Diet 1 & $5.6 \mathrm{E}-06$ & $1.4 \mathrm{E}-04$ & $3.9 \mathrm{E}-03$ \\
Diet 2 & $3.3 \mathrm{E}-06$ & $6.3 \mathrm{E}-05$ & $1.2 \mathrm{E}-03$ \\
Diet 3 & $4.2 \mathrm{E}-06$ & $7.1 \mathrm{E}-05$ & $1.2 \mathrm{E}-03$ \\
Diet 4 & $1.6 \mathrm{E}-05$ & $5.8 \mathrm{E}-04$ & $1.6 \mathrm{E}-02$ \\
\hline
\end{tabular}

\section{Relative Risk [ ]}

\begin{tabular}{lccc}
\hline Diet 1 & 1.0023 & 1.035 & 1.86 \\
Diet 2 & 1.00094 & 1.015 & 1.3 \\
Diet 3 & 1.00095 & 1.017 & 1.37 \\
Diet 4 & 1.0053 & 1.12 & 4 \\
\hline
\end{tabular}

\begin{tabular}{lccc}
\hline Diet 1 & 0.23 & 3.3 & 46 \\
Diet 2 & 0.09 & 1.5 & 23 \\
Diet 3 & 0.10 & 1.7 & 27 \\
Diet 4 & 0.52 & 10.9 & 75 \\
\hline
\end{tabular}

Diet 1 - Backyard cow milk + all other locally produced non-milk exposure pathways

Diet 2 - Locally produced commercial milk + all other locally produced non-milk exposure pathways

Diet 3 - Regionally mixed commercial milk + inhalation (other regionally mixed food items are minor contributors to the total)

Diet 4 - Goat milk (all other exposure pathways are of negligible importance compared to goat milk) 


\section{Location: Oliver Springs}

\section{Receptor: Male born in 1956}

\begin{tabular}{lccc}
\hline & \multicolumn{3}{c}{ Thyroid Dose [cGy] } \\
& \multicolumn{2}{c}{$95 \%$ Subjective Confidence Interval } \\
Exposure Pathway & lower limit & central estimate & upper limit \\
\hline Backyard Cow Milk & 0.027 & 0.45 & 6.3 \\
Commercial Milk (locally produced) & 0.01 & 0.16 & 2 \\
Commercial Milk (regionally mixed) & 0.017 & 0.23 & 2.2 \\
Goat Milk (locally produced) & 0.11 & 1.7 & 34 \\
Beef (locally produced) & 0.000016 & 0.00036 & 0.01 \\
Leafy Vegetables (locally produced) & 0.000018 & 0.00029 & 0.004 \\
Eggs (locally produced) & 0.0013 & 0.018 & 0.27 \\
Cottage Cheese (locally produced) & 0.000068 & 0.0014 & 0.026 \\
Inhalation & 0.00075 & 0.004 & 0.022 \\
Mother's milk (mother on Diet 1) & 0.00085 & 0.025 & 0.96 \\
Prenatal exposure (mother on Diet 1) & 0.0085 & 0.088 & 1.3 \\
Diet 1 & 0.032 & 0.48 & 6.7 \\
Diet 2 & 0.019 & 0.2 & 2.2 \\
Diet 3 & 0.019 & 0.24 & 2.2 \\
\hline
\end{tabular}

Excess Lifetime Risk [ ]

\begin{tabular}{llll}
\hline Diet 1 & $1.0 \mathrm{E}-06$ & $3.5 \mathrm{E}-05$ & $1.4 \mathrm{E}-03$ \\
Diet 2 & $4.7 \mathrm{E}-07$ & $1.5 \mathrm{E}-05$ & $4.3 \mathrm{E}-04$ \\
Diet 3 & $5.8 \mathrm{E}-07$ & $1.7 \mathrm{E}-05$ & $5.4 \mathrm{E}-04$ \\
Diet 4 & $3.3 \mathrm{E}-06$ & $1.3 \mathrm{E}-04$ & $5.1 \mathrm{E}-03$ \\
\hline
\end{tabular}

\section{Relative Risk [ ]}

\begin{tabular}{lccc}
\hline Diet 1 & 1.0011 & 1.028 & 1.98 \\
Diet 2 & 1.0005 & 1.011 & 1.3 \\
Diet 3 & 1.00053 & 1.012 & 1.36 \\
Diet 4 & 1.0025 & 1.098 & 4.9 \\
\hline
\end{tabular}

\section{Probability of Causation [\%]}

\begin{tabular}{llll}
\hline Diet 1 & 0.11 & 2.7 & 49 \\
Diet 2 & 0.05 & 1.0 & 23 \\
Diet 3 & 0.05 & 1.2 & 26 \\
Diet 4 & 0.25 & 8.9 & 79 \\
\hline
\end{tabular}

Diet 1 - Backyard cow milk + all other locally produced non-milk exposure pathways

Diet 2 - Locally produced commercial milk + all other locally produced non-milk exposure pathways

Diet 3 - Regionally mixed commercial milk + inhalation (other regionally mixed food items are minor contributors to the total)

Diet 4 - Goat milk (all other exposure pathways are of negligible importance compared to goat milk) 


\section{Location: Solway}

Receptor: Female born in 1956

\begin{tabular}{lccc}
\hline & \multicolumn{3}{c}{ Thyroid Dose [cGy] } \\
Exposure Pathway & \multicolumn{2}{c}{$95 \%$ Subjective Confidence Interval } \\
\hline Backyard Cow Milk & lower limit & central estimate & upper limit \\
Commercial Milk (locally produced) & 0.18 & 2.5 & 31 \\
Commercial Milk (regionally mixed) & 0.073 & 0.94 & 9.5 \\
Goat Milk (locally produced) & 0.018 & 0.22 & 2.4 \\
Beef (locally produced) & 0.54 & 10 & 150 \\
Leafy Vegetables (locally produced) & 0.000098 & 0.0022 & 0.052 \\
Eggs (locally produced) & 0.00014 & 0.0018 & 0.019 \\
Cottage Cheese (locally produced) & 0.0063 & 0.11 & 1.3 \\
Inhalation & 0.00034 & 0.0084 & 0.18 \\
Mother's milk (mother on Diet 1) & 0.0041 & 0.024 & 0.13 \\
Prenatal exposure (mother on Diet 1) & 0.0052 & 0.15 & 4.6 \\
Diet 1 & 0.047 & 0.51 & 5.9 \\
Diet 2 & 0.21 & 2.7 & 33 \\
Diet 3 & 0.13 & 1.2 & 11 \\
\hline
\end{tabular}

Excess Lifetime Risk [ ]

\begin{tabular}{llll}
\hline Diet 1 & $3.5 \mathrm{E}-05$ & $8.1 \mathrm{E}-04$ & $1.8 \mathrm{E}-02$ \\
Diet 2 & $1.9 \mathrm{E}-05$ & $3.7 \mathrm{E}-04$ & $6.4 \mathrm{E}-03$ \\
Diet 3 & $5.0 \mathrm{E}-06$ & $8.1 \mathrm{E}-05$ & $1.3 \mathrm{E}-03$ \\
Diet 4 & $1.1 \mathrm{E}-04$ & $3.4 \mathrm{E}-03$ & $8.7 \mathrm{E}-02$ \\
\hline
\end{tabular}

\section{Relative Risk [ ]}

\begin{tabular}{lccc}
\hline Diet 1 & 1.014 & 1.21 & 5.8 \\
Diet 2 & 1.0062 & 1.085 & 2.8 \\
Diet 3 & 1.0013 & 1.019 & 1.38 \\
Diet 4 & 1.033 & 1.73 & 19 \\
\hline
\end{tabular}

Probability of Causation [\%]

\begin{tabular}{lccc}
\hline Diet 1 & 1.37 & 17.1 & 83 \\
Diet 2 & 0.61 & 7.8 & 64 \\
Diet 3 & 0.13 & 1.9 & 28 \\
Diet 4 & 3.20 & 42.2 & 95 \\
\hline
\end{tabular}

Diet 1 - Backyard cow milk + all other locally produced non-milk exposure pathways

Diet 2 - Locally produced commercial milk + all other locally produced non-milk exposure pathways

Diet 3 - Regionally mixed commercial milk + inhalation (other regionally mixed food items are minor contributors to the total)

Diet 4 - Goat milk (all other exposure pathways are of negligible importance compared to goat milk) 


\section{Location: Solway}

\section{Receptor: Male born in 1956}

\begin{tabular}{lccc}
\hline & \multicolumn{3}{c}{ Thyroid Dose [cGy] } \\
& \multicolumn{2}{c}{$95 \%$ Subjective Confidence Interval } \\
Exposure Pathway & lower limit & central estimate & upper limit \\
\hline Backyard Cow Milk & 0.18 & 2.7 & 30 \\
Commercial Milk (locally produced) & 0.068 & 0.99 & 9 \\
Commercial Milk (regionally mixed) & 0.017 & 0.23 & 2.2 \\
Goat Milk (locally produced) & 0.73 & 9.8 & 170 \\
Beef (locally produced) & 0.000097 & 0.0021 & 0.052 \\
Leafy Vegetables (locally produced) & 0.00011 & 0.0017 & 0.019 \\
Eggs (locally produced) & 0.0078 & 0.11 & 1.5 \\
Cottage Cheese (locally produced) & 0.00041 & 0.0086 & 0.15 \\
Inhalation & 0.0047 & 0.024 & 0.12 \\
Mother's milk (mother on Diet 1) & 0.0052 & 0.15 & 4.6 \\
Prenatal exposure (mother on Diet 1) & 0.047 & 0.51 & 5.9 \\
Diet 1 & 0.2 & 2.8 & 32 \\
Diet 2 & 0.12 & 1.2 & 10 \\
Diet 3 & 0.03 & 0.26 & 2.2 \\
\hline
\end{tabular}

Excess Lifetime Risk [ ]

\begin{tabular}{llll}
\hline Diet 1 & $6.7 \mathrm{E}-06$ & $2.0 \mathrm{E}-04$ & $7.0 \mathrm{E}-03$ \\
Diet 2 & $2.8 \mathrm{E}-06$ & $8.8 \mathrm{E}-05$ & $2.4 \mathrm{E}-03$ \\
Diet 3 & $6.6 \mathrm{E}-07$ & $1.9 \mathrm{E}-05$ & $5.7 \mathrm{E}-04$ \\
Diet 4 & $1.9 \mathrm{E}-05$ & $7.7 \mathrm{E}-04$ & $3.3 \mathrm{E}-02$ \\
\hline
\end{tabular}

\section{Relative Risk [ ]}

\begin{tabular}{lccc}
\hline Diet 1 & 1.0059 & 1.16 & 5.9 \\
Diet 2 & 1.0029 & 1.061 & 2.8 \\
Diet 3 & 1.0007 & 1.013 & 1.37 \\
Diet 4 & 1.018 & 1.58 & 25 \\
\hline
\end{tabular}

\section{Probability of Causation [\%]}

\begin{tabular}{lccc}
\hline Diet 1 & 0.59 & 13.8 & 83 \\
Diet 2 & 0.29 & 5.8 & 64 \\
Diet 3 & 0.07 & 1.3 & 27 \\
Diet 4 & 1.74 & 36.6 & 96 \\
\hline
\end{tabular}

Diet 1 - Backyard cow milk + all other locally produced non-milk exposure pathways

Diet 2 - Locally produced commercial milk + all other locally produced non-milk exposure pathways

Diet 3 - Regionally mixed commercial milk + inhalation (other regionally mixed food items are minor contributors to the total)

Diet 4 - Goat milk (all other exposure pathways are of negligible importance compared to goat milk) 
Location: Sugar Grove

Receptor: Female born in 1956

\begin{tabular}{lccc}
\hline & \multicolumn{3}{c}{ Thyroid Dose [cGy] } \\
Exposure Pathway & \multicolumn{2}{c}{$95 \%$ Subjective Confidence Interval } \\
\hline Backyard Cow Milk & lower limit & central estimate & upper limit \\
Commercial Milk (locally produced) & 0.079 & 1.1 & 13 \\
Commercial Milk (regionally mixed) & 0.032 & 0.4 & 4.4 \\
Goat Milk (locally produced) & 0.018 & 0.22 & 2.4 \\
Beef (locally produced) & 0.23 & 4.3 & 65 \\
Leafy Vegetables (locally produced) & 0.000042 & 0.00091 & 0.022 \\
Eggs (locally produced) & 0.000057 & 0.00072 & 0.0082 \\
Cottage Cheese (locally produced) & 0.0027 & 0.045 & 0.59 \\
Inhalation & 0.00014 & 0.0036 & 0.08 \\
Mother's milk (mother on Diet 1) & 0.0017 & 0.01 & 0.054 \\
Prenatal exposure (mother on Diet 1) & 0.0023 & 0.063 & 2.1 \\
Diet 1 & 0.02 & 0.21 & 2.7 \\
Diet 2 & 0.09 & 1.2 & 14 \\
Diet 3 & 0.052 & 0.5 & 5.1 \\
\hline
\end{tabular}

Excess Lifetime Risk [ ]

\begin{tabular}{llll}
\hline Diet 1 & $1.4 \mathrm{E}-05$ & $3.4 \mathrm{E}-04$ & $8.0 \mathrm{E}-03$ \\
Diet 2 & $7.7 \mathrm{E}-06$ & $1.5 \mathrm{E}-04$ & $2.9 \mathrm{E}-03$ \\
Diet 3 & $4.3 \mathrm{E}-06$ & $7.3 \mathrm{E}-05$ & $1.2 \mathrm{E}-03$ \\
Diet 4 & $4.5 \mathrm{E}-05$ & $1.4 \mathrm{E}-03$ & $3.6 \mathrm{E}-02$ \\
\hline
\end{tabular}

\section{Relative Risk [ ]}

\begin{tabular}{lccc}
\hline Diet 1 & 1.0057 & 1.088 & 3.1 \\
Diet 2 & 1.0025 & 1.036 & 1.71 \\
Diet 3 & 1.001 & 1.018 & 1.38 \\
Diet 4 & 1.014 & 1.31 & 7.9 \\
\hline
\end{tabular}

\section{Probability of Causation [\%]}

\begin{tabular}{lccc}
\hline Diet 1 & 0.57 & 8.0 & 66 \\
Diet 2 & 0.25 & 3.4 & 40 \\
Diet 3 & 0.10 & 1.7 & 27 \\
Diet 4 & 1.35 & 23.2 & 86 \\
\hline
\end{tabular}

Diet 1 - Backyard cow milk + all other locally produced non-milk exposure pathways

Diet 2 - Locally produced commercial milk + all other locally produced non-milk exposure pathways

Diet 3 - Regionally mixed commercial milk + inhalation (other regionally mixed food items are minor contributors to the total)

Diet 4 - Goat milk (all other exposure pathways are of negligible importance compared to goat milk) 
Location: Sugar Grove

Receptor: Male born in 1956

\begin{tabular}{lccc}
\hline & \multicolumn{3}{c}{ Thyroid Dose [cGy] } \\
& \multicolumn{2}{c}{$95 \%$ Subjective Confidence Interval } \\
Exposure Pathway & lower limit & central estimate & upper limit \\
\hline Backyard Cow Milk & 0.073 & 1.1 & 13 \\
Commercial Milk (locally produced) & 0.028 & 0.41 & 4 \\
Commercial Milk (regionally mixed) & 0.017 & 0.23 & 2.2 \\
Goat Milk (locally produced) & 0.31 & 4.2 & 74 \\
Beef (locally produced) & 0.000044 & 0.00089 & 0.022 \\
Leafy Vegetables (locally produced) & 0.000043 & 0.0007 & 0.0087 \\
Eggs (locally produced) & 0.0031 & 0.046 & 0.61 \\
Cottage Cheese (locally produced) & 0.00018 & 0.0035 & 0.067 \\
Inhalation & 0.0019 & 0.0098 & 0.052 \\
Mother's milk (mother on Diet 1) & 0.0023 & 0.063 & 2.1 \\
Prenatal exposure (mother on Diet 1) & 0.02 & 0.21 & 2.7 \\
Diet 1 & 0.083 & 1.2 & 14 \\
Diet 2 & 0.051 & 0.5 & 4.6 \\
Diet 3 & 0.023 & 0.24 & 2.2 \\
\hline
\end{tabular}

Excess Lifetime Risk [ ]

\begin{tabular}{llll}
\hline Diet 1 & $2.9 \mathrm{E}-06$ & $8.6 \mathrm{E}-05$ & $2.9 \mathrm{E}-03$ \\
Diet 2 & $1.3 \mathrm{E}-06$ & $3.6 \mathrm{E}-05$ & $1.0 \mathrm{E}-03$ \\
Diet 3 & $6.1 \mathrm{E}-07$ & $1.8 \mathrm{E}-05$ & $5.5 \mathrm{E}-04$ \\
Diet 4 & $8.7 \mathrm{E}-06$ & $3.2 \mathrm{E}-04$ & $1.4 \mathrm{E}-02$ \\
\hline
\end{tabular}

\section{Relative Risk [ ]}

\begin{tabular}{lccc}
\hline Diet 1 & 1.0027 & 1.068 & 3.1 \\
Diet 2 & 1.0013 & 1.027 & 1.78 \\
Diet 3 & 1.00057 & 1.012 & 1.36 \\
Diet 4 & 1.007 & 1.24 & 10.6 \\
\hline
\end{tabular}

\section{Probability of Causation [\%]}

\begin{tabular}{lccc}
\hline Diet 1 & 0.27 & 6.3 & 65 \\
Diet 2 & 0.13 & 2.6 & 43 \\
Diet 3 & 0.06 & 1.2 & 26 \\
Diet 4 & 0.69 & 19.1 & 89 \\
\hline
\end{tabular}

Diet 1 - Backyard cow milk + all other locally produced non-milk exposure pathways

Diet 2 - Locally produced commercial milk + all other locally produced non-milk exposure pathways

Diet 3 - Regionally mixed commercial milk + inhalation (other regionally mixed food items are minor contributors to the total)

Diet 4 - Goat milk (all other exposure pathways are of negligible importance compared to goat milk) 


\section{Location: OR Townsite}

Receptor: Female born in 1956

\begin{tabular}{lccc}
\hline & \multicolumn{3}{c}{ Thyroid Dose [cGy] } \\
Exposure Pathway & \multicolumn{2}{c}{$9 \%$ Subjective Confidence Interval } \\
\hline Backyard Cow Milk & lower limit & central estimate & upper limit \\
Commercial Milk (locally produced) & -- & -- & -- \\
Commercial Milk (regionally mixed) & -- & -- & -- \\
Goat Milk (locally produced) & 0.018 & 0.22 & 2.4 \\
Beef (locally produced) & -- & -- & -- \\
Leafy Vegetables (locally produced) & -- & -- & -- \\
Eggs (locally produced) & -- & -- & -- \\
Cottage Cheese (locally produced) & -- & -- & -- \\
Inhalation & 0.00015 & 0.0038 & 0.084 \\
Mother's milk (mother on Diet 3) & 0.0018 & 0.011 & 0.057 \\
Prenatal exposure (mother on Diet 3) & 0.00066 & 0.012 & 0.19 \\
Diet 1 & 0.0051 & 0.035 & 0.28 \\
Diet 2 & -- & -- & -- \\
Diet 3 & -- & -- & -- \\
\hline
\end{tabular}

Excess Lifetime Risk [ ]

\begin{tabular}{lccc}
\hline Diet 1 & -- & -- & -- \\
Diet 2 & -- & -- & -- \\
Diet 3 & $4.4 \mathrm{E}-06$ & $7.4 \mathrm{E}-05$ & $1.2 \mathrm{E}-03$ \\
Diet 4 & -- & -- & -- \\
\hline
\end{tabular}

\section{Relative Risk [ ]}

\begin{tabular}{lccc}
\hline Diet 1 & -- & -- & -- \\
Diet 2 & -- & -- & -- \\
Diet 3 & 1.001 & 1.018 & 1.38 \\
Diet 4 & -- & -- & -- \\
\hline
\end{tabular}

\begin{tabular}{lccc}
\hline Diet 1 & -- & -- & -- \\
Diet 2 & -- & -- & -- \\
Diet 3 & 0.10 & 1.8 & -- \\
Diet 4 & -- & -- \\
\hline Diet 1 - Backyard cow milk + all other locally produced non-milk exposure pathways & \\
Diet 2 - Locally produced commercial milk + all other locally produced non-milk exposure pathways \\
Diet 3 - Regionally mixed commercial milk + inhalation (other regionally mixed food items are minor contributors to the total) \\
Diet 4 - Goat milk (all other exposure pathways are of negligible importance compared to goat milk)
\end{tabular}




\section{Location: OR Townsite}

\section{Receptor: Male born in 1956}

\begin{tabular}{lccc}
\hline & \multicolumn{3}{c}{ Thyroid Dose [cGy] } \\
Exposure Pathway & \multicolumn{2}{c}{$95 \%$ Subjective Confidence Interval } \\
\hline Backyard Cow Milk & lower limit & central estimate & upper limit \\
Commercial Milk (locally produced) & -- & -- & -- \\
Commercial Milk (regionally mixed) & -- & -- & -- \\
Goat Milk (locally produced) & 0.017 & 0.23 & 2.2 \\
Beef (locally produced) & -- & -- & -- \\
Leafy Vegetables (locally produced) & -- & -- & -- \\
Eggs (locally produced) & -- & -- & -- \\
Cottage Cheese (locally produced) & -- & -- & -- \\
Inhalation & 0.00018 & 0.0039 & 0.07 \\
Mother's milk (mother on Diet 3) & 0.0021 & 0.011 & 0.056 \\
Prenatal exposure (mother on Diet 3) & 0.00066 & 0.012 & 0.19 \\
Diet 1 & 0.0051 & 0.035 & 0.28 \\
Diet 2 & -- & -- & - \\
Diet 3 & -- & -- & - \\
\hline
\end{tabular}

Excess Lifetime Risk [ ]

\begin{tabular}{lccc}
\hline Diet 1 & -- & -- & -- \\
Diet 2 & -- & -- & -- \\
Diet 3 & $6.1 \mathrm{E}-07$ & $1.8 \mathrm{E}-05$ & $5.5 \mathrm{E}-04$ \\
Diet 4 & -- & -- & -- \\
\hline
\end{tabular}

\section{Relative Risk [ ]}

\begin{tabular}{lccc}
\hline Diet 1 & -- & -- & -- \\
Diet 2 & -- & -- & -- \\
Diet 3 & 1.00059 & 1.012 & 1.36 \\
Diet 4 & -- & -- & -- \\
\hline
\end{tabular}

\begin{tabular}{lccc}
\hline Diet 1 & -- & -- & -- \\
Diet 2 & -- & -- & -- \\
Diet 3 & 0.06 & 1.2 & 27 \\
Diet 4 & -- & -- & - \\
\hline Diet 1 - Backyard cow milk + all other locally produced non-milk exposure pathways & \\
Diet 2 - Locally produced commercial milk + all other locally produced non-milk exposure pathways \\
Diet 3 - Regionally mixed commercial milk + inhalation (other regionally mixed food items are minor contributors to the total) \\
Diet 4 - Goat milk (all other exposure pathways are of negligible importance compared to goat milk)
\end{tabular}


Location: Hines Valley

Receptor: Female born in 1956

\begin{tabular}{lccc}
\hline & \multicolumn{3}{c}{ Thyroid Dose [cGy] } \\
Exposure Pathway & $95 \%$ Subjective Confidence Interval \\
\hline Backyard Cow Milk & lower limit & central estimate & upper limit \\
Commercial Milk (locally produced) & 0.087 & 1.3 & 14 \\
Commercial Milk (regionally mixed) & 0.037 & 0.48 & 5.7 \\
Goat Milk (locally produced) & 0.018 & 0.22 & 2.4 \\
Beef (locally produced) & 0.28 & 5 & 79 \\
Leafy Vegetables (locally produced) & 0.00005 & 0.0011 & 0.025 \\
Eggs (locally produced) & 0.000073 & 0.00091 & 0.0094 \\
Cottage Cheese (locally produced) & 0.0036 & 0.055 & 0.69 \\
Inhalation & 0.00019 & 0.0042 & 0.098 \\
Mother's milk (mother on Diet 1) & 0.0021 & 0.013 & 0.067 \\
Prenatal exposure (mother on Diet 1) & 0.0027 & 0.082 & 2.5 \\
Diet 1 & 0.025 & 0.26 & 3.2 \\
Diet 2 & 0.1 & 1.4 & 15 \\
Diet 3 & 0.063 & 0.6 & 6.3 \\
\hline
\end{tabular}

Excess Lifetime Risk [ ]

\begin{tabular}{llll}
\hline Diet 1 & $1.8 \mathrm{E}-05$ & $4.3 \mathrm{E}-04$ & $9.9 \mathrm{E}-03$ \\
Diet 2 & $9.9 \mathrm{E}-06$ & $1.9 \mathrm{E}-04$ & $3.3 \mathrm{E}-03$ \\
Diet 3 & $4.5 \mathrm{E}-06$ & $7.5 \mathrm{E}-05$ & $1.2 \mathrm{E}-03$ \\
Diet 4 & $5.2 \mathrm{E}-05$ & $1.7 \mathrm{E}-03$ & $4.5 \mathrm{E}-02$ \\
\hline
\end{tabular}

\section{Relative Risk [ ]}

\begin{tabular}{lccc}
\hline Diet 1 & 1.0067 & 1.1 & 3.1 \\
Diet 2 & 1.003 & 1.044 & 1.76 \\
Diet 3 & 1.0011 & 1.018 & 1.38 \\
Diet 4 & 1.019 & 1.39 & 9.7 \\
\hline
\end{tabular}

\section{Probability of Causation [\%]}

\begin{tabular}{lccc}
\hline Diet 1 & 0.67 & 9.4 & 67 \\
Diet 2 & 0.30 & 4.2 & 43 \\
Diet 3 & 0.11 & 1.8 & 27 \\
Diet 4 & 1.84 & 27.8 & 90 \\
\hline
\end{tabular}

Diet 1 - Backyard cow milk + all other locally produced non-milk exposure pathways

Diet 2 - Locally produced commercial milk + all other locally produced non-milk exposure pathways

Diet 3 - Regionally mixed commercial milk + inhalation (other regionally mixed food items are minor contributors to the total)

Diet 4 - Goat milk (all other exposure pathways are of negligible importance compared to goat milk) 
Location: Hines Valley

Receptor: Male born in 1956

\begin{tabular}{lccc}
\hline & \multicolumn{3}{c}{ Thyroid Dose [cGy] } \\
& \multicolumn{2}{c}{$95 \%$ Subjective Confidence Interval } \\
Exposure Pathway & lower limit & central estimate & upper limit \\
\hline Backyard Cow Milk & 0.093 & 1.4 & 16 \\
Commercial Milk (locally produced) & 0.038 & 0.49 & 4.7 \\
Commercial Milk (regionally mixed) & 0.017 & 0.23 & 2.2 \\
Goat Milk (locally produced) & 0.38 & 5 & 90 \\
Beef (locally produced) & 0.000046 & 0.0011 & 0.026 \\
Leafy Vegetables (locally produced) & 0.000051 & 0.00083 & 0.011 \\
Eggs (locally produced) & 0.0039 & 0.057 & 0.78 \\
Cottage Cheese (locally produced) & 0.00022 & 0.0043 & 0.086 \\
Inhalation & 0.0025 & 0.013 & 0.067 \\
Mother's milk (mother on Diet 1) & 0.0027 & 0.082 & 2.5 \\
Prenatal exposure (mother on Diet 1) & 0.025 & 0.26 & 3.2 \\
Diet 1 & 0.11 & 1.5 & 18 \\
Diet 2 & 0.06 & 0.6 & 5.5 \\
Diet 3 & 0.026 & 0.25 & 2.2 \\
\hline
\end{tabular}

Excess Lifetime Risk [ ]

\begin{tabular}{llll}
\hline Diet 1 & $3.2 \mathrm{E}-06$ & $1.0 \mathrm{E}-04$ & $3.9 \mathrm{E}-03$ \\
Diet 2 & $1.5 \mathrm{E}-06$ & $4.5 \mathrm{E}-05$ & $1.2 \mathrm{E}-03$ \\
Diet 3 & $6.2 \mathrm{E}-07$ & $1.8 \mathrm{E}-05$ & $5.5 \mathrm{E}-04$ \\
Diet 4 & $1.0 \mathrm{E}-05$ & $3.8 \mathrm{E}-04$ & $1.4 \mathrm{E}-02$ \\
\hline
\end{tabular}

\section{Relative Risk [ ]}

\begin{tabular}{lccc}
\hline Diet 1 & 1.0031 & 1.084 & 3.7 \\
Diet 2 & 1.0014 & 1.031 & 1.99 \\
Diet 3 & 1.00061 & 1.012 & 1.36 \\
Diet 4 & 1.0086 & 1.29 & 13 \\
\hline
\end{tabular}

\section{Probability of Causation [\%]}

\begin{tabular}{lccc}
\hline Diet 1 & 0.31 & 7.8 & 73 \\
Diet 2 & 0.14 & 3.0 & 50 \\
Diet 3 & 0.06 & 1.2 & 27 \\
Diet 4 & 0.85 & 22.4 & 92 \\
\hline
\end{tabular}

Diet 1 - Backyard cow milk + all other locally produced non-milk exposure pathways

Diet 2 - Locally produced commercial milk + all other locally produced non-milk exposure pathways

Diet 3 - Regionally mixed commercial milk + inhalation (other regionally mixed food items are minor contributors to the total)

Diet 4 - Goat milk (all other exposure pathways are of negligible importance compared to goat milk) 


\section{Location: Farragut}

Receptor: Female born in 1956

\begin{tabular}{lccc}
\hline & \multicolumn{3}{c}{ Thyroid Dose [cGy] } \\
& \multicolumn{2}{c}{$95 \%$ Subjective Confidence Interval } \\
Exposure Pathway & lower limit & central estimate & upper limit \\
\hline Backyard Cow Milk & 0.1 & 1.5 & 17 \\
Commercial Milk (locally produced) & 0.043 & 0.55 & 6.4 \\
Commercial Milk (regionally mixed) & 0.018 & 0.22 & 2.4 \\
Goat Milk (locally produced) & 0.33 & 5.8 & 86 \\
Beef (locally produced) & 0.000061 & 0.0012 & 0.03 \\
Leafy Vegetables (locally produced) & 0.000077 & 0.001 & 0.01 \\
Eggs (locally produced) & 0.0036 & 0.063 & 0.84 \\
Cottage Cheese (locally produced) & 0.0002 & 0.0048 & 0.097 \\
Inhalation & 0.0024 & 0.015 & 0.081 \\
Mother's milk (mother on Diet 1) & 0.0031 & 0.087 & 3.3 \\
Prenatal exposure (mother on Diet 1) & 0.027 & 0.3 & 3.5 \\
Diet 1 & 0.12 & 1.6 & 18 \\
Diet 2 & 0.073 & 0.67 & 6.9 \\
Diet 3 & 0.028 & 0.24 & 2.4 \\
\hline
\end{tabular}

Excess Lifetime Risk [ ]

\begin{tabular}{llll}
\hline Diet 1 & $2.1 \mathrm{E}-05$ & $4.6 \mathrm{E}-04$ & $1.2 \mathrm{E}-02$ \\
Diet 2 & $1.1 \mathrm{E}-05$ & $2.1 \mathrm{E}-04$ & $3.8 \mathrm{E}-03$ \\
Diet 3 & $4.4 \mathrm{E}-06$ & $7.6 \mathrm{E}-05$ & $1.2 \mathrm{E}-03$ \\
Diet 4 & $6.0 \mathrm{E}-05$ & $2.0 \mathrm{E}-03$ & $5.3 \mathrm{E}-02$ \\
\hline
\end{tabular}

\section{Relative Risk [ ]}

\begin{tabular}{lccc}
\hline Diet 1 & 1.0079 & 1.12 & 3.6 \\
Diet 2 & 1.0031 & 1.051 & 2 \\
Diet 3 & 1.0011 & 1.018 & 1.38 \\
Diet 4 & 1.017 & 1.43 & 12 \\
\hline
\end{tabular}

\section{Probability of Causation [\%]}

\begin{tabular}{lccc}
\hline Diet 1 & 0.79 & 10.5 & 72 \\
Diet 2 & 0.31 & 4.8 & 50 \\
Diet 3 & 0.11 & 1.8 & 27 \\
Diet 4 & 1.63 & 30.1 & 91 \\
\hline
\end{tabular}

Diet 1 - Backyard cow milk + all other locally produced non-milk exposure pathways

Diet 2 - Locally produced commercial milk + all other locally produced non-milk exposure pathways

Diet 3 - Regionally mixed commercial milk + inhalation (other regionally mixed food items are minor contributors to the total)

Diet 4 - Goat milk (all other exposure pathways are of negligible importance compared to goat milk) 


\section{Location: Farragut}

\section{Receptor: Male born in 1956}

\begin{tabular}{lccc}
\hline & \multicolumn{3}{c}{ Thyroid Dose [cGy] } \\
& \multicolumn{2}{c}{$95 \%$ Subjective Confidence Interval } \\
Exposure Pathway & lower limit & central estimate & upper limit \\
\hline Backyard Cow Milk & 0.11 & 1.5 & 18 \\
Commercial Milk (locally produced) & 0.041 & 0.55 & 5.1 \\
Commercial Milk (regionally mixed) & 0.017 & 0.23 & 2.2 \\
Goat Milk (locally produced) & 0.4 & 5.8 & 110 \\
Beef (locally produced) & 0.000057 & 0.0012 & 0.031 \\
Leafy Vegetables (locally produced) & 0.000057 & 0.00097 & 0.012 \\
Eggs (locally produced) & 0.0048 & 0.064 & 0.83 \\
Cottage Cheese (locally produced) & 0.00024 & 0.0049 & 0.085 \\
Inhalation & 0.0027 & 0.015 & 0.077 \\
Mother's milk (mother on Diet 1) & 0.0031 & 0.087 & 3.3 \\
Prenatal exposure (mother on Diet 1) & 0.027 & 0.3 & 3.5 \\
Diet 1 & 0.13 & 1.7 & 19 \\
Diet 2 & 0.064 & 0.68 & 5.9 \\
Diet 3 & 0.026 & 0.25 & 2.2 \\
\hline
\end{tabular}

Excess Lifetime Risk [ ]

\begin{tabular}{llll}
\hline Diet 1 & $3.8 \mathrm{E}-06$ & $1.1 \mathrm{E}-04$ & $4.5 \mathrm{E}-03$ \\
Diet 2 & $1.7 \mathrm{E}-06$ & $5.2 \mathrm{E}-05$ & $1.5 \mathrm{E}-03$ \\
Diet 3 & $6.3 \mathrm{E}-07$ & $1.8 \mathrm{E}-05$ & $5.6 \mathrm{E}-04$ \\
Diet 4 & $1.1 \mathrm{E}-05$ & $4.1 \mathrm{E}-04$ & $1.8 \mathrm{E}-02$ \\
\hline
\end{tabular}

\section{Relative Risk [ ]}

\begin{tabular}{lccc}
\hline Diet 1 & 1.0038 & 1.089 & 3.9 \\
Diet 2 & 1.0017 & 1.035 & 2.1 \\
Diet 3 & 1.00062 & 1.012 & 1.36 \\
Diet 4 & 1.0088 & 1.33 & 15 \\
\hline
\end{tabular}

\section{Probability of Causation [\%]}

\begin{tabular}{lccc}
\hline Diet 1 & 0.38 & 8.2 & 74 \\
Diet 2 & 0.17 & 3.4 & 51 \\
Diet 3 & 0.06 & 1.2 & 27 \\
Diet 4 & 0.87 & 24.7 & 93 \\
\hline
\end{tabular}

Diet 1 - Backyard cow milk + all other locally produced non-milk exposure pathways

Diet 2 - Locally produced commercial milk + all other locally produced non-milk exposure pathways

Diet 3 - Regionally mixed commercial milk + inhalation (other regionally mixed food items are minor contributors to the total)

Diet 4 - Goat milk (all other exposure pathways are of negligible importance compared to goat milk) 
Location: Lenoir City

Receptor: Female born in 1956

\begin{tabular}{lccc}
\hline & \multicolumn{3}{c}{ Thyroid Dose [cGy] } \\
Exposure Pathway & \multicolumn{2}{c}{$95 \%$ Subjective Confidence Interval } \\
\hline Backyard Cow Milk & lower limit & central estimate & upper limit \\
Commercial Milk (locally produced) & 0.071 & 0.96 & 9.8 \\
Commercial Milk (regionally mixed) & 0.026 & 0.33 & 4.2 \\
Goat Milk (locally produced) & 0.018 & 0.22 & 2.4 \\
Beef (locally produced) & -- & -- & -- \\
Leafy Vegetables (locally produced) & 0.000032 & 0.0008 & 0.02 \\
Eggs (locally produced) & 0.000048 & 0.00064 & 0.0063 \\
Cottage Cheese (locally produced) & 0.0023 & 0.04 & 0.41 \\
Inhalation & 0.00012 & 0.0029 & 0.073 \\
Mother's milk (mother on Diet 1) & 0.0016 & 0.009 & 0.052 \\
Prenatal exposure (mother on Diet 1) & 0.0019 & 0.053 & 1.7 \\
Diet 1 & 0.02 & 0.18 & 2.2 \\
Diet 2 & 0.082 & 1 & 10 \\
Diet 3 & 0.039 & 0.41 & 4.4 \\
\hline
\end{tabular}

Excess Lifetime Risk [ ]

\begin{tabular}{lccc}
\hline Diet 1 & $1.2 \mathrm{E}-05$ & $2.9 \mathrm{E}-04$ & $6.3 \mathrm{E}-03$ \\
Diet 2 & $8.2 \mathrm{E}-06$ & $1.3 \mathrm{E}-04$ & $2.5 \mathrm{E}-03$ \\
Diet 3 & $4.3 \mathrm{E}-06$ & $7.2 \mathrm{E}-05$ & $1.2 \mathrm{E}-03$ \\
Diet 4 & -- & -- & -- \\
\hline
\end{tabular}

Relative Risk [ ]

\begin{tabular}{lccc}
\hline Diet 1 & 1.0047 & 1.073 & 2.6 \\
Diet 2 & 1.0022 & 1.03 & 1.6 \\
Diet 3 & 1.001 & 1.018 & 1.38 \\
Diet 4 & -- & -- & -- \\
\hline
\end{tabular}

\begin{tabular}{lccc}
\hline Diet 1 & 0.46 & 6.8 & 62 \\
Diet 2 & 0.22 & 2.9 & 37 \\
Diet 3 & 0.10 & 1.7 & 27 \\
Diet 4 & -- & -- & -- \\
\hline Diet 1 - Backyard cow milk + all other locally produced non-milk exposure pathways & \\
Diet 2 - Locally produced commercial milk + all other locally produced non-milk exposure pathways \\
Diet 3 - Regionally mixed commercial milk + inhalation (other regionally mixed food items are minor contributors to the total) \\
Diet 4 - Goat milk (all other exposure pathways are of negligible importance compared to goat milk)
\end{tabular}




\section{Location: Lenoir City}

\section{Receptor: Male born in 1956}

\begin{tabular}{lccc}
\hline & \multicolumn{3}{c}{ Thyroid Dose [cGy] } \\
& \multicolumn{2}{c}{$95 \%$ Subjective Confidence Interval } \\
Exposure Pathway & lower limit & central estimate & upper limit \\
\hline Backyard Cow Milk & 0.058 & 0.94 & 10 \\
Commercial Milk (locally produced) & 0.028 & 0.34 & 3.5 \\
Commercial Milk (regionally mixed) & 0.017 & 0.23 & 2.2 \\
Goat Milk (locally produced) & -- & -- & -- \\
Beef (locally produced) & 0.000034 & 0.00072 & 0.02 \\
Leafy Vegetables (locally produced) & 0.000036 & 0.00063 & 0.0078 \\
Eggs (locally produced) & 0.0029 & 0.037 & 0.46 \\
Cottage Cheese (locally produced) & 0.00014 & 0.0029 & 0.053 \\
Inhalation & 0.0017 & 0.009 & 0.053 \\
Mother's milk (mother on Diet 1) & 0.0019 & 0.053 & 1.7 \\
Prenatal exposure (mother on Diet 1) & 0.02 & 0.18 & 2.2 \\
Diet 1 & 0.074 & 1 & 11 \\
Diet 2 & 0.043 & 0.42 & 3.8 \\
Diet 3 & 0.023 & 0.24 & 2.2 \\
\hline
\end{tabular}

Excess Lifetime Risk [ ]

\begin{tabular}{lccc}
\hline Diet 1 & $2.1 \mathrm{E}-06$ & $7.5 \mathrm{E}-05$ & $2.6 \mathrm{E}-03$ \\
Diet 2 & $1.1 \mathrm{E}-06$ & $3.0 \mathrm{E}-05$ & $9.0 \mathrm{E}-04$ \\
Diet 3 & $6.1 \mathrm{E}-07$ & $1.7 \mathrm{E}-05$ & $5.5 \mathrm{E}-04$ \\
Diet 4 & -- & -- & -- \\
\hline
\end{tabular}

\section{Relative Risk [ ]}

\begin{tabular}{lccc}
\hline Diet 1 & 1.0023 & 1.058 & 2.7 \\
Diet 2 & 1.00098 & 1.022 & 1.55 \\
Diet 3 & 1.0006 & 1.012 & 1.36 \\
Diet 4 & -- & -- & -- \\
\hline
\end{tabular}

\begin{tabular}{lccc}
\hline Diet 1 & 0.23 & 5.5 & 63 \\
Diet 2 & 0.10 & 2.2 & 35 \\
Diet 3 & 0.06 & 1.2 & 26 \\
Diet 4 & -- & -- \\
\hline Diet 1 - Backyard cow milk + all other locally produced non-milk exposure pathways & \\
Diet 2 - Locally produced commercial milk + all other locally produced non-milk exposure pathways \\
Diet 3 - Regionally mixed commercial milk + inhalation (other regionally mixed food items are minor contributors to the total) \\
Diet 4 - Goat milk (all other exposure pathways are of negligible importance compared to goat milk)
\end{tabular}




\section{Location: Kingston}

Receptor: Female born in 1956

\begin{tabular}{lccc}
\hline & \multicolumn{3}{c}{ Thyroid Dose [cGy] } \\
& \multicolumn{2}{c}{$95 \%$ Subjective Confidence Interval } \\
Exposure Pathway & lower limit & central estimate & upper limit \\
\hline Backyard Cow Milk & 0.086 & 1.1 & 12 \\
Commercial Milk (locally produced) & 0.032 & 0.42 & 5.4 \\
Commercial Milk (regionally mixed) & 0.018 & 0.22 & 2.4 \\
Goat Milk (locally produced) & 0.27 & 4.6 & 69 \\
Beef (locally produced) & 0.000045 & 0.001 & 0.024 \\
Leafy Vegetables (locally produced) & 0.00006 & 0.00076 & 0.0077 \\
Eggs (locally produced) & 0.003 & 0.049 & 0.6 \\
Cottage Cheese (locally produced) & 0.00015 & 0.0038 & 0.085 \\
Inhalation & 0.0021 & 0.012 & 0.066 \\
Mother's milk (mother on Diet 1) & 0.0026 & 0.068 & 2.2 \\
Prenatal exposure (mother on Diet 1) & 0.021 & 0.23 & 3 \\
Diet 1 & 0.097 & 1.2 & 13 \\
Diet 2 & 0.055 & 0.53 & 6.2 \\
Diet 3 & 0.026 & 0.24 & 2.4 \\
\hline
\end{tabular}

Excess Lifetime Risk [ ]

\begin{tabular}{llll}
\hline Diet 1 & $1.6 \mathrm{E}-05$ & $3.9 \mathrm{E}-04$ & $8.8 \mathrm{E}-03$ \\
Diet 2 & $8.5 \mathrm{E}-06$ & $1.6 \mathrm{E}-04$ & $3.0 \mathrm{E}-03$ \\
Diet 3 & $4.4 \mathrm{E}-06$ & $7.4 \mathrm{E}-05$ & $1.2 \mathrm{E}-03$ \\
Diet 4 & $5.0 \mathrm{E}-05$ & $1.5 \mathrm{E}-03$ & $3.7 \mathrm{E}-02$ \\
\hline
\end{tabular}

\section{Relative Risk [ ]}

\begin{tabular}{lccc}
\hline Diet 1 & 1.0065 & 1.091 & 3 \\
Diet 2 & 1.0027 & 1.039 & 1.79 \\
Diet 3 & 1.0011 & 1.018 & 1.38 \\
Diet 4 & 1.015 & 1.33 & 8.7 \\
\hline
\end{tabular}

\section{Probability of Causation [\%]}

\begin{tabular}{lccc}
\hline Diet 1 & 0.64 & 8.3 & 66 \\
Diet 2 & 0.27 & 3.8 & 44 \\
Diet 3 & 0.11 & 1.8 & 27 \\
Diet 4 & 1.47 & 24.9 & 88 \\
\hline
\end{tabular}

Diet 1 - Backyard cow milk + all other locally produced non-milk exposure pathways

Diet 2 - Locally produced commercial milk + all other locally produced non-milk exposure pathways

Diet 3 - Regionally mixed commercial milk + inhalation (other regionally mixed food items are minor contributors to the total)

Diet 4 - Goat milk (all other exposure pathways are of negligible importance compared to goat milk) 


\section{Location: Kingston}

\section{Receptor: Male born in 1956}

\begin{tabular}{lccc}
\hline & \multicolumn{3}{c}{ Thyroid Dose [cGy] } \\
& \multicolumn{2}{c}{$95 \%$ Subjective Confidence Interval } \\
Exposure Pathway & lower limit & central estimate & upper limit \\
\hline Backyard Cow Milk & 0.081 & 1.2 & 14 \\
Commercial Milk (locally produced) & 0.032 & 0.43 & 4.5 \\
Commercial Milk (regionally mixed) & 0.017 & 0.23 & 2.2 \\
Goat Milk (locally produced) & 0.35 & 4.5 & 78 \\
Beef (locally produced) & 0.000043 & 0.00091 & 0.023 \\
Leafy Vegetables (locally produced) & 0.000044 & 0.00075 & 0.0097 \\
Eggs (locally produced) & 0.0032 & 0.049 & 0.64 \\
Cottage Cheese (locally produced) & 0.00019 & 0.0039 & 0.063 \\
Inhalation & 0.0023 & 0.012 & 0.066 \\
Mother's milk (mother on Diet 1) & 0.0026 & 0.068 & 2.2 \\
Prenatal exposure (mother on Diet 1) & 0.021 & 0.23 & 3 \\
Diet 1 & 0.09 & 1.3 & 15 \\
Diet 2 & 0.051 & 0.54 & 5.1 \\
Diet 3 & 0.025 & 0.25 & 2.2 \\
\hline
\end{tabular}

Excess Lifetime Risk [ ]

\begin{tabular}{llll}
\hline Diet 1 & $2.9 \mathrm{E}-06$ & $9.4 \mathrm{E}-05$ & $2.9 \mathrm{E}-03$ \\
Diet 2 & $1.2 \mathrm{E}-06$ & $3.9 \mathrm{E}-05$ & $1.1 \mathrm{E}-03$ \\
Diet 3 & $6.2 \mathrm{E}-07$ & $1.8 \mathrm{E}-05$ & $5.5 \mathrm{E}-04$ \\
Diet 4 & $9.6 \mathrm{E}-06$ & $3.3 \mathrm{E}-04$ & $1.3 \mathrm{E}-02$ \\
\hline
\end{tabular}

\section{Relative Risk [ ]}

\begin{tabular}{lccc}
\hline Diet 1 & 1.0028 & 1.069 & 3.4 \\
Diet 2 & 1.0013 & 1.027 & 1.83 \\
Diet 3 & 1.0006 & 1.012 & 1.36 \\
Diet 4 & 1.0067 & 1.26 & 10.2 \\
\hline
\end{tabular}

\section{Probability of Causation [\%]}

\begin{tabular}{lccc}
\hline Diet 1 & 0.28 & 6.5 & 70 \\
Diet 2 & 0.13 & 2.6 & 45 \\
Diet 3 & 0.06 & 1.2 & 27 \\
Diet 4 & 0.67 & 20.4 & 90 \\
\hline
\end{tabular}

Diet 1 - Backyard cow milk + all other locally produced non-milk exposure pathways

Diet 2 - Locally produced commercial milk + all other locally produced non-milk exposure pathways

Diet 3 - Regionally mixed commercial milk + inhalation (other regionally mixed food items are minor contributors to the total)

Diet 4 - Goat milk (all other exposure pathways are of negligible importance compared to goat milk) 


\section{Location: Karns}

Receptor: Female born in 1956

\begin{tabular}{lccc}
\hline & \multicolumn{3}{c}{ Thyroid Dose [cGy] } \\
Exposure Pathway & \multicolumn{2}{c}{$95 \%$ Subjective Confidence Interval } \\
\hline Backyard Cow Milk & lower limit & central estimate & upper limit \\
Commercial Milk (locally produced) & 0.11 & 1.6 & 17 \\
Commercial Milk (regionally mixed) & 0.045 & 0.58 & 6.2 \\
Goat Milk (locally produced) & 0.018 & 0.22 & 2.4 \\
Beef (locally produced) & 0.36 & 6.2 & 84 \\
Leafy Vegetables (locally produced) & 0.000065 & 0.0013 & 0.028 \\
Eggs (locally produced) & 0.000087 & 0.0011 & 0.01 \\
Cottage Cheese (locally produced) & 0.004 & 0.066 & 0.85 \\
Inhalation & 0.0002 & 0.0052 & 0.11 \\
Mother's milk (mother on Diet 1) & 0.0027 & 0.016 & 0.088 \\
Prenatal exposure (mother on Diet 1) & 0.0032 & 0.093 & 3.1 \\
Diet 1 & 0.029 & 0.31 & 3.7 \\
Diet 2 & 0.13 & 1.7 & 18 \\
Diet 3 & 0.074 & 0.74 & 7 \\
\hline
\end{tabular}

Excess Lifetime Risk [ ]

\begin{tabular}{llll}
\hline Diet 1 & $2.2 \mathrm{E}-05$ & $4.9 \mathrm{E}-04$ & $1.2 \mathrm{E}-02$ \\
Diet 2 & $1.3 \mathrm{E}-05$ & $2.2 \mathrm{E}-04$ & $3.9 \mathrm{E}-03$ \\
Diet 3 & $4.5 \mathrm{E}-06$ & $7.7 \mathrm{E}-05$ & $1.2 \mathrm{E}-03$ \\
Diet 4 & $6.4 \mathrm{E}-05$ & $2.0 \mathrm{E}-03$ & $5.4 \mathrm{E}-02$ \\
\hline
\end{tabular}

\section{Relative Risk [ ]}

\begin{tabular}{lccc}
\hline Diet 1 & 1.0092 & 1.12 & 3.6 \\
Diet 2 & 1.0032 & 1.054 & 2 \\
Diet 3 & 1.0011 & 1.018 & 1.38 \\
Diet 4 & 1.02 & 1.46 & 12 \\
\hline
\end{tabular}

\section{Probability of Causation [\%]}

\begin{tabular}{lccc}
\hline Diet 1 & 0.91 & 10.9 & 72 \\
Diet 2 & 0.32 & 5.1 & 50 \\
Diet 3 & 0.11 & 1.8 & 27 \\
Diet 4 & 1.99 & 31.4 & 92 \\
\hline
\end{tabular}

Diet 1 - Backyard cow milk + all other locally produced non-milk exposure pathways

Diet 2 - Locally produced commercial milk + all other locally produced non-milk exposure pathways

Diet 3 - Regionally mixed commercial milk + inhalation (other regionally mixed food items are minor contributors to the total)

Diet 4 - Goat milk (all other exposure pathways are of negligible importance compared to goat milk) 


\section{Location: Karns}

\section{Receptor: Male born in 1956}

\begin{tabular}{lccc}
\hline & \multicolumn{3}{c}{ Thyroid Dose [cGy] } \\
& \multicolumn{2}{c}{$95 \%$ Subjective Confidence Interval } \\
Exposure Pathway & lower limit & central estimate & upper limit \\
\hline Backyard Cow Milk & 0.12 & 1.7 & 19 \\
Commercial Milk (locally produced) & 0.044 & 0.58 & 5.3 \\
Commercial Milk (regionally mixed) & 0.017 & 0.23 & 2.2 \\
Goat Milk (locally produced) & 0.44 & 6.1 & 99 \\
Beef (locally produced) & 0.000058 & 0.0013 & 0.03 \\
Leafy Vegetables (locally produced) & 0.000065 & 0.001 & 0.012 \\
Eggs (locally produced) & 0.0051 & 0.069 & 0.89 \\
Cottage Cheese (locally produced) & 0.00025 & 0.0052 & 0.091 \\
Inhalation & 0.0031 & 0.016 & 0.084 \\
Mother's milk (mother on Diet 1) & 0.0032 & 0.093 & 3.1 \\
Prenatal exposure (mother on Diet 1) & 0.029 & 0.31 & 3.7 \\
Diet 1 & 0.14 & 1.8 & 20 \\
Diet 2 & 0.074 & 0.73 & 6.1 \\
Diet 3 & 0.027 & 0.25 & 2.2 \\
\hline
\end{tabular}

Excess Lifetime Risk [ ]

\begin{tabular}{llll}
\hline Diet 1 & $4.2 \mathrm{E}-06$ & $1.2 \mathrm{E}-04$ & $4.5 \mathrm{E}-03$ \\
Diet 2 & $1.9 \mathrm{E}-06$ & $5.4 \mathrm{E}-05$ & $1.5 \mathrm{E}-03$ \\
Diet 3 & $6.4 \mathrm{E}-07$ & $1.8 \mathrm{E}-05$ & $5.6 \mathrm{E}-04$ \\
Diet 4 & $1.2 \mathrm{E}-05$ & $4.4 \mathrm{E}-04$ & $1.9 \mathrm{E}-02$ \\
\hline
\end{tabular}

Relative Risk [ ]

\begin{tabular}{lccc}
\hline Diet 1 & 1.0038 & 1.096 & 4 \\
Diet 2 & 1.0018 & 1.037 & 2.1 \\
Diet 3 & 1.00064 & 1.013 & 1.36 \\
Diet 4 & 1.01 & 1.36 & 15 \\
\hline
\end{tabular}

\section{Probability of Causation [\%]}

\begin{tabular}{lccc}
\hline Diet 1 & 0.38 & 8.7 & 75 \\
Diet 2 & 0.18 & 3.6 & 51 \\
Diet 3 & 0.06 & 1.2 & 27 \\
Diet 4 & 0.99 & 26.6 & 93 \\
\hline
\end{tabular}

Diet 1 - Backyard cow milk + all other locally produced non-milk exposure pathways

Diet 2 - Locally produced commercial milk + all other locally produced non-milk exposure pathways

Diet 3 - Regionally mixed commercial milk + inhalation (other regionally mixed food items are minor contributors to the total)

Diet 4 - Goat milk (all other exposure pathways are of negligible importance compared to goat milk) 


\section{Location: Loudon}

Receptor: Female born in 1956

\begin{tabular}{lccc}
\hline & \multicolumn{3}{c}{ Thyroid Dose [cGy] } \\
& \multicolumn{2}{c}{$95 \%$ Subjective Confidence Interval } \\
Exposure Pathway & lower limit & central estimate & upper limit \\
\hline Backyard Cow Milk & 0.062 & 0.85 & 10 \\
Commercial Milk (locally produced) & 0.026 & 0.32 & 3.6 \\
Commercial Milk (regionally mixed) & 0.018 & 0.22 & 2.4 \\
Goat Milk (locally produced) & 0.19 & 3.6 & 50 \\
Beef (locally produced) & 0.000032 & 0.00073 & 0.017 \\
Leafy Vegetables (locally produced) & 0.000048 & 0.0006 & 0.0063 \\
Eggs (locally produced) & 0.0024 & 0.037 & 0.5 \\
Cottage Cheese (locally produced) & 0.00012 & 0.0029 & 0.066 \\
Inhalation & 0.0015 & 0.0094 & 0.053 \\
Mother's milk (mother on Diet 1) & 0.0017 & 0.052 & 1.7 \\
Prenatal exposure (mother on Diet 1) & 0.017 & 0.18 & 2 \\
Diet 1 & 0.072 & 0.92 & 11 \\
Diet 2 & 0.044 & 0.4 & 4.2 \\
Diet 3 & 0.025 & 0.23 & 2.4 \\
\hline
\end{tabular}

Excess Lifetime Risk [ ]

\begin{tabular}{llll}
\hline Diet 1 & $1.2 \mathrm{E}-05$ & $2.8 \mathrm{E}-04$ & $6.3 \mathrm{E}-03$ \\
Diet 2 & $6.6 \mathrm{E}-06$ & $1.3 \mathrm{E}-04$ & $2.2 \mathrm{E}-03$ \\
Diet 3 & $4.3 \mathrm{E}-06$ & $7.3 \mathrm{E}-05$ & $1.2 \mathrm{E}-03$ \\
Diet 4 & $3.6 \mathrm{E}-05$ & $1.2 \mathrm{E}-03$ & $2.9 \mathrm{E}-02$ \\
\hline
\end{tabular}

\section{Relative Risk [ ]}

\begin{tabular}{lccc}
\hline Diet 1 & 1.0044 & 1.07 & 2.6 \\
Diet 2 & 1.0019 & 1.029 & 1.59 \\
Diet 3 & 1.001 & 1.018 & 1.38 \\
Diet 4 & 1.011 & 1.25 & 7 \\
\hline
\end{tabular}

\section{Probability of Causation [\%]}

\begin{tabular}{lccc}
\hline Diet 1 & 0.44 & 6.5 & 61 \\
Diet 2 & 0.19 & 2.8 & 37 \\
Diet 3 & 0.10 & 1.7 & 27 \\
Diet 4 & 1.09 & 19.9 & 85 \\
\hline
\end{tabular}

Diet 1 - Backyard cow milk + all other locally produced non-milk exposure pathways

Diet 2 - Locally produced commercial milk + all other locally produced non-milk exposure pathways

Diet 3 - Regionally mixed commercial milk + inhalation (other regionally mixed food items are minor contributors to the total)

Diet 4 - Goat milk (all other exposure pathways are of negligible importance compared to goat milk) 


\section{Location: Loudon}

\section{Receptor: Male born in 1956}

\begin{tabular}{lccc}
\hline & \multicolumn{3}{c}{ Thyroid Dose [cGy] } \\
& \multicolumn{2}{c}{$95 \%$ Subjective Confidence Interval } \\
Exposure Pathway & lower limit & central estimate & upper limit \\
\hline Backyard Cow Milk & 0.064 & 0.91 & 11 \\
Commercial Milk (locally produced) & 0.023 & 0.33 & 3.1 \\
Commercial Milk (regionally mixed) & 0.017 & 0.23 & 2.2 \\
Goat Milk (locally produced) & 0.26 & 3.4 & 58 \\
Beef (locally produced) & 0.000036 & 0.00071 & 0.018 \\
Leafy Vegetables (locally produced) & 0.000035 & 0.00057 & 0.0072 \\
Eggs (locally produced) & 0.0026 & 0.038 & 0.5 \\
Cottage Cheese (locally produced) & 0.00014 & 0.0029 & 0.052 \\
Inhalation & 0.0018 & 0.0095 & 0.052 \\
Mother's milk (mother on Diet 1) & 0.0017 & 0.052 & 1.7 \\
Prenatal exposure (mother on Diet 1) & 0.017 & 0.18 & 2 \\
Diet 1 & 0.075 & 0.99 & 12 \\
Diet 2 & 0.041 & 0.41 & 3.6 \\
Diet 3 & 0.023 & 0.24 & 2.2 \\
\hline
\end{tabular}

Excess Lifetime Risk [ ]

\begin{tabular}{llll}
\hline Diet 1 & $2.2 \mathrm{E}-06$ & $6.8 \mathrm{E}-05$ & $2.4 \mathrm{E}-03$ \\
Diet 2 & $1.1 \mathrm{E}-06$ & $3.0 \mathrm{E}-05$ & $8.6 \mathrm{E}-04$ \\
Diet 3 & $6.1 \mathrm{E}-07$ & $1.8 \mathrm{E}-05$ & $5.4 \mathrm{E}-04$ \\
Diet 4 & $6.6 \mathrm{E}-06$ & $2.6 \mathrm{E}-04$ & $1.0 \mathrm{E}-02$ \\
\hline
\end{tabular}

\section{Relative Risk [ ]}

\begin{tabular}{lccc}
\hline Diet 1 & 1.0022 & 1.056 & 2.7 \\
Diet 2 & 1.001 & 1.022 & 1.63 \\
Diet 3 & 1.00056 & 1.012 & 1.36 \\
Diet 4 & 1.0058 & 1.2 & 8.2 \\
\hline
\end{tabular}

\section{Probability of Causation [\%]}

\begin{tabular}{lccc}
\hline Diet 1 & 0.22 & 5.3 & 62 \\
Diet 2 & 0.10 & 2.1 & 38 \\
Diet 3 & 0.06 & 1.2 & 26 \\
Diet 4 & 0.57 & 16.5 & 88 \\
\hline
\end{tabular}

Diet 1 - Backyard cow milk + all other locally produced non-milk exposure pathways

Diet 2 - Locally produced commercial milk + all other locally produced non-milk exposure pathways

Diet 3 - Regionally mixed commercial milk + inhalation (other regionally mixed food items are minor contributors to the total)

Diet 4 - Goat milk (all other exposure pathways are of negligible importance compared to goat milk) 
Location: Harriman

Receptor: Female born in 1956

\begin{tabular}{lccc}
\hline & \multicolumn{3}{c}{ Thyroid Dose [cGy] } \\
Exposure Pathway & \multicolumn{2}{c}{$95 \%$ Subjective Confidence Interval } \\
\hline Backyard Cow Milk & lower limit & central estimate & upper limit \\
Commercial Milk (locally produced) & 0.039 & 0.54 & 5.6 \\
Commercial Milk (regionally mixed) & 0.015 & 0.19 & 2.2 \\
Goat Milk (locally produced) & 0.018 & 0.22 & 2.4 \\
Beef (locally produced) & 0.13 & 2 & 31 \\
Leafy Vegetables (locally produced) & 0.000019 & 0.00046 & 0.012 \\
Eggs (locally produced) & 0.000026 & 0.00037 & 0.0036 \\
Cottage Cheese (locally produced) & 0.0013 & 0.023 & 0.24 \\
Inhalation & 0.000068 & 0.0017 & 0.04 \\
Mother's milk (mother on Diet 1) & 0.00087 & 0.005 & 0.028 \\
Prenatal exposure (mother on Diet 1) & 0.0011 & 0.031 & 0.99 \\
Diet 1 & 0.012 & 0.1 & 1.3 \\
Diet 2 & 0.045 & 0.57 & 5.9 \\
Diet 3 & 0.023 & 0.24 & 2.4 \\
\hline
\end{tabular}

Excess Lifetime Risk [ ]

\begin{tabular}{lllc}
\hline Diet 1 & $7.3 \mathrm{E}-06$ & $1.7 \mathrm{E}-04$ & $3.6 \mathrm{E}-03$ \\
Diet 2 & $4.7 \mathrm{E}-06$ & $7.4 \mathrm{E}-05$ & $1.4 \mathrm{E}-03$ \\
Diet 3 & $4.2 \mathrm{E}-06$ & $7.1 \mathrm{E}-05$ & $1.2 \mathrm{E}-03$ \\
Diet 4 & $2.1 \mathrm{E}-05$ & $6.7 \mathrm{E}-04$ & $2.0 \mathrm{E}-02$ \\
\hline
\end{tabular}

\section{Relative Risk [ ]}

\begin{tabular}{lccc}
\hline Diet 1 & 1.0027 & 1.042 & 1.95 \\
Diet 2 & 1.0012 & 1.017 & 1.35 \\
Diet 3 & 1.00098 & 1.017 & 1.37 \\
Diet 4 & 1.0074 & 1.15 & 4.6 \\
\hline
\end{tabular}

\section{Probability of Causation [\%]}

\begin{tabular}{lccc}
\hline Diet 1 & 0.27 & 4.0 & 49 \\
Diet 2 & 0.13 & 1.7 & 26 \\
Diet 3 & 0.10 & 1.7 & 27 \\
Diet 4 & 0.74 & 13.2 & 78 \\
\hline
\end{tabular}

Diet 1 - Backyard cow milk + all other locally produced non-milk exposure pathways

Diet 2 - Locally produced commercial milk + all other locally produced non-milk exposure pathways

Diet 3 - Regionally mixed commercial milk + inhalation (other regionally mixed food items are minor contributors to the total)

Diet 4 - Goat milk (all other exposure pathways are of negligible importance compared to goat milk) 


\section{Location: Harriman}

\section{Receptor: Male born in 1956}

\begin{tabular}{lccc}
\hline & \multicolumn{3}{c}{ Thyroid Dose [cGy] } \\
& \multicolumn{2}{c}{$95 \%$ Subjective Confidence Interval } \\
Exposure Pathway & lower limit & central estimate & upper limit \\
\hline Backyard Cow Milk & 0.033 & 0.53 & 5.9 \\
Commercial Milk (locally produced) & 0.016 & 0.2 & 2 \\
Commercial Milk (regionally mixed) & 0.017 & 0.23 & 2.2 \\
Goat Milk (locally produced) & 0.15 & 2.1 & 37 \\
Beef (locally produced) & 0.00002 & 0.00041 & 0.011 \\
Leafy Vegetables (locally produced) & 0.00002 & 0.00036 & 0.0044 \\
Eggs (locally produced) & 0.0016 & 0.021 & 0.27 \\
Cottage Cheese (locally produced) & 0.000083 & 0.0017 & 0.03 \\
Inhalation & 0.00097 & 0.005 & 0.029 \\
Mother's milk (mother on Diet 1) & 0.0011 & 0.031 & 0.99 \\
Prenatal exposure (mother on Diet 1) & 0.012 & 0.1 & 1.3 \\
Diet 1 & 0.042 & 0.57 & 6.4 \\
Diet 2 & 0.024 & 0.24 & 2.2 \\
Diet 3 & 0.02 & 0.24 & 2.2 \\
\hline
\end{tabular}

Excess Lifetime Risk [ ]

\begin{tabular}{llll}
\hline Diet 1 & $1.2 \mathrm{E}-06$ & $4.2 \mathrm{E}-05$ & $1.5 \mathrm{E}-03$ \\
Diet 2 & $6.1 \mathrm{E}-07$ & $1.7 \mathrm{E}-05$ & $5.1 \mathrm{E}-04$ \\
Diet 3 & $5.8 \mathrm{E}-07$ & $1.7 \mathrm{E}-05$ & $5.4 \mathrm{E}-04$ \\
Diet 4 & $4.0 \mathrm{E}-06$ & $1.5 \mathrm{E}-04$ & $7.0 \mathrm{E}-03$ \\
\hline
\end{tabular}

\section{Relative Risk [ ]}

\begin{tabular}{lccc}
\hline Diet 1 & 1.0013 & 1.033 & 2 \\
Diet 2 & 1.00058 & 1.012 & 1.32 \\
Diet 3 & 1.00055 & 1.012 & 1.36 \\
Diet 4 & 1.0035 & 1.12 & 5.1 \\
\hline
\end{tabular}

\section{Probability of Causation [\%]}

\begin{tabular}{lccc}
\hline Diet 1 & 0.13 & 3.2 & 50 \\
Diet 2 & 0.06 & 1.2 & 24 \\
Diet 3 & 0.06 & 1.2 & 26 \\
Diet 4 & 0.35 & 10.5 & 80 \\
\hline
\end{tabular}

Diet 1 - Backyard cow milk + all other locally produced non-milk exposure pathways

Diet 2 - Locally produced commercial milk + all other locally produced non-milk exposure pathways

Diet 3 - Regionally mixed commercial milk + inhalation (other regionally mixed food items are minor contributors to the total)

Diet 4 - Goat milk (all other exposure pathways are of negligible importance compared to goat milk) 


\section{Location: Cedar Bluff}

Receptor: Female born in 1956

\begin{tabular}{lccc}
\hline & \multicolumn{3}{c}{ Thyroid Dose [cGy] } \\
Exposure Pathway & \multicolumn{2}{c}{$95 \%$ Subjective Confidence Interval } \\
\hline Backyard Cow Milk & lower limit & central estimate & upper limit \\
Commercial Milk (locally produced) & 0.085 & 1.2 & 12 \\
Commercial Milk (regionally mixed) & 0.035 & 0.46 & 5 \\
Goat Milk (locally produced) & 0.018 & 0.22 & 2.4 \\
Beef (locally produced) & 0.26 & 4.8 & 74 \\
Leafy Vegetables (locally produced) & 0.000049 & 0.0011 & 0.024 \\
Eggs (locally produced) & 0.000069 & 0.00084 & 0.0085 \\
Cottage Cheese (locally produced) & 0.0034 & 0.052 & 0.61 \\
Inhalation & 0.00017 & 0.004 & 0.09 \\
Mother's milk (mother on Diet 1) & 0.0021 & 0.013 & 0.069 \\
Prenatal exposure (mother on Diet 1) & 0.0025 & 0.076 & 2.4 \\
Diet 1 & 0.024 & 0.25 & 3.1 \\
Diet 2 & 0.098 & 1.3 & 13 \\
Diet 3 & 0.06 & 0.57 & 5.6 \\
\hline
\end{tabular}

Excess Lifetime Risk [ ]

\begin{tabular}{llcc}
\hline Diet 1 & $1.6 \mathrm{E}-05$ & $4.1 \mathrm{E}-04$ & $9.1 \mathrm{E}-03$ \\
Diet 2 & $9.5 \mathrm{E}-06$ & $1.8 \mathrm{E}-04$ & $3.0 \mathrm{E}-03$ \\
Diet 3 & $4.6 \mathrm{E}-06$ & $7.5 \mathrm{E}-05$ & $1.2 \mathrm{E}-03$ \\
Diet 4 & $5.1 \mathrm{E}-05$ & $1.6 \mathrm{E}-03$ & $4.1 \mathrm{E}-02$ \\
\hline
\end{tabular}

Relative Risk [ ]

\begin{tabular}{lccc}
\hline Diet 1 & 1.0065 & 1.097 & 2.9 \\
Diet 2 & 1.0029 & 1.041 & 1.72 \\
Diet 3 & 1.0011 & 1.018 & 1.38 \\
Diet 4 & 1.017 & 1.36 & 9.1 \\
\hline
\end{tabular}

\section{Probability of Causation [\%]}

\begin{tabular}{lccc}
\hline Diet 1 & 0.65 & 8.8 & 66 \\
Diet 2 & 0.29 & 3.9 & 42 \\
Diet 3 & 0.11 & 1.8 & 27 \\
Diet 4 & 1.70 & 26.2 & 89 \\
\hline
\end{tabular}

Diet 1 - Backyard cow milk + all other locally produced non-milk exposure pathways

Diet 2 - Locally produced commercial milk + all other locally produced non-milk exposure pathways

Diet 3 - Regionally mixed commercial milk + inhalation (other regionally mixed food items are minor contributors to the total)

Diet 4 - Goat milk (all other exposure pathways are of negligible importance compared to goat milk) 


\section{Location: Cedar Bluff}

Receptor: Male born in 1956

\begin{tabular}{lccc}
\hline & \multicolumn{3}{c}{ Thyroid Dose [cGy] } \\
& \multicolumn{2}{c}{$95 \%$ Subjective Confidence Interval } \\
Exposure Pathway & lower limit & central estimate & upper limit \\
\hline Backyard Cow Milk & 0.093 & 1.4 & 15 \\
Commercial Milk (locally produced) & 0.036 & 0.46 & 4.3 \\
Commercial Milk (regionally mixed) & 0.017 & 0.23 & 2.2 \\
Goat Milk (locally produced) & 0.36 & 4.7 & 79 \\
Beef (locally produced) & 0.000043 & 0.001 & 0.024 \\
Leafy Vegetables (locally produced) & 0.000051 & 0.00078 & 0.01 \\
Eggs (locally produced) & 0.0037 & 0.054 & 0.69 \\
Cottage Cheese (locally produced) & 0.0002 & 0.0042 & 0.076 \\
Inhalation & 0.0025 & 0.013 & 0.068 \\
Mother's milk (mother on Diet 1) & 0.0025 & 0.076 & 2.4 \\
Prenatal exposure (mother on Diet 1) & 0.024 & 0.25 & 3.1 \\
Diet 1 & 0.11 & 1.5 & 16 \\
Diet 2 & 0.057 & 0.58 & 4.8 \\
Diet 3 & 0.025 & 0.25 & 2.2 \\
\hline
\end{tabular}

Excess Lifetime Risk [ ]

\begin{tabular}{llll}
\hline Diet 1 & $3.0 \mathrm{E}-06$ & $9.5 \mathrm{E}-05$ & $3.5 \mathrm{E}-03$ \\
Diet 2 & $1.4 \mathrm{E}-06$ & $4.3 \mathrm{E}-05$ & $1.1 \mathrm{E}-03$ \\
Diet 3 & $6.3 \mathrm{E}-07$ & $1.8 \mathrm{E}-05$ & $5.5 \mathrm{E}-04$ \\
Diet 4 & $9.8 \mathrm{E}-06$ & $3.6 \mathrm{E}-04$ & $1.2 \mathrm{E}-02$ \\
\hline
\end{tabular}

\section{Relative Risk [ ]}

\begin{tabular}{lccc}
\hline Diet 1 & 1.003 & 1.079 & 3.5 \\
Diet 2 & 1.0014 & 1.029 & 1.97 \\
Diet 3 & 1.00061 & 1.012 & 1.36 \\
Diet 4 & 1.0084 & 1.28 & 11 \\
\hline
\end{tabular}

\section{Probability of Causation [\%]}

\begin{tabular}{lccc}
\hline Diet 1 & 0.30 & 7.3 & 71 \\
Diet 2 & 0.14 & 2.8 & 49 \\
Diet 3 & 0.06 & 1.2 & 27 \\
Diet 4 & 0.83 & 21.8 & 91 \\
\hline
\end{tabular}

Diet 1 - Backyard cow milk + all other locally produced non-milk exposure pathways

Diet 2 - Locally produced commercial milk + all other locally produced non-milk exposure pathways

Diet 3 - Regionally mixed commercial milk + inhalation (other regionally mixed food items are minor contributors to the total)

Diet 4 - Goat milk (all other exposure pathways are of negligible importance compared to goat milk) 


\section{Location: Oakdale}

Receptor: Female born in 1956

\begin{tabular}{lccc}
\hline & \multicolumn{3}{c}{ Thyroid Dose [cGy] } \\
Exposure Pathway & \multicolumn{2}{c}{$95 \%$ Subjective Confidence Interval } \\
\hline Backyard Cow Milk & lower limit & central estimate & upper limit \\
Commercial Milk (locally produced) & 0.02 & 0.3 & 3.4 \\
Commercial Milk (regionally mixed) & 0.0073 & 0.11 & 1.3 \\
Goat Milk (locally produced) & 0.018 & 0.22 & 2.4 \\
Beef (locally produced) & 0.06 & 1.1 & 18 \\
Leafy Vegetables (locally produced) & 0.000011 & 0.00025 & 0.0056 \\
Eggs (locally produced) & 0.000014 & 0.0002 & 0.0022 \\
Cottage Cheese (locally produced) & 0.00072 & 0.012 & 0.15 \\
Inhalation & 0.000039 & 0.00093 & 0.021 \\
Mother's milk (mother on Diet 1) & 0.00048 & 0.003 & 0.017 \\
Prenatal exposure (mother on Diet 1) & 0.00057 & 0.018 & 0.55 \\
Diet 1 & 0.006 & 0.057 & 0.72 \\
Diet 2 & 0.022 & 0.32 & 3.5 \\
Diet 3 & 0.012 & 0.14 & 1.4 \\
\hline
\end{tabular}

Excess Lifetime Risk [ ]

\begin{tabular}{lllc}
\hline Diet 1 & $3.6 \mathrm{E}-06$ & $9.1 \mathrm{E}-05$ & $2.1 \mathrm{E}-03$ \\
Diet 2 & $2.2 \mathrm{E}-06$ & $4.2 \mathrm{E}-05$ & $7.1 \mathrm{E}-04$ \\
Diet 3 & $4.1 \mathrm{E}-06$ & $7.0 \mathrm{E}-05$ & $1.2 \mathrm{E}-03$ \\
Diet 4 & $1.0 \mathrm{E}-05$ & $3.7 \mathrm{E}-04$ & $1.0 \mathrm{E}-02$ \\
\hline
\end{tabular}

\section{Relative Risk [ ]}

\begin{tabular}{lccc}
\hline Diet 1 & 1.0015 & 1.023 & 1.51 \\
Diet 2 & 1.00066 & 1.01 & 1.21 \\
Diet 3 & 1.00093 & 1.017 & 1.37 \\
Diet 4 & 1.0036 & 1.087 & 3.2 \\
\hline
\end{tabular}

\section{Probability of Causation [\%]}

\begin{tabular}{llll}
\hline Diet 1 & 0.15 & 2.2 & 33 \\
Diet 2 & 0.07 & 1.0 & 17 \\
Diet 3 & 0.09 & 1.7 & 27 \\
Diet 4 & 0.36 & 7.9 & 67 \\
\hline
\end{tabular}

Diet 1 - Backyard cow milk + all other locally produced non-milk exposure pathways

Diet 2 - Locally produced commercial milk + all other locally produced non-milk exposure pathways

Diet 3 - Regionally mixed commercial milk + inhalation (other regionally mixed food items are minor contributors to the total)

Diet 4 - Goat milk (all other exposure pathways are of negligible importance compared to goat milk) 


\section{Location: Oakdale}

\section{Receptor: Male born in 1956}

\begin{tabular}{lccc}
\hline & \multicolumn{3}{c}{ Thyroid Dose [cGy] } \\
Exposure Pathway & \multicolumn{2}{c}{$95 \%$ Subjective Confidence Interval } \\
\hline Backyard Cow Milk & lower limit & central estimate & upper limit \\
Commercial Milk (locally produced) & 0.019 & 0.31 & 3.7 \\
Commercial Milk (regionally mixed) & 0.0081 & 0.11 & 1.1 \\
Goat Milk (locally produced) & 0.017 & 0.23 & 2.2 \\
Beef (locally produced) & 0.076 & 1.1 & 20 \\
Leafy Vegetables (locally produced) & 0.000011 & 0.00024 & 0.0058 \\
Eggs (locally produced) & 0.000011 & 0.00019 & 0.0024 \\
Cottage Cheese (locally produced) & 0.00085 & 0.012 & 0.15 \\
Inhalation & 0.000044 & 0.00097 & 0.017 \\
Mother's milk (mother on Diet 1) & 0.00055 & 0.0029 & 0.016 \\
Prenatal exposure (mother on Diet 1) & 0.00057 & 0.018 & 0.55 \\
Diet 1 & 0.006 & 0.057 & 0.72 \\
Diet 2 & 0.022 & 0.32 & 3.9 \\
Diet 3 & 0.012 & 0.14 & 1.2 \\
\hline
\end{tabular}

Excess Lifetime Risk [ ]

\begin{tabular}{llll}
\hline Diet 1 & $6.2 \mathrm{E}-07$ & $2.3 \mathrm{E}-05$ & $8.4 \mathrm{E}-04$ \\
Diet 2 & $3.1 \mathrm{E}-07$ & $1.0 \mathrm{E}-05$ & $2.8 \mathrm{E}-04$ \\
Diet 3 & $5.7 \mathrm{E}-07$ & $1.7 \mathrm{E}-05$ & $5.3 \mathrm{E}-04$ \\
Diet 4 & $2.1 \mathrm{E}-06$ & $8.2 \mathrm{E}-05$ & $3.5 \mathrm{E}-03$ \\
\hline
\end{tabular}

\section{Relative Risk [ ]}

\begin{tabular}{lccc}
\hline Diet 1 & 1.00063 & 1.019 & 1.55 \\
Diet 2 & 1.00031 & 1.007 & 1.2 \\
Diet 3 & 1.00053 & 1.012 & 1.36 \\
Diet 4 & 1.0019 & 1.068 & 3.8 \\
\hline
\end{tabular}

\section{Probability of Causation [\%]}

\begin{tabular}{llcc}
\hline Diet 1 & 0.06 & 1.8 & 35 \\
Diet 2 & 0.03 & 0.7 & 17 \\
Diet 3 & 0.05 & 1.2 & 26 \\
Diet 4 & 0.19 & 6.3 & 73 \\
\hline
\end{tabular}

Diet 1 - Backyard cow milk + all other locally produced non-milk exposure pathways

Diet 2 - Locally produced commercial milk + all other locally produced non-milk exposure pathways

Diet 3 - Regionally mixed commercial milk + inhalation (other regionally mixed food items are minor contributors to the total)

Diet 4 - Goat milk (all other exposure pathways are of negligible importance compared to goat milk) 


\section{Location: Claxton}

Receptor: Female born in 1956

\begin{tabular}{lccc}
\hline & \multicolumn{3}{c}{ Thyroid Dose [cGy] } \\
Exposure Pathway & \multicolumn{2}{c}{$95 \%$ Subjective Confidence Interval } \\
\hline Backyard Cow Milk & lower limit & central estimate & upper limit \\
Commercial Milk (locally produced) & 0.07 & 1 & 11 \\
Commercial Milk (regionally mixed) & 0.03 & 0.37 & 4.1 \\
Goat Milk (locally produced) & 0.018 & 0.22 & 2.4 \\
Beef (locally produced) & 0.22 & 4 & 60 \\
Leafy Vegetables (locally produced) & 0.00004 & 0.00088 & 0.02 \\
Eggs (locally produced) & 0.000057 & 0.00071 & 0.0074 \\
Cottage Cheese (locally produced) & 0.0027 & 0.043 & 0.52 \\
Inhalation & 0.00014 & 0.0033 & 0.074 \\
Mother's milk (mother on Diet 1) & 0.0018 & 0.011 & 0.056 \\
Prenatal exposure (mother on Diet 1) & 0.0021 & 0.062 & 2 \\
Diet 1 & 0.02 & 0.21 & 2.4 \\
Diet 2 & 0.082 & 1.1 & 11 \\
Diet 3 & 0.051 & 0.47 & 4.6 \\
\hline
\end{tabular}

Excess Lifetime Risk [ ]

\begin{tabular}{lllc}
\hline Diet 1 & $1.4 \mathrm{E}-05$ & $3.4 \mathrm{E}-04$ & $7.7 \mathrm{E}-03$ \\
Diet 2 & $7.9 \mathrm{E}-06$ & $1.5 \mathrm{E}-04$ & $2.5 \mathrm{E}-03$ \\
Diet 3 & $4.3 \mathrm{E}-06$ & $7.4 \mathrm{E}-05$ & $1.2 \mathrm{E}-03$ \\
Diet 4 & $4.2 \mathrm{E}-05$ & $1.3 \mathrm{E}-03$ & $3.4 \mathrm{E}-02$ \\
\hline
\end{tabular}

Relative Risk [ ]

\begin{tabular}{lccc}
\hline Diet 1 & 1.0055 & 1.082 & 2.7 \\
Diet 2 & 1.0024 & 1.034 & 1.63 \\
Diet 3 & 1.001 & 1.018 & 1.38 \\
Diet 4 & 1.014 & 1.29 & 7.6 \\
\hline
\end{tabular}

\section{Probability of Causation [\%]}

\begin{tabular}{lccc}
\hline Diet 1 & 0.55 & 7.5 & 62 \\
Diet 2 & 0.24 & 3.3 & 39 \\
Diet 3 & 0.11 & 1.8 & 27 \\
Diet 4 & 1.39 & 22.7 & 87 \\
\hline
\end{tabular}

Diet 1 - Backyard cow milk + all other locally produced non-milk exposure pathways

Diet 2 - Locally produced commercial milk + all other locally produced non-milk exposure pathways

Diet 3 - Regionally mixed commercial milk + inhalation (other regionally mixed food items are minor contributors to the total)

Diet 4 - Goat milk (all other exposure pathways are of negligible importance compared to goat milk) 


\section{Location: Claxton}

\section{Receptor: Male born in 1956}

\begin{tabular}{lccc}
\hline & \multicolumn{3}{c}{ Thyroid Dose [cGy] } \\
& \multicolumn{2}{c}{$95 \%$ Subjective Confidence Interval } \\
Exposure Pathway & lower limit & central estimate & upper limit \\
\hline Backyard Cow Milk & 0.077 & 1.1 & 12 \\
Commercial Milk (locally produced) & 0.03 & 0.39 & 3.6 \\
Commercial Milk (regionally mixed) & 0.017 & 0.23 & 2.2 \\
Goat Milk (locally produced) & 0.3 & 3.9 & 67 \\
Beef (locally produced) & 0.000037 & 0.00083 & 0.02 \\
Leafy Vegetables (locally produced) & 0.000043 & 0.00065 & 0.0079 \\
Eggs (locally produced) & 0.0031 & 0.044 & 0.59 \\
Cottage Cheese (locally produced) & 0.00017 & 0.0035 & 0.062 \\
Inhalation & 0.0021 & 0.011 & 0.055 \\
Mother's milk (mother on Diet 1) & 0.0021 & 0.062 & 2 \\
Prenatal exposure (mother on Diet 1) & 0.02 & 0.21 & 2.4 \\
Diet 1 & 0.087 & 1.2 & 13 \\
Diet 2 & 0.049 & 0.48 & 4 \\
Diet 3 & 0.024 & 0.24 & 2.2 \\
\hline
\end{tabular}

Excess Lifetime Risk [ ]

\begin{tabular}{llll}
\hline Diet 1 & $2.7 \mathrm{E}-06$ & $7.9 \mathrm{E}-05$ & $2.8 \mathrm{E}-03$ \\
Diet 2 & $1.2 \mathrm{E}-06$ & $3.6 \mathrm{E}-05$ & $9.5 \mathrm{E}-04$ \\
Diet 3 & $6.2 \mathrm{E}-07$ & $1.8 \mathrm{E}-05$ & $5.5 \mathrm{E}-04$ \\
Diet 4 & $8.4 \mathrm{E}-06$ & $3.0 \mathrm{E}-04$ & $1.1 \mathrm{E}-02$ \\
\hline
\end{tabular}

\section{Relative Risk [ ]}

\begin{tabular}{lccc}
\hline Diet 1 & 1.0025 & 1.065 & 3 \\
Diet 2 & 1.0012 & 1.024 & 1.76 \\
Diet 3 & 1.00059 & 1.012 & 1.36 \\
Diet 4 & 1.0068 & 1.23 & 9.9 \\
\hline
\end{tabular}

\section{Probability of Causation [\%]}

\begin{tabular}{lccc}
\hline Diet 1 & 0.25 & 6.1 & 67 \\
Diet 2 & 0.12 & 2.4 & 43 \\
Diet 3 & 0.06 & 1.2 & 27 \\
Diet 4 & 0.68 & 18.7 & 90 \\
\hline
\end{tabular}

Diet 1 - Backyard cow milk + all other locally produced non-milk exposure pathways

Diet 2 - Locally produced commercial milk + all other locally produced non-milk exposure pathways

Diet 3 - Regionally mixed commercial milk + inhalation (other regionally mixed food items are minor contributors to the total)

Diet 4 - Goat milk (all other exposure pathways are of negligible importance compared to goat milk) 
Location: Dutch Valley

Receptor: Female born in 1956

\begin{tabular}{lccc}
\hline & \multicolumn{3}{c}{ Thyroid Dose [cGy] } \\
Exposure Pathway & \multicolumn{2}{c}{$95 \%$ Subjective Confidence Interval } \\
\hline Backyard Cow Milk & lower limit & central estimate & upper limit \\
Commercial Milk (locally produced) & 0.037 & 0.52 & 5.3 \\
Commercial Milk (regionally mixed) & 0.014 & 0.18 & 2.1 \\
Goat Milk (locally produced) & 0.018 & 0.22 & 2.4 \\
Beef (locally produced) & 0.12 & 2 & 29 \\
Leafy Vegetables (locally produced) & 0.000019 & 0.00042 & 0.0099 \\
Eggs (locally produced) & 0.000027 & 0.00035 & 0.0034 \\
Cottage Cheese (locally produced) & 0.0013 & 0.021 & 0.25 \\
Inhalation & 0.000064 & 0.0016 & 0.037 \\
Mother's milk (mother on Diet 1) & 0.00084 & 0.0051 & 0.028 \\
Prenatal exposure (mother on Diet 1) & 0.001 & 0.03 & 0.92 \\
Diet 1 & 0.01 & 0.1 & 1.2 \\
Diet 2 & 0.044 & 0.56 & 5.5 \\
Diet 3 & 0.022 & 0.23 & 2.3 \\
\hline
\end{tabular}

Excess Lifetime Risk [ ]

\begin{tabular}{llll}
\hline Diet 1 & $6.9 \mathrm{E}-06$ & $1.6 \mathrm{E}-04$ & $3.7 \mathrm{E}-03$ \\
Diet 2 & $4.2 \mathrm{E}-06$ & $7.0 \mathrm{E}-05$ & $1.3 \mathrm{E}-03$ \\
Diet 3 & $4.2 \mathrm{E}-06$ & $7.1 \mathrm{E}-05$ & $1.2 \mathrm{E}-03$ \\
Diet 4 & $1.9 \mathrm{E}-05$ & $6.3 \mathrm{E}-04$ & $1.8 \mathrm{E}-02$ \\
\hline
\end{tabular}

\section{Relative Risk [ ]}

\begin{tabular}{lccc}
\hline Diet 1 & 1.0028 & 1.039 & 1.82 \\
Diet 2 & 1.0011 & 1.017 & 1.32 \\
Diet 3 & 1.00097 & 1.017 & 1.37 \\
Diet 4 & 1.0069 & 1.15 & 4.4 \\
\hline
\end{tabular}

\section{Probability of Causation [\%]}

\begin{tabular}{lccc}
\hline Diet 1 & 0.28 & 3.8 & 45 \\
Diet 2 & 0.11 & 1.7 & 24 \\
Diet 3 & 0.10 & 1.7 & 27 \\
Diet 4 & 0.68 & 12.8 & 77 \\
\hline
\end{tabular}

Diet 1 - Backyard cow milk + all other locally produced non-milk exposure pathways

Diet 2 - Locally produced commercial milk + all other locally produced non-milk exposure pathways

Diet 3 - Regionally mixed commercial milk + inhalation (other regionally mixed food items are minor contributors to the total)

Diet 4 - Goat milk (all other exposure pathways are of negligible importance compared to goat milk) 


\section{Location: Dutch Valley}

\section{Receptor: Male born in 1956}

\begin{tabular}{lccc}
\hline & \multicolumn{3}{c}{ Thyroid Dose [cGy] } \\
& \multicolumn{2}{c}{$95 \%$ Subjective Confidence Interval } \\
Exposure Pathway & lower limit & central estimate & upper limit \\
\hline Backyard Cow Milk & 0.035 & 0.52 & 5.8 \\
Commercial Milk (locally produced) & 0.014 & 0.18 & 1.8 \\
Commercial Milk (regionally mixed) & 0.017 & 0.23 & 2.2 \\
Goat Milk (locally produced) & 0.14 & 2 & 32 \\
Beef (locally produced) & 0.000018 & 0.00039 & 0.01 \\
Leafy Vegetables (locally produced) & 0.00002 & 0.00033 & 0.004 \\
Eggs (locally produced) & 0.0016 & 0.021 & 0.27 \\
Cottage Cheese (locally produced) & 0.00008 & 0.0016 & 0.03 \\
Inhalation & 0.00095 & 0.0051 & 0.028 \\
Mother's milk (mother on Diet 1) & 0.001 & 0.03 & 0.92 \\
Prenatal exposure (mother on Diet 1) & 0.01 & 0.1 & 1.2 \\
Diet 1 & 0.044 & 0.55 & 6.3 \\
Diet 2 & 0.023 & 0.23 & 2 \\
Diet 3 & 0.02 & 0.24 & 2.2 \\
\hline
\end{tabular}

Excess Lifetime Risk [ ]

\begin{tabular}{llll}
\hline Diet 1 & $1.2 \mathrm{E}-06$ & $4.0 \mathrm{E}-05$ & $1.3 \mathrm{E}-03$ \\
Diet 2 & $6.1 \mathrm{E}-07$ & $1.7 \mathrm{E}-05$ & $4.9 \mathrm{E}-04$ \\
Diet 3 & $5.8 \mathrm{E}-07$ & $1.7 \mathrm{E}-05$ & $5.4 \mathrm{E}-04$ \\
Diet 4 & $3.8 \mathrm{E}-06$ & $1.4 \mathrm{E}-04$ & $6.0 \mathrm{E}-03$ \\
\hline
\end{tabular}

\section{Relative Risk [ ]}

\begin{tabular}{lccc}
\hline Diet 1 & 1.0012 & 1.031 & 1.96 \\
Diet 2 & 1.00052 & 1.012 & 1.31 \\
Diet 3 & 1.00054 & 1.012 & 1.36 \\
Diet 4 & 1.0031 & 1.12 & 5.1 \\
\hline
\end{tabular}

\section{Probability of Causation [\%]}

\begin{tabular}{lccc}
\hline Diet 1 & 0.12 & 3.0 & 48 \\
Diet 2 & 0.05 & 1.2 & 23 \\
Diet 3 & 0.05 & 1.2 & 26 \\
Diet 4 & 0.31 & 10.7 & 80 \\
\hline
\end{tabular}

Diet 1 - Backyard cow milk + all other locally produced non-milk exposure pathways

Diet 2 - Locally produced commercial milk + all other locally produced non-milk exposure pathways

Diet 3 - Regionally mixed commercial milk + inhalation (other regionally mixed food items are minor contributors to the total)

Diet 4 - Goat milk (all other exposure pathways are of negligible importance compared to goat milk) 


\section{Location: Clinton}

Receptor: Female born in 1956

\begin{tabular}{lccc}
\hline & \multicolumn{3}{c}{ Thyroid Dose [cGy] } \\
Exposure Pathway & \multicolumn{2}{c}{$95 \%$ Subjective Confidence Interval } \\
\hline Backyard Cow Milk & lower limit & central estimate & upper limit \\
Commercial Milk (locally produced) & 0.056 & 0.8 & 8.5 \\
Commercial Milk (regionally mixed) & 0.022 & 0.3 & 3 \\
Goat Milk (locally produced) & 0.018 & 0.22 & 2.4 \\
Beef (locally produced) & 0.16 & 3.1 & 47 \\
Leafy Vegetables (locally produced) & 0.000032 & 0.00069 & 0.015 \\
Eggs (locally produced) & 0.000044 & 0.00056 & 0.0056 \\
Cottage Cheese (locally produced) & 0.0022 & 0.034 & 0.39 \\
Inhalation & 0.00011 & 0.0026 & 0.056 \\
Mother's milk (mother on Diet 1) & 0.0014 & 0.0084 & 0.044 \\
Prenatal exposure (mother on Diet 1) & 0.0016 & 0.048 & 1.4 \\
Diet 1 & 0.016 & 0.16 & 1.9 \\
Diet 2 & 0.064 & 0.88 & 8.9 \\
Diet 3 & 0.038 & 0.37 & 3.6 \\
\hline
\end{tabular}

Excess Lifetime Risk [ ]

\begin{tabular}{lllc}
\hline Diet 1 & $1.0 \mathrm{E}-05$ & $2.5 \mathrm{E}-04$ & $5.6 \mathrm{E}-03$ \\
Diet 2 & $6.1 \mathrm{E}-06$ & $1.1 \mathrm{E}-04$ & $1.9 \mathrm{E}-03$ \\
Diet 3 & $4.3 \mathrm{E}-06$ & $7.2 \mathrm{E}-05$ & $1.2 \mathrm{E}-03$ \\
Diet 4 & $3.2 \mathrm{E}-05$ & $1.0 \mathrm{E}-03$ & $2.6 \mathrm{E}-02$ \\
\hline
\end{tabular}

\section{Relative Risk [ ]}

\begin{tabular}{lccc}
\hline Diet 1 & 1.0043 & 1.064 & 2.3 \\
Diet 2 & 1.0019 & 1.026 & 1.49 \\
Diet 3 & 1.001 & 1.018 & 1.38 \\
Diet 4 & 1.011 & 1.24 & 6.5 \\
\hline
\end{tabular}

\section{Probability of Causation [\%]}

\begin{tabular}{lccc}
\hline Diet 1 & 0.43 & 6.0 & 56 \\
Diet 2 & 0.19 & 2.5 & 32 \\
Diet 3 & 0.10 & 1.7 & 27 \\
Diet 4 & 1.05 & 18.9 & 84 \\
\hline
\end{tabular}

Diet 1 - Backyard cow milk + all other locally produced non-milk exposure pathways

Diet 2 - Locally produced commercial milk + all other locally produced non-milk exposure pathways

Diet 3 - Regionally mixed commercial milk + inhalation (other regionally mixed food items are minor contributors to the total)

Diet 4 - Goat milk (all other exposure pathways are of negligible importance compared to goat milk) 


\section{Location: Clinton}

\section{Receptor: Male born in 1956}

\begin{tabular}{lccc}
\hline & \multicolumn{3}{c}{ Thyroid Dose [cGy] } \\
& \multicolumn{2}{c}{$95 \%$ Subjective Confidence Interval } \\
Exposure Pathway & lower limit & central estimate & upper limit \\
\hline Backyard Cow Milk & 0.055 & 0.85 & 9.3 \\
Commercial Milk (locally produced) & 0.022 & 0.31 & 2.7 \\
Commercial Milk (regionally mixed) & 0.017 & 0.23 & 2.2 \\
Goat Milk (locally produced) & 0.23 & 3.1 & 49 \\
Beef (locally produced) & 0.000028 & 0.00065 & 0.016 \\
Leafy Vegetables (locally produced) & 0.000033 & 0.00051 & 0.006 \\
Eggs (locally produced) & 0.0024 & 0.034 & 0.41 \\
Cottage Cheese (locally produced) & 0.00013 & 0.0026 & 0.047 \\
Inhalation & 0.0016 & 0.0085 & 0.043 \\
Mother's milk (mother on Diet 1) & 0.0016 & 0.048 & 1.4 \\
Prenatal exposure (mother on Diet 1) & 0.016 & 0.16 & 1.9 \\
Diet 1 & 0.063 & 0.91 & 10 \\
Diet 2 & 0.036 & 0.37 & 3 \\
Diet 3 & 0.022 & 0.24 & 2.2 \\
\hline
\end{tabular}

Excess Lifetime Risk [ ]

\begin{tabular}{llll}
\hline Diet 1 & $1.9 \mathrm{E}-06$ & $6.3 \mathrm{E}-05$ & $2.2 \mathrm{E}-03$ \\
Diet 2 & $8.8 \mathrm{E}-07$ & $2.8 \mathrm{E}-05$ & $7.1 \mathrm{E}-04$ \\
Diet 3 & $6.1 \mathrm{E}-07$ & $1.7 \mathrm{E}-05$ & $5.4 \mathrm{E}-04$ \\
Diet 4 & $5.9 \mathrm{E}-06$ & $2.3 \mathrm{E}-04$ & $8.4 \mathrm{E}-03$ \\
\hline
\end{tabular}

\section{Relative Risk [ ]}

\begin{tabular}{lccc}
\hline Diet 1 & 1.0018 & 1.05 & 2.5 \\
Diet 2 & 1.00089 & 1.019 & 1.61 \\
Diet 3 & 1.00056 & 1.012 & 1.36 \\
Diet 4 & 1.0054 & 1.18 & 8.1 \\
\hline
\end{tabular}

\section{Probability of Causation [\%]}

\begin{tabular}{lccc}
\hline Diet 1 & 0.18 & 4.8 & 59 \\
Diet 2 & 0.09 & 1.8 & 37 \\
Diet 3 & 0.06 & 1.2 & 26 \\
Diet 4 & 0.54 & 15.2 & 87 \\
\hline
\end{tabular}

Diet 1 - Backyard cow milk + all other locally produced non-milk exposure pathways

Diet 2 - Locally produced commercial milk + all other locally produced non-milk exposure pathways

Diet 3 - Regionally mixed commercial milk + inhalation (other regionally mixed food items are minor contributors to the total)

Diet 4 - Goat milk (all other exposure pathways are of negligible importance compared to goat milk) 
Location: Friendsville

Receptor: Female born in 1956

\begin{tabular}{lccc}
\hline & \multicolumn{3}{c}{ Thyroid Dose [cGy] } \\
Exposure Pathway & $95 \%$ Subjective Confidence Interval \\
\hline Backyard Cow Milk & lower limit & central estimate & upper limit \\
Commercial Milk (locally produced) & 0.033 & 0.46 & 5.6 \\
Commercial Milk (regionally mixed) & 0.012 & 0.17 & 1.9 \\
Goat Milk (locally produced) & 0.018 & 0.22 & 2.4 \\
Beef (locally produced) & 0.11 & 1.8 & 26 \\
Leafy Vegetables (locally produced) & 0.000018 & 0.00037 & 0.0079 \\
Eggs (locally produced) & 0.000023 & 0.00032 & 0.003 \\
Cottage Cheese (locally produced) & 0.0012 & 0.02 & 0.26 \\
Inhalation & 0.000058 & 0.0015 & 0.03 \\
Mother's milk (mother on Diet 1) & 0.00092 & 0.0057 & 0.034 \\
Prenatal exposure (mother on Diet 1) & 0.00095 & 0.028 & 1 \\
Diet 1 & 0.0084 & 0.094 & 1.1 \\
Diet 2 & 0.038 & 0.51 & 5.7 \\
Diet 3 & 0.02 & 0.22 & 2.1 \\
\hline
\end{tabular}

Excess Lifetime Risk [ ]

\begin{tabular}{llll}
\hline Diet 1 & $6.4 \mathrm{E}-06$ & $1.4 \mathrm{E}-04$ & $4.5 \mathrm{E}-03$ \\
Diet 2 & $3.5 \mathrm{E}-06$ & $6.7 \mathrm{E}-05$ & $1.1 \mathrm{E}-03$ \\
Diet 3 & $4.2 \mathrm{E}-06$ & $7.1 \mathrm{E}-05$ & $1.2 \mathrm{E}-03$ \\
Diet 4 & $1.8 \mathrm{E}-05$ & $6.4 \mathrm{E}-04$ & $1.5 \mathrm{E}-02$ \\
\hline
\end{tabular}

\section{Relative Risk [ ]}

\begin{tabular}{lccc}
\hline Diet 1 & 1.0028 & 1.035 & 1.74 \\
Diet 2 & 1.00092 & 1.016 & 1.29 \\
Diet 3 & 1.00097 & 1.018 & 1.37 \\
Diet 4 & 1.0056 & 1.13 & 4.3 \\
\hline
\end{tabular}

\begin{tabular}{lccc}
\hline Diet 1 & 0.28 & 3.4 & 43 \\
Diet 2 & 0.09 & 1.6 & 23 \\
Diet 3 & 0.10 & 1.7 & 27 \\
Diet 4 & 0.56 & 11.7 & 77 \\
\hline
\end{tabular}

Diet 1 - Backyard cow milk + all other locally produced non-milk exposure pathways

Diet 2 - Locally produced commercial milk + all other locally produced non-milk exposure pathways

Diet 3 - Regionally mixed commercial milk + inhalation (other regionally mixed food items are minor contributors to the total)

Diet 4 - Goat milk (all other exposure pathways are of negligible importance compared to goat milk) 
Location: Friendsville

Receptor: Male born in 1956

\begin{tabular}{lccc}
\hline & \multicolumn{3}{c}{ Thyroid Dose [cGy] } \\
& \multicolumn{2}{c}{$95 \%$ Subjective Confidence Interval } \\
Exposure Pathway & lower limit & central estimate & upper limit \\
\hline Backyard Cow Milk & 0.033 & 0.47 & 5.6 \\
Commercial Milk (locally produced) & 0.012 & 0.17 & 1.6 \\
Commercial Milk (regionally mixed) & 0.017 & 0.23 & 2.2 \\
Goat Milk (locally produced) & 0.13 & 1.8 & 30 \\
Beef (locally produced) & 0.000018 & 0.00035 & 0.0091 \\
Leafy Vegetables (locally produced) & 0.000019 & 0.00032 & 0.0033 \\
Eggs (locally produced) & 0.0015 & 0.02 & 0.26 \\
Cottage Cheese (locally produced) & 0.000073 & 0.0015 & 0.029 \\
Inhalation & 0.0011 & 0.0057 & 0.03 \\
Mother's milk (mother on Diet 1) & 0.00095 & 0.028 & 1 \\
Prenatal exposure (mother on Diet 1) & 0.0084 & 0.094 & 1.1 \\
Diet 1 & 0.041 & 0.51 & 6.1 \\
Diet 2 & 0.02 & 0.21 & 1.8 \\
Diet 3 & 0.02 & 0.24 & 2.2 \\
\hline
\end{tabular}

Excess Lifetime Risk [ ]

\begin{tabular}{llll}
\hline Diet 1 & $1.2 \mathrm{E}-06$ & $3.6 \mathrm{E}-05$ & $1.5 \mathrm{E}-03$ \\
Diet 2 & $5.8 \mathrm{E}-07$ & $1.6 \mathrm{E}-05$ & $4.6 \mathrm{E}-04$ \\
Diet 3 & $5.9 \mathrm{E}-07$ & $1.7 \mathrm{E}-05$ & $5.4 \mathrm{E}-04$ \\
Diet 4 & $3.4 \mathrm{E}-06$ & $1.3 \mathrm{E}-04$ & $5.6 \mathrm{E}-03$ \\
\hline
\end{tabular}

\section{Relative Risk [ ]}

\begin{tabular}{lccc}
\hline Diet 1 & 1.0011 & 1.027 & 2 \\
Diet 2 & 1.0005 & 1.011 & 1.28 \\
Diet 3 & 1.00054 & 1.012 & 1.36 \\
Diet 4 & 1.0026 & 1.11 & 5.1 \\
\hline
\end{tabular}

\section{Probability of Causation [\%]}

\begin{tabular}{llll}
\hline Diet 1 & 0.11 & 2.7 & 50 \\
Diet 2 & 0.05 & 1.1 & 22 \\
Diet 3 & 0.05 & 1.2 & 26 \\
Diet 4 & 0.26 & 9.6 & 80 \\
\hline
\end{tabular}

Diet 1 - Backyard cow milk + all other locally produced non-milk exposure pathways

Diet 2 - Locally produced commercial milk + all other locally produced non-milk exposure pathways

Diet 3 - Regionally mixed commercial milk + inhalation (other regionally mixed food items are minor contributors to the total)

Diet 4 - Goat milk (all other exposure pathways are of negligible importance compared to goat milk) 
Location: Wartburg

Receptor: Female born in 1956

\begin{tabular}{lccc}
\hline & \multicolumn{3}{c}{ Thyroid Dose [cGy] } \\
Exposure Pathway & \multicolumn{2}{c}{$95 \%$ Subjective Confidence Interval } \\
\hline Backyard Cow Milk & lower limit & central estimate & upper limit \\
Commercial Milk (locally produced) & 0.0092 & 0.13 & 1.5 \\
Commercial Milk (regionally mixed) & 0.0034 & 0.044 & 0.6 \\
Goat Milk (locally produced) & 0.018 & 0.22 & 2.4 \\
Beef (locally produced) & 0.026 & 0.51 & 9.3 \\
Leafy Vegetables (locally produced) & 0.0000048 & 0.0001 & 0.0031 \\
Eggs (locally produced) & 0.0000054 & 0.000083 & 0.00087 \\
Cottage Cheese (locally produced) & 0.0003 & 0.0052 & 0.08 \\
Inhalation & 0.00002 & 0.00042 & 0.0085 \\
Mother's milk (mother on Diet 1) & 0.0002 & 0.0013 & 0.0082 \\
Prenatal exposure (mother on Diet 1) & 0.00024 & 0.0071 & 0.29 \\
Diet 1 & 0.0023 & 0.025 & 0.38 \\
Diet 2 & 0.011 & 0.14 & 1.5 \\
Diet 3 & 0.0058 & 0.055 & 0.69 \\
\hline
\end{tabular}

Excess Lifetime Risk [ ]

\begin{tabular}{llll}
\hline Diet 1 & $1.6 \mathrm{E}-06$ & $4.3 \mathrm{E}-05$ & $1.2 \mathrm{E}-03$ \\
Diet 2 & $8.7 \mathrm{E}-07$ & $1.8 \mathrm{E}-05$ & $3.9 \mathrm{E}-04$ \\
Diet 3 & $4.1 \mathrm{E}-06$ & $7.0 \mathrm{E}-05$ & $1.2 \mathrm{E}-03$ \\
Diet 4 & $4.7 \mathrm{E}-06$ & $1.6 \mathrm{E}-04$ & $4.3 \mathrm{E}-03$ \\
\hline
\end{tabular}

\section{Relative Risk [ ]}

\begin{tabular}{lccc}
\hline Diet 1 & 1.00064 & 1.0095 & 1.25 \\
Diet 2 & 1.00028 & 1.0042 & 1.091 \\
Diet 3 & 1.00084 & 1.017 & 1.37 \\
Diet 4 & 1.0017 & 1.036 & 1.96 \\
\hline
\end{tabular}

\section{Probability of Causation [\%]}

\begin{tabular}{llcc}
\hline Diet 1 & 0.06 & 0.9 & 20 \\
Diet 2 & 0.03 & 0.4 & 8 \\
Diet 3 & 0.08 & 1.7 & 27 \\
Diet 4 & 0.17 & 3.5 & 49 \\
\hline
\end{tabular}

Diet 1 - Backyard cow milk + all other locally produced non-milk exposure pathways

Diet 2 - Locally produced commercial milk + all other locally produced non-milk exposure pathways

Diet 3 - Regionally mixed commercial milk + inhalation (other regionally mixed food items are minor contributors to the total)

Diet 4 - Goat milk (all other exposure pathways are of negligible importance compared to goat milk) 


\section{Location: Wartburg}

\section{Receptor: Male born in 1956}

\begin{tabular}{lccc}
\hline & \multicolumn{3}{c}{ Thyroid Dose [cGy] } \\
Exposure Pathway & 95\% Subjective Confidence Interval \\
\hline Backyard Cow Milk & 0.0089 & 0.13 & 1.7 \\
Commercial Milk (locally produced) & 0.003 & 0.046 & 0.52 \\
Commercial Milk (regionally mixed) & 0.017 & 0.23 & 2.2 \\
Goat Milk (locally produced) & 0.036 & 0.46 & 10 \\
Beef (locally produced) & 0.0000049 & 0.000093 & 0.0031 \\
Leafy Vegetables (locally produced) & 0.0000051 & 0.000086 & 0.0011 \\
Eggs (locally produced) & 0.0003 & 0.0052 & 0.075 \\
Cottage Cheese (locally produced) & 0.000021 & 0.00042 & 0.0074 \\
Inhalation & 0.00023 & 0.0013 & 0.0078 \\
Mother's milk (mother on Diet 1) & 0.00024 & 0.0071 & 0.29 \\
Prenatal exposure (mother on Diet 1) & 0.0023 & 0.025 & 0.38 \\
Diet 1 & 0.011 & 0.14 & 1.8 \\
Diet 2 & 0.0056 & 0.059 & 0.6 \\
Diet 3 & 0.018 & 0.23 & 2.2 \\
\hline
\end{tabular}

Excess Lifetime Risk [ ]

\begin{tabular}{llll}
\hline Diet 1 & $2.6 \mathrm{E}-07$ & $1.0 \mathrm{E}-05$ & $3.7 \mathrm{E}-04$ \\
Diet 2 & $1.1 \mathrm{E}-07$ & $3.9 \mathrm{E}-06$ & $1.3 \mathrm{E}-04$ \\
Diet 3 & $5.5 \mathrm{E}-07$ & $1.7 \mathrm{E}-05$ & $5.3 \mathrm{E}-04$ \\
Diet 4 & $8.8 \mathrm{E}-07$ & $3.6 \mathrm{E}-05$ & $1.5 \mathrm{E}-03$ \\
\hline
\end{tabular}

\section{Relative Risk [ ]}

\begin{tabular}{lccc}
\hline Diet 1 & 1.00027 & 1.0072 & 1.27 \\
Diet 2 & 1.00013 & 1.003 & 1.088 \\
Diet 3 & 1.00052 & 1.012 & 1.36 \\
Diet 4 & 1.00075 & 1.029 & 2 \\
\hline
\end{tabular}

\section{Probability of Causation [\%]}

\begin{tabular}{lccc}
\hline Diet 1 & 0.03 & 0.7 & 21 \\
Diet 2 & 0.01 & 0.3 & 8 \\
Diet 3 & 0.05 & 1.2 & 26 \\
Diet 4 & 0.08 & 2.8 & 50 \\
\hline
\end{tabular}

Diet 1 - Backyard cow milk + all other locally produced non-milk exposure pathways

Diet 2 - Locally produced commercial milk + all other locally produced non-milk exposure pathways

Diet 3 - Regionally mixed commercial milk + inhalation (other regionally mixed food items are minor contributors to the total)

Diet 4 - Goat milk (all other exposure pathways are of negligible importance compared to goat milk) 
Location: Rockwood

Receptor: Female born in 1956

\begin{tabular}{lccc}
\hline & \multicolumn{3}{c}{ Thyroid Dose [cGy] } \\
& \multicolumn{2}{c}{$95 \%$ Subjective Confidence Interval } \\
Exposure Pathway & lower limit & central estimate & upper limit \\
\hline Backyard Cow Milk & 0.038 & 0.51 & 5.8 \\
Commercial Milk (locally produced) & 0.016 & 0.19 & 2.3 \\
Commercial Milk (regionally mixed) & 0.018 & 0.22 & 2.4 \\
Goat Milk (locally produced) & 0.11 & 2.1 & 31 \\
Beef (locally produced) & 0.00002 & 0.00044 & 0.011 \\
Leafy Vegetables (locally produced) & 0.000028 & 0.00035 & 0.0037 \\
Eggs (locally produced) & 0.0014 & 0.022 & 0.28 \\
Cottage Cheese (locally produced) & 0.00007 & 0.0017 & 0.037 \\
Inhalation & 0.00095 & 0.0059 & 0.032 \\
Mother's milk (mother on Diet 1) & 0.0011 & 0.031 & 1 \\
Prenatal exposure (mother on Diet 1) & 0.01 & 0.1 & 1.3 \\
Diet 1 & 0.045 & 0.56 & 6 \\
Diet 2 & 0.026 & 0.24 & 2.5 \\
Diet 3 & 0.023 & 0.23 & 2.4 \\
\hline
\end{tabular}

Excess Lifetime Risk [ ]

\begin{tabular}{llll}
\hline Diet 1 & $7.1 \mathrm{E}-06$ & $1.7 \mathrm{E}-04$ & $4.3 \mathrm{E}-03$ \\
Diet 2 & $3.8 \mathrm{E}-06$ & $7.4 \mathrm{E}-05$ & $1.4 \mathrm{E}-03$ \\
Diet 3 & $4.2 \mathrm{E}-06$ & $7.2 \mathrm{E}-05$ & $1.2 \mathrm{E}-03$ \\
Diet 4 & $2.1 \mathrm{E}-05$ & $6.9 \mathrm{E}-04$ & $1.6 \mathrm{E}-02$ \\
\hline
\end{tabular}

\section{Relative Risk [ ]}

\begin{tabular}{lccc}
\hline Diet 1 & 1.0026 & 1.042 & 1.9 \\
Diet 2 & 1.0012 & 1.017 & 1.35 \\
Diet 3 & 1.00096 & 1.017 & 1.37 \\
Diet 4 & 1.0061 & 1.15 & 4.5 \\
\hline
\end{tabular}

\begin{tabular}{llcc} 
& \multicolumn{3}{c}{ Probability of Causation [\%] } \\
\hline Diet 1 & 0.26 & 4.0 & 46 \\
Diet 2 & 0.12 & 1.7 & 25 \\
Diet 3 & 0.10 & 1.7 & 27 \\
Diet 4 & 0.61 & 12.7 & 76 \\
\hline
\end{tabular}

Diet 1 - Backyard cow milk + all other locally produced non-milk exposure pathways

Diet 2 - Locally produced commercial milk + all other locally produced non-milk exposure pathways

Diet 3 - Regionally mixed commercial milk + inhalation (other regionally mixed food items are minor contributors to the total)

Diet 4 - Goat milk (all other exposure pathways are of negligible importance compared to goat milk) 


\section{Location: Rockwood}

\section{Receptor: Male born in 1956}

\begin{tabular}{lccc}
\hline & \multicolumn{3}{c}{ Thyroid Dose [cGy] } \\
Exposure Pathway & \multicolumn{2}{c}{$95 \%$ Subjective Confidence Interval } \\
\hline Backyard Cow Milk & lower limit & central estimate & upper limit \\
Commercial Milk (locally produced) & 0.039 & 0.53 & 6.3 \\
Commercial Milk (regionally mixed) & 0.014 & 0.19 & 1.9 \\
Goat Milk (locally produced) & 0.017 & 0.23 & 2.2 \\
Beef (locally produced) & 0.15 & 2 & 32 \\
Leafy Vegetables (locally produced) & 0.00002 & 0.00042 & 0.011 \\
Eggs (locally produced) & 0.000021 & 0.00034 & 0.0044 \\
Cottage Cheese (locally produced) & 0.0015 & 0.022 & 0.28 \\
Inhalation & 0.000091 & 0.0017 & 0.031 \\
Mother's milk (mother on Diet 1) & 0.0011 & 0.0059 & 0.031 \\
Prenatal exposure (mother on Diet 1) & 0.0011 & 0.031 & 1 \\
Diet 1 & 0.01 & 0.1 & 1.3 \\
Diet 2 & 0.043 & 0.57 & 6.7 \\
Diet 3 & 0.024 & 0.24 & 2.2 \\
\hline
\end{tabular}

Excess Lifetime Risk [ ]

\begin{tabular}{llll}
\hline Diet 1 & $1.4 \mathrm{E}-06$ & $4.1 \mathrm{E}-05$ & $1.4 \mathrm{E}-03$ \\
Diet 2 & $6.9 \mathrm{E}-07$ & $1.8 \mathrm{E}-05$ & $5.2 \mathrm{E}-04$ \\
Diet 3 & $5.9 \mathrm{E}-07$ & $1.7 \mathrm{E}-05$ & $5.4 \mathrm{E}-04$ \\
Diet 4 & $4.2 \mathrm{E}-06$ & $1.5 \mathrm{E}-04$ & $5.8 \mathrm{E}-03$ \\
\hline
\end{tabular}

\section{Relative Risk [ ]}

\begin{tabular}{lccc}
\hline Diet 1 & 1.0012 & 1.032 & 1.99 \\
Diet 2 & 1.00057 & 1.013 & 1.39 \\
Diet 3 & 1.00054 & 1.012 & 1.36 \\
Diet 4 & 1.0032 & 1.12 & 5.3 \\
\hline
\end{tabular}

\section{Probability of Causation [\%]}

\begin{tabular}{lccc}
\hline Diet 1 & 0.12 & 3.1 & 49 \\
Diet 2 & 0.06 & 1.3 & 28 \\
Diet 3 & 0.05 & 1.2 & 26 \\
Diet 4 & 0.32 & 10.3 & 80 \\
\hline
\end{tabular}

Diet 1 - Backyard cow milk + all other locally produced non-milk exposure pathways

Diet 2 - Locally produced commercial milk + all other locally produced non-milk exposure pathways

Diet 3 - Regionally mixed commercial milk + inhalation (other regionally mixed food items are minor contributors to the total)

Diet 4 - Goat milk (all other exposure pathways are of negligible importance compared to goat milk) 


\section{Location: Louisville}

Receptor: Female born in 1956

\begin{tabular}{lccc}
\hline & \multicolumn{3}{c}{ Thyroid Dose [cGy] } \\
Exposure Pathway & \multicolumn{2}{c}{$95 \%$ Subjective Confidence Interval } \\
\hline Backyard Cow Milk & lower limit & central estimate & upper limit \\
Commercial Milk (locally produced) & 0.038 & 0.49 & 5 \\
Commercial Milk (regionally mixed) & 0.013 & 0.17 & 2.2 \\
Goat Milk (locally produced) & 0.018 & 0.22 & 2.4 \\
Beef (locally produced) & 0.11 & 1.9 & 29 \\
Leafy Vegetables (locally produced) & 0.000018 & 0.00041 & 0.0096 \\
Eggs (locally produced) & 0.000025 & 0.00033 & 0.0031 \\
Cottage Cheese (locally produced) & 0.0013 & 0.021 & 0.26 \\
Inhalation & 0.000066 & 0.0016 & 0.034 \\
Mother's milk (mother on Diet 1) & 0.00096 & 0.0058 & 0.033 \\
Prenatal exposure (mother on Diet 1) & 0.001 & 0.029 & 0.9 \\
Diet 1 & 0.0097 & 0.098 & 1.2 \\
Diet 2 & 0.044 & 0.52 & 5.2 \\
Diet 3 & 0.022 & 0.22 & 2.4 \\
\hline
\end{tabular}

Excess Lifetime Risk [ ]

\begin{tabular}{llll}
\hline Diet 1 & $6.6 \mathrm{E}-06$ & $1.6 \mathrm{E}-04$ & $3.9 \mathrm{E}-03$ \\
Diet 2 & $3.6 \mathrm{E}-06$ & $6.8 \mathrm{E}-05$ & $1.3 \mathrm{E}-03$ \\
Diet 3 & $4.2 \mathrm{E}-06$ & $7.2 \mathrm{E}-05$ & $1.2 \mathrm{E}-03$ \\
Diet 4 & $2.0 \mathrm{E}-05$ & $6.2 \mathrm{E}-04$ & $1.6 \mathrm{E}-02$ \\
\hline
\end{tabular}

\section{Relative Risk [ ]}

\begin{tabular}{lccc}
\hline Diet 1 & 1.0028 & 1.038 & 1.8 \\
Diet 2 & 1.0012 & 1.016 & 1.32 \\
Diet 3 & 1.00098 & 1.017 & 1.37 \\
Diet 4 & 1.0063 & 1.14 & 4.3 \\
\hline
\end{tabular}

\begin{tabular}{lccc}
\hline Diet 1 & 0.28 & 3.6 & 44 \\
Diet 2 & 0.12 & 1.6 & 24 \\
Diet 3 & 0.10 & 1.7 & 27 \\
Diet 4 & 0.63 & 12.4 & 76 \\
\hline
\end{tabular}

Diet 1 - Backyard cow milk + all other locally produced non-milk exposure pathways

Diet 2 - Locally produced commercial milk + all other locally produced non-milk exposure pathways

Diet 3 - Regionally mixed commercial milk + inhalation (other regionally mixed food items are minor contributors to the total)

Diet 4 - Goat milk (all other exposure pathways are of negligible importance compared to goat milk) 


\section{Location: Louisville}

\section{Receptor: Male born in 1956}

\begin{tabular}{lccc}
\hline & \multicolumn{3}{c}{ Thyroid Dose [cGy] } \\
& \multicolumn{2}{c}{$95 \%$ Subjective Confidence Interval } \\
Exposure Pathway & lower limit & central estimate & upper limit \\
\hline Backyard Cow Milk & 0.035 & 0.5 & 5.8 \\
Commercial Milk (locally produced) & 0.013 & 0.18 & 1.8 \\
Commercial Milk (regionally mixed) & 0.017 & 0.23 & 2.2 \\
Goat Milk (locally produced) & 0.13 & 1.9 & 32 \\
Beef (locally produced) & 0.000018 & 0.00038 & 0.01 \\
Leafy Vegetables (locally produced) & 0.00002 & 0.00032 & 0.0039 \\
Eggs (locally produced) & 0.0014 & 0.02 & 0.26 \\
Cottage Cheese (locally produced) & 0.00008 & 0.0016 & 0.028 \\
Inhalation & 0.0011 & 0.0059 & 0.033 \\
Mother's milk (mother on Diet 1) & 0.001 & 0.029 & 0.9 \\
Prenatal exposure (mother on Diet 1) & 0.0097 & 0.098 & 1.2 \\
Diet 1 & 0.041 & 0.53 & 6.2 \\
Diet 2 & 0.023 & 0.22 & 2 \\
Diet 3 & 0.021 & 0.24 & 2.2 \\
\hline
\end{tabular}

Excess Lifetime Risk [ ]

\begin{tabular}{llll}
\hline Diet 1 & $1.2 \mathrm{E}-06$ & $4.0 \mathrm{E}-05$ & $1.3 \mathrm{E}-03$ \\
Diet 2 & $5.5 \mathrm{E}-07$ & $1.6 \mathrm{E}-05$ & $4.7 \mathrm{E}-04$ \\
Diet 3 & $5.9 \mathrm{E}-07$ & $1.7 \mathrm{E}-05$ & $5.4 \mathrm{E}-04$ \\
Diet 4 & $3.7 \mathrm{E}-06$ & $1.4 \mathrm{E}-04$ & $5.7 \mathrm{E}-03$ \\
\hline
\end{tabular}

\section{Relative Risk [ ]}

\begin{tabular}{lccc}
\hline Diet 1 & 1.0011 & 1.029 & 1.94 \\
Diet 2 & 1.00051 & 1.012 & 1.31 \\
Diet 3 & 1.00055 & 1.012 & 1.36 \\
Diet 4 & 1.0028 & 1.11 & 4.8 \\
\hline
\end{tabular}

\section{Probability of Causation [\%]}

\begin{tabular}{llcc}
\hline Diet 1 & 0.11 & 2.8 & 48 \\
Diet 2 & 0.05 & 1.1 & 23 \\
Diet 3 & 0.06 & 1.2 & 26 \\
Diet 4 & 0.28 & 9.8 & 79 \\
\hline
\end{tabular}

Diet 1 - Backyard cow milk + all other locally produced non-milk exposure pathways

Diet 2 - Locally produced commercial milk + all other locally produced non-milk exposure pathways

Diet 3 - Regionally mixed commercial milk + inhalation (other regionally mixed food items are minor contributors to the total)

Diet 4 - Goat milk (all other exposure pathways are of negligible importance compared to goat milk) 
Location: Barnardville

Receptor: Female born in 1956

\begin{tabular}{lccc}
\hline & \multicolumn{3}{c}{ Thyroid Dose [cGy] } \\
Exposure Pathway & $95 \%$ Subjective Confidence Interval \\
\hline Backyard Cow Milk & lower limit & central estimate & upper limit \\
Commercial Milk (locally produced) & 0.04 & 0.54 & 6 \\
Commercial Milk (regionally mixed) & 0.015 & 0.2 & 2.5 \\
Goat Milk (locally produced) & 0.018 & 0.22 & 2.4 \\
Beef (locally produced) & 0.13 & 2.2 & 31 \\
Leafy Vegetables (locally produced) & 0.000021 & 0.00046 & 0.01 \\
Eggs (locally produced) & 0.000028 & 0.00037 & 0.0036 \\
Cottage Cheese (locally produced) & 0.0015 & 0.023 & 0.33 \\
Inhalation & 0.000067 & 0.0018 & 0.04 \\
Mother's milk (mother on Diet 1) & 0.0011 & 0.0068 & 0.039 \\
Prenatal exposure (mother on Diet 1) & 0.0012 & 0.032 & 1.1 \\
Diet 1 & 0.01 & 0.11 & 1.4 \\
Diet 2 & 0.047 & 0.59 & 6.3 \\
Diet 3 & 0.026 & 0.25 & 2.9 \\
\hline
\end{tabular}

Excess Lifetime Risk [ ]

\begin{tabular}{llll}
\hline Diet 1 & $7.4 \mathrm{E}-06$ & $1.8 \mathrm{E}-04$ & $4.1 \mathrm{E}-03$ \\
Diet 2 & $4.0 \mathrm{E}-06$ & $7.8 \mathrm{E}-05$ & $1.3 \mathrm{E}-03$ \\
Diet 3 & $4.2 \mathrm{E}-06$ & $7.2 \mathrm{E}-05$ & $1.2 \mathrm{E}-03$ \\
Diet 4 & $2.3 \mathrm{E}-05$ & $7.3 \mathrm{E}-04$ & $1.7 \mathrm{E}-02$ \\
\hline
\end{tabular}

\section{Relative Risk [ ]}

\begin{tabular}{lccc}
\hline Diet 1 & 1.003 & 1.043 & 1.84 \\
Diet 2 & 1.0013 & 1.018 & 1.37 \\
Diet 3 & 1.00099 & 1.017 & 1.37 \\
Diet 4 & 1.0071 & 1.16 & 4.7 \\
\hline
\end{tabular}

\section{Probability of Causation [\%]}

\begin{tabular}{lccc}
\hline Diet 1 & 0.30 & 4.1 & 46 \\
Diet 2 & 0.13 & 1.8 & 27 \\
Diet 3 & 0.10 & 1.7 & 27 \\
Diet 4 & 0.71 & 13.6 & 79 \\
\hline
\end{tabular}

Diet 1 - Backyard cow milk + all other locally produced non-milk exposure pathways

Diet 2 - Locally produced commercial milk + all other locally produced non-milk exposure pathways

Diet 3 - Regionally mixed commercial milk + inhalation (other regionally mixed food items are minor contributors to the total)

Diet 4 - Goat milk (all other exposure pathways are of negligible importance compared to goat milk) 


\section{Location: Barnardville}

Receptor: Male born in 1956

\begin{tabular}{lccc}
\hline & \multicolumn{3}{c}{ Thyroid Dose [cGy] } \\
Exposure Pathway & \multicolumn{2}{c}{$95 \%$ Subjective Confidence Interval } \\
\hline Backyard Cow Milk & lower limit & central estimate & upper limit \\
Commercial Milk (locally produced) & 0.039 & 0.57 & 6.8 \\
Commercial Milk (regionally mixed) & 0.015 & 0.21 & 1.9 \\
Goat Milk (locally produced) & 0.017 & 0.23 & 2.2 \\
Beef (locally produced) & 0.16 & 2.2 & 33 \\
Leafy Vegetables (locally produced) & 0.000021 & 0.00044 & 0.01 \\
Eggs (locally produced) & 0.000023 & 0.00036 & 0.0043 \\
Cottage Cheese (locally produced) & 0.0015 & 0.024 & 0.31 \\
Inhalation & 0.000084 & 0.0018 & 0.031 \\
Mother's milk (mother on Diet 1) & 0.0013 & 0.007 & 0.037 \\
Prenatal exposure (mother on Diet 1) & 0.0012 & 0.032 & 1.1 \\
Diet 1 & 0.01 & 0.11 & 1.4 \\
Diet 2 & 0.046 & 0.62 & 7.3 \\
Diet 3 & 0.024 & 0.26 & 2.4 \\
\hline
\end{tabular}

Excess Lifetime Risk [ ]

\begin{tabular}{llcc}
\hline Diet 1 & $1.4 \mathrm{E}-06$ & $4.4 \mathrm{E}-05$ & $1.5 \mathrm{E}-03$ \\
Diet 2 & $6.1 \mathrm{E}-07$ & $1.9 \mathrm{E}-05$ & $5.2 \mathrm{E}-04$ \\
Diet 3 & $5.9 \mathrm{E}-07$ & $1.7 \mathrm{E}-05$ & $5.4 \mathrm{E}-04$ \\
Diet 4 & $4.3 \mathrm{E}-06$ & $1.6 \mathrm{E}-04$ & $5.7 \mathrm{E}-03$ \\
\hline
\end{tabular}

\section{Relative Risk [ ]}

\begin{tabular}{lccc}
\hline Diet 1 & 1.0013 & 1.033 & 2.2 \\
Diet 2 & 1.00061 & 1.013 & 1.38 \\
Diet 3 & 1.00054 & 1.012 & 1.36 \\
Diet 4 & 1.0034 & 1.12 & 5.8 \\
\hline
\end{tabular}

\section{Probability of Causation [\%]}

\begin{tabular}{lccc}
\hline Diet 1 & 0.13 & 3.2 & 55 \\
Diet 2 & 0.06 & 1.3 & 27 \\
Diet 3 & 0.05 & 1.2 & 26 \\
Diet 4 & 0.34 & 10.8 & 82 \\
\hline
\end{tabular}

Diet 1 - Backyard cow milk + all other locally produced non-milk exposure pathways

Diet 2 - Locally produced commercial milk + all other locally produced non-milk exposure pathways

Diet 3 - Regionally mixed commercial milk + inhalation (other regionally mixed food items are minor contributors to the total)

Diet 4 - Goat milk (all other exposure pathways are of negligible importance compared to goat milk) 
Location: Greenback

Receptor: Female born in 1956

\begin{tabular}{lccc}
\hline & \multicolumn{3}{c}{ Thyroid Dose [cGy] } \\
& \multicolumn{2}{c}{$95 \%$ Subjective Confidence Interval } \\
Exposure Pathway & lower limit & central estimate & upper limit \\
\hline Backyard Cow Milk & 0.021 & 0.3 & 3.7 \\
Commercial Milk (locally produced) & 0.0089 & 0.11 & 1.2 \\
Commercial Milk (regionally mixed) & 0.018 & 0.22 & 2.4 \\
Goat Milk (locally produced) & 0.069 & 1.3 & 18 \\
Beef (locally produced) & 0.0000097 & 0.00025 & 0.0062 \\
Leafy Vegetables (locally produced) & 0.000015 & 0.0002 & 0.0023 \\
Eggs (locally produced) & 0.00086 & 0.013 & 0.18 \\
Cottage Cheese (locally produced) & 0.000041 & 0.001 & 0.022 \\
Inhalation & 0.00068 & 0.0039 & 0.025 \\
Mother's milk (mother on Diet 1) & 0.00062 & 0.018 & 0.56 \\
Prenatal exposure (mother on Diet 1) & 0.0064 & 0.063 & 0.75 \\
Diet 1 & 0.024 & 0.33 & 4 \\
Diet 2 & 0.017 & 0.14 & 1.4 \\
Diet 3 & 0.021 & 0.23 & 2.4 \\
\hline
\end{tabular}

Excess Lifetime Risk [ ]

\begin{tabular}{lllc}
\hline Diet 1 & $3.7 \mathrm{E}-06$ & $9.7 \mathrm{E}-05$ & $2.3 \mathrm{E}-03$ \\
Diet 2 & $2.4 \mathrm{E}-06$ & $4.4 \mathrm{E}-05$ & $7.7 \mathrm{E}-04$ \\
Diet 3 & $4.1 \mathrm{E}-06$ & $7.1 \mathrm{E}-05$ & $1.2 \mathrm{E}-03$ \\
Diet 4 & $1.2 \mathrm{E}-05$ & $4.2 \mathrm{E}-04$ & $1.1 \mathrm{E}-02$ \\
\hline
\end{tabular}

\section{Relative Risk [ ]}

\begin{tabular}{lccc}
\hline Diet 1 & 1.0018 & 1.023 & 1.55 \\
Diet 2 & 1.00074 & 1.0099 & 1.2 \\
Diet 3 & 1.00094 & 1.017 & 1.37 \\
Diet 4 & 1.0036 & 1.087 & 3.2 \\
\hline
\end{tabular}

\section{Probability of Causation [\%]}

\begin{tabular}{llcc}
\hline Diet 1 & 0.18 & 2.3 & 36 \\
Diet 2 & 0.07 & 1.0 & 16 \\
Diet 3 & 0.09 & 1.7 & 27 \\
Diet 4 & 0.36 & 8.0 & 68 \\
\hline
\end{tabular}

Diet 1 - Backyard cow milk + all other locally produced non-milk exposure pathways

Diet 2 - Locally produced commercial milk + all other locally produced non-milk exposure pathways

Diet 3 - Regionally mixed commercial milk + inhalation (other regionally mixed food items are minor contributors to the total)

Diet 4 - Goat milk (all other exposure pathways are of negligible importance compared to goat milk) 


\section{Location: Greenback}

\section{Receptor: Male born in 1956}

\begin{tabular}{lccc}
\hline & \multicolumn{3}{c}{ Thyroid Dose [cGy] } \\
Exposure Pathway & 95\% Subjective Confidence Interval \\
\hline Backyard Cow Milk & 0.02 & 0.31 & 4.1 \\
Commercial Milk (locally produced) & 0.0077 & 0.11 & 1 \\
Commercial Milk (regionally mixed) & 0.017 & 0.23 & 2.2 \\
Goat Milk (locally produced) & 0.087 & 1.1 & 20 \\
Beef (locally produced) & 0.000011 & 0.00024 & 0.0066 \\
Leafy Vegetables (locally produced) & 0.000012 & 0.00019 & 0.0026 \\
Eggs (locally produced) & 0.00097 & 0.013 & 0.16 \\
Cottage Cheese (locally produced) & 0.000055 & 0.00098 & 0.02 \\
Inhalation & 0.00074 & 0.0039 & 0.022 \\
Mother's milk (mother on Diet 1) & 0.00062 & 0.018 & 0.56 \\
Prenatal exposure (mother on Diet 1) & 0.0064 & 0.063 & 0.75 \\
Diet 1 & 0.027 & 0.34 & 4.3 \\
Diet 2 & 0.014 & 0.14 & 1.1 \\
Diet 3 & 0.019 & 0.24 & 2.2 \\
\hline
\end{tabular}

Excess Lifetime Risk [ ]

\begin{tabular}{llll}
\hline Diet 1 & $7.4 \mathrm{E}-07$ & $2.3 \mathrm{E}-05$ & $8.1 \mathrm{E}-04$ \\
Diet 2 & $3.7 \mathrm{E}-07$ & $1.0 \mathrm{E}-05$ & $2.9 \mathrm{E}-04$ \\
Diet 3 & $5.7 \mathrm{E}-07$ & $1.7 \mathrm{E}-05$ & $5.3 \mathrm{E}-04$ \\
Diet 4 & $2.2 \mathrm{E}-06$ & $9.1 \mathrm{E}-05$ & $3.8 \mathrm{E}-03$ \\
\hline
\end{tabular}

\section{Relative Risk [ ]}

\begin{tabular}{lccc}
\hline Diet 1 & 1.00081 & 1.02 & 1.53 \\
Diet 2 & 1.00036 & 1.0079 & 1.21 \\
Diet 3 & 1.00053 & 1.012 & 1.36 \\
Diet 4 & 1.0019 & 1.072 & 3.5 \\
\hline
\end{tabular}

\section{Probability of Causation [\%]}

\begin{tabular}{llll}
\hline Diet 1 & 0.08 & 1.9 & 34 \\
Diet 2 & 0.04 & 0.8 & 17 \\
Diet 3 & 0.05 & 1.2 & 26 \\
Diet 4 & 0.19 & 6.7 & 71 \\
\hline
\end{tabular}

Diet 1 - Backyard cow milk + all other locally produced non-milk exposure pathways

Diet 2 - Locally produced commercial milk + all other locally produced non-milk exposure pathways

Diet 3 - Regionally mixed commercial milk + inhalation (other regionally mixed food items are minor contributors to the total)

Diet 4 - Goat milk (all other exposure pathways are of negligible importance compared to goat milk) 


\section{Location: Rockford}

Receptor: Female born in 1956

\begin{tabular}{lccc}
\hline & \multicolumn{3}{c}{ Thyroid Dose [cGy] } \\
& \multicolumn{2}{c}{$95 \%$ Subjective Confidence Interval } \\
Exposure Pathway & lower limit & central estimate & upper limit \\
\hline Backyard Cow Milk & 0.032 & 0.47 & 5.2 \\
Commercial Milk (locally produced) & 0.014 & 0.17 & 1.8 \\
Commercial Milk (regionally mixed) & 0.018 & 0.22 & 2.4 \\
Goat Milk (locally produced) & 0.1 & 1.9 & 26 \\
Beef (locally produced) & 0.000017 & 0.00039 & 0.0092 \\
Leafy Vegetables (locally produced) & 0.000025 & 0.00033 & 0.0034 \\
Eggs (locally produced) & 0.0012 & 0.02 & 0.26 \\
Cottage Cheese (locally produced) & 0.000067 & 0.0015 & 0.032 \\
Inhalation & 0.001 & 0.006 & 0.033 \\
Mother's milk (mother on Diet 1) & 0.001 & 0.028 & 0.83 \\
Prenatal exposure (mother on Diet 1) & 0.0099 & 0.096 & 1.1 \\
Diet 1 & 0.038 & 0.51 & 5.6 \\
Diet 2 & 0.024 & 0.22 & 2.1 \\
Diet 3 & 0.022 & 0.23 & 2.4 \\
\hline
\end{tabular}

Excess Lifetime Risk [ ]

\begin{tabular}{llll}
\hline Diet 1 & $6.5 \mathrm{E}-06$ & $1.5 \mathrm{E}-04$ & $3.6 \mathrm{E}-03$ \\
Diet 2 & $3.5 \mathrm{E}-06$ & $6.8 \mathrm{E}-05$ & $1.2 \mathrm{E}-03$ \\
Diet 3 & $4.2 \mathrm{E}-06$ & $7.2 \mathrm{E}-05$ & $1.2 \mathrm{E}-03$ \\
Diet 4 & $1.8 \mathrm{E}-05$ & $6.4 \mathrm{E}-04$ & $1.6 \mathrm{E}-02$ \\
\hline
\end{tabular}

\section{Relative Risk [ ]}

\begin{tabular}{lccc}
\hline Diet 1 & 1.0026 & 1.037 & 1.81 \\
Diet 2 & 1.0012 & 1.016 & 1.31 \\
Diet 3 & 1.00099 & 1.017 & 1.37 \\
Diet 4 & 1.0057 & 1.14 & 4.3 \\
\hline
\end{tabular}

\begin{tabular}{lccc}
\hline Diet 1 & 0.26 & 3.6 & 45 \\
Diet 2 & 0.12 & 1.6 & 24 \\
Diet 3 & 0.10 & 1.7 & 27 \\
Diet 4 & 0.57 & 12.1 & 76 \\
\hline
\end{tabular}

Diet 1 - Backyard cow milk + all other locally produced non-milk exposure pathways

Diet 2 - Locally produced commercial milk + all other locally produced non-milk exposure pathways

Diet 3 - Regionally mixed commercial milk + inhalation (other regionally mixed food items are minor contributors to the total)

Diet 4 - Goat milk (all other exposure pathways are of negligible importance compared to goat milk) 


\section{Location: Rockford}

\section{Receptor: Male born in 1956}

\begin{tabular}{lccc}
\hline & \multicolumn{3}{c}{ Thyroid Dose [cGy] } \\
& \multicolumn{2}{c}{$95 \%$ Subjective Confidence Interval } \\
Exposure Pathway & lower limit & central estimate & upper limit \\
\hline Backyard Cow Milk & 0.032 & 0.49 & 5.4 \\
Commercial Milk (locally produced) & 0.012 & 0.18 & 1.5 \\
Commercial Milk (regionally mixed) & 0.017 & 0.23 & 2.2 \\
Goat Milk (locally produced) & 0.13 & 1.9 & 29 \\
Beef (locally produced) & 0.000017 & 0.00038 & 0.0093 \\
Leafy Vegetables (locally produced) & 0.00002 & 0.00031 & 0.0036 \\
Eggs (locally produced) & 0.0015 & 0.02 & 0.24 \\
Cottage Cheese (locally produced) & 0.000081 & 0.0016 & 0.027 \\
Inhalation & 0.0011 & 0.0058 & 0.031 \\
Mother's milk (mother on Diet 1) & 0.001 & 0.028 & 0.83 \\
Prenatal exposure (mother on Diet 1) & 0.0099 & 0.096 & 1.1 \\
Diet 1 & 0.038 & 0.53 & 5.8 \\
Diet 2 & 0.023 & 0.23 & 1.7 \\
Diet 3 & 0.02 & 0.24 & 2.2 \\
\hline
\end{tabular}

Excess Lifetime Risk [ ]

\begin{tabular}{llll}
\hline Diet 1 & $1.2 \mathrm{E}-06$ & $3.6 \mathrm{E}-05$ & $1.2 \mathrm{E}-03$ \\
Diet 2 & $5.5 \mathrm{E}-07$ & $1.7 \mathrm{E}-05$ & $4.5 \mathrm{E}-04$ \\
Diet 3 & $5.9 \mathrm{E}-07$ & $1.7 \mathrm{E}-05$ & $5.4 \mathrm{E}-04$ \\
Diet 4 & $3.6 \mathrm{E}-06$ & $1.4 \mathrm{E}-04$ & $5.6 \mathrm{E}-03$ \\
\hline
\end{tabular}

\section{Relative Risk [ ]}

\begin{tabular}{lccc}
\hline Diet 1 & 1.0012 & 1.03 & 1.82 \\
Diet 2 & 1.00054 & 1.012 & 1.32 \\
Diet 3 & 1.00054 & 1.012 & 1.36 \\
Diet 4 & 1.003 & 1.11 & 5.2 \\
\hline
\end{tabular}

\section{Probability of Causation [\%]}

\begin{tabular}{llcc}
\hline Diet 1 & 0.12 & 2.9 & 45 \\
Diet 2 & 0.05 & 1.1 & 24 \\
Diet 3 & 0.05 & 1.2 & 26 \\
Diet 4 & 0.30 & 9.9 & 80 \\
\hline
\end{tabular}

Diet 1 - Backyard cow milk + all other locally produced non-milk exposure pathways

Diet 2 - Locally produced commercial milk + all other locally produced non-milk exposure pathways

Diet 3 - Regionally mixed commercial milk + inhalation (other regionally mixed food items are minor contributors to the total)

Diet 4 - Goat milk (all other exposure pathways are of negligible importance compared to goat milk) 
Location: Lake City

Receptor: Female born in 1956

\begin{tabular}{lccc}
\hline & \multicolumn{3}{c}{ Thyroid Dose [cGy] } \\
Exposure Pathway & \multicolumn{2}{c}{$95 \%$ Subjective Confidence Interval } \\
\hline Backyard Cow Milk & lower limit & central estimate & upper limit \\
Commercial Milk (locally produced) & 0.022 & 0.28 & 2.8 \\
Commercial Milk (regionally mixed) & 0.0092 & 0.11 & 1.2 \\
Goat Milk (locally produced) & 0.018 & 0.22 & 2.4 \\
Beef (locally produced) & 0.068 & 1.2 & 17 \\
Leafy Vegetables (locally produced) & 0.000011 & 0.00025 & 0.0062 \\
Eggs (locally produced) & 0.000016 & 0.0002 & 0.0019 \\
Cottage Cheese (locally produced) & 0.00077 & 0.012 & 0.15 \\
Inhalation & 0.000045 & 0.00094 & 0.02 \\
Mother's milk (mother on Diet 1) & 0.00051 & 0.0032 & 0.017 \\
Prenatal exposure (mother on Diet 1) & 0.00059 & 0.018 & 0.57 \\
Diet 1 & 0.006 & 0.057 & 0.7 \\
Diet 2 & 0.026 & 0.3 & 3 \\
Diet 3 & 0.015 & 0.13 & 1.4 \\
\hline
\end{tabular}

Excess Lifetime Risk [ ]

\begin{tabular}{lllc}
\hline Diet 1 & $4.3 \mathrm{E}-06$ & $9.5 \mathrm{E}-05$ & $2.4 \mathrm{E}-03$ \\
Diet 2 & $2.2 \mathrm{E}-06$ & $3.9 \mathrm{E}-05$ & $7.9 \mathrm{E}-04$ \\
Diet 3 & $4.1 \mathrm{E}-06$ & $7.0 \mathrm{E}-05$ & $1.2 \mathrm{E}-03$ \\
Diet 4 & $1.2 \mathrm{E}-05$ & $3.7 \mathrm{E}-04$ & $9.9 \mathrm{E}-03$ \\
\hline
\end{tabular}

\section{Relative Risk [ ]}

\begin{tabular}{lccc}
\hline Diet 1 & 1.0017 & 1.023 & 1.48 \\
Diet 2 & 1.00073 & 1.0097 & 1.18 \\
Diet 3 & 1.00095 & 1.017 & 1.37 \\
Diet 4 & 1.0041 & 1.083 & 2.9 \\
\hline
\end{tabular}

\section{Probability of Causation [\%]}

\begin{tabular}{llll}
\hline Diet 1 & 0.17 & 2.2 & 32 \\
Diet 2 & 0.07 & 1.0 & 15 \\
Diet 3 & 0.10 & 1.7 & 27 \\
Diet 4 & 0.41 & 7.6 & 65 \\
\hline
\end{tabular}

Diet 1 - Backyard cow milk + all other locally produced non-milk exposure pathways

Diet 2 - Locally produced commercial milk + all other locally produced non-milk exposure pathways

Diet 3 - Regionally mixed commercial milk + inhalation (other regionally mixed food items are minor contributors to the total)

Diet 4 - Goat milk (all other exposure pathways are of negligible importance compared to goat milk) 
Location: Lake City

Receptor: Male born in 1956

\begin{tabular}{lccc}
\hline & \multicolumn{3}{c}{ Thyroid Dose [cGy] } \\
& \multicolumn{2}{c}{$95 \%$ Subjective Confidence Interval } \\
Exposure Pathway & lower limit & central estimate & upper limit \\
\hline Backyard Cow Milk & 0.023 & 0.31 & 3.3 \\
Commercial Milk (locally produced) & 0.0087 & 0.11 & 1 \\
Commercial Milk (regionally mixed) & 0.017 & 0.23 & 2.2 \\
Goat Milk (locally produced) & 0.086 & 1.1 & 19 \\
Beef (locally produced) & 0.000011 & 0.00023 & 0.006 \\
Leafy Vegetables (locally produced) & 0.000012 & 0.00019 & 0.0024 \\
Eggs (locally produced) & 0.00087 & 0.012 & 0.15 \\
Cottage Cheese (locally produced) & 0.00005 & 0.00095 & 0.016 \\
Inhalation & 0.00061 & 0.0032 & 0.017 \\
Mother's milk (mother on Diet 1) & 0.00059 & 0.018 & 0.57 \\
Prenatal exposure (mother on Diet 1) & 0.006 & 0.057 & 0.7 \\
Diet 1 & 0.026 & 0.33 & 3.6 \\
Diet 2 & 0.015 & 0.13 & 1.1 \\
Diet 3 & 0.019 & 0.24 & 2.2 \\
\hline
\end{tabular}

Excess Lifetime Risk [ ]

\begin{tabular}{lllc}
\hline Diet 1 & $6.8 \mathrm{E}-07$ & $2.3 \mathrm{E}-05$ & $8.1 \mathrm{E}-04$ \\
Diet 2 & $3.0 \mathrm{E}-07$ & $9.5 \mathrm{E}-06$ & $2.8 \mathrm{E}-04$ \\
Diet 3 & $5.7 \mathrm{E}-07$ & $1.7 \mathrm{E}-05$ & $5.3 \mathrm{E}-04$ \\
Diet 4 & $2.3 \mathrm{E}-06$ & $8.4 \mathrm{E}-05$ & $3.5 \mathrm{E}-03$ \\
\hline
\end{tabular}

\section{Relative Risk [ ]}

\begin{tabular}{lccc}
\hline Diet 1 & 1.00077 & 1.018 & 1.56 \\
Diet 2 & 1.00033 & 1.0068 & 1.19 \\
Diet 3 & 1.00053 & 1.012 & 1.36 \\
Diet 4 & 1.0018 & 1.067 & 3.3 \\
\hline
\end{tabular}

\section{Probability of Causation [\%]}

\begin{tabular}{llll}
\hline Diet 1 & 0.08 & 1.7 & 35 \\
Diet 2 & 0.03 & 0.7 & 16 \\
Diet 3 & 0.05 & 1.2 & 26 \\
Diet 4 & 0.18 & 6.2 & 69 \\
\hline
\end{tabular}

Diet 1 - Backyard cow milk + all other locally produced non-milk exposure pathways

Diet 2 - Locally produced commercial milk + all other locally produced non-milk exposure pathways

Diet 3 - Regionally mixed commercial milk + inhalation (other regionally mixed food items are minor contributors to the total)

Diet 4 - Goat milk (all other exposure pathways are of negligible importance compared to goat milk) 
Location: Sweetwater

Receptor: Female born in 1956

\begin{tabular}{lccc}
\hline & \multicolumn{3}{c}{ Thyroid Dose [cGy] } \\
Exposure Pathway & \multicolumn{2}{c}{$95 \%$ Subjective Confidence Interval } \\
\hline Backyard Cow Milk & lower limit & central estimate & upper limit \\
Commercial Milk (locally produced) & 0.026 & 0.37 & 3.8 \\
Commercial Milk (regionally mixed) & 0.0094 & 0.13 & 1.5 \\
Goat Milk (locally produced) & 0.018 & 0.22 & 2.4 \\
Beef (locally produced) & 0.082 & 1.4 & 21 \\
Leafy Vegetables (locally produced) & 0.000014 & 0.0003 & 0.0065 \\
Eggs (locally produced) & 0.000019 & 0.00025 & 0.0023 \\
Cottage Cheese (locally produced) & 0.00099 & 0.015 & 0.19 \\
Inhalation & 0.000048 & 0.0012 & 0.024 \\
Mother's milk (mother on Diet 1) & 0.00073 & 0.0047 & 0.026 \\
Prenatal exposure (mother on Diet 1) & 0.00075 & 0.022 & 0.66 \\
Diet 1 & 0.0069 & 0.071 & 0.84 \\
Diet 2 & 0.032 & 0.39 & 4 \\
Diet 3 & 0.015 & 0.17 & 1.6 \\
\hline
\end{tabular}

Excess Lifetime Risk [ ]

\begin{tabular}{lllc}
\hline Diet 1 & $4.8 \mathrm{E}-06$ & $1.1 \mathrm{E}-04$ & $2.9 \mathrm{E}-03$ \\
Diet 2 & $2.6 \mathrm{E}-06$ & $5.1 \mathrm{E}-05$ & $8.4 \mathrm{E}-04$ \\
Diet 3 & $4.2 \mathrm{E}-06$ & $7.1 \mathrm{E}-05$ & $1.2 \mathrm{E}-03$ \\
Diet 4 & $1.4 \mathrm{E}-05$ & $4.4 \mathrm{E}-04$ & $1.1 \mathrm{E}-02$ \\
\hline
\end{tabular}

Relative Risk [ ]

\begin{tabular}{lccc}
\hline Diet 1 & 1.0022 & 1.027 & 1.58 \\
Diet 2 & 1.00079 & 1.012 & 1.22 \\
Diet 3 & 1.00096 & 1.017 & 1.37 \\
Diet 4 & 1.0048 & 1.11 & 3.5 \\
\hline
\end{tabular}

\begin{tabular}{llcc}
\hline Diet 1 & 0.22 & 2.6 & 37 \\
Diet 2 & 0.08 & 1.2 & 18 \\
Diet 3 & 0.10 & 1.7 & 27 \\
Diet 4 & 0.48 & 9.7 & 71 \\
\hline
\end{tabular}

Diet 1 - Backyard cow milk + all other locally produced non-milk exposure pathways

Diet 2 - Locally produced commercial milk + all other locally produced non-milk exposure pathways

Diet 3 - Regionally mixed commercial milk + inhalation (other regionally mixed food items are minor contributors to the total)

Diet 4 - Goat milk (all other exposure pathways are of negligible importance compared to goat milk) 
Location: Sweetwater

Receptor: Male born in 1956

\begin{tabular}{lccc}
\hline & \multicolumn{3}{c}{ Thyroid Dose [cGy] } \\
Exposure Pathway & \multicolumn{2}{c}{$95 \%$ Subjective Confidence Interval } \\
\hline Backyard Cow Milk & lower limit & central estimate & upper limit \\
Commercial Milk (locally produced) & 0.027 & 0.36 & 4.3 \\
Commercial Milk (regionally mixed) & 0.01 & 0.13 & 1.3 \\
Goat Milk (locally produced) & 0.017 & 0.23 & 2.2 \\
Beef (locally produced) & 0.091 & 1.4 & 22 \\
Leafy Vegetables (locally produced) & 0.000013 & 0.00028 & 0.0072 \\
Eggs (locally produced) & 0.000015 & 0.00023 & 0.0027 \\
Cottage Cheese (locally produced) & 0.0011 & 0.015 & 0.2 \\
Inhalation & 0.000062 & 0.0012 & 0.023 \\
Mother's milk (mother on Diet 1) & 0.00081 & 0.0047 & 0.026 \\
Prenatal exposure (mother on Diet 1) & 0.00075 & 0.022 & 0.66 \\
Diet 1 & 0.0069 & 0.071 & 0.84 \\
Diet 2 & 0.032 & 0.39 & 4.5 \\
Diet 3 & 0.017 & 0.16 & 1.4 \\
\hline
\end{tabular}

Excess Lifetime Risk [ ]

\begin{tabular}{llll}
\hline Diet 1 & $8.7 \mathrm{E}-07$ & $2.8 \mathrm{E}-05$ & $9.2 \mathrm{E}-04$ \\
Diet 2 & $4.9 \mathrm{E}-07$ & $1.2 \mathrm{E}-05$ & $3.7 \mathrm{E}-04$ \\
Diet 3 & $5.8 \mathrm{E}-07$ & $1.7 \mathrm{E}-05$ & $5.4 \mathrm{E}-04$ \\
Diet 4 & $2.8 \mathrm{E}-06$ & $1.0 \mathrm{E}-04$ & $4.2 \mathrm{E}-03$ \\
\hline
\end{tabular}

\section{Relative Risk [ ]}

\begin{tabular}{lccc}
\hline Diet 1 & 1.00076 & 1.021 & 1.66 \\
Diet 2 & 1.00034 & 1.0084 & 1.2 \\
Diet 3 & 1.00054 & 1.012 & 1.36 \\
Diet 4 & 1.002 & 1.081 & 3.8 \\
\hline
\end{tabular}

\begin{tabular}{llcc} 
& \multicolumn{3}{c}{ Probability of Causation [\%] } \\
\hline Diet 1 & 0.08 & 2.1 & 40 \\
Diet 2 & 0.03 & 0.8 & 17 \\
Diet 3 & 0.05 & 1.2 & 26 \\
Diet 4 & 0.20 & 7.5 & 73 \\
\hline
\end{tabular}

Diet 1 - Backyard cow milk + all other locally produced non-milk exposure pathways

Diet 2 - Locally produced commercial milk + all other locally produced non-milk exposure pathways

Diet 3 - Regionally mixed commercial milk + inhalation (other regionally mixed food items are minor contributors to the total)

Diet 4 - Goat milk (all other exposure pathways are of negligible importance compared to goat milk) 


\section{Location: Knoxville}

Receptor: Female born in 1956

\begin{tabular}{lccc}
\hline & \multicolumn{3}{c}{ Thyroid Dose [cGy] } \\
& \multicolumn{2}{c}{$95 \%$ Subjective Confidence Interval } \\
Exposure Pathway & lower limit & central estimate & upper limit \\
\hline Backyard Cow Milk & 0.043 & 0.61 & 6.7 \\
Commercial Milk (locally produced) & 0.016 & 0.22 & 2.3 \\
Commercial Milk (regionally mixed) & 0.018 & 0.22 & 2.4 \\
Goat Milk (locally produced) & 0.12 & 2.4 & 35 \\
Beef (locally produced) & 0.000024 & 0.00051 & 0.011 \\
Leafy Vegetables (locally produced) & 0.000032 & 0.00041 & 0.0042 \\
Eggs (locally produced) & 0.0016 & 0.026 & 0.32 \\
Cottage Cheese (locally produced) & 0.000076 & 0.0019 & 0.042 \\
Inhalation & 0.0013 & 0.0074 & 0.04 \\
Mother's milk (mother on Diet 1) & 0.0013 & 0.035 & 1.1 \\
Prenatal exposure (mother on Diet 1) & 0.012 & 0.12 & 1.5 \\
Diet 1 & 0.05 & 0.66 & 7.1 \\
Diet 2 & 0.028 & 0.28 & 2.7 \\
Diet 3 & 0.023 & 0.23 & 2.4 \\
\hline
\end{tabular}

Excess Lifetime Risk [ ]

\begin{tabular}{llll}
\hline Diet 1 & $7.9 \mathrm{E}-06$ & $1.9 \mathrm{E}-04$ & $4.3 \mathrm{E}-03$ \\
Diet 2 & $4.4 \mathrm{E}-06$ & $8.7 \mathrm{E}-05$ & $1.5 \mathrm{E}-03$ \\
Diet 3 & $4.2 \mathrm{E}-06$ & $7.2 \mathrm{E}-05$ & $1.2 \mathrm{E}-03$ \\
Diet 4 & $2.2 \mathrm{E}-05$ & $7.9 \mathrm{E}-04$ & $2.0 \mathrm{E}-02$ \\
\hline
\end{tabular}

\section{Relative Risk [ ]}

\begin{tabular}{lccc}
\hline Diet 1 & 1.0033 & 1.047 & 1.97 \\
Diet 2 & 1.0015 & 1.02 & 1.35 \\
Diet 3 & 1.001 & 1.018 & 1.38 \\
Diet 4 & 1.0074 & 1.17 & 4.8 \\
\hline
\end{tabular}

\section{Probability of Causation [\%]}

\begin{tabular}{lccc}
\hline Diet 1 & 0.32 & 4.5 & 49 \\
Diet 2 & 0.15 & 2.0 & 26 \\
Diet 3 & 0.10 & 1.7 & 27 \\
Diet 4 & 0.73 & 14.8 & 79 \\
\hline
\end{tabular}

Diet 1 - Backyard cow milk + all other locally produced non-milk exposure pathways

Diet 2 - Locally produced commercial milk + all other locally produced non-milk exposure pathways

Diet 3 - Regionally mixed commercial milk + inhalation (other regionally mixed food items are minor contributors to the total)

Diet 4 - Goat milk (all other exposure pathways are of negligible importance compared to goat milk) 


\section{Location: Knoxville}

\section{Receptor: Male born in 1956}

\begin{tabular}{lccc}
\hline & \multicolumn{3}{c}{ Thyroid Dose [cGy] } \\
Exposure Pathway & \multicolumn{2}{c}{$95 \%$ Subjective Confidence Interval } \\
\hline Backyard Cow Milk & lower limit & central estimate & upper limit \\
Commercial Milk (locally produced) & 0.04 & 0.63 & 6.7 \\
Commercial Milk (regionally mixed) & 0.016 & 0.23 & 2 \\
Goat Milk (locally produced) & 0.017 & 0.23 & 2.2 \\
Beef (locally produced) & 0.16 & 2.3 & 35 \\
Leafy Vegetables (locally produced) & 0.000023 & 0.00049 & 0.012 \\
Eggs (locally produced) & 0.000025 & 0.00038 & 0.0047 \\
Cottage Cheese (locally produced) & 0.0018 & 0.025 & 0.3 \\
Inhalation & 0.000096 & 0.002 & 0.036 \\
Mother's milk (mother on Diet 1) & 0.0014 & 0.0073 & 0.039 \\
Prenatal exposure (mother on Diet 1) & 0.0013 & 0.035 & 1.1 \\
Diet 1 & 0.012 & 0.12 & 1.5 \\
Diet 2 & 0.045 & 0.68 & 7.2 \\
Diet 3 & 0.028 & 0.28 & 2.2 \\
\hline
\end{tabular}

Excess Lifetime Risk [ ]

\begin{tabular}{llll}
\hline Diet 1 & $1.5 \mathrm{E}-06$ & $4.6 \mathrm{E}-05$ & $1.6 \mathrm{E}-03$ \\
Diet 2 & $6.8 \mathrm{E}-07$ & $2.1 \mathrm{E}-05$ & $5.3 \mathrm{E}-04$ \\
Diet 3 & $6.0 \mathrm{E}-07$ & $1.7 \mathrm{E}-05$ & $5.4 \mathrm{E}-04$ \\
Diet 4 & $4.7 \mathrm{E}-06$ & $1.8 \mathrm{E}-04$ & $6.8 \mathrm{E}-03$ \\
\hline
\end{tabular}

\section{Relative Risk [ ]}

\begin{tabular}{lccc}
\hline Diet 1 & 1.0014 & 1.038 & 2.1 \\
Diet 2 & 1.00069 & 1.014 & 1.41 \\
Diet 3 & 1.00055 & 1.012 & 1.36 \\
Diet 4 & 1.0038 & 1.14 & 6.1 \\
\hline
\end{tabular}

\section{Probability of Causation [\%]}

\begin{tabular}{lccc}
\hline Diet 1 & 0.14 & 3.6 & 53 \\
Diet 2 & 0.07 & 1.4 & 29 \\
Diet 3 & 0.06 & 1.2 & 26 \\
Diet 4 & 0.38 & 12.2 & 84 \\
\hline
\end{tabular}

Diet 1 - Backyard cow milk + all other locally produced non-milk exposure pathways

Diet 2 - Locally produced commercial milk + all other locally produced non-milk exposure pathways

Diet 3 - Regionally mixed commercial milk + inhalation (other regionally mixed food items are minor contributors to the total)

Diet 4 - Goat milk (all other exposure pathways are of negligible importance compared to goat milk) 


\section{Location: Maryville}

Receptor: Female born in 1956

\begin{tabular}{lccc}
\hline & \multicolumn{3}{c}{ Thyroid Dose [cGy] } \\
Exposure Pathway & \multicolumn{2}{c}{$95 \%$ Subjective Confidence Interval } \\
\hline Backyard Cow Milk & lower limit & central estimate & upper limit \\
Commercial Milk (locally produced) & 0.02 & 0.28 & 3.4 \\
Commercial Milk (regionally mixed) & 0.0086 & 0.11 & 1.3 \\
Goat Milk (locally produced) & 0.018 & 0.22 & 2.4 \\
Beef (locally produced) & 0.07 & 1.1 & 16 \\
Leafy Vegetables (locally produced) & 0.000011 & 0.00024 & 0.0052 \\
Eggs (locally produced) & 0.000015 & 0.0002 & 0.002 \\
Cottage Cheese (locally produced) & 0.00083 & 0.012 & 0.18 \\
Inhalation & 0.000038 & 0.00094 & 0.022 \\
Mother's milk (mother on Diet 1) & 0.00062 & 0.004 & 0.023 \\
Prenatal exposure (mother on Diet 1) & 0.00057 & 0.018 & 0.59 \\
Diet 1 & 0.0055 & 0.061 & 0.58 \\
Diet 2 & 0.025 & 0.31 & 3.6 \\
Diet 3 & 0.014 & 0.13 & 1.6 \\
\hline
\end{tabular}

Excess Lifetime Risk [ ]

\begin{tabular}{llll}
\hline Diet 1 & $3.8 \mathrm{E}-06$ & $9.5 \mathrm{E}-05$ & $2.0 \mathrm{E}-03$ \\
Diet 2 & $2.1 \mathrm{E}-06$ & $4.2 \mathrm{E}-05$ & $6.9 \mathrm{E}-04$ \\
Diet 3 & $4.1 \mathrm{E}-06$ & $7.1 \mathrm{E}-05$ & $1.2 \mathrm{E}-03$ \\
Diet 4 & $1.1 \mathrm{E}-05$ & $4.0 \mathrm{E}-04$ & $9.7 \mathrm{E}-03$ \\
\hline
\end{tabular}

\section{Relative Risk [ ]}

\begin{tabular}{lccc}
\hline Diet 1 & 1.0015 & 1.023 & 1.47 \\
Diet 2 & 1.00066 & 1.0091 & 1.18 \\
Diet 3 & 1.00094 & 1.017 & 1.37 \\
Diet 4 & 1.0038 & 1.084 & 2.9 \\
\hline
\end{tabular}

\section{Probability of Causation [\%]}

\begin{tabular}{llll}
\hline Diet 1 & 0.15 & 2.2 & 32 \\
Diet 2 & 0.07 & 0.9 & 15 \\
Diet 3 & 0.09 & 1.7 & 27 \\
Diet 4 & 0.38 & 7.8 & 65 \\
\hline
\end{tabular}

Diet 1 - Backyard cow milk + all other locally produced non-milk exposure pathways

Diet 2 - Locally produced commercial milk + all other locally produced non-milk exposure pathways

Diet 3 - Regionally mixed commercial milk + inhalation (other regionally mixed food items are minor contributors to the total)

Diet 4 - Goat milk (all other exposure pathways are of negligible importance compared to goat milk) 


\section{Location: Maryville}

\section{Receptor: Male born in 1956}

\begin{tabular}{lccc}
\hline & \multicolumn{3}{c}{ Thyroid Dose [cGy] } \\
& \multicolumn{2}{c}{$95 \%$ Subjective Confidence Interval } \\
Exposure Pathway & lower limit & central estimate & upper limit \\
\hline Backyard Cow Milk & 0.02 & 0.31 & 3.8 \\
Commercial Milk (locally produced) & 0.0083 & 0.11 & 1.2 \\
Commercial Milk (regionally mixed) & 0.017 & 0.23 & 2.2 \\
Goat Milk (locally produced) & 0.089 & 1.2 & 18 \\
Beef (locally produced) & 0.000012 & 0.00024 & 0.0058 \\
Leafy Vegetables (locally produced) & 0.000013 & 0.0002 & 0.0023 \\
Eggs (locally produced) & 0.00091 & 0.012 & 0.16 \\
Cottage Cheese (locally produced) & 0.000045 & 0.00094 & 0.017 \\
Inhalation & 0.00071 & 0.004 & 0.022 \\
Mother's milk (mother on Diet 1) & 0.00057 & 0.018 & 0.59 \\
Prenatal exposure (mother on Diet 1) & 0.0055 & 0.061 & 0.58 \\
Diet 1 & 0.024 & 0.34 & 3.9 \\
Diet 2 & 0.013 & 0.14 & 1.3 \\
Diet 3 & 0.019 & 0.24 & 2.2 \\
\hline
\end{tabular}

Excess Lifetime Risk [ ]

\begin{tabular}{llll}
\hline Diet 1 & $6.3 \mathrm{E}-07$ & $2.3 \mathrm{E}-05$ & $8.9 \mathrm{E}-04$ \\
Diet 2 & $3.3 \mathrm{E}-07$ & $1.0 \mathrm{E}-05$ & $2.8 \mathrm{E}-04$ \\
Diet 3 & $5.7 \mathrm{E}-07$ & $1.7 \mathrm{E}-05$ & $5.3 \mathrm{E}-04$ \\
Diet 4 & $2.1 \mathrm{E}-06$ & $8.9 \mathrm{E}-05$ & $3.0 \mathrm{E}-03$ \\
\hline
\end{tabular}

\section{Relative Risk [ ]}

\begin{tabular}{lccc}
\hline Diet 1 & 1.00075 & 1.018 & 1.65 \\
Diet 2 & 1.00034 & 1.0075 & 1.2 \\
Diet 3 & 1.00053 & 1.012 & 1.36 \\
Diet 4 & 1.002 & 1.065 & 3.7 \\
\hline
\end{tabular}

\section{Probability of Causation [\%]}

\begin{tabular}{llcc}
\hline Diet 1 & 0.08 & 1.8 & 39 \\
Diet 2 & 0.03 & 0.7 & 17 \\
Diet 3 & 0.05 & 1.2 & 26 \\
Diet 4 & 0.20 & 6.1 & 73 \\
\hline
\end{tabular}

Diet 1 - Backyard cow milk + all other locally produced non-milk exposure pathways

Diet 2 - Locally produced commercial milk + all other locally produced non-milk exposure pathways

Diet 3 - Regionally mixed commercial milk + inhalation (other regionally mixed food items are minor contributors to the total)

Diet 4 - Goat milk (all other exposure pathways are of negligible importance compared to goat milk) 
Location: Cedar Grove

Receptor: Female born in 1956

\begin{tabular}{lccc}
\hline & \multicolumn{3}{c}{ Thyroid Dose [cGy] } \\
Exposure Pathway & $95 \%$ Subjective Confidence Interval \\
\hline Backyard Cow Milk & lower limit & central estimate & upper limit \\
Commercial Milk (locally produced) & 0.037 & 0.54 & 6 \\
Commercial Milk (regionally mixed) & 0.016 & 0.19 & 2 \\
Goat Milk (locally produced) & 0.018 & 0.22 & 2.4 \\
Beef (locally produced) & 0.12 & 2.2 & 31 \\
Leafy Vegetables (locally produced) & 0.00002 & 0.00046 & 0.011 \\
Eggs (locally produced) & 0.000029 & 0.00037 & 0.004 \\
Cottage Cheese (locally produced) & 0.0014 & 0.023 & 0.29 \\
Inhalation & 0.000074 & 0.0018 & 0.038 \\
Mother's milk (mother on Diet 1) & 0.0011 & 0.0065 & 0.033 \\
Prenatal exposure (mother on Diet 1) & 0.0012 & 0.033 & 1 \\
Diet 1 & 0.011 & 0.11 & 1.2 \\
Diet 2 & 0.043 & 0.59 & 6.4 \\
Diet 3 & 0.028 & 0.25 & 2.4 \\
\hline
\end{tabular}

Excess Lifetime Risk [ ]

\begin{tabular}{llll}
\hline Diet 1 & $7.6 \mathrm{E}-06$ & $1.7 \mathrm{E}-04$ & $4.1 \mathrm{E}-03$ \\
Diet 2 & $4.2 \mathrm{E}-06$ & $7.8 \mathrm{E}-05$ & $1.3 \mathrm{E}-03$ \\
Diet 3 & $4.2 \mathrm{E}-06$ & $7.2 \mathrm{E}-05$ & $1.2 \mathrm{E}-03$ \\
Diet 4 & $2.2 \mathrm{E}-05$ & $7.4 \mathrm{E}-04$ & $1.9 \mathrm{E}-02$ \\
\hline
\end{tabular}

\section{Relative Risk [ ]}

\begin{tabular}{lccc}
\hline Diet 1 & 1.0031 & 1.043 & 1.92 \\
Diet 2 & 1.0013 & 1.018 & 1.35 \\
Diet 3 & 1.001 & 1.018 & 1.37 \\
Diet 4 & 1.0069 & 1.15 & 4.6 \\
\hline
\end{tabular}

\section{Probability of Causation [\%]}

\begin{tabular}{lccc}
\hline Diet 1 & 0.31 & 4.1 & 48 \\
Diet 2 & 0.13 & 1.8 & 26 \\
Diet 3 & 0.10 & 1.7 & 27 \\
Diet 4 & 0.69 & 13.4 & 78 \\
\hline
\end{tabular}

Diet 1 - Backyard cow milk + all other locally produced non-milk exposure pathways

Diet 2 - Locally produced commercial milk + all other locally produced non-milk exposure pathways

Diet 3 - Regionally mixed commercial milk + inhalation (other regionally mixed food items are minor contributors to the total)

Diet 4 - Goat milk (all other exposure pathways are of negligible importance compared to goat milk) 


\section{Location: Cedar Grove}

Receptor: Male born in 1956

\begin{tabular}{lccc}
\hline & \multicolumn{3}{c}{ Thyroid Dose [cGy] } \\
& \multicolumn{2}{c}{$95 \%$ Subjective Confidence Interval } \\
Exposure Pathway & lower limit & central estimate & upper limit \\
\hline Backyard Cow Milk & 0.039 & 0.56 & 6 \\
Commercial Milk (locally produced) & 0.015 & 0.21 & 1.8 \\
Commercial Milk (regionally mixed) & 0.017 & 0.23 & 2.2 \\
Goat Milk (locally produced) & 0.15 & 2.1 & 34 \\
Beef (locally produced) & 0.000021 & 0.00044 & 0.011 \\
Leafy Vegetables (locally produced) & 0.000023 & 0.00035 & 0.004 \\
Eggs (locally produced) & 0.0017 & 0.023 & 0.3 \\
Cottage Cheese (locally produced) & 0.000093 & 0.0018 & 0.031 \\
Inhalation & 0.0012 & 0.0064 & 0.034 \\
Mother's milk (mother on Diet 1) & 0.0012 & 0.033 & 1 \\
Prenatal exposure (mother on Diet 1) & 0.011 & 0.11 & 1.2 \\
Diet 1 & 0.044 & 0.61 & 6.4 \\
Diet 2 & 0.027 & 0.26 & 2.1 \\
Diet 3 & 0.02 & 0.24 & 2.2 \\
\hline
\end{tabular}

Excess Lifetime Risk [ ]

\begin{tabular}{llcc}
\hline Diet 1 & $1.5 \mathrm{E}-06$ & $4.2 \mathrm{E}-05$ & $1.4 \mathrm{E}-03$ \\
Diet 2 & $6.2 \mathrm{E}-07$ & $1.9 \mathrm{E}-05$ & $5.0 \mathrm{E}-04$ \\
Diet 3 & $5.9 \mathrm{E}-07$ & $1.7 \mathrm{E}-05$ & $5.4 \mathrm{E}-04$ \\
Diet 4 & $4.3 \mathrm{E}-06$ & $1.6 \mathrm{E}-04$ & $6.7 \mathrm{E}-03$ \\
\hline
\end{tabular}

\section{Relative Risk [ ]}

\begin{tabular}{lccc}
\hline Diet 1 & 1.0014 & 1.034 & 1.98 \\
Diet 2 & 1.00064 & 1.013 & 1.36 \\
Diet 3 & 1.00054 & 1.012 & 1.36 \\
Diet 4 & 1.0035 & 1.13 & 5.6 \\
\hline
\end{tabular}

\section{Probability of Causation [\%]}

\begin{tabular}{lccc}
\hline Diet 1 & 0.14 & 3.3 & 49 \\
Diet 2 & 0.06 & 1.3 & 26 \\
Diet 3 & 0.05 & 1.2 & 26 \\
Diet 4 & 0.35 & 11.1 & 82 \\
\hline
\end{tabular}

Diet 1 - Backyard cow milk + all other locally produced non-milk exposure pathways

Diet 2 - Locally produced commercial milk + all other locally produced non-milk exposure pathways

Diet 3 - Regionally mixed commercial milk + inhalation (other regionally mixed food items are minor contributors to the total)

Diet 4 - Goat milk (all other exposure pathways are of negligible importance compared to goat milk) 
APPENDIX 12A

MILK PRODUCTION DATA 
TASK 1 REPORT

July 1999

${ }^{131}$ I Releases from X-10 Radioactive Lanthanum Processing-

Page 12A-2

Milk Production Data

This page intentionally left blank. 


\section{APPENDIX 12A: MILK PRODUCTION DATA}

Milk production for the state of Tennessee has been recorded since 1929 (NASS, 1998b). Available milk production records represent the entire state and not specific counties or other regions of interest (metropolitan areas, geographic regions, etc.). Appendix 12-A presents the data used to determine the volume of milk produced within a 38-km radius of the X-10 facility. Appendix 12-A also provides the areas of the six counties of interest: Anderson, Blount, Knox, Loudon, Morgan, and Roane. The areas of these counties were used to determine the fraction of each county that was within $38 \mathrm{~km}$ of X-10. Table 12-A.1 provides the volume of backyard cow's milk produced in the state of Tennessee from 1944 to 1956 . The volume of commercial milk produced in the state of Tennessee from 1944 to 1956 is listed in Table 12-A.2. The number of cows in Anderson, Blount, Knox, Loudon, Morgan, and Roane counties during the years 1975 to 1991 are provided in Table 12-A.3. Tables 12-A.4 and 12-A.5 list the estimated volume of backyard cow's milk and commercial milk respectively, available for use in the six counties of interest from 1944 to 1956 . The estimated values are based on the average number of cows in each county during the period of 1975 to 1991 . The yearly milk production and utilization patterns (fed to calves, used to produce cream or butter, marketed, etc.) are provided in Table 12-A.6. This information was used to determine the range for the fraction of fluid milk available for human consumption $\left(f_{1}\right)$ between 1944 and 1956. Table 12-A.7 lists the areas of the counties affected by the atmospheric releases of ${ }^{131} \mathrm{I}$. The areas were used to determine the fraction of the counties within a $38-\mathrm{km}$ radius of $\mathrm{X}-10\left(f_{3}\right)$.

Table 12-A.1 The volume of backyard cow's milk produced in the state of Tennessee from 1944 to 1956 (NASS, 1998b).

\begin{tabular}{cccc}
\hline Year & $\begin{array}{c}\text { Fluid Milk/Cream Consumed } \\
\text { on Farm (L) }\end{array}$ & $\begin{array}{c}\text { Milk/Cream Sold by } \\
\text { Farmers to } \\
\text { Consumers (L) }\end{array}$ & $\begin{array}{c}\text { Total Amount of Backyard } \\
\text { Cow's Milk (L) }\end{array}$ \\
\hline 1944 & $200,584,599$ & $37,389,673$ & \\
1945 & $184,309,094$ & $37,389,673$ & $237,974,272$ \\
1946 & $190,467,393$ & $37,389,673$ & $221,698,767$ \\
1947 & $195,745,935$ & $36,509,916$ & $227,857,066$ \\
1948 & $189,587,636$ & $35,630,159$ & $232,255,851$ \\
1949 & $183,429,337$ & $35,190,280$ & $225,217,795$ \\
1950 & $189,587,636$ & $35,190,280$ & $218,619,617$ \\
1951 & $190,907,271$ & $33,430,766$ & $224,777,916$ \\
1952 & $186,948,365$ & $30,791,495$ & $224,338,038$ \\
1953 & $181,229,944$ & $27,272,467$ & $217,739,860$ \\
1954 & $176,831,159$ & $26,392,710$ & $208,502,412$ \\
1955 & $169,793,103$ & $23,313,561$ & $203,223,870$ \\
1956 & $167,153,832$ & $19,794,533$ & $193,106,664$ \\
\hline
\end{tabular}


TASK 1 REPORT

July 1999

Page 12A-4

${ }^{131}$ I Releases from X-10 Radioactive Lanthanum Processing-

Milk Production Data

Table 12-A.2 The volume of commercial milk produced in the state of Tennessee from 1944 to 1956 (NASS, 1998b).

\begin{tabular}{cccc}
\hline Year & Cream Sold to Dealers (L) & Milk Sold to Dealers (L) & $\begin{array}{c}\text { Total Commercial Milk } \\
\text { Produced in TN (L) }\end{array}$ \\
\hline & & & \\
1944 & $98,972,664$ & $437,679,113$ & $536,651,777$ \\
1945 & $87,975,701$ & $455,274,253$ & $543,249,954$ \\
1946 & $67,741,290$ & $468,470,608$ & $536,211,898$ \\
1947 & $61,582,991$ & $483,866,356$ & $545,449,347$ \\
1948 & $61,582,991$ & $489,144,898$ & $550,727,889$ \\
1949 & $68,181,168$ & $538,851,169$ & $607,032,338$ \\
1950 & $61,582,991$ & $560,845,095$ & $622,428,085$ \\
1951 & $56,304,449$ & $565,243,880$ & $621,548,328$ \\
1952 & $52,785,421$ & $589,437,197$ & $642,222,618$ \\
1953 & $48,386,636$ & $686,210,469$ & $734,597,104$ \\
1954 & $23,313,561$ & $703,805,609$ & $727,119,170$ \\
1955 & $21,993,925$ & $718,761,478$ & $740,755,403$ \\
1956 & $19,794,533$ & $779,904,590$ & $799,699,123$ \\
\hline
\end{tabular}

Table 12-A.3 The number of dairy cows in Anderson, Blount, Knox, Loudon, Morgan, and Roane counties from 1975 to 1991 (NASS, 1998a).

\begin{tabular}{ccccccc}
\hline Year & & & \multicolumn{2}{c}{ County } & \\
& Anderson & Blount & Knox & Loudon & Morgan & Roane \\
\hline & & & & & & \\
1975 & 800 & 2,900 & 1,400 & 3,500 & 550 & 1,000 \\
1976 & 700 & 2,800 & 1,300 & 3,500 & 500 & 1,200 \\
1977 & 600 & 2,900 & 1,300 & 4,000 & 550 & 1,300 \\
1978 & 550 & 2,600 & 1,300 & 4,000 & 550 & 1,200 \\
1979 & 600 & 2,700 & 1,300 & 3,750 & 650 & 1,250 \\
1980 & 600 & 2,850 & 1,250 & 3,800 & 600 & 1,350 \\
1981 & 700 & 3,200 & 1,200 & 4,300 & 500 & 1,450 \\
1982 & 700 & 3,300 & 1,400 & 4,800 & 500 & 1,400 \\
1983 & 700 & 3,100 & 1,500 & 4,700 & 650 & 900 \\
1984 & 700 & 3,000 & 1,600 & 4,700 & 600 & 700 \\
1985 & 700 & 2,900 & 1,500 & 4,400 & 600 & 750 \\
1986 & 700 & 2,800 & 1,400 & 4,300 & 600 & 700 \\
1987 & 700 & 2,700 & 1,300 & 4,100 & 500 & 700 \\
1988 & 700 & 2,800 & 1,400 & 3,900 & 500 & 700 \\
1989 & 500 & 2,400 & 1,500 & 3,500 & 500 & 500 \\
1990 & 500 & 2,400 & 1,500 & 3,300 & 400 & 400 \\
1991 & 500 & 2,400 & 1,500 & 3,300 & 400 & 400 \\
\hline
\end{tabular}


Table 12-A.4 The volume of backyard cow's milk (L) produced from 1944 to 1956 in Anderson, Blount, Knox, Loudon, Morgan, and Roane counties based on the average number of cows in each county.

\begin{tabular}{ccccccc}
\hline Year & & \multicolumn{5}{c}{ County } \\
& Anderson & Blount & Knox & Loudon & Morgan & Roane \\
\hline & & & & & & \\
1944 & 313,356 & 676,791 & $1,941,661$ & 455,010 & 261,737 & $1,366,460$ \\
1945 & 291,925 & 630,504 & $1,808,867$ & 423,891 & 243,836 & $1,273,005$ \\
1946 & 300,034 & 648,018 & $1,859,114$ & 435,666 & 250,609 & $1,308,367$ \\
1947 & 305,826 & 660,528 & $1,895,004$ & 444,076 & 255,447 & $1,333,625$ \\
1948 & 296,559 & 640,512 & $1,837,580$ & 430,619 & 247,706 & $1,293,212$ \\
1949 & 287,870 & 621,747 & $1,783,744$ & 418,003 & 240,449 & $1,255,325$ \\
1950 & 295,979 & 639,261 & $1,833,991$ & 429,778 & 247,223 & $1,290,686$ \\
1951 & 295,400 & 638,010 & $1,830,402$ & 428,937 & 246,739 & $1,288,160$ \\
1952 & 286,712 & 619,245 & $1,776,566$ & 416,321 & 239,482 & $1,250,273$ \\
1953 & 274,548 & 592,974 & $1,701,197$ & 398,659 & 229,322 & $1,197,231$ \\
1954 & 267,598 & 577,962 & $1,658,128$ & 388,567 & 223,516 & $1,166,922$ \\
1955 & 254,276 & 549,189 & $1,575,581$ & 369,222 & 212,389 & $1,108,828$ \\
1956 & 246,167 & 531,675 & $1,525,335$ & 357,448 & 205,616 & $1,073,467$ \\
\hline
\end{tabular}

Table 12-A.5 The volume of commercial milk (L) produced from 1944 to 1956 in Anderson, Blount, Knox, Loudon, Morgan, and Roane counties based on the average number of cows in each county.

\begin{tabular}{ccccccc}
\hline Year & & \multicolumn{5}{c}{ County } \\
& Anderson & Blount & Knox & Loudon & Morgan & Roane \\
\hline & & & & & & \\
1944 & 706,643 & $1,526,221$ & $4,378,608$ & $1,026,085$ & 590,238 & $3,081,481$ \\
1945 & 715,332 & $1,544,986$ & $4,432,443$ & $1,038,701$ & 597,495 & $3,119,368$ \\
1946 & 706,064 & $1,524,970$ & $4,375,019$ & $1,025,244$ & 589,754 & $3,078,956$ \\
1947 & 718,228 & $1,551,241$ & $4,450,388$ & $1,042,906$ & 599,914 & $3,131,998$ \\
1948 & 725,178 & $1,566,253$ & $4,493,456$ & $1,052,999$ & 605,719 & $3,162,307$ \\
1949 & 799,318 & $1,726,381$ & $4,952,851$ & $1,160,653$ & 667,646 & $3,485,610$ \\
1950 & 819,590 & $1,770,166$ & $5,078,467$ & $1,190,090$ & 684,579 & $3,574,013$ \\
1951 & 818,432 & $1,767,664$ & $5,071,289$ & $1,188,408$ & 683,611 & $3,568,962$ \\
1952 & 845,655 & $1,826,461$ & $5,239,973$ & $1,227,938$ & 706,350 & $3,687,674$ \\
1953 & 967,291 & $2,089,171$ & $5,993,668$ & $1,404,559$ & 807,948 & $4,218,093$ \\
1954 & 957,444 & $2,067,904$ & $5,932,654$ & $1,390,261$ & 799,724 & $4,175,155$ \\
1955 & 975,400 & $2,106,685$ & $6,043,914$ & $1,416,334$ & 814,722 & $4,253,455$ \\
1956 & $1,053,014$ & $2,274,319$ & $6,524,843$ & $1,529,035$ & 879,551 & $4,591,912$ \\
\hline
\end{tabular}


TASK 1 REPORT

July 1999

Page 12A-6

${ }^{131}$ I Releases from X-10 Radioactive Lanthanum ProcessingMilk Production Data

Table 12-A.6 The yearly production and utilization of milk (millions of pounds) in the state of Tennessee during 1950-1969 and 1975-1996 (NASS, 1998b).

\begin{tabular}{|c|c|c|c|c|c|}
\hline Year & $\begin{array}{c}\text { Milk } \\
\text { Produced } \\
\end{array}$ & $\begin{array}{c}\text { Milk Fed to } \\
\text { Calves }\end{array}$ & $\begin{array}{c}\text { Milk Used for } \\
\text { Cream and Butter }\end{array}$ & $\begin{array}{c}\text { Milk } \\
\text { Marketed } \\
\end{array}$ & $\begin{array}{c}\text { Fraction of Milk } \\
\text { Available } \\
\end{array}$ \\
\hline 1950 & 2,329 & 40 & 794 & 1,495 & 0.64 \\
\hline 1951 & 2,311 & 44 & 778 & 1,489 & 0.64 \\
\hline 1952 & 2,294 & 42 & 727 & 1,525 & 0.66 \\
\hline 1953 & 2,421 & 42 & 674 & 1,705 & 0.70 \\
\hline 1954 & 2,416 & 43 & 660 & 1,713 & 0.71 \\
\hline 1955 & 2,372 & 40 & 599 & 1,733 & 0.73 \\
\hline 1956 & 2,445 & 39 & 549 & 1,857 & 0.76 \\
\hline 1957 & 2,422 & 36 & 489 & 1,897 & 0.78 \\
\hline 1958 & 2,279 & 34 & 431 & 1,814 & 0.80 \\
\hline 1959 & 2,200 & 33 & 365 & 1,802 & 0.82 \\
\hline 1960 & 2,194 & 32 & 328 & 1,834 & 0.84 \\
\hline 1961 & 2,277 & 32 & 294 & 1,951 & 0.86 \\
\hline 1962 & 2,262 & 31 & 263 & 1,968 & 0.87 \\
\hline 1963 & 2,173 & 31 & 236 & 1,906 & 0.88 \\
\hline 1964 & 2,106 & 30 & 212 & 1,864 & 0.89 \\
\hline 1965 & 2,171 & 30 & 189 & 1,952 & 0.90 \\
\hline 1966 & 2,122 & 30 & 165 & 1,927 & 0.91 \\
\hline 1967 & 2,144 & 29 & 154 & 1,961 & 0.91 \\
\hline 1968 & 2,115 & 28 & 143 & 1,944 & 0.92 \\
\hline 1969 & 2,104 & 28 & 132 & 1,944 & 0.92 \\
\hline 1975 & 2,031 & 54 & 60 & 1,917 & 0.94 \\
\hline 1976 & 2,096 & 51 & 58 & 1,987 & 0.95 \\
\hline 1977 & 2,181 & 49 & 55 & 2,077 & 0.95 \\
\hline $\begin{array}{l}1978 \\
\end{array}$ & 2,073 & 48 & 49 & 1,976 & 0.95 \\
\hline $\begin{array}{l}1979 \\
\end{array}$ & 2,091 & 48 & 47 & 1,996 & 0.95 \\
\hline 1980 & 2,241 & 40 & 45 & 2,156 & 0.96 \\
\hline 1981 & 2,300 & 40 & 44 & 2,216 & 0.96 \\
\hline 1982 & 2,326 & 44 & 46 & 2,236 & 0.96 \\
\hline 1983 & 2,250 & 41 & 43 & 2,166 & 0.96 \\
\hline 1984 & 2,152 & 110 & 35 & 2,007 & 0.93 \\
\hline 1985 & 2,235 & 118 & 35 & 2,082 & 0.93 \\
\hline 1986 & 2,221 & 104 & 30 & 2,087 & 0.94 \\
\hline 1987 & 2,207 & 145 & 30 & 2,032 & 0.92 \\
\hline 1988 & 2,137 & 123 & 25 & 1,989 & 0.93 \\
\hline 1989 & 2,040 & 90 & 20 & 1,930 & 0.95 \\
\hline 1990 & 2,022 & 90 & 25 & 1,907 & 0.94 \\
\hline 1991 & 1,993 & 90 & 20 & 1,883 & 0.94 \\
\hline 1992 & 1,968 & 90 & 15 & 1,863 & 0.95 \\
\hline 1993 & 1,945 & 90 & 15 & 1,840 & 0.95 \\
\hline 1994 & 1,780 & 45 & 5 & 1,730 & 0.97 \\
\hline 1995 & 1,745 & 9 & 1 & 1,735 & 0.99 \\
\hline 1996 & 1,617 & 11 & 1 & 1,605 & 0.99 \\
\hline
\end{tabular}


Table 12-A.7 The areas of the counties affected by the atmospheric releases of ${ }^{131}$ I from X-10 (USDA, 1950).

\begin{tabular}{lcc}
\hline \multicolumn{1}{c}{ County of Interest } & $\begin{array}{c}\text { Size of County } \\
\text { (Acres) }\end{array}$ & $\begin{array}{c}\text { Size of County } \\
\text { (Square Kilometers) }\end{array}$ \\
\hline Anderson & 216,320 & 875 \\
Knox & 327,040 & 1,324 \\
Loudon & 153,600 & 622 \\
Roane & 226,560 & 917 \\
Morgan & 344,960 & 1,396 \\
Blount & 370,560 & 1,500 \\
\hline & Total Area & $\mathbf{1 , 6 3 9 , 0 4 0}$ \\
\hline
\end{tabular}




\section{KEY TECH NICAL REPORT SOFTHE OAK RIDGE DOSE RECON STRUCTION PROJECT}

- Volume 1 .

I odine-131 $R$ eleases from $R$ adioactive $L$ anthanum Processing at the $X-10$ Site in $O$ ak R idge, T ennessee (1944-1956)- an A ssessment of $Q$ uantities $R$ eleased, 0 ff-Site R adiation D oses, and Potential Excess $R$ isks of T hyroid C ancer

The report of project T ask 1

- Volume $1 \mathrm{~A}$.

A ppendices to the lodine-131 R eport

- Volume 2 .

Mercury R eleases from Lithium Enrichment at the 0 ak R idge $Y-12$ Planta $R$ econstruction of $H$ istorical $R$ eleases and $O$ ff-Site $D$ oses and $H$ ealth $R$ isks

The report of project T ask 2

- V olume $2 \mathrm{~A}$.

A ppendices to the Mercury R eport

- Volume 3 .

PC Bs in the Environment near the 0 ak $R$ idge $R$ eservationa R econstruction of $H$ istorical $D$ oses and $H$ ealth $R$ isks

The report of project T ask 3

- Volume 4 -

$R$ adionuclide $R$ eleases to the $C$ linch $R$ iver from $W$ hite $\mathbf{O}$ ak $C$ reek on the $O$ ak $R$ idge $R$ eservation- an $A$ ssessment of $H$ istorical $Q$ uantities $R$ eleased, O ff-Site $\mathbf{R}$ adiation $D$ oses, and $H$ ealth $R$ isks

The report of project Task 4

- $V$ olume $4 \mathrm{~A}$.

A ppendices to the $\mathrm{W}$ hite $\mathrm{O}$ ak $\mathrm{C}$ reek $\mathrm{R}$ eport

- Volume 5 .

$U$ ranium $R$ eleases from the 0 ak $R$ idge $R$ eservation-

a $R$ eview of the $Q$ uality of $H$ istorical Effluent M onitoring $D$ ata and a Screening Evaluation of Potential O ff-Site Exposures

The report of project Task 6

- V olume 6 .

Screening-L evel Evaluation of A dditional Potential M aterials of $C$ oncern The report of project T ask 7

- Volume 7 .

$\mathrm{O}$ ak R idge D ose R econstruction Project Summary R eport 